\title{
Water Resources Data
}

\section{for}

\author{
Minnesota
}

Part 1. Surface Water Records

Part 2. Water Quality Records

\author{
UNITED STATES \\ DEPARTMENT OF THE INTERIOR \\ GEOLOGICAL SURVEY
}

Prepared in cooperation with the Minnesota Department of Conservation, Division of Waters, Soils and Minerals; the Minnesota Department of Highways; and with other State, municipal, and Federal agencies 
United States Department of the Interior Geological Survey - Water Resources Division

\author{
WATER RESOURCES DATA \\ FOR \\ MINNESOTA
}

1968

Part 1: Surface Water Records

Prepared in cooperation with

Minnesota Department of Conservation, Division of Waters, Soils and Minerals

Through the Division of Waters, Soils and Minerals

Nine Mile Creek Watershed District

City of Austin

City of Rochester

Erie Mining Company

Eveleth Taconite Company

Hanna Mining Company

United States Steel Corporation

Minnesota Department of Highways

Corps of Engineers, U.S. Army

U.S. Department of State

U.S. Department of Interior

Federal Water Pollution Control Administration

Bureau of Sport Fisheries and Wildlife

Copies of this report may be obtained from

District Chief, Water Resources Division

U.S. Geological Survey

1033 Post. Office Building

st. Paul, Minnesota 55101 
CALENDAR FOR WATER YEAR 1968

\section{OCTOBER 1967}

$\begin{array}{lllllll}S & M & T & W & T & F & S\end{array}$

$\begin{array}{lllllll}1 & 2 & 3 & 4 & 5 & 6 & 7\end{array}$

$\begin{array}{lllllll}8 & 9 & 10 & 11 & 12 & 13 & 14\end{array}$

$\begin{array}{lllllll}15 & 16 & 17 & 18 & 19 & 20 & 21\end{array}$

$\begin{array}{lllllll}22 & 23 & 24 & 25 & 26 & 27 & 28\end{array}$

$2930 \quad 31$
NOVEMBER 1967

$\begin{array}{lllllll}S & M & T & W & T & F & S\end{array}$

$\begin{array}{llll}1 & 2 & 3 & 4\end{array}$

$\begin{array}{lllllll}5 & 6 & 7 & 8 & 9 & 10 & 11\end{array}$

$\begin{array}{lllllll}12 & 13 & 14 & 15 & 16 & 17 & 18\end{array}$

$\begin{array}{lllllll}19 & 20 & 21 & 22 & 23 & 24 & 25\end{array}$

$\begin{array}{lllll}26 & 27 & 28 & 29 & 30\end{array}$
DECEMBER 1967

$\begin{array}{lllllll}S & M & T & W & T & F & S\end{array}$

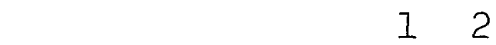

$\begin{array}{lllllll}3 & 4 & 5 & 6 & 7 & 8 & 9\end{array}$

$\begin{array}{lllllll}10 & 11 & 12 & 13 & 14 & 15 & 16\end{array}$

$\begin{array}{lllllll}17 & 18 & 19 & 20 & 21 & 22 & 23\end{array}$

$\begin{array}{lllllll}24 & 25 & 26 & 27 & 28 & 29 & 30\end{array}$ 31

\section{MARCH 1968}

$\begin{array}{ccccccccccccccc}S & M & T & W & T & F & S & & S & M & T & W & T & F & S \\ & 1 & 2 & 3 & 4 & 5 & 6 & & & & & & 1 & 2 & 3\end{array}$

$\begin{array}{lllllll}4 & 5 & 6 & 7 & 8 & 9 & 10\end{array}$

$\begin{array}{lllllll}11 & 12 & 13 & 14 & 15 & 16 & 17\end{array}$

$\begin{array}{lllllll}18 & 19 & 20 & 21 & 22 & 23 & 24\end{array}$

$\begin{array}{lllll}25 & 26 & 27 & 28 & 29\end{array}$

$\begin{array}{lllllll}S & M & T & W & T & F & S\end{array}$ $\begin{array}{lll}1 & 12\end{array}$ $\begin{array}{lllllll}3 & 4 & 5 & 6 & 7 & 8 & 9\end{array}$ $\begin{array}{lllllll}10 & 11 & 12 & 13 & 14 & 15 & 16\end{array}$ $\begin{array}{lllllll}17 & 18 & 19 & 20 & 21 & 22 & 23\end{array}$ $\begin{array}{lllllll}24 & 25 & 26 & 27 & 28 & 29 & 30\end{array}$ 31

\section{JUNE 1968}

$\begin{array}{lllllll}S & M & T & W & T & F & S \\ & 1 & 2 & 3 & 4 & 5 & 6\end{array}$

$\begin{array}{lllllll}7 & 8 & 9 & 10 & 11 & 12 & 13\end{array}$

$\begin{array}{lllllll}14 & 15 & 16 & 17 & 18 & 19 & 20\end{array}$

$\begin{array}{lllllll}21 & 22 & 23 & 24 & 25 & 26 & 27\end{array}$

$\begin{array}{lll}28 & 29 & 30\end{array}$

\section{MAY 1968}

$\begin{array}{lllllll}S & M & T & W & T & F & S\end{array}$

$\begin{array}{lllll} & 1 & 2 & 3 & 4\end{array}$

$\begin{array}{lllllll}5 & 6 & 7 & 8 & 9 & 10 & 11\end{array}$

$\begin{array}{lllllll}12 & 13 & 14 & 15 & 16 & 17 & 18\end{array}$

$\begin{array}{llllllll}19 & 20 & 21 & 22 & 23 & 24 & 25\end{array}$

$\begin{array}{llllll}26 & 27 & 28 & 29 & 30 & 31\end{array}$
$\begin{array}{lllllll}S & M & T & W & T & F & S\end{array}$

$\begin{array}{lllllll}2 & 3 & 4 & 5 & 6 & 7 & 8\end{array}$

$\begin{array}{lllllll}16 & 17 & 18 & 19 & 20 & 21 & 22\end{array}$

$\begin{array}{lllllll}23 & 24 & 25 & 26 & 27 & 28 & 29\end{array}$ 30 $\begin{array}{llllllll}9 & 10 & 11 & 12 & 13 & 14 & 15\end{array}$
JULY 1968

$\begin{array}{lllllll}S & M & T & W & T & F & S\end{array}$ $\begin{array}{llllll}1 & 2 & 3 & 4 & 5 & 6\end{array}$

$\begin{array}{lllllll}7 & 8 & 9 & 10 & 11 & 12 & 13\end{array}$

$\begin{array}{lllllll}14 & 15 & 16 & 17 & 18 & 19 & 20\end{array}$

$\begin{array}{lllllll}21 & 22 & 23 & 24 & 25 & 26 & 27\end{array}$

$\begin{array}{llll}28 & 29 & 30 & 31\end{array}$

\section{AUGUST 1968}

$\begin{array}{lllllll}S & M & T & W & T & F & S\end{array}$

$\begin{array}{lll}1 & 2 & 3\end{array}$

$\begin{array}{lllllll}4 & 5 & 6 & 7 & 8 & 9 & 10\end{array}$

$\begin{array}{lllllll}11 & 12 & 13 & 14 & 15 & 16 & 17\end{array}$

$\begin{array}{lllllll}18 & 19 & 20 & 21 & 22 & 23 & 24\end{array}$

$\begin{array}{lllllll}25 & 26 & 27 & 28 & 29 & 30 & 31\end{array}$

\section{SEPTEMBER 1968}

$\begin{array}{lllllll}S & M & T & W & T & F & S\end{array}$

$\begin{array}{lllllll}1 & 2 & 3 & 4 & 5 & 6 & 7\end{array}$

$\begin{array}{lllllll}8 & 9 & 10 & 11 & 12 & 13 & 14\end{array}$

$\begin{array}{lllllll}15 & 16 & 17 & 18 & 19 & 20 & 21\end{array}$

$\begin{array}{lllllll}22 & 23 & 24 & 25 & 26 & 27 & 28\end{array}$

$29 \cdot 30$ 
Part 1. Surface Water Records

(For Part 2 Table of Contents see p. 193)

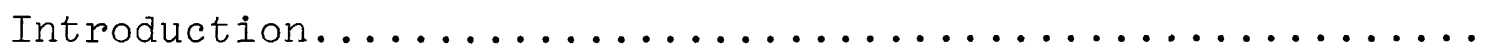

Page

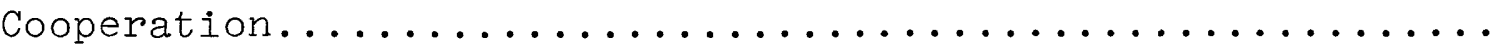

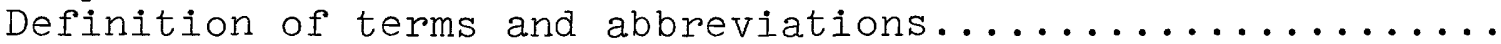

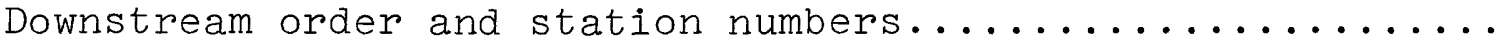

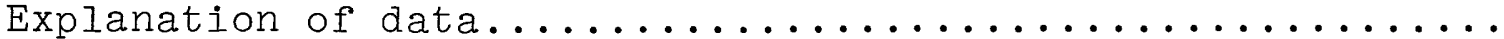

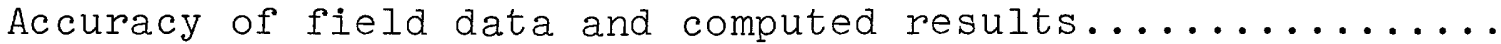

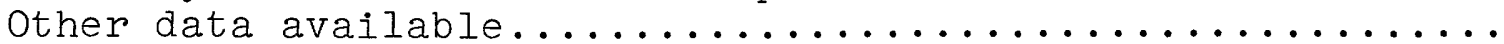

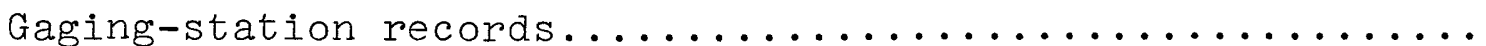

Streams tributary to Lake Superior

Pigeon River at Middle Falls, near Grand Portage.......

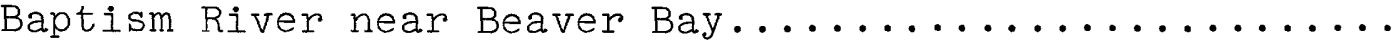

St. Louis River:

Partridge River:

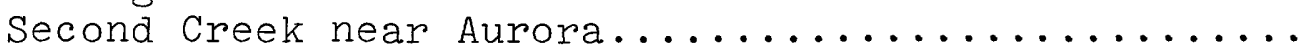

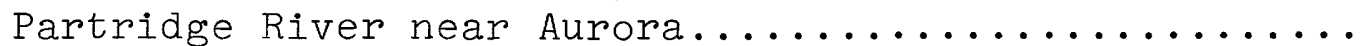

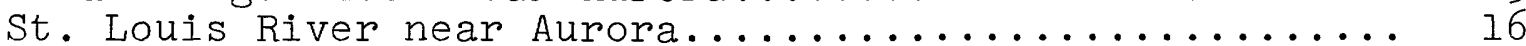

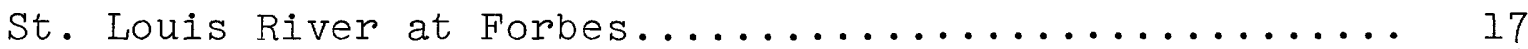

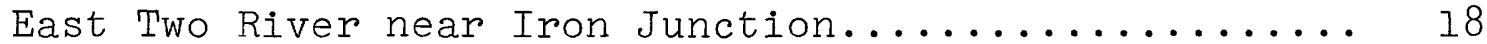

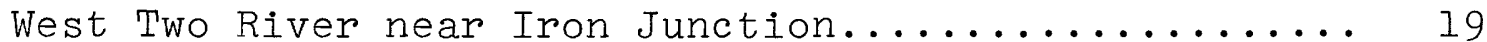

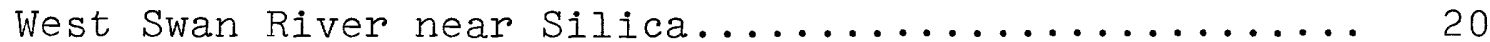

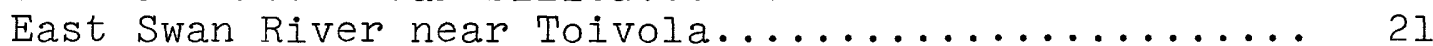

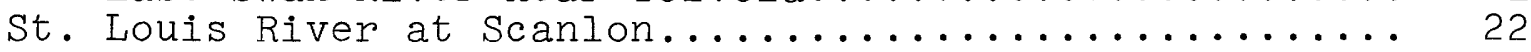

Hudson Bay basin

Lake Winnipeg (head of NeIson River):

Red River of the North basin

Otter Tail River (head of Red River of the North)

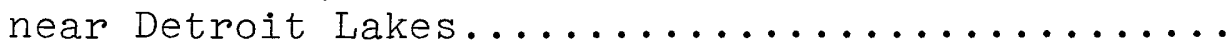

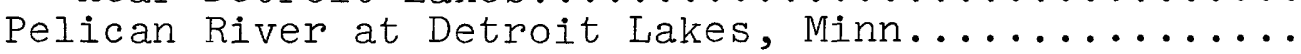

23

Pelican River at Detroit Lake Outlet, near Detroit

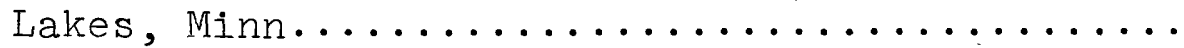

St. Clair Lake:

Long Lake Outlet near Detroit Lakes, Minn.......

West Branch County ditch No. 14 near Detroit

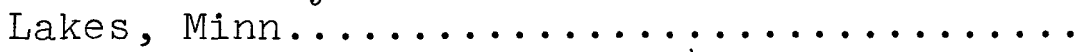

East Branch County ditch No. 14 near

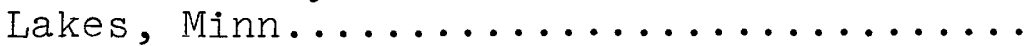

St. Clair Lake near Detroit Lakes, Minn.........

St. Clair Lake Outlet near Detroit Lakes, Minn....

Pelican River at Muskrat Lake Outlet, near Detroit

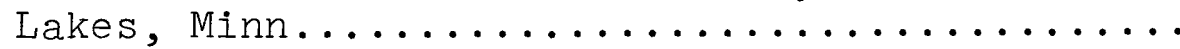


Gaging-station records--Continued

Hudson Bay basin--Continued

Lake Winnipeg (head of Nelson River)--Continued

Red River of the North basin--Continued

Pelican River at Sallie Lake Outlet, near Detroit

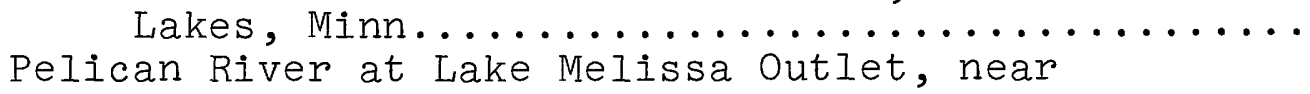

Page

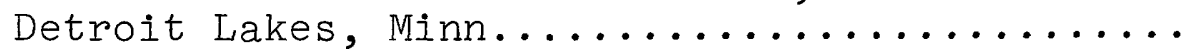

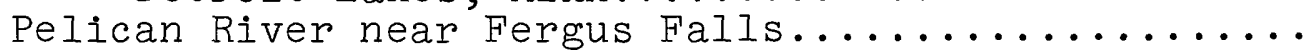

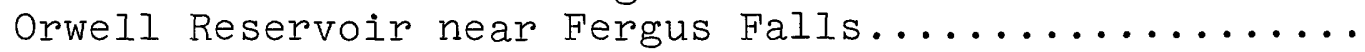

Otter Tail River below Orwell Dam, near Fergus Falls..

Bois de Sioux River near White Rock, S. Dak........

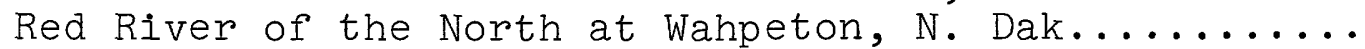

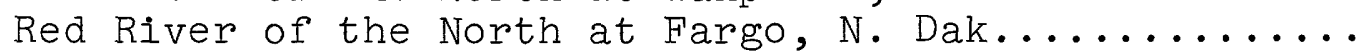

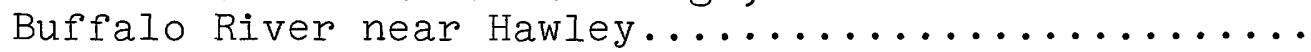

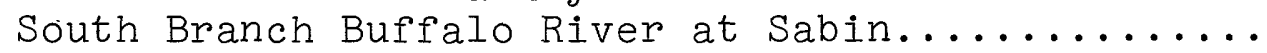

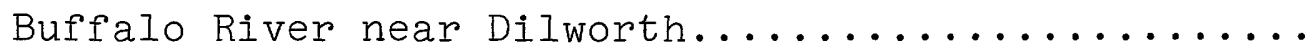

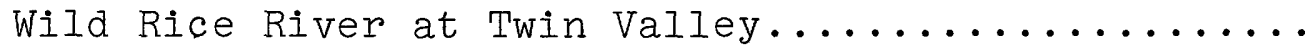

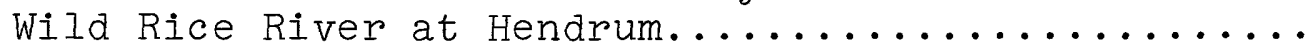

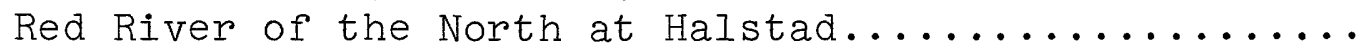

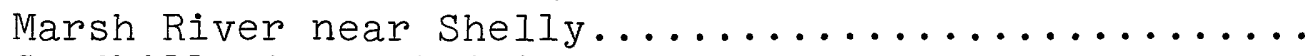

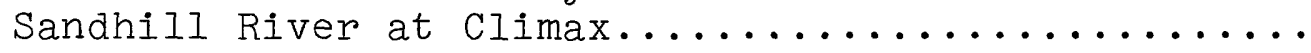

Red Lake River:

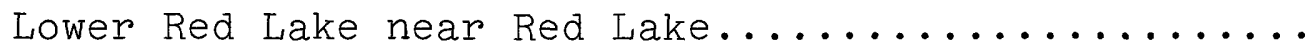

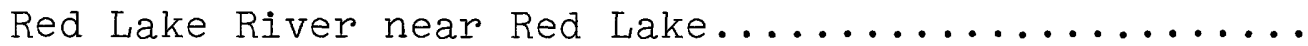

Red Lake River at Highlanding, near Goodridge......

Thief River near Thief River Falls............

Clearwater River:

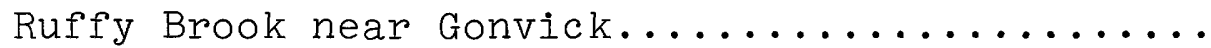

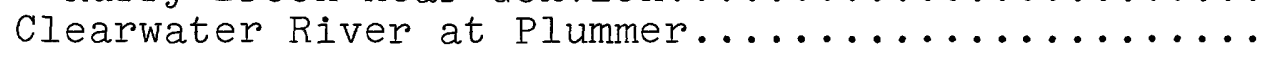

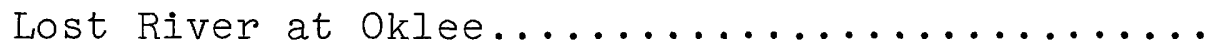

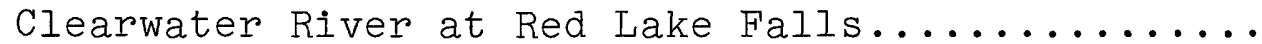

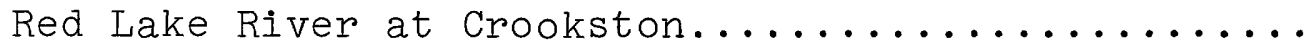

Red River of the North at Grand Forks, N. Dak........

Snake River:

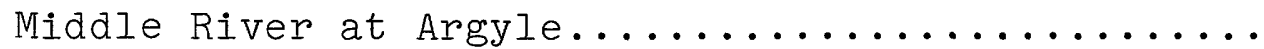

Red River of the North at Drayton, N. Dak............

Two Rivers:

South Branch Two Rivers at Lake Bronson..........

Red River of the North at Emerson, Manitoba...........

Roseau River below South Fork, near Malung.........

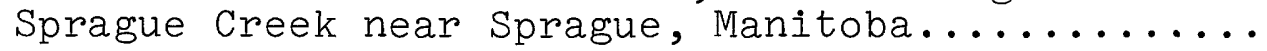

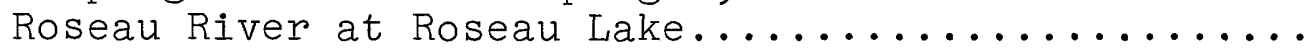

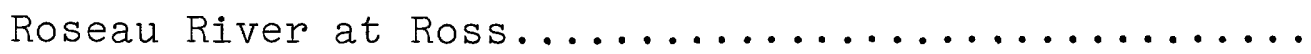

Roseau River near Badger...................

Roseau River below State ditch 51, near Caribou.....

Roseau River at international boundary, near.

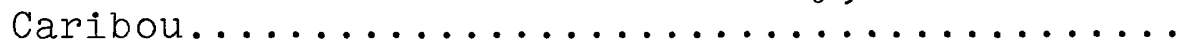


Gaging-station records--Continued

Hudson Bay basin--Continued

Lake Winnipeg (head of Nelson River)--Continued

Lake of the Woods basin (head of Winnipeg River)

Namakan River (head of Rainy River):

Basswood River:

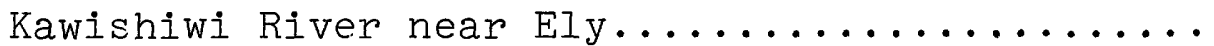

Page

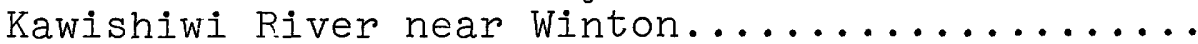

Burntside River (head of Shagawa River):

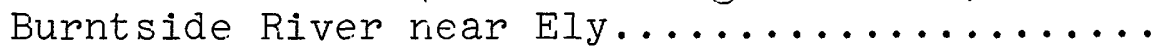

Shagawa Irake:

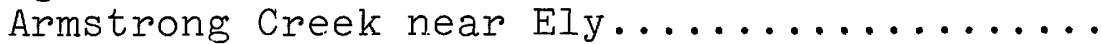

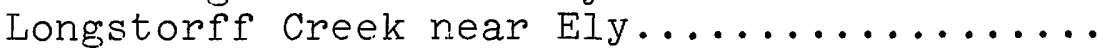

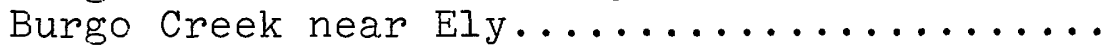

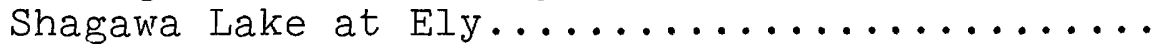

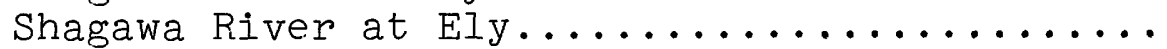

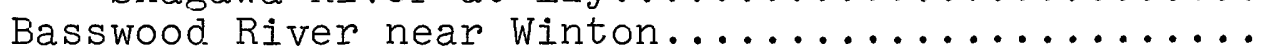

Namakan River at outlet of Iac la Croix, Ontario....

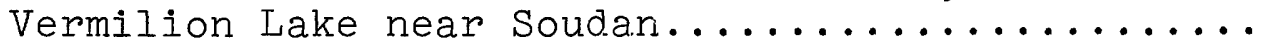

Vermilion River below Vermilion Lake near Tower...

Rainy Lake near Fort Frances, Ontario............ Rainy River:

Little Fork River:

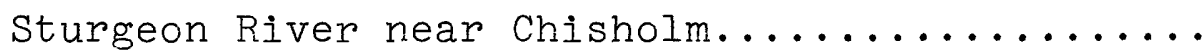

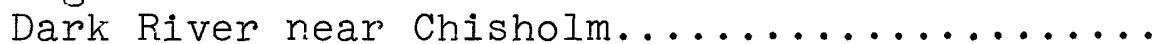

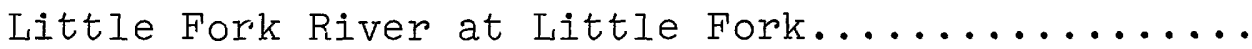

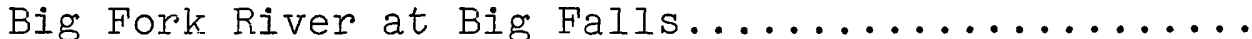

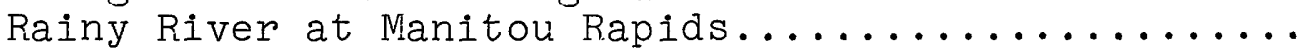

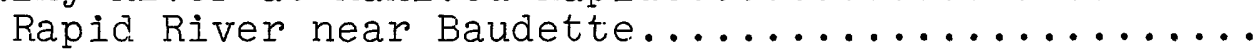

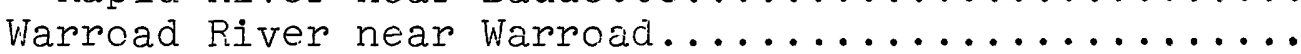

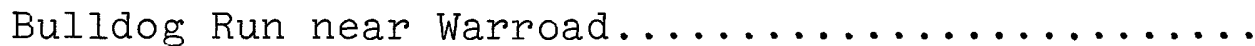

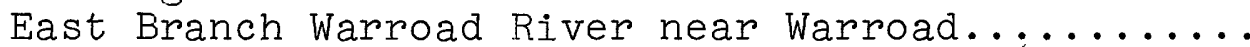

Upper Mississippi River basin

Mississippi River:

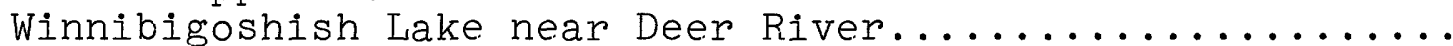
Mississippi River at Winnibigoshish Dam, near Deer River..

Leech Lake River basin

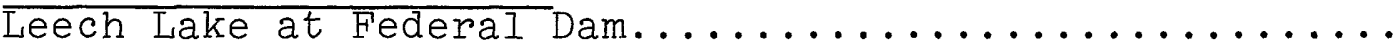

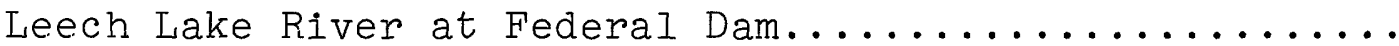

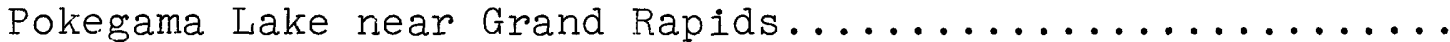

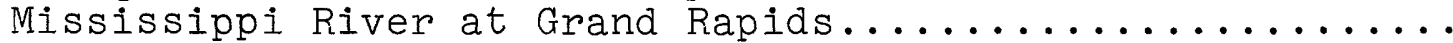

Prairie River basin

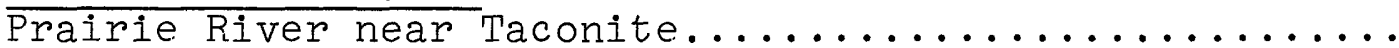

Swan River basin

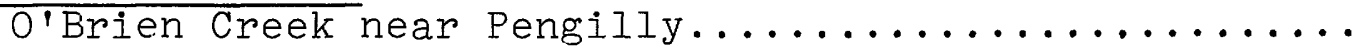

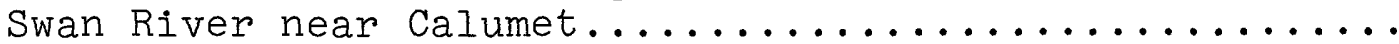

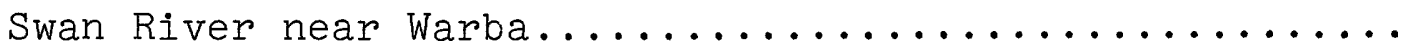


Gaging-station records--Continued

Upper Mississippi River basin--Continued

Page

Sandy River basin

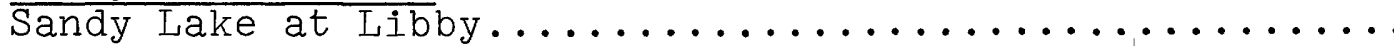

101

Sandy River at Sandy Lake Dam, at Libby.............. 102

Mississippi River below Sandy River, near Libby......... 103

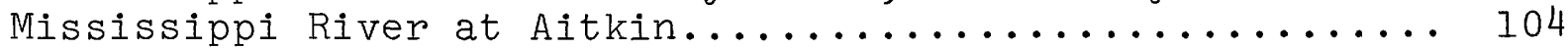

Pine River basin

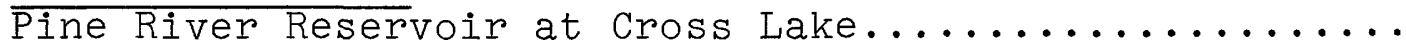

Pine River at Cross Lake Dam, at Cross Lake............

Pelican Brook:

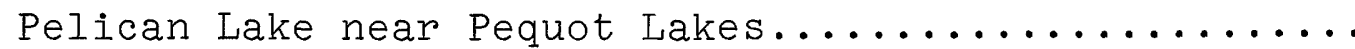
Crow Wing River basin

Crow Wing River at Nimrod.................... 108

Gull River:

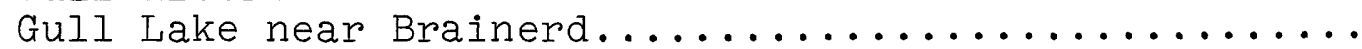

Gull River at Gull Lake Dam, near Brainerd...........

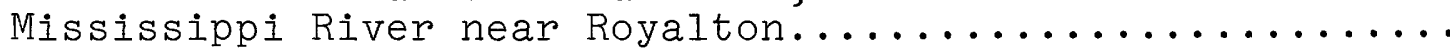
Sauk River basin

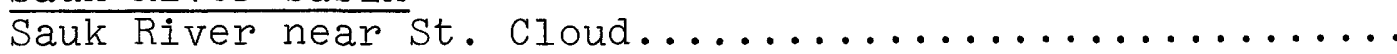
Elk River basin

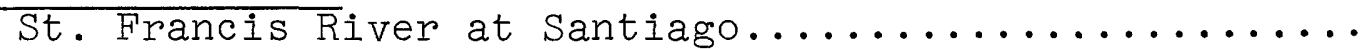

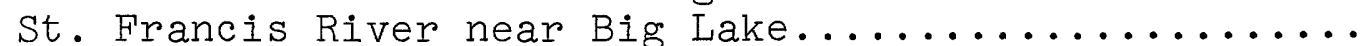

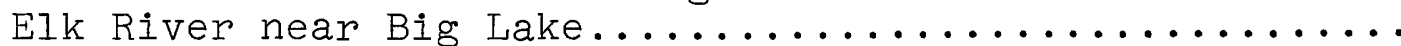
Crow River basin

North Fork Crow River:

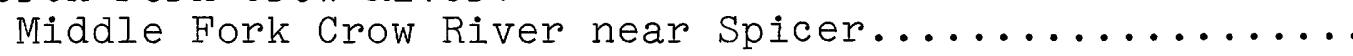

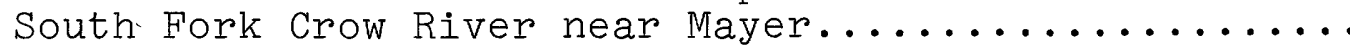

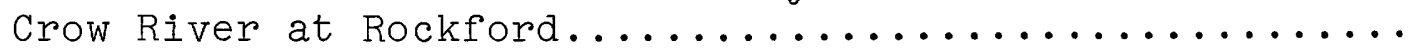
Rum River basin

Mille Lacs Lake (head of Rum River) at Garrison.........

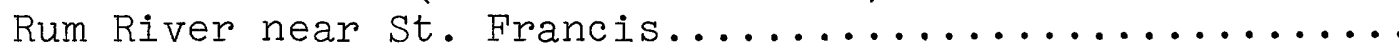

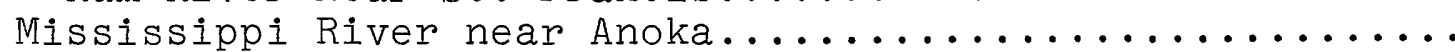
Minnesota River basin

Little Minnesota River (head of Minnesota River) near

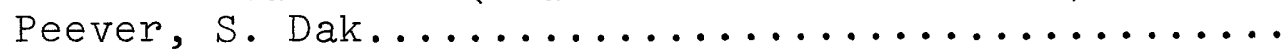

Whetstone River near Big Stone City, S. Dak..........

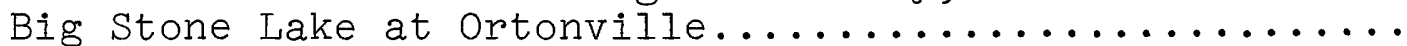

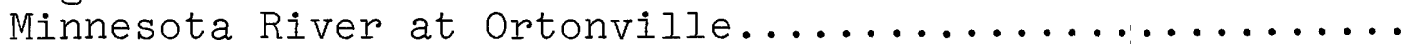

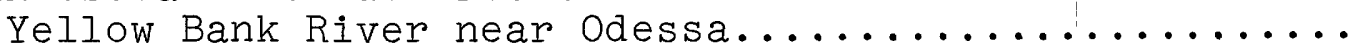

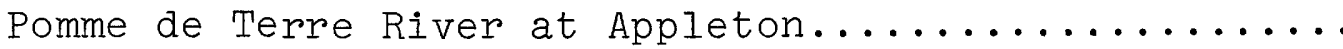

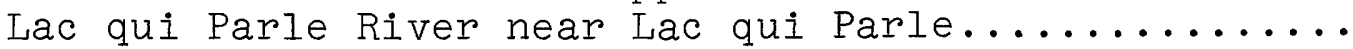

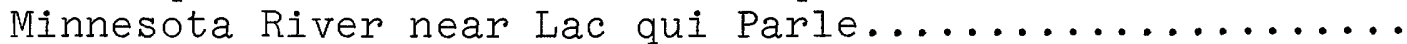

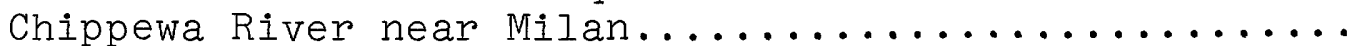

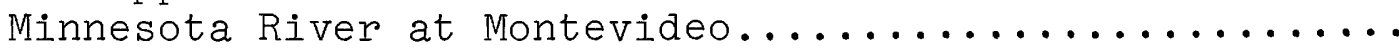
Yellow Medicine River:

South Branch Yellow Medicine River at Minneota......

105

106

107

109

110

111

112

113

114

115

116

117

118

119

120

121

122

123

124

125

126

127

128

129

130

131

132 
Gaging-station records--Continued

Upper Mississippi River basin--Continued

Page

Minnesota River basin--Continued

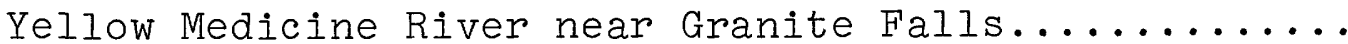

133

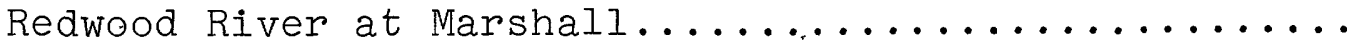

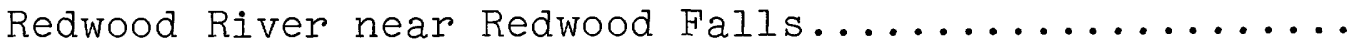

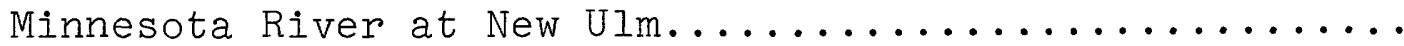

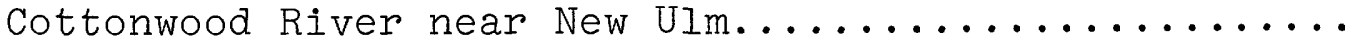

Blue Earth River:

East Branch Blue Earth River near Bricelyn........

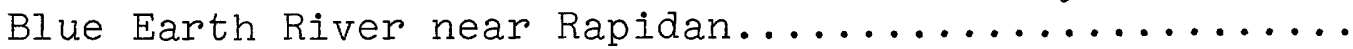

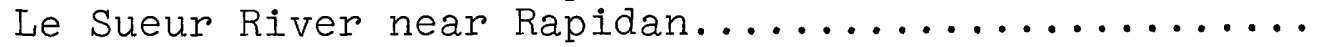

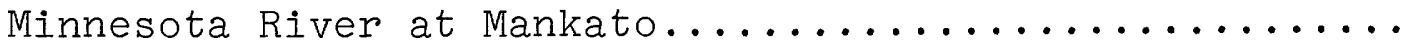

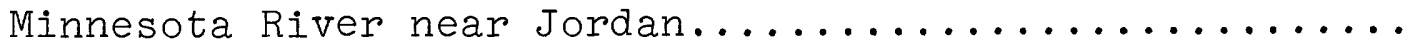

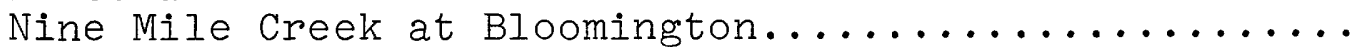

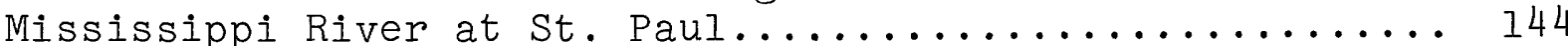

$\frac{\text { St. Croix River basin }}{\text { St. Croix River: }}$

Kettle River:

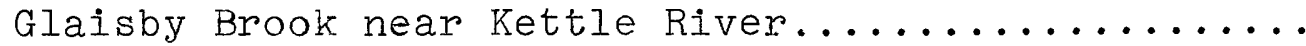

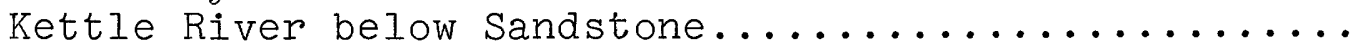

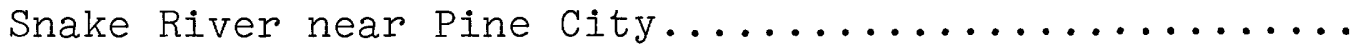

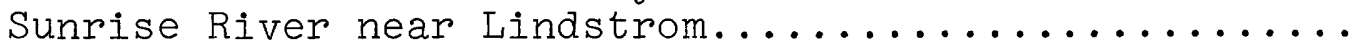

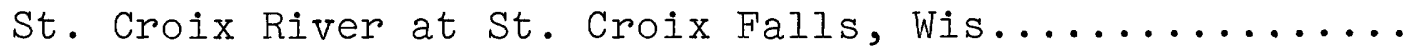

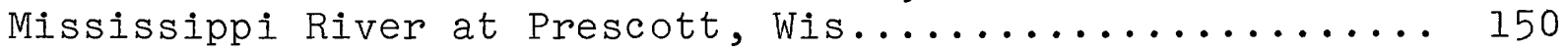

Cannon River basin

Cannon River:

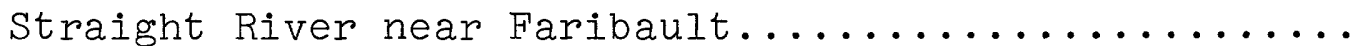

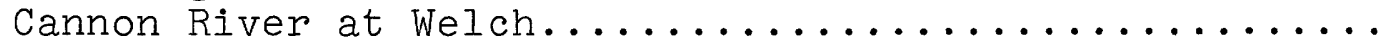

Zumbro River basin

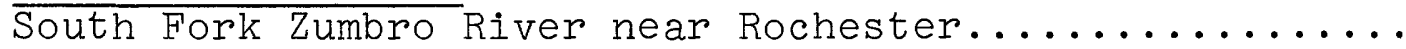

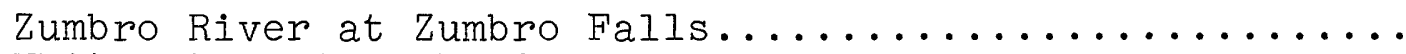

Whitewater River basin

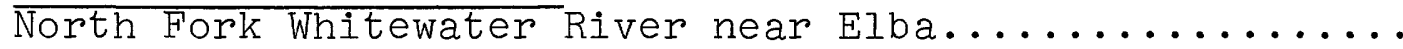

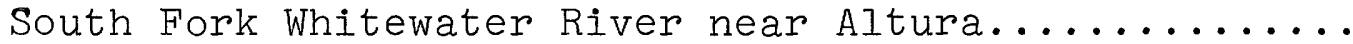

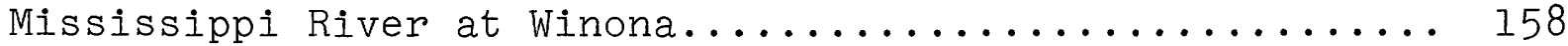

Root River basin

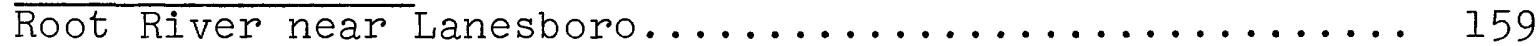

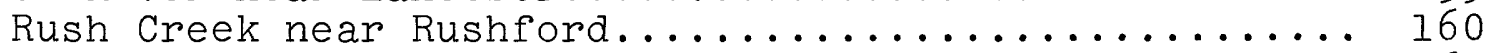

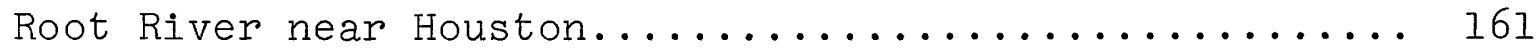

South Fork Root River near Houston............... 162

Iowa River basin

Iowa River:

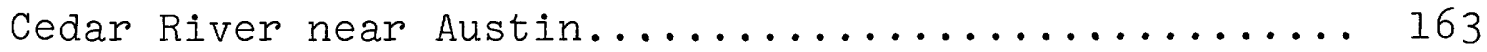

Des Moines River basin

West Fork Des Moines River at Jackson.............. 164 
Discharge at partial-record stations and miscellaneous sites:

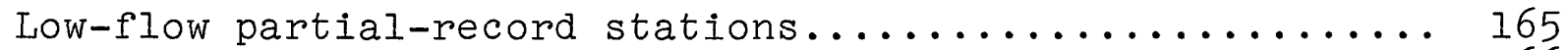

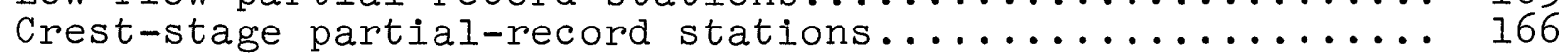

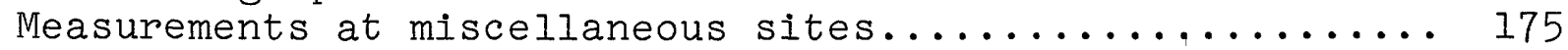

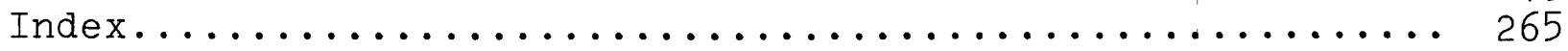

ILLUSTRATIONS

Figure 1. Map of Minnesota showing location of lake and

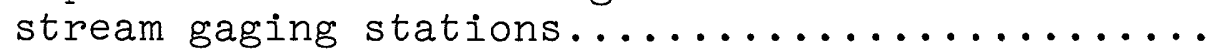


WATER RESOURCES DATA FOR MINNESOTA, 1968

Part 1. Surface Water Records

\section{INTRODUCTION}

The surface-water records for the 1968 water year for gaging stations, partial-record stations, and miscellaneous sites within the state of Minnesota are given in this report. For convenience there are also included records for a few pertinent gaging stations in bordering states. The records were collected and computed by the Water Resources Division of the U.S. Geological survey under the direction of C. R. Collier, district chief.

Through september 30, 1960, the records of discharge and stage of streams and contents and stage of lakes or reservoirs were published in an annual series of U.S. Geological Survey water-supply papers entitled "Surface Water Supply of the United States". Since 1951 there have been 20 volumes in the series; each volume covered an area whose boundaries coincided with those of certain natural drainage areas. The records in Minnesota were contained in Parts 4,5 and 6 of that series.

Beginning with the 1961 water year, streamflow records and related data will be released by the Geological Survey in annual reports on a state-boundary basis. Distribution of these basic-data reports will be limited and primarily for local needs. The records later will be published in Geological survey water-supply papers at 5-year intervals. 


\section{COOPERATION}

Cooperative agreements between the U. S. Geological Survey and organizations of the state of Minnesota for the systematic collection of streamflow records began in 1909. Organizations that supplied data are acknow1edged in station descriptions. Organizations that assisted in collecting data through cooperative agreements with the survey are:

Minnesota Department of Conservation, Division of Waters, Soils and Minerals, Eugene R.Gere, Director.

Minnesota Department of Highways, N. T. Waldor, Commissioner.

Assistance in the form of funds or services was given by Corps of Engineers, U. S. Army, in collecting records for 30 gaging stations published in this report.

Several gaging stations in the Hudson Bay and st. Lawrence River basins were maintained by funds appropriated to the United States Department of State.

On waters adjacent to the international boundary, certain gaging stations are maintained by the United States (or Canada) under agreement with Canada (or the United States), and the records are obtained and compiled in a manner equally acceptable in both countries. These stations are designated herein as "International gaging stations."

\section{DEFINITION OF TERMS AND ABBREVIATIONS}

The terms of streamflow and other hydrologic data, as used in this report, are defined as follows:

Gaging station is a particular site on a stream, canal, lake or reservoir where systematic observations of gage height or discharge are obtained. When used in connection with a discharge record, the term is applied only to those gaging stations where a continuous record of discharge is obtained.

Hydrologic bench-mark station is one that provides hydrologic data for a basin in which the hydrologic regimes will likely be governed solely by natural conditions. Data 
collected at a bench-mark station may be used to separate effects of natural from manmade changes in other basins which have been developed and in which the physiography, climate, and geology are similar to those in the underdeveloped bench-mark basin.

Partial-record station is a particular site where limited streamflow data are collected systematically over a period of years for use in hydrologic analyses.

Cubic foot per second (cfs) is the rate of discharge of a stream whose channel is 1 square foot in crosssectional area and whose average velocity is 1 foot per second.

Cubic feet per second per square mile (cfsm) is the average number of cubic feet of water flowing per second from each square mile of area drained, assuming that the runoff is distributed uniformly in time and area.

Runoff in inches (in.) shows the depth to which the drainage area would be covered if all the runoff for a given time period were uniformly distributed on it.

Acre-foot $(a c-f t)$ is the quantity of water required to cover an acre to the depth of 1 foot and is equivalent to 43,560 cubic feet.

Cfs-day is the volume of water represented by a flow of 1 cubic foot per second for 24 hours. It is equivalent to 86,400 cubic feet, 1.983471 acre-feet, or 646,317 gallons and represents a runoff of 0.0372 inch from 1 square mile.

Stage-discharge relation is the relation between gage height and the amount of water flowing in a channel, expressed as volume per unit of time.

Gage height is the water-surface elevation referred to some arbitrary gage datum. Gage height is often used interchangeably with the more general term "stage," although gage height is more appropriate when used with a reading on a gage.

Control designates a feature downstream from the gage that determines the stage-discharge relation at the gage. 
This feature may be a natural constriction of the channel, a long reach of the channel, or an artificial structure.

Contents is the volume of water in a reservoir or lake. Unless otherwise indicated, volume is computed on the basis of a level pool and does not include bank storage.

The drainage area of a stream at a specified location is that area, measured in a horizontal plane, which is so enclosed by a topographic divide that direct surface runoff from precipitation normally would drain by gravity into the river above the specified point. Figures of drainage area given herein include all closed basins, or noncontributing areas, within the area unless otherwise noted.

WSP is used as an abbreviation for "Water-Supply Paper" in references to previously published reports.

Water year in Geological Survey reports dealing with surface-water supply is the 12-month period, October 1 through September 30. The water year is designated by the calendar year in which it ends and which includes 9 of the 12 months. Thus, the year ending september 30, 1964, is called the "1964 water year."

\section{DOWNSTREAM ORDER AND STATION NUMBERS}

Stations are listed in the same downstream order used in the water-supply papers. Records are listed in a downstream direction along the main stem with all stations on a tributary entering above a main-stem station listed before that station. If a tributary enters between two main-stem stations, it is listed between them. A similar order is followed listing stations on first rank, second rank, and other ranks of tributaries. To indicate the rank of any tributary on which a gaging station is situated and the stream to which it is immediately tributary, each indention in the listing of gaging stations in the table of contents of this report represents one rank. This downstream order and system of indention shows which gaging stations are on tributaries between any two stations on a main stem and the rank of the tributary on which each gaging station is situated. 
As an added means of identification, each gaging station and partial-record station has been assigned a station number. The numbers have been assigned in the same downstream order used in the annual series of water-supply papers. In assigning station numbers, no distinction is made between partialrecord stations and continuous-record gaging stations, so that the station number for a partial-record station indicates downstream order position in a list made up of both types of stations. Gaps are left in the numbers to allow for new stations that may be established; hence the numbers are not consecutive.

The complete 8-digit number for each station, such as 05-0615.00, includes the part number "05" and a 6-digit station number. In this report, the part number and only the essential digits of the station number are shown. For example, the complete number 05-0615.00 would appear as 5-0615, just to the left of the station name. In this report, the records are listed in downstream order by parts. Al1 records for a drainage basin encompassing more than one state could be arranged in downstream order by assembling pages from the various state reports by station number to include all records in the basin.

\section{EXPLANATION OF DATA}

The base data collected at gaging stations consist of records of stage and measurements of discharge. In addition, observations of factors affecting the stage-discharge relation, weather records, and other information are used to supplement base data in determing the daily flow. The records of stage are obtained from a water-stage recorder that gives a continuous record of fluctuations or from direct readings on a nonrecording gage. Measurements of discharge are made with a current meter by the general methods adopted by the Geological Survey on the basis of experience in stream gaging since 1888 . These methods are described in water-Supply Paper 888 and are also outlined in standard textbooks on the measurement of stream discharge.

Rating tables giving the discharge for any stage are prepared from stage-discharge relation curves defined by discharge measurements. If extensions to the rating curves are necessary to define the extremes of discharge, they are made on the basis of indirect measurements of peak discharge (such as slope-area or contracted-opening measurements, or computation of flow over dams or weirs), velocity-area studies, and logarithmic plotting. The application of the daily mean 
gage height to those rating tables gives the daily mean discharge, from which the monthly and the yearly mean discharge are computed. If the stage-discharge relation is subject to change because of frequent or continual change in the physical features that form the control, the daily mean discharge is determined by the shifting-control method, in which correction factors based on individual discharge measurements and notes by engineers and observers are used in applying the gage heights to the rating tables. If the stage-discharge relation for a station is temporarily changed by the presence of aquatic growth or debris on the control, the daily mean discharge is computed by what is in effect the shifting-control method.

At some gaging stations the stage-discharge relation is affected by backwater from reservoirs, tributary streams, or other sources. This necessitates the use of the slope method in which the slope or fall in a reach of the stream is a factor in determining discharge. Information required for determining the slope or fall is obtained by means of an auxiliary gage set at some distance from the base gage. At some stations the stage-discharge relation is affected by changing stage. For such stations, the rate of change in stage is used as a factor in determining discharge.

At some gaging stations the stage-discharge relation is affected by ice during the winter, and it becomes impossible to compute the discharge in the usual manner. Discharge for periods of ice effect is computed on the basis of the gage-height record and occasional winter discharge measurements, consideration being given to the available information on temperature and precipitation, notes by gage observers and engineers, and comparable records of discharge for other stations in the same or nearby basins.

For some gaging stations there are periods when no gage-height record is obtained or the recorded gage height is so faulty that it cannot be used to compute the daily discharge. This happens when the recorder stops or otherwise fails to operate properly, intakes are plugged, float is frozen in the well, or for various other reasons. For such periods the daily discharges are estimated on the basis of recorded range in stage, adjoining good record, discharge measurements, weather 
records, and comparison with other station records from the same or nearby basins.

The data in this report generally comprise a description of the station, and a table showing the daily discharge and monthly and yearly discharge of the stream. Tables of mean daily gage height are included for some stations. Records are published for the water year which begins on October 1 and ends on september 30 . A calendar for the 1968 water year is shown on page II to facilitate finding the day of the week for any date.

The description of the station gives the location, drainage area, records available, type and history of gages, average discharge, extremes of discharge, and general remarks. The location of the gaging station and the drainage area are obtained from the most accurate maps available. River mileage given under "Location" for some stations, is that determined and used by the Corps of Engineers unless otherwise noted. Under "Records available" are given periods for which there are published records for the present station or for stations generally equivalent to the present one. Under "Gage" are given the type of gage currently in use and the datum of the gage above mean sea level, and a condensed history of the types, locations, and datums of previous gages used during the period of records available. The references to "datum of 1929" and adjustments of other years are to the datum and adjustments of the U. S. Coast and Geodetic Survey. Under "Average discharge" is given the average discharge for the number of years indicated. It is not given for stations having fewer than five complete years of record or for stations where changes in water development during the period of record cause the figure to have little significance. Under "Extremes" are given the maximum discharge and gage height; the minimum discharge if there is little or no regulation; the minimum daily discharge if there is extensive regulation (also the minimum discharge if useful); and the minimum gage height if it is significant. In the first paragraph, the data given are for the complete current water year unless otherwise specified. In the second paragraph, the data given are for the periods of record within the calendar year dates in the heading (not necessarily those for the complete years indicated by the heading dates). Reliable information concerning major floods that have occurred outside the period of 
record are given in the third or last paragraph under "Extremes". Unless otherwise qualified, the maximum discharge corresponds to the crest stage obtained by use of a water-stage recorder, a crest-stage indicator, or a nonrecording gage read at the time of the crest. If the maximum gage height did not occur at the same time as the maximum discharge, it is given separately. Information pertaining to the accuracy of the records and to conditions which affect the natural flow at the gaging station is given under "Remarks".

The daily table gives the discharge corresponding to the daily mean gage height unless there are large or rapid changes in discharge during a day. For days having large or rapid changes, discharge for the day is computed by averaging the mean discharges for several parts of a day. For digital recorders, the daily mean discharge is always the average of the discharges at each punched reading. For stations equipped with nonrecording gages, the daily discharge corresponds to once-daily readings of the gage or to the mean of twice-daily readings; but for periods of rapidly changing stage the discharge is determined from a gage-height graph based on gage readings.

In the monthly summary below the daily table, the line headed "Total" gives the sum of the daily figures; it is the total cfs-days for the month. The line headed "Mean" gives the average flow in cubic feet per second during the month. The maximum day and the minimum day for each month are shown in the line with the respective headings. Discharge for the month may be expressed in cubic feet per second per square mile (line headed "Cfsm"), or in inches (line headed "In."), or in acre-feet (line headed "Ac-ft"). Figures for cubic feet per second per square mile and runoff in inches are omitted if the drainage area includes large noncontributing areas, or if the average annual rainfall on the drainage basin is usually less than 20 inches.

In the yearly summary below the monthly summary, the figures of maximum are the maximum daily discharges, not the momentary discharges when the water was at crest stage. Likewise, the minimums in this summary are the minimum daily discharges.

Peak discharges and the times of their occurrence and corresponding gage heights for most stations are listed below the table of daily and monthly discharge. All independent peaks above the selected base are given. The base discharge, which is given in parentheses, is selected so that an average 
of about three peaks a year can be presented. Peak discharges are not published for any stream for which the peaks are subject to substantial control by man. Time of day is expressed in 24-hour local standard time, for example, 12:30 a.m. is $0030,1: 30$ p.m. is 1330 .

In a general footnote, introduced by the word "Note", certain periods are indicated for which the discharge is computed or estimated by special methods because of no gageheight record, backwater from various sources, or other unusual conditions. Periods of no gage-height record are indicated if the period is continuous for a month or more or includes the maximum discharge for the year. Periods of backwater from an unusual source, of indefinite stage-discharge relation, or of any other unusual condition at the gage are indicated only if they are a month or more in length and the accuracy of the records is affected. Days on which the stage-discharge relation is affected by ice are not indicated. The methods used in computing discharge for various unusual conditions have been explained in preceding paragraphs.

For most gaging stations on lakes and reservoirs the data presented comprise a description of the station and a monthly summary table of stage and contents. For some reservoirs a table showing daily contents or stage is given. A skeleton table of capacity at given stages is published each year for all reservoirs for which records are published on a daily basis, but it is not published for reservoirs for which only monthly data are given.

\section{ACCURACY OF FIEID DATA AND COMPUTED RESULTS}

The accuracy of streamflow data depends primarily on (1) the stability of the stage-discharge relation or, if the control is unstable, the frequency of discharge measurements, and (2) the accuracy of observations of stage, measurements of discharge, and interpretation of records.

The station description under "Remarks" states the degree of accuracy of the records. "Excellent" means that about 95 percent of the daily discharges are within 5 percent; "good" within 10 percent; and "fair" within 15 percent. "Poor" means that daily discharges have less than "fair" accuracy.

For most gaging stations equipped with digital recorders the figures of mean daily discharge are shown to the nearest hundredth of a cfs for discharges less than 1 cfs. This has been done as a matter of uniformity in the computer program and should not be construed to indicate an accuracy greater than that used in the past. 
Discharge at some stations, as indicated by the monthly mean, may vary widely from natural runoff, owing to diversion, consumption, regulation by storage, increase or decrease in evaporation due to artificial causes, or to other factors. For such stations, figures of cubic feet per second per square mile and of runoff in inches are not published unless satisfactory adjustments can be made for diversions, for changes in contents of reservoirs, or for other changes incident to use and control. Evaporation from a reservoir is not included in the adjustments for changes in reservoir contents, unless it is so stated. Even at those stations where adjustments are made, large errors in computed runoff may occur when relatively large negative adjustments are made or when evaporation is large in comparison with the observed discharge.

\section{OTHER DATA AVAILABLE}

Data collected at partial-record stations and at miscellaneous sites are given at the end of this report. Data for partial-record stations are presented in two tables. The first is a table of discharge measurements at low-flow partialrecord stations, and the second is a table of annual maximum stage and discharge at crest-stage stations. Discharge measurements at miscellaneous sites are given in a third table.

Information of a more detailed nature than that published for most of the gaging stations is on file in the district office, such as discharge measurements and recorder charts or nonrecording-gage readings. Most gaging-station records in the State through 1967 have been analyzed with an electronic computer to give: (1) the number of days in each year that the daily discharge was between selected limits (duration tables); (2) the lowest mean discharge for selected numbers of consecutive days in each year; and (3) the highest mean discharge for selected numbers of consecutive days in each year.

At or near some gaging stations, water-quality records also are collected. Data are obtained on the chemical quality of the stream water, on water temperature, on suspended-sediment concentration, and on the particle-size distribution of suspended sediment and bed material. These data are given in Part 2 of this report. Under the "Remarks" paragraph of the gagingstation description, reference is made to water-quality records collected on a regular basis. 


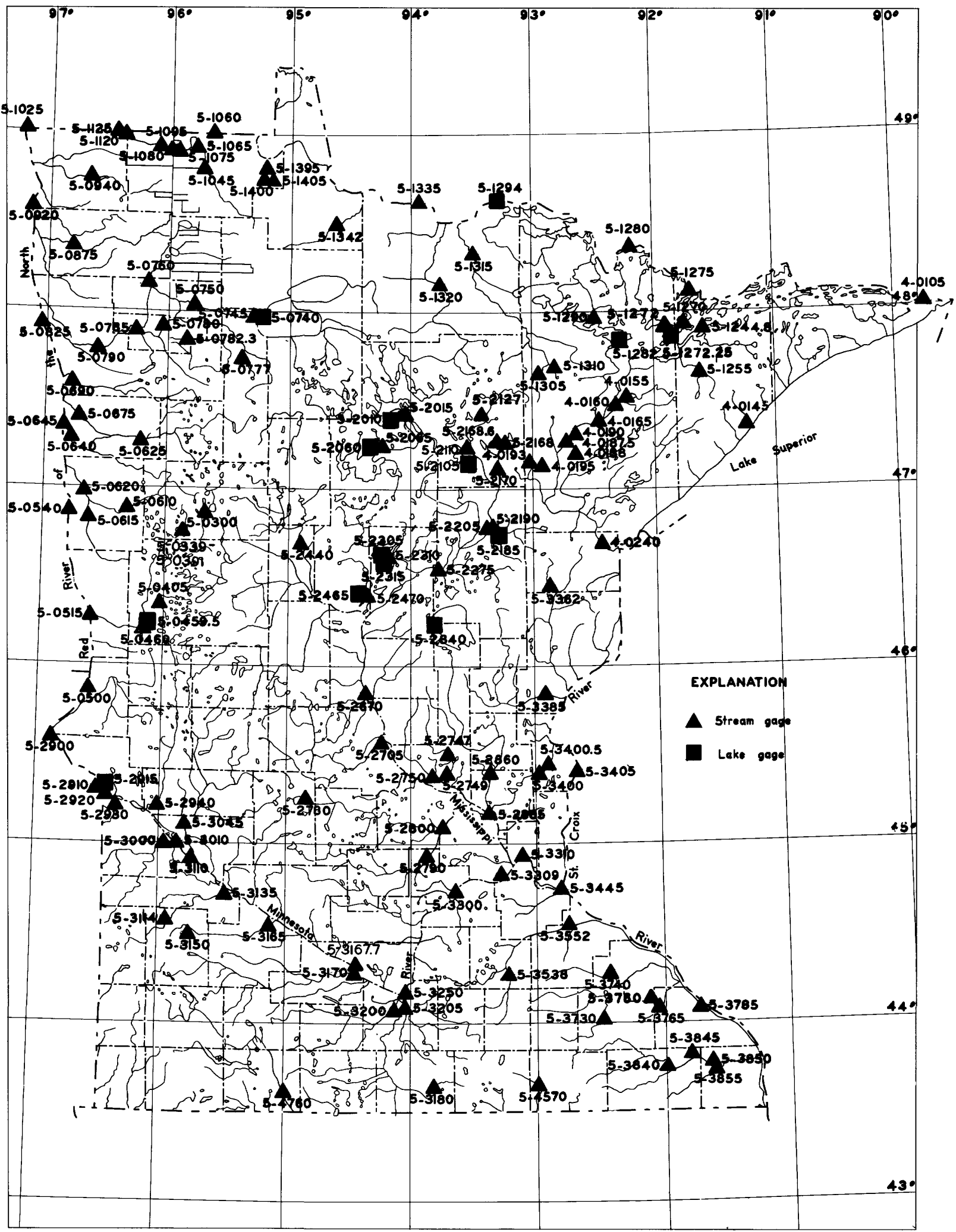

Figure 1.-- Map of Minnesota showing location of lake and stream gaging stations. 
4-0105. Pigeon River at Middle Falls, near Grand Portage, Minn.

(International gaging station)

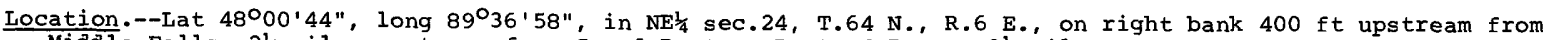

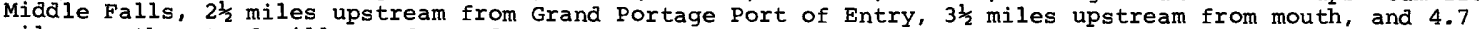
miles northeast of village of Grand portage.

Drainage area. $--600 \mathrm{sq} \mathrm{mi}$.

Records available.--June to October 1921, April to November 1922, March 1923 to september 1968. Published as "at International Bridge" April 1924 to September 1940; as "below International Bridge" October 1940 to September 1965. Monthly discharge only for some periods, published in WSP 1307.

Gaqe.--Digital water-stage recorder. Datum of gage is $789.58 \mathrm{ft}$ above mean sea level, datum of 1929 . Prior to sept. 2, 1936, staff gage and Sept. 2, 1936, to sept. 30, 1940, wire-weight gage at International Bridge, $5 \frac{3}{4}$ miles upstream at datum $100.24 \mathrm{ft}$ higher. Oct. 1, 1940 to Aug. 13, 1968, graphic water-stage recorder at same site and datum.

Average discharge. --45 years $(1923-68), 484 \mathrm{cfs}$.

Extremes.--Maximum discharge during year, $3,630 \mathrm{cfs} J u 1 y 17$ (gage height, 6.71 ft); minimum daily, 30 cfs Feb. 15-22; minimum gage height, $0.12 \mathrm{ft}$ Nov. 15.

1923-68: Maximum discharge, 11,000 cfs May 5, 1934 (gage height, 7.6 ft, site and datum then in use), from rating curve extended above $7,000 \mathrm{cfs}$; minimum, $27 \mathrm{cfs}$ Nov. 4, 1945 (gage height, $-0.08 \mathrm{ft}$ ).

Remarks.--Records good except those for winter months, which are fair. Records of chemical analyses for the water year 1968 are published in Part 2 of this report.

Cooperation.--This station is one of the international gaging stations maintained by the United states under agreement with Canada.

DISCHARGE, IN CFS, WATER YEAR OCTOBER 1967 TO SEPTEMBER 1968

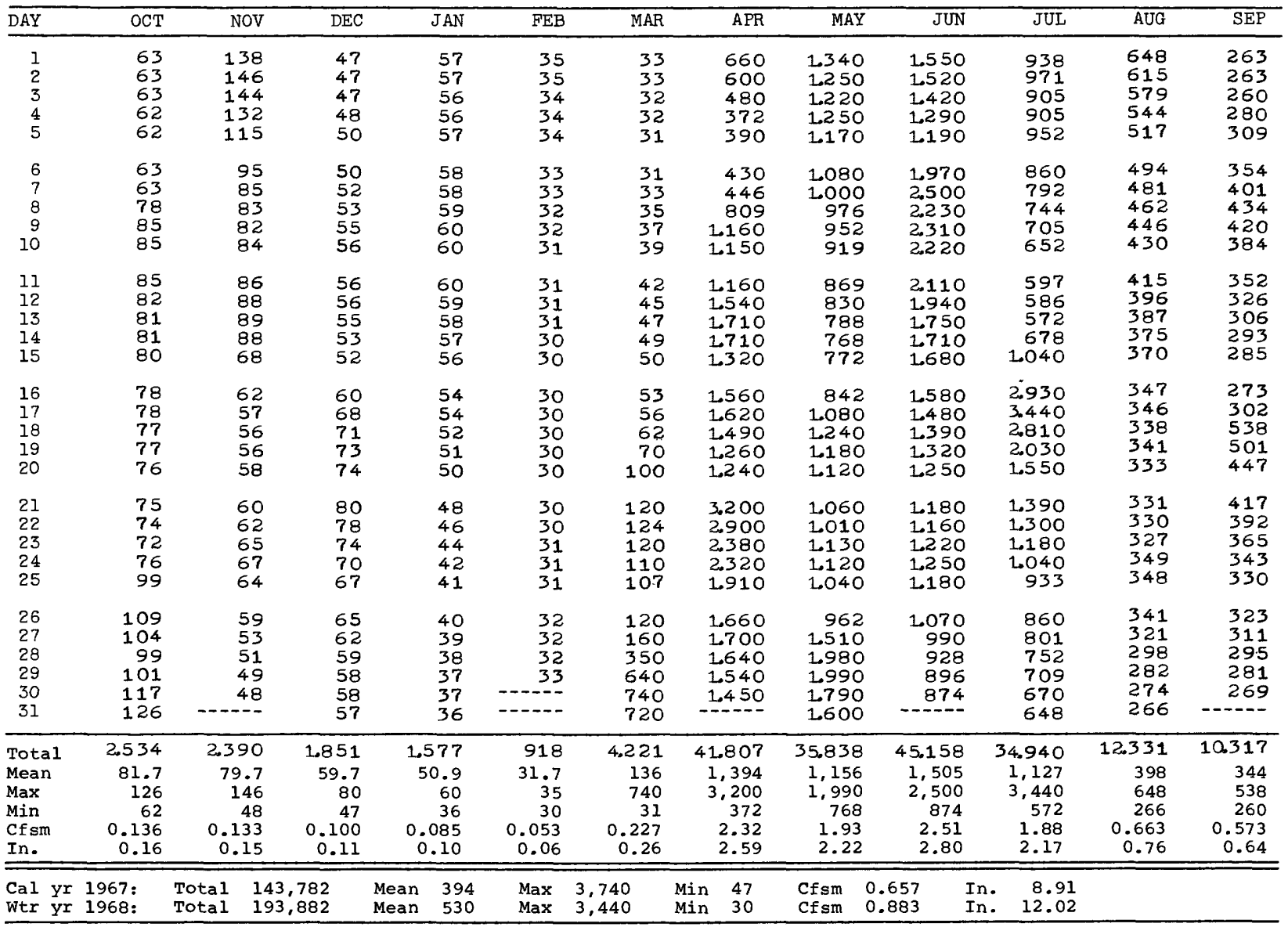

Peak discharge (base 3,000 cfs)

\begin{tabular}{|c|c|c|c|c|c|c|c|}
\hline Date & Time & $\begin{array}{c}\text { Gage } \\
\text { height }\end{array}$ & Discharge & Date & Time & $\begin{array}{c}\text { Gage } \\
\text { height }\end{array}$ & Discharge \\
\hline $4-21$ & $\begin{array}{r}\text { about } \\
1200\end{array}$ & $\begin{array}{r}\text { about } \\
6.50\end{array}$ & $\begin{array}{l}\text { about } \\
3,420\end{array}$ & $7-17$ & 0100 & 6.71 & 3,630 \\
\hline
\end{tabular}


4-0145. Baptism River near Beaver Bay, Minn.

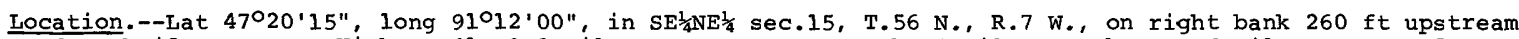
from bridge on U.S. Highway $61,0.2$ mile upstream from mouth, 4 miles northeast of Silver Bay, and 7

miles northeast of village of Beaver Bay.

Drainage area. $--140 \mathrm{sq} \mathrm{mi}$.

Records available.--October 1927 to September 1968. Monthly discharge only for some periods, published in WSP 1307.

Gage.--Water-stage recorder. Datum of gage is $609.97 \mathrm{ft}$ above mean sea level (Corps of Engineers bench mark). Prior to Oct. 5, 1934, staff gage at same site and datum.

Average discharge. -41 years, $160 \mathrm{cfs}$.

Extremes.--Maximum discharge during year, 2,840 cfs Aug. 22 (gage height, $5.19 \mathrm{ft}$ ); minimum daily, $6.2 \mathrm{cfs}$ Feb. 20-24.

1927-68: Maximum discharge recorded, 9,350 cfs Aug. 9, 1939 (gage height, $8.11 \mathrm{ft}$ ), from rating curve extended above $4,000 \mathrm{cfs}$; maximum gage height, 11.06 ft Apr. 12, 1965 (from floodmark, backwater from ice); minimum daily discharge, 0.4 cfs Jan. 5, 6, 1940.

Remarks.--Records good except those for winter months and period of backwater from bridge construction, which are fair. Records for chemical analyses and suspended sediment loads for water year 1968 are published in Part 2 of this report.

DISCHARGE, IN CFS, WATER YEAR OCTOBER 1967 TO SEPTEMBER 1968

\begin{tabular}{|c|c|c|c|c|c|c|c|c|c|c|c|c|}
\hline DAY & $\mathrm{OCT}$ & NoV & $\mathrm{DEC}$ & JAN & $\mathrm{FEB}$ & MAR & APR & MAY & JUN & JUL & $\mathrm{AUG}$ & SEP \\
\hline $\begin{array}{l}1 \\
2 \\
3 \\
4 \\
5\end{array}$ & $\begin{array}{l}28 \\
27 \\
26 \\
26 \\
24\end{array}$ & $\begin{array}{l}63 \\
64 \\
62 \\
55 \\
56\end{array}$ & $\begin{array}{l}30 \\
30 \\
29 \\
29 \\
28\end{array}$ & $\begin{array}{l}11 \\
11 \\
10 \\
10 \\
10\end{array}$ & $\begin{array}{l}11 \\
12 \\
12 \\
13 \\
13\end{array}$ & $\begin{array}{l}6.8 \\
6.8 \\
7.0 \\
7.2 \\
7.4\end{array}$ & $\begin{array}{l}326 \\
270 \\
192 \\
158 \\
155\end{array}$ & $\begin{array}{l}525 \\
458 \\
385 \\
421 \\
358\end{array}$ & $\begin{array}{l}582 \\
534 \\
430 \\
367 \\
312\end{array}$ & $\begin{array}{l}155 \\
164 \\
146 \\
167 \\
141\end{array}$ & $\begin{array}{l}40 \\
35 \\
31 \\
29 \\
26\end{array}$ & $\begin{array}{l}200 \\
176 \\
149 \\
505 \\
600\end{array}$ \\
\hline $\begin{array}{r}6 \\
7 \\
8 \\
9 \\
10\end{array}$ & $\begin{array}{r}25 \\
34 \\
170 \\
158 \\
136\end{array}$ & $\begin{array}{l}71 \\
72 \\
52 \\
53 \\
51\end{array}$ & $\begin{array}{l}28 \\
30 \\
32 \\
33 \\
34\end{array}$ & $\begin{array}{l}10 \\
10 \\
10 \\
10 \\
10\end{array}$ & $\begin{array}{l}13 \\
13 \\
12 \\
12 \\
11\end{array}$ & $\begin{array}{l}7.6 \\
7.9 \\
8.9 \\
10 \\
11\end{array}$ & $\begin{array}{l}161 \\
188 \\
412 \\
440 \\
412\end{array}$ & $\begin{array}{l}305 \\
291 \\
305 \\
319 \\
284\end{array}$ & $\begin{array}{r}630 \\
658 \\
1.030 \\
1110 \\
1070\end{array}$ & $\begin{array}{r}117 \\
95 \\
80 \\
68 \\
59\end{array}$ & $\begin{array}{l}25 \\
24 \\
25 \\
26 \\
22\end{array}$ & $\begin{array}{l}690 \\
750 \\
730 \\
580 \\
370\end{array}$ \\
\hline $\begin{array}{l}11 \\
12 \\
13 \\
14 \\
15\end{array}$ & $\begin{array}{r}113 \\
97 \\
92 \\
83 \\
78\end{array}$ & $\begin{array}{l}52 \\
53 \\
51 \\
46 \\
35\end{array}$ & $\begin{array}{l}34 \\
34 \\
32 \\
30 \\
27\end{array}$ & $\begin{array}{l}9.7 \\
9.5 \\
9.5 \\
9.5 \\
9.5\end{array}$ & $\begin{array}{c}10 \\
9.5 \\
9.0 \\
8.0 \\
7.2\end{array}$ & $\begin{array}{l}11 \\
12 \\
13 \\
13 \\
14\end{array}$ & $\begin{array}{l}412 \\
487 \\
478 \\
554 \\
421\end{array}$ & $\begin{array}{l}243 \\
227 \\
212 \\
216 \\
265\end{array}$ & $\begin{array}{r}1040 \\
772 \\
582 \\
582 \\
516\end{array}$ & $\begin{array}{r}67 \\
138 \\
183 \\
705 \\
421\end{array}$ & $\begin{array}{l}18 \\
16 \\
14 \\
13 \\
25\end{array}$ & $\begin{array}{r}230 \\
140 \\
105 \\
85 \\
70\end{array}$ \\
\hline $\begin{array}{l}16 \\
17 \\
18 \\
19 \\
20\end{array}$ & $\begin{array}{l}72 \\
65 \\
63 \\
59 \\
56\end{array}$ & $\begin{array}{l}33 \\
31 \\
31 \\
32 \\
36\end{array}$ & $\begin{array}{l}25 \\
28 \\
30 \\
32 \\
33\end{array}$ & $\begin{array}{l}9.5 \\
9.5 \\
9.7 \\
10 \\
10\end{array}$ & $\begin{array}{l}6.8 \\
6.6 \\
6.4 \\
6.4 \\
6.2\end{array}$ & $\begin{array}{r}17 \\
32 \\
260 \\
400 \\
238\end{array}$ & $\begin{array}{l}449 \\
487 \\
458 \\
385 \\
635\end{array}$ & $\begin{array}{l}630 \\
706 \\
610 \\
468 \\
385\end{array}$ & $\begin{array}{l}430 \\
367 \\
305 \\
265 \\
212\end{array}$ & $\begin{array}{l}312 \\
277 \\
221 \\
208 \\
158\end{array}$ & $\begin{array}{l}63 \\
52 \\
42 \\
54 \\
53\end{array}$ & $\begin{array}{r}60 \\
170 \\
770 \\
660 \\
500\end{array}$ \\
\hline $\begin{array}{l}21 \\
22 \\
23 \\
24 \\
25\end{array}$ & $\begin{array}{l}54 \\
52 \\
52 \\
60 \\
68\end{array}$ & $\begin{array}{l}40 \\
42 \\
41 \\
39 \\
36\end{array}$ & $\begin{array}{l}33 \\
34 \\
30 \\
27 \\
25\end{array}$ & $\begin{array}{ll}1 & 1 \\
11 \\
10 \\
10 \\
10\end{array}$ & $\begin{array}{l}6.2 \\
6.2 \\
6.2 \\
6.2 \\
6.3\end{array}$ & $\begin{array}{r}136 \\
113 \\
102 \\
100 \\
92\end{array}$ & $\begin{array}{r}1.180 \\
896 \\
1.100 \\
1060 \\
734\end{array}$ & $\begin{array}{l}312 \\
259 \\
265 \\
277 \\
238\end{array}$ & $\begin{array}{l}221 \\
248 \\
421 \\
376 \\
284\end{array}$ & $\begin{array}{r}134 \\
100 \\
80 \\
68 \\
60\end{array}$ & $\begin{array}{r}151 \\
2.480 \\
2.380 \\
2.100 \\
1.370\end{array}$ & $\begin{array}{l}310 \\
230 \\
210 \\
175 \\
140\end{array}$ \\
\hline $\begin{array}{l}26 \\
27 \\
28 \\
29 \\
30 \\
31\end{array}$ & $\begin{array}{l}67 \\
64 \\
60 \\
60 \\
64 \\
64\end{array}$ & $\begin{array}{r}34 \\
32 \\
31 \\
31 \\
30 \\
-\end{array}$ & $\begin{array}{l}21 \\
19 \\
17 \\
14 \\
13 \\
12\end{array}$ & $\begin{array}{l}10 \\
10 \\
10 \\
10 \\
11 \\
11\end{array}$ & $\begin{array}{r}6.5 \\
6.6 \\
6.7 \\
68 \\
- \\
-\end{array}$ & $\begin{array}{l}150 \\
367 \\
563 \\
677 \\
554 \\
478\end{array}$ & $\begin{array}{r}610 \\
630 \\
610 \\
620 \\
601 \\
\end{array}$ & $\begin{array}{r}255 \\
1.020 \\
1060 \\
896 \\
668 \\
534\end{array}$ & $\begin{array}{r}216 \\
180 \\
161 \\
152 \\
144 \\
\end{array}$ & $\begin{array}{l}55 \\
55 \\
50 \\
43 \\
42 \\
48\end{array}$ & $\begin{array}{l}924 \\
620 \\
458 \\
319 \\
232 \\
192\end{array}$ & $\begin{array}{r}120 \\
105 \\
95 \\
90 \\
85 \\
\end{array}$ \\
\hline $\begin{array}{l}\text { Tota } 1 \\
\text { Mean } \\
\text { Max } \\
\text { Min } \\
\text { Cfsm } \\
\text { In. }\end{array}$ & $\begin{array}{r}2.097 \\
67.6 \\
170 \\
24 \\
0.483 \\
+\quad 0.56\end{array}$ & $\begin{array}{r}1355 \\
45.2 \\
72 \\
30 \\
0.323 \\
0.36\end{array}$ & $\begin{array}{r}853 \\
27.5 \\
34 \\
12 \\
0.196 \\
0.23\end{array}$ & $\begin{array}{r}312.4 \\
10.1 \\
11 \\
9.5 \\
0.072 \\
0.08\end{array}$ & $\begin{array}{r}2558 \\
8.82 \\
13 \\
6.2 \\
0.063 \\
0.07\end{array}$ & $\begin{array}{r}4.4226 \\
143 \\
677 \\
6.8 \\
1.02 \\
1.17\end{array}$ & $\begin{array}{r}12.521 \\
517 \\
1.180 \\
155 \\
3.69 \\
4.12\end{array}$ & $\begin{array}{r}13.397 \\
432 \\
1.060 \\
212 \\
3.09 \\
3.56\end{array}$ & $\begin{array}{r}14.197 \\
473 \\
1.110 \\
144 \\
3.38 \\
3.77\end{array}$ & $\begin{array}{r}4.617 \\
149 \\
705 \\
42 \\
1.06 \\
1.23\end{array}$ & $\begin{array}{r}11859 \\
383 \\
2,480 \\
13 \\
2.74 \\
3.15\end{array}$ & $\begin{array}{r}9.100 \\
303 \\
770 \\
160 \\
2.16 \\
2.42\end{array}$ \\
\hline $\begin{array}{l}\text { Cal y } \\
\text { wtr ys }\end{array}$ & $\begin{array}{l}r \text { 1967: } \\
r \text { 1968: }\end{array}$ & $\begin{array}{l}\text { Total } \\
\text { Total }\end{array}$ & $\begin{array}{l}637 \\
986.8\end{array}$ & $\begin{array}{l}\text { Mean } \\
\text { Mean }\end{array}$ & $\begin{array}{l}\operatorname{Max} \\
\operatorname{Max}\end{array}$ & $\begin{array}{l}1,980 \\
2,480\end{array}$ & $\begin{array}{l}\operatorname{Min} \\
\operatorname{Min}\end{array}$ & $\begin{array}{l}\text { Cfsm } \\
\text { Cfsm }\end{array}$ & $\begin{array}{l}1.09 \\
1.52\end{array}$ & $\begin{array}{ll}\text { In. } & 14 \\
\text { In. } & 20\end{array}$ & & \\
\hline
\end{tabular}

PEAK DISCHARGE (BASE, 1,300 CFS)

DATE TIME G.HT. DISCHARGE DATE TTME G.HT. DISCHARGE

$\begin{array}{lllllll}4-23 & 1830 & 4.55 & 1,580 & 8-22 & 0500 & 5.19\end{array}$
Note.--Backwater from bridge construction sept. 5-30. 
Location.--Lat $47^{\circ} 31^{\prime} 25^{\prime \prime}$, long $92^{\circ} 11^{\prime} 35^{\prime \prime}$, in SW/4 sec.12, T.58 N., R.15 W., on left bank 0.1 mile downstream from First Creek, 0.4 mile upstream from mouth, and 2.1 miles east of Aurora.

Drainage area.-- $-26.3 \mathrm{sq} \mathrm{mi}$.

Records available.--March 1955 to september 1968.

Gage.--Water-stage recorder. Datum of gage is $1,410.36$ ft above mean sea level, datum of 1929 (levels by Erie Mining Company).

Average discharge.--13 years, $19.2 \mathrm{cfs}$.

Extremes.--Maximum discharge during year, 164 cfs about Aug. 26 (gage height, 5.23 ft, from recorded range in stage); minimum, $7.8 \mathrm{cfs}$ Jan. $8,9$.

1955-68: Maximum discharge, 213 cfs Apr. 22, 1961; maximum gage height, 5.75 ft Mar. 28,1957 (backwater from ice); minimum daily discharge, 1.5 cfs Jan. 26 to Feb. 4,1963 ; minimum gage height, 3.10 ft Feb. 2 , 3 , 4, 1963.

Remarks.--Records good except those for winter months and those for periods of no gage-height record, which are fair.

DISCHARGE, IN CFS, WATER YEAR OCTOBER 1967 TO SEPTEMBER 1968

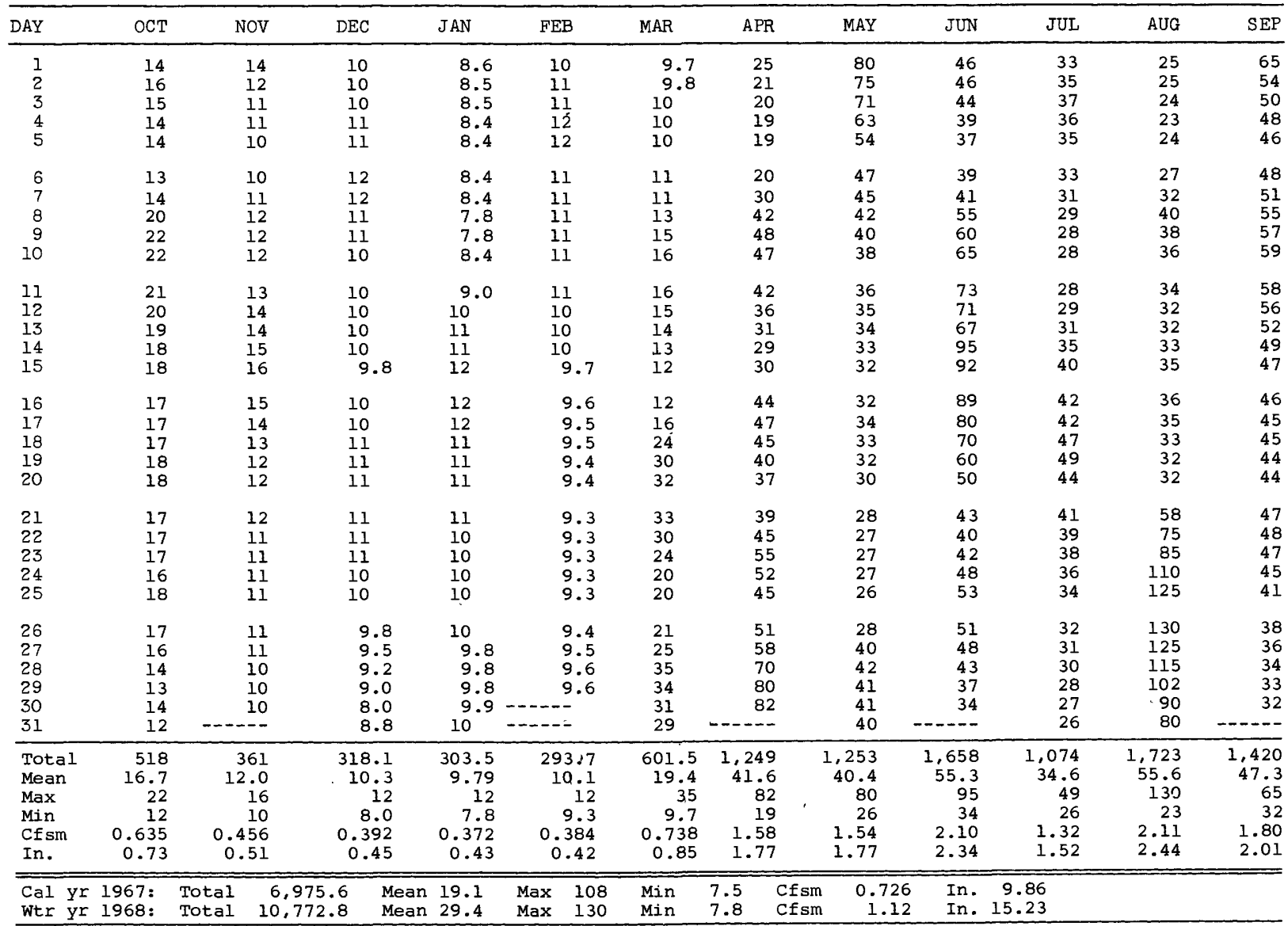

Peak discharge (base, 60 cfs)

\begin{tabular}{|r|c|c|c||c|c|c|c|}
\hline Date & Time & $\begin{array}{c}\text { Gage } \\
\text { height }\end{array}$ & Discharge & Date & Time & $\begin{array}{c}\text { Gage } \\
\text { height }\end{array}$ & Discharge \\
\hline about & & & about & about & & & \\
$4-30$ & Unkn & Unkn & 82 & $8-26$ & Unkn & 5.23 & 164 \\
$6-14$ & Unkn & 4.83 & 116 & & & & \\
\hline
\end{tabular}

Note.--No gage-height record June 14 to July 15 and Aug. 22 to sept. 19. 
4-0160. Partridge River near Aurora, Minn.

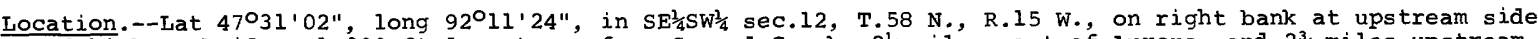
of highway bridge, 1, 000 ft downstream from Second Creek, $2 \frac{1}{2}$ miles east of Aurora, and $2 \frac{3}{4}$ miles upstream from mouth.

Drainage area.--156 sq $\mathrm{mi}$.

Records available.--August 1942 to september 1968 .

Gage.--Water-stage recorder. Datum of gage is $1,402.30 \mathrm{ft}$ above mean sea level, datum of 1929 . Aug. 5, 1942, to Aug. 25, 1944, staff gage and Aug. 26, 1944, to July 1, 1956, water-stage recorder at site 45 ft downstream at same datum.

Average discharge.--26 years, 122 cfs (adjusted for storage and diversion).

Extremes.--Maximum discharge during year, 1,010 cfs Aug. 27 (gage height, 5.11 ft); maximum gage height, 5.15 ft June 14; minimum discharge 12 cfs about Jan. 6 (gage height, 1.38 ft, from recorded range in stage). 1942-68: Maximum discharge, 3,230 cfs May 10, 1950 (gage height, $7.86 \mathrm{ft}$ ); minimum, $2.2 \mathrm{cfs}$ Jan. 30 , 31, 1961; minimum gage height, $0.88 \mathrm{ft}$ Mar. 2, 1963.

Remarks.--Records good except those for winter months, which are fair. Flow regulated at' times by storage in off-channel Partridge Reservoir, formerly known as Whitewater Lake. Reservoir formed from lake by levees around marsh areas and natural outlet. Available capacity, 20,000 acre-ft between elevations 1 , 410 (natural lake level) and 1,440 ft. Storage began Apr. 9, 1955. Storage in reservoir obtained from Colby Lake during periods of high flow; release from storage returned to colby Lake to maintain lake elevation during diversion for iron-ore processing. Diversion began Feb. 7, 1956 Some seepage losses from reservoir bypass station.

DISCHARGE, IN CFS, WATER YEAR OCTOBER 1967 TO SEPTEMBER 1968

\begin{tabular}{|c|c|c|c|c|c|c|c|c|c|c|c|c|}
\hline DAY & OCT & NOV & $\mathrm{DEC}$ & JAN & $F E B$ & MAR & APR & MAY & JUN & JUL & AUG & SEP \\
\hline $\begin{array}{l}1 \\
2 \\
3 \\
4 \\
5\end{array}$ & $\begin{array}{l}27 \\
29 \\
28 \\
28 \\
26\end{array}$ & $\begin{array}{l}31 \\
28 \\
27 \\
26 \\
27\end{array}$ & $\begin{array}{l}23 \\
23 \\
24 \\
25 \\
26\end{array}$ & $\begin{array}{l}15 \\
14 \\
14 \\
13 \\
13\end{array}$ & $\begin{array}{l}15 \\
15 \\
16 \\
16 \\
15\end{array}$ & $\begin{array}{l}14 \\
14 \\
15 \\
15 \\
15\end{array}$ & $\begin{array}{l}35 \\
32 \\
30 \\
29 \\
28\end{array}$ & $\begin{array}{l}410 \\
390 \\
370 \\
350 \\
320\end{array}$ & $\begin{array}{r}275 \\
275 \\
272 \\
244 \\
203\end{array}$ & $\begin{array}{l}247 \\
252 \\
258 \\
252 \\
220\end{array}$ & $\begin{array}{l}56 \\
56 \\
54 \\
53 \\
52\end{array}$ & $\begin{array}{l}275 \\
244 \\
222 \\
205 \\
190\end{array}$ \\
\hline $\begin{array}{r}6 \\
7 \\
8 \\
9 \\
10\end{array}$ & $\begin{array}{l}26 \\
28 \\
36 \\
36 \\
36\end{array}$ & $\begin{array}{l}28 \\
29 \\
31 \\
31 \\
31\end{array}$ & $\begin{array}{l}27 \\
29 \\
30 \\
29 \\
27\end{array}$ & $\begin{array}{l}13 \\
13 \\
13 \\
13 \\
13\end{array}$ & $\begin{array}{l}15 \\
15 \\
15 \\
15 \\
15\end{array}$ & $\begin{array}{l}16 \\
16 \\
17 \\
19 \\
20\end{array}$ & $\begin{array}{l}31 \\
39 \\
46 \\
49 \\
51\end{array}$ & $\begin{array}{l}300 \\
280 \\
270 \\
260 \\
250\end{array}$ & $\begin{array}{l}201 \\
194 \\
236 \\
347 \\
485\end{array}$ & $\begin{array}{l}196 \\
179 \\
164 \\
146 \\
130\end{array}$ & $\begin{array}{l}53 \\
56 \\
74 \\
69 \\
65\end{array}$ & $\begin{array}{l}201 \\
208 \\
220 \\
225 \\
220\end{array}$ \\
\hline $\begin{array}{l}11 \\
12 \\
13 \\
14 \\
15\end{array}$ & $\begin{array}{l}36 \\
37 \\
38 \\
39 \\
40\end{array}$ & $\begin{array}{l}34 \\
34 \\
33 \\
31 \\
28\end{array}$ & $\begin{array}{l}25 \\
24 \\
23 \\
22 \\
21\end{array}$ & $\begin{array}{l}13 \\
14 \\
14 \\
15 \\
16\end{array}$ & $\begin{array}{l}15 \\
15 \\
14 \\
14 \\
14\end{array}$ & $\begin{array}{l}21 \\
20 \\
19 \\
17 \\
17\end{array}$ & $\begin{array}{l}56 \\
52 \\
44 \\
44 \\
39\end{array}$ & $\begin{array}{l}243 \\
238 \\
230 \\
222 \\
212\end{array}$ & $\begin{array}{l}665 \\
794 \\
824 \\
951 \\
930\end{array}$ & $\begin{array}{l}122 \\
123 \\
117 \\
120 \\
123\end{array}$ & $\begin{array}{l}62 \\
61 \\
60 \\
58 \\
61\end{array}$ & $\begin{array}{l}203 \\
183 \\
168 \\
151 \\
142\end{array}$ \\
\hline $\begin{array}{l}16 \\
17 \\
18 \\
19 \\
20\end{array}$ & $\begin{array}{l}41 \\
42 \\
43 \\
44 \\
44\end{array}$ & $\begin{array}{l}30 \\
31 \\
30 \\
29 \\
28\end{array}$ & $\begin{array}{l}21 \\
22 \\
22 \\
23 \\
24\end{array}$ & $\begin{array}{l}17 \\
18 \\
18 \\
18 \\
17\end{array}$ & $\begin{array}{l}14 \\
14 \\
14 \\
13 \\
13\end{array}$ & $\begin{array}{l}18 \\
20 \\
22 \\
30 \\
37\end{array}$ & $\begin{array}{l}58 \\
71 \\
68 \\
60 \\
54\end{array}$ & $\begin{array}{l}205 \\
210 \\
220 \\
201 \\
201\end{array}$ & $\begin{array}{l}895 \\
830 \\
722 \\
610 \\
500\end{array}$ & $\begin{array}{l}142 \\
146 \\
162 \\
179 \\
185\end{array}$ & $\begin{array}{l}62 \\
58 \\
54 \\
55 \\
53\end{array}$ & $\begin{array}{l}132 \\
128 \\
133 \\
151 \\
160\end{array}$ \\
\hline $\begin{array}{l}21 \\
22 \\
23 \\
24 \\
25\end{array}$ & $\begin{array}{l}43 \\
43 \\
43 \\
41 \\
39\end{array}$ & $\begin{array}{l}28 \\
29 \\
30 \\
30 \\
30\end{array}$ & $\begin{array}{l}24 \\
23 \\
22 \\
21 \\
20\end{array}$ & $\begin{array}{l}17 \\
17 \\
16 \\
16 \\
16\end{array}$ & $\begin{array}{l}13 \\
13 \\
13 \\
13 \\
13\end{array}$ & $\begin{array}{l}36 \\
34 \\
32 \\
31 \\
31\end{array}$ & $\begin{array}{l}59 \\
59 \\
90 \\
89 \\
85\end{array}$ & $\begin{array}{l}194 \\
177 \\
168 \\
162 \\
150\end{array}$ & $\begin{array}{l}406 \\
356 \\
379 \\
396 \\
425\end{array}$ & $\begin{array}{l}181 \\
166 \\
151 \\
133 \\
115\end{array}$ & $\begin{array}{l}112 \\
203 \\
225 \\
356 \\
565\end{array}$ & $\begin{array}{l}170 \\
168 \\
170 \\
162 \\
155\end{array}$ \\
\hline $\begin{array}{l}26 \\
27 \\
28 \\
29 \\
30 \\
31\end{array}$ & $\begin{array}{l}39 \\
38 \\
36 \\
34 \\
33 \\
30\end{array}$ & $\begin{array}{r}29 \\
28 \\
26 \\
24 \\
23 \\
\end{array}$ & $\begin{array}{l}18 \\
17 \\
16 \\
16 \\
15 \\
15\end{array}$ & $\begin{array}{l}15 \\
14 \\
14 \\
14 \\
14 \\
14\end{array}$ & $\begin{array}{r}13 \\
14 \\
14 \\
14 \\
\hdashline-1\end{array}$ & $\begin{array}{l}31 \\
34 \\
43 \\
47 \\
43 \\
39\end{array}$ & $\begin{array}{r}106 \\
168 \\
250 \\
350 \\
400 \\
-\end{array}$ & $\begin{array}{l}142 \\
160 \\
185 \\
201 \\
220 \\
247\end{array}$ & $\begin{array}{r}387 \\
360 \\
334 \\
280 \\
255\end{array}$ & $\begin{array}{r}105 \\
90 \\
79 \\
71 \\
64 \\
60\end{array}$ & $\begin{array}{l}916 \\
951 \\
806 \\
640 \\
440 \\
328\end{array}$ & $\begin{array}{r}146 \\
137 \\
123 \\
115 \\
106 \\
-\end{array}$ \\
\hline $\begin{array}{l}\text { Total } \\
\text { Mean } \\
(f) \\
\text { Mean } \neq \\
\text { Max } \\
\text { Min } \\
\text { Cfsm } \neq \\
\text { In. } \neq\end{array}$ & $\begin{array}{r}1,123 \\
36.2 \\
+2.23 \\
38.4 \\
44 \\
26 \\
0.246 \\
0.28 \\
\end{array}$ & $\begin{array}{r}874 \\
29.1 \\
+0.39 \\
29.5 \\
34 \\
23 \\
0.189 \\
0.21 \\
\end{array}$ & $\begin{array}{r}697 \\
22.5 \\
+0.31 \\
22.8 \\
30 \\
15 \\
0.146 \\
0.17\end{array}$ & $\begin{array}{r}461 \\
14.9 \\
+1.86 \\
16.8 \\
18 \\
13 \\
0.108 \\
0.12 \\
\end{array}$ & $\begin{array}{r}412 \\
14.2 \\
-0.05 \\
14.2 \\
16 \\
13 \\
0.091 \\
0.10\end{array}$ & $\begin{array}{r}783 \\
25.3 \\
+8.83 \\
34.1 \\
47 \\
14 \\
0.219 \\
0.25 \\
\end{array}$ & $\begin{array}{r}2,572 \\
85.7 \\
+155 \\
241 \\
400 \\
28 \\
1.54 \\
1.72 \\
\end{array}$ & $\begin{array}{r}7.388 \\
238 \\
+23.3 \\
261 \\
410 \\
142 \\
1.67 \\
1.93 \\
\end{array}$ & $\begin{array}{r}14.031 \\
468 \\
+21.2 \\
489 \\
951 \\
194 \\
3.13 \\
3.50 \\
\end{array}$ & $\begin{array}{r}4.678 \\
151 \\
+20.3 \\
171 \\
258 \\
60 \\
1.10 \\
1.27 \\
\end{array}$ & $\begin{array}{r}6.714 \\
217 \\
+42.9 \\
260 \\
+951 \\
52 \\
1.67 \\
1.92 \\
\end{array}$ & $\begin{array}{r}5,213 \\
174 \\
+21.3 \\
195 \\
275 \\
106 \\
1.25 \\
1.40 \\
\end{array}$ \\
\hline $\begin{array}{l}\text { Cal yr } \\
\text { Wtr yr }\end{array}$ & 7: & $\begin{array}{lr}\text { Mean } & 75.6 \\
\text { Mean } & 123\end{array}$ & $\begin{array}{l}\text { Mean } \neq \\
\text { Mean } \neq\end{array}$ & $\begin{array}{r}98.0 \\
148\end{array}$ & $\begin{array}{l}\text { Max } \\
\operatorname{Max}\end{array}$ & $\begin{array}{l}\operatorname{Min} \\
\operatorname{Min}\end{array}$ & $\begin{array}{l}\text { Cfsm } \\
\text { Cfsm }\end{array}$ & $\begin{array}{l}0.628 \\
0.949\end{array}$ & $\begin{array}{l}\text { In. } \\
\text { In. }\end{array}$ & $\begin{array}{l}52 \\
87\end{array}$ & & \\
\hline
\end{tabular}

f Change in contents in Partridge Reservoir and diversion to iron-ore processing plant, equivalent in cubic

feet per second; furnished by Erie Mining Co.

$\neq$ Adjusted for change in contents and diversion. 
4-0165. St. Louis River near Aurora, Minn.

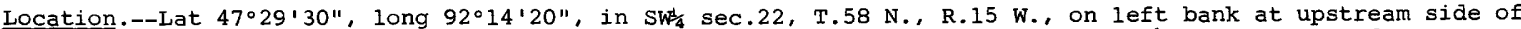
highway bridge, three-quarters of a mile downstream from Partridge River and $1 \frac{1}{2}$ miles south of Aurora.

Drainage area.--312 sq $\mathrm{mi}$.

Records available.--August 1942 to September 1968.

Gage.--Digital water-stage recorder. Datum of gage is 1,371.24 ft above mean sea level, datum of 1929 . Prior to Aug, 26, 1944, chain gage at same site and datum. Aug. 26, 1944 to Sept. 27, 1967, graphic water-stage recorder at present site and datum.

Average discharge.--26 years, $237 \mathrm{cfs}$ (adjusted for storage and diversion).

Extremes.--Maximum discharge during year, 1,560 cfs Aug. 26 (gage height, 4.48 ft); minimum daily, 23 cfs Feb. 20-26; minimum gage height, $0.95 \mathrm{ft}$ Feb. 22-26.

1942-68: Maximum discharge, 5,380 cfs May 14, 1950 (gage height, 8.37 ft); minimum, 4.0 cfs Oct. 2, 3, 1948 (gage height, $0.30 \mathrm{ft}$ ).

Remarks.--Records good except those for winter months, which are fair. Flow regulated at times by storage in off-channel Partridge Reservoir, formerly known as Whitewater lake. Reservoir formed from lake by levees around marsh areas and natural outlet. Available capacity 20,000 acre-ft between elevations 1,410 ft

(natural lake level) and $1,440 \mathrm{ft}$. Storage began Apr. 9, 1955. Storage in reservoir obtained from Colby

Lake during periods of high flow; release from storage returned to Colby Lake to maintain lake elevation

during diversion for iron-ore processing. Diversion began Feb. 7, 1956. Some seepage losses from reser-

voir enter above station.

DISCHARGE, IN CFS, WATER YEAR OCTOBER 1967 TO SEPTEMBER 1968

\begin{tabular}{|c|c|c|c|c|c|c|c|c|c|c|c|c|c|c|}
\hline DAY & OCT & NOV & $\mathrm{DEC}$ & & $\mathrm{JAN}$ & FEB & MAR & & APR & MAY & JUN & JUL & AUG & SEP \\
\hline $\begin{array}{l}1 \\
2 \\
3 \\
4 \\
5\end{array}$ & $\begin{array}{l}47 \\
49 \\
50 \\
49 \\
45\end{array}$ & $\begin{array}{l}58 \\
58 \\
54 \\
51 \\
48\end{array}$ & $\begin{array}{l}52 \\
53 \\
54 \\
55 \\
56\end{array}$ & & $\begin{array}{l}41 \\
40 \\
40 \\
39 \\
38\end{array}$ & $\begin{array}{l}31 \\
31 \\
30 \\
30 \\
30\end{array}$ & $\begin{array}{l}25 \\
26 \\
26 \\
27 \\
27\end{array}$ & & $\begin{array}{l}130 \\
132 \\
125 \\
118 \\
112\end{array}$ & $\begin{array}{l}1,340 \\
1,440 \\
1,420 \\
1,350 \\
1,250\end{array}$ & $\begin{array}{l}594 \\
594 \\
579 \\
542 \\
478\end{array}$ & $\begin{array}{l}523 \\
520 \\
480 \\
425 \\
370\end{array}$ & $\begin{array}{l}134 \\
130 \\
126 \\
122 \\
121\end{array}$ & $\begin{array}{l}714 \\
651 \\
594 \\
560 \\
509\end{array}$ \\
\hline $\begin{array}{r}6 \\
7 \\
8 \\
9 \\
10\end{array}$ & $\begin{array}{l}43 \\
46 \\
60 \\
58 \\
58\end{array}$ & $\begin{array}{l}47 \\
46 \\
47 \\
47 \\
48\end{array}$ & $\begin{array}{l}56 \\
55 \\
53 \\
51 \\
48\end{array}$ & & $\begin{array}{l}38 \\
38 \\
39 \\
39 \\
39\end{array}$ & $\begin{array}{l}29 \\
28 \\
27 \\
27 \\
26\end{array}$ & $\begin{array}{l}28 \\
29 \\
31 \\
37 \\
41\end{array}$ & & $\begin{array}{l}110 \\
115 \\
137 \\
174 \\
196\end{array}$ & $\begin{array}{r}1,120 \\
1,030 \\
956 \\
887 \\
800\end{array}$ & $\begin{array}{l}486 \\
496 \\
651 \\
838 \\
992\end{array}$ & $\begin{array}{l}325 \\
305 \\
290 \\
280 \\
270\end{array}$ & $\begin{array}{l}119 \\
126 \\
154 \\
139 \\
132\end{array}$ & $\begin{array}{l}509 \\
504 \\
532 \\
504 \\
482\end{array}$ \\
\hline $\begin{array}{l}11 \\
12 \\
13 \\
14 \\
15\end{array}$ & $\begin{array}{l}58 \\
57 \\
57 \\
58 \\
59\end{array}$ & $\begin{array}{l}50 \\
51 \\
51 \\
52 \\
50\end{array}$ & $\begin{array}{l}46 \\
45 \\
44 \\
43 \\
43\end{array}$ & & $\begin{array}{l}40 \\
40 \\
39 \\
38 \\
37\end{array}$ & $\begin{array}{l}26 \\
26 \\
25 \\
25 \\
25\end{array}$ & $\begin{array}{l}43 \\
41 \\
39 \\
37 \\
36\end{array}$ & & $\begin{array}{l}210 \\
222 \\
216 \\
213 \\
208\end{array}$ & $\begin{array}{l}748 \\
695 \\
632 \\
584 \\
551\end{array}$ & $\begin{array}{l}1,200 \\
1,320 \\
1,340 \\
1,500 \\
1,460\end{array}$ & $\begin{array}{l}255 \\
245 \\
248 \\
242 \\
240\end{array}$ & $\begin{array}{l}124 \\
122 \\
121 \\
114 \\
117\end{array}$ & $\begin{array}{l}444 \\
409 \\
378 \\
359 \\
344\end{array}$ \\
\hline $\begin{array}{l}16 \\
17 \\
18 \\
19 \\
20\end{array}$ & $\begin{array}{l}59 \\
58 \\
59 \\
60 \\
62\end{array}$ & $\begin{array}{l}52 \\
53 \\
54 \\
53 \\
51\end{array}$ & $\begin{array}{l}44 \\
45 \\
47 \\
48 \\
48\end{array}$ & & $\begin{array}{l}37 \\
37 \\
37 \\
37 \\
36\end{array}$ & $\begin{array}{l}25 \\
24 \\
24 \\
24 \\
23\end{array}$ & $\begin{array}{l}38 \\
43 \\
55 \\
75 \\
98\end{array}$ & & $\begin{array}{l}245 \\
318 \\
355 \\
351 \\
336\end{array}$ & $\begin{array}{l}527 \\
514 \\
514 \\
491 \\
486\end{array}$ & $\begin{array}{r}1,410 \\
1,330 \\
1,200 \\
1,060 \\
914\end{array}$ & $\begin{array}{l}242 \\
242 \\
255 \\
286 \\
296\end{array}$ & $\begin{array}{l}122 \\
110 \\
105 \\
109 \\
104\end{array}$ & $\begin{array}{l}322 \\
311 \\
333 \\
333 \\
340\end{array}$ \\
\hline $\begin{array}{l}21 \\
22 \\
23 \\
24 \\
25\end{array}$ & $\begin{array}{l}60 \\
60 \\
60 \\
60 \\
62\end{array}$ & $\begin{array}{l}52 \\
53 \\
53 \\
54 \\
54\end{array}$ & $\begin{array}{l}48 \\
48 \\
49 \\
49 \\
48\end{array}$ & & $\begin{array}{l}36 \\
36 \\
36 \\
35 \\
34\end{array}$ & $\begin{array}{l}23 \\
23 \\
23 \\
23 \\
23\end{array}$ & $\begin{array}{r}102 \\
105 \\
100 \\
94 \\
88\end{array}$ & & $\begin{array}{l}363 \\
424 \\
594 \\
656 \\
623\end{array}$ & $\begin{array}{l}469 \\
444 \\
424 \\
405 \\
389\end{array}$ & $\begin{array}{l}816 \\
753 \\
784 \\
784 \\
794\end{array}$ & $\begin{array}{l}296 \\
279 \\
255 \\
242 \\
222\end{array}$ & $\begin{array}{r}232 \\
374 \\
716 \\
1,130 \\
1,370\end{array}$ & $\begin{array}{l}344 \\
333 \\
336 \\
318 \\
300\end{array}$ \\
\hline $\begin{array}{l}26 \\
27 \\
28 \\
29 \\
30 \\
31 \\
\end{array}$ & $\begin{array}{l}63 \\
63 \\
63 \\
62 \\
60 \\
58 \\
\end{array}$ & $\begin{array}{r}53 \\
51 \\
50 \\
50 \\
51 \\
\end{array}$ & $\begin{array}{l}47 \\
46 \\
45 \\
44 \\
43 \\
42 \\
\end{array}$ & & $\begin{array}{l}33 \\
32 \\
32 \\
32 \\
31 \\
31 \\
\end{array}$ & \begin{tabular}{r}
23 \\
24 \\
24 \\
25 \\
\\
\hdashline \\
\end{tabular} & $\begin{array}{r}86 \\
86 \\
92 \\
105 \\
118 \\
123 \\
\end{array}$ & & $\begin{array}{r}671 \\
800 \\
903 \\
986 \\
1,140 \\
\end{array}$ & $\begin{array}{l}382 \\
420 \\
469 \\
500 \\
514 \\
542 \\
\end{array}$ & $\begin{array}{r}748 \\
709 \\
661 \\
589 \\
556 \\
--- \\
\end{array}$ & $\begin{array}{l}205 \\
188 \\
169 \\
158 \\
149 \\
141 \\
\end{array}$ & $\begin{array}{r}1,540 \\
1,480 \\
1,300 \\
1,130 \\
920 \\
794 \\
\end{array}$ & $\begin{array}{r}286 \\
265 \\
251 \\
238 \\
220 \\
\end{array}$ \\
\hline $\begin{array}{l}\text { Total } \\
\text { Mean } \\
(\not) \\
\text { Mean } \neq \\
\text { Max } \\
\text { Min } \\
\text { Cfsm } \neq \\
\text { In. } \neq\end{array}$ & $\begin{array}{r}1,763 \\
56.9 \\
+2.23 \\
59.1 \\
63 \\
43 \\
0.189 \\
0.22\end{array}$ & $\begin{array}{r}1.542 \\
51.4 \\
+0.39 \\
51.8 \\
58 \\
46 \\
0.166 \\
0.19\end{array}$ & $\begin{array}{r}1,495 \\
48.2 \\
+0.31 \\
48.5 \\
56 \\
42 \\
0.155 \\
0.18\end{array}$ & & $\begin{array}{r}1.137 \\
36.7 \\
+1.86 \\
38.6 \\
41 \\
31 \\
0.124 \\
0.14\end{array}$ & $\begin{array}{r}747 \\
25.8 \\
-0.05 \\
25.8 \\
31 \\
23 \\
0.083 \\
0.09\end{array}$ & $\begin{array}{r}1,901 \\
61.3 \\
+8.83 \\
70.1 \\
123 \\
25 \\
0.225 \\
0.26\end{array}$ & & $\begin{array}{r}1,183 \\
373 \\
+155 \\
528 \\
1,140 \\
110 \\
1.69 \\
1.89\end{array}$ & $\begin{array}{r}22,293 \\
719 \\
+23.3 \\
742 \\
1,440 \\
382 \\
2.38 \\
2.74\end{array}$ & $\begin{array}{r}26,178 \\
873 \\
+21.2 \\
894 \\
1,500 \\
478 \\
2.87 \\
3.20\end{array}$ & $\begin{array}{r}8,643 \\
27.9 \\
+20.3 \\
299 \\
523 \\
141 \\
0.958 \\
1.11\end{array}$ & $\begin{array}{r}13,437 \\
433 \\
+42.9 \\
476 \\
1,540 \\
104 \\
1.53 \\
1.76\end{array}$ & $\begin{array}{r}12,023 \\
401 \\
+21.3 \\
422 \\
714 \\
220 \\
1.35 \\
1.51\end{array}$ \\
\hline $\begin{array}{l}\text { Cal yr } \\
\text { Wtr yr }\end{array}$ & $\begin{array}{l}1967: \\
1968:\end{array}$ & $\begin{array}{lr}\operatorname{Max} & 898 \\
\operatorname{Max} & 1,540\end{array}$ & $\begin{array}{l}\text { Min } \\
\text { Min }\end{array}$ & $\begin{array}{l}17 \\
23\end{array}$ & $\begin{array}{l}\text { Mean } \\
\text { Mean }\end{array}$ & $\begin{array}{l}156 \\
280\end{array}$ & $\begin{array}{l}\text { Mean } \neq \\
\text { Mean } \neq\end{array}$ & $\begin{array}{l}178 \\
305\end{array}$ & $\begin{array}{l}\text { Cfsm } \\
\text { Cfsm }\end{array}$ & $\begin{array}{l}0.571 \\
0.978\end{array}$ & $\begin{array}{l}\text { In. } \neq \\
\text { In. } \neq\end{array}$ & $\begin{array}{r}7.78 \\
13.31\end{array}$ & & \\
\hline
\end{tabular}

t Change in contents in Partridge Reservoir and diversion to iron-ore processing plant, equivalent in cubic feet per second, furnished by Erie Mining Company.

$\neq$ Adjusted for change in contents and diversion. 
4-0187.5 St. Louis River at Forbes, Minn.

Location.--Lat $47^{\circ} 21^{\prime} 48^{\prime \prime}$, long $92^{\circ} 35^{\prime} 56^{\prime \prime}$, in NE $\frac{1}{4} \mathrm{SE}_{4}^{\frac{l}{4}}$ sec.3, T.56 N., R.18 W., on right bank at downstream side of highway bridge, $0.5 \mathrm{mile}$ downstream from Eveleth Taconite Company dam, $0.6 \mathrm{mile}$ south of Forbes, 1.8 miles upstream from Elbow Creek.

Records available.--August 1964 to September 1968.

Gage.--Digital water-stage recorder. Datum of gage is 1,293.11 ft above mean sea level, datum of 1929. Prior to Oct. 28, 1964, wire-weight gage Oct. 28, 1964, to sept. 1, 1967, graphic water-stage recorder at same site and datum.

Extremes.--Maximum discharge during year, 2,770 cfs June 18 (gage height, $12.27 \mathrm{ft}$ ) ; minimum daily, $34 \mathrm{cfs}$ Feb. 18-25.

1964-68: Maximum discharge, 4,140 cfs Apr. 23, 1966 (gage height, 14.60 ft); minimum, 24 cfs Nov. 26, 1966 (gage height, $5.23 \mathrm{ft}$ ).

Flood of June 1964 reached a stage of about $12.3 \mathrm{ft}$, from information furnished by Eveleth Taconite Company (discharge, 2,780 cfs).

Remarks.--Records good except those for winter months, which are fair. There is some regulation at medium and low flows and diversion for iron-ore processing at Eveleth Taconite Company dam $\frac{1}{2}$ mile upstream. Diversion began Dec. 5, 1965. Records of suspended sediment loads for the water year 1968 are published in Part 2 of this report.

DISCHARGE, IN CUBIC FEET PER SECOND, WATER YEAR OCTOBER 1967 TO SEPTEMBER 1968

\begin{tabular}{|c|c|c|c|c|c|c|c|c|c|c|c|c|}
\hline DAY & OCT & NUV & DEC & JAN & FEB & MAP & $A P R$ & MAY & JUN & JUL & AUG & SEP \\
\hline $\begin{array}{l}1 \\
2 \\
3 \\
4 \\
5\end{array}$ & $\begin{array}{l}89 \\
89 \\
91 \\
91 \\
89\end{array}$ & $\begin{array}{r}98 \\
108 \\
112 \\
100 \\
98\end{array}$ & $\begin{array}{l}67 \\
68 \\
70 \\
72 \\
74\end{array}$ & $\begin{array}{l}50 \\
48 \\
47 \\
45 \\
44\end{array}$ & $\begin{array}{l}38 \\
39 \\
39 \\
37 \\
37\end{array}$ & $\begin{array}{l}37 \\
38 \\
40 \\
43 \\
47\end{array}$ & $\begin{array}{l}490 \\
465 \\
430 \\
400 \\
390\end{array}$ & $\begin{array}{l}2.220 \\
2.310 \\
2.340 \\
2.340 \\
2.290\end{array}$ & $\begin{array}{r}1.030 \\
1.090 \\
1.070 \\
1.040 \\
934\end{array}$ & $\begin{array}{r}1.200 \\
1.130 \\
1.060 \\
1.010 \\
957\end{array}$ & $\begin{array}{l}225 \\
19 B \\
250 \\
237 \\
228\end{array}$ & $\begin{array}{r}1.220 \\
1,090 \\
968 \\
888 \\
802\end{array}$ \\
\hline $\begin{array}{r}6 \\
7 \\
8 \\
9 \\
10\end{array}$ & $\begin{array}{r}87 \\
91 \\
115 \\
135 \\
129\end{array}$ & $\begin{array}{l}36 \\
84 \\
92 \\
79 \\
74\end{array}$ & $\begin{array}{l}74 \\
68 \\
42 \\
66 \\
42\end{array}$ & $\begin{array}{l}44 \\
43 \\
43 \\
44 \\
45\end{array}$ & $\begin{array}{l}37 \\
37 \\
36 \\
36 \\
36\end{array}$ & $\begin{array}{l}53 \\
60 \\
64 \\
66 \\
63\end{array}$ & $\begin{array}{l}360 \\
350 \\
385 \\
460 \\
515\end{array}$ & $\begin{array}{l}2.170 \\
2.030 \\
1.920 \\
1.820 \\
1.700\end{array}$ & $\begin{array}{l}1.020 \\
1.060 \\
1.200 \\
1.470 \\
1.620\end{array}$ & $\begin{array}{l}881 \\
809 \\
614 \\
702 \\
636\end{array}$ & $\begin{array}{l}219 \\
213 \\
222 \\
250 \\
231\end{array}$ & $\begin{array}{l}794 \\
813 \\
820 \\
816 \\
791\end{array}$ \\
\hline $\begin{array}{l}11 \\
12 \\
13 \\
14 \\
15\end{array}$ & $\begin{array}{r}120 \\
117 \\
115 \\
87 \\
80\end{array}$ & $\begin{array}{l}63 \\
76 \\
84 \\
86 \\
76\end{array}$ & $\begin{array}{l}55 \\
65 \\
47 \\
58 \\
66\end{array}$ & $\begin{array}{l}45 \\
44 \\
44 \\
43 \\
42\end{array}$ & $\begin{array}{l}36 \\
36 \\
35 \\
35 \\
35\end{array}$ & $\begin{array}{l}58 \\
54 \\
52 \\
51 \\
52\end{array}$ & $\begin{array}{l}550 \\
543 \\
515 \\
504 \\
498\end{array}$ & $\begin{array}{l}1.560 \\
1.450 \\
1.280 \\
1.240 \\
1.070\end{array}$ & $\begin{array}{l}1,900 \\
2.070 \\
2,180 \\
2,480 \\
2,630\end{array}$ & $\begin{array}{l}585 \\
512 \\
540 \\
529 \\
508\end{array}$ & $\begin{array}{l}213 \\
204 \\
204 \\
198 \\
187\end{array}$ & $\begin{array}{l}754 \\
713 \\
568 \\
605 \\
573\end{array}$ \\
\hline $\begin{array}{l}16 \\
17 \\
18 \\
19 \\
20\end{array}$ & $\begin{array}{l}98 \\
117 \\
117 \\
120 \\
103\end{array}$ & $\begin{array}{l}72 \\
93 \\
92 \\
90 \\
83\end{array}$ & $\begin{array}{l}50 \\
47 \\
63 \\
72 \\
55\end{array}$ & $\begin{array}{l}41 \\
41 \\
41 \\
41 \\
41\end{array}$ & $\begin{array}{l}35 \\
35 \\
34 \\
34 \\
34\end{array}$ & $\begin{array}{r}56 \\
85 \\
150 \\
250 \\
290\end{array}$ & $\begin{array}{l}592 \\
750 \\
794 \\
776 \\
765\end{array}$ & $\begin{array}{r}1.030 \\
1.010 \\
976 \\
953 \\
907\end{array}$ & $\begin{array}{l}2.700 \\
2.710 \\
2.650 \\
2.470 \\
2.240\end{array}$ & $\begin{array}{l}508 \\
501 \\
442 \\
377 \\
508\end{array}$ & $\begin{array}{l}143 \\
17 B \\
181 \\
146 \\
172\end{array}$ & $\begin{array}{l}530 \\
513 \\
581 \\
672 \\
658\end{array}$ \\
\hline $\begin{array}{l}21 \\
2 ? \\
23 \\
24 \\
25\end{array}$ & $\begin{array}{l}123 \\
123 \\
123 \\
123 \\
120\end{array}$ & $\begin{array}{l}79 \\
82 \\
134 \\
86 \\
86\end{array}$ & $\begin{array}{l}62 \\
68 \\
53 \\
57 \\
63\end{array}$ & $\begin{array}{l}41 \\
40 \\
40 \\
40 \\
40\end{array}$ & $\begin{array}{l}34 \\
34 \\
34 \\
34 \\
34\end{array}$ & $\begin{array}{l}30 C \\
285 \\
260 \\
250 \\
240\end{array}$ & $\begin{array}{r}816 \\
889 \\
1.120 \\
1.360 \\
1.440\end{array}$ & $\begin{array}{l}794 \\
B 54 \\
813 \\
783 \\
742\end{array}$ & $\begin{array}{l}2.000 \\
1.820 \\
1,770 \\
1.750 \\
1.680\end{array}$ & $\begin{array}{l}543 \\
518 \\
512 \\
487 \\
456\end{array}$ & $\begin{array}{r}201 \\
384 \\
709 \\
1.350 \\
1.650\end{array}$ & $\begin{array}{l}634 \\
620 \\
648 \\
651 \\
611\end{array}$ \\
\hline $\begin{array}{l}26 \\
27 \\
28 \\
29 \\
30 \\
31\end{array}$ & $\begin{array}{l}115 \\
112 \\
112 \\
112 \\
112 \\
109\end{array}$ & $\begin{array}{r}83 \\
90 \\
75 \\
72 \\
69 \\
\end{array}$ & $\begin{array}{l}57 \\
48 \\
46 \\
52 \\
57 \\
52\end{array}$ & $\begin{array}{l}40 \\
39 \\
39 \\
39 \\
38 \\
38\end{array}$ & $\begin{array}{r}35 \\
35 \\
36 \\
37 \\
\\
\end{array}$ & $\begin{array}{l}260 \\
290 \\
340 \\
390 \\
460 \\
480\end{array}$ & $\begin{array}{r}1.570 \\
1.760 \\
1.930 \\
2.110 \\
2.140 \\
\end{array}$ & $\begin{array}{l}706 \\
761 \\
846 \\
892 \\
907 \\
877\end{array}$ & $\begin{array}{r}1.580 \\
1.490 \\
1.340 \\
1.260 \\
1.200 \\
-\end{array}$ & $\begin{array}{l}394 \\
290 \\
367 \\
240 \\
344 \\
340\end{array}$ & $\begin{array}{l}1.720 \\
1.800 \\
1.820 \\
1.750 \\
1.580 \\
1.360\end{array}$ & $\begin{array}{r}573 \\
458 \\
519 \\
606 \\
313 \\
\end{array}$ \\
\hline $\begin{array}{l}\text { TOTAL } \\
\text { MEAN } \\
\text { MAX } \\
\text { MIN }\end{array}$ & $\begin{array}{r}3.364 \\
109 \\
135 \\
80\end{array}$ & $\begin{array}{r}2.517 \\
83.9 \\
112 \\
68\end{array}$ & $\begin{array}{r}1+836 \\
59.2 \\
74 \\
42\end{array}$ & $\begin{array}{r}1.310 \\
42.3 \\
50 \\
38\end{array}$ & $\begin{array}{r}1.032 \\
35.6 \\
38 \\
34\end{array}$ & $\begin{array}{r}5,164 \\
167 \\
480 \\
37\end{array}$ & $\begin{array}{r}25,716 \\
857 \\
2,140 \\
350\end{array}$ & $\begin{array}{r}41,591 \\
1,342 \\
2,340 \\
706\end{array}$ & $\begin{array}{r}51.454 \\
1.715 \\
2.710 \\
934\end{array}$ & $\begin{array}{r}1 B .600 \\
600 \\
1.200 \\
240\end{array}$ & $\begin{array}{r}18.423 \\
594 \\
1.820 \\
143\end{array}$ & $\begin{array}{r}20.802 \\
693 \\
1.220 \\
313\end{array}$ \\
\hline
\end{tabular}

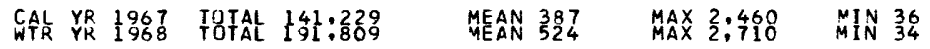


4-0189. East Two River near Iron Junction, Minn.

Location.--Lat $47^{\circ} 24^{\prime} 04^{\prime \prime}$, long $92^{\circ} 39^{\prime} 52^{\prime \prime}$, in NW/1 NW' sec.29, T.57 N., R.18 W., on right bank downstream from bridge on State Highway 37 and 2.2 miles southwest of Iron Junction.

Drainage area. $-40.0 \mathrm{sq} \mathrm{mi}$.

Records available.--June 1966 to September 1968. Occasional low-flow measurements, water years 1957-62.

Gage.--Water-stage recorder. Altitude of gage is 1,335 ft (from topographic map).

Extremes.--Maximum discharge during year, 395 cfs Aug. 25 (gage height, 9.43 ft); minimum daily, 5.5 cfs Dec. $27,28,29$; minimum gage height, $2.93 \mathrm{ft}$ oct. 1

1966-68: Maximum discharge, that of Aug. 25, 1968; maximum gage height, $9.48 \mathrm{ft}$ Apr. 1, 1967 (backwater

from ice); minimum daily discharge, that of Dec. $27,28,29,1967$.

Remarks.--Records good except those for winter months, which are fair.

Cooperation.--Recorás computed by U. S. Steel Corporation and reviewed by Geological Survey.

DISCHARGE, IN CFS, WATER YEAR OCTOBER 1967 TO SEPTEMBER 1968

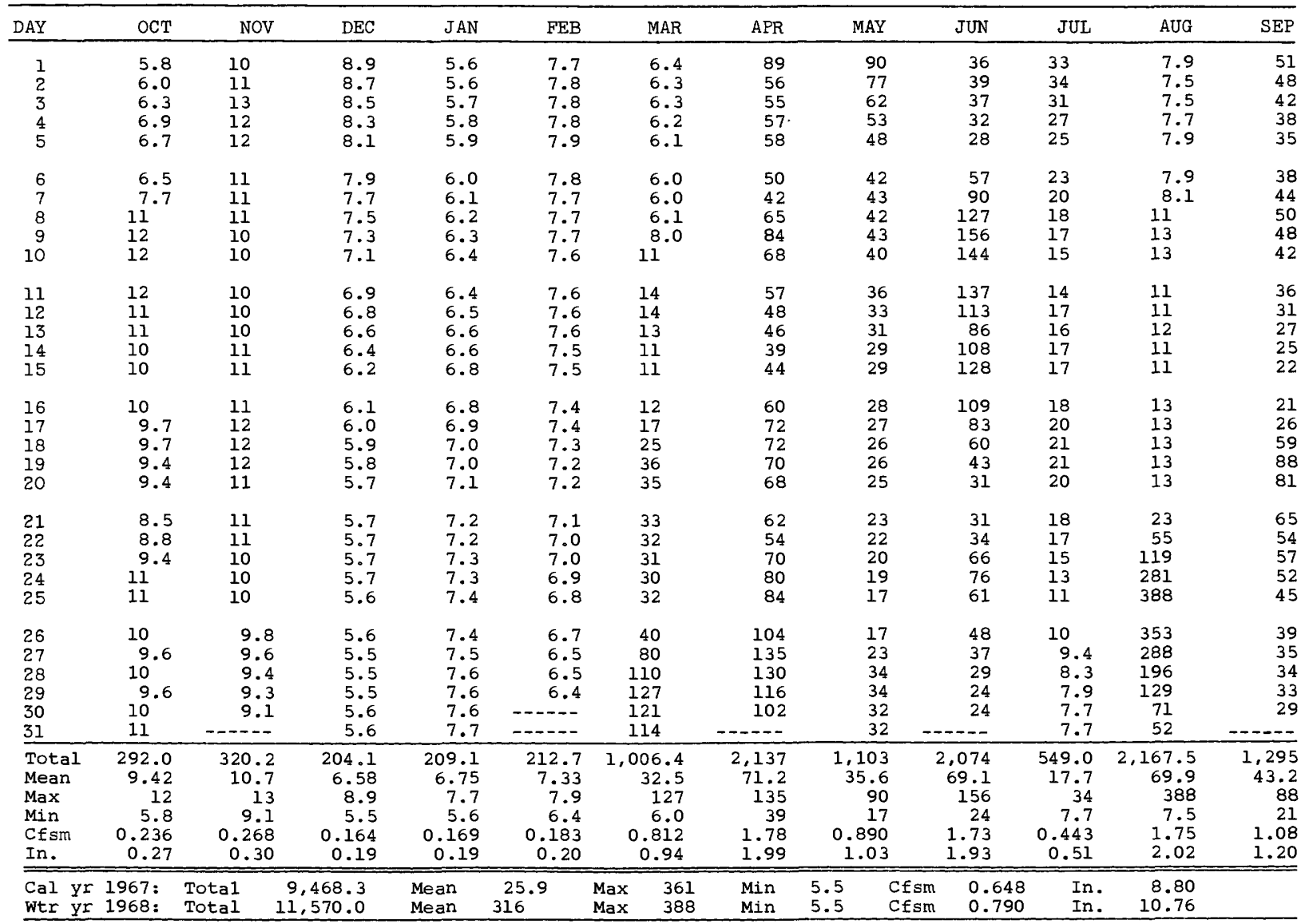


4-0190. West Two River near Iron Junction, Minn.

Location.--Lat $47^{\circ} 24^{\prime} 05^{\prime \prime}$, long $92^{\circ} 42^{\prime} 10^{\prime \prime}$, in SWlsw sec. 24, T.57 N., R.19 W. on right bank 40 ft upstream from bridge on State Highway 37, 5 miles southwest of Iron Junction, and 91/4 miles upstream from St. Louis River.

Drainage area. $--68.4 \mathrm{sq} \mathrm{mi}$.

Records available.--October 1953 to September 1962, October 1965 to September 1968 .

Gage.--Water-stage recorder. Datum of gage is 1,322.05 ft above mean sea level, datum of 1929 (Minnegota Highway Department bench mark).

Average discharge.--12 years, $44.4 \mathrm{cfs}$.

Extremes.--Maximum discharge during year, 345 cfs Aug. 26 (gage height, 7.72 ft); minimum daily, 5.2 cfs Oct. 31. Nov. 1 (gage height, $2.34 \mathrm{ft}$ ).

1953-62, 1966-68: Maximum discharge, $916 \mathrm{cfs}$ Apr. 17, 1954 (gage height, 9.85 ft); minimum daily, 3.0 cfs Jan. 22 to Feb. 6, 1957; minimum gage height, 2.34 ft Aug. 15-17, 24-28, 1961, 0ct. 31, Nov. 1, 1967.

Remarks.--Records good except those for winter months, which are fair. Flow regulated at times by storage in West Two Rivers Reservoir for taconite processing at U. S. Steel Mountain Iron Taconite Plant. The reservoir impounds water from the upper $27.9 \mathrm{sq}$ miles of the drainage area and has an available capacity of

8,500 acre-ft between elevations $1370 \mathrm{ft}$ (natural inlet) and 1395 ft (crest of spillway at outlet dam). Storage began July 28, 1966. Some seepage losses from reservoir enter above station.

Cooperation.--Records for 1968 water year computed by U. S. Steel Corporation and reviewed by Geological Survey.

DISCHARGE, IN CFS, WATER YEAR OCTOBER 1967 TO SEPTEMBER 1968

\begin{tabular}{|c|c|c|c|c|c|c|c|c|c|c|c|c|}
\hline$\overline{\mathrm{DAY}}$ & OCT & NOV & $\mathrm{DEC}$ & JAN & FEB & MAR & APR & MAY & JUN & JUL & AUG & SEP \\
\hline $\begin{array}{l}1 \\
2 \\
3 \\
4 \\
5\end{array}$ & $\begin{array}{l}6.3 \\
5.7 \\
5.5 \\
5.5 \\
5.5\end{array}$ & $\begin{array}{l}5.2 \\
5.5 \\
5.7 \\
7.1 \\
6.7\end{array}$ & $\begin{array}{l}5.8 \\
5.8 \\
5.8 \\
5.8 \\
5.8\end{array}$ & $\begin{array}{l}5.6 \\
5.6 \\
5.7 \\
5.8 \\
5.9\end{array}$ & $\begin{array}{l}6.1 \\
6.1 \\
6.2 \\
6.2 \\
6.2\end{array}$ & $\begin{array}{l}6.0 \\
6.1 \\
6.1 \\
6.2 \\
6.5\end{array}$ & $\begin{array}{l}79 \\
72 \\
67 \\
63 \\
60\end{array}$ & $\begin{array}{r}111 \\
84 \\
70 \\
59 \\
54\end{array}$ & $\begin{array}{l}42 \\
45 \\
45 \\
39 \\
29\end{array}$ & $\begin{array}{r}79 \\
138 \\
188 \\
187 \\
152\end{array}$ & $\begin{array}{l}16 \\
16 \\
15 \\
14 \\
14\end{array}$ & $\begin{array}{l}70 \\
69 \\
68 \\
64 \\
59\end{array}$ \\
\hline $\begin{array}{r}6 \\
7 \\
8 \\
9 \\
10\end{array}$ & $\begin{array}{r}6.7 \\
5.9 \\
9.2 \\
11 \\
10\end{array}$ & $\begin{array}{l}5.9 \\
6.3 \\
6.3 \\
6.7 \\
6.3\end{array}$ & $\begin{array}{l}5.8 \\
5.7 \\
5.7 \\
5.7 \\
5.7\end{array}$ & $\begin{array}{l}6.0 \\
6.0 \\
6.0 \\
6.0 \\
6.0\end{array}$ & $\begin{array}{l}6.2 \\
6.2 \\
6.2 \\
6.2 \\
6.2\end{array}$ & $\begin{array}{r}6.8 \\
7.0 \\
8.5 \\
9.5 \\
11\end{array}$ & $\begin{array}{l}60 \\
63 \\
69 \\
86 \\
87\end{array}$ & $\begin{array}{l}48 \\
40 \\
44 \\
49 \\
48\end{array}$ & $\begin{array}{r}82 \\
169 \\
245 \\
299 \\
307\end{array}$ & $\begin{array}{r}110 \\
75 \\
58 \\
42 \\
33\end{array}$ & $\begin{array}{l}14 \\
13 \\
14 \\
16 \\
17\end{array}$ & $\begin{array}{l}65 \\
71 \\
79 \\
76 \\
67\end{array}$ \\
\hline $\begin{array}{l}11 \\
12 \\
13 \\
14 \\
15\end{array}$ & $\begin{array}{l}9.6 \\
8.4 \\
8.0 \\
18 \\
10\end{array}$ & $\begin{array}{l}8.0 \\
7.5 \\
7.1 \\
6.8 \\
6.6\end{array}$ & $\begin{array}{l}5.7 \\
5.7 \\
5.7 \\
5.7 \\
5.7\end{array}$ & $\begin{array}{l}6.0 \\
6.0 \\
6.0 \\
6.0 \\
6.0\end{array}$ & $\begin{array}{l}6.2 \\
6.2 \\
6.3 \\
6.3 \\
6.3\end{array}$ & $\begin{array}{l}11 \\
11 \\
10 \\
10 \\
11\end{array}$ & $\begin{array}{l}79 \\
68 \\
54 \\
53 \\
52\end{array}$ & $\begin{array}{l}43 \\
40 \\
36 \\
33 \\
34\end{array}$ & $\begin{array}{l}278 \\
229 \\
185 \\
201 \\
222\end{array}$ & $\begin{array}{l}31 \\
43 \\
36 \\
38 \\
37\end{array}$ & $\begin{array}{l}16 \\
17 \\
18 \\
18 \\
18\end{array}$ & $\begin{array}{l}57 \\
48 \\
40 \\
35 \\
29\end{array}$ \\
\hline $\begin{array}{l}16 \\
17 \\
18 \\
19 \\
20\end{array}$ & $\begin{array}{l}7.5 \\
6.7 \\
6.7 \\
6.3 \\
5.9\end{array}$ & $\begin{array}{l}6.4 \\
6.1 \\
5.9 \\
5.9 \\
5.9\end{array}$ & $\begin{array}{l}5.6 \\
5.6 \\
5.6 \\
5.6 \\
5.6\end{array}$ & $\begin{array}{l}6.0 \\
6.0 \\
6.0 \\
6.0 \\
6.0\end{array}$ & $\begin{array}{l}6.3 \\
6.3 \\
6.2 \\
6.2 \\
6.2\end{array}$ & $\begin{array}{l}13 \\
14 \\
16 \\
23 \\
32\end{array}$ & $\begin{array}{l}67 \\
80 \\
82 \\
76 \\
70\end{array}$ & $\begin{array}{l}34 \\
34 \\
32 \\
31 \\
30\end{array}$ & $\begin{array}{r}221 \\
195 \\
155 \\
118 \\
85\end{array}$ & $\begin{array}{l}42 \\
50 \\
54 \\
56 \\
49\end{array}$ & $\begin{array}{l}24 \\
22 \\
20 \\
20 \\
20\end{array}$ & $\begin{array}{r}25 \\
45 \\
121 \\
161 \\
181\end{array}$ \\
\hline $\begin{array}{l}21 \\
22 \\
23 \\
24 \\
25\end{array}$ & $\begin{array}{l}6.7 \\
6.3 \\
6.3 \\
5.9 \\
5.7\end{array}$ & $\begin{array}{l}5.9 \\
5.8 \\
5.8 \\
5.8 \\
5.8\end{array}$ & $\begin{array}{l}5.6 \\
5.6 \\
5.6 \\
5.6 \\
5.6\end{array}$ & $\begin{array}{l}6.0 \\
6.0 \\
6.0 \\
6.0 \\
6.0\end{array}$ & $\begin{array}{l}6.1 \\
6.1 \\
0.0 \\
6.0 \\
6.0\end{array}$ & $\begin{array}{l}37 \\
38 \\
38 \\
35 \\
32\end{array}$ & $\begin{array}{r}68 \\
66 \\
81 \\
100 \\
109\end{array}$ & $\begin{array}{l}27 \\
27 \\
26 \\
25 \\
26\end{array}$ & $\begin{array}{l}70 \\
62 \\
91 \\
88 \\
82\end{array}$ & $\begin{array}{l}43 \\
36 \\
32 \\
28 \\
25\end{array}$ & $\begin{array}{r}25 \\
53 \\
112 \\
236 \\
317\end{array}$ & $\begin{array}{l}185 \\
169 \\
170 \\
156 \\
142\end{array}$ \\
\hline $\begin{array}{l}26 \\
27 \\
28 \\
29 \\
30 \\
31\end{array}$ & $\begin{array}{l}5.7 \\
5.5 \\
5.7 \\
5.7 \\
5.7 \\
5.2\end{array}$ & $\begin{array}{r}5.8 \\
5.8 \\
5.8 \\
5.8 \\
5.8 \\
- \\
\end{array}$ & $\begin{array}{l}5.6 \\
5.6 \\
5.6 \\
5.6 \\
5.6 \\
5.6\end{array}$ & $\begin{array}{l}6.0 \\
6.0 \\
6.1 \\
6.1 \\
6.1 \\
6.1\end{array}$ & $\begin{array}{r}6.0 \\
6.0 \\
6.0 \\
6.0 \\
\\
\end{array}$ & $\begin{array}{l}30 \\
35 \\
60 \\
85 \\
83 \\
80\end{array}$ & $\begin{array}{r}128 \\
149 \\
148 \\
142 \\
138 \\
- \\
\end{array}$ & $\begin{array}{l}29 \\
30 \\
38 \\
45 \\
50 \\
39\end{array}$ & $\begin{array}{r}68 \\
56 \\
42 \\
30 \\
34 \\
- \\
\end{array}$ & $\begin{array}{l}24 \\
22 \\
20 \\
18 \\
18 \\
18\end{array}$ & $\begin{array}{r}341 \\
310 \\
174 \\
123 \\
100 \\
72\end{array}$ & $\begin{array}{r}114 \\
89 \\
67 \\
52 \\
40 \\
-\end{array}$ \\
\hline $\begin{array}{l}\text { Total } \\
\text { Mean } \\
\text { Max } \\
\text { Min } \\
\text { Cfsm } \\
\text { In. }\end{array}$ & $\begin{array}{r}222.8 \\
7.19 \\
18 \\
5.2 \\
0.105 \\
0.12\end{array}$ & $\begin{array}{r}186.0 \\
6.20 \\
8.0 \\
5.2 \\
0.091 \\
0.10\end{array}$ & $\begin{array}{r}175.7 \\
5.67 \\
5.8 \\
5.6 \\
0.083 \\
0.10\end{array}$ & $\begin{array}{r}185.0 \\
5.97 \\
6.1 \\
5.6 \\
0.087 \\
0.10\end{array}$ & $\begin{array}{r}178.5 \\
6.16 \\
6.3 \\
6.0 \\
0.091 \\
0.10\end{array}$ & $\begin{array}{r}777.7 \\
25.1 \\
85 \\
6.0 \\
0.367 \\
0.42\end{array}$ & $\begin{array}{r}2,516 \\
83.9 \\
149 \\
52 \\
1.23 \\
1.37\end{array}$ & $\begin{array}{r}1,316 \\
42.5 \\
111 \\
25 \\
0.621 \\
0.72\end{array}$ & $\begin{array}{r}3.814 \\
127 \\
307 \\
29 \\
1.86 \\
2.07\end{array}$ & $\begin{array}{r}1,782 \\
57.5 \\
188 \\
18 \\
0.841 \\
0.97\end{array}$ & $\begin{array}{r}2,205 \\
71.1 \\
341 \\
13 \\
1.04 \\
1.20\end{array}$ & $\begin{array}{r}2,614 \\
87.1 \\
185 \\
25 \\
1.27 \\
1.42\end{array}$ \\
\hline $\begin{array}{l}\text { Cal yr } \\
\text { Wtr yr }\end{array}$ & $\begin{array}{l}1967: \\
1968:\end{array}$ & $\begin{array}{l}\text { tal } \\
\text { tal }\end{array}$ & $\begin{array}{l}19.7 \\
72.7\end{array}$ & $\begin{array}{l}\text { Mean } \\
\text { Mean }\end{array}$ & $\begin{array}{l}26.1 \\
43.6\end{array}$ & $\begin{array}{l}500 \\
341\end{array}$ & $\begin{array}{l}\text { Min } \\
\text { Min }\end{array}$ & $\begin{array}{l}5.2 \\
5.2\end{array}$ & $\begin{array}{l}0.382 \\
0.637\end{array}$ & $\begin{array}{l}\text { In. } \\
\text { In. }\end{array}$ & $\begin{array}{l}5.18 \\
8.68\end{array}$ & \\
\hline
\end{tabular}


4-0193. West Swan River near Silica, Minn.

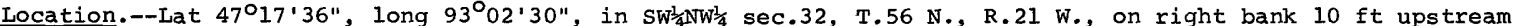
from pilings of dismantled bridge and railroad bed of Great Northern Railroad, 2 miles northwest of silica, 9 miles southwest of Hibbing and 20 miles upstream from confluence of East Swan and West Swan.

Records available.--April 1963 to September 1968

Gage.-Water-stage recorder. Altitude of gage is 1, $360 \mathrm{ft}$ (from topographic map). Prior to Aug. 2, 1963, reference point at same site and datum.

Average discharge. --5 years, $12.0 \mathrm{cfs}$.

Extremes.--Maximum discharge during year, 97 cfs July 1 (gage height, 3.12 ft); maximum gage height recorded, 5.17 ft Mar. 28 (backwater from ice); minimum daily discharge, 0.5 cfs Nov. 29, 30, Dec. 1-4; minimum gage height, $0.89 \mathrm{ft} \mathrm{Aug.} 14$ 1963.

Remarks:--Records good except those for period of no gage-height record and those for winter months, which are fair.

Cooperation.--Additional discharge measurements furnished by M. A. Hanna Mining Co.

DISCHARGE, IN CFS, WATER YEAR OCTOBER 1967 TO SEPTEMBER 1968

\begin{tabular}{|c|c|c|c|c|c|c|c|c|c|c|c|c|}
\hline DAY & OCT & NOV & DEC & JAN & FEB & MAR & APR & MAY & JUN & JUL & AUG & SEP \\
\hline $\begin{array}{l}1 \\
2 \\
3 \\
4 \\
5\end{array}$ & $\begin{array}{l}1.9 \\
2.0 \\
2.1 \\
2.2 \\
2.0\end{array}$ & $\begin{array}{l}3.6 \\
3.7 \\
3.5 \\
3.1 \\
2.6\end{array}$ & $\begin{array}{r}0.5 \\
.5 \\
.5 \\
.5 \\
.6\end{array}$ & $\begin{array}{l}2.6 \\
2.5 \\
2.5 \\
2.5 \\
2.5\end{array}$ & $\begin{array}{l}3.1 \\
3.3 \\
3.4 \\
3.5 \\
3.6\end{array}$ & $\begin{array}{l}4.6 \\
4.9 \\
5.5 \\
6.2 \\
7.2\end{array}$ & $\begin{array}{l}28 \\
23 \\
20 \\
18 \\
16\end{array}$ & $\begin{array}{r}14 \\
9.9 \\
6.8 \\
5.3 \\
4.6\end{array}$ & $\begin{array}{r}22 \\
19 \\
15 \\
9.9 \\
8.6\end{array}$ & $\begin{array}{l}87 \\
73 \\
46 \\
16 \\
5.4\end{array}$ & $\begin{array}{l}1.4 \\
1.3 \\
1.4 \\
1.4 \\
1.4\end{array}$ & $\begin{array}{l}2.5 \\
2.6 \\
2.3 \\
2.3 \\
2.2\end{array}$ \\
\hline $\begin{array}{r}6 \\
7 \\
8 \\
9 \\
10\end{array}$ & $\begin{array}{l}1.9 \\
2.4 \\
3.3 \\
3.3 \\
3.1\end{array}$ & $\begin{array}{l}2.2 \\
2.1 \\
2.0 \\
1.9 \\
1.9\end{array}$ & $\begin{array}{r}.7 \\
.8 \\
.9 \\
2.0 \\
6.0\end{array}$ & $\begin{array}{l}2.5 \\
2.4 \\
2.4 \\
2.4 \\
2.4\end{array}$ & $\begin{array}{l}3.6 \\
3.6 \\
3.6 \\
3.5 \\
3.5\end{array}$ & $\begin{array}{l}8.5 \\
10 \\
11 \\
11 \\
10\end{array}$ & $\begin{array}{l}22 \\
40 \\
58 \\
65 \\
60\end{array}$ & $\begin{array}{l}3.9 \\
4.1 \\
5.3 \\
7.1 \\
5.7\end{array}$ & $\begin{array}{l}34 \\
44 \\
41 \\
38 \\
30\end{array}$ & $\begin{array}{l}3.6 \\
2.8 \\
2.4 \\
2.0 \\
1.6\end{array}$ & $\begin{array}{l}1.4 \\
1.5 \\
1.4 \\
1.5 \\
1.4\end{array}$ & $\begin{array}{l}2.7 \\
2.9 \\
2.9 \\
2.9 \\
2.7\end{array}$ \\
\hline $\begin{array}{l}11 \\
12 \\
13 \\
14 \\
15\end{array}$ & $\begin{array}{l}3.4 \\
3.3 \\
3.4 \\
3.4 \\
3.5\end{array}$ & $\begin{array}{l}1.9 \\
1.9 \\
1.8 \\
1.7 \\
1.6\end{array}$ & $\begin{array}{l}6.5 \\
7.0 \\
6.5 \\
5.0 \\
4.2\end{array}$ & $\begin{array}{l}2.4 \\
2.3 \\
2.3 \\
2.3 \\
2.2\end{array}$ & $\begin{array}{l}3.4 \\
3.3 \\
3.2 \\
3.2 \\
3.1\end{array}$ & $\begin{array}{l}9.0 \\
8.5 \\
8.0 \\
8.0 \\
8.5\end{array}$ & $\begin{array}{l}51 \\
36 \\
20 \\
18 \\
26\end{array}$ & $\begin{array}{l}5.0 \\
4.6 \\
4.0 \\
4.4 \\
6.5\end{array}$ & $\begin{array}{l}31 \\
23 \\
15 \\
46 \\
77\end{array}$ & $\begin{array}{l}1.6 \\
1.5 \\
1.5 \\
2.0 \\
1.9\end{array}$ & $\begin{array}{l}1.2 \\
1.1 \\
1.1 \\
1.0 \\
1.4\end{array}$ & $\begin{array}{l}2.3 \\
2.1 \\
2.0 \\
2.0 \\
1.8\end{array}$ \\
\hline $\begin{array}{l}16 \\
17 \\
18 \\
19 \\
20\end{array}$ & $\begin{array}{l}3.3 \\
3.1 \\
3.0 \\
3.1 \\
2.8\end{array}$ & $\begin{array}{l}1.5 \\
1.5 \\
1.5 \\
1.4 \\
1.4\end{array}$ & $\begin{array}{l}3.7 \\
3.5 \\
3.3 \\
3.1 \\
3.0\end{array}$ & $\begin{array}{l}2.2 \\
2.2 \\
2.2 \\
2.2 \\
2.2\end{array}$ & $\begin{array}{l}3.1 \\
3.0 \\
3.0 \\
3.0 \\
3.0\end{array}$ & $\begin{array}{l}11 \\
15 \\
25 \\
32 \\
30\end{array}$ & $\begin{array}{l}38 \\
45 \\
39 \\
24 \\
16\end{array}$ & $\begin{array}{r}6.5 \\
7.1 \\
12 \\
9.9 \\
7.6\end{array}$ & $\begin{array}{l}45 \\
23 \\
13 \\
6.8 \\
4.4\end{array}$ & $\begin{array}{l}2.3 \\
2.3 \\
2.2 \\
1.8 \\
1.4\end{array}$ & $\begin{array}{l}2.6 \\
2.4 \\
2.2 \\
2.8 \\
2.4\end{array}$ & $\begin{array}{l}2.0 \\
3.4 \\
13 \\
29 \\
34\end{array}$ \\
\hline $\begin{array}{l}21 \\
22 \\
23 \\
24 \\
25\end{array}$ & $\begin{array}{l}2.8 \\
2.9 \\
2.9 \\
3.0 \\
3.1\end{array}$ & $\begin{array}{r}1.2 \\
1.1 \\
1.0 \\
.9 \\
.8\end{array}$ & $\begin{array}{l}3.0 \\
3.0 \\
2.9 \\
2.8 \\
2.8\end{array}$ & $\begin{array}{l}2.3 \\
2.3 \\
2.3 \\
2.4 \\
2.4\end{array}$ & $\begin{array}{l}3.0 \\
3.1 \\
3.1 \\
3.2 \\
3.4\end{array}$ & $\begin{array}{r}17 \\
12 \\
9.5 \\
9.0 \\
8.7\end{array}$ & $\begin{array}{l}25 \\
22 \\
38 \\
50 \\
48\end{array}$ & $\begin{array}{l}7.4 \\
5.7 \\
4.4 \\
3.9 \\
3.6\end{array}$ & $\begin{array}{l}3.7 \\
3.5 \\
7.9 \\
6.8 \\
4.0\end{array}$ & $\begin{array}{l}1.3 \\
1.2 \\
1.2 \\
1.1 \\
1.2\end{array}$ & $\begin{array}{l}2.5 \\
2.6 \\
3.7 \\
6.0 \\
6.2\end{array}$ & $\begin{array}{l}22 \\
22 \\
31 \\
37 \\
26\end{array}$ \\
\hline $\begin{array}{l}26 \\
27 \\
28 \\
29 \\
30 \\
31 \\
\end{array}$ & $\begin{array}{l}2.9 \\
3.1 \\
3.0 \\
3.3 \\
3.3 \\
3.4 \\
\end{array}$ & $\begin{array}{r}.8 \\
.7 \\
.6 \\
.5 \\
.5 \\
- \\
\end{array}$ & $\begin{array}{l}2.7 \\
2.7 \\
2.6 \\
2.6 \\
2.6 \\
2.6 \\
\end{array}$ & $\begin{array}{l}2.4 \\
2.5 \\
2.5 \\
2.6 \\
2.7 \\
3.0 \\
\end{array}$ & $\begin{array}{r}3.7 \\
3.9 \\
4.0 \\
4.2 \\
- \\
\\
\end{array}$ & $\begin{array}{l}10 \\
20 \\
33 \\
35 \\
34 \\
31 \\
\end{array}$ & $\begin{array}{r}59 \\
66 \\
56 \\
35 \\
20 \\
\\
\end{array}$ & $\begin{array}{l}3.6 \\
5.1 \\
7.9 \\
7.1 \\
6.2 \\
7.9 \\
\end{array}$ & $\begin{array}{r}3.1 \\
2.7 \\
2.0 \\
1.8 \\
16 \\
\end{array}$ & $\begin{array}{l}1.5 \\
1.5 \\
1.3 \\
1.2 \\
1.5 \\
1.5\end{array}$ & $\begin{array}{l}5.4 \\
4.7 \\
4.0 \\
3.6 \\
3.1 \\
3.1 \\
\end{array}$ & $\begin{array}{r}16 \\
12 \\
8.2 \\
15.4 \\
4.6 \\
- \\
- \\
\end{array}$ \\
\hline $\begin{array}{l}\text { Total } \\
\text { Mean } \\
\text { Max } \\
\text { Min } \\
\end{array}$ & $\begin{array}{r}90.2 \\
2.91 \\
3.5 \\
1.9 \\
\end{array}$ & $\begin{array}{r}50.9 \\
1.70 \\
3.7 \\
0.5 \\
\end{array}$ & $\begin{array}{r}89.1 \\
2.87 \\
7.0 \\
0.5 \\
\end{array}$ & $\begin{array}{r}74.6 \\
2.41 \\
3.0 \\
2.2 \\
\end{array}$ & $\begin{array}{r}97.6 \\
3.37 \\
4.2 \\
3.0 \\
\end{array}$ & $\begin{array}{r}453.1 \\
14.6 \\
35 \\
4.6 \\
\end{array}$ & $\begin{array}{r}1,082 \\
36.1 \\
66 \\
16 \\
\end{array}$ & $\begin{array}{r}197.1 \\
6.36 \\
14 \\
3.6 \\
\end{array}$ & $\begin{array}{r}597.2 \\
19.9 \\
77 \\
1.8 \\
\end{array}$ & $\begin{array}{r}272.8 \\
8.80 \\
87 \\
1.1 \\
\end{array}$ & $\begin{array}{r}77.2 \\
2.49 \\
6.2 \\
1.0 \\
\end{array}$ & $\begin{array}{r}301.8 \\
10.1 \\
37 \\
1.8 \\
\end{array}$ \\
\hline $\begin{array}{l}\text { Cal yr } \\
\text { Wtr yr }\end{array}$ & $\begin{array}{l}967: \\
968:\end{array}$ & $\begin{array}{l}\text { Total } \\
\text { Total }\end{array}$ & & $\begin{array}{l}\text { Mean } \\
\text { Mean }\end{array}$ & $\begin{array}{l}\text { Max } \\
\text { Max }\end{array}$ & $\begin{array}{r}290 \\
87\end{array}$ & $\begin{array}{l}\operatorname{Min} \\
\operatorname{Min}\end{array}$ & & & & & \\
\hline
\end{tabular}

Note.--No gage-height record Feb. 10 to Mar. 22. 
4-0195. East Swan River near Toivola, Minn.

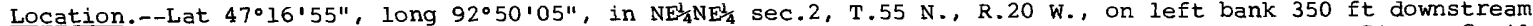
from bridge on St. Louis County Road $442,4.8$ miles upstream from confluence with West Swan River, 8 miles northwest of Toivola, and $8 \frac{3}{4}$ miles upstream from St. Louis River.

Drainage area. --112 sq $\mathrm{mi}$.

Records available.--September 1953 to September 1962, October 1964 to September 1968.

Gage.-Digital water-stage recorder. Datum of gage is 1,260.46 ft above mean sea level, datum of 1929 . Prior to Sept. 11, 1968, graphic water-stage recorder at same site and datum.

Average discharge.--13 years $(1953-62,1964-68), 90.5$ cfs .

Extremes.--Maximum discharge during year, 661 cfs July 2 (gage height, 12.57 ft); minimum daily, 9.4 cfs Jan. 3-6; minimum gage height, $3.29 \mathrm{ft}$ Nov. 15.

1953-62, 1964-68: Maximum discharge, 1,690 cfs Apr. 15, 1956 (gage height, 17.94 ft); maximum gage height, 18.45 ft Apr. 12, 1954 (backwater from ice); minimum daily discharge, 12 cfs Feb. 22-26, 1965, Feb. 18-24, 1967, minimum gage height, 3.15 ft Aug. 24, 1961.

Flood in May 1950 reached a stage of about $20.0 \mathrm{ft}$, from information by local residents.

Remarks.--Records good except those for winter months, which are fair.

DISCHARGE, IN CFS, WATER YEAR OCTOBER 1967 TO SEPTEMBER 1968

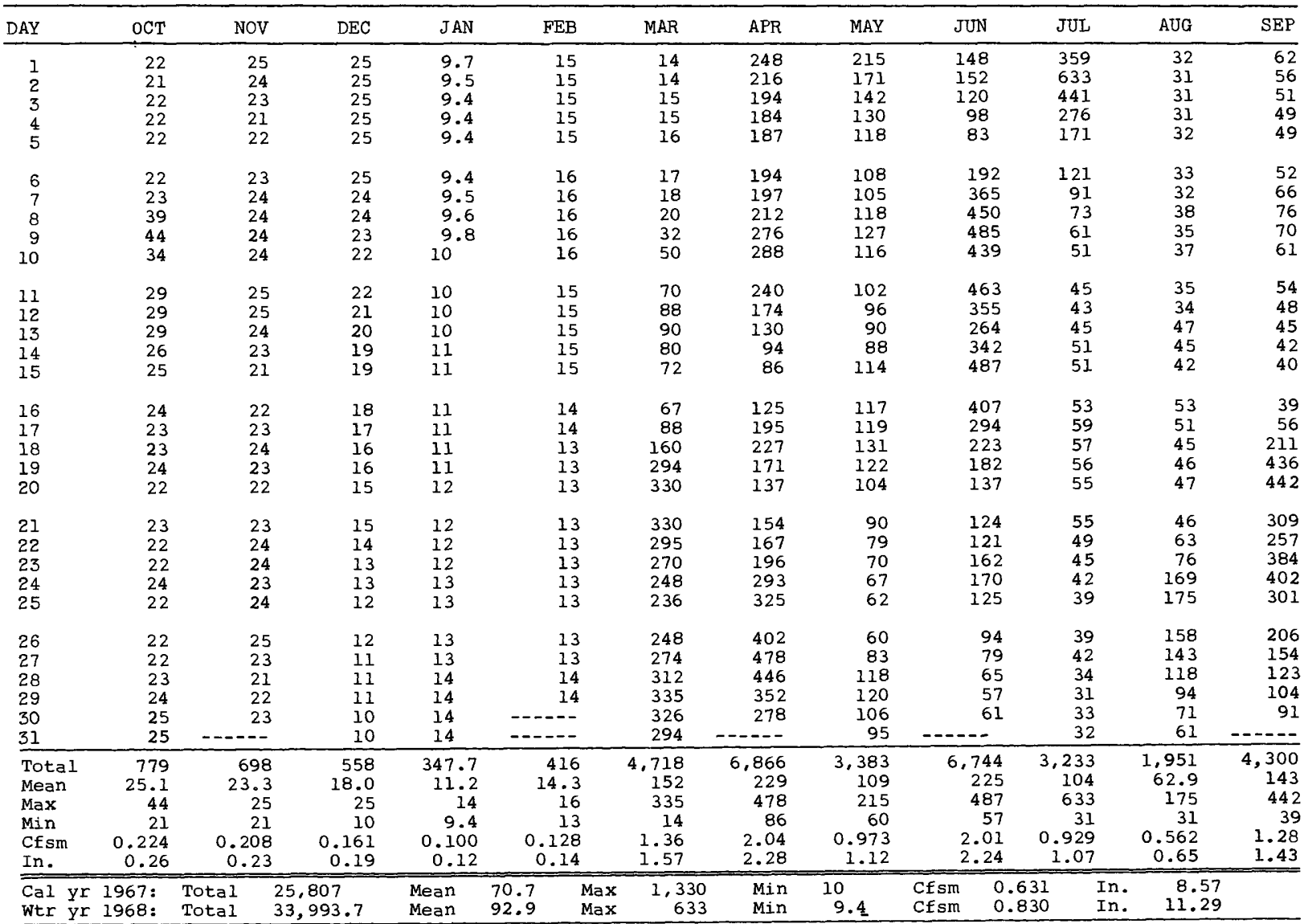

PEAK DISCHARGE (BASE，400 CFS)

$\begin{array}{lccccccc}\text { DATE } & \text { TIME } & \text { G.HT. } & \text { DISCHARGE } & \text { DATE } & \text { TIME } & \text { G.HT. } & \text { DISCHARGE } \\ 3-20 & 2100 & 10.87 & 402 & 7-2 & 0830 & 12.57 & 661 \\ 4-27 & 1200 & 10.65 & 489 & 9-20 & 0215 & 10.18 & 480 \\ 6-15 & 1600 & 10.82 & 518 & & & & \end{array}$




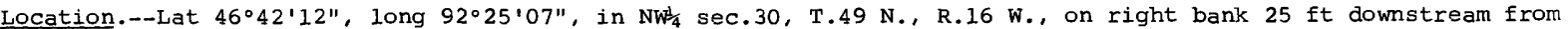
lower bridge on U. S. Highway 61 at Scanlon, 0.6 mile downstream from Minnesota Power \& Light Co. powerplant, 3 miles upstream from Thomson Reservoir, and 3.2 miles upstream from Midway River.

Drainage area. $--3,430 \mathrm{sq} \mathrm{mi}$, approximately.

Records available.--January 1908 to September 1968. Monthly discharge only for some periods published in wsP 1307. Published as "near Thomson" 1908-50.

Gage.--Digital water-stage recorder. Datum of gage is 1,101.23 ft above mean sea level, datum of 1929. Oct. 5, 1909, to Sept. 5, 1914, chain gage 3 miles downstream and 50 ft below powerplant at datum about 420 ft lower. Sept. 6, 1914, to Aug. 4, 1953, powerplant record at Thomson hydroelectric plant. Aug. 4, 1953, to May 13,

1966, graphic water-stage recorder at present site and datum.

Average discharge.--60 years, 2,200 cfs (unadjusted).

Extremes.--Maximum discharge during year, 11,500 cfs June 12 (gage height, 7.90 ft); minimum, 195 cfs Nov. 28 (gage height, $1.94 \mathrm{ft}$ ).

1908-68: Maximum daily discharge, 37,900 cfs May 9, 1950; maximum gage height, 15.8 ft, May 9, 1950, from Minnesota Highway Department (discharge uncertain); minimum discharge, 80 cfs Aug. 29, 1963 ; minimum daily, 109 cfs Feb. 7, 1924.

Remarks.--Records good. Diurnal fluctuation caused by powerplant upstream. Flow regulated by Whiteface Reservoir and Boulder. Island, Rice and Fish Lakes (combined capacity, 332,160 acre-ft). Records of chemical analyses for the water year 1968 are published in Part 2 of this report.

DISCHARGE. IN CUBIC FEFT PER SECOVD, WATER YEAR OCTOBER 1967 TO SEPTEMRER 1969

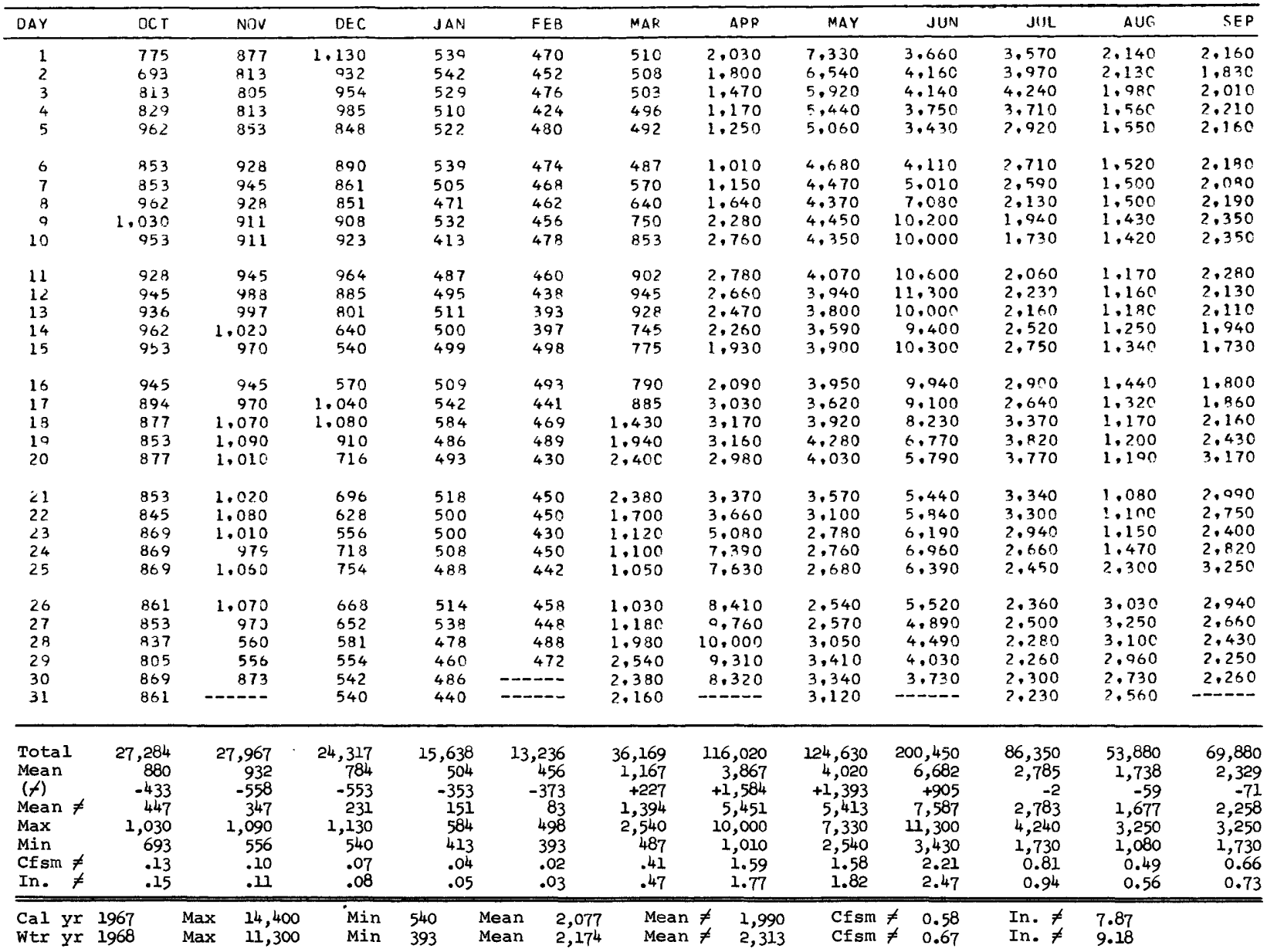

f Change in contents, equivalent in cubic feet per second, in Whiteface Reservoir and Boulder, Island, Rice and Fish Lakes; records furnished by Minnesota Power and Light Co.

$\neq$ Adjusted for change in contents. 
5-0300. Otter Tail River near Detroit Lakes, Minn.

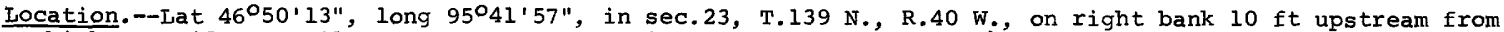
highway bridge, 5 miles downstream from Height of Land Lake, and $7 i_{2}$ miles east of city of Detroit Lakes.

Drainage area. $--270 \mathrm{sq} \mathrm{mi}$.

Records available.--March 1937 to september 1968.

Gage.--Water-stage recorder and concrete control. Datum of gage is $1,409.49$ ft above mean sea level, datum of 1929.

Average discharge.--31 years, $54.7 \mathrm{cfs}(39,600$ acre-ft per year).

Extremes.--Maximum discharge during year, $188 \mathrm{cfs}$ May 29 (gage height, $4.18 \mathrm{ft}$ ); minimum, $1.5 \mathrm{cfs}$ Oct. 6 (gage height, $2.67 \mathrm{ft}$ ).

1937-68: Maximum discharge, 371 cfs June 26, 1943 (gage height, 4.78 ft, from graph based on partial record); maximum gage height, $6.96 \mathrm{ft} J a n .27,1950$ (backwater from ice); minimum daily discharge, 0.1 cfs Mar. 23, 1940

Remarks.--Records good except those for periods of no gage-helght record and those for winter months, which are fair. Flow partly regulated by dams of Minnesota Department of Conservation on several lakes above station.

DISCHARGE, IN CFS, WATER YEAR OCTOBER 1967 TO SEPTEMBER 1968

\begin{tabular}{|c|c|c|c|c|c|c|c|c|c|c|c|c|}
\hline DAY & OCT & NOV & $\mathrm{DEC}$ & JAN & FEB & MAR & APR & MAY & JUN & JUL & AUG & SEP \\
\hline $\begin{array}{l}1 \\
2 \\
3 \\
4 \\
5\end{array}$ & $\begin{array}{l}1.6 \\
1.6 \\
1.9 \\
2.1 \\
1.6\end{array}$ & $\begin{array}{l}12 \\
12 \\
12 \\
12 \\
12\end{array}$ & $\begin{array}{l}14 \\
14 \\
14 \\
14 \\
14\end{array}$ & $\begin{array}{l}13 \\
13 \\
12 \\
12 \\
12\end{array}$ & $\begin{array}{l}7.4 \\
6.8 \\
6.2 \\
5.6 \\
5.1\end{array}$ & $\begin{array}{l}2.2 \\
2.2 \\
2.3 \\
2.4 \\
2.6\end{array}$ & $\begin{array}{l}11 \\
10 \\
10 \\
10 \\
10\end{array}$ & $\begin{array}{l}105 \\
104 \\
103 \\
103 \\
103\end{array}$ & $\begin{array}{l}149 \\
146 \\
144 \\
137 \\
146\end{array}$ & $\begin{array}{l}121 \\
119 \\
116 \\
112 \\
110\end{array}$ & $\begin{array}{l}44 \\
46 \\
47 \\
47 \\
46\end{array}$ & $\begin{array}{c}15 \\
15 \\
15 \\
11 \\
6.2\end{array}$ \\
\hline $\begin{array}{r}6 \\
7 \\
8 \\
9 \\
10\end{array}$ & $\begin{array}{l}1.5 \\
2.6 \\
3.0 \\
3.0 \\
2.8\end{array}$ & $\begin{array}{l}12 \\
12 \\
13 \\
13 \\
14\end{array}$ & $\begin{array}{l}14 \\
14 \\
14 \\
14 \\
14\end{array}$ & $\begin{array}{l}12 \\
12 \\
12 \\
12 \\
12\end{array}$ & $\begin{array}{l}4.7 \\
4.3 \\
3.9 \\
3.7 \\
3.4\end{array}$ & $\begin{array}{l}4.7 \\
6.8 \\
22 \\
51 \\
53\end{array}$ & $\begin{array}{l}11 \\
18 \\
30 \\
40 \\
42\end{array}$ & $\begin{array}{l}105 \\
107 \\
113 \\
121 \\
132\end{array}$ & $\begin{array}{l}151 \\
151 \\
154 \\
149 \\
149\end{array}$ & $\begin{array}{r}106 \\
104 \\
102 \\
97 \\
80\end{array}$ & $\begin{array}{l}47 \\
48 \\
54 \\
53 \\
51\end{array}$ & $\begin{array}{l}6.5 \\
7.2 \\
7.5 \\
7.2 \\
6.8\end{array}$ \\
\hline $\begin{array}{l}11 \\
12 \\
13 \\
14 \\
15\end{array}$ & $\begin{array}{l}2.3 \\
2.1 \\
2.1 \\
2.3 \\
2.3\end{array}$ & $\begin{array}{l}14 \\
13 \\
13 \\
13 \\
13\end{array}$ & $\begin{array}{l}17 \\
20 \\
21 \\
21 \\
21\end{array}$ & $\begin{array}{l}13 \\
13 \\
13 \\
13 \\
13\end{array}$ & $\begin{array}{l}3.2 \\
3.0 \\
2.8 \\
2.7 \\
2.5\end{array}$ & $\begin{array}{l}40 \\
31 \\
25 \\
23 \\
18\end{array}$ & $\begin{array}{l}41 \\
39 \\
37 \\
35 \\
34\end{array}$ & $\begin{array}{l}141 \\
152 \\
159 \\
166 \\
170\end{array}$ & $\begin{array}{l}149 \\
141 \\
137 \\
146 \\
144\end{array}$ & $\begin{array}{l}71 \\
80 \\
80 \\
85 \\
88\end{array}$ & $\begin{array}{l}48 \\
45 \\
41 \\
39 \\
41\end{array}$ & $\begin{array}{l}6.5 \\
5.9 \\
5.9 \\
6.5 \\
5.9\end{array}$ \\
\hline $\begin{array}{l}16 \\
17 \\
18 \\
19 \\
20\end{array}$ & $\begin{array}{l}2.3 \\
2.1 \\
2.1 \\
2.1 \\
1.9\end{array}$ & $\begin{array}{l}14 \\
14 \\
14 \\
15 \\
15\end{array}$ & $\begin{array}{l}21 \\
21 \\
22 \\
22 \\
22\end{array}$ & $\begin{array}{l}13 \\
14 \\
14 \\
14 \\
14\end{array}$ & $\begin{array}{l}2.4 \\
2.3 \\
2.2 \\
2.1 \\
2.0\end{array}$ & $\begin{array}{l}16 \\
15 \\
15 \\
14 \\
14\end{array}$ & $\begin{array}{l}33 \\
32 \\
31 \\
31 \\
32\end{array}$ & $\begin{array}{l}171 \\
173 \\
176 \\
178 \\
180\end{array}$ & $\begin{array}{l}144 \\
139 \\
137 \\
132 \\
132\end{array}$ & $\begin{array}{l}88 \\
83 \\
82 \\
78 \\
75\end{array}$ & $\begin{array}{l}41 \\
39 \\
37 \\
37 \\
29\end{array}$ & $\begin{array}{l}7.5 \\
9.3 \\
9.3 \\
9.3 \\
8.9\end{array}$ \\
\hline $\begin{array}{l}21 \\
22 \\
23 \\
24 \\
25\end{array}$ & $\begin{array}{l}2.3 \\
3.0 \\
4.0 \\
4.7 \\
5.3\end{array}$ & $\begin{array}{l}15 \\
15 \\
15 \\
15 \\
15\end{array}$ & $\begin{array}{l}21 \\
21 \\
20 \\
19 \\
18\end{array}$ & $\begin{array}{l}14 \\
14 \\
14 \\
14 \\
13\end{array}$ & $\begin{array}{l}2.0 \\
2.0 \\
2.1 \\
2.1 \\
2.1\end{array}$ & $\begin{array}{l}14 \\
14 \\
14 \\
13 \\
13\end{array}$ & $\begin{array}{l}34 \\
40 \\
51 \\
62 \\
76\end{array}$ & $\begin{array}{l}180 \\
180 \\
181 \\
183 \\
178\end{array}$ & $\begin{array}{l}156 \\
161 \\
156 \\
149 \\
141\end{array}$ & $\begin{array}{l}69 \\
66 \\
65 \\
58 \\
52\end{array}$ & $\begin{array}{l}23 \\
25 \\
26 \\
25 \\
24\end{array}$ & $\begin{array}{l}7.8 \\
15 \\
18 \\
15 \\
14\end{array}$ \\
\hline $\begin{array}{l}26 \\
27 \\
28 \\
29 \\
30 \\
31 \\
\end{array}$ & $\begin{array}{r}6.5 \\
7.8 \\
8.6 \\
10 \\
11 \\
11 \\
\end{array}$ & $\begin{array}{r}14 \\
16 \\
17 \\
15 \\
15 \\
- \\
\end{array}$ & $\begin{array}{l}18 \\
17 \\
16 \\
15 \\
15 \\
14\end{array}$ & $\begin{array}{l}12 \\
11 \\
11 \\
9.7 \\
8.9 \\
8.1 \\
\end{array}$ & $\begin{array}{r}2.2 \\
2.2 \\
2.2 \\
2.2 \\
- \\
- \\
\end{array}$ & $\begin{array}{l}13 \\
13 \\
12 \\
12 \\
11 \\
11\end{array}$ & $\begin{array}{r}92 \\
107 \\
112 \\
110 \\
108 \\
\end{array}$ & $\begin{array}{l}170 \\
160 \\
154 \\
172 \\
174 \\
156\end{array}$ & $\begin{array}{r}134 \\
129 \\
125 \\
119 \\
123 \\
-\end{array}$ & $\begin{array}{l}56 \\
58 \\
57 \\
57 \\
45 \\
42 \\
\end{array}$ & $\begin{array}{l}23 \\
22 \\
20 \\
18 \\
18 \\
17\end{array}$ & $\begin{array}{r}13 \\
12 \\
11 \\
9.7 \\
9.3 \\
-\end{array}$ \\
\hline $\begin{array}{l}\text { Total } \\
\text { Mean } \\
\text { Max } \\
\text { Min } \\
\text { Ac-ft }\end{array}$ & $\begin{array}{r}117.5 \\
3.79 \\
11 \\
1.5 \\
233\end{array}$ & $\begin{array}{r}414 \\
13.8 \\
17 \\
12 \\
821\end{array}$ & $\begin{array}{r}542 \\
17.5 \\
22 \\
14 \\
1.080\end{array}$ & $\begin{array}{r}385.7 \\
12.4 \\
14 \\
8.1 \\
765\end{array}$ & $\begin{array}{r}95.4 \\
3.29 \\
7.4 \\
2.0 \\
189\end{array}$ & $\begin{array}{r}500.2 \\
16.1 \\
53 \\
2.2 \\
992\end{array}$ & $\begin{array}{r}1,329 \\
44.3 \\
112 \\
10 \\
2,640\end{array}$ & $\begin{array}{r}4,650 \\
150 \\
183 \\
103 \\
9,220\end{array}$ & $\begin{array}{r}4,270 \\
142 \\
161 \\
119 \\
8,470\end{array}$ & $\begin{array}{r}2,502 \\
80.7 \\
121 \\
42 \\
4,960\end{array}$ & $\begin{array}{r}1,121 \\
36.2 \\
54 \\
17 \\
2,220\end{array}$ & $\begin{array}{r}297.2 \\
9.91 \\
18 \\
5.9 \\
589\end{array}$ \\
\hline $\begin{array}{l}\text { Cal yr } \\
\text { wtr yr }\end{array}$ & $\begin{array}{l}1967: \\
1968:\end{array}$ & $\begin{array}{l}\text { Eal } \\
\text { cal }\end{array}$ & 9.9 & $n$ & $\begin{array}{l}2.2 \\
4.3\end{array}$ & $\begin{array}{l}264 \\
183\end{array}$ & $\begin{array}{l}\text { Min } \\
\text { Min }\end{array}$ & $\begin{array}{l}1.0 \\
1.5\end{array}$ & $\begin{array}{l}52 \\
32\end{array}$ & & & \\
\hline
\end{tabular}


05-0339. Pelican River at Detroit Lakes, Minn.

Location.--Lat 46\%48.37", long 95049'42", in SW $\frac{1}{4}$ sec.35, T.139 N., R.41 W., on left bank 60 ft upstream from culvert, 's mile upstream from Detroit' Lake, at Detroit Lakes, Becker County.

Records available.--July to september 1968.

Gage.--Staff gage read once daily. Altitude of gage is $1,337 \mathrm{ft}$ (from topographic map).

Extremes.--Maximum daily discharge during period July to September, 5.8 cfs July 23, 24; maximum gage height observed, $2.20 \mathrm{ft}$ July 24 ; minimum daily discharge, $1.0 \mathrm{cfs}$ Sept. 15.

Remarks.--Records fair. Records of chemical analyses for the water year 1968 are published in Part 2 of this report.

Cooperation.--Gage-height record furnished by Pelican River Watershed District.

DISCHARGE, IN CUBIC FEET PER SECOND, PERIOD JULY TO SEPTEMBER 1968

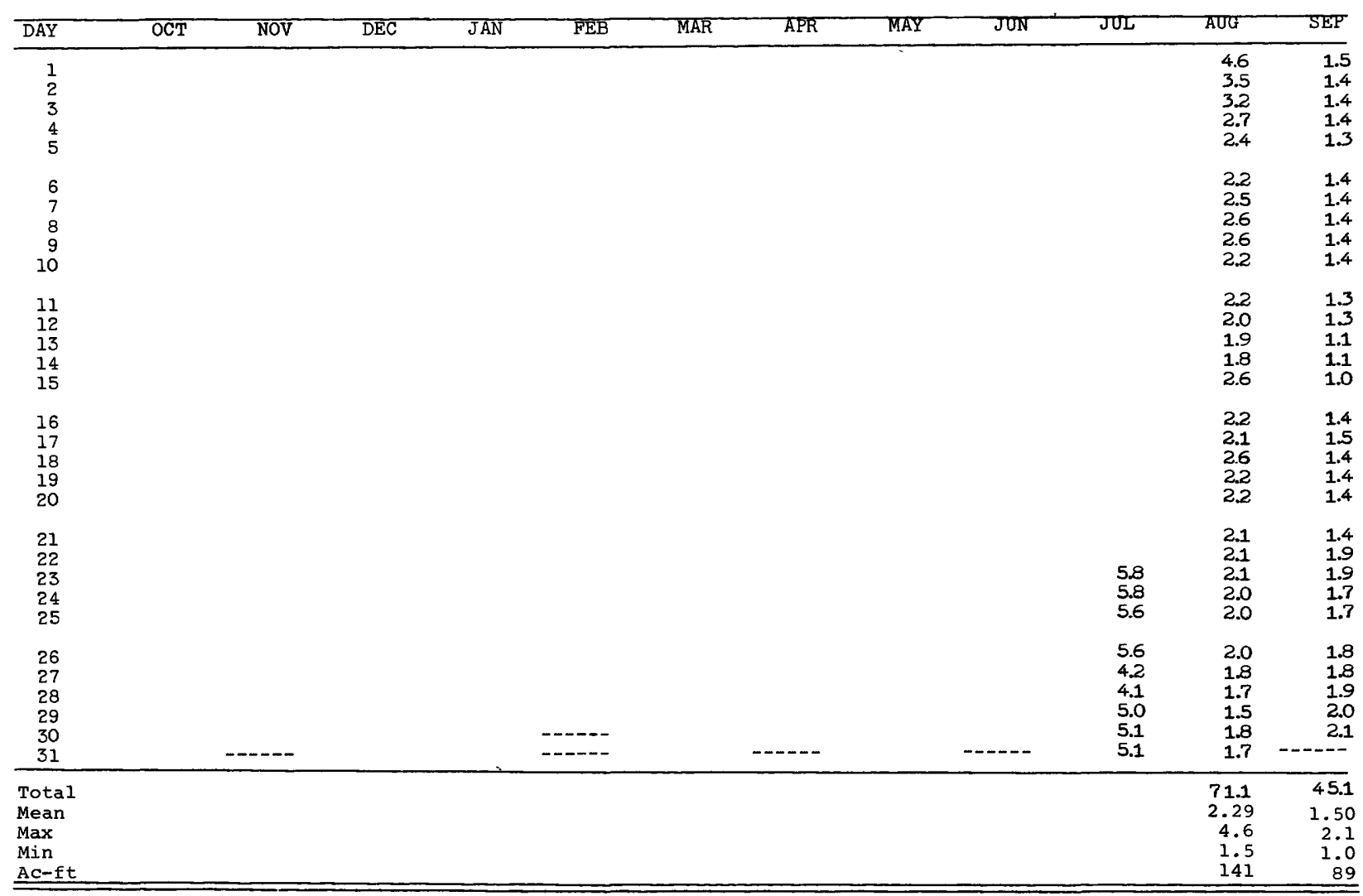


5-0341. Pelican River at Detroit Lake Outlet near Detroit Lakes, Minn.

Location.--Iat $46^{\circ} 47^{\prime} 22^{\prime \prime}$, long 95'52'00", on line between secs. 4 and 9, T.138 N., R.41 W., on left wíngwall of remains of old dam at outlet of Detroit Lake, $60 \mathrm{ft}$ upstream from bridge on' County Highway 22 and $2 \frac{1}{2}$ miles southwest of Detroit Lakes.

Records available.--July to september 1968.

Gage.--Staff gage read once daily. Datum of gage is 1,330.01 ft above mean sea level, datum of 1929 .

Extremes.--Maximum discharge during period July to september, 15 cfs July 23 (gage height, 4.11 ft); no flow sept. $6,9-16,25,31$.

Remarks.--Records fair.

Cooperation.--Gage readings furnished by Pelican River Watershed District.

DISCHARGE, IN CUBIC FEET PER SECOND, JULY TO SEPTEMBER 1968

\begin{tabular}{|c|c|c|c|c|c|c|c|c|c|c|c|c|}
\hline DAY & OCT & NOV & $\mathrm{DEC}$ & JAN & FEB & MAR & APR & MAY & JUN & JUL & AUG & SEP \\
\hline $\begin{array}{l}1 \\
2 \\
3 \\
4 \\
5\end{array}$ & & & & & & & & & & & $\begin{array}{l}9.2 \\
8.5 \\
8.5 \\
7.5 \\
7.5\end{array}$ & $\begin{array}{r}0.5 \\
.6 \\
.4 \\
.1 \\
.1\end{array}$ \\
\hline $\begin{array}{r}6 \\
7 \\
8 \\
9 \\
10\end{array}$ & . & & & & & & & & & & $\begin{array}{l}7.8 \\
7.8 \\
8.8 \\
7.8 \\
7.5\end{array}$ & $\begin{array}{l}0 \\
.1 \\
.1 \\
0 \\
0\end{array}$ \\
\hline $\begin{array}{l}11 \\
12 \\
13 \\
14 \\
15\end{array}$ & & & & & & & & - & & & $\begin{array}{l}6.2 \\
4.8 \\
3.8 \\
4.3 \\
4.8\end{array}$ & $\begin{array}{l}0 \\
0 \\
0 \\
0 \\
0\end{array}$ \\
\hline $\begin{array}{l}16 \\
17 \\
18 \\
19 \\
20\end{array}$ & & & & & & & & & & & $\begin{array}{l}4.3 \\
3.8 \\
4.8 \\
4.0 \\
3.8\end{array}$ & $\begin{array}{l}0 \\
.1 \\
.1 \\
.1 \\
.1\end{array}$ \\
\hline $\begin{array}{l}26 \\
27 \\
28 \\
29 \\
30 \\
31 \\
\end{array}$ & & ------ & & & $-\cdots$ & & --- & & ---- & $\begin{array}{c}13 \\
12 \\
11 \\
11 \\
10 \\
8.8\end{array}$ & $\begin{array}{r}3.0 \\
2.2 \\
1.5 \\
.9 \\
.9 \\
.9\end{array}$ & $\begin{array}{c}.1 \\
.1 \\
.1 \\
-1 \\
-0 .-1\end{array}$ \\
\hline Total & & & & & & & & & & & 152.7 & 4.40 \\
\hline $\begin{array}{l}\text { Mean } \\
\text { Max } \\
\text { Min } \\
\text { Ac-ft }\end{array}$ & & & . & & & & & & & & $\begin{array}{r}4.92 \\
9.2 \\
0.9 \\
303\end{array}$ & $\begin{array}{r}0.147 \\
0.7 \\
0 \\
9\end{array}$ \\
\hline
\end{tabular}


5-0351. Long Lake Outlet near Detroit Lakes, Minn.

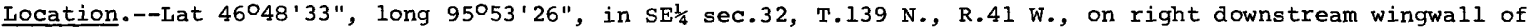
abandoned Minnesota Conservation Department dam, $200 \mathrm{ft}$ downstream from Long Lake, 2 , 500 ft upstream from St. Clair Lake and $2 \frac{1}{4}$ miles west of Detroit Lakes, Becker County.

Records available.--July to september 1968.

Gage.--Staff gage read once daily. Altitude of gage is 1,350 ft (from topographic map).

Extremes.--Maximum daily discharge during period, 1.2 cfs July $23-25$ (gage height, 3.68 ft); no flow for several days.

Remarks.--Records fair. Flow affected by natural storage in Long Lake.

Cooperation.--Gage-height record furnished by Pelican River Watershed District.

DISCHARGE, IN CUBIC FEET PER SECOND, WATER YEAR OCTOBER 1967 TO SEPTEMBER 1968

\begin{tabular}{|c|c|c|c|c|c|c|c|c|c|c|c|c|}
\hline DAY & $\mathrm{OCT}$ & Nov & $\mathrm{DEC}$ & JAN & FEB & MAR & APR & MAY & JUN & JUL & AUG & $\overline{S E P}$ \\
\hline $\begin{array}{l}1 \\
2 \\
3 \\
4 \\
5\end{array}$ & & & & & & & & & & & $\begin{array}{r}0.5 \\
.2 \\
.2 \\
.2 \\
.2\end{array}$ & $\begin{array}{r}0.1 \\
.2 \\
.2 \\
.2 \\
.1\end{array}$ \\
\hline $\begin{array}{r}6 \\
7 \\
8 \\
9 \\
10\end{array}$ & & & & & & & & & & & $\begin{array}{l}.2 \\
.2 \\
.5 \\
.4 \\
.3\end{array}$ & $\begin{array}{l}.1 \\
.1 \\
.1 \\
0 \\
0\end{array}$ \\
\hline $\begin{array}{l}11 \\
12 \\
13 \\
14 \\
15\end{array}$ & & & & & & & & & & & $\begin{array}{l}.2 \\
.2 \\
.2 \\
0 \\
.2\end{array}$ & $\begin{array}{l}0^{.1} \\
0 \\
0 \\
0\end{array}$ \\
\hline $\begin{array}{l}16 \\
17 \\
18 \\
19 \\
20\end{array}$ & & & & & & & & & & & $\begin{array}{l}.2 \\
0.1 \\
.1 \\
.1\end{array}$ & $\begin{array}{l}.1 \\
.2 \\
.2 \\
.2 \\
.2\end{array}$ \\
\hline $\begin{array}{l}26 \\
27 \\
28 \\
29 \\
30 \\
31\end{array}$ & & - - & & & $-\cdots$ & & $-\cdots-$ & & ---- & $\begin{array}{r}1.0 \\
.4 \\
.7 \\
.6 \\
.6 \\
.5\end{array}$ & $\begin{array}{l}0 \\
.2 \\
.2 \\
.1 \\
.2 \\
.2\end{array}$ & $\begin{array}{r}.2 \\
2 \\
.2 \\
.1 \\
.1 \\
\end{array}$ \\
\hline $\begin{array}{l}\text { Total } \\
\text { Mean } \\
\text { Max } \\
\text { Min } \\
\text { Ac-ft }\end{array}$ & & & & & & & & & & & $\begin{array}{r}5.8 \\
0.19 \\
0.5 \\
0 \\
12\end{array}$ & $\begin{array}{r}4.7 \\
0.16 \\
0.5 \\
0 \\
9.3\end{array}$ \\
\hline
\end{tabular}


5-0352. West Branch County Ditch No. 14 near Detroit Lakes, Minn.

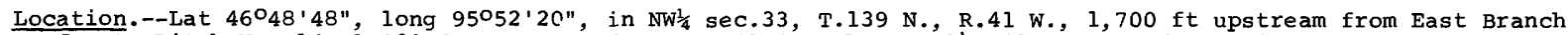
County Ditch No. 14, 1,900 ft upstream from st. Clair Lake and $1 \frac{1}{2}$ miles west of Detroit Lakes, Becker County. Records available.--July to september 1968.

Gage.--Staff gage read once-daily.

Extremes.--Maximum discharge during period, $3.6 \mathrm{cfs}$ sept. 3 (gage height, $3.46 \mathrm{ft}$, backwater from aquatic growth); no flow Aug. 27-29.

Remarks.--Records poor.

Cooperation.--Gage-height record furnished by Pelican River Watershed District.

Discharge in cubic feet per second, August to september 1968

\begin{tabular}{|c|c|c|c|c|c|c|c|c|c|c|c|c|}
\hline$\overline{D A Y}$ & OCT & NOV & DEC & JAN & FEB & MAR & APR & MAY & JUN & JUL & AUG & SEP \\
\hline $\begin{array}{l}1 \\
2 \\
3 \\
4 \\
5\end{array}$ & & & & & & & & & & & $\begin{array}{r}0.3 \\
.3 \\
.3 \\
2 \\
.3\end{array}$ & $\begin{array}{r}0.1 \\
.3 \\
1.9 \\
.1 \\
.1\end{array}$ \\
\hline $\begin{array}{r}6 \\
7 \\
8 \\
9 \\
10\end{array}$ & & & & & & & & & & & $\begin{array}{l}.3 \\
.3 \\
.5 \\
.3 \\
.2\end{array}$ & $\begin{array}{l}.1 \\
.1 \\
.1 \\
.1 \\
1\end{array}$ \\
\hline $\begin{array}{l}11 \\
12 \\
13 \\
14 \\
15\end{array}$ & & & & & & & & & & & $\begin{array}{l}2 \\
.2 \\
.1 \\
.1 \\
.2\end{array}$ & $\begin{array}{l}1 \\
.1 \\
.1 \\
.1 \\
.1\end{array}$ \\
\hline $\begin{array}{l}16 \\
17 \\
18 \\
19 \\
20\end{array}$ & & & & & & & & & & & $\begin{array}{l}.1 \\
.1 \\
.1 \\
.1 \\
.1\end{array}$ & $\begin{array}{l}.2 \\
.2 \\
.2 \\
.3 \\
.2\end{array}$ \\
\hline $\begin{array}{l}21 \\
22 \\
23 \\
24 \\
25\end{array}$ & & & & & & & & & & $\begin{array}{r}0.3 \\
2 \\
2\end{array}$ & $\begin{array}{l}.1 \\
.1 \\
.1 \\
.1\end{array}$ & $\begin{array}{l}.2 \\
.7 \\
.7 \\
.6 \\
.5\end{array}$ \\
\hline $\begin{array}{l}26 \\
27 \\
28 \\
29 \\
30 \\
31\end{array}$ & & ----- & & & - & & -....- & & ------ & $\begin{array}{l}.3 \\
.3 \\
.3 \\
.3 \\
.3 \\
.3\end{array}$ & $\begin{array}{l}.1 \\
0 \\
0 \\
0 \\
.1 \\
.1\end{array}$ & $\begin{array}{r}.4 \\
.3 \\
.2 \\
.2 \\
.2 \\
- \\
\end{array}$ \\
\hline $\begin{array}{l}\text { Total } \\
\text { Mean } \\
\text { Max } \\
\text { Min } \\
\text { Ac-ft }\end{array}$ & & & & & & & & & & & $\begin{array}{r}5.1 \\
0.16 \\
0.5 \\
0 \\
10\end{array}$ & $\begin{array}{r}8.6 \\
0.29 \\
1.9 \\
0.1 \\
17\end{array}$ \\
\hline
\end{tabular}


5-0353. East Branch County Ditch No. 14 near Detroit Lakes, Minn.

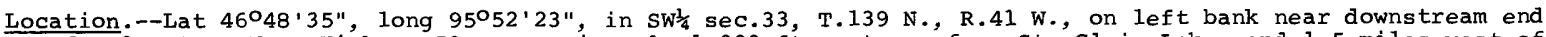
of culvert on U.S. Highway 59, approximately 1,000 ft upstream from st. Clair Lake, and 1.5 miles west of city of Detroit Lakes.

Records available.--July to september 1968.

Gage.--Digital water-stage recorder. Datum of gage is $1,333.96$ ft above mean sea level, datum of 1929.

Extremes.--Maximum discharge during period, 2.0 cfs July 22 (gage height, 2.87 ft); maximum gage height, 2.90 ft July 20; minimum daily discharge, 0.50 cfs sept. 11 .

Remarks. -Records fair.

DISCHARGE, IN CUBIC FEET PER SECOND, WATER YEAR JULY TO SEPTEMBER 1968

\begin{tabular}{|c|c|c|c|c|c|c|c|c|c|c|c|c|}
\hline DAY & OCT & NOV & $\mathrm{DEC}$ & JAN & FEB & MAR & APR & MAY & JUN & JUL & AUG & SEP \\
\hline $\begin{array}{l}1 \\
2 \\
3 \\
4 \\
5\end{array}$ & & & & & & & & & & & $\begin{array}{r}0.78 \\
.77 \\
.77 \\
.72 \\
.73\end{array}$ & $\begin{array}{r}0.85 \\
.86 \\
.86 \\
.84 \\
.93\end{array}$ \\
\hline $\begin{array}{r}6 \\
7 \\
8 \\
9 \\
10\end{array}$ & & & & & & & & & & $\begin{array}{l}1.1 \\
1.0\end{array}$ & $\begin{array}{l}.79 \\
.77 \\
.93 \\
.88 \\
.77\end{array}$ & $\begin{array}{l}.98 \\
.92 \\
.73 \\
.61 \\
.53\end{array}$ \\
\hline $\begin{array}{l}11 \\
12 \\
13 \\
14 \\
15\end{array}$ & & & & & & & & & & $\begin{array}{l}1.0 \\
.99 \\
1.0 \\
1.0 \\
1.1\end{array}$ & $\begin{array}{l}.68 \\
.64 \\
.63 \\
.57 \\
.70\end{array}$ & $\begin{array}{l}.5 \\
.72 \\
.82 \\
.89 \\
.90\end{array}$ \\
\hline $\begin{array}{l}16 \\
17 \\
18 \\
19 \\
20\end{array}$ & & & & & & & & & & $\begin{array}{l}1.1 \\
1.1 \\
1.1 \\
1.1 \\
1.1\end{array}$ & $\begin{array}{l}.81 \\
.74 \\
.74 \\
.84 \\
.78\end{array}$ & $\begin{array}{c}1.1 \\
.97 \\
.86 \\
.78 \\
.87\end{array}$ \\
\hline $\begin{array}{l}26 \\
27 \\
28 \\
29 \\
30 \\
31\end{array}$ & & - - - & & & ---- & & $-\cdots$ & & $-\ldots$ & $\begin{array}{l}.94 \\
.93 \\
.83 \\
.80 \\
.82 \\
.82\end{array}$ & $\begin{array}{l}.75 \\
.76 \\
.82 \\
.84 \\
.89 \\
.90\end{array}$ & $\begin{array}{r}.89 \\
.83 \\
81 \\
.80 \\
.79 \\
\end{array}$ \\
\hline $\begin{array}{l}\text { Tota } 1 \\
\text { Mean } \\
\text { Max } \\
\text { Min } \\
\text { Ac-ft }\end{array}$ & & & & & & & & & & & $\begin{array}{r}23.91 \\
0.77 \\
.93 \\
.57 \\
47\end{array}$ & $\begin{array}{r}26.96 \\
0.90 \\
1.9 \\
.50 \\
53\end{array}$ \\
\hline
\end{tabular}


5-0354. St. Clair Lake near Detroit Lakes, Minn.

Location.--Iat 46047'52", long 95052'49", in NE/ sec.5, T.138 N., R.41 W., at south end of lake, 2, 000 ft south at St. Clair Lake outlet and $2 \frac{1}{4}$ miles southwest of Detroit Lakes, Becker County.

Records available.--July to September 1968.

Gage.--Staff gage read once daily. Datum of gage is 1,330.653 ft above mean sea level, datum of 1929 (Minnesota Highway Department benchmark).

Extremes.--Maximum gage height observed during period, $4.91 \mathrm{ft}$ July 23 ; minimum observed, 4.22 ft sept. 15.

Remarks.--Water level subject to fluctuation caused by wind action.

Cooperation.--Gage reading furnished by Pelican River Watershed District.

Gage height, in feet, July to september 1968

July $31 \ldots .4 .66$

Aug. $31 \ldots .4 .37$

sept. $30 \ldots . .4 .66$

Note.--Gage-height record other than that shown above is available. 
Location.--Lat $46^{\circ} 48^{\prime} 03^{\prime \prime}$, long $95^{\circ} 52^{\prime} 37^{\prime \prime}$, in NW'/ sec.4, T.138 N., R.4l w. near right bank 25 ft upstream from culvert on U.S. Highway 59, 500 ft downstream from outlet of St. Clair Lake, 1 mile upstream from Pelican River and 2 miles southwest of Detroit Lakes.

Records available.--July to september 1968.

Gage.--Staff gage read once daily. Datum of gage is $1,333.35 \mathrm{ft}$ above mean sea level, datum of 1929 .

Extremes.--Maximum daily discharge during period July to september, 6.0 cfs July 23 , 26; minimum daily, 1.3 cfs, Sept. 15 .

Remarks.--Record fair.

Cooperation.--Gage reading furnished by Pelican River Watershed District.

DISCHARGE, IN CUBIC FEET PER SECOND, PERIOD JULY TO SEPTEMBER 1968

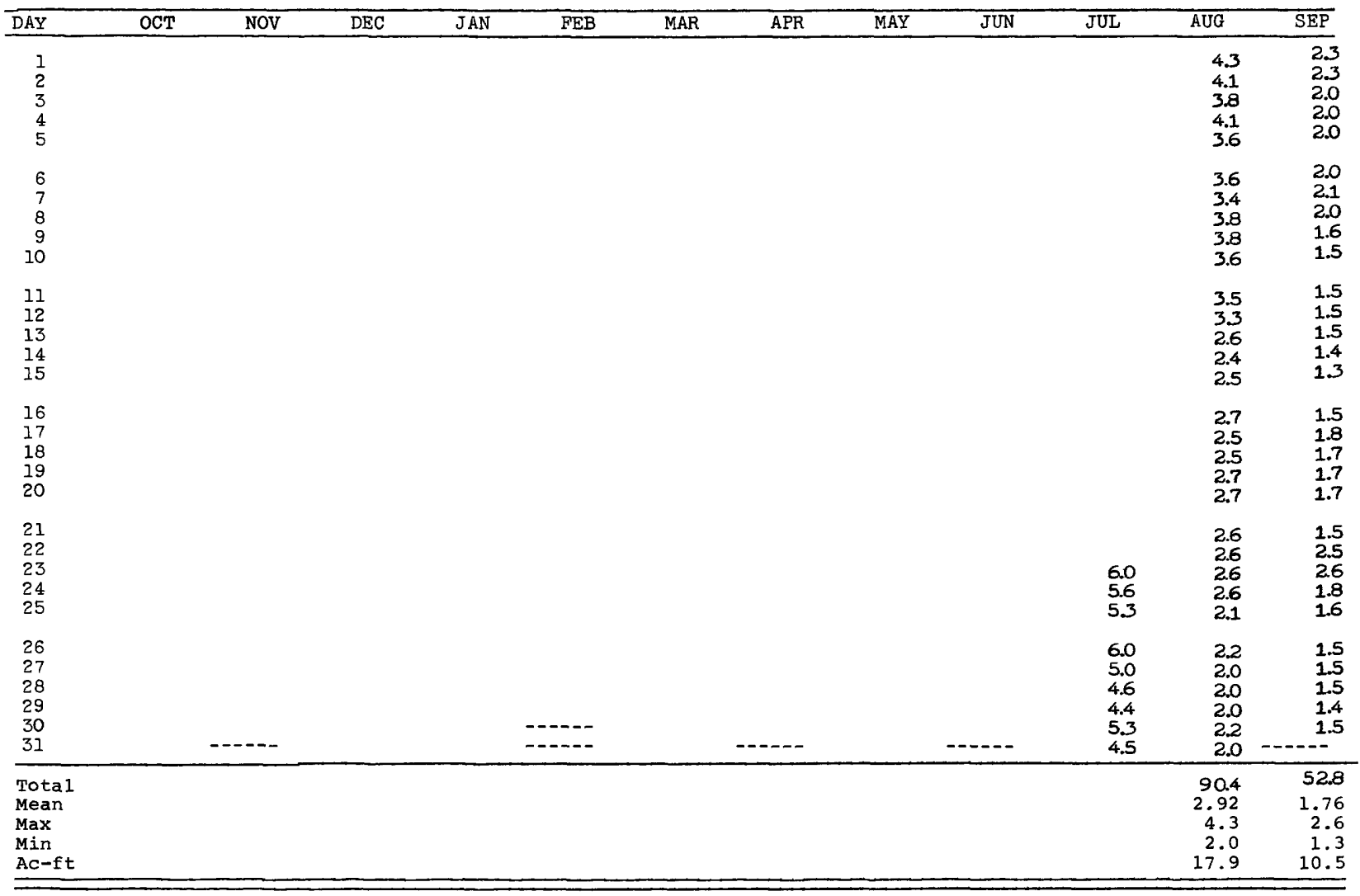


5-0356. Pelican River at Muskrat Lake Outlet near Detroit Lakes, Minn.

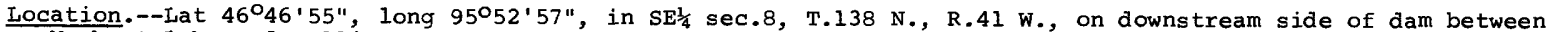
Muskrat Lake and Sallie Lake at State Fish Hatchery, 3 miles southwest of Detroit Lakes.

Records available.--July to september 1968.

Gage.--Boat lock: Staff gage read once daily. Datum of gage is $1,327.63 \mathrm{ft}$ above mean sea level, datum of 1929. Spillway: Staff gage read once daily. Datum of gage is $1,328.98 \mathrm{ft}$ above mean sea level, datum of 1929.

Extremes.--Maximum daily discharge during period July to september, 22 cfs July 23; no flow on many days.

Remarks.--Records fair. These records include flow in boat lock, spillway, and small bypass channel around gages.

Cooperation.--Gage readings and record of stoplog changes in dam furnished by Pelican River watershed District.

DISCHARGE, IN CUBIC FEET PER SECOND, PERIOD JULY TO SEPTEMBER 1968

\begin{tabular}{|c|c|c|c|c|c|c|c|c|c|c|c|c|}
\hline DAY & OCT & NOV & DEC & JAN & FEB & MAR & APR & MAY & JUN & JUL & AUG & SEP \\
\hline $\begin{array}{l}1 \\
2 \\
3 \\
4 \\
5\end{array}$ & & & & & & & & & & & $\begin{array}{l}12 \\
12 \\
12 \\
12 \\
12\end{array}$ & $\begin{array}{l}0 \\
0 \\
0 \\
0 \\
0\end{array}$ \\
\hline $\begin{array}{r}6 \\
7 \\
8 \\
9 \\
10\end{array}$ & & & & & & & & & & & $\begin{array}{l}12 \\
12 \\
12 \\
11 \\
11\end{array}$ & $\begin{array}{l}0 \\
0 \\
0 \\
0 \\
0\end{array}$ \\
\hline $\begin{array}{l}11 \\
12 \\
13 \\
14 \\
15\end{array}$ & & & & & & & & & & & $\begin{array}{l}11 \\
11 \\
11 \\
11 \\
11\end{array}$ & $\begin{array}{l}0 \\
0 \\
0 \\
0 \\
0\end{array}$ \\
\hline $\begin{array}{l}16 \\
17 \\
18 \\
19 \\
20\end{array}$ & & & & & & & & & & & $\begin{array}{l}11 \\
11 \\
11 \\
11 \\
11\end{array}$ & $\begin{array}{l}0 \\
0 \\
0 \\
0 \\
0\end{array}$ \\
\hline $\begin{array}{l}21 \\
22 \\
23 \\
24 \\
25\end{array}$ & & & & & & & & & & $\begin{array}{l}22 \\
19 \\
19\end{array}$ & $\begin{array}{l}11 \\
11 \\
11 \\
13 \\
11\end{array}$ & $\begin{array}{l}0 \\
0 \\
.3 \\
3 \\
.2\end{array}$ \\
\hline $\begin{array}{l}26 \\
27 \\
28 \\
29 \\
30 \\
31 \\
\end{array}$ & & ------ & & & ----- & & ----- & & ----- & $\begin{array}{l}18 \\
17 \\
14 \\
12 \\
12 \\
12\end{array}$ & $\begin{array}{r}11 \\
11 \\
11 \\
0 \\
0 \\
0 \\
\end{array}$ & $\begin{array}{r}.2 \\
0^{.1} \\
0 \\
0 \\
\end{array}$ \\
\hline $\begin{array}{l}\text { Total } \\
\text { Mean } \\
\text { Max } \\
\text { Min } \\
\text { Ac-ft } \\
\end{array}$ & & & & & & & & & & & $\begin{array}{r}318 \\
10.3 \\
13 \\
0 \\
631 \\
\end{array}$ & $\begin{array}{r}1.1 \\
0.037 \\
0.3 \\
0 \\
2 \\
\end{array}$ \\
\hline
\end{tabular}


5-0371. Pelican River at Sallie Lake Outlet, near Detroit Iakes, Minn.

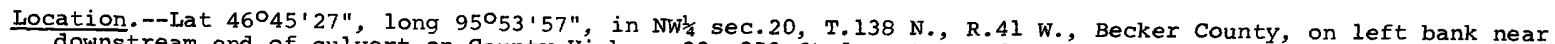
downstream end of culvert on County Highway 22, $250 \mathrm{ft}$ downstream from sallie Irake, 800 ft upstream from Minnesota Department of Conservation dam and 5 miles southwest of city of Detroit Lakes.

Records available.--July to september 1968.

Gage.--Digital water-stage recorder. Datum of gage is 1,327.58 ft above mean sea level, datum of 1929 .

Extremes.--Maximum daily discharge during period, 20 cfs July 25 ; minimum daily, 0.88 cfs sept. 14.

Remarks.--Records good.

DISCHARGE, IN CUBIC FEET PER SECOND, JulY to september 1968

\begin{tabular}{|c|c|c|c|c|c|c|c|c|c|c|c|c|}
\hline DAY & OCT & NOV & DEC & JAN & FEB & MAR & APR & MAY & JUN & JUL & AUG & $\overline{S E P}$ \\
\hline $\begin{array}{l}1 \\
2 \\
3 \\
4 \\
5\end{array}$ & & & & & & & & & & & $\begin{array}{c}11 \\
10 \\
10 \\
9.8 \\
10\end{array}$ & $\begin{array}{l}2.9 \\
2.5 \\
2.0 \\
1.8 \\
1.5\end{array}$ \\
\hline $\begin{array}{r}6 \\
7 \\
8 \\
9 \\
10\end{array}$ & & & & & & & & & & & $\begin{array}{l}11 \\
11 \\
14 \\
13 \\
12\end{array}$ & $\begin{array}{l}1.5 \\
1.5 \\
1.5 \\
1.5 \\
1.4\end{array}$ \\
\hline $\begin{array}{l}11 \\
12 \\
13 \\
14 \\
15\end{array}$ & & & & & & & & & & & $\begin{array}{c}10 \\
8.9 \\
8.0 \\
7.1 \\
8.2\end{array}$ & $\begin{array}{c}1.3 \\
1.2 \\
1.0 \\
.88 \\
1.0\end{array}$ \\
\hline $\begin{array}{l}16 \\
17 \\
18 \\
19 \\
20\end{array}$ & & & & & & & & & & & $\begin{array}{l}9.2 \\
8.5 \\
7.5 \\
8.3 \\
7.5\end{array}$ & $\begin{array}{l}1.6 \\
2.1 \\
2.2 \\
2.2 \\
2.0\end{array}$ \\
\hline $\begin{array}{l}26 \\
27 \\
28 \\
29 \\
30 \\
31 \\
\end{array}$ & & - & & & $\cdots$ & & - - & & $-\cdots$ & $\begin{array}{l}19 \\
19 \\
16 \\
14 \\
13 \\
14\end{array}$ & $\begin{array}{l}4.9 \\
4.1 \\
4.0 \\
3.6 \\
4.0 \\
3.9\end{array}$ & $\begin{array}{r}2.6 \\
2.6 \\
2.5 \\
2.2 \\
2.0\end{array}$ \\
\hline $\begin{array}{l}\text { Total } \\
\text { Mean } \\
\text { Max } \\
\text { Min }\end{array}$ & & & & & & & & & & & $\begin{array}{r}251.4 \\
8.11 \\
14 \\
3.6\end{array}$ & $\begin{array}{r}62.18 \\
2.07 \\
4.2 \\
0.88\end{array}$ \\
\hline
\end{tabular}


5-0391. Pelican River at Lake Melissa Outlet near Detroit Lakes, Minn.

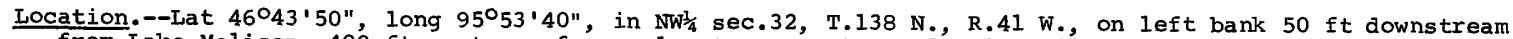
from Lake Melissa, 400 ft upstream from culvert on county road and 6 miles southwest of Detroit Lakes.

Records available.--July to september 1968.

Gage.--Staff gage read once daily. Altitude of gage is 1,330 ft (from topographic map).

Extremes.--Maximum discharge during period July to september, 35 cfs July 23 (gage height, 1.85 ft); minimum daily, 5.0 cfs sept. 10

Remarks.--Records fair. Records of chemical analyses for the water year 1968 are published in Part 2 of this report. Flow affected by storage in lakes above station.

Cooperation.--Gage readings furnished by Pelican River watershed District.

DISCHARGE, IN CUBIC FEET PER SECOND, PERIOD JULY TO SEPTEMBER 1968

\begin{tabular}{|c|c|c|c|c|c|c|c|c|c|c|c|c|}
\hline DAY & OCT & NOV & $\mathrm{DEC}$ & JAN & FEB & MAR & APR & MAY & JUN & JUL & AUG & SEP \\
\hline $\begin{array}{l}1 \\
2 \\
3 \\
4 \\
5\end{array}$ & & & & & & & & & & & $\begin{array}{l}25 \\
24 \\
24 \\
23 \\
23\end{array}$ & $\begin{array}{l}7.2 \\
6.2 \\
6.0 \\
5.4 \\
5.7\end{array}$ \\
\hline $\begin{array}{r}6 \\
7 \\
8 \\
9 \\
10\end{array}$ & & & & & & & & & & & $\begin{array}{l}22 \\
22 \\
24 \\
23 \\
20\end{array}$ & $\begin{array}{l}5.7 \\
5.7 \\
5.7 \\
5.4 \\
5.0\end{array}$ \\
\hline $\begin{array}{l}11 \\
12 \\
13 \\
14 \\
15\end{array}$ & & & & & & & & & & & $\begin{array}{l}18 \\
15 \\
14 \\
13 \\
12\end{array}$ & $\begin{array}{l}5.4 \\
5.7 \\
6.0 \\
6.4 \\
6.2\end{array}$ \\
\hline $\begin{array}{l}16 \\
17 \\
18 \\
19 \\
20\end{array}$ & & & & & & & & & & & $\begin{array}{l}13 \\
12 \\
12 \\
12 \\
12\end{array}$ & $\begin{array}{l}8.7 \\
10 \\
11 \\
12 \\
11\end{array}$ \\
\hline $\begin{array}{l}26 \\
27 \\
278 \\
29 \\
30 \\
31\end{array}$ & & -- & & & $-\cdots$ & & $-\ldots$ & & ----- & $\begin{array}{l}31 \\
31 \\
33 \\
28 \\
27 \\
27\end{array}$ & $\begin{array}{l}8.2 \\
7.2 \\
7.2 \\
7.0 \\
7.7 \\
7.4\end{array}$ & $\begin{array}{r}14 \\
14 \\
13 \\
13 \\
12 \\
\end{array}$ \\
\hline $\begin{array}{l}\text { Total } \\
\text { Mean } \\
\text { Max } \\
\text { Min } \\
\text { Ac-ft }\end{array}$ & & & & & & & & & & & $\begin{array}{r}458.5 \\
14.8 \\
25 \\
7.0 \\
\end{array}$ & $\begin{array}{r}278.4 \\
9.28 \\
16 \\
5.4 \\
\end{array}$ \\
\hline
\end{tabular}


Location.--Lat $46^{\circ} 20^{\prime} 10^{\prime \prime}$, long $96^{\circ} 07^{\prime} 00^{\prime \prime}$, in NE⿺ sec.17, T.133 N., R.43 W., on left bank 990 ft downstream from bridge on U. S. Highway 52, 3 miles northwest of Fergus Falls and $7 \frac{1}{2}$ miles upstream from mouth.

Drainage area.--482 sq $\mathrm{mi}$.

Records available.--June 1909 to December 1912, July 1942 to September 1968.

Gage.--Water-stage recorder. Datum of gage is 1,176.98 ft above mean sea level, datum of 1929 (levels by Minnesota Highway Department). June 19, 1909, to Dec. 31, 1912, staff gage at site 1 mile downstream at different datum. July 1, 1942, to Nov. 6, 1955, staff gage and Nov. 7, 1955, to Sept. 30, 1963, waterstage recorder at site $900 \mathrm{ft}$ upstream at datum $3.00 \mathrm{ft}$ higher.

Average discharge.--29 years $(1909-12,1942-68), 76.6$ cfs $(55,460$ acre-ft per year).

Extremes.--Maximum discharge during year, 194 cfs May 15 (gage height, 4.05 ft); maximum gage height recorded, $6.88 \mathrm{ft} \mathrm{Feb} .20$ (backwater from ice); minimum daily discharge, $1.1 \mathrm{cfs}$ Feb. 17-23.

1909-12, 1942-68: Maximum discharge, 756 cfs Mar. 29, 1943 (gage height, 8.53 ft, present datum); maximum gage height, $8.99 \mathrm{ft} \mathrm{Mar.} \mathrm{21,} 1966$ (backwater from ice); no flow on many days in 1946, 1949-50.

Remarks.--iRecords good except those for winter months, which are fair. Records of suspended-sediment loads

for water year 1968 published in Part 2 of this report. Flow affected by storage in lakes above station.

DISCHARGE, IN CFS, WATER YEAR OCTOBER 1967 TO SEPTEMBER 1968

\begin{tabular}{|c|c|c|c|c|c|c|c|c|c|c|c|c|}
\hline DAY & $\mathrm{OCT}$ & NoV & $\mathrm{DEC}$ & JAN & FEB & MAR & APR & MAY & JUN & JUL & AUG & SEP \\
\hline $\begin{array}{l}1 \\
2 \\
3 \\
4 \\
5\end{array}$ & $\begin{array}{l}22 \\
21 \\
20 \\
20 \\
19\end{array}$ & $\begin{array}{l}15 \\
15 \\
15 \\
14 \\
14\end{array}$ & $\begin{array}{l}14 \\
14 \\
15 \\
16 \\
17\end{array}$ & $\begin{array}{r}12 \\
11 \\
10 \\
8.0 \\
6.8\end{array}$ & $\begin{array}{l}2.1 \\
2.1 \\
2.1 \\
2.0 \\
2.0\end{array}$ & $\begin{array}{l}1.4 \\
1.5 \\
1.6 \\
1.8 \\
2.0\end{array}$ & $\begin{array}{l}78 \\
73 \\
78 \\
80 \\
72\end{array}$ & $\begin{array}{l}129 \\
126 \\
123 \\
120 \\
117\end{array}$ & $\begin{array}{l}145 \\
138 \\
138 \\
135 \\
132\end{array}$ & $\begin{array}{l}111 \\
108 \\
108 \\
104 \\
101\end{array}$ & $\begin{array}{l}67 \\
67 \\
64 \\
63 \\
61\end{array}$ & $\begin{array}{l}50 \\
50 \\
49 \\
49 \\
46\end{array}$ \\
\hline $\begin{array}{r}6 \\
7 \\
8 \\
9 \\
10\end{array}$ & $\begin{array}{l}19 \\
19 \\
19 \\
19 \\
19\end{array}$ & $\begin{array}{l}14 \\
14 \\
14 \\
15 \\
15\end{array}$ & $\begin{array}{l}19 \\
21 \\
20 \\
20 \\
19\end{array}$ & $\begin{array}{l}6.2 \\
5.7 \\
5.2 \\
4.6 \\
4.3\end{array}$ & $\begin{array}{l}1.9 \\
1.8 \\
1.7 \\
1.6 \\
1.6\end{array}$ & $\begin{array}{l}2.3 \\
2.8 \\
3.0 \\
2.9 \\
2.6\end{array}$ & $\begin{array}{r}97 \\
108 \\
138 \\
142 \\
120\end{array}$ & $\begin{array}{l}117 \\
117 \\
129 \\
138 \\
142\end{array}$ & $\begin{array}{l}142 \\
149 \\
160 \\
160 \\
174\end{array}$ & $\begin{array}{l}97 \\
94 \\
99 \\
90 \\
78\end{array}$ & $\begin{array}{l}63 \\
61 \\
59 \\
57 \\
57\end{array}$ & $\begin{array}{l}48 \\
48 \\
48 \\
46 \\
45\end{array}$ \\
\hline $\begin{array}{l}11 \\
12 \\
13 \\
14 \\
15\end{array}$ & $\begin{array}{l}20 \\
19 \\
18 \\
18 \\
18\end{array}$ & $\begin{array}{l}15 \\
15 \\
15 \\
14 \\
14\end{array}$ & $\begin{array}{l}19 \\
18 \\
18 \\
19 \\
20\end{array}$ & $\begin{array}{l}4.1 \\
3.9 \\
3.7 \\
3.6 \\
3.5\end{array}$ & $\begin{array}{l}1.5 \\
1.4 \\
1.3 \\
1.3 \\
1.2\end{array}$ & $\begin{array}{l}2.4 \\
2.5 \\
3.2 \\
4.7 \\
6.2\end{array}$ & $\begin{array}{l}108 \\
106 \\
104 \\
104 \\
101\end{array}$ & $\begin{array}{l}145 \\
152 \\
149 \\
170 \\
186\end{array}$ & $\begin{array}{l}182 \\
178 \\
178 \\
167 \\
149\end{array}$ & $\begin{array}{r}73 \\
86 \\
90 \\
111 \\
106\end{array}$ & $\begin{array}{l}57 \\
56 \\
54 \\
54 \\
56\end{array}$ & $\begin{array}{l}45 \\
44 \\
42 \\
42 \\
41\end{array}$ \\
\hline $\begin{array}{l}16 \\
17 \\
18 \\
19 \\
20\end{array}$ & $\begin{array}{l}18 \\
17 \\
16 \\
16 \\
15\end{array}$ & $\begin{array}{l}14 \\
14 \\
14 \\
15 \\
15\end{array}$ & $\begin{array}{l}24 \\
26 \\
25 \\
25 \\
25\end{array}$ & $\begin{array}{l}3.3 \\
3.1 \\
3.0 \\
2.8 \\
2.7\end{array}$ & $\begin{array}{l}1.2 \\
1.1 \\
1.1 \\
1.1 \\
1.1\end{array}$ & $\begin{array}{l}8.8 \\
11 \\
12 \\
10 \\
9.0\end{array}$ & $\begin{array}{r}101 \\
101 \\
101 \\
99 \\
106\end{array}$ & $\begin{array}{l}190 \\
186 \\
182 \\
163 \\
156\end{array}$ & $\begin{array}{l}142 \\
135 \\
126 \\
120 \\
117\end{array}$ & $\begin{array}{l}97 \\
94 \\
94 \\
92 \\
92\end{array}$ & $\begin{array}{l}56 \\
54 \\
56 \\
56 \\
56\end{array}$ & $\begin{array}{l}48 \\
46 \\
46 \\
48 \\
49\end{array}$ \\
\hline $\begin{array}{l}21 \\
22 \\
23 \\
24 \\
25\end{array}$ & $\begin{array}{l}16 \\
14 \\
14 \\
14 \\
14\end{array}$ & $\begin{array}{l}15 \\
15 \\
16 \\
16 \\
16\end{array}$ & $\begin{array}{l}24 \\
23 \\
21 \\
19 \\
18\end{array}$ & $\begin{array}{l}2.6 \\
2.5 \\
2.4 \\
2.3 \\
2.3\end{array}$ & $\begin{array}{l}1.1 \\
1.1 \\
1.1 \\
1.2 \\
1.2\end{array}$ & $\begin{array}{l}8.0 \\
7.2 \\
7.0 \\
7.6 \\
9.5\end{array}$ & $\begin{array}{l}120 \\
132 \\
160 \\
174 \\
163\end{array}$ & $\begin{array}{l}152 \\
145 \\
142 \\
138 \\
138\end{array}$ & $\begin{array}{l}132 \\
145 \\
142 \\
135 \\
129\end{array}$ & $\begin{array}{l}97 \\
94 \\
92 \\
90 \\
88\end{array}$ & $\begin{array}{l}53 \\
53 \\
53 \\
52 \\
51\end{array}$ & $\begin{array}{l}49 \\
67 \\
78 \\
73 \\
70\end{array}$ \\
\hline $\begin{array}{l}26 \\
27 \\
28 \\
29 \\
30 \\
31\end{array}$ & $\begin{array}{l}14 \\
14 \\
14 \\
14 \\
14 \\
15\end{array}$ & $\begin{array}{r}15 \\
15 \\
15 \\
14 \\
14 \\
\end{array}$ & $\begin{array}{l}17 \\
16 \\
15 \\
14 \\
13 \\
13\end{array}$ & $\begin{array}{l}2.2 \\
2.2 \\
2.2 \\
2.1 \\
2.1 \\
2.1\end{array}$ & \begin{tabular}{r}
1.3 \\
1.3 \\
1.4 \\
1.4 \\
\hdashline \\
\end{tabular} & $\begin{array}{l}17 \\
40 \\
65 \\
78 \\
82 \\
80\end{array}$ & $\begin{array}{r}142 \\
132 \\
135 \\
135 \\
129 \\
\end{array}$ & $\begin{array}{l}138 \\
142 \\
142 \\
145 \\
142 \\
149\end{array}$ & $\begin{array}{r}126 \\
120 \\
117 \\
111 \\
114 \\
\end{array}$ & $\begin{array}{l}84 \\
82 \\
77 \\
73 \\
72 \\
68 \\
\end{array}$ & $\begin{array}{l}50 \\
48 \\
46 \\
46 \\
51 \\
50\end{array}$ & $\begin{array}{r}68 \\
64 \\
60 \\
56 \\
52 \\
- \\
\end{array}$ \\
\hline $\begin{array}{l}\text { Total } \\
\text { Mean } \\
\text { Max } \\
\text { Min } \\
\text { Ac-ft } \\
\end{array}$ & $\begin{array}{r}529 \\
17.1 \\
22 \\
14 \\
1.050 \\
\end{array}$ & $\begin{array}{r}441 \\
14.7 \\
16 \\
14 \\
875 \\
\end{array}$ & $\begin{array}{r}587 \\
18.9 \\
26 \\
13 \\
1.160 \\
\end{array}$ & $\begin{array}{r}132.5 \\
4.27 \\
12 \\
2.1 \\
263\end{array}$ & $\begin{array}{r}42.3 \\
1.46 \\
2.1 \\
1.1 \\
84\end{array}$ & $\begin{array}{r}493.0 \\
15.9 \\
82 \\
1.4 \\
978\end{array}$ & $\begin{array}{r}3,439 \\
115 \\
174 \\
72 \\
6,820 \\
\end{array}$ & $\begin{array}{r}4,510 \\
145 \\
190 \\
117 \\
8,940 \\
\end{array}$ & $\begin{array}{r}4,238 \\
141 \\
182 \\
111 \\
8,410 \\
\end{array}$ & $\begin{array}{r}2,842 \\
91.7 \\
111 \\
68 \\
5,640 \\
\end{array}$ & $\begin{array}{r}1,727 \\
55.7 \\
67 \\
46 \\
3,420 \\
\end{array}$ & $\begin{array}{r}1,567 \\
52.2 \\
78 \\
41 \\
3,110 \\
\end{array}$ \\
\hline $\begin{array}{l}\text { Cal yr } \\
\text { wtr yr }\end{array}$ & $\begin{array}{l}967: \\
968:\end{array}$ & $\begin{array}{l}\text { tal } \\
\text { tal }\end{array}$ & 7.8 & $\begin{array}{l}\text { Mean } \\
\text { Mean }\end{array}$ & $\begin{array}{l}\text { Max } \\
\text { Max }\end{array}$ & $\begin{array}{l}600 \\
190\end{array}$ & $\begin{array}{ll}\mathrm{n} & 13 \\
\mathrm{n} & 1.1\end{array}$ & $\begin{array}{l}\text { Ac-ft } \\
\text { Ac-ft }\end{array}$ & $\begin{array}{l}800 \\
760 \\
\end{array}$ & & & \\
\hline
\end{tabular}


5-0459.5 Orwell Reservoir near Fergus Falls, Minn.

Location.--Lat $46^{\circ} 12^{\prime} 55^{\prime \prime}$, long 96 $10^{\prime} 40^{\prime \prime}$, in SW $\frac{1}{4}$ sec.26, T.132 N., R.44 W., at dam on otter Tail River at outlet of Orwell Reservoir, 7 miles southwest of Fergus Falls, Minn.

Drainage area. $--1,830 \mathrm{sq} \mathrm{mi}$, approximately.

Records available.--March 1953 to september 1968.

Gage.--Water-stage recorder. Datum of gage is 1,000.00 ft above mean sea level, adjustment of 1912 . Gage readings reduced to elevations above mean sea level.

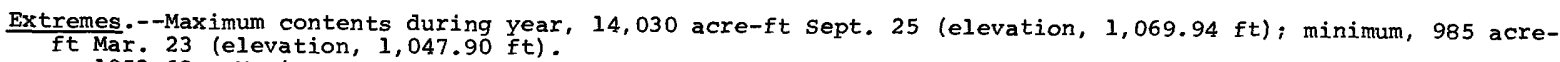

1953-68: Maximum contents, 16,920 acre-ft June 17, 1962, May 23, 1966 (elevation, 1,072.38 ft); minimum (after initial filling), 844 acre-ft Aug. 26, 27, 1953 (elevation, 1,046.96 ft).

Remarks.--Reservoir is formed by earth dam with concrete spillway with one taintor gate; storage began in March 1953. Capacity to elevation, 1,070 ft (maximum operating stage) is 14, 100 acre-ft of which 13,100 acre-ft is controlled storage above elevation 1,048 ft (minimum operating stage). Dead storage, 210 acre-ft. Figures given herein represent total contents. Reservoir is used for flood control and to increase low flow for water supply and pollution abatement.

Cooperation.--Records furnished by Corps of Engineers.

Month-end elevation and contents, water year October 1967 to september 1968

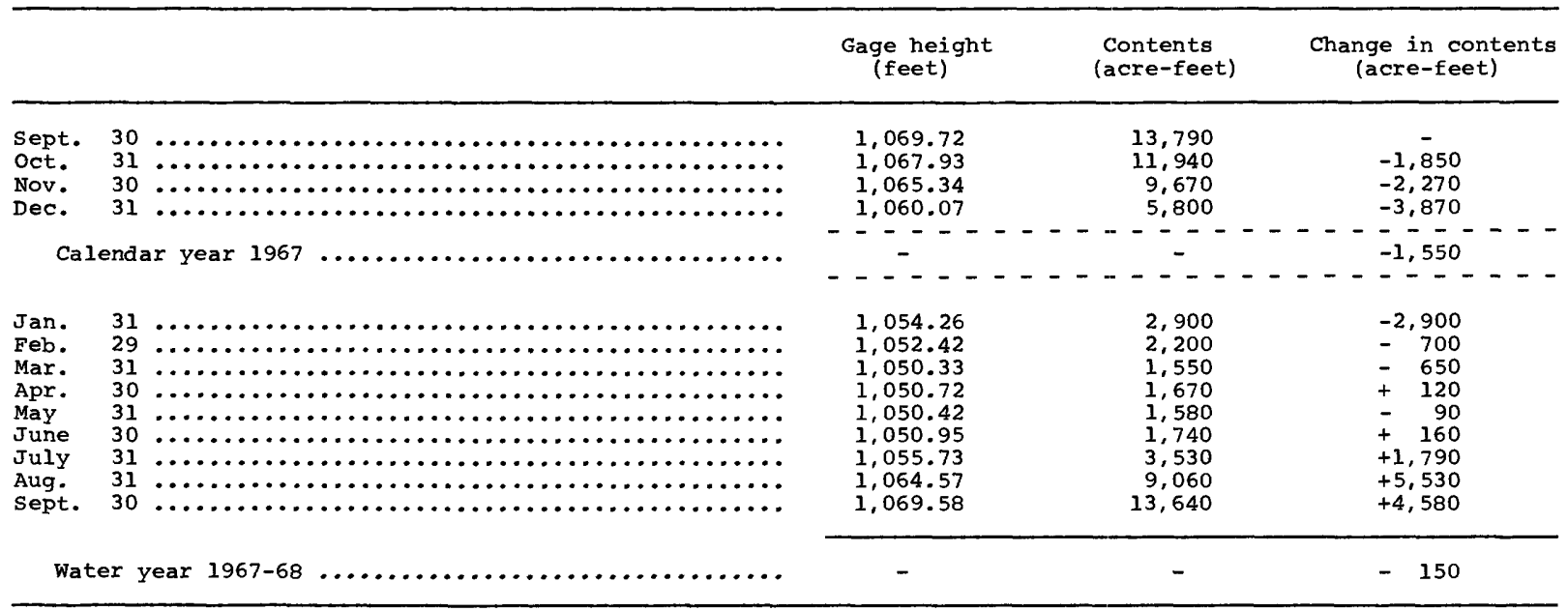


5-0460. Otter Tail River below Orwell Dam, near Fergus Falls, Minn.

Location.--Lat $46^{\circ} 12^{\prime} 35^{\prime \prime}$, long $96^{\circ} 11^{\prime} 05^{\prime \prime}$, in NE⿱ from Orwell Dam, 6.1 miles downstream from Dayton Hollow Dam, 8 miles southwest of Fergus Falls, and 1l.1 miles downstream from Pelican River.

Drainage area.--1,830 sq $\mathrm{mi}$, approximately.

Records available.--October 1930 to September 1968. Prior to October 1952, published as Otter Tail River below Pelican River, near Fergus Falls. Monthly discharge only for some periods, published in wSP 1308.

Gage.-Water-stage recorder. Datum of gage is $1,029.65 \mathrm{ft}$ above mean sea level, adjustment of 1912 (levels by Corps of Engineers). Oct. 11, 1930, to Nov. 17, 1933, at same site at datum 2.00 ft higher; Nov. 18, 1933, to Mar. 21, 1953, at site 6.1 miles upstream at datum $40.30 \mathrm{ft}$ higher.

Average discharge.-- 38 years, 282 cfs $(204,200$ acre-ft per year).

Extremes.--Maximum discharge during year, 714 cfs May 14 (gage height, 3.37 ft); minimum, 107 cfs Sept. 9, 10 , 11 (gage height, $2.32 \mathrm{ft}$ ).

1930-68: Maximum discharge, 1,710 cfs June 17, 1953 (gage height, 5.60 ft, backwater from aquatic vegetation); minimum, 1.0 cfs May 2, 1934, Sept. 30, 1935; minimum daily, 1.6 cfs Feb. 7, 1937.

Remarks.--Records good. Flow regulated by orwell Reservoir beginning Mar. 21 , 1953 (see preceding page) and powerplants upstream.

DISCHARGE, IN CFS, WATER YEAR OCTOBER 1967 TO SEPTEMBER 1968

\begin{tabular}{|c|c|c|c|c|c|c|c|c|c|c|c|c|}
\hline DAY & OCT & NOV & DEC & JAN & FEB & MAR & APR & MAY & JUN & JUL & AUG & SEP \\
\hline $\begin{array}{l}1 \\
2 \\
3 \\
4 \\
5\end{array}$ & $\begin{array}{l}173 \\
191 \\
201 \\
201 \\
205\end{array}$ & $\begin{array}{l}201 \\
201 \\
196 \\
196 \\
196\end{array}$ & $\begin{array}{l}168 \\
164 \\
163 \\
162 \\
161\end{array}$ & $\begin{array}{l}149 \\
149 \\
149 \\
149 \\
145\end{array}$ & $\begin{array}{l}133 \\
133 \\
133 \\
137 \\
137\end{array}$ & $\begin{array}{l}161 \\
161 \\
161 \\
161 \\
161\end{array}$ & $\begin{array}{l}322 \\
283 \\
272 \\
283 \\
283\end{array}$ & $\begin{array}{l}507 \\
559 \\
553 \\
519 \\
496\end{array}$ & $\begin{array}{l}599 \\
593 \\
593 \\
587 \\
553\end{array}$ & $\begin{array}{l}536 \\
530 \\
530 \\
507 \\
440\end{array}$ & $\begin{array}{l}300 \\
235 \\
240 \\
246 \\
251\end{array}$ & $\begin{array}{l}173 \\
173 \\
153 \\
130 \\
133\end{array}$ \\
\hline $\begin{array}{r}6 \\
7 \\
8 \\
9 \\
10\end{array}$ & $\begin{array}{l}205 \\
205 \\
201 \\
201 \\
201\end{array}$ & $\begin{array}{l}196 \\
196 \\
191 \\
187 \\
187\end{array}$ & $\begin{array}{l}160 \\
160 \\
159 \\
159 \\
158\end{array}$ & $\begin{array}{l}145 \\
145 \\
141 \\
141 \\
137\end{array}$ & $\begin{array}{l}137 \\
137 \\
141 \\
141 \\
141\end{array}$ & $\begin{array}{l}157 \\
182 \\
278 \\
322 \\
311\end{array}$ & $\begin{array}{l}283 \\
278 \\
289 \\
289 \\
390\end{array}$ & $\begin{array}{l}496 \\
457 \\
502 \\
587 \\
616\end{array}$ & $\begin{array}{l}536 \\
542 \\
542 \\
547 \\
559\end{array}$ & $\begin{array}{l}423 \\
446 \\
457 \\
457 \\
440\end{array}$ & $\begin{array}{l}251 \\
251 \\
256 \\
261 \\
256\end{array}$ & $\begin{array}{l}133 \\
133 \\
133 \\
123 \\
107\end{array}$ \\
\hline $\begin{array}{l}11 \\
12 \\
13 \\
14 \\
15\end{array}$ & $\begin{array}{l}201 \\
201 \\
201 \\
201 \\
201\end{array}$ & $\begin{array}{l}187 \\
182 \\
182 \\
182 \\
182\end{array}$ & $\begin{array}{l}158 \\
158 \\
157 \\
157 \\
157\end{array}$ & $\begin{array}{l}137 \\
141 \\
141 \\
141 \\
137\end{array}$ & $\begin{array}{l}141 \\
145 \\
145 \\
145 \\
149\end{array}$ & $\begin{array}{l}306 \\
300 \\
300 \\
246 \\
240\end{array}$ & $\begin{array}{l}440 \\
451 \\
446 \\
446 \\
395\end{array}$ & $\begin{array}{l}616 \\
610 \\
616 \\
674 \\
709\end{array}$ & $\begin{array}{l}627 \\
656 \\
662 \\
656 \\
645\end{array}$ & $\begin{array}{l}395 \\
390 \\
390 \\
395 \\
401\end{array}$ & $\begin{array}{l}256 \\
261 \\
261 \\
256 \\
261\end{array}$ & $\begin{array}{l}107 \\
110 \\
110 \\
110 \\
110\end{array}$ \\
\hline $\begin{array}{l}16 \\
17 \\
18 \\
19 \\
20\end{array}$ & $\begin{array}{l}201 \\
205 \\
201 \\
201 \\
205\end{array}$ & $\begin{array}{l}182 \\
178 \\
178 \\
178 \\
178\end{array}$ & $\begin{array}{l}155 \\
155 \\
154 \\
153 \\
153\end{array}$ & $\begin{array}{l}137 \\
137 \\
133 \\
133 \\
133\end{array}$ & $\begin{array}{l}153 \\
153 \\
141 \\
141 \\
153\end{array}$ & $\begin{array}{l}267 \\
294 \\
328 \\
350 \\
339\end{array}$ & $\begin{array}{l}317 \\
300 \\
362 \\
401 \\
401\end{array}$ & $\begin{array}{l}703 \\
697 \\
685 \\
674 \\
622\end{array}$ & $\begin{array}{l}616 \\
553 \\
530 \\
542 \\
547\end{array}$ & $\begin{array}{l}412 \\
423 \\
429 \\
440 \\
446\end{array}$ & $\begin{array}{l}261 \\
261 \\
251 \\
251 \\
240\end{array}$ & $\begin{array}{l}116 \\
120 \\
113 \\
113 \\
133\end{array}$ \\
\hline $\begin{array}{l}21 \\
22 \\
23 \\
24 \\
25\end{array}$ & $\begin{array}{l}205 \\
201 \\
205 \\
205 \\
205\end{array}$ & $\begin{array}{l}178 \\
178 \\
178 \\
178 \\
178\end{array}$ & $\begin{array}{l}153 \\
153 \\
153 \\
153 \\
153\end{array}$ & $\begin{array}{l}133 \\
130 \\
126 \\
126 \\
126\end{array}$ & $\begin{array}{l}157 \\
161 \\
161 \\
165 \\
165\end{array}$ & $\begin{array}{l}334 \\
289 \\
256 \\
235 \\
251\end{array}$ & $\begin{array}{l}423 \\
446 \\
451 \\
479 \\
547\end{array}$ & $\begin{array}{l}593 \\
599 \\
604 \\
610 \\
610\end{array}$ & $\begin{array}{l}553 \\
564 \\
559 \\
570 \\
587\end{array}$ & $\begin{array}{l}451 \\
423 \\
418 \\
423 \\
429\end{array}$ & $\begin{array}{l}224 \\
201 \\
205 \\
210 \\
214\end{array}$ & $\begin{array}{l}161 \\
205 \\
219 \\
224 \\
261\end{array}$ \\
\hline $\begin{array}{l}26 \\
27 \\
28 \\
29 \\
30 \\
31 \\
\end{array}$ & $\begin{array}{l}205 \\
205 \\
205 \\
205 \\
201 \\
201 \\
\end{array}$ & $\begin{array}{r}178 \\
177 \\
176 \\
174 \\
172 \\
\\
\end{array}$ & $\begin{array}{l}153 \\
153 \\
153 \\
149 \\
149 \\
153 \\
\end{array}$ & $\begin{array}{l}126 \\
126 \\
126 \\
130 \\
130 \\
133 \\
\end{array}$ & $\begin{array}{r}161 \\
161 \\
161 \\
161 \\
- \\
\end{array}$ & $\begin{array}{l}256 \\
267 \\
306 \\
322 \\
328 \\
339 \\
\end{array}$ & $\begin{array}{r}576 \\
564 \\
553 \\
496 \\
457 \\
---- \\
\end{array}$ & $\begin{array}{l}610 \\
610 \\
610 \\
604 \\
599 \\
593 \\
\end{array}$ & $\begin{array}{r}582 \\
547 \\
536 \\
530 \\
536 \\
---- \\
\end{array}$ & $\begin{array}{l}423 \\
434 \\
429 \\
372 \\
339 \\
345 \\
\end{array}$ & $\begin{array}{l}210 \\
191 \\
169 \\
165 \\
173 \\
173 \\
\end{array}$ & $\begin{array}{r}283 \\
283 \\
283 \\
283 \\
283 \\
- \\
\end{array}$ \\
\hline $\begin{array}{l}\text { Total } \\
\text { Mean } \\
\text { Max } \\
\text { Min } \\
\text { Ac-ft } \\
\end{array}$ & $\begin{array}{r}6,245 \\
201 \\
205 \\
173 \\
12,390 \\
\end{array}$ & $\begin{array}{r}5,523 \\
184 \\
201 \\
172 \\
10,950 \\
\end{array}$ & $\begin{array}{r}4,846 \\
156 \\
168 \\
149 \\
9,610 \\
\end{array}$ & $\begin{array}{r}4,232 \\
137 \\
149 \\
126 \\
8,390 \\
\end{array}$ & $\begin{array}{r}4,289 \\
148 \\
165 \\
133 \\
8,510 \\
\end{array}$ & $\begin{array}{r}8,208 \\
265 \\
350 \\
157 \\
16,280 \\
\end{array}$ & $\begin{array}{r}11,923 \\
397 \\
576 \\
272 \\
23,650 \\
\end{array}$ & $\begin{array}{r}18,540 \\
598 \\
709 \\
457 \\
36,770 \\
\end{array}$ & $\begin{array}{r}17,249 \\
575 \\
662 \\
530 \\
34,210 \\
\end{array}$ & $\begin{array}{r}13,373 \\
431 \\
536 \\
339 \\
26,520 \\
\end{array}$ & $\begin{array}{r}7,241 \\
234 \\
300 \\
165 \\
14,360 \\
\end{array}$ & $\begin{array}{r}5,018 \\
167 \\
283 \\
107 \\
9,950 \\
\end{array}$ \\
\hline $\begin{array}{l}\text { Cal yr } \\
\text { wtr yr }\end{array}$ & $\begin{array}{l}1967: \\
1968:\end{array}$ & $\begin{array}{l}\text { Total } \\
\text { Total }\end{array}$ & $\begin{array}{l}168,858 \\
106,687\end{array}$ & $\begin{array}{l}\text { Mean } \\
\text { Mean }\end{array}$ & $\begin{array}{l}463 \\
291 \\
\end{array}$ & $\begin{array}{r}1.120 \\
709\end{array}$ & $\begin{array}{l}\text { Min } \\
\text { Min }\end{array}$ & $\begin{array}{r}35 \\
107 \\
\end{array}$ & & $\begin{array}{l}300 \\
000\end{array}$ & & \\
\hline
\end{tabular}


5-0500. Bois de Sioux River near White Rock, S. Dak.

Location.--Lat $45^{\circ} 51^{\prime} 45^{\prime \prime}$, long $96^{\circ} 34^{\prime} 25^{\prime \prime}$, in SW $\frac{1}{4} W^{\prime} \frac{1}{4}$ sec. 27, T.128 N., R.47 W., on left bank just downstream from Big slough outlet, $300 \mathrm{ft}$ downstream from White Rock Dam, 4 miles south of White Rock, and $5 \mathrm{miles}$ northwest of Wheaton, Minn.

Drainage area. $-1,160 \mathrm{sq} \mathrm{mi}$, approximately.

Records available.--October 1941 to September 1968.

Gage.--Water-stage recorder. Datum of gage is $960.00 \mathrm{ft}$ above mean sea level, adjustment of 1912 (levels by Corps of Engineers). Prior to Jan. 14, 1943, staff gage at same site at datum 0.11 ft lower. Jan. 15,

1943, to sept. 30, 1963, water stage recorder at same site at datum 0.11 ft lower.

Average discharge.--27 years, 82.0 cfs $(59,360$ acre-ft per year).

Extremes.--Maximum discharge during year, 58 cfs Apr. 23 (gage height, 3.94 ft); no flow for many days.

1941-68: Maximum discharge, 1,620 cfs Aug. 6, 1962 (gage height, 11.41 ft, present datum); no flow at times in most years.

Remarks.--Records good except those for winter months, which are fair. Flow regulated by Lake Traverse-

Bois de Sioux Flood Control and Water Conservation project (available capacity for flood control, 137,000 acre-ft).

DISCHARGE, IN CFS, WATER YEAR OCTOBER 1967 TO SEPTEMBER 1968

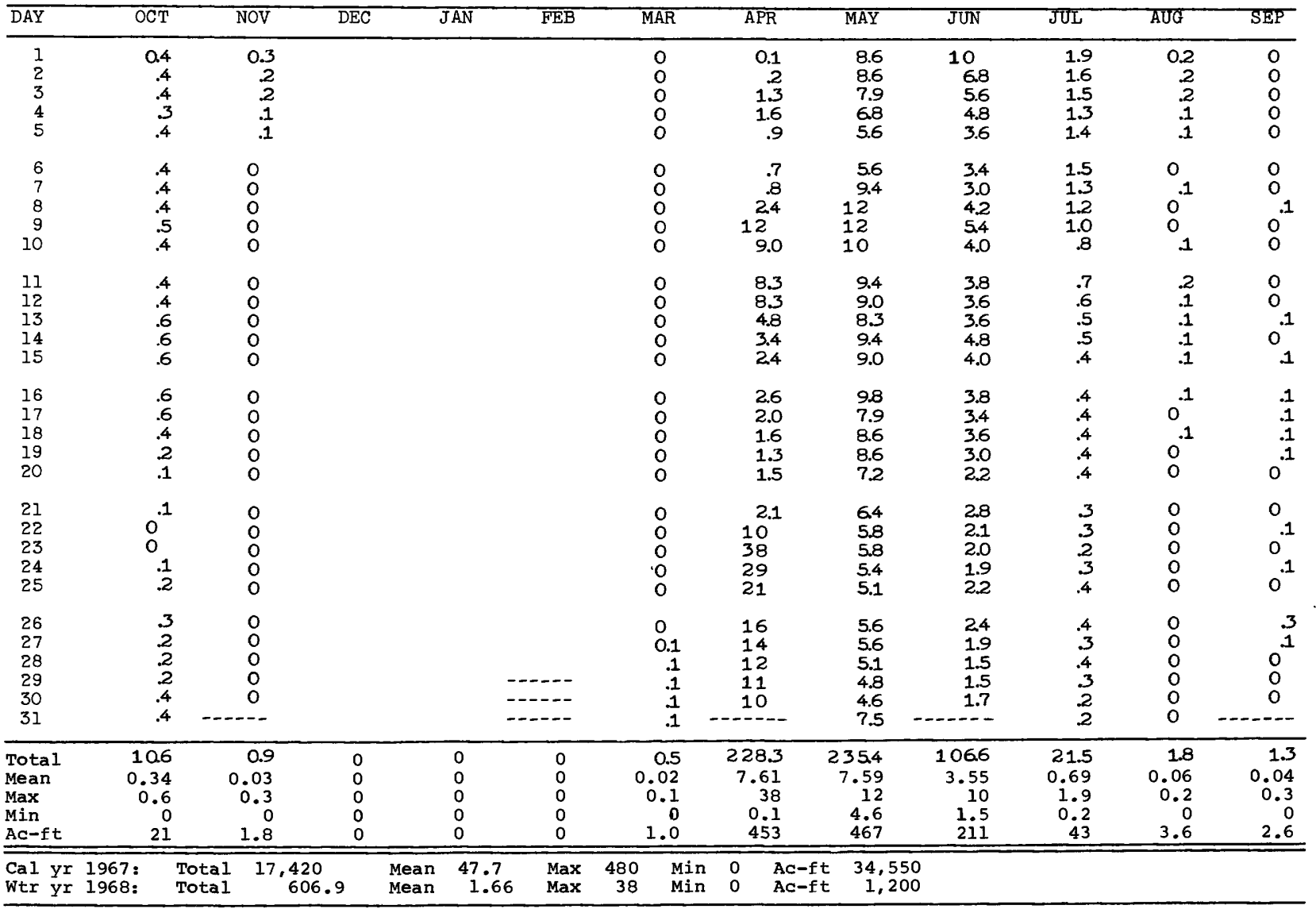




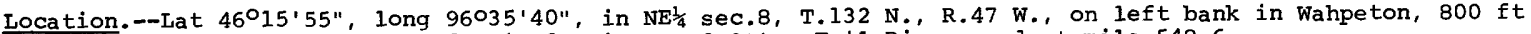
downstream from confluence of Bois de'sioux and otter Tail Rivers and at mile 548.6 .

Drainage area.--4,010 sq $\mathrm{mi}$, approximately.

Records available.--April 1942 to september 1968. Gage-height records collected in this vicinity since 1917 are contained in reports of U.S. Weather Bureau.

Gage.--Water-stage recorder and concrete and wooden dam. Datum of gage is $943 \mathrm{ft}$ above mean sea level, datum of 1929. Prior to Aug, 6, 1943, U.S. Weather Bureau staff gage 800 ft upstream, converted to present datum. Aug. 6, 1943, to Oct. 27, 1950, chain gage at present site and datum.

Average discharge.--25 years $(1943-68), 529 \mathrm{cfs}(383,000$ acre-ft per year).

Extremes.--Maximum discharge during year, 708 cfs May 18 (gage height, 4.95 ft); maximum gage height, 4.97 ft Mar. 19 (backwater from ice); minimum discharge, $39 \mathrm{cfs}$ Nov. 27 (gage height, $2.29 \mathrm{ft}$ ). 1942-68: Maximum discharge, 7,130 cfs Apr. 12, 1952 (gage height, 14.99 ft); minimum, 8 cfs Aug. 25, 1961 (gage height, $2.26 \mathrm{ft}$ ); minimum gage height, $2.05 \mathrm{ft}$ sept. 17, 18, 1967. Maximum stage known, $17.0 \mathrm{ft}$ in spring of 1897.

Remarks.--Records good. Flow regulated by Orwell Reservoir (capacity, 14,100 acre-ft at elevation 1,070 ft above mean sea level, adjustment of 1912); Lake Traverse (capacity, 137,000 acre-ft), available for flood control; numerous other controlled lakes and ponds, and several powerplants.

DISCHARGE, IN CFS, WATER YEAR OCTOBER 1967 TO SEPTEMBER 1968

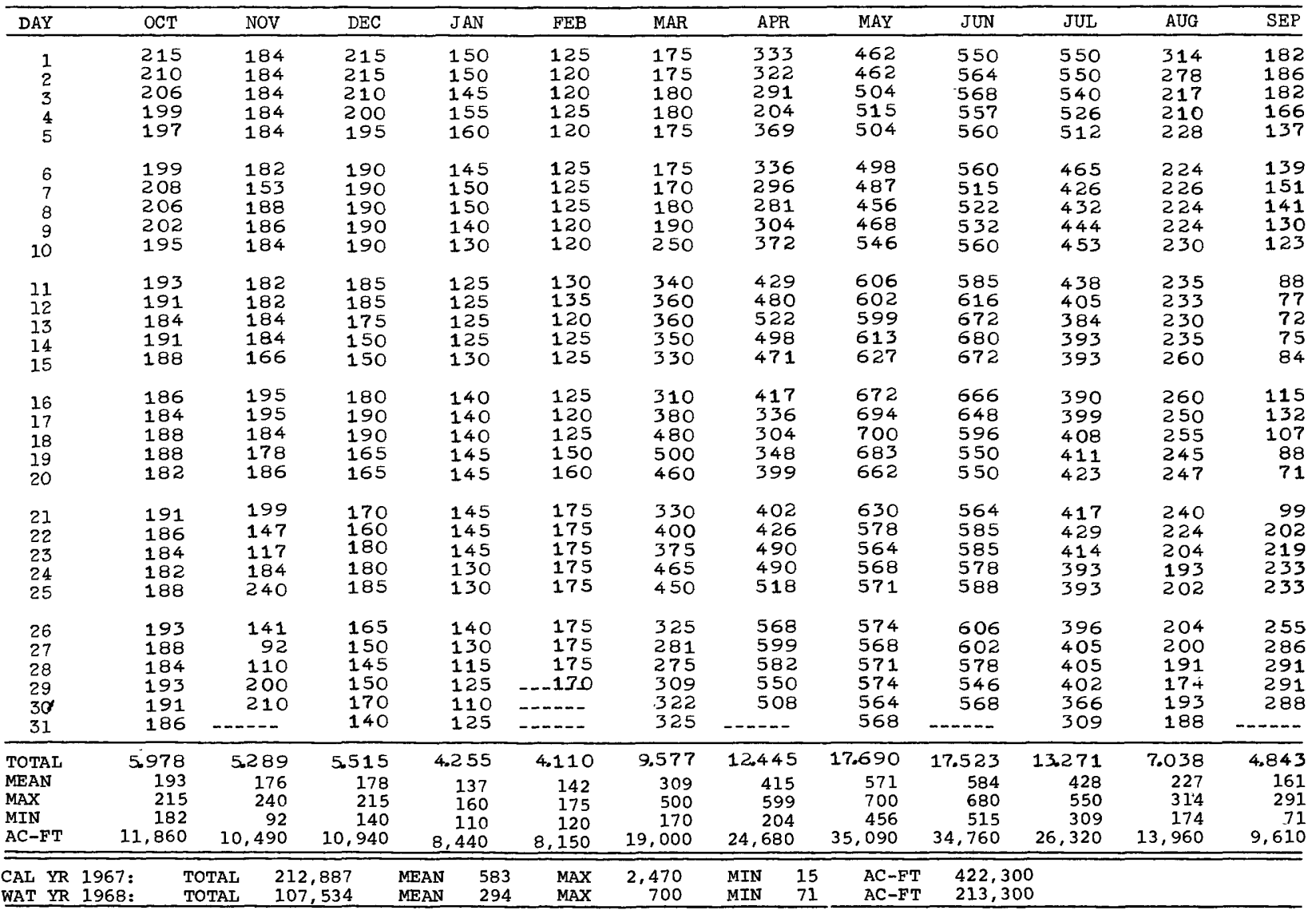


5-0540. Red River of the North at Fargo, N. Dak.

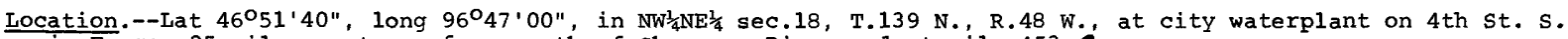
in Fargo, 25 miles upstream from mouth of sheyenne River and at mile 453.0

Drainage area.,--6,800 sq $\mathrm{mi}$, approximately.

Records available.--May 1901 to september 1968. Published as "at Moorhead, Minn." 1901. Monthly discharge only for some periods, published in WSP 1308.

Gage.--Water-stage recorder and concrete control. Datum of gage is $861.8 \mathrm{ft}$ above mean sea level, datum of

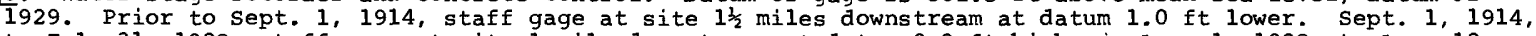
to July 31, 1928, staff gage at site 1 mile downstream at datum 9.3 ft higher: Aug. 1, 1928, to Apr. 12, 1959, staff gage at site 1 mile downstream at datum $5.6 \mathrm{ft}$ higher. Affi 13, 1959, to Sept. 30, 1960, waterstage recorder at site 2 miles upstream at datum $5.6 \mathrm{ft}$ higher. oct. 1, 1960, to sept. 30, 1962, waterstage recorder at present site at datum $5.6 \mathrm{ft}$ higher. Since oct. 1, 1960, auxiliary water-stage recorder 2 miles upstream.

Average discharge (unadjusted) .--67 years, 525 cfs $(380,100$ acre-ft per year); median of yearly mean discharges, 430 cfs $(311,000$ acre-ft per year).

Extremes.--Maximum discharge during year, 788 cfs Apr. 30 (gage height, 14.71 ft); maximum gage height, 14.77 ft May 20 (backwater from debris); minimum discharge, 88 cfs sept. 16 (gage height, $13.52 \mathrm{ft}$ ).

1901-68: Maximum discharge, 16,300 cfs Apr. 15, 16, 1952; maximum gage height, 28.79 ft Apr. 16, 1952, datum then in use; no flow for many days in each year for period 1932-41.

Maximum stage known, 40.1 ft Apr. 7, 1897, site and datum in use prior to 1914 (discharge, 25,000 cfs) .

Remarks.--Records good. Flow regulated by Orwell Reservoir (capacity, 14,100 acre-ft at elevation 1,070 ft above mean sea level, adjustment of 1912); Lake Traverse (capacity, 137,000 acre-ft, available for flood control); other controlled lakes and ponds and several powerplants. Some small diversions for municipal supply. Figures of daily discharge do not include diversion by cities of Fargo and Moorhead. Records of chemical analyses for water year 1968 are published in Part 2 of this report.

DISCHARGE, IN CFS, WATER YEAR OCTOBER 1967 TO SEPTEMBER 1968

\begin{tabular}{|c|c|c|c|c|c|c|c|c|c|c|c|c|}
\hline DAY & $\mathrm{OCT}$ & NOV & $\mathrm{DEC}$ & JAN & FEB & MAR & APR & MAY & JUN & JUL & AUG & SEP \\
\hline $\begin{array}{l}1 \\
2 \\
3 \\
4 \\
5\end{array}$ & $\begin{array}{l}133 \\
141 \\
152 \\
141 \\
133\end{array}$ & $\begin{array}{l}190 \\
190 \\
190 \\
186 \\
186\end{array}$ & $\begin{array}{l}148 \\
169 \\
194 \\
203 \\
194\end{array}$ & $\begin{array}{l}160 \\
152 \\
141 \\
152 \\
165\end{array}$ & $\begin{array}{l}107 \\
101 \\
111 \\
114 \\
118\end{array}$ & $\begin{array}{l}177 \\
177 \\
177 \\
208 \\
203\end{array}$ & $\begin{array}{l}362 \\
362 \\
374 \\
380 \\
374\end{array}$ & $\begin{array}{l}740 \\
700 \\
628 \\
612 \\
636\end{array}$ & $\begin{array}{l}620 \\
620 \\
612 \\
605 \\
620\end{array}$ & $\begin{array}{l}684 \\
644 \\
612 \\
582 \\
582\end{array}$ & $\begin{array}{l}356 \\
299 \\
283 \\
272 \\
218\end{array}$ & $\begin{array}{l}177 \\
177 \\
169 \\
165 \\
169\end{array}$ \\
\hline $\begin{array}{r}6 \\
7 \\
8 \\
9 \\
10\end{array}$ & $\begin{array}{l}141 \\
160 \\
165 \\
173 \\
169\end{array}$ & $\begin{array}{l}186 \\
160 \\
129 \\
129 \\
186\end{array}$ & $\begin{array}{l}190 \\
181 \\
181 \\
181 \\
186\end{array}$ & $\begin{array}{l}165 \\
165 \\
165 \\
152 \\
156\end{array}$ & $\begin{array}{l}122 \\
114 \\
114 \\
114 \\
114\end{array}$ & $\begin{array}{l}203 \\
222 \\
251 \\
257 \\
257\end{array}$ & $\begin{array}{l}304 \\
316 \\
398 \\
398 \\
392\end{array}$ & $\begin{array}{l}644 \\
676 \\
644 \\
620 \\
590\end{array}$ & $\begin{array}{l}628 \\
636 \\
636 \\
598 \\
590\end{array}$ & $\begin{array}{l}545 \\
517 \\
456 \\
423 \\
404\end{array}$ & $\begin{array}{l}194 \\
203 \\
236 \\
203 \\
199\end{array}$ & $\begin{array}{l}173 \\
165 \\
144 \\
144 \\
148\end{array}$ \\
\hline $\begin{array}{l}11 \\
12 \\
13 \\
14 \\
15\end{array}$ & $\begin{array}{l}169 \\
165 \\
169 \\
173 \\
177\end{array}$ & $\begin{array}{l}222 \\
190 \\
173 \\
160 \\
173\end{array}$ & $\begin{array}{l}190 \\
190 \\
186 \\
177 \\
165\end{array}$ & $\begin{array}{l}156 \\
152 \\
144 \\
141 \\
133\end{array}$ & $\begin{array}{l}114 \\
114 \\
118 \\
125 \\
129\end{array}$ & $\begin{array}{l}262 \\
277 \\
333 \\
392 \\
423\end{array}$ & $\begin{array}{l}462 \\
552 \\
590 \\
620 \\
628\end{array}$ & $\begin{array}{l}598 \\
628 \\
676 \\
740 \\
740\end{array}$ & $\begin{array}{l}598 \\
636 \\
660 \\
700 \\
740\end{array}$ & $\begin{array}{l}398 \\
410 \\
410 \\
398 \\
374\end{array}$ & $\begin{array}{l}199 \\
199 \\
203 \\
213 \\
208\end{array}$ & $\begin{array}{r}144 \\
137 \\
129 \\
107 \\
94\end{array}$ \\
\hline $\begin{array}{l}16 \\
17 \\
18 \\
19 \\
20\end{array}$ & $\begin{array}{l}177 \\
177 \\
173 \\
177 \\
169\end{array}$ & $\begin{array}{l}177 \\
169 \\
177 \\
190 \\
181\end{array}$ & $\begin{array}{l}141 \\
137 \\
144 \\
156 \\
160\end{array}$ & $\begin{array}{l}125 \\
125 \\
129 \\
129 \\
125\end{array}$ & $\begin{array}{l}129 \\
129 \\
141 \\
144 \\
137\end{array}$ & $\begin{array}{l}430 \\
442 \\
468 \\
510 \\
482\end{array}$ & $\begin{array}{l}617 \\
582 \\
517 \\
436 \\
416\end{array}$ & $\begin{array}{l}724 \\
732 \\
756 \\
772 \\
772\end{array}$ & $\begin{array}{l}756 \\
748 \\
732 \\
708 \\
660\end{array}$ & $\begin{array}{l}380 \\
380 \\
362 \\
362 \\
374\end{array}$ & $\begin{array}{l}232 \\
236 \\
251 \\
232 \\
232\end{array}$ & $\begin{array}{r}98 \\
111 \\
114 \\
125 \\
129\end{array}$ \\
\hline $\begin{array}{l}21 \\
22 \\
23 \\
24 \\
25\end{array}$ & $\begin{array}{l}173 \\
169 \\
181 \\
190 \\
194\end{array}$ & $\begin{array}{l}160 \\
169 \\
129 \\
125 \\
118\end{array}$ & $\begin{array}{l}169 \\
148 \\
152 \\
144 \\
137\end{array}$ & $\begin{array}{l}129 \\
125 \\
125 \\
125 \\
118\end{array}$ & $\begin{array}{l}137 \\
148 \\
152 \\
173 \\
177\end{array}$ & $\begin{array}{l}524 \\
545 \\
482 \\
416 \\
436\end{array}$ & $\begin{array}{l}398 \\
442 \\
510 \\
524 \\
582\end{array}$ & $\begin{array}{l}764 \\
740 \\
708 \\
668 \\
628\end{array}$ & $\begin{array}{l}605 \\
598 \\
605 \\
612 \\
620\end{array}$ & $\begin{array}{l}380 \\
386 \\
386 \\
398 \\
386\end{array}$ & $\begin{array}{l}232 \\
222 \\
232 \\
213 \\
203\end{array}$ & $\begin{array}{l}122 \\
144 \\
122 \\
148 \\
181\end{array}$ \\
\hline $\begin{array}{l}26 \\
27 \\
28 \\
29 \\
30 \\
31\end{array}$ & $\begin{array}{l}190 \\
190 \\
208 \\
203 \\
194 \\
194\end{array}$ & $\begin{array}{r}133 \\
122 \\
101 \\
98 \\
133 \\
\end{array}$ & $\begin{array}{l}148 \\
160 \\
152 \\
152 \\
144 \\
152\end{array}$ & $\begin{array}{r}114 \\
104 \\
104 \\
101 \\
98 \\
98\end{array}$ & $\begin{array}{r}186 \\
186 \\
181 \\
177 \\
- \\
\end{array}$ & $\begin{array}{l}496 \\
560 \\
575 \\
582 \\
449 \\
368\end{array}$ & $\begin{array}{r}620 \\
660 \\
716 \\
764 \\
780 \\
-\end{array}$ & $\begin{array}{l}620 \\
620 \\
636 \\
636 \\
628 \\
636\end{array}$ & $\begin{array}{r}605 \\
598 \\
598 \\
590 \\
660\end{array}$ & $\begin{array}{l}374 \\
368 \\
374 \\
380 \\
380 \\
380\end{array}$ & $\begin{array}{l}190 \\
190 \\
190 \\
186 \\
199 \\
181\end{array}$ & $\begin{array}{r}208 \\
208 \\
222 \\
257 \\
272 \\
-\end{array}$ \\
\hline $\begin{array}{l}\text { TOTAL } \\
\text { MEAN } \\
\text { MAX } \\
\text { MIN } \\
\text { AC-FT } \\
\quad+ \\
\end{array}$ & $\begin{array}{r}5320 \\
172 \\
208 \\
133 \\
10,550 \\
716 \\
\end{array}$ & $\begin{array}{r}4.832 \\
161 \\
222 \\
98 \\
9.580 \\
688 \\
\end{array}$ & $\begin{array}{r}5131 \\
166 \\
203 \\
137 \\
10,180 \\
703\end{array}$ & $\begin{array}{r}4.173 \\
135 \\
165 \\
98 \\
8,280 \\
707\end{array}$ & $\begin{array}{r}3.926 \\
135 \\
186 \\
101 \\
7,790 \\
668\end{array}$ & $\begin{array}{r}11.584 \\
374 \\
582 \\
177 \\
22.980 \\
670\end{array}$ & $\begin{array}{r}15076 \\
503 \\
780 \\
304 \\
29,900 \\
659\end{array}$ & $\begin{array}{r}20.912 \\
675 \\
772 \\
590 \\
41,480 \\
728\end{array}$ & $\begin{array}{r}19.194 \\
640 \\
756 \\
590 \\
38,070 \\
784\end{array}$ & $\begin{array}{r}13,489 \\
435 \\
684 \\
362 \\
26,760 \\
1,020 \\
\end{array}$ & $\begin{array}{r}6,906 \\
223 \\
356 \\
181 \\
13,700 \\
1,030\end{array}$ & $\begin{array}{r}4.703 \\
157 \\
272 \\
94 \\
9,330 \\
736 \\
\end{array}$ \\
\hline
\end{tabular}

ADJUSTED FOR DIVERSION IN ACRE-FEET AT FARGO AND MOORHEAD

\begin{tabular}{|c|c|c|c|c|c|c|c|c|c|c|c|c|}
\hline $\begin{array}{l}\text { MEAN } \\
\mathrm{AC}-\mathrm{FT}\end{array}$ & $\begin{array}{r}183 \\
11,270\end{array}$ & $\begin{array}{r}173 \\
10,880\end{array}$ & $\begin{array}{r}177 \\
10,880\end{array}$ & $\begin{array}{r}146 \\
8,990\end{array}$ & $\begin{array}{r}147 \\
8,460\end{array}$ & $\begin{array}{r}385 \\
23,650\end{array}$ & $\begin{array}{r}514 \\
30,560\end{array}$ & $\begin{array}{r}686 \\
42,210\end{array}$ & $\begin{array}{r}653 \\
38,850\end{array}$ & $\begin{array}{r}452 \\
27,780\end{array}$ & $\begin{array}{r}240 \\
14,730\end{array}$ & $\begin{array}{r}169 \\
10,070\end{array}$ \\
\hline \multicolumn{9}{|c|}{ OBSERVED } & \multicolumn{4}{|c|}{ ADJUSTED } \\
\hline
\end{tabular}

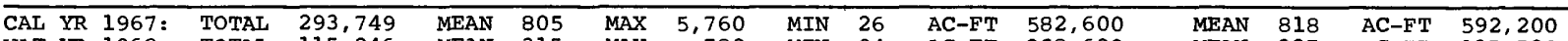

WAT YR 1968: TOTAL 115,246 MEAN 315 MAX 780 MIN 94 AC-FT 228,600




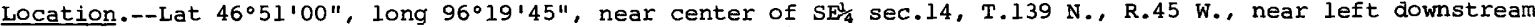
end of bridge on farm lane, 2 miles southwest of Hawley.

Drainage area. $--322 \mathrm{sq} \mathrm{mi}$.

Records available.--March 1945 to September 1968.

Gage.--Digital water-stage recorder. Datum of gage is $1,111.91$ ft above mean sea level, datum of 1929 . Prior to Jan. 29, 1953, chain gage at bridge $1,800 \mathrm{ft}$ upstream at datum 3.17 ft lower. Jan. 29,1953 to Aug. 29, 1968, graphic water-stage recorder at same site and datum.

Average discharge. -23 years, 72.8 cfs $(52,710$ acre-ft per year)

Extremes.--Maximum discharge during year, 202 cfs Mar. 28 (gage height, $4.97 \mathrm{ft}$ ); maximum gage height, $6.16 \mathrm{ft}$ Mar. 23 (backwater from ice); minimum daily discharge, 12 cfs Jan. 18-27, Feb. 5-27. 1945-68: Maximum discharge, $1,590 \mathrm{cfs}$ Aug. 5, 1955 (gage height, $9.31 \mathrm{ft}$ ); minimum observed, 6.8 cfs July 28, 1945; minimum gage height, $2.55 \mathrm{ft}$ Sept. 5, 1961.

Maximum stage known, about $11.3 \mathrm{ft}$, present datum, spring of 1921 , from information by local resident.

Remarks.--Records good except those for winter months, which are fair.

Discharge, in cubic feet per second, water year October 1967 to September 1968

\begin{tabular}{|c|c|c|c|c|c|c|c|c|c|c|c|c|}
\hline Day & Oct. & Nov. & Dec. & Jan. & Feb. & Mar. & Apr. & May & June & July & Aug. & Sept. \\
\hline $\begin{array}{l}1 \\
2 \\
3 \\
4 \\
5\end{array}$ & $\begin{array}{l}17 \\
17 \\
17 \\
18 \\
17\end{array}$ & $\begin{array}{l}18 \\
18 \\
19 \\
19 \\
19\end{array}$ & $\begin{array}{l}16 \\
16 \\
17 \\
17 \\
17\end{array}$ & $\begin{array}{l}17 \\
16 \\
16 \\
16 \\
16\end{array}$ & $\begin{array}{l}13 \\
13 \\
13 \\
13 \\
12\end{array}$ & $\begin{array}{l}13 \\
14 \\
15 \\
16 \\
18\end{array}$ & $\begin{array}{l}68 \\
59 \\
55 \\
40 \\
35\end{array}$ & $\begin{array}{l}99 \\
91 \\
83 \\
78 \\
74\end{array}$ & $\begin{array}{l}56 \\
54 \\
50 \\
48 \\
45\end{array}$ & $\begin{array}{l}64 \\
69 \\
56 \\
47 \\
44\end{array}$ & $\begin{array}{l}23 \\
22 \\
20 \\
20 \\
20\end{array}$ & $\begin{array}{l}17 \\
17 \\
16 \\
16 \\
16\end{array}$ \\
\hline $\begin{array}{r}6 \\
7 \\
8 \\
9 \\
10\end{array}$ & $\begin{array}{l}16 \\
18 \\
19 \\
21 \\
21\end{array}$ & $\begin{array}{l}19 \\
19 \\
20 \\
20 \\
20\end{array}$ & $\begin{array}{l}17 \\
18 \\
18 \\
18 \\
19\end{array}$ & $\begin{array}{l}16 \\
15 \\
15 \\
15 \\
14\end{array}$ & $\begin{array}{l}12 \\
1.2 \\
12 \\
12 \\
12\end{array}$ & $\begin{array}{l}21 \\
24 \\
25 \\
25 \\
24\end{array}$ & $\begin{array}{r}41 \\
77 \\
116 \\
136 \\
132\end{array}$ & $\begin{array}{l}71 \\
73 \\
78 \\
82 \\
80\end{array}$ & $\begin{array}{l}45 \\
48 \\
59 \\
70 \\
82\end{array}$ & $\begin{array}{l}47 \\
43 \\
37 \\
34 \\
32\end{array}$ & $\begin{array}{l}20 \\
19 \\
23 \\
20 \\
20\end{array}$ & $\begin{array}{l}16 \\
17 \\
17 \\
17 \\
17\end{array}$ \\
\hline $\begin{array}{l}11 \\
12 \\
13 \\
14 \\
15\end{array}$ & $\begin{array}{l}20 \\
19 \\
19 \\
18 \\
18\end{array}$ & $\begin{array}{l}20 \\
20 \\
20 \\
20 \\
19\end{array}$ & $\begin{array}{l}20 \\
20 \\
21 \\
21 \\
21\end{array}$ & $\begin{array}{l}14 \\
14 \\
13 \\
13 \\
13\end{array}$ & $\begin{array}{l}12 \\
12 \\
12 \\
12 \\
12\end{array}$ & $\begin{array}{l}24 \\
25 \\
26 \\
30 \\
35\end{array}$ & $\begin{array}{r}115 \\
102 \\
89 \\
79 \\
75\end{array}$ & $\begin{array}{r}94 \\
114 \\
112 \\
121 \\
124\end{array}$ & $\begin{array}{r}106 \\
98 \\
84 \\
84 \\
80\end{array}$ & $\begin{array}{l}30 \\
29 \\
31 \\
41 \\
41\end{array}$ & $\begin{array}{l}19 \\
20 \\
20 \\
18 \\
20\end{array}$ & $\begin{array}{l}16 \\
15 \\
15 \\
15 \\
14\end{array}$ \\
\hline $\begin{array}{l}16 \\
17 \\
18 \\
19 \\
20\end{array}$ & $\begin{array}{l}17 \\
17 \\
17 \\
16 \\
16\end{array}$ & $\begin{array}{r}19 \\
18 \\
18 \\
18 \\
.8\end{array}$ & $\begin{array}{l}21 \\
20 \\
20 \\
20 \\
20\end{array}$ & $\begin{array}{l}13 \\
13 \\
12 \\
12 \\
12\end{array}$ & $\begin{array}{l}12 \\
12 \\
12 \\
12 \\
12\end{array}$ & $\begin{array}{l}38 \\
39 \\
40 \\
41 \\
40\end{array}$ & $\begin{array}{l}72 \\
69 \\
64 \\
60 \\
62\end{array}$ & $\begin{array}{r}107 \\
97 \\
93 \\
92 \\
87\end{array}$ & $\begin{array}{l}75 \\
68 \\
60 \\
56 \\
51\end{array}$ & $\begin{array}{l}51 \\
68 \\
59 \\
46 \\
46\end{array}$ & $\begin{array}{l}19 \\
20 \\
20 \\
20 \\
19\end{array}$ & $\begin{array}{l}33 \\
29 \\
25 \\
24 \\
23\end{array}$ \\
\hline $\begin{array}{l}21 \\
22 \\
23 \\
24 \\
25\end{array}$ & $\begin{array}{l}16 \\
17 \\
16 \\
17 \\
15\end{array}$ & $\begin{array}{l}18 \\
18 \\
18 \\
18 \\
18\end{array}$ & $\begin{array}{l}20 \\
20 \\
19 \\
19 \\
18\end{array}$ & $\begin{array}{l}12 \\
12 \\
12 \\
12 \\
12\end{array}$ & $\begin{array}{l}12 \\
12 \\
12 \\
12 \\
12\end{array}$ & $\begin{array}{r}43 \\
50 \\
75 \\
125 \\
92\end{array}$ & $\begin{array}{r}71 \\
79 \\
95 \\
116 \\
122\end{array}$ & $\begin{array}{l}81 \\
78 \\
73 \\
69 \\
66\end{array}$ & $\begin{array}{l}48 \\
50 \\
51 \\
52 \\
50\end{array}$ & $\begin{array}{l}38 \\
35 \\
32 \\
30 \\
29\end{array}$ & $\begin{array}{l}19 \\
18 \\
21 \\
20 \\
19\end{array}$ & $\begin{array}{l}21 \\
34 \\
50 \\
50 \\
38\end{array}$ \\
\hline $\begin{array}{l}26 \\
27 \\
28 \\
29 \\
30 \\
31\end{array}$ & $\begin{array}{l}15 \\
15 \\
15 \\
17 \\
17 \\
18\end{array}$ & $\begin{array}{r}17 \\
16 \\
16 \\
16 \\
16 \\
-\end{array}$ & $\begin{array}{l}18 \\
18 \\
17 \\
17 \\
17 \\
17\end{array}$ & $\begin{array}{l}12 \\
12 \\
13 \\
13 \\
13 \\
13\end{array}$ & $\begin{array}{r}12 \\
12 \\
13 \\
13 \\
-\ldots-- \\
-\ldots\end{array}$ & $\begin{array}{r}104 \\
162 \\
189 \\
123 \\
92 \\
78\end{array}$ & $\begin{array}{r}116 \\
112 \\
118 \\
114 \\
107 \\
-\end{array}$ & $\begin{array}{l}65 \\
64 \\
62 \\
61 \\
60 \\
59\end{array}$ & $\begin{array}{r}46 \\
42 \\
39 \\
36 \\
43 \\
-\ldots---\end{array}$ & $\begin{array}{l}28 \\
27 \\
26 \\
24 \\
23 \\
23\end{array}$ & $\begin{array}{l}18 \\
17 \\
16 \\
16 \\
18 \\
19\end{array}$ & $\begin{array}{r}31 \\
26 \\
24 \\
22 \\
21 \\
-\quad-\quad--\end{array}$ \\
\hline $\begin{array}{l}\text { Total } \\
\text { Mean } \\
\text { Max } \\
\text { Min } \\
\text { Ac-ft }\end{array}$ & $\begin{array}{r}536 \\
17.3 \\
21 \\
15 \\
1,060\end{array}$ & $\begin{array}{r}552 \\
18.4 \\
20 \\
1.6 \\
1,090\end{array}$ & $\begin{array}{r}577 \\
18.6 \\
21 \\
1.6 \\
1,140\end{array}$ & $\begin{array}{r}421 \\
13.6 \\
17 \\
12 \\
906\end{array}$ & $\begin{array}{r}354 \\
12.2 \\
13 \\
12 \\
714\end{array}$ & $\begin{array}{r}1,666 \\
53.7 \\
189 \\
13 \\
3,300\end{array}$ & $\begin{array}{r}2,596 \\
86.5 \\
136 \\
35 \\
5,150\end{array}$ & $\begin{array}{r}2,588 \\
83.5 \\
124 \\
59 \\
5,130\end{array}$ & $\begin{array}{r}1,776 \\
59.2 \\
106 \\
36 \\
3,520\end{array}$ & $\begin{array}{r}1,230 \\
39.7 \\
69 \\
23 \\
2,440\end{array}$ & $\begin{array}{r}603 \\
19.5 \\
23 \\
16 \\
1,200\end{array}$ & $\begin{array}{r}692 \\
23.1 \\
50 \\
14 \\
1.370\end{array}$ \\
\hline $\begin{array}{l}\text { Cal yr } \\
\text { Wtr yr }\end{array}$ & 67: $\mathrm{T}$ & $\begin{array}{l}29,5 \\
13,5\end{array}$ & $\mathrm{Me}$ & $\begin{array}{l}81.1 \\
37.1\end{array}$ & $\begin{array}{l}\text { Max } \\
\text { Max }\end{array}$ & $\begin{array}{l}\text { Min } \\
\text { Min }\end{array}$ & $\begin{array}{l}1.2 \\
12\end{array}$ & & $\begin{array}{l}700 \\
960\end{array}$ & & & \\
\hline
\end{tabular}


5-0615. South Branch Buffalo River at Sabin, Minn.

Location.--Lat $46^{\circ} 46^{\prime} 20^{\prime \prime}$, long $96^{\circ} 37^{\prime} 40^{\prime \prime}$, in SWhSW/4 sec.9, T.138 N., R.47 W., near center of span on downstream side of highway bridge, a quarter of a mile downstream from Whisky creek and 1 mile east of Sabin.

Drainage area.--522 sq $\mathrm{mi}$.

Records available.--March 1945 to september 1968.

Gage.--Wire-weight gage read once or twice daily and crest-stage gage. Datum of gage is 902.39 ft above mean sea level, datum of 1929 (levels by Soil Conservation Service). Prior to Aug. 17, 1948, at site 1 mile downstream at different datum. Aug. 17, 1948, to July 26, 1965, chain gage at same site and datum.

Average discharge.--23 years, 55.6 cfs $(40,250$ acre-ft per year).

Extremes.--Maximum discharge during year, $230 \mathrm{cfs}$ Apr. 10 (gage height, 8.25 ft, from graph based on gage readings); no flow Jan. 28 to Mar. 4 .

1945-68: Maximum discharge, 6,340 cfs June 9, 1962 (gage height, 17.04 ft); no flow for many days in most years.

Remarks.--Records fair.

DISCHARGE, IN CUBIC FEET PER SECOND, WATER YEAR OCTOBER 1967 TO SEPTEMBER 1968

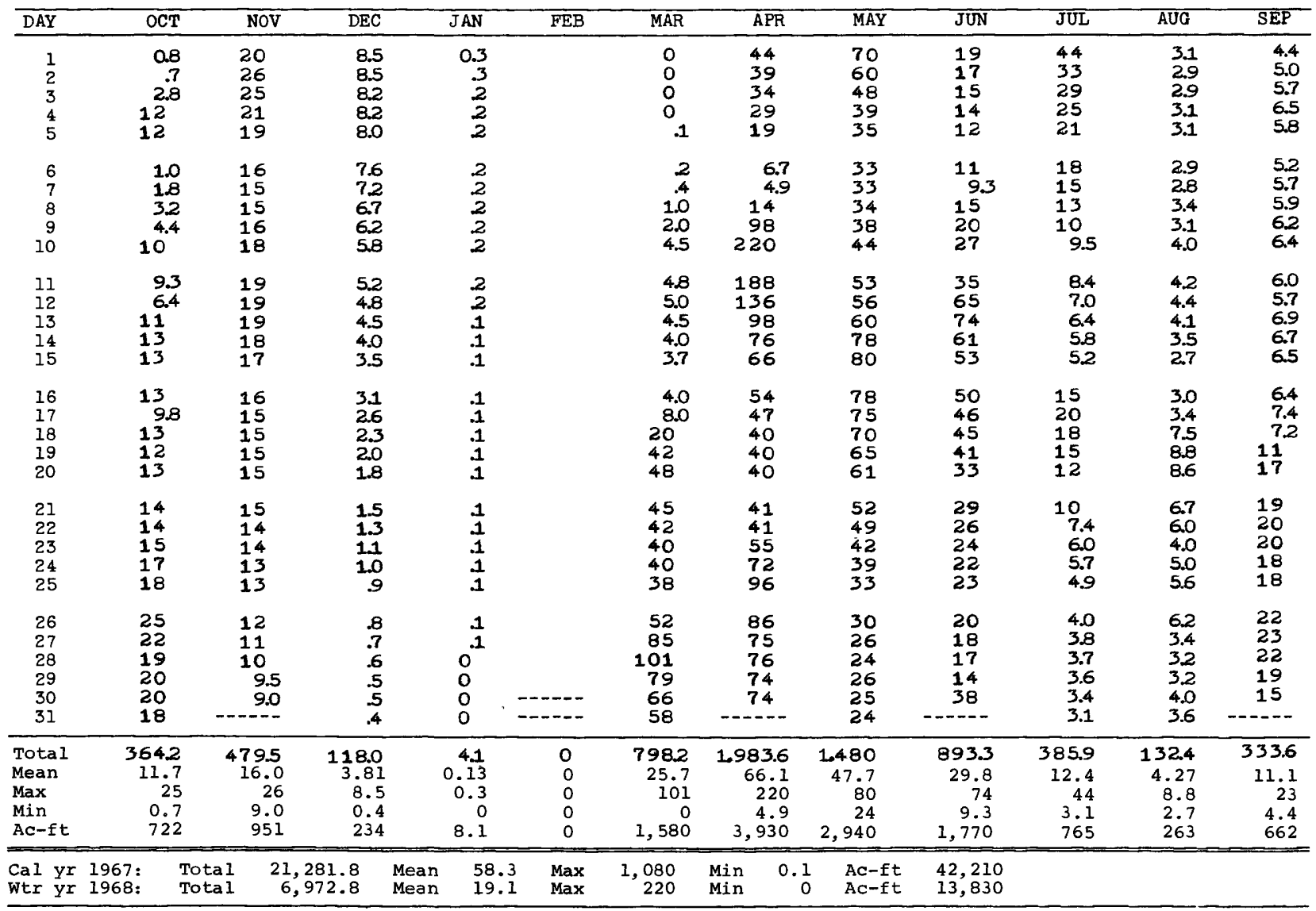




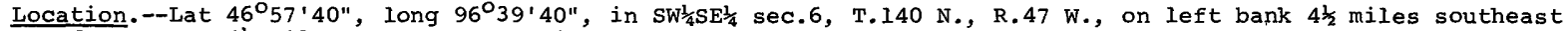
of Kragnes, $6 \frac{1}{2}$ miles northeast of Dilworth, and 9 miles downstream from South Branch.

Drainaqe area. $--1,040 \mathrm{sq} \mathrm{mi}$, approximately.

Records available.--March 1931 to September 1968 . Monthly discharge only for some periods, published in Wsp 1308 .

Gage.--Water-stage recorder. Datum of gage is $878.31 \mathrm{ft}$ above mean sea level, datum of 1929 (levels by Corps of Engineers). Prior to Apr. 5, 1937, chain gage at same site and datum.

Average discharge. -37 years, 122 cfs $(88,320$ acre-ft per year).

Extremes.--Maximum discharge during year, $406 \mathrm{cfs}$ Mar. 30 (gage height, 8.92 ft); minimum daily, 9.4 cfs Jan. 21-25; minimum gage height, $2.30 \mathrm{ft}$ sept. 15 .

1931-68: Maximum discharge, 6, $140 \mathrm{cfs} J u n e$ 11, 1962 (gage height, $23.56 \mathrm{ft}$ ) no flow at times in 1936.

Remarks.--Record fair. Records of chemical analyses for the water year 1968 are published in Part 2 of this report.

DISCHARGE, IN CUBIC FEET PER SECOND, WATER YEAR OCTOBER 1967 TO SEPTEMBER 1968

\begin{tabular}{|c|c|c|c|c|c|c|c|c|c|c|c|c|c|}
\hline DAY & $\mathrm{OCT}$ & NOV & $\mathrm{DEC}$ & & JAN & FEB & MAR & APR & MAY & JUN & JUL & AUG & SEP \\
\hline $\begin{array}{l}1 \\
2 \\
3 \\
4 \\
5\end{array}$ & $\begin{array}{l}19 \\
20 \\
18 \\
16 \\
16\end{array}$ & $\begin{array}{l}25 \\
23 \\
23 \\
23 \\
23\end{array}$ & $\begin{array}{l}18 \\
18 \\
18 \\
19 \\
19\end{array}$ & & $\begin{array}{l}14 \\
13 \\
13 \\
12 \\
12\end{array}$ & $\begin{array}{l}10 \\
10 \\
11 \\
11 \\
11\end{array}$ & $\begin{array}{l}11 \\
12 \\
12 \\
13 \\
15\end{array}$ & $\begin{array}{r}210 \\
171 \\
143 \\
116 \\
87\end{array}$ & $\begin{array}{l}177 \\
164 \\
148 \\
132 \\
118\end{array}$ & $\begin{array}{l}74 \\
72 \\
70 \\
64 \\
59\end{array}$ & $\begin{array}{l}165 \\
226 \\
195 \\
156 \\
122\end{array}$ & $\begin{array}{l}27 \\
26 \\
24 \\
23 \\
21\end{array}$ & $\begin{array}{l}25 \\
24 \\
23 \\
21 \\
21\end{array}$ \\
\hline $\begin{array}{r}6 \\
7 \\
8 \\
9 \\
10\end{array}$ & $\begin{array}{l}16 \\
19 \\
20 \\
20 \\
20\end{array}$ & $\begin{array}{l}21 \\
20 \\
18 \\
19 \\
19\end{array}$ & $\begin{array}{l}20 \\
20 \\
21 \\
21 \\
22\end{array}$ & & $\begin{array}{l}12 \\
11 \\
11 \\
11 \\
11\end{array}$ & $\begin{array}{l}11 \\
11 \\
11 \\
10 \\
10\end{array}$ & $\begin{array}{l}19 \\
25 \\
27 \\
28 \\
27\end{array}$ & $\begin{array}{r}100 \\
79 \\
82 \\
133 \\
192\end{array}$ & $\begin{array}{r}109 \\
101 \\
97 \\
98 \\
102\end{array}$ & $\begin{array}{l}57 \\
55 \\
55 \\
71 \\
87\end{array}$ & $\begin{array}{l}99 \\
89 \\
80 \\
68 \\
58\end{array}$ & $\begin{array}{l}19 \\
19 \\
18 \\
21 \\
25\end{array}$ & $\begin{array}{l}19 \\
19 \\
20 \\
21 \\
21\end{array}$ \\
\hline $\begin{array}{l}11 \\
12 \\
13 \\
14 \\
15\end{array}$ & $\begin{array}{l}20 \\
21 \\
21 \\
21 \\
19\end{array}$ & $\begin{array}{l}20 \\
20 \\
20 \\
21 \\
20\end{array}$ & $\begin{array}{l}22 \\
22 \\
22 \\
22 \\
21\end{array}$ & & $\begin{array}{l}11 \\
11 \\
10 \\
10 \\
9.8\end{array}$ & $\begin{array}{l}10 \\
10 \\
10 \\
10 \\
10\end{array}$ & $\begin{array}{l}26 \\
26 \\
28 \\
34 \\
45\end{array}$ & $\begin{array}{l}299 \\
332 \\
294 \\
242 \\
201\end{array}$ & $\begin{array}{l}110 \\
139 \\
155 \\
172 \\
192\end{array}$ & $\begin{array}{l}147 \\
241 \\
226 \\
209 \\
204\end{array}$ & $\begin{array}{l}52 \\
46 \\
42 \\
41 \\
47\end{array}$ & $\begin{array}{l}22 \\
19 \\
18 \\
17 \\
19\end{array}$ & $\begin{array}{l}21 \\
20 \\
19 \\
18 \\
17\end{array}$ \\
\hline $\begin{array}{l}16 \\
17 \\
18 \\
19 \\
20\end{array}$ & $\begin{array}{l}20 \\
19 \\
19 \\
20 \\
20\end{array}$ & $\begin{array}{l}20 \\
20 \\
20 \\
20 \\
19\end{array}$ & $\begin{array}{l}21 \\
21 \\
20 \\
20 \\
20\end{array}$ & & $\begin{array}{l}9.8 \\
9.8 \\
9.6 \\
9.6 \\
9.6\end{array}$ & $\begin{array}{l}10 \\
10 \\
10 \\
10 \\
10\end{array}$ & $\begin{array}{l}56 \\
63 \\
68 \\
71 \\
72\end{array}$ & $\begin{array}{l}172 \\
153 \\
141 \\
129 \\
120\end{array}$ & $\begin{array}{l}197 \\
188 \\
174 \\
164 \\
153\end{array}$ & $\begin{array}{l}180 \\
163 \\
148 \\
129 \\
116\end{array}$ & $\begin{array}{l}56 \\
58 \\
64 \\
79 \\
81\end{array}$ & $\begin{array}{l}22 \\
34 \\
28 \\
24 \\
24\end{array}$ & $\begin{array}{l}19 \\
25 \\
41 \\
43 \\
38\end{array}$ \\
\hline $\begin{array}{l}21 \\
22 \\
23 \\
24 \\
25\end{array}$ & $\begin{array}{l}21 \\
21 \\
21 \\
21 \\
22\end{array}$ & $\begin{array}{l}19 \\
19 \\
19 \\
19 \\
18\end{array}$ & $\begin{array}{l}19 \\
19 \\
19 \\
18 \\
17\end{array}$ & & $\begin{array}{l}9.4 \\
9.4 \\
9.4 \\
9.4 \\
9.4\end{array}$ & $\begin{array}{l}10 \\
10 \\
10 \\
10 \\
10\end{array}$ & $\begin{array}{r}73 \\
77 \\
85 \\
95 \\
115\end{array}$ & $\begin{array}{l}118 \\
118 \\
127 \\
150 \\
171\end{array}$ & $\begin{array}{l}142 \\
132 \\
121 \\
112 \\
104\end{array}$ & $\begin{array}{r}106 \\
95 \\
88 \\
86 \\
84\end{array}$ & $\begin{array}{l}73 \\
71 \\
57 \\
42 \\
35\end{array}$ & $\begin{array}{l}22 \\
21 \\
22 \\
21 \\
22\end{array}$ & $\begin{array}{l}34 \\
35 \\
43 \\
56 \\
64\end{array}$ \\
\hline $\begin{array}{l}26 \\
27 \\
28 \\
29 \\
30 \\
31\end{array}$ & $\begin{array}{l}22 \\
22 \\
22 \\
21 \\
26 \\
26\end{array}$ & $\begin{array}{r}18 \\
17 \\
17 \\
17 \\
18 \\
\end{array}$ & $\begin{array}{l}16 \\
16 \\
15 \\
15 \\
14 \\
14\end{array}$ & & $\begin{array}{l}9.6 \\
9.6 \\
9.8 \\
9.8 \\
10 \\
10\end{array}$ & $\begin{array}{r}10 \\
10 \\
11 \\
11 \\
-\end{array}$ & $\begin{array}{l}148 \\
220 \\
295 \\
360 \\
397 \\
322\end{array}$ & $\begin{array}{r}196 \\
211 \\
206 \\
194 \\
186 \\
\end{array}$ & $\begin{array}{l}96 \\
90 \\
87 \\
83 \\
79 \\
76\end{array}$ & $\begin{array}{r}81 \\
76 \\
71 \\
64 \\
64 \\
-\end{array}$ & $\begin{array}{l}32 \\
43 \\
55 \\
42 \\
35 \\
30\end{array}$ & $\begin{array}{l}24 \\
23 \\
22 \\
21 \\
22 \\
23\end{array}$ & $\begin{array}{r}68 \\
61 \\
53 \\
46 \\
43 \\
\end{array}$ \\
\hline $\begin{array}{l}\text { Total } \\
\text { Mean } \\
\text { Max } \\
\text { Min } \\
\text { Ac-ft } \\
\end{array}$ & $\begin{array}{r}629 \\
20.3 \\
26 \\
16 \\
1,250 \\
\end{array}$ & $\begin{array}{r}595 \\
19.8 \\
25 \\
17 \\
1,180 \\
\end{array}$ & $\begin{array}{r}589 \\
19.0 \\
22 \\
14 \\
1,170 \\
\end{array}$ & & $\begin{array}{r}326.0 \\
10.5 \\
14 \\
9.4 \\
647 \\
\end{array}$ & $\begin{array}{r}298 \\
10.3 \\
11 \\
10 \\
591 \\
\end{array}$ & $\begin{array}{r}2.865 \\
92.4 \\
397 \\
11 \\
5,680 \\
\end{array}$ & $\begin{array}{r}5073 \\
169 \\
332 \\
79 \\
1,010 \\
\end{array}$ & $\begin{array}{r}4.012 \\
129 \\
197 \\
76 \\
7,960 \\
\end{array}$ & $\begin{array}{r}3.242 \\
108 \\
241 \\
55 \\
6,430 \\
\end{array}$ & $\begin{array}{r}2339 \\
75.5 \\
226 \\
30 \\
4,640 \\
\end{array}$ & $\begin{array}{r}693 \\
22.4 \\
34 \\
17 \\
1,370 \\
\end{array}$ & $\begin{array}{r}978 \\
32.6 \\
68 \\
17 \\
1,940 \\
\end{array}$ \\
\hline $\begin{array}{ll}\text { Cal yr } \\
\text { Wtr yr }\end{array}$ & $\begin{array}{l}1967: \\
1968:\end{array}$ & al & .0 & $\begin{array}{l}\text { Mean } \\
\text { Mean }\end{array}$ & $\begin{array}{r}159 \\
59.1\end{array}$ & $\begin{array}{l}\operatorname{Max} \\
\operatorname{Max}\end{array}$ & $\begin{array}{r}1,710 \\
397\end{array}$ & $\begin{array}{ll}\min & 11 \\
\operatorname{Min} & 9.4\end{array}$ & $\begin{array}{l}A c-f t \\
A c-f t\end{array}$ & $\begin{array}{r}114, \\
42,\end{array}$ & & & \\
\hline
\end{tabular}


5-0625. Wild Rice River at Twin Valley, Minn.

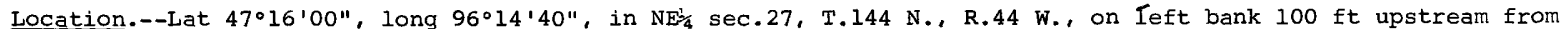
highway bridge, three-quarters of a mile northeast of village of Twin Valley, and 2 miles upstream from small tributary.

Drainage area. $--888 \mathrm{sq} \mathrm{mi}$.

Records available.--June 1909 to September 1917, July 1930 to September 1968 . Monthly discharge only for some periods, published in wSP 1308.

Gage.--Digital water-stage recorder. Datum of gage is 1,008.16 ft above mean sea level, datum of 1929 (Corps of Engineers bench mark). June 1909 to september 1917, staff gage at site a quarter of a mile downstreak at different datum. July 23, 1930, to Nov. 24, 1934, chain gage at highway bridge 100 ft downstream from present site at present datum. Nov, 25, 1934 to Aug. 2, 1950, graphic water-stage recorder 80 ft upstream from present site at present datum, and Aug. 3, 1950, to Aug. 14, 1967, graphic water-stage recorder at present site and datum.

Average discharge.--46 years, $164 \mathrm{cfs}(118,700$ acre-ft per year).

Extremes.--Maximum discharge during year, 594 cfs Mar. 30 (gage height, 4.33 ft); maximum gage height, 4.62 ft Mar. 28 (backwater from ice); minimum daily discharge, 13 cfs oct. 1.

1909-17, 1930-68: Maximum discharge, 9,200 cfs July 22, 1909 (gage height, 20.0 ft, site and datum then in use), from rating curve extended above $3,300 \mathrm{cfs}$; minimum, $0.5 \mathrm{cfs}$ Nov. 4 , 1939.

Remarks.--Records good except those for period of no gage-height record and those for winter months, which are fair. Flow slightly regulated by Rice Lake and many other small lakes above station. Diurnal fluctuation caused by operation of mill at Faith, 17 miles above station.

DISCHARGE, IN CUBIC FEET PER SECOND, WATER YEAR OCTCBER 1 O67 TO SEPTEMBER 196B

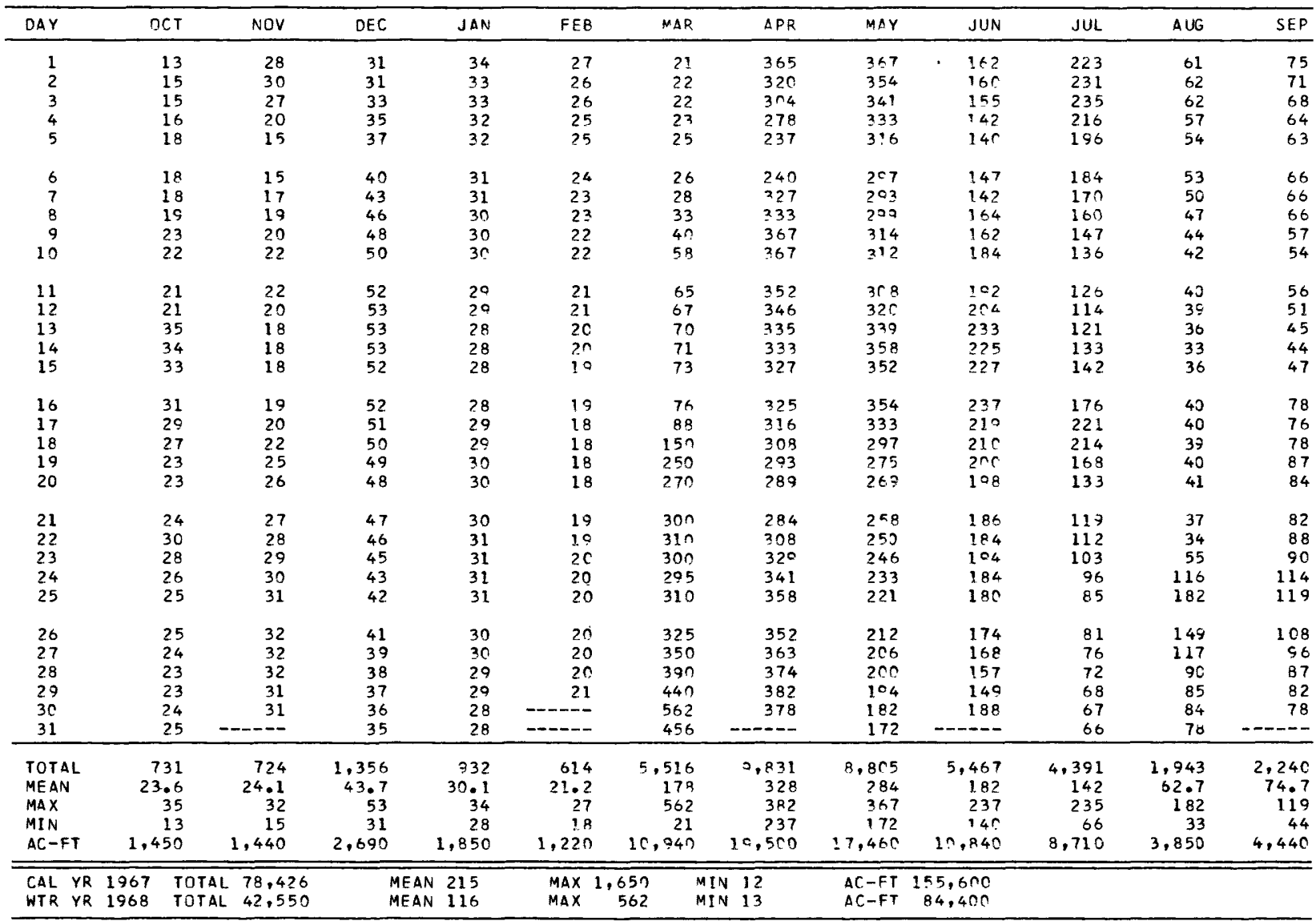

Note.--No gage-height record Oct. 1 to Nov, 27. 
Location.--Lat $47^{\circ} 16^{\prime} 05^{\prime \prime}$, long $96^{\circ} 47^{\prime} 50^{\prime \prime}$, in SEl sec.19, T.144 N., R.48 W., near center of span on downstream side of highway bridge, half a mile east of Hendrum and 4 miles upstream from mouth.

Drainage area.--1,600 sq $\mathrm{mi}$, approximately.

Records available.--March 1944 to September 1968.

Gage.--Wire-weight gage read once or twice daily and crest stage gage. Datum of gage is 836.75 ft above mean sea level, datum of 1929 (levels by Corps of Engineers). Prior to Aug. 2, 1949, chain gage at same site and datum.

Average discharge.--24 years, 227 cfs $(164,300$ acre-ft per year).

Extremes.--Maximum discharge during year, 726 cfs Mar. 29 (gage height, 11.26 ft, from graph based on gage reading, backwater from ice); minimum, 12 cfs oct. 1 .

1944-68: Maximum discharge 6,800 cfs Apr. 14, 1965; maximum gage height, 29.52 ft Apr. 15, 1965 (from floodmark, backwater from Red River of the North); no flow some days in $1948-49$.

Remarks.--Records good except those for winter months, which are fair. Large part of high flow diverted into Marsh River basin at overflow section $3 \frac{1}{2}$ miles east of Ada. Another diversion into Marsh River basin,

formed $1 \frac{1}{2}$ miles southeast of Ada, diverted water at all stages 1947-5l, after which it was closed except

for small regulated flow diverted at same point. Amount of diversion not known. Records of chemical analyses for the water year 1968 are published in Part 2 of this report.

DISCHARGE, IN CFS, WATER YEAR OCTOBER 1967 TO SEPTEMBER 1968

\begin{tabular}{|c|c|c|c|c|c|c|c|c|c|c|c|c|}
\hline DAY & $\mathrm{OCT}$ & NOV & $\mathrm{DEC}$ & JAN & $F E B$ & MAR & APR & MAY & JUN & JUL & AUG & SEP \\
\hline $\begin{array}{l}1 \\
2 \\
3 \\
4 \\
5\end{array}$ & $\begin{array}{l}12 \\
17 \\
17 \\
17 \\
17\end{array}$ & $\begin{array}{l}29 \\
32 \\
28 \\
22 \\
16\end{array}$ & $\begin{array}{l}29 \\
31 \\
34 \\
37 \\
39\end{array}$ & $\begin{array}{l}38 \\
37 \\
37 \\
36 \\
35\end{array}$ & $\begin{array}{l}29 \\
28 \\
28 \\
28 \\
27\end{array}$ & $\begin{array}{l}21 \\
22 \\
24 \\
25 \\
26\end{array}$ & $\begin{array}{l}500 \\
427 \\
369 \\
360 \\
393\end{array}$ & $\begin{array}{l}420 \\
396 \\
378 \\
357 \\
346\end{array}$ & $\begin{array}{l}195 \\
188 \\
183 \\
180 \\
173\end{array}$ & $\begin{array}{l}221 \\
304 \\
350 \\
321 \\
300\end{array}$ & $\begin{array}{l}65 \\
65 \\
64 \\
66 \\
61\end{array}$ & $\begin{array}{l}95 \\
86 \\
93 \\
84 \\
73\end{array}$ \\
\hline $\begin{array}{r}6 \\
7 \\
8 \\
9 \\
10\end{array}$ & $\begin{array}{l}19 \\
20 \\
20 \\
20 \\
26\end{array}$ & $\begin{array}{l}15 \\
16 \\
18 \\
21 \\
23\end{array}$ & $\begin{array}{l}43 \\
47 \\
50 \\
53 \\
55\end{array}$ & $\begin{array}{l}35 \\
34 \\
34 \\
33 \\
33\end{array}$ & $\begin{array}{l}26 \\
26 \\
25 \\
24 \\
24\end{array}$ & $\begin{array}{l}27 \\
29 \\
30 \\
34 \\
50\end{array}$ & $\begin{array}{l}305 \\
300 \\
330 \\
401 \\
400\end{array}$ & $\begin{array}{l}333 \\
318 \\
308 \\
314 \\
324\end{array}$ & $\begin{array}{l}182 \\
200 \\
230 \\
243 \\
267\end{array}$ & $\begin{array}{l}275 \\
251 \\
224 \\
201 \\
183\end{array}$ & $\begin{array}{l}55 \\
53 \\
56 \\
50 \\
44\end{array}$ & $\begin{array}{l}72 \\
71 \\
71 \\
71 \\
66\end{array}$ \\
\hline $\begin{array}{l}11 \\
12 \\
13 \\
14 \\
15\end{array}$ & $\begin{array}{l}25 \\
26 \\
26 \\
41 \\
39\end{array}$ & $\begin{array}{l}23 \\
22 \\
21 \\
20 \\
19\end{array}$ & $\begin{array}{l}58 \\
60 \\
60 \\
60 \\
60\end{array}$ & $\begin{array}{l}32 \\
32 \\
32 \\
32 \\
31\end{array}$ & $\begin{array}{l}23 \\
22 \\
22 \\
21 \\
21\end{array}$ & $\begin{array}{l}60 \\
78 \\
80 \\
78 \\
75\end{array}$ & $\begin{array}{l}518 \\
495 \\
450 \\
398 \\
379\end{array}$ & $\begin{array}{l}340 \\
360 \\
374 \\
390 \\
405\end{array}$ & $\begin{array}{l}287 \\
314 \\
328 \\
337 \\
345\end{array}$ & $\begin{array}{l}169 \\
156 \\
149 \\
170 \\
171\end{array}$ & $\begin{array}{l}43 \\
42 \\
40 \\
38 \\
43\end{array}$ & $\begin{array}{l}59 \\
56 \\
56 \\
50 \\
44\end{array}$ \\
\hline $\begin{array}{l}16 \\
17 \\
18 \\
19 \\
20\end{array}$ & $\begin{array}{l}38 \\
33 \\
31 \\
29 \\
26\end{array}$ & $\begin{array}{l}19 \\
19 \\
21 \\
23 \\
25\end{array}$ & $\begin{array}{l}59 \\
59 \\
59 \\
58 \\
57\end{array}$ & $\begin{array}{l}31 \\
31 \\
31 \\
32 \\
32\end{array}$ & $\begin{array}{l}20 \\
19 \\
19 \\
18 \\
18\end{array}$ & $\begin{array}{l}105 \\
140 \\
175 \\
205 \\
230\end{array}$ & $\begin{array}{l}372 \\
361 \\
347 \\
333 \\
334\end{array}$ & $\begin{array}{l}410 \\
395 \\
369 \\
329 \\
303\end{array}$ & $\begin{array}{l}333 \\
317 \\
291 \\
273 \\
260\end{array}$ & $\begin{array}{l}177 \\
218 \\
244 \\
237 \\
200\end{array}$ & $\begin{array}{l}46 \\
46 \\
54 \\
53 \\
44\end{array}$ & $\begin{array}{r}47 \\
66 \\
110 \\
92 \\
93\end{array}$ \\
\hline $\begin{array}{l}21 \\
22 \\
23 \\
24 \\
25\end{array}$ & $\begin{array}{l}29 \\
26 \\
34 \\
30 \\
28\end{array}$ & $\begin{array}{l}27 \\
29 \\
30 \\
30 \\
30\end{array}$ & $\begin{array}{l}56 \\
54 \\
52 \\
50 \\
48\end{array}$ & $\begin{array}{l}32 \\
32 \\
31 \\
31 \\
30\end{array}$ & $\begin{array}{l}18 \\
17 \\
17 \\
17 \\
18\end{array}$ & $\begin{array}{l}250 \\
280 \\
285 \\
320 \\
350\end{array}$ & $\begin{array}{l}324 \\
323 \\
343 \\
373 \\
389\end{array}$ & $\begin{array}{l}292 \\
280 \\
267 \\
275 \\
250\end{array}$ & $\begin{array}{l}247 \\
230 \\
231 \\
232 \\
211\end{array}$ & $\begin{array}{l}178 \\
168 \\
152 \\
141 \\
122\end{array}$ & $\begin{array}{l}44 \\
46 \\
45 \\
50 \\
78\end{array}$ & $\begin{array}{r}92 \\
97 \\
99 \\
115 \\
133\end{array}$ \\
\hline $\begin{array}{l}26 \\
27 \\
28 \\
29 \\
30 \\
31\end{array}$ & $\begin{array}{l}26 \\
26 \\
25 \\
24 \\
24 \\
26\end{array}$ & $\begin{array}{r}30 \\
29 \\
29 \\
28 \\
28 \\
- \\
-\end{array}$ & $\begin{array}{l}47 \\
46 \\
44 \\
43 \\
41 \\
39\end{array}$ & $\begin{array}{l}30 \\
30 \\
29 \\
29 \\
29 \\
29\end{array}$ & $\begin{array}{r}18 \\
19 \\
19 \\
20 \\
- \\
-\end{array}$ & $\begin{array}{l}420 \\
510 \\
570 \\
690 \\
675 \\
580\end{array}$ & $\begin{array}{r}401 \\
402 \\
407 \\
430 \\
450 \\
- \\
---\end{array}$ & $\begin{array}{l}238 \\
230 \\
225 \\
220 \\
214 \\
199\end{array}$ & $\begin{array}{r}211 \\
204 \\
207 \\
192 \\
185\end{array}$ & $\begin{array}{r}109 \\
97 \\
91 \\
85 \\
79 \\
77\end{array}$ & $\begin{array}{l}166 \\
162 \\
152 \\
118 \\
109 \\
105\end{array}$ & $\begin{array}{r}141 \\
132 \\
123 \\
115 \\
110 \\
-\end{array}$ \\
\hline $\begin{array}{l}\text { Total } \\
\text { Mean } \\
\text { Max } \\
\text { Min } \\
\text { Ac-ft }\end{array}$ & $\begin{array}{r}797 \\
25.7 \\
41 \\
12 \\
1,580\end{array}$ & $\begin{array}{r}722 \\
24.1 \\
32 \\
15 \\
1.430\end{array}$ & $\begin{array}{r}1,528 \\
49.3 \\
60 \\
29 \\
3,030\end{array}$ & $\begin{array}{r}1,000 \\
32.3 \\
38 \\
29 \\
1,980\end{array}$ & $\begin{array}{r}631 \\
21.8 \\
29 \\
17 \\
1,250\end{array}$ & $\begin{array}{r}6,444 \\
208 \\
690 \\
21 \\
12,780\end{array}$ & $\begin{array}{r}11,614 \\
387 \\
518 \\
300 \\
23,040\end{array}$ & $\begin{array}{r}9,859 \\
318 \\
420 \\
199 \\
19,560\end{array}$ & $\begin{array}{r}7,276 \\
-\quad 243 \\
345 \\
173 \\
14,430 \\
\end{array}$ & $\begin{array}{r}5.820 \\
188 \\
350 \\
77 \\
11.540 \\
\end{array}$ & $\begin{array}{r}2,103 \\
67.8 \\
166 \\
38 \\
4,170\end{array}$ & $\begin{array}{r}2,612 \\
87.1 \\
141 \\
44 \\
5,180 \\
\end{array}$ \\
\hline
\end{tabular}

Cal yr 1967: Total 108,863 Mean 298 Max 3,170 Min 12 Ac-ft 215,900

Wtr yr 1968: Total 50,406 Mean 138 Max 690 Min 12 Ac-ft 99,980 
5-0645. Red River of the North at Halstad, Minn.

Location.--Iat 47021'10", long 96050'50", on line between secs. 24 and 25 , T.145 N., R.49 W., on left bank on upstream side of highway bridge, half a mile west of Halstad, $2 \frac{1}{2}$ miles downstream from wild Rice River, and at mile 375.2

Drainage area.--21,800 sq $\mathrm{mi}$, approximately (includes 3,800 $\mathrm{sq} \mathrm{mi}$ in closed basins).

Records available.--April 1936 to June 1937 (no winter records), April 1942 to September 1960 (spring and summer months only), May 1961 to September 1968.

Gage.--Water-stage recorder. Datum of gage is $826.65 \mathrm{ft}$ above mean sea level, datum of 1929 . Prior to July 17 , 1961, wire-weight gage at same site and datum.

Average discharge. --7 years, 1,808 cfs $(1,309,000$ acre-ft per year).

Extremes.--Maximum discharge during year, 2,350 cfs June 19 (gage height, 7.80 ft); maximum gage height, 9.82

ft Mar. 28 (backwater from ice); minimum discharge, $215 \mathrm{cfs}$ Feb. 20, 21 (gage height, 3.82 ft); minimum gage height, 2.52 ft sept. 16

1936-37, 1942-68: Maximum discharge, 26,800 cfs Mar. 27, 1966 (gage height, 35.35 ft, backwater from

ice); minimum discharge observed, 5.4 cfs oct. $8,9,12-14,1936$.

Flood in 1897 reached a stage of about $38.5 \mathrm{ft}$.

Remarks.--Records good. Some regulation by many controlled lakes and reservoirs on tributaries.

DISCHARGE, IN CFS, WATER YEAR OCTOBER 1967 TO SEPTEMBER 1968

\begin{tabular}{|c|c|c|c|c|c|c|c|c|c|c|c|c|c|}
\hline$\overline{D A Y}$ & OCT & NOV & $\mathrm{DEC}$ & & JAN & FEB & MAR & APR & MAY & JUN & JUL & $\overline{A U G}$ & $\overline{\mathrm{SEP}}$ \\
\hline $\begin{array}{l}1 \\
2 \\
3 \\
4 \\
5\end{array}$ & $\begin{array}{l}255 \\
248 \\
244 \\
242 \\
246\end{array}$ & $\begin{array}{l}325 \\
320 \\
322 \\
320 \\
312\end{array}$ & $\begin{array}{l}290 \\
250 \\
255 \\
290 \\
305\end{array}$ & & $\begin{array}{l}280 \\
280 \\
275 \\
275 \\
275\end{array}$ & $\begin{array}{l}240 \\
240 \\
240 \\
240 \\
245\end{array}$ & $\begin{array}{l}250 \\
255 \\
255 \\
255 \\
260\end{array}$ & $\begin{array}{l}1.880 \\
1.640 \\
1.500 \\
1.380 \\
1.160\end{array}$ & $\begin{array}{l}1.650 \\
1.610 \\
1.550 \\
1.450 \\
1.370\end{array}$ & $\begin{array}{l}1.100 \\
1.090 \\
1.070 \\
1.050 \\
1.020\end{array}$ & $\begin{array}{l}1.340 \\
1.440 \\
1.540 \\
1.580 \\
1.520\end{array}$ & $\begin{array}{l}616 \\
596 \\
576 \\
537 \\
498\end{array}$ & $\begin{array}{l}378 \\
361 \\
358 \\
352 \\
341\end{array}$ \\
\hline $\begin{array}{r}6 \\
7 \\
8 \\
9 \\
10\end{array}$ & $\begin{array}{l}238 \\
233 \\
236 \\
253 \\
266\end{array}$ & $\begin{array}{l}297 \\
300 \\
305 \\
305 \\
275\end{array}$ & $\begin{array}{l}315 \\
315 \\
315 \\
315 \\
320\end{array}$ & & $\begin{array}{l}270 \\
270 \\
265 \\
265 \\
260\end{array}$ & $\begin{array}{l}245 \\
245 \\
245 \\
245 \\
245\end{array}$ & $\begin{array}{l}270 \\
285 \\
310 \\
350 \\
375\end{array}$ & $\begin{array}{l}1.130 \\
1.120 \\
1.070 \\
1.200 \\
1.420\end{array}$ & $\begin{array}{l}1.300 \\
1.300 \\
1.320 \\
1.330 \\
1.340\end{array}$ & $\begin{array}{l}1.000 \\
1.080 \\
1.290 \\
1.370 \\
1.400\end{array}$ & $\begin{array}{l}1.400 \\
1.290 \\
1.200 \\
1.110 \\
1.020\end{array}$ & $\begin{array}{l}474 \\
438 \\
387 \\
355 \\
366\end{array}$ & $\begin{array}{l}320 \\
312 \\
314 \\
307 \\
282\end{array}$ \\
\hline $\begin{array}{l}11 \\
12 \\
13 \\
14 \\
15\end{array}$ & $\begin{array}{l}273 \\
280 \\
280 \\
285 \\
300\end{array}$ & $\begin{array}{l}275 \\
280 \\
300 \\
305 \\
285\end{array}$ & $\begin{array}{l}320 \\
325 \\
335 \\
290 \\
280\end{array}$ & & $\begin{array}{l}260 \\
260 \\
255 \\
255 \\
250\end{array}$ & $\begin{array}{l}240 \\
240 \\
235 \\
235 \\
235\end{array}$ & $\begin{array}{l}415 \\
430 \\
480 \\
540 \\
620\end{array}$ & $\begin{array}{l}1.660 \\
1.880 \\
2.000 \\
2.000 \\
1.920\end{array}$ & $\begin{array}{l}1.350 \\
1.360 \\
1.400 \\
1.480 \\
1.580\end{array}$ & $\begin{array}{l}1.520 \\
1.700 \\
1.860 \\
2.090 \\
2.160\end{array}$ & $\begin{array}{l}936 \\
879 \\
849 \\
845 \\
860\end{array}$ & $\begin{array}{l}361 \\
336 \\
325 \\
320 \\
317\end{array}$ & $\begin{array}{l}266 \\
259 \\
253 \\
246 \\
238\end{array}$ \\
\hline $\begin{array}{l}16 \\
17 \\
18 \\
19 \\
20\end{array}$ & $\begin{array}{l}310 \\
317 \\
312 \\
307 \\
292\end{array}$ & $\begin{array}{l}265 \\
250 \\
275 \\
265 \\
250\end{array}$ & $\begin{array}{l}275 \\
270 \\
280 \\
270 \\
275\end{array}$ & & $\begin{array}{l}250 \\
250 \\
250 \\
245 \\
245\end{array}$ & $\begin{array}{l}235 \\
230 \\
230 \\
225 \\
220\end{array}$ & $\begin{array}{r}750 \\
890 \\
990 \\
1150 \\
1.260\end{array}$ & $\begin{array}{l}1.850 \\
1.740 \\
1.610 \\
1.480 \\
1.380\end{array}$ & $\begin{array}{l}1.680 \\
1.700 \\
1.680 \\
1.690 \\
1.680\end{array}$ & $\begin{array}{l}2.190 \\
2.270 \\
2.330 \\
2.340 \\
2.340\end{array}$ & $\begin{array}{l}849 \\
841 \\
883 \\
902 \\
868\end{array}$ & $\begin{array}{l}328 \\
352 \\
393 \\
393 \\
396\end{array}$ & $\begin{array}{l}231 \\
236 \\
257 \\
294 \\
287\end{array}$ \\
\hline $\begin{array}{l}21 \\
22 \\
23 \\
24 \\
25\end{array}$ & $\begin{array}{l}287 \\
285 \\
285 \\
292 \\
290\end{array}$ & $\begin{array}{l}260 \\
275 \\
260 \\
305 \\
310\end{array}$ & $\begin{array}{l}280 \\
280 \\
285 \\
300 \\
300\end{array}$ & & $\begin{array}{l}240 \\
240 \\
240 \\
240 \\
240\end{array}$ & $\begin{array}{l}220 \\
225 \\
225 \\
225 \\
225\end{array}$ & $\begin{array}{l}1.310 \\
1.300 \\
1.300 \\
1.330 \\
1.420\end{array}$ & $\begin{array}{l}1.290 \\
1.220 \\
1.180 \\
1.230 \\
1.300\end{array}$ & $\begin{array}{l}1.640 \\
1.580 \\
1.540 \\
1.480 \\
1.420\end{array}$ & $\begin{array}{l}2.340 \\
2.290 \\
2.140 \\
1.940 \\
1.780\end{array}$ & $\begin{array}{l}830 \\
826 \\
857 \\
841 \\
798\end{array}$ & $\begin{array}{l}387 \\
366 \\
366 \\
364 \\
360\end{array}$ & $\begin{array}{l}292 \\
310 \\
314 \\
372 \\
375\end{array}$ \\
\hline $\begin{array}{l}26 \\
27 \\
28 \\
29 \\
30 \\
31\end{array}$ & $\begin{array}{l}287 \\
282 \\
290 \\
290 \\
292 \\
325\end{array}$ & $\begin{array}{r}315 \\
315 \\
295 \\
290 \\
305\end{array}$ & $\begin{array}{l}285 \\
270 \\
270 \\
280 \\
285 \\
285\end{array}$ & 2 & $\begin{array}{l}240 \\
240 \\
240 \\
240 \\
240 \\
240\end{array}$ & $\begin{array}{r}230 \\
230 \\
240 \\
250 \\
- \\
-\end{array}$ & $\begin{array}{l}1.560 \\
1.900 \\
2.200 \\
2.180 \\
2.100 \\
2.090\end{array}$ & $\begin{array}{l}1.360 \\
1.440 \\
1.490 \\
1.580 \\
1.640 \\
-\end{array}$ & $\begin{array}{l}1.360 \\
1.280 \\
1.240 \\
1.210 \\
1.180 \\
1.140\end{array}$ & $\begin{array}{r}1.660 \\
1.550 \\
1.450 \\
1.380 \\
1.330 \\
-\end{array}$ & $\begin{array}{l}744 \\
704 \\
661 \\
633 \\
619 \\
623\end{array}$ & $\begin{array}{l}365 \\
380 \\
405 \\
366 \\
358 \\
364\end{array}$ & $\begin{array}{r}372 \\
414 \\
447 \\
453 \\
444 \\
-\end{array}$ \\
\hline $\begin{array}{l}\text { TOTAL } \\
\text { MEAN } \\
\text { MAX } \\
\text { MIN } \\
\text { AC-ET }\end{array}$ & $\begin{array}{r}8622 \\
278 \\
325 \\
233 \\
17.100 \\
\end{array}$ & $\begin{array}{r}8761 \\
292 \\
325 \\
250 \\
17,380\end{array}$ & $\begin{array}{r}9.010 \\
291 \\
335 \\
250 \\
17,870\end{array}$ & $\begin{array}{r}7.8 \\
15 .\end{array}$ & $\begin{array}{r}875 \\
254 \\
280 \\
240 \\
.620\end{array}$ & $\begin{array}{r}6.825 \\
235 \\
250 \\
220 \\
13,540\end{array}$ & $\begin{array}{r}29.080 \\
938 \\
2,200 \\
250 \\
57,680\end{array}$ & $\begin{array}{r}44,750 \\
1,492 \\
2,000 \\
1,070 \\
88,760\end{array}$ & $\begin{array}{r}44.890 \\
1,448 \\
1,700 \\
1,140 \\
89,040\end{array}$ & $\begin{array}{r}50.130 \\
1,671 \\
2,340 \\
1,000 \\
99,430\end{array}$ & $\begin{array}{c}30288 \\
977 \\
1,580 \\
619 \\
60,080\end{array}$ & $\begin{array}{r}12.445 \\
401 \\
616 \\
317 \\
24,680 \\
\end{array}$ & $\begin{array}{r}9.685 \\
323 \\
453 \\
231 \\
19,210 \\
\end{array}$ \\
\hline $\begin{array}{l}\text { CAL YR } \\
\text { WAT YR }\end{array}$ & $\begin{array}{l}1967: \\
1968:\end{array}$ & aL & $\begin{array}{l}11 \\
61\end{array}$ & $\begin{array}{l}\text { MEAN } \\
\text { MEAN }\end{array}$ & 1 & $\begin{array}{l}\text { MAX } \\
\text { MAX }\end{array}$ & $\begin{array}{r}13,700 \\
2,340\end{array}$ & $\begin{array}{l}\text { MIN } \\
\text { MIN }\end{array}$ & $\begin{array}{l}134 \\
220\end{array}$ & $\begin{array}{l}A C-F T \\
A C-F T\end{array}$ & $\begin{array}{r}1,203,000 \\
520,400\end{array}$ & & \\
\hline
\end{tabular}




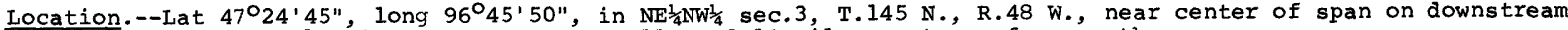
truss of bridge, $3 \frac{3}{4}$ miles southeast of shelly and 10 miles upstream from mouth.

Drainage area. $--151 \mathrm{sq} \mathrm{mi}$.

Records available.--March 1944 to september 1968. Monthly discharge only for March 1944, published in WsP 1308 .

Gage.--Wire-weight gage read once or twice daily and crest-stage gage. Datum of gage is 841.14 ft above mean sea level, datum of 1929 (levels by Corps of Engineers). Prior to oct. 1 , 1965 , chain gage at datum 3.0 ft higher. Oct. 1, 1965, to June 27, 1966, chain gage at same datum.

Average discharqe. --24 years, 80.4 cfs $(58,210$ acre-ft per year).

Extremes.--Maximum discharge during year, 221 cfs Mar. 27 (gage height, 7.35 ft); no flow for many days. 1944-68: Maximum discharge, 4,660 cfs May 11, ig50 (gage height, 18.96 ft, from floodmark); no flow for many days most years.

Remarks.--Records good. Large part of high flow of Wild Rice River diverted into Marsh River basin at overflow section $3 \frac{1}{2}$ miles east of Ada. Another diversion from wild Rice River formed 1 mile southeast of Ada supplemented flow at all stages 1947-51, after which it was closed except for small regulated flow diverted at same point for abatement of pollution from Ada sewage plant effluent.

DISCHARGE, IN CFS, WATER YEAR OCTOBER 1967 TO SEPTEMBER 1968

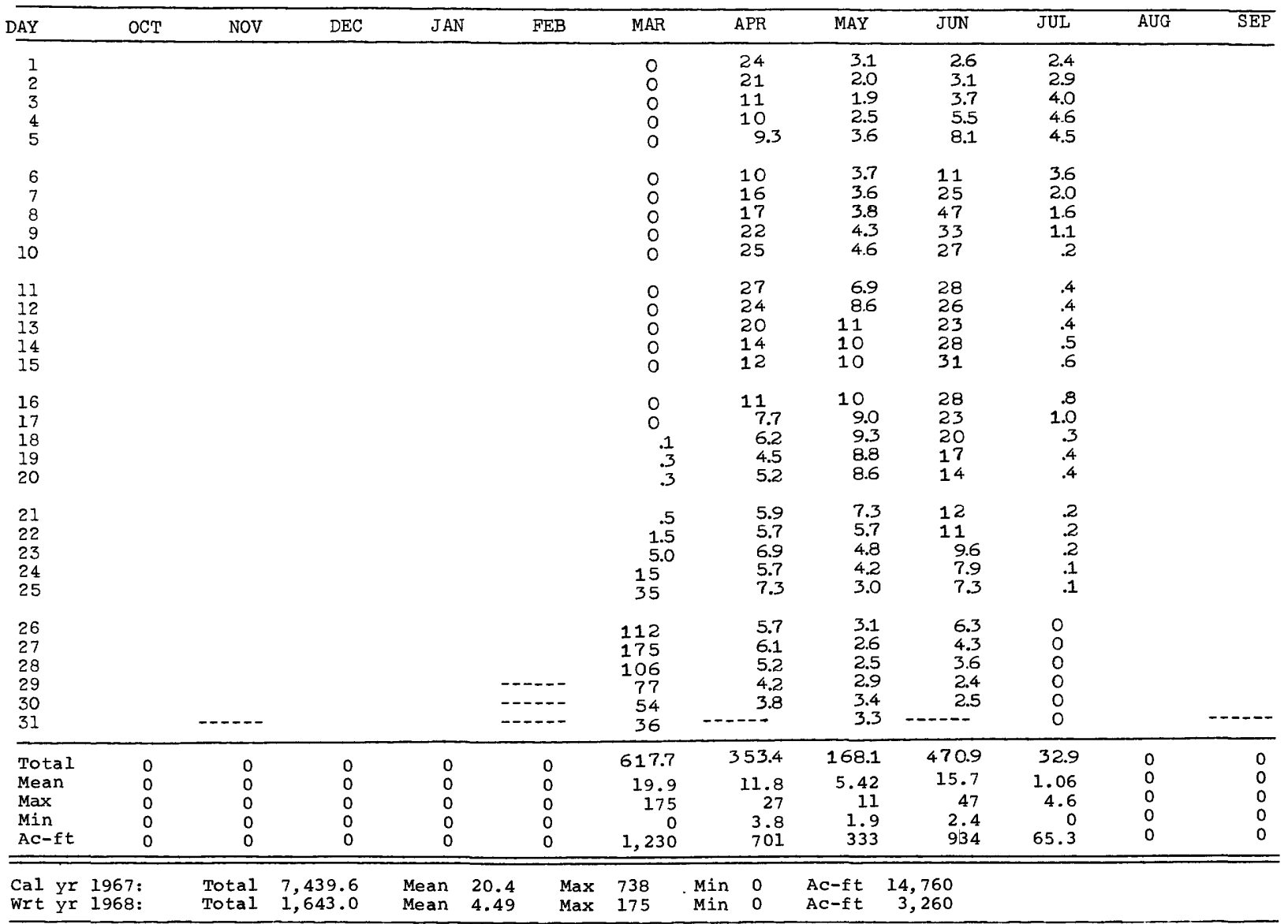


5-0690. Sandhill River at Climax, Minn.

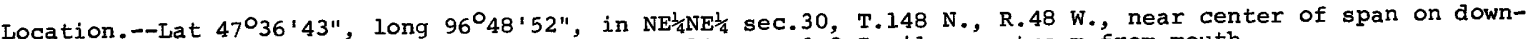
stream side of bridge on U.S. Highway' 75 in Climax and 3.7 miles upstream from mouth.

Records available.--March 1943 to September 1968 (winter records incomplete in some years). Monthly discharge only for some periods, published in WSP 1308.

Gage.-Wire-weight gage and crest-stage gage; gage read once or twice daily. Datum of gage is 820.10 ft above mean sea level, datum of 1929 (levels by corps of Engineers). Prior to oct. 1 , 1966, chain gage at site 3.2 miles upstream at datum $12.78 \mathrm{ft}$ higher.

Average discharge.--22 years $(1946-68), 63.7$ cfs $(46,120$ acre-ft per year).

Extremes.--Maximum discharge during year, 1,400 cfs June 7 (gage height, $12.71 \mathrm{ft}$ ); minimum daily, 7.0 cfs Feb. $21-25$.

1943-68: Maximum discharge, 4,560 cfs Apr. 14, 1965 (gage height 17.81 ft, site and datum then in use); minimum not determined.

Remarks.--Records fair.

DISCHARGE, IN CFS, WATER YEAR OCTOBER 1967 TO SEPTEMBER 1968

\begin{tabular}{|c|c|c|c|c|c|c|c|c|c|c|c|c|}
\hline DAY & OCT & NOV & $\mathrm{DEC}$ & JAN & FEB & MAR & APR & MAY & JUN & JUL & AUG & SEP \\
\hline $\begin{array}{l}1 \\
2 \\
3 \\
4 \\
5\end{array}$ & $\begin{array}{l}13 \\
14 \\
14 \\
14 \\
14\end{array}$ & $\begin{array}{l}14 \\
15 \\
14 \\
13 \\
12\end{array}$ & $\begin{array}{l}11 \\
11 \\
12 \\
12 \\
12\end{array}$ & $\begin{array}{l}10 \\
10 \\
10 \\
9.8 \\
9.5\end{array}$ & $\begin{array}{l}9.5 \\
9.4 \\
9.3 \\
9.2 \\
9.0\end{array}$ & $\begin{array}{l}8.5 \\
9.2 \\
10 \\
13 \\
15\end{array}$ & $\begin{array}{r}135 \\
115 \\
100 \\
85 \\
75\end{array}$ & $\begin{array}{l}35 \\
33 \\
32 \\
31 \\
32\end{array}$ & $\begin{array}{r}35 \\
45 \\
65 \\
160 \\
346\end{array}$ & $\begin{array}{r}71 \\
82 \\
90 \\
100 \\
103\end{array}$ & $\begin{array}{l}17 \\
16 \\
16 \\
16 \\
15\end{array}$ & $\begin{array}{l}13 \\
12 \\
11 \\
12 \\
12\end{array}$ \\
\hline $\begin{array}{r}6 \\
7 \\
8 \\
9 \\
10\end{array}$ & $\begin{array}{l}15 \\
14 \\
14 \\
15 \\
15\end{array}$ & $\begin{array}{l}11 \\
11 \\
11 \\
12 \\
13\end{array}$ & $\begin{array}{l}13 \\
13 \\
13 \\
14 \\
14\end{array}$ & $\begin{array}{r}9.5 \\
9.5 \\
9.5 \\
9.8 \\
10\end{array}$ & $\begin{array}{l}9.0 \\
9.0 \\
8.8 \\
8.8 \\
8.5\end{array}$ & $\begin{array}{l}19 \\
24 \\
28 \\
31 \\
34\end{array}$ & $\begin{array}{r}95 \\
108 \\
92 \\
80 \\
73\end{array}$ & $\begin{array}{l}33 \\
36 \\
37 \\
39 \\
42\end{array}$ & $\begin{array}{r}652 \\
1,320 \\
1,080 \\
590 \\
354\end{array}$ & $\begin{array}{r}100 \\
97 \\
90 \\
75 \\
60\end{array}$ & $\begin{array}{l}15 \\
14 \\
13 \\
12 \\
11\end{array}$ & $\begin{array}{l}12 \\
12 \\
12 \\
13 \\
12\end{array}$ \\
\hline $\begin{array}{l}11 \\
12 \\
13 \\
14 \\
15\end{array}$ & $\begin{array}{l}15 \\
15 \\
14 \\
13 \\
12\end{array}$ & $\begin{array}{l}14 \\
14 \\
13 \\
12 \\
12\end{array}$ & $\begin{array}{l}15 \\
15 \\
15 \\
14 \\
14\end{array}$ & $\begin{array}{l}10 \\
10 \\
10 \\
10 \\
11\end{array}$ & $\begin{array}{l}8.2 \\
8.0 \\
7.7 \\
7.5 \\
7.3\end{array}$ & $\begin{array}{l}36 \\
38 \\
40 \\
42 \\
43\end{array}$ & $\begin{array}{l}75 \\
62 \\
57 \\
55 \\
54\end{array}$ & $\begin{array}{l}43 \\
45 \\
48 \\
50 \\
48\end{array}$ & $\begin{array}{l}478 \\
380 \\
310 \\
250 \\
230\end{array}$ & $\begin{array}{l}45 \\
37 \\
36 \\
38 \\
40\end{array}$ & $\begin{array}{l}11 \\
10 \\
10 \\
9.5 \\
9.0\end{array}$ & $\begin{array}{l}13 \\
13 \\
13 \\
14 \\
13\end{array}$ \\
\hline $\begin{array}{l}16 \\
17 \\
18 \\
19 \\
20\end{array}$ & $\begin{array}{l}12 \\
12 \\
12 \\
12 \\
13\end{array}$ & $\begin{array}{l}12 \\
12 \\
13 \\
13 \\
13\end{array}$ & $\begin{array}{l}14 \\
14 \\
14 \\
14 \\
14\end{array}$ & $\begin{array}{l}11 \\
11 \\
11 \\
11 \\
11\end{array}$ & $\begin{array}{l}7.3 \\
7.2 \\
7.2 \\
7.2 \\
7.1\end{array}$ & $\begin{array}{l}44 \\
46 \\
49 \\
54 \\
62\end{array}$ & $\begin{array}{l}53 \\
50 \\
45 \\
38 \\
32\end{array}$ & $\begin{array}{l}46 \\
44 \\
43 \\
40 \\
38\end{array}$ & $\begin{array}{r}220 \\
180 \\
145 \\
110 \\
96\end{array}$ & $\begin{array}{l}45 \\
48 \\
46 \\
42 \\
35\end{array}$ & $\begin{array}{l}12 \\
18 \\
23 \\
19 \\
18\end{array}$ & $\begin{array}{l}13 \\
13 \\
14 \\
14 \\
13\end{array}$ \\
\hline $\begin{array}{l}21 \\
22 \\
23 \\
24 \\
25\end{array}$ & $\begin{array}{l}13 \\
13 \\
14 \\
13 \\
13\end{array}$ & $\begin{array}{l}14 \\
14 \\
14 \\
14 \\
14\end{array}$ & $\begin{array}{l}14 \\
14 \\
13 \\
13 \\
13\end{array}$ & $\begin{array}{l}11 \\
11 \\
11 \\
11 \\
11\end{array}$ & $\begin{array}{l}7.0 \\
7.0 \\
7.0 \\
7.0 \\
7.0\end{array}$ & $\begin{array}{r}75 \\
95 \\
130 \\
175 \\
225\end{array}$ & $\begin{array}{l}35 \\
45 \\
60 \\
71 \\
74\end{array}$ & $\begin{array}{l}37 \\
35 \\
33 \\
31 \\
30\end{array}$ & $\begin{array}{r}92 \\
98 \\
112 \\
140 \\
128\end{array}$ & $\begin{array}{l}28 \\
25 \\
23 \\
22 \\
20\end{array}$ & $\begin{array}{l}16 \\
15 \\
14 \\
14 \\
18\end{array}$ & $\begin{array}{l}12 \\
12 \\
12 \\
12 \\
12\end{array}$ \\
\hline $\begin{array}{l}26 \\
27 \\
28 \\
29 \\
30 \\
31\end{array}$ & $\begin{array}{l}13 \\
13 \\
12 \\
13 \\
13 \\
14\end{array}$ & $\begin{array}{r}14 \\
13 \\
12 \\
12 \\
12 \\
- \\
-\end{array}$ & $\begin{array}{l}12 \\
12 \\
12 \\
11 \\
11 \\
11\end{array}$ & $\begin{array}{l}10 \\
10 \\
10 \\
9.8 \\
9.5 \\
9.5\end{array}$ & $\begin{array}{r}7.1 \\
7.6 \\
7.8 \\
8.2 \\
- \\
-\end{array}$ & $\begin{array}{l}340 \\
380 \\
350 \\
300 \\
230 \\
180\end{array}$ & $\begin{array}{r}69 \\
62 \\
55 \\
47 \\
39 \\
- \\
-\end{array}$ & $\begin{array}{l}28 \\
27 \\
30 \\
38 \\
42 \\
41\end{array}$ & $\begin{array}{r}118 \\
109 \\
96 \\
76 \\
64 \\
- \\
\end{array}$ & $\begin{array}{l}20 \\
18 \\
17 \\
18 \\
17 \\
17\end{array}$ & $\begin{array}{l}20 \\
18 \\
17 \\
16 \\
17 \\
14\end{array}$ & $\begin{array}{r}11 \\
10 \\
10 \\
10 \\
10 \\
- \\
\end{array}$ \\
\hline $\begin{array}{l}\text { Total } \\
\text { Mean } \\
\text { Max } \\
\text { Min } \\
\text { Ac-ft }\end{array}$ & $\begin{array}{r}416 \\
13.4 \\
15 \\
12 \\
825 \\
\end{array}$ & $\begin{array}{r}387 \\
12.9 \\
15 \\
11 \\
768 \\
\end{array}$ & $\begin{array}{r}404 \\
13.0 \\
15 \\
11 \\
801\end{array}$ & $\begin{array}{r}317.4 \\
10.2 \\
11 \\
9.5 \\
630\end{array}$ & $\begin{array}{r}230.9 \\
7.96 \\
9.5 \\
7.0 \\
458\end{array}$ & $\begin{array}{r}3,125.7 \\
101 \\
380 \\
8.5 \\
6,200 \\
\end{array}$ & $\begin{array}{r}2,036 \\
67.9 \\
135 \\
32 \\
4,040 \\
\end{array}$ & $\begin{array}{r}1,167 \\
37,6 \\
50 \\
27 \\
2,310 \\
\end{array}$ & $\begin{array}{r}8,079 \\
269 \\
1,320 \\
35 \\
16,020 \\
\end{array}$ & $\begin{array}{r}1,505 \\
48.5 \\
103 \\
17 \\
2,990 \\
\end{array}$ & $\begin{array}{r}463.5 \\
15.0 \\
23 \\
9.0 \\
919 \\
\end{array}$ & $\begin{array}{r}365 \\
12.2 \\
14 \\
10 \\
724 \\
\end{array}$ \\
\hline $\begin{array}{l}\text { Cal yr } \\
\text { wtr yr }\end{array}$ & $\begin{array}{l}1967: \\
1968:\end{array}$ & $\begin{array}{l}\text { Total } \\
\text { Total }\end{array}$ & .4 & $\begin{array}{ll}\text { Mean } 60 \\
\text { Mean } & 50\end{array}$ & $\begin{array}{l}\operatorname{Max} \\
\operatorname{Max}\end{array}$ & $\begin{array}{l}1,080 \\
1,320\end{array}$ & $\begin{array}{ll}\operatorname{Min} & 9.8 \\
\operatorname{Min} & 7.0\end{array}$ & $\begin{array}{l}\text { Ac-ft } \\
\text { Ac-ft }\end{array}$ & $\begin{array}{l}43,550 \\
36,690\end{array}$ & & & \\
\hline
\end{tabular}


Iocation.--Lat $47^{\circ} 57^{\prime}$, long $95^{\circ} 17^{\prime}$, in NW/ sec. 28 , T.152 N., R.36 w., on left bank just upstream from dam at outlet, 13 miles northwest of village of Red lake.

Drainage area.--1,950 sq $\mathrm{mi}$, approximately.

Records available.--June 1930 to November 1932 (published as Red Lake at Redby), May 1933 to September 1968 (published as Red Lake near Red Lake 1933-40); records on Upper Red Lake published as Red Lake at Waskish, April 1930 to September 1933, all in reports of Geological Survey. October 1921 to September 1929 gage heights at Redby and on Upper Red Lake at Waskish in files of Minnesota Department of Conservation (fragmentary).

Gage.-Water-stage recorder. Datum of gage is $1,169.00 \mathrm{ft}$ above mean sea level, adjustment of 1912 (levels by Corps of Engineers). May 1933 to Sept. 6, 1934, staff gage at same site and datum. staff gages at Waskish and Redby at datum $69.00 \mathrm{ft}$ lower.

Extremes.--Maximum gage height during year, $6.77 \mathrm{ft} \mathrm{July} 16$ (affected by wind action); maximum daily, 5.92 ft July 16; minimum, $3.61 \mathrm{ft}$ Nov. 15 (result of freeze-up); minimum daily, $4.05 \mathrm{ft}$ Nov. 15.

1930-68: Maximum gage height, $9.53 \mathrm{ft} J u n e ~ 25,1950$; minimum recorded, $0.80 \mathrm{ft}$ Nov. 20 , 1936.

Remarks:--Water level subject to fluctuation caused by change in direction and velocity of wind and by seiches.

Month-end gage height, in feet, October 1967 to september 1968
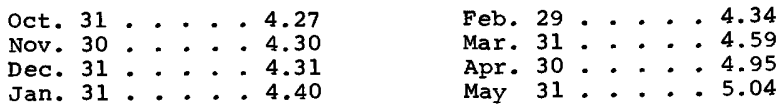

June $30 . . .5 .50$

July 31 ... 5.60

Aug. 31 ... 5.29

Jan. $31 . . .4 .40$

Sept. $30 . . .5 .22$

Note.--Mean daily gage heights are available. 
5-0745. Red Lake River near Red Lake, Minn.

Location.--Lat $47^{\circ} 57^{\prime}$, long $95^{\circ} 17^{\prime}$, in NWh sec.28, T.152 N., R. 36 w., on left bank 50 ft downstream from dam at outlet of Lower Red Lake and 13 miles northwest of village of Red Lake.

Drainage area.--1,950 sq $\mathrm{mi}$, approximately.

Records available.--May 1933 to September 1968. Monthly discharge only for May 1933 , published in wSP 1.308.

Gage.--Water-stage recorder. Datum of gage is $1.167 .00 \mathrm{ft}$ above mean sea level, adjustment of 1912 (1evels by Corps of Engineers). Prior to Sept. 7, 1934, staff gage at site 50 ft upstream at datum 2.00 ft higher.

Sept. 7, 1934, to Nov. 26, 1951, water-stage recorder at present site at datum 2.00 ft higher.

Average discharge.--35 years, 428 cfs $(309,900$ acre-ft per year)'.

Extremes.--Maximum daily discharge during year, 705 cfs July 16; maximum gage height, 6.41 ft Aug. 9 (affected by backwater from aquatic vegetation); minimum discharge, 2.3 cfs May 26,27 (gage height, 0.79 ft)

1933-68: Maximum discharge, 3,600 cfs June 25, 1950 (gage height, 11.19 ft, affected by seiches and backwater from aquatic vegetation, present datum), from rating curve extended above 1,400 cfs; no flow at times.

Remarks.--Records fair. Flow completely regulated by outlet dam on Lower Red Lake.

DISCHARGE, IN CFS, WATER YEAR OCTOBER 1967 TO SEPTEMBER 1968

\begin{tabular}{|c|c|c|c|c|c|c|c|c|c|c|c|c|}
\hline DAY & $\mathrm{OCT}$ & NOV & $\mathrm{DEC}$ & JAN & FEB & MAR & APR & MAY & JUN & JUL & AUG & SEP \\
\hline $\begin{array}{l}1 \\
2 \\
3 \\
4 \\
5\end{array}$ & $\begin{array}{l}539 \\
539 \\
532 \\
488 \\
390\end{array}$ & $\begin{array}{l}325 \\
325 \\
325 \\
328 \\
325\end{array}$ & $\begin{array}{l}280 \\
255 \\
240 \\
235 \\
235\end{array}$ & $\begin{array}{l}210 \\
210 \\
210 \\
210 \\
210\end{array}$ & $\begin{array}{l}195 \\
195 \\
195 \\
195 \\
195\end{array}$ & $\begin{array}{l}215 \\
215 \\
215 \\
215 \\
210\end{array}$ & $\begin{array}{l}38 \\
38 \\
37 \\
37 \\
38\end{array}$ & $\begin{array}{l}15 \\
23 \\
26 \\
22 \\
22\end{array}$ & $\begin{array}{l}10 \\
10 \\
2.5 \\
2.4 \\
2.5\end{array}$ & $\begin{array}{l}658 \\
612 \\
609 \\
612 \\
606\end{array}$ & $\begin{array}{l}474 \\
468 \\
471 \\
471 \\
471\end{array}$ & $\begin{array}{l}550 \\
553 \\
553 \\
550 \\
556\end{array}$ \\
\hline $\begin{array}{r}6 \\
7 \\
8 \\
9 \\
10\end{array}$ & $\begin{array}{l}354 \\
345 \\
357 \\
360 \\
354\end{array}$ & $\begin{array}{l}325 \\
312 \\
328 \\
312 \\
312\end{array}$ & $\begin{array}{l}230 \\
225 \\
220 \\
215 \\
215\end{array}$ & $\begin{array}{l}210 \\
215 \\
215 \\
220 \\
220\end{array}$ & $\begin{array}{l}190 \\
190 \\
190 \\
190 \\
195\end{array}$ & $\begin{array}{l}210 \\
210 \\
190 \\
145 \\
120\end{array}$ & $\begin{array}{l}41 \\
41 \\
60 \\
33 \\
26\end{array}$ & $\begin{array}{l}8.0 \\
3.6 \\
9.2 \\
22 \\
20\end{array}$ & $\begin{array}{l}2.5 \\
2.4 \\
3.6 \\
3.0 \\
10\end{array}$ & $\begin{array}{l}592 \\
595 \\
602 \\
584 \\
578\end{array}$ & $\begin{array}{l}536 \\
595 \\
595 \\
595 \\
588\end{array}$ & $\begin{array}{l}570 \\
584 \\
606 \\
595 \\
602\end{array}$ \\
\hline $\begin{array}{l}11 \\
12 \\
13 \\
14 \\
15\end{array}$ & $\begin{array}{l}345 \\
345 \\
360 \\
342 \\
342\end{array}$ & $\begin{array}{l}312 \\
314 \\
312 \\
304 \\
345\end{array}$ & $\begin{array}{l}220 \\
230 \\
235 \\
235 \\
230\end{array}$ & $\begin{array}{l}220 \\
220 \\
220 \\
220 \\
220\end{array}$ & $\begin{array}{l}195 \\
195 \\
195 \\
200 \\
200\end{array}$ & $\begin{array}{l}115 \\
110 \\
108 \\
106 \\
105\end{array}$ & $\begin{array}{l}26 \\
31 \\
26 \\
36 \\
30\end{array}$ & $\begin{array}{c}20 \\
18 \\
8.0 \\
5.8 \\
20\end{array}$ & $\begin{array}{l}26 \\
30 \\
48 \\
82 \\
96\end{array}$ & $\begin{array}{l}570 \\
564 \\
567 \\
570 \\
588\end{array}$ & $\begin{array}{l}570 \\
564 \\
553 \\
550 \\
546\end{array}$ & $\begin{array}{l}522 \\
381 \\
354 \\
337 \\
325\end{array}$ \\
\hline $\begin{array}{l}16 \\
17 \\
18 \\
19 \\
20\end{array}$ & $\begin{array}{l}331 \\
339 \\
334 \\
328 \\
354\end{array}$ & $\begin{array}{l}325 \\
310 \\
310 \\
320 \\
340\end{array}$ & $\begin{array}{l}230 \\
230 \\
230 \\
230 \\
230\end{array}$ & $\begin{array}{l}215 \\
215 \\
215 \\
215 \\
215\end{array}$ & $\begin{array}{l}200 \\
200 \\
205 \\
205 \\
205\end{array}$ & $\begin{array}{l}102 \\
101 \\
100 \\
100 \\
100\end{array}$ & $\begin{array}{l}36 \\
36 \\
28 \\
26 \\
26\end{array}$ & $\begin{array}{l}28 \\
26 \\
23 \\
22 \\
12\end{array}$ & $\begin{array}{r}90 \\
77 \\
87 \\
73 \\
211\end{array}$ & $\begin{array}{l}705 \\
623 \\
602 \\
522 \\
425\end{array}$ & $\begin{array}{l}536 \\
532 \\
525 \\
508 \\
512\end{array}$ & $\begin{array}{l}331 \\
345 \\
357 \\
369 \\
400\end{array}$ \\
\hline $\begin{array}{l}21 \\
22 \\
23 \\
24 \\
25\end{array}$ & $\begin{array}{l}312 \\
312 \\
314 \\
325 \\
328\end{array}$ & $\begin{array}{l}315 \\
305 \\
310 \\
325 \\
335\end{array}$ & $\begin{array}{l}230 \\
225 \\
220 \\
220 \\
220\end{array}$ & $\begin{array}{l}215 \\
210 \\
210 \\
210 \\
210\end{array}$ & $\begin{array}{l}210 \\
210 \\
210 \\
210 \\
210\end{array}$ & $\begin{array}{l}95 \\
85 \\
74 \\
68 \\
60\end{array}$ & $\begin{array}{l}41 \\
33 \\
58 \\
30 \\
26\end{array}$ & $\begin{array}{l}9.2 \\
9.2 \\
6.8 \\
2.5 \\
2.5\end{array}$ & $\begin{array}{l}462 \\
529 \\
546 \\
560 \\
570\end{array}$ & $\begin{array}{l}387 \\
307 \\
419 \\
564 \\
584\end{array}$ & $\begin{array}{l}522 \\
525 \\
532 \\
539 \\
542\end{array}$ & $\begin{array}{l}505 \\
539 \\
567 \\
578 \\
584\end{array}$ \\
\hline $\begin{array}{l}26 \\
27 \\
28 \\
29 \\
30 \\
31 \\
\end{array}$ & $\begin{array}{l}317 \\
320 \\
301 \\
323 \\
328 \\
323 \\
\end{array}$ & $\begin{array}{r}350 \\
350 \\
350 \\
345 \\
330 \\
- \\
\end{array}$ & $\begin{array}{l}215 \\
215 \\
215 \\
210 \\
210 \\
210 \\
\end{array}$ & $\begin{array}{l}205 \\
205 \\
205 \\
205 \\
200 \\
200 \\
\end{array}$ & $\begin{array}{r}215 \\
215 \\
215 \\
215 \\
----- \\
- \\
\end{array}$ & $\begin{array}{l}53 \\
47 \\
43 \\
41 \\
40 \\
38 \\
\end{array}$ & $\begin{array}{r}23 \\
23 \\
23 \\
23 \\
9.2 \\
---- \\
\end{array}$ & $\begin{array}{l}2.4 \\
2.5 \\
3.0 \\
4.2 \\
3.0 \\
3.0 \\
\end{array}$ & $\begin{array}{c}567 \\
560 \\
560 \\
570 \\
592 \\
----- \\
\end{array}$ & $\begin{array}{l}584 \\
560 \\
542 \\
522 \\
505 \\
481 \\
\end{array}$ & $\begin{array}{l}550 \\
550 \\
536 \\
539 \\
542 \\
546 \\
\end{array}$ & $\begin{array}{r}581 \\
578 \\
578 \\
578 \\
581 \\
- \\
\end{array}$ \\
\hline $\begin{array}{l}\text { Total } \\
\text { Mean } \\
\text { Max } \\
\text { Min } \\
\text { Ac ft }\end{array}$ & $\begin{array}{r}11,181 \\
361 \\
539 \\
301 \\
22,180 \\
\end{array}$ & $\begin{array}{r}9,724 \\
324 \\
350 \\
304 \\
19,290\end{array}$ & $\begin{array}{r}7.040 \\
227 \\
280 \\
210 \\
13,960 \\
\end{array}$ & $\begin{array}{r}6,580 \\
212 \\
220 \\
200 \\
13,050 \\
\end{array}$ & $\begin{array}{r}5,840 \\
201 \\
215 \\
190 \\
11,580\end{array}$ & $\begin{array}{r}3,636 \\
117 \\
215 \\
38 \\
7,210 \\
\end{array}$ & $\begin{array}{r}979.2 \\
32.6 \\
60 \\
9.2 \\
1,940\end{array}$ & $\begin{array}{r}401.9 \\
13.0 \\
28 \\
2.4 \\
797\end{array}$ & $\begin{array}{r}6,384.9 \\
213 \\
592 \\
2.4 \\
12,660 \\
\end{array}$ & $\begin{array}{r}17,239 \\
556 \\
705 \\
307 \\
34,190 \\
\end{array}$ & $\begin{array}{r}16,583 \\
535 \\
595 \\
468 \\
32,890 \\
\end{array}$ & $\begin{array}{r}15,109 \\
504 \\
606 \\
325 \\
29,970 \\
\end{array}$ \\
\hline $\begin{array}{l}\text { Cal yr } \\
\text { Wtr yr }\end{array}$ & $\begin{array}{l}967: \\
968:\end{array}$ & $\begin{array}{l}\operatorname{tal} \\
\operatorname{tal}\end{array}$ & $\begin{array}{l}87 \\
97.0\end{array}$ & $\begin{array}{ll}\text { ean } & 691 \\
\text { ean } & 275\end{array}$ & $\begin{array}{l}\operatorname{Max} \\
\operatorname{Max}\end{array}$ & $\begin{array}{r}1,240 \\
705\end{array}$ & $\begin{array}{ll}\operatorname{lin} & 82 \\
\operatorname{lin} & 2.4\end{array}$ & $\begin{array}{l}A C \text { ft } \\
\text { AC ft }\end{array}$ & $\begin{array}{l}500,000 \\
199,700\end{array}$ & & & \\
\hline
\end{tabular}


5-0750. Red Lake River at High Landing, near Goodridge, Minn.

Location.--Lat $48^{\circ} 03^{\prime}$, long $95^{\circ} 48^{\prime}$, on line between secs. 28 and 29, T.153 N., R.40 W., on left bank at upstream side of highway bridge at High Landing, 7 miles south of Goodridge and 33 miles upstream from Thief River.

Drainage area.--2,300 sq mi, approximately.

Records available.--September 1929 to September 1968. Prior to October 1930, published as "at Kratka."

Gage.--Water-stage recorder. Datum of gage is 1,141.57 ft above mean sea level, adjustment of 1912 (levels by Corps of Engineers). Prior to Oct. 1, 1930, staff gage at site 10 miles downstream at different datum.

oct. 1, 1930, to sept. 30, 1932, staff gage at datum 5.00 ft higher and oct. 1, 1932, to Dec. 8, 1938, staff gage at datum $4.00 \mathrm{ft}$ higher at site $50 \mathrm{ft}$ downstream. Dec. 9, 1938, to Sept. 30, 1949, water-stage record-

er at present site at datum $4.00 \mathrm{ft}$ higher.

Average discharge.--39 years, 457 cfs $(330,900$ acre-ft per year).

Extremes.--Maximum discharge during year, 2,770 cfs July 18 (gage height, 11:98 ft); minimum, 16 cfs June 5 (gage height, $0.53 \mathrm{ft}$ )

1929-68: Maximum discharge, 3,720 cfs May 11, 1950 (gage height, 13.42 ft); no flow during infrequent periods in 1931-34, 1936-37.

Remarks.--Records good except those for winter months, which are fair. Flow regulated by outlet dam on Lower Red Lake.

DISCHARGE, IN CFS, WATER YEAR OCTOBER 1967 TO SEPTEMBER 1968

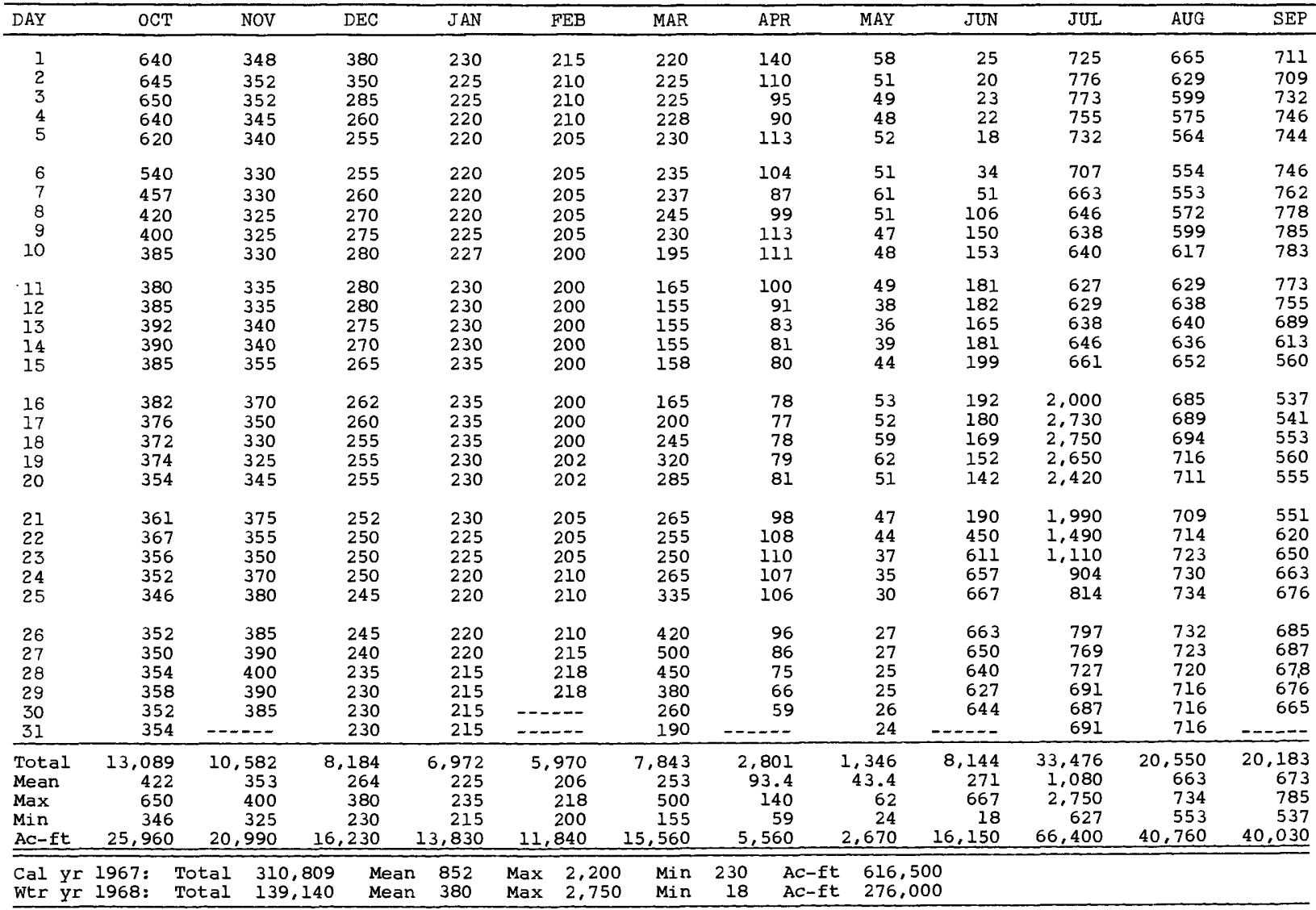


5-0760. Thief River near Thief River Falls, Minn.

Location.--Lat $48^{\circ} 11^{\prime}$, long $96^{\circ} 10^{\prime}$, in sec.3, T.154 N., R.43 W., on right bank a quarter of a mile upstream from highway bridge, 5 miles north of city of Thief River Falls, 7 miles upstream from mouth, and 9 miles downstream from Mud Lake National Wild Life Refuge.

Drainage area.--959 sq $\mathrm{mi}$.

Records available.--July 1909 to September 1917, April 1920 to September 1921, October 1922 to September 1924, October 1928 to september 1968 . Monthly discharge only for some periods, annual maximums for water years $1919,1922,1925,1926$, published in WSP 1308

Gage.--Digital water-stage recorder and control of grouted boulders. Datum of gage is $1,112.33$ ft above mean sea level, datum of 1929 (levels by Minnesota Highway Department). Prior to May 4 , 1939 , staff or chain gage at same site and datum. May 4, 1939 to Aug. 20, 1968, graphic water-stage recorder at present site and datum.

Average discharge.--51 years, 149 cfs $(107,900$ acre-ft per year).

Extremes.--Maximum discharge during year, 1,950 cfs July 18 (gage height, 10.84 ft); no flow on many days.

1909-17, 1919-26, 1928-68: Maximum discharge, 5,610 cfs May 13, 1950 (gage height, 17.38 ft); no flow at times in some years.

Remarks.--Records good except those for winter months, which are fair. Some regulation by Thief and Mud Lakes.

DISCHARGE, IN CFS, WATER YEAR OCTOBER 1967 TO SEPTEMBER 1968

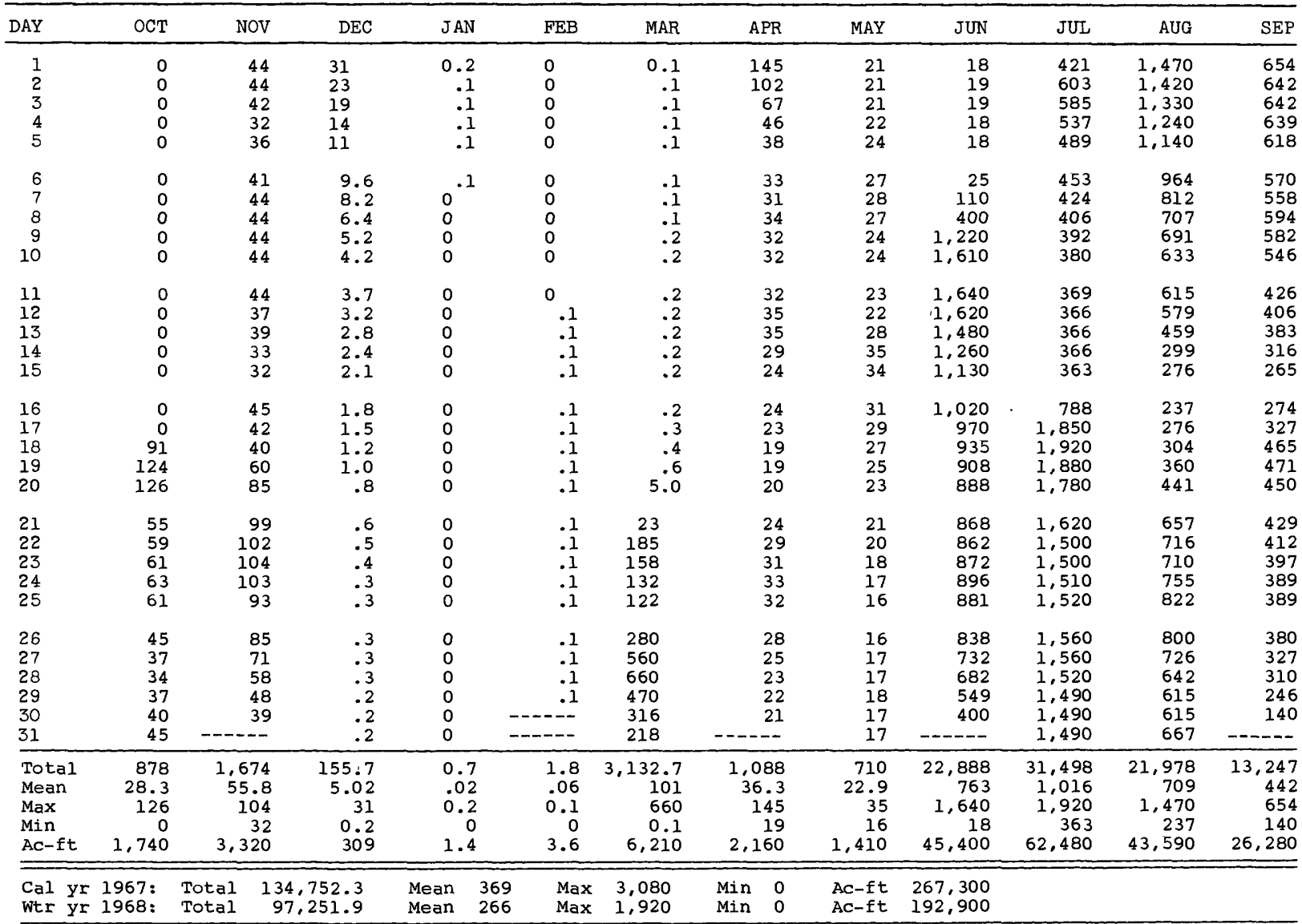


Location:--Lat $47^{\circ} 44^{\prime} 50^{\prime \prime}$, long $95^{\circ} 24^{\prime} 45^{\prime \prime}$, on line between secs. 5 and 8 , T.149 N., R.37 W., on downstream side of bridge on County Highway 17,4 miles upstream from mouth, and $4 \frac{3}{4}$ miles east of Gonvick.

Drainage area. $--45.2 \mathrm{sq} \mathrm{mi}$.

Records available.--April 1960 to September 1968. Monthly and daily figures for April 1, 1960 to June 30 , 1960 published in WSP 1913.

Gage.--Wire-weight gage read once daily and crest-stage gage. Datum of gage is $1,227.93$ ft above mean sea level, adjustment of 1912 (levels by corps of Engineers). Prior to sept. 9, 1960, reference point at same site and datum.

Average discharge. --8 years, $14.7 \mathrm{cfs}(10,640$ acre-ft per year).

Extremes.--Maximum discharge during year, $76 \mathrm{cfs}$ June 10 (gage height, $2.70 \mathrm{ft}$, from graph based on gage readings); maximum gage height, $5.65 \mathrm{ft}$ Mar. 19 (from graph based on gage readings, backwater from ice); no flow Feb. 20 to Mar. 6 .

1960-68: Maximum discharge, 453 cfs Mar. 30, 1967 (gage height, 6.35 ft, from graph based on gage readings): maximum gage height, $6.55 \mathrm{ft}$ Mar. 29, 1967 (from graph based on gage readings, backwater from ice);

no flow Feb. 20 to Mar. 6, 1968.

Remarks.--Records good except those for winter months, which are fair.

DISCHARGE, IN CFS, WATER YEAR OCTOBER 1967 TO SEPTEMBER 1968

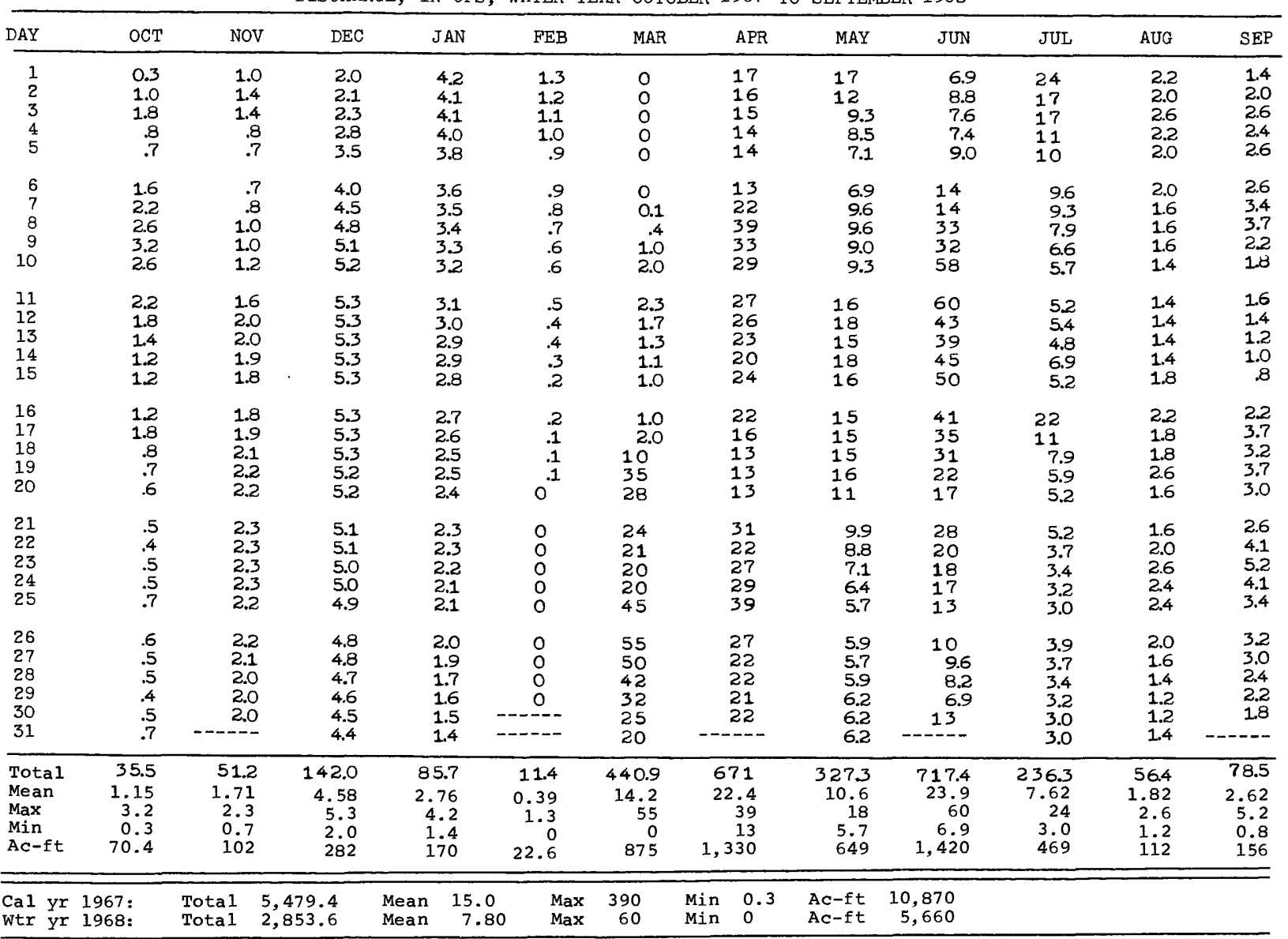

\footnotetext{
Peak discharge (base, 65 cfs)

Date Time height Discharge

$\begin{array}{cccc}6-10 & 2000 & 2.70 & 76\end{array}$
} 
5-0780. Clearwater River at Plumer, Minn.

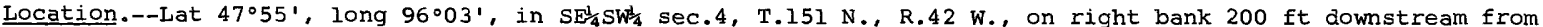
Soo Line Railroad bridge, $300 \mathrm{ft}$ downstream from bridge on U. S. Highway 59 , $0.9 \mathrm{mile}$ northwest of railroad depot in Plummer, and 8 miles upstream from Hill River.

Drainage area.--512 sq $\mathrm{mi}$.

Records available.--April 1939 to september 1968.

Gage.--Digital water-stage recorder. Datum of gage is 1,099.12 ft above mean sea level, adjustment of 1912

(levels by Corps of Engineers). Prior to Nov. 10, 1939, staff gage at site loo ft upstream at same datum.

Nov. 11, 1939 to Aug. 20, 1968, graphic water-stage recorder at same site and datum.

Average discharge.--29 years, 175 cfs $(126,690$ acre-ft per year).

Extremes.--Maximum discharge during year, 3,000 cfs July 18 (gage height, 11.00 ft); minimum, 26 cfs Nov. 5 (gage height, $2.31 \mathrm{ft}$ ).

1939-68: Maximum discharge, 3,640 cfs June 9, 1962 (gage height, 11.90 ft); maximum gage height, 11.97

ft Apr. 11, 1965 (backwater from ice); minimum discharge, 7.9 cfs July 8 , 1940 .

Remarks.--Records good except those for winter months, which are fair.

DISCHARGE, IN CUBIC FEET PER SECOND, WATER YEAR OCTOBER 1967 TO SEPTEMBER 1968

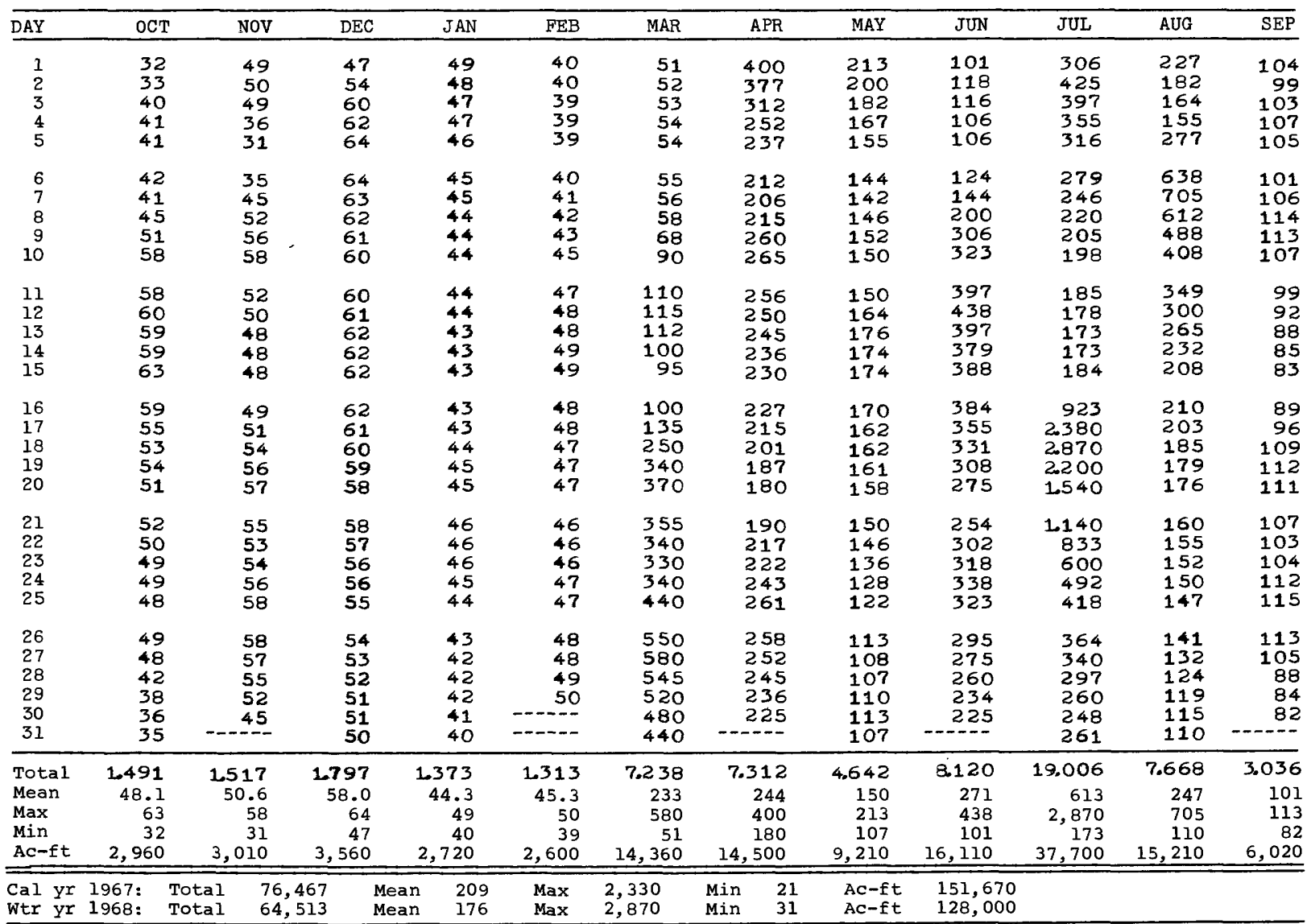

Peak discharge (base, 500 cfs)

\begin{tabular}{|c|c|r|r||l|l|l|c|}
\hline Date & Time & $\begin{array}{r}\text { Gage } \\
\text { height }\end{array}$ & Discharge & Date & Time & $\begin{array}{c}\text { Gage } \\
\text { height }\end{array}$ & Discharge \\
\hline $3-27$ & 0300 & 7.15 & 610 & $8-7$ & 1600 & 5.96 & 718 \\
$7-18$ & 0430 & 11.00 & 3,000 & & & & \\
\hline
\end{tabular}




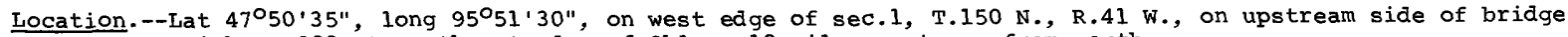
on State Highway 222 at northwest edge of oklee, 12 miles upstream from mouth.

Drainage area.--266 sq $\mathrm{mi}$.

Records available.--April 1960 to September 1968. Monthly and daily figures for April 1 , 1960 to June 30 , 1960 published in WSP 1913.

Gage.--Wire-weight gage read once or twice daily and crest-stage gage. Datum of gage is 1 , 126.94 ft above mean sea level, adjustment of 1912 (levels by Corps of Engineers). Prior to sept. 9, 1960, reference points at same site at datum $8.00 \mathrm{ft}$ higher. September 9, 1960, to sept. 30, 1964, wire-weight gage at same site at datum $8.00 \mathrm{ft}$ higher.

Average discharge. -8 years, 75.1 cfs $(54,370$ acre-ft per year).

Extremes.--Maximum discharge during year, 551 cfs July 17 (gage height, $7.35 \mathrm{ft}$, from graph based on gage readings); maximum gage height, $9.05 \mathrm{ft}$ Mar. 19 (from graph based on gage readings, backwater from ice); minimum daily discharge, 0.2 cfs Feb. 13 to Mar. 3; minimum gage height, $2.00 \mathrm{ft}$ Oct. 1.

1960-68: Maximum discharge, 2, $880 \mathrm{cfs}$ Mar. 31 , 1967 (gage height, $14.17 \mathrm{ft}$, from graph based on gage readings) no flow Feb. 16 to Mar. 21, 1963, Feb. 15 to Mar. 2, 1964.

Maximum stage known since at least 1897, $18.39 \mathrm{ft}$ Apr. 21, 1950 (present datum), from floodmarks.

Remarks.--Records good except those for winter months, which are fair.

\begin{tabular}{|c|c|c|c|c|c|c|c|c|c|c|c|c|}
\hline & & & DISCHARGE, & IN CFS, & WATER & YEAR OCTOBER & $\mathrm{R} \quad 1967 \mathrm{TO}$ & SEPTEMBER & 1968 & & & \\
\hline DAY & OCT & NOV & $\mathrm{DEC}$ & JAN & FEB & MAR & APR & MAY & JUN & JUL & $\mathrm{AUG}$ & SEP \\
\hline $\begin{array}{l}1 \\
2 \\
3 \\
4 \\
5\end{array}$ & $\begin{array}{l}1.1 \\
1.2 \\
2.7 \\
2.7 \\
1.2\end{array}$ & $\begin{array}{l}3.2 \\
2.4 \\
2.0 \\
1.9 \\
1.8\end{array}$ & $\begin{array}{l}3.0 \\
3.1 \\
3.3 \\
3.5 \\
3.7\end{array}$ & $\begin{array}{l}3.4 \\
3.2 \\
3.0 \\
2.8 \\
2.5\end{array}$ & $\begin{array}{r}0.5 \\
.4 \\
.4 \\
.4 \\
.4\end{array}$ & $\begin{array}{r}0.2 \\
.2 \\
.2 \\
.3 \\
.8\end{array}$ & $\begin{array}{l}188 \\
152 \\
150 \\
147 \\
162\end{array}$ & $\begin{array}{l}69 \\
65 \\
57 \\
54 \\
49\end{array}$ & $\begin{array}{l}32 \\
38 \\
40 \\
38 \\
39\end{array}$ & $\begin{array}{l}264 \\
300 \\
226 \\
179 \\
139\end{array}$ & $\begin{array}{c}12 \\
11 \\
7.5 \\
6.8 \\
6.4\end{array}$ & $\begin{array}{c}7.3 \\
7.5 \\
8.1 \\
16 \\
16\end{array}$ \\
\hline $\begin{array}{r}6 \\
7 \\
8 \\
9 \\
10\end{array}$ & $\begin{array}{l}1.2 \\
1.8 \\
1.5 \\
4.7 \\
2.7\end{array}$ & $\begin{array}{l}2.1 \\
2.4 \\
2.3 \\
2.4 \\
3.3\end{array}$ & $\begin{array}{l}4.2 \\
4.5 \\
4.7 \\
4.9 \\
5.0\end{array}$ & $\begin{array}{l}2.3 \\
2.1 \\
1.9 \\
1.7 \\
1.4\end{array}$ & $\begin{array}{l}.4 \\
.4 \\
.4 \\
.4 \\
.3\end{array}$ & $\begin{array}{l}1.7 \\
3.0 \\
6.0 \\
13 \\
19\end{array}$ & $\begin{array}{l}127 \\
128 \\
127 \\
133 \\
123\end{array}$ & $\begin{array}{l}51 \\
57 \\
59 \\
60 \\
54\end{array}$ & $\begin{array}{r}42 \\
47 \\
70 \\
128 \\
129\end{array}$ & $\begin{array}{r}107 \\
79 \\
71 \\
61 \\
54\end{array}$ & $\begin{array}{l}5.6 \\
5.1 \\
4.7 \\
4.7 \\
4.7\end{array}$ & $\begin{array}{l}16 \\
14 \\
14 \\
14 \\
12\end{array}$ \\
\hline $\begin{array}{l}11 \\
12 \\
13 \\
14 \\
15\end{array}$ & $\begin{array}{l}2.2 \\
1.6 \\
1.4 \\
2.1 \\
2.2\end{array}$ & $\begin{array}{l}3.7 \\
3.5 \\
2.9 \\
2.8 \\
2.7\end{array}$ & $\begin{array}{l}5.1 \\
5.1 \\
5.0 \\
5.0 \\
4.9\end{array}$ & $\begin{array}{l}1.3 \\
1.2 \\
1.1 \\
1.1 \\
1.0\end{array}$ & $\begin{array}{l}3 \\
.3 \\
2 \\
2 \\
2\end{array}$ & $\begin{array}{l}18 \\
16 \\
13 \\
11 \\
10\end{array}$ & $\begin{array}{r}112 \\
105 \\
96 \\
87 \\
89\end{array}$ & $\begin{array}{l}69 \\
97 \\
93 \\
95 \\
96\end{array}$ & $\begin{array}{l}153 \\
157 \\
122 \\
169 \\
161\end{array}$ & $\begin{array}{l}49 \\
47 \\
48 \\
49 \\
54\end{array}$ & $\begin{array}{l}4.5 \\
4.2 \\
4.1 \\
3.7 \\
3.6\end{array}$ & $\begin{array}{c}9.6 \\
10 \\
8.1 \\
7.5 \\
9.1\end{array}$ \\
\hline $\begin{array}{l}16 \\
17 \\
18 \\
19 \\
20\end{array}$ & $\begin{array}{l}2.6 \\
3.0 \\
4.1 \\
3.7 \\
3.3\end{array}$ & $\begin{array}{l}2.9 \\
3.1 \\
3.3 \\
3.4 \\
3.4\end{array}$ & $\begin{array}{l}4.9 \\
4.9 \\
4.8 \\
4.8 \\
4.8\end{array}$ & $\begin{array}{r}1.0 \\
.9 \\
.9 \\
.9 \\
.8\end{array}$ & $\begin{array}{l}.2 \\
.2 \\
.2 \\
2 \\
.2\end{array}$ & $\begin{array}{r}10 \\
25 \\
190 \\
270 \\
350\end{array}$ & $\begin{array}{r}104 \\
92 \\
81 \\
71 \\
68\end{array}$ & $\begin{array}{l}86 \\
78 \\
67 \\
66 \\
55\end{array}$ & $\begin{array}{r}117 \\
100 \\
92 \\
89 \\
61\end{array}$ & $\begin{array}{l}327 \\
506 \\
383 \\
258 \\
173\end{array}$ & $\begin{array}{l}6.6 \\
7.3 \\
7.5 \\
7.9 \\
7.0\end{array}$ & $\begin{array}{l}13 \\
14 \\
19 \\
26 \\
21\end{array}$ \\
\hline $\begin{array}{l}21 \\
22 \\
23 \\
24 \\
25\end{array}$ & $\begin{array}{l}2.8 \\
3.0 \\
3.1 \\
3.0 \\
2.8\end{array}$ & $\begin{array}{l}3.5 \\
3.5 \\
3.5 \\
3.5 \\
3.4\end{array}$ & $\begin{array}{l}4.7 \\
4.6 \\
4.6 \\
4.5 \\
4.5\end{array}$ & $\begin{array}{l}.8 \\
.8 \\
.8 \\
.8 \\
.7\end{array}$ & $\begin{array}{l}.2 \\
2 \\
.2 \\
.2 \\
.2\end{array}$ & $\begin{array}{l}375 \\
310 \\
260 \\
240 \\
290\end{array}$ & $\begin{array}{l}115 \\
140 \\
162 \\
167 \\
149\end{array}$ & $\begin{array}{l}49 \\
44 \\
39 \\
38 \\
37\end{array}$ & $\begin{array}{r}59 \\
79 \\
125 \\
131 \\
112\end{array}$ & $\begin{array}{r}124 \\
88 \\
67 \\
49 \\
37\end{array}$ & $\begin{array}{l}8.8 \\
9.6 \\
9.6 \\
9.1 \\
8.4\end{array}$ & $\begin{array}{l}21 \\
17 \\
16 \\
16 \\
14\end{array}$ \\
\hline $\begin{array}{l}26 \\
27 \\
28 \\
29 \\
30 \\
31\end{array}$ & $\begin{array}{l}2.8 \\
2.7 \\
2.6 \\
2.5 \\
2.5 \\
2.7\end{array}$ & $\begin{array}{r}3.2 \\
3.1 \\
3.1 \\
3.0 \\
3.0 \\
\end{array}$ & $\begin{array}{l}4.4 \\
4.3 \\
4.1 \\
3.9 \\
3.7 \\
3.6\end{array}$ & $\begin{array}{l}.7 \\
.6 \\
.6 \\
.6 \\
.5 \\
.5\end{array}$ & $\begin{array}{r}2 \\
2 \\
2 \\
.2 \\
-.-2 \\
\end{array}$ & $\begin{array}{l}350 \\
330 \\
300 \\
304 \\
238 \\
192\end{array}$ & $\begin{array}{r}132 \\
123 \\
104 \\
92 \\
78 \\
-\end{array}$ & $\begin{array}{l}36 \\
34 \\
33 \\
32 \\
31 \\
31\end{array}$ & $\begin{array}{r}91 \\
74 \\
63 \\
61 \\
134 \\
\end{array}$ & $\begin{array}{l}32 \\
28 \\
19 \\
17 \\
13 \\
13\end{array}$ & $\begin{array}{l}7.9 \\
7.9 \\
7.9 \\
7.0 \\
6.8 \\
6.6\end{array}$ & $\begin{array}{r}14 \\
11 \\
11 \\
10 \\
10 \\
-\end{array}$ \\
\hline $\begin{array}{l}\text { Total } \\
\text { Mean } \\
\text { Max } \\
\text { Min } \\
\text { Ac-ft }\end{array}$ & $\begin{array}{r}77.5 \\
2.50 \\
4.7 \\
1.1 \\
154\end{array}$ & $\begin{array}{r}88.3 \\
2.94 \\
3.7 \\
1.8 \\
175\end{array}$ & $\begin{array}{r}136.1 \\
4.39 \\
5.1 \\
3.0 \\
270 \\
\end{array}$ & $\begin{array}{r}41.9 \\
1.35 \\
3.4 \\
0.5 \\
83 \\
\end{array}$ & $\begin{array}{r}8.0 \\
0.28 \\
0.5 \\
0.2 \\
16 \\
\end{array}$ & $\begin{array}{r}4.146 .4 \\
134 \\
375 \\
0.2 \\
8,220 \\
\end{array}$ & $\begin{array}{r}3.604 \\
120 \\
188 \\
68 \\
7,150\end{array}$ & $\begin{array}{r}1,781 \\
57.5 \\
97 \\
31 \\
3,530\end{array}$ & $\begin{array}{r}2.753 \\
91.8 \\
169 \\
32 \\
5,460\end{array}$ & $\begin{array}{r}3.861 \\
125 \\
506 \\
13 \\
7,660\end{array}$ & $\begin{array}{r}214.5 \\
6.92 \\
12 \\
3.6 \\
425\end{array}$ & $\begin{array}{r}402.2 \\
13.4 \\
26 \\
7.3 \\
798\end{array}$ \\
\hline $\begin{array}{l}\text { Cal yr } \\
\text { Wat yr }\end{array}$ & & $\begin{array}{l}\text { Total } \\
\text { Total }\end{array}$ & $\begin{array}{l}24,782 \\
17,113.9\end{array}$ & $\begin{array}{ll}\text { Mean } & 67 \\
\text { Mean } & 46\end{array}$ & .9 & $\begin{array}{r}2,560 \\
506\end{array}$ & $\begin{array}{ll}\operatorname{Min} & 0.4 \\
\operatorname{Min} & 0.2\end{array}$ & $\begin{array}{l}A c-f t \\
A c-f t\end{array}$ & $\begin{array}{l}49, \\
33,\end{array}$ & & & \\
\hline
\end{tabular}


5-0785. Clearwater River at Red Lake Falls, Minn.

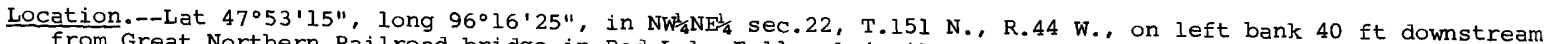
from Great Northern Railroad bridge in Red Lake Falls, 1.4 miles upstream from mouth, and 3 miles downstream from Badger Creek.

Drainage area.--1,370 sq mi, approximately.

Records available.--June 1909 to September 1917, October 1934 to September 1968 . Monthly discharge only for October, November 1934 , published in WSP 1308 .

Gage.--Water-stage recorder. Datum of gage is $949.49 \mathrm{ft}$ above mean sea level, adjustment of 1912 (levels' by Corps of Engineers) . Prior to Sept. 12, 1911, staff gage at site half a mile upstream and Sept. 12, 1911. to Sept. 30, 1917, staff gage at site 40 ft upstream at different datum.

Average discharge.--42 years, 299 cfs $(216,500$ acre-ft per year).

Extremes:--Maximum discharge during year, 3,550 cfs July 18 (gage height, 7.30 ft); maximum gage height rece.ded, $9.36 \mathrm{ft} \mathrm{Mar.} 16$ (backwater from ice); minimum discharge, 21 cfs Nov. 6 (gage height, $1.65 \mathrm{ft}$ ).

1909-17, 1934-68: Maximum discharge, 9, 310 cfs May 6, 1950 (gage height, 11.28 ft); maximum gage height observed, $17.5 \mathrm{ft}$ Apr. 5, 1913, site and datum then in use (backwater from ice); no flow sept. 15 , 1936 , sept. 14, 1939, Aug. 19-22, 1940 .

Remarks.--Records good except those for winter months, which are fair. Slight regulation by clearwater Lake and several smaller lakes.

DISCHARGE, IN CUBIC FEET PER SECOND, WATER YEAR OCTOBER 1967 TO SEPTEMBER 1968

\begin{tabular}{|c|c|c|c|c|c|c|c|c|c|c|c|c|}
\hline DAY & OCT & NOV & DEC & JAN & FEB & MAR & APR & MAY & JUN & JUL & AUG & $\overline{\mathrm{SEP}}$ \\
\hline $\begin{array}{l}1 \\
2 \\
3 \\
4 \\
5\end{array}$ & $\begin{array}{l}36 \\
36 \\
40 \\
40 \\
44\end{array}$ & $\begin{array}{l}58 \\
64 \\
64 \\
58 \\
44\end{array}$ & $\begin{array}{l}43 \\
46 \\
60 \\
72 \\
74\end{array}$ & $\begin{array}{l}64 \\
63 \\
63 \\
62 \\
62\end{array}$ & $\begin{array}{l}64 \\
64 \\
64 \\
65 \\
65\end{array}$ & $\begin{array}{l}62 \\
64 \\
66 \\
68 \\
73\end{array}$ & $\begin{array}{l}730 \\
610 \\
490 \\
426 \\
384\end{array}$ & $\begin{array}{l}355 \\
335 \\
301 \\
273 \\
254\end{array}$ & $\begin{array}{l}166 \\
159 \\
166 \\
164 \\
164\end{array}$ & $\begin{array}{l}485 \\
935 \\
947 \\
769 \\
634\end{array}$ & $\begin{array}{l}276 \\
233 \\
196 \\
181 \\
166\end{array}$ & $\begin{array}{l}121 \\
117 \\
119 \\
119 \\
123\end{array}$ \\
\hline $\begin{array}{r}6 \\
7 \\
8 \\
9 \\
10\end{array}$ & $\begin{array}{l}46 \\
50 \\
55 \\
54 \\
62\end{array}$ & $\begin{array}{l}30 \\
40 \\
71 \\
73 \\
78\end{array}$ & $\begin{array}{l}75 \\
75 \\
75 \\
74 \\
73\end{array}$ & $\begin{array}{l}62 \\
61 \\
61 \\
61 \\
61\end{array}$ & $\begin{array}{l}66 \\
66 \\
67 \\
68 \\
69\end{array}$ & $\begin{array}{r}81 \\
93 \\
110 \\
140 \\
164\end{array}$ & $\begin{array}{l}400 \\
359 \\
363 \\
417 \\
455\end{array}$ & $\begin{array}{l}239 \\
233 \\
236 \\
248 \\
248\end{array}$ & $\begin{array}{l}176 \\
257 \\
590 \\
606 \\
644\end{array}$ & $\begin{array}{l}538 \\
451 \\
380 \\
320 \\
294\end{array}$ & $\begin{array}{l}478 \\
697 \\
702 \\
570 \\
472\end{array}$ & $\begin{array}{l}132 \\
130 \\
130 \\
137 \\
137\end{array}$ \\
\hline $\begin{array}{l}11 \\
12 \\
13 \\
14 \\
15\end{array}$ & $\begin{array}{l}71 \\
71 \\
73 \\
73 \\
67\end{array}$ & $\begin{array}{l}82 \\
80 \\
74 \\
72 \\
68\end{array}$ & $\begin{array}{l}73 \\
74 \\
75 \\
76 \\
77\end{array}$ & $\begin{array}{l}61 \\
61 \\
61 \\
61 \\
62\end{array}$ & $\begin{array}{l}70 \\
70 \\
70 \\
69 \\
68\end{array}$ & $\begin{array}{l}163 \\
159 \\
146 \\
140 \\
140\end{array}$ & $\begin{array}{l}434 \\
413 \\
392 \\
380 \\
359\end{array}$ & $\begin{array}{l}260 \\
276 \\
332 \\
355 \\
351\end{array}$ & $\begin{array}{l}658 \\
732 \\
697 \\
649 \\
753\end{array}$ & $\begin{array}{l}270 \\
251 \\
245 \\
251 \\
242\end{array}$ & $\begin{array}{l}396 \\
335 \\
287 \\
254 \\
230\end{array}$ & $\begin{array}{l}130 \\
119 \\
111 \\
106 \\
100\end{array}$ \\
\hline $\begin{array}{l}16 \\
17 \\
18 \\
19 \\
20\end{array}$ & $\begin{array}{l}71 \\
71 \\
69 \\
65 \\
67\end{array}$ & $\begin{array}{l}68 \\
69 \\
71 \\
73 \\
76\end{array}$ & $\begin{array}{l}77 \\
76 \\
75 \\
75 \\
74\end{array}$ & $\begin{array}{l}62 \\
62 \\
62 \\
63 \\
64\end{array}$ & $\begin{array}{l}68 \\
67 \\
66 \\
64 \\
63\end{array}$ & $\begin{array}{l}158 \\
340 \\
600 \\
740 \\
760\end{array}$ & $\begin{array}{l}372 \\
367 \\
335 \\
312 \\
308\end{array}$ & $\begin{array}{l}347 \\
320 \\
305 \\
294 \\
283\end{array}$ & $\begin{array}{r}687 \\
658 \\
1030 \\
658 \\
520\end{array}$ & $\begin{array}{r}649 \\
2.120 \\
2.460 \\
3.200 \\
2240\end{array}$ & $\begin{array}{l}219 \\
219 \\
213 \\
194 \\
186\end{array}$ & $\begin{array}{l}111 \\
117 \\
123 \\
143 \\
152\end{array}$ \\
\hline $\begin{array}{l}21 \\
22 \\
23 \\
24 \\
25\end{array}$ & $\begin{array}{l}65 \\
62 \\
64 \\
57 \\
57\end{array}$ & $\begin{array}{l}82 \\
75 \\
66 \\
64 \\
64\end{array}$ & $\begin{array}{l}72 \\
71 \\
70 \\
70 \\
69\end{array}$ & $\begin{array}{l}64 \\
65 \\
65 \\
65 \\
66\end{array}$ & $\begin{array}{l}62 \\
61 \\
60 \\
60 \\
60\end{array}$ & $\begin{array}{r}710 \\
665 \\
680 \\
1.100 \\
1.350\end{array}$ & $\begin{array}{l}312 \\
392 \\
434 \\
451 \\
502\end{array}$ & $\begin{array}{l}276 \\
260 \\
242 \\
222 \\
208\end{array}$ & $\begin{array}{l}434 \\
434 \\
498 \\
542 \\
538\end{array}$ & $\begin{array}{r}1.490 \\
1.110 \\
822 \\
639 \\
542\end{array}$ & $\begin{array}{l}183 \\
186 \\
176 \\
169 \\
164\end{array}$ & $\begin{array}{l}152 \\
143 \\
137 \\
130 \\
132\end{array}$ \\
\hline $\begin{array}{l}26 \\
27 \\
28 \\
29 \\
30 \\
31\end{array}$ & $\begin{array}{l}55 \\
55 \\
54 \\
55 \\
54 \\
52\end{array}$ & $\begin{array}{r}63 \\
62 \\
60 \\
55 \\
47 \\
\end{array}$ & $\begin{array}{l}68 \\
67 \\
66 \\
65 \\
64 \\
64\end{array}$ & $\begin{array}{l}66 \\
65 \\
65 \\
65 \\
65 \\
64\end{array}$ & $\begin{array}{r}60 \\
60 \\
61 \\
61 \\
\end{array}$ & $\begin{array}{r}1.490 \\
1.500 \\
1.330 \\
1.220 \\
1.080 \\
940\end{array}$ & $\begin{array}{r}498 \\
472 \\
451 \\
409 \\
384 \\
\end{array}$ & $\begin{array}{l}194 \\
183 \\
176 \\
174 \\
176 \\
174\end{array}$ & $\begin{array}{r}489 \\
434 \\
392 \\
347 \\
332 \\
\end{array}$ & $\begin{array}{l}459 \\
413 \\
367 \\
316 \\
287 \\
287\end{array}$ & $\begin{array}{l}162 \\
152 \\
143 \\
132 \\
132 \\
126\end{array}$ & $\begin{array}{r}130 \\
130 \\
123 \\
119 \\
108\end{array}$ \\
\hline $\begin{array}{l}\text { Total } \\
\text { Mean } \\
\text { Max } \\
\text { Min } \\
\text { Ac-ft }\end{array}$ & $\begin{array}{r}1.791 \\
57.8 \\
73 \\
36 \\
3,550 \\
\end{array}$ & $\begin{array}{r}1.951 \\
65.0 \\
82 \\
30 \\
3,870\end{array}$ & $\begin{array}{r}2.165 \\
69.8 \\
77 \\
43 \\
4,290\end{array}$ & $\begin{array}{r}1.954 \\
63.0 \\
66 \\
61 \\
3,880\end{array}$ & $\begin{array}{r}1.878 \\
64.8 \\
70 \\
60 \\
3,720 \\
\end{array}$ & $\begin{array}{r}16.332 \\
527 \\
1,500 \\
62 \\
32,390\end{array}$ & $\begin{array}{r}12611 \\
420 \\
730 \\
308 \\
25,010\end{array}$ & $\begin{array}{r}8130 \\
262 \\
355 \\
174 \\
16,130\end{array}$ & $\begin{array}{r}14.574 \\
486 \\
1,030 \\
159 \\
28,910\end{array}$ & $\begin{array}{r}24,413 \\
820 \\
3,460 \\
242 \\
50,410\end{array}$ & $\begin{array}{r}8229 \\
265 \\
702 \\
126 \\
16,320\end{array}$ & $\begin{array}{r}3.781 \\
126 \\
152 \\
100 \\
7,500 \\
\end{array}$ \\
\hline $\begin{array}{l}\text { Cal yr } \\
\text { Wtr yr }\end{array}$ & $\begin{array}{l}67: \\
68:\end{array}$ & $\begin{array}{l}\text { al } \\
\text { al }\end{array}$ & 6 & $\begin{array}{l}382 \\
270\end{array}$ & $\begin{array}{l}\text { Max } \\
\text { Max }\end{array}$ & $\begin{array}{l}5,210 \\
3,460\end{array}$ & $\begin{array}{l}23 \\
30\end{array}$ & $\begin{array}{l}A c-f t \\
A c-f t\end{array}$ & $\begin{array}{l}276,550 \\
196,000\end{array}$ & & & \\
\hline
\end{tabular}




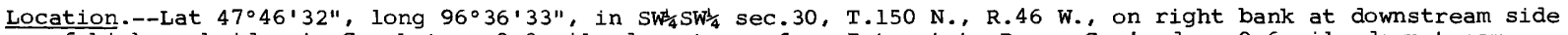
of highway bridge in Crookston, 0.3 mile downstream from Interstate Power Co.'s dam, $0.6 \mathrm{mile}$ downstream from bridge on U. S. Highway 75, and 53 miles above mouth.

Drainage area.--5,280 sq $\mathrm{mi}$, approximately.

Records available.--May 1901 to September 1968. Monthly discharge only for some periods, published in WSP 1308. Figures of daily discharge for Apr. 3-30, 1904, published in WSP 130, have been found unreliable and should not be used.

Gage.--Digital water-stage recorder. Datum of gage is 832.72 ft above mean sea level, datum of 1929 . May 18 , 1901 , to June 30,1909 , chain gage at bridge $300 \mathrm{ft}$ upstream at same datum. July 1, 1909, to Sept. 25, 1911, chain gage, Sept. 26,1911 , to sept. 30, 1919, graphic water-stage recorder. Oct. 1.1919 , to Sept. 30, 1930, chain gage and oct. 1,1930 , to June 10, 1965, graphic water stage recorder, at present site and datum.

Average discharge.--67 years, 1,041 cfs $(753,700$ acre-ft per year).

Extremes.--Maximum discharge during year, 11,100 cfs July 19 (gage height, 17.17 ft); minimum, 75 cfs Nov. 20 (gage height, $2.69 \mathrm{ft}$ ).

1901-68: Maximum discharge, 27,400 cfs May 7, 1950 (gage height, 25.70 ft); maximum gage height, 25.82 ft Apr. 12, 1965 (backwater from ice); no flow for part of July 13, 1960 (caused by regulation of power plant upstream)

Remarks.--Records good except those for winter months, which are fair. Diurnal fluctuation caused by powerplant upstream. Records of chemical analyses. for the water year 1968 published in Part 2 of this report.

DISCHARGE, IN CUBIC FEET PER SECOND, WATER YEAR OCTOBER 1967 TO SEPTFMBFR 1968

\begin{tabular}{|c|c|c|c|c|c|c|c|c|c|c|c|c|}
\hline$\overline{\mathrm{DAY}}$ & OCT & NJV & $\overline{D E C}$ & JAN & $\overline{F E B}$ & $\widehat{M A R}$ & $\overline{A P R}$ & MAY & JUN & JUL & AUG & SEP \\
\hline $\begin{array}{l}1 \\
2 \\
3 \\
4 \\
5\end{array}$ & $\begin{array}{l}667 \\
663 \\
663 \\
663 \\
654\end{array}$ & $\begin{array}{l}471 \\
500 \\
475 \\
500 \\
407\end{array}$ & $\begin{array}{l}325 \\
335 \\
350 \\
360 \\
370\end{array}$ & $\begin{array}{l}315 \\
315 \\
310 \\
310 \\
310\end{array}$ & $\begin{array}{l}300 \\
300 \\
295 \\
290 \\
285\end{array}$ & $\begin{array}{l}255 \\
260 \\
270 \\
270 \\
275\end{array}$ & $\begin{array}{r}2,630 \\
2,190 \\
1.540 \\
1.090 \\
883\end{array}$ & $\begin{array}{l}512 \\
496 \\
491 \\
434 \\
399\end{array}$ & $\begin{array}{l}265 \\
265 \\
243 \\
257 \\
250\end{array}$ & $\begin{array}{l}1,650 \\
2.210 \\
3,090 \\
2.820 \\
2.430\end{array}$ & $\begin{array}{l}2.740 \\
2.690 \\
2.520 \\
2.320 \\
2.230\end{array}$ & $\begin{array}{l}1.610 \\
1.570 \\
1.600 \\
1.600 \\
1.600\end{array}$ \\
\hline $\begin{array}{r}6 \\
7 \\
8 \\
9 \\
10\end{array}$ & $\begin{array}{l}667 \\
650 \\
623 \\
589 \\
559\end{array}$ & $\begin{array}{l}360 \\
350 \\
340 \\
430 \\
490\end{array}$ & $\begin{array}{l}370 \\
375 \\
370 \\
360 \\
350\end{array}$ & $\begin{array}{l}310 \\
310 \\
305 \\
305 \\
305\end{array}$ & $\begin{array}{l}280 \\
275 \\
270 \\
270 \\
265\end{array}$ & $\begin{array}{l}280 \\
290 \\
305 \\
350 \\
380\end{array}$ & $\begin{array}{l}780 \\
756 \\
775 \\
775 \\
771\end{array}$ & $\begin{array}{l}375 \\
355 \\
367 \\
386 \\
394\end{array}$ & $\begin{array}{r}272 \\
584 \\
3.760 \\
5.690 \\
3.950\end{array}$ & $\begin{array}{l}? .150 \\
1.990 \\
1.710 \\
1.520 \\
1.380\end{array}$ & $\begin{array}{l}2.150 \\
2.340 \\
2.300 \\
2.100 \\
1.950\end{array}$ & $\begin{array}{l}1.620 \\
1,540 \\
1.550 \\
1,590 \\
1.600\end{array}$ \\
\hline $\begin{array}{l}11 \\
12 \\
13 \\
14 \\
15\end{array}$ & $\begin{array}{l}559 \\
546 \\
529 \\
533 \\
517\end{array}$ & $\begin{array}{l}500 \\
460 \\
415 \\
330 \\
345\end{array}$ & $\begin{array}{l}340 \\
340 \\
340 \\
340 \\
345\end{array}$ & $\begin{array}{l}305 \\
305 \\
310 \\
310 \\
310\end{array}$ & $\begin{array}{l}265 \\
260 \\
260 \\
260 \\
255\end{array}$ & $\begin{array}{l}395 \\
410 \\
425 \\
450 \\
480\end{array}$ & $\begin{array}{l}775 \\
752 \\
725 \\
689 \\
658\end{array}$ & $\begin{array}{l}410 \\
422 \\
434 \\
500 \\
546\end{array}$ & $\begin{array}{l}3.890 \\
4.260 \\
3.510 \\
2.920 \\
2.610\end{array}$ & $\begin{array}{l}1.330 \\
1.280 \\
1.290 \\
1.370 \\
1.360\end{array}$ & $\begin{array}{l}1.820 \\
1.750 \\
1.680 \\
1.500 \\
1.290\end{array}$ & $\begin{array}{l}1.540 \\
1.390 \\
1.310 \\
1.250 \\
1.100\end{array}$ \\
\hline $\begin{array}{l}16 \\
17 \\
19 \\
19 \\
20\end{array}$ & $\begin{array}{l}491 \\
475 \\
487 \\
475 \\
538\end{array}$ & $\begin{array}{l}330 \\
410 \\
430 \\
440 \\
360\end{array}$ & $\begin{array}{l}355 \\
360 \\
360 \\
355 \\
350\end{array}$ & $\begin{array}{l}310 \\
315 \\
315 \\
315 \\
320\end{array}$ & $\begin{array}{l}255 \\
250 \\
250 \\
245 \\
245\end{array}$ & $\begin{array}{r}550 \\
620 \\
800 \\
1.150 \\
1.550\end{array}$ & $\begin{array}{l}538 \\
610 \\
567 \\
559 \\
571\end{array}$ & $\begin{array}{l}517 \\
508 \\
446 \\
430 \\
446\end{array}$ & $\begin{array}{l}2.440 \\
2.230 \\
2.670 \\
2.530 \\
2.020\end{array}$ & $\begin{array}{r}1.920 \\
5.640 \\
0.920 \\
11.000 \\
9.770\end{array}$ & $\begin{array}{l}1.260 \\
1.170 \\
1.280 \\
1.360 \\
1.360\end{array}$ & $\begin{array}{r}995 \\
985 \\
1.000 \\
1.220 \\
1.280\end{array}$ \\
\hline $\begin{array}{l}21 \\
22 \\
23 \\
24 \\
25\end{array}$ & $\begin{array}{l}517 \\
483 \\
403 \\
467 \\
475\end{array}$ & $\begin{array}{l}320 \\
330 \\
235 \\
300 \\
320\end{array}$ & $\begin{array}{l}345 \\
340 \\
340 \\
335 \\
330\end{array}$ & $\begin{array}{l}320 \\
320 \\
320 \\
320 \\
320\end{array}$ & $\begin{array}{l}240 \\
240 \\
240 \\
240 \\
240\end{array}$ & $\begin{array}{l}1.450 \\
1.400 \\
1.380 \\
1.480 \\
1.800\end{array}$ & $\begin{array}{l}576 \\
600 \\
667 \\
695 \\
716\end{array}$ & $\begin{array}{l}418 \\
414 \\
378 \\
348 \\
322\end{array}$ & $\begin{array}{l}1.780 \\
1.690 \\
1.800 \\
2.180 \\
2.360\end{array}$ & $\begin{array}{l}8.060 \\
6.550 \\
5.280 \\
4.310 \\
3.700\end{array}$ & $\begin{array}{l}1.430 \\
1.680 \\
1.840 \\
2.070 \\
2.060\end{array}$ & $\begin{array}{l}1.220 \\
1.190 \\
1.140 \\
1.140 \\
1,170\end{array}$ \\
\hline $\begin{array}{l}26 \\
27 \\
28 \\
29 \\
30 \\
31\end{array}$ & $\begin{array}{l}463 \\
463 \\
458 \\
467 \\
471 \\
487\end{array}$ & $\begin{array}{r}330 \\
335 \\
340 \\
335 \\
325 \\
\end{array}$ & $\begin{array}{l}330 \\
325 \\
320 \\
320 \\
315 \\
315\end{array}$ & $\begin{array}{l}320 \\
320 \\
315 \\
310 \\
310 \\
305\end{array}$ & $\begin{array}{r}240 \\
245 \\
245 \\
250 \\
\\
\end{array}$ & $\begin{array}{l}2,500 \\
3,200 \\
3,700 \\
3,800 \\
3.820 \\
3.280\end{array}$ & $\begin{array}{r}734 \\
720 \\
685 \\
630 \\
571 \\
\end{array}$ & $\begin{array}{l}303 \\
284 \\
272 \\
235 \\
261 \\
269\end{array}$ & $\begin{array}{r}2.310 \\
2.190 \\
1.990 \\
1.880 \\
1.740 \\
-\end{array}$ & $\begin{array}{l}3.400 \\
3.300 \\
3.170 \\
2.090 \\
2.840 \\
2.770\end{array}$ & $\begin{array}{l}2.040 \\
1.970 \\
1.920 \\
1.670 \\
1.520 \\
1.560\end{array}$ & $\begin{array}{l}1.190 \\
1.210 \\
1.140 \\
1.110 \\
1.050\end{array}$ \\
\hline $\begin{array}{l}\text { JOTAL } \\
\text { MEAN } \\
\text { MAX } \\
\text { MIN } \\
\text { AC-FT }\end{array}$ & $\begin{array}{r}16.762 \\
541 \\
667 \\
458 \\
33.250\end{array}$ & $\begin{array}{r}11.713 \\
390 \\
505 \\
285 \\
23.230\end{array}$ & $\begin{array}{r}10.665 \\
344 \\
375 \\
315 \\
21.150\end{array}$ & $\begin{array}{r}9.690 \\
313 \\
320 \\
305 \\
19.220\end{array}$ & $\begin{array}{r}7.555 \\
261 \\
300 \\
240 \\
14.990\end{array}$ & $\begin{array}{r}37,575 \\
1,212 \\
3,820 \\
255 \\
74,530\end{array}$ & $\begin{array}{r}25.238 \\
841 \\
2,630 \\
538 \\
50,060\end{array}$ & $\begin{array}{r}12.371 \\
399 \\
546 \\
235 \\
24.540\end{array}$ & $\begin{array}{r}64.536 \\
2,151 \\
5.690 \\
243 \\
128.000\end{array}$ & $\begin{array}{r}112,100 \\
3,616 \\
11.000 \\
1,290 \\
222.300\end{array}$ & $\begin{array}{r}57.570 \\
1,857 \\
2.740 \\
1,170 \\
114.200\end{array}$ & $\begin{array}{r}39.490 \\
1.316 \\
1.620 \\
985 \\
78.330\end{array}$ \\
\hline $\begin{array}{l}\text { CAL YR } \\
\text { WTR YR. }\end{array}$ & $\begin{array}{l}1967 \\
1968\end{array}$ & $\begin{array}{l}667 \\
405\end{array}$ & & $N=\frac{1.828}{N} 1.107$ & $\underset{\text { MAXX }}{\operatorname{MAX}}$ & $\begin{array}{l}100 \\
00\end{array}$ & $\begin{array}{l}285 \\
235\end{array}$ & $\begin{array}{l}A C-F T \\
A C-F T\end{array}$ & $\begin{array}{r}1.323,000 \\
803,800\end{array}$ & & & \\
\hline
\end{tabular}


5-0825. Red River of the North at Grand Forks, N. Dak.

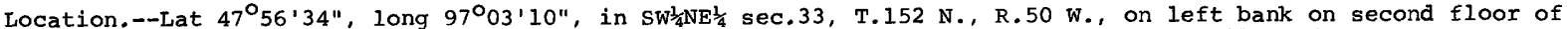
old sewage plant in Grand Forks, 2.3 miles downstream from Red Lake River, and at mile 296.0 .

Drainage area. $--30,100 \mathrm{sq} \mathrm{mi}$, approximately (includes 3,800 $\mathrm{sq} \mathrm{mi}$ in closed basins).

Records available.--April 1882 to september 1968. Monthly discharge only prior to May 1901, published in WSP 1308.

Gage.-Water-stage recorder. Datum of gage is $778.35 \mathrm{ft}$ above mean sea level, datum of 1929 . $1882-92$, gages near site of Northern Pacific Railway bridge, $1 \frac{1}{2}$ miles upstream (history not available, datum apparently the same as following gage) 1892 to Oct. 15, 1926, staff and chain gages on Northern Pacific Railway bridge, at datum about $5 \frac{1}{2} \mathrm{ft}$ higher, but published records referred to datum only half a foot higher than present datum. oct. 16, 1926, to Nov. 2, 1933, staff gages near present site, at datum 5 ft higher than present datum but published at present datum. Nov. 3, 1933, to Apr. 13, 1965, water-stage recorder 0.3 mile upstream at present datum.

Average discharge. --86 years, 2,390 cfs $(1,730,000$ acre-ft per year).

Extremes.--Maximum discharge during year, 9,420 cfs June 11 (gage height, 20.03 ft); minimum, 420 cfs Noy. 24 (gage height, $3.70 \mathrm{ft}$ ).

1882-1968: Maximum discharge, about 80,000 cfs Apr. 10, 1897 (gage height, 50.2 ft, site and datum then in use), from rating curve extended above 54,000 cfs; minimum, 2.4 cfs Feb. 3-5, 12, 14 ; 16-19, 1937 (caused by unusual regulation during repair of dam at Grand Forks).

Remarks.--Records good. Flow regulated by many lakes and reservoirs on tributaries. Records of chemical analyses and water temperatures for the water year 1968 are published in Part 2 of this report.

DISCHARGE, IN CFS, WATER YEAR OCTOBER 1967 TO SEPTEMBER 1968

\begin{tabular}{|c|c|c|c|c|c|c|c|c|c|c|c|c|}
\hline DAY & OCT & NOV & $\mathrm{DEC}$ & JAN & FEB & MAR & APR & MAY & JUN & JUL & AUG & SEP \\
\hline $\begin{array}{l}1 \\
2 \\
3 \\
4 \\
5\end{array}$ & $\begin{array}{r}1050 \\
952 \\
896 \\
819 \\
805\end{array}$ & $\begin{array}{l}735 \\
763 \\
766 \\
770 \\
760\end{array}$ & $\begin{array}{l}637 \\
770 \\
917 \\
952 \\
938\end{array}$ & $\begin{array}{l}630 \\
630 \\
620 \\
610 \\
600\end{array}$ & $\begin{array}{l}630 \\
620 \\
610 \\
610 \\
600\end{array}$ & $\begin{array}{l}510 \\
520 \\
540 \\
540 \\
560\end{array}$ & $\begin{array}{l}6.250 \\
5.050 \\
4.200 \\
3.250 \\
2.820\end{array}$ & $\begin{array}{l}2.230 \\
2.200 \\
2.120 \\
2.060 \\
1.940\end{array}$ & $\begin{array}{l}1.350 \\
1.320 \\
1.270 \\
1.240 \\
1.230\end{array}$ & $\begin{array}{l}3.030 \\
2.900 \\
3.110 \\
3.860 \\
4.130\end{array}$ & $\begin{array}{l}3.090 \\
3.030 \\
2.970 \\
2.840 \\
2.640\end{array}$ & $\begin{array}{l}1.780 \\
1.790 \\
1.820 \\
1.820 \\
1.810\end{array}$ \\
\hline $\begin{array}{r}6 \\
7 \\
8 \\
9 \\
10\end{array}$ & $\begin{array}{l}794 \\
794 \\
802 \\
794 \\
774\end{array}$ & $\begin{array}{l}735 \\
676 \\
573 \\
618 \\
721\end{array}$ & $\begin{array}{l}914 \\
864 \\
812 \\
780 \\
756\end{array}$ & $\begin{array}{l}590 \\
580 \\
580 \\
580 \\
580\end{array}$ & $\begin{array}{l}600 \\
590 \\
580 \\
580 \\
580\end{array}$ & $\begin{array}{l}580 \\
580 \\
630 \\
710 \\
760\end{array}$ & $\begin{array}{l}2.350 \\
2.070 \\
2.020 \\
1.950 \\
1.940\end{array}$ & $\begin{array}{l}1.820 \\
1.730 \\
1.700 \\
1.690 \\
1.700\end{array}$ & $\begin{array}{l}1.800 \\
3.250 \\
5.600 \\
7.930 \\
9.220\end{array}$ & $\begin{array}{l}3.890 \\
3.520 \\
3.200 \\
2.910 \\
2.620\end{array}$ & $\begin{array}{l}2.500 \\
2.380 \\
2.390 \\
2.390 \\
2.250\end{array}$ & $\begin{array}{l}1.820 \\
1.800 \\
1.760 \\
1.730 \\
1.720\end{array}$ \\
\hline $\begin{array}{l}11 \\
12 \\
13 \\
14 \\
15\end{array}$ & $\begin{array}{r}752 \\
738 \\
742 \\
756 \\
738\end{array}$ & $\begin{array}{l}833 \\
868 \\
844 \\
805 \\
777\end{array}$ & $\begin{array}{l}749 \\
746 \\
746 \\
756 \\
724\end{array}$ & $\begin{array}{l}580 \\
580 \\
580 \\
570 \\
570\end{array}$ & $\begin{array}{l}580 \\
570 \\
560 \\
550 \\
530\end{array}$ & $\begin{array}{l}800 \\
810 \\
850 \\
940 \\
960\end{array}$ & $\begin{array}{l}2.060 \\
2.280 \\
2.470 \\
2.600 \\
2.630\end{array}$ & $\begin{array}{l}1.740 \\
1.770 \\
1.780 \\
1.820 \\
1.940\end{array}$ & $\begin{array}{l}9.310 \\
7.650 \\
7.200 \\
6.700 \\
6.350\end{array}$ & $\begin{array}{l}2.400 \\
2.250 \\
2.140 \\
2.110 \\
2.160\end{array}$ & $\begin{array}{l}2.100 \\
2.000 \\
1.940 \\
1.900 \\
1.820\end{array}$ & $\begin{array}{l}1.730 \\
1.680 \\
1.580 \\
1.500 \\
1.430\end{array}$ \\
\hline $\begin{array}{l}16 \\
17 \\
18 \\
19 \\
20\end{array}$ & $\begin{array}{l}742 \\
728 \\
738 \\
735 \\
721\end{array}$ & $\begin{array}{l}735 \\
752 \\
756 \\
777 \\
836\end{array}$ & $\begin{array}{l}662 \\
618 \\
612 \\
640 \\
720\end{array}$ & $\begin{array}{l}570 \\
570 \\
570 \\
570 \\
570\end{array}$ & $\begin{array}{l}520 \\
510 \\
500 \\
500 \\
510\end{array}$ & $\begin{array}{r}990 \\
1.100 \\
1.200 \\
1.450 \\
1.600\end{array}$ & $\begin{array}{l}2.570 \\
2.470 \\
2.340 \\
2.250 \\
2.160\end{array}$ & $\begin{array}{l}2.060 \\
2.160 \\
2.220 \\
2.200 \\
2.130\end{array}$ & $\begin{array}{l}5.900 \\
5.590 \\
5.160 \\
5.160 \\
5.150\end{array}$ & $\begin{array}{l}2.470 \\
3.120 \\
4.850 \\
6.980 \\
8.540\end{array}$ & $\begin{array}{l}1.740 \\
1.650 \\
1.640 \\
1.690 \\
1.730\end{array}$ & $\begin{array}{l}1.350 \\
1.300 \\
1.220 \\
1.180 \\
1.280\end{array}$ \\
\hline $\begin{array}{l}21 \\
22 \\
23 \\
24 \\
25\end{array}$ & $\begin{array}{l}738 \\
738 \\
756 \\
735 \\
735\end{array}$ & $\begin{array}{l}798 \\
615 \\
486 \\
462 \\
570\end{array}$ & $\begin{array}{l}870 \\
750 \\
650 \\
650 \\
640\end{array}$ & $\begin{array}{l}570 \\
570 \\
580 \\
580 \\
590\end{array}$ & $\begin{array}{l}500 \\
480 \\
470 \\
460 \\
460\end{array}$ & $\begin{array}{l}1.900 \\
2.500 \\
2.700 \\
2.700 \\
2.600\end{array}$ & $\begin{array}{l}2.080 \\
1.980 \\
1.910 \\
1.900 \\
1.920\end{array}$ & $\begin{array}{l}2.100 \\
2.060 \\
1.970 \\
1.900 \\
1.820\end{array}$ & $\begin{array}{l}4.720 \\
4.340 \\
4.120 \\
3.970 \\
3.970\end{array}$ & $\begin{array}{l}9.200 \\
9.160 \\
7.250 \\
6.350 \\
5.300\end{array}$ & $\begin{array}{l}1.760 \\
1.810 \\
1.960 \\
2.050 \\
2.100\end{array}$ & $\begin{array}{l}1.420 \\
1.450 \\
1.410 \\
1.370 \\
1.380\end{array}$ \\
\hline $\begin{array}{l}26 \\
27 \\
28 \\
29 \\
30 \\
31\end{array}$ & $\begin{array}{l}752 \\
749 \\
735 \\
710 \\
728 \\
732\end{array}$ & $\begin{array}{r}662 \\
662 \\
540 \\
600 \\
624 \\
-\end{array}$ & $\begin{array}{l}630 \\
630 \\
630 \\
630 \\
630 \\
630\end{array}$ & $\begin{array}{l}610 \\
610 \\
620 \\
640 \\
630 \\
620\end{array}$ & $\begin{array}{r}470 \\
480 \\
500 \\
510 \\
- \\
-0--\end{array}$ & $\begin{array}{l}2.700 \\
3.350 \\
4.500 \\
5.500 \\
6.350 \\
6.750\end{array}$ & $\begin{array}{r}2.010 \\
2.100 \\
2.160 \\
2.200 \\
2.230 \\
\end{array}$ & $\begin{array}{l}1.760 \\
1.670 \\
1.590 \\
1.510 \\
1.460 \\
1.420\end{array}$ & $\begin{array}{r}3.970 \\
3.820 \\
3.590 \\
3.340 \\
3.170 \\
\end{array}$ & $\begin{array}{l}4.600 \\
4.030 \\
3.740 \\
3.570 \\
3.380 \\
3.220\end{array}$ & $\begin{array}{l}2.160 \\
2.150 \\
2.140 \\
2.110 \\
2.020 \\
1.860\end{array}$ & $\begin{array}{r}1.430 \\
1.460 \\
1.520 \\
1.470 \\
1.480 \\
-\end{array}$ \\
\hline $\begin{array}{l}\text { TOTAL } \\
\text { MEAN } \\
\text { MAX } \\
\text { MIN } \\
\text { AC-FT } \\
\end{array}$ & $\begin{array}{r}23,978 \\
773 \\
1,050 \\
710 \\
47,560 \\
\end{array}$ & $\begin{array}{r}21119 \\
704 \\
868 \\
462 \\
41,890 \\
\end{array}$ & $\begin{array}{r}22.653 \\
731 \\
952 \\
612 \\
44,930 \\
\end{array}$ & $\begin{array}{r}18.350 \\
592 \\
640 \\
570 \\
36,400 \\
\end{array}$ & $\begin{array}{r}15.660 \\
540 \\
630 \\
460 \\
31,060 \\
\end{array}$ & $\begin{array}{r}58,180 \\
1,877 \\
6,750 \\
510 \\
115,400\end{array}$ & $\begin{array}{r}76,220 \\
2,541 \\
6,250 \\
1,900 \\
151,200\end{array}$ & $\begin{array}{r}53,270 \\
1,880 \\
2,230 \\
1,420 \\
115,600\end{array}$ & $\begin{array}{r}137.390 \\
4,580 \\
9,310 \\
1,230 \\
272,500\end{array}$ & $\begin{array}{r}129,990 \\
4,193 \\
9,200 \\
2,110 \\
257,800\end{array}$ & $\begin{array}{r}66,810 \\
2,155 \\
3,090 \\
1,640 \\
132,500\end{array}$ & $\begin{array}{r}46,490 \\
1,550 \\
1,820 \\
1,180 \\
92,210\end{array}$ \\
\hline $\begin{array}{l}\text { CAL YR } \\
\text { WAT YR }\end{array}$ & $\begin{array}{l}1967: \\
1968:\end{array}$ & $\begin{array}{l}\text { TOTAI } \\
\text { TOTAI }\end{array}$ & $\begin{array}{r}383,878 \\
675,110\end{array}$ & $\begin{array}{l}\text { MEAN } \\
\text { MEAN }\end{array}$ & $\begin{array}{l}3,791 \\
1,845\end{array}$ & $\begin{array}{l}\text { MAX } \\
\text { MAX }\end{array}$ & $\begin{array}{r}28,000 \\
9,310\end{array}$ & $\begin{array}{l}\text { MIN } \\
\text { MIN }\end{array}$ & $\mathrm{AC}$ & 1,33 & $\begin{array}{l}00 \\
00\end{array}$ & \\
\hline
\end{tabular}




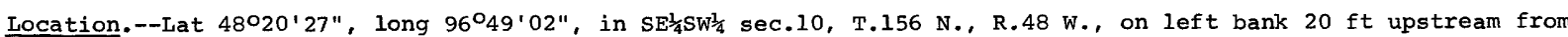
bridge on U.S. Highway 75 in Argyle and 14 miles upstream from mouth.

Drainage area. $--265 \mathrm{sq} \mathrm{mi}$.

Records available.--March to september 1945, October 1950 to September 1968. Monthly discharge only for some periods, published in WSP 1728.

Gage.-Water-stage recorder. Datum of gage is $828.53 \mathrm{ft}$ above mean sea level, datum of 1929 . Prior to Nov. 8 ,

1951, chain or wire-weight gage at bridge $20 \mathrm{ft}$ upstream at datum 1.0 ft higher. Nov. 8 , 1951 , to Sept. 18,

1952, water-stage recorder at present site at datum $1.0 \mathrm{ft}$ higher.

Average discharge.--18 years $(1950-68), 41.4 \mathrm{cfs}(29,970$ acre-ft per year) .

Extremes.--Maximum discharge during year, 1,120 cfs July 20 (gage height, 12.87 ft); no flow for many days. 1945; 1950-68: Maximum discharge, 2,590 cfs Apr. 12, 1965 (gage height, 15.29 ft); maximum gage height, $16.00 \mathrm{ft}$ Apr. 3, 1966 (backwater from ice); no flow at times in most years.

Flood of April 1950 reached a stage of $15.25 \mathrm{ft}$, present datum, from floodmarks (discharge, 2,790 cfs).

Remarks.--Records good except those for winter months, which are fair. Records of chemical analysis and suspended sediment loads for the water year 1968 are published in Part 2 of this report.

DISCHARGE, IN CFS, WATER YEAR OCTOBER 1967 TO SEPTEMBER 1968

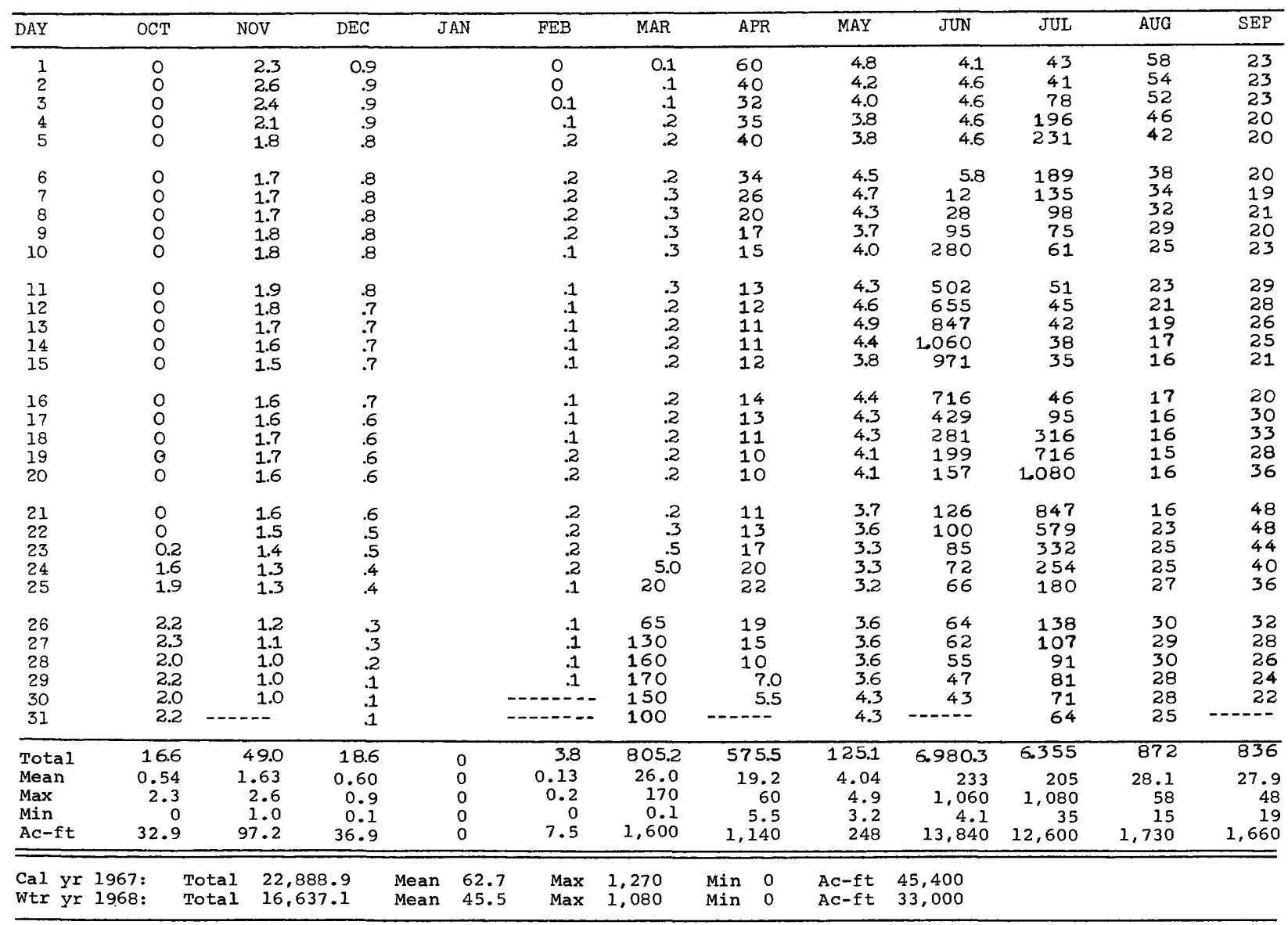


5-0920. Red River of the North at Drayton, N. Dak.

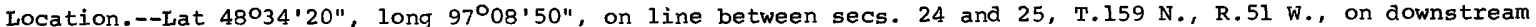

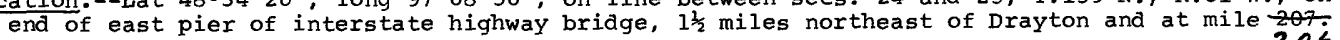

Drainage area.--34,800 sq $\mathrm{mi}$, approximately (includes 3,800 sq $\mathrm{mi}$ in closed basins).

Records available.--April 1936 to June 1937, April 1941 to september 1968 (fragmentary prior to April 1949).

Gage.--Water-stage recorder and concrete control. Datum of gage is $755.00 \mathrm{ft}$ above mean sea level, datum of 1929 (Minnesota highway bench mark). Prior to Nov. 30, 1954, wire-weight gage at site li/2 miles upstream at datum $1.59 \mathrm{ft}$ higher.

Average discharge.--19 years (1949-68) 3,549 cfs $(2,569,000$ acre-ft per year); median of yearly mean discharges, $2,940 \mathrm{cfs}(2,130,000$ acre-ft per year).

Extremes.--Maximum discharge during year, 12,500 cfs July 23; maximum gage height, 20.41 ft July 23 ; minimum discharge, 437 cfs Nov. 28 (gage height, $9.18 \mathrm{ft}$ ).

1936-37, 1941-68: Maximum discharge, $86,500 \mathrm{cfs}$ May 12, 1950 (gage height, $41.58 \mathrm{ft}$, former site and datum); minimum observed, $7.7 \mathrm{cfs}$ Oct. 16, 1936 (gage height, 1.75 ft, former site and datum).

Maximum discharge known since 1860, that of May 12, 1950. Flood of April 1897 reached a stage of about

$41 \mathrm{ft}$, at site and datum in use prior to Nov. $30,1954$.

Remarks.--Records good. Some regulation by reservoirs on tributaries. Records of water temperatures for the water year 1968 are published in Part 2 of this report.

DISCHARGE, IN CFS, WATER YEAR OCTOBER 1967 TO SEPTEMBER 1968

\begin{tabular}{|c|c|c|c|c|c|c|c|c|c|c|c|c|}
\hline DAY & OCT & NOV & $\mathrm{DEC}$ & JAN & FEB & MAR & APR & MAY & JUN & JUL & AUG & SEP \\
\hline $\begin{array}{l}1 \\
2 \\
3 \\
4 \\
5\end{array}$ & $\begin{array}{l}850 \\
805 \\
814 \\
823 \\
880\end{array}$ & $\begin{array}{l}814 \\
814 \\
814 \\
823 \\
823\end{array}$ & $\begin{array}{l}535 \\
572 \\
595 \\
611 \\
667\end{array}$ & $\begin{array}{l}580 \\
560 \\
550 \\
540 \\
530\end{array}$ & $\begin{array}{l}530 \\
530 \\
540 \\
540 \\
540\end{array}$ & $\begin{array}{l}500 \\
500 \\
520 \\
540 \\
560\end{array}$ & $\begin{array}{l}6.700 \\
7.000 \\
5.900 \\
6.450 \\
5.800\end{array}$ & $\begin{array}{l}2.580 \\
2.590 \\
2.550 \\
2.480 \\
2.370\end{array}$ & $\begin{array}{l}1.680 \\
1.640 \\
1.550 \\
1.480 \\
1.460\end{array}$ & $\begin{array}{l}3.810 \\
3.630 \\
3.390 \\
3.420 \\
4.010\end{array}$ & $\begin{array}{l}4.130 \\
3.840 \\
3.650 \\
3.510 \\
3.360\end{array}$ & $\begin{array}{l}2.330 \\
2.180 \\
2.140 \\
2.140 \\
2.120\end{array}$ \\
\hline $\begin{array}{r}6 \\
7 \\
8 \\
9 \\
10\end{array}$ & $\begin{array}{l}890 \\
890 \\
890 \\
880 \\
900\end{array}$ & $\begin{array}{l}814 \\
814 \\
805 \\
787 \\
752\end{array}$ & $\begin{array}{l}718 \\
752 \\
760 \\
760 \\
752\end{array}$ & $\begin{array}{l}520 \\
510 \\
500 \\
500 \\
500\end{array}$ & $\begin{array}{l}540 \\
540 \\
540 \\
540 \\
540\end{array}$ & $\begin{array}{l}560 \\
600 \\
600 \\
610 \\
640\end{array}$ & $\begin{array}{l}5.200 \\
4.500 \\
4.000 \\
3.460 \\
2.740\end{array}$ & $\begin{array}{l}2.230 \\
2.120 \\
2.000 \\
1.970 \\
1.890\end{array}$ & $\begin{array}{l}1.470 \\
1.560 \\
1.780 \\
3.370 \\
6.610\end{array}$ & $\begin{array}{l}4.660 \\
4.740 \\
4.430 \\
4.010 \\
3.580\end{array}$ & $\begin{array}{l}3.190 \\
3.040 \\
2.870 \\
2.790 \\
2.770\end{array}$ & $\begin{array}{l}2.120 \\
2.120 \\
2.110 \\
2.100 \\
2.050\end{array}$ \\
\hline $\begin{array}{l}11 \\
12 \\
13 \\
14 \\
15\end{array}$ & $\begin{array}{l}890 \\
850 \\
841 \\
860 \\
850\end{array}$ & $\begin{array}{l}692 \\
675 \\
692 \\
718 \\
734\end{array}$ & $\begin{array}{l}734 \\
726 \\
718 \\
709 \\
692\end{array}$ & $\begin{array}{l}530 \\
520 \\
510 \\
510 \\
510\end{array}$ & $\begin{array}{l}540 \\
540 \\
540 \\
540 \\
540\end{array}$ & $\begin{array}{r}660 \\
700 \\
750 \\
850 \\
1.000\end{array}$ & $\begin{array}{l}2.540 \\
2.510 \\
2.740 \\
3.150 \\
3.160\end{array}$ & $\begin{array}{l}1.860 \\
1.890 \\
1.910 \\
1.920 \\
1.970\end{array}$ & $\begin{array}{r}9.380 \\
10.800 \\
10.900 \\
10.600 \\
10.500\end{array}$ & $\begin{array}{l}3.230 \\
2.940 \\
2.740 \\
2.570 \\
2.590\end{array}$ & $\begin{array}{l}2.700 \\
2.550 \\
2.380 \\
2.270 \\
2.180\end{array}$ & $\begin{array}{l}2.000 \\
1.950 \\
1.900 \\
1.850 \\
1.810\end{array}$ \\
\hline $\begin{array}{l}16 \\
17 \\
18 \\
19 \\
20\end{array}$ & $\begin{array}{l}850 \\
850 \\
850 \\
850 \\
832\end{array}$ & $\begin{array}{l}800 \\
823 \\
805 \\
814 \\
787\end{array}$ & $\begin{array}{l}684 \\
675 \\
667 \\
651 \\
635\end{array}$ & $\begin{array}{l}510 \\
520 \\
520 \\
520 \\
520\end{array}$ & $\begin{array}{l}550 \\
540 \\
540 \\
540 \\
540\end{array}$ & $\begin{array}{l}1.200 \\
1.350 \\
1.450 \\
1.550 \\
1.600\end{array}$ & $\begin{array}{l}3.110 \\
3.020 \\
2.940 \\
2.800 \\
2.750\end{array}$ & $\begin{array}{l}2.070 \\
2.200 \\
2.450 \\
2.530 \\
2.570\end{array}$ & $\begin{array}{r}10.000 \\
9.400 \\
3.750 \\
8.080 \\
7.320\end{array}$ & $\begin{array}{l}2.590 \\
3.000 \\
4.300 \\
6.270 \\
8.850\end{array}$ & $\begin{array}{l}2.100 \\
2.010 \\
1.910 \\
1.850 \\
1.820\end{array}$ & $\begin{array}{l}1.780 \\
1.750 \\
1.720 \\
1.800 \\
1.840\end{array}$ \\
\hline $\begin{array}{l}21 \\
22 \\
23 \\
24 \\
25\end{array}$ & $\begin{array}{l}823 \\
814 \\
814 \\
814 \\
814\end{array}$ & $\begin{array}{l}778 \\
760 \\
720 \\
680 \\
640\end{array}$ & $\begin{array}{l}635 \\
625 \\
620 \\
620 \\
620\end{array}$ & $\begin{array}{l}530 \\
530 \\
530 \\
520 \\
530\end{array}$ & $\begin{array}{l}540 \\
540 \\
540 \\
540 \\
540\end{array}$ & $\begin{array}{l}1.650 \\
1.750 \\
2.100 \\
2.500 \\
2.750\end{array}$ & $\begin{array}{l}2.710 \\
2.600 \\
2.480 \\
2.400 \\
2.310\end{array}$ & $\begin{array}{l}2.510 \\
2.440 \\
2.380 \\
2.310 \\
2.240\end{array}$ & $\begin{array}{l}6.720 \\
6.090 \\
5.460 \\
4.970 \\
4.680\end{array}$ & $\begin{array}{l}11.300 \\
12.200 \\
12.500 \\
11.600 \\
10.400\end{array}$ & $\begin{array}{l}1.840 \\
1.880 \\
1.920 \\
1.980 \\
2.190\end{array}$ & $\begin{array}{l}1.760 \\
1.770 \\
1.820 \\
1.780 \\
1.710\end{array}$ \\
\hline $\begin{array}{l}26 \\
27 \\
28 \\
29 \\
30 \\
31\end{array}$ & $\begin{array}{l}814 \\
805 \\
805 \\
796 \\
796 \\
814\end{array}$ & $\begin{array}{r}595 \\
478 \\
450 \\
506 \\
535 \\
\end{array}$ & $\begin{array}{l}620 \\
620 \\
620 \\
620 \\
620 \\
605\end{array}$ & $\begin{array}{l}530 \\
520 \\
520 \\
530 \\
530 \\
530\end{array}$ & $\begin{array}{r}530 \\
510 \\
500 \\
500 \\
- \\
-\end{array}$ & $\begin{array}{l}2.900 \\
3.100 \\
3.300 \\
4.500 \\
5.600 \\
6.200\end{array}$ & $\begin{array}{r}2.270 \\
2.310 \\
2.400 \\
2.460 \\
2.530 \\
-\end{array}$ & $\begin{array}{l}2.190 \\
2.080 \\
2.000 \\
1.900 \\
1.790 \\
1.740\end{array}$ & $\begin{array}{r}4.560 \\
4.500 \\
4.380 \\
4.160 \\
3.960 \\
-\end{array}$ & $\begin{array}{l}9.500 \\
8.680 \\
7.320 \\
6.060 \\
5.080 \\
4.520\end{array}$ & $\begin{array}{l}2.330 \\
2.510 \\
2.570 \\
2.550 \\
2.510 \\
2.480\end{array}$ & $\begin{array}{r}1.650 \\
1.620 \\
1.660 \\
1.700 \\
1.700 \\
-\end{array}$ \\
\hline $\begin{array}{l}\text { TOTAL } \\
\text { MEAN } \\
\text { MAX } \\
\text { MIN } \\
\text { AC-FT } \\
\end{array}$ & $\begin{array}{r}26.054 \\
840 \\
900 \\
796 \\
51,680 \\
\end{array}$ & $\begin{array}{r}21,742 \\
725 \\
823 \\
450 \\
43,120 \\
\end{array}$ & $\begin{array}{r}20.438 \\
659 \\
760 \\
535 \\
40,540 \\
\end{array}$ & $\begin{array}{r}16.260 \\
525 \\
580 \\
500 \\
32,250 \\
\end{array}$ & $\begin{array}{r}15.530 \\
536 \\
550 \\
500 \\
30,800\end{array}$ & $\begin{array}{r}53.090 \\
1,713 \\
6,200 \\
500 \\
105,300\end{array}$ & $\begin{array}{r}105,940 \\
3,531 \\
7,000 \\
2,270 \\
210,100\end{array}$ & $\begin{array}{r}67.730 \\
2,185 \\
2,590 \\
1,740 \\
134,300\end{array}$ & $\begin{array}{r}167,810 \\
5,594 \\
10,900 \\
1,460 \\
332,800\end{array}$ & $\begin{array}{r}177.920 \\
5,739 \\
12,500 \\
2,570 \\
352,900\end{array}$ & $\begin{array}{r}79,680 \\
2,570 \\
4,130 \\
1,820 \\
158,000\end{array}$ & $\begin{array}{r}56,980 \\
1,899 \\
2,330 \\
1,620 \\
113,000\end{array}$ \\
\hline $\begin{array}{l}\text { :AL YR } \\
\text { IAT YR }\end{array}$ & $\begin{array}{l}967: \\
968: \\
\end{array}$ & $\begin{array}{l}\text { TOTAL } \\
\text { TOTAL }\end{array}$ & $\begin{array}{r}716,831 \\
809,174 \\
\end{array}$ & $\begin{array}{l}\text { MEAN } \\
\text { MEAN }\end{array}$ & $\begin{array}{l}704 \\
211 \\
\end{array}$ & $\begin{array}{l}A X \\
A X\end{array}$ & $\begin{array}{l}00 \\
00\end{array}$ & $\begin{array}{l}450 \\
450\end{array}$ & $\begin{array}{l}A C-F T \\
A C-F T\end{array}$ & $\begin{array}{l}3,405 \\
1,605\end{array}$ & & \\
\hline
\end{tabular}


5-0940. South Branch Two Rivers at Lake Bronson, Minn.

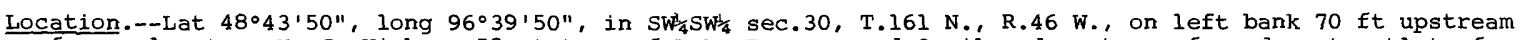
from culvert on U. S. Highway 59 at town of Lake Bronson and 2 miles downstream from dam at outlet of Bronson Lake.

Drainage area. $--444 \mathrm{sq} \mathrm{mi}$.

Records available.--September 1928 to November 1936, April to September 1937, April 1941 to October 1943, April to December 1944, April 1945 to september 1947, October 1953 to September 1968. Monthly discharge only for some periods, published in WSP 1308. Published as South Fork Two Rivers at Bronson prior to 194l.

Gage.--Digital water-stage recorder. Datum of gage is $928.46 \mathrm{ft}$ above mean sea level, adjustment of 1928 (levels by Geodetic Survey of Canada). Prior to Nov. 23, 1953, chain gage at bridge loo ft downstream at datum 2.00 ft higher. Nov 23,1953 , to Oct. 5, 1963, graphic water-stage recorder at same site at datum $2.00 \mathrm{ft}$ higher and Oct. 6, 1963 to July 19, 1967, graphic water-stage recorder at same site and datum.

Average discharge.--27 years (1928-36, 1941-43, 1945-47, 1953-68), 86.6 cfs (62,700 acre-ft per year).

Extremes.--Maximum discharge during year, 2,290 cfs July 19 (gage height, 11.08 ft); minimum daily, 1.3 cfs Feb. $10-26$

1928-37, 1941-47, 1953-68: Maximum discharge, 5,410 cfs Apr. 5, 1966 (gage height 18.23 ft); no flow at times in 1937, 1941, 1960

Remarks.--Records good except those for period of no gage-height record and those for winter months, which are fair. Flow partly regulated since 1937 by Bronson Lake (usable capacity, 3,700 acre-ft). Records of chemical analyses for the water year 1968 are published in Part 2 of this report.

OISCHARGE, IN CUBIC FEET PER SECONO, WATER YEAR OCTOBER 1967 TO SEPTEMBER 1968

\begin{tabular}{|c|c|c|c|c|c|c|c|c|c|c|c|c|}
\hline DAY & OCT & NOV & DEC & JAN & FEB & MAR & APR & MAY & JUN & JUL & AUG & SEP \\
\hline $\begin{array}{l}1 \\
2 \\
3 \\
4 \\
5\end{array}$ & $\begin{array}{l}2.5 \\
2.8 \\
2.5 \\
1.8 \\
2.0\end{array}$ & $\begin{array}{l}2.0 \\
2.3 \\
2.0 \\
2.3 \\
2.3\end{array}$ & $\begin{array}{l}2.4 \\
2.4 \\
2.4 \\
2.3 \\
2.3\end{array}$ & $\begin{array}{l}1.8 \\
1.7 \\
1.7 \\
1.6 \\
1.6\end{array}$ & $\begin{array}{l}1.5 \\
1.5 \\
1.4 \\
1.4 \\
1.4\end{array}$ & $\begin{array}{l}1.4 \\
1.5 \\
1.5 \\
1.6 \\
1.6\end{array}$ & $\begin{array}{r}221 \\
35 \\
237 \\
223 \\
159\end{array}$ & $\begin{array}{l}15 \\
14 \\
9.0 \\
2.0 \\
2.0\end{array}$ & $\begin{array}{l}4.9 \\
4.3 \\
5.2 \\
5.5 \\
4.9\end{array}$ & $\begin{array}{r}28 \\
586 \\
732 \\
678 \\
605\end{array}$ & $\begin{array}{r}219 \\
135 \\
66 \\
99 \\
101\end{array}$ & $\begin{array}{l}158 \\
173 \\
227 \\
243 \\
219\end{array}$ \\
\hline $\begin{array}{r}6 \\
7 \\
8 \\
9 \\
10\end{array}$ & $\begin{array}{l}2.3 \\
2.8 \\
2.5 \\
2.3 \\
1.8\end{array}$ & $\begin{array}{l}2.3 \\
2.5 \\
2.5 \\
2.8 \\
2.8\end{array}$ & $\begin{array}{l}2.3 \\
2.2 \\
2.2 \\
2.2 \\
2.2\end{array}$ & $\begin{array}{l}1.6 \\
1.5 \\
1.5 \\
1.5 \\
1.5\end{array}$ & $\begin{array}{l}1.4 \\
1.4 \\
1.4 \\
1.4 \\
1.3\end{array}$ & $\begin{array}{l}1.7 \\
1.7 \\
1.8 \\
1.9 \\
1.9\end{array}$ & $\begin{array}{c}173 \\
165 \\
84 \\
5.0 \\
30\end{array}$ & $\begin{array}{l}4.9 \\
5.9 \\
4.9 \\
4.6 \\
5.2\end{array}$ & $\begin{array}{r}11 \\
19 \\
249 \\
729 \\
997\end{array}$ & $\begin{array}{r}385 \\
219 \\
158 \\
74 \\
154\end{array}$ & $\begin{array}{l}110 \\
229 \\
273 \\
265 \\
251\end{array}$ & $\begin{array}{l}217 \\
217 \\
267 \\
453 \\
463\end{array}$ \\
\hline $\begin{array}{l}11 \\
12 \\
13 \\
14 \\
15\end{array}$ & $\begin{array}{l}2.0 \\
2.0 \\
2.5 \\
2.3 \\
1.6\end{array}$ & $\begin{array}{l}2.8 \\
3.1 \\
2.8 \\
2.8 \\
3.1\end{array}$ & $\begin{array}{l}2.2 \\
2.3 \\
2.3 \\
2.3 \\
2.4\end{array}$ & $\begin{array}{l}1.5 \\
1.5 \\
1.5 \\
1.5 \\
1.5\end{array}$ & $\begin{array}{l}1.3 \\
1.3 \\
1.3 \\
1.3 \\
1.3\end{array}$ & $\begin{array}{l}1.8 \\
1.8 \\
1.7 \\
1.7 \\
1.7\end{array}$ & $\begin{array}{r}201 \\
217 \\
53 \\
8.5 \\
8.5\end{array}$ & $\begin{array}{l}5.9 \\
5.9 \\
6.3 \\
6.7 \\
5.9\end{array}$ & $\begin{array}{l}1,350 \\
1,690 \\
1,890 \\
2,080 \\
1,850\end{array}$ & $\begin{array}{l}175 \\
177 \\
110 \\
146 \\
463\end{array}$ & $\begin{array}{l}154 \\
132 \\
277 \\
257 \\
158\end{array}$ & $\begin{array}{l}311 \\
241 \\
196 \\
201 \\
201\end{array}$ \\
\hline $\begin{array}{l}16 \\
17 \\
18 \\
19 \\
20\end{array}$ & $\begin{array}{l}1.5 \\
1.5 \\
1.5 \\
1.5 \\
1.8\end{array}$ & $\begin{array}{l}3.4 \\
3.4 \\
3.7 \\
3.4 \\
3.4\end{array}$ & $\begin{array}{l}2.4 \\
2.4 \\
2.5 \\
2.5 \\
2.5\end{array}$ & $\begin{array}{l}1.5 \\
1.6 \\
1.6 \\
1.6 \\
1.6\end{array}$ & $\begin{array}{l}1.3 \\
1.3 \\
1.3 \\
1.3 \\
1.3\end{array}$ & $\begin{array}{l}1.7 \\
1.7 \\
1.7 \\
1.7 \\
1.8\end{array}$ & $\begin{array}{l}6.3 \\
2.3 \\
2.0 \\
2.0 \\
2.3\end{array}$ & $\begin{array}{l}5.2 \\
4.9 \\
5.2 \\
4.9 \\
5.2\end{array}$ & $\begin{array}{r}1.270 \\
848 \\
569 \\
178 \\
307\end{array}$ & $\begin{array}{r}473 \\
1,130 \\
1,600 \\
2,070 \\
2,220\end{array}$ & $\begin{array}{r}42 \\
91 \\
213 \\
277 \\
28\end{array}$ & $\begin{array}{l}201 \\
292 \\
349 \\
351 \\
353\end{array}$ \\
\hline $\begin{array}{l}21 \\
22 \\
23 \\
24 \\
25\end{array}$ & $\begin{array}{l}2.0 \\
1.8 \\
2.0 \\
1.6 \\
1.8\end{array}$ & $\begin{array}{l}3.7 \\
3.7 \\
3.7 \\
3.2 \\
2.4\end{array}$ & $\begin{array}{l}2.4 \\
2.3 \\
2.3 \\
2.2 \\
2.2\end{array}$ & $\begin{array}{l}1.6 \\
1.6 \\
1.5 \\
1.5 \\
1.5\end{array}$ & $\begin{array}{l}1.3 \\
1.3 \\
1.3 \\
1.3 \\
1.3\end{array}$ & $\begin{array}{c}1.9 \\
3.5 \\
10 \\
150 \\
350\end{array}$ & $\begin{array}{l}2.0 \\
7.1 \\
21 \\
36 \\
39\end{array}$ & $\begin{array}{l}5.2 \\
5.2 \\
5.2 \\
4.9 \\
4.0\end{array}$ & $\begin{array}{r}263 \\
82 \\
96 \\
111 \\
108\end{array}$ & $\begin{array}{r}2,240 \\
2,030 \\
1,510 \\
997 \\
895\end{array}$ & $\begin{array}{r}25 \\
27 \\
173 \\
289 \\
327\end{array}$ & $\begin{array}{l}349 \\
338 \\
255 \\
201 \\
199\end{array}$ \\
\hline $\begin{array}{l}26 \\
27 \\
28 \\
29 \\
30 \\
31\end{array}$ & $\begin{array}{l}2.3 \\
2.3 \\
2.0 \\
2.3 \\
2.3 \\
2.3\end{array}$ & $\begin{array}{r}1.9 \\
2.2 \\
2.3 \\
2.4 \\
2.4 \\
\end{array}$ & $\begin{array}{l}2.1 \\
2.1 \\
2.0 \\
2.0 \\
1.9 \\
1.8\end{array}$ & $\begin{array}{l}1.5 \\
1.5 \\
1.5 \\
1.5 \\
1.5 \\
1.5\end{array}$ & $\begin{array}{r}1.3 \\
1.4 \\
1.4 \\
1.4 \\
- \\
\end{array}$ & $\begin{array}{l}450 \\
520 \\
558 \\
572 \\
617 \\
617\end{array}$ & $\begin{array}{l}34 \\
28 \\
24 \\
21 \\
18 \\
-\end{array}$ & $\begin{array}{l}3.7 \\
3.7 \\
4.9 \\
4.0 \\
5.2 \\
5.9\end{array}$ & $\begin{array}{r}96 \\
134 \\
108 \\
11 \\
12 \\
\end{array}$ & $\begin{array}{l}600 \\
342 \\
257 \\
322 \\
320 \\
221\end{array}$ & $\begin{array}{l}400 \\
314 \\
235 \\
277 \\
314 \\
251\end{array}$ & $\begin{array}{r}123 \\
63 \\
66 \\
86 \\
178 \\
\end{array}$ \\
\hline $\begin{array}{l}\text { TOTAL } \\
\text { MEAN } \\
\text { MAX } \\
\text { MIN } \\
\text { AC-FT }\end{array}$ & $\begin{array}{r}64.2 \\
2.07 \\
2.8 \\
1.5 \\
127\end{array}$ & $\begin{array}{r}83.6 \\
2.79 \\
3.7 \\
1.9 \\
166\end{array}$ & $\begin{array}{r}70.0 \\
2.26 \\
2.5 \\
1.8 \\
139\end{array}$ & $\begin{array}{r}48.1 \\
1.55 \\
1.8 \\
1.5 \\
95\end{array}$ & $\begin{array}{r}39.1 \\
1.35 \\
1.5 \\
1.3 \\
78\end{array}$ & $\begin{array}{r}3,883.3 \\
125 \\
617 \\
1.4 \\
7,700\end{array}$ & $\begin{array}{r}2,065.9 \\
69.0 \\
237 \\
2.0 \\
4,110\end{array}$ & $\begin{array}{r}175.5 \\
5.66 \\
15 \\
2.0 \\
348\end{array}$ & $\begin{array}{r}15,082.8 \\
503 \\
2,080 \\
4.3 \\
29,920\end{array}$ & $\begin{array}{r}21,917 \\
707 \\
2,240 \\
28 \\
43,470\end{array}$ & $\begin{array}{r}6,011 \\
194 \\
400 \\
25 \\
11,920\end{array}$ & $\begin{array}{r}7,191 \\
240 \\
463 \\
63 \\
14,260\end{array}$ \\
\hline
\end{tabular}

CAL YR 1967 TOTAL $60,016.40$ MEAN 164 MAX 2,210 MIN .90 AC-FT 119,000

WTR YR 1968 TOTAL $56,635.5$ MEAN 155 MAX $2,240 \quad$ MIN $1.3 \quad$ AC-FT 112,300

Note.--No gage-height record Feb. 18 to Mar. 23. 
5-1025. Red River of the North at Emerson, Manitoba

(International Gaging station)

Location.--Lat 49000'30", long 97012'40", in sec.2, T.1, R.2 E., on right bank 1,500 ft downstream from Canadian National Railway bridge in Emerson, three-quarters of a mile downstream from international boundary, 3.6 miles downstream from Pembina River, and at mile 154.3

Drainage area.--40,200 sq $\mathrm{mi}$, approximately (includes $3,800 \mathrm{sq} \mathrm{mi}$ in closed basins).

Records available.--March to November 1902 (gage heights only), May 1912 to september 1929 (monthly discharge only, published in ysp 1308$)$, October 1929 to september $1968 ., \ldots, 1)$

Gaqe.--Water-stage recorder. Datum of gage is $700.00 \mathrm{ft}$ above mean sea level, datumbf lozo, by Geodetic survey of Canada. Prior to 1912, staff gage at different datum. May 3, 1912, to Apr. 10, 1953, chain gage and/or * staff gage on Canadian National Railway bridge, 1,500 ft upstream. May 3, 1912, to sept. 30, 1923 , at datum $2.55 \mathrm{ft}$ lower than present datum; Oct. 1,1923 , to sept. 30 , 1925, at datum $1.14 \mathrm{ft}$ lower than present datum; Oct. 1, 1925, to sept. 30, 1947, at datum $0.57 \mathrm{ft}$ higher than present datum; and Oct. 1, 1947, to Sept. 30, 1948, at datum $0.21 \mathrm{ft}$ higher than present datum.

Average discharge.--56 years (1912-68) 2,970 cfs (2,150,000 acre-ft per year); median of yearly mean discharges, 2,500 cfs $(1,810,000$ acre-ft per year)

Extremes.--Maximum daily discharge during year, 13, $900 \mathrm{cfs} J u l y ~ 24,25 ;$ maximum gage height, 64.12 ft July 25; minimum daily discharge, $404 \mathrm{cfs}$ Mar. 23; minimum gage height, $47.48 \mathrm{ft} \mathrm{Jan}$. 10.

1912-68: Maximum discharge, 95, 500 cfs May 13, 1950 (gage height, $90.89 \mathrm{ft}$ ); minimum observed, 0.9 cfs

Feb. 6-8, 1937 (gage height, 44.00 ft).

Remarks.--Records good. Discharge partially regulated by reservoirs on tributaries.

Cooperation.--This station is one of the international gaging stations maintained by Canada under agreement with the United States.

DISCHARGE, IN CUBIC FEET PER SECOND, WATER YEAR OCTOBER 1967 TO SEPTEMBER 1968

\begin{tabular}{|c|c|c|c|c|c|c|c|c|c|c|c|c|}
\hline DAY & OCT & NOV & DEC & JAN & FEB & MAR & APR & MAY & JUN & JUL & AUG & SEP \\
\hline $\begin{array}{l}1 \\
2 \\
3 \\
4 \\
5\end{array}$ & $\begin{array}{l}980 \\
872 \\
840 \\
824 \\
832\end{array}$ & $\begin{array}{l}950 \\
960 \\
960 \\
975 \\
980\end{array}$ & $\begin{array}{l}792 \\
772 \\
752 \\
748 \\
752\end{array}$ & $\begin{array}{l}616 \\
620 \\
612 \\
588 \\
564\end{array}$ & $\begin{array}{l}508 \\
508 \\
508 \\
520 \\
528\end{array}$ & $\begin{array}{l}412 \\
404 \\
404 \\
412 \\
416\end{array}$ & $\begin{array}{l}3.240 \\
8.270 \\
3.630 \\
3.350 \\
3.560\end{array}$ & $\begin{array}{l}2.660 \\
2.670 \\
2.670 \\
2.660 \\
2.620\end{array}$ & $\begin{array}{l}2.020 \\
1.940 \\
1.900 \\
1.860 \\
1.790\end{array}$ & $\begin{array}{l}4.750 \\
4.760 \\
4.670 \\
4.640 \\
4.820\end{array}$ & $\begin{array}{l}5.260 \\
4.880 \\
4.590 \\
4.360 \\
4.190\end{array}$ & $\begin{array}{l}3.120 \\
2.980 \\
2.800 \\
2.790 \\
2.830\end{array}$ \\
\hline $\begin{array}{r}6 \\
7 \\
8 \\
9 \\
10\end{array}$ & $\begin{array}{l}880 \\
915 \\
930 \\
935 \\
930\end{array}$ & $\begin{array}{l}995 \\
990 \\
965 \\
945 \\
930\end{array}$ & $\begin{array}{l}744 \\
760 \\
788 \\
784 \\
796\end{array}$ & $\begin{array}{l}544 \\
528 \\
500 \\
460 \\
436\end{array}$ & $\begin{array}{l}536 \\
536 \\
528 \\
528 \\
520\end{array}$ & $\begin{array}{l}436 \\
456 \\
472 \\
492 \\
512\end{array}$ & $\begin{array}{l}8.580 \\
3.080 \\
7.630 \\
7.400 \\
6.860\end{array}$ & $\begin{array}{l}2.570 \\
2.490 \\
2.400 \\
2.300 \\
2.260\end{array}$ & $\begin{array}{l}1.740 \\
1.730 \\
1.760 \\
1.890 \\
3.280\end{array}$ & $\begin{array}{l}5.250 \\
5.570 \\
5.530 \\
5.300 \\
4.940\end{array}$ & $\begin{array}{l}4.060 \\
3.930 \\
3.780 \\
3.680 \\
3.620\end{array}$ & $\begin{array}{l}2.880 \\
3.020 \\
3.280 \\
3.370 \\
3.300\end{array}$ \\
\hline $\begin{array}{l}11 \\
12 \\
13 \\
14 \\
15\end{array}$ & $\begin{array}{l}935 \\
935 \\
925 \\
915 \\
935\end{array}$ & $\begin{array}{r}892 \\
844 \\
832 \\
876 \\
1010\end{array}$ & $\begin{array}{l}789 \\
772 \\
752 \\
728 \\
704\end{array}$ & $\begin{array}{l}444 \\
460 \\
472 \\
476 \\
464\end{array}$ & $\begin{array}{l}512 \\
504 \\
504 \\
504 \\
508\end{array}$ & $\begin{array}{l}528 \\
552 \\
588 \\
636 \\
688\end{array}$ & $\begin{array}{l}5.710 \\
4.430 \\
3.680 \\
3.540 \\
3.680\end{array}$ & $\begin{array}{l}2.200 \\
2.210 \\
2.200 \\
2.180 \\
2.280\end{array}$ & $\begin{array}{r}6.370 \\
9.030 \\
10.300 \\
11.200 \\
11.700\end{array}$ & $\begin{array}{l}4.540 \\
4.230 \\
3.970 \\
4.060 \\
4.070\end{array}$ & $\begin{array}{l}3.550 \\
3.400 \\
3.180 \\
2.910 \\
2.750\end{array}$ & $\begin{array}{l}3.280 \\
3.240 \\
3.130 \\
2.960 \\
2.760\end{array}$ \\
\hline $\begin{array}{l}16 \\
17 \\
18 \\
19 \\
20\end{array}$ & $\begin{array}{l}935 \\
925 \\
910 \\
915 \\
905\end{array}$ & $\begin{array}{r}1.120 \\
1.120 \\
1.040 \\
995 \\
980\end{array}$ & $\begin{array}{l}664 \\
632 \\
604 \\
588 \\
576\end{array}$ & $\begin{array}{l}456 \\
448 \\
444 \\
444 \\
444\end{array}$ & $\begin{array}{l}516 \\
516 \\
516 \\
508 \\
492\end{array}$ & $\begin{array}{r}776 \\
884 \\
985 \\
1.060 \\
1.100\end{array}$ & $\begin{array}{l}3.760 \\
3.610 \\
3.470 \\
3.310 \\
3.170\end{array}$ & $\begin{array}{l}2.500 \\
2.530 \\
2.600 \\
2.750 \\
2.850\end{array}$ & $\begin{array}{r}12.000 \\
11.700 \\
11.200 \\
10400 \\
9.280\end{array}$ & $\begin{array}{l}4.040 \\
4.270 \\
4.780 \\
5.880 \\
8.070\end{array}$ & $\begin{array}{l}2.650 \\
2.530 \\
2.380 \\
2.370 \\
2.420\end{array}$ & $\begin{array}{l}2.770 \\
2.980 \\
3.040 \\
3.050 \\
3.130\end{array}$ \\
\hline $\begin{array}{l}21 \\
22 \\
23 \\
24 \\
25\end{array}$ & $\begin{array}{l}900 \\
905 \\
905 \\
896 \\
892\end{array}$ & $\begin{array}{r}995 \\
1.000 \\
995 \\
1.040 \\
1.030\end{array}$ & $\begin{array}{l}572 \\
564 \\
556 \\
552 \\
564\end{array}$ & $\begin{array}{l}452 \\
448 \\
452 \\
468 \\
468\end{array}$ & $\begin{array}{l}472 \\
460 \\
452 \\
452 \\
448\end{array}$ & $\begin{array}{l}1.120 \\
1.140 \\
1.330 \\
1.480 \\
1.840\end{array}$ & $\begin{array}{l}3.100 \\
3.020 \\
2.920 \\
2.830 \\
2.710\end{array}$ & $\begin{array}{l}2.880 \\
2.860 \\
2.790 \\
2.720 \\
2.640\end{array}$ & $\begin{array}{l}8.160 \\
7.260 \\
6.540 \\
5.850 \\
5.260\end{array}$ & $\begin{array}{r}9.650 \\
11.800 \\
13.600 \\
13.900 \\
13.900\end{array}$ & $\begin{array}{l}2.380 \\
2.260 \\
2.130 \\
2.190 \\
2.500\end{array}$ & $\begin{array}{l}3.190 \\
3.160 \\
3.120 \\
3.090 \\
3.020\end{array}$ \\
\hline $\begin{array}{l}26 \\
27 \\
28 \\
29 \\
30 \\
31\end{array}$ & $\begin{array}{l}905 \\
910 \\
896 \\
892 \\
892 \\
915 \\
\end{array}$ & $\begin{array}{r}940 \\
900 \\
940 \\
884 \\
816 \\
\end{array}$ & $\begin{array}{l}576 \\
584 \\
584 \\
592 \\
604 \\
616 \\
\end{array}$ & $\begin{array}{l}468 \\
480 \\
488 \\
492 \\
496 \\
504 \\
\end{array}$ & $\begin{array}{r}444 \\
436 \\
428 \\
416 \\
- \\
\end{array}$ & $\begin{array}{l}2.360 \\
2.870 \\
3.540 \\
4.290 \\
5.630 \\
6.630 \\
\end{array}$ & $\begin{array}{r}2.600 \\
2.550 \\
2.560 \\
2.600 \\
2.640 \\
-\end{array}$ & $\begin{array}{l}2.550 \\
2.500 \\
2.400 \\
2.310 \\
2210 \\
2110 \\
\end{array}$ & $\begin{array}{r}5130 \\
5120 \\
5020 \\
4.900 \\
4.790 \\
\end{array}$ & $\begin{array}{r}12.100 \\
10.700 \\
8.880 \\
8180 \\
6.360 \\
5.640 \\
\end{array}$ & $\begin{array}{l}2.890 \\
3.310 \\
3.630 \\
3.610 \\
3.420 \\
3.240 \\
\end{array}$ & $\begin{array}{r}2.910 \\
2.780 \\
2.690 \\
2.610 \\
2.550 \\
-\end{array}$ \\
\hline $\begin{array}{l}\text { Tota } 1 \\
\text { Mean } \\
\text { Max } \\
\text { Min } \\
\text { Ac-ft }\end{array}$ & $\begin{array}{r}28081 \\
906 \\
980 \\
824 \\
55,700\end{array}$ & $\begin{array}{r}28.899 \\
963 \\
1,120 \\
816 \\
57,320\end{array}$ & $\begin{array}{r}20860 \\
673 \\
792 \\
552 \\
41,380\end{array}$ & $\begin{array}{r}15236 \\
491 \\
620 \\
436 \\
30,200\end{array}$ & $\begin{array}{r}14.308 \\
493 \\
536 \\
416 \\
28,400\end{array}$ & $\begin{array}{r}44,443 \\
1,430 \\
6,630 \\
404 \\
88,200\end{array}$ & $\begin{array}{r}146,490 \\
4,880 \\
8,630 \\
2,550 \\
291,000\end{array}$ & $\begin{array}{r}77.570 \\
2,500 \\
2,880 \\
2,110 \\
154,000\end{array}$ & $\begin{array}{r}181,120 \\
6,040 \\
12,000 \\
1,730 \\
359,000\end{array}$ & $\begin{array}{r}212,850 \\
6,870 \\
13,900 \\
3,970 \\
422,000\end{array}$ & $\begin{array}{r}102050 \\
3,290 \\
5,260 \\
2,130 \\
202,000\end{array}$ & $\begin{array}{r}89.830 \\
2,990 \\
3,370 \\
2,550 \\
178,000\end{array}$ \\
\hline $\begin{array}{ll}\text { al yr } \\
\text { rtr yr }\end{array}$ & 1968: & & $\begin{array}{l}194 \\
737\end{array}$ & $\begin{array}{l}\text { Mean } \\
\text { Mean }\end{array}$ & $\begin{array}{l}\operatorname{Max} \\
\operatorname{Max}\end{array}$ & $\begin{array}{l}33,200 \\
13,900\end{array}$ & $\begin{array}{l}\text { Min } \\
\text { Min }\end{array}$ & $\begin{array}{l}552 \\
404\end{array}$ & $\begin{array}{l}f t \\
f t\end{array}$ &, 000 & & \\
\hline
\end{tabular}




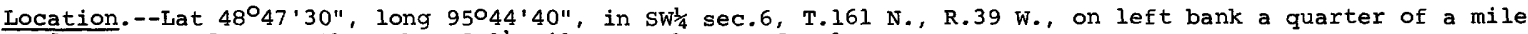
downstream from South Fork and $1 \frac{1}{2}$ miles northwest of Malung.

Drainage area. $--573 \mathrm{sq} \mathrm{mi}$.

Records available.--October 1946 to september 1968 .

Gage.--Water-stage recorder and concrete control. Datum of gage is $1,029.67 \mathrm{ft}$ above mean sea level, adjustment of 1912 .

Average discharge.--22 years, $150 \mathrm{cfs}(108,600$ acre-ft per year).

Extremes.--Maximum discharge during year, 4,920 cfs July 18 (gage height, 22.32 ft); minimum, 0.4 cfs Oct. 1, 2 . 1946-68: Maximum discharge, that of July 18, 1968; no flow for part of Jan. 15, 1952 (caused by construction of concrete control) and July 23 to sept. 8, 1961.

Remarks.--Records good except those for winter months, which are fair.

DISCHARGE, IN CFS, WATER YEAR OCTOBER 1967 TO SEPTEMBER I968

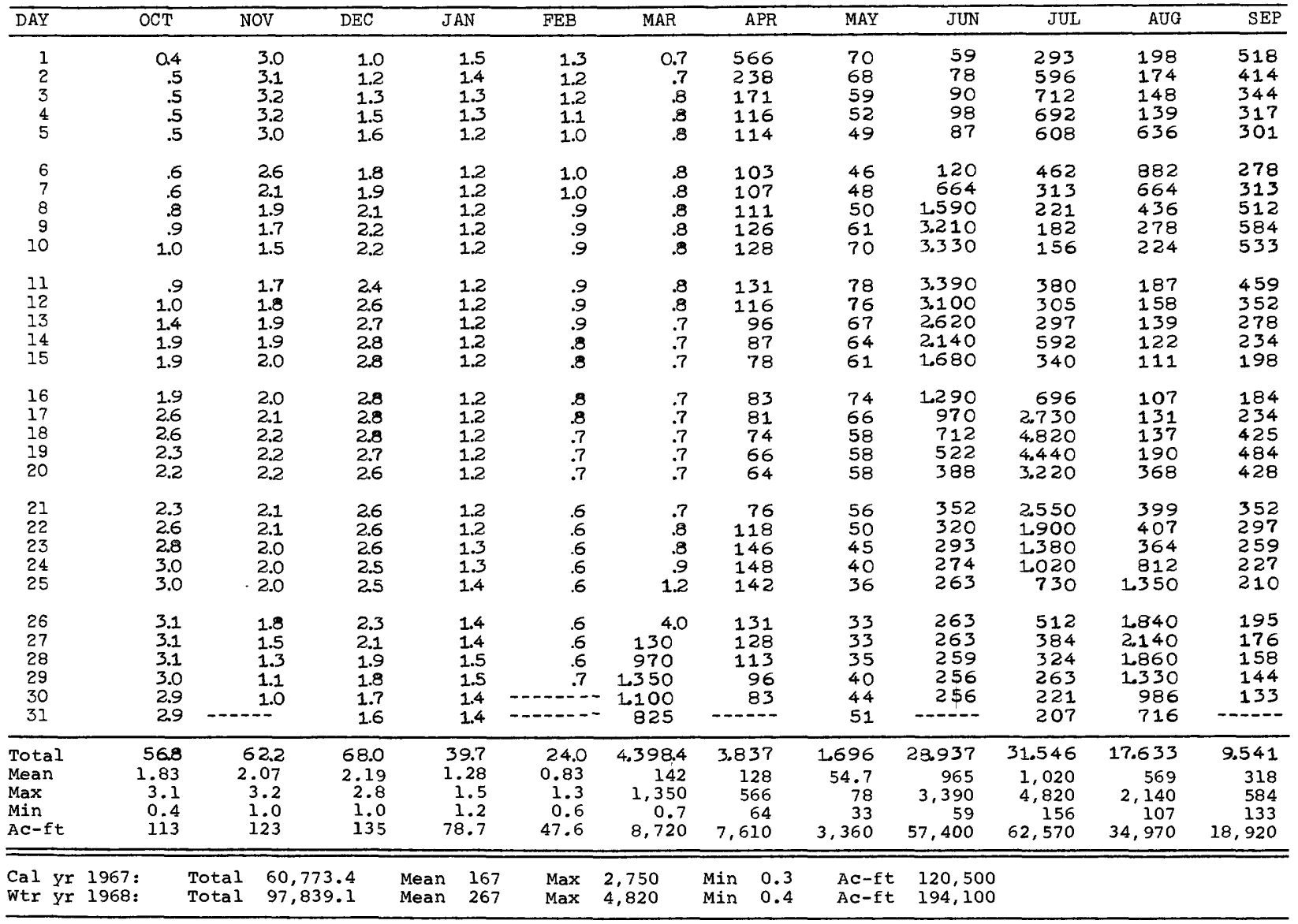


5-1060. Sprague Creek near Sprague, Manitoba

(International gaging station)

Location.--Lat $48^{\circ} 59^{\prime} 33^{\prime \prime}$, long 95\%39'43", in NE $\frac{1}{4}$ sec.34, T.164 N., R.39 W., on left bank half a mile south of international boundary, $3 \frac{1}{2}$ miles south of Sprague, Manitoba, 8 miles upstream from mouth, and $10 \frac{1}{2}$ miles (revised) northeast of Roseau, Minn.

Drainage area.--169 sq mi. Prior to october 1958, $151 \mathrm{sq}$ mi; change due to construction of drainage ditch within basin.

Records available.--September 1928 to September 1968 (winter records incomplete prior to 1941 ). Prior to September 1951, published as Mud Creek near sprague.

Gage.-Water-stage recorder and concrete control. Datum of gage is $1,038.4 \mathrm{ft}$ above mean sea level, adjustment of 1928 by Geodetic Survey of Canada. Prior to Mar. 15, 1929, staff at same site and datum.

Average discharge.--29 years $(1928-29,1940-68), 61.3$ cfs $(44,380$ acre-ft per year).

Extremes.--Maximum discharge during year, 861 cfs June 11 (gage height, 11.79 ft, backwater from Roseau River); minimum daily, 0.6 cfs Jan. 18 to Feb. 24 .

1928-68: Maximum discharge, 2,070 cfs sept. 1, 1942 (gage height, $15.31 \mathrm{ft}$ ), from rating curve extended above 960 cfs; no flow at times in some years.

Remarks.--Records good except those for winter months, which are fair.

Cooperation.--This station is maintained by the United states under agreement with Canada.

DISCHARGE, IN CFS, WATER YEAR OCTOBER 1967 TO SEPTEMBER 1968

\begin{tabular}{|c|c|c|c|c|c|c|c|c|c|c|c|c|}
\hline DAY & $\mathrm{OCT}$ & NOV & DEC & JAN & FEB & MAR & APR & MAY & JUN & JUL & AUG & SEP \\
\hline $\begin{array}{l}1 \\
2 \\
3 \\
4 \\
5\end{array}$ & $\begin{array}{r}0.7 \\
.8 \\
1.2 \\
.8 \\
.9\end{array}$ & $\begin{array}{r}0.9 \\
.8 \\
.8 \\
.8 \\
.9\end{array}$ & $\begin{array}{l}1.1 \\
1.1 \\
1.1 \\
1.1 \\
1.1\end{array}$ & $\begin{array}{r}0.9 \\
.9 \\
.8 \\
.8 \\
.7\end{array}$ & $\begin{array}{r}0.6 \\
.6 \\
.6 \\
.6 \\
.6\end{array}$ & $\begin{array}{r}0.8 \\
.8 \\
.8 \\
.9 \\
1.0\end{array}$ & $\begin{array}{l}95 \\
80 \\
68 \\
64 \\
62\end{array}$ & $\begin{array}{l}52 \\
47 \\
43 \\
42 \\
40\end{array}$ & $\begin{array}{l}50 \\
52 \\
50 \\
54 \\
70\end{array}$ & $\begin{array}{l}209 \\
379 \\
387 \\
369 \\
297\end{array}$ & $\begin{array}{r}40 \\
33 \\
25 \\
20 \\
276\end{array}$ & $\begin{array}{l}142 \\
120 \\
109 \\
125 \\
114\end{array}$ \\
\hline $\begin{array}{r}6 \\
7 \\
8 \\
9 \\
10\end{array}$ & $\begin{array}{r}.9 \\
1.0 \\
1.3 \\
1.3 \\
1.2\end{array}$ & $\begin{array}{r}.9 \\
1.0 \\
1.1 \\
1.2 \\
1.3\end{array}$ & $\begin{array}{l}1.2 \\
1.4 \\
1.4 \\
1.5 \\
1.5\end{array}$ & $\begin{array}{l}.7 \\
.7 \\
.7 \\
.7 \\
.7\end{array}$ & $\begin{array}{l}.6 \\
.6 \\
.6 \\
.6 \\
.6\end{array}$ & $\begin{array}{l}1.1 \\
1.1 \\
1.2 \\
1.2 \\
1.4\end{array}$ & $\begin{array}{l}63 \\
67 \\
69 \\
71 \\
72\end{array}$ & $\begin{array}{l}37 \\
46 \\
62 \\
74 \\
70\end{array}$ & $\begin{array}{l}104 \\
195 \\
555 \\
720 \\
725\end{array}$ & $\begin{array}{r}229 \\
164 \\
115 \\
83 \\
64\end{array}$ & $\begin{array}{r}282 \\
180 \\
110 \\
83 \\
68\end{array}$ & $\begin{array}{l}98 \\
96 \\
97 \\
84 \\
72\end{array}$ \\
\hline $\begin{array}{l}11 \\
12 \\
13 \\
14 \\
15\end{array}$ & $\begin{array}{l}1.2 \\
1.7 \\
1.8 \\
1.6 \\
1.5\end{array}$ & $\begin{array}{l}1.3 \\
1.3 \\
1.3 \\
1.3 \\
1.2\end{array}$ & $\begin{array}{l}1.5 \\
1.5 \\
1.4 \\
1.4 \\
1.4\end{array}$ & $\begin{array}{l}.7 \\
.7 \\
.7 \\
.7 \\
.7\end{array}$ & $\begin{array}{l}.6 \\
.6 \\
.6 \\
.6 \\
.6\end{array}$ & $\begin{array}{l}1.6 \\
1.7 \\
1.6 \\
1.4 \\
1.3\end{array}$ & $\begin{array}{l}70 \\
67 \\
62 \\
59 \\
56\end{array}$ & $\begin{array}{l}67 \\
61 \\
54 \\
49 \\
46\end{array}$ & $\begin{array}{l}840 \\
800 \\
725 \\
594 \\
462\end{array}$ & $\begin{array}{r}54 \\
48 \\
61 \\
190 \\
206\end{array}$ & $\begin{array}{l}49 \\
37 \\
31 \\
26 \\
22\end{array}$ & $\begin{array}{l}62 \\
53 \\
45 \\
39 \\
34\end{array}$ \\
\hline $\begin{array}{l}16 \\
17 \\
18 \\
19 \\
20\end{array}$ & $\begin{array}{l}1.4 \\
1.3 \\
1.3 \\
1.3 \\
1.7\end{array}$ & $\begin{array}{l}1.2 \\
1.3 \\
1.3 \\
1.4 \\
1.5\end{array}$ & $\begin{array}{l}1.3 \\
1.3 \\
1.3 \\
1.3 \\
1.3\end{array}$ & $\begin{array}{l}.7 \\
.7 \\
.6 \\
.6 \\
.6\end{array}$ & $\begin{array}{l}.6 \\
.6 \\
.6 \\
.6 \\
.6\end{array}$ & $\begin{array}{l}1.2 \\
1.1 \\
1.2 \\
1.4 \\
1.8\end{array}$ & $\begin{array}{l}54 \\
52 \\
51 \\
53 \\
54\end{array}$ & $\begin{array}{l}46 \\
43 \\
46 \\
48 \\
48\end{array}$ & $\begin{array}{l}353 \\
284 \\
240 \\
192 \\
150\end{array}$ & $\begin{array}{l}189 \\
224 \\
185 \\
140 \\
108\end{array}$ & $\begin{array}{l}33 \\
36 \\
34 \\
54 \\
56\end{array}$ & $\begin{array}{r}43 \\
83 \\
132 \\
125 \\
106\end{array}$ \\
\hline $\begin{array}{l}21 \\
22 \\
23 \\
24 \\
25\end{array}$ & $\begin{array}{l}1.6 \\
1.6 \\
1.6 \\
1.4 \\
1.6\end{array}$ & $\begin{array}{l}1.5 \\
1.5 \\
1.5 \\
1.6 \\
1.6\end{array}$ & $\begin{array}{l}1.3 \\
1.2 \\
1.2 \\
1.2 \\
1.2\end{array}$ & $\begin{array}{l}.6 \\
.6 \\
.6 \\
.6 \\
.6\end{array}$ & $\begin{array}{l}.6 \\
.6 \\
.6 \\
.6 \\
.7\end{array}$ & $\begin{array}{r}2.3 \\
3.5 \\
6.0 \\
8.0 \\
17\end{array}$ & $\begin{array}{l}73 \\
87 \\
91 \\
87 \\
78\end{array}$ & $\begin{array}{l}46 \\
41 \\
36 \\
31 \\
28\end{array}$ & $\begin{array}{r}127 \\
142 \\
115 \\
89 \\
72\end{array}$ & $\begin{array}{l}96 \\
91 \\
79 \\
75 \\
67\end{array}$ & $\begin{array}{r}45 \\
48 \\
58 \\
489 \\
758\end{array}$ & $\begin{array}{l}90 \\
79 \\
75 \\
65 \\
57\end{array}$ \\
\hline $\begin{array}{l}26 \\
27 \\
28 \\
29 \\
30 \\
31\end{array}$ & $\begin{array}{l}1.6 \\
1.6 \\
1.5 \\
1.4 \\
1.2 \\
1.0\end{array}$ & $\begin{array}{r}1.5 \\
1.5 \\
1.3 \\
1.2 \\
1.2 \\
- \\
\end{array}$ & $\begin{array}{l}1.2 \\
1.1 \\
1.1 \\
1.0 \\
1.0 \\
1.0\end{array}$ & $\begin{array}{l}.6 \\
.6 \\
.6 \\
.6 \\
.6 \\
.6\end{array}$ & $\begin{array}{r}.7 \\
.7 \\
.8 \\
.8 \\
\\
-\end{array}$ & $\begin{array}{r}23 \\
110 \\
200 \\
190 \\
160 \\
120\end{array}$ & $\begin{array}{r}73 \\
68 \\
64 \\
60 \\
57 \\
\end{array}$ & $\begin{array}{l}28 \\
30 \\
34 \\
43 \\
47 \\
49\end{array}$ & $\begin{array}{r}59 \\
46 \\
40 \\
34 \\
38 \\
\end{array}$ & $\begin{array}{l}54 \\
48 \\
39 \\
31 \\
31 \\
43\end{array}$ & $\begin{array}{l}508 \\
458 \\
351 \\
271 \\
207 \\
167\end{array}$ & $\begin{array}{r}57 \\
47 \\
40 \\
34 \\
31 \\
\end{array}$ \\
\hline $\begin{array}{l}\text { Total } \\
\text { Mean } \\
\text { Max } \\
\text { Min } \\
\text { Ac-ft }\end{array}$ & $\begin{array}{r}41.0 \\
1.32 \\
1.8 \\
0.7 \\
81.3\end{array}$ & $\begin{array}{r}37.2 \\
1.24 \\
1.6 \\
0.8 \\
73.8\end{array}$ & $\begin{array}{r}38.7 \\
1.25 \\
1.5 \\
1.0 \\
76.8\end{array}$ & $\begin{array}{r}20.9 \\
0.67 \\
0.9 \\
0.6 \\
41.5\end{array}$ & $\begin{array}{r}18.1 \\
0.62 \\
0.8 \\
0.6 \\
35.9\end{array}$ & $\begin{array}{r}864.4 \\
27.9 \\
200 \\
0.8 \\
1,710\end{array}$ & $\begin{array}{r}2.027 \\
67.6 \\
95 \\
51 \\
4,020\end{array}$ & $\begin{array}{r}1.434 \\
46.3 \\
74 \\
28 \\
2,840\end{array}$ & $\begin{array}{r}7.977 \\
266 \\
840 \\
34 \\
15,820\end{array}$ & $\begin{array}{r}4.355 \\
140 \\
387 \\
31 \\
8,640\end{array}$ & $\begin{array}{r}4.965 \\
160 \\
758 \\
20 \\
9,850\end{array}$ & $\begin{array}{r}2.354 \\
78.5 \\
142 \\
31 \\
4,670\end{array}$ \\
\hline
\end{tabular}

Cal yr 1967: Total 20,242.5 Mean 55.5 Max 1,060 Min $0.1 \quad$ Ac-ft 40,150

Wtr yr 1968: Total 24,132.3 Mean 65.9 $\quad$ Max $840 \quad$ Min $0.6 \quad$ Ac-ft 47,870 


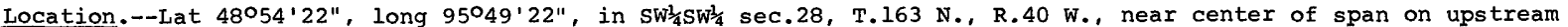
side of bridge (revised) at Roseau Lake, $3 \frac{1}{2}$ miles upstream from Pine Creek, $3 \frac{3}{4}$ miles downstream from sprague creek, and 7 miles northwest of Roseau.

Records available.--November 1939 to september 1968 (incomplete).

Gage.--Wire-weight gage read once daily. Datum of gage is 1,018.59 ft above mean sea level, adjustment of 1928 by Geodetic survey of Canada. Prior to Sept. 6,1967 , staff gage at same site and datum. Gage readings have been reduced to elevations above mean sea level.

Extremes.--Maximum elevation observed during year, 1,033.68 ft June 16; minimum observed, $1,021.02 \mathrm{ft}$ Oct. 5. 1941 .

1939-68: Maximum elevation observed, 1,036.86 ft May 13, 1950; minimum observed, 1, 019.75 ft Aug. 16,

Flood of July 1919 reached an elevation of about 1,034 ft.

Elevation, in feet, water year October 1967 to September 1968

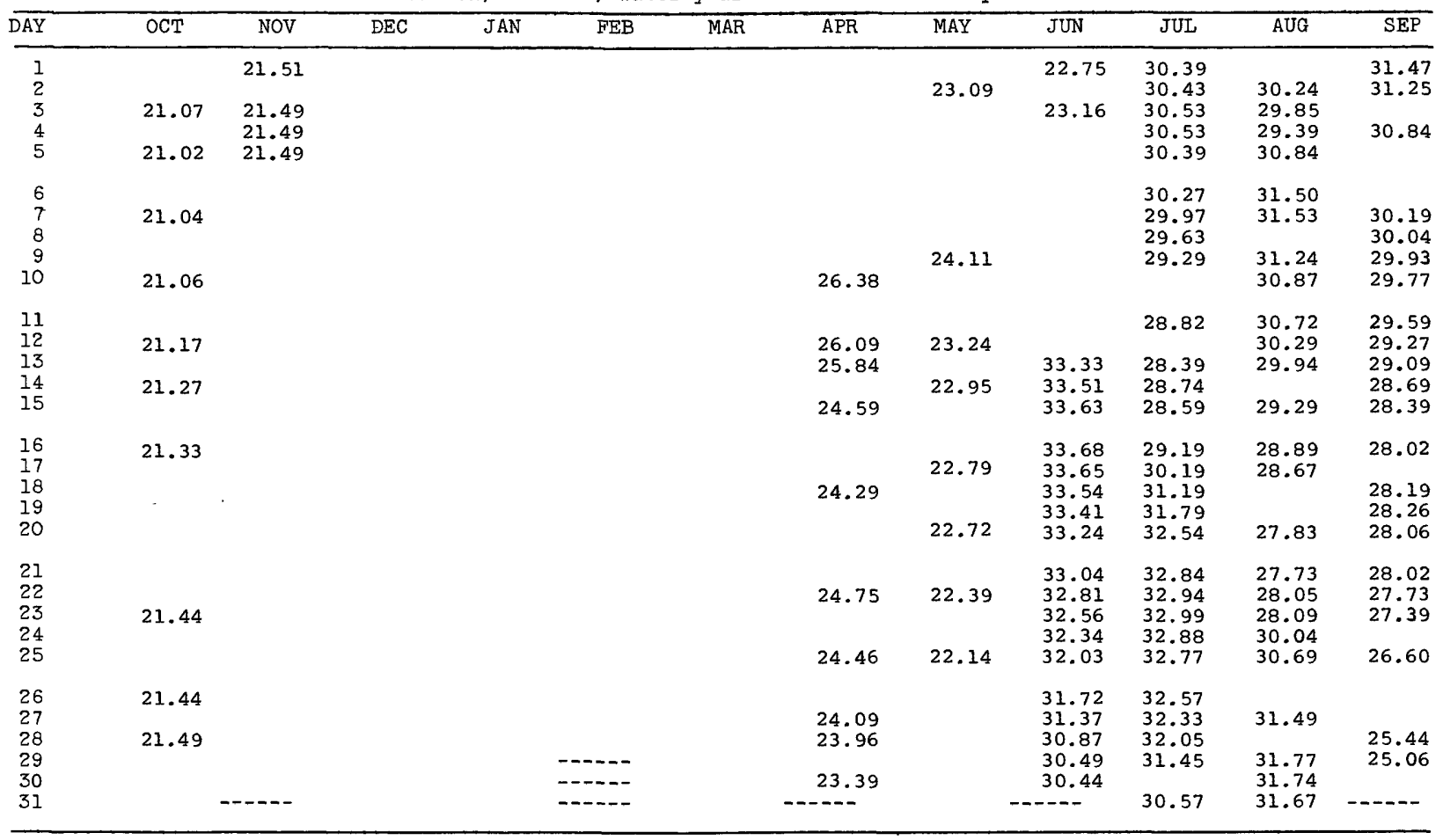

Note.--Add 1,000.00 ft to obtain elevation above mean sea level. 
5-1075. Roseau River at Ross, Minn.

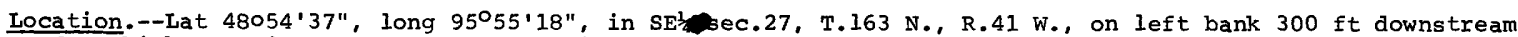
from highway bridge, a quarter of a mile north of Ross, and 2.3 miles downstream from Pine Creek.

Drainage area.--1,220 sq $\mathrm{mi}$, approximately.

Records available.--July 1928 to september 1968.

Gage.-Water-stage recorder. Datum of gage is $1,018.44 \mathrm{ft}$ above mean sea level, adjustment of 1928 by Geodetic Survey of Canada. Prior to Mar. 13, 1929, staff gage at same site and datum.

Average discharge.--40 years, 259 cfs $(187,500$ acre-ft per year) .

Extremes--Maximum discharge during year, 2,370 cfs June 18 (gage height, $14.35 \mathrm{ft}$ ); minimum, $0.9 \mathrm{cfs}$ Oct. 8 , 9. 10 (gage height, $0.84 \mathrm{ft}$ ).

1928-68: Maximum discharge, 6,560 cfs May 12, 1950 (gage height, 18.25 ft); no flow Aug. 29, 30, 1961. Maximum stage known, about $19 \mathrm{ft}$ in 1896. Other outstanding floods reached the following stages (from information by local residents): flood of July 1919, $17.5 \mathrm{ft}$; flood of 1927 , about 16 ft.

Remarks.--Records good except those for winter months, which are fair. High flow regulated by natural storage in Roseau Lake. Records of chemical analyses for the water year 1968 are published in Part 2 of this report.

DISCHARGE, IN CFS, WATER YEAR OCTOBER 1967 TO SEPTEMBER 1968

\begin{tabular}{|c|c|c|c|c|c|c|c|c|c|c|c|c|}
\hline DAY & OCT & NOV & $\mathrm{DEC}$ & JAN & FEB & MAR & APR & MAY & JUN & JUL & AUG & SEP \\
\hline $\begin{array}{l}1 \\
2 \\
3 \\
4 \\
5\end{array}$ & $\begin{array}{l}1.0 \\
1.0 \\
1.4 \\
5.9 \\
2.7\end{array}$ & $\begin{array}{l}3.5 \\
3.6 \\
3.2 \\
2.7 \\
2.2\end{array}$ & $\begin{array}{l}1.9 \\
1.9 \\
2.1 \\
2.4 \\
2.6\end{array}$ & $\begin{array}{l}4.8 \\
4.7 \\
4.5 \\
4.2 \\
3.9\end{array}$ & $\begin{array}{l}5.1 \\
5.0 \\
5.0 \\
4.9 \\
4.9\end{array}$ & $\begin{array}{l}5.1 \\
5.4 \\
5.7 \\
5.9 \\
6.0\end{array}$ & $\begin{array}{r}1.050 \\
1.000 \\
950 \\
870 \\
800\end{array}$ & $\begin{array}{l}199 \\
182 \\
169 \\
158 \\
149\end{array}$ & $\begin{array}{l}149 \\
160 \\
165 \\
178 \\
193\end{array}$ & $\begin{array}{l}1.400 \\
1.420 \\
1.420 \\
1.430 \\
1.420\end{array}$ & $\begin{array}{l}1.540 \\
1.440 \\
1.360 \\
1.270 \\
1.480\end{array}$ & $\begin{array}{l}1.710 \\
1.660 \\
1.610 \\
1.550 \\
1.490\end{array}$ \\
\hline $\begin{array}{r}6 \\
7 \\
8 \\
9 \\
10\end{array}$ & $\begin{array}{l}1.1 \\
1.0 \\
1.0 \\
1.0 \\
1.0\end{array}$ & $\begin{array}{l}1.9 \\
1.8 \\
2.0 \\
2.8 \\
3.3\end{array}$ & $\begin{array}{l}2.8 \\
3.0 \\
3.3 \\
3.6 \\
4.3\end{array}$ & $\begin{array}{l}3.6 \\
3.3 \\
3.2 \\
3.2 \\
3.1\end{array}$ & $\begin{array}{l}4.8 \\
4.8 \\
4.9 \\
5.0 \\
5.0\end{array}$ & $\begin{array}{l}6.1 \\
6.2 \\
6.4 \\
6.6 \\
6.7\end{array}$ & $\begin{array}{l}736 \\
648 \\
619 \\
613 \\
582\end{array}$ & $\begin{array}{l}140 \\
143 \\
158 \\
180 \\
194\end{array}$ & $\begin{array}{r}233 \\
473 \\
1.100 \\
1.270 \\
1.400\end{array}$ & $\begin{array}{l}1.380 \\
1.340 \\
1.280 \\
1.210 \\
1.130\end{array}$ & $\begin{array}{l}1.600 \\
1.680 \\
1.700 \\
1.660 \\
1.600\end{array}$ & $\begin{array}{l}1.420 \\
1.380 \\
1.340 \\
1.310 \\
1.280\end{array}$ \\
\hline $\begin{array}{l}11 \\
12 \\
13 \\
14 \\
15\end{array}$ & $\begin{array}{l}1.0 \\
1.2 \\
2.0 \\
2.1 \\
2.4\end{array}$ & $\begin{array}{l}3.5 \\
3.5 \\
3.1 \\
2.9 \\
2.8\end{array}$ & $\begin{array}{l}5.0 \\
5.5 \\
5.8 \\
6.0 \\
6.2\end{array}$ & $\begin{array}{l}3.1 \\
3.2 \\
3.3 \\
3.4 \\
3.4\end{array}$ & $\begin{array}{l}5.0 \\
5.0 \\
5.0 \\
5.0 \\
5.0\end{array}$ & $\begin{array}{l}7.1 \\
7.6 \\
8.0 \\
8.5 \\
8.8\end{array}$ & $\begin{array}{l}570 \\
552 \\
490 \\
425 \\
388\end{array}$ & $\begin{array}{l}195 \\
192 \\
181 \\
167 \\
157\end{array}$ & $\begin{array}{l}1.380 \\
1.850 \\
1.980 \\
2.160 \\
2.280\end{array}$ & $\begin{array}{r}1.070 \\
1.030 \\
980 \\
1.020 \\
1.020\end{array}$ & $\begin{array}{l}1.530 \\
1.450 \\
1.370 \\
1.290 \\
1.210\end{array}$ & $\begin{array}{r}1.230 \\
1.180 \\
1120 \\
1.050 \\
979\end{array}$ \\
\hline $\begin{array}{l}16 \\
17 \\
18 \\
19 \\
20\end{array}$ & $\begin{array}{l}2.7 \\
2.9 \\
2.7 \\
2.4 \\
2.7\end{array}$ & $\begin{array}{l}2.8 \\
3.1 \\
3.3 \\
3.4 \\
3.4\end{array}$ & $\begin{array}{l}6.2 \\
6.3 \\
6.4 \\
6.4 \\
6.4\end{array}$ & $\begin{array}{l}3.5 \\
3.6 \\
3.6 \\
3.7 \\
3.7\end{array}$ & $\begin{array}{l}5.0 \\
5.1 \\
5.2 \\
5.3 \\
5.3\end{array}$ & $\begin{array}{l}9.1 \\
9.4 \\
9.7 \\
10 \\
11\end{array}$ & $\begin{array}{l}360 \\
347 \\
319 \\
278 \\
236\end{array}$ & $\begin{array}{l}152 \\
156 \\
153 \\
147 \\
145\end{array}$ & $\begin{array}{l}2.350 \\
2.350 \\
2.340 \\
2.300 \\
2.240\end{array}$ & $\begin{array}{l}1.040 \\
1.210 \\
1.380 \\
1.540 \\
1.700\end{array}$ & $\begin{array}{r}1.120 \\
1.040 \\
980 \\
909 \\
880\end{array}$ & $\begin{array}{l}919 \\
901 \\
927 \\
931 \\
919\end{array}$ \\
\hline $\begin{array}{l}21 \\
22 \\
23 \\
24 \\
25\end{array}$ & $\begin{array}{l}2.7 \\
3.1 \\
3.3 \\
3.3 \\
3.3\end{array}$ & $\begin{array}{l}3.4 \\
3.3 \\
3.2 \\
3.2 \\
3.1\end{array}$ & $\begin{array}{l}6.3 \\
6.2 \\
6.1 \\
5.9 \\
5.7\end{array}$ & $\begin{array}{l}3.8 \\
4.0 \\
4.4 \\
5.0 \\
5.4\end{array}$ & $\begin{array}{l}5.2 \\
5.1 \\
5.0 \\
5.0 \\
4.8\end{array}$ & $\begin{array}{ll}1 & 1 \\
1 & 1 \\
1 & 1 \\
1 & 2 \\
13\end{array}$ & $\begin{array}{l}264 \\
302 \\
343 \\
356 \\
341\end{array}$ & $\begin{array}{l}142 \\
136 \\
124 \\
110 \\
100\end{array}$ & $\begin{array}{l}2.170 \\
2.100 \\
2.020 \\
1.940 \\
1.860\end{array}$ & $\begin{array}{l}1.810 \\
1.970 \\
2.070 \\
2.090 \\
2.060\end{array}$ & $\begin{array}{r}850 \\
867 \\
911 \\
1.140 \\
1.300\end{array}$ & $\begin{array}{l}889 \\
846 \\
787 \\
726 \\
659\end{array}$ \\
\hline $\begin{array}{l}26 \\
27 \\
28 \\
29 \\
30 \\
31\end{array}$ & $\begin{array}{l}3.3 \\
3.1 \\
2.9 \\
2.8 \\
2.8 \\
3.1\end{array}$ & $\begin{array}{r}2.9 \\
2.7 \\
2.4 \\
2.1 \\
2.0 \\
---\end{array}$ & $\begin{array}{l}5.5 \\
5.3 \\
5.2 \\
5.1 \\
5.1 \\
5.0\end{array}$ & $\begin{array}{l}5.6 \\
5.4 \\
5.3 \\
5.2 \\
5.1 \\
5.1\end{array}$ & $\begin{array}{r}4.8 \\
4.8 \\
4.8 \\
5.0 \\
\\
\end{array}$ & $\begin{array}{r}16 \\
50 \\
250 \\
550 \\
780 \\
960\end{array}$ & $\begin{array}{r}314 \\
286 \\
269 \\
243 \\
216\end{array}$ & $\begin{array}{r}93 \\
90 \\
104 \\
105 \\
118 \\
129\end{array}$ & $\begin{array}{r}1.780 \\
1.690 \\
1.590 \\
1.500 \\
1430 \\
-\end{array}$ & $\begin{array}{l}2.000 \\
1.930 \\
1.860 \\
1.780 \\
1.710 \\
1.620\end{array}$ & $\begin{array}{l}1.420 \\
1.540 \\
1.630 \\
1.700 \\
1.730 \\
1.730\end{array}$ & $\begin{array}{r}595 \\
536 \\
479 \\
423 \\
375\end{array}$ \\
\hline $\begin{array}{l}\text { Total } \\
\text { Mean } \\
\text { Max } \\
\text { Min } \\
\text { Ac-ft }\end{array}$ & $\begin{array}{r}72.9 \\
2.35 \\
5.9 \\
1.0 \\
145\end{array}$ & $\begin{array}{r}87.1 \\
2.90 \\
3.6 \\
1.8 \\
173\end{array}$ & $\begin{array}{r}149.5 \\
4.82 \\
6.4 \\
1.9 \\
297\end{array}$ & $\begin{array}{r}127.3 \\
4.11 \\
5.6 \\
3.1 \\
252\end{array}$ & $\begin{array}{r}144.8 \\
4.99 \\
5.3 \\
4.8 \\
287\end{array}$ & $\begin{array}{r}2813.3 \\
90.8 \\
960 \\
5.1 \\
5.580\end{array}$ & $\begin{array}{r}14.767 \\
492 \\
1,050 \\
216 \\
29,290\end{array}$ & $\begin{array}{r}4.568 \\
147 \\
199 \\
90 \\
9,060\end{array}$ & $\begin{array}{r}44,631 \\
1,488 \\
2,350 \\
149 \\
88,520\end{array}$ & $\begin{array}{r}46,320 \\
1,494 \\
2,090 \\
980 \\
91,870\end{array}$ & $\begin{array}{r}41,927 \\
1,352 \\
1,730 \\
850 \\
83,160\end{array}$ & $\begin{array}{r}31,221 \\
1,041 \\
1,710 \\
375 \\
61,930\end{array}$ \\
\hline $\begin{array}{l}\text { Cal yr } \\
\text { wtr yr }\end{array}$ & & $\begin{array}{l}\text { Total } \\
\text { Total }\end{array}$ & $\begin{array}{l}62.3 \\
28.9\end{array}$ & $\begin{array}{l}\text { lean } \\
\text { lean }\end{array}$ & $\begin{array}{l}\text { Max } \\
\text { Max }\end{array}$ & $\begin{array}{l}2,760 \\
2,350\end{array}$ & $\begin{array}{ll}\text { in } & 0.7 \\
\text { in } & 1.0\end{array}$ & $\begin{array}{l}A c-f t \\
A c-f t\end{array}$ & $\begin{array}{l}6,500 \\
0,600\end{array}$ & & & \\
\hline
\end{tabular}


Location.--Lat $48^{\circ} 54^{\prime} 4^{\prime \prime}$, long $96^{\circ} 00^{\prime} 24^{\prime \prime}$, in SW/ sec. 30, T.163 highway bridge and 9 miles north of Badger.

Records available.--August 1928 to september 1968 (incomplete).

Gage.--Water-stage recorder. Datum of gage is $1,016.90 \mathrm{ft}$ above mean sea level, adjustment of 1928 by Geodetic survey of Canada. Gage readings have been reduced to elevations above mean sea level.

Extremes.--Maximum elevation during year, 1,030.11 ft about June 18 (from recorded range in stage); minimum recorded, $1,017.47 \mathrm{ft}$ Nov. $8,9$.

1928-68: Maximum elevation, 1,023.65 ft May 13, 1950; minimum recorded, 1,017.42 ft Aug. $30,1961$. Maximum elevation known, about 1,034 ft in 1896.

Mean elevation, in feet, water year October 1967 to September 1968

\begin{tabular}{|c|c|c|c|c|c|c|c|c|c|c|c|c|}
\hline DAY & $\mathrm{OCT}$ & NOV & DEC & JAN & FEB & MAR & APR & MAY & JUN & JUL & AUG & SEP \\
\hline $\begin{array}{l}1 \\
2 \\
3 \\
4 \\
5\end{array}$ & & $\begin{array}{l}17.58 \\
17.57 \\
17.57 \\
17.56 \\
17.55\end{array}$ & & & & & & $\begin{array}{l}21.23 \\
21.09 \\
20.87 \\
20.71 \\
20.57\end{array}$ & & & $\begin{array}{l}27.95 \\
27.68 \\
27.40 \\
27.12 \\
28.16\end{array}$ & \\
\hline $\begin{array}{r}6 \\
7 \\
8 \\
9 \\
10\end{array}$ & & $\begin{array}{l}17.51 \\
17.48 \\
17.47 \\
17.51 \\
17.64\end{array}$ & & & & & & $\begin{array}{l}20.46 \\
20.38 \\
20.50 \\
20.73 \\
20.96\end{array}$ & & & $\begin{array}{l}28.46 \\
28.64 \\
28.66 \\
28.55 \\
28.31\end{array}$ & \\
\hline $\begin{array}{l}11 \\
12 \\
13 \\
14 \\
15\end{array}$ & & $\begin{array}{l}17.71 \\
17.76 \\
17.76 \\
17.75 \\
17.76\end{array}$ & & & & & $\begin{array}{l}23.95 \\
23.81 \\
23.45 \\
23.03 \\
22.73\end{array}$ & $\begin{array}{l}21.03 \\
21.02 \\
20.95 \\
20.80\end{array}$ & 29.23 & & 28.02 & \\
\hline $\begin{array}{l}16 \\
17 \\
18 \\
19 \\
20\end{array}$ & $\begin{array}{l}17.53 \\
17.59\end{array}$ & $\begin{array}{l}17.76 \\
17.74 \\
17.77 \\
17.80 \\
17.81\end{array}$ & & & & & $\begin{array}{l}22.51 \\
22.37 \\
22.19 \\
21.95 \\
21.56\end{array}$ & & & & & $\begin{array}{l}25.76 \\
25.68 \\
25.67\end{array}$ \\
\hline $\begin{array}{l}26 \\
27 \\
28 \\
29 \\
30 \\
31\end{array}$ & $\begin{array}{l}17.51 \\
17.51 \\
17.51 \\
17.51 \\
17.55 \\
17.59\end{array}$ & $\begin{array}{r}17.79 \\
17.80 \\
17.83 \\
17.84\end{array}$ & & & 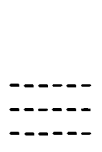 & $\begin{array}{l}19.48 \\
20.26\end{array}$ & $\begin{array}{r}22.21 \\
22.02 \\
21.85 \\
21.70 \\
21.47 \\
-\end{array}$ & & ---- & $\begin{array}{l}29.36 \\
29.17 \\
28.96 \\
28.76 \\
28.52 \\
28.24\end{array}$ & & $\begin{array}{r}24.19 \\
23.85 \\
23.47 \\
23.09 \\
22.74 \\
-\end{array}$ \\
\hline
\end{tabular}

Note.--Add 1,000.00 ft to obtain elevation above mean sea level. 
5-1120. Roseau River below state ditch 51, near Caribou, Minn.

(International gaging station)

Iocation.--Lat $48^{\circ} 58^{\prime} 54^{\prime \prime}$, long $96^{\circ} 27^{\prime} 46^{\prime \prime}$, in SE $\frac{1}{4} S^{\frac{1}{4}}$ sec. 34, T. 164 N., R.45 W., on left bank 400 ft downstream from state ditch 51 (known locally as Caribou cutoff ditch) and 0.6 mile west of Caribou.

Drainage area. $--1,570 \mathrm{sq} \mathrm{mi}$, approximately.

Records available.--April to October 1917, April 1920 to september 1968 (some winter records incomplete). Published as "at Caribou", prior to April 1929; as "below Cutoff ditch, near Caribou" April I929 to September 1936. Records published for both sites April 1929 to september 1930. Monthly discharge only for some periods, published in WSP 1308.

Gage.--Water-stage recorder. Datum of gage is 1,002.14 ft above mean sea level, adjustment of 1928 , by Geo-

detic survey of Canada. Prior to Apr. 1, 1929, chain gage at site at Caribou 0.6 mile upstream at datum 0.95 ft lower.

Average discharge.--15 years (1920-30, 1932-33, 1936-37, 1940-43), 298 cfs (215,700 acre-ft per year).

Extremes.--Maximum discharge during year, 1,860 cfs July 2 (gage height, 8.16 ft); maximum gage height, about 8.40 ft Apr. 7 (backwater from ice); minimum daily discharge recorded, $0.8 \mathrm{cfs} 0 \mathrm{ct}$. 6,7 .

1917, 1920-68: Maximum discharge, 4,080 cfs May 19, 1950 (gage height, 11.81 ft); no flow Aug. 13, 1936.

Flood of 1916 is reported to have reached a stage of about $15.5 \mathrm{ft}$ at former site.

Remarks.--Records good. Occasionally, at high stages, there is some natural diversion of flow above station

to headwaters of Two Rivers. Station not operated during winter period.

Cooperation.--This station is maintained by the United states under agreement with Canada.

DISCHARGE, IN CFS, WATER YEAR OCTOBER 1967 TO SEPTEMBER 1968

\begin{tabular}{|c|c|c|c|c|c|c|c|c|c|c|c|c|c|}
\hline $\overrightarrow{D A Y}$ & OCT & NOV & $\overline{D E C}$ & & JAN & FEB & MAR & APR & MAY & JUN & JUL & AUG & $\overline{S E P}$ \\
\hline $\begin{array}{l}1 \\
2 \\
3 \\
4 \\
5\end{array}$ & $\begin{array}{l}1.4 \\
1.4 \\
1.5 \\
1.2 \\
1.0\end{array}$ & $\begin{array}{l}4.2 \\
4.3 \\
4.4 \\
4.4 \\
4.4\end{array}$ & & & & & & $\begin{array}{r}650 \\
800 \\
920 \\
1000 \\
1.070\end{array}$ & $\begin{array}{l}239 \\
214 \\
196 \\
184 \\
169\end{array}$ & $\begin{array}{l}134 \\
141 \\
152 \\
173 \\
179\end{array}$ & $\begin{array}{l}1.830 \\
1.860 \\
1.840 \\
1.800 \\
1.770\end{array}$ & $\begin{array}{l}1.730 \\
1.720 \\
1.710 \\
1.700 \\
1.750\end{array}$ & $\begin{array}{l}1.460 \\
1.490 \\
1.530 \\
1.560 \\
1.570\end{array}$ \\
\hline $\begin{array}{r}6 \\
7 \\
8 \\
9 \\
10\end{array}$ & $\begin{array}{r}.9 \\
.8 \\
1.0 \\
1.2 \\
1.4\end{array}$ & $\begin{array}{l}4.2 \\
4.1 \\
3.9 \\
3.8 \\
3.8\end{array}$ & & & & & & $\begin{array}{l}1.120 \\
1.140 \\
1160 \\
1.130 \\
1090\end{array}$ & $\begin{array}{l}158 \\
152 \\
150 \\
154 \\
162\end{array}$ & $\begin{array}{r}214 \\
290 \\
546 \\
941 \\
1.070\end{array}$ & $\begin{array}{l}1.740 \\
1.710 \\
1.680 \\
1.640 \\
1.610\end{array}$ & $\begin{array}{l}1.750 \\
1.730 \\
1.710 \\
1.700 \\
1.690\end{array}$ & $\begin{array}{l}1.580 \\
1.620 \\
1.640 \\
1.630 \\
1.620\end{array}$ \\
\hline $\begin{array}{l}11 \\
12 \\
13 \\
14 \\
15\end{array}$ & $\begin{array}{l}1.6 \\
1.6 \\
1.8 \\
1.8 \\
1.6\end{array}$ & $\begin{array}{l}3.8 \\
3.9 \\
4.0 \\
4.0 \\
3.8\end{array}$ & & & & & & $\begin{array}{r}1040 \\
934 \\
840 \\
730 \\
640\end{array}$ & $\begin{array}{l}177 \\
188 \\
192 \\
194 \\
192\end{array}$ & $\begin{array}{l}1.150 \\
1.190 \\
1230 \\
1.270 \\
1.310\end{array}$ & $\begin{array}{l}1.620 \\
1.610 \\
1.610 \\
1.640 \\
1.600\end{array}$ & $\begin{array}{l}1.680 \\
1.670 \\
1.660 \\
1.640 \\
1.630\end{array}$ & $\begin{array}{l}1.600 \\
1.590 \\
1.570 \\
1.550 \\
1.530\end{array}$ \\
\hline $\begin{array}{l}16 \\
17 \\
18 \\
19 \\
20\end{array}$ & $\begin{array}{l}1.5 \\
2.0 \\
1.6 \\
1.5 \\
1.5\end{array}$ & $\begin{array}{l}3.6 \\
3.5 \\
3.4 \\
3.3 \\
3.3\end{array}$ & & & & & 6.0 & $\begin{array}{l}562 \\
497 \\
453 \\
408 \\
370\end{array}$ & $\begin{array}{l}175 \\
167 \\
167 \\
173 \\
162\end{array}$ & $\begin{array}{l}1.360 \\
1.400 \\
1.470 \\
1.510 \\
1.560\end{array}$ & $\begin{array}{l}1.620 \\
1.670 \\
1.600 \\
1.580 \\
1.540\end{array}$ & $\begin{array}{l}1.620 \\
1.600 \\
1.580 \\
1.560 \\
1.530\end{array}$ & $\begin{array}{l}1.560 \\
1.570 \\
1.540 \\
1.510 \\
1.480\end{array}$ \\
\hline $\begin{array}{l}21 \\
22 \\
23 \\
24 \\
25\end{array}$ & $\begin{array}{l}1.6 \\
1.6 \\
1.8 \\
2.2 \\
3.0\end{array}$ & $\begin{array}{l}3.3 \\
3.4 \\
3.5 \\
3.5 \\
3.4\end{array}$ & & & & & $\begin{array}{l}6.4 \\
7.5 \\
9.0 \\
13 \\
18\end{array}$ & $\begin{array}{l}340 \\
340 \\
365 \\
389 \\
406\end{array}$ & $\begin{array}{l}143 \\
140 \\
132 \\
123 \\
110\end{array}$ & $\begin{array}{l}1.630 \\
1.670 \\
1.710 \\
1.740 \\
1.750\end{array}$ & $\begin{array}{l}1.530 \\
1.520 \\
1.530 \\
1.560 \\
1.580\end{array}$ & $\begin{array}{l}1.480 \\
1.480 \\
1.410 \\
1.430 \\
1.400\end{array}$ & $\begin{array}{l}1.440 \\
1.420 \\
1.390 \\
1.350 \\
1.310\end{array}$ \\
\hline $\begin{array}{l}26 \\
27 \\
28 \\
29 \\
30 \\
31\end{array}$ & $\begin{array}{l}3.2 \\
3.4 \\
3.3 \\
3.2 \\
3.2 \\
4.0\end{array}$ & $\begin{array}{r}3.2 \\
3.1 \\
3.0 \\
2.8 \\
2.7 \\
\end{array}$ & & & & - & $\begin{array}{l}45 \\
100 \\
170 \\
250 \\
350 \\
500\end{array}$ & $\begin{array}{r}389 \\
360 \\
326 \\
299 \\
269 \\
\end{array}$ & $\begin{array}{r}100 \\
94 \\
94 \\
97 \\
110 \\
125\end{array}$ & $\begin{array}{r}1.750 \\
1.750 \\
1.740 \\
1.730 \\
1.730 \\
\end{array}$ & $\begin{array}{l}1600 \\
1630 \\
1640 \\
1670 \\
1690 \\
1.720\end{array}$ & $\begin{array}{l}1380 \\
1.370 \\
1370 \\
1380 \\
1.400 \\
1430\end{array}$ & $\begin{array}{r}1260 \\
1.190 \\
1110 \\
997 \\
868 \\
\end{array}$ \\
\hline Tota 1 & 58.8 & 110.0 & & & & & & 20.037 & 4.833 & 34.490 & 51.240 & 48.890 & 43.535 \\
\hline $\begin{array}{l}\text { Mean } \\
\text { Max } \\
\text { Min } \\
\text { Ac-ft }\end{array}$ & $\begin{array}{r}1.90 \\
4.0 \\
0.8 \\
-\end{array}$ & $\begin{array}{r}3.67 \\
4.4 \\
2.7 \\
-\end{array}$ & & & & & & $\begin{array}{r}668 \\
1,160 \\
269 \\
-\end{array}$ & $\begin{array}{r}156 \\
239 \\
94 \\
-\end{array}$ & $\begin{array}{r}1,150 \\
1,750 \\
134 \\
-\end{array}$ & $\begin{array}{r}1,653 \\
1,860 \\
1,520\end{array}$ & $\begin{array}{l}1,577 \\
1,750 \\
1,370\end{array}$ & $\begin{array}{r}1,451 \\
1,640 \\
868\end{array}$ \\
\hline $\begin{array}{l}\text { Cal yr } \\
\text { Wtr yr }\end{array}$ & $\begin{array}{l}1967: \\
1968:\end{array}$ & $\begin{array}{l}\text { Total - } \\
\text { Total - }\end{array}$ & $\begin{array}{l}\text { Mean } \\
\text { Mean }\end{array}$ & - & $\begin{array}{l}\operatorname{Max} \\
\operatorname{Max}\end{array}$ & $\begin{array}{l}\text { Min } \\
\operatorname{Min}\end{array}$ & - & $\begin{array}{l}\mathrm{ft}- \\
\mathrm{ft}-\end{array}$ & & & & & \\
\hline
\end{tabular}


5-1125. Roseau River at international boundary, near Caribou, Minn.

Location.--Iat $48^{\circ} 59^{\prime} 57$, long 96030'20", near center of sec.29, T.164 N., R.45 W., on left bank 400 ft upstream from international boundary crossing and 3 miles northwest of Caribou.

Drainage area. $--1,590 \mathrm{sq} \mathrm{mi}$, approximately.

Records available.--May 1933 to September 1968 (incomplete).

Gage.--Water-stage recorder. Datum of gage is 1,002.59 ft above mean sea level, adjustment of 1928, by Geodetic Survey of Canada. Gage readings have been reduced to elevations above mean sea level.

Extremes.--Maximum elevation during year, 1,006.98 ft July 4; minimum recorded, 1,002.33 ft 0ct. 5-8. 1933.

1933-68: Maximum elevation recorded, 1,007.43 ft Apr. 14, 1960; minimum recorded, 1,001.97 ft Aug. 14,

Mean elevation, in feet, water year October 1967 to september 1968

\begin{tabular}{|c|c|c|c|c|c|c|c|c|c|c|c|c|}
\hline DAY & OCT & NOV & DEC & JAN & FEB & MAR & APR & MAY & JUN & JUL & AUG & SEP \\
\hline $\begin{array}{l}1 \\
2 \\
3 \\
4 \\
5\end{array}$ & $\begin{array}{l}2.43 \\
2.42 \\
2.43 \\
2.36 \\
2.35\end{array}$ & $\begin{array}{l}2.83 \\
2.85 \\
2.82 \\
2.78 \\
2.85\end{array}$ & & & & & $\begin{array}{l}5.73 \\
5.88 \\
6.11 \\
6.16 \\
6.16\end{array}$ & $\begin{array}{l}3.85 \\
3.77 \\
3.76 \\
3.71 \\
3.66\end{array}$ & $\begin{array}{l}3.67 \\
3.71 \\
3.71 \\
3.79 \\
3.82\end{array}$ & $\begin{array}{l}6.82 \\
6.84 \\
6.91 \\
6.94 \\
6.92\end{array}$ & $\begin{array}{l}6.72 \\
6.71 \\
6.70 \\
6.69 \\
6.69\end{array}$ & $\begin{array}{l}6.60 \\
6.61 \\
6.65 \\
6.68 \\
6.71\end{array}$ \\
\hline $\begin{array}{r}6 \\
7 \\
8 \\
9 \\
10\end{array}$ & $\begin{array}{l}2.34 \\
2.33 \\
2.35 \\
2.48 \\
2.54\end{array}$ & $\begin{array}{l}2.83 \\
2.84 \\
2.84 \\
2.84 \\
2.86\end{array}$ & & & & & $\begin{array}{l}6.18 \\
6.21 \\
6.14 \\
5.87 \\
5.77\end{array}$ & $\begin{array}{l}3.66 \\
3.66 \\
3.64 \\
3.66 \\
3.68\end{array}$ & $\begin{array}{l}3.91 \\
4.04 \\
4.69 \\
5.53 \\
5.86\end{array}$ & $\begin{array}{l}6.91 \\
6.89 \\
6.86 \\
6.82 \\
6.81\end{array}$ & $\begin{array}{l}6.75 \\
6.78 \\
6.76 \\
6.74 \\
6.82\end{array}$ & $\begin{array}{l}6.71 \\
6.73 \\
6.73 \\
6.73 \\
6.73\end{array}$ \\
\hline $\begin{array}{l}11 \\
12 \\
13 \\
14 \\
15\end{array}$ & $\begin{array}{l}2.53 \\
2.48 \\
2.54 \\
2.55 \\
2.48\end{array}$ & $\begin{array}{l}2.87 \\
2.96 \\
3.03 \\
2.94 \\
3.04\end{array}$ & & & & & $\begin{array}{l}5.59 \\
5.36 \\
5.13 \\
4.86 \\
4.66\end{array}$ & $\begin{array}{l}3.74 \\
3.75 \\
3.76 \\
3.75 \\
3.75\end{array}$ & $\begin{array}{l}6.04 \\
6.14 \\
6.24 \\
6.32 \\
6.36\end{array}$ & $\begin{array}{l}6.82 \\
4.80 \\
4.80 \\
6.82 \\
6.77\end{array}$ & $\begin{array}{l}6.87 \\
6.90 \\
6.92 \\
6.92 \\
6.93\end{array}$ & $\begin{array}{l}6.73 \\
6.73 \\
6.72 \\
6.71 \\
6.71\end{array}$ \\
\hline $\begin{array}{l}16 \\
17 \\
18 \\
19 \\
20\end{array}$ & $\begin{array}{l}2.50 \\
2.61 \\
2.50 \\
2.44 \\
2.44\end{array}$ & 3.09 & & & & & $\begin{array}{l}4.46 \\
4.30 \\
4.21 \\
4.14 \\
3.99\end{array}$ & $\begin{array}{l}3.73 \\
3.71 \\
3.73 \\
3.71 \\
3.71\end{array}$ & $\begin{array}{l}6.44 \\
6.51 \\
6.57 \\
6.65 \\
6.70\end{array}$ & $\begin{array}{l}6.78 \\
6.84 \\
6.81 \\
6.76 \\
6.73\end{array}$ & $\begin{array}{l}6.92 \\
6.90\end{array}$ & $\begin{array}{l}6.73 \\
6.74 \\
6.73 \\
6.69 \\
6.65\end{array}$ \\
\hline $\begin{array}{l}21 \\
22 \\
23 \\
24 \\
25\end{array}$ & $\begin{array}{l}2.46 \\
2.49 \\
2.56 \\
2.55 \\
2.54\end{array}$ & & & & & & $\begin{array}{l}4.00 \\
4.01 \\
4.07 \\
4.13 \\
4.15\end{array}$ & $\begin{array}{l}3.70 \\
3.70 \\
3.69 \\
3.64 \\
3.61\end{array}$ & $\begin{array}{l}6.78 \\
6.81 \\
6.83 \\
6.89 \\
6.89\end{array}$ & $\begin{array}{l}6.72 \\
6.71 \\
6.73 \\
6.75 \\
6.79\end{array}$ & $\begin{array}{l}6.61 \\
6.57 \\
6.54 \\
6.56 \\
6.53\end{array}$ & $\begin{array}{l}6.61 \\
6.57 \\
6.54 \\
6.49 \\
6.42\end{array}$ \\
\hline $\begin{array}{l}26 \\
27 \\
28 \\
29 \\
30 \\
31\end{array}$ & $\begin{array}{l}2.58 \\
2.77 \\
2.75 \\
2.84 \\
2.83 \\
2.85\end{array}$ & 3.02 & & & $\begin{array}{l}----- \\
------ \\
------\end{array}$ & $\begin{array}{l}4.57 \\
5.18 \\
5.55\end{array}$ & $\begin{array}{l}4.12 \\
4.06 \\
4.02 \\
3.96 \\
3.91 \\
---\end{array}$ & $\begin{array}{l}3.57 \\
3.56 \\
3.54 \\
3.56 \\
3.60 \\
3.64\end{array}$ & $\begin{array}{l}6.82 \\
6.79 \\
6.75 \\
6.75 \\
6.80 \\
----\end{array}$ & $\begin{array}{l}6.81 \\
6.82 \\
6.81 \\
6.74 \\
6.73 \\
6.71\end{array}$ & $\begin{array}{l}6.51 \\
6.51 \\
6.51 \\
6.52 \\
6.54 \\
6.56\end{array}$ & $\begin{array}{l}6.34 \\
6.25 \\
6.07 \\
5.83 \\
5.51\end{array}$ \\
\hline
\end{tabular}

Note.--Add $1,000.00 \mathrm{ft}$ to obtain elevation above mean sea level. 
5-1244.8 Kawishiwi River near Ely, Minn.

(Hydrologic bench-mark station)

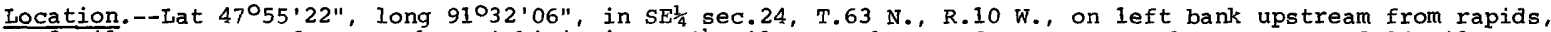
2 miles upstream from South Kawishiwi' River, $2 \frac{1}{4}$ miles southwest of Fernberg Lookout Tower and l4 miles east of Ely.

Drainage area. $--253 \mathrm{sq} \mathrm{mi}$.

Records available.--June 1966 to september 1968.

Gage.--Water-stage recorder. Altitude of gage is 1,450 ft (from topographic map).

Extremes.--Maximum discharge during year, 1,200 cfs June 18 (gage height, 5.54 ft); minimum, 29 cfs Feb. 23-29 (gage height, $2.70 \mathrm{ft}$ ).

1966-68: Maximum discharge, that of June 18, 1968; minimum, 24 cfs Sept. 28, 29, 1966; minimum gage height that of Feb. 23-29, 1968.

Remarks.--Records good. Records of chemical analyses, water temperatures and suspended sediment loads for the water year 1968 are published in Part 2 of this report.

DISCHARGE, IN GFS, WATER YEAR OCTOBER 1967 TO SEPTEMBER 1968

\begin{tabular}{|c|c|c|c|c|c|c|c|c|c|c|c|c|}
\hline DAY & OCT & NOV & DEC & JAN & FEB & MAR & APR & MAY & JUN & JUL & AUG & SEP \\
\hline $\begin{array}{l}1 \\
2 \\
3 \\
4 \\
5\end{array}$ & $\begin{array}{l}39 \\
39 \\
39 \\
39 \\
39\end{array}$ & $\begin{array}{l}34 \\
33 \\
33 \\
33 \\
33\end{array}$ & $\begin{array}{l}31 \\
31 \\
30 \\
30 \\
30\end{array}$ & $\begin{array}{l}33 \\
33 \\
34 \\
34 \\
36\end{array}$ & $\begin{array}{l}39 \\
39 \\
39 \\
38 \\
38\end{array}$ & $\begin{array}{l}30 \\
31 \\
31 \\
31 \\
31\end{array}$ & $\begin{array}{l}59 \\
59 \\
60 \\
68 \\
68\end{array}$ & $\begin{array}{l}1.020 \\
1.040 \\
1.070 \\
1.070 \\
1.060\end{array}$ & $\begin{array}{l}525 \\
525 \\
513 \\
508 \\
502\end{array}$ & $\begin{array}{l}822 \\
788 \\
735 \\
728 \\
702\end{array}$ & $\begin{array}{l}247 \\
236 \\
225 \\
222 \\
213\end{array}$ & $\begin{array}{l}111 \\
108 \\
105 \\
102 \\
102\end{array}$ \\
\hline $\begin{array}{r}6 \\
7 \\
8 \\
9 \\
10\end{array}$ & $\begin{array}{l}38 \\
37 \\
40 \\
40 \\
41\end{array}$ & $\begin{array}{l}32 \\
32 \\
31 \\
31 \\
31\end{array}$ & $\begin{array}{l}30 \\
30 \\
30 \\
30 \\
30\end{array}$ & $\begin{array}{l}36 \\
36 \\
37 \\
38 \\
38\end{array}$ & $\begin{array}{l}38 \\
38 \\
39 \\
39 \\
38\end{array}$ & $\begin{array}{l}31 \\
30 \\
30 \\
31 \\
31\end{array}$ & $\begin{array}{r}70 \\
73 \\
92 \\
100 \\
103\end{array}$ & $\begin{array}{r}1.030 \\
1.020 \\
996 \\
960 \\
932\end{array}$ & $\begin{array}{l}525 \\
536 \\
664 \\
735 \\
795\end{array}$ & $\begin{array}{l}664 \\
633 \\
620 \\
565 \\
530\end{array}$ & $\begin{array}{l}203 \\
197 \\
186 \\
182 \\
169\end{array}$ & $\begin{array}{l}103 \\
103 \\
105 \\
105 \\
103\end{array}$ \\
\hline $\begin{array}{l}11 \\
12 \\
13 \\
14 \\
15\end{array}$ & $\begin{array}{l}41 \\
40 \\
40 \\
40 \\
40\end{array}$ & $\begin{array}{l}31 \\
31 \\
31 \\
32 \\
32\end{array}$ & $\begin{array}{l}31 \\
31 \\
31 \\
30 \\
30\end{array}$ & $\begin{array}{l}38 \\
38 \\
38 \\
38 \\
38\end{array}$ & $\begin{array}{l}38 \\
38 \\
38 \\
37 \\
37\end{array}$ & $\begin{array}{l}31 \\
31 \\
31 \\
31 \\
31\end{array}$ & $\begin{array}{l}110 \\
118 \\
125 \\
150 \\
180\end{array}$ & $\begin{array}{l}897 \\
863 \\
829 \\
795 \\
755\end{array}$ & $\begin{array}{r}904 \\
953 \\
1.000 \\
1.100 \\
1.140\end{array}$ & $\begin{array}{l}508 \\
486 \\
464 \\
448 \\
422\end{array}$ & $\begin{array}{l}161 \\
154 \\
154 \\
148 \\
144\end{array}$ & $\begin{array}{r}102 \\
99 \\
97 \\
97 \\
97\end{array}$ \\
\hline $\begin{array}{l}16 \\
17 \\
18 \\
19 \\
20\end{array}$ & $\begin{array}{l}39 \\
39 \\
39 \\
38 \\
37\end{array}$ & $\begin{array}{l}32 \\
32 \\
32 \\
32 \\
32\end{array}$ & $\begin{array}{l}30 \\
30 \\
30 \\
30 \\
31\end{array}$ & $\begin{array}{l}38 \\
38 \\
38 \\
38 \\
38\end{array}$ & $\begin{array}{l}36 \\
33 \\
33 \\
33 \\
31\end{array}$ & $\begin{array}{l}31 \\
32 \\
32 \\
39 \\
44\end{array}$ & $\begin{array}{l}225 \\
261 \\
304 \\
331 \\
387\end{array}$ & $\begin{array}{l}748 \\
728 \\
709 \\
683 \\
645\end{array}$ & $\begin{array}{l}1.160 \\
1.180 \\
1.190 \\
1.180 \\
1.160\end{array}$ & $\begin{array}{l}412 \\
402 \\
387 \\
373 \\
368\end{array}$ & $\begin{array}{l}146 \\
143 \\
135 \\
134 \\
132\end{array}$ & $\begin{array}{r}97 \\
97 \\
99 \\
100 \\
100\end{array}$ \\
\hline $\begin{array}{l}21 \\
22 \\
23 \\
24 \\
25\end{array}$ & $\begin{array}{l}37 \\
37 \\
37 \\
39 \\
39\end{array}$ & $\begin{array}{l}32 \\
31 \\
32 \\
32 \\
32\end{array}$ & $\begin{array}{l}31 \\
31 \\
31 \\
31 \\
31\end{array}$ & $\begin{array}{l}38 \\
38 \\
39 \\
39 \\
39\end{array}$ & $\begin{array}{l}31 \\
31 \\
30 \\
29 \\
29\end{array}$ & $\begin{array}{l}44 \\
42 \\
41 \\
40 \\
40\end{array}$ & $\begin{array}{l}443 \\
491 \\
590 \\
696 \\
735\end{array}$ & $\begin{array}{l}639 \\
608 \\
596 \\
583 \\
565\end{array}$ & $\begin{array}{l}1.140 \\
1.130 \\
1.130 \\
1.110 \\
1.070\end{array}$ & $\begin{array}{l}363 \\
344 \\
344 \\
336 \\
322\end{array}$ & $\begin{array}{l}130 \\
128 \\
134 \\
134 \\
132\end{array}$ & $\begin{array}{r}100 \\
100 \\
100 \\
97 \\
94\end{array}$ \\
\hline $\begin{array}{l}26 \\
27 \\
28 \\
29 \\
30 \\
31\end{array}$ & $\begin{array}{l}39 \\
38 \\
37 \\
37 \\
37 \\
36\end{array}$ & $\begin{array}{r}32 \\
32 \\
31 \\
30 \\
30 \\
\end{array}$ & $\begin{array}{l}31 \\
31 \\
31 \\
32 \\
33 \\
33\end{array}$ & $\begin{array}{l}39 \\
39 \\
39 \\
38 \\
38 \\
38\end{array}$ & $\begin{array}{r}29 \\
29 \\
29 \\
30 \\
-\end{array}$ & $\begin{array}{l}40 \\
47 \\
56 \\
59 \\
59 \\
59\end{array}$ & $\begin{array}{r}768 \\
808 \\
849 \\
904 \\
974 \\
\end{array}$ & $\begin{array}{l}548 \\
542 \\
542 \\
542 \\
536 \\
536\end{array}$ & $\begin{array}{r}1020 \\
981 \\
925 \\
890 \\
856 \\
\end{array}$ & $\begin{array}{l}322 \\
304 \\
287 \\
270 \\
258 \\
255\end{array}$ & $\begin{array}{l}128 \\
125 \\
122 \\
116 \\
113 \\
110\end{array}$ & $\begin{array}{r}94 \\
92 \\
91 \\
90 \\
88 \\
\end{array}$ \\
\hline $\begin{array}{l}\text { Total } \\
\text { Mean } \\
\text { Max } \\
\text { Min } \\
\text { Cfsm } \\
\text { In. }\end{array}$ & $\begin{array}{r}1.197 \\
38.6 \\
41 \\
36 \\
0.153 \\
0.18\end{array}$ & $\begin{array}{r}954 \\
31.8 \\
34 \\
30 \\
0.126 \\
0.14\end{array}$ & $\begin{array}{r}952 \\
30.7 \\
33 \\
30 \\
0.121 \\
0.14\end{array}$ & $\begin{array}{r}1.159 \\
37.4 \\
39 \\
33 \\
0.148 \\
0.17\end{array}$ & $\begin{array}{r}1.006 \\
34.7 \\
39 \\
29 \\
0.137 \\
0.15\end{array}$ & $\begin{array}{r}1167 \\
37.6 \\
59 \\
30 \\
0.149 \\
0.17\end{array}$ & $\begin{array}{r}10.201 \\
340 \\
974 \\
59 \\
1.34 \\
1.50\end{array}$ & $\begin{array}{r}24.087 \\
777 \\
1.070 \\
536 \\
3.07 \\
3.54\end{array}$ & $\begin{array}{r}27.047 \\
902 \\
1.190 \\
502 \\
3.57 \\
3.98\end{array}$ & $\begin{array}{r}14.462 \\
467 \\
822 \\
255 \\
1.85 \\
2.13\end{array}$ & $\begin{array}{r}4.903 \\
158 \\
247 \\
110 \\
0.624 \\
0.72\end{array}$ & $\begin{array}{r}2.978 \\
99.3 \\
111 \\
88 \\
0.392 \\
0.44\end{array}$ \\
\hline $\begin{array}{l}\text { Cal yr } \\
\text { wtr yr }\end{array}$ & $\begin{array}{l}967: \\
968:\end{array}$ & $\begin{array}{l}\operatorname{ta} 1 \\
\operatorname{ta} 1\end{array}$ & & $\begin{array}{l}169 \\
246\end{array}$ & $\begin{array}{l}\text { Max } \\
\text { Max }\end{array}$ & & $\begin{array}{l}30 \\
29\end{array}$ & $\begin{array}{l}\mathrm{fsm} \\
\mathrm{fsm}\end{array}$ & $I 1$ & $\begin{array}{r}9.07 \\
13.25\end{array}$ & & \\
\hline
\end{tabular}




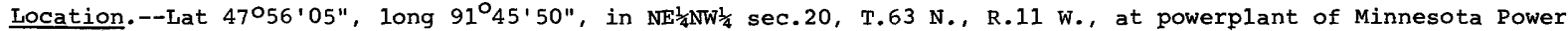
$\&$ Light Co., just upstream from Fall Lake, and 1.8 miles east of winton.

Drainaqe area. $--1,200 \mathrm{sq} \mathrm{mi}$, approximately.

Records available.--June 1905 to June 1907, October 1912 to september 1919 (fragmentary), September 1923 to September 1968. Monthly discharge only for some periods, published in wSP 1308.

Averaqe discharge.--49 years (1905-06, 1915-17, 1918-19, 1923-68), 979 cfs (unadjusted).

Extremes.--Maximum daily discharge during year, 8,030 cfs June 17; no flow Feb. 11.

1905-07, 1912-19, 1923-68: Maximum daily discharge, 16,000 cfs May 18, 1950; no flow at times.

Remarks.--Records good. Daily discharge computed from powerplant records. Flow regulated by powerplant and by Camp Six, Bald Eagle, Gabbro, Little Gabbro, Birch, White Iron, South Farm, and Garden Lakes.

Cooperation.--Records collected by Minnesota Power \& Light Co., under general supervision of Geological Survey, in connection with a Federal Power Commission project.

DISCHARGE, IN CFS, WATER YEAR OCTOBER 1967 TO SEPTEMBER 1968

\begin{tabular}{|c|c|c|c|c|c|c|c|c|c|c|c|c|}
\hline$\overline{D A Y}$ & $\overline{\mathrm{OCT}}$ & NOV & DEC & JAN & $\overline{F E B}$ & MAR & APR & MAY & JUN & JUL & AUG & SEP \\
\hline $\begin{array}{l}1 \\
2 \\
3 \\
4 \\
5\end{array}$ & $\begin{array}{l}348 \\
348 \\
348 \\
366 \\
344\end{array}$ & $\begin{array}{r}368 \\
303 \\
303 \\
65 \\
161\end{array}$ & $\begin{array}{l}438 \\
438 \\
438 \\
411 \\
470\end{array}$ & $\begin{array}{l}206 \\
265 \\
206 \\
206 \\
206\end{array}$ & $\begin{array}{l}174 \\
174 \\
103 \\
32 \\
303\end{array}$ & $\begin{array}{l}174 \\
206 \\
206 \\
174 \\
238\end{array}$ & $\begin{array}{l}523 \\
490 \\
523 \\
652 \\
555\end{array}$ & $\begin{array}{l}5.770 \\
5.980 \\
6.050 \\
6.000 \\
5.940\end{array}$ & $\begin{array}{l}2.950 \\
2.950 \\
2.950 \\
2.950 \\
2.930\end{array}$ & $\begin{array}{l}4.520 \\
4.050 \\
3.950 \\
3.340 \\
3.020\end{array}$ & $\begin{array}{l}960 \\
928 \\
960 \\
960 \\
960\end{array}$ & $\begin{array}{l}1.760 \\
1.760 \\
1.750 \\
1.340 \\
1.220\end{array}$ \\
\hline $\begin{array}{r}6 \\
7 \\
8 \\
9 \\
10\end{array}$ & $\begin{array}{l}344 \\
376 \\
473 \\
408 \\
344\end{array}$ & $\begin{array}{l}368 \\
336 \\
303 \\
336 \\
368\end{array}$ & $\begin{array}{l}348 \\
382 \\
350 \\
172 \\
205\end{array}$ & $\begin{array}{l}206 \\
206 \\
206 \\
206 \\
206\end{array}$ & $\begin{array}{l}174 \\
205 \\
174 \\
174 \\
141\end{array}$ & $\begin{array}{l}206 \\
238 \\
206 \\
271 \\
310\end{array}$ & $\begin{array}{l}523 \\
620 \\
686 \\
801 \\
675\end{array}$ & $\begin{array}{l}5.910 \\
5.760 \\
5.610 \\
5.110 \\
4.700\end{array}$ & $\begin{array}{l}2.960 \\
3.040 \\
3.630 \\
4.090 \\
5.360\end{array}$ & $\begin{array}{l}2.490 \\
3.040 \\
3.010 \\
2.760 \\
2.030\end{array}$ & $\begin{array}{l}960 \\
928 \\
895 \\
863 \\
799\end{array}$ & $\begin{array}{l}1.020 \\
1.140 \\
1.160 \\
1.220 \\
1.310\end{array}$ \\
\hline $\begin{array}{l}11 \\
12 \\
13 \\
14 \\
15\end{array}$ & $\begin{array}{l}311 \\
311 \\
280 \\
345 \\
280\end{array}$ & $\begin{array}{l}129 \\
129 \\
369 \\
337 \\
368\end{array}$ & $\begin{array}{l}318 \\
318 \\
315 \\
228 \\
261\end{array}$ & $\begin{array}{l}206 \\
174 \\
118 \\
150 \\
206\end{array}$ & $\begin{array}{rr} & 0 \\
2 & 00 \\
1 & 03 \\
1 & 03 \\
1 & 03\end{array}$ & $\begin{array}{l}271 \\
238 \\
174 \\
206 \\
271\end{array}$ & $\begin{array}{l}443 \\
361 \\
297 \\
297 \\
496\end{array}$ & $\begin{array}{l}4.260 \\
4.140 \\
3.940 \\
3.520 \\
3.200\end{array}$ & $\begin{array}{l}6.490 \\
6.820 \\
6.990 \\
7.530 \\
7.650\end{array}$ & $\begin{array}{l}1.520 \\
1.630 \\
1.590 \\
1.860 \\
1.910\end{array}$ & $\begin{array}{l}827 \\
797 \\
673 \\
673 \\
673\end{array}$ & $\begin{array}{l}1.400 \\
1.460 \\
1.370 \\
1.360 \\
1.320\end{array}$ \\
\hline $\begin{array}{l}16 \\
17 \\
18 \\
19 \\
20\end{array}$ & $\begin{array}{l}312 \\
312 \\
491 \\
281 \\
249\end{array}$ & $\begin{array}{l}368 \\
368 \\
368 \\
534 \\
388\end{array}$ & $\begin{array}{l}241 \\
176 \\
261 \\
149 \\
117\end{array}$ & $\begin{array}{l}174 \\
131 \\
271 \\
174 \\
118\end{array}$ & $\begin{array}{l}103 \\
168 \\
103 \\
103 \\
135\end{array}$ & $\begin{array}{l}206 \\
206 \\
238 \\
421 \\
539\end{array}$ & $\begin{array}{r}464 \\
525 \\
631 \\
857 \\
1.320\end{array}$ & $\begin{array}{l}3.190 \\
2.800 \\
3.200 \\
2.860 \\
2.840\end{array}$ & $\begin{array}{l}7.860 \\
8.030 \\
7.980 \\
7.920 \\
7.920\end{array}$ & $\begin{array}{l}1.870 \\
2.020 \\
1.720 \\
1.860 \\
1.790\end{array}$ & $\begin{array}{l}570 \\
295 \\
226 \\
397 \\
397\end{array}$ & $\begin{array}{l}1.220 \\
1.220 \\
1.330 \\
1.400 \\
1.220\end{array}$ \\
\hline $\begin{array}{l}21 \\
22 \\
23 \\
24 \\
25\end{array}$ & $\begin{array}{l}313 \\
281 \\
352 \\
337 \\
304\end{array}$ & $\begin{array}{l}420 \\
508 \\
271 \\
420 \\
388\end{array}$ & $\begin{array}{r}182 \\
285 \\
149 \\
124 \\
91\end{array}$ & $\begin{array}{r}59 \\
238 \\
174 \\
206 \\
174\end{array}$ & $\begin{array}{r}135 \\
134 \\
276 \\
71 \\
103\end{array}$ & $\begin{array}{l}569 \\
536 \\
536 \\
536 \\
503\end{array}$ & $\begin{array}{l}1.800 \\
2.060 \\
3.320 \\
3.500 \\
3.640\end{array}$ & $\begin{array}{l}2.870 \\
2.910 \\
3.070 \\
2.980 \\
3.000\end{array}$ & $\begin{array}{l}7.470 \\
7.240 \\
7.180 \\
6.670 \\
6.320\end{array}$ & $\begin{array}{l}1.900 \\
1.840 \\
1.910 \\
1.910 \\
1.770\end{array}$ & $\begin{array}{r}397 \\
487 \\
659 \\
831 \\
1020\end{array}$ & $\begin{array}{r}1.060 \\
1.020 \\
960 \\
992 \\
1.020\end{array}$ \\
\hline $\begin{array}{l}26 \\
27 \\
28 \\
29 \\
30 \\
31\end{array}$ & $\begin{array}{r}337 \\
336 \\
97 \\
220 \\
401 \\
336\end{array}$ & $\begin{array}{r}355 \\
495 \\
284 \\
419 \\
406 \\
-\end{array}$ & $\begin{array}{l}221 \\
183 \\
118 \\
242 \\
206 \\
206\end{array}$ & $\begin{array}{r}206 \\
71 \\
32 \\
271 \\
206 \\
190\end{array}$ & $\begin{array}{r}206 \\
206 \\
174 \\
206 \\
-\end{array}$ & $\begin{array}{l}536 \\
598 \\
566 \\
613 \\
491 \\
490\end{array}$ & $\begin{array}{r}4.100 \\
4.530 \\
4.970 \\
5.180 \\
5.370 \\
- \\
----\end{array}$ & $\begin{array}{l}3.000 \\
3.060 \\
2.990 \\
2.980 \\
2.990 \\
2.920\end{array}$ & $\begin{array}{r}5.090 \\
5.280 \\
4.850 \\
5.090 \\
4.640 \\
-\end{array}$ & $\begin{array}{l}1.800 \\
1480 \\
1560 \\
1.380 \\
1040 \\
1080\end{array}$ & $\begin{array}{l}1210 \\
1.150 \\
1.120 \\
1.480 \\
1600 \\
1570\end{array}$ & $\begin{array}{r}1020 \\
992 \\
960 \\
960 \\
960 \\
---\end{array}$ \\
\hline $\begin{array}{l}\text { Total } \\
\text { Mean } \\
\quad(f) \\
\text { Mean } \neq \\
\text { Max } \\
\text { Min } \\
\text { Cfsm } \neq \\
\text { In. } \neq\end{array}$ & $\begin{array}{r}10.185 \\
329 \\
-87 \\
242 \\
491 \\
97 \\
0.202 \\
0.23\end{array}$ & $\begin{array}{r}10.235 \\
341 \\
-48 \\
293 \\
534 \\
65 \\
0.244 \\
0.27\end{array}$ & $\begin{array}{r}8043 \\
259 \\
-8 \\
251 \\
470 \\
91 \\
0.209 \\
0.24\end{array}$ & $\begin{array}{r}5668 \\
183 \\
-23 \\
160 \\
271 \\
32 \\
0.133 \\
0.15\end{array}$ & $\begin{array}{r}4.286 \\
148 \\
-29 \\
119 \\
303 \\
0 \\
0.099 \\
0.11\end{array}$ & $\begin{array}{r}10973 \\
354 \\
-133 \\
221 \\
613 \\
174 \\
0.184 \\
0.21\end{array}$ & $\begin{array}{r}50.209 \\
1,674 \\
+460 \\
2,134 \\
5,370 \\
297 \\
1.78 \\
1.98\end{array}$ & $\begin{array}{r}123.550 \\
3,985 \\
+65 \\
4,050 \\
6,050 \\
2,800 \\
3.38 \\
3.89\end{array}$ & $\begin{array}{r}169,830 \\
5,661 \\
-47 \\
5,614 \\
8,030 \\
2,930 \\
4.68 \\
5.22\end{array}$ & $\begin{array}{r}67.650 \\
2,182 \\
+91 \\
2,273 \\
4,520 \\
1,040 \\
1.89 \\
2.18\end{array}$ & $\begin{array}{r}26.265 \\
847 \\
+26 \\
873 \\
1,600 \\
226 \\
0.728 \\
0.84\end{array}$ & $\begin{array}{r}36.924 \\
1,231 \\
-69 \\
1,162 \\
1,760 \\
960 \\
0.968 \\
1.08\end{array}$ \\
\hline $\begin{array}{l}\text { Cal yr } \\
\text { wtr yr }\end{array}$ & $\begin{array}{l}\text { 67: } \mathrm{T} \\
\text { 68: TC }\end{array}$ & 523 & $\begin{array}{l}\text { Max } \\
\operatorname{Max}\end{array}$ & $\begin{array}{l}240 \\
30\end{array}$ & $\begin{array}{l}0 \\
0\end{array}$ & $\begin{array}{r}870 \\
1,431\end{array}$ & $\begin{array}{l}\text { Mean } \neq \\
\text { Mean } \neq\end{array}$ & $\begin{array}{l}866 \\
447\end{array}$ & $\neq 1.21$ & $\begin{array}{l}\text { In. } \neq \\
\text { In. } \neq 1\end{array}$ & & \\
\hline
\end{tabular}

f Change in contents, equivalent in cubic feet per second, in Camp six, Bald Eagle, Gabbro, Little Gabbro, Birch, White Iron, South Farm, Farm and Garden Lakes.

$\neq$ Adjusted for change in reservoir contents. 
5-1272.05 Burntside River near Ely, Minn.

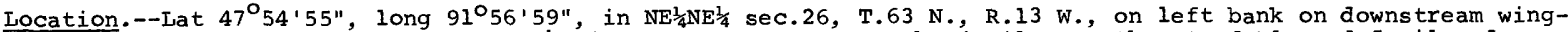
wall of bridge on County Road $88,2 \frac{1}{2}$ miles upstream from mouth, 4 miles northwest of $E l y$ and 5 miles downstream from outlet on Burntside Lake.

Records available.--May 1967 to September 1968.

Gage.--Staff gage read once daily. Altitude of gage is $1,340 \mathrm{ft}$ (from topographic map).

Extremes.--Maximum discharge during year, $300 \mathrm{cfs}$ June 14 (gage height, 7.85 ft, from graph based on gage readings); minimum daily, $0.8 \mathrm{cfs}$ Oct. 6,7

1967-68: Maximum discharge, that of June 14, 1968; minimum daily discharge, that of Oct. 6, 7 , 1967.

Remarks.--Records good except those for winter months, which are fair.

Cooperation.--Gage readings furnished by the Federal Water Pollution Control Administration.

DISCHARGE, IN CFS, WATER YEAR OCTOBER 1967 TO SEPTEMBER 1968

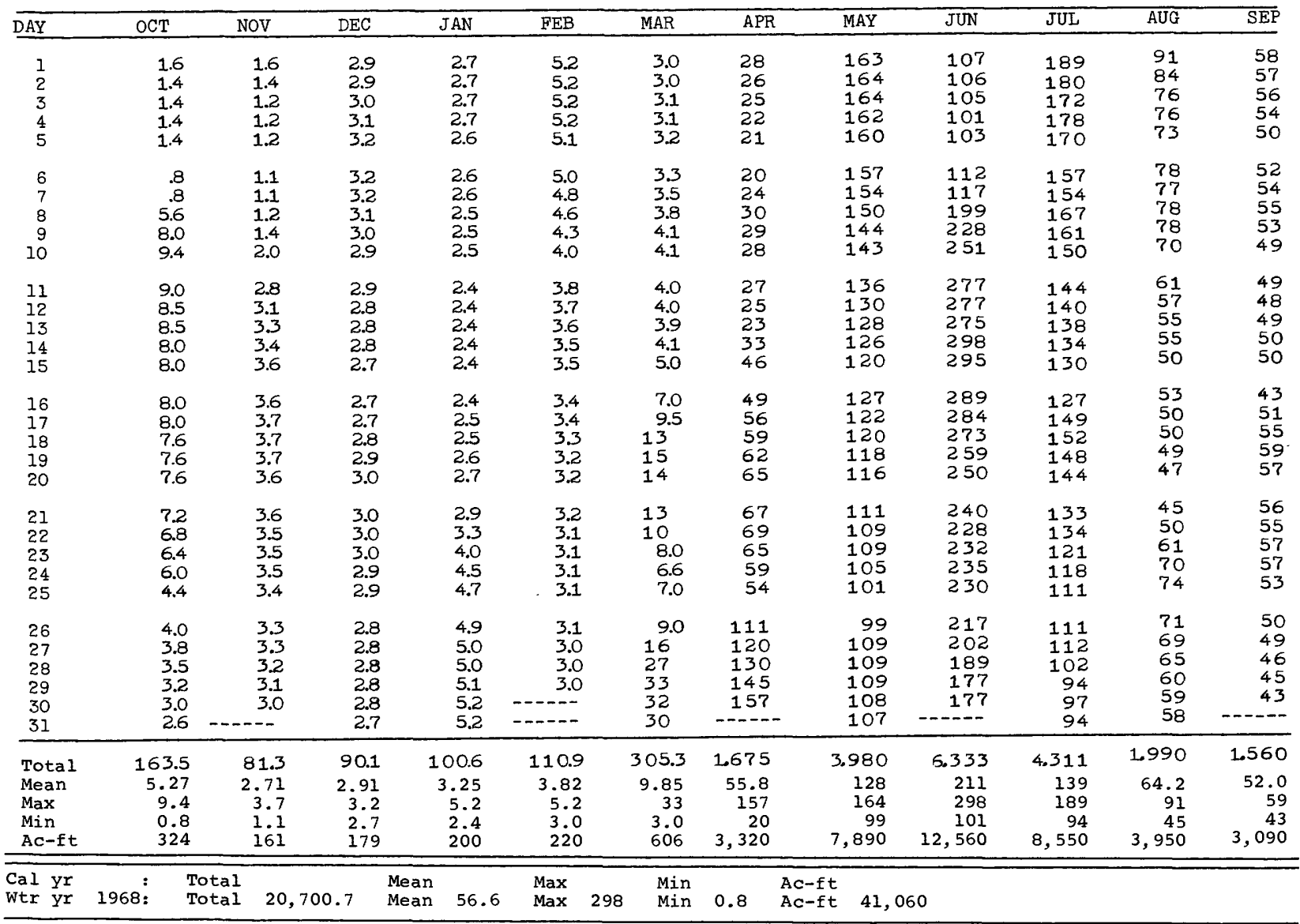


5-1272.10 Armstrong Creek near Ely, Minn.

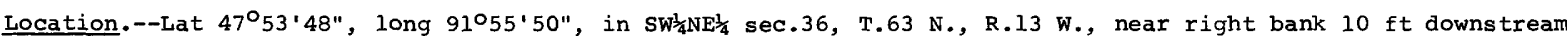
from culvert on County Road $88,1 \frac{1}{4}$ miles upstream from mouth and $2 \frac{1}{2}$ miles southwest of Ely.

Records available.--May 1967 to september 1968.

Gage.--Staff gage read once daily. Altitude of gage is 1,365 ft (from topographic map).

Extremes.--Maximum discharge during year, 41 cfs June 9 (gage height 4.51 ft, from floodmark); minimum, no flow Feb. 28 to Mar. 6 .

1967-68: Maximum discharge, that of June 9, 1968; minimum, that of Feb. 28 to Mar. 6, 1968.

Remarks.--Records fair.

Cooperation.--Gage readings furnished by Federal Water Pollution Control Administration.

DISCHARGE, IN CUBIC FEET PER SECOND, WATER YEAR OCTOBER 1967 TO SEPTEMBER 1968

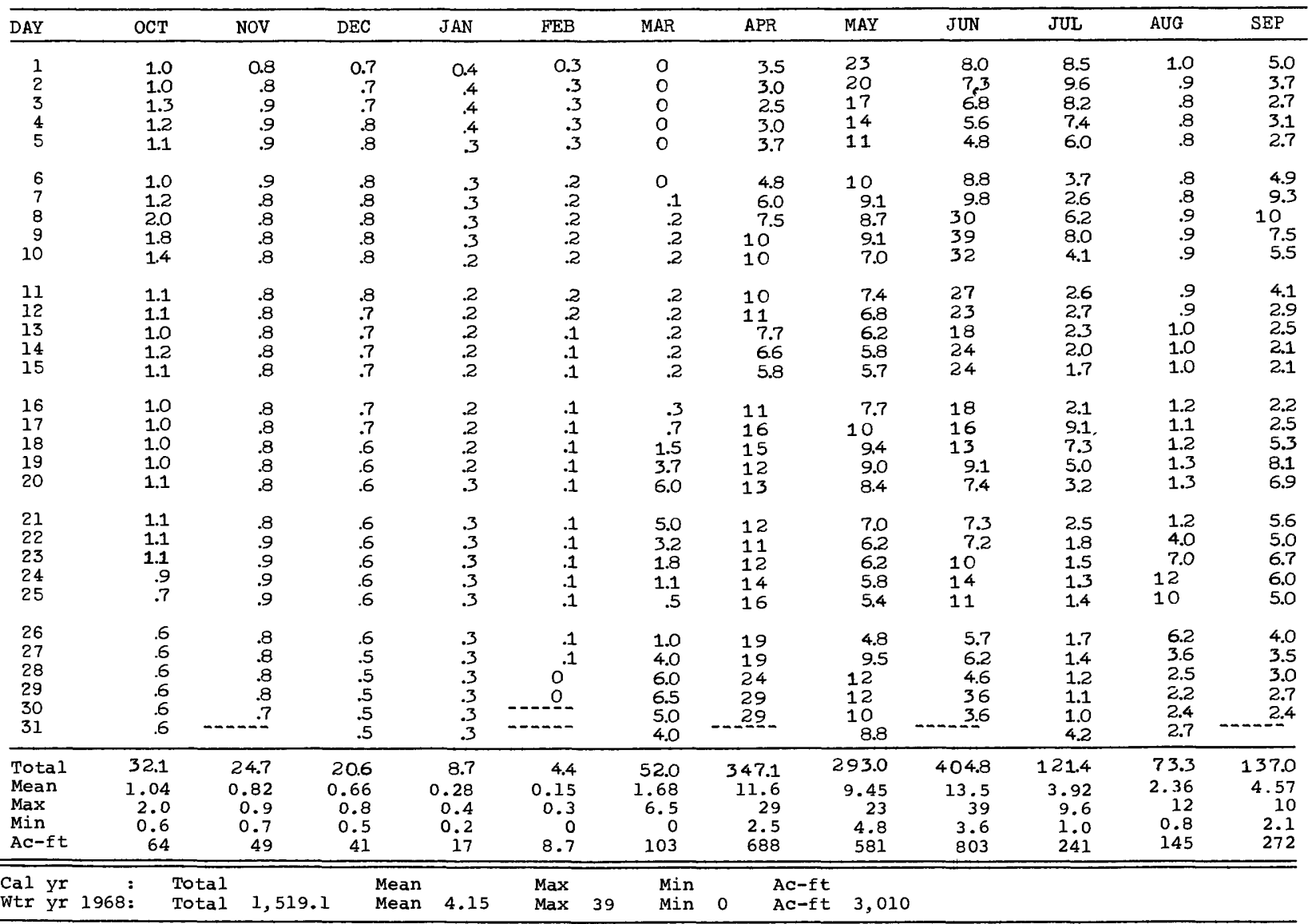


5-1272.15 Longstorff Creek near Ely, Minn.

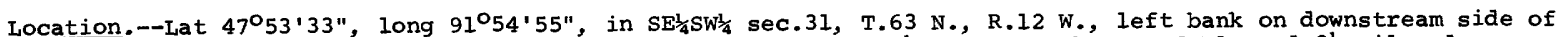
culvert of U.S. Highway $169,0.7$ mile upstream from mouth, $1 \frac{1}{2}$ miles southwest of Ely and $2 \frac{1}{2}$ miles downstream from outlet of Mitchell Lake.

Records available.--May 1967 to September 1968.

Gage.--Staff gage read once daily. Datum of gage is $1,360.67 \mathrm{ft}$ above mean sea level, datum of 1929 (levels by Minnesota Highway Department).

Extremes.--Maximum discharge during year, $111 \mathrm{cfs} J u n e ~ 8$ (gage height $4.34 \mathrm{ft}$, from floodmark) no flow on many days.

1967-68: Maximum discharge, that of June 8,1968 ; no flow on many days in 1968.

Remarks. --Records good.

Cooperation.--Gage readings furnished by the Federal water Pollution Control Administration.

DISCHARGE, IN CFS, WATER YEAR OCTOBER 1967 TO SEPTEMBER 1968

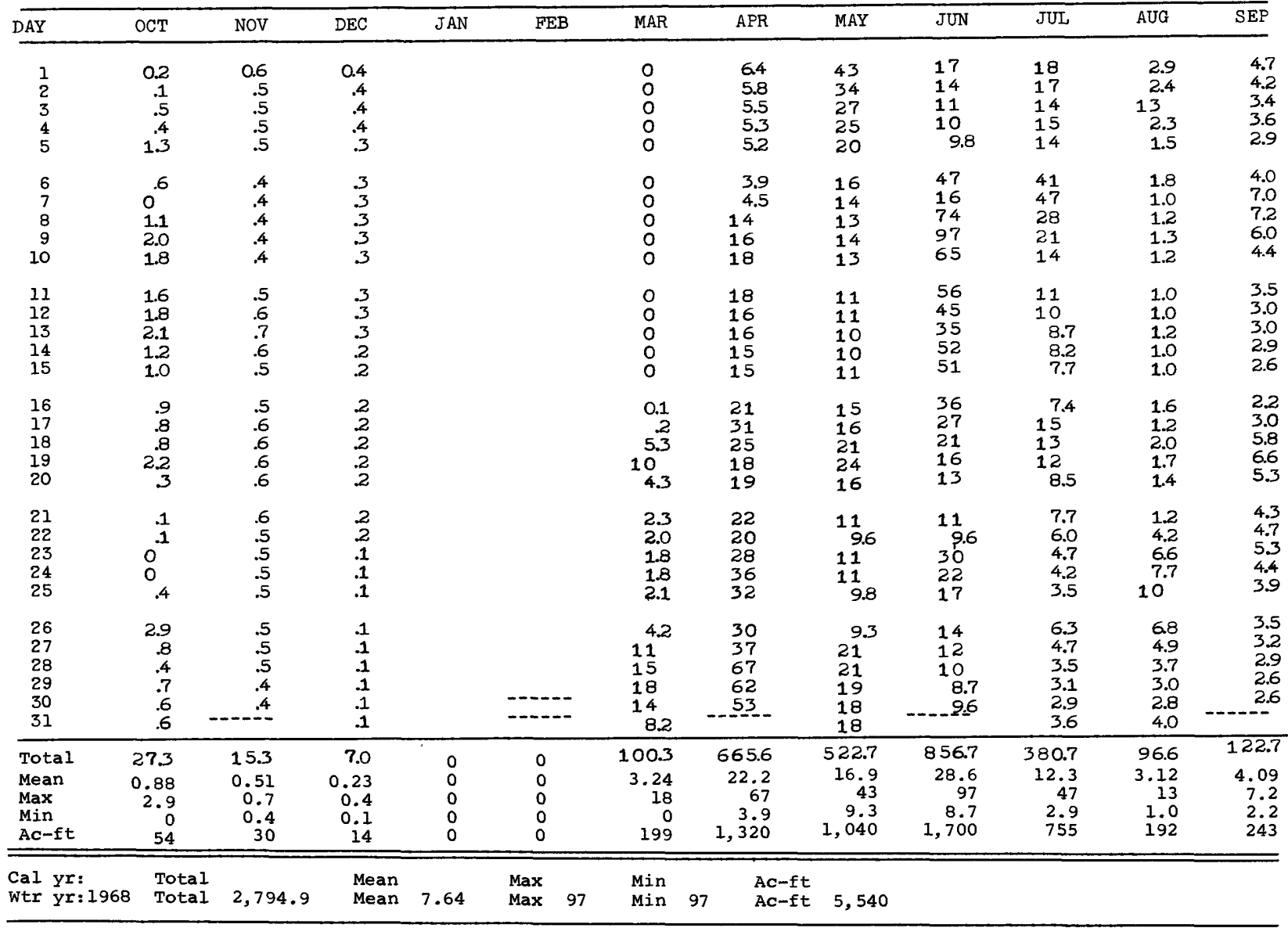


5-1272.20 Burgo Creek near Ely, Minn.

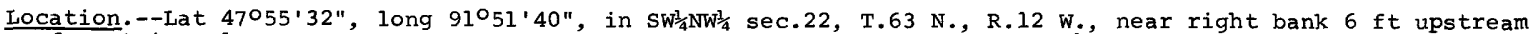
from twin culverts on County Road $88,0.5 \mathrm{mile}$ upstream from mouth and lis miles north of Ely.

Records available. --May 1967 to september 1968.

Gage.--Staff gage and crest-stage gage; gage read once daily. Altitude of gage is 1 , 340 ft (from topographic map).

Extremes.--Maximum discharge during year, 139 cfs June 8 (gage height 9.77 ft), from rating curve extended above $35 \mathrm{cfs}$; no flow on many days.

1967-68: Maximum discharge, that of June 8, 1968; no flow at times each year.

Remarks.--Records fair.

Cooperation.--Gage readings furnished by Federal water Pollution Control Administration.

DISCHARGE, IN CUBIC FEET PER SECOND, WATER YEAR OCTOBER 1967 TO SEPTEMBER 1968

\begin{tabular}{|c|c|c|c|c|c|c|c|c|c|c|c|c|}
\hline DAY & OCT & NOV & $\overline{D E C}$ & JAN & FEB & MAR & APR & MAY & JUN & JUL & AUG & SEP \\
\hline $\begin{array}{l}1 \\
2 \\
3 \\
4 \\
5\end{array}$ & $\begin{array}{r}0.1 \\
.1 \\
.1 \\
.1 \\
.1\end{array}$ & $\begin{array}{r}0.3 \\
.2 \\
.2 \\
.3 \\
.3\end{array}$ & $\begin{array}{r}0.4 \\
.2 \\
.3 \\
.3 \\
.3\end{array}$ & $\begin{array}{l}0.1 \\
.1 \\
.1 \\
0 \\
0\end{array}$ & $\begin{array}{r}0.4 \\
.4 \\
.4 \\
.4 \\
.4\end{array}$ & $\begin{array}{l}0 \\
0 \\
0 \\
0 \\
0\end{array}$ & $\begin{array}{l}6.3 \\
6.0 \\
5.8 \\
5.2 \\
5.0\end{array}$ & $\begin{array}{c}21 \\
16 \\
11 \\
9.6 \\
8.4\end{array}$ & $\begin{array}{l}4.2 \\
3.4 \\
2.8 \\
2.4 \\
2.7\end{array}$ & $\begin{array}{l}4.7 \\
3.6 \\
2.5 \\
2.7 \\
2.0\end{array}$ & $\begin{array}{r}0.8 \\
.6 \\
.5 \\
.5 \\
.5\end{array}$ & $\begin{array}{r}1.9 \\
1.2 \\
1.0 \\
1.1 \\
.8\end{array}$ \\
\hline $\begin{array}{r}6 \\
7 \\
8 \\
9 \\
10\end{array}$ & $\begin{array}{l}.1 \\
.1 \\
.5 \\
.5 \\
.4\end{array}$ & $\begin{array}{l}.3 \\
.2 \\
.2 \\
.2 \\
.3\end{array}$ & $\begin{array}{l}.3 \\
.5 \\
.3 \\
.3 \\
.2\end{array}$ & $\begin{array}{l}0 \\
0 \\
0 \\
0 \\
0\end{array}$ & $\begin{array}{l}.4 \\
.3 \\
.3 \\
.2 \\
.2\end{array}$ & $\begin{array}{l}0 \\
0 \\
0 \\
0 \\
0\end{array}$ & $\begin{array}{l}7.5 \\
7.2 \\
15 \\
24 \\
23\end{array}$ & $\begin{array}{l}7.2 \\
7.2 \\
6.5 \\
5.5 \\
4.7\end{array}$ & $\begin{array}{l}6.1 \\
6.3 \\
64 \\
38 \\
24\end{array}$ & $\begin{array}{r}1.5 \\
1.8 \\
11 \\
9.3 \\
5.3\end{array}$ & $\begin{array}{l}.6 \\
.5 \\
.4 \\
.3 \\
.2\end{array}$ & $\begin{array}{l}2.3 \\
2.6 \\
2.4 \\
2.0 \\
1.6\end{array}$ \\
\hline $\begin{array}{l}11 \\
12 \\
13 \\
14 \\
15\end{array}$ & $\begin{array}{l}.3 \\
.3 \\
.4 \\
.7 \\
.5\end{array}$ & $\begin{array}{l}.4 \\
.3 \\
.2 \\
.2 \\
.3\end{array}$ & $\begin{array}{l}.2 \\
.2 \\
.2 \\
.2 \\
.2\end{array}$ & $\begin{array}{l}0 \\
0 \\
0 \\
0 \\
0\end{array}$ & $\begin{array}{l}.2 \\
.2 \\
.2 \\
.2 \\
.2\end{array}$ & $\begin{array}{l}0 \\
0 \\
0 \\
.1 \\
.1\end{array}$ & $\begin{array}{c}18 \\
15 \\
12 \\
11 \\
8.9\end{array}$ & $\begin{array}{l}3.9 \\
3.7 \\
3.4 \\
3.2 \\
2.9\end{array}$ & $\begin{array}{l}26 \\
20 \\
18 \\
28 \\
22\end{array}$ & $\begin{array}{l}1.7 \\
3.2 \\
2.9 \\
2.8 \\
2.7\end{array}$ & $\begin{array}{l}.1 \\
.4 \\
.2 \\
.1 \\
.1\end{array}$ & $\begin{array}{r}1.4 \\
1.1 \\
.9 \\
.8 \\
.7\end{array}$ \\
\hline $\begin{array}{l}16 \\
17 \\
18 \\
19 \\
20\end{array}$ & $\begin{array}{l}.3 \\
.5 \\
.9 \\
.6 \\
.6\end{array}$ & $\begin{array}{l}.4 \\
.5 \\
.5 \\
.5 \\
.5\end{array}$ & $\begin{array}{l}.3 \\
.3 \\
.3 \\
.3 \\
.5\end{array}$ & $\begin{array}{l}0 \\
0 \\
0 \\
.1 \\
.1\end{array}$ & $\begin{array}{l}.1 \\
.1 \\
.1 \\
.1 \\
.1\end{array}$ & 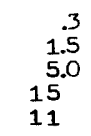 & $\begin{array}{l}17 \\
22 \\
18 \\
13 \\
10\end{array}$ & $\begin{array}{r}7.4 \\
8.0 \\
13 \\
9.0 \\
5.5\end{array}$ & $\begin{array}{c}15 \\
11 \\
8.0 \\
5.3 \\
3.2\end{array}$ & $\begin{array}{l}4.1 \\
17 \\
12 \\
7.6 \\
2.0\end{array}$ & $\begin{array}{l}.2 \\
.1 \\
.1 \\
.2 \\
.2\end{array}$ & $\begin{array}{r}.6 \\
.7 \\
1.1 \\
1.5 \\
1.1\end{array}$ \\
\hline $\begin{array}{l}21 \\
22 \\
23 \\
24 \\
25\end{array}$ & $\begin{array}{l}.6 \\
.5 \\
.5 \\
.5 \\
.6\end{array}$ & $\begin{array}{l}.6 \\
.8 \\
.8 \\
.8 \\
.8\end{array}$ & $\begin{array}{l}.3 \\
.3 \\
.3 \\
.3 \\
.2\end{array}$ & $\begin{array}{l}.2 \\
.3 \\
.4 \\
.4 \\
.3\end{array}$ & $\begin{array}{l}.1 \\
.1 \\
.1 \\
.1 \\
.1\end{array}$ & $\begin{array}{l}8.5 \\
6.0 \\
4.0 \\
2.8 \\
3.0\end{array}$ & $\begin{array}{l}10 \\
11 \\
12 \\
13 \\
14\end{array}$ & $\begin{array}{l}3.6 \\
2.9 \\
2.7 \\
2.2 \\
2.1\end{array}$ & $\begin{array}{l}2.8 \\
2.5 \\
2.2 \\
4.9 \\
3.3\end{array}$ & $\begin{array}{l}3.0 \\
2.3 \\
2.0 \\
1.6 \\
1.3\end{array}$ & $\begin{array}{r}.2 \\
.3 \\
.9 \\
1.7 \\
2.1\end{array}$ & $\begin{array}{l}.8 \\
1.0 \\
1.4 \\
1.2 \\
1.0\end{array}$ \\
\hline $\begin{array}{l}26 \\
27 \\
28 \\
29 \\
30 \\
31\end{array}$ & $\begin{array}{l}.4 \\
.4 \\
.4 \\
.3 \\
.3 \\
.3\end{array}$ & $\begin{array}{r}.8 \\
.6 \\
.6 \\
.6 \\
.6 \\
---\end{array}$ & $\begin{array}{l}.1 \\
.1 \\
.1 \\
.1 \\
.1 \\
.1\end{array}$ & $\begin{array}{l}.3 \\
.3 \\
.3 \\
.3 \\
.3 \\
.4\end{array}$ & $0_{0}^{.1}$ & $\begin{array}{l}9.0 \\
12 \\
11 \\
9.0 \\
7.5 \\
7.0\end{array}$ & $\begin{array}{r}15 \\
21 \\
28 \\
39 \\
29 \\
\end{array}$ & $\begin{array}{l}1.8 \\
5.5 \\
6.7 \\
7.6 \\
6.4 \\
5.1\end{array}$ & $\begin{array}{r}2.5 \\
2.2 \\
1.6 \\
1.6 \\
1.8\end{array}$ & $\begin{array}{r}1.7 \\
1.2 \\
.9 \\
.8 \\
.6 \\
.9\end{array}$ & $\begin{array}{r}1.4 \\
1.0 \\
2.8 \\
.7 \\
1.0 \\
1.6\end{array}$ & $\begin{array}{r}1.0 \\
1.0 \\
.9 \\
.6 \\
.6\end{array}$ \\
\hline $\begin{array}{l}\text { Total } \\
\text { Mean } \\
\text { Max } \\
\text { Min } \\
\text { Ac-ft }\end{array}$ & $\begin{array}{r}12.0 \\
0.39 \\
0.9 \\
0.1 \\
24\end{array}$ & $\begin{array}{r}13.3 \\
0.44 \\
0.8 \\
0.2 \\
26\end{array}$ & $\begin{array}{r}7.8 \\
0.25 \\
0.5 \\
0.1 \\
15\end{array}$ & $\begin{array}{r}4.0 \\
0.13 \\
0.4 \\
0 \\
7.9\end{array}$ & $\begin{array}{r}56 \\
0.19 \\
0.4 \\
0 \\
11\end{array}$ & $\begin{array}{r}112.8 \\
3.64 \\
15 \\
0 \\
224\end{array}$ & $\begin{array}{r}441.9 \\
14.7 \\
39 \\
5.0 \\
876\end{array}$ & $\begin{array}{r}203.7 \\
6.57 \\
21 \\
1.8 \\
404\end{array}$ & $\begin{array}{r}335.8 \\
11.2 \\
64 \\
1.6 \\
666\end{array}$ & $\begin{array}{r}116.7 \\
3.76 \\
17 \\
0.6 \\
231\end{array}$ & $\begin{array}{r}20.3 \\
0.65 \\
2.8 \\
0.1 \\
40\end{array}$ & $\begin{array}{r}36.3 \\
1.21 \\
2.6 \\
0.6 \\
72\end{array}$ \\
\hline
\end{tabular}

$\begin{array}{llllllll}\text { Cal yr } & \text { Total } & \text { Mean } & \text { Max } & \text { Min } & \text { Ac-ft } \\ \text { Wtr yr 1968: } & \text { Total 1,310.2 } & \text { Mean 3.58 } & \text { Max } 64 & \text { Min } 0 & \text { Ac-ft } 2,600\end{array}$ 
5-1272.25 Shagawa Lake at Ely, Minn.

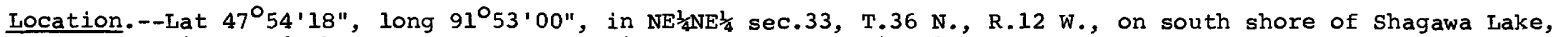
on east pier of dock at U.S. Forest Service Seaplane Base in Ely.

Records available.--April 1967 to September 1968. April 1962 to July 1966 (fragmentary) in files of Minnesota Department of Conservation, Division of Waters, Soils and Minerals.

Gage.--Staff gage read once daily. Datum of gage is 1,330.00 ft above mean sea level, adjustment of 1929 (levels by Minnesota Department of Conservation). Gage readings have been reduced to elevations above mean sea level.

Extremes.--Maximum elevation observed during year, 1,339.55 ft June 16; minimum observed, 1336.91 ft Feb. 26. 1967-68: Maximum elevation observed, that of June 16, 1968; minimum observed, that of Feb. 26, 1968. Maximum elevation observed April 1962 to July 1966, 1,339.95 ft April 27, 28, 1966, from Minnesota Department of Conservation, Division of Waters, Soils and Minerals.

Mean daily elevation, in feet, water year october 1967 to september 1968

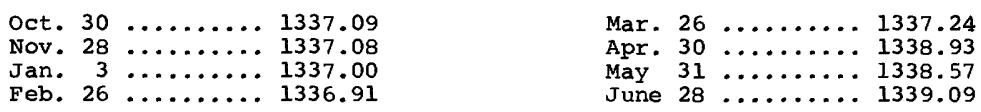

July $29 \ldots \ldots \ldots 1338.37$

Aug. $30 \ldots \ldots \ldots .1337 .89$

Sept. $30 \ldots \ldots \ldots \ldots 1337.81$

Note.--Elevations other than those shown are available. 
5-1272.30 Shagawa River at Ely, Minn.

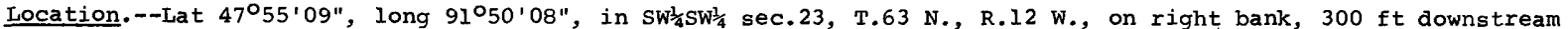
from outlet of Shagawa Lake, $150 \mathrm{ft}$ north of the viliage limits of Ely, $\frac{3}{4}$ mile upstream from County Road 88 and 3 miles upstream from Fall Lake.

Records available.--May 1967 to September 1968.

Gage.--Water-stage recorder. Altitude of gage is 1,335 ft (from topographic map). Prior to Aug. 2, 1967, staff gage at same site and datum.

Extremes.--Maximum discharge during year, 385 cfs June 15 (gage height, 6.33 ft); minimum, 6.2 cfs Mar. 4 (gage height, $3.74 \mathrm{ft}$ ).

1967-68: Maximum discharge, that of June 15, 1968; minimum, that of Mar. 4, 1968.

Remarks.--Records good.

DISCHARGE, IN CUBIC FEET PER SECOND, WATER YEAR OCTOBER 1967 TO SEPTEMMER 1968

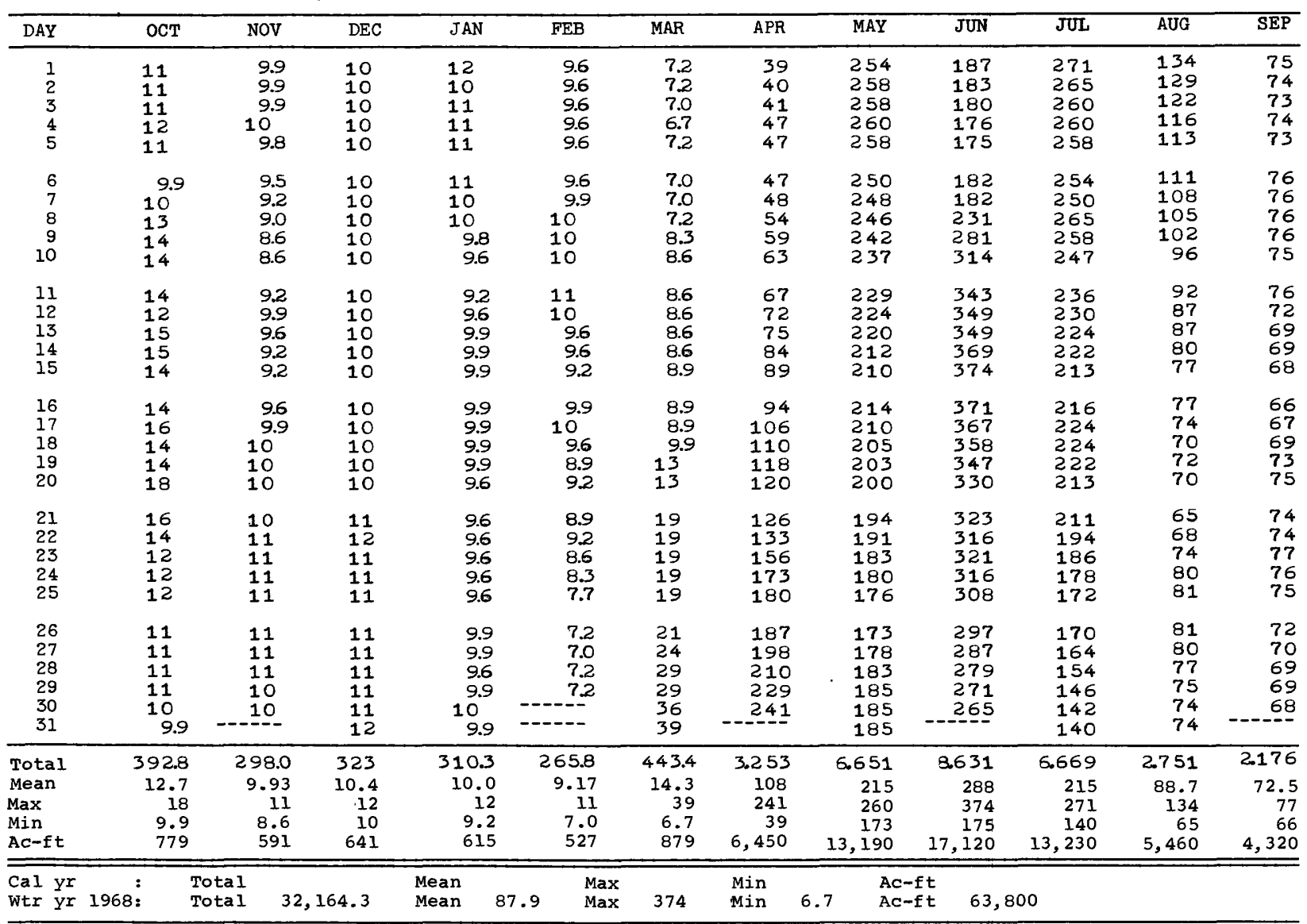


5-1275. Basswood River near Winton, Minn.

(International gaging station)

Location.--Lat 48004'55", long 91\%39'10", in sec.30, T.65 N., R.10 W., on Jackfish Bay of Basswood Lake, used to determine discharge at outlet (lat $48^{\circ} 06^{\prime}$, long $91^{\circ} 39^{\circ}$, in sec. 19, T.65 N., R.10 W., on international boundary 14 miles northeast of winton).

Drainage area.--1,740 sq mi, approximately (above outlet of Basswood Lake).

Records available.--March to June 1924, September 1925 to March 1928, January 1930 to September 1968. Monthly discharge only for some periods, published in wSP 1308.

Gage.--Digital water-stage recorder. Datum of gage is 1,296.80 ft (revised) above mean sea level, adjustment of 1928, by Geodetic Survey of Canada. Prior to June 2, 1938, staff gages at several sites on Jackfish Bay, at datum $3.0 \mathrm{ft}$ higher. June 2 to oct. 27 , 1938 , staff gage at Williams Island half a mile northeast of present gage at datum $3.0 \mathrm{ft}$ higher. Oct. 28, 1938 to sept. 30, 1966, graphic water-stage recorder at datum $3.0 \mathrm{ft}$ higher and Oct. 1, 1966 to June 28, 1967, graphic water-stage recorder at present datum.

Average discharge.--40 years $(1925-27,1930-68), 1,330$ cfs.

Extremes.--Maximum discharge during year, 9,040 cfs June 23 (gage height, 7.73 ft); minimum, 257 cfs Mar. 2 , $3,4,5$ (gage height, $2.41 \mathrm{ft}$ ).

1924, 1925-27, 1930-68: Maximum discharge, 15,600 cfs May 24, 1950 (gage height, 9.94 ft, present

datum); minimum, 73 cfs Dec. 5, 1948

Remarks.--Records excellent. Flow affected by storage on Kawishiwi River.

Cooperation.--This station is maintained by the United states under agreement with Canada.

DISCHARGE, IN CUBIC FEET PER SECOND, WATER YEAR OCTOBER 1967 TO SEPTEMBER 1968

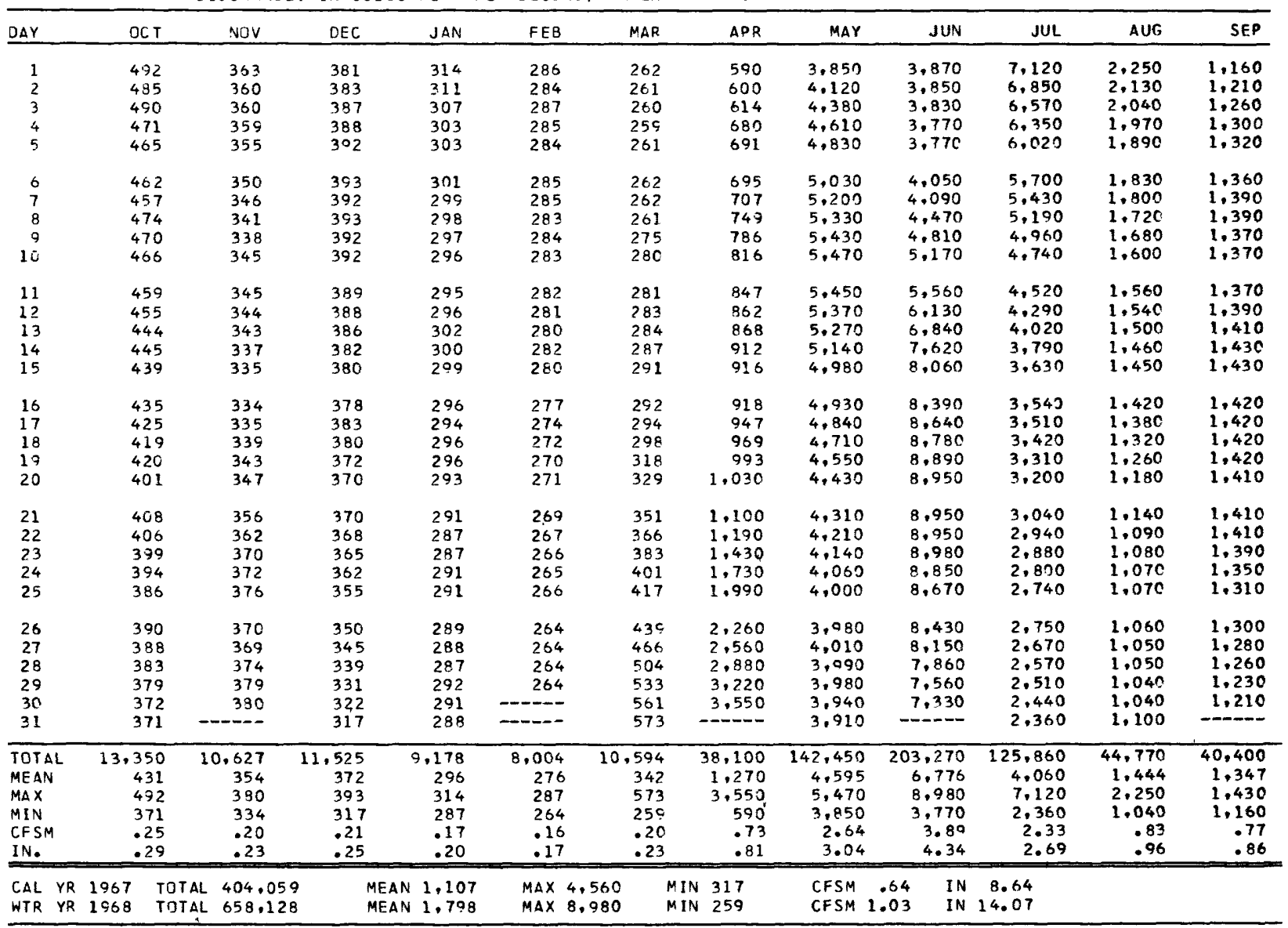




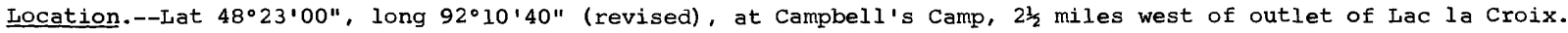
Drainage area.--5,170 sq $\mathrm{mi}$ (revised).

Records available.--September 1921 to January 1922, April 1922 to September 1968, in reports of Geological Survey. Monthly discharge only for some periods, published in wSP 1308 . August 1921 to September 1968 in reports of Inland Waters Branch, Department of Energy, Mines and Resources, Canada.

Gage.--Water-stage recorder. Gage readings have been reduced to elevations above mean sea level, United states and Canada Boundary Survey datum. Prior to October 1933, staff gages at various sites on Lac la Croix. October 1933 to March 13, 1963, staff gage at present site and datum.

Average discharge.--46 years $(1922-68), 3,644$ cfs.

Extremes.--Maximum discharge during year, 20,000 cfs June 26 (elevation, 1,190.83 ft); minimum, 843 cfs Mar. 4 , 5,8 (elevation, $1,182.01 \mathrm{ft}$ ).

1921-68: Maximum discharge, 28,200 cfs May 31 to June 2, 1950 (elevation, 1,193.30 ft); minimum, 535 cfs at times in February, March and April 1924 (elevation, 1,181.50 ft).

Remarks.--Records excellent.

Cooperation.--This station is maintained by Canada under agreement with the United States.

DISCHARGE, IN CFS, WATER YEAR OCTOBER 1967 TO SEPTEMBER 1968

\begin{tabular}{|c|c|c|c|c|c|c|c|c|c|c|c|c|}
\hline DAY & $\mathrm{OCT}$ & NOV & $\mathrm{DEC}$ & JAN & FEB & MAR & APR & MAY & JUN & JUL & AUG & SEP \\
\hline $\begin{array}{l}1 \\
2 \\
3 \\
4 \\
5\end{array}$ & $\begin{array}{l}1,980 \\
1,950 \\
1,980 \\
1,900 \\
1,870\end{array}$ & $\begin{array}{l}1,440 \\
1,410 \\
1,390 \\
1,380 \\
1,380\end{array}$ & $\begin{array}{l}1,140 \\
1,130 \\
1,130 \\
1,130 \\
1,120\end{array}$ & $\begin{array}{l}1,120 \\
1,110 \\
1,110 \\
1,100 \\
1,100\end{array}$ & $\begin{array}{l}999 \\
999 \\
990 \\
982 \\
974\end{array}$ & $\begin{array}{l}858 \\
850 \\
850 \\
843 \\
843\end{array}$ & $\begin{array}{l}1,150 \\
1,180 \\
1,200 \\
1,270 \\
1,310\end{array}$ & $\begin{array}{l}5,820 \\
6,190 \\
6,610 \\
7,030 \\
7,350\end{array}$ & $\begin{array}{l}9,890 \\
9,790 \\
9,760 \\
9,660 \\
9,610\end{array}$ & $\begin{array}{l}19,800 \\
19,700 \\
19,500 \\
19,400 \\
19,100\end{array}$ & $\begin{array}{l}11,600 \\
11,300 \\
11,100 \\
10,800 \\
10,500\end{array}$ & $\begin{array}{l}5,360 \\
5,290 \\
5,190 \\
5,110 \\
5,050\end{array}$ \\
\hline $\begin{array}{r}6 \\
7 \\
8 \\
9 \\
10\end{array}$ & $\begin{array}{l}1,880 \\
1,870 \\
1,900 \\
1,880 \\
1,870\end{array}$ & $\begin{array}{l}1,370 \\
1,350 \\
1,300 \\
1,300 \\
1,310\end{array}$ & $\begin{array}{l}1,120 \\
1,110 \\
1,100 \\
1,100 \\
1,090\end{array}$ & $\begin{array}{l}1,100 \\
1,100 \\
1,100 \\
1,100 \\
1,100\end{array}$ & $\begin{array}{l}982 \\
982 \\
974 \\
966 \\
958\end{array}$ & $\begin{array}{l}850 \\
850 \\
843 \\
873 \\
888\end{array}$ & $\begin{array}{l}1,360 \\
1,440 \\
1,530 \\
1,630 \\
1,720\end{array}$ & $\begin{array}{l}7,690 \\
7,990 \\
8,290 \\
8,590 \\
9,000\end{array}$ & $\begin{array}{r}9,890 \\
10,100 \\
10,800 \\
11,700 \\
12,500\end{array}$ & $\begin{array}{l}18,800 \\
18,400 \\
18,000 \\
17,600 \\
17,200\end{array}$ & $\begin{array}{r}10,200 \\
9,810 \\
9,510 \\
9,140 \\
8,850\end{array}$ & $\begin{array}{l}5,050 \\
4,970 \\
4,870 \\
4,830 \\
4,770\end{array}$ \\
\hline $\begin{array}{l}11 \\
12 \\
13 \\
14 \\
15\end{array}$ & $\begin{array}{l}1,860 \\
1,840 \\
1,800 \\
1,800 \\
1,770\end{array}$ & $\begin{array}{l}1,290 \\
1,250 \\
1,240 \\
1,240 \\
1,240\end{array}$ & $\begin{array}{l}1,100 \\
1,110 \\
1,100 \\
1,090 \\
1,090\end{array}$ & $\begin{array}{l}1,100 \\
1,100 \\
1,100 \\
1,100 \\
1,100\end{array}$ & $\begin{array}{l}950 \\
950 \\
926 \\
918 \\
918\end{array}$ & $\begin{array}{l}880 \\
873 \\
865 \\
873 \\
873\end{array}$ & $\begin{array}{l}1,800 \\
1,890 \\
2,000 \\
2,170 \\
2,280\end{array}$ & $\begin{array}{r}9,310 \\
9,590 \\
9,760 \\
9,990 \\
10,100\end{array}$ & $\begin{array}{l}13,100 \\
13,600 \\
14,100 \\
14,500 \\
15,200\end{array}$ & $\begin{array}{l}16,700 \\
16,300 \\
15,800 \\
15,300 \\
15,600\end{array}$ & $\begin{array}{l}9,000 \\
8,640 \\
8,520 \\
8,410 \\
8,290\end{array}$ & $\begin{array}{l}4,720 \\
4,680 \\
4,850 \\
4,810 \\
4,750\end{array}$ \\
\hline $\begin{array}{l}16 \\
17 \\
18 \\
19 \\
20\end{array}$ & $\begin{array}{l}1,770 \\
1,720 \\
1,720 \\
1,740 \\
1,630\end{array}$ & $\begin{array}{l}1,220 \\
1,210 \\
1,190 \\
1,200 \\
1,200\end{array}$ & $\begin{array}{l}1,090 \\
1,120 \\
1,120 \\
1,110 \\
1,120\end{array}$ & $\begin{array}{l}1,110 \\
1,100 \\
1,090 \\
1,070 \\
1,050\end{array}$ & $\begin{array}{l}918 \\
918 \\
910 \\
903 \\
895\end{array}$ & $\begin{array}{l}873 \\
873 \\
880 \\
910 \\
934\end{array}$ & $\begin{array}{l}2,390 \\
2,520 \\
2,640 \\
2,770 \\
2,920\end{array}$ & $\begin{array}{l}10,200 \\
10,500 \\
10,600 \\
10,600 \\
10,600\end{array}$ & $\begin{array}{l}15,800 \\
16,400 \\
16,900 \\
17,500 \\
18,000\end{array}$ & $\begin{array}{l}15,700 \\
15,900 \\
15,700 \\
15,500 \\
15,100\end{array}$ & $\begin{array}{l}8,060 \\
7,830 \\
7,230 \\
7,000 \\
6,890\end{array}$ & $\begin{array}{l}4,720 \\
4,680 \\
4,660 \\
4,640 \\
4,590\end{array}$ \\
\hline $\begin{array}{l}21 \\
22 \\
23 \\
24 \\
25\end{array}$ & $\begin{array}{l}1,690 \\
1,680 \\
1,640 \\
1,590 \\
1,540\end{array}$ & $\begin{array}{l}1,200 \\
1,190 \\
1,190 \\
1,180 \\
1,180\end{array}$ & $\begin{array}{l}1,140 \\
1,140 \\
1,150 \\
1,150 \\
1,150\end{array}$ & $\begin{array}{l}1,040 \\
1,040 \\
1,040 \\
1,040 \\
1,040\end{array}$ & $\begin{array}{l}895 \\
895 \\
888 \\
880 \\
873\end{array}$ & $\begin{array}{l}958 \\
958 \\
966 \\
966 \\
982\end{array}$ & $\begin{array}{l}3,060 \\
3,210 \\
3,500 \\
3,810 \\
4,020\end{array}$ & $\begin{array}{l}10,600 \\
10,600 \\
10,500 \\
10,400 \\
10,300\end{array}$ & $\begin{array}{l}18,500 \\
19,000 \\
19,400 \\
19,700 \\
19,900\end{array}$ & $\begin{array}{l}14,900 \\
14,700 \\
14,400 \\
14,100 \\
13,900\end{array}$ & $\begin{array}{l}6,730 \\
6,680 \\
6,540 \\
6,390 \\
6,230\end{array}$ & $\begin{array}{l}4,530 \\
4,480 \\
4,300 \\
4,240 \\
4,230\end{array}$ \\
\hline $\begin{array}{l}26 \\
27 \\
28 \\
29 \\
30 \\
31 \\
\end{array}$ & $\begin{array}{l}1,540 \\
1,530 \\
1,530 \\
1,500 \\
1,480 \\
1,460 \\
\end{array}$ & $\begin{array}{r}1,150 \\
1,140 \\
1,150 \\
1,140 \\
1,140 \\
- \\
\end{array}$ & $\begin{array}{l}1,140 \\
1,140 \\
1,140 \\
1,120 \\
1,120 \\
1,120 \\
\end{array}$ & $\begin{array}{r}1,030 \\
1,020 \\
1,020 \\
1,020 \\
1,020 \\
999 \\
\end{array}$ & $\begin{array}{r}865 \\
865 \\
865 \\
873 \\
----- \\
--- \\
\end{array}$ & $\begin{array}{r}982 \\
999 \\
1,030 \\
1,040 \\
1,100 \\
1,120 \\
\end{array}$ & $\begin{array}{r}4,190 \\
4,440 \\
4,740 \\
5,050 \\
5,440 \\
- \\
\end{array}$ & $\begin{array}{r}10,200 \\
10,200 \\
10,200 \\
10,100 \\
10,000 \\
9,960 \\
\end{array}$ & $\begin{array}{l}19,900 \\
19,900 \\
19,700 \\
19,600 \\
19,900 \\
-\end{array}$ & $\begin{array}{l}13,600 \\
13,200 \\
13,000 \\
12,600 \\
12,300 \\
12,000 \\
\end{array}$ & $\begin{array}{l}6,060 \\
5,930 \\
5,760 \\
5,610 \\
5,520 \\
5,460 \\
\end{array}$ & $\begin{array}{r}4,230 \\
4,190 \\
4,140 \\
4,100 \\
4,050 \\
-=-=\end{array}$ \\
\hline $\begin{array}{l}\text { Total } \\
\text { Mean } \\
\text { Max } \\
\text { Min } \\
\text { Cfsm } \\
\text { In. } \\
\text { Ac-ft } \\
\end{array}$ & $\begin{array}{r}53,910 \\
1,739 \\
1,980 \\
1,460 \\
0.336 \\
0.39 \\
106,900 \\
\end{array}$ & $\begin{array}{r}37,570 \\
1,252 \\
1,440 \\
1,140 \\
0.242 \\
0.27 \\
74,520 \\
\end{array}$ & $\begin{array}{r}34,730 \\
1,120 \\
1,150 \\
1,090 \\
0.217 \\
0.25 \\
68,890 \\
\end{array}$ & $\begin{array}{r}33,269 \\
1,073 \\
1,120 \\
999 \\
0.208 \\
0.24 \\
65,990 \\
\end{array}$ & $\begin{array}{r}26,911 \\
928 \\
999 \\
865 \\
0.180 \\
0.19 \\
53,380 \\
\end{array}$ & $\begin{array}{r}28,483 \\
919 \\
1,120 \\
843 \\
0.178 \\
0.20 \\
56,500 \\
\end{array}$ & $\begin{array}{r}78,630 \\
2,621 \\
5,440 \\
1,150 \\
0.507 \\
0.57 \\
156,000 \\
\end{array}$ & $\begin{array}{r}288,870 \\
9,318 \\
10,600 \\
5,820 \\
1.80 \\
2.08 \\
573,000 \\
\end{array}$ & $\begin{array}{r}454,300 \\
15,143 \\
19,900 \\
9,610 \\
2.93 \\
3.27 \\
901,100 \\
\end{array}$ & $\begin{array}{r}493,800 \\
15,929 \\
19,800 \\
12,000 \\
3,08 \\
3.55 \\
979,400 \\
\end{array}$ & $\begin{array}{r}249,590 \\
8,051 \\
11,600 \\
5,460 \\
1.56 \\
1.80 \\
495,100 \\
\end{array}$ & $\begin{array}{r}140,080 \\
4,669 \\
5,360 \\
4,050 \\
0.903 \\
1.01 \\
277,800 \\
\end{array}$ \\
\hline wtr yr & $\begin{array}{l}1967= \\
1968:\end{array}$ & $\begin{array}{l}\text { Total } 1,2 \\
\text { Total } 1,9\end{array}$ & 143 & $\begin{array}{l}\text { Mean } 3,473 \\
\text { Mean } 5,246\end{array}$ & $\operatorname{Max}$ & 900 & $\begin{array}{r}\text { Min } 1,090 \\
\text { Min } \\
\end{array}$ & $\begin{array}{l}\text { Cfsm } 0 . \\
\text { Cfsm } 1 .\end{array}$ & $\begin{array}{l}\text { In } \\
\text { In }\end{array}$ & $\begin{array}{r}9.12 \\
13.81\end{array}$ & $\begin{array}{l}\text { c-ft } 2, \\
\text { c-ft } 3,\end{array}$ & $\begin{array}{l}000 \\
000\end{array}$ \\
\hline
\end{tabular}


5-1282. Vermilion Lake near Soudan, Minn.

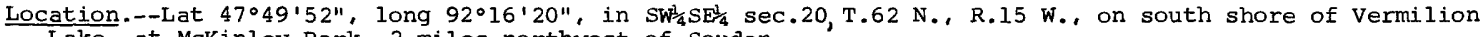
Lake, at McKinley Park, 2 miles northwest of Soudan.

Records available.--October 1913 to July 1915, July 1941 to November 1942 , June 1946 to September 1968 (fragmentary during 1947).

Gage.-Water-stage recorder. Datum of gage is 1,355.10 ft above mean sea level, datum of 1929 . October 1913 to July 1915, staff gage 2 miles southwest of present gage at Tower, at datum about 0.5 ft lower. July 1941 to November 1942 and June 1946 to June 1951, staff gage approximately 13 miles northwest at Vermilion Dam near Tower, at same datum. All gage readings have been reduced to elevations above mean sea level, datum of 1929.

Extremes.--Maximum elevation during year, 1,358.98 ft June 14 (affected by wind action); maximum daily, 1,358.81 ft June 15; minimum, $1,356.46 \mathrm{ft}$ Nov. 10 .

1913-15, 1941-42, 1946-68: Maximum elevation observed, 1,359.52 ft May 16, 1950; minimum observed, $1,356.02 \mathrm{ft}$ Jan. 29,1942 .

Elevation on June 6,1913 , was $1,359,94 \mathrm{ft}$ (determined from reference point set by locà observers). Daily mean elevation, in feet, October 1967 to september 1968

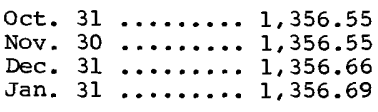

Note.--Elevations other than those shown above are available.

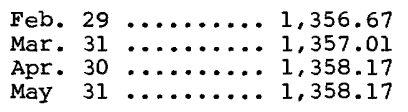

June $30 \ldots \ldots \ldots . . \ldots 1,358.69$

July $31 \ldots \ldots \ldots \ldots \ldots 1,358.19$

Aug. $31 \ldots \ldots \ldots \ldots . . .1,357.87$

Sept. $30 \ldots \ldots \ldots \ldots . . .1,357.75$ 
Location.--Lat $47^{\circ} 57^{\prime} 41^{\prime \prime}$, long $92^{\circ} 28^{\prime} 33^{\prime \prime}$, in SEl $\frac{1}{4} S_{\frac{1}{4}}$ sec.2, T.63 N., R.17 W., on left bank 200 ft downstream from dam at outlet of Vermilion Lake, 4.4 miles upstream from Twomile Creek, and 14.2 miles northwest of Tower.

Drainage area.--483 sq $\mathrm{mi}$.

Records available.--May 1911 to September 1917, June 1928 to September 1968.

Gage.--Digital water-stage recorder. Datum of gage is $1,347.36 \mathrm{ft}$ above mean sea level, datum of 1929 . May 17, 1911, to Sept. 30, 1917, and July 9, 1931, to Apr. 11, 1939, staff gage at same site, at datum $3.0 \mathrm{ft}$ higher. June 26, 1928, to July 8, 1931, staff gage at datum $3.05 \mathrm{ft}$ higher. Apr. 12, 1939, to Sept. 6, 1967, graphic water-stage recorder, and Sept. 7-30, 1967, digital water-stage recorder at same site, at datum $3.0 \mathrm{ft}$ higher.

Average discharge.--46 years, 305 cfs.

Extremes.--Maximum discharge during year, 1,420 cfs July 1 (gage height, $6.40 \mathrm{ft}$ ); minimum, $1.7 \mathrm{cfs}$ Nov. 12 (gage height, $1.95 \mathrm{ft}$, affected by seiche action).

1911-17, 1928-68: Maximum discharge, 2,710 cfs May 23, 1950 (gage height, 7.68 ft); no flow Oct. 25-29, 1955, caused by temporary storage behind new concrete dam at outlet of Vermilion Lake.

Remarks.--Records good except those for period of doubtful or no gage-height record, which are fair.

OISCHARGE, IN CUBIC FEET PER SECOND, WATER YEAR OCTOBER 1967 TD SEPTEMBER 1968

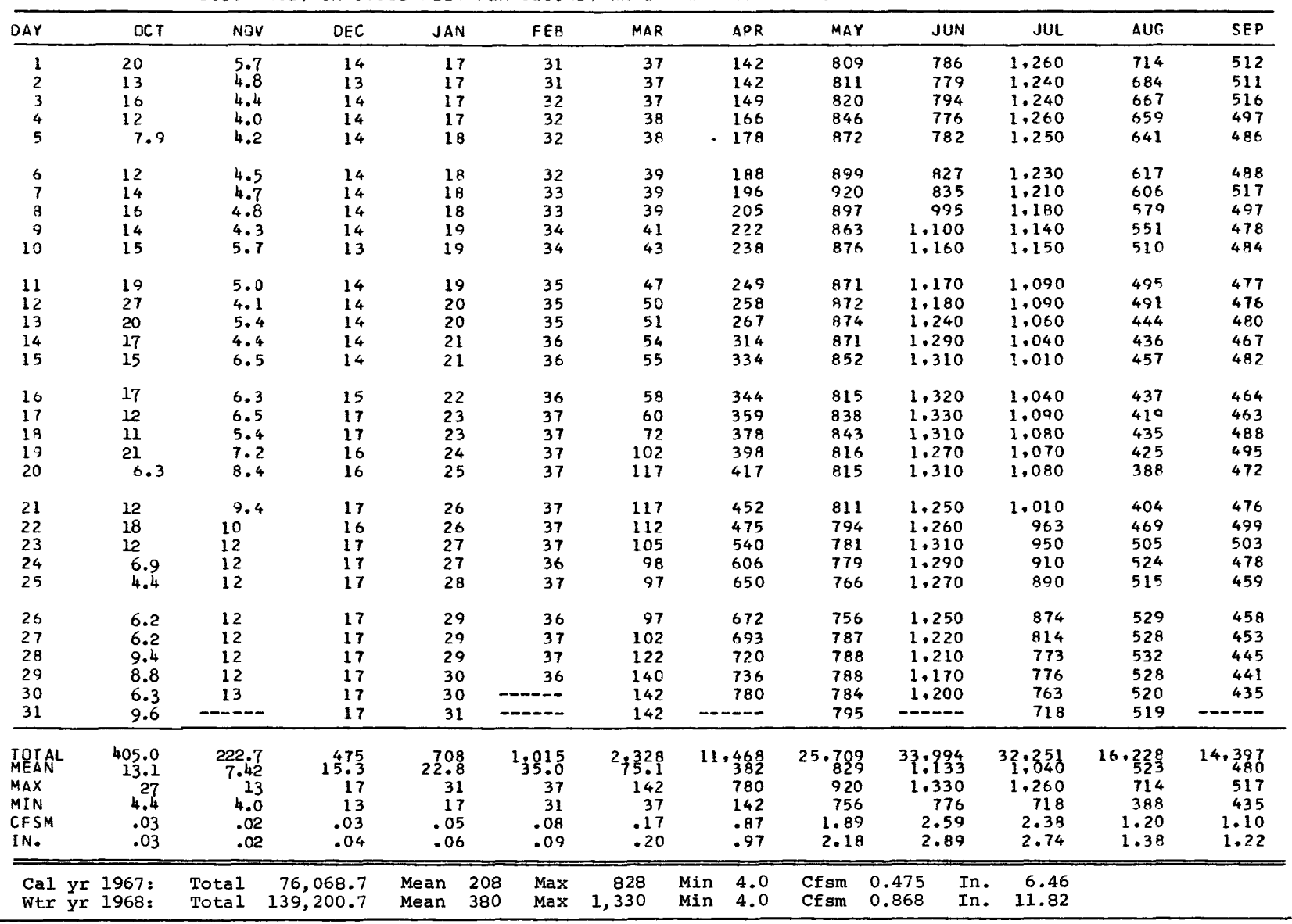

Note.--Doubtful or no gage-height record Jan. 10 to Apr. 12 . 
5-1294. Rainy Lake near Fort Frances, Ontario

(International gaging station)

Location.--Lat 48038'30", long 93020'00", at Five Mile dock, approximately 5 miles northeast of town of Fort Frances.

Records available.--January 1910 to september 1917 and October 1934 to september 1968 in reports of Geological Survey, August 1911 to September 1968 in reports of Inland Waters Branch, Department of Energy, Mines and Resources, Canada. Prior to October 1949, published as "at Ranier, Minn.", and as "at Fort Frences, Ontario" October 1949 to September 1964

Gage.--Water-stage recorder. Datum of gage is at mean sea level (United States and Canadian Boundary Survey). January 1910 to December 1949, staff gage 3 miles northeast at Ranier, Minn., at same datum. January 1950 to October 1964, water-stage recorder on Government dock at Pither's Point, in Fort Frances and supplementary gage in town pumping station, half a mile south, used during winter months, as same datum.

Extremes.--Maximum elevation during year 1,110.19 ft July 20; minimum, 1,105.08 ft Mar. 17.

1910-17, 1934-68: Maximum elevation observed, 1,112.97 ft July 5, 1950; minimum observed, 1, 101.26 ft Apr. 17, 1923, Apr. 2, 1930.

Cooperation.--This station is maintained by Canada under agreement with the United states.

Month-end elevation, in feet, water year October 1967 to september 1968
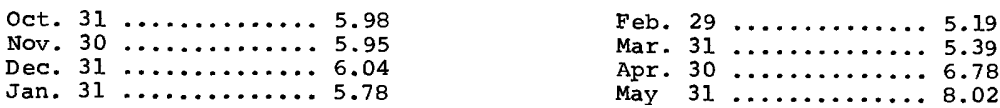

June $30 \ldots \ldots \ldots \ldots .9 .40$

July $31 \ldots \ldots \ldots \ldots . . .9 .62$

Aug. $31 \ldots \ldots \ldots \ldots . . . . . .13$

Jan. $31 \ldots \ldots \ldots \ldots \ldots . . . . .58$

May $31 \ldots . . . . . . . . . . .02$

sept. $30 \ldots \ldots \ldots \ldots \ldots .8 .09$

Note.--Add $1,100 \mathrm{ft}$ to obtain elevation above mean sea level. Elevations other than those shown are available. 


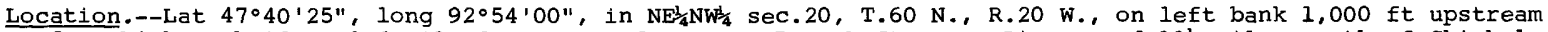

from highway bridge, $0.6 \mathrm{mile}$ downstream from East Branch Sturgeon River, and $11 \frac{1}{2} \mathrm{miles}$ north of Chisholm.

Drainage area.--187 sq $\mathrm{mi}$.

Records available.--August 1942 to september 1968.

Gage.--Water-stage recorder. Datum of gage is 1,306.7 ft above mean sea level, datum of 1929 . Prior to Aug.

24, 1944, staff gage at site $1,000 \mathrm{ft}$ downstream at different datum.

Average discharge.--26 years, 120 cfs.

Extremes.--Maximum discharge during year, 599 cfs June 16 (gage height, 2.95 ft); minimum daily, 8.5 cfs Nov. 29,30 .

1942-68: Maximum discharge, 3,630 cfs May 7, 1950 (gage height, 6.41 ft), from rating curve extended above $1,600 \mathrm{cfs}$ on basis of slope-area measurement of peak flow; minimum daily, 6.0 cfs Feb. 18-27, 1944; minimum gage height, $0.08 \mathrm{ft}$ Jan. 28 to Feb. $1,1963$.

Remarks.--Records good except those for winter months, which are fair.

DISCHARGE, IN CFS, WATER YEAR OCTOBER 1967 TO SEPTEMBER 1968

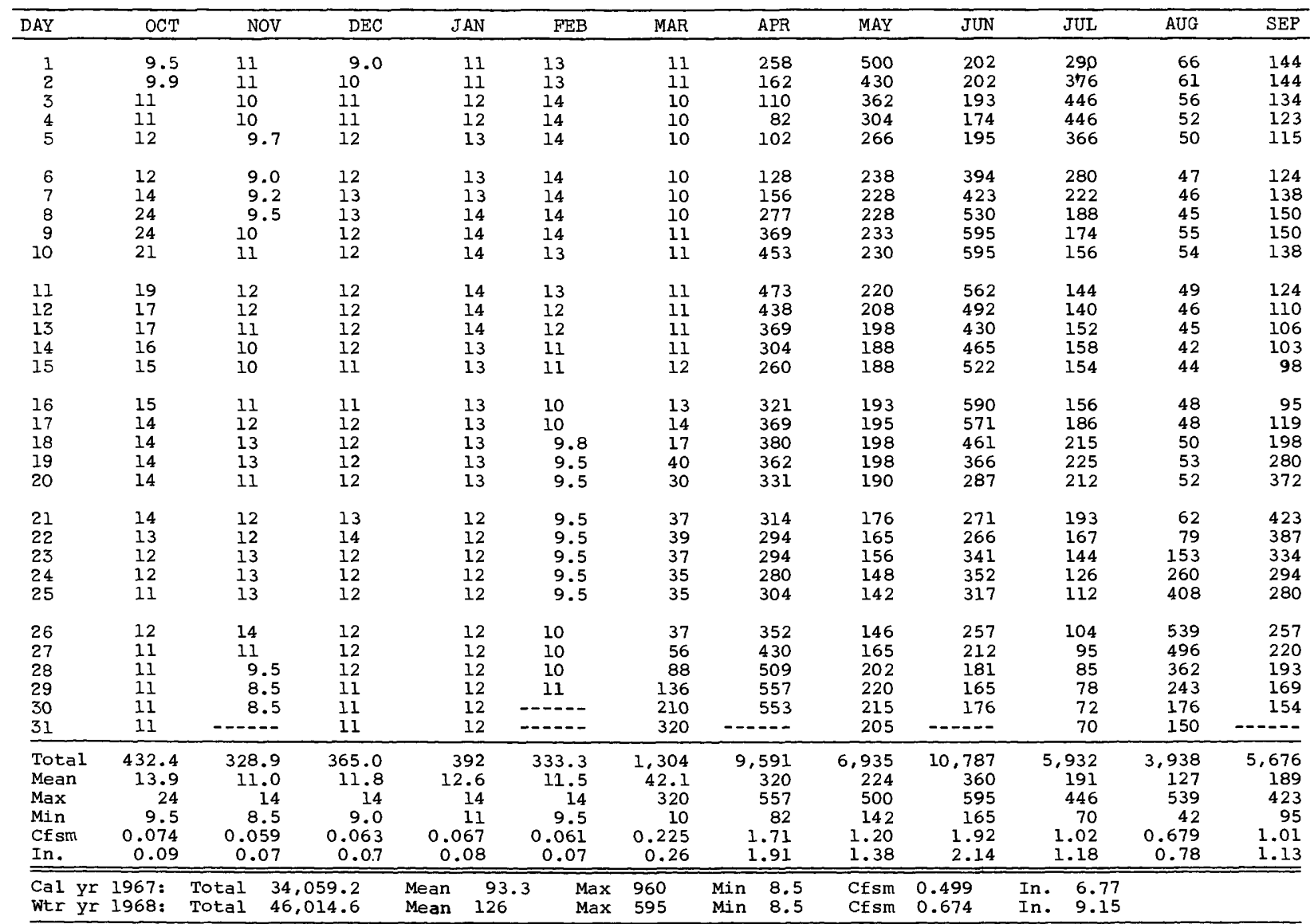

PEAK DISCHARGE (BASE, 500 CFS)

$\begin{array}{lllccccc}\text { DATE } & \text { TIME } & \text { G.HT. } & \text { DISCHARGE } & \text { DATE } & \text { TTME } & \text { G.HT. } & \text { DISCHARGE } \\ 4-30 & 0100 & 2.95 & 562 & 6-16 & 2100 & 2.95 & 599 \\ 8-26 & 1600 & 2.93 & 553 & & & & \end{array}$


5-1310. Dark River near Chisholm, Minn.

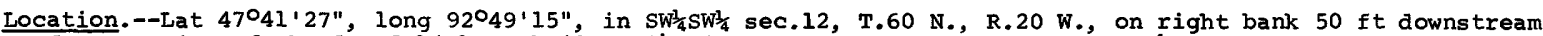
from remains of abandoned highway bridge, $3 \frac{1}{2}$ miles upstream from mouth, and $12 \frac{1}{4}$ miles northeast of Chisholm.

Drainage area. $--50.6 \mathrm{sq} \mathrm{mi}$.

Records available.--August 1942 to September 1961, October 1965 to September 1968.

Gage.-Water-stage recorder. Datum of gage is $1,316.8 \mathrm{ft}$ above mean sea level, datum of 1929 (surveyed by Topographic Division). Prior to Aug. 24, 1944, staff gage at site 50 ft upstream at same datum.

Average discharge. -22 years, 36.1 cfs.

Extremes.--Maximum discharge during year, 200 cfs June 8 (gage height, 3.11 ft); minimum daily, 3.0 cfs Feb. 15-23.

1942-61, 1966-68: Maximum discharge, 1,170 cfs May 7, 1950 (gage height, 7.10 ft); minimum, 0.3 cfs

Aug. 3, 1956; minimum gage height, 0.87 ft Mar. 22, 23, 1949, Aug. 16, 17, 1961.

Remarks.--Records good.

Cooperation.--Records for 1968 water year computed by U.S. Steel Corporation and reviewed by Geological Survey.

DISCHARGE, IN CFS, WATER YEAR OCTOBER 1967 TO SEPTEMBER 1968

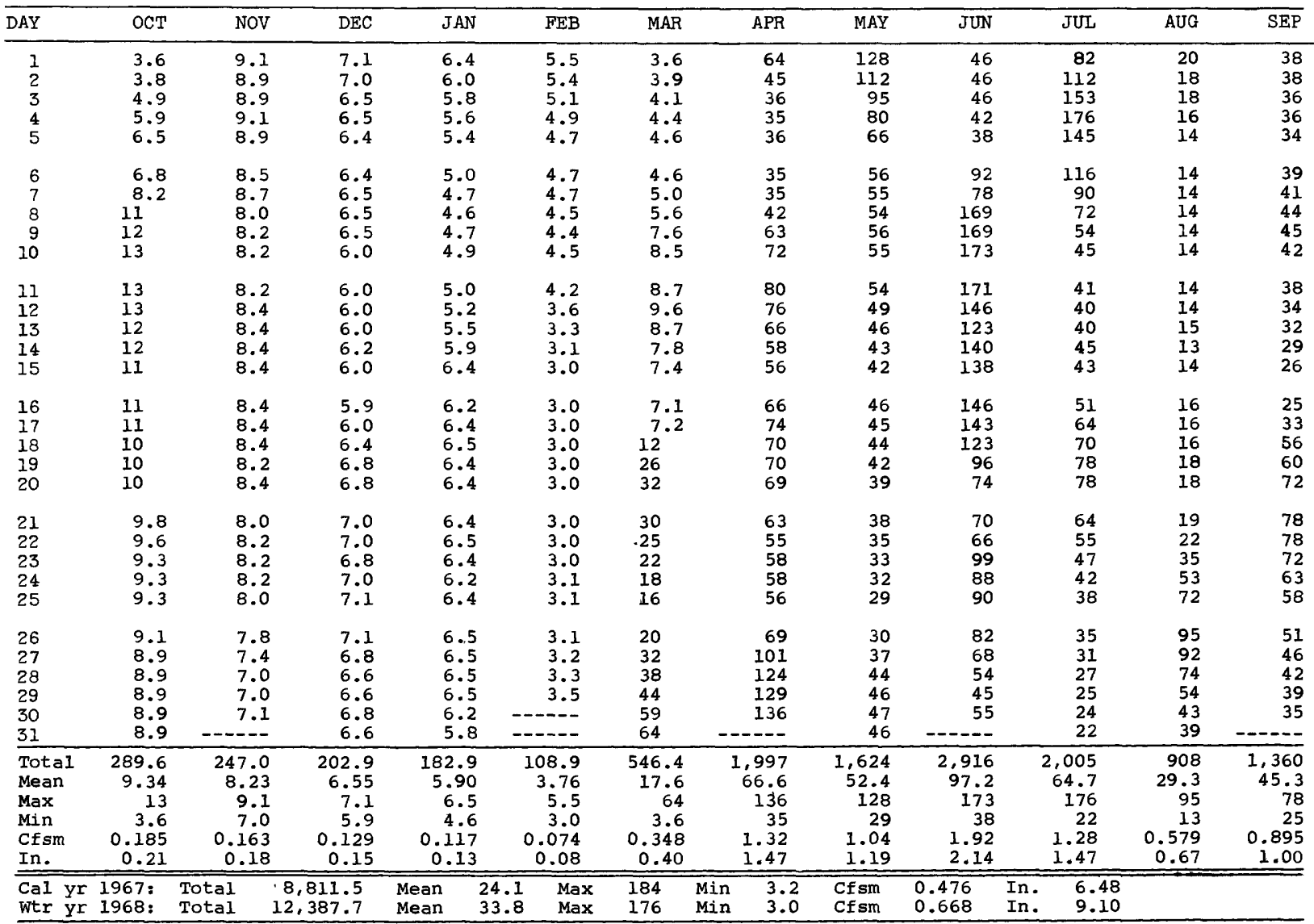


5-1315. Little Fork River at Little Fork, Minn.

Location.--Lat $48^{\circ} 24^{\prime}$, long $93^{\circ} 34^{\prime}$, in NW/ sec.9, T.68 N., R. 25 W., on left bank 100 ft downstream from highway bridge at town of Little Fork, 0.3 mile downstream from bridge on state Highway 217 , 1/2 miles upstream from Beaver Creek, and 18 miles upstream from mouth.

Drainage area.--1,730 sq $\mathrm{mi}$, approximately.

Records available.--June to November 1909, April to November 1910, April 1911 to June 1917, September 1917, October 1917 to March 1919 (gage heights only), June 1928 to september 1968.

Gage.-Water-stage recorder. Datum of gage is 1,073.06 ft above mean sea level, datum of 1929. June 23, 1909, to Mar. 4, 1917, staff gage at same site and datum. Mar. 5 to Sept. 30, 1917, June 22, 1928 , to June 21, 1936, chain gage and June 22, 1936, to July 20, 1937, wire-weight gage, at site 100 ft upstream at same datum.

Average discharge. - -45 years $(1911-16,1928-68), 991$ cfs.

Extremes.--Maximum discharge during year, 5,790 cfs June 10 (gage height, 18.51 ft, backwater from Rainy

River): minimum daily discharge, 41 cfs Feb. 17-23.

1909-17, 1928-68: Maximum discharge, 25,000 cfi Apr. 18, 1916, May 11, 1950 (gage height, 37.00 ft);

minimum daily, 15 cfs Feb. 9-22, 1963.

Remarks---Records good except those for winter months or period of backwater from Rainy River, which are fair.

DISCHARGE, IN CFS, WATER YEAR OCTOBER 1967 TO SEPTEMBER 1968

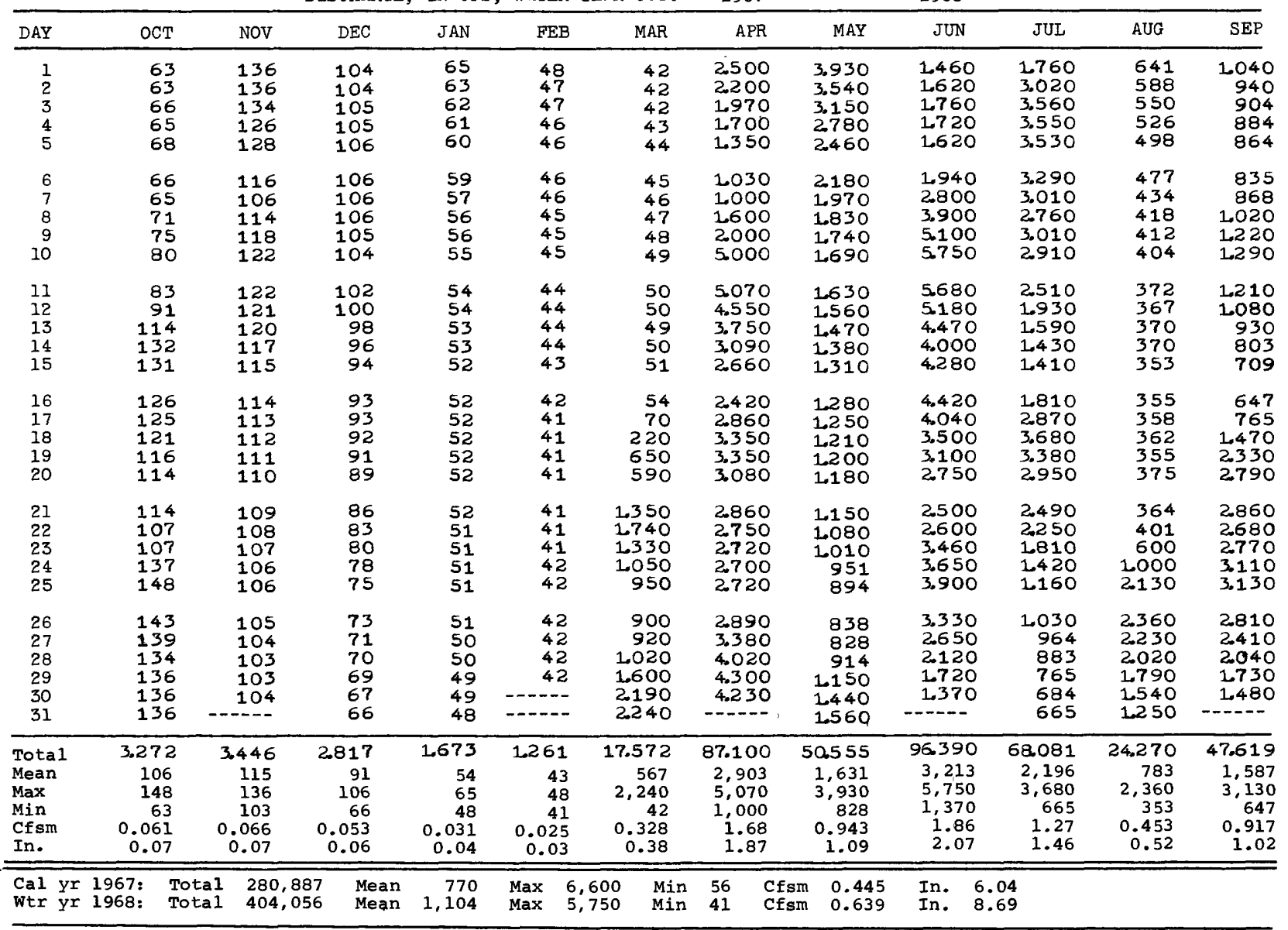

Note.--Backwater from Rainy River May 29 to Aug. 19. 
5-1320. Big Fork River at Big Falls, Ninn.

Location.--Lat $48^{\circ} 12^{\prime}$, long $93^{\circ} 48^{\prime}$, in sec.35, T.155 N., R.25 W., on left bank at village of Big Falls, 700 ft downstream from falls, $0.3 \mathrm{mile}$ downstream from bridge on U.S. Highway 71 , and $4 \frac{3}{4} \mathrm{miles}$ upstream from Sturgeon River.

Drajnage area.--1,460 sq $\mathrm{mi}$, approximately.

Records available.--August to November 1909, April to November 1910, April 1911 to September 1912 (gage heights and discharge measurements only), June 1928 to september 1968.

Gage.-Digital water-stage recorder. Datum of gage is 1,144.71 ft above mean sea level, datum of 1929 . Prior to June 10, 1911, staff gage at railroad bridge about 0.4 mile upstream at different datum. June 10 , 1911 , , 17, 1937, to Sept. 6, 1967, graphic water-stage recorder at same site and datum.

Average discharge. --40 years $(1928-1968), 656$ cfs.

Extremes.--Maximum discharge during year, 2,620 cfs Apr. 11 (gage height, 6.33 ft); minimum daily, 54 cfs Feb, 16-24; minimum gage height, $2.75 \mathrm{ft}$ Nov, 15 . 1950; minimum discharge recorded, 7 cfs Aug. 7, 1939.

Remarks.--Records good except those for period of no gage-height record and those for winter months, which are fair. Some diurnal fluctuation at low flow caused by powerplant a quarter of a mile upstream. Records of chemical analyses for the water year 1968 are published in Part 2 of this report.

DISCHARGE, IN CUBIC FEET PFR SECOND, WATEF YEAR DCTOBER 1967 TO SEPTEMBER 1968

\begin{tabular}{|c|c|c|c|c|c|c|c|c|c|c|c|c|}
\hline DAY & $O C T$ & NoV & $D E C$ & JAN & FEB & MAR & $A P R$ & MAY & JUN & JUL & AUG & SEP \\
\hline $\begin{array}{l}1 \\
2 \\
3 \\
4 \\
5\end{array}$ & $\begin{array}{l}129 \\
132 \\
137 \\
138 \\
135\end{array}$ & $\begin{array}{l}138 \\
201 \\
164 \\
214 \\
187\end{array}$ & $\begin{array}{l}165 \\
166 \\
167 \\
168 \\
169\end{array}$ & $\begin{array}{l}111 \\
109 \\
106 \\
104 \\
103\end{array}$ & $\begin{array}{l}72 \\
71 \\
70 \\
67 \\
68\end{array}$ & $\begin{array}{l}59 \\
60 \\
62 \\
64 \\
67\end{array}$ & $\begin{array}{l}616 \\
552 \\
520 \\
480 \\
460\end{array}$ & $\begin{array}{l}1.600 \\
1,460 \\
1,320 \\
1.210 \\
1.120\end{array}$ & $\begin{array}{l}839 \\
921 \\
981 \\
946 \\
878\end{array}$ & $\begin{array}{r}981 \\
1,890 \\
2,040 \\
2,010 \\
1,830\end{array}$ & $\begin{array}{l}298 \\
282 \\
260 \\
248 \\
236\end{array}$ & $\begin{array}{l}450 \\
410 \\
394 \\
383 \\
372\end{array}$ \\
\hline $\begin{array}{r}6 \\
7 \\
8 \\
9 \\
10\end{array}$ & $\begin{array}{l}134 \\
139 \\
153 \\
158 \\
163\end{array}$ & $\begin{array}{l}169 \\
167 \\
178 \\
243 \\
258\end{array}$ & $\begin{array}{l}170 \\
172 \\
171 \\
169 \\
166\end{array}$ & $\begin{array}{r}101 \\
99 \\
97 \\
94 \\
92\end{array}$ & $\begin{array}{l}66 \\
65 \\
64 \\
62 \\
60\end{array}$ & $\begin{array}{l}71 \\
74 \\
77 \\
78 \\
76\end{array}$ & $\begin{array}{r}445 \\
512 \\
661 \\
1,050 \\
1.640\end{array}$ & $\begin{array}{r}1,040 \\
998 \\
1,000 \\
1,050 \\
1,100\end{array}$ & $\begin{array}{l}1.060 \\
1.100 \\
1.400 \\
2.170 \\
2.060\end{array}$ & $\begin{array}{r}1.590 \\
1.320 \\
1.100 \\
935 \\
791\end{array}$ & $\begin{array}{l}223 \\
213 \\
200 \\
196 \\
193\end{array}$ & $\begin{array}{l}364 \\
400 \\
470 \\
542 \\
563\end{array}$ \\
\hline $\begin{array}{l}11 \\
12 \\
13 \\
14 \\
15\end{array}$ & $\begin{array}{l}168 \\
171 \\
177 \\
173 \\
172\end{array}$ & $\begin{array}{l}251 \\
241 \\
219 \\
195 \\
130\end{array}$ & $\begin{array}{l}164 \\
162 \\
160 \\
157 \\
156\end{array}$ & $\begin{array}{l}91 \\
89 \\
88 \\
87 \\
86\end{array}$ & $\begin{array}{l}59 \\
58 \\
57 \\
56 \\
55\end{array}$ & $\begin{array}{r}75 \\
79 \\
89 \\
103 \\
113\end{array}$ & $\begin{array}{l}2.150 \\
2.010 \\
1.480 \\
1.360 \\
1.280\end{array}$ & $\begin{array}{r}1,110 \\
1,080 \\
1,030 \\
991 \\
984\end{array}$ & $\begin{array}{l}1.790 \\
1.560 \\
1,370 \\
1.400 \\
1,720\end{array}$ & $\begin{array}{l}680 \\
622 \\
558 \\
552 \\
564\end{array}$ & $\begin{array}{l}184 \\
175 \\
1.74 \\
173 \\
170\end{array}$ & $\begin{array}{l}524 \\
441 \\
415 \\
376 \\
349\end{array}$ \\
\hline $\begin{array}{l}16 \\
17 \\
18 \\
19 \\
20\end{array}$ & $\begin{array}{l}177 \\
214 \\
1 \geqslant 9 \\
193 \\
175\end{array}$ & $\begin{array}{l}145 \\
160 \\
168 \\
172 \\
172\end{array}$ & $\begin{array}{l}157 \\
156 \\
154 \\
152 \\
148\end{array}$ & $\begin{array}{l}86 \\
85 \\
84 \\
84 \\
84\end{array}$ & $\begin{array}{l}54 \\
54 \\
54 \\
54 \\
54\end{array}$ & $\begin{array}{l}113 \\
121 \\
147 \\
20 \mathrm{C} \\
36 \mathrm{C}\end{array}$ & $\begin{array}{l}1,310 \\
1,460 \\
1,510 \\
1.460 \\
1.380\end{array}$ & $\begin{array}{l}970 \\
970 \\
935 \\
893 \\
830\end{array}$ & $\begin{array}{l}1.730 \\
1.570 \\
1.380 \\
1.200 \\
1.050\end{array}$ & $\begin{array}{r}872 \\
1.370 \\
1.820 \\
1.670 \\
1.430\end{array}$ & $\begin{array}{l}172 \\
174 \\
176 \\
173 \\
172\end{array}$ & $\begin{array}{r}334 \\
510 \\
1,190 \\
2.130 \\
2.290\end{array}$ \\
\hline $\begin{array}{l}21 \\
22 \\
23 \\
24 \\
25\end{array}$ & $\begin{array}{l}185 \\
186 \\
191 \\
192 \\
186\end{array}$ & $\begin{array}{l}170 \\
169 \\
167 \\
166 \\
165\end{array}$ & $\begin{array}{l}143 \\
138 \\
135 \\
132 \\
128\end{array}$ & $\begin{array}{l}84 \\
83 \\
82 \\
81 \\
80\end{array}$ & $\begin{array}{l}54 \\
54 \\
54 \\
54 \\
55\end{array}$ & $\begin{array}{r}1.300 \\
1.280 \\
1.170 \\
1.620 \\
878\end{array}$ & $\begin{array}{l}1.350 \\
1.340 \\
1,370 \\
1.420 \\
1.510\end{array}$ & $\begin{array}{l}804 \\
772 \\
726 \\
694 \\
654\end{array}$ & $\begin{array}{r}998 \\
1.020 \\
1,090 \\
1.180 \\
1,190\end{array}$ & $\begin{array}{r}1.260 \\
1.110 \\
880 \\
690 \\
560\end{array}$ & $\begin{array}{r}170 \\
176 \\
208 \\
460 \\
1,070\end{array}$ & $\begin{array}{l}2,080 \\
1.860 \\
1.900 \\
2,20 . \\
2.230\end{array}$ \\
\hline $\begin{array}{l}26 \\
27 \\
28 \\
27 \\
30 \\
31\end{array}$ & $\begin{array}{l}190 \\
200 \\
204 \\
201 \\
198 \\
204\end{array}$ & $\begin{array}{r}164 \\
164 \\
163 \\
163 \\
164 \\
\end{array}$ & $\begin{array}{l}125 \\
123 \\
120 \\
117 \\
115 \\
113\end{array}$ & $\begin{array}{l}80 \\
79 \\
78 \\
77 \\
75 \\
74\end{array}$ & $\begin{array}{r}56 \\
57 \\
57 \\
58 \\
\\
\end{array}$ & $\begin{array}{l}800 \\
748 \\
728 \\
724 \\
650 \\
638\end{array}$ & $\begin{array}{l}1,690 \\
1.870 \\
1.900 \\
1.840 \\
1.720 \\
-\end{array}$ & $\begin{array}{l}616 \\
622 \\
689 \\
758 \\
790 \\
791\end{array}$ & $\begin{array}{r}1,110 \\
963 \\
817 \\
721 \\
680 \\
\end{array}$ & $\begin{array}{l}408 \\
438 \\
392 \\
350 \\
321 \\
310\end{array}$ & $\begin{array}{r}1.200 \\
1.110 \\
1.000 \\
860 \\
720 \\
560\end{array}$ & $\begin{array}{l}2,090 \\
1,880 \\
1,660 \\
1,470 \\
1,300 \\
-\end{array}$ \\
\hline $\begin{array}{l}\text { TOTAL } \\
\text { MEAN } \\
\text { MAX } \\
\text { MIN } \\
\text { CFSM } \\
\text { IN. }\end{array}$ & $\begin{array}{r}5,364 \\
173 \\
214 \\
129 \\
.12 \\
.14 \\
\end{array}$ & $\begin{array}{r}5.507 \\
184 \\
258 \\
130 \\
.13 \\
.14 \\
\end{array}$ & $\begin{array}{r}4,638 \\
150 \\
172 \\
113 \\
.10 \\
.12 \\
\end{array}$ & $\begin{array}{r}2.753 \\
88.8 \\
111 \\
74 \\
.06 \\
.07 \\
\end{array}$ & $\begin{array}{r}1.721 \\
59.3 \\
72 \\
54 \\
.04 \\
.04 \\
\end{array}$ & $\begin{array}{r}12, .024 \\
388 \\
1.306 \\
50 \\
.27 \\
.31 \\
\end{array}$ & $\begin{array}{r}38,346 \\
1.278 \\
2,150 \\
445 \\
.88 \\
.98\end{array}$ & $\begin{array}{r}29,607 \\
955 \\
1,600 \\
616 \\
.65 \\
.75\end{array}$ & $\begin{array}{r}36.894 \\
1.230 \\
2.170 \\
680 \\
.84 \\
.94\end{array}$ & $\begin{array}{r}31,434 \\
1,014 \\
2,040 \\
310 \\
.69 \\
.80\end{array}$ & $\begin{array}{r}11.626 \\
375 \\
1.200 \\
170 \\
.26 \\
.30\end{array}$ & $\begin{array}{r}31.577 \\
1,053 \\
2.290 \\
334 \\
.72 \\
.80\end{array}$ \\
\hline $\begin{array}{ll}\text { CAL YR } \\
\text { WTR YR }\end{array}$ & $\begin{array}{l}967 \\
968\end{array}$ & 211.4 & & $\begin{array}{l}700 \\
578\end{array}$ & $\begin{array}{l}\text { MAX } \\
\text { MAX }\end{array}$ & $\begin{array}{l}20 \\
90\end{array}$ & $\begin{array}{r}106 \\
54\end{array}$ & $\begin{array}{l}\text { CFSM } \\
\text { CFSM }\end{array}$ & $\begin{array}{l}\text { IN } \\
\text { IN }\end{array}$ & $\begin{array}{l}51 \\
39\end{array}$ & & \\
\hline
\end{tabular}

Note.--No gage-height record July 17 to sept. 11. 


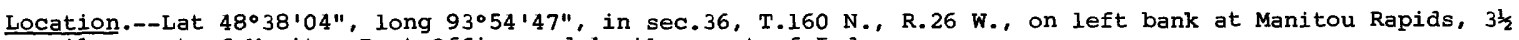
miles east of Manitou Post Office and 4 miles west of Indus.

Drainage area.--19,400 sq $\mathrm{mi}$, approximately.

Records available.--July 1928 to September 1968. Monthly discharge only for some periods, published in wSP 1308. October 1911 to October 1924 (gage heights only) at site near Birchdale in files of Corps of Engineers. Published as "near Birchdale" 1932-34.

Gage.--Digital water-stage recorder. Datum of gage is $1,062.48$ ft above mean sea level, datum of 1929. Prior to Nov. 10, 1934, chain gage at site near Birchdale 7 miles downstream at different datum. Nov. 10, 1934 , to sept. 20, 1967, graphic water-stage recorder at present site and datum.

Average discharge.-- 40 years, $12,310 \mathrm{cfs}$.

Extremes.--Maximum discharge during year, 46,200 cfs July 19 (gage height, 15.78 ft); minimum daily, 3,470 cfs Mar. 4; minimum gage height, 2.06 ft Nov. 3. 1928-68: Maximum discharge, 71,600 cfs May 12, 1950 (gage height, 21.04 ft); minimum daily, 928 cfs Dec. $26,1929$.

Remarks.--Records good except those for winter months, which are fair. Diurnal fluctuation caused by powerplant at International Falls. Some regulation at low and medium flows by Rainy and Namakan Lakes. Records of chemical analyses for the water year 1968 are published in Part 2 of this report.

Cooperation.--This station is maintained by the United States under agreement with Canada.

DISCHARGE, IN CUBIC FEET PER SECOND, WATER YEAR OCTOBER 1967 TO SEPTEMBER 1968

\begin{tabular}{|c|c|c|c|c|c|c|c|c|c|c|c|c|}
\hline DAY & OCT & NOV & DEC & JAN & FEB & MAR & APR & MAY & JUN & JUL & AUG & SEP \\
\hline $\begin{array}{l}1 \\
2 \\
3 \\
4 \\
5\end{array}$ & $\begin{array}{l}9,710 \\
9,020 \\
8,720 \\
8,220 \\
7,560\end{array}$ & $\begin{array}{l}4,980 \\
4,940 \\
4,340 \\
5,240 \\
6,130\end{array}$ & $\begin{array}{l}4, \\
5, \\
4, \\
4, \\
4,\end{array}$ & $\begin{array}{l}4, \\
4, \\
4, \\
4, \\
4,\end{array}$ & $\begin{array}{l}40 \\
10 \\
60 \\
60 \\
70\end{array}$ & $\begin{array}{l}940 \\
970 \\
300 \\
+70 \\
210\end{array}$ & $\begin{array}{l}7,110 \\
8,430 \\
9,850 \\
9,510 \\
9,040\end{array}$ & $\begin{array}{l}13,300 \\
13,100 \\
12,900 \\
13,000 \\
12,600\end{array}$ & $\begin{array}{l}22,500 \\
22,800 \\
23,000 \\
23,500 \\
22,800\end{array}$ & $\begin{array}{l}37,400 \\
39,500 \\
42,200 \\
43,200 \\
43,400\end{array}$ & $\begin{array}{l}36,200 \\
35,800 \\
35,400 \\
35,100 \\
34,800\end{array}$ & $\begin{array}{l}14,900 \\
14,300 \\
12,500 \\
13,400 \\
13,900\end{array}$ \\
\hline $\begin{array}{r}6 \\
7 \\
8 \\
9 \\
10\end{array}$ & $\begin{array}{l}8,320 \\
8,640 \\
9,040 \\
8,360 \\
7,810\end{array}$ & $\begin{array}{l}5,690 \\
4,790 \\
4,420 \\
4,660 \\
4,620\end{array}$ & $\begin{array}{l}4,920 \\
4,620 \\
4,610 \\
4,460 \\
4,510\end{array}$ & $\begin{array}{l}5,270 \\
5,360 \\
4,780 \\
5,770 \\
6,120\end{array}$ & $\begin{array}{l}6,610 \\
6,300 \\
6,430 \\
6,880 \\
7,440\end{array}$ & $\begin{array}{l}4,270 \\
3,840 \\
4,670 \\
4,320 \\
5,340\end{array}$ & $\begin{array}{r}8,500 \\
7,580 \\
7,640 \\
8,720 \\
10,400\end{array}$ & $\begin{array}{l}11,900 \\
12,300 \\
12,600 \\
14,800 \\
16,900\end{array}$ & $\begin{array}{l}22,900 \\
34,700 \\
37,600 \\
42,700 \\
43,100\end{array}$ & $\begin{array}{l}42,900 \\
42,000 \\
41,100 \\
40,400 \\
40,200\end{array}$ & $\begin{array}{l}34,500 \\
34,200 \\
33,700 \\
33,300 \\
32,600\end{array}$ & $\begin{array}{l}14,000 \\
14,000 \\
14,200 \\
14,700 \\
14,900\end{array}$ \\
\hline $\begin{array}{l}11 \\
12 \\
13 \\
14 \\
15\end{array}$ & $\begin{array}{l}6,740 \\
7,040 \\
7,720 \\
8,150 \\
7,750\end{array}$ & $\begin{array}{l}4,430 \\
4,660 \\
4,700 \\
4,940 \\
5,250\end{array}$ & $\begin{array}{l}4,650 \\
4,690 \\
4,580 \\
4,730 \\
4,770\end{array}$ & $\begin{array}{l}6,710 \\
6,540 \\
6,480 \\
6,060 \\
5,070\end{array}$ & $\begin{array}{l}6,870 \\
5,040 \\
7,370 \\
5,670 \\
5,110\end{array}$ & $\begin{array}{l}3,840 \\
4,620 \\
4,240 \\
4,970 \\
4,830\end{array}$ & $\begin{array}{l}13,400 \\
14,300 \\
13,700 \\
12,300 \\
11,600\end{array}$ & $\begin{array}{l}18,500 \\
19,000 \\
18,900 \\
18,700 \\
18,500\end{array}$ & $\begin{array}{l}43,800 \\
43,600 \\
43,400 \\
42,900 \\
42,100\end{array}$ & $\begin{array}{l}39,600 \\
39,200 \\
38,800 \\
38,500 \\
38,600\end{array}$ & $\begin{array}{l}32,000 \\
31,500 \\
30,300 \\
29,500 \\
29,000\end{array}$ & $\begin{array}{l}14,900 \\
14,600 \\
14,300 \\
14,000 \\
13,800\end{array}$ \\
\hline $\begin{array}{l}16 \\
17 \\
18 \\
19 \\
20\end{array}$ & $\begin{array}{l}7,380 \\
7,670 \\
8,220 \\
8,570 \\
8,350\end{array}$ & $\begin{array}{l}4,940 \\
4,740 \\
4,740 \\
4,850 \\
4,720\end{array}$ & $\begin{array}{l}4,720 \\
4,690 \\
4,750 \\
5,410 \\
4,800\end{array}$ & $\begin{array}{l}5,340 \\
5,680 \\
6,110 \\
6,730 \\
6,080\end{array}$ & $\begin{array}{l}5,490 \\
5,850 \\
5,270 \\
4,360 \\
6,290\end{array}$ & $\begin{array}{l}4,800 \\
4,630 \\
4,180 \\
5,100 \\
5,690\end{array}$ & $\begin{array}{l}10,900 \\
10,700 \\
11,300 \\
11,700 \\
12,100\end{array}$ & $\begin{array}{l}18,500 \\
18,500 \\
18,400 \\
18,200 \\
18,200\end{array}$ & $\begin{array}{l}42,000 \\
41,500 \\
40,400 \\
39,400 \\
38,500\end{array}$ & $\begin{array}{l}41,300 \\
44,400 \\
45,500 \\
46,100 \\
45,300\end{array}$ & $\begin{array}{l}26,200 \\
24,200 \\
23,500 \\
22,800 \\
18,500\end{array}$ & $\begin{array}{l}13,500 \\
14,400 \\
19,400 \\
23,200 \\
24,800\end{array}$ \\
\hline $\begin{array}{l}21 \\
22 \\
23 \\
24 \\
25\end{array}$ & $\begin{array}{l}7,690 \\
7,410 \\
6,760 \\
7,080 \\
7,040\end{array}$ & $\begin{array}{l}4,610 \\
4,790 \\
4,870 \\
5,050 \\
5,130\end{array}$ & $\begin{array}{l}4,560 \\
4,350 \\
4,810 \\
4,750 \\
4,270\end{array}$ & $\begin{array}{l}5,220 \\
4,400 \\
5,240 \\
6,580 \\
6,190\end{array}$ & $\begin{array}{l}6,610 \\
6,130 \\
6,200 \\
6,240 \\
6,160\end{array}$ & $\begin{array}{l}5,790 \\
7,650 \\
8,260 \\
7,260 \\
6,210\end{array}$ & $\begin{array}{l}12,000 \\
12,200 \\
12,700 \\
12,800 \\
13,200\end{array}$ & $\begin{array}{l}18,200 \\
18,200 \\
18,200 \\
18,000 \\
17,900\end{array}$ & $\begin{array}{l}38,000 \\
38,100 \\
38,900 \\
39,500 \\
40,300\end{array}$ & $\begin{array}{l}43,900 \\
42,400 \\
41,600 \\
40,300 \\
39,400\end{array}$ & $\begin{array}{l}14,500 \\
13,200 \\
13,300 \\
15,000 \\
16,300\end{array}$ & $\begin{array}{l}24,200 \\
24,500 \\
26,000 \\
26,200 \\
25,200\end{array}$ \\
\hline $\begin{array}{l}26 \\
27 \\
28 \\
29 \\
30 \\
31\end{array}$ & $\begin{array}{l}6,100 \\
5,130 \\
5,070 \\
4,950 \\
4,850 \\
4,930\end{array}$ & $\begin{array}{r}5,130 \\
4,690 \\
4,710 \\
4,510 \\
4,680 \\
\end{array}$ & $\begin{array}{l}4,420 \\
4,510 \\
4,490 \\
4,490 \\
4,710 \\
4,430\end{array}$ & $\begin{array}{l}5,530 \\
6,020 \\
5,580 \\
4,560 \\
4,780 \\
5,370\end{array}$ & $\begin{array}{r}4,540 \\
6,810 \\
5,830 \\
5,460 \\
\\
\end{array}$ & $\begin{array}{l}7,020 \\
7,120 \\
6.030 \\
5,500 \\
7.220 \\
7,640\end{array}$ & $\begin{array}{l}12,800 \\
12,700 \\
12,800 \\
13,500 \\
13,600\end{array}$ & $\begin{array}{l}17,700 \\
17,400 \\
17,400 \\
17,700 \\
19,600 \\
20,800\end{array}$ & $\begin{array}{l}39,800 \\
39,200 \\
38,300 \\
37,500 \\
37,100\end{array}$ & $\begin{array}{l}38,900 \\
38,400 \\
37,900 \\
37,400 \\
36,900 \\
36,400\end{array}$ & $\begin{array}{l}17,700 \\
17,800 \\
17,300 \\
16,600 \\
15,900 \\
15,400\end{array}$ & $\begin{array}{l}23,400 \\
21,600 \\
20,200 \\
18,900 \\
17,800 \\
\end{array}$ \\
\hline $\begin{array}{l}\text { TOTAL } \\
\text { MEAN } \\
\text { MAX } \\
\text { MIN } \\
\text { CFSM } \\
\text { IN. }\end{array}$ & $\begin{array}{r}230,000 \\
7,419 \\
9,710 \\
4,850 \\
.38 \\
.44\end{array}$ & $\begin{array}{r}145,950 \\
4,865 \\
6,130 \\
4,340 \\
.25 \\
.28\end{array}$ & $\begin{array}{r}144,440 \\
4,659 \\
5,420 \\
4,270 \\
.24 \\
.28\end{array}$ & $\begin{array}{r}170,250 \\
5,492 \\
6,730 \\
4,330 \\
.28 \\
.33\end{array}$ & $\begin{array}{r}172,700 \\
5,955 \\
7,440 \\
4,360 \\
.31 \\
.33\end{array}$ & $\begin{array}{r}167,930 \\
5,417 \\
8,260 \\
3,470 \\
.28 \\
.32\end{array}$ & $\begin{array}{r}337,080 \\
11,240 \\
14,300 \\
7,110 \\
.58 \\
.65\end{array}$ & $\begin{array}{r}519,900 \\
16,770 \\
20,800 \\
11,900 \\
.86 \\
1.00\end{array}$ & $\begin{array}{r}1,099.9 M \\
36,660 \\
43,800 \\
22,500 \\
1.89 \\
2.11\end{array}$ & $\begin{array}{r}1,263.1 \mathrm{M} \\
40,750 \\
46,100 \\
36,400 \\
2.10 \\
2.42\end{array}$ & $\begin{array}{r}786,100 \\
25,360 \\
36,200 \\
13,200 \\
1.31 \\
1.51\end{array}$ & $\begin{array}{r}535,700 \\
17,860 \\
26,200 \\
12,500 \\
.92 \\
1.03\end{array}$ \\
\hline
\end{tabular}

CAL YR 1967 TOTAL 4,053,570 MEAN 11,110 MAX 33,600 MIN 3,160 CFSM .57 IN 7.77

WTR YR 1968 TOTAL $5,573,050$ MEAN 15,230 MAX 46,100 MIN 3,470 
5-1342. Rapid River near Baudette, Minn.

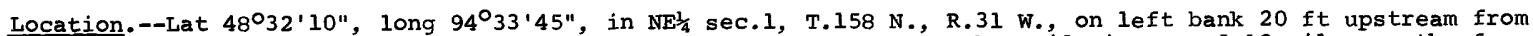
bridge on State Highway 72 , 1.2 miles downstream from North Branch Rapid River, and 12 miles south of Baudette.

Drainage area. $--543 \mathrm{sq} \mathrm{mi}$.

Records available.--October 1956 to september 1968 .

Gage.--Water-stage recorder. Datum of gage is 1,093.92 ft above mean sea level, datum of 1929 (Minnesota Highway Department bench mark).

Average discharge. - - 12 years, $335 \mathrm{cfs}$.

Extremes.--Maximum discharge during year, 4,310 cfs July 17 (gage height, 15.08 ft); minimum, 1.0 cfs Oct. 4. (gage height, $1.50 \mathrm{ft}$ ).

1956-68: Maximum discharge, $5,160 \mathrm{cfs}$ (revised) May 24, 1962 (gage height, $17.13 \mathrm{ft}$ ); minimum, $0.1 \mathrm{cfs}$ Aug. 13, 1961 (gage height, $1.18 \mathrm{ft}$ ).

Flood of May 11, 1950 reached a stage of $21.1 \mathrm{ft}$, from information by local residents and Minnesota Highway Department (discharge, about $7,000 \mathrm{cfs}$, revised).

Remarks.--Records good except those for winter months, which are fair.

DISCHARGE, IN CUBIC FEET PER SECOND, WATER YEAR OCTOBER 1967 TO SEPTEMBER 1968

\begin{tabular}{|c|c|c|c|c|c|c|c|c|c|c|c|c|}
\hline DAY & OCT & NOV & $\mathrm{DEC}$ & JAN & FEB & MAR & APR & MAY & JUN & JUL & AUG & SEP \\
\hline $\begin{array}{l}1 \\
2 \\
3 \\
4 \\
5\end{array}$ & $\begin{array}{l}1.5 \\
1.5 \\
1.4 \\
1.2 \\
1.3\end{array}$ & $\begin{array}{l}2.9 \\
2.9 \\
3.0 \\
3.2 \\
3.2\end{array}$ & $\begin{array}{l}3.6 \\
3.7 \\
3.7 \\
3.7 \\
3.8\end{array}$ & $\begin{array}{l}3.3 \\
3.1 \\
3.0 \\
2.9 \\
2.8\end{array}$ & $\begin{array}{l}2.5 \\
2.4 \\
2.4 \\
2.3 \\
2.3\end{array}$ & $\begin{array}{l}1.9 \\
2.0 \\
2.0 \\
2.1 \\
2.2\end{array}$ & $\begin{array}{l}310 \\
260 \\
220 \\
180 \\
155\end{array}$ & $\begin{array}{l}244 \\
219 \\
200 \\
199 \\
228\end{array}$ & $\begin{array}{l}392 \\
476 \\
446 \\
365 \\
305\end{array}$ & $\begin{array}{l}572 \\
926 \\
916 \\
903 \\
967\end{array}$ & $\begin{array}{l}622 \\
544 \\
495 \\
504 \\
461\end{array}$ & $\begin{array}{l}464 \\
425 \\
422 \\
550 \\
516\end{array}$ \\
\hline $\begin{array}{r}6 \\
7 \\
8 \\
9 \\
10\end{array}$ & $\begin{array}{l}1.6 \\
1.8 \\
2.4 \\
2.6 \\
2.6\end{array}$ & $\begin{array}{l}3.2 \\
2.8 \\
2.8 \\
3.0 \\
3.0\end{array}$ & $\begin{array}{l}3.8 \\
3.7 \\
3.7 \\
3.6 \\
3.6\end{array}$ & $\begin{array}{l}2.8 \\
2.7 \\
2.7 \\
2.6 \\
2.6\end{array}$ & $\begin{array}{l}2.3 \\
2.2 \\
2.2 \\
2.1 \\
2.1\end{array}$ & $\begin{array}{l}3.0 \\
6.0 \\
12 \\
14 \\
13\end{array}$ & $\begin{array}{l}140 \\
135 \\
140 \\
180 \\
220\end{array}$ & $\begin{array}{l}219 \\
264 \\
541 \\
603 \\
550\end{array}$ & $\begin{array}{r}662 \\
1.050 \\
1.330 \\
1.430 \\
1.450\end{array}$ & $\begin{array}{l}789 \\
606 \\
498 \\
440 \\
362\end{array}$ & $\begin{array}{l}428 \\
386 \\
350 \\
314 \\
268\end{array}$ & $\begin{array}{l}485 \\
702 \\
970 \\
922 \\
798\end{array}$ \\
\hline $\begin{array}{l}11 \\
12 \\
13 \\
14 \\
15\end{array}$ & $\begin{array}{l}4.2 \\
3.2 \\
2.4 \\
1.8 \\
1.5\end{array}$ & $\begin{array}{l}3.0 \\
3.4 \\
3.4 \\
3.2 \\
3.1\end{array}$ & $\begin{array}{l}3.6 \\
3.6 \\
3.6 \\
3.7 \\
3.7\end{array}$ & $\begin{array}{l}2.6 \\
2.5 \\
2.5 \\
2.5 \\
2.5\end{array}$ & $\begin{array}{l}2.1 \\
2.0 \\
2.0 \\
1.9 \\
1.9\end{array}$ & $\begin{array}{l}10 \\
9.0 \\
7.5 \\
6.3 \\
6.0\end{array}$ & $\begin{array}{l}214 \\
211 \\
187 \\
175 \\
195\end{array}$ & $\begin{array}{l}476 \\
446 \\
422 \\
374 \\
336\end{array}$ & $\begin{array}{l}1.890 \\
1.880 \\
1.650 \\
1.740 \\
1.670\end{array}$ & $\begin{array}{l}669 \\
907 \\
755 \\
808 \\
926\end{array}$ & $\begin{array}{l}228 \\
195 \\
173 \\
159 \\
148\end{array}$ & $\begin{array}{l}681 \\
569 \\
464 \\
401 \\
347\end{array}$ \\
\hline $\begin{array}{l}16 \\
17 \\
18 \\
19 \\
20\end{array}$ & $\begin{array}{l}1.5 \\
1.8 \\
2.0 \\
2.2 \\
2.4\end{array}$ & $\begin{array}{l}3.3 \\
3.4 \\
3.5 \\
3.5 \\
3.4\end{array}$ & $\begin{array}{l}3.8 \\
3.8 \\
3.9 \\
4.0 \\
4.0\end{array}$ & $\begin{array}{l}2.5 \\
2.5 \\
2.5 \\
2.6 \\
2.6\end{array}$ & $\begin{array}{l}1.9 \\
1.8 \\
1.8 \\
1.8 \\
1.8\end{array}$ & $\begin{array}{l}6.5 \\
11 \\
17 \\
50 \\
40\end{array}$ & $\begin{array}{l}246 \\
252 \\
234 \\
214 \\
211\end{array}$ & $\begin{array}{l}311 \\
282 \\
264 \\
246 \\
225\end{array}$ & $\begin{array}{r}1.340 \\
1.100 \\
973 \\
870 \\
730\end{array}$ & $\begin{array}{l}2.270 \\
4.530 \\
4.540 \\
4.100 \\
3.600\end{array}$ & $\begin{array}{l}194 \\
248 \\
234 \\
236 \\
266\end{array}$ & $\begin{array}{l}324 \\
588 \\
901 \\
882 \\
820\end{array}$ \\
\hline $\begin{array}{l}21 \\
22 \\
23 \\
24 \\
25\end{array}$ & $\begin{array}{l}2.8 \\
2.7 \\
2.7 \\
2.8 \\
2.8\end{array}$ & $\begin{array}{l}3.3 \\
3.3 \\
3.2 \\
3.2 \\
3.2\end{array}$ & $\begin{array}{l}3.9 \\
3.8 \\
3.7 \\
3.6 \\
3.6\end{array}$ & $\begin{array}{l}2.7 \\
2.7 \\
2.8 \\
2.8 \\
2.8\end{array}$ & $\begin{array}{l}1.8 \\
1.8 \\
1.8 \\
1.8 \\
1.8\end{array}$ & $\begin{array}{l}38 \\
34 \\
32 \\
33 \\
60\end{array}$ & $\begin{array}{l}268 \\
350 \\
374 \\
470 \\
510\end{array}$ & $\begin{array}{l}207 \\
202 \\
183 \\
159 \\
147\end{array}$ & $\begin{array}{l}712 \\
995 \\
992 \\
935 \\
789\end{array}$ & $\begin{array}{l}2.970 \\
2.480 \\
1.920 \\
1.490 \\
1.220\end{array}$ & $\begin{array}{r}240 \\
356 \\
736 \\
986 \\
1060\end{array}$ & $\begin{array}{r}718 \\
817 \\
1.760 \\
1.680 \\
1.430\end{array}$ \\
\hline $\begin{array}{l}26 \\
27 \\
28 \\
29 \\
30 \\
31\end{array}$ & $\begin{array}{l}2.8 \\
2.9 \\
3.0 \\
2.9 \\
2.7 \\
2.5\end{array}$ & $\begin{array}{l}3.2 \\
3.2 \\
3.3 \\
3.4 \\
3.5 \\
\end{array}$ & $\begin{array}{l}3.5 \\
3.5 \\
3.5 \\
3.4 \\
3.4 \\
3.3\end{array}$ & $\begin{array}{l}2.7 \\
2.7 \\
2.7 \\
2.6 \\
2.6 \\
2.5\end{array}$ & $\begin{array}{r}1.8 \\
1.8 \\
1.8 \\
1.9 \\
\\
\end{array}$ & $\begin{array}{l}150 \\
350 \\
430 \\
420 \\
405 \\
360\end{array}$ & $\begin{array}{r}455 \\
404 \\
350 \\
305 \\
278 \\
\end{array}$ & $\begin{array}{l}132 \\
129 \\
145 \\
181 \\
242 \\
278\end{array}$ & $\begin{array}{r}643 \\
519 \\
425 \\
362 \\
330\end{array}$ & $\begin{array}{r}1.140 \\
1.110 \\
935 \\
789 \\
696 \\
681\end{array}$ & $\begin{array}{l}963 \\
829 \\
708 \\
616 \\
538 \\
488\end{array}$ & $\begin{array}{r}1120 \\
922 \\
774 \\
662 \\
584 \\
\end{array}$ \\
\hline Total & 71.5 & 96.0 & 113.8 & 83.4 & 58.1 & 2.533 .5 & 7.843 & 8654 & 28451 & 45515 & 13.973 & 226698 \\
\hline $\begin{array}{l}\text { Mean } \\
\text { Max } \\
\text { Min } \\
\text { Cfsm } \\
\text { In. } \\
\end{array}$ & $\begin{array}{r}2.31 \\
4.2 \\
1.2 \\
.0043 \\
.005 \\
\end{array}$ & $\begin{array}{r}3.20 \\
3.5 \\
2.8 \\
.0059 \\
.007 \\
\end{array}$ & $\begin{array}{r}3.67 \\
4.0 \\
3.3 \\
.0068 \\
.008 \\
\end{array}$ & $\begin{array}{r}2.69 \\
3.3 \\
2.5 \\
.0050 \\
.006 \\
\end{array}$ & $\begin{array}{r}2.00 \\
2.5 \\
1.8 \\
.0037 \\
.004 \\
\end{array}$ & $\begin{array}{r}81.7 \\
430 \\
1.9 \\
.150 \\
.17 \\
\end{array}$ & $\begin{array}{r}261 \\
510 \\
135 \\
.481 \\
.54 \\
\end{array}$ & $\begin{array}{r}279 \\
603 \\
129 \\
.514 \\
0.59 \\
\end{array}$ & $\begin{array}{r}948 \\
1,890 \\
305 \\
1.75 \\
1.95\end{array}$ & $\begin{array}{r}1,470 \\
4,540 \\
362 \\
2.71 \\
3.12\end{array}$ & $\begin{array}{r}451 \\
1,060 \\
148 \\
.831 \\
0.96\end{array}$ & $\begin{array}{r}757 \\
1,760 \\
324 \\
1.39 \\
1.55\end{array}$ \\
\hline $\begin{array}{l}\text { Cal yr } \\
\text { wtr yr }\end{array}$ & & $\begin{array}{l}\text { Total } \\
\text { Total }\end{array}$ & $\begin{array}{l}430.4 \\
090.3\end{array}$ & $\begin{array}{l}\text { Mean } \\
\text { Mean }\end{array}$ & $\begin{array}{l}349 \\
356\end{array}$ & $\begin{array}{l}3,310 \\
4,540\end{array}$ & $\begin{array}{l}\text { Min } \\
\text { Min }\end{array}$ & $\begin{array}{l}1.2 \\
1.2\end{array}$ & $\begin{array}{l}\mathrm{fsm} \\
\mathrm{fsm}\end{array}$ & $\begin{array}{l}\text { In } \\
\text { In }\end{array}$ & $\begin{array}{l}8.73 \\
8.91\end{array}$ & \\
\hline
\end{tabular}


5-1395. Warroad River near Warroad, Minn.

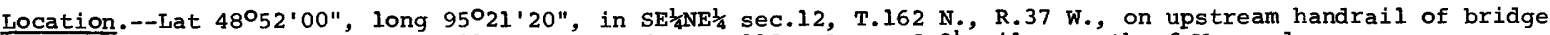
near center of span, half a mile upstream from Bulldog Run and $2 \frac{1}{2}$ miles south of Warroad.

Drainage area.--110 sq $\mathrm{mi}$, approximately.

Records available.--March 1946 to September 1968. Monthly discharge only for some periods, published in wsP 1308 .

Gage.--Wire-weight gage read once daily, and crest-stage gage. Datum of gage is $1,070.74$ ft above mean sea level, datum of 1929 (levels by Stanley Johnson, consulting engineer and instructor at University of North Dakota). Prior to Sept. 6, 1967, chain gage at same site and datum.

Average discharge. --22 years, $43.6 \mathrm{cfs}$.

Extremes.--Maximum discharge during year, 1,530 cfs June 11 (gage height, 9.32 ft); no flow Oct. $1-11$. 1946-68: Maximum discharge, 1,780 cfs Apr. 15, 1965 (gage height, 9.95 ft); no flow at times,

Remarks.--Records good except those for winter months, which are fair.

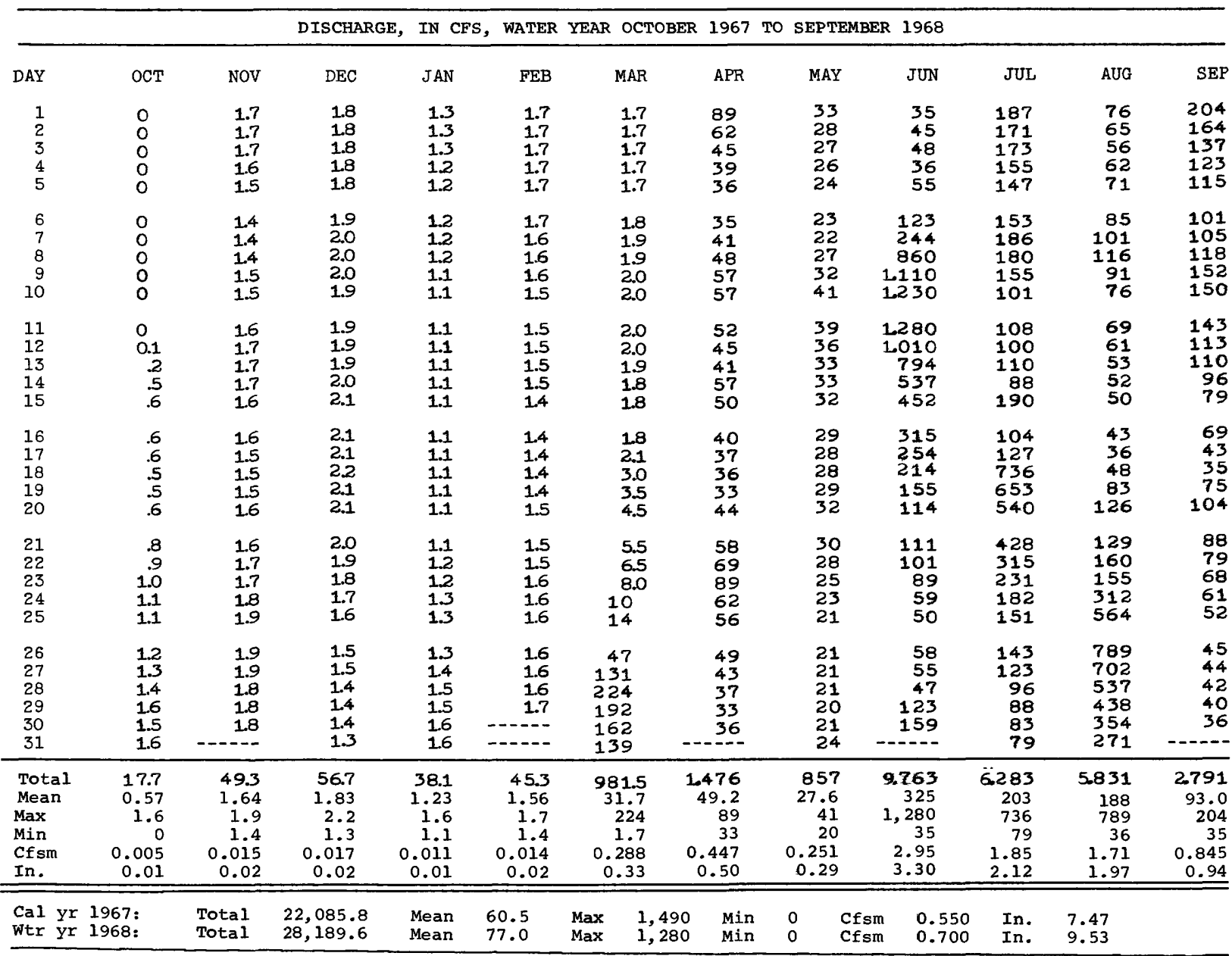


5-1400. Bulldog Run near Warroad, Minn.

Location.--Lat 48051'30", long 95020'20", in SE⿺ sec.7, T.162 N., R.36 w., near right bank 5 ft downstream from culvert on county highway, three-quarters of a mile upstream from mouth and $3 \mathrm{miles}$ south of warroad.

Drainage area. $--14.2 \mathrm{sq} \mathrm{mi}$.

Records available.--March 1946 to November 1951, June 1966 to September 1968.

Gage.--Staff gage read once daily. Altitude of gage is 1,090 ft (from topographic map).

Average discharge. --7 years $(1946-51,1967-68), 3.87 \mathrm{cfs}$.

Extremes.--Maximum discharge during year, 171 cfs June 8 (gage height, 6.06 ft, from floodmark); no flow for many days .

1946-51, 1966-68: Maximum discharge, 420 cfs June 10, 1947 (gage height, 6.91 ft); no flow for many days in most years.

Remarks.--Records fair.

DISCHARGE, IN CFS, WATER YEAR OCTOBER 1967 TO SEPTEMBER 1968

\begin{tabular}{|c|c|c|c|c|c|c|c|c|c|c|c|c|}
\hline OCT & NOV & DEC & & JAN & FEB & MAR & APR & MAY & JUN & JUL & AUG & SEP \\
\hline $\begin{array}{l}1 \\
2 \\
3 \\
4 \\
5\end{array}$ & & & & & $:$ & $\begin{array}{l}0 \\
0 \\
0 \\
0 \\
0\end{array}$ & $\begin{array}{l}6.4 \\
4.5 \\
3.9 \\
3.7 \\
3.2\end{array}$ & $\begin{array}{l}0.2 \\
.1 \\
.1 \\
0 \\
0\end{array}$ & $\begin{array}{l}0 \\
0 \\
0 \\
0 \\
0\end{array}$ & $\begin{array}{c}5.5 \\
24 \\
5.5 \\
3.2 \\
4.7\end{array}$ & $\begin{array}{r}1.1 \\
.9 \\
.6 \\
.8 \\
1.1\end{array}$ & $\begin{array}{r}3.8 \\
2.3 \\
.8 \\
.6 \\
.6\end{array}$ \\
\hline $\begin{array}{r}6 \\
7 \\
8 \\
9 \\
10\end{array}$ & & & & & & $\begin{array}{l}0 \\
0 \\
0 \\
0 \\
0\end{array}$ & $\begin{array}{l}2.9 \\
2.9 \\
3.3 \\
3.5 \\
3.0\end{array}$ & $\begin{array}{l}0 \\
0 \\
.1 \\
.1\end{array}$ & $\begin{aligned} & 1.8 \\
& 48 \\
& 131 \\
& 35 \\
& 52\end{aligned}$ & $\begin{array}{r}3.2 \\
1.8 \\
1.2 \\
1.0 \\
.7\end{array}$ & $\begin{array}{r}1.3 \\
1.0 \\
.6 \\
.3 \\
.1\end{array}$ & $\begin{array}{l}.6 \\
4 \\
.4 \\
.4 \\
2\end{array}$ \\
\hline $\begin{array}{l}11 \\
12 \\
13 \\
14 \\
15\end{array}$ & & & & & . & $\begin{array}{l}0 \\
0 \\
0 \\
0 . \\
0\end{array}$ & $\begin{array}{l}2.9 \\
2.5 \\
2.0 \\
2.0 \\
1.8\end{array}$ & $\begin{array}{l}0 \\
0 \\
0 \\
0 \\
0\end{array}$ & $\begin{array}{l}82 \\
44 \\
20 \\
9.5 \\
5.5\end{array}$ & $\begin{array}{r}.7 \\
.7 \\
1.8 \\
1.8 \\
2.2\end{array}$ & $\begin{array}{l}0 \\
0 \\
0 \\
0 \\
0\end{array}$ & $\begin{array}{l}2 \\
.2 \\
.1 \\
0\end{array}$ \\
\hline $\begin{array}{l}16 \\
17 \\
18 \\
19 \\
20\end{array}$ & & & & & & $\begin{array}{l}0 \\
0 \\
0 \\
0 \\
0\end{array}$ & $\begin{array}{l}1.7 \\
1.3 \\
1.2 \\
1.2 \\
1.0\end{array}$ & $\begin{array}{l}0 \\
0 \\
0 \\
0 \\
0\end{array}$ & $\begin{array}{l}4.6 \\
3.7 \\
2.6 \\
1.8 \\
1.1\end{array}$ & $\begin{array}{l}5.7 \\
50 \\
27 \\
11 \\
8.4\end{array}$ & $\begin{array}{r}.1 \\
.4 \\
.4 \\
.7 \\
1.6\end{array}$ & $\begin{array}{l}0 \\
0 \\
.1 \\
2 \\
2\end{array}$ \\
\hline $\begin{array}{l}21 \\
22 \\
23 \\
24 \\
25\end{array}$ & & & & & & $\begin{array}{l}0 \\
0 \\
2.2 \\
7.2 \\
11\end{array}$ & $\begin{array}{r}1.9 \\
1.7 \\
1.4 \\
1.0 \\
.7\end{array}$ & $\begin{array}{l}0 \\
.1 \\
.1 \\
0 \\
0\end{array}$ & $\begin{array}{l}1.1 \\
1.1 \\
1.0 \\
1.0 \\
2.1\end{array}$ & $\begin{array}{l}8.2 \\
6.8 \\
6.0 \\
5.5 \\
5.0\end{array}$ & $\begin{array}{r}1.3 \\
1.5 \\
2.7 \\
85 \\
106\end{array}$ & $\begin{array}{l}.2 \\
.2 \\
.2 \\
.1 \\
.1\end{array}$ \\
\hline $\begin{array}{l}26 \\
27 \\
28 \\
29 \\
30 \\
31\end{array}$ & 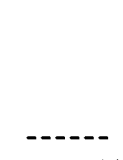 & & & & ----- & $\begin{array}{l}18 \\
50 \\
61 \\
43 \\
32 \\
11\end{array}$ & $\begin{array}{r}.5 \\
.4 \\
.3 \\
.2 \\
.2 \\
-\end{array}$ & $\begin{array}{l}0 \\
0 \\
0 \\
0 \\
0 \\
0\end{array}$ & $\begin{array}{r}1.8 \\
.9 \\
1.4 \\
1.9 \\
1.1\end{array}$ & $\begin{array}{l}3.5 \\
2.5 \\
2.0 \\
1.6 \\
1.3 \\
1.2\end{array}$ & $\begin{array}{l}41 \\
22 \\
10 \\
7.8 \\
6.4 \\
4.9\end{array}$ & $\begin{array}{c}0 \\
0 \\
0 \\
0 \\
0 \\
--\end{array}$ \\
\hline $\begin{array}{l}\text { Total } \\
\text { Mean } \\
\text { Max } \\
\text { Min } \\
\text { Cfsm } \\
\text { In. }\end{array}$ & & & & & & $\begin{array}{r}235.4 \\
7.59 \\
61 \\
0 \\
0.535\end{array}$ & $\begin{array}{r}63.2 \\
2.11 \\
6.4 \\
0.2 \\
0.149 \\
0.17\end{array}$ & $\begin{array}{r}0.8 \\
0.03 \\
0.2 \\
0 \\
0.0021 \\
0.002 \\
\end{array}$ & $\begin{array}{r}456.0 \\
15.2 \\
131 \\
0 \\
1.07 \\
1.19 \\
\end{array}$ & $\begin{array}{r}203.7 \\
6.57 \\
50 \\
0.7 \\
0.463 \\
0.53\end{array}$ & $\begin{array}{r}299.6 \\
9.66 \\
106 \\
0 \\
0.680 \\
0.78\end{array}$ & $\begin{array}{r}11.9 \\
0.40 \\
3.8 \\
0 \\
0.0028 \\
0.003 \\
\end{array}$ \\
\hline $\begin{array}{l}\text { Cal yr 1967: } \\
\text { wtr yr 1968: }\end{array}$ & $\begin{array}{l}\text { Total } \\
\text { Total }\end{array}$ & $\begin{array}{l}1,273 \\
1,270.6\end{array}$ & $\begin{array}{l}\text { Mean } \\
\text { Mean }\end{array}$ & $\begin{array}{l}3.49 \\
3.47\end{array}$ & $\begin{array}{l}\text { Max } \\
\operatorname{Max}\end{array}$ & $\begin{array}{l}305 \\
131\end{array}$ & $\begin{array}{l}0 \\
0\end{array}$ & $\begin{array}{ll}\mathrm{sm} & 0.246 \\
\mathrm{sm} & 0.244\end{array}$ & $\begin{array}{l}\text { In. } \\
\text { In. }\end{array}$ & $\begin{array}{l}3.33 \\
3.33\end{array}$ & & \\
\hline
\end{tabular}


5-1405. East Branch Warroad River near Warroad, Minn.

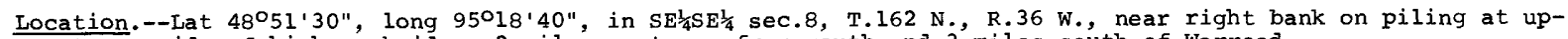
stream side of highway bridge, 2 miles upstream from mouth and 3 miles south of Warroad.

Drainage area.--102 sq $\mathrm{mi}$.

Records available.--March 1946 to September 1954, June 1966 to September 1968 (fragmentary prior to April 1947). Gage.--Staff gage read once daily. Altitude of gage is $1,080 \mathrm{ft}$ (from topographic map).

Average discharge. --9 years $(1947-54,1967-68), 22.6$ cfs.

Extremes.--Maximum discharge during year, $430 \mathrm{cfs}$ June 11 (gage height, $8.40 \mathrm{ft}$, from graph based on gage readings); maximum gage height, $8.60 \mathrm{ft}$ Aug. 25 (from graph based on gage readings, backwater from aquatic growth); no flow for many days.

1946-54, 1966-68: Maximum discharge, 1, $340 \mathrm{cfs} J u n e ~ 11,1947$ (gage height $9.36 \mathrm{ft}$, from floodmark); no flow at times in most years.

Remarks.--Records fair.

DISCHARGE, IN CFS, WATER YEAR OCTOBER 1967 TO SEPTEMBER 1968

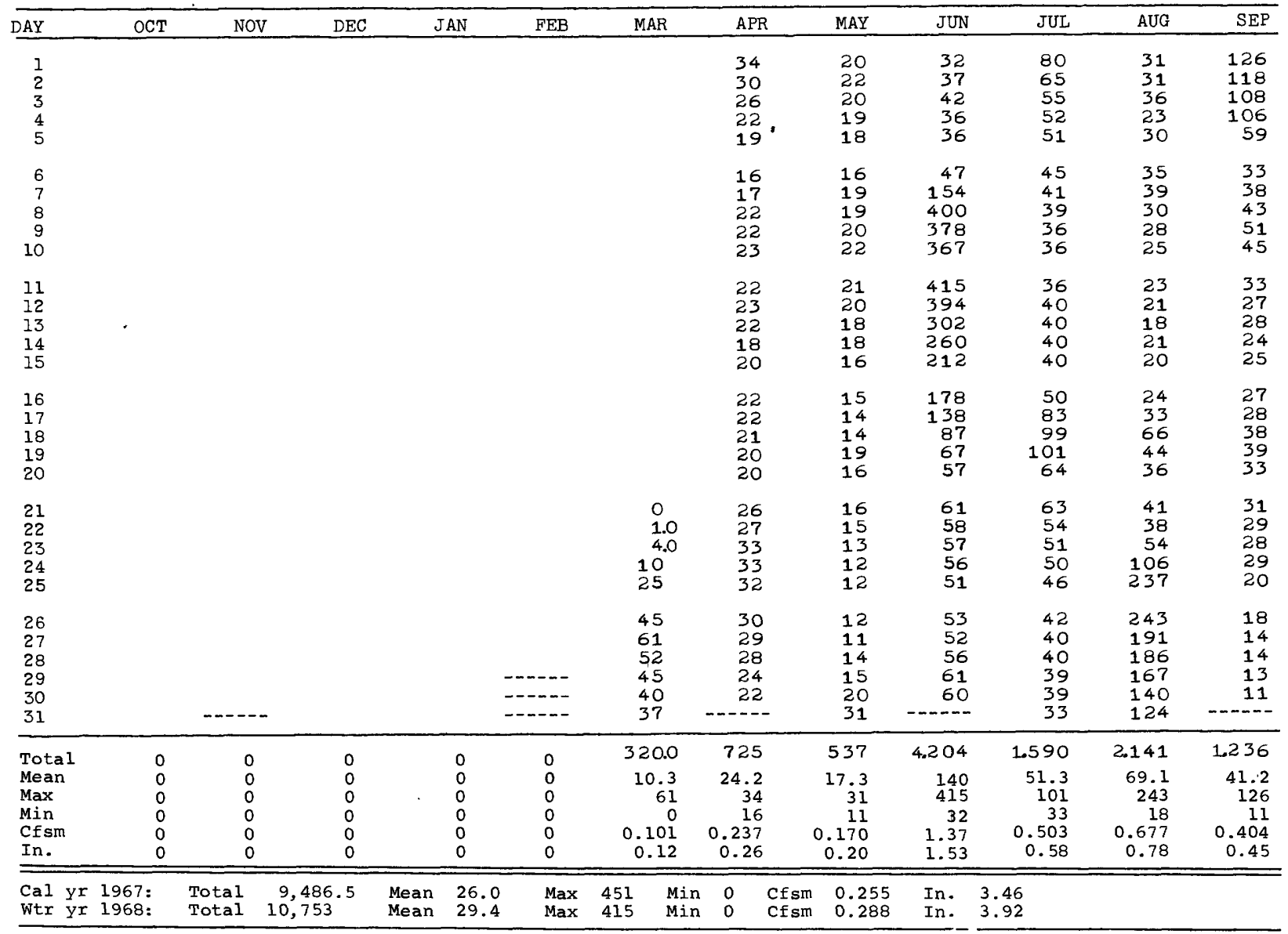


MISSISSIPPI RIVER MAIN STEM

5-2010. Winnibigoshish Lake near Deer River, Minn.

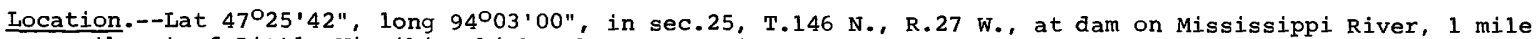
northwest of Little Winnibigoshish Lake and 14 miles northwest of town of Deer River.

Drainage area. $-1,442 \mathrm{sq} \mathrm{mi}$.

Records available.--April 1884 to September 1968. Prior to October 1941 month-end contents only, published in WSP 1308. Published as Winnibigoshish Reservoir near Deer River October 1941 to September 1956.

Gage.-Water-stage recorder. Datum of gage is 1,289.47 ft above mean sea level, adjustment of 1912 . Prior to July 8, 1949, staff gage at same site and datum.

Extremes:--Maximum contents during year, 622,800 acre-ft July 1 (gage height, 10.82 ft); minimum, 437,800 acre-ft Mar. 1, 3, 4, 7, 8, 17 (gage height, $8.06 \mathrm{ft}$ ).

1884-1968: 'Maximum contents observed, 996,500 acre-ft July 30, 1905 (gage height, 14.45 ft); minimum observed, 33,680 acre-ft below zero of capacity table oct. 20, 1931 (gage height, -0.69 ft).

Remarks.--Reservoir is formed by winnibigoshish Lake and several other natural lakes controlled by a concrete and timber dam, completed in 1884; storage began in 1884. Capacity between gage heights $6.00 \mathrm{ft}$ and 14.2 $\mathrm{ft}$ (maximum allowable range) is 653,570 acre-ft of which 416,270 acre-ft is controlled storage between gage heights $6.00 \mathrm{ft}$ and $12.0 \mathrm{ft}$ (normal operating range). Contents shown herein are contents above gage height $0.00 \mathrm{ft}$. Water is used to benefit navigation on Mississippi River below Minneapolis.

Cooperation.--Records furnished by Corps of Engineers in terms of cfs-days and converted to acre-feet by Geological survey.

Month-end gage height and contents, water year october 1967 to september 1968

\begin{tabular}{|c|c|c|c|c|}
\hline & & $\begin{array}{l}\text { Gage height } \\
\text { (feet) }\end{array}$ & $\begin{array}{l}\text { Contents } \\
\text { (acre-feet) }\end{array}$ & $\begin{array}{c}\text { Change in contents } \\
\text { (acre-feet) }\end{array}$ \\
\hline $\begin{array}{l}\text { Sept. } \\
\text { Oct. } \\
\text { Nov. } \\
\text { Dec. }\end{array}$ & 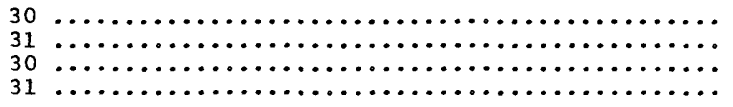 & $\begin{array}{l}8.79 \\
8.35 \\
8.24 \\
8.26\end{array}$ & $\begin{array}{l}485,800 \\
456,800 \\
449,500 \\
450,800\end{array}$ & $\begin{array}{r}- \\
-29,000 \\
-7,300 \\
+1,300\end{array}$ \\
\hline \multicolumn{2}{|r|}{ 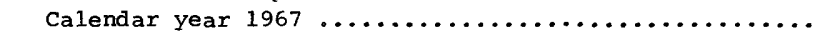 } & - & - & $-35,200$ \\
\hline \multirow{2}{*}{\multicolumn{2}{|c|}{ 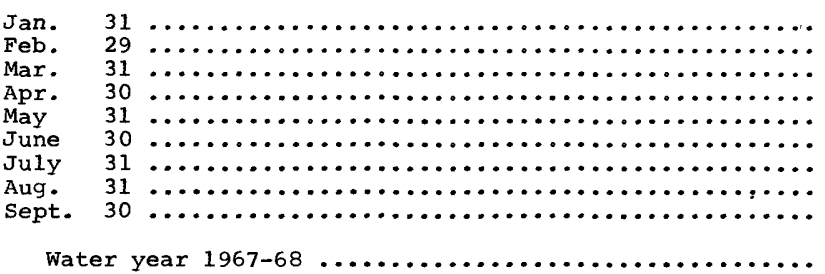 }} & $\begin{array}{r}8.20 \\
8.07 \\
8.31 \\
8.85 \\
9.80 \\
10.60 \\
10.19 \\
9.87 \\
9.90\end{array}$ & $\begin{array}{l}446,900 \\
438,300 \\
454,200 \\
489,500 \\
551,800 \\
607,500 \\
578,800 \\
556,600 \\
558,500\end{array}$ & $\begin{array}{l}-3,900 \\
-8,600 \\
+15,900 \\
+35,300 \\
+62,300 \\
+55,700 \\
-28,700 \\
-22,200 \\
+1,900\end{array}$ \\
\hline & & - & - & $+72,700$ \\
\hline
\end{tabular}


5-2015. Mississippi River at Winnibigoshish Dam near Deer River, Minn.

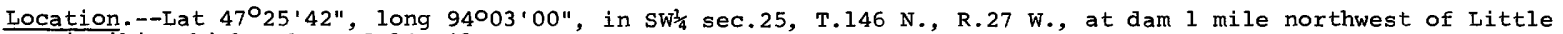
Winnibigoshish Lake and 14 miles northwest of town of Deer River.

Drainage area.--1, $442 \mathrm{sq} \mathrm{mi}$.

Records available.--May 1884 to September 1968. Monthly discharge only for some periods, published in wSP 1308 .

Gage.--Water-stage recorder on headwater and staff gage on tailwater. Tailwater gage read twice daily. Datum of gage is $1,289.47 \mathrm{ft}$ above mean sea level, adjustment of 1912 . Prior to July 8 , 1949, staff headwater gage at same site and datur.

Average discharge.--84 years, $504 \mathrm{cfs}$, unadjusted.

Extremes.--Maximum daily discharge during year, 1, 010 cfs July 4, 12, 25-27; minimum daily, 100 cfs Mar. 27 to Apr. 22.

1884-1968: Maximum daily discharge, 4,370 cfs Aug. 6, 1905; no flow at times in several years.

Remarks.-Daily discharge is computed on the basis of modified weir formula and corrected to conform with discharge measurements, the head being determined from readings of headwater and tailwater gages. Flow completely regulated by Winnibigoshish Lake (see preceding page).

Cooperation.--Computations of daily discharge furnished by Corps of Engineers; two discharge measurements made and records reviewed by Geological Survey.

DISCHARGE, IN CFS, WATER YEAR OCTOBER 1967 TO SEPTEMBER 1968

\begin{tabular}{|c|c|c|c|c|c|c|c|c|c|c|c|c|}
\hline DAY & OCT & NOV & DEC & JAN & FEB & MAR & APR & MAY & JUN & JUL & AUG & SEP \\
\hline $\begin{array}{l}1 \\
2 \\
3 \\
4 \\
5\end{array}$ & $\begin{array}{l}369 \\
443 \\
455 \\
455 \\
460\end{array}$ & $\begin{array}{l}491 \\
491 \\
496 \\
496 \\
496\end{array}$ & $\begin{array}{l}490 \\
490 \\
490 \\
490 \\
490\end{array}$ & $\begin{array}{l}490 \\
490 \\
489 \\
489 \\
489\end{array}$ & $\begin{array}{l}525 \\
525 \\
540 \\
540 \\
540\end{array}$ & $\begin{array}{l}412 \\
415 \\
415 \\
415 \\
415\end{array}$ & $\begin{array}{l}100 \\
100 \\
100 \\
100 \\
100\end{array}$ & $\begin{array}{l}103 \\
103 \\
104 \\
104 \\
104\end{array}$ & $\begin{array}{l}106 \\
106 \\
106 \\
106 \\
107\end{array}$ & $\begin{array}{r}905 \\
900 \\
900 \\
1.010 \\
1000\end{array}$ & $\begin{array}{l}803 \\
802 \\
605 \\
532 \\
526\end{array}$ & $\begin{array}{l}306 \\
306 \\
306 \\
306 \\
306\end{array}$ \\
\hline $\begin{array}{r}6 \\
7 \\
8 \\
9 \\
10\end{array}$ & $\begin{array}{l}478 \\
497 \\
506 \\
506 \\
505\end{array}$ & $\begin{array}{l}491 \\
491 \\
491 \\
496 \\
491\end{array}$ & $\begin{array}{l}490 \\
495 \\
490 \\
490 \\
491\end{array}$ & $\begin{array}{l}480 \\
480 \\
480 \\
480 \\
480\end{array}$ & $\begin{array}{l}540 \\
540 \\
535 \\
535 \\
525\end{array}$ & $\begin{array}{l}415 \\
415 \\
415 \\
417 \\
417\end{array}$ & $\begin{array}{l}100 \\
100 \\
100 \\
100 \\
100\end{array}$ & $\begin{array}{l}103 \\
103 \\
104 \\
104 \\
104\end{array}$ & $\begin{array}{l}107 \\
107 \\
107 \\
107 \\
107\end{array}$ & $\begin{array}{r}1.000 \\
1000 \\
1.000 \\
1000 \\
995\end{array}$ & $\begin{array}{l}502 \\
502 \\
507 \\
511 \\
511\end{array}$ & $\begin{array}{l}306 \\
306 \\
306 \\
306 \\
306\end{array}$ \\
\hline $\begin{array}{l}11 \\
12 \\
13 \\
14 \\
15\end{array}$ & $\begin{array}{l}505 \\
493 \\
505 \\
505 \\
493\end{array}$ & $\begin{array}{l}491 \\
496 \\
496 \\
496 \\
491\end{array}$ & $\begin{array}{l}491 \\
495 \\
495 \\
495 \\
491\end{array}$ & $\begin{array}{l}480 \\
480 \\
480 \\
480 \\
480\end{array}$ & $\begin{array}{l}525 \\
525 \\
525 \\
520 \\
520\end{array}$ & $\begin{array}{l}417 \\
417 \\
417 \\
415 \\
415\end{array}$ & $\begin{array}{l}100 \\
100 \\
100 \\
100 \\
100\end{array}$ & $\begin{array}{l}104 \\
104 \\
104 \\
104 \\
105\end{array}$ & $\begin{array}{l}107 \\
107 \\
107 \\
108 \\
108\end{array}$ & $\begin{array}{r}990 \\
1.010 \\
1000 \\
1000 \\
1000\end{array}$ & $\begin{array}{l}511 \\
512 \\
516 \\
300 \\
300\end{array}$ & $\begin{array}{l}306 \\
306 \\
306 \\
306 \\
303\end{array}$ \\
\hline $\begin{array}{l}16 \\
17 \\
18 \\
19 \\
20\end{array}$ & $\begin{array}{l}493 \\
497 \\
505 \\
492 \\
506\end{array}$ & $\begin{array}{l}491 \\
496 \\
496 \\
496 \\
491\end{array}$ & $\begin{array}{l}491 \\
495 \\
495 \\
495 \\
495\end{array}$ & $\begin{array}{l}489 \\
530 \\
525 \\
525 \\
525\end{array}$ & $\begin{array}{l}525 \\
525 \\
520 \\
520 \\
515\end{array}$ & $\begin{array}{l}310 \\
315 \\
315 \\
315 \\
310\end{array}$ & $\begin{array}{l}100 \\
100 \\
100 \\
100 \\
100\end{array}$ & $\begin{array}{l}105 \\
105 \\
105 \\
105 \\
105\end{array}$ & $\begin{array}{l}108 \\
166 \\
382 \\
565 \\
675\end{array}$ & $\begin{array}{r}1000 \\
1000 \\
1.000 \\
1000 \\
996\end{array}$ & $\begin{array}{l}300 \\
300 \\
300 \\
300 \\
300\end{array}$ & $\begin{array}{l}303 \\
303 \\
306 \\
306 \\
306\end{array}$ \\
\hline $\begin{array}{l}21 \\
22 \\
23 \\
24 \\
25\end{array}$ & $\begin{array}{l}495 \\
493 \\
496 \\
497 \\
497\end{array}$ & $\begin{array}{l}491 \\
496 \\
491 \\
496 \\
491\end{array}$ & $\begin{array}{l}496 \\
496 \\
495 \\
496 \\
496\end{array}$ & $\begin{array}{l}535 \\
535 \\
535 \\
535 \\
535\end{array}$ & $\begin{array}{l}510 \\
510 \\
510 \\
510 \\
515\end{array}$ & $\begin{array}{l}310 \\
192 \\
102 \\
104 \\
104\end{array}$ & $\begin{array}{l}100 \\
100 \\
102 \\
102 \\
102\end{array}$ & $\begin{array}{l}105 \\
105 \\
105 \\
105 \\
106\end{array}$ & $\begin{array}{l}713 \\
707 \\
707 \\
701 \\
701\end{array}$ & $\begin{array}{r}996 \\
1.000 \\
986 \\
986 \\
1010\end{array}$ & $\begin{array}{l}300 \\
300 \\
300 \\
303 \\
306\end{array}$ & $\begin{array}{l}306 \\
306 \\
306 \\
306 \\
300\end{array}$ \\
\hline $\begin{array}{l}26 \\
27 \\
28 \\
29 \\
30 \\
31\end{array}$ & $\begin{array}{l}492 \\
496 \\
491 \\
491 \\
491 \\
492\end{array}$ & $\begin{array}{r}496 \\
492 \\
491 \\
491 \\
490\end{array}$ & $\begin{array}{l}495 \\
494 \\
495 \\
491 \\
490 \\
490\end{array}$ & $\begin{array}{l}525 \\
525 \\
525 \\
525 \\
525 \\
525\end{array}$ & $\begin{array}{r}515 \\
515 \\
400 \\
415 \\
- \\
---\end{array}$ & $\begin{array}{l}104 \\
100 \\
100 \\
100 \\
100 \\
100\end{array}$ & $\begin{array}{r}102 \\
102 \\
103 \\
103 \\
103 \\
-\end{array}$ & $\begin{array}{l}106 \\
106 \\
106 \\
106 \\
106 \\
106\end{array}$ & $\begin{array}{r}850 \\
915 \\
910 \\
910 \\
905 \\
\end{array}$ & $\begin{array}{r}1010 \\
1010 \\
1000 \\
1000 \\
990 \\
935\end{array}$ & $\begin{array}{l}306 \\
306 \\
306 \\
306 \\
306 \\
306\end{array}$ & $\begin{array}{r}300 \\
300 \\
300 \\
300 \\
300 \\
\end{array}$ \\
\hline $\begin{array}{l}\text { Total } \\
\text { Mean } \\
\text { Max } \\
\text { Min } \\
\text { Cfsm } \\
\text { In. }\end{array}$ & $\begin{array}{r}15099 \\
487 \\
506 \\
369 \\
0.338 \\
0.39\end{array}$ & $\begin{array}{r}1.795 \\
493 \\
496 \\
490 \\
0.342 \\
0.38\end{array}$ & $\begin{array}{r}15278 \\
493 \\
496 \\
490 \\
0.342 \\
0.39\end{array}$ & $\begin{array}{r}15.666 \\
505 \\
535 \\
480 \\
0.350 \\
0.40\end{array}$ & $\begin{array}{r}14.965 \\
516 \\
540 \\
400 \\
0.358 \\
0.39\end{array}$ & $\begin{array}{r}9.213 \\
297 \\
417 \\
100 \\
0.206 \\
0.24\end{array}$ & $\begin{array}{r}3.019 \\
101 \\
103 \\
100 \\
0.070 \\
0.08\end{array}$ & $\begin{array}{r}3.244 \\
105 \\
106 \\
103 \\
0.073 \\
0.08\end{array}$ & $\begin{array}{r}11.518 \\
384 \\
915 \\
106 \\
0.266 \\
0.30\end{array}$ & $\begin{array}{r}30.629 \\
988 \\
1,010 \\
900 \\
0.685 \\
0.79\end{array}$ & $\begin{array}{r}12.785 \\
412 \\
803 \\
300 \\
0.286 \\
0.33\end{array}$ & $\begin{array}{r}9.135 \\
304 \\
306 \\
300 \\
0.211 \\
0.24\end{array}$ \\
\hline $\begin{array}{ll}\text { Cal yr } \\
\text { wtr yr }\end{array}$ & $\begin{array}{l}\text { 1967: } \\
1968:\end{array}$ & $\begin{array}{l}\text { Total } \\
\text { Total }\end{array}$ & $\begin{array}{l}221,203 \\
155,346\end{array}$ & $\begin{array}{l}\text { Mean } \\
\text { Mean }\end{array}$ & $\begin{array}{l}\operatorname{Max} \\
\operatorname{Max}\end{array}$ & $\begin{array}{r}935 \\
1,010\end{array}$ & $\begin{array}{ll}\text { Min } & 100 \\
\text { Min } & 100\end{array}$ & $\begin{array}{l}\text { Cfsm } \\
\text { Cfsm }\end{array}$ & $\begin{array}{l}0.420 \\
0.294\end{array}$ & $\begin{array}{ll}\text { In. } & 5.70 \\
\text { In. } & 4.01\end{array}$ & & \\
\hline
\end{tabular}


5-2060. Leech Lake at Federal Dam, Minn.

Location.--Lat $47^{\circ} 12^{\prime} 23^{\prime \prime}$, long $94^{\circ} 18^{\prime} 31^{\prime \prime}$, in lot 2 , sec.14, T.143 N., R. 29 W., at head of Leech Lake River on Waboose Bay, 5 miles southwest of town of Federal $\mathrm{Dam}$.

Drainage area. $--1,163 \mathrm{sq} \mathrm{mi}$.

Records available.--April 1884 to September 1968 . Month-end contents only for some periods, published in wsP 1308. Prior to October 1956, published as "Leech Lake Reservoir".

Gage.--Water-stage recorder. Datum of gage is $1,293.23 \mathrm{ft}$ above mean sea level, adjustment of 1912 . Prior to Dec. 31, 1884 , staff gage half a mile north of outlet to Leech Lake River at datum 5.76 ft lower. Dec. 31, 1884, to May 24, 1931, staff gage half a mile north of outlet to Leech Lake River at present datum. Extremes:--Maximum contents during year, 305, 200 acre-ft July 1 (gage height, 2.17 ft); minimum, 124,500 acre-ft

1884-1968: Maximum contents observed, 734, 300 acre-ft June 30, 1916 (gage height, 5.18 ft); minimum observed, 72,830 acre-ft below zero of capacity table Sept. 30, Nov. 19, 1934, Jan. 9, 1935 (gage height, $-1.18 \mathrm{ft}$ ).

Remarks.--Reservoir is formed by Leech Lake and several other natural lakes controlled by concrete and timber dam; storage began in 1884; original timber structure completed in 1884, replaced by present dam in 1902 . Capacity between gage heights $0.00 \mathrm{ft}$ and $5.24 \mathrm{ft}$ (maximum allowable range) is 689,780 acre-ft, of which 356,570 acre-ft is controlled storage between gage heights 0.00 and $3.00 \mathrm{ft}$ (normal operating range). Contents shown herein are contents above gage height $-0.50 \mathrm{ft}$. Water is used to benefit navigation on Mississippi River below Minneapolis. Cooperation.--Records furnished by Corps of Engineers in terms of cfs-days and converted to acre-feet by Geo-
logical survey.

Month-end gage height and contents, water year October 1967 to September 1968

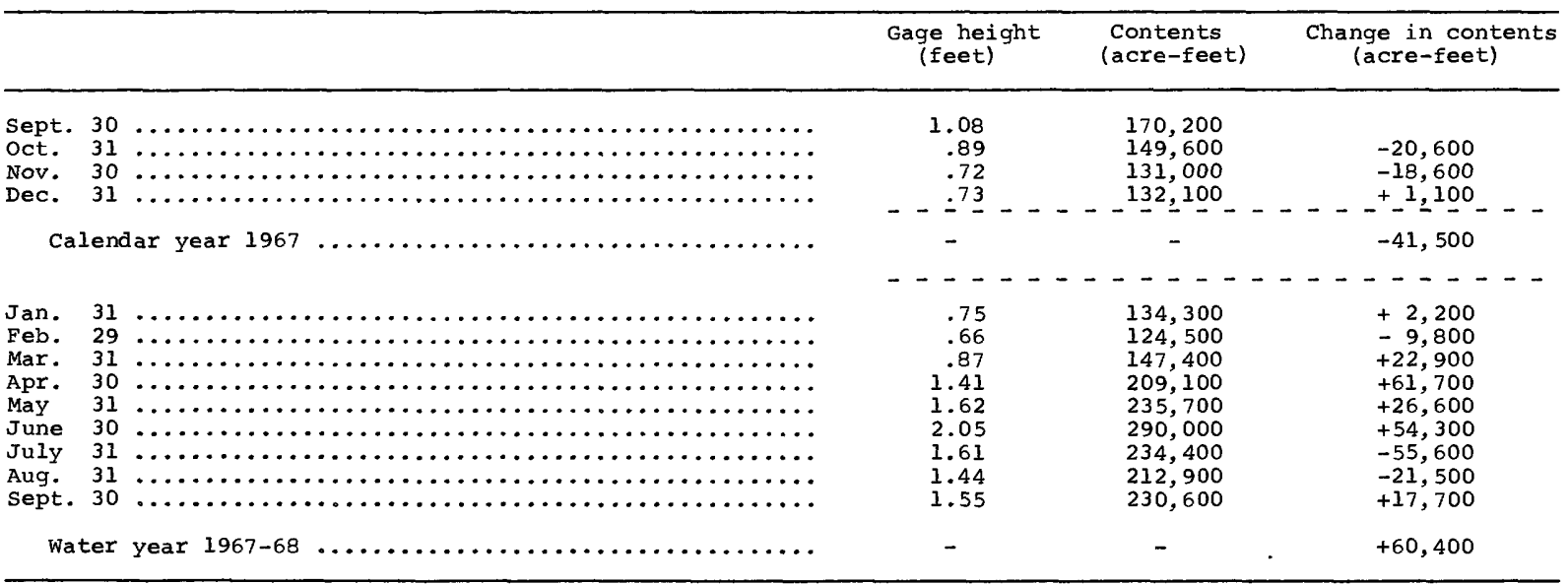


5-2065. Leech Lake River at Federal Dam, Minn.

Location.--Lat $47^{\circ} 14^{\prime} 45^{\prime \prime}$, long $94^{\circ} 13^{\prime} 12^{\prime \prime}$, in sec.29, T.144 N., R. 28 w., on right bank at dam on Leech Lake River at town of Federal Dam, 2 miles downstream from natural outlet of Leech Lake.

Drainage area. $--1,163 \mathrm{sq} \mathrm{mi}$

Records available.--May 1884 to september 1968. Monthly discharge only for some periods, published in wSP 1308 .

Gage.--Water-stage recorder, headwater gage, and staff tailwater gage, read twice daily. Datum of gage is $1,293.23 \mathrm{ft}$ above mean sea level, adjustment of 1912. Prior to July 3, 1948, staff headwater gage at same datum. May 27 to Nov. 30, 1929, staff gage at site 600 ft downstream at different datum.

Average discharge. --84 years, 345 cfs, unadjusted.

Extremes.--Maximum daily discharge during year, 943 cfs July 1; minimum daily, 77 cfs sept. 9.

1884-1968: Maximum daily discharge, 2,520 cfs June 7, 1957 (result of dam failure); no flow at times.

Remarks.-Discharge computed on basis of modified weir formula, the head being obtained from readings on tailwater gage and mean gage height from recording headwater gage. Flow completely regulated by Leech Lake (see preceding page).

Cooperation.--Computations of daily discharge furnished by Corps of Engineers; five discharge measurements made and records reviewed by Geological survey.

DISCHARGE, IN CFS, WATER YEAR OCTOBER 1967 TO SEPTEMBER 1968

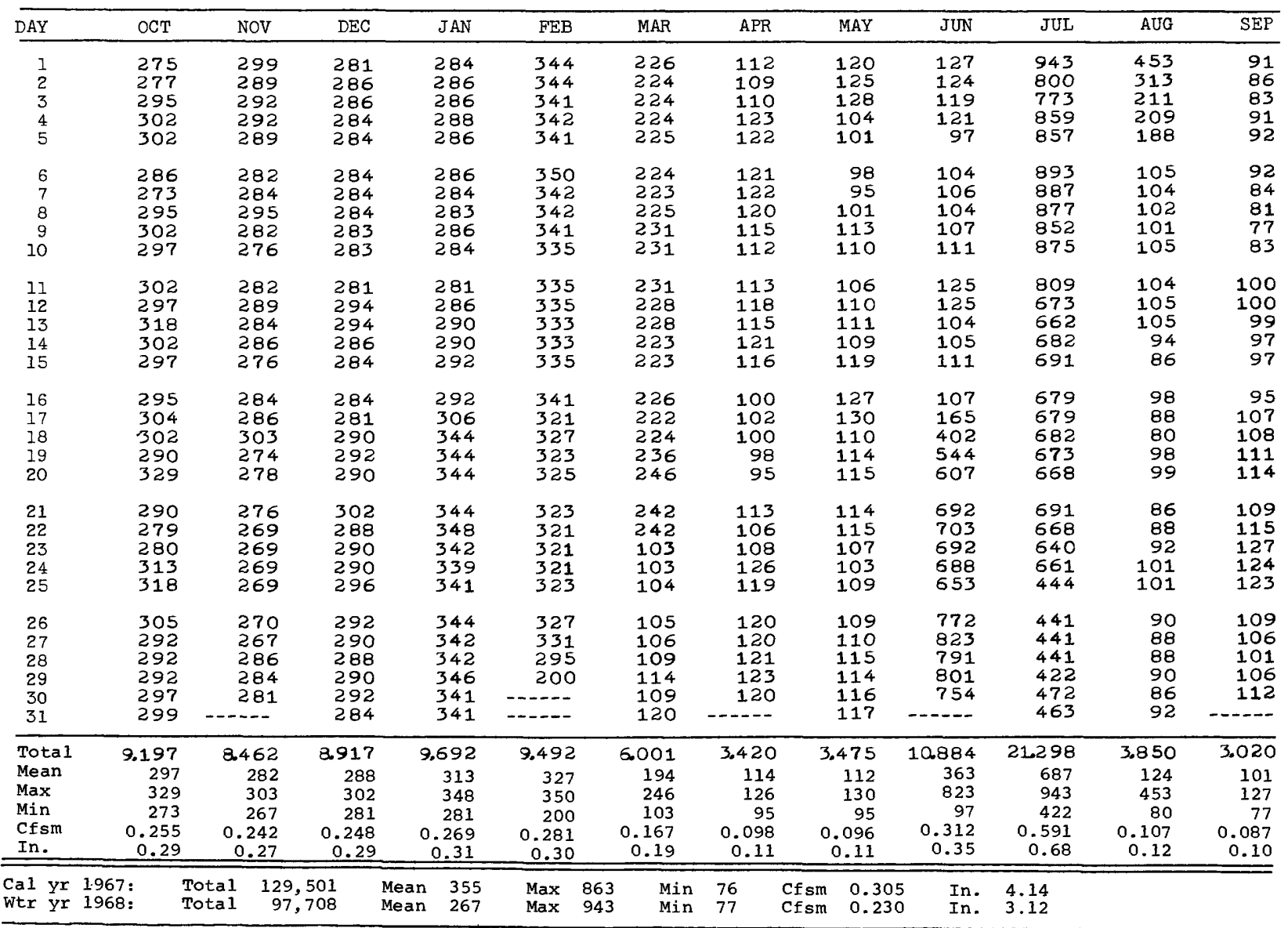


5-2105. Pokegama Lake near Grand Rapids, Minn.

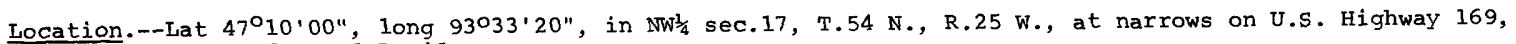
4 miles south of Grand Rapids.

Drainage area. $--3,265 \mathrm{sq} \mathrm{mi}$.

Records available.--April 1884 to September 1968. Prior to October 1941 month-end contents only, published in WSP 1308. Published as Pokegama Reservoir near Grand Rapids October 1941 to september 1956.

Gage.-Water-stage recorder. Datum of gage is $1,264.89 \mathrm{ft}$ above mean sea level, adjustment of 1912 . Prior to May 30, 1949, staff gage at Pooles Arm of Pokegama Iake, 5 miles northwest at same datum.

Extremes.--Maximum contents during year, 61,490 acre-ft June 11 (gage height, $9.30 \mathrm{ft}$ ); minimum, 27, 570 acre-ft Mar. 14 (gage height, $6.74 \mathrm{ft}$ ).

1884-1968: Maximum contents, 121,400 acre-ft May 8, 1897 (gage height, 13.50 ft)'; minimum observed, 4,520 acre-ft below zero of capacity table sept. 30, 1934 (gage height, $4.12 \mathrm{ft}$ ).

Remarks.--Reservoir is formed by Pokegama Lake and several other natural lakes controlled by concrete dam; storage began in 1884; original timber dam completed in 1884, replaced by present structure in $1888-89$. storage began in 1884; original timber dam completed in 1884, replaced by present structure in 1888-89. 53,150 acre-ft is controlled storage between gage heights 6.00 ft and 10.00 ft (normal operating range) Contents shown herein are contents above gage height $4.50 \mathrm{ft}$. Water is used to benefit navigation on Mississippi River below Minneapolis.

Cooperation.--Records furnished by Corps of Engineers in terms of cfs-days and converted to acre-feet by Geological survey.

Month-end gage height and contents, water year October 1967 to September 1968

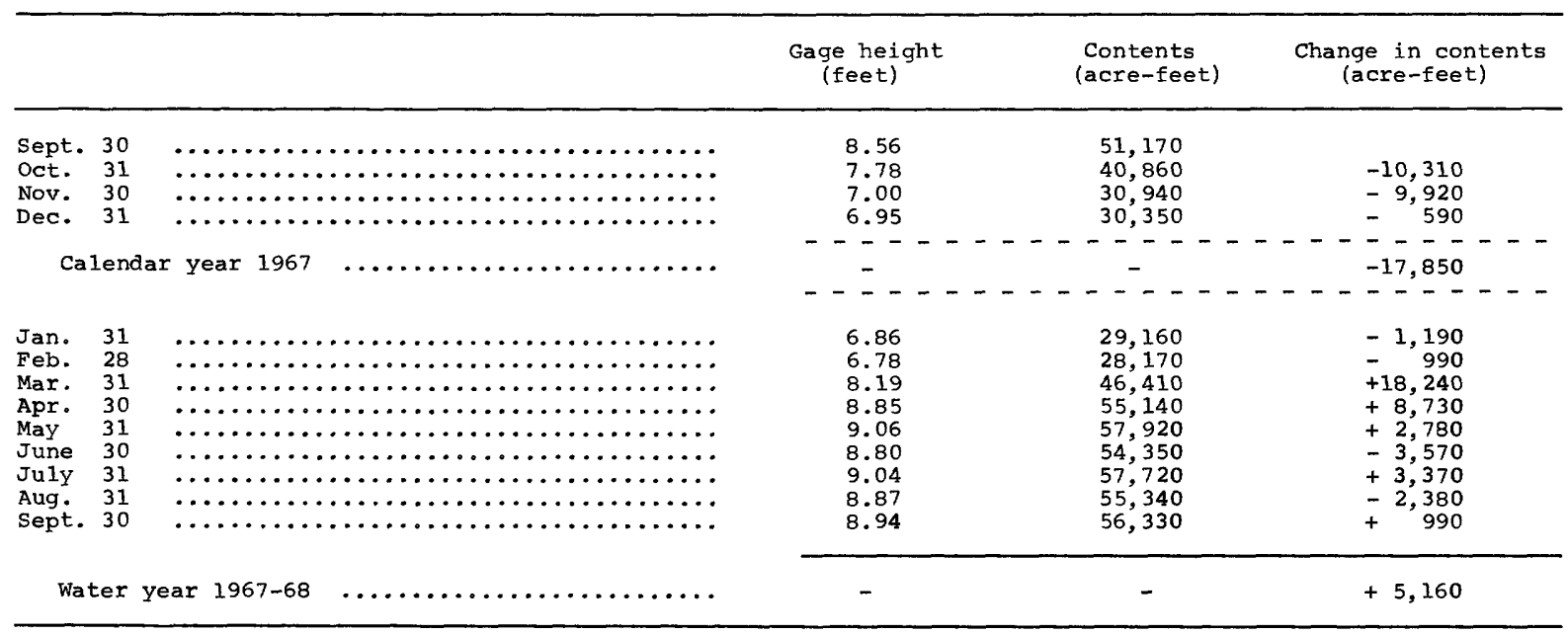




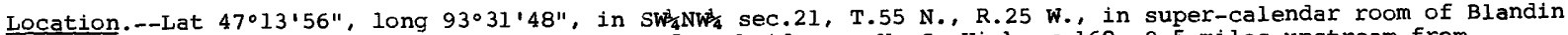
Paper Mill in Grand Rapids, 400 ft upstream from bridge on U. S. Highway $169,2.5$ miles upstream from Prairie River, and at mile 1,182 above Onio River.

Drainage area.--3,370 sq $\mathrm{mi}$, approximately.

Records available.--October 1883 to september 1968. Monthly discharge only for some periods, published in WSP 1308. Pubiished as "at Pokegama Dam near Grand Rapids" 1942-44.

Gage.--Digital water-stage recorder. Datum of gage is 1,242.00 ft above mean sea level, adjustment of 1912 . Prior to Feb. 17, 1945, staff gages operated by Corps of Engineers at Pokegama Dam $3 \frac{1}{2}$ miles upstream at datum $22.89 \mathrm{ft}$ higher. Feb. 17, 1945, to sept. 3, 1948, water-stage recorder at site 300 ft upstream, within $0.10 \mathrm{ft}$ of present datum. Sept. 9, 1948, to Jan. 6, 1949, staff gage at site 400 ft downstream at present datum. Jan. 7, 1949, to Jan. 16, 1951, tape float and inside staff gages at present site and datum. Jan. 17, 1951, to July 11, 1966, graphic water-stage recorder at present site and datum.

Average discharge. --85 years, $1,127 \mathrm{cfs}$.

Extremes.--Maximum discharge during year, 1,880 cfs July 8 (gage height, 6.68 ft); maximum gage height, 6.75 ft June 25 (backwater from Prairie River); minimum discharge, 212 cfs May 18 (gage height, 2.61 ft), result of regulation.

1883-1968: Maximum discharge, 12,500 cfs sept. 3, 1948 (gage height, 15.2 ft, from floodmark), caused by dam failure at gage, from rating curve extended above 4,500 cfs by logarithmic plotting; maximum daily, 5,250 cfs sept. 5, 8, 1905; no flow at times in several years.

Remarks.--Records fair. Flow completely regulated by Pokegama Lake (see preceding page). Backwater from Prairie River occurs at times in most years. Records of chemical analyses for the water year 1968 are published in Part 2 of this report.

Cooperation.--Records furnished by Corps of Engineers, 1883-1944, 1959-64.

DISCHARGE, IN CUBIC FEET PER SECOND, WATER YEAR OCTORER 1967 TO SEPTFMBER 19GA

\begin{tabular}{|c|c|c|c|c|c|c|c|c|c|c|c|c|}
\hline DAY & $\mathrm{OCT}$ & Nov & $\mathrm{NEC}$ & JAN & FEA & MAR & $A P R$ & MAY & JUN & $\mathrm{JIIL}$ & AUG & SEP \\
\hline 1 & 754 & $1.0 ? 0$ & 820 & 750 & 986 & 834 & 312 & 643 & 630 & 1.810 & 1.440 & 772 \\
\hline 2 & 721 & 798 & 814 & 754 & 986 & 822 & 305 & 584 & 734 & 1.690 & 1,500 & 606 \\
\hline 3 & 764 & $9 \ni 1$ & 827 & 750 & $85 h$ & 810 & 306 & 443 & 598 & 1.760 & 1.470 & 761 \\
\hline 4 & 735 & 904 & 827 & 750 & 854 & 787 & 360 & 499 & 510 & 1.810 & 1.410 & 702 \\
\hline 5 & 733 & 968 & 850 & 746 & 878 & 768 & 348 & 501 & 538 & 1.760 & 1,470 & 677 \\
\hline 6 & 700 & 957 & 836 & 742 & 877 & 764 & 299 & 504 & 552 & 1.710 & 1.460 & 687 \\
\hline 7 & 729 & 934 & 836 & 742 & 983 & 759 & 306 & 483 & 556 & 1.820 & 1,440 & 579 \\
\hline 3 & 734 & 953 & 918 & 742 & 371 & 727 & 509 & 516 & 573 & 1.830 & 1.400 & 554 \\
\hline$\varphi$ & 748 & 937 & 805 & 742 & 856 & 745 & 758 & 512 & 587 & 1.800 & 1.340 & 822 \\
\hline 10 & 756 & 1.050 & 814 & 738 & 989 & 731 & 676 & 512 & 800 & 1.790 & 1.300 & 698 \\
\hline 11 & 915 & 1.010 & 814 & 738 & 879 & 715 & 692 & 535 & 1.260 & 1.7 คO & 1.220 & 578 \\
\hline $\begin{array}{l}12 \\
13\end{array}$ & $\begin{array}{l}1.070 \\
1.090\end{array}$ & $\begin{array}{r}1.030 \\
970\end{array}$ & $\begin{array}{l}823 \\
809\end{array}$ & $\begin{array}{l}742 \\
746\end{array}$ & $\begin{array}{l}843 \\
873\end{array}$ & $\begin{array}{l}706 \\
690\end{array}$ & $\begin{array}{l}605 \\
527\end{array}$ & $\begin{array}{l}527 \\
508\end{array}$ & $\begin{array}{l}1.460 \\
1.570\end{array}$ & $\begin{array}{l}1.780 \\
1.750\end{array}$ & $\begin{array}{l}93 R \\
962\end{array}$ & $\begin{array}{l}554 \\
592\end{array}$ \\
\hline 14 & 1.040 & 973 & 796 & 750 & 859 & 652 & 532 & 565 & 1.590 & 1.780 & 906 & 663 \\
\hline 15 & 992 & 936 & 809 & 730 & 854 & 568 & 731 & 536 & 1.490 & 1.810 & 906 & 592 \\
\hline 16 & 1.000 & 938 & 762 & 754 & 864 & 622 & 844 & 542 & 1.090 & 1,790 & 986 & 582 \\
\hline 17 & 1.040 & 944 & 792 & 742 & 896 & 566 & 812 & 534 & 718 & 1.810 & 858 & 858 \\
\hline 19 & 1.050 & 951 & 832 & 775 & 871 & 624 & 811 & 567 & 734 & 1.830 & 846 & 603 \\
\hline 17 & 1.100 & 951 & 779 & 827 & 349 & 571 & 785 & 538 & 854 & 1,790 & 822 & 593 \\
\hline 20 & 1.080 & 907 & 796 & 836 & 861 & 359 & 700 & 526 & 750 & 1,780 & 649 & 709 \\
\hline 21 & $1,0 \leqslant 0$ & 905 & 809 & 832 & 341 & 393 & 758 & 570 & 955 & 1.760 & 677 & $6 ? .4$ \\
\hline 22 & 1.060 & 903 & 792 & 827 & 843 & 400 & 678 & 539 & 1.090 & $1,7 \geq 0$ & 694 & 702 \\
\hline 23 & 1.040 & 907 & 800 & 832 & 857 & 403 & 896 & 553 & 1.400 & 1.720 & 691 & 764 \\
\hline 24 & 1.030 & 859 & 775 & 850 & 841 & 405 & 1.280 & 587 & 1.570 & 1.700 & 694 & 795 \\
\hline 25 & 996 & 829 & 814 & 845 & 947 & 387 & 1.510 & 697 & 1.780 & 1.690 & 720 & 670 \\
\hline 26 & 975 & 852 & 800 & 850 & 843 & 280 & 1,250 & 683 & 1.740 & 1.720 & 691 & 822 \\
\hline 27 & 999 & 859 & 771 & 850 & 947 & 310 & 871 & 742 & 1.710 & 1.710 & 680 & 1.040 \\
\hline 28 & 1.110 & 793 & 766 & 854 & 847 & 302 & 807 & 644 & 1.680 & 1.670 & 735 & 998 \\
\hline 29 & 1.050 & 785 & 758 & 854 & 852 & 297 & 708 & 754 & 1.630 & 1,630 & 673 & 926 \\
\hline 30 & 1.040 & 795 & 758 & 872 & -ーーーー & 278 & 591 & 623 & 1.700 & 1,500 & 572 & 958 \\
\hline 31 & 1.040 & ---- & 754 & 863 & ---- & 300 & $\cdots$ & 707 & $-\infty-\infty$ & 1.420 & 738 & $-\cdots$ \\
\hline $\begin{array}{l}\text { TOTAL } \\
\text { MEAN }\end{array}$ & $29 \cdot 150$ & $\begin{array}{r}27.859 \\
929\end{array}$ & $\begin{array}{r}24.856 \\
902\end{array}$ & 24.425 & $\begin{array}{r}25.002 \\
862\end{array}$ & $\begin{array}{r}17.584 \\
567\end{array}$ & $\begin{array}{r}20,556 \\
686\end{array}$ & 17.674 & $\begin{array}{r}32,839 \\
1,095\end{array}$ & $\begin{array}{r}53,930 \\
1: 740\end{array}$ & $\begin{array}{r}30,988 \\
996\end{array}$ & $21 \cdot 4816$ \\
\hline $\operatorname{MAX}$ & 1,110 & $1,050^{\circ}$ & 850 & 872 & 896 & 834 & 1.510 & 754 & 1.780 & 1,830 & 1.500 & 1,040 \\
\hline MIN & 700 & 795 & 754 & 730 & 841 & 278 & 299 & 443 & 510 & 1.420 & 572 & 554 \\
\hline
\end{tabular}

\begin{tabular}{llllll} 
CAL YR 1967 & TOTAL 488.966 & MEAN 1.340 & MAX 2,950 & MIN 700 \\
WTR YR 1968 & TOTAL 326.264 & MEAN 891 & MAX 1930 & MIN 278 \\
\hline
\end{tabular} 
5-2127. Prairie River near Taconite, Minn.

Location.--Lat $47^{\circ} 23^{\prime} 20^{\prime \prime}$, long $93^{\circ} 22^{\prime} 50^{\prime \prime}$, in NWtsw sec. 27, T.57 N., R.24 W., on left bank 125 ft upstream

from highway bridge, $1 \frac{1}{2}$ miles downstream from outlet of Lawrence Lake and 5 miles north of Taconite.

Records available.--April 1967 to September 1968.

Gage.--Graphic water-stage recorder. Prior to Aug. 31, 1967, staff gage at site 125 ft downstream at same datum.

Extremes.--Maximum discharge during year, 836 cfs June 16 (gage height, 7.10 ft); minimum, 26 cfs Oct. 6 , 7,22 ; minimum gage height, 1.91 ft oct. 22

1967-68: Maximum discharge observed, 1,020 cfs Apr. 22, 23, 1967; maximum gage height observed, 7.96

ft Apr. 23, 1967; minimum discharge, 25 cfs sept. 12, 13, 1967; minimum gage height, that of oct. 22 , 1967.

Remarks.--Records good except those for winter months, which are fair.

Cooperation.--Additional discharge measurements and gage readings furnished by M. A. Hanna Mining Co.

DISCHARGE, IN CFS, WATER YEAR OCTOBER 1967 TO SEPTEMBER 1968

\begin{tabular}{|c|c|c|c|c|c|c|c|c|c|c|c|c|}
\hline DAY & OCT & NOV & DEC & JAN & FEB & MAR & APR & MAY & JUN & JUL & AUG & SEP \\
\hline $\begin{array}{l}1 \\
2 \\
3 \\
4 \\
5\end{array}$ & $\begin{array}{l}27 \\
28 \\
30 \\
30 \\
30\end{array}$ & $\begin{array}{l}36 \\
38 \\
38 \\
38 \\
37\end{array}$ & $\begin{array}{l}41 \\
38 \\
36 \\
34 \\
33\end{array}$ & $\begin{array}{l}41 \\
40 \\
40 \\
39 \\
38\end{array}$ & $\begin{array}{l}37 \\
37 \\
38 \\
38 \\
38\end{array}$ & $\begin{array}{l}48 \\
48 \\
48 \\
49 \\
52\end{array}$ & $\begin{array}{l}185 \\
190 \\
193 \\
202 \\
195\end{array}$ & $\begin{array}{l}588 \\
586 \\
578 \\
566 \\
542\end{array}$ & $\begin{array}{l}269 \\
280 \\
276 \\
272 \\
275\end{array}$ & $\begin{array}{l}368 \\
379 \\
387 \\
404 \\
411\end{array}$ & $\begin{array}{r}114 \\
109 \\
102 \\
95 \\
89\end{array}$ & $\begin{array}{l}299 \\
282 \\
263 \\
248 \\
227\end{array}$ \\
\hline $\begin{array}{r}6 \\
7 \\
8 \\
9 \\
10\end{array}$ & $\begin{array}{l}26 \\
28 \\
33 \\
34 \\
33\end{array}$ & $\begin{array}{l}36 \\
34 \\
32 \\
31 \\
31\end{array}$ & $\begin{array}{l}33 \\
33 \\
33 \\
34 \\
35\end{array}$ & $\begin{array}{l}38 \\
37 \\
36 \\
35 \\
34\end{array}$ & $\begin{array}{l}38 \\
38 \\
37 \\
37 \\
36\end{array}$ & $\begin{array}{l}56 \\
58 \\
59 \\
60 \\
60\end{array}$ & $\begin{array}{l}190 \\
200 \\
257 \\
275 \\
296\end{array}$ & $\begin{array}{l}512 \\
482 \\
458 \\
441 \\
411\end{array}$ & $\begin{array}{l}320 \\
376 \\
460 \\
518 \\
576\end{array}$ & $\begin{array}{l}416 \\
414 \\
408 \\
387 \\
355\end{array}$ & $\begin{array}{l}89 \\
84 \\
83 \\
78 \\
76\end{array}$ & $\begin{array}{l}220 \\
210 \\
206 \\
199 \\
190\end{array}$ \\
\hline $\begin{array}{l}11 \\
12 \\
13 \\
14 \\
15\end{array}$ & $\begin{array}{l}32 \\
29 \\
29 \\
32 \\
32\end{array}$ & $\begin{array}{l}32 \\
33 \\
35 \\
35 \\
35\end{array}$ & $\begin{array}{l}36 \\
37 \\
39 \\
42 \\
46\end{array}$ & $\begin{array}{l}33 \\
33 \\
33 \\
32 \\
32\end{array}$ & $\begin{array}{l}36 \\
35 \\
35 \\
35 \\
34\end{array}$ & $\begin{array}{l}58 \\
57 \\
57 \\
58 \\
60\end{array}$ & $\begin{array}{l}320 \\
358 \\
385 \\
423 \\
438\end{array}$ & $\begin{array}{l}389 \\
371 \\
350 \\
352 \\
366\end{array}$ & $\begin{array}{l}644 \\
682 \\
704 \\
777 \\
819\end{array}$ & $\begin{array}{l}329 \\
299 \\
274 \\
260 \\
244\end{array}$ & $\begin{array}{l}70 \\
67 \\
72 \\
68 \\
66\end{array}$ & $\begin{array}{l}187 \\
182 \\
176 \\
170 \\
159\end{array}$ \\
\hline $\begin{array}{l}16 \\
17 \\
18 \\
19 \\
20\end{array}$ & $\begin{array}{l}30 \\
31 \\
33 \\
31 \\
32\end{array}$ & $\begin{array}{l}34 \\
35 \\
37 \\
39 \\
41\end{array}$ & $\begin{array}{l}50 \\
51 \\
52 \\
52 \\
51\end{array}$ & $\begin{array}{l}32 \\
32 \\
32 \\
32 \\
32\end{array}$ & $\begin{array}{l}34 \\
33 \\
33 \\
33 \\
33\end{array}$ & $\begin{array}{l}63 \\
66 \\
75 \\
80 \\
84\end{array}$ & $\begin{array}{l}475 \\
494 \\
498 \\
498 \\
510\end{array}$ & $\begin{array}{l}366 \\
353 \\
352 \\
347 \\
335\end{array}$ & $\begin{array}{l}832 \\
830 \\
826 \\
809 \\
781\end{array}$ & $\begin{array}{l}239 \\
225 \\
218 \\
216 \\
210\end{array}$ & $\begin{array}{l}68 \\
66 \\
63 \\
66 \\
66\end{array}$ & $\begin{array}{l}155 \\
182 \\
211 \\
228 \\
248\end{array}$ \\
\hline $\begin{array}{l}21 \\
22 \\
23 \\
24 \\
25\end{array}$ & $\begin{array}{l}31 \\
29 \\
32 \\
34 \\
36\end{array}$ & $\begin{array}{l}43 \\
44 \\
44 \\
46 \\
46\end{array}$ & $\begin{array}{l}56 \\
49 \\
48 \\
47 \\
46\end{array}$ & $\begin{array}{l}32 \\
32 \\
32 \\
33 \\
33\end{array}$ & $\begin{array}{l}34 \\
35 \\
36 \\
37 \\
39\end{array}$ & $\begin{array}{r}90 \\
96 \\
103 \\
109 \\
115\end{array}$ & $\begin{array}{l}526 \\
530 \\
562 \\
590 \\
596\end{array}$ & $\begin{array}{l}320 \\
304 \\
288 \\
272 \\
252\end{array}$ & $\begin{array}{l}752 \\
708 \\
678 \\
630 \\
574\end{array}$ & $\begin{array}{l}202 \\
193 \\
184 \\
173 \\
159\end{array}$ & $\begin{array}{r}74 \\
81 \\
94 \\
110 \\
133\end{array}$ & $\begin{array}{l}274 \\
310 \\
348 \\
368 \\
387\end{array}$ \\
\hline $\begin{array}{l}26 \\
27 \\
28 \\
29 \\
30 \\
31 \\
\end{array}$ & $\begin{array}{l}33 \\
33 \\
30 \\
35 \\
35 \\
34 \\
\end{array}$ & $\begin{array}{r}46 \\
46 \\
45 \\
45 \\
43 \\
- \\
\end{array}$ & $\begin{array}{l}46 \\
45 \\
45 \\
44 \\
43 \\
42 \\
\end{array}$ & $\begin{array}{l}33 \\
34 \\
34 \\
35 \\
36 \\
36 \\
\end{array}$ & \begin{tabular}{r}
41 \\
43 \\
45 \\
47 \\
- \\
\hdashline- \\
\end{tabular} & $\begin{array}{l}123 \\
132 \\
145 \\
156 \\
167 \\
180 \\
\end{array}$ & $\begin{array}{r}600 \\
594 \\
586 \\
586 \\
588 \\
- \\
\end{array}$ & $\begin{array}{l}241 \\
239 \\
237 \\
232 \\
230 \\
234 \\
\end{array}$ & $\begin{array}{r}520 \\
467 \\
416 \\
379 \\
355 \\
- \\
\end{array}$ & $\begin{array}{l}156 \\
147 \\
139 \\
132 \\
127 \\
125 \\
\end{array}$ & $\begin{array}{l}167 \\
210 \\
248 \\
276 \\
290 \\
300 \\
\end{array}$ & $\begin{array}{r}401 \\
411 \\
414 \\
408 \\
396 \\
- \\
\end{array}$ \\
\hline $\begin{array}{l}\text { Total } \\
\text { Mean } \\
\text { Max } \\
\text { Min }\end{array}$ & $\begin{array}{r}972 \\
31.4 \\
36 \\
26\end{array}$ & $\begin{array}{r}1,157 \\
38.6 \\
46 \\
31\end{array}$ & $\begin{array}{r}1,311 \\
42.3 \\
52 \\
33\end{array}$ & $\begin{array}{r}1,071 \\
34.5 \\
41 \\
32\end{array}$ & $\begin{array}{r}1,072 \\
37.0 \\
47 \\
33\end{array}$ & $\begin{array}{r}2,612 \\
84.3 \\
180 \\
48 \\
\end{array}$ & $\begin{array}{r}12,340 \\
411 \\
600 \\
185 \\
\end{array}$ & $\begin{array}{r}11,594 \\
374 \\
588 \\
230 \\
\end{array}$ & $\begin{array}{r}16,805 \\
560 \\
832 \\
269 \\
\end{array}$ & $\begin{array}{r}8,180 \\
264 \\
416 \\
125 \\
\end{array}$ & $\begin{array}{r}3,574 \\
115 \\
300 \\
63 \\
\end{array}$ & $\begin{array}{r}7,958 \\
265 \\
414 \\
155 \\
\end{array}$ \\
\hline
\end{tabular}


5-2168. O'Brien Creek near Pengilly, Minn.

Location.--Lat $47^{\circ} 18^{\prime} 56^{\prime \prime}$, long $9^{\circ} 09^{\prime} 26^{\prime \prime}$, in SEl/ sec.20, T.56 N., R.22 w., on right bank 200 ft upstream from Duluth, Missabe and Iron Range Railroad bridge, 1.0 mile upstream from mouth and 2 miles southeast of Pengilly.

Records available.--April 1963 to September 1968 (discontinued) .

Gage.-Water-stage recorder. Datum of gage is 1,337.92 ft (revised) above mean sea, level, datum of 1929 (Levels by Hanna Mining Co.). Prior to July 24, 1963, reference point at same site and datum.

Average discharge.--5 years, $20.4 \mathrm{cfs}$.

Extremes.--Maximum discharge during year, 184 cfs June 8 (gage height, 3.71 ft); minimum, 1.2 cfs Oct. 1, 2, $4,5,6,7$

1963-68: Maximum discharge, 265 cfs Apț. 19, 1965 (gage height, 4.29 ft); minimum, 0.9 cfs Sept. 27, 1966 .

Remarks.--Records good. Flow affected by natural storage in lakes above station.

Cooperation.--Additional discharge measurements furnished by M. A. Hanna Mining Co.

DISCHARGE, IN CFS, WATER YEAR OCTOBER 1967 TO SEPTEMBER 1968

\begin{tabular}{|c|c|c|c|c|c|c|c|c|c|c|c|c|}
\hline DAY & $\mathrm{OCT}$ & NOV & DEC & JAN & FEB & MAR & APR & MAY & JUN & JUL & AUG & SEP \\
\hline $\begin{array}{l}1 \\
2 \\
3 \\
4 \\
5\end{array}$ & $\begin{array}{l}1.2 \\
1.2 \\
1.4 \\
1.4 \\
1.2\end{array}$ & $\begin{array}{l}5.9 \\
6.1 \\
6.1 \\
6.1 \\
6.1\end{array}$ & $\begin{array}{l}6.1 \\
6.1 \\
5.0 \\
4.2 \\
3.4\end{array}$ & $\begin{array}{l}1.6 \\
1.6 \\
1.6 \\
1.6 \\
1.6\end{array}$ & $\begin{array}{l}2.4 \\
2.4 \\
2.4 \\
2.3 \\
2.3\end{array}$ & $\begin{array}{l}1.6 \\
1.6 \\
1.5 \\
1.5 \\
1.6\end{array}$ & $\begin{array}{r}17 \\
14 \\
11 \\
8.2 \\
6.5\end{array}$ & $\begin{array}{l}27 \\
22 \\
19 \\
18 \\
19\end{array}$ & $\begin{array}{l}19 \\
24 \\
25 \\
20 \\
16\end{array}$ & $\begin{array}{l}53 \\
50 \\
33 \\
23 \\
16\end{array}$ & $\begin{array}{l}3.1 \\
2.8 \\
2.8 \\
2.7 \\
2.7\end{array}$ & $\begin{array}{l}2.6 \\
2.5 \\
2.4 \\
2.5 \\
2.5\end{array}$ \\
\hline $\begin{array}{r}6 \\
7 \\
8 \\
9 \\
10\end{array}$ & $\begin{array}{l}1.2 \\
1.5 \\
2.1 \\
4.8 \\
5.0\end{array}$ & $\begin{array}{l}5.6 \\
5.2 \\
5.2 \\
5.6 \\
6.1\end{array}$ & $\begin{array}{l}3.1 \\
2.8 \\
2.7 \\
2.7 \\
2.7\end{array}$ & $\begin{array}{l}1.6 \\
1.6 \\
1.6 \\
1.6 \\
1.6\end{array}$ & $\begin{array}{l}2.3 \\
2.2 \\
2.2 \\
2.3 \\
2.6\end{array}$ & $\begin{array}{l}1.6 \\
1.6 \\
1.9 \\
2.6 \\
3.5\end{array}$ & $\begin{array}{l}11 \\
25 \\
51 \\
75 \\
62\end{array}$ & $\begin{array}{l}18 \\
17 \\
19 \\
20 \\
20\end{array}$ & $\begin{array}{r}39 \\
118 \\
178 \\
127 \\
71\end{array}$ & $\begin{array}{r}11 \\
9.3 \\
8.0 \\
7.2 \\
6.1\end{array}$ & $\begin{array}{l}2.8 \\
2.7 \\
2.8 \\
3.0 \\
2.7\end{array}$ & $\begin{array}{l}3.0 \\
4.0 \\
5.0 \\
5.2 \\
4.0\end{array}$ \\
\hline $\begin{array}{l}11 \\
12 \\
13 \\
14 \\
15\end{array}$ & $\begin{array}{l}4.6 \\
4.0 \\
3.6 \\
4.0 \\
4.6\end{array}$ & $\begin{array}{l}6.4 \\
6.4 \\
6.1 \\
6.1 \\
5.9\end{array}$ & $\begin{array}{l}2.5 \\
2.5 \\
2.5 \\
2.4 \\
2.2\end{array}$ & $\begin{array}{l}1.6 \\
1.6 \\
1.7 \\
1.7 \\
1.7\end{array}$ & $\begin{array}{l}3.3 \\
2.6 \\
2.3 \\
2.2 \\
2.1\end{array}$ & $\begin{array}{l}5.0 \\
7.5 \\
5.0 \\
3.8 \\
2.8\end{array}$ & $\begin{array}{l}50 \\
38 \\
28 \\
23 \\
18\end{array}$ & $\begin{array}{l}18 \\
16 \\
14 \\
15 \\
16\end{array}$ & $\begin{array}{r}55 \\
46 \\
36 \\
75 \\
119\end{array}$ & $\begin{array}{l}5.2 \\
5.2 \\
5.4 \\
5.9 \\
6.4\end{array}$ & $\begin{array}{l}2.5 \\
2.3 \\
2.1 \\
2.1 \\
2.4\end{array}$ & $\begin{array}{l}3.4 \\
3.1 \\
2.1 \\
1.5 \\
1.7\end{array}$ \\
\hline $\begin{array}{l}16 \\
17 \\
18 \\
19 \\
20\end{array}$ & $\begin{array}{l}4.8 \\
4.8 \\
5.0 \\
5.0 \\
5.0\end{array}$ & $\begin{array}{l}5.9 \\
5.9 \\
6.4 \\
6.7 \\
6.1\end{array}$ & $\begin{array}{l}2.2 \\
2.3 \\
2.4 \\
2.1 \\
2.1\end{array}$ & $\begin{array}{l}1.8 \\
1.8 \\
1.8 \\
1.9 \\
2.1\end{array}$ & $\begin{array}{l}2.0 \\
2.0 \\
2.0 \\
2.0 \\
2.0\end{array}$ & $\begin{array}{l}4.5 \\
7.0 \\
10 \\
14 \\
19\end{array}$ & $\begin{array}{l}35 \\
52 \\
45 \\
33 \\
26\end{array}$ & $\begin{array}{l}19 \\
18 \\
19 \\
20 \\
19\end{array}$ & $\begin{array}{l}86 \\
46 \\
31 \\
23 \\
17\end{array}$ & $\begin{array}{l}7.8 \\
8.3 \\
8.0 \\
7.2 \\
5.9\end{array}$ & $\begin{array}{l}3.8 \\
4.6 \\
3.8 \\
3.6 \\
3.8\end{array}$ & $\begin{array}{l}1.7 \\
1.9 \\
5.9 \\
13 \\
17\end{array}$ \\
\hline $\begin{array}{l}21 \\
22 \\
23 \\
24 \\
25\end{array}$ & $\begin{array}{l}5.2 \\
5.0 \\
5.2 \\
5.0 \\
4.8\end{array}$ & $\begin{array}{l}5.9 \\
6.4 \\
8.0 \\
8.6 \\
8.0\end{array}$ & $\begin{array}{l}2.1 \\
2.1 \\
2.0 \\
1.9 \\
1.9\end{array}$ & $\begin{array}{l}2.1 \\
2.1 \\
2.3 \\
2.3 \\
2.4\end{array}$ & $\begin{array}{l}1.9 \\
1.7 \\
1.6 \\
1.6 \\
1.6\end{array}$ & $\begin{array}{r}20 \\
16 \\
12 \\
9.5 \\
9.0\end{array}$ & $\begin{array}{l}31 \\
31 \\
35 \\
42 \\
42\end{array}$ & $\begin{array}{l}16 \\
14 \\
12 \\
10 \\
8.3\end{array}$ & $\begin{array}{l}13 \\
12 \\
18 \\
22 \\
17\end{array}$ & $\begin{array}{l}5.2 \\
4.4 \\
3.6 \\
3.3 \\
2.8\end{array}$ & $\begin{array}{l}3.6 \\
3.3 \\
5.9 \\
18 \\
19\end{array}$ & $\begin{array}{l}14 \\
13 \\
17 \\
21 \\
19\end{array}$ \\
\hline $\begin{array}{l}26 \\
27 \\
28 \\
29 \\
30 \\
31 \\
\end{array}$ & $\begin{array}{l}4.6 \\
4.6 \\
4.8 \\
5.2 \\
5.6 \\
5.6 \\
\end{array}$ & $\begin{array}{r}7.8 \\
7.0 \\
6.4 \\
5.9 \\
6.1 \\
\end{array}$ & $\begin{array}{l}1.8 \\
1.8 \\
1.7 \\
1.7 \\
1.7 \\
1.6 \\
\end{array}$ & $\begin{array}{l}2.3 \\
2.3 \\
2.5 \\
2.4 \\
2.5 \\
2.5 \\
\end{array}$ & $\begin{array}{r}1.6 \\
1.6 \\
1.6 \\
1.6 \\
-.-.- \\
\end{array}$ & $\begin{array}{l}10 \\
15 \\
20 \\
23 \\
25 \\
21 \\
\end{array}$ & $\begin{array}{r}52 \\
57 \\
54 \\
44 \\
35 \\
------ \\
\end{array}$ & $\begin{array}{l}7.8 \\
10 \\
14 \\
15 \\
15 \\
14 \\
\end{array}$ & $\begin{array}{c}13 \\
10 \\
8.6 \\
7.8 \\
12 \\
\\
\end{array}$ & $\begin{array}{l}3.3 \\
3.4 \\
3.3 \\
3.3 \\
3.4 \\
3.3 \\
\end{array}$ & $\begin{array}{l}15 \\
11 \\
7.8 \\
5.6 \\
3.8 \\
3.0 \\
\end{array}$ & $\begin{array}{r}15 \\
11 \\
7.5 \\
5.4 \\
4.4 \\
- \\
\end{array}$ \\
\hline $\begin{array}{l}\text { Total } \\
\text { Mean } \\
\text { Max } \\
\text { Min }\end{array}$ & $\begin{array}{r}122.0 \\
3.94 \\
5.6 \\
1.2\end{array}$ & $\begin{array}{r}190.0 \\
6.33 \\
8.6 \\
5.2\end{array}$ & $\begin{array}{r}82.3 \\
2.65 \\
6.1 \\
1.6\end{array}$ & $\begin{array}{r}59.4 \\
1.92 \\
2.5 \\
1.6\end{array}$ & $\begin{array}{r}60.7 \\
2.09 \\
3.3 \\
1.6\end{array}$ & $\begin{array}{r}278.1 \\
8.97 \\
25 \\
1.5\end{array}$ & $\begin{array}{r}1,051.7 \\
35.1 \\
75 \\
6.5\end{array}$ & $\begin{array}{r}509.1 \\
16.4 \\
27 \\
7.8\end{array}$ & $\begin{array}{r}1,304.4 \\
43.5 \\
178 \\
7.8\end{array}$ & $\begin{array}{r}321.2 \\
10.4 \\
53 \\
2.8\end{array}$ & $\begin{array}{r}155.1 \\
5.00 \\
19 \\
2.1\end{array}$ & $\begin{array}{r}212.3 \\
7.07 \\
21 \\
1.5\end{array}$ \\
\hline $\begin{array}{l}\text { Cal yr } \\
\text { wtr yr } \\
\end{array}$ & 1967: & $\begin{array}{ll}\text { tal } & 3, \\
\text { tal } & 4 \\
\end{array}$ & & $\begin{array}{l}10.2 \\
11.9 \\
\end{array}$ & $\begin{array}{l}\text { Max } \\
\text { Max }\end{array}$ & $\begin{array}{l}241 \\
178 \\
\end{array}$ & in 1.0 & & & & & \\
\hline
\end{tabular}


5-2168.6 Swan River near Calumet, Minn.

Location.--Lat $4^{\circ} 17^{\prime} 20^{\prime \prime}$, long $93^{\circ} 1^{\prime} 3^{\prime} 54^{\prime \prime}$, in SW/4 sec.35, T.56 N., R.23 W., on left bank 1.0 mile downstream from Snowball Creek, 2.1.miles downstream from bridge on U. S. Highway 65 at outlet of Swan Lake and 3.1 miles southeast of Calumet.

Records available.--January 1964 to September 1968.

Gage.--Water-stage recorder. Datum of gage is $1,331.19 \mathrm{ft}$ above mean sea level, datum of 1929 . Prior to June 5, 1964, reference point at same site and datum.

Extremes.--Maximum discharge during year, $193 \mathrm{cfs} J u n e ~ 17$ (gage height, 5.07 ft); minimum daily, $4.9 \mathrm{cfs}$ Jan. 4-6; minimum gage height, $3.50 \mathrm{ft}$ Oct. 20.

1964-68: Maximum discharge $540 \mathrm{cfs}$ Apr. 19, 1966 (gage height, 5.01 ft); maximum gage height, 5.11 ft Apr. 3, 1967; minimum discharge, 3.0 cfs Sept. 26, 27, 1966 (result of weir construction).

Remarks.--Records good. Flow affected by natural storage in swan Lake.

Cooperation.--Additional discharge measurements and gage readings furnished by M. A. Hanna Mining Company.

DISCHARGE, IN CFS, WATER YEAR OCTOBER 1967 TO SEPTEMBER 1968

\begin{tabular}{|c|c|c|c|c|c|c|c|c|c|c|c|c|}
\hline DAY & OCT & NOV & $\mathrm{DEC}$ & JAN & FEB & MAR & APR & MAY & JUN & JUL & AUG & SEP \\
\hline $\begin{array}{l}1 \\
2 \\
3 \\
4 \\
5\end{array}$ & $\begin{array}{l}6.0 \\
5.9 \\
6.0 \\
5.8 \\
5.8\end{array}$ & $\begin{array}{l}8.2 \\
8.2 \\
7.9 \\
7.8 \\
7.6\end{array}$ & $\begin{array}{l}5.6 \\
5.6 \\
5.6 \\
5.6 \\
5.7\end{array}$ & $\begin{array}{l}5.1 \\
5.0 \\
5.0 \\
4.9 \\
4.9\end{array}$ & $\begin{array}{l}6.8 \\
6.8 \\
6.8 \\
6.8 \\
6.9\end{array}$ & $\begin{array}{l}7.0 \\
6.9 \\
6.8 \\
6.7 \\
6.7\end{array}$ & $\begin{array}{l}56 \\
58 \\
61 \\
73 \\
73\end{array}$ & $\begin{array}{l}150 \\
135 \\
129 \\
123 \\
120\end{array}$ & $\begin{array}{l}70 \\
75 \\
78 \\
78 \\
78\end{array}$ & $\begin{array}{l}100 \\
118 \\
123 \\
129 \\
123\end{array}$ & $\begin{array}{l}18 \\
18 \\
16 \\
15 \\
15\end{array}$ & $\begin{array}{l}36 \\
36 \\
34 \\
32 \\
32\end{array}$ \\
\hline $\begin{array}{r}6 \\
7 \\
8 \\
9 \\
10\end{array}$ & $\begin{array}{l}5.8 \\
6.0 \\
6.2 \\
6.2 \\
6.2\end{array}$ & $\begin{array}{l}7.5 \\
7.4 \\
7.2 \\
7.1 \\
7.0\end{array}$ & $\begin{array}{l}5.7 \\
5.7 \\
5.7 \\
5.6 \\
5.7\end{array}$ & $\begin{array}{l}4.9 \\
5.0 \\
5.0 \\
5.1 \\
5.2\end{array}$ & $\begin{array}{l}6.9 \\
6.9 \\
6.9 \\
6.9 \\
7.0\end{array}$ & $\begin{array}{l}6.6 \\
6.6 \\
6.6 \\
6.7 \\
6.7\end{array}$ & $\begin{array}{r}75 \\
78 \\
81 \\
89 \\
106\end{array}$ & $\begin{array}{l}115 \\
109 \\
109 \\
103 \\
103\end{array}$ & $\begin{array}{r}75 \\
103 \\
129 \\
147 \\
159\end{array}$ & $\begin{array}{r}115 \\
112 \\
103 \\
100 \\
92\end{array}$ & $\begin{array}{l}16 \\
16 \\
16 \\
15 \\
12\end{array}$ & $\begin{array}{l}32 \\
36 \\
36 \\
34 \\
32\end{array}$ \\
\hline $\begin{array}{l}11 \\
12 \\
13 \\
14 \\
15\end{array}$ & $\begin{array}{l}6.2 \\
6.4 \\
6.3 \\
6.5 \\
6.6\end{array}$ & $\begin{array}{l}7.0 \\
6.9 \\
6.8 \\
6.8 \\
6.7\end{array}$ & $\begin{array}{l}5.7 \\
5.7 \\
5.7 \\
5.7 \\
5.7\end{array}$ & $\begin{array}{l}5.2 \\
5.3 \\
5.4 \\
5.4 \\
5.5\end{array}$ & $\begin{array}{l}7.0 \\
7.0 \\
7.0 \\
7.0 \\
7.0\end{array}$ & $\begin{array}{l}6.6 \\
6.6 \\
6.5 \\
6.5 \\
6.4\end{array}$ & $\begin{array}{l}118 \\
123 \\
126 \\
129 \\
126\end{array}$ & $\begin{array}{r}103 \\
100 \\
100 \\
100 \\
97\end{array}$ & $\begin{array}{l}159 \\
156 \\
156 \\
171 \\
181\end{array}$ & $\begin{array}{l}81 \\
78 \\
73 \\
73 \\
70\end{array}$ & $\begin{array}{l}11 \\
11 \\
9.9 \\
9.9 \\
11\end{array}$ & $\begin{array}{l}30 \\
30 \\
28 \\
28 \\
25\end{array}$ \\
\hline $\begin{array}{l}16 \\
17 \\
18 \\
19 \\
20\end{array}$ & $\begin{array}{l}6.7 \\
6.6 \\
6.8 \\
6.9 \\
6.6\end{array}$ & $\begin{array}{l}6.7 \\
6.6 \\
6.6 \\
6.6 \\
6.5\end{array}$ & $\begin{array}{l}5.7 \\
5.8 \\
5.9 \\
5.9 \\
5.9\end{array}$ & $\begin{array}{l}5.6 \\
5.6 \\
5.7 \\
5.8 \\
5.8\end{array}$ & $\begin{array}{l}7.0 \\
7.0 \\
7.0 \\
7.0 \\
7.0\end{array}$ & $\begin{array}{l}6.4 \\
6.4 \\
6.6 \\
6.9 \\
7.1\end{array}$ & $\begin{array}{l}126 \\
132 \\
141 \\
141 \\
144\end{array}$ & $\begin{array}{l}92 \\
95 \\
97 \\
92 \\
86\end{array}$ & $\begin{array}{l}190 \\
190 \\
184 \\
174 \\
165\end{array}$ & $\begin{array}{l}68 \\
63 \\
58 \\
56 \\
53\end{array}$ & $\begin{array}{l}11 \\
15 \\
16 \\
16 \\
16\end{array}$ & $\begin{array}{l}27 \\
36 \\
45 \\
49 \\
51\end{array}$ \\
\hline $\begin{array}{l}21 \\
22 \\
23 \\
24 \\
25\end{array}$ & $\begin{array}{l}7.0 \\
7.2 \\
7.4 \\
7.2 \\
7.2\end{array}$ & $\begin{array}{l}6.4 \\
6.3 \\
6.2 \\
6.1 \\
6.0\end{array}$ & $\begin{array}{l}5.8 \\
5.8 \\
5.7 \\
5.6 \\
5.5\end{array}$ & $\begin{array}{l}5.9 \\
6.0 \\
6.0 \\
6.2 \\
6.3\end{array}$ & $\begin{array}{l}7.0 \\
7.0 \\
7.0 \\
7.0 \\
7.1\end{array}$ & $\begin{array}{l}7.5 \\
8.0 \\
8.4 \\
8.7 \\
9.3\end{array}$ & $\begin{array}{l}144 \\
144 \\
150 \\
162 \\
165\end{array}$ & $\begin{array}{l}81 \\
78 \\
73 \\
73 \\
68\end{array}$ & $\begin{array}{l}150 \\
141 \\
144 \\
135 \\
126\end{array}$ & $\begin{array}{l}43 \\
40 \\
40 \\
36 \\
34\end{array}$ & $\begin{array}{l}20 \\
20 \\
28 \\
32 \\
34\end{array}$ & $\begin{array}{l}51 \\
58 \\
65 \\
63 \\
65\end{array}$ \\
\hline $\begin{array}{l}26 \\
27 \\
28 \\
29 \\
30 \\
31 \\
\end{array}$ & $\begin{array}{l}7.5 \\
7.6 \\
7.8 \\
8.0 \\
8.3 \\
8.4 \\
\end{array}$ & $\begin{array}{r}6.0 \\
5.9 \\
5.8 \\
5.7 \\
5.6 \\
\end{array}$ & $\begin{array}{l}5.4 \\
5.4 \\
5.3 \\
5.2 \\
5.2 \\
5.1 \\
\end{array}$ & $\begin{array}{l}6.4 \\
6.6 \\
6.7 \\
6.8 \\
6.8 \\
6.8 \\
\end{array}$ & $\begin{array}{r}7.1 \\
7.1 \\
7.1 \\
7.1 \\
-0 .- \\
\end{array}$ & $\begin{array}{l}11 \\
23 \\
32 \\
40 \\
47 \\
53 \\
\end{array}$ & $\begin{array}{r}165 \\
165 \\
165 \\
156 \\
153 \\
- \\
\end{array}$ & $\begin{array}{l}65 \\
70 \\
70 \\
70 \\
68 \\
70 \\
\end{array}$ & $\begin{array}{r}118 \\
106 \\
95 \\
86 \\
92 \\
\end{array}$ & $\begin{array}{l}34 \\
30 \\
25 \\
23 \\
22 \\
20 \\
\end{array}$ & $\begin{array}{l}36 \\
36 \\
36 \\
34 \\
34 \\
36 \\
\end{array}$ & $\begin{array}{r}65 \\
65 \\
63 \\
58 \\
56 \\
- \\
\end{array}$ \\
\hline $\begin{array}{l}\text { Total } \\
\text { Mean } \\
\text { Max } \\
\text { Min } \\
\end{array}$ & $\begin{array}{r}209.1 \\
6.75 \\
8.4 \\
5.8 \\
\end{array}$ & $\begin{array}{r}203.1 \\
6.77 \\
8.2 \\
5.6 \\
\end{array}$ & $\begin{array}{r}174.2 \\
5.62 \\
5.9 \\
5.1 \\
\end{array}$ & $\begin{array}{r}175.9 \\
5.67 \\
6.8 \\
4.9 \\
\end{array}$ & $\begin{array}{r}202.2 \\
6.97 \\
7.1 \\
6.8 \\
\end{array}$ & $\begin{array}{r}381.2 \\
12.3 \\
53 \\
6.4 \\
\end{array}$ & $\begin{array}{r}3,625 \\
121 \\
165 \\
56\end{array}$ & $\begin{array}{r}2,944 \\
95,0 \\
150 \\
65 \\
\end{array}$ & $\begin{array}{r}3,911 \\
130 \\
190 \\
70 \\
\end{array}$ & $\begin{array}{r}2,135 \\
68.9 \\
129 \\
20 \\
\end{array}$ & $\begin{array}{r}629.8 \\
20.3 \\
36 \\
9.9 \\
\end{array}$ & $\begin{array}{r}1,298 \\
43.3 \\
65 \\
25 \\
\end{array}$ \\
\hline $\begin{array}{l}\text { Cal yr } \\
\text { Wtr yr }\end{array}$ & $\begin{array}{l}1967: \\
1968:\end{array}$ & $\begin{array}{l}\operatorname{tal} 1 \\
\operatorname{tal} 1\end{array}$ & $\begin{array}{l}48 \\
88.5\end{array}$ & $\begin{array}{l}3 \\
4\end{array}$ & $\begin{array}{l}\text { Max } \\
\text { Max }\end{array}$ & $\begin{array}{l}184 \\
190 \\
\end{array}$ & $\begin{array}{l}5.1 \\
4.9 \\
\end{array}$ & & & & & \\
\hline
\end{tabular}


Location.--Lat $47^{\circ} 06^{\prime} 40^{\prime \prime}$, long $93^{\circ} 15^{\prime} 50^{\prime \prime}$, in SE⿺辶 sec.33, T.54 N., R.23 W., on left bank 75 ft upstream from highway bridge, $1 \frac{3}{4}$ miles south of Warba, $3 \frac{3}{4}$ miles northwest of Swan River, and 22 miles upstream from mouth.

Drainage area.--254 sq $\mathrm{mi}$.

Records available.--October 1953 to september 1968.

Gage.--Digital water-stage recorder. Datum of gage is 1,259.80 ft above mean sea level (Minnesota State High-

way Department bench mark). Prior to Sept. 10, 1968, graphic water-stage recorder at same site and datum.

Average discharge.--15 years, $128 \mathrm{cfs}$.

Extremes.--Maximum discharge during year, $429 \mathrm{cfs}$ Apr. 26 (gage height, $6.44 \mathrm{ft}$ ); minimum daily, $21 \mathrm{cfs}$ Jan.

2-11; minimum gage height, $2.06 \mathrm{ft}$ Nov. 13.

1953-68: Maximum discharge, 1,080 cfs Apr. 17, 1965 (gage height, $9.49 \mathrm{ft}$, backwater from ice) ; maximum

gage height, $9.53 \mathrm{ft}$ Apr. 16, 1965 (backwater from ice); minimum discharge, $15 \mathrm{cfs}$ Sept. 4, 5, 1961 (gage

height, $1.65 \mathrm{ft}$ )

Flood of May 1950 reached a stage of about $11.5 \mathrm{ft}$ from information by local residents.

Remarks.--Records good except those for winter months, which are fair.

DISCHARGE, IN CFS, WATER YEAR OCTOBER 1967 TO SEPTEMBER 1968

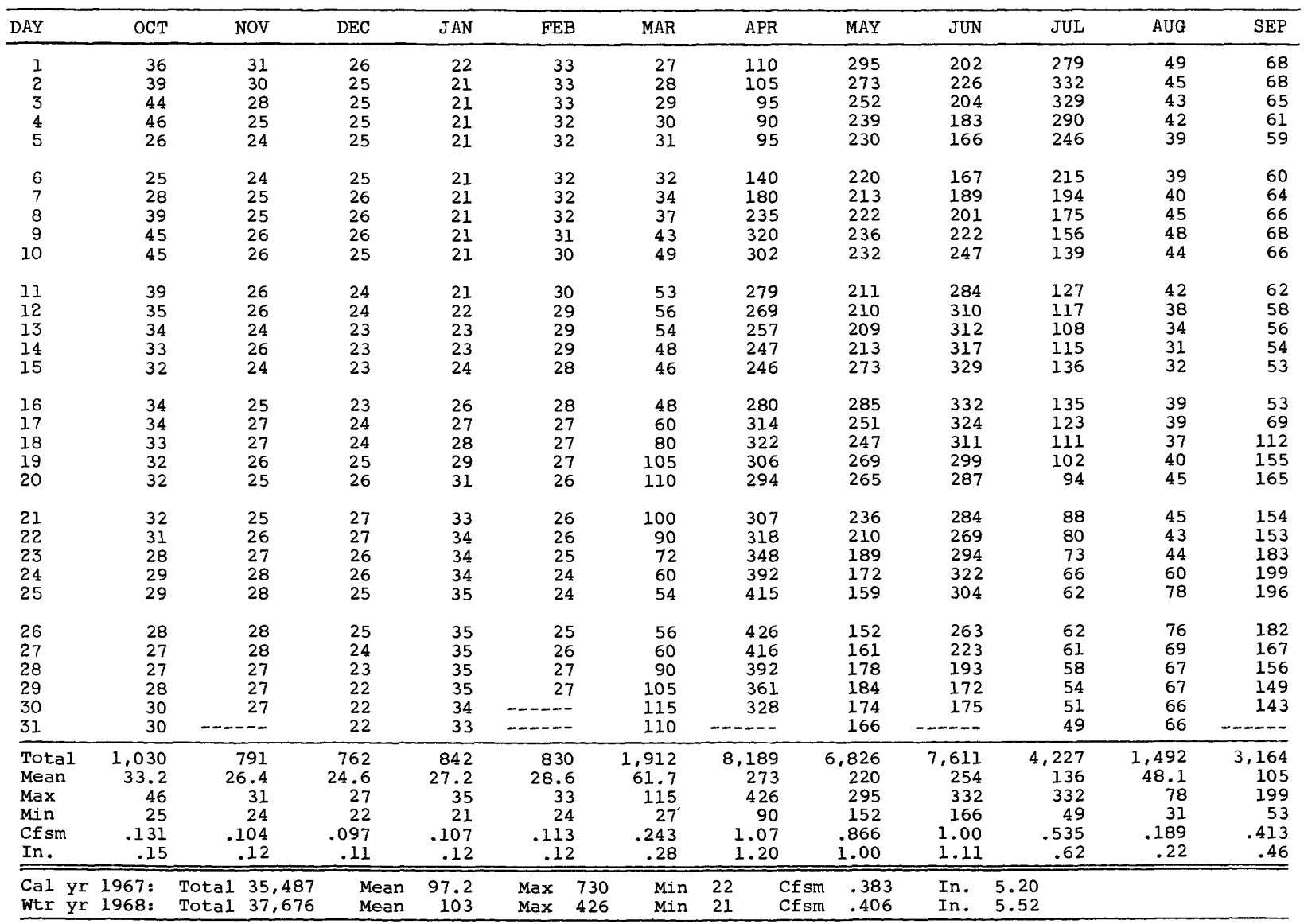


5-2185. Sandy Lake at Libby, Minn.

Location.--Lat $46047^{\prime} 20$, long $93^{\circ} 19^{\prime} 10^{\prime \prime}$, in sec. 25 , T.50 N., R.24 W., on dam on Sandy River at Libby, 1.2 miles above mouth, and 14 miles north of McGregor.

Drainage area. $--421 \mathrm{sq} \mathrm{mi}$.

Records available.--July to December 1893, October to December 1894, July 1895 to September 1968 . Month-end contents only for some pexiods, published in WSP 1308. Published as Sandy Lake Reservoir at Libby October 1941 to september 1956.

Gage.-Water-stage recorder. Datum of gage is $1,207.71$ ft above mean sea level, adjustment of 1912 . Prior to Sept. 23, 1949, float gage at same site and datum.

Extremes.--Maximum contents during year, 55,640 acre-ft June 12 (gage height, $9.33 \mathrm{ft}$ ); minimum, 33,360 acreft Feb. 27 (gage height $6.80 \mathrm{ft}$ ).

1895-1968: Maximum contents, 167, 200 acre-ft May 19, 1950 (gage height, $17.51 \mathrm{ft}$ ); minimum observed, 5,950 acre-ft below zero of capacity table Jan. 20, 1921 (gage height, $0.65 \mathrm{ft}$ ).

Remarks.--Reservoir is formed by Sandy, Flowage, Snake, and Aitkin Lakes controlled by concrete dam. Storage began in 1893; original timber crib dam completed in 1895, replaced by present structure in 1911. Capacity between gage heights $7.00 \mathrm{ft}$ and $14.00 \mathrm{ft}$ (minimum allowable limit to top of structure) is 73,330 acre-ft, of which 37,550 acre-ft is controlled storage between gage heights $7.00 \mathrm{ft}$ and $11.00 \mathrm{ft}$ (normal operating range). Contents shown herein are contents above gage height 1.72 ft. Water is used to benefit navigation on Mississippi River below Minneapolis.

Cooperation.--Records furnished by Corps of Engineers in terms of cfs-days and converted to acre-feet by Geological survey.

Month-end gage height and contents, water year October 1967 to September 1968

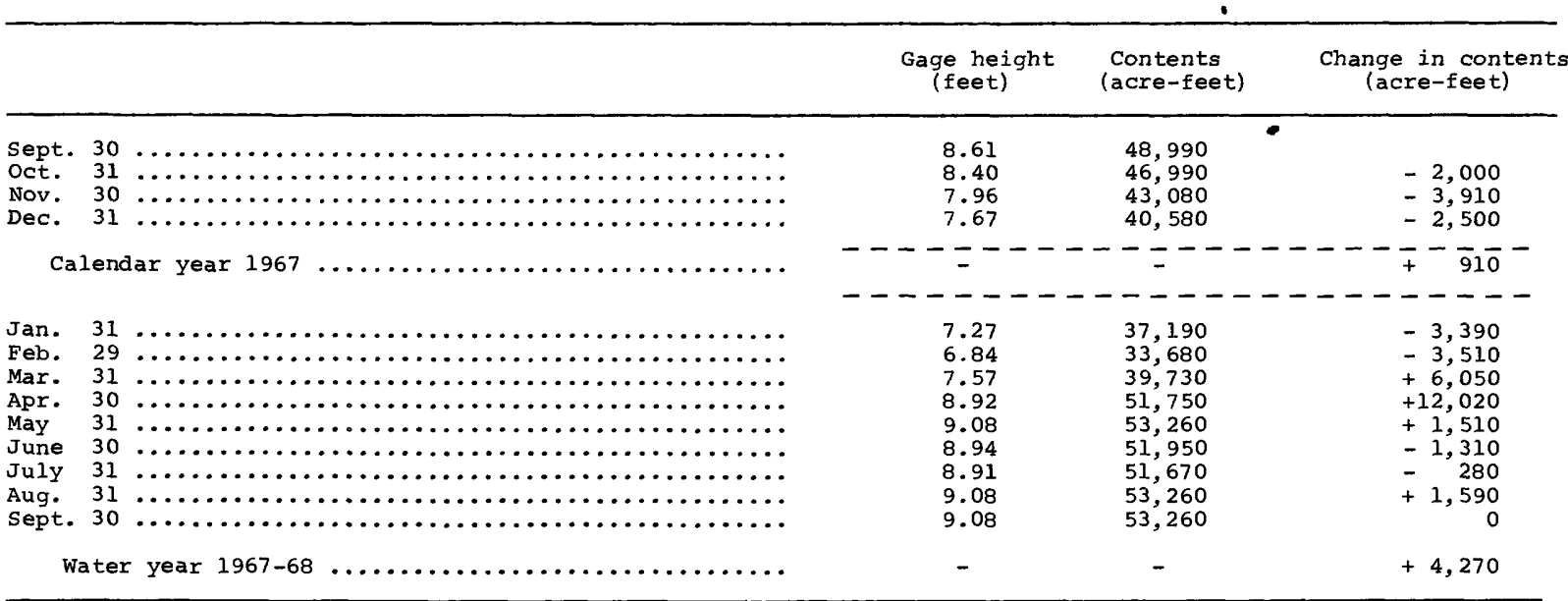


5-2190. Sandy River at Sandy Lake Dam, at Libby, Minn.

Location.--Lat $46047^{\prime} 20^{\prime \prime}$, long $93^{\circ} 19^{\prime} 10^{\prime \prime}$, in sec. 25 , T.50 N., R. 24 W., on dam on Sandy River at Libby, 1.2 miles above mouth, and 14 miles north of McGregor.

Drainage area. $--421 \mathrm{sq} \mathrm{mi}$.

Records available.--July 1893 to March 1894, July 1894, November 1894 to March 1895 , August 1895 to September 1968. Monthly discharge only for some periods, published in wSP 1308 . Published as "below Sandy Lake Reservoir" 1893-1916.

Gage.--Water-stage recorders on headwater and tailwater. Datum of gages is $1,207.71 \mathrm{ft}$ above mean sea level, adjustment of 1912. Prior to June 20, 1949, staff gages at same site and datum.

Average discharge. - -73 years (1895-1968), 206 cfs, unadjusted.

Extremes.--Maximum daily discharge during year, 1,680 cfs June 11; minimum daily, 12 cfs many days in October, March, April, July, August and September.

1893-1968: Maximum daily discharge, 3,740 cfs July 12, 1897; no flow at times.

Remarks.--Discharge computed on basis of head over dam, using modified weir formula, head being obtained from headwater and tailwater recorder records. Flow completely regulated by sandy Lake (see preceding page).

Cooperation.--One discharge measurement made and records reviewed by Geological survey. Computations of daily discharge furnished by Corps of Engineers.

DISCHARGE, IN CFS, WATER YEAR OCTOBER 1967 TO SEPTEMBER 1968

\begin{tabular}{|c|c|c|c|c|c|c|c|c|c|c|c|c|}
\hline $\mathrm{DAY}$ & OCT & NOV & DEC & JAN & FEB & MAR & APR & MAY & JUN & JUL & AUG & SEP \\
\hline $\begin{array}{l}1 \\
2 \\
3 \\
4 \\
5\end{array}$ & $\begin{array}{l}12 \\
12 \\
12 \\
12 \\
12\end{array}$ & $\begin{array}{l}83 \\
83 \\
83 \\
83 \\
83\end{array}$ & $\begin{array}{r}81 \\
80 \\
80 \\
-\quad 80 \\
80\end{array}$ & $\begin{array}{l}76 \\
76 \\
76 \\
76 \\
76\end{array}$ & $\begin{array}{l}71 \\
69 \\
69 \\
69 \\
70\end{array}$ & $\begin{array}{l}21 \\
21 \\
21 \\
21 \\
21\end{array}$ & $\begin{array}{l}12 \\
12 \\
12 \\
12 \\
12\end{array}$ & $\begin{array}{c}1.380 \\
645 \\
315 \\
74 \\
78\end{array}$ & $\begin{array}{l}540 \\
535 \\
775 \\
755 \\
775\end{array}$ & $\begin{array}{l}320 \\
315 \\
310 \\
310 \\
315\end{array}$ & $\begin{array}{l}12 \\
12 \\
12 \\
12 \\
12\end{array}$ & $\begin{array}{l}12 \\
12 \\
12 \\
12 \\
12\end{array}$ \\
\hline $\begin{array}{r}6 \\
7 \\
8 \\
9 \\
10\end{array}$ & $\begin{array}{l}12 \\
12 \\
12 \\
12 \\
12\end{array}$ & $\begin{array}{l}83 \\
83 \\
83 \\
83 \\
83\end{array}$ & $\begin{array}{l}80 \\
80 \\
79 \\
79 \\
79\end{array}$ & $\begin{array}{l}76 \\
76 \\
76 \\
76 \\
76\end{array}$ & $\begin{array}{l}69 \\
69 \\
69 \\
69 \\
70\end{array}$ & $\begin{array}{l}21 \\
21 \\
20 \\
12 \\
12\end{array}$ & $\begin{array}{l}12 \\
12 \\
12 \\
12 \\
12\end{array}$ & $\begin{array}{l}216 \\
300 \\
331 \\
375 \\
370\end{array}$ & $\begin{array}{r}848 \\
915 \\
910 \\
900 \\
1380\end{array}$ & $\begin{array}{l}75 \\
12 \\
12 \\
12 \\
12\end{array}$ & $\begin{array}{l}12 \\
12 \\
12 \\
12 \\
12\end{array}$ & $\begin{array}{l}12 \\
12 \\
12 \\
12 \\
12\end{array}$ \\
\hline $\begin{array}{l}11 \\
12 \\
13 \\
14 \\
15\end{array}$ & $\begin{array}{l}12 \\
12 \\
12 \\
12 \\
12\end{array}$ & $\begin{array}{l}83 \\
82 \\
82 \\
81 \\
82\end{array}$ & $\begin{array}{l}80 \\
80 \\
80 \\
80 \\
80\end{array}$ & $\begin{array}{l}76 \\
75 \\
75 \\
75 \\
75\end{array}$ & $\begin{array}{l}71 \\
71 \\
70 \\
70 \\
70\end{array}$ & $\begin{array}{l}12 \\
12 \\
12 \\
12 \\
12\end{array}$ & $\begin{array}{l}12 \\
12 \\
12 \\
12 \\
12\end{array}$ & $\begin{array}{l}375 \\
375 \\
375 \\
440 \\
535\end{array}$ & $\begin{array}{l}1.680 \\
1.600 \\
1.580 \\
1.620 \\
1.330\end{array}$ & $\begin{array}{r}12 \\
82 \\
350 \\
340 \\
345\end{array}$ & $\begin{array}{l}12 \\
12 \\
12 \\
12 \\
12\end{array}$ & $\begin{array}{ll}12 & 12 \\
12 & 12 \\
12 \\
12\end{array}$ \\
\hline $\begin{array}{l}16 \\
17 \\
18 \\
19 \\
20\end{array}$ & $\begin{array}{l}12 \\
12 \\
12 \\
12 \\
12\end{array}$ & $\begin{array}{l}82 \\
82 \\
82 \\
82 \\
82\end{array}$ & $\begin{array}{l}80 \\
80 \\
79 \\
79 \\
79\end{array}$ & $\begin{array}{l}74 \\
74 \\
74 \\
74 \\
72\end{array}$ & $\begin{array}{l}69 \\
69 \\
69 \\
69 \\
69\end{array}$ & $\begin{array}{l}12 \\
12 \\
12 \\
12 \\
12\end{array}$ & $\begin{array}{l}12 \\
12 \\
12 \\
12 \\
12\end{array}$ & $\begin{array}{l}520 \\
520 \\
525 \\
745 \\
730\end{array}$ & $\begin{array}{l}1.400 \\
1.430 \\
1.390 \\
1.580 \\
1.420\end{array}$ & $\begin{array}{l}335 \\
695 \\
680 \\
330 \\
345\end{array}$ & $\begin{array}{l}12 \\
12 \\
12 \\
12 \\
12\end{array}$ & $\begin{array}{r}12 \\
12 \\
180 \\
370 \\
360\end{array}$ \\
\hline $\begin{array}{l}21 \\
22 \\
23 \\
24 \\
25\end{array}$ & $\begin{array}{l}84 \\
84 \\
84 \\
84 \\
84\end{array}$ & $\begin{array}{l}82 \\
82 \\
82 \\
81 \\
82\end{array}$ & $\begin{array}{l}79 \\
79 \\
79 \\
80 \\
79\end{array}$ & $\begin{array}{l}72 \\
71 \\
71 \\
71 \\
71\end{array}$ & $\begin{array}{l}68 \\
68 \\
68 \\
68 \\
68\end{array}$ & $\begin{array}{l}12 \\
12 \\
12 \\
12 \\
12\end{array}$ & $\begin{array}{r}12 \\
12 \\
12 \\
320 \\
1.030\end{array}$ & $\begin{array}{l}875 \\
875 \\
875 \\
410 \\
465\end{array}$ & $\begin{array}{r}1.360 \\
640 \\
675 \\
670 \\
655\end{array}$ & $\begin{array}{l}345 \\
345 \\
350 \\
355 \\
355\end{array}$ & $\begin{array}{l}12 \\
12 \\
12 \\
12 \\
12\end{array}$ & $\begin{array}{l}360 \\
246 \\
240 \\
240 \\
160\end{array}$ \\
\hline $\begin{array}{l}26 \\
27 \\
28 \\
29 \\
30 \\
31\end{array}$ & $\begin{array}{l}84 \\
84 \\
84 \\
83 \\
83 \\
83\end{array}$ & $\begin{array}{r}82 \\
82 \\
83 \\
83 \\
82 \\
\end{array}$ & $\begin{array}{l}79 \\
78 \\
78 \\
77 \\
77 \\
77\end{array}$ & $\begin{array}{l}71 \\
71 \\
71 \\
70 \\
70 \\
71\end{array}$ & $\begin{array}{r}68 \\
21 \\
21 \\
21 \\
- \\
2-\end{array}$ & $\begin{array}{l}12 \\
12 \\
12 \\
12 \\
12 \\
12\end{array}$ & $\begin{array}{r}1.280 \\
1.190 \\
1.200 \\
1.260 \\
1.320 \\
\end{array}$ & $\begin{array}{l}465 \\
470 \\
465 \\
445 \\
455 \\
545\end{array}$ & $\begin{array}{r}635 \\
630 \\
635 \\
517 \\
315 \\
\end{array}$ & $\begin{array}{r}360 \\
360 \\
360 \\
360 \\
190 \\
40\end{array}$ & $\begin{array}{l}12 \\
12 \\
12 \\
12 \\
12 \\
12\end{array}$ & $\begin{array}{r}160 \\
160 \\
80 \\
80 \\
80 \\
-\end{array}$ \\
\hline Total & 1.161 & 2.471 & 2.457 & 2.284 & 1.862 & 443 & 7.876 & 15.569 & 30.095 & 3627 & 372 & 2.920 \\
\hline $\begin{array}{l}\text { Mean } \\
\text { Max } \\
\text { Min } \\
\text { Cfsm } \\
\text { In. }\end{array}$ & $\begin{array}{r}37.5 \\
84 \\
12 \\
0.089 \\
0.10\end{array}$ & $\begin{array}{r}82.4 \\
83 \\
81 \\
0.196 \\
0.22\end{array}$ & $\begin{array}{r}79.3 \\
81 \\
77 \\
0.188 \\
0.22\end{array}$ & $\begin{array}{r}73.7 \\
76 \\
70 \\
0.175 \\
0.20\end{array}$ & $\begin{array}{r}64.2 \\
71 \\
21 \\
0.152 \\
0.16\end{array}$ & $\begin{array}{r}14.3 \\
21 \\
12 \\
0.034 \\
0.04\end{array}$ & $\begin{array}{r}263 \\
1,320 \\
12 \\
0.625 \\
0.70\end{array}$ & $\begin{array}{r}502 \\
1,380 \\
74 \\
1.19 \\
1.38\end{array}$ & $\begin{array}{r}1,003 \\
1,680 \\
315 \\
2.38 \\
2.66\end{array}$ & $\begin{array}{r}278 \\
695 \\
12 \\
0.660 \\
0.76\end{array}$ & $\begin{array}{r}12.0 \\
12 \\
12 \\
0.029 \\
0.03\end{array}$ & $\begin{array}{r}97.3 \\
370 \\
12 \\
0.231 \\
0.26\end{array}$ \\
\hline $\begin{array}{l}\text { Cal yr } \\
\text { wtr yr } \\
\end{array}$ & $\begin{array}{l}1967: \\
1968:\end{array}$ & $\begin{array}{l}\text { Total } \\
\text { Tota } 1\end{array}$ & $\begin{array}{l}109 \\
137 \\
\end{array}$ & $\begin{array}{ll}\text { lean } & 206 \\
\text { lean } & 208\end{array}$ & $\begin{array}{l}\text { Max } \\
\operatorname{Max}\end{array}$ & $\begin{array}{l}1,280 \\
1,680\end{array}$ & $\begin{array}{lr}\text { Min } & 0 \\
\text { Min } & 12\end{array}$ & $\begin{array}{l}\text { Cfsm } \\
\text { Cfsm }\end{array}$ & $\begin{array}{l}489 \\
494 \\
\end{array}$ & $\begin{array}{l}6.63 \\
6.73 \\
\end{array}$ & & \\
\hline
\end{tabular}


5-2205. Mississippi River below Sandy River, near Libby, Minn.

Location.--Lat $46^{\circ} 47^{\prime}$, long $93^{\circ} 20^{\prime}$, in sec.25, T.50 N., R.24 W., on right bank 600 ft downstream from Sandy River, three-quarters of a mile northwest of Libby, and at mile 1,106 upstream from ohio River.

Drainage area.--5,060 sq mi, approximately.

Records available.--April 1930 to september 1968.

Gage.-Water-stage recorder. Datum of gage is $1,204.55 \mathrm{ft}$ above mean sea level, adjustment of 1912 . Prior to July 28, 1931, staff gage at site $600 \mathrm{ft}$ upstream at datum $3.16 \mathrm{ft}$ higher.

Average discharge.--38 years, $1,917 \mathrm{cfs}$.

Extremes.--Maximum discharge during year, 4,590 cfs June 15 (gage height, 10.25 ft); minimum, 772 cfs Oct. 1 (gage height, $3.12 \mathrm{ft}$ )

1930-68: Maximum discharge, 16,000 cfs May 17, 1950 (gage height, $20.02 \mathrm{ft}$ ); minimum, 83 cfs Nov. 16, 1936 (gage height, $1.44 \mathrm{ft}$ ).

Remarks.--Records good except those for winter months, which are fair. Flow regulated by powerplants and Winnibigoshish, Leech, Pokegama, and Sandy Lakes (see p. 91,93,95,101).

DISCHARGE, IN CUBIC FEET PER SECOND, WATER YEAR OCTOBER 1967 TO SEPTEMBER 1968

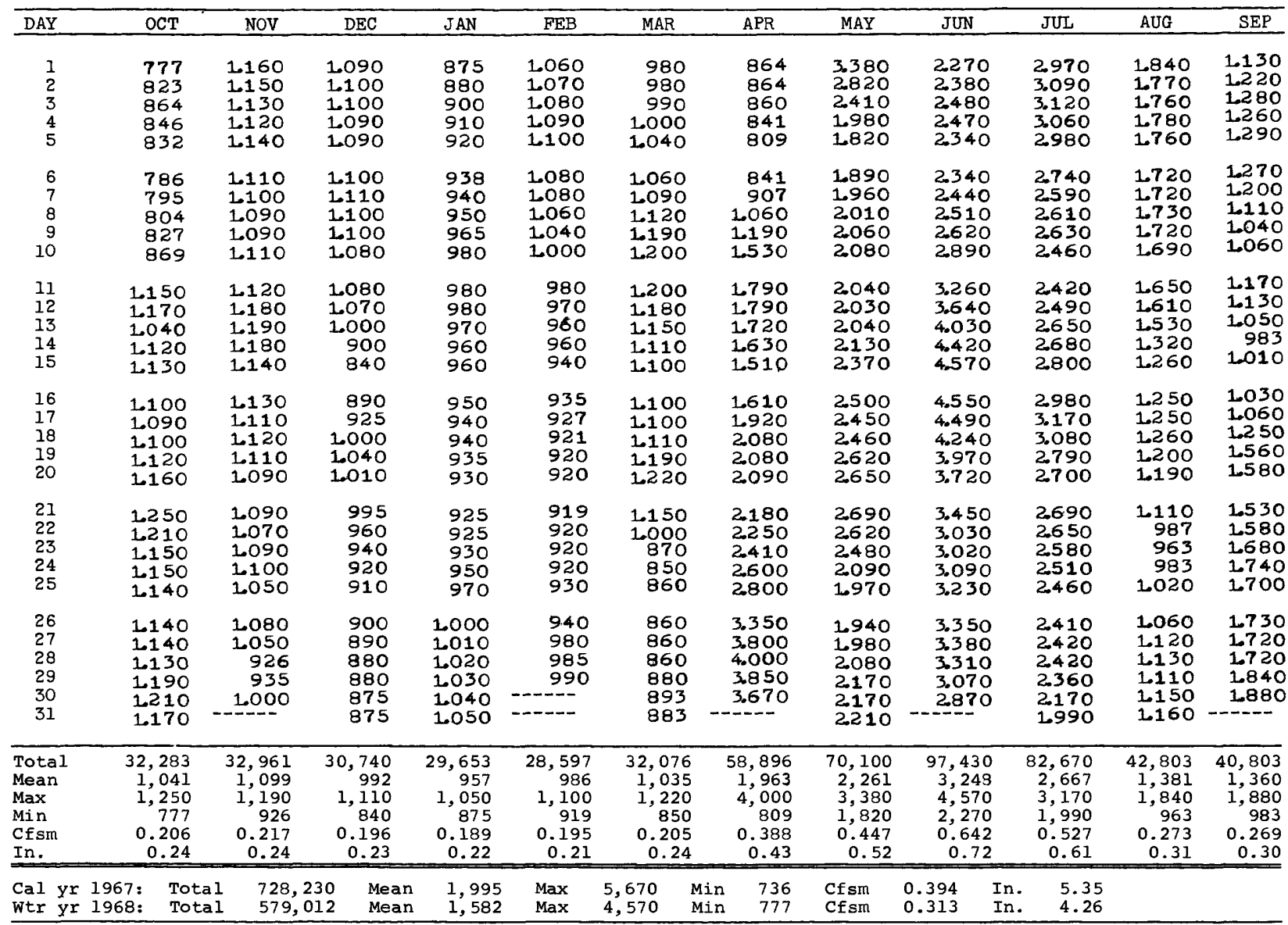


Location.--Lat $46^{\circ} 32^{\prime}$ 26" $^{\prime \prime}$, long $93^{\circ} 42^{\prime} 26^{\prime \prime}$, in W/s sec.24, T.47 N., R.27 W., at upstream side of highway bridge at north edge of Aitkin, 1 mile downstream from Mud River and at mile 1,055.9 upstream from ohio River.

Drainage area.--6,140 sq $\mathrm{mi}$, approximately.

Records available.--March 1945 to September 1968.

Gage.--Water-stage recorder. Datum of gage is 1,182.41 ft above mean sea level, datum of 1929 (levels by Corps of Engineers). Mar. 1, 1945, to July 12, 1954, staff gage, July 13, 1954, to June 23, 1955, chain gage, June 24, 1955, to Mar. 14, 1961, wire-weight gage, and Mar. 15, 1961, to Sept. 30, 1967, water-stage recorder at same site at datum $3.0 \mathrm{ft}$ higher. Diversion channel: wire-weight gage. Datum of gage is $1,182.02 \mathrm{ft}$ above mean sea level, datum of 1929. Apr. 9, 1955, to Apr. 10, 1956, staff gage at site 4 miles downstream at different datum. Apr. 11, 1956, to sept. 6, 1960, staff gage, and Sept. 7, 1960, to Sept. 30, 1967, wire-weight gage at same site at datum $3.0 \mathrm{ft}$ higher.

Average discharge.--23 years, $2,840 \mathrm{cfs}$.

Extremes.--Maximum discharge during year, 5,850 cfs June 17; minimum, 848 cfs Oct. 1. River gage: Maximum discharge during year, 4,160 cfs June 17 (gage height, $10.90 \mathrm{ft}$ ); minimum, 848 cfs oct. I (gage height,
$2.71 \mathrm{ft}$ ). Diversion gage: Maximum discharge during year, 1,690 cfs June 17 (gage height, $9.70 \mathrm{ft}$, from graph based on gage readings); no flow on many days.

1945-68: Maximum discharge, 20,000 cfs May 20, 1950 (gage height, $22.49 \mathrm{ft}$, present datum); minimum, 151 cfs Sept. 1, 1961 (gage height, $0.60 \mathrm{ft}$ ).

Remarks.--Records good except those for winter months, which are fair. slight regulation by powerplants and by Winnibigoshish, Leech, Pokegama, and Sandy Lakes (see p. 91,93,95, 101). Water diverted at medium and high stages into Aitkin diversion channel $6 \frac{1}{2}$ miles above station, bypasses station and returns to river 15 $\frac{1}{2}$ miles below station. Diversion began Apr. 2, 1955. These records include flow in diversion channel. Records of chemical analyses for water year 1968 are published in Part 2 of this report.

DISCHARGE, IN CUBIC FEET PER SECOND, WATER YEAR OCTOBER 1967 TO SEPTEMBER 1968

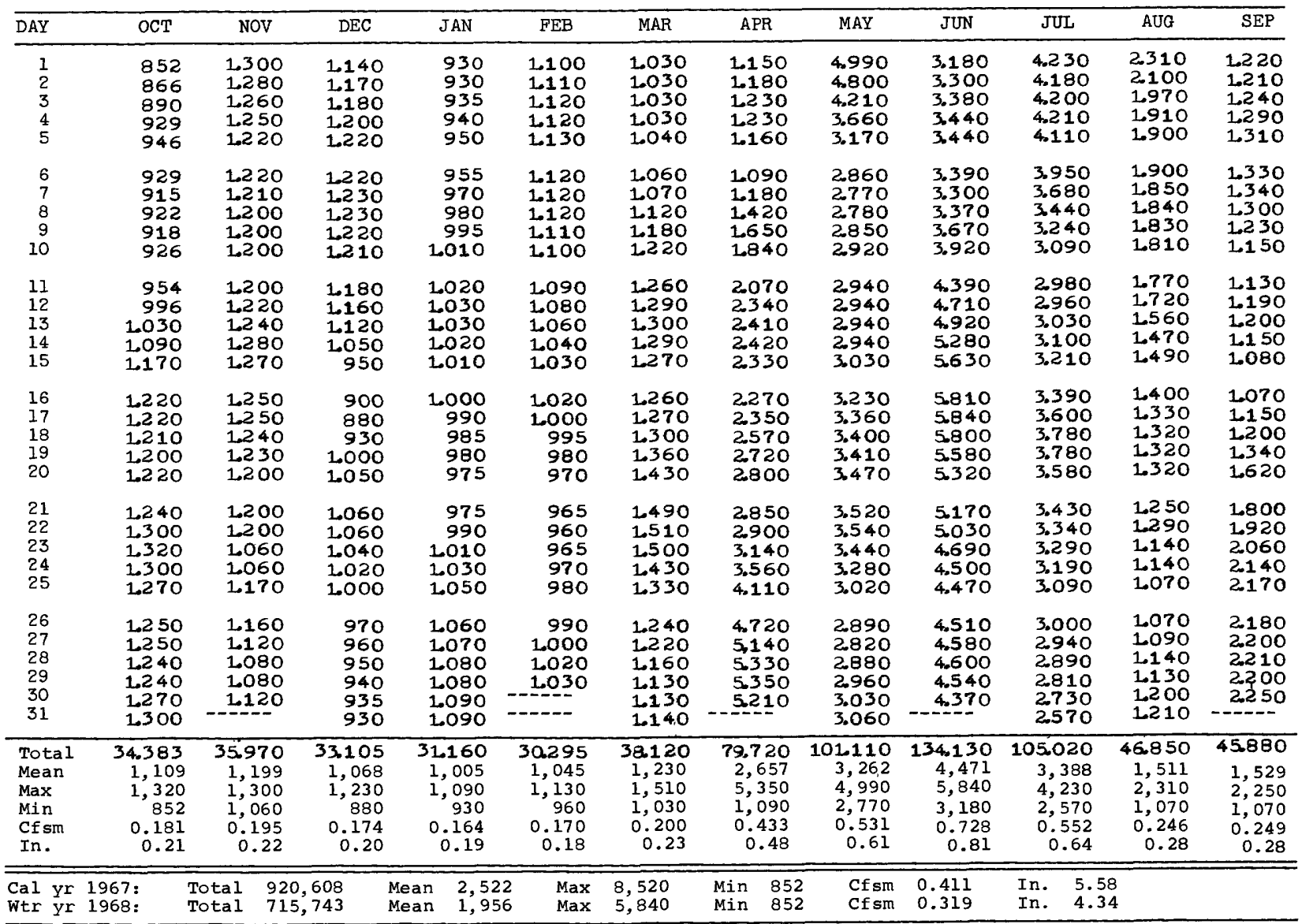


5-2305. Pine River Reservoir at Cross Lake, Minn.

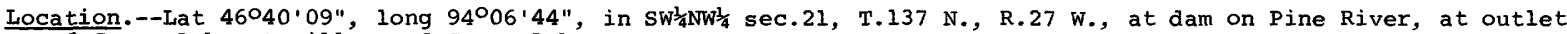
of Cross Lake at village of Cross Lake.

Drainaqe area. $--562 \mathrm{sq} \mathrm{mi}$.

Records available.--March 1886 to september 1968. Month-end contents only for some periods, published in wsP 1308.

Gaqe.--Water-stage recorder. Datum of gage is $1,216.32 \mathrm{ft}$ above mean sea level, datum of 1929 (levels by Corps of Engineers). Prior to May 3, 1949, staff gage at same site and datum.

Extremes.--Maximum contents during year, 102,300 acre-ft Apr. 24 (gage height, 13.29 ft); minimum, 78,900 acreft Mar. 13-17 (gage height, $11.55 \mathrm{ft}$ ).

1886-1968: Maximum contents observed, 173,600 acre-ft July 10, 1916 (gage height, 18.24 ft); minimum observed, 1,310 acre-ft below zero of capacity table Aug. 20, 1918 (gage height, 1.35 ft).

Remarks.--Reservoir is formed by Trout, Whitefish, Rush, and Cross Lakes and several other natural lakes controlled by timber crib dams; storage began in 1886; dam completed in 1886. Capacity between gage heights $10.00 \mathrm{ft}$ and $18.5 \mathrm{ft}$ (maximum allowable range) is 118,710 acre-ft of which 53,280 acre-ft is controlled storage between gage heights $10.00 \mathrm{ft}$ and $14.00 \mathrm{ft}$ (normal operating range). Contents shown herein are contents above a gage height of $2.35 \mathrm{ft}$. Water is used to benefit navigation on Mississippi River below Minneapolis.

Cooperation.--Records furnished by Corps of Engineers in terms of cfs-days and converted to acre-feet by Geological survey.

Month-end gage height and contents, water year October 1967 to september 1968

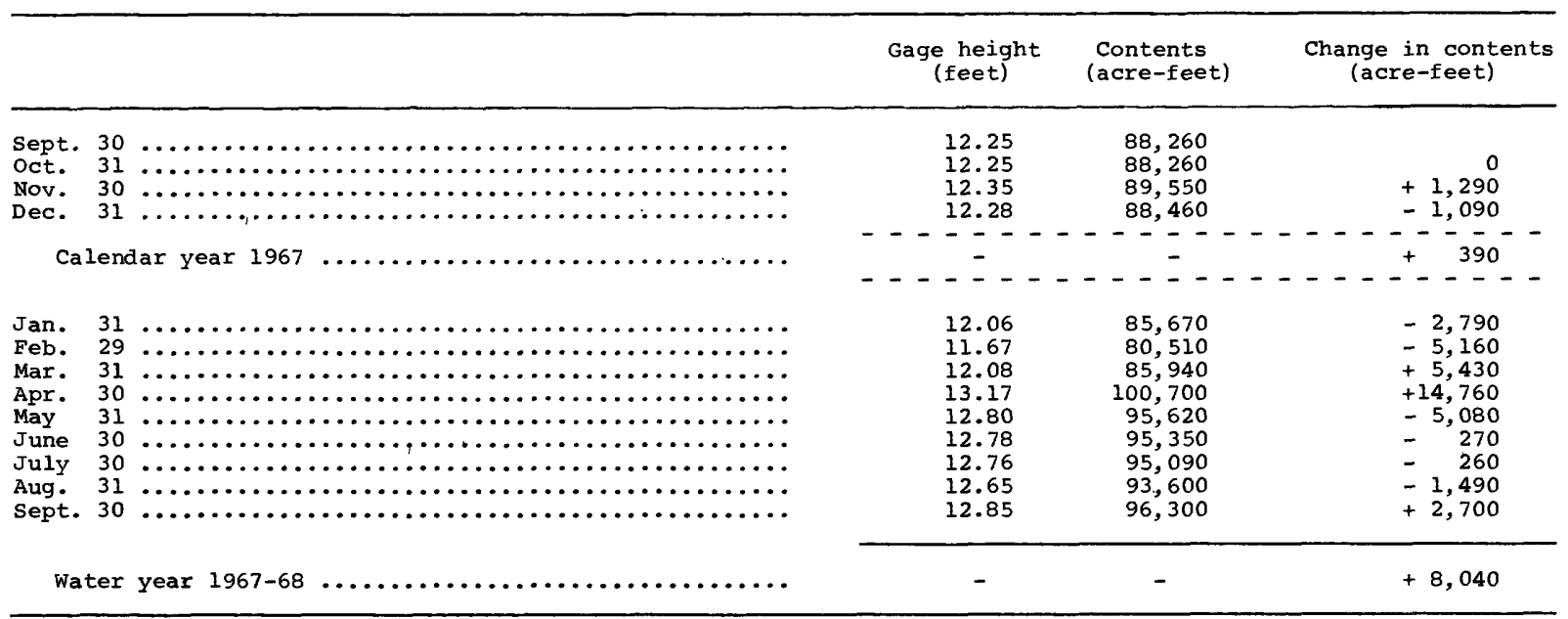


Location.--Lat $46^{\circ} 40^{\prime} 09^{\prime \prime}$, long $94^{\circ} 06^{\prime} 44^{\prime \prime}$, in SW' $\times$ NW/ $\frac{1}{4}$ sec. 21 , T.137 N., R. 27 w., at dam at outlet of Cross Lake at village of Cross Lake.

Drainage area. $--562 \mathrm{sq} \mathrm{mi}$.

Records available.--April 1886 to september 1968 . Monthly discharge only for some periods, published in wsP 1308. Published as "below Pine River Reservoir" 1895-1916, 1929, and as "at Pine River Dam, at Cross Lake" $1941-56$.

Gage.--Water-stage recorder, headwater gage, and tape float tailwater gage, read twice daily. Datum of gages is $1,216.32 \mathrm{ft}$ above mean sea level, datum of 1929 . Mar. 26, 1886, to May 31, 1929, staff gages on headwater and tailwater at same sites and datum. June 1 to Nov. 30, 1929, staff gage in tailwater at datum $1.60 \mathrm{ft}$ lower. Dec. 1, 1929, to May 2, 1949, staff gage on headwater and Dec. 1 , 1929, to August 1949, staff gage on tailwater at present sites and datum.

Average discharge.--82 years, $211 \mathrm{cfs}$, unadjusted.

Extremes.--Maximum daily discharge during year, 710 cfs July 20, 21 ; minimum daily, 15 cfs Oct. 1 to Nov. 28. 1886-1968: Maximum daily discharge, 2,250 cfs in June 1896 (does not include flow bypassing dam through crevasse); no flow at times.

Remarks.--Discharge computed principally on basis of modified weir formula, the head being obtained from twicedaily readings on tailwater gage and from headwater recorder. Flow completely regulated by Pine River Reservoir (see preceding page).

Cooperation.--Computations of daily discharge furnished by Corps of Engineers; two discharge measurements made and records reviewed by Geological survey.

DISCHARGE, IN CFS, WATER YEAR OCTOBER 1967 TO SEPTEMBER 1968

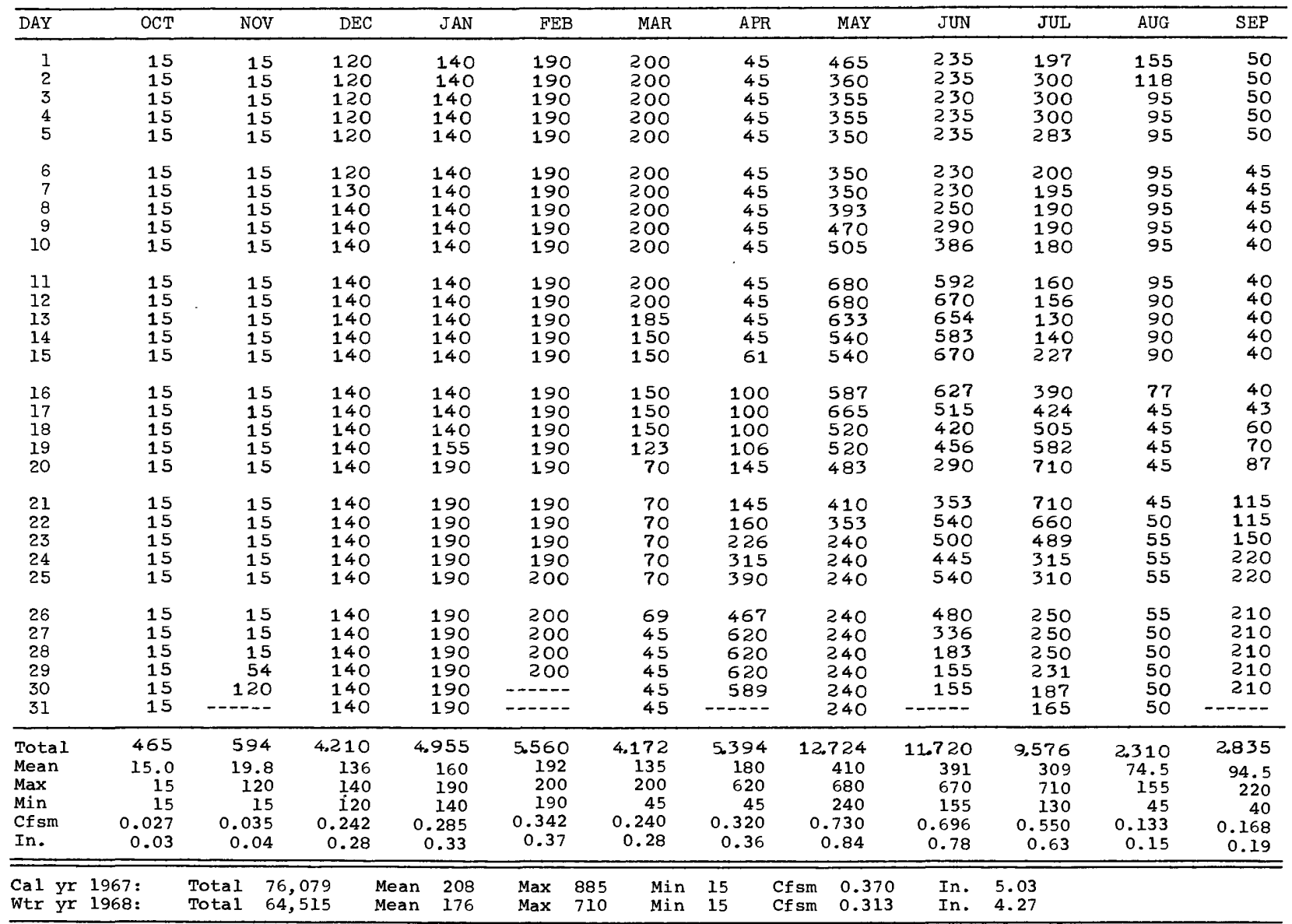


5-2315. Pelican Lake near Pequot Lakes, Minn.

Location.--Lat $46^{\circ} 37^{\prime}$, long $94^{\circ} 11^{\prime}$, in NE $\frac{1}{4} \mathrm{NE}_{4}^{\frac{1}{4}}$ sec.10, T.136 N., R.28 W., on downstream side of right abutment of dam and bridge on channel between Ossawinnamakee and Pelican Lakes, 0.5 mile upstream from Pelican Lake and 6 miles east of town of Pequot Lakes.

Records available.--April 1938 to September 1968 (fragmentary). Prior to October 1956, published as Pelican Lake diversion near Pequot Lakes.

Gage.--Staff gage read about three times weekly during open-water period. Datum of gage is $1,203.69$ ft above mean sea level, datum of 1929 (levels by Minnesota Department of Conservation).

Extremes.--Maximum gage height observed during year, $3.70 \mathrm{ft}$ June 22 ; minimum observed, $3.12 \mathrm{ft}$ Sept. 6 , 11. 1938-68: Maximum gage height observed, 4.28 ft May 23, 1966; minimum observed, 1.36 ft May 2, 1938.

Remarks.--Crest of stoplogs on dam is normally fixed at 1,207.19 ft, but may be lowered to $1,203.69$ ft (elevation of sill and apron) by removal of stoplogs.

Gage height, in feet, October 1967 to September 1968

May $31 \ldots \ldots \ldots .3 .36$

July $30 \ldots \ldots \ldots \ldots . . . .36$

Aug. $30 \ldots \ldots \ldots \ldots \ldots 3.20$

Sept.29 $\ldots \ldots \ldots \ldots .3 .24$

June $29 \ldots \ldots \ldots \ldots \ldots .3 .34$

Note.--Gage readings other than those shown are available. 
5-2440. Crow Wing River at Nimrod, Minn.

Location.--Lat $46^{\circ} 39^{\prime}$, long $94^{\circ} 53^{\prime}$, in sec.32, T.137 N., R. 33 W., on right bank 200 ft upstream from highway

bridge, $0.2 \mathrm{mile}$ north of Nimrod, and $0.7 \mathrm{mile}$ upstream from Cat River.

Drainage area.--1,010 sq $\mathrm{mi}$, approximately.

Records available.--April 1910 to September 1914, July 1930 to September 1968 (winter records incomplete prior to 1940).

Gage.--Digital water-stage recorder. Datum of gage is 1,313.27 ft above mean sea level, datum of 1929 (levels

by Wadena County Highway Department from Minnesota Highway Department bench mark). Apr. 15, 19l0, to Sept. 30, 1914, chain gage at bridge $10 \mathrm{ft}$ downstream at datum 2.2 ft lower. July 28, 1930, to Aug. 19, 1948,

chain gage and Aug. 20, 1948, to Nov. 4, 1949, wire-weight gage, at bridge 10 ft downstream at same datum.

Nov. 5, 1949, to Aug. 27, 1968, graphic water-stage recorder at same site and datum.

Average discharge.--29 years $(1939-68), 472$ cfs.

Extremes.--Maximum discharge during year, 2,750 cfs June 9 (gage height, 6.07 ft, from recorded range-in-stage) minimum, 196 cfs Nov. 23, 24 (gage height, $2.53 \mathrm{ft}$, result of freezeup).

1910-14, 1930-68: Maximum discharge, 2,890 cfs Apr. 13, 1965 (gage height, 7.57 ft, backwater from ice); maximum gage height, $7.64 \mathrm{ft}$, backwater from ice; minimum discharge observed, $45 \mathrm{cfs}$ Aug. 7 , 1936 .

Remarks.--Records good except those for winter months, which are fair. Flow affected by natural storage in many lakes. Records of chemical analysis and suspended sediment loads for the water year 1968 are published

DISCHARGE, IN CUBIC FEET PER SECOND, WATER YEAR OCTOBER 1967 TO SEPTEMBER 1968

\begin{tabular}{|c|c|c|c|c|c|c|c|c|c|c|c|c|c|}
\hline $\mathrm{DAY}$ & $\overline{\mathrm{OCT}}$ & NOV & $\mathrm{DEC}$ & & JAN & $\overline{F E B}$ & MAR & APR & MAY & JUN & JUL & AUG & SEP \\
\hline $\begin{array}{l}1 \\
2 \\
3 \\
4 \\
5\end{array}$ & $\begin{array}{l}300 \\
295 \\
300 \\
305 \\
305\end{array}$ & $\begin{array}{l}397 \\
397 \\
397 \\
397 \\
393\end{array}$ & $\begin{array}{l}370 \\
370 \\
380 \\
395 \\
410\end{array}$ & & $\begin{array}{l}290 \\
285 \\
280 \\
275 \\
270\end{array}$ & $\begin{array}{l}265 \\
265 \\
265 \\
260 \\
260\end{array}$ & $\begin{array}{l}260 \\
260 \\
265 \\
270 \\
270\end{array}$ & $\begin{array}{l}649 \\
616 \\
610 \\
598 \\
630\end{array}$ & $\begin{array}{l}912 \\
870 \\
857 \\
838 \\
824\end{array}$ & $\begin{array}{l}610 \\
600 \\
598 \\
600 \\
610\end{array}$ & $\begin{array}{l}955 \\
919 \\
870 \\
824 \\
805\end{array}$ & $\begin{array}{l}592 \\
568 \\
544 \\
532 \\
508\end{array}$ & $\begin{array}{l}419 \\
413 \\
421 \\
424 \\
418\end{array}$ \\
\hline $\begin{array}{r}6 \\
7 \\
8 \\
9 \\
10\end{array}$ & $\begin{array}{l}305 \\
331 \\
358 \\
370 \\
370\end{array}$ & $\begin{array}{l}390 \\
385 \\
390 \\
392 \\
386\end{array}$ & $\begin{array}{l}415 \\
420 \\
420 \\
420 \\
415\end{array}$ & & $\begin{array}{l}260 \\
260 \\
255 \\
250 \\
250\end{array}$ & $\begin{array}{l}255 \\
250 \\
250 \\
245 \\
245\end{array}$ & $\begin{array}{l}280 \\
290 \\
305 \\
320 \\
340\end{array}$ & $\begin{array}{l}604 \\
649 \\
766 \\
818 \\
812\end{array}$ & $\begin{array}{l}805 \\
818 \\
818 \\
824 \\
824\end{array}$ & $\begin{array}{r}630 \\
792 \\
1.040 \\
2.650 \\
2.620\end{array}$ & $\begin{array}{l}772 \\
746 \\
727 \\
688 \\
668\end{array}$ & $\begin{array}{l}490 \\
484 \\
502 \\
490 \\
484\end{array}$ & $\begin{array}{l}426 \\
435 \\
441 \\
441 \\
437\end{array}$ \\
\hline $\begin{array}{l}11 \\
12 \\
13 \\
14 \\
15\end{array}$ & $\begin{array}{l}370 \\
375 \\
375 \\
370 \\
370\end{array}$ & $\begin{array}{l}386 \\
386 \\
386 \\
386 \\
386\end{array}$ & $\begin{array}{l}410 \\
410 \\
405 \\
405 \\
405\end{array}$ & & $\begin{array}{l}250 \\
245 \\
245 \\
245 \\
240\end{array}$ & $\begin{array}{l}245 \\
240 \\
240 \\
240 \\
240\end{array}$ & $\begin{array}{l}355 \\
360 \\
360 \\
360 \\
365\end{array}$ & $\begin{array}{l}805 \\
798 \\
792 \\
798 \\
818\end{array}$ & $\begin{array}{l}798 \\
805 \\
798 \\
818 \\
824\end{array}$ & $\begin{array}{l}2.380 \\
2.020 \\
1.880 \\
1.850 \\
1.760\end{array}$ & $\begin{array}{r}642 \\
884 \\
844 \\
827 \\
1.720\end{array}$ & $\begin{array}{l}472 \\
466 \\
454 \\
442 \\
454\end{array}$ & $\begin{array}{l}422 \\
408 \\
397 \\
393 \\
384\end{array}$ \\
\hline $\begin{array}{l}16 \\
17 \\
18 \\
19 \\
20\end{array}$ & $\begin{array}{l}364 \\
358 \\
358 \\
358 \\
358\end{array}$ & $\begin{array}{l}380 \\
375 \\
375 \\
375 \\
386\end{array}$ & $\begin{array}{l}410 \\
415 \\
425 \\
435 \\
440\end{array}$ & & $\begin{array}{l}240 \\
240 \\
240 \\
240 \\
240\end{array}$ & $\begin{array}{l}240 \\
245 \\
245 \\
245 \\
245\end{array}$ & $\begin{array}{l}370 \\
375 \\
385 \\
410 \\
425\end{array}$ & $\begin{array}{l}792 \\
772 \\
766 \\
740 \\
805\end{array}$ & $\begin{array}{l}824 \\
805 \\
798 \\
792 \\
760\end{array}$ & $\begin{array}{l}1.660 \\
1.560 \\
1.420 \\
1.290 \\
1.220\end{array}$ & $\begin{array}{r}1.550 \\
1.370 \\
1.270 \\
1.100 \\
948\end{array}$ & $\begin{array}{l}478 \\
466 \\
472 \\
490 \\
484\end{array}$ & $\begin{array}{l}400 \\
495 \\
497 \\
496 \\
489\end{array}$ \\
\hline $\begin{array}{l}21 \\
22 \\
23 \\
24 \\
25\end{array}$ & $\begin{array}{l}358 \\
358 \\
358 \\
358 \\
358\end{array}$ & $\begin{array}{l}380 \\
375 \\
256 \\
326 \\
419\end{array}$ & $\begin{array}{l}440 \\
430 \\
410 \\
390 \\
370\end{array}$ & & $\begin{array}{l}245 \\
245 \\
250 \\
255 \\
255\end{array}$ & $\begin{array}{l}250 \\
250 \\
250 \\
250 \\
250\end{array}$ & $\begin{array}{l}420 \\
415 \\
410 \\
410 \\
420\end{array}$ & $\begin{array}{r}940 \\
970 \\
1.060 \\
1.170 \\
1.150\end{array}$ & $\begin{array}{l}746 \\
714 \\
705 \\
727 \\
670\end{array}$ & $\begin{array}{r}1190 \\
1120 \\
1050 \\
978 \\
962\end{array}$ & $\begin{array}{l}877 \\
838 \\
792 \\
753 \\
714\end{array}$ & $\begin{array}{l}472 \\
454 \\
454 \\
454 \\
448\end{array}$ & $\begin{array}{l}482 \\
520 \\
567 \\
566 \\
561\end{array}$ \\
\hline $\begin{array}{l}26 \\
27 \\
28 \\
29 \\
30 \\
31\end{array}$ & $\begin{array}{l}358 \\
370 \\
375 \\
386 \\
397 \\
397\end{array}$ & $\begin{array}{l}408 \\
390 \\
380 \\
370 \\
370\end{array}$ & $\begin{array}{l}350 \\
340 \\
325 \\
315 \\
305 \\
295\end{array}$ & & $\begin{array}{l}255 \\
260 \\
260 \\
260 \\
260 \\
260\end{array}$ & $\begin{array}{r}255 \\
255 \\
260 \\
260 \\
\end{array}$ & $\begin{array}{l}480 \\
640 \\
760 \\
649 \\
662 \\
649\end{array}$ & $\begin{array}{r}1120 \\
1060 \\
1020 \\
1000 \\
955\end{array}$ & $\begin{array}{l}642 \\
640 \\
642 \\
600 \\
598 \\
600\end{array}$ & $\begin{array}{r}926 \\
891 \\
850 \\
831 \\
850 \\
\end{array}$ & $\begin{array}{l}682 \\
682 \\
636 \\
616 \\
616 \\
616\end{array}$ & $\begin{array}{l}454 \\
430 \\
412 \\
404 \\
404 \\
419\end{array}$ & $\begin{array}{r}554 \\
543 \\
537 \\
525 \\
514 \\
\end{array}$ \\
\hline $\begin{array}{l}\text { Total } \\
\text { Mean } \\
\text { Max } \\
\text { Min } \\
\text { Cfsm } \\
\text { In. }\end{array}$ & $\begin{array}{r}10.968 \\
354 \\
397 \\
295 \\
0.350 \\
0.40\end{array}$ & $\begin{array}{r}11.19 \\
381 \\
419 \\
256 \\
0.377 \\
0.42\end{array}$ & $\begin{array}{r}12.145 \\
392 \\
440 \\
295 \\
0.388 \\
0.45\end{array}$ & & $\begin{array}{r}7.905 \\
255 \\
290 \\
240 \\
0.252 \\
0.29\end{array}$ & $\begin{array}{r}7.265 \\
251 \\
265 \\
240 \\
0.249 \\
0.27\end{array}$ & $\begin{array}{r}12540 \\
405 \\
760 \\
260 \\
0.401 \\
0.46\end{array}$ & $\begin{array}{r}25083 \\
836 \\
1,170 \\
598 \\
0.828 \\
0.92\end{array}$ & $\begin{array}{r}23.696 \\
764 \\
912 \\
598 \\
0.756 \\
0.87\end{array}$ & $\begin{array}{r}37.438 \\
1,248 \\
2,650 \\
598 \\
1.24 \\
1.38\end{array}$ & $\begin{array}{r}26.931 \\
869 \\
1,720 \\
616 \\
0.860 \\
0.99\end{array}$ & $\begin{array}{r}14.677 \\
473 \\
592 \\
404 \\
0.468 \\
0.54\end{array}$ & $\begin{array}{r}14.025 \\
468 \\
567 \\
384 \\
0.463 \\
0.52\end{array}$ \\
\hline $\begin{array}{l}\text { Cal yr } \\
\text { wtr yr }\end{array}$ & $\begin{array}{l}1967: \\
1968:\end{array}$ & $\begin{array}{l}a 1 \\
a 1\end{array}$ & $\begin{array}{l}51 \\
92\end{array}$ & $\begin{array}{l}\text { Mean } \\
\text { Mean }\end{array}$ & $\begin{array}{l}603 \\
558\end{array}$ & $\begin{array}{l}\text { Max } \\
\operatorname{Max}\end{array}$ & $\begin{array}{l}40 \\
50\end{array}$ & $\begin{array}{l}\text { Min } \\
\text { Min }\end{array}$ & $\begin{array}{l}\text { Cfsm } \\
\text { Cfsm }\end{array}$ & $\begin{array}{l}97 \\
52\end{array}$ & $\begin{array}{l}8.11 \\
7.52\end{array}$ & & \\
\hline
\end{tabular}


5-2465. Gull Lake near Brainerd, Minn.

Location.--Lat $46^{\circ} 24^{\prime} 40^{\prime \prime}$, long $94^{\circ} 21^{\prime} 26^{\prime \prime}$, in N $\frac{1}{2}$ sec. 20 , T.134 N., R. 29 W., in pool of dam on Gull River, 800 ft south of outlet of Gull Lake, a quarter of a mile upstream from Gull Lake Dam, and 8 miles northwest of Brainerd.

Drainage area. $--287 \mathrm{sq} \mathrm{mi}$.

Records available.--August 1911 to September 1968 . Prior to October 1941 month-end contents only, published in WSP 1308. Published as Gull Lake Reservoir October 1941 to september 1956.

Gage.-Water-stage recorder. Datum of gage is $1,188.14 \mathrm{ft}$ above mean sea level, adjustment of 1912 . Prior to Aug. 10, 1949, staff gage $800 \mathrm{ft}$ north of present site at same datum.

Extremes.--Maximum contents during year, 63,630 acre-ft June 23 (gage height, 6.45 ft); minimum, 46,890 acreft Feb. 23 (gage height, $5.16 \mathrm{ft}$ ).

1911-68: Maximum contents,74,800 acre-ft June 30, 1914 (gage height, $7.30 \mathrm{ft}$ ); minimum observed, 22, 250 acre-ft Mar. 20, 1924 (gage height, $3.00 \mathrm{ft}$ ).

Remarks.--Reservoir is formed by Gull Lake and several other natural lakes controlled by concrete dam compleţed in 1913; storage began in 1912. Capacity between gage heights 5.00 ft and $7.00 \mathrm{ft}$ (maximum allowable range and normal operating range) is 26,020 acre-ft. Contents shown herein are contents above gage height 1.00

ft. Water is used to benefit navigation on Mississippi River below Minneapolis.

Cooperation.--Records furnished by Corps of Engineers, in terms of cfs-days and converted to acre-feet by Geological survey.

Month-end gage height and contents, water year October 1967 to September 1968

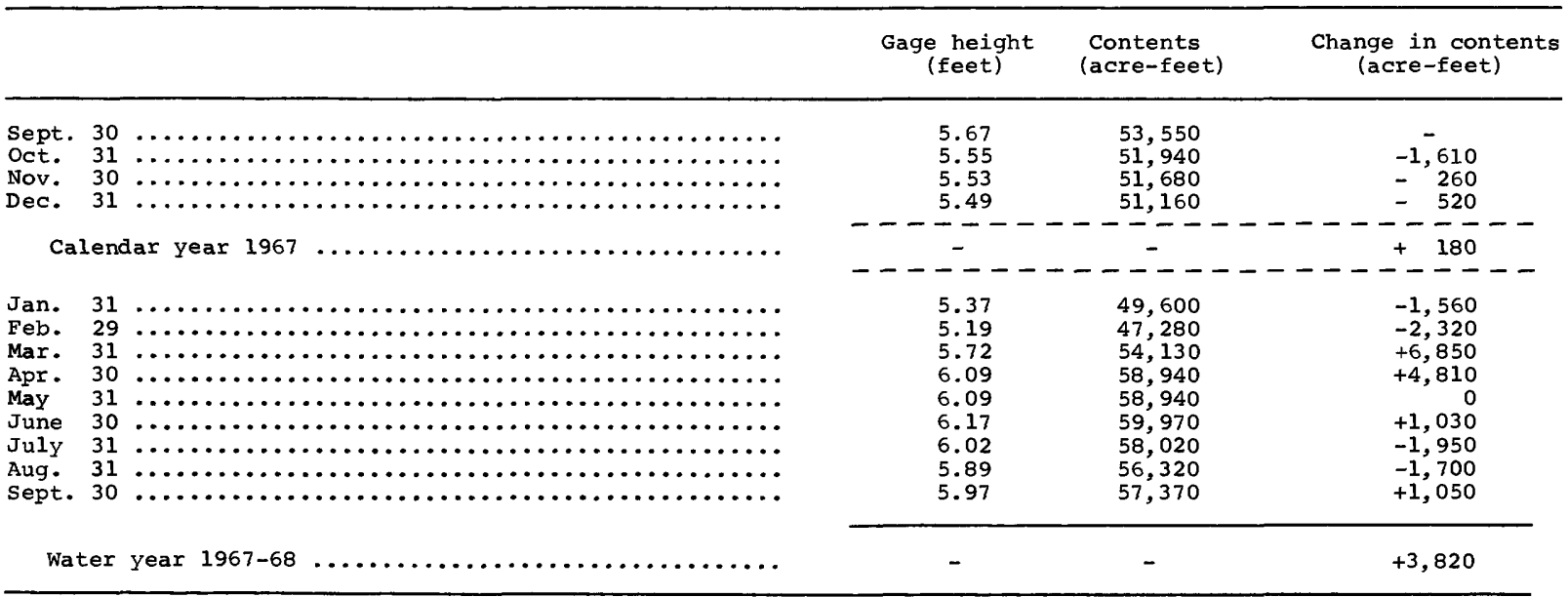


Location.--Lat 46024'40", long $94^{\circ} 21^{\prime} 12^{\prime \prime}$, in sec.20, T.134 N., R.29 W., in headwater and tailwater of dam at outlet of Gull Lake, 8 miles northwest of Brainerd.

Drainage area. $-287 \mathrm{sq} \mathrm{mi}$.

Records available.--August 1911 to September 1968. Monthly discharge only for some periods, published in wSP 1308. Published as "at Gull Lake Reservoir" 1929.

Gage.--Water-stage recorder on headwater and staff gage on tailwater. Datum of gages is $1,188.14 \mathrm{ft}$ above mean sea level, adjustment of 1912. August 1911 to May 23, 1929, and Dec. 1, 1929 to Aug. 1, 1949, both gages were staff gages at same site and datum. May 24 to Nov. 30, 1929, staff gage $500 \mathrm{ft}$ downstream at different datum.

Average discharge. - -57 years, $103 \mathrm{cfs}$, unadjusted.

Extremes.--Maximum daily discharge during year, 650 cfs June 23-26; minimum daily, 3 cfs Aug. 17-19, 26-30, Sept. $2,3,5,6,13-16$

1911-68: Maximum daily discharge, 1,120 cfs May 15, 1938; no flow at times.

Remarks.--Discharge computed at dam on basis of modified weir formulas, the head being obtained from twicedaily readings on tailwater gage and from headwater recorder. Flow completely regulated by Gull lake (see preceding page.

Cooperation.--Computations of daily discharge furnished by Corps of Engineers; two discharge measurements made and records reviewed by Geological survey.

DISCHARGE, IN CFS, WATER YEAR OCTOBER 1967 TO SEPTEMBER 1968

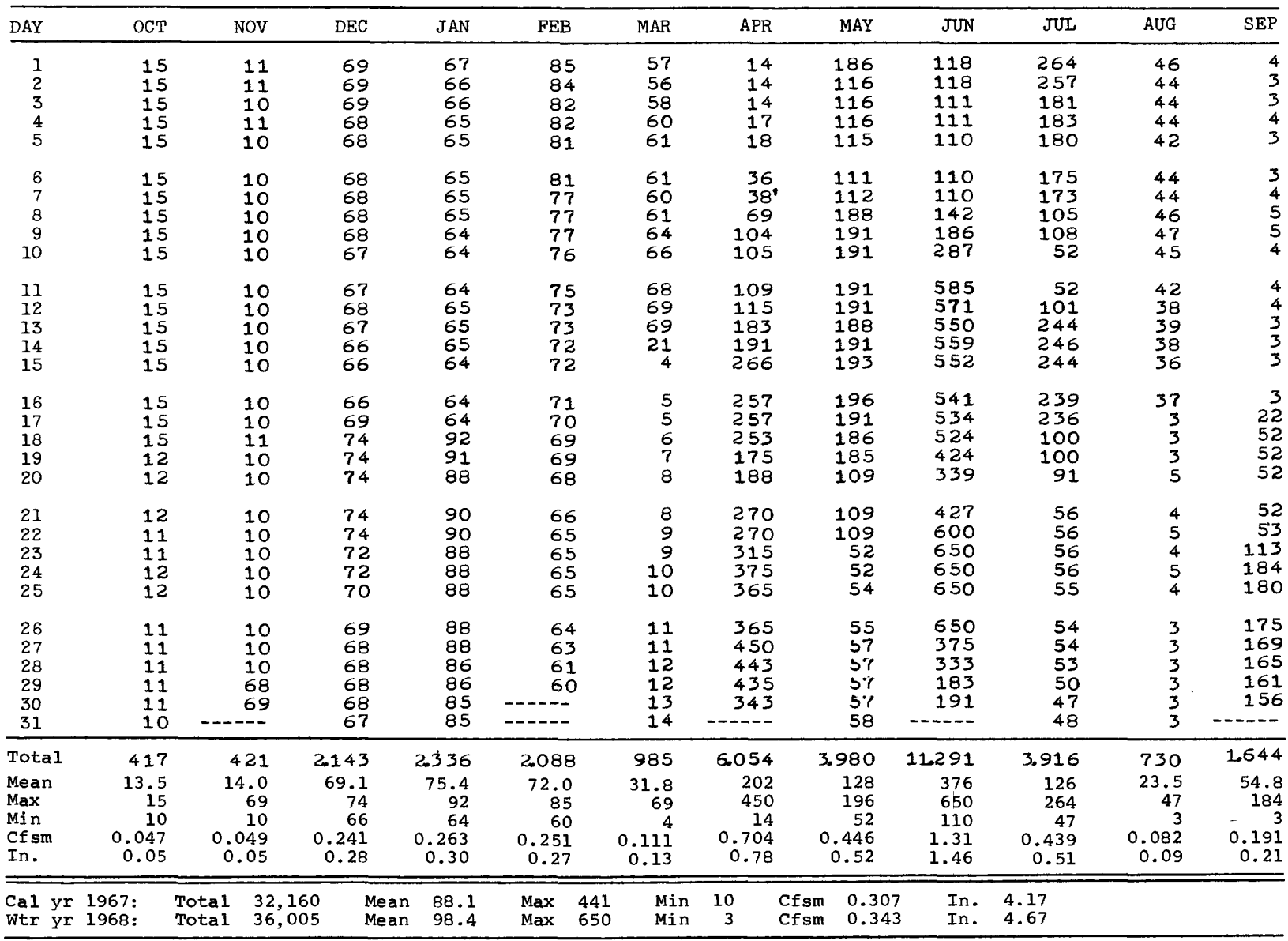


5-2670. Mississippi River near Royalton, Minn.

Location.--Lat $45^{\circ} 51^{\prime} 40^{\prime \prime}$, long $94021^{\prime} 30^{\prime \prime}$, in lot 2, sec.20, T.39 N., R.32 W., at plant of Minnesota Power \& Light Co., 4 miles northwest of Royalton, and 4.5 miles downstream from Swan River, and at mile 956 upstream from ohio River.

Drainage area.--11,600 sq $\mathrm{mi}$, approximately.

Records available.--March 1924 to september 1968.

Average discharge. - -44 years, $4,106 \mathrm{cfs}$.

Extremes.--Maximum daily discharge during year, 16,400 cfs June 14; minimum daily, 955 cfs Nov. 28.

1924-68: Maximum daily discharge, 37,700 cfs Apr. 16, 1965; minimum daily, 254 cfs Nov. $25,1936$.

Remarks.--Records fair. Discharge computed on basis of powerplant records. Flow partly regulated by powerplants and Winnibigoshish, Leech, Pokegama, Sandy and Gull Lakes and by Pine River Reservoir (see p. 91,93 , 95, 105, 109).

Cooperation.--Records collected by Minnesota Power \& Light Co. under general supervision of Geological survey, in connection with a Federal Power Commission project.

DISCHARGE, IN CFS, WATER YEAR OCTOBER 1967 TO SEPTEMBER 1968

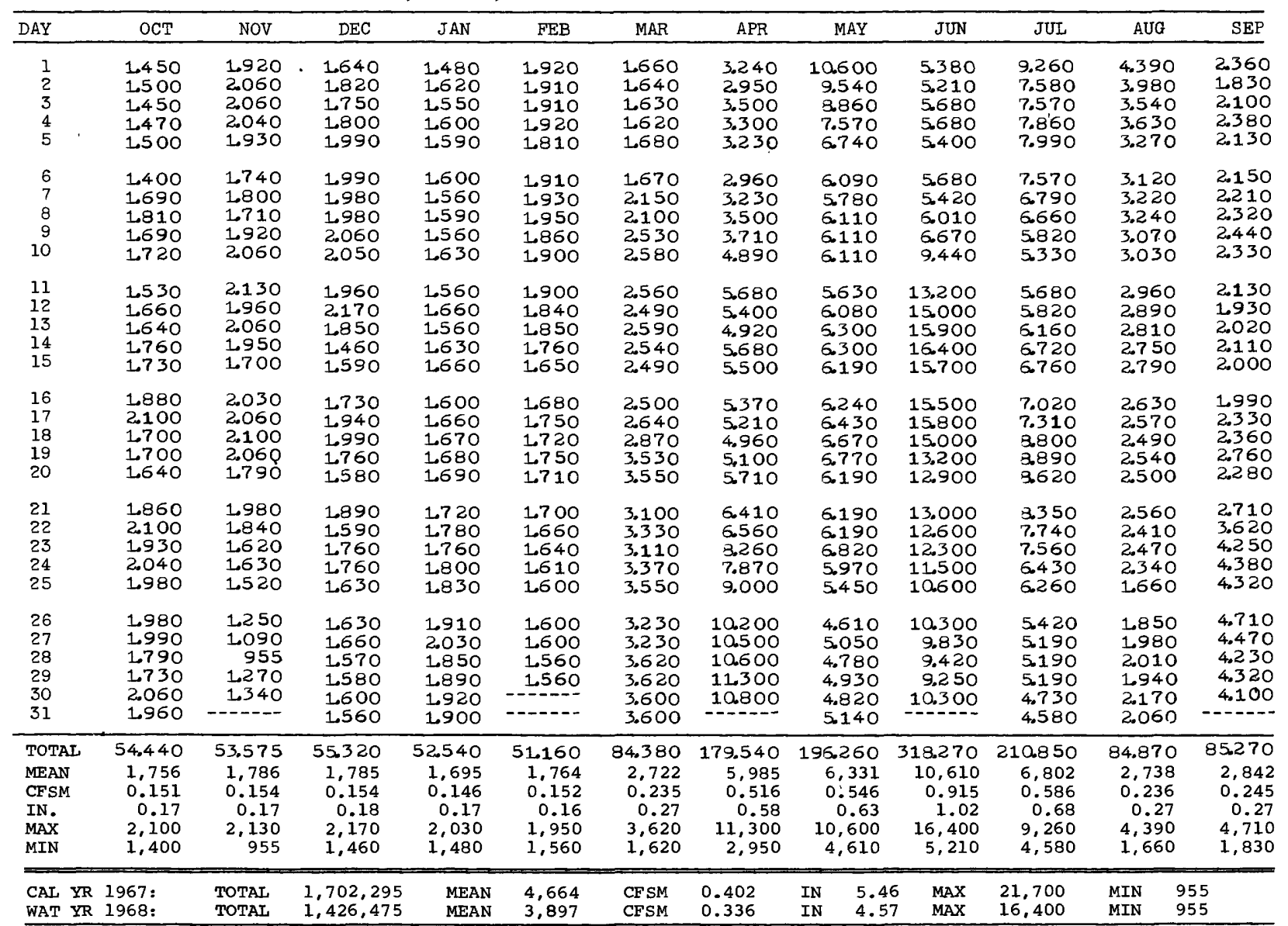


5-2705. Sauk River near st. Cloud, Minn.

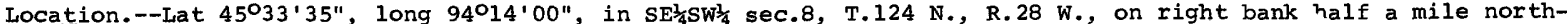
west of Waite Park, 3 miles west of $s t$. Cloud, and 5 miles upstream from mouth.

Drainage area. $--925 \mathrm{sq} \mathrm{mi}$.

Records available.--July 1909 to December 1912, April to December 1913, May to November 1929 , March 1930 to September 1931, April to November 1932, March to November 1933, March 1934 to September 1968. Month1y discharge only for some periods, published in WSP 1308.

Gage.--Water-stage recorder. Datum of gage is 1,034.95 ft above mean sea level, adjustment of 1912 . Prior to Nov. 22, 1934, chain gage on highway bridge 1 mile downstream at datum $6.77 \mathrm{ft} 10 \mathrm{fer}$

Average discharge.--38 years $(1909-12,1930-31,1934-68), 249$ cfs.

Extremes.--Maximum discharge during year, $443 \mathrm{cfs}$ Apr. 24 (gage height, $2.47 \mathrm{ft}$ ); minimum, 13 cfs Nov. 27 (gage height, $0.62 \mathrm{ft}$ )

1909-13, 1929-68: Maximum discharge, 9,100 cfs Apr. 13, 1965 (gage height, 10.68 ft); minimum, 0.3 cfs Nov. 25, 1936.

Remarks.--Records good except those for winter months, which are fair. Flow regulated by powerplants and reservoirs above station. Records of chemical analyses for the water year 1968 are published in Part 2 of this report.

DISCHARGE, IN CFS, WATER YEAR OCTOBER 1967 TO SEPTEMBER 1968

\begin{tabular}{|c|c|c|c|c|c|c|c|c|c|c|c|c|}
\hline DAY & $\mathrm{OCT}$ & NOV & $\mathrm{DEC}$ & JAN & FEB & MAR & APR & MAY & JUN & JUL & AUG & SEP \\
\hline $\begin{array}{l}1 \\
2 \\
3 \\
4 \\
5\end{array}$ & $\begin{array}{l}64 \\
62 \\
66 \\
70 \\
70\end{array}$ & $\begin{array}{l}62 \\
62 \\
62 \\
62 \\
61\end{array}$ & $\begin{array}{l}58 \\
58 \\
58 \\
57 \\
56\end{array}$ & $\begin{array}{l}38 \\
38 \\
38 \\
37 \\
37\end{array}$ & $\begin{array}{l}37 \\
37 \\
37 \\
38 \\
38\end{array}$ & $\begin{array}{r}74 \\
83 \\
91 \\
102 \\
122\end{array}$ & $\begin{array}{l}180 \\
167 \\
154 \\
186 \\
202\end{array}$ & $\begin{array}{l}390 \\
377 \\
368 \\
346 \\
312\end{array}$ & $\begin{array}{l}178 \\
181 \\
180 \\
175 \\
170\end{array}$ & $\begin{array}{l}292 \\
257 \\
222 \\
199 \\
180\end{array}$ & $\begin{array}{l}94 \\
92 \\
89 \\
86 \\
83\end{array}$ & $\begin{array}{l}44 \\
43 \\
45 \\
55 \\
48\end{array}$ \\
\hline $\begin{array}{r}6 \\
7 \\
8 \\
9 \\
10\end{array}$ & $\begin{array}{l}70 \\
81 \\
79 \\
76 \\
76\end{array}$ & $\begin{array}{l}60 \\
60 \\
60 \\
60 \\
60\end{array}$ & $\begin{array}{l}56 \\
55 \\
54 \\
54 \\
54\end{array}$ & $\begin{array}{l}37 \\
37 \\
37 \\
37 \\
37\end{array}$ & $\begin{array}{l}38 \\
38 \\
38 \\
38 \\
38\end{array}$ & $\begin{array}{l}167 \\
160 \\
160 \\
158 \\
147\end{array}$ & $\begin{array}{l}199 \\
202 \\
260 \\
254 \\
254\end{array}$ & $\begin{array}{l}289 \\
272 \\
295 \\
292 \\
251\end{array}$ & $\begin{array}{l}167 \\
162 \\
160 \\
170 \\
216\end{array}$ & $\begin{array}{l}172 \\
164 \\
157 \\
150 \\
138\end{array}$ & $\begin{array}{l}98 \\
99 \\
94 \\
90 \\
86\end{array}$ & $\begin{array}{l}44 \\
45 \\
54 \\
61 \\
55\end{array}$ \\
\hline $\begin{array}{l}11 \\
12 \\
13 \\
14 \\
15\end{array}$ & $\begin{array}{l}75 \\
75 \\
73 \\
75 \\
72\end{array}$ & $\begin{array}{l}60 \\
58 \\
57 \\
58 \\
60\end{array}$ & $\begin{array}{l}54 \\
54 \\
54 \\
54 \\
54\end{array}$ & $\begin{array}{l}37 \\
37 \\
37 \\
37 \\
37\end{array}$ & $\begin{array}{l}39 \\
39 \\
39 \\
40 \\
40\end{array}$ & $\begin{array}{l}150 \\
152 \\
154 \\
157 \\
160\end{array}$ & $\begin{array}{l}260 \\
257 \\
237 \\
277 \\
280\end{array}$ & $\begin{array}{l}240 \\
228 \\
216 \\
214 \\
219\end{array}$ & $\begin{array}{l}306 \\
320 \\
337 \\
371 \\
387\end{array}$ & $\begin{array}{l}132 \\
125 \\
123 \\
127 \\
127\end{array}$ & $\begin{array}{l}83 \\
81 \\
78 \\
76 \\
75\end{array}$ & $\begin{array}{l}52 \\
52 \\
51 \\
51 \\
51\end{array}$ \\
\hline $\begin{array}{l}16 \\
17 \\
18 \\
19 \\
20\end{array}$ & $\begin{array}{l}68 \\
67 \\
67 \\
67 \\
67\end{array}$ & $\begin{array}{l}61 \\
61 \\
61 \\
58 \\
61\end{array}$ & $\begin{array}{l}54 \\
54 \\
54 \\
53 \\
52\end{array}$ & $\begin{array}{l}37 \\
37 \\
37 \\
37 \\
37\end{array}$ & $\begin{array}{l}40 \\
41 \\
42 \\
43 \\
45\end{array}$ & $\begin{array}{l}162 \\
163 \\
164 \\
165 \\
166\end{array}$ & $\begin{array}{l}277 \\
272 \\
257 \\
248 \\
274\end{array}$ & $\begin{array}{l}222 \\
205 \\
199 \\
196 \\
191\end{array}$ & $\begin{array}{l}374 \\
371 \\
352 \\
330 \\
320\end{array}$ & $\begin{array}{l}125 \\
121 \\
119 \\
119 \\
112\end{array}$ & $\begin{array}{l}73 \\
73 \\
72 \\
71 \\
70\end{array}$ & $\begin{array}{l}61 \\
96 \\
78 \\
73 \\
72\end{array}$ \\
\hline $\begin{array}{l}21 \\
22 \\
23 \\
24 \\
25\end{array}$ & $\begin{array}{l}66 \\
64 \\
62 \\
66 \\
64\end{array}$ & $\begin{array}{l}60 \\
60 \\
60 \\
58 \\
58\end{array}$ & $\begin{array}{l}50 \\
48 \\
47 \\
46 \\
45\end{array}$ & $\begin{array}{l}37 \\
37 \\
37 \\
37 \\
37\end{array}$ & $\begin{array}{l}46 \\
47 \\
49 \\
51 \\
52\end{array}$ & $\begin{array}{l}167 \\
170 \\
175 \\
180 \\
190\end{array}$ & $\begin{array}{l}324 \\
298 \\
380 \\
436 \\
410\end{array}$ & $\begin{array}{l}186 \\
180 \\
176 \\
173 \\
170\end{array}$ & $\begin{array}{l}310 \\
295 \\
277 \\
254 \\
242\end{array}$ & $\begin{array}{l}132 \\
121 \\
114 \\
117 \\
110\end{array}$ & $\begin{array}{l}69 \\
68 \\
68 \\
64 \\
62\end{array}$ & $\begin{array}{r}73 \\
83 \\
121 \\
119 \\
136\end{array}$ \\
\hline $\begin{array}{l}26 \\
27 \\
28 \\
29 \\
30 \\
31\end{array}$ & $\begin{array}{l}62 \\
62 \\
62 \\
62 \\
64 \\
64\end{array}$ & $\begin{array}{r}49 \\
43 \\
54 \\
58 \\
58 \\
\end{array}$ & $\begin{array}{l}44 \\
42 \\
41 \\
40 \\
40 \\
39\end{array}$ & $\begin{array}{l}37 \\
37 \\
37 \\
37 \\
37 \\
37\end{array}$ & $\begin{array}{r}56 \\
60 \\
63 \\
66 \\
- \\
-\end{array}$ & $\begin{array}{l}205 \\
205 \\
216 \\
208 \\
199 \\
199\end{array}$ & $\begin{array}{r}407 \\
403 \\
403 \\
410 \\
407 \\
\end{array}$ & $\begin{array}{l}167 \\
162 \\
159 \\
159 \\
165 \\
170\end{array}$ & $\begin{array}{r}242 \\
234 \\
225 \\
214 \\
263 \\
\end{array}$ & $\begin{array}{r}110 \\
108 \\
101 \\
96 \\
94 \\
94\end{array}$ & $\begin{array}{l}62 \\
57 \\
44 \\
40 \\
41 \\
43 \\
\end{array}$ & $\begin{array}{r}134 \\
134 \\
129 \\
125 \\
125 \\
\end{array}$ \\
\hline $\begin{array}{l}\text { Tota } 1 \\
\text { Mean } \\
\text { Max } \\
\text { Min } \\
\text { Cfsm } \\
\text { In. } \\
\end{array}$ & $\begin{array}{r}2118 \\
68.3 \\
81 \\
62 \\
0.074 \\
0.09\end{array}$ & $\begin{array}{r}1.762 \\
58.7 \\
62 \\
43 \\
0.063 \\
0.07\end{array}$ & $\begin{array}{r}1.579 \\
50.9 \\
58 \\
39 \\
0.055 \\
0.06 \\
\end{array}$ & $\begin{array}{r}1.150 \\
37.1 \\
38 \\
37 \\
0.040 \\
0.05 \\
\end{array}$ & $\begin{array}{r}1.275 \\
44.0 \\
66 \\
37 \\
0.048 \\
0.05 \\
\end{array}$ & $\begin{array}{r}4.971 \\
160 \\
216 \\
74 \\
0.173 \\
0.20 \\
\end{array}$ & $\begin{array}{r}8.575 \\
286 \\
436 \\
154 \\
0.309 \\
0.34 \\
\end{array}$ & $\begin{array}{r}7.189 \\
232 \\
390 \\
159 \\
0.251 \\
0.29 \\
\end{array}$ & $\begin{array}{r}7.783 \\
259 \\
387 \\
160 \\
0.0280 \\
0.31 \\
\end{array}$ & $\begin{array}{r}4.358 \\
141 \\
292 \\
94 \\
0.152 \\
0.18 \\
\end{array}$ & $\begin{array}{r}2.281 \\
73.6 \\
99 \\
40 \\
0.080 \\
0.09 \\
\end{array}$ & $\begin{array}{r}2.310 \\
77.0 \\
136 \\
43 \\
0.083 \\
0.09 \\
\end{array}$ \\
\hline
\end{tabular}

Ca1 yr 1967: Total 85,654 Mean $235 \quad$ Max 2, 290 Min $39 \quad$ Cfsm $0.254 \quad$ In. 3.44

Wtr yr 1968: Total 45,351 Mean $124 \quad$ Max $436 \quad$ Min $37 \quad$ Cfsm $0.134 \quad$ In. 1.82 
5-2747. St. Francis River at Santiago, Minn.

Location.--Lat $45^{\circ} 32^{\prime} 30^{\prime \prime}$, long $93^{\circ} 48^{\prime} 50^{\prime \prime}$, in NE⿺ sec.10, T.35 N., R.28 W., on right bank, 0.2 mile east of Santiago and $0.4 \mathrm{mile}$ upstream from bridge on county road.

Records available.--June 1965 to September 1968.

Gage.--Water-stage recorder.

Extremes.--Maximum discharge during year, 389 cfs Sept. 24 (gage height, 6.96 ft); minimum daily, 0.3 cfs

Feb. 15-25; minimum gage height $3.11 \mathrm{ft}$ Nov. 26

1965-68: Maximum discharge 1,930 cfs Mar. 30, 1967 (gage height, 10.38 ft); minimum, 0.1 cfs July 10, 1966, minimum gage height, 3.11 ft Nov. 26, 1967.

Flood of Apr. 14, 1965 reached a stage of $12.17 \mathrm{ft}$, backwater from ice (discharge, 2,940 cfs).

Remarks.--Records good except those for winter months, which are fair.

DISCHARGE, IN CFS, WATER YEAR OCTOBER 1967 TO SEPTEMBER 1968

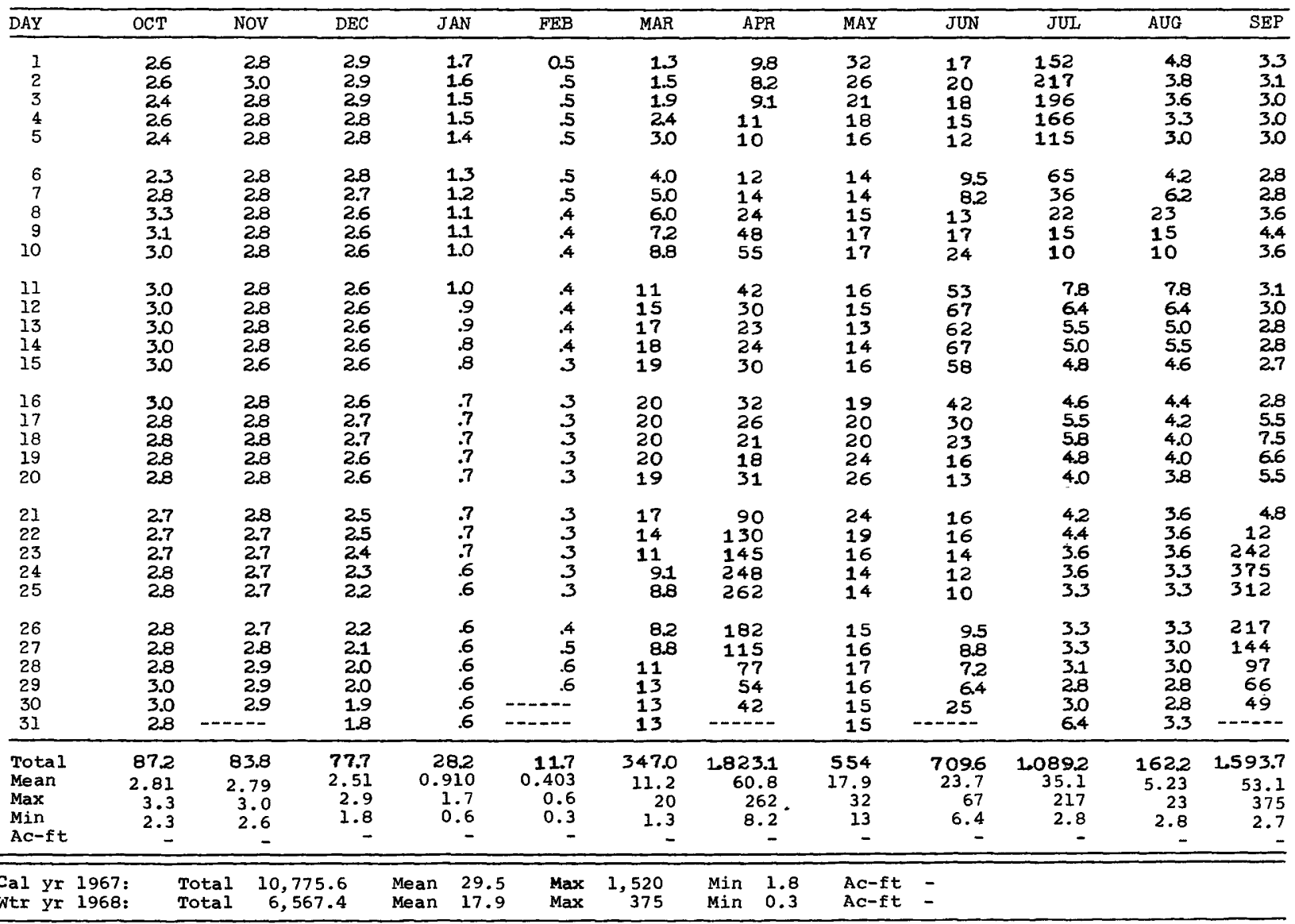


5-2749. St. Francis River near Big Lake, Minn.

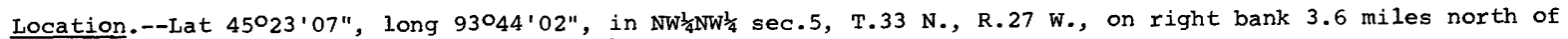

Big Lake and 4 miles upstream from mouth.

Records available.--May 1965 to september 1968.

Gage.-Water-stage recorder. Altitude of gage is $925 \mathrm{ft}$ (from topographic map).

Extremes.--Maximum discharge during year, $309 \mathrm{cfs}$ sept. 30 (gage height, $7.54 \mathrm{ft}$ ); minimum daily, 20 cfs Feb. 19-22; minimum gage height, 4.71 ft Aug. 30 , Sept. 15.

1965-68: Maximum discharge, 992 cfs Apr. 4, 1967 (gage height, 9.49 ft); maximum gage height, 10.15 ft

about Feb. 14, 1966 (from floodmark, backwater from ice): minimum daily discharge, 20 cfs Feb. 19-22, 1968; minimum gage height, $4.67 \mathrm{ft} \mathrm{Aug.} \mathrm{22,} 1967$.

Flood of Apr. 16, 1965 reached a stage of $11.34 \mathrm{ft}$, from floodmarks (discharge 2,700 cfs).

Remarks.--Records good except those for the periods of no gage-height record and those for winter months, which are fair.

DISCHARGE, IN CFS, WATER YEAR OCTOBER 1967 TO SEPTEMBER 1968

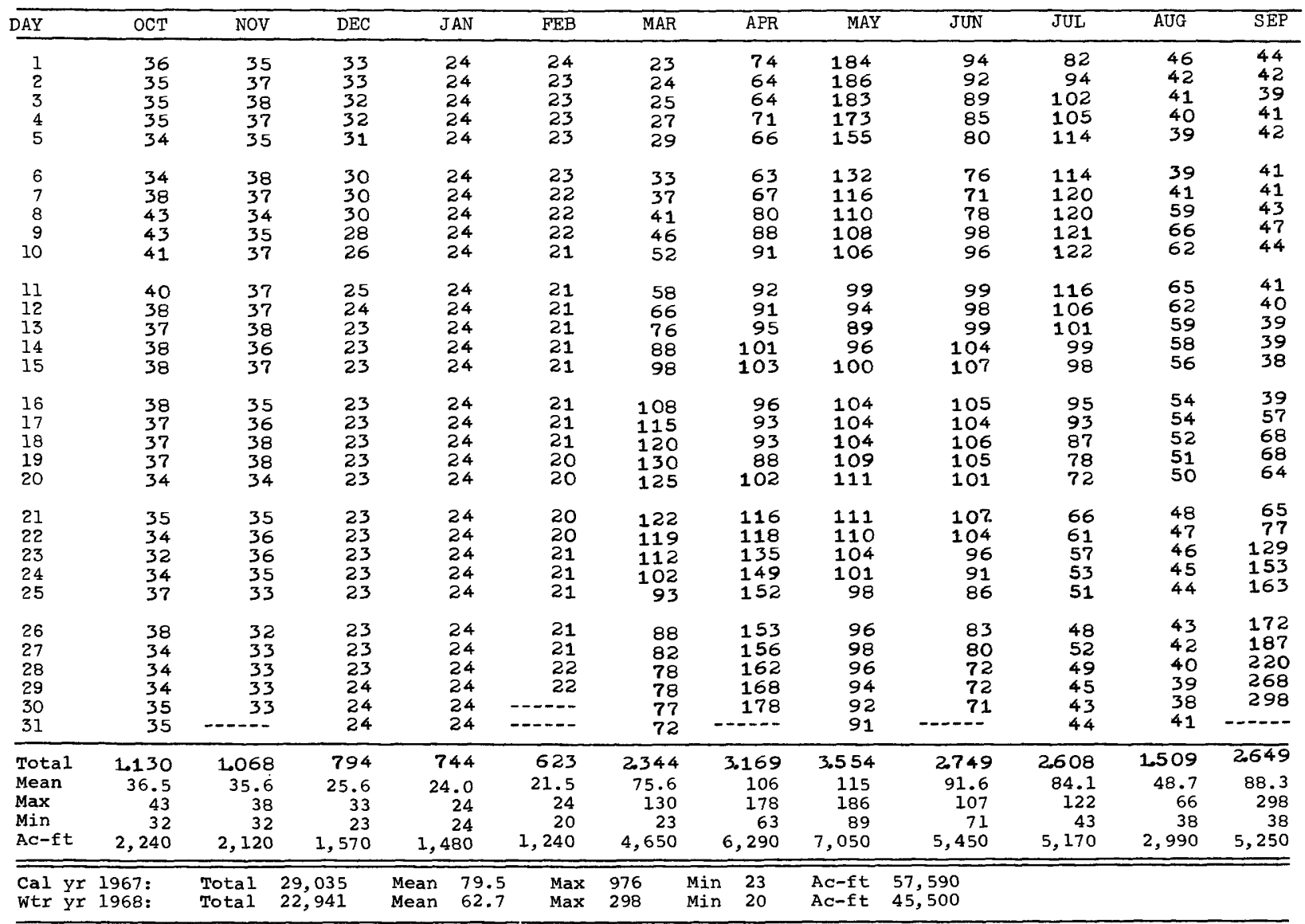


5-2750. Elk River near Big Lake, Minn.

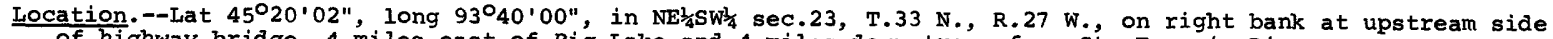
of highway bridge, 4 miles east of Big lake and 4 miles downstream from st. Francis River.

Drainage area. $--615 \mathrm{sq} \mathrm{mi}$.

Records available.--April 1911 to September 1917, April to September 1931, April to November 1932, March to November 1933, March 1934 to september 1968.

Gage.--Digital water-stage recorder. Datum of gage is $899.60 \mathrm{ft}$ above mean sea level, datum of 1929 . April 1911 to Sept. 30, 1917, staff gage, Apr. 1, 1931, to July 26, 1934, chain gage, July 27 , 1934, to Oct. 20,

1967 graphic water-stage recorder at same site and datum.

Average discharqe. -40 years $(1911-17,1934-68), 241$ cfs.

Extremes.--Maximum discharge during year, 633 cfs Mar. 18 (gage height, 2.64 ft); minimum, 25 cfs Nov. 26 (gage height, $0.54 \mathrm{ft}$, result of freezeup) 1911-17, 1931-68: Maximum discharge, 7, 360 cfs Apr. 16, 1965 (gage height, 10.86 ft); minimum, $3.6 \mathrm{cfs}$
July 31, 1934.

Remarks.--Records good except those for winter months, which are fair.

OISCHARGE, IN CUBIC FEFT PER SECOND, WATER YEAR CCTOBER 1967 TO SEPTEMBER 1968

\begin{tabular}{|c|c|c|c|c|c|c|c|c|c|c|c|c|}
\hline D $t Y$ & $D C T$ & NOV & TEC & JAN & $F E B$ & MAR & $A P R$ & MAY & JUN & JUL & AUG & SEP \\
\hline $\begin{array}{l}1 \\
2 \\
3 \\
4 \\
5\end{array}$ & $\begin{array}{l}68 \\
66 \\
68 \\
98 \\
63\end{array}$ & $\begin{array}{l}102 \\
103 \\
108 \\
106 \\
106\end{array}$ & $\begin{array}{l}107 \\
107 \\
106 \\
105 \\
104\end{array}$ & $\begin{array}{l}83 \\
83 \\
82 \\
81 \\
80\end{array}$ & $\begin{array}{l}66 \\
65 \\
65 \\
64 \\
63\end{array}$ & $\begin{array}{l}58 \\
59 \\
60 \\
61 \\
62\end{array}$ & $\begin{array}{l}217 \\
203 \\
200 \\
214 \\
210\end{array}$ & $\begin{array}{l}483 \\
455 \\
422 \\
300 \\
354\end{array}$ & $\begin{array}{l}260 \\
257 \\
242 \\
235 \\
224\end{array}$ & $\begin{array}{l}264 \\
271 \\
293 \\
325 \\
365\end{array}$ & $\begin{array}{r}113 \\
105 \\
102 \\
99 \\
96\end{array}$ & $\begin{array}{r}105 \\
99 \\
94 \\
99 \\
102\end{array}$ \\
\hline $\begin{array}{r}6 \\
7 \\
8 \\
4 \\
10\end{array}$ & $\begin{array}{r}c 1 \\
100 \\
125 \\
125 \\
114\end{array}$ & $\begin{array}{r}91 \\
193 \\
103 \\
114 \\
112\end{array}$ & $\begin{array}{r}102 \\
102 \\
101 \\
89 \\
95\end{array}$ & $\begin{array}{l}79 \\
79 \\
79 \\
79 \\
78\end{array}$ & $\begin{array}{l}52 \\
62 \\
61 \\
50 \\
50\end{array}$ & $\begin{array}{l}63 \\
64 \\
66 \\
72 \\
78\end{array}$ & $\begin{array}{l}200 \\
210 \\
260 \\
267 \\
275\end{array}$ & $\begin{array}{l}314 \\
289 \\
282 \\
282 \\
275\end{array}$ & $\begin{array}{l}214 \\
200 \\
203 \\
242 \\
249\end{array}$ & $\begin{array}{l}375 \\
365 \\
332 \\
300 \\
278\end{array}$ & $\begin{array}{r}96 \\
110 \\
142 \\
193 \\
182\end{array}$ & $\begin{array}{r}99 \\
99 \\
105 \\
126 \\
122\end{array}$ \\
\hline $\begin{array}{l}11 \\
12 \\
13 \\
14 \\
15\end{array}$ & $\begin{array}{l}108 \\
103 \\
160 \\
100 \\
103\end{array}$ & $\begin{array}{lll}1 & 1 & 1 \\
1 & 1 & 4 \\
1 & 1 & 5 \\
1 & 05 \\
1 & 05\end{array}$ & $\begin{array}{l}95 \\
90 \\
80 \\
80 \\
80\end{array}$ & $\begin{array}{l}78 \\
78 \\
78 \\
78 \\
78\end{array}$ & $\begin{array}{l}57 \\
58 \\
57 \\
57 \\
56\end{array}$ & $\begin{array}{r}88 \\
105 \\
130 \\
165 \\
220\end{array}$ & $\begin{array}{l}296 \\
303 \\
293 \\
300 \\
296\end{array}$ & $\begin{array}{l}264 \\
264 \\
253 \\
271 \\
275\end{array}$ & $\begin{array}{l}264 \\
260 \\
264 \\
303 \\
318\end{array}$ & $\begin{array}{l}257 \\
239 \\
235 \\
235 \\
235\end{array}$ & $\begin{array}{l}168 \\
165 \\
155 \\
148 \\
148\end{array}$ & $\begin{array}{r}113 \\
108 \\
105 \\
99 \\
94\end{array}$ \\
\hline $\begin{array}{l}16 \\
17 \\
18 \\
13 \\
20\end{array}$ & $\begin{array}{r}100 \\
56 \\
100 \\
58 \\
93\end{array}$ & $\begin{array}{l}108 \\
109 \\
111 \\
113 \\
106\end{array}$ & $\begin{array}{l}82 \\
84 \\
86 \\
86 \\
86\end{array}$ & $\begin{array}{l}77 \\
77 \\
76 \\
76 \\
75\end{array}$ & $\begin{array}{l}56 \\
56 \\
55 \\
54 \\
54\end{array}$ & $\begin{array}{l}257 \\
209 \\
447 \\
481 \\
354\end{array}$ & $\begin{array}{l}282 \\
271 \\
264 \\
257 \\
293\end{array}$ & $\begin{array}{l}278 \\
278 \\
278 \\
293 \\
293\end{array}$ & $\begin{array}{l}318 \\
318 \\
325 \\
307 \\
293\end{array}$ & $\begin{array}{l}217 \\
207 \\
200 \\
182 \\
172\end{array}$ & $\begin{array}{l}155 \\
142 \\
138 \\
138 \\
135\end{array}$ & $\begin{array}{l}96 \\
142 \\
168 \\
172 \\
168\end{array}$ \\
\hline $\begin{array}{l}21 \\
22 \\
23 \\
24 \\
25\end{array}$ & $\begin{array}{l}94 \\
99 \\
53 \\
93 \\
93\end{array}$ & $\begin{array}{r}110 \\
112 \\
87 \\
116 \\
111\end{array}$ & $\begin{array}{l}86 \\
86 \\
85 \\
85 \\
85\end{array}$ & $\begin{array}{l}75 \\
74 \\
73 \\
72 \\
71\end{array}$ & $\begin{array}{l}53 \\
53 \\
53 \\
54 \\
54\end{array}$ & $\begin{array}{l}285 \\
264 \\
271 \\
242 \\
214\end{array}$ & $\begin{array}{l}329 \\
329 \\
411 \\
494 \\
519\end{array}$ & $\begin{array}{l}282 \\
275 \\
267 \\
257 \\
249\end{array}$ & $\begin{array}{l}303 \\
300 \\
278 \\
260 \\
246\end{array}$ & $\begin{array}{l}158 \\
145 \\
135 \\
129 \\
126\end{array}$ & $\begin{array}{l}126 \\
119 \\
113 \\
108 \\
102\end{array}$ & $\begin{array}{l}168 \\
200 \\
368 \\
458 \\
523\end{array}$ \\
\hline $\begin{array}{l}26 \\
27 \\
28 \\
29 \\
30 \\
31\end{array}$ & $\begin{array}{r}09 \\
95 \\
92 \\
98 \\
98 \\
103\end{array}$ & $\begin{array}{r}87 \\
95 \\
100 \\
105 \\
107 \\
\end{array}$ & $\begin{array}{l}84 \\
84 \\
84 \\
84 \\
84 \\
84\end{array}$ & $\begin{array}{l}70 \\
70 \\
69 \\
69 \\
68 \\
67\end{array}$ & $\begin{array}{r}55 \\
56 \\
57 \\
57 \\
- \\
\end{array}$ & $\begin{array}{l}210 \\
221 \\
231 \\
224 \\
224 \\
224\end{array}$ & $\begin{array}{r}516 \\
548 \\
573 \\
555 \\
516 \\
\end{array}$ & $\begin{array}{l}246 \\
253 \\
246 \\
242 \\
242 \\
242\end{array}$ & $\begin{array}{r}239 \\
228 \\
210 \\
200 \\
217 \\
\end{array}$ & $\begin{array}{l}119 \\
129 \\
129 \\
116 \\
110 \\
113\end{array}$ & $\begin{array}{l}99 \\
94 \\
88 \\
86 \\
84 \\
91\end{array}$ & $\begin{array}{r}563 \\
537 \\
498 \\
487 \\
498 \\
\end{array}$ \\
\hline $\begin{array}{l}\text { TOTAL } \\
\text { MEAN } \\
\text { MAX } \\
\text { MIN } \\
\text { CFSM } \\
\text { IN. }\end{array}$ & $\begin{array}{r}3,096 \\
99.9 \\
125 \\
91 \\
.16 \\
.19\end{array}$ & $\begin{array}{r}3.179 \\
106 \\
116 \\
87 \\
.17 \\
.19\end{array}$ & $\begin{array}{r}2,809 \\
90.6 \\
107 \\
80 \\
.15 \\
.17\end{array}$ & $\begin{array}{r}2,352 \\
75.9 \\
83 \\
67 \\
.12 \\
.14\end{array}$ & $\begin{array}{r}1,682 \\
58.0 \\
66 \\
53 \\
.09 \\
.10\end{array}$ & $\begin{array}{r}5,799 \\
187 \\
481 \\
58 \\
.30 \\
.35\end{array}$ & $\begin{array}{r}9,001 \\
330 \\
573 \\
200 \\
.54 \\
.60\end{array}$ & $\begin{array}{r}9,094 \\
203 \\
483 \\
242 \\
.48 \\
.55\end{array}$ & $\begin{array}{r}7.777 \\
259 \\
325 \\
200 \\
.42 \\
.47\end{array}$ & $\begin{array}{r}6,756 \\
218 \\
375 \\
110 \\
.35 \\
.41\end{array}$ & $\begin{array}{r}3.840 \\
124 \\
193 \\
84 \\
.20 \\
.23\end{array}$ & $\begin{array}{r}0,615 \\
221 \\
563 \\
94 \\
.36 \\
.40\end{array}$ \\
\hline $\begin{array}{ll}\text { CAL YR } \\
\text { WTR }\end{array}$ & $\begin{array}{l}967 \\
968\end{array}$ & $\begin{array}{l}\mathrm{L} 1,8 \\
\mathrm{~L} \quad 62,90\end{array}$ & & $\begin{array}{l}224 \\
172\end{array}$ & $\begin{array}{l}\text { MAX } \\
\text { MAX }\end{array}$ & & $\begin{array}{l}78 \\
53\end{array}$ & $\begin{array}{l}\text { CFSM } \\
\text { CFSM }\end{array}$ & $\begin{array}{l}\text { IN } \\
\text { IN }\end{array}$ & & & \\
\hline
\end{tabular}


5-2780. Middle Fork Crow River near spicer, Minn.

Location.--Iat $45^{\circ} 15^{\prime} 45^{\prime \prime}$, long $94^{\circ} 48^{\prime} 10^{\prime \prime}$, in NE $\frac{1}{4}$ sec. 27 , T.121 N., R.33 W., on right bank 75 ft upstream from highway bridge, $1 \frac{1}{2}$ miles downstream from Lake Calhoun, 3 miles downstream from Green Lake, and 6.8 miles northeast of spicer.

Drainage area. $--179 \mathrm{sq} \mathrm{mi}$.

Records available.--March 1949 to September 1968.

Gaqe.--Water-stage recorder and concrete and steel V-notch sharp-crested weir. Datum of gage is 1 , 147.93 ft above mean sea level, datum of 1929 (Kandiyohi County Highway Department bench mark). Prior to July 20, 1950, chain gage at bridge 75 ft downstream at same datum.

Average discharge. -19 years, 46.9 cfs.

Extremes.--Maximum discharge during year, 90 cfs June 14 (gage height $3.31 \mathrm{ft}$ ); no flow Feb. $19-28$.

1949-68: Maximum discharge, 408 cfs June 29, 1953; maximum gage height, 6.67 ft June 25 , 1957; no flow Mar. 15-24, 1949, Feb. 26 to Mar. 26, 1960, Dec. 8, 1963, Feb. 10-21, 1965, Feb. 19-28, 1968.

Remarks.--Records good except those for periods of no gage-height record and for winter months, which are fair. Flow affected by natural storage and some regulation from lakes above station.

DISCHARGE, IN CFS, WATER YEAR OCTOBER 1967 TO SEPTEMBER 1968

\begin{tabular}{|c|c|c|c|c|c|c|c|c|c|c|c|c|}
\hline DAY & OCT & NOV & $\mathrm{DEC}$ & JAN & FEB & MAR & APR & MAY & JUN & JUL & AUG & $\overline{\mathrm{SEP}}$ \\
\hline $\begin{array}{l}1 \\
2 \\
3 \\
4 \\
5\end{array}$ & $\begin{array}{l}2.0 \\
3.6 \\
3.8 \\
3.6 \\
3.4\end{array}$ & $\begin{array}{l}1.5 \\
1.5 \\
1.5 \\
1.5 \\
1.5\end{array}$ & $\begin{array}{r}1.0 \\
1.0 \\
.9 \\
.9 \\
.9\end{array}$ & $\begin{array}{r}0.2 \\
.2 \\
.2 \\
.2 \\
.2\end{array}$ & $\begin{array}{r}0.2 \\
.2 \\
.2 \\
2 \\
.2\end{array}$ & $\begin{array}{r}0.1 \\
.1 \\
.1 \\
.2 \\
.2\end{array}$ & $\begin{array}{l}3.2 \\
2.0 \\
2.3 \\
2.6 \\
2.1\end{array}$ & $\begin{array}{l}9.3 \\
10 \\
14 \\
15 \\
12\end{array}$ & $\begin{array}{l}48 \\
46 \\
42 \\
43 \\
43\end{array}$ & $\begin{array}{l}72 \\
70 \\
64 \\
59 \\
56\end{array}$ & $\begin{array}{l}31 \\
29 \\
28 \\
30 \\
30\end{array}$ & $\begin{array}{l}9.3 \\
7.8 \\
7.5 \\
7.8 \\
7.1\end{array}$ \\
\hline $\begin{array}{r}6 \\
7 \\
8 \\
9 \\
10\end{array}$ & $\begin{array}{l}3.0 \\
3.8 \\
4.5 \\
4.5 \\
3.8\end{array}$ & $\begin{array}{l}1.5 \\
1.5 \\
1.4 \\
1.3 \\
1.4\end{array}$ & $\begin{array}{l}.9 \\
.9 \\
.8 \\
.8 \\
.7\end{array}$ & $\begin{array}{l}.2 \\
.2 \\
.2 \\
2 \\
2 \\
2\end{array}$ & $\begin{array}{l}.2 \\
.2 \\
.2 \\
.2 \\
.2\end{array}$ & $\begin{array}{r}.2 \\
.3 \\
.4 \\
.7 \\
1.0\end{array}$ & $\begin{array}{l}1.9 \\
3.1 \\
3.2 \\
2.7 \\
2.3\end{array}$ & $\begin{array}{l}8.6 \\
6.7 \\
15 \\
20 \\
15\end{array}$ & $\begin{array}{l}44 \\
42 \\
42 \\
37 \\
56\end{array}$ & $\begin{array}{l}54 \\
52 \\
51 \\
48 \\
43\end{array}$ & $\begin{array}{l}30 \\
30 \\
29 \\
28 \\
26\end{array}$ & $\begin{array}{l}6.7 \\
5.4 \\
7.5 \\
9.7 \\
7.8\end{array}$ \\
\hline $\begin{array}{l}11 \\
12 \\
13 \\
14 \\
15\end{array}$ & $\begin{array}{l}3.4 \\
3.4 \\
3.2 \\
3.4 \\
3.4\end{array}$ & $\begin{array}{l}1.4 \\
1.2 \\
1.3 \\
1.2 \\
1.3\end{array}$ & $\begin{array}{l}.7 \\
.6 \\
.6 \\
.6 \\
.5\end{array}$ & $\begin{array}{l}.2 \\
2 \\
.2 \\
2 \\
.2\end{array}$ & $\begin{array}{l}.1 \\
.1 \\
.1 \\
.1 \\
.1\end{array}$ & $\begin{array}{l}1.3 \\
1.7 \\
2.5 \\
3.8 \\
5.2\end{array}$ & $\begin{array}{l}2.1 \\
2.0 \\
2.1 \\
3.4 \\
1.9\end{array}$ & $\begin{array}{l}16 \\
17 \\
12 \\
13 \\
18\end{array}$ & $\begin{array}{l}65 \\
61 \\
62 \\
89 \\
86\end{array}$ & $\begin{array}{l}41 \\
40 \\
41 \\
46 \\
46\end{array}$ & $\begin{array}{l}23 \\
21 \\
19 \\
17 \\
16\end{array}$ & $\begin{array}{l}6.7 \\
6.0 \\
5.7 \\
5.7 \\
4.2\end{array}$ \\
\hline $\begin{array}{l}16 \\
17 \\
18 \\
19 \\
20\end{array}$ & $\begin{array}{l}3.2 \\
3.4 \\
3.2 \\
2.8 \\
3.0\end{array}$ & $\begin{array}{l}1.3 \\
1.3 \\
1.3 \\
1.3 \\
1.2\end{array}$ & $\begin{array}{l}.5 \\
.5 \\
.5 \\
.4 \\
.4\end{array}$ & $\begin{array}{l}.2 \\
.2 \\
2 \\
.2 \\
.2\end{array}$ & $0_{0}^{.1}$ & $\begin{array}{l}7.8 \\
11 \\
14 \\
15 \\
14\end{array}$ & $\begin{array}{l}2.4 \\
2.4 \\
2.1 \\
2.1 \\
4.0\end{array}$ & $\begin{array}{l}21 \\
18 \\
18 \\
17 \\
15\end{array}$ & $\begin{array}{l}82 \\
78 \\
81 \\
79 \\
73\end{array}$ & $\begin{array}{l}44 \\
44 \\
44 \\
38 \\
38\end{array}$ & $\begin{array}{l}18 \\
17 \\
14 \\
15 \\
15\end{array}$ & $\begin{array}{l}4.8 \\
8.6 \\
10 \\
7.8 \\
7.5\end{array}$ \\
\hline $\begin{array}{l}21 \\
22 \\
23 \\
24 \\
25\end{array}$ & $\begin{array}{l}2.6 \\
2.4 \\
2.0 \\
2.4 \\
2.4\end{array}$ & $\begin{array}{l}1.3 \\
1.3 \\
1.2 \\
1.3 \\
1.3\end{array}$ & $\begin{array}{l}.4 \\
.4 \\
.4 \\
.4 \\
.4\end{array}$ & $\begin{array}{l}.2 \\
.2 \\
.2 \\
2 \\
.2\end{array}$ & $\begin{array}{l}0 \\
0 \\
0 \\
0 \\
0\end{array}$ & $\begin{array}{l}13 \\
12 \\
11 \\
11 \\
10\end{array}$ & $\begin{array}{l}3.6 \\
3.6 \\
9.0 \\
7.5 \\
6.4\end{array}$ & $\begin{array}{l}14 \\
13 \\
13 \\
9.3 \\
12\end{array}$ & $\begin{array}{l}75 \\
74 \\
72 \\
70 \\
69\end{array}$ & $\begin{array}{l}38 \\
37 \\
36 \\
39 \\
37\end{array}$ & $\begin{array}{l}13 \\
15 \\
13 \\
15 \\
13\end{array}$ & $\begin{array}{l}7.5 \\
15 \\
28 \\
26 \\
25\end{array}$ \\
\hline $\begin{array}{l}26 \\
27 \\
28 \\
29 \\
30 \\
31 \\
\end{array}$ & $\begin{array}{l}2.0 \\
1.9 \\
1.6 \\
1.7 \\
1.6 \\
1.6 \\
\end{array}$ & $\begin{array}{r}1.3 \\
1.4 \\
1.2 \\
1.1 \\
1.0 \\
-0 . \\
\end{array}$ & $\begin{array}{l}3 \\
3 \\
3 \\
3 \\
3 \\
2 \\
\end{array}$ & $\begin{array}{l}2 \\
2 \\
2 \\
2 \\
2 \\
2 \\
.2 \\
\end{array}$ & \begin{tabular}{c}
0 \\
0 \\
0 \\
.1 \\
\hdashline- \\
\end{tabular} & $\begin{array}{l}9.2 \\
8.5 \\
7.6 \\
6.8 \\
5.4 \\
4.1 \\
\end{array}$ & $\begin{array}{r}6.7 \\
6.7 \\
7.8 \\
9.3 \\
10 \\
\end{array}$ & $\begin{array}{l}28 \\
32 \\
32 \\
32 \\
32 \\
38 \\
\end{array}$ & $\begin{array}{r}68 \\
66 \\
62 \\
62 \\
62 \\
\end{array}$ & $\begin{array}{l}38 \\
45 \\
40 \\
36 \\
35 \\
36\end{array}$ & $\begin{array}{l}9.3 \\
7.8 \\
7.5 \\
7.1 \\
7.8 \\
9.3\end{array}$ & $\begin{array}{r}22 \\
20 \\
19 \\
17 \\
17 \\
\end{array}$ \\
\hline $\begin{array}{l}\text { Tota } 1 \\
\text { Mean } \\
\text { Max } \\
\text { Min } \\
\text { Cfsm } \\
\text { In. } \\
\end{array}$ & $\begin{array}{r}90.6 \\
2.92 \\
4.5 \\
1.6 \\
0.016 \\
0.02 \\
\end{array}$ & $\begin{array}{r}39.8 \\
1.33 \\
1.5 \\
1.0 \\
0.0074 \\
0.008 \\
\end{array}$ & $\begin{array}{r}17.8 \\
0.574 \\
1.0 \\
0.2 \\
0.0032 \\
0.004 \\
\end{array}$ & $\begin{array}{r}6.2 \\
0.20 \\
0.2 \\
0.2 \\
0.0011 \\
0.001 \\
\end{array}$ & $\begin{array}{r}2.9 \\
0.10 \\
0.2 \\
0 \\
0.00056 \\
0.0006 \\
\end{array}$ & $\begin{array}{r}178.2 \\
5.75 \\
15 \\
0.1 \\
0.032 \\
0.04 \\
\end{array}$ & $\begin{array}{r}120.5 \\
4.02 \\
10 \\
1.9 \\
0.022 \\
0.02 \\
\end{array}$ & $\begin{array}{r}545.9 \\
17.6 \\
38 \\
6.7 \\
0.098 \\
0.11 \\
\end{array}$ & $\begin{array}{r}1879 \\
62.6 \\
89 \\
37 \\
0.350 \\
0.39 \\
\end{array}$ & $\begin{array}{r}1.408 \\
45.4 \\
72 \\
35 \\
0.254 \\
0.29 \\
\end{array}$ & $\begin{array}{r}583.8 \\
18.8 \\
31 \\
7.1 \\
0.105 \\
0.12 \\
\end{array}$ & $\begin{array}{r}340.1 \\
11.3 \\
28 \\
4.2 \\
0.063 \\
0.07 \\
\end{array}$ \\
\hline $\begin{array}{l}\text { Cal yr } \\
\text { wtr yr }\end{array}$ & $\begin{array}{l}967: \\
968:\end{array}$ & $\begin{array}{l}\text { Tota } 1 \\
\text { Tota } 1\end{array}$ & $\begin{array}{l}640.5 \\
212.8\end{array}$ & $\begin{array}{l}\text { Mean } \\
\text { Mean }\end{array}$ & $\begin{array}{l}.9 \\
. .2\end{array}$ & $\begin{array}{r}156 \\
89\end{array}$ & $\begin{array}{ll}\operatorname{Min} & 0.2 \\
\operatorname{Min} & 0\end{array}$ & $\begin{array}{l}\text { Cfsm } \\
\text { Cfsm }\end{array}$ & $\begin{array}{r}0.178 \\
.079\end{array}$ & $\begin{array}{l}\text { n. } \\
\text { n. }\end{array}$ & & \\
\hline
\end{tabular}


5-2790. South Fork Crow River near Mayer, Minn.

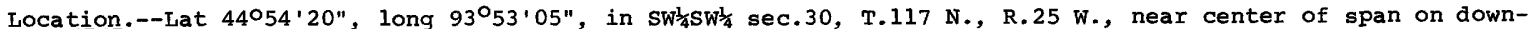
stream side of bridge on State Highway $7,1.3 \mathrm{miles}$ north of Mayer, $4.3 \mathrm{miles}$ southwest of watertown, and 16 miles upstream from confluence with North Fork.

Drainaqe area.--1,170 sq $\mathrm{mi}$, approximately.

Records available.--April 1934 to September 1968. Monthly discharge only for some periods, published in WSP 1308 .

Gage.--Wire-weight gage read once or twice daily. Datum of gage is $926.00 \mathrm{ft}$ above mean sea level (1evels by Hennepin County Park Board Survey). Prior to June 14, 1940, chain gage at same site and datum.

Average discharge. --34 years, $228 \mathrm{cfs}$.

Extremes.--Maximum discharge during year, 1,460 cfs July 16 (gage height, 7.14 ft, from graph based on gage readings); no flow Feb. 18-23; minimum gage height, 0.72 ft Feb. 13, 14, $15,16$.

1934-68: Maximum discharge, $16,100 \mathrm{cfs}$ Apr. 13, 1965 (gage height, 19.23 ft, from floodmark); no flow at times.

Remarks.--Records good except those for winter months, which are fair.

DISCHARGE, IN CFS, WATER YEAR OCTOBER 1967 TO SEPTEMBER 1968

\begin{tabular}{|c|c|c|c|c|c|c|c|c|c|c|c|c|}
\hline DAY & OCT & NOV & $\mathrm{DEC}$ & JAN & FEB & MAR & APR & MAY & JUN & JUL & AUG & SEP \\
\hline $\begin{array}{l}1 \\
2 \\
3 \\
4 \\
5\end{array}$ & $\begin{array}{c}11 \\
9.4 \\
8.4 \\
8.4 \\
20\end{array}$ & $\begin{array}{l}6.7 \\
6.7 \\
6.3 \\
6.3 \\
5.5\end{array}$ & $\begin{array}{l}5.8 \\
6.5 \\
7.4 \\
8.2 \\
8.7\end{array}$ & $\begin{array}{r}1.7 \\
1.4 \\
1.2 \\
.9 \\
.7\end{array}$ & $\begin{array}{r}0.1 \\
.1 \\
.1 \\
.1 \\
.1\end{array}$ & $\begin{array}{r}0.4 \\
.7 \\
1.4 \\
2.9 \\
5.3\end{array}$ & $\begin{array}{l}61 \\
58 \\
57 \\
57 \\
58\end{array}$ & $\begin{array}{r}126 \\
104 \\
90 \\
80 \\
76\end{array}$ & $\begin{array}{r}98 \\
118 \\
106 \\
87 \\
69\end{array}$ & $\begin{array}{l}230 \\
209 \\
179 \\
151 \\
145\end{array}$ & $\begin{array}{l}206 \\
194 \\
185 \\
168 \\
138\end{array}$ & $\begin{array}{l}18 \\
18 \\
17 \\
17 \\
20\end{array}$ \\
\hline $\begin{array}{r}6 \\
7 \\
8 \\
9 \\
10\end{array}$ & $\begin{array}{l}12 \\
13 \\
16 \\
16 \\
14\end{array}$ & $\begin{array}{l}5.9 \\
6.7 \\
5.9 \\
5.9 \\
5.9\end{array}$ & $\begin{array}{l}9.1 \\
9.2 \\
9.4 \\
9.5 \\
9.5\end{array}$ & $\begin{array}{l}.6 \\
.5 \\
.4 \\
.3 \\
.3\end{array}$ & $\begin{array}{l}.1 \\
.1 \\
.1 \\
.1 \\
.1\end{array}$ & $\begin{array}{l}9.4 \\
16 \\
28 \\
50 \\
58\end{array}$ & $\begin{array}{l}56 \\
44 \\
61 \\
60 \\
58\end{array}$ & $\begin{array}{l}70 \\
67 \\
57 \\
64 \\
69\end{array}$ & $\begin{array}{l}52 \\
46 \\
40 \\
40 \\
70\end{array}$ & $\begin{array}{r}128 \\
110 \\
92 \\
80 \\
70\end{array}$ & $\begin{array}{r}140 \\
126 \\
117 \\
102 \\
89\end{array}$ & $\begin{array}{l}19 \\
16 \\
32 \\
51 \\
41\end{array}$ \\
\hline $\begin{array}{l}11 \\
12 \\
13 \\
14 \\
15\end{array}$ & $\begin{array}{c}11 \\
10 \\
9.4 \\
8.0 \\
8.0\end{array}$ & $\begin{array}{l}7.6 \\
8.4 \\
7.6 \\
8.4 \\
8.4\end{array}$ & $\begin{array}{l}9.6 \\
9.4 \\
9.2 \\
9.0 \\
8.6\end{array}$ & $\begin{array}{l}.2 \\
.2 \\
.2 \\
.2 \\
.2\end{array}$ & $\begin{array}{l}.1 \\
.1 \\
.1 \\
.1 \\
.1\end{array}$ & $\begin{array}{l}59 \\
59 \\
63 \\
70 \\
86\end{array}$ & $\begin{array}{l}51 \\
45 \\
41 \\
53 \\
53\end{array}$ & $\begin{array}{l}65 \\
57 \\
46 \\
52 \\
45\end{array}$ & $\begin{array}{r}115 \\
182 \\
214 \\
726 \\
1.050\end{array}$ & $\begin{array}{r}66 \\
57 \\
230 \\
698 \\
1.140\end{array}$ & $\begin{array}{l}77 \\
65 \\
60 \\
46 \\
44\end{array}$ & $\begin{array}{l}35 \\
29 \\
26 \\
22 \\
19\end{array}$ \\
\hline $\begin{array}{l}16 \\
17 \\
18 \\
19 \\
20\end{array}$ & $\begin{array}{l}7.1 \\
7.1 \\
6.7 \\
6.3 \\
5.9\end{array}$ & $\begin{array}{l}8.4 \\
9.4 \\
8.9 \\
8.0 \\
8.0\end{array}$ & $\begin{array}{l}8.5 \\
8.5 \\
8.5 \\
8.5 \\
8.1\end{array}$ & $\begin{array}{l}.2 \\
.2 \\
2 \\
2 \\
2 \\
2\end{array}$ & $\begin{array}{l}.1 \\
0 \\
0 \\
0\end{array}$ & $\begin{array}{l}100 \\
109 \\
116 \\
111 \\
106\end{array}$ & $\begin{array}{l}51 \\
42 \\
39 \\
38 \\
64\end{array}$ & $\begin{array}{l}53 \\
53 \\
54 \\
50 \\
51\end{array}$ & $\begin{array}{l}860 \\
670 \\
648 \\
747 \\
560\end{array}$ & $\begin{array}{r}1.430 \\
1.350 \\
1.190 \\
1.030 \\
936\end{array}$ & $\begin{array}{l}43 \\
42 \\
36 \\
37 \\
35\end{array}$ & $\begin{array}{l}17 \\
34 \\
87 \\
89 \\
83\end{array}$ \\
\hline $\begin{array}{l}21 \\
22 \\
23 \\
24 \\
25\end{array}$ & $\begin{array}{l}5.1 \\
5.5 \\
5.5 \\
5.1 \\
10\end{array}$ & $\begin{array}{l}9.4 \\
8.9 \\
8.9 \\
8.4 \\
9.3\end{array}$ & $\begin{array}{l}7.6 \\
6.7 \\
6.0 \\
5.3 \\
4.6\end{array}$ & $\begin{array}{l}.2 \\
.2 \\
.2 \\
.2 \\
.1\end{array}$ & $\begin{array}{l}0 \\
0 \\
0 \\
.1 \\
.1\end{array}$ & $\begin{array}{l}98 \\
92 \\
89 \\
88 \\
88\end{array}$ & $\begin{array}{l}110 \\
125 \\
214 \\
433 \\
381\end{array}$ & $\begin{array}{l}44 \\
44 \\
40 \\
38 \\
44\end{array}$ & $\begin{array}{l}622 \\
572 \\
498 \\
419 \\
362\end{array}$ & $\begin{array}{l}844 \\
737 \\
610 \\
486 \\
383\end{array}$ & $\begin{array}{l}34 \\
32 \\
26 \\
24 \\
23\end{array}$ & $\begin{array}{r}74 \\
102 \\
135 \\
130 \\
118\end{array}$ \\
\hline $\begin{array}{l}26 \\
27 \\
28 \\
29 \\
30 \\
31 \\
\end{array}$ & $\begin{array}{l}6.3 \\
5.5 \\
5.1 \\
6.3 \\
6.7 \\
6.7 \\
\end{array}$ & $\begin{array}{r}5.5 \\
4.9 \\
4.8 \\
5.0 \\
5.4 \\
\end{array}$ & $\begin{array}{l}4.0 \\
3.6 \\
3.2 \\
2.7 \\
2.3 \\
1.9\end{array}$ & $\begin{array}{l}.1 \\
.1 \\
.1 \\
.1 \\
.1 \\
.1\end{array}$ & $\begin{array}{r}.1 \\
.1 \\
.2 \\
.2 \\
\hdashline- \\
\hdashline\end{array}$ & $\begin{array}{r}92 \\
100 \\
109 \\
87 \\
80 \\
67\end{array}$ & $\begin{array}{r}305 \\
247 \\
201 \\
179 \\
153 \\
\end{array}$ & $\begin{array}{r}43 \\
81 \\
102 \\
81 \\
80 \\
74\end{array}$ & $\begin{array}{r}346 \\
358 \\
330 \\
296 \\
252 \\
\end{array}$ & $\begin{array}{l}336 \\
312 \\
280 \\
238 \\
216 \\
226\end{array}$ & $\begin{array}{l}21 \\
21 \\
21 \\
19 \\
17 \\
17\end{array}$ & $\begin{array}{r}123 \\
107 \\
90 \\
81 \\
73 \\
\end{array}$ \\
\hline $\begin{array}{l}\text { Total } \\
\text { Mean } \\
\text { Max } \\
\text { Min } \\
\text { Cfsm } \\
\text { In. }\end{array}$ & $\begin{array}{r}275.5 \\
8.89 \\
20 \\
5.1 \\
0.0076 \\
0.009\end{array}$ & $\begin{array}{r}215.4 \\
7.18 \\
9.4 \\
4.8 \\
0.0061 \\
0.007\end{array}$ & $\begin{array}{r}219.1 \\
7.07 \\
9.6 \\
1.9 \\
0.0060 \\
0.007\end{array}$ & $\begin{array}{r}11.5 \\
0.37 \\
1.7 \\
0.1 \\
0.00032 \\
0.0004\end{array}$ & $\begin{array}{r}2.5 \\
0.086 \\
0.2 \\
0 \\
0.000074 \\
0.00008\end{array}$ & $\begin{array}{r}2041.1 \\
65.8 \\
116 \\
0.4 \\
0.056 \\
0.06\end{array}$ & $\begin{array}{r}3.395 \\
113 \\
433 \\
38 \\
0.097 \\
0.11\end{array}$ & $\begin{array}{r}2.000 \\
64.5 \\
126 \\
38 \\
0.055 \\
0.06\end{array}$ & $\begin{array}{r}10.553 \\
352 \\
1,050 \\
40 \\
0.301 \\
0.34\end{array}$ & $\begin{array}{r}14.189 \\
458 \\
1,430 \\
57 \\
0.391 \\
0.45\end{array}$ & $\begin{array}{r}2.205 \\
71.1 \\
206 \\
17 \\
0.061 \\
0.07\end{array}$ & $\begin{array}{r}1.723 \\
57.4 \\
135 \\
16 \\
0.049 \\
0.055\end{array}$ \\
\hline $\begin{array}{l}\text { Cal yr } \\
\text { Wtr yr }\end{array}$ & $\begin{array}{l}\text { 1967: } \\
1968:\end{array}$ & $\begin{array}{l}\text { Total } \\
\text { Tota1 }\end{array}$ & $\begin{array}{r}112,381.5 \\
36,830.1\end{array}$ & $\begin{array}{l}\text { Mean } \\
\text { Mean }\end{array}$ & $\begin{array}{l}308 \\
101\end{array}$ & $\begin{array}{l}3,100 \\
1,430\end{array}$ & $\begin{array}{l}\operatorname{Min} \\
\operatorname{Min}\end{array}$ & $\begin{array}{ll}1.8 & \text { Cfsm } \\
0 & \text { Cfsm }\end{array}$ & $\begin{array}{l}0.263 \\
0.086\end{array}$ & $\begin{array}{ll}\text { In. } & 3 \\
\text { In. } & 1\end{array}$ & & \\
\hline
\end{tabular}


Location.--Lat $45^{\circ} 05^{\prime} 15^{\prime \prime}$, long $93^{\circ} 44^{\prime} 00^{\prime \prime}$, in sec.29, T.119 N., R. 24 w., on right bank at Rockford, 150 ft downstream from bridge on' State Highway 55 and 1 mile downstream from confluence of North and South Forks.

Drainage area $:-2,520 \mathrm{sq} \mathrm{mi}$, approximately.

Records available.--April to July 1906 (published as "near Dayton"), June 1909 to September 1917 , April to November 1929, March 1930 to September 1931, April to November 1932, March to November 1933, March 1934 to september 1968. Monthly discharge only for some periods, published in wSP 1308 .

Gage.--Water-stage recorder. Datum of gage is $893.65 \mathrm{ft}$ above mean sea level, adjustment of 1912 . Apr. 13 to $J u l y ~ 21,1906$, staff gage at Berning Mill $14 \mathrm{miles}$ downstream at different datum.' June 4 , 1909 , to Sept. 30, 1917, staff gage at site $600 \mathrm{ft}$ downstream at different datum. Apr. 23, 1929, to Aug. 21, 1934, chain gage at site $600 \mathrm{ft}$ downstream at present datum.

Average discharge. -43 years $(1909-17,1930-31,1934-68), 573$ cfs.

Extremes.--Maximum discharge during year, 1,820 cfs July 18 (gage height, 5.00 ft); minimum daily, 23 cfs Jan. 15-22; minimum gage height, 1.43 ft Nov. 27.

1909-17, 1929-68: Maximum discharge, 22,400 cfs Apr. 16, 1965 (gage height, 19.27 ft, from floodmark); minimum, 1.8 cfs Nov. 15, 1936 (gage height, $1.05 \mathrm{ft}$ ), caused by ice jam upstream.

Remarks.--Records good except those for winter months, which are fair. Records of chemical analyses for the water year 1968 are published in Part 2 of this report.

DISCHARGE, IN CUBIC FEET PER SECOND, WATER YEAR OCTOBER 1967 TO SEPTEMBER 1968

\begin{tabular}{|c|c|c|c|c|c|c|c|c|c|c|c|c|}
\hline DAY & OCT & NOV & DEC & JAN & FEB & MAR & APR & MAY & JUN & JUL & AUG & SEP \\
\hline $\begin{array}{l}1 \\
2 \\
3 \\
4 \\
5\end{array}$ & $\begin{array}{l}51 \\
50 \\
50 \\
49 \\
68\end{array}$ & $\begin{array}{l}51 \\
53 \\
53 \\
50 \\
50\end{array}$ & $\begin{array}{l}40 \\
44 \\
46 \\
47 \\
47\end{array}$ & $\begin{array}{l}32 \\
30 \\
30 \\
30 \\
30\end{array}$ & $\begin{array}{l}26 \\
26 \\
26 \\
26 \\
26\end{array}$ & $\begin{array}{l}28 \\
28 \\
28 \\
28 \\
28\end{array}$ & $\begin{array}{l}220 \\
209 \\
218 \\
229 \\
229\end{array}$ & $\begin{array}{l}450 \\
402 \\
374 \\
334 \\
316\end{array}$ & $\begin{array}{l}350 \\
362 \\
362 \\
342 \\
302\end{array}$ & $\begin{array}{l}805 \\
750 \\
712 \\
646 \\
586\end{array}$ & $\begin{array}{l}487 \\
450 \\
406 \\
370 \\
342\end{array}$ & $\begin{array}{r}88 \\
80 \\
80 \\
88 \\
134\end{array}$ \\
\hline $\begin{array}{r}6 \\
7 \\
8 \\
9 \\
10\end{array}$ & $\begin{array}{l}66 \\
63 \\
66 \\
68 \\
69\end{array}$ & $\begin{array}{l}45 \\
48 \\
49 \\
54 \\
58\end{array}$ & $\begin{array}{l}49 \\
51 \\
54 \\
58 \\
62\end{array}$ & $\begin{array}{l}30 \\
28 \\
26 \\
26 \\
25\end{array}$ & $\begin{array}{l}26 \\
27 \\
27 \\
27 \\
27\end{array}$ & $\begin{array}{l}29 \\
32 \\
38 \\
48 \\
84\end{array}$ & $\begin{array}{l}226 \\
232 \\
251 \\
257 \\
266\end{array}$ & $\begin{array}{l}306 \\
302 \\
298 \\
292 \\
289\end{array}$ & $\begin{array}{l}273 \\
248 \\
238 \\
229 \\
238\end{array}$ & $\begin{array}{l}538 \\
482 \\
445 \\
392 \\
358\end{array}$ & $\begin{array}{l}330 \\
312 \\
302 \\
279 \\
263\end{array}$ & $\begin{array}{r}80 \\
73 \\
78 \\
82 \\
109\end{array}$ \\
\hline $\begin{array}{l}11 \\
12 \\
13 \\
14 \\
15\end{array}$ & $\begin{array}{l}66 \\
66 \\
64 \\
61 \\
58\end{array}$ & $\begin{array}{l}60 \\
60 \\
60 \\
56 \\
54\end{array}$ & $\begin{array}{l}64 \\
66 \\
70 \\
60 \\
58\end{array}$ & $\begin{array}{l}25 \\
25 \\
25 \\
24 \\
23\end{array}$ & $\begin{array}{l}27 \\
27 \\
27 \\
28 \\
28\end{array}$ & $\begin{array}{l}114 \\
120 \\
130 \\
150 \\
164\end{array}$ & $\begin{array}{l}266 \\
251 \\
235 \\
244 \\
248\end{array}$ & $\begin{array}{l}292 \\
269 \\
257 \\
269 \\
266\end{array}$ & $\begin{array}{r}266 \\
358 \\
445 \\
805 \\
1270\end{array}$ & $\begin{array}{r}338 \\
334 \\
397 \\
854 \\
1320\end{array}$ & $\begin{array}{l}241 \\
220 \\
203 \\
186 \\
175\end{array}$ & $\begin{array}{r}113 \\
109 \\
96 \\
86 \\
74\end{array}$ \\
\hline $\begin{array}{l}16 \\
17 \\
18 \\
19 \\
20\end{array}$ & $\begin{array}{l}56 \\
54 \\
53 \\
51 \\
51\end{array}$ & $\begin{array}{l}51 \\
54 \\
54 \\
56 \\
54\end{array}$ & $\begin{array}{l}55 \\
55 \\
55 \\
54 \\
54\end{array}$ & $\begin{array}{l}23 \\
23 \\
23 \\
23 \\
23\end{array}$ & $\begin{array}{l}28 \\
28 \\
28 \\
28 \\
28\end{array}$ & $\begin{array}{l}180 \\
194 \\
220 \\
285 \\
200\end{array}$ & $\begin{array}{l}260 \\
263 \\
254 \\
241 \\
279\end{array}$ & $\begin{array}{l}273 \\
273 \\
276 \\
285 \\
276\end{array}$ & $\begin{array}{l}1.440 \\
1.420 \\
1.370 \\
1.480 \\
1.490\end{array}$ & $\begin{array}{l}1.600 \\
1.770 \\
1.810 \\
1.690 \\
1.510\end{array}$ & $\begin{array}{l}172 \\
162 \\
151 \\
146 \\
143\end{array}$ & $\begin{array}{r}73 \\
94 \\
115 \\
183 \\
197\end{array}$ \\
\hline $\begin{array}{l}21 \\
22 \\
23 \\
24 \\
25\end{array}$ & $\begin{array}{l}49 \\
50 \\
50 \\
50 \\
48\end{array}$ & $\begin{array}{l}54 \\
51 \\
44 \\
41 \\
39\end{array}$ & $\begin{array}{l}54 \\
54 \\
51 \\
49 \\
47\end{array}$ & $\begin{array}{l}23 \\
23 \\
24 \\
24 \\
24\end{array}$ & $\begin{array}{l}28 \\
28 \\
28 \\
28 \\
28\end{array}$ & $\begin{array}{l}175 \\
210 \\
215 \\
230 \\
266\end{array}$ & $\begin{array}{l}316 \\
388 \\
509 \\
770 \\
875\end{array}$ & $\begin{array}{l}269 \\
257 \\
238 \\
232 \\
244\end{array}$ & $\begin{array}{l}1.480 \\
1.500 \\
1.450 \\
1.360 \\
1.240\end{array}$ & $\begin{array}{r}1.390 \\
1.250 \\
1.110 \\
973 \\
847\end{array}$ & $\begin{array}{l}141 \\
134 \\
124 \\
117 \\
100\end{array}$ & $\begin{array}{l}200 \\
209 \\
241 \\
276 \\
312\end{array}$ \\
\hline $\begin{array}{l}26 \\
27 \\
28 \\
29 \\
30 \\
31\end{array}$ & $\begin{array}{l}47 \\
49 \\
49 \\
51 \\
51 \\
51 \\
\end{array}$ & $\begin{array}{r}37 \\
36 \\
36 \\
36 \\
38 \\
\end{array}$ & $\begin{array}{l}45 \\
42 \\
40 \\
37 \\
35 \\
33 \\
\end{array}$ & $\begin{array}{l}26 \\
28 \\
27 \\
26 \\
25 \\
25 \\
\end{array}$ & $\begin{array}{r}28 \\
28 \\
28 \\
28 \\
-- \\
\end{array}$ & $\begin{array}{l}292 \\
316 \\
330 \\
298 \\
279 \\
248 \\
\end{array}$ & $\begin{array}{r}819 \\
705 \\
598 \\
532 \\
487 \\
\end{array}$ & $\begin{array}{l}260 \\
306 \\
338 \\
350 \\
334 \\
323\end{array}$ & $\begin{array}{r}1.180 \\
1.120 \\
1.040 \\
959 \\
882 \\
\\
\end{array}$ & $\begin{array}{l}750 \\
712 \\
646 \\
586 \\
532 \\
509\end{array}$ & $\begin{array}{l}98 \\
96 \\
96 \\
92 \\
86 \\
84\end{array}$ & $\begin{array}{r}326 \\
312 \\
285 \\
263 \\
248 \\
- \\
\end{array}$ \\
\hline $\begin{array}{l}\text { Tota } 1 \\
\text { Mean } \\
\text { Max } \\
\text { Min } \\
\text { Cfsm } \\
\text { In. } \\
\end{array}$ & $\begin{array}{r}1.725 \\
55.6 \\
69 \\
47 \\
0.022 \\
0.03 \\
\end{array}$ & $\begin{array}{r}1.482 \\
49.4 \\
60 \\
36 \\
0.020 \\
0.02 \\
\end{array}$ & $\begin{array}{r}1.576 \\
50.8 \\
70 \\
33 \\
0.020 \\
0.02 \\
\end{array}$ & $\begin{array}{r}799 \\
25.8 \\
32 \\
23 \\
0.010 \\
0.01 \\
\end{array}$ & $\begin{array}{r}793 \\
27.3 \\
28 \\
26 \\
0.011 \\
0.01 \\
\end{array}$ & $\begin{array}{r}4.987 \\
161 \\
330 \\
28 \\
0.064 \\
0.07 \\
\end{array}$ & $\begin{array}{r}10877 \\
363 \\
875 \\
209 \\
0.144 \\
0.16\end{array}$ & $\begin{array}{r}9.250 \\
298 \\
450 \\
232 \\
0.118 \\
0.14\end{array}$ & $\begin{array}{r}25.499 \\
850 \\
1,500 \\
229 \\
0.337 \\
0.38\end{array}$ & $\begin{array}{r}26642 \\
859 \\
1,810 \\
334 \\
0.341 \\
0.39\end{array}$ & $\begin{array}{r}6508 \\
210 \\
487 \\
84 \\
0.083 \\
0.10\end{array}$ & $\begin{array}{r}4.704 \\
157 \\
326 \\
73 \\
0.062 \\
0.07\end{array}$ \\
\hline $\begin{array}{ll}1 & \mathrm{yr} \\
\mathrm{r} & \mathrm{yr}\end{array}$ & 3: & 19 & & $\begin{array}{l}592 \\
259\end{array}$ & $\begin{array}{l}\operatorname{Max} \\
\operatorname{Max}\end{array}$ & $\begin{array}{l}90 \\
10\end{array}$ & $\begin{array}{ll}\text { in } & 33 \\
\text { in } & 23\end{array}$ & $\begin{array}{ll}\text { Cfsm } & 0.2 \\
\text { Cfsm } & 0.1\end{array}$ & $\begin{array}{l}\text { In. } \\
\text { In. }\end{array}$ & $\begin{array}{l}3.19 \\
1.40\end{array}$ & & \\
\hline
\end{tabular}


5-2840. Mille Lacs Lake at Garrison, Minn.

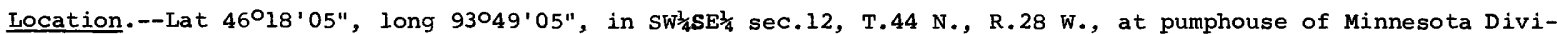
sion of Game and Fish, a quarter of a mile southwest of Borden Lake outlet and three-quarters mile northeast of Garrison.

Records available.--June 1931 to September 1968. Prior to October 1939, published as "at Wealthwood."

Gage.-Water-stage recorder. Datum of gage is 1,240.40 ft above mean sea level, datum of 1929 . Prior to Oct. 1, 1941, staff gage at Wealthwood at various datums; gage readings have been reduced to elevations above mean sea level, adjustment of 1912. Oct. I, 1941, to Sept. 30, 1958, water-stage recorder at datum $1,240.50 \mathrm{ft}$ above mean sea level, adjustment of 1912. To convert these records to datum of 1929 , subtract $0.10 \mathrm{ft}$.

Extremes.--Maximum elevation recorded during year, 1,252.99 ft June 30 (affected by wind action); maximum daily recorded, 1,252.05 ft June 30 (may have been higher during period of no gage-height record July 4 to Aug. 8); minimum recorded, $1,250.18 \mathrm{ft}$ Nov. 17 (affected by wind action).

1931-68: Maximum elevation, 1,253.51 ft July 25, 1952; minimum observed, 1,245.74 ft 0ct. 16-19, 1936.

Remarks.--Water level affected by fixed-crest spillway at outlet of Ogechie Lake with crest at elevation $1,250.50 \mathrm{ft}$. Water level subject to fluctuation caused by change in direction and velocity of wind and by seiches.

Daily mean elevation, in feet, October 1967 to September 1968

oct. $31 \ldots . .1,250.69$

Nov. $30 \ldots \ldots$ 1, 250.53

Dec. $31 \ldots \ldots$ 1,250.63

Jan. $31 \ldots \ldots .1,250.69$
Feb. $29 \ldots \ldots 1,250.69$

Mar. $31 \ldots \ldots 1,250.77$

Apr. $30 \ldots \ldots 1,251.18$
May $31 \ldots \ldots 1,251.39$

June $30 \ldots \ldots .1,252.05$

July 3 .... $1,252.04$ 
5-2860. Rum River near st. Francis, Minn.

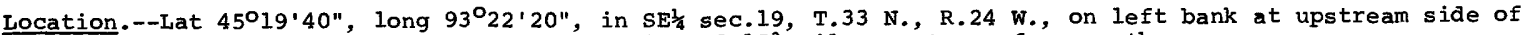
highway bridge, $4 \mathrm{miles}$ south of $\mathrm{St}$. Francis and $15 \frac{3}{4}$ miles upstream from mouth.

Drainage area. $-1,360 \mathrm{sq} \mathrm{mi}$, approximately.

Records available.--May to November 1929, March 1930 to September 1931, April to November 1932 , March 1933 to September 1968 .

Gage.-Water-stage recorder. Datum of gage is $861.12 \mathrm{ft}$ above mean sea level, adjustment of 1912 . Prior to Nov. 9, 1933, chain gage at site $50 \mathrm{ft}$ downstream at same datum.

Average discharge. --36 years $(1930-31,1933-68), 549$ cfs.

Extremes.--Maximum discharge during year, 2,150 cfs Sept. 30 (gage height, $5.17 \mathrm{ft}$ ); maximum gage height, 5.28 ft July 8 ; minimum discharge, $48 \mathrm{cfs}$ Nov. 26 (gage height, $2.06 \mathrm{ft}$ )

1929-68: Maximum discharge, 10, $100 \mathrm{cfs}$ Apr. 20, 1965 (gage height, 11.57 ft); minimum, 29 cfs Aug. 18, 1934 (gage height, $1.91 \mathrm{ft}$ ).

Remarks.--Records good except those for winter months, which are fair. Records of chemical analyses for the water year 1968 are published in Part 2 of this report. Occasional regulation by Ogechie (also controls Mille Lacs Lake) and Onamia Lakes.

DISCHARGE, IN CFS, WATER YEAR OCTOBER 1967 TO SEPTEMBER 1968

\begin{tabular}{|c|c|c|c|c|c|c|c|c|c|c|c|c|}
\hline$\overline{\mathrm{DAY}}$ & OCT & NOV & DEC & JAN & FEB & MAR & APR & MAY & JUN & JUL & AUG & SEP \\
\hline $\begin{array}{l}1 \\
2 \\
3 \\
4 \\
5\end{array}$ & $\begin{array}{l}216 \\
212 \\
206 \\
199 \\
195\end{array}$ & $\begin{array}{l}151 \\
157 \\
157 \\
157 \\
151\end{array}$ & $\begin{array}{l}150 \\
160 \\
157 \\
157 \\
157\end{array}$ & $\begin{array}{r}102 \\
100 \\
96 \\
94 \\
93\end{array}$ & $\begin{array}{l}94 \\
94 \\
94 \\
94 \\
94\end{array}$ & $\begin{array}{l}95 \\
95 \\
95 \\
95 \\
95\end{array}$ & $\begin{array}{l}424 \\
397 \\
366 \\
358 \\
345\end{array}$ & $\begin{array}{r}1060 \\
931 \\
808 \\
716 \\
629\end{array}$ & $\begin{array}{l}716 \\
723 \\
685 \\
666 \\
666\end{array}$ & $\begin{array}{l}1130 \\
1.140 \\
1.220 \\
1.350 \\
1.540\end{array}$ & $\begin{array}{l}429 \\
429 \\
434 \\
424 \\
415\end{array}$ & $\begin{array}{l}325 \\
325 \\
325 \\
333 \\
325\end{array}$ \\
\hline $\begin{array}{r}6 \\
7 \\
8 \\
9 \\
10\end{array}$ & $\begin{array}{l}192 \\
192 \\
195 \\
202 \\
202\end{array}$ & $\begin{array}{l}148 \\
134 \\
138 \\
148 \\
163\end{array}$ & $\begin{array}{l}157 \\
157 \\
157 \\
156 \\
156\end{array}$ & $\begin{array}{l}93 \\
93 \\
93 \\
93 \\
93\end{array}$ & $\begin{array}{l}94 \\
95 \\
95 \\
95 \\
95\end{array}$ & $\begin{array}{r}96 \\
98 \\
104 \\
117 \\
140\end{array}$ & $\begin{array}{l}333 \\
329 \\
354 \\
392 \\
454\end{array}$ & $\begin{array}{l}563 \\
523 \\
517 \\
517 \\
517\end{array}$ & $\begin{array}{l}678 \\
580 \\
534 \\
534 \\
580\end{array}$ & $\begin{array}{l}1.750 \\
1.930 \\
2.060 \\
2.050 \\
1.780\end{array}$ & $\begin{array}{l}410 \\
406 \\
415 \\
454 \\
469\end{array}$ & $\begin{array}{l}325 \\
325 \\
325 \\
333 \\
329\end{array}$ \\
\hline $\begin{array}{l}11 \\
12 \\
13 \\
14 \\
15\end{array}$ & $\begin{array}{l}209 \\
209 \\
206 \\
206 \\
209\end{array}$ & $\begin{array}{l}160 \\
157 \\
160 \\
160 \\
157\end{array}$ & $\begin{array}{l}155 \\
154 \\
149 \\
145 \\
140\end{array}$ & $\begin{array}{l}93 \\
93 \\
93 \\
93 \\
93\end{array}$ & $\begin{array}{l}95 \\
95 \\
95 \\
95 \\
95\end{array}$ & $\begin{array}{l}160 \\
190 \\
220 \\
250 \\
300\end{array}$ & $\begin{array}{l}604 \\
697 \\
678 \\
604 \\
569\end{array}$ & $\begin{array}{l}523 \\
545 \\
557 \\
540 \\
545\end{array}$ & $\begin{array}{r}782 \\
995 \\
1160 \\
1290 \\
1350\end{array}$ & $\begin{array}{r}1340^{\circ} \\
1040 \\
896 \\
840 \\
861\end{array}$ & $\begin{array}{l}469 \\
434 \\
410 \\
388 \\
375\end{array}$ & $\begin{array}{l}329 \\
329 \\
325 \\
321 \\
314\end{array}$ \\
\hline $\begin{array}{l}16 \\
17 \\
18 \\
19 \\
20\end{array}$ & $\begin{array}{l}202 \\
195 \\
192 \\
186 \\
182\end{array}$ & $\begin{array}{l}154 \\
151 \\
151 \\
151 \\
154\end{array}$ & $\begin{array}{l}140 \\
140 \\
139 \\
138 \\
138\end{array}$ & $\begin{array}{l}93 \\
93 \\
93 \\
93 \\
93\end{array}$ & $\begin{array}{l}95 \\
95 \\
95 \\
95 \\
95\end{array}$ & $\begin{array}{l}360 \\
362 \\
360 \\
352 \\
350\end{array}$ & $\begin{array}{l}534 \\
528 \\
534 \\
517 \\
545\end{array}$ & $\begin{array}{l}563 \\
569 \\
604 \\
653 \\
685\end{array}$ & $\begin{array}{l}1.420 \\
1.450 \\
1.440 \\
1.360 \\
1.270\end{array}$ & $\begin{array}{l}875 \\
854 \\
827 \\
794 \\
730\end{array}$ & $\begin{array}{l}375 \\
379 \\
375 \\
366 \\
362\end{array}$ & $\begin{array}{l}310 \\
329 \\
388 \\
420 \\
454\end{array}$ \\
\hline $\begin{array}{l}21 \\
22 \\
23 \\
24 \\
25\end{array}$ & $\begin{array}{l}182 \\
170 \\
170 \\
163 \\
151\end{array}$ & $\begin{array}{l}154 \\
154 \\
151 \\
141 \\
132\end{array}$ & $\begin{array}{l}137 \\
133 \\
130 \\
125 \\
122\end{array}$ & $\begin{array}{l}93 \\
93 \\
93 \\
93 \\
93\end{array}$ & $\begin{array}{l}95 \\
95 \\
95 \\
95 \\
95\end{array}$ & $\begin{array}{l}298 \\
392 \\
384 \\
392 \\
388\end{array}$ & $\begin{array}{r}635 \\
710 \\
834 \\
1010 \\
1100\end{array}$ & $\begin{array}{l}685 \\
672 \\
666 \\
641 \\
616\end{array}$ & $\begin{array}{r}1200 \\
1080 \\
995 \\
995 \\
1120\end{array}$ & $\begin{array}{l}666 \\
616 \\
569 \\
551 \\
528\end{array}$ & $\begin{array}{l}358 \\
358 \\
362 \\
358 \\
354\end{array}$ & $\begin{array}{r}469 \\
496 \\
889 \\
1110 \\
1.330\end{array}$ \\
\hline $\begin{array}{l}26 \\
27 \\
28 \\
29 \\
30 \\
31 \\
\end{array}$ & $\begin{array}{l}148 \\
144 \\
144 \\
144 \\
144 \\
151 \\
\end{array}$ & $\begin{array}{r}128 \\
131 \\
135 \\
138 \\
145 \\
-\end{array}$ & $\begin{array}{l}119 \\
117 \\
113 \\
110 \\
107 \\
104\end{array}$ & $\begin{array}{l}93 \\
93 \\
93 \\
93 \\
93 \\
93\end{array}$ & $\begin{array}{r}95 \\
95 \\
95 \\
95 \\
\end{array}$ & $\begin{array}{l}375 \\
379 \\
424 \\
449 \\
444 \\
449\end{array}$ & $\begin{array}{r}1150 \\
1190 \\
1270 \\
1300 \\
1.220 \\
- \\
\end{array}$ & $\begin{array}{l}598 \\
592 \\
629 \\
672 \\
678 \\
697\end{array}$ & $\begin{array}{r}1220 \\
1330 \\
1320 \\
1210 \\
1100 \\
-\end{array}$ & $\begin{array}{l}506 \\
506 \\
469 \\
449 \\
434 \\
434\end{array}$ & $\begin{array}{l}341 \\
329 \\
325 \\
318 \\
318 \\
321\end{array}$ & $\begin{array}{r}1.540 \\
1.740 \\
1.970 \\
21120 \\
2130 \\
-\end{array}$ \\
\hline $\begin{array}{l}\text { Total } \\
\text { Mean } \\
\text { Max } \\
\text { Min } \\
\text { Cfsm } \\
\text { In. }\end{array}$ & $\begin{array}{r}5718 \\
184 \\
216 \\
144 \\
0.135 \\
0.16 \\
\end{array}$ & $\begin{array}{r}4.468 \\
149 \\
163 \\
128 \\
0.110 \\
0.12\end{array}$ & $\begin{array}{r}4.319 \\
139 \\
160 \\
104 \\
0.102 \\
0.12\end{array}$ & $\begin{array}{r}2903 \\
93.6 \\
102 \\
93 \\
0.069 \\
0.08\end{array}$ & $\begin{array}{r}2.749 \\
94.8 \\
95 \\
94 \\
0.070 \\
0.08\end{array}$ & $\begin{array}{r}8.308 \\
268 \\
449 \\
95 \\
0.197 \\
0.23\end{array}$ & $\begin{array}{r}19.981 \\
666 \\
1,300 \\
329 \\
0.490 \\
0.55\end{array}$ & $\begin{array}{r}19.711 \\
636 \\
1,060 \\
517 \\
0.468 \\
0.54\end{array}$ & $\begin{array}{r}30.449 \\
1,015 \\
1,450 \\
534 \\
0.746 \\
0.83\end{array}$ & $\begin{array}{r}30735 \\
991 \\
2,060 \\
434 \\
0.729 \\
0.84\end{array}$ & $\begin{array}{r}11960 \\
386 \\
469 \\
318 \\
0.284 \\
0.33 \\
\end{array}$ & $\begin{array}{r}20.583 \\
686 \\
2,130 \\
310 \\
0.504 \\
0.56 \\
\end{array}$ \\
\hline
\end{tabular}

Cal yr 1967: Total 227,555 Mean 623 Max 5,290 Min 104, Cfsm 0.458 In. 6.22

Wtr yr 1968: Total 161,884 Mean 442 Max 2,130 Min 93 Cfsm $0.325 \quad$ In. 4.43 
5-2885. Mississippi River near Anoka, Minn.

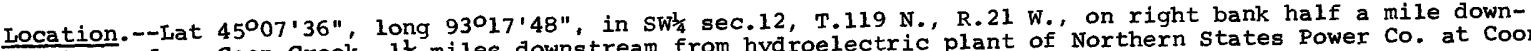
stream from Coon Creek, $1 \frac{1}{2}$ miles downstream from hydroelectric plant of Northern States Power Co. at Coon strom Ohio River.

Drainage area, $--19,100 \mathrm{sq} \mathrm{mi}$, approximately.

Records available.--June 1931 to september 1968. Prior to october 1931 published as "at Coon Rapids, near Anoka".

Gage.--Digital water-stage recorder. Datum of gage is 805.02 ft above mean sea level, adjustment of 1912 .

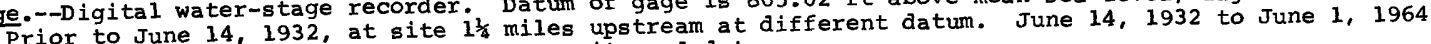
graphic water-stage recorder at present site and datum.

Average discharge. - - 37 years, $7,044 \mathrm{cfs}$.

Extremes.--Maximum discharge during year, 20,900 cfs June 16 (gage height, $7.38 \mathrm{ft}$ ); minimum 641 cfs Nov. 28 (gage height, $0.13 \mathrm{ft}$ ). 1931-68: Maximum discharge, $91,000 \mathrm{cfs}$ Apr. 17 , 1965 (gage height, 1967.

Flow slightly regulated by six reservoirs on headwaters; total usable capacity,

Remarks.--Records good. Flow slightly regulated by six reservoirs of station. Records of chemical analyses for the water year 1968 are published in Part 2 of this report.

OISCHARGE, IN CUBIC FEET PER SECJND, WATER YEAR OCTOBER 1967 TO SEPTEMBER 1968

\begin{tabular}{|c|c|c|c|c|c|c|c|c|c|c|c|c|}
\hline DAY & $\mathrm{OCT}$ & NOV & DEC & JAN & FEB & $M A P$ & $A P R$ & MAY & JUN & JUL & AUG & SEP \\
\hline $\begin{array}{l}1 \\
2 \\
3 \\
4 \\
5\end{array}$ & $\begin{array}{l}2.500 \\
2.480 \\
2.310 \\
2.560 \\
2.290\end{array}$ & $\begin{array}{l}2.770 \\
3.140 \\
2.980 \\
3.240 \\
3,140\end{array}$ & $\begin{array}{l}2,180 \\
2.400 \\
2.780 \\
2.940 \\
2.810\end{array}$ & $\begin{array}{l}2,000 \\
2,020 \\
1,700 \\
2,000 \\
2,080\end{array}$ & $\begin{array}{l}2,850 \\
2,800 \\
2,700 \\
2,650 \\
2,650\end{array}$ & $\begin{array}{l}2.220 \\
2.250 \\
2.300 \\
2.406 \\
2.600\end{array}$ & $\begin{array}{l}5.730 \\
5.520 \\
5.140 \\
5.330 \\
6.020\end{array}$ & $\begin{array}{l}14,300 \\
13.500 \\
12.800 \\
11,500 \\
10,400\end{array}$ & $\begin{array}{l}8,320 \\
8,330 \\
8,080 \\
8,510 \\
8,150\end{array}$ & $\begin{array}{l}14,700 \\
13.600 \\
12.500 \\
12.100 \\
12.200\end{array}$ & $\begin{array}{l}6.800 \\
6.630 \\
6,010 \\
5.260 \\
5.290\end{array}$ & $\begin{array}{l}3,010 \\
3,070 \\
2,690 \\
2,930 \\
3,240\end{array}$ \\
\hline $\begin{array}{r}6 \\
7 \\
3 \\
9 \\
10\end{array}$ & $\begin{array}{l}2.290 \\
2.310 \\
2.560 \\
2.960 \\
2.950\end{array}$ & $\begin{array}{l}2.890 \\
2.920 \\
2.710 \\
2.330 \\
2.990\end{array}$ & $\begin{array}{l}2,840 \\
3,180 \\
3,090 \\
3,080 \\
3,000\end{array}$ & $\begin{array}{l}2.100 \\
2.150 \\
2.180 \\
2.200 \\
2.200\end{array}$ & $\begin{array}{l}2.750 \\
2.750 \\
2.800 \\
2.800 \\
2.900\end{array}$ & $\begin{array}{l}2,50 C \\
3,000 \\
3,10 \mathrm{C} \\
3,40 \mathrm{C} \\
4, \mathrm{CCO}\end{array}$ & $\begin{array}{l}5,150 \\
5,080 \\
5,620 \\
6,260 \\
6,490\end{array}$ & $\begin{array}{l}9,300 \\
8.940 \\
8.780 \\
8.610 \\
8.870\end{array}$ & $\begin{array}{l}7,880 \\
8,580 \\
7,460 \\
8,060 \\
9,280\end{array}$ & $\begin{array}{l}12,600 \\
11,900 \\
11,300 \\
11,300 \\
10,100\end{array}$ & $\begin{array}{l}5,440 \\
5.320 \\
5.510 \\
5.220 \\
4.990\end{array}$ & $\begin{array}{l}3,060 \\
3,080 \\
2,940 \\
3,200 \\
3,530\end{array}$ \\
\hline $\begin{array}{l}11 \\
12 \\
13 \\
14 \\
15\end{array}$ & $\begin{array}{l}2.970 \\
2.610 \\
2.850 \\
2.470 \\
2.890\end{array}$ & $\begin{array}{l}3.260 \\
2,650 \\
3.270 \\
3.260 \\
2,380\end{array}$ & $\begin{array}{l}3.090 \\
2,880 \\
2.700 \\
2,520 \\
2,410\end{array}$ & $\begin{array}{l}2,220 \\
2,250 \\
2,280 \\
2,300 \\
2,300\end{array}$ & $\begin{array}{l}2,500 \\
2,700 \\
3,100 \\
2,600 \\
2,300\end{array}$ & $\begin{array}{l}4,700 \\
4,700 \\
5,000 \\
5,000 \\
5,000\end{array}$ & $\begin{array}{l}7,400 \\
8,300 \\
8,130 \\
7,860 \\
8,180\end{array}$ & $\begin{array}{l}8.660 \\
8,370 \\
8.790 \\
9,080 \\
0.110\end{array}$ & $\begin{array}{l}12,600 \\
16.000 \\
18.000 \\
19,700 \\
20.400\end{array}$ & $\begin{array}{r}8,900 \\
8,350 \\
8,940 \\
9,140 \\
10,800\end{array}$ & $\begin{array}{l}4,840 \\
4,460 \\
4,440 \\
4,230 \\
4,330\end{array}$ & $\begin{array}{l}3,410 \\
3,060 \\
2,760 \\
2,710 \\
2,540\end{array}$ \\
\hline $\begin{array}{l}16 \\
17 \\
18 \\
19 \\
20\end{array}$ & $\begin{array}{l}2,760 \\
2,890 \\
3,050 \\
3,510 \\
2,810\end{array}$ & $\begin{array}{l}2.830 \\
2.690 \\
2.890 \\
3.100 \\
3.190\end{array}$ & $\begin{array}{l}2,120 \\
2,220 \\
2,700 \\
2,900 \\
2,900\end{array}$ & $\begin{array}{l}2,320 \\
2,380 \\
2,400 \\
2,420 \\
2,450\end{array}$ & $\begin{array}{l}2,100 \\
2,000 \\
1,900 \\
2.100 \\
2,050\end{array}$ & $\begin{array}{l}5,000 \\
5,500 \\
5,800 \\
6,190 \\
6,190\end{array}$ & $\begin{array}{l}8,060 \\
8,020 \\
7,640 \\
7.720 \\
7.740\end{array}$ & $\begin{array}{l}9,280 \\
8,940 \\
8,710 \\
9.250 \\
9.630\end{array}$ & $\begin{array}{l}20,600 \\
20,100 \\
20,300 \\
19,100 \\
17,700\end{array}$ & $\begin{array}{l}11.100 \\
11,300 \\
11,200 \\
12,400 \\
12,300\end{array}$ & $\begin{array}{l}4.240 \\
4.270 \\
4.090 \\
3.860 \\
3.590\end{array}$ & $\begin{array}{l}2,830 \\
3,300 \\
3,590 \\
4,130 \\
4,100\end{array}$ \\
\hline $\begin{array}{l}21 \\
22 \\
23 \\
24 \\
25\end{array}$ & $\begin{array}{l}2.150 \\
2.630 \\
2.680 \\
3.450 \\
3.010\end{array}$ & $\begin{array}{l}3,380 \\
2.860 \\
2.310 \\
2.600 \\
2.630\end{array}$ & $\begin{array}{l}2,700 \\
2,000 \\
1,800 \\
2.100 \\
2,400\end{array}$ & $\begin{array}{l}2,500 \\
2,500 \\
2,550 \\
2,550 \\
2,600\end{array}$ & $\begin{array}{l}2.080 \\
2.080 \\
2.090 \\
2.100 \\
2.120\end{array}$ & $\begin{array}{l}6.386 \\
5.830 \\
5.380 \\
6.320 \\
6.580\end{array}$ & $\begin{array}{r}8.640 \\
6.110 \\
10.690 \\
12,400 \\
12,400\end{array}$ & $\begin{array}{l}8.970 \\
8.860 \\
8,840 \\
9.260 \\
8.800\end{array}$ & $\begin{array}{l}17,600 \\
17,400 \\
17,100 \\
16.500 \\
16,000\end{array}$ & $\begin{array}{r}11,800 \\
11,700 \\
10,600 \\
10,000 \\
9,130\end{array}$ & $\begin{array}{l}3,580 \\
3,660 \\
3,830 \\
3,580 \\
3,360\end{array}$ & $\begin{array}{l}4,160 \\
3,880 \\
5,500 \\
7,060 \\
7,340\end{array}$ \\
\hline $\begin{array}{l}25 \\
27 \\
28 \\
29 \\
30 \\
31\end{array}$ & $\begin{array}{l}2,840 \\
2,970 \\
2,750 \\
2,990 \\
2,960 \\
2,820\end{array}$ & $\begin{array}{r}2.510 \\
1.810 \\
1,730 \\
1,400 \\
1,600 \\
\end{array}$ & $\begin{array}{l}2,900 \\
2,600 \\
2,400, \\
2,600 \\
2,400 \\
2,000\end{array}$ & $\begin{array}{l}2,700 \\
2,700 \\
2,700 \\
2,800 \\
2,700 \\
2,900\end{array}$ & $\begin{array}{r}2,150 \\
2.150 \\
2,150 \\
2,150 \\
2 \\
\end{array}$ & $\begin{array}{l}6,010 \\
5,980 \\
5,950 \\
5,920 \\
6,030 \\
5,000\end{array}$ & $\begin{array}{l}13,600 \\
14,400 \\
14,700 \\
15,000 \\
15,100\end{array}$ & $\begin{array}{r}8,010 \\
7,460 \\
7,560 \\
7.610 \\
7,620 \\
7,600\end{array}$ & $\begin{array}{l}15,100 \\
14,900 \\
14,100 \\
13,600 \\
13,700 \\
\end{array}$ & $\begin{array}{l}8,810 \\
8,410 \\
7,630 \\
7,480 \\
7,140 \\
6,870\end{array}$ & $\begin{array}{l}3,150 \\
2,530 \\
2,480 \\
2,660 \\
2,720 \\
2,810\end{array}$ & $\begin{array}{r}7.570 \\
8,130 \\
7,810 \\
7,780 \\
7,840 \\
\end{array}$ \\
\hline $\begin{array}{l}\text { TOTAL } \\
\text { MEAN } \\
\text { MAX } \\
\text { MIN } \\
\text { CFSM } \\
\text { IN. } \\
\end{array}$ & $\begin{array}{r}85,350 \\
2,753 \\
3,510 \\
2,170 \\
.14 \\
.17 \\
\end{array}$ & $\begin{array}{r}82,580 \\
2,753 \\
3,230 \\
1,400 \\
.14 \\
.16 \\
\end{array}$ & $\begin{array}{r}80,640 \\
2,601 \\
3,180 \\
1.800 \\
.14 \\
.16 \\
\end{array}$ & $\begin{array}{r}73,150 \\
2,360 \\
2,900 \\
1,700 \\
.12 \\
.14 \\
\end{array}$ & $\begin{array}{r}70,070 \\
2.416 \\
3,100 \\
1.900 \\
.13 \\
.14 \\
\end{array}$ & $\begin{array}{r}147.130 \\
4.746 \\
6.586 \\
2.220 \\
.25 \\
.29 \\
\end{array}$ & $\begin{array}{r}261,340 \\
8,711 \\
15,100 \\
5,080 \\
.46 \\
.51 \\
\end{array}$ & $\begin{array}{r}287,410 \\
9,271 \\
14,300 \\
7,460 \\
.49 \\
.56 \\
\end{array}$ & $\begin{array}{r}423,450 \\
14,120 \\
20,600 \\
7,460 \\
.74 \\
.82 \\
\end{array}$ & $\begin{array}{r}326,300 \\
10,530 \\
14,700 \\
6,870 \\
.55 \\
.64 \\
\end{array}$ & $\begin{array}{r}133,180 \\
4,296 \\
6,800 \\
2,480 \\
.22 \\
.26 \\
\end{array}$ & $\begin{array}{r}130,250 \\
4,342 \\
8,130 \\
2,540 \\
.23 \\
.25 \\
\end{array}$ \\
\hline $\begin{array}{ll}\text { CAL } & Y R \\
\text { WTR } & Y R \\
\end{array}$ & $\begin{array}{ll}1967^{\circ} & \mathrm{T} 0 \\
1968 & \mathrm{TO} \\
\end{array}$ & $L \quad 2,100$ & $\begin{array}{l}50 \\
50\end{array}$ & $\begin{array}{l}7.388 \\
N \quad 5.740 \\
\end{array}$ & $\begin{array}{l}\text { MAX } \\
\text { MAX }\end{array}$ & $\begin{array}{l}.600 \\
.600 \\
\end{array}$ & $\begin{array}{ll}N & 1.400 \\
N & 1.400 \\
\end{array}$ & $\begin{array}{l}\text { CFSM } \\
\text { CFSM }\end{array}$ & $\begin{array}{l}\text { IN } \\
\text { IN }\end{array}$ & $\begin{array}{r}.25 \\
.09 \\
\end{array}$ & & \\
\hline
\end{tabular}


5-2900. Little Minnesota River near Peever, S. Dak.

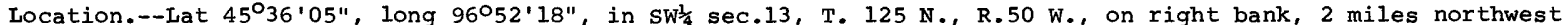

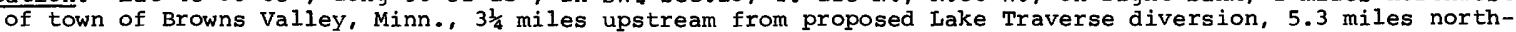
east of Peever, $7 \frac{1}{4}$ miles downstream from Jorgenson River, and 8 miles upstream from Big Stone Lake.

Drainage area. $--447 \mathrm{sq} \mathrm{mi}$.

Records available.--October 1939 to september 1968.

Gage.--Water-stage recorder. Altitude of gage is 1,000 ft (from topographic map). Oct. 1, 1939, to Mar. 20, 1940, staff gage at site $4 \frac{1}{2}$ miles downstream at different datum. Mar. 21 to Apr. 12, 1940, staff gage at site $100 \mathrm{ft}$ downstream at present datum. April 13 to Aug. 27, 1940, staff gage at present site and datum.

Average discharge. --29 years, $45.8 \mathrm{cfs}(33,160$ acre-ft per year).

Extremes.--Maximum discharge during year, 152 cfs May 9 (gage height, 3.55 ft); no flow Jan. 3-13.

1939-68: Maximum discharge, 4,730 cfs Apr. 8, 1952 (gage height, 12.16 ft); maximum gage height, 13.35 ft Mar. 25, 1943, from floodmark (backwater from ice); no flow at times in 1940, 1942, 1950, 1954, 1957, $1959,1963,1968$.

Remarks.--Records good except those for winter months, which are fair.

DISCHARGE, IN CFS, WATER YEAR OCTOBER 1967 TO SEPTEMBER 1968

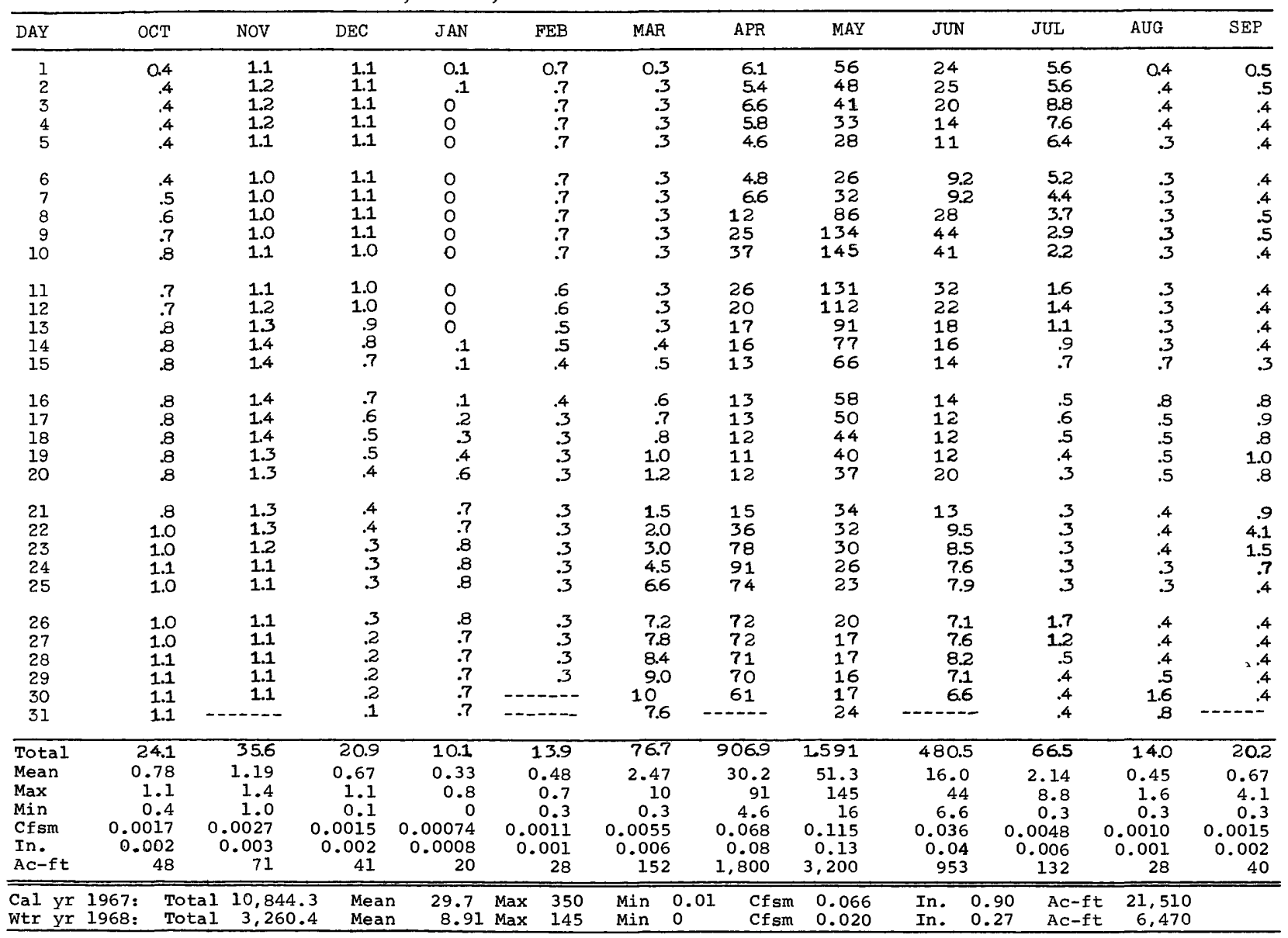

Peak discharge (base, 450 cfs).--No peak above base. 
5-2910. Whetstone River near Big stone City, S. Dak.

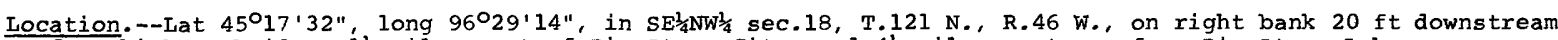

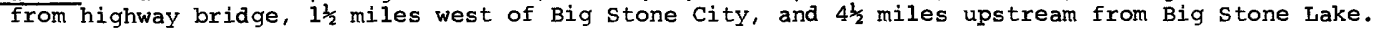

Drainage area. $--389 \mathrm{sq} \mathrm{mi}$.

Records available.--March 1910 to November 1912 (no winter records) and March 1931 to September 1968. Monthly discharge only for some periods, published in WSP 1308.

Gage.--Water-stage recorder. Datum of gage is $996.96 \mathrm{ft}$ above mean sea leve1, adjustment of 1912 . Mar. 8 , 1910 , to Nov. 30, 1912, staff gage 2 miles downstream at different datum. Mar. 18, 1931, to Aug. 27, 1938, chain gage and Aug. 28, 1938, to May 3, 1939, wire-weight gage, at site 20 ft upstream at present datum.

May 4, 1939 to Nov. 8, 1952, water-stage recorder at site 80 ft downstream at present datum.

Average discharge.--37 years $(1931-68), 44.3 \mathrm{cfs}(32,070$ acre-ft per year).

Extremes.--Maximum discharge during year, 153 cfs Apr. 24 (gage height, $3.67 \mathrm{ft}$ ); maximum gage height, 4.12 ft Mar. 11 (backwater from ice); minimum daily discharge, 0.2 cfs Jan. 4-20; minimum observed gage height, $1.73 \mathrm{ft}$ Jan. 9

1910-12, 1931-68: Maximum discharge, 5,710 cfs Apr. 8, 1952 (gage height, 13.64 ft, from floodmark); maximum gage height, $13.95 \mathrm{ft}$ Apr. 11, 1947; no flow at times in most years.

Maximum stage known, about $26 \mathrm{ft}$ in June 1919, present site and datum, from information by local resident.

Remarks.--Records good except those for winter months, which are fair. Records of chemical analyses for the water year 1968 are published in Part 2 of this report.

DISCHARGE, IN CFS, WATER YEAR OCTOBER 1967 TO SEPTEMBER 1968

\begin{tabular}{|c|c|c|c|c|c|c|c|c|c|c|c|c|}
\hline DAY & OCT & NOV & $\mathrm{DEC}$ & $\mathrm{JAN}$ & FEB & MAR & APR & MAY & JINN & JUL & AUG & $\overline{S E P}$ \\
\hline $\begin{array}{l}1 \\
2 \\
3 \\
4 \\
5\end{array}$ & $\begin{array}{l}1.7 \\
1.8 \\
1.8 \\
1.9 \\
2.0\end{array}$ & $\begin{array}{l}4.4 \\
4.4 \\
4.3 \\
4.4 \\
4.4\end{array}$ & $\begin{array}{l}5.1 \\
5.0 \\
4.9 \\
4.8 \\
4.5\end{array}$ & $\begin{array}{r}0.3 \\
.3 \\
.3 \\
.2 \\
.2\end{array}$ & $\begin{array}{l}1.3 \\
1.6 \\
1.8 \\
2.0 \\
2.1\end{array}$ & $\begin{array}{l}2.9 \\
3.8 \\
4.5 \\
5.4 \\
6.6\end{array}$ & $\begin{array}{r}6.6 \\
6.6 \\
8.2 \\
8.2 \\
11\end{array}$ & $\begin{array}{l}23 \\
21 \\
17 \\
15 \\
15\end{array}$ & $\begin{array}{l}9.2 \\
7.5 \\
6.4 \\
5.6 \\
5.1\end{array}$ & $\begin{array}{l}5.1 \\
5.4 \\
4.8 \\
4.4 \\
4.1\end{array}$ & $\begin{array}{l}3.4 \\
3.0 \\
3.0 \\
2.8 \\
2.7\end{array}$ & $\begin{array}{l}2.8 \\
2.8 \\
2.6 \\
2.2 \\
2.2\end{array}$ \\
\hline $\begin{array}{r}6 \\
7 \\
8 \\
9 \\
10\end{array}$ & $\begin{array}{l}2.2 \\
2.8 \\
3.0 \\
3.0 \\
3.2\end{array}$ & $\begin{array}{l}4.6 \\
4.6 \\
4.8 \\
4.8 \\
4.8\end{array}$ & $\begin{array}{l}4.4 \\
4.3 \\
4.2 \\
4.1 \\
4.0\end{array}$ & $\begin{array}{l}.2 \\
2 \\
2 \\
.2 \\
.2\end{array}$ & $\begin{array}{l}2.1 \\
2.2 \\
2.2 \\
2.3 \\
2.3\end{array}$ & $\begin{array}{l}7.7 \\
8.5 \\
9.1 \\
9.4 \\
9.7\end{array}$ & $\begin{array}{l}9.6 \\
9.9 \\
12 \\
16 \\
21\end{array}$ & $\begin{array}{l}15 \\
18 \\
21 \\
34 \\
34\end{array}$ & $\begin{array}{l}4.6 \\
4.8 \\
34 \\
34 \\
20\end{array}$ & $\begin{array}{l}3.8 \\
3.5 \\
3.5 \\
3.2 \\
3.1\end{array}$ & $\begin{array}{l}2.6 \\
2.6 \\
2.3 \\
2.3 \\
2.3\end{array}$ & $\begin{array}{l}2.2 \\
2.0 \\
2.3 \\
2.7 \\
2.6\end{array}$ \\
\hline $\begin{array}{l}11 \\
12 \\
13 \\
14 \\
15\end{array}$ & $\begin{array}{l}3.0 \\
3.0 \\
3.0 \\
3.5 \\
3.6\end{array}$ & $\begin{array}{l}5.1 \\
5.4 \\
4.9 \\
5.3 \\
5.6\end{array}$ & $\begin{array}{l}3.9 \\
3.8 \\
3.6 \\
3.5 \\
3.2\end{array}$ & $\begin{array}{l}.2 \\
2 \\
2 \\
.2 \\
2\end{array}$ & $\begin{array}{l}2.3 \\
2.3 \\
2.3 \\
2.4 \\
2.4\end{array}$ & $\begin{array}{l}9.7 \\
9.8 \\
10 \\
10 \\
11\end{array}$ & $\begin{array}{l}19 \\
16 \\
14 \\
12 \\
9.2\end{array}$ & $\begin{array}{l}31 \\
2 \% \\
23 \\
21 \\
19\end{array}$ & $\begin{array}{l}14 \\
11 \\
9.6 \\
7.5 \\
6.4\end{array}$ & $\begin{array}{l}2.8 \\
3.0 \\
3.0 \\
3.1 \\
3.1\end{array}$ & $\begin{array}{l}2.2 \\
2.2 \\
2.2 \\
2.2 \\
2.5\end{array}$ & $\begin{array}{l}2.6 \\
2.5 \\
2.2 \\
2.2 \\
2.3\end{array}$ \\
\hline $\begin{array}{l}16 \\
17 \\
18 \\
19 \\
20\end{array}$ & $\begin{array}{l}3.9 \\
4.1 \\
4.4 \\
4.1 \\
3.8\end{array}$ & $\begin{array}{l}5.4 \\
5.3 \\
5.4 \\
5.8 \\
5.4\end{array}$ & $\begin{array}{l}3.0 \\
2.7 \\
2.5 \\
2.2 \\
2.0\end{array}$ & $\begin{array}{l}.2 \\
.2 \\
.2 \\
.2 \\
.2\end{array}$ & $\begin{array}{l}2.4 \\
2.5 \\
2.5 \\
2.5 \\
2.5\end{array}$ & $\begin{array}{l}12 \\
12 \\
12 \\
13 \\
12\end{array}$ & $\begin{array}{l}7.2 \\
6.2 \\
5.4 \\
4.9 \\
5.8\end{array}$ & $\begin{array}{l}17 \\
16 \\
15 \\
15 \\
13\end{array}$ & $\begin{array}{l}6.0 \\
5.4 \\
5.3 \\
4.9 \\
4.9\end{array}$ & $\begin{array}{l}2.8 \\
3.4 \\
3.2 \\
2.8 \\
2.8\end{array}$ & $\begin{array}{l}2.8 \\
2.3 \\
2.1 \\
2.3 \\
2.1\end{array}$ & $\begin{array}{l}3.1 \\
3.2 \\
3.4 \\
3.5 \\
3.5\end{array}$ \\
\hline $\begin{array}{l}21 \\
22 \\
23 \\
24 \\
25\end{array}$ & $\begin{array}{l}4.1 \\
4.1 \\
4.3 \\
4.3 \\
4.3\end{array}$ & $\begin{array}{l}5.6 \\
5.4 \\
5.4 \\
5.3 \\
5.1\end{array}$ & $\begin{array}{l}1.8 \\
1.7 \\
1.5 \\
1.4 \\
1.2\end{array}$ & $\begin{array}{l}.3 \\
.3 \\
.3 \\
.3 \\
.3\end{array}$ & $\begin{array}{l}2.5 \\
2.5 \\
2.5 \\
2.5 \\
2.6\end{array}$ & $\begin{array}{l}12 \\
12 \\
12 \\
13 \\
15\end{array}$ & $\begin{array}{c}6.4 \\
20 \\
73 \\
140 \\
94\end{array}$ & $\begin{array}{c}12 \\
9.9 \\
9.2 \\
8.8 \\
6.9\end{array}$ & $\begin{array}{l}5.4 \\
5.8 \\
6.0 \\
5.3 \\
5.8\end{array}$ & $\begin{array}{l}2.8 \\
2.7 \\
2.6 \\
2.6 \\
2.8\end{array}$ & $\begin{array}{l}2.0 \\
1.8 \\
1.8 \\
1.6 \\
1.7\end{array}$ & $\begin{array}{l}3.2 \\
3.6 \\
3.6 \\
3.2 \\
5.4\end{array}$ \\
\hline $\begin{array}{l}26 \\
27 \\
28 \\
29 \\
30 \\
31\end{array}$ & $\begin{array}{l}4.1 \\
4.3 \\
4.3 \\
4.4 \\
4.6 \\
4.3\end{array}$ & $\begin{array}{r}5.3 \\
5.1 \\
5.3 \\
5.2 \\
5.1 \\
- \\
-\end{array}$ & $\begin{array}{r}1.1 \\
.7 \\
.6 \\
.5 \\
.4 \\
.3\end{array}$ & $\begin{array}{r}.3 \\
.4 \\
.5 \\
.7 \\
.9 \\
1.1\end{array}$ & $\begin{array}{r}2.6 \\
2.7 \\
2.7 \\
2.8 \\
- \\
-\end{array}$ & $\begin{array}{c}16 \\
17 \\
18 \\
17 \\
14 \\
8.5\end{array}$ & $\begin{array}{r}56 \\
41 \\
52 \\
28 \\
24 \\
\end{array}$ & $\begin{array}{l}8.2 \\
7.8 \\
9.6 \\
9.2 \\
8.5 \\
9.6\end{array}$ & $\begin{array}{r}5.8 \\
5.8 \\
5.3 \\
4.9 \\
5.1 \\
-\end{array}$ & $\begin{array}{l}18 \\
12 \\
5.6 \\
4.1 \\
3.8 \\
3.6\end{array}$ & $\begin{array}{l}1.6 \\
1.7 \\
1.7 \\
1.7 \\
3.4 \\
3.0\end{array}$ & $\begin{array}{r}3.1 \\
2.8 \\
2.6 \\
2.0 \\
1.8 \\
\end{array}$ \\
\hline $\begin{array}{l}\text { Total } \\
\text { Mean } \\
\text { Max } \\
\text { Min } \\
\text { Cfsm } \\
\text { In. } \\
\text { Ac-ft }\end{array}$ & $\begin{array}{r}106.9 \\
3.45 \\
4.6 \\
1.7 \\
0.0089 \\
0.01 \\
212\end{array}$ & $\begin{array}{r}151.9 \\
5.06 \\
5.8 \\
4.3 \\
0.013 \\
0.01 \\
301\end{array}$ & $\begin{array}{r}86.9 \\
2.80 \\
5.1 \\
0.3 \\
0.0072 \\
0.008 \\
172\end{array}$ & $\begin{array}{r}9.7 \\
0.31 \\
1.1 \\
0.2 \\
0.00080 \\
0.0009 \\
19\end{array}$ & $\begin{array}{r}67.4 \\
2.32 \\
2.8 \\
1.3 \\
0.0060 \\
0.006 \\
134\end{array}$ & $\begin{array}{r}333.6 \\
10.8 \\
18 \\
2.9 \\
0.028 \\
0.03 \\
662\end{array}$ & $\begin{array}{r}723.2 \\
24.1 \\
140 \\
4.9 \\
0.062 \\
0.07 \\
1.430\end{array}$ & $\begin{array}{r}509.7 \\
16.4 \\
34 \\
6.9 \\
0.042 \\
0.05 \\
1,010\end{array}$ & $\begin{array}{r}2614 \\
8.71 \\
34 \\
4.6 \\
0.022 \\
0.02 \\
518\end{array}$ & $\begin{array}{r}131.5 \\
4.24 \\
18 \\
2.6 \\
0.011 \\
0.01 \\
261\end{array}$ & $\begin{array}{r}71.9 \\
2.32 \\
3.4 \\
1.6 \\
0.0060 \\
0.007 \\
143\end{array}$ & $\begin{array}{r}82.2 \\
2.74 \\
3.6 \\
1.8 \\
0.0070 \\
0.008 \\
163\end{array}$ \\
\hline $\begin{array}{ll}\text { al } & y r \\
\text { tr } & y^{\prime}\end{array}$ & $\begin{array}{l}967: \\
968:\end{array}$ & $\begin{array}{l}9,3 \\
2,5\end{array}$ & $\begin{array}{l}\text { Mea } \\
\text { Mea }\end{array}$ & $\begin{array}{l}25.6 \\
6.93\end{array}$ & $\begin{array}{ll}\operatorname{Max} & 420 \\
\operatorname{Max} & 140\end{array}$ & $\begin{array}{l}\text { Min } \\
\text { Min }\end{array}$ & $\begin{array}{l}\text { Cfsm } \\
\text { Cfsm }\end{array}$ & $\begin{array}{l}0.066 \\
0.018\end{array}$ & $\begin{array}{ll}\text { In. } & 0.8 \\
\text { In. } & 0.2\end{array}$ & $\begin{array}{l}A c-f t \\
A c-f t\end{array}$ & $\begin{array}{r}18,550 \\
5,030\end{array}$ & \\
\hline
\end{tabular}

Note.--Peak discharge (base, $200 \mathrm{cfs}$ ). No peak above base. 
5-2915. Big Stone Lake at Ortonville, Minn.

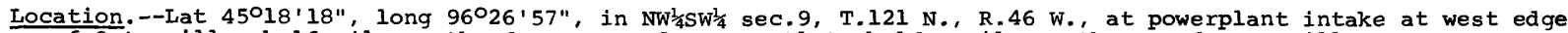
of Ortonville, half mile north of concrete dam at outlet, half a mile southwest of ortonville.

Records available.--March 1937 to September 1968.

Gage.--Wire-weight gage read once a day. Datum of gage is 957.69 ft above mean sea level, datum of 1929. Prior to Sept. 17, 1947, staff gage at site $\frac{1}{2}$ mile south at same datum. Sept. 18, 1947 , to June 30 , 1963, water-stage recorder at site $\frac{1}{2}$ mile south at same datum. Sept. 21, 1959, to June 30, 1963, supplementary wire-weight gage read once daily, at present site and datum.

Extremes.--Maximum gage height observed during year, $7.62 \mathrm{ft}$ June 11 ; minimum observed, 6.00 ft Oct. 28 .

1937-68: Maximum gage height, 12.73 ft Apr. 17, 1952; minimum observed, 2.20 ft Nov. 20 , 1940.

Remarks.--Reservoir is formed by natural lake with concrete dam at outlet. Fixed crest of dam is at elevation $963.64 \mathrm{ft}$, with one 5-foot gate and two $2 \frac{1}{2}$-foot gates with lowest sill at elevation 958.40 ft (all elevations are referred to datum of 1929). Changes in gate openings are not made.

Silt barrier dam $700 \mathrm{ft}$ upstream in outlet channel of lake completed July 7 , 1958; crest elevation

$963.6 \mathrm{ft}$. Supplementary wire-weight gage readings used for stages below crest of silt barrier to June 30 , 1963. Water level subject to fluctuation caused by wind action.

Gage height, in teet, October 1967 to september 1968

\begin{tabular}{|c|c|c|c|c|}
\hline $\begin{array}{l}\text { Oct. } \\
\text { Nov. }\end{array}$ & $\begin{array}{l}31 \ldots \ldots \ldots \ldots \ldots \ldots \ldots \ldots \ldots .6 .15 \\
30 \ldots \ldots \ldots \ldots \ldots \ldots \ldots \ldots \\
31 \ldots \ldots \ldots \ldots \ldots \ldots\end{array}$ & $\begin{array}{l}\text { Feb. } \\
\text { Mar. } \\
\text { Apr. } \\
\text { May }\end{array}$ & $\begin{array}{l}29 \ldots \ldots \ldots \ldots \ldots \\
31 \\
30 \ldots \ldots \ldots \ldots \ldots \ldots\end{array}$ & $\begin{array}{ll}\text { June } & 30 \ldots \ldots \ldots \ldots \ldots \ldots \ldots \ldots .7 .20 \\
\text { July } & 31 \ldots \ldots \ldots \ldots \ldots \ldots \ldots \ldots .7 .20 \\
\text { Aug. } & 31 \ldots \ldots \ldots \ldots \ldots \ldots \ldots \ldots .3 .32 \\
\text { Sept. } & 30 \ldots \ldots \ldots \ldots \ldots \ldots \ldots .16\end{array}$ \\
\hline
\end{tabular}

Note.--Gage-height record other than that shown above is available. 
5-2920. Minnesota River at ortonville, Minn.

Location.--Lat $45^{\circ} 17^{\prime} 44^{\prime \prime}$, long $96^{\circ} 26^{\prime} 38^{\prime \prime}$, in NE $\frac{1}{4} \mathrm{NW}^{\frac{1}{4}}$ sec.16, T.121 N., R.46 W., on left bank $400 \mathrm{ft}$ downstream from bridge on U.S. Highway 12 and $1,300 \mathrm{ft}$ downstream from dam at outlet of Big stone Lake, at ortonville.

Drainage area.--1, $160 \mathrm{sq} \mathrm{mi}$, approximately.

Records available.--February 1938 to september 1968.

Gage.--Water-stage recorder. Datum of gage is $956.38 \mathrm{ft}$ above mean sea level, datum of 1929. Prior to Mar. 31 , 1939, staff gage on downstream side of dam 1,300 ft upstream at datum $1.31 \mathrm{ft}$ higher.

Average discharge.--30 years, 111 cfs $(80,360$ acre-ft per year).

Extremes.--Maximum discharge during year, 248 cfs Apr. 23 (gage height, $4.91 \mathrm{ft}$ ); minimum, 1.2 cfs Sept. 27 (gage height, $1.14 \mathrm{ft}$ ).

1938-68: Maximum discharge, 3,060 cfs Apr. 13, 1952 (gage height, $12.92 \mathrm{ft}$ ); no flow Dec. $13,1940$.

Remarks.--Records fair. Flow affected by natural storage in Big stone Lake (see preceding page). Records of chemical analyses for the water year 1968 are published in Part 2 of this report.

OISCHARGE, IN CUBIC FEET PER SECOND, WATER YEAR OCTOBER 19G7 TO SEPTFMBER 1968

\begin{tabular}{|c|c|c|c|c|c|c|c|c|c|c|c|c|}
\hline DAY & $O C T$ & vov & DEC & JAN & FEB & MAR & $A D R$ & MAY & JUN & JUt & AUG & SEP \\
\hline $\begin{array}{l}1 \\
2 \\
3 \\
4 \\
5\end{array}$ & $\begin{array}{l}13 \\
15 \\
13 \\
13 \\
12\end{array}$ & $\begin{array}{l}4.6 \\
4.6 \\
4.5 \\
4.2 \\
4.4\end{array}$ & $\begin{array}{l}5.5 \\
5.5 \\
5.6 \\
5.6 \\
5.6\end{array}$ & $\begin{array}{l}3.3 \\
3.2 \\
3.2 \\
3.1 \\
3.1\end{array}$ & $\begin{array}{l}3.0 \\
3.0 \\
3.0 \\
3.0 \\
3.0\end{array}$ & $\begin{array}{l}2.4 \\
3.6 \\
3.8 \\
4.0 \\
4.2\end{array}$ & $\begin{array}{c}11 \\
8.8 \\
15 \\
28 \\
9.4\end{array}$ & $\begin{array}{l}12 \\
13 \\
20 \\
15 \\
12\end{array}$ & $\begin{array}{l}35 \\
28 \\
27 \\
25 \\
23\end{array}$ & $\begin{array}{l}42 \\
26 \\
25 \\
25 \\
25\end{array}$ & $\begin{array}{l}26 \\
26 \\
27 \\
26 \\
26\end{array}$ & $\begin{array}{l}14 \\
13 \\
14 \\
14 \\
13\end{array}$ \\
\hline $\begin{array}{r}6 \\
7 \\
9 \\
3 \\
10\end{array}$ & $\begin{array}{l}10 \\
12 \\
11 \\
10 \\
9.1\end{array}$ & $\begin{array}{l}4.5 \\
4.3 \\
5.2 \\
4.9 \\
5.1\end{array}$ & $\begin{array}{l}5.5 \\
5.5 \\
5.5 \\
5.5 \\
5.5\end{array}$ & $\begin{array}{l}3.0 \\
3.0 \\
3.0 \\
3.0 \\
3.0\end{array}$ & $\begin{array}{l}3.0 \\
3.0 \\
3.0 \\
3.0 \\
3.0\end{array}$ & $\begin{array}{l}4.6 \\
4.7 \\
4.9 \\
5.1 \\
5.2\end{array}$ & $\begin{array}{l}11 \\
18 \\
29 \\
14 \\
12\end{array}$ & $\begin{array}{l}11 \\
14 \\
25 \\
20 \\
17\end{array}$ & $\begin{array}{l}23 \\
23 \\
25 \\
23 \\
23\end{array}$ & $\begin{array}{l}25 \\
25 \\
27 \\
25 \\
24\end{array}$ & $\begin{array}{l}25 \\
25 \\
24 \\
23 \\
23\end{array}$ & $\begin{array}{l}13 \\
13 \\
20 \\
18 \\
12\end{array}$ \\
\hline $\begin{array}{l}11 \\
12 \\
13 \\
14 \\
15\end{array}$ & $\begin{array}{l}8.5 \\
8.4 \\
8.4 \\
7.4 \\
7.9\end{array}$ & $\begin{array}{l}6.1 \\
5.7 \\
5.4 \\
5.4 \\
5.4\end{array}$ & $\begin{array}{l}5.5 \\
5.5 \\
5.5 \\
5.4 \\
5.3\end{array}$ & $\begin{array}{l}3.0 \\
3.0 \\
3.0 \\
3.0 \\
3.0\end{array}$ & $\begin{array}{l}3.0 \\
3.0 \\
3.0 \\
3.0 \\
3.0\end{array}$ & $\begin{array}{l}5.2 \\
5.1 \\
5.1 \\
5.6 \\
5.7\end{array}$ & $\begin{array}{l}12 \\
17 \\
14 \\
15 \\
13\end{array}$ & $\begin{array}{l}19 \\
19 \\
21 \\
30 \\
30\end{array}$ & $\begin{array}{l}31 \\
24 \\
26 \\
27 \\
21\end{array}$ & $\begin{array}{l}23 \\
22 \\
22 \\
22 \\
22\end{array}$ & $\begin{array}{l}22 \\
23 \\
24 \\
21 \\
21\end{array}$ & $\begin{array}{l}9.9 \\
7.7 \\
8.6 \\
6.4 \\
3.5\end{array}$ \\
\hline $\begin{array}{l}16 \\
17 \\
18 \\
19 \\
20\end{array}$ & $\begin{array}{l}7.6 \\
8.2 \\
6.6 \\
6.4 \\
7.5\end{array}$ & $\begin{array}{l}5.8 \\
6.5 \\
4 \cdot 7 \\
4 \cdot 3 \\
4 \cdot 4\end{array}$ & $\begin{array}{l}5 . C \\
5 . C \\
5.1 \\
5.1 \\
5.0\end{array}$ & $\begin{array}{l}3.0 \\
3.0 \\
3.0 \\
3.0 \\
3.0\end{array}$ & $\begin{array}{l}3.0 \\
3.0 \\
3.0 \\
3.0 \\
3.0\end{array}$ & $\begin{array}{l}6.1 \\
6.5 \\
7.0 \\
7.6 \\
8.5\end{array}$ & $\begin{array}{l}15 \\
15 \\
13 \\
11 \\
16\end{array}$ & $\begin{array}{l}69 \\
31 \\
47 \\
49 \\
31\end{array}$ & $\begin{array}{l}23 \\
23 \\
23 \\
22 \\
22\end{array}$ & $\begin{array}{l}23 \\
26 \\
26 \\
25 \\
23\end{array}$ & $\begin{array}{l}24 \\
26 \\
25 \\
23 \\
18\end{array}$ & $\begin{array}{l}5.4 \\
6.4 \\
7.0 \\
5.1 \\
4.8\end{array}$ \\
\hline $\begin{array}{l}21 \\
22 \\
23 \\
24 \\
25\end{array}$ & $\begin{array}{l}5.6 \\
6.4 \\
6.9 \\
7.8 \\
6.0\end{array}$ & $\begin{array}{l}5.4 \\
5.1 \\
5.1 \\
5.2 \\
5.2\end{array}$ & $\begin{array}{l}4.7 \\
4.5 \\
4.2 \\
4.2 \\
4.2\end{array}$ & $\begin{array}{l}3.0 \\
3.0 \\
3.0 \\
3.0 \\
3.0\end{array}$ & $\begin{array}{l}3.0 \\
3.0 \\
3.0 \\
3.0 \\
3.1\end{array}$ & $\begin{array}{l}9.1 \\
9.8 \\
10 \\
11 \\
10\end{array}$ & $\begin{array}{l}16 \\
31 \\
92 \\
22 \\
13\end{array}$ & $\begin{array}{l}30 \\
29 \\
30 \\
30 \\
31\end{array}$ & $\begin{array}{l}27 \\
21 \\
22 \\
21 \\
22\end{array}$ & $\begin{array}{l}27 \\
25 \\
25 \\
25 \\
25\end{array}$ & $\begin{array}{l}18 \\
19 \\
18 \\
25 \\
14\end{array}$ & $\begin{array}{l}3.8 \\
4.8 \\
4.4 \\
5.6 \\
3.4\end{array}$ \\
\hline $\begin{array}{l}26 \\
27 \\
28 \\
29 \\
30 \\
31\end{array}$ & $\begin{array}{l}5.0 \\
5.2 \\
3.8 \\
4.5 \\
4.4 \\
4.4\end{array}$ & $\begin{array}{r}5.4 \\
5.5 \\
5.5 \\
5.5 \\
5.5 \\
\end{array}$ & $\begin{array}{l}4.0 \\
3.8 \\
3.6 \\
3.6 \\
3.6 \\
3.4\end{array}$ & $\begin{array}{l}3.0 \\
3.0 \\
3.0 \\
3.0 \\
3.0 \\
3.0\end{array}$ & $\begin{array}{r}3.2 \\
3.2 \\
3.2 \\
3.3 \\
- \\
\end{array}$ & $\begin{array}{l}10 \\
11 \\
11 \\
11 \\
13 \\
19\end{array}$ & $\begin{array}{r}13 \\
14 \\
14 \\
14 \\
13 \\
-\end{array}$ & $\begin{array}{l}32 \\
34 \\
35 \\
32 \\
31 \\
30\end{array}$ & $\begin{array}{r}22 \\
22 \\
21 \\
25 \\
32 \\
- \\
\end{array}$ & $\begin{array}{l}28 \\
27 \\
25 \\
24 \\
25 \\
27\end{array}$ & $\begin{array}{l}13 \\
12 \\
12 \\
12 \\
13 \\
14\end{array}$ & $\begin{array}{r}2.2 \\
3.1 \\
5.9 \\
5.8 \\
5.3 \\
\end{array}$ \\
\hline $\begin{array}{l}\text { TOTAL } \\
\text { MEAN } \\
\text { MAX } \\
\text { MIN } \\
\text { AC-FT }\end{array}$ & $\begin{array}{r}255.0 \\
8.23 \\
15 \\
3.8 \\
506 \\
\end{array}$ & $\begin{array}{r}153.4 \\
5.11 \\
6.5 \\
4.2 \\
304 \\
\end{array}$ & $\begin{array}{r}151.5 \\
4.89 \\
5.6 \\
3.4 \\
301 \\
\end{array}$ & $\begin{array}{r}93.9 \\
3.03 \\
3.3 \\
3.0 \\
186 \\
\end{array}$ & $\begin{array}{r}88.0 \\
3.03 \\
3.3 \\
3.0 \\
175 \\
\end{array}$ & $\begin{array}{r}230.8 \\
7.45 \\
19 \\
3.4 \\
458 \\
\end{array}$ & $\begin{array}{r}539.2 \\
18.0 \\
92 \\
8.8 \\
1.070 \\
\end{array}$ & $\begin{array}{r}858 \\
27.7 \\
69 \\
11 \\
1.700 \\
\end{array}$ & $\begin{array}{r}732 \\
24.4 \\
35 \\
21 \\
1.450 \\
\end{array}$ & $\begin{array}{r}786 \\
25.4 \\
42 \\
22 \\
1.560 \\
\end{array}$ & $\begin{array}{r}648 \\
20.9 \\
27 \\
12 \\
1.290 \\
\end{array}$ & $\begin{array}{r}253.1 \\
8.44 \\
20 \\
2.2 \\
502 \\
\end{array}$ \\
\hline $\begin{array}{ll}\text { CAL } & Y R \\
W T R & Y R\end{array}$ & $\begin{array}{l}1967 \\
1968\end{array}$ & $\begin{array}{r}26.89 \\
4.78\end{array}$ & & $\begin{array}{l}73.7 \\
13.1\end{array}$ & $\begin{array}{rr}\text { MAX } & 639 \\
\text { MAX } & 92\end{array}$ & & $\begin{array}{ll}N & 3.4 \\
N & 2.2\end{array}$ & $\begin{array}{l}A C-F T \\
A C-F T\end{array}$ & $\begin{array}{r}53,340 \\
9.500\end{array}$ & & & \\
\hline
\end{tabular}


5-2930. Yellow Bank River near Odessa, Minn.

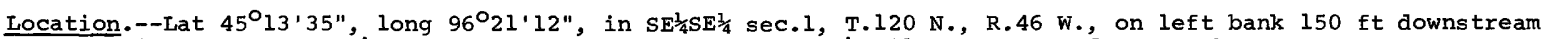
from highway bridge, $2 \frac{1}{2}$ miles southwest of Odessa, and $4 \frac{1}{2}$ miles upstream from mouth.

Drainage area. $--398 \mathrm{sq} \mathrm{mi}$.

Records available.--October 1939 to september 1968.

Gage.--Water-stage recorder. Datum of gage is $953.34 \mathrm{ft}$ above mean sea level, datum of 1929 (Corps of Engineers bench mark). Prior to Aug. 28, 1940, wire-weight gage at site 150 ft upstream at same datum.

Average discharge.--29 years, 54.6 cfs $(39,530$ acre-ft per year).

Extremes.--Maximum discharge during year, 211 cfs July 26 (gage height, 3.78 ft); minimum, 0.3 cfs Jan. $6-25$; minimum gage height, $1.70 \mathrm{ft} \mathrm{Aug.} \mathrm{28,} \mathrm{Sept.} \mathrm{6,} 7$.

1939-68: Maximum discharge, 6, 260 cfs Apr. 4, I952 (gage height, 17.06 ft); maximum gage height, 17.98

ft Mar. 25, 1943, from floodmark (backwater from ice); no flow Jan. 26 to Feb. 8, 1940, Jan. 8, 9, 1942,

Jan. 25 to Feb. 25, 1959, Feb. 11 to Mar. 9, 1965.

Remarks.--Records good except those for period of no gage-height record and those for winter months, which are fair.

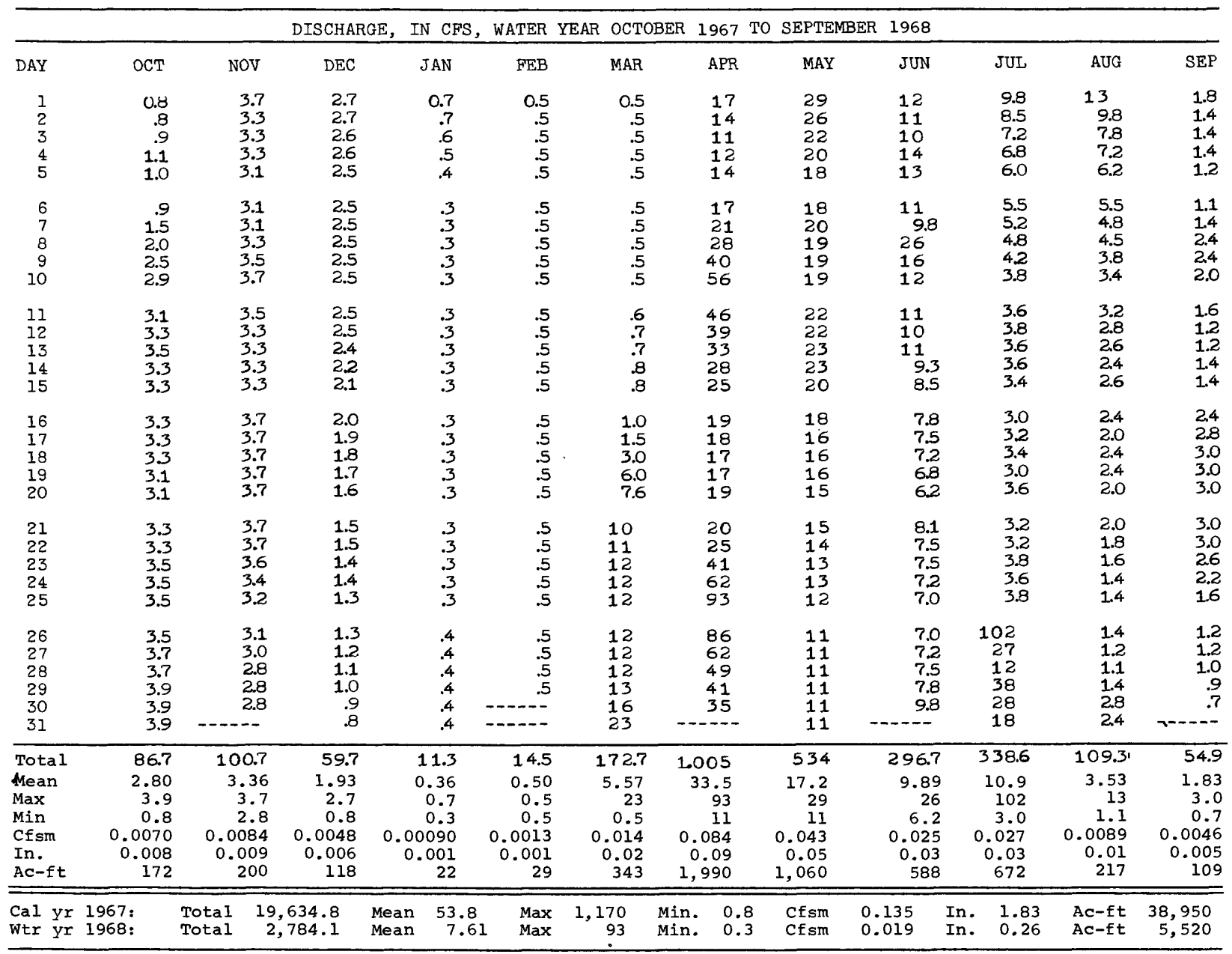

Peak discharge (base, $300 \mathrm{cfs}$ ).--No peak above base. 
5-2940. Pomme de Terre River at Appleton, Minn.

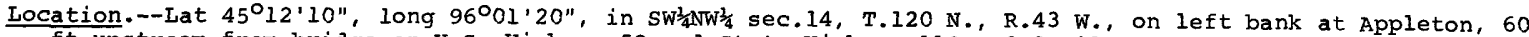
ft upstream from bridge on U.S. Highway 59 and state Highway 119 and 8 miles upstream from mouth.

Drainage area. $--905 \mathrm{sq} \mathrm{mi}$, approximately.

Records available.--March 1931 to september 1935 (no winter records), October 1935 to September 1968. Prior to October 1953, published as "near Appleton".

Gage.--Water-stage recorder and concrete control. Datum of gage is $978.00 \mathrm{ft}$ above mean sea level, datum of 1929. Prior to Dec. 22, 1952, staff gage at site 4 miles upstream at datum 25.17 ft higher.

Average discharge.--33 years $(1935-68), 97.8$ cfs $(70,800$ acre-ft per year).

Extremes.--Maximum discharge during year, 193 cfs June 21 (gage height, 5.40 ft); minimum daily, 2.8 cfs Jan. 11-17, minimum gage height, 3.75 ft Jan. 15.

1931-68: Maximum discharge, 5,050 cfs Apr. 8, 1952 (gage height, 10.13 ft, site and datum then in use) no flow for several periods.

Remarks.--Records good except those for winter months, which are fair. Flow affected by lakes above station. occasional regulation at low flow by old milldam $500 \mathrm{ft}$ upstream.

DISCHARGE, IN CFS, WATER YEAR OCTOBER 1967 TO SEPTEMBER 1968

\begin{tabular}{|c|c|c|c|c|c|c|c|c|c|c|c|c|}
\hline DAY & OCT & NOV & DEC & JAN & FEB & MAR & APR & MAY & JUN & JUL & AUG & SEP \\
\hline $\begin{array}{l}1 \\
2 \\
3 \\
4 \\
5\end{array}$ & $\begin{array}{l}21 \\
20 \\
19 \\
20 \\
20\end{array}$ & $\begin{array}{l}25 \\
24 \\
24 \\
24 \\
21\end{array}$ & $\begin{array}{l}18 \\
18 \\
18 \\
18 \\
17\end{array}$ & $\begin{array}{l}4.0 \\
3.8 \\
3.5 \\
3.2 \\
3.1\end{array}$ & $\begin{array}{l}7.0 \\
7.4 \\
8.0 \\
8.4 \\
8.6\end{array}$ & $\begin{array}{c}8.6 \\
8.8 \\
9.2 \\
10 \\
12\end{array}$ & $\begin{array}{l}83 \\
80 \\
80 \\
83 \\
93\end{array}$ & $\begin{array}{l}96 \\
87 \\
76 \\
73 \\
71\end{array}$ & $\begin{array}{l}62 \\
73 \\
73 \\
68 \\
65\end{array}$ & $\begin{array}{l}55 \\
58 \\
60 \\
58 \\
52\end{array}$ & $\begin{array}{l}20 \\
18 \\
18 \\
18 \\
16\end{array}$ & $\begin{array}{l}11 \\
12 \\
12 \\
12 \\
11\end{array}$ \\
\hline $\begin{array}{r}6 \\
7 \\
8 \\
9 \\
10\end{array}$ & $\begin{array}{l}19 \\
23 \\
23 \\
28 \\
28\end{array}$ & $\begin{array}{l}20 \\
19 \\
20 \\
25 \\
25\end{array}$ & $\begin{array}{l}17 \\
17 \\
17 \\
17 \\
16\end{array}$ & $\begin{array}{l}3.0 \\
3.0 \\
3.0 \\
2.9 \\
2.9\end{array}$ & $\begin{array}{l}8.9 \\
8.9 \\
8.9 \\
8.8 \\
8.8\end{array}$ & $\begin{array}{l}14 \\
20 \\
30 \\
29 \\
28\end{array}$ & $\begin{array}{r}91 \\
83 \\
87 \\
100 \\
102\end{array}$ & $\begin{array}{l}71 \\
70 \\
68 \\
70 \\
75\end{array}$ & $\begin{array}{l}62 \\
62 \\
64 \\
71 \\
73\end{array}$ & $\begin{array}{l}48 \\
46 \\
43 \\
41 \\
40\end{array}$ & $\begin{array}{l}21 \\
18 \\
16 \\
15 \\
14\end{array}$ & $\begin{array}{l}9.9 \\
11 \\
20 \\
24 \\
20\end{array}$ \\
\hline $\begin{array}{l}11 \\
12 \\
13 \\
14 \\
15\end{array}$ & $\begin{array}{l}28 \\
27 \\
27 \\
26 \\
25\end{array}$ & $\begin{array}{l}24 \\
25 \\
24 \\
26 \\
24\end{array}$ & $\begin{array}{l}16 \\
16 \\
15 \\
15 \\
14\end{array}$ & $\begin{array}{l}2.9 \\
2.8 \\
2.8 \\
2.8 \\
2.8\end{array}$ & $\begin{array}{l}8.6 \\
8.6 \\
8.6 \\
8.6 \\
8.4\end{array}$ & $\begin{array}{l}26 \\
26 \\
26 \\
27 \\
28\end{array}$ & $\begin{array}{l}91 \\
89 \\
91 \\
93 \\
93\end{array}$ & $\begin{array}{l}80 \\
80 \\
76 \\
75 \\
78\end{array}$ & $\begin{array}{l}67 \\
67 \\
65 \\
67 \\
68\end{array}$ & $\begin{array}{l}40 \\
39 \\
36 \\
34 \\
33\end{array}$ & $\begin{array}{l}13 \\
12 \\
12 \\
12 \\
14\end{array}$ & $\begin{array}{c}18 \\
14 \\
12 \\
10 \\
9.3\end{array}$ \\
\hline $\begin{array}{l}16 \\
17 \\
18 \\
19 \\
20\end{array}$ & $\begin{array}{l}24 \\
23 \\
24 \\
22 \\
24\end{array}$ & $\begin{array}{l}23 \\
22 \\
24 \\
23 \\
25\end{array}$ & $\begin{array}{l}14 \\
13 \\
12 \\
12 \\
12\end{array}$ & $\begin{array}{l}2.8 \\
2.8 \\
2.9 \\
3.0 \\
3.1\end{array}$ & $\begin{array}{l}8.4 \\
8.4 \\
8.2 \\
8.2 \\
8.2\end{array}$ & $\begin{array}{l}29 \\
30 \\
35 \\
50 \\
40\end{array}$ & $\begin{array}{r}91 \\
83 \\
82 \\
83 \\
100\end{array}$ & $\begin{array}{l}76 \\
83 \\
85 \\
78 \\
76\end{array}$ & $\begin{array}{l}67 \\
65 \\
67 \\
67 \\
65\end{array}$ & $\begin{array}{l}32 \\
35 \\
37 \\
33 \\
31\end{array}$ & $\begin{array}{l}13 \\
14 \\
17 \\
18 \\
18\end{array}$ & $\begin{array}{l}12 \\
13 \\
14 \\
15 \\
16\end{array}$ \\
\hline $\begin{array}{l}21 \\
22 \\
23 \\
24 \\
25\end{array}$ & $\begin{array}{l}26 \\
25 \\
26 \\
25 \\
25\end{array}$ & $\begin{array}{l}27 \\
23 \\
21 \\
24 \\
19\end{array}$ & $\begin{array}{l}11 \\
10 \\
9.0 \\
9.0 \\
9.0\end{array}$ & $\begin{array}{l}3.3 \\
3.7 \\
4.1 \\
4.1 \\
4.2\end{array}$ & $\begin{array}{l}8.0 \\
8.0 \\
8.0 \\
8.0 \\
8.2\end{array}$ & $\begin{array}{r}35 \\
50 \\
80 \\
90 \\
120\end{array}$ & $\begin{array}{l}114 \\
131 \\
148 \\
173 \\
148\end{array}$ & $\begin{array}{l}73 \\
68 \\
65 \\
64 \\
61\end{array}$ & $\begin{array}{l}93 \\
70 \\
70 \\
70 \\
68\end{array}$ & $\begin{array}{l}29 \\
28 \\
28 \\
28 \\
28\end{array}$ & $\begin{array}{l}16 \\
16 \\
14 \\
11 \\
13\end{array}$ & $\begin{array}{l}18 \\
18 \\
20 \\
25 \\
22\end{array}$ \\
\hline $\begin{array}{l}26 \\
27 \\
28 \\
29 \\
30 \\
31\end{array}$ & $\begin{array}{l}24 \\
24 \\
24 \\
27 \\
26 \\
25\end{array}$ & $\begin{array}{r}16 \\
16 \\
18 \\
18 \\
18 \\
\end{array}$ & $\begin{array}{l}8.0 \\
7.2 \\
6.5 \\
6.0 \\
5.2 \\
4.5\end{array}$ & $\begin{array}{l}4.5 \\
5.0 \\
6.0 \\
6.0 \\
6.0 \\
6.4\end{array}$ & $\begin{array}{r}8.2 \\
8.4 \\
8.5 \\
8.5 \\
\\
--.--\end{array}$ & $\begin{array}{r}105 \\
99 \\
98 \\
93 \\
87 \\
87\end{array}$ & $\begin{array}{r}126 \\
114 \\
111 \\
111 \\
104 \\
-\end{array}$ & $\begin{array}{r}60 \\
58 \\
60 \\
60 \\
60 \\
64\end{array}$ & $\begin{array}{r}70 \\
67 \\
62 \\
60 \\
58 \\
-\end{array}$ & $\begin{array}{l}28 \\
19 \\
24 \\
24 \\
22 \\
21\end{array}$ & $\begin{array}{l}12 \\
11 \\
10 \\
9.6 \\
13 \\
12\end{array}$ & $\begin{array}{r}20 \\
17 \\
15 \\
14 \\
14 \\
\end{array}$ \\
\hline $\begin{array}{l}\text { Total } \\
\text { Mean } \\
\text { Max } \\
\text { Min } \\
\text { Cfsm } \\
\text { In. } \\
\text { Ac-ft }\end{array}$ & $\begin{array}{r}748 \\
24.1 \\
28 \\
19 \\
0.027 \\
0.03 \\
1,480\end{array}$ & $\begin{array}{r}667 \\
22.2 \\
27 \\
16 \\
0.024 \\
0.03 \\
1,320\end{array}$ & $\begin{array}{r}397.4 \\
12.8 \\
18 \\
4.5 \\
0.014 \\
0.02 \\
788\end{array}$ & $\begin{array}{r}114.4 \\
3.69 \\
6.4 \\
2.8 \\
0.0041 \\
0.005 \\
227\end{array}$ & $\begin{array}{r}241.7 \\
8.33 \\
8.9 \\
7.0 \\
0.0092 \\
0.001 \\
479\end{array}$ & $\begin{array}{r}1.430 .6 \\
46.1 \\
120 \\
8.6 \\
0.051 \\
0.06 \\
2.840\end{array}$ & $\begin{array}{r}3.058 \\
102 \\
173 \\
80 \\
0.113 \\
0.12 \\
6.060\end{array}$ & $\begin{array}{r}2.237 \\
72.2 \\
96 \\
58 \\
0.080 \\
0.09 \\
4.440\end{array}$ & $\begin{array}{r}2.026 \\
67.5 \\
93 \\
58 \\
0.075 \\
0.08 \\
4,020\end{array}$ & $\begin{array}{r}1.130 \\
36.4 \\
60 \\
19 \\
0.040 \\
0.05 \\
2,240\end{array}$ & $\begin{array}{r}454.6 \\
14.7 \\
21 \\
9.6 \\
0.016 \\
0.02 \\
902\end{array}$ & $\begin{array}{r}459.2 \\
15.3 \\
25 \\
9.3 \\
0.017 \\
0.02 \\
911\end{array}$ \\
\hline $\begin{array}{l}\text { Cal yr } \\
\text { wtr yr }\end{array}$ & $\begin{array}{l}967: \\
968:\end{array}$ & $\begin{array}{l}\text { otal } \\
\text { otal }\end{array}$ & $\begin{array}{l}6,069.4 \\
2,963.9\end{array}$ & $\begin{array}{l}\text { lean } \\
\text { lean }\end{array}$ & 4. $\begin{array}{r}\operatorname{Max} \\
\operatorname{Max}\end{array}$ & $\begin{array}{r}1,010 \\
173\end{array}$ & Min & $\begin{array}{l}\text { Cfs } \\
\text { Cfs }\end{array}$ & $\begin{array}{l}0.139 \\
0.039\end{array}$ & $\begin{array}{ll}\text { In. } & 1.89 \\
\text { In. } & 0.53\end{array}$ & $\begin{array}{l}\text { Ac-ft } \\
\text { Ac-ft }\end{array}$ & $\begin{array}{l}91,340 \\
25,710\end{array}$ \\
\hline
\end{tabular}

Note.--Peak discharge (base, $200 \mathrm{cfs}$ ). No peak above base. 
Location.--Lat $45^{\circ} 00^{\prime}$, long $95^{\circ} 55^{\prime}$, in SW $\frac{1}{4} S w^{\frac{1}{4}}$ sec. 27 , T.118 N., R.42 W., on right bank 40 ft downstream from highway bridge and half a mile southwest of village of Lac qui Parle.

Drainage area.--983 sq $\mathrm{mi}$.

Records available.--April 1910 to November 1914; March 1931 to September 1968 (winter records incomplete prior to 1934). Published as "at Lac qui Parle", 1910-14.

Gage.--Water-stage recorder. Datum of gage is $951.98 \mathrm{ft}$ above mean sea level (Minnesota Highway Department bench mark). Apr. 27, 1910, to Nov. 15, 1914, staff gage at site 2 miles downstream at different datum. Mar. 17, 1931, to Mar. 9, 1937, staff gage at site $40 \mathrm{ft}$ upstream at present datum.

Average discharge.--37 years (1912-13, 1931-32, 1933-68), 113 cfs (81,810 acre-ft per year).

Extremes.--Maximum discharge during year, 241 cfs July 28 (gage height, 2.21 ft); no flow Jan. 3 to Mar. 2 . 1910-14, 1931-68: Maximum discharge, 11,100 cfs Apr. 6, 1952 (gage height, 18.18 ft); maximum gage

height, $19.37 \mathrm{ft}$ Apr. 9, 1965, from floodmark, backwater from ice; no flow at times in several years.

Remarks.--Records fair.

DISCHARGE, IN CFS, WATER YEAR OCTOBER 1967 TO SEPTEMBER 1968

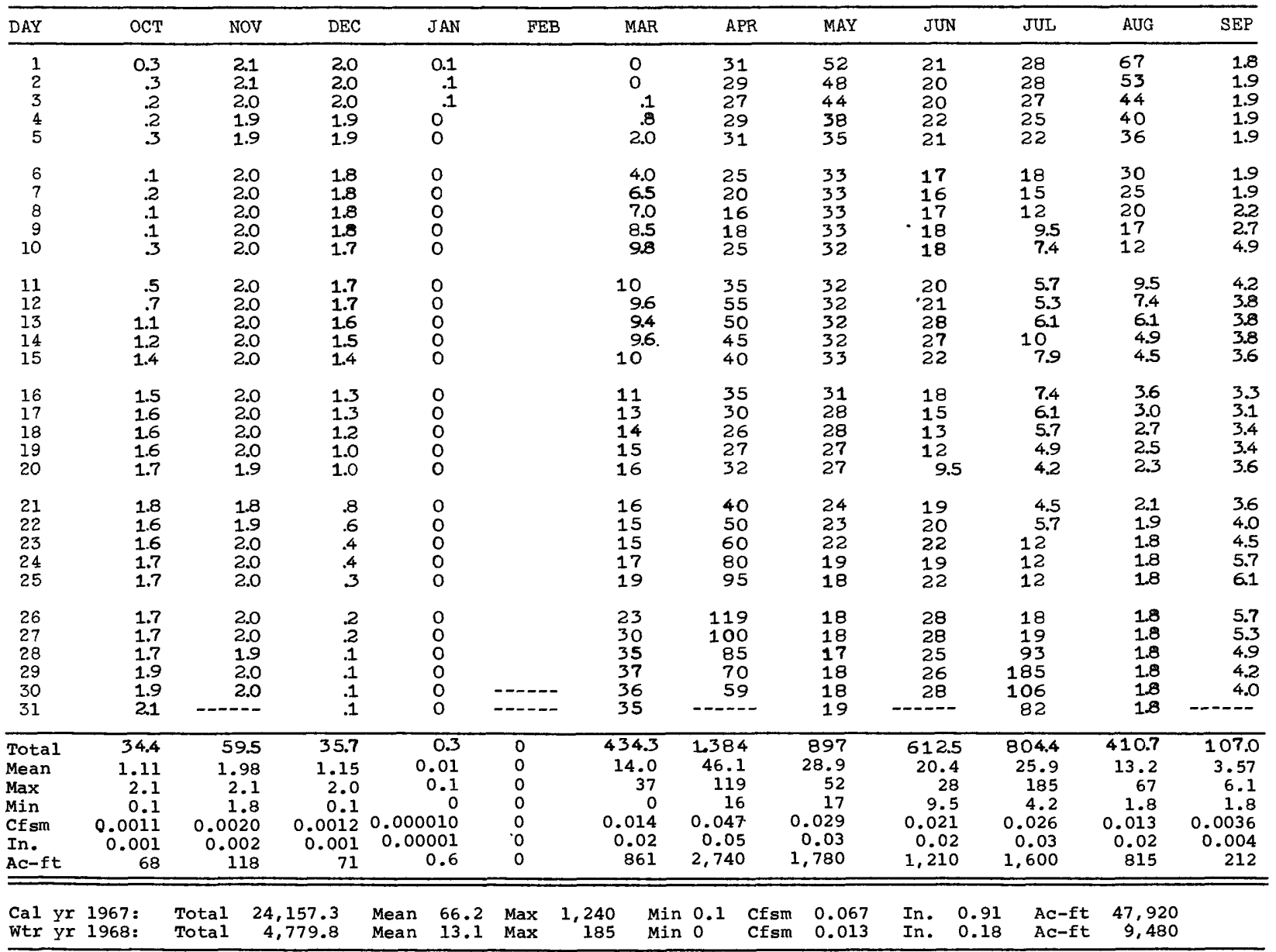


5-3010. Minnesota River near Lac qui Parle, Minn.

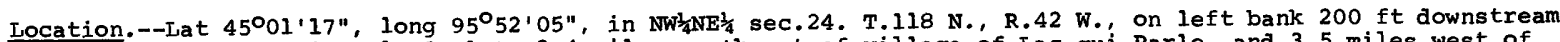
from dam at Lac qui Parle outlet, 2.4 miles northeast of village of Lac qui Parle, and 3.5 miles west of Watson.

Drainage area. $-4,050 \mathrm{sq} \mathrm{mi}$, approximately.

Records available.--October 1942 to September 1968.

Gage.--Digital water-stage recorder. Datum of gage is $900.00 \mathrm{ft}$ above mean sea level, datum of 1929 (levels by Corps of Engineers). Oct. 1, 1942 to Nov. 10, 1944, graphic water-stage recorder at same site at datum $0.20 \mathrm{ft}$ lower. Nov. 11,1944 to Aug. 4, 1967, graphic water-stage recorder at same site and datum.

Average discharge.--26 years, 616 cfs $(446,000$ acre-ft per year).

Extremes:--Maximum discharge during year, $590 \mathrm{cfs}$ Apr. 18 (gage height, $23.06 \mathrm{ft}$ ); minimum daily, $3.7 \mathrm{cfs}$ oct. 3 .

1942-68: Maximum discharge, 19,700 cfs Apr. 10, 1952 (gage height,
. 17, 1942, Sept. 29, 1947, Oct. 19 to Nov. 18, 1951, Nov. 24, 1952.

Remarks.--Records good except those for winter months, which are fair. Part of flow from 2,050 square miles of Chippewa River basin at times diverted into Minnesota River above station. Some regulation by Big

stone Lake since Apr. 17, 1927, Lac qui Parle Lake since January 1938, and Marsh Lake since Nov. 1, 1939.

DISCHARGE, IN CUBIC FEET PER SECOND, WATER YEAR OCTOBER 1967 TO SEPTEMBER 1968

\begin{tabular}{|c|c|c|c|c|c|c|c|c|c|c|c|c|}
\hline DAY & OCT & NOV & $D E C$ & JAN & FEB & MAR & APR & MAY & JUN & JUL & AUG & SEP \\
\hline $\begin{array}{l}1 \\
2 \\
3 \\
4 \\
5\end{array}$ & $\begin{array}{c}3.8 \\
3.8 \\
3.7 \\
15 \\
34\end{array}$ & $\begin{array}{l}19 \\
19 \\
19 \\
19 \\
19\end{array}$ & $\begin{array}{l}18 \\
18 \\
18 \\
18 \\
18\end{array}$ & $\begin{array}{l}18 \\
1 B \\
18 \\
18 \\
18\end{array}$ & $\begin{array}{l}17 \\
17 \\
17 \\
16 \\
16\end{array}$ & $\begin{array}{l}16 \\
17 \\
17 \\
17 \\
17\end{array}$ & $\begin{array}{l}145 \\
144 \\
142 \\
142 \\
145\end{array}$ & $\begin{array}{l}331 \\
284 \\
285 \\
284 \\
280\end{array}$ & $\begin{array}{l}131 \\
130 \\
130 \\
129 \\
130\end{array}$ & $\begin{array}{l}296 \\
172 \\
125 \\
124 \\
124\end{array}$ & $\begin{array}{l}52 \\
52 \\
52 \\
52 \\
52\end{array}$ & $\begin{array}{l}24 \\
24 \\
24 \\
24 \\
25\end{array}$ \\
\hline $\begin{array}{r}6 \\
7 \\
8 \\
9 \\
10\end{array}$ & $\begin{array}{l}34 \\
35 \\
35 \\
34 \\
33\end{array}$ & $\begin{array}{l}19 \\
20 \\
20 \\
20 \\
20\end{array}$ & $\begin{array}{l}18 \\
18 \\
18 \\
18 \\
18\end{array}$ & $\begin{array}{l}19 \\
19 \\
19 \\
19 \\
19\end{array}$ & $\begin{array}{l}16 \\
16 \\
16 \\
16 \\
16\end{array}$ & $\begin{array}{l}18 \\
18 \\
18 \\
18 \\
18\end{array}$ & $\begin{array}{l}145 \\
143 \\
143 \\
144 \\
146\end{array}$ & $\begin{array}{l}207 \\
165 \\
164 \\
160 \\
159\end{array}$ & $\begin{array}{l}129 \\
129 \\
129 \\
129 \\
129\end{array}$ & $\begin{array}{r}122 \\
122 \\
119 \\
119 \\
81\end{array}$ & $\begin{array}{l}52 \\
52 \\
52 \\
52 \\
52\end{array}$ & $\begin{array}{l}25 \\
25 \\
25 \\
25 \\
23\end{array}$ \\
\hline $\begin{array}{l}11 \\
12 \\
13 \\
14 \\
15\end{array}$ & $\begin{array}{l}32 \\
32 \\
31 \\
30 \\
29\end{array}$ & $\begin{array}{l}20 \\
20 \\
20 \\
20 \\
20\end{array}$ & $\begin{array}{l}18 \\
18 \\
18 \\
18 \\
18\end{array}$ & $\begin{array}{l}19 \\
19 \\
19 \\
19 \\
19\end{array}$ & $\begin{array}{l}16 \\
16 \\
16 \\
16 \\
16\end{array}$ & $\begin{array}{l}18 \\
18 \\
18 \\
18 \\
18\end{array}$ & $\begin{array}{l}147 \\
147 \\
150 \\
150 \\
152\end{array}$ & $\begin{array}{l}158 \\
156 \\
155 \\
154 \\
149\end{array}$ & $\begin{array}{l}129 \\
129 \\
129 \\
129 \\
129\end{array}$ & $\begin{array}{l}57 \\
56 \\
56 \\
55 \\
55\end{array}$ & $\begin{array}{l}52 \\
53 \\
53 \\
53 \\
53\end{array}$ & $\begin{array}{l}24 \\
24 \\
23 \\
24 \\
24\end{array}$ \\
\hline $\begin{array}{l}16 \\
17 \\
18 \\
19 \\
20\end{array}$ & $\begin{array}{l}29 \\
29 \\
28 \\
27 \\
27\end{array}$ & $\begin{array}{l}20 \\
20 \\
19 \\
19 \\
19\end{array}$ & $\begin{array}{l}18 \\
18 \\
18 \\
18 \\
18\end{array}$ & $\begin{array}{l}19 \\
19 \\
19 \\
19 \\
19\end{array}$ & $\begin{array}{l}16 \\
16 \\
16 \\
16 \\
16\end{array}$ & $\begin{array}{r}19 \\
19 \\
19 \\
90 \\
300\end{array}$ & $\begin{array}{l}233 \\
497 \\
462 \\
273 \\
362\end{array}$ & $\begin{array}{l}147 \\
146 \\
144 \\
143 \\
141\end{array}$ & $\begin{array}{l}130 \\
130 \\
129 \\
130 \\
130\end{array}$ & $\begin{array}{l}53 \\
53 \\
52 \\
52 \\
52\end{array}$ & $\begin{array}{l}53 \\
52 \\
53 \\
53 \\
53\end{array}$ & $\begin{array}{l}24 \\
24 \\
24 \\
24 \\
24\end{array}$ \\
\hline $\begin{array}{l}21 \\
22 \\
23 \\
24 \\
25\end{array}$ & $\begin{array}{l}26 \\
25 \\
25 \\
24 \\
22\end{array}$ & $\begin{array}{l}19 \\
19 \\
18 \\
18 \\
18\end{array}$ & $\begin{array}{l}18 \\
18 \\
18 \\
18 \\
18\end{array}$ & $\begin{array}{l}19 \\
19 \\
18 \\
18 \\
18\end{array}$ & $\begin{array}{l}16 \\
16 \\
16 \\
16 \\
16\end{array}$ & $\begin{array}{l}260 \\
165 \\
151 \\
150 \\
149\end{array}$ & $\begin{array}{l}514 \\
518 \\
533 \\
532 \\
486\end{array}$ & $\begin{array}{l}140 \\
138 \\
135 \\
134 \\
133\end{array}$ & $\begin{array}{l}132 \\
133 \\
132 \\
132 \\
188\end{array}$ & $\begin{array}{l}51 \\
51 \\
51 \\
51 \\
51\end{array}$ & $\begin{array}{l}53 \\
53 \\
53 \\
53 \\
53\end{array}$ & $\begin{array}{l}24 \\
24 \\
24 \\
24 \\
24\end{array}$ \\
\hline $\begin{array}{l}26 \\
27 \\
28 \\
27 \\
30 \\
31\end{array}$ & $\begin{array}{l}22 \\
21 \\
21 \\
20 \\
19 \\
19\end{array}$ & $\begin{array}{r}18 \\
18 \\
18 \\
18 \\
18 \\
\end{array}$ & $\begin{array}{l}18 \\
18 \\
18 \\
18 \\
18 \\
18\end{array}$ & $\begin{array}{l}18 \\
17 \\
17 \\
17 \\
17 \\
17\end{array}$ & $\begin{array}{r}16 \\
16 \\
16 \\
16 \\
\end{array}$ & $\begin{array}{l}149 \\
149 \\
150 \\
148 \\
147 \\
145\end{array}$ & $\begin{array}{r}384 \\
385 \\
386 \\
387 \\
390 \\
\end{array}$ & $\begin{array}{l}131 \\
129 \\
129 \\
130 \\
130 \\
131\end{array}$ & $\begin{array}{r}299 \\
298 \\
297 \\
295 \\
294 \\
\end{array}$ & $\begin{array}{l}51 \\
51 \\
51 \\
51 \\
52 \\
51\end{array}$ & $\begin{array}{l}53 \\
35 \\
24 \\
24 \\
24 \\
24\end{array}$ & $\begin{array}{r}24 \\
24 \\
24 \\
24 \\
24 \\
\end{array}$ \\
\hline $\begin{array}{l}\text { TOTAL } \\
\text { MEAN } \\
\text { MAX } \\
\text { MIN } \\
\text { AC-FT }\end{array}$ & $\begin{array}{r}769.3 \\
24.8 \\
35 \\
3.7 \\
1.530\end{array}$ & $\begin{array}{r}573 \\
19.1 \\
20 \\
18 \\
1,140\end{array}$ & $\begin{array}{r}558 \\
18.0 \\
18 \\
18 \\
1,110\end{array}$ & $\begin{array}{r}570 \\
18.4 \\
19 \\
17 \\
1.130\end{array}$ & $\begin{array}{r}467 \\
16.1 \\
17 \\
16 \\
926\end{array}$ & $\begin{array}{r}2.474 \\
79.8 \\
300 \\
16 \\
4.910\end{array}$ & $\begin{array}{r}8.547 \\
285 \\
533 \\
142 \\
16.950\end{array}$ & $\begin{array}{r}5,272 \\
170 \\
331 \\
129 \\
10,460\end{array}$ & $\begin{array}{r}4.789 \\
160 \\
299 \\
129 \\
9.500\end{array}$ & $\begin{array}{r}2.507 \\
80.9 \\
296 \\
51 \\
4.970\end{array}$ & $\begin{array}{r}1,497 \\
48.3 \\
53 \\
24 \\
2,970\end{array}$ & $\begin{array}{r}723 \\
24.1 \\
25 \\
23 \\
1.430\end{array}$ \\
\hline
\end{tabular}

CAL YR 1967 TOTAL 151.193 .9 MEAN $414 \quad$.MAX 3.070 MIN 3.7\% AC-FT 299.900

WTR YR 1968 TOTAL 28.746 .3 MEAN 78.5 MAX 533 MIN 3.7 $\quad$ AC-FT 57.020 
5-3045. Chippewa River near Milan, Minn.

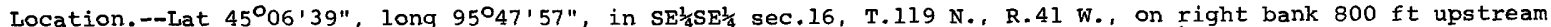
from bridge on State Highway $40,2.0$ miles upstream from small tributary, and $5 \frac{1}{2}$ miles east of Milan.

Drainage area. $--1,870 \mathrm{sq} \mathrm{mi}$, approximately.

Records available.--March 1937 to September 1968.

Gage.-Water-stage recorder. Datum of gage is $959.69 \mathrm{ft}$ above mean sea level, datum of 1929 . Prior to Mar. 23, 1940, chain gage and Mar. 23, 1940, to June 14, 1942, wire-weight gage, on bridge 800 ft downstream at same datum.

Average discharge. --31 years, $242 \operatorname{cfs}(175,200$ acre-ft per year).

Extremes.--Maximum discharge during year, $283 \mathrm{cfs}$ Apr. 26 (gage height, $2.50 \mathrm{ft}$ ); maximum gage height, 3.33 ft Mar. 21 (backwater from ice); minimum daily discharge, 2.2 cfs Jan. 11-18; minimum gage height, 1.22 ft

Aug. 29.

1937-68: Maximum discharge, $6,930 \mathrm{cfs}$ Apr. 9, 1952 (gage height, 12.12 ft); maximum gage height, 12.29

ft Apr. 7, 1952 (backwater from ice); no flow at times during 1940.

Remarks.--Records good except those for winter months, which are fair. Records of chemical analyses for the water year 1968 are published in part 2 of this report. Flow regulated by several small lakes above gage.

DISCHARGE, IN CFS, WATER YEAR OCTOBER 1967 TO SEPTEMBER 1968

\begin{tabular}{|c|c|c|c|c|c|c|c|c|c|c|c|c|}
\hline DAY & $\mathrm{OCT}$ & NOV & $\mathrm{DEC}$ & JAN & FEB & MAR & APR & MAY & JUN & JUL & AUG & SEP \\
\hline $\begin{array}{l}1 \\
2 \\
3 \\
4 \\
5\end{array}$ & $\begin{array}{l}32 \\
30 \\
30 \\
30 \\
29\end{array}$ & $\begin{array}{l}40 \\
41 \\
44 \\
42 \\
31\end{array}$ & $\begin{array}{l}29 \\
28 \\
28 \\
28 \\
28\end{array}$ & $\begin{array}{l}3.5 \\
3.2 \\
3.0 \\
3.0 \\
3.0\end{array}$ & $\begin{array}{l}7.5 \\
9.0 \\
11 \\
14 \\
15\end{array}$ & $\begin{array}{c}10 \\
9.8 \\
9.8 \\
9.6 \\
9.6\end{array}$ & $\begin{array}{l}134 \\
127 \\
127 \\
141 \\
161\end{array}$ & $\begin{array}{l}228 \\
213 \\
190 \\
180 \\
170\end{array}$ & $\begin{array}{l}138 \\
136 \\
141 \\
134 \\
127\end{array}$ & $\begin{array}{l}125 \\
123 \\
119 \\
115 \\
108\end{array}$ & $\begin{array}{l}39 \\
35 \\
34 \\
39 \\
46\end{array}$ & $\begin{array}{l}23 \\
23 \\
24 \\
25 \\
24\end{array}$ \\
\hline $\begin{array}{r}6 \\
7 \\
8 \\
9 \\
10\end{array}$ & $\begin{array}{l}30 \\
32 \\
40 \\
44 \\
49\end{array}$ & $\begin{array}{l}31 \\
31 \\
32 \\
36 \\
35\end{array}$ & $\begin{array}{l}27 \\
27 \\
27 \\
26 \\
26\end{array}$ & $\begin{array}{l}2.9 \\
2.8 \\
2.8 \\
2.5 \\
2.3\end{array}$ & $\begin{array}{l}16 \\
16 \\
16 \\
16 \\
16\end{array}$ & $\begin{array}{l}9.6 \\
10 \\
11 \\
17 \\
25\end{array}$ & $\begin{array}{l}168 \\
183 \\
213 \\
234 \\
225\end{array}$ & $\begin{array}{l}168 \\
168 \\
166 \\
170 \\
161\end{array}$ & $\begin{array}{l}117 \\
117 \\
130 \\
134 \\
138\end{array}$ & $\begin{array}{r}100 \\
91 \\
83 \\
76 \\
71\end{array}$ & $\begin{array}{l}61 \\
55 \\
49 \\
43 \\
38\end{array}$ & $\begin{array}{l}21 \\
21 \\
36 \\
47 \\
49\end{array}$ \\
\hline $\begin{array}{l}11 \\
12 \\
13 \\
14 \\
15\end{array}$ & $\begin{array}{l}47 \\
46 \\
44 \\
44 \\
44\end{array}$ & $\begin{array}{l}34 \\
34 \\
34 \\
34 \\
33\end{array}$ & $\begin{array}{l}26 \\
25 \\
25 \\
24 \\
20\end{array}$ & $\begin{array}{l}2.2 \\
2.2 \\
2.2 \\
2.2 \\
2.2\end{array}$ & $\begin{array}{l}15 \\
15 \\
14 \\
13 \\
12\end{array}$ & $\begin{array}{l}26 \\
30 \\
35 \\
40 \\
42\end{array}$ & $\begin{array}{l}219 \\
210 \\
199 \\
196 \\
193\end{array}$ & $\begin{array}{l}156 \\
159 \\
156 \\
156 \\
154\end{array}$ & $\begin{array}{l}213 \\
202 \\
186 \\
170 \\
193\end{array}$ & $\begin{array}{l}64 \\
61 \\
60 \\
60 \\
60\end{array}$ & $\begin{array}{l}33 \\
30 \\
26 \\
25 \\
26\end{array}$ & $\begin{array}{l}45 \\
38 \\
34 \\
32 \\
30\end{array}$ \\
\hline $\begin{array}{l}16 \\
17 \\
18 \\
19 \\
20\end{array}$ & $\begin{array}{l}41 \\
38 \\
38 \\
38 \\
36\end{array}$ & $\begin{array}{l}33 \\
33 \\
33 \\
31 \\
31\end{array}$ & $\begin{array}{l}19 \\
17 \\
17 \\
16 \\
16\end{array}$ & $\begin{array}{l}2.2 \\
2.2 \\
2.2 \\
2.5 \\
4.0\end{array}$ & $\begin{array}{l}12 \\
12 \\
12 \\
11 \\
11\end{array}$ & $\begin{array}{r}44 \\
48 \\
65 \\
90 \\
110\end{array}$ & $\begin{array}{l}188 \\
178 \\
166 \\
161 \\
180\end{array}$ & $\begin{array}{l}150 \\
145 \\
147 \\
147 \\
145\end{array}$ & $\begin{array}{l}234 \\
237 \\
231 \\
213 \\
196\end{array}$ & $\begin{array}{l}60 \\
66 \\
94 \\
92 \\
73\end{array}$ & $\begin{array}{l}24 \\
25 \\
29 \\
38 \\
46\end{array}$ & $\begin{array}{l}28 \\
32 \\
34 \\
36 \\
41\end{array}$ \\
\hline $\begin{array}{l}21 \\
22 \\
23 \\
24 \\
25\end{array}$ & $\begin{array}{l}37 \\
40 \\
38 \\
38 \\
41\end{array}$ & $\begin{array}{l}31 \\
31 \\
30 \\
30 \\
30\end{array}$ & $\begin{array}{l}15 \\
14 \\
13 \\
10 \\
10\end{array}$ & $\begin{array}{l}4.0 \\
4.3 \\
5.0 \\
5.5 \\
6.0\end{array}$ & $\begin{array}{ll}1 & 1 \\
1 & 1 \\
1 & 1 \\
1 & 0 \\
1 & 0\end{array}$ & $\begin{array}{l}180 \\
155 \\
150 \\
165 \\
200\end{array}$ & $\begin{array}{l}196 \\
219 \\
237 \\
252 \\
274\end{array}$ & $\begin{array}{l}143 \\
138 \\
136 \\
127 \\
125\end{array}$ & $\begin{array}{l}196 \\
188 \\
183 \\
173 \\
143\end{array}$ & $\begin{array}{l}61 \\
61 \\
55 \\
49 \\
46\end{array}$ & $\begin{array}{l}39 \\
33 \\
33 \\
26 \\
24\end{array}$ & $\begin{array}{l}41 \\
41 \\
47 \\
46 \\
46\end{array}$ \\
\hline $\begin{array}{l}26 \\
27 \\
28 \\
29 \\
30 \\
31\end{array}$ & $\begin{array}{l}41 \\
41 \\
40 \\
37 \\
44 \\
41\end{array}$ & $\begin{array}{r}30 \\
29 \\
29 \\
29 \\
29 \\
\end{array}$ & $\begin{array}{l}8.5 \\
7.5 \\
6.0 \\
5.5 \\
5.2 \\
4.5\end{array}$ & $\begin{array}{l}6.5 \\
6.5 \\
7.0 \\
7.0 \\
7.0 \\
7.0\end{array}$ & $\begin{array}{r}10 \\
10 \\
10 \\
10 \\
- \\
-\end{array}$ & $\begin{array}{l}240 \\
196 \\
176 \\
168 \\
156 \\
145\end{array}$ & $\begin{array}{r}277 \\
264 \\
255 \\
252 \\
240 \\
- \\
\end{array}$ & $\begin{array}{l}125 \\
121 \\
119 \\
119 \\
117 \\
127\end{array}$ & $\begin{array}{r}161 \\
156 \\
147 \\
143 \\
134 \\
\end{array}$ & $\begin{array}{l}53 \\
56 \\
50 \\
47 \\
45 \\
43\end{array}$ & $\begin{array}{l}25 \\
21 \\
19 \\
19 \\
23 \\
24\end{array}$ & $\begin{array}{r}46 \\
45 \\
42 \\
41 \\
41 \\
\end{array}$ \\
\hline $\begin{array}{l}\text { Total } \\
\text { Mean } \\
\text { Max } \\
\text { Min } \\
\text { Cfsm } \\
\text { In. } \\
\text { Ac-ft }\end{array}$ & $\begin{array}{r}1.200 \\
38.7 \\
49 \\
29 \\
0.021 \\
0.02 \\
2,380\end{array}$ & $\begin{array}{r}991 \\
33.0 \\
44 \\
29 \\
0.018 \\
0.02 \\
1,960\end{array}$ & $\begin{array}{r}578.2 \\
18.7 \\
29 \\
4.5 \\
0.010 \\
0.01 \\
1,150\end{array}$ & $\begin{array}{r}118.9 \\
3.83 \\
7.0 \\
2.2 \\
0.0020 \\
0.002 \\
236\end{array}$ & $\begin{array}{r}356.5 \\
12.3 \\
16 \\
7.5 \\
0.0066 \\
0.007 \\
707\end{array}$ & $\begin{array}{r}2.5824 \\
83.3 \\
240 \\
9.6 \\
0.045 \\
0.05 \\
5,120\end{array}$ & $\begin{array}{r}6.069 \\
202 \\
277 \\
127 \\
0.108 \\
0.12 \\
12,040\end{array}$ & $\begin{array}{r}4.726 \\
152 \\
228 \\
117 \\
0.081 \\
0.09 \\
9.370\end{array}$ & $\begin{array}{r}5011 \\
167 \\
237 \\
117 \\
0.089 \\
0.10 \\
9,940\end{array}$ & $\begin{array}{r}2.267 \\
73.1 \\
125 \\
43 \\
0.039 \\
0.05 \\
4,500\end{array}$ & $\begin{array}{r}1027 \\
33.1 \\
61 \\
19 \\
0.018 \\
0.02 \\
2,040\end{array}$ & $\begin{array}{r}1079 \\
36.0 \\
49 \\
21 \\
0.019 \\
0.02 \\
2,140\end{array}$ \\
\hline $\begin{array}{l}\text { Cal yr } \\
\text { Wtr yr }\end{array}$ & $\begin{array}{l}\text { 1967: } \\
1968:\end{array}$ & $\begin{array}{ll}\text { al } & 96 \\
\text { al } & 26\end{array}$ & & $\begin{array}{r}263 \\
71.1\end{array}$ & $\begin{array}{l}\operatorname{Max} \\
\operatorname{Max}\end{array}$ & $\begin{array}{l}930 \\
277\end{array}$ & $\begin{array}{l}4.5 \\
2.2\end{array}$ & $\begin{array}{l}\text { Cfsm } \\
\text { Cfsm }\end{array}$ & $\begin{array}{l}\text { In. } \\
\text { In. }\end{array}$ & $\begin{array}{l}1.91 \\
0.52\end{array}$ & $\begin{array}{l}-f t \\
-f t\end{array}$ & $\begin{array}{l}500 \\
.580\end{array}$ \\
\hline
\end{tabular}

Peak discharge (base, $400 \mathrm{cfs}$ ).--No peak above base. 
5-3110. Minnesota River at Montevideo, Minn.

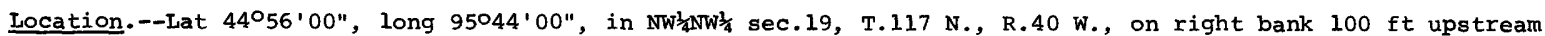
from bridge on U.S. Highway 212, at Montevideo, and $400 \mathrm{ft}$ downstream from Chippewa River.

Drainage area. $--6,180 \mathrm{sq} \mathrm{mi}$, approximately.

Records available.--July 1909 to september 1917, October 1917 to September 1929 (no winter records), October 1929 to September 1968. Prior to October 1939, published as "near Montevideo". Monthly discharge only for some periods, published in WSP 1308.

Gage.-Water-stage recorder. Datum of gage is $910.87 \mathrm{ft}$ above mean sea level, adjustment of 1912 . July 22 , 1909, to Feb. 4, 1932, chain gage at bridge $600 \mathrm{ft}$ downstream at present datum. Feb. 5, 1932, to Nov. 26, 1934, chain gage at bridge $100 \mathrm{ft}$ downstream at present datum.

Average discharqe. --47 years $(1909-17,1929-68), 635$ ofs $(459,700$ acre-ft per year).

Extremes.--Maximum discharge during year, 829 cfs Apr. 25 (gage height, 5.10 ft); minimum, 14 cfs Oct. 5 (gage height, $1.21 \mathrm{ft}$ )

1909-68: Maximum discharge, 24,500 cfs Apr. 10, 1952 (gage height, 20.02 ft, from floodmark); no flow for several days in 1933-34, 1936 .

Remarks.--Records good except those for winter months, which are fair. Flow regulated by Big stone Lake since Apr. 17, 1937, Lac qui Parle Lake since January 1938 and Marsh Lake since Nov. 1, 1939.

DISCHARGE, IN CUBIC FEET PER SECOND, WATER YEAR OCTDBER 1967 TO SEPTEMBER 1968

\begin{tabular}{|c|c|c|c|c|c|c|c|c|c|c|c|c|}
\hline OAY & OCT & NOV & DEC & JAN & FEB & MAQ & $\triangle P R$ & MAY & JUN & JUL & AUG & SEP \\
\hline $\begin{array}{l}1 \\
2 \\
3 \\
4 \\
5\end{array}$ & $\begin{array}{l}51 \\
48 \\
47 \\
35 \\
20\end{array}$ & $\begin{array}{l}67 \\
66 \\
67 \\
67 \\
63\end{array}$ & $\begin{array}{l}52 \\
52 \\
52 \\
51 \\
50\end{array}$ & $\begin{array}{l}21 \\
20 \\
20 \\
20 \\
19\end{array}$ & $\begin{array}{l}21 \\
22 \\
22 \\
22 \\
22\end{array}$ & $\begin{array}{l}28 \\
23 \\
29 \\
35 \\
45\end{array}$ & $\begin{array}{l}278 \\
270 \\
274 \\
281 \\
285\end{array}$ & $\begin{array}{l}673 \\
578 \\
547 \\
526 \\
517\end{array}$ & $\begin{array}{l}265 \\
258 \\
260 \\
256 \\
248\end{array}$ & $\begin{array}{l}425 \\
405 \\
282 \\
259 \\
247\end{array}$ & $\begin{array}{r}96 \\
94 \\
92 \\
89 \\
91\end{array}$ & $\begin{array}{l}49 \\
50 \\
51 \\
51 \\
50\end{array}$ \\
\hline $\begin{array}{r}6 \\
7 \\
8 \\
9 \\
10\end{array}$ & $\begin{array}{l}45 \\
63 \\
66 \\
65 \\
73\end{array}$ & $\begin{array}{l}56 \\
53 \\
54 \\
58 \\
65\end{array}$ & $\begin{array}{l}50 \\
50 \\
49 \\
49 \\
49\end{array}$ & $\begin{array}{l}19 \\
19 \\
19 \\
19 \\
19\end{array}$ & $\begin{array}{l}22 \\
22 \\
23 \\
23 \\
24\end{array}$ & $\begin{array}{l}56 \\
58 \\
61 \\
62 \\
64\end{array}$ & $\begin{array}{l}303 \\
321 \\
348 \\
382 \\
385\end{array}$ & $\begin{array}{l}455 \\
382 \\
357 \\
349 \\
351\end{array}$ & $\begin{array}{l}238 \\
231 \\
248 \\
253 \\
272\end{array}$ & $\begin{array}{l}238 \\
231 \\
219 \\
210 \\
200\end{array}$ & $\begin{array}{r}95 \\
103 \\
100 \\
95 \\
90\end{array}$ & $\begin{array}{l}49 \\
49 \\
55 \\
65 \\
70\end{array}$ \\
\hline $\begin{array}{l}11 \\
12 \\
13 \\
14 \\
15\end{array}$ & $\begin{array}{l}75 \\
75 \\
73 \\
72 \\
69\end{array}$ & $\begin{array}{l}64 \\
63 \\
62 \\
61 \\
59\end{array}$ & $\begin{array}{l}48 \\
48 \\
47 \\
46 \\
45\end{array}$ & $\begin{array}{l}19 \\
19 \\
19 \\
19 \\
19\end{array}$ & $\begin{array}{l}24 \\
24 \\
24 \\
24 \\
24\end{array}$ & $\begin{array}{l}66 \\
68 \\
7 n \\
72 \\
76\end{array}$ & $\begin{array}{l}374 \\
261 \\
256 \\
356 \\
354\end{array}$ & $\begin{array}{l}336 \\
330 \\
331 \\
323 \\
316\end{array}$ & $\begin{array}{l}318 \\
401 \\
371 \\
340 \\
330\end{array}$ & $\begin{array}{l}142 \\
130 \\
125 \\
149 \\
129\end{array}$ & $\begin{array}{l}87 \\
85 \\
82 \\
80 \\
80\end{array}$ & $\begin{array}{l}72 \\
69 \\
64 \\
59 \\
56\end{array}$ \\
\hline $\begin{array}{l}16 \\
17 \\
18 \\
19 \\
20\end{array}$ & $\begin{array}{l}69 \\
68 \\
6 t \\
67 \\
66\end{array}$ & $\begin{array}{l}58 \\
57 \\
56 \\
55 \\
54\end{array}$ & $\begin{array}{l}44 \\
43 \\
42 \\
41 \\
39\end{array}$ & $\begin{array}{l}10 \\
20 \\
20 \\
20 \\
20\end{array}$ & $\begin{array}{l}25 \\
25 \\
25 \\
25 \\
26\end{array}$ & $\begin{array}{r}82 \\
86 \\
37 \\
180 \\
310\end{array}$ & $\begin{array}{l}352 \\
561 \\
707 \\
520 \\
524\end{array}$ & $\begin{array}{l}307 \\
302 \\
300 \\
297 \\
294\end{array}$ & $\begin{array}{l}365 \\
391 \\
390 \\
377 \\
359\end{array}$ & $\begin{array}{l}121 \\
120 \\
122 \\
146 \\
154\end{array}$ & $\begin{array}{l}78 \\
77 \\
80 \\
83 \\
86\end{array}$ & $\begin{array}{l}55 \\
63 \\
60 \\
61 \\
62\end{array}$ \\
\hline $\begin{array}{l}21 \\
22 \\
23 \\
24 \\
25\end{array}$ & $\begin{array}{l}63 \\
65 \\
65 \\
63 \\
61\end{array}$ & $\begin{array}{l}53 \\
52 \\
52 \\
52 \\
52\end{array}$ & $\begin{array}{l}36 \\
33 \\
31 \\
28 \\
27\end{array}$ & $\begin{array}{l}20 \\
20 \\
20 \\
21 \\
21\end{array}$ & $\begin{array}{l}26 \\
26 \\
26 \\
27 \\
27\end{array}$ & $\begin{array}{l}520 \\
490 \\
439 \\
340 \\
360\end{array}$ & $\begin{array}{l}696 \\
751 \\
807 \\
811 \\
824\end{array}$ & $\begin{array}{l}291 \\
286 \\
278 \\
268 \\
262\end{array}$ & $\begin{array}{l}380 \\
351 \\
341 \\
339 \\
342\end{array}$ & $\begin{array}{l}130 \\
116 \\
118 \\
111 \\
107\end{array}$ & $\begin{array}{l}93 \\
88 \\
84 \\
80 \\
78\end{array}$ & $\begin{array}{l}67 \\
72 \\
78 \\
71 \\
69\end{array}$ \\
\hline $\begin{array}{l}26 \\
27 \\
28 \\
29 \\
30 \\
31\end{array}$ & $\begin{array}{l}62 \\
65 \\
66 \\
66 \\
63 \\
68\end{array}$ & $\begin{array}{r}52 \\
52 \\
52 \\
52 \\
52 \\
\end{array}$ & $\begin{array}{l}26 \\
25 \\
24 \\
23 \\
22 \\
22\end{array}$ & $\begin{array}{l}21 \\
21 \\
21 \\
21 \\
21 \\
21\end{array}$ & $\begin{array}{r}27 \\
27 \\
27 \\
28 \\
\\
\end{array}$ & $\begin{array}{l}300 \\
430 \\
380 \\
312 \\
304 \\
283\end{array}$ & $\begin{array}{r}747 \\
715 \\
699 \\
693 \\
692 \\
\end{array}$ & $\begin{array}{l}258 \\
253 \\
246 \\
245 \\
242 \\
253\end{array}$ & $\begin{array}{r}456 \\
463 \\
452 \\
443 \\
436 \\
\end{array}$ & $\begin{array}{r}122 \\
114 \\
110 \\
105 \\
104 \\
99\end{array}$ & $\begin{array}{l}76 \\
75 \\
57 \\
49 \\
53 \\
51\end{array}$ & $\begin{array}{r}69 \\
68 \\
67 \\
66 \\
65 \\
\end{array}$ \\
\hline $\begin{array}{l}\text { TOTAL } \\
\text { MEAN } \\
\text { MAX } \\
\text { MIN } \\
\text { AC-FT }\end{array}$ & $\begin{array}{r}1.924 \\
62.1 \\
75 \\
2 \mathrm{C} \\
3.82 \mathrm{C}\end{array}$ & $\begin{array}{r}1,726 \\
.57 .5 \\
67 \\
52 \\
3,420\end{array}$ & $\begin{array}{r}1,244 \\
40.1 \\
52 \\
22 \\
2,470\end{array}$ & $\begin{array}{r}617 \\
19.9 \\
21 \\
19 \\
1.220\end{array}$ & $\begin{array}{r}710 \\
24.5 \\
28 \\
21 \\
1,410\end{array}$ & $\begin{array}{r}5,810 \\
187 \\
520 \\
28 \\
11,520\end{array}$ & $\begin{array}{r}15,025 \\
501 \\
824 \\
270 \\
29,800\end{array}$ & $\begin{array}{r}10,793 \\
348 \\
673 \\
242 \\
21,410\end{array}$ & $\begin{array}{r}10,174 \\
339 \\
463 \\
231 \\
20,18 \mathrm{C}\end{array}$ & $\begin{array}{r}5,290 \\
171 \\
425 \\
99 \\
10,490\end{array}$ & $\begin{array}{r}2,547 \\
82.2 \\
103 \\
49 \\
5,050\end{array}$ & $\begin{array}{r}1,852 \\
61.7 \\
78 \\
49 \\
3,670\end{array}$ \\
\hline
\end{tabular}

CAL YR 1967 TOTAL $232,979 \quad$ MEAN $638 \quad$ MAX 3.980 MIN 20 AC-FT 462.100

WTR YR 1968 TOTAL 57,712 MEAN 158 MAX $824 \quad$ MIN 19 AC-FT 114,500 
5-3114. South Branch Yellow Medicine River at Minneota, Minn.

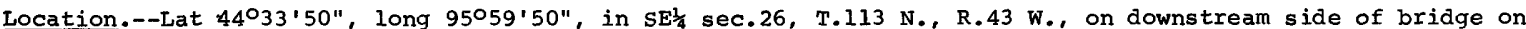
state Highway $68,0.5 \mathrm{mile}$ northwest of Minneota, and 6 miles upstream from confluence with North Branch Yellow Medicine River.

Drainage area.--111 sq $\mathrm{mi}$, approximately.

Records available.--April 1960 to september 1968 . Month1y and daily discharge for the period Apr. 1 , 1960 to June 30, 1960, published in WSP 1914.

Gage.--Wire-weight gage read once daily. Datum of gage is $1,150.00$ ft above mean sea level, datum of 1929 .

Prior to Mar. 21, 1963, staff gage at same site and datum.

Average discharge. --8 years, 19.6 cfs $(14,190$ acre-ft per year) .

Extremes.--Maximum discharge during year, 272 cfs July 26 (gage height, 7.50 ft); no flow for many days.

1960-68: Maximum discharge, 1,830 efs Apx. 6, 1960 (gage height, 11.10 ft); no flow at times.

Remarks.--Records good except those for winter months, which are fair.

DISCHARGE, IN CFS, WATER YEAR OCTOBER 1967 TO SEPTEMBER 1968

\begin{tabular}{|c|c|c|c|c|c|c|c|c|c|c|c|c|}
\hline DAY & $\mathrm{OCT}$ & NOV & DEC & JAN & FEB & MAR & APR & MAY & JUN & JUL & AUG & $\overline{\mathrm{SEP}}$ \\
\hline $\begin{array}{l}1 \\
2 \\
3 \\
4 \\
5\end{array}$ & & $\begin{array}{l}0 \\
0 \\
0 \\
0 \\
0\end{array}$ & $\begin{array}{r}0.3 \\
.3 \\
.2 \\
.2 \\
.2\end{array}$ & $\begin{array}{l}0 \\
0 \\
0 \\
0 \\
0\end{array}$ & & $\begin{array}{l}0 \\
0 \\
0.2 \\
.4 \\
.6\end{array}$ & $\begin{array}{l}4.6 \\
3.2 \\
3.6 \\
64 \\
10\end{array}$ & $\begin{array}{l}5.1 \\
4.5 \\
3.8 \\
3.1 \\
3.1\end{array}$ & $\begin{array}{l}3.9 \\
3.1 \\
2.4 \\
1.4 \\
1.2\end{array}$ & $\begin{array}{c}21 \\
9.0 \\
6.6 \\
8.0 \\
20\end{array}$ & $\begin{array}{c}14 \\
9.5 \\
6.4 \\
3.6 \\
3.6\end{array}$ & $\begin{array}{r}0.8 \\
.6 \\
.4 \\
.3 \\
.1\end{array}$ \\
\hline $\begin{array}{r}6 \\
7 \\
8 \\
9 \\
10\end{array}$ & & $\begin{array}{l}0 \\
0 \\
0 \\
0 \\
0\end{array}$ & $\begin{array}{l}.2 \\
.2 \\
.2 \\
.1 \\
.1\end{array}$ & $\begin{array}{l}0 \\
0 \\
0 \\
0 \\
0\end{array}$ & & $\begin{array}{r}.7 \\
.8 \\
.9 \\
1.0 \\
1.0\end{array}$ & $\begin{array}{c}9.7 \\
9.5 \\
10 \\
7.9 \\
6.6\end{array}$ & $\begin{array}{l}3.2 \\
3.9 \\
5.0 \\
4.6 \\
3.9\end{array}$ & $\begin{array}{r}1.0 \\
.6 \\
.8 \\
1.0 \\
1.4\end{array}$ & $\begin{array}{l}12 \\
66 \\
3.2 \\
1.9 \\
1.5\end{array}$ & $\begin{array}{l}4.1 \\
2.4 \\
3.1 \\
7.1 \\
4.2\end{array}$ & $\begin{array}{l}0 \\
0 \\
1.3 \\
7.1 \\
2.8\end{array}$ \\
\hline $\begin{array}{l}11 \\
12 \\
13 \\
14 \\
15\end{array}$ & & $\begin{array}{l}0 \\
0 \\
0 \\
0 \\
0.1\end{array}$ & $\begin{array}{l}.1 \\
.1 \\
.1 \\
.1 \\
.1\end{array}$ & $\begin{array}{l}0 \\
0 \\
0 \\
0 \\
0\end{array}$ & & $\begin{array}{l}1.0 \\
1.1 \\
1.1 \\
1.2 \\
1.2\end{array}$ & $\begin{array}{l}4.5 \\
4.4 \\
3.5 \\
6.8 \\
5.7\end{array}$ & $\begin{array}{l}3.5 \\
3.2 \\
3.1 \\
2.8 \\
2.6\end{array}$ & $\begin{array}{r}1.7 \\
1.7 \\
1.2 \\
.8 \\
.7\end{array}$ & $\begin{array}{r}1.0 \\
.6 \\
.7 \\
.8 \\
.7\end{array}$ & $\begin{array}{l}4.4 \\
4.2 \\
3.1 \\
2.6 \\
2.4\end{array}$ & $\begin{array}{r}2.0 \\
1.7 \\
1.0 \\
.6 \\
.4\end{array}$ \\
\hline $\begin{array}{l}16 \\
17 \\
18 \\
19 \\
20\end{array}$ & & $\begin{array}{l}.2 \\
.3 \\
.4 \\
.4 \\
.4\end{array}$ & $\begin{array}{l}0 \\
0 \\
0 \\
0 \\
0\end{array}$ & $\begin{array}{l}0 \\
0 \\
0 \\
0 \\
0\end{array}$ & & $\begin{array}{l}1.3 \\
1.4 \\
5.0 \\
20 \\
34\end{array}$ & $\begin{array}{l}3.8 \\
3.1 \\
2.6 \\
2.7 \\
6.2\end{array}$ & $\begin{array}{l}2.4 \\
1.9 \\
2.3 \\
2.4 \\
2.3\end{array}$ & $\begin{array}{l}.5 \\
.4 \\
.3 \\
.2 \\
.1\end{array}$ & $\begin{array}{l}.5 \\
.3 \\
.3 \\
.5 \\
.3\end{array}$ & $\begin{array}{l}2.0 \\
1.8 \\
1.6 \\
1.4 \\
1.7\end{array}$ & $\begin{array}{r}.3 \\
.3 \\
1.2 \\
1.5 \\
1.2\end{array}$ \\
\hline $\begin{array}{l}21 \\
22 \\
23 \\
24 \\
25\end{array}$ & & $\begin{array}{l}.4 \\
.4 \\
.4 \\
.4 \\
.4\end{array}$ & $\begin{array}{l}0 \\
0 \\
0 \\
0 \\
0\end{array}$ & $\begin{array}{l}0 \\
0 \\
0 \\
0 \\
0\end{array}$ & & $\begin{array}{l}16 \\
16 \\
10 \\
8.8 \\
6.1\end{array}$ & $\begin{array}{l}7.0 \\
10 \\
56 \\
23 \\
15\end{array}$ & $\begin{array}{l}2.1 \\
2.1 \\
2.1 \\
1.8 \\
1.5\end{array}$ & $\begin{array}{l}.3 \\
.1 \\
.1 \\
.3 \\
.8\end{array}$ & $\begin{array}{l}.1 \\
0 \\
0 \\
0 \\
5.5\end{array}$ & $\begin{array}{r}1.4 \\
1.0 \\
.4 \\
.1 \\
.1\end{array}$ & $\begin{array}{l}2.0 \\
15 \\
26 \\
19 \\
13\end{array}$ \\
\hline $\begin{array}{l}26 \\
27 \\
28 \\
29 \\
30 \\
31\end{array}$ & & $\begin{array}{r}3 \\
3 \\
3 \\
3 \\
.3 \\
-\end{array}$ & $\begin{array}{l}0 \\
0 \\
0 \\
0 \\
0 \\
0\end{array}$ & $\begin{array}{r}0.2 \\
.1 \\
.2 \\
.2 \\
.2 \\
.1\end{array}$ & - & $\begin{array}{l}7.1 \\
7.9 \\
7.0 \\
5.7 \\
5.7 \\
5.2\end{array}$ & $\begin{array}{r}11 \\
9.3 \\
7.5 \\
6.4 \\
5.9 \\
\end{array}$ & $\begin{array}{l}1.7 \\
1.7 \\
1.5 \\
2.1 \\
1.9 \\
2.3\end{array}$ & $\begin{array}{r}9.6 \\
5.4 \\
2.6 \\
2.9 \\
2.5 \\
\end{array}$ & $\begin{array}{r}186 \\
196 \\
83 \\
35 \\
27 \\
21\end{array}$ & $\begin{array}{r}.2 \\
.1 \\
1 \\
2 \\
1.0 \\
1.0\end{array}$ & $\begin{array}{r}10 \\
7.9 \\
5.6 \\
5.0 \\
4.1 \\
-\end{array}$ \\
\hline $\begin{array}{l}\text { Total } \\
\text { Mean } \\
\text { Max } \\
\text { Min } \\
\text { Ac-ft }\end{array}$ & $\begin{array}{l}0 \\
0 \\
0 \\
0 \\
0\end{array}$ & $\begin{array}{r}5.3 \\
0.18 \\
0.4 \\
0 \\
11\end{array}$ & $\begin{array}{r}2.5 \\
0.08 \\
0.3 \\
0 \\
5.0\end{array}$ & $\begin{array}{r}1.0 \\
0.03 \\
0.2 \\
0 \\
2.0\end{array}$ & $\begin{array}{l}0 \\
0 \\
0 \\
0 \\
0\end{array}$ & $\begin{array}{r}168.4 \\
5.43 \\
34 \\
0 \\
334\end{array}$ & $\begin{array}{r}265.9 \\
8.86 \\
56 \\
2.6 \\
527\end{array}$ & $\begin{array}{r}87.5 \\
2.82 \\
5.1 \\
1.5 \\
174\end{array}$ & $\begin{array}{r}71.5 \\
2.38 \\
25 \\
0.1 \\
142\end{array}$ & $\begin{array}{r}649.1 \\
20.9 \\
196 \\
0 \\
1,290\end{array}$ & $\begin{array}{r}88.8 \\
2.86 \\
14 \\
0.1 \\
176\end{array}$ & $\begin{array}{r}131.2 \\
4.37 \\
26 \\
0 \\
260\end{array}$ \\
\hline $\begin{array}{l}\text { Cal yr } \\
\text { wtr yr }\end{array}$ & & $\begin{array}{l}\operatorname{tal} \\
\operatorname{tal}\end{array}$ & & $\begin{array}{r}10 \\
4\end{array}$ & $\begin{array}{l}\operatorname{Max} \\
\operatorname{Max}\end{array}$ & $\begin{array}{l}578 \\
196\end{array}$ & $\begin{array}{ll}\operatorname{Min} & 0 \\
\operatorname{Min} & 0\end{array}$ & $\begin{array}{l}A c-f t \\
A c-f t\end{array}$ & $\begin{array}{l}7,370 \\
2,920\end{array}$ & & & \\
\hline
\end{tabular}


5-3135. Yellow Medicine River near Granite Falls, Minn.

Location.--Lat 44\%43', long 95031', in sec.35, T.115 N., R.39 W., on right bank 50 ft downstream from highway bridge, 6 miles upstream from mouth, and 8 miles south of town of Granite Falls.

Drainage area. $--653 \mathrm{sq} \mathrm{mi}$.

Records available.--March 1931 to september 1935 (no winter records), October 1935 to September 1938 , October 1939, to september 1968. Monthly discharge only for some periods, published in WSP 1308.

Gaqe.-Water-stage recorder. Datum of gage is $971.59 \mathrm{ft}$ above mean sea level, datum of 1929 . Mar. 16, 1931, to June 10, 1936, chain gage and June 11, 1936, to June 13, 1938, wire-weight gage, on bridge 50 ft upstream at same datum. Oct. 12, 1939, to Nov. 30, 1952, staff gage 500 ft downstream at same datum.

Average discharge. -32 years $(1935-38,1939-68), 98.3$ cfs $(71,170$ acre-ft per year).

Extremes.--Maximum discharge during year, 634 cfs July 29 (gage height, 4.32 ft); minimum, 1.9 cfs Oct. ${ }^{\prime} 2$ (gage height, $2.20 \mathrm{ft}$ ).

1931-38, 1939-68: Maximum discharge, 11,800 cfs June 18, 1957 (gage height, l2.41 ft); no flow at times in $1931,1933,1948,1959$.

Flood in June 1919 reached a stage of $17.5 \mathrm{ft}$, from information by local residents.

Remarks.--Records good except those for winter months, which are fair.

DISCHARGE, IN CFS, WATER YEAR OCTOBER 1967 TO SEPTEMBER 1968

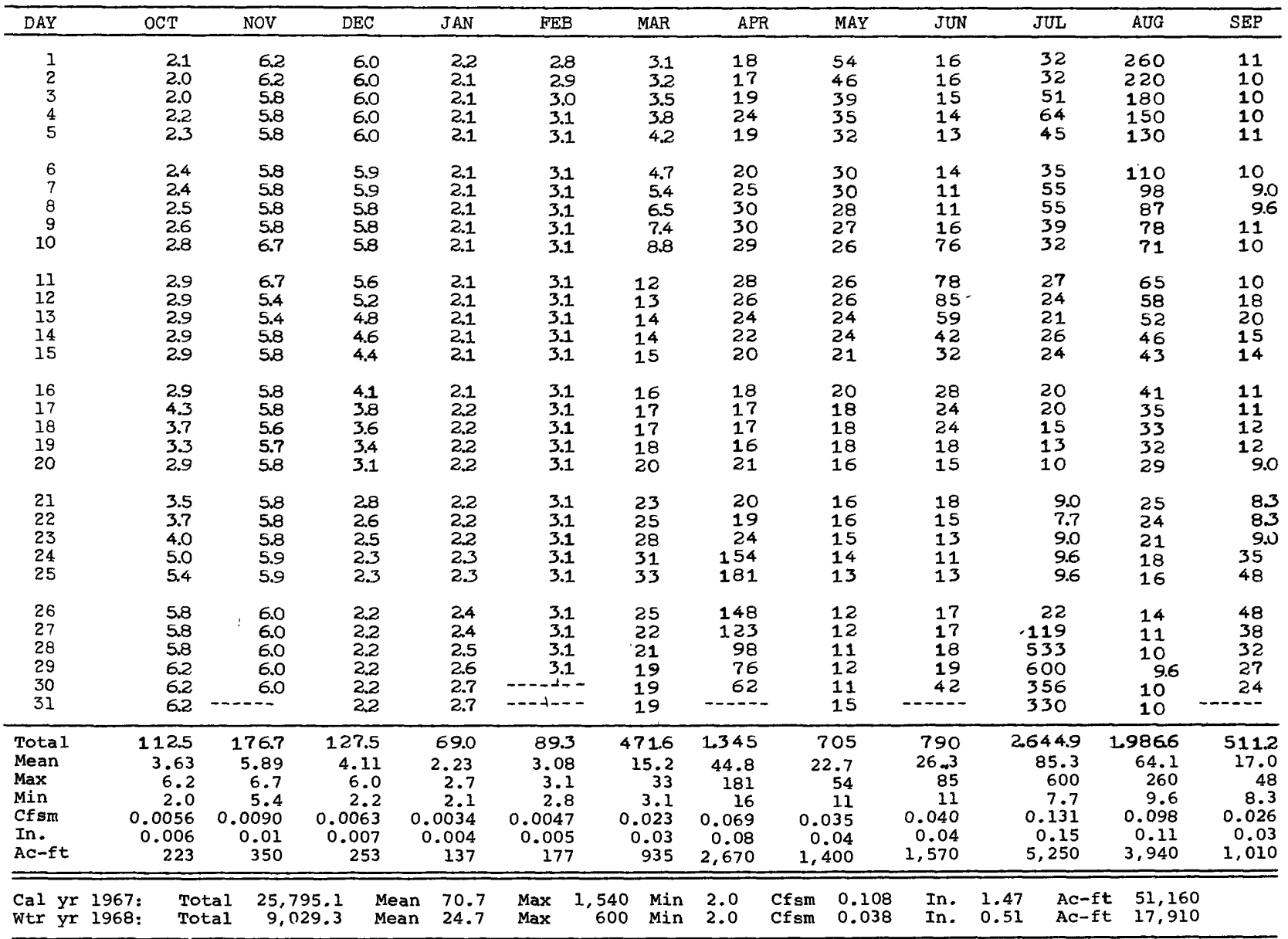

Peak discharge (base, $300 \mathrm{cfs}$ )

$\begin{array}{cccc}\text { Date } & \text { Time } & \begin{array}{c}\text { Gage } \\ \text { height }\end{array} & \text { Discharge } \\ 7-29 & 0800 & 4.32 & 634\end{array}$


5-3150. Redwood River at Marsha11, Minn.

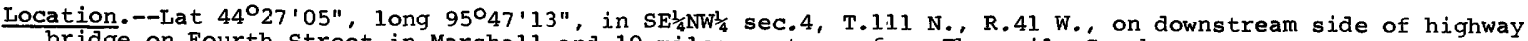
bridge on Fourth street in Marshall and 10 miles upstream from Threemile Creek.

Drainage area. $--307 \mathrm{sq} \mathrm{mi}$.

Records available.--March 1940 to September 1968. Monthly discharge only for some periods, published in wsP 1308.

Gage.--Wire-weight gage read once or twice daily. Datum of gage is $1,144.88$ ft above mean sea level, datum of 1929. Prior to July 12, 1966, chain gage at same site and datum. Staff gage on diversion channel. Datum of gage is $1,100.00 \mathrm{ft}$ above mean sea level, datum of 1929 (levels by Corps of Engineers).

Average discharge.-- -28 years, $45.6 \mathrm{cfs}(33,010$ acre-ft per year).

Extremes.--Maximum discharge during year, 162 cfs sept. 21. River channel: Maximum discharge during year,

$162 \mathrm{cfs}$ sept. 21 (gage height, $1.98 \mathrm{ft}$ ); maximum gage height, $2.40 \mathrm{ft}$ Jan. 20 (backwater from ice); minimum daily discharge 0.1 cfs Oct. 6, Jan. 3-13. Diversion channel: no flow.

1940-68: Maximum discharge, 5, 370 cfs June 17, 1957 (gage height, 10.14 ft); maximum gage height, 11.05 ft Apr. 6, 1951 (from floodmark); no flow at times.

Remarks.--Records good except those for periods of no gage-height record and the winter months, which are fair. Water diverted at medium and high stages into diversion channel 3 miles above station, bypasses station and returns to river $1 \mathrm{mile}$ below station. Diversion began Mar. 18, 1964 . These records include flow in diversion channel. Unknown amount of natural diversion into cottonwood River basin at extremely high stages. Records of suspended sediment loads for the water year 1968 are published in Part 2 of this report.

DISCHARGE, IN CFS, WATER YEAR OCTOBER 1967 TO SEPTEMBER 1968

\begin{tabular}{|c|c|c|c|c|c|c|c|c|c|c|c|c|}
\hline DAY & OCT & NOV & DEC & JAN & FEB & MAR & $\mathrm{APR}$ & MAY & JUN & JUL & AUG & SEP \\
\hline $\begin{array}{l}1 \\
2 \\
3 \\
4 \\
5\end{array}$ & $\begin{array}{r}0.8 \\
.3 \\
.2 \\
.2 \\
.2\end{array}$ & $\begin{array}{l}2.2 \\
2.4 \\
2.2 \\
2.2 \\
2.2\end{array}$ & $\begin{array}{l}3.1 \\
3.1 \\
3.1 \\
3.1 \\
3.1\end{array}$ & $\begin{array}{r}0.2 \\
.2 \\
.1 \\
.1 \\
.1\end{array}$ & $\begin{array}{r}0.8 \\
.9 \\
.9 \\
.9 \\
1.0\end{array}$ & $\begin{array}{l}2.9 \\
3.3 \\
4.0 \\
4.3 \\
4.7\end{array}$ & $\begin{array}{l}30 \\
30 \\
31 \\
45 \\
26\end{array}$ & $\begin{array}{l}7.3 \\
6.2 \\
5.1 \\
4.1 \\
4.1\end{array}$ & $\begin{array}{l}8.5 \\
7.9 \\
5.1 \\
5.6 \\
4.6\end{array}$ & $\begin{array}{l}9.0 \\
12 \\
13 \\
9.2 \\
8.0\end{array}$ & $\begin{array}{l}13 \\
12 \\
14 \\
14 \\
22\end{array}$ & $\begin{array}{l}2.6 \\
1.8 \\
1.8 \\
2.6 \\
2.2\end{array}$ \\
\hline $\begin{array}{r}6 \\
7 \\
8 \\
9 \\
10\end{array}$ & $\begin{array}{r}.1 \\
.4 \\
1.4 \\
1.4 \\
1.6\end{array}$ & $\begin{array}{l}2.5 \\
3.0 \\
3.3 \\
2.9 \\
2.9\end{array}$ & $\begin{array}{l}3.1 \\
3.1 \\
3.1 \\
3.1 \\
3.1\end{array}$ & $\begin{array}{l}.1 \\
.1 \\
.1 \\
.1 \\
.1\end{array}$ & $\begin{array}{l}1.0 \\
1.1 \\
1.1 \\
1.1 \\
1.1\end{array}$ & $\begin{array}{l}5.2 \\
5.6 \\
6.1 \\
6.8 \\
7.5\end{array}$ & $\begin{array}{l}24 \\
30 \\
33 \\
30 \\
11\end{array}$ & $\begin{array}{r}4.1 \\
8.5 \\
9.1 \\
12 \\
6.2\end{array}$ & $\begin{array}{l}5.6 \\
3.2 \\
3.6 \\
3.8 \\
4.2\end{array}$ & $\begin{array}{l}7.1 \\
6.4 \\
5.7 \\
5.0 \\
4.6\end{array}$ & $\begin{array}{l}20 \\
18 \\
21 \\
16 \\
16\end{array}$ & $\begin{array}{l}2.0 \\
1.6 \\
1.4 \\
12 \\
17\end{array}$ \\
\hline $\begin{array}{l}11 \\
12 \\
13 \\
14 \\
15\end{array}$ & $\begin{array}{l}1.6 \\
1.4 \\
2.2 \\
2.6 \\
4.1\end{array}$ & $\begin{array}{l}3.2 \\
4.1 \\
4.1 \\
4.6 \\
4.6\end{array}$ & $\begin{array}{l}3.1 \\
3.1 \\
3.0 \\
2.9 \\
2.8\end{array}$ & $\begin{array}{l}.1 \\
.1 \\
.1 \\
.2 \\
.2\end{array}$ & $\begin{array}{l}1.2 \\
1.2 \\
1.2 \\
1.2 \\
1.2\end{array}$ & $\begin{array}{l}8.0 \\
8.2 \\
8.4 \\
8.7 \\
9.1\end{array}$ & $\begin{array}{c}9.8 \\
10 \\
9.8 \\
9.1 \\
8.5\end{array}$ & $\begin{array}{l}5.1 \\
5.6 \\
5.6 \\
7.3 \\
7.9\end{array}$ & $\begin{array}{l}5.0 \\
4.1 \\
3.9 \\
3.6 \\
3.7\end{array}$ & $\begin{array}{l}3.2 \\
2.9 \\
5.6 \\
2.6 \\
2.2\end{array}$ & $\begin{array}{r}12 \\
11 \\
9.8 \\
8.5 \\
8.5\end{array}$ & $\begin{array}{l}7.9 \\
4.1 \\
3.2 \\
3.2 \\
2.9\end{array}$ \\
\hline $\begin{array}{l}16 \\
17 \\
18 \\
19 \\
20\end{array}$ & $\begin{array}{l}3.6 \\
4.1 \\
4.1 \\
5.1 \\
4.6\end{array}$ & $\begin{array}{l}4.1 \\
3.6 \\
4.6 \\
4.5 \\
4.1\end{array}$ & $\begin{array}{l}2.7 \\
2.5 \\
2.4 \\
2.3 \\
2.2\end{array}$ & $\begin{array}{l}.2 \\
.2 \\
.3 \\
.3 \\
.4\end{array}$ & $\begin{array}{l}1.3 \\
1.4 \\
1.4 \\
1.5 \\
1.6\end{array}$ & $\begin{array}{l}9.5 \\
10 \\
11 \\
12 \\
13\end{array}$ & $\begin{array}{r}7.3 \\
1.4 \\
6.2 \\
9.8 \\
1.5\end{array}$ & $\begin{array}{l}7.9 \\
8.5 \\
7.3 \\
6.4 \\
5.6\end{array}$ & $\begin{array}{l}3.8 \\
3.6 \\
3.2 \\
3.2 \\
3.2\end{array}$ & $\begin{array}{l}2.4 \\
2.4 \\
2.2 \\
1.8 \\
1.8\end{array}$ & $\begin{array}{l}8.5 \\
6.7 \\
6.7 \\
5.1 \\
4.1\end{array}$ & $\begin{array}{c}9.8 \\
4.6 \\
17 \\
6.2 \\
4.6\end{array}$ \\
\hline $\begin{array}{l}21 \\
22 \\
23 \\
24 \\
25\end{array}$ & $\begin{array}{l}4.1 \\
3.0 \\
1.6 \\
2.0 \\
2.0\end{array}$ & $\begin{array}{l}3.7 \\
3.5 \\
3.5 \\
3.5 \\
3.5\end{array}$ & $\begin{array}{l}2.2 \\
2.1 \\
2.0 \\
1.9 \\
1.7\end{array}$ & $\begin{array}{l}.4 \\
.4 \\
.4 \\
.5 \\
.5\end{array}$ & $\begin{array}{l}1.6 \\
1.7 \\
1.8 \\
1.9 \\
1.9\end{array}$ & $\begin{array}{l}14 \\
16 \\
18 \\
20 \\
23\end{array}$ & $\begin{array}{l}15 \\
18 \\
38 \\
33 \\
18\end{array}$ & $\begin{array}{l}5.6 \\
4.6 \\
4.6 \\
5.6 \\
4.6\end{array}$ & $\begin{aligned} & 5.1 \\
& 2.6 \\
& 3.2 \\
& 11 \\
& 12\end{aligned}$ & $\begin{array}{l}2.2 \\
2.4 \\
7.3 \\
6.2 \\
5.1\end{array}$ & $\begin{array}{l}4.1 \\
3.2 \\
2.6 \\
2.2 \\
2.2\end{array}$ & $\begin{array}{l}90 \\
73 \\
48 \\
45 \\
39\end{array}$ \\
\hline $\begin{array}{l}26 \\
27 \\
28 \\
29 \\
30 \\
31\end{array}$ & $\begin{array}{l}2.2 \\
2.4 \\
2.6 \\
2.6 \\
2.9 \\
2.6\end{array}$ & $\begin{array}{r}3.5 \\
3.4 \\
3.4 \\
3.3 \\
3.2 \\
\end{array}$ & $\begin{array}{r}1.4 \\
1.2 \\
.9 \\
.7 \\
.5 \\
.4\end{array}$ & $\begin{array}{l}.6 \\
.6 \\
.7 \\
.7 \\
.7 \\
.7\end{array}$ & $\begin{array}{r}2.0 \\
2.2 \\
2.4 \\
2.5 \\
- \\
- \\
-\end{array}$ & $\begin{array}{l}27 \\
30 \\
34 \\
30 \\
29 \\
28\end{array}$ & $\begin{array}{r}15 \\
14 \\
11 \\
9.8 \\
8.5 \\
\end{array}$ & $\begin{array}{l}5.6 \\
4.6 \\
4.6 \\
5.6 \\
5.6 \\
7.3\end{array}$ & $\begin{array}{r}18 \\
12 \\
9.8 \\
7.3 \\
6.7 \\
\end{array}$ & $\begin{array}{l}51 \\
9.8 \\
28 \\
18 \\
15 \\
14\end{array}$ & $\begin{array}{l}2.0 \\
1.8 \\
1.6 \\
1.6 \\
2.2 \\
2.9\end{array}$ & $\begin{array}{r}35 \\
38 \\
43 \\
56 \\
68 \\
\end{array}$ \\
\hline $\begin{array}{l}\text { Total } \\
\text { Mean } \\
\text { Max } \\
\text { Min } \\
\text { Cfsm } \\
\text { In. } \\
\text { Ac-ft }\end{array}$ & $\begin{array}{r}68.0 \\
2.19 \\
5.1 \\
0.1 \\
0.0071 \\
0.01 \\
135 \\
\end{array}$ & $\begin{array}{r}101.8 \\
3.39 \\
4.6 \\
2.2 \\
0.011 \\
0.01 \\
202 \\
\end{array}$ & $\begin{array}{r}73.0 \\
2.35 \\
3.1 \\
0.4 \\
0.0076 \\
0.01 \\
145 \\
\end{array}$ & $\begin{array}{r}9.5 \\
0.31 \\
0.7 \\
0.1 \\
0.00099 \\
0.001 \\
19\end{array}$ & $\begin{array}{r}41.1 \\
1.42 \\
2.5 \\
0.8 \\
0.0046 \\
0.005 \\
81\end{array}$ & $\begin{array}{r}417.3 \\
13.5 \\
34 \\
2.9 \\
0.044 \\
0.05 \\
828\end{array}$ & $\begin{array}{r}557.2 \\
18.6 \\
45 \\
1.4 \\
0.061 \\
0.07 \\
1.105\end{array}$ & $\begin{array}{r}192.2 \\
6.20 \\
12 \\
4.1 \\
0.020 \\
0.02 \\
381\end{array}$ & $\begin{array}{r}177.1 \\
5.90 \\
18 \\
2.6 \\
0.019 \\
0.02 \\
351\end{array}$ & $\begin{array}{r}266.1 \\
8.58 \\
51 \\
1.8 \\
0.028 \\
0.03 \\
528\end{array}$ & $\begin{array}{r}273.3 \\
8.82 \\
22 \\
1.6 \\
0.029 \\
0.03 \\
542\end{array}$ & $\begin{array}{r}643.5 \\
21.4 \\
90 \\
1.4 \\
0.070 \\
0.08 \\
1,280\end{array}$ \\
\hline $\begin{array}{l}\text { Cal yr } \\
\text { Wtr yr }\end{array}$ & $\begin{array}{l}1967: \\
1968:\end{array}$ & $\begin{array}{ll}1 & 8 \\
1 & 2\end{array}$ & $\begin{array}{l}4 \\
1 \\
\end{array}$ & $\begin{array}{r}23.3 \\
7.71\end{array}$ & $\begin{array}{l}\operatorname{Max} \\
\operatorname{Max}\end{array}$ & $\begin{array}{l}\operatorname{Min} \\
\operatorname{Min}\end{array}$ & $\begin{array}{l}1 \\
.1\end{array}$ & $\begin{array}{l}0.076 \\
0.025\end{array}$ & $\begin{array}{l}\text { In. } \\
\text { In. }\end{array}$ & $\begin{array}{l}\text { AC } \\
\text { AC }\end{array}$ & $\begin{array}{r}16,8 \\
5,5\end{array}$ & \\
\hline
\end{tabular}


5-3165. Redwood River near Redwood Falls, Minn.

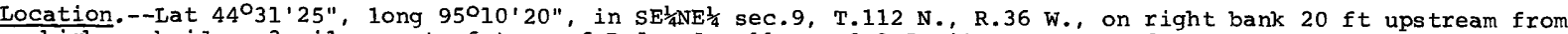
highway bridge, 3 miles west of town of Redwood Falls, and 8.5 miles upstream from mouth.

Drainage area. $--697 \mathrm{sq} \mathrm{mi}$.

Records available.--July 1909 to september 1911 (no winter records), October 1911 to september 1912 , October 1912 to september 1914 and August 1930 to september 1935 (no winter records), October 1935 to september 1968.

Gage.--Water-stage recorder. Datum of gage is $972.33 \mathrm{ft}$ above mean sea 1 eve1, datum of 1929 . Ju1y 1909 to September 1914, chain gage at bridge $20 \mathrm{ft}$ downstream at datum 0.22 ft lower. August 1930 to Mar. 25 , 1940 , chain gage and Mar. 26, 1940, to Oct. 25, 1949, wire-weight gage, at bridge 20 ft downstream at present datum.

Average discharge.--34 years (1911-12, 1935-68) 96.5 cfs $(69,860$ acre-feet per year).

Extremes.--Maximum discharge during year, 722 cfs July 27 (gage height, 3.61 ft); minimum daily, 0.7 cfs Jan. 8-17; minimum gage height, 1.37 ft Nov. 27.

1909-14, 1930-68: Maximum discharge, 19,700 cfs June 18, 1957 (gage height, 15.92 ft, from floodmark); no flow for several days in January 1940 and for part of each day Aug. 19, 20, 1959.

Remarks.--Records good except those for periods of no gage-height record and those for winter months, which are fair. Records of suspended sediment loads for the water year 1968 are published in Part 2 of this report. Natural discharge affected by unknown amount of interbasin flow between Yellow Medicine, Redwood and Cottonwood River basins during extreme floods.

DISCHARGE, IN CFS, WATER YEAR OCTOBER 1967 TO SEPTEMBER 1968

\begin{tabular}{|c|c|c|c|c|c|c|c|c|c|c|c|c|}
\hline DAY & $\mathrm{OCT}$ & $\mathrm{NOV}$ & DEC & JAN & FEB & MAR & APR & MAY & JUN & JUL & AUG & SEP \\
\hline $\begin{array}{l}1 \\
2 \\
3 \\
4 \\
5\end{array}$ & $\begin{array}{r}5.9 \\
8.2 \\
10 \\
9.7 \\
9.2\end{array}$ & $\begin{array}{l}14 \\
13 \\
16 \\
13 \\
12\end{array}$ & $\begin{array}{l}11 \\
11 \\
11 \\
11 \\
12\end{array}$ & $\begin{array}{l}2.5 \\
2.1 \\
1.7 \\
1.3 \\
1.1\end{array}$ & $\begin{array}{l}1.4 \\
1.5 \\
1.5 \\
1.6 \\
1.6\end{array}$ & $\begin{array}{l}2.5 \\
2.9 \\
3.3 \\
3.7 \\
4.5\end{array}$ & $\begin{array}{l}44 \\
42 \\
43 \\
69 \\
76\end{array}$ & $\begin{array}{l}39 \\
33 \\
29 \\
27 \\
24\end{array}$ & $\begin{array}{l}20 \\
21 \\
22 \\
20 \\
17\end{array}$ & $\begin{array}{l}29 \\
44 \\
47 \\
44 \\
38\end{array}$ & $\begin{array}{r}201 \\
150 \\
116 \\
96 \\
89\end{array}$ & $\begin{array}{l}14 \\
16 \\
14 \\
14 \\
14\end{array}$ \\
\hline $\begin{array}{r}6 \\
7 \\
8 \\
9 \\
10\end{array}$ & $\begin{array}{l}8.2 \\
8.2 \\
7.7 \\
7.7 \\
7.2\end{array}$ & $\begin{array}{l}12 \\
11 \\
11 \\
13 \\
20\end{array}$ & $\begin{array}{l}12 \\
13 \\
13 \\
14 \\
14\end{array}$ & $\begin{array}{l}.9 \\
.8 \\
.7 \\
.7 \\
.7\end{array}$ & $\begin{array}{l}1.6 \\
1.7 \\
1.7 \\
1.8 \\
1.8\end{array}$ & $\begin{array}{r}5.4 \\
6.6 \\
8.0 \\
10 \\
11\end{array}$ & $\begin{array}{l}66 \\
59 \\
57 \\
55 \\
51\end{array}$ & $\begin{array}{l}23 \\
23 \\
24 \\
27 \\
26\end{array}$ & $\begin{array}{l}14 \\
14 \\
14 \\
16 \\
47\end{array}$ & $\begin{array}{l}31 \\
28 \\
24 \\
19 \\
20\end{array}$ & $\begin{array}{r}84 \\
87 \\
102 \\
98 \\
89\end{array}$ & $\begin{array}{l}12 \\
12 \\
14 \\
16 \\
20\end{array}$ \\
\hline $\begin{array}{l}11 \\
12 \\
13 \\
14 \\
15\end{array}$ & $\begin{array}{l}7.2 \\
6.3 \\
5.9 \\
6.8 \\
6.8\end{array}$ & $\begin{array}{l}20 \\
19 \\
19 \\
19 \\
17\end{array}$ & $\begin{array}{ll}1 & 4 \\
14 \\
14 \\
13 \\
13\end{array}$ & $\begin{array}{l}.7 \\
.7 \\
.7 \\
.7 \\
.7\end{array}$ & $\begin{array}{l}1.8 \\
1.8 \\
1.8 \\
1.8 \\
1.8\end{array}$ & $\begin{array}{l}12 \\
13 \\
14 \\
15 \\
16\end{array}$ & $\begin{array}{l}44 \\
39 \\
35 \\
36 \\
33\end{array}$ & $\begin{array}{l}27 \\
26 \\
24 \\
23 \\
21\end{array}$ & $\begin{array}{l}89 \\
87 \\
84 \\
74 \\
55\end{array}$ & $\begin{array}{l}15 \\
14 \\
20 \\
19 \\
15\end{array}$ & $\begin{array}{l}79 \\
68 \\
59 \\
54 \\
47\end{array}$ & $\begin{array}{l}33 \\
35 \\
28 \\
23 \\
18\end{array}$ \\
\hline $\begin{array}{l}16 \\
17 \\
18 \\
19 \\
20\end{array}$ & $\begin{array}{l}6.8 \\
6.8 \\
7.2 \\
7.7 \\
7.2\end{array}$ & $\begin{array}{l}16 \\
15 \\
18 \\
16 \\
14\end{array}$ & $\begin{array}{l}12 \\
12 \\
11 \\
11 \\
10\end{array}$ & $\begin{array}{l}.7 \\
.7 \\
.8 \\
.8 \\
.8\end{array}$ & $\begin{array}{l}1.8 \\
1.9 \\
1.9 \\
1.9 \\
1.9\end{array}$ & $\begin{array}{l}17 \\
19 \\
20 \\
21 \\
22\end{array}$ & $\begin{array}{l}33 \\
29 \\
28 \\
27 \\
33\end{array}$ & $\begin{array}{l}22 \\
22 \\
23 \\
22 \\
22\end{array}$ & $\begin{array}{l}41 \\
29 \\
23 \\
19 \\
16\end{array}$ & $\begin{array}{l}14 \\
13 \\
14 \\
11 \\
11\end{array}$ & $\begin{array}{l}42 \\
38 \\
35 \\
33 \\
32\end{array}$ & $\begin{array}{l}19 \\
21 \\
21 \\
23 \\
21\end{array}$ \\
\hline $\begin{array}{l}21 \\
22 \\
23 \\
24 \\
25\end{array}$ & $\begin{array}{r}8.2 \\
9.2 \\
16 \\
17 \\
16\end{array}$ & $\begin{array}{l}13 \\
12 \\
11 \\
11 \\
11\end{array}$ & $\begin{array}{l}10 \\
10 \\
10 \\
9.7 \\
9.4\end{array}$ & $\begin{array}{r}.9 \\
.9 \\
1.0 \\
1.0 \\
1.1\end{array}$ & $\begin{array}{l}1.9 \\
1.9 \\
1.9 \\
2.0 \\
2.0\end{array}$ & $\begin{array}{l}23 \\
24 \\
25 \\
27 \\
29\end{array}$ & $\begin{array}{l}35 \\
42 \\
51 \\
54 \\
89\end{array}$ & $\begin{array}{l}21 \\
20 \\
20 \\
18 \\
17\end{array}$ & $\begin{array}{l}15 \\
14 \\
13 \\
14 \\
14\end{array}$ & $\begin{array}{r}11 \\
11 \\
11 \\
9.7 \\
9.7\end{array}$ & $\begin{array}{l}28 \\
24 \\
21 \\
19 \\
18\end{array}$ & $\begin{array}{r}24 \\
56 \\
102 \\
114 \\
110\end{array}$ \\
\hline $\begin{array}{l}26 \\
27 \\
28 \\
29 \\
30 \\
31\end{array}$ & $\begin{array}{l}15 \\
14 \\
14 \\
16 \\
14 \\
14\end{array}$ & $\begin{array}{c}11 \\
9.8 \\
10 \\
11 \\
11 \\
\end{array}$ & $\begin{array}{l}9.2 \\
8.5 \\
7.5 \\
6.2 \\
4.8 \\
3.4\end{array}$ & $\begin{array}{l}1.1 \\
1.2 \\
1.2 \\
1.3 \\
1.3 \\
1.4\end{array}$ & $\begin{array}{r}2.1 \\
2.1 \\
2.2 \\
2.4 \\
- \\
-\end{array}$ & $\begin{array}{l}31 \\
34 \\
38 \\
46 \\
51 \\
45\end{array}$ & $\begin{array}{r}89 \\
80 \\
68 \\
57 \\
47 \\
- \\
-\end{array}$ & $\begin{array}{l}17 \\
17 \\
16 \\
16 \\
17 \\
19\end{array}$ & $\begin{array}{r}16 \\
19 \\
27 \\
28 \\
24 \\
-\end{array}$ & $\begin{array}{l}114 \\
608 \\
460 \\
377 \\
321 \\
257\end{array}$ & $\begin{array}{l}16 \\
14 \\
12 \\
12 \\
13 \\
14\end{array}$ & $\begin{array}{r}108 \\
96 \\
87 \\
80 \\
79 \\
- \\
-\end{array}$ \\
\hline $\begin{array}{l}\text { Total } \\
\text { Mean } \\
\text { Max } \\
\text { Min } \\
\text { Cfsm } \\
\text { In. } \\
\text { Ac-ft }\end{array}$ & $\begin{array}{r}304.1 \\
9.81 \\
17 \\
5.9 \\
0.014 \\
0.02 \\
603\end{array}$ & $\begin{array}{r}418.8 \\
14.0 \\
20 \\
9.8 \\
0.020 \\
0.02 \\
831\end{array}$ & $\begin{array}{r}334.7 \\
10.8 \\
14 \\
3.4 \\
0.015 \\
0.02 \\
664\end{array}$ & $\begin{array}{r}32.2 \\
1.04 \\
2.5 \\
0.7 \\
0.0015 \\
0.002 \\
64\end{array}$ & $\begin{array}{r}53.1 \\
1.83 \\
2.4 \\
1.4 \\
0.0026 \\
0.003 \\
105\end{array}$ & $\begin{array}{r}599.9 \\
19.4 \\
51 \\
2.5 \\
0.028 \\
0.03 \\
1.190\end{array}$ & $\begin{array}{r}1.511 \\
50.4 \\
89 \\
27 \\
0.072 \\
0.08 \\
3,000\end{array}$ & $\begin{array}{r}705 \\
22.7 \\
39 \\
16 \\
0.033 \\
0.04 \\
1,400\end{array}$ & $\begin{array}{r}906 \\
30.2 \\
89 \\
13 \\
0.043 \\
0.05 \\
1.800\end{array}$ & $\begin{array}{r}2659.4 \\
85.8 \\
608 \\
9.7 \\
0.123 \\
0.14 \\
5,270\end{array}$ & $\begin{array}{r}1790 \\
57.7 \\
201 \\
12 \\
0.083 \\
0.10 \\
3,550\end{array}$ & $\begin{array}{r}1244 \\
41.5 \\
114 \\
12 \\
0.060 \\
0.07 \\
2,470\end{array}$ \\
\hline
\end{tabular}

Ca1 yr 1967: Tota1 26,796.4 Mean 73.4 Max 1,090 Min $2.7 \quad$ Cfsm $0.105 \quad$ In. $1.43 \quad$ Ac-ft 53,150 wtr yr 1968: Total 10,558.2 Mean 28.8 Max 608 Min 0.7 Cfsm 0.041 In. 0.56 Ac-ft 20,940

PEAK DISCHARGE (BASE, 150 CFS)

DATE TIME G.HT. DISCHARGE TATE TTME G.HT. DISCHARGE

$\begin{array}{llll}7-27 & 0830 \quad 722 & 3.61\end{array}$

Note.--No gage-height record Feb. 14 to Mar. 12, Mar. 14 to Apr. 7. 
5-3167.7 Minnesota River at New Ulm, Minn.

Location.--Lat $44^{\circ} 19^{\prime} 29^{\prime \prime}$, long $94^{\circ} 27^{\prime} 09^{\prime \prime}$, in NE $\frac{1}{4} \mathrm{NE}^{\frac{1}{4}}$ sec.20, T.110 N., R.30 W., on left bank $30 \mathrm{ft}$ downstream from U.S. Highway 14, at New Ulm and 6.1 miles upstream from Cottonwood River.

Drainage area. $--9,536 \mathrm{sq} \mathrm{mi}$ (at mouth of Cottonwood River).

Records available.--October 1967 to september 1968.

Gage.--Digital water-stage recorder. Datum of gage is $778.72 \mathrm{ft}$ above mean sea level, datum of 1929.

Extremes.--Maximum discharge during year, $2,810 \mathrm{cfs} J \mathrm{Ju}$ (y 28 (gage height, $14.18 \mathrm{ft}$, backwater from Cottonwood River); minimum daily discharge, 42 cfs Jan. 15, 19.

Remarks.--Records good except those for periods of no gage-height record or backwater from Cottonwood River and those for winter months, which are fair. Records of suspended sediment loads for the water year 1968 are published in Part 2 of this report.

DISCHARGE, IN CUBIC FEET PER SECOND, WATER YEAR OCTOBER 1967 TO SEPTEMBER 1968

\begin{tabular}{|c|c|c|c|c|c|c|c|c|c|c|c|c|}
\hline DAY & $\mathrm{OCT}$ & NOV & $D E C$ & JAN & FEB & MAR & $\triangle P R$ & MAY & JUN & JUL & AUG & SEP \\
\hline $\begin{array}{l}1 \\
2 \\
3 \\
4 \\
5\end{array}$ & $\begin{array}{l}128 \\
128 \\
128 \\
128 \\
128\end{array}$ & $\begin{array}{l}148 \\
148 \\
148 \\
148 \\
148\end{array}$ & $\begin{array}{l}110 \\
121 \\
123 \\
125 \\
127\end{array}$ & $\begin{array}{l}64 \\
62 \\
59 \\
57 \\
54\end{array}$ & $\begin{array}{l}56 \\
58 \\
59 \\
60 \\
61\end{array}$ & $\begin{array}{l}60 \\
60 \\
60 \\
60 \\
62\end{array}$ & $\begin{array}{l}551 \\
512 \\
449 \\
464 \\
479\end{array}$ & $\begin{array}{l}934 \\
914 \\
892 \\
850 \\
791\end{array}$ & $\begin{array}{l}428 \\
467 \\
527 \\
527 \\
491\end{array}$ & $\begin{array}{l}704 \\
714 \\
742 \\
732 \\
700\end{array}$ & $\begin{array}{r}2,170 \\
1.960 \\
1.350 \\
930 \\
611\end{array}$ & $\begin{array}{l}168 \\
164 \\
162 \\
160 \\
164\end{array}$ \\
\hline $\begin{array}{r}6 \\
7 \\
8 \\
9 \\
10\end{array}$ & $\begin{array}{l}128 \\
128 \\
128 \\
130 \\
130\end{array}$ & $\begin{array}{l}149 \\
148 \\
146 \\
142 \\
141\end{array}$ & $\begin{array}{l}125 \\
123 \\
128 \\
134 \\
136\end{array}$ & $\begin{array}{l}53 \\
52 \\
51 \\
49 \\
48\end{array}$ & $\begin{array}{l}62 \\
62 \\
64 \\
64 \\
64\end{array}$ & $\begin{array}{r}66 \\
74 \\
90 \\
110 \\
140\end{array}$ & $\begin{array}{l}563 \\
539 \\
509 \\
494 \\
539\end{array}$ & $\begin{array}{l}710 \\
696 \\
693 \\
650 \\
635\end{array}$ & $\begin{array}{l}452 \\
419 \\
417 \\
410 \\
533\end{array}$ & $\begin{array}{l}644 \\
596 \\
524 \\
461 \\
437\end{array}$ & $\begin{array}{r}506 \\
87 B \\
1.050 \\
1.030 \\
728\end{array}$ & $\begin{array}{l}162 \\
156 \\
150 \\
222 \\
292\end{array}$ \\
\hline $\begin{array}{l}11 \\
12 \\
13 \\
14 \\
15\end{array}$ & $\begin{array}{l}130 \\
130 \\
130 \\
131 \\
132\end{array}$ & $\begin{array}{l}140 \\
138 \\
136 \\
145 \\
155\end{array}$ & $\begin{array}{l}144 \\
146 \\
123 \\
107 \\
116\end{array}$ & $\begin{array}{l}46 \\
45 \\
44 \\
43 \\
42\end{array}$ & $\begin{array}{l}64 \\
63 \\
63 \\
62 \\
62\end{array}$ & $\begin{array}{l}180 \\
250 \\
310 \\
305 \\
30 C\end{array}$ & $\begin{array}{l}575 \\
596 \\
656 \\
638 \\
611\end{array}$ & $\begin{array}{l}590 \\
548 \\
530 \\
518 \\
491\end{array}$ & $\begin{array}{l}1.040 \\
1.170 \\
1.110 \\
1.120 \\
1.070\end{array}$ & $\begin{array}{l}410 \\
395 \\
458 \\
605 \\
840\end{array}$ & $\begin{array}{l}672 \\
503 \\
440 \\
353 \\
295\end{array}$ & $\begin{array}{l}275 \\
240 \\
226 \\
212 \\
188\end{array}$ \\
\hline $\begin{array}{l}16 \\
17 \\
19 \\
19 \\
20\end{array}$ & $\begin{array}{l}138 \\
139 \\
140 \\
160 \\
200\end{array}$ & $\begin{array}{l}161 \\
150 \\
152 \\
154 \\
152\end{array}$ & $\begin{array}{l}119 \\
128 \\
130 \\
130 \\
136\end{array}$ & $\begin{array}{l}43 \\
44 \\
44 \\
42 \\
43\end{array}$ & $\begin{array}{l}61 \\
60 \\
59 \\
57 \\
57\end{array}$ & $\begin{array}{l}290 \\
330 \\
355 \\
363 \\
355\end{array}$ & $\begin{array}{l}569 \\
578 \\
554 \\
542 \\
566\end{array}$ & $\begin{array}{l}470 \\
464 \\
467 \\
458 \\
446\end{array}$ & $\begin{array}{l}942 \\
822 \\
763 \\
728 \\
718\end{array}$ & $\begin{array}{l}732 \\
6 B 6 \\
679 \\
696 \\
608\end{array}$ & $\begin{array}{l}285 \\
258 \\
230 \\
234 \\
226\end{array}$ & $\begin{array}{l}174 \\
344 \\
410 \\
392 \\
347\end{array}$ \\
\hline $\begin{array}{l}21 \\
22 \\
23 \\
24 \\
25\end{array}$ & $\begin{array}{l}160 \\
140 \\
138 \\
138 \\
128\end{array}$ & $\begin{array}{l}150 \\
144 \\
142 \\
138 \\
140\end{array}$ & $\begin{array}{r}136 \\
114 \\
109 \\
114 \\
99\end{array}$ & $\begin{array}{l}43 \\
43 \\
44 \\
45 \\
46\end{array}$ & $\begin{array}{l}57 \\
57 \\
57 \\
58 \\
60\end{array}$ & $\begin{array}{l}355 \\
360 \\
365 \\
566 \\
756\end{array}$ & $\begin{array}{r}728 \\
822 \\
875 \\
868 \\
1.010\end{array}$ & $\begin{array}{l}437 \\
428 \\
422 \\
419 \\
410\end{array}$ & $\begin{array}{l}686 \\
656 \\
653 \\
679 \\
710\end{array}$ & $\begin{array}{l}521 \\
485 \\
506 \\
700 \\
728\end{array}$ & $\begin{array}{l}206 \\
218 \\
198 \\
194 \\
174\end{array}$ & $\begin{array}{r}431 \\
672 \\
950 \\
1.250 \\
1.060\end{array}$ \\
\hline $\begin{array}{l}26 \\
27 \\
28 \\
29 \\
30 \\
31\end{array}$ & $\begin{array}{l}132 \\
138 \\
142 \\
143 \\
149 \\
150\end{array}$ & $\begin{array}{r}142 \\
92 \\
105 \\
112 \\
105 \\
\end{array}$ & $\begin{array}{l}84 \\
68 \\
73 \\
68 \\
65 \\
64\end{array}$ & $\begin{array}{l}47 \\
48 \\
50 \\
51 \\
52 \\
54\end{array}$ & $\begin{array}{r}60 \\
60 \\
60 \\
60 \\
\\
\end{array}$ & $\begin{array}{l}794 \\
707 \\
641 \\
629 \\
623 \\
569\end{array}$ & $\begin{array}{l}1,120 \\
1,190 \\
1,210 \\
1,150 \\
1,050 \\
-\end{array}$ & $\begin{array}{l}407 \\
419 \\
416 \\
413 \\
392 \\
404\end{array}$ & $\begin{array}{r}714 \\
690 \\
700 \\
696 \\
710 \\
\end{array}$ & $\begin{array}{r}836 \\
2,010 \\
2,770 \\
2,540 \\
1,960 \\
2,140 \\
\end{array}$ & $\begin{array}{l}180 \\
174 \\
162 \\
168 \\
156 \\
164\end{array}$ & $\begin{array}{r}730 \\
467 \\
371 \\
270 \\
247 \\
\end{array}$ \\
\hline $\begin{array}{l}\text { TOTAL } \\
\text { MEAN } \\
\text { MAX } \\
\text { MIN } \\
A C-F T\end{array}$ & $\begin{array}{r}4.272 \\
138 \\
200 \\
128 \\
8.470\end{array}$ & $\begin{array}{r}4.229 \\
141 \\
161 \\
92 \\
8.390\end{array}$ & $\begin{array}{r}3.525 \\
114 \\
146 \\
64 \\
6.990\end{array}$ & $\begin{array}{r}1,508 \\
48.6 \\
64 \\
42 \\
2,990\end{array}$ & $\begin{array}{r}1.747 \\
60.2 \\
64 \\
56 \\
3,470\end{array}$ & $\begin{array}{r}10.185 \\
329 \\
794 \\
60 \\
20,200\end{array}$ & $\begin{array}{r}21,007 \\
700 \\
1.210 \\
449 \\
41.670\end{array}$ & $\begin{array}{r}17,314 \\
559 \\
934 \\
392 \\
34.340\end{array}$ & $\begin{array}{r}21.048 \\
702 \\
1.170 \\
410 \\
41.750\end{array}$ & $\begin{array}{r}27.559 \\
889 \\
2.770 \\
395 \\
54.660\end{array}$ & $\begin{array}{r}16,703 \\
539 \\
2,170 \\
156 \\
33,130\end{array}$ & $\begin{array}{r}11.056 \\
369 \\
1.250 \\
150 \\
21.930\end{array}$ \\
\hline
\end{tabular}

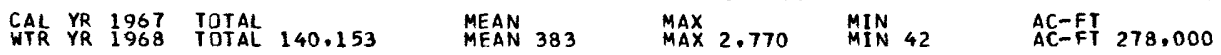


5-3170. Cottonwood River near New U1m, Minn.

Location.-Lat $44^{\circ} 17^{\prime} 40^{\prime \prime}$, long $94^{\circ} 26^{\prime} 40^{\prime \prime}$, in Nk sec.33, T.110 N.., R.30 W., on left bank 600 ft upstream from highway bridge, 1.8 miles south of New U1m, and 2 miles upstream from mouth.

Drainage area. $-1,280 \mathrm{sq} \mathrm{mi}$, approximately.

Records available.--July 1909 to December 1913, March 1931 to March 1938, August 1938 to September 1968 (winter records incomplete prior to 1936.

Gaqe.--Water-stage recorder. Datum of gage is $799.09 \mathrm{ft}$ above mean sea level, adjustment of 1912. July 1 , 1909, to Dec. 13, 1913, chain gage at site 2.7 miles upstream at different datum. Mar. 15, 1931, to Mar. 31, 1938, chain or wire-weight gage $2 \frac{1}{4}$ miles upstream at datum 11.41 ft higher. Aug. 23, 1938, to June 25, 1948, staff gage at present site and datum.

Average discharge. --34 years $(1911-13,1935-37,1938-68), 253$ cfs $(183,200$ acre-ft per year).

Extremes.--Maximum discharge during year, 4,550 cfs sept. 23 (gage height, $11.98 \mathrm{ft}$ ); minimum $1.8 \mathrm{cfs}$ May 17 , (gage height, $2.17 \mathrm{ft}$ ).

1909-13, 1931-68: Maximum discharge, 26,000 cfs Apr. 8, 1965 (gage height, 20.86 ft, from floodmark,

backwater from ice); minimum observed, $0.5 \mathrm{cfs}$ Nov. 27,1952 ; minimum gage height, 0.72 ft Nov. 20 , 1964 .

Remarks.--Records good except those for winter months, which are fair. Records of chemical analyses and suspended sediment loads for the water year 1968 are published in Part 2 of this report. Some regulation by dam at cottonwood Lake and several other small lakes above station.

DISCHARGE, IN CFS, WATER YEAR OCTOBER 1967 TO SEPTEMBER 1968

\begin{tabular}{|c|c|c|c|c|c|c|c|c|c|c|c|c|}
\hline$\overline{D A Y}$ & $\mathrm{OCT}$ & NOV & $\overline{D E C}$ & JAN & FEB & MAR & APR & MAY & JUN & JUL & AUG & SEP \\
\hline $\begin{array}{l}1 \\
2 \\
3 \\
4 \\
5\end{array}$ & $\begin{array}{l}20 \\
20 \\
16 \\
18 \\
24\end{array}$ & $\begin{array}{l}31 \\
29 \\
30 \\
30 \\
28\end{array}$ & $\begin{array}{l}12 \\
12 \\
13 \\
13 \\
13\end{array}$ & $\begin{array}{l}5.0 \\
4.9 \\
4.9 \\
4.9 \\
4.8\end{array}$ & $\begin{array}{l}6.3 \\
6.5 \\
6.6 \\
6.8 \\
6.8\end{array}$ & $\begin{array}{l}7.7 \\
7.8 \\
8.2 \\
8.5 \\
9.0\end{array}$ & $\begin{array}{c}46 \\
32 \\
11 \\
8.5 \\
108\end{array}$ & $\begin{array}{l}83 \\
79 \\
72 \\
58 \\
43\end{array}$ & $\begin{array}{l}32 \\
35 \\
35 \\
34 \\
28\end{array}$ & $\begin{array}{l}60 \\
56 \\
54 \\
52 \\
49\end{array}$ & $\begin{array}{r}2.510 \\
2.400 \\
1970 \\
1090 \\
981\end{array}$ & $\begin{array}{l}95 \\
92 \\
87 \\
90 \\
89\end{array}$ \\
\hline $\begin{array}{r}6 \\
7 \\
8 \\
9 \\
10\end{array}$ & $\begin{array}{l}17 \\
37 \\
28 \\
21 \\
26\end{array}$ & $\begin{array}{l}25 \\
22 \\
22 \\
27 \\
27\end{array}$ & $\begin{array}{l}13 \\
13 \\
13 \\
13 \\
13\end{array}$ & $\begin{array}{l}4.8 \\
4.7 \\
4.7 \\
4.6 \\
4.6\end{array}$ & $\begin{array}{l}6.9 \\
6.9 \\
7.0 \\
7.0 \\
7.0\end{array}$ & $\begin{array}{l}9.5 \\
10 \\
11 \\
14 \\
19\end{array}$ & $\begin{array}{r}136 \\
20 \\
17 \\
16 \\
32\end{array}$ & $\begin{array}{c}83 \\
30 \\
5.0 \\
3.5 \\
3.2\end{array}$ & $\begin{array}{l}26 \\
23 \\
29 \\
33 \\
71\end{array}$ & $\begin{array}{l}47 \\
44 \\
42 \\
41 \\
40\end{array}$ & $\begin{array}{r}668 \\
743 \\
1040 \\
488 \\
864\end{array}$ & $\begin{array}{r}84 \\
79 \\
108 \\
229 \\
211\end{array}$ \\
\hline $\begin{array}{l}11 \\
12 \\
13 \\
14 \\
15\end{array}$ & $\begin{array}{l}27 \\
28 \\
28 \\
26 \\
26\end{array}$ & $\begin{array}{l}29 \\
29 \\
30 \\
30 \\
28\end{array}$ & $\begin{array}{l}13 \\
12 \\
12 \\
12 \\
12\end{array}$ & $\begin{array}{l}4.6 \\
4.6 \\
4.6 \\
4.6 \\
4.6\end{array}$ & $\begin{array}{l}7.1 \\
7.2 \\
7.2 \\
7.2 \\
7.2\end{array}$ & $\begin{array}{l}30 \\
50 \\
80 \\
90 \\
88\end{array}$ & $\begin{array}{r}127 \\
195 \\
185 \\
119 \\
89\end{array}$ & $\begin{array}{l}3.2 \\
3.8 \\
4.2 \\
3.8 \\
3.8\end{array}$ & $\begin{array}{r}87 \\
179 \\
209 \\
177 \\
146\end{array}$ & $\begin{array}{l}39 \\
53 \\
78 \\
60 \\
72\end{array}$ & $\begin{array}{l}622 \\
584 \\
490 \\
415 \\
368\end{array}$ & $\begin{array}{l}233 \\
253 \\
213 \\
185 \\
163\end{array}$ \\
\hline $\begin{array}{l}16 \\
17 \\
18 \\
19 \\
20\end{array}$ & $\begin{array}{r}23 \\
42 \\
331 \\
210 \\
61\end{array}$ & $\begin{array}{l}28 \\
26 \\
25 \\
26 \\
28\end{array}$ & $\begin{array}{l}12 \\
12 \\
12 \\
12 \\
12\end{array}$ & $\begin{array}{l}4.6 \\
4.6 \\
4.6 \\
4.6 \\
4.6\end{array}$ & $\begin{array}{l}7.3 \\
7.4 \\
7.5 \\
7.7 \\
7.7\end{array}$ & $\begin{array}{r}90 \\
95 \\
114 \\
127 \\
106\end{array}$ & $\begin{array}{r}117 \\
92 \\
70 \\
68 \\
73\end{array}$ & $\begin{array}{l}3.0 \\
2.5 \\
4.0 \\
2.5 \\
3.3\end{array}$ & $\begin{array}{r}123 \\
106 \\
92 \\
80 \\
82\end{array}$ & $\begin{array}{l}174 \\
189 \\
151 \\
142 \\
119\end{array}$ & $\begin{array}{l}342 \\
302 \\
170 \\
225 \\
219\end{array}$ & $\begin{array}{l}143 \\
253 \\
253 \\
237 \\
249\end{array}$ \\
\hline $\begin{array}{l}21 \\
22 \\
23 \\
24 \\
25\end{array}$ & $\begin{array}{l}41 \\
38 \\
65 \\
35 \\
32\end{array}$ & $\begin{array}{c}26 \\
24 \\
9.5 \\
25 \\
25\end{array}$ & $\begin{array}{c}11 \\
10 \\
9.5 \\
9.0 \\
7.3\end{array}$ & $\begin{array}{l}4.6 \\
4.7 \\
4.7 \\
4.8 \\
4.9\end{array}$ & $\begin{array}{l}7.7 \\
7.7 \\
7.7 \\
7.7 \\
7.7\end{array}$ & $\begin{array}{r}101 \\
94 \\
100 \\
98 \\
108\end{array}$ & $\begin{array}{r}74 \\
107 \\
114 \\
108 \\
107\end{array}$ & $\begin{array}{l}14 \\
37 \\
36 \\
18 \\
24\end{array}$ & $\begin{array}{l}63 \\
53 \\
48 \\
54 \\
59\end{array}$ & $\begin{array}{r}96 \\
78 \\
126 \\
443 \\
472\end{array}$ & $\begin{array}{l}199 \\
181 \\
166 \\
148 \\
130\end{array}$ & $\begin{array}{r}555 \\
2.340 \\
3.430 \\
4.170 \\
3.240\end{array}$ \\
\hline $\begin{array}{l}26 \\
27 \\
28 \\
29 \\
30 \\
31\end{array}$ & $\begin{array}{l}31 \\
31 \\
31 \\
33 \\
31 \\
31\end{array}$ & $\begin{array}{l}18 \\
11 \\
12 \\
12 \\
12 \\
---\end{array}$ & $\begin{array}{l}6.4 \\
5.8 \\
5.4 \\
5.2 \\
5.1 \\
5.0\end{array}$ & $\begin{array}{l}5.1 \\
5.3 \\
5.6 \\
5.8 \\
6.0 \\
6.1\end{array}$ & $\begin{array}{r}7.7 \\
7.7 \\
7.7 \\
7.7 \\
- \\
\end{array}$ & $\begin{array}{l}96 \\
94 \\
88 \\
82 \\
78 \\
74\end{array}$ & $\begin{array}{r}108 \\
102 \\
99 \\
95 \\
89 \\
\end{array}$ & $\begin{array}{l}34 \\
43 \\
87 \\
50 \\
12 \\
25\end{array}$ & $\begin{array}{r}64 \\
62 \\
60 \\
66 \\
64 \\
\end{array}$ & $\begin{array}{l}1.040 \\
3.530 \\
3.540 \\
2.970 \\
2.000 \\
2.130\end{array}$ & $\begin{array}{l}119 \\
113 \\
107 \\
102 \\
104 \\
102\end{array}$ & $\begin{array}{l}2.310 \\
1.740 \\
1.470 \\
1.300 \\
1.220 \\
-----\end{array}$ \\
\hline $\begin{array}{l}\text { Total } \\
\text { Mean } \\
\text { Max } \\
\text { Min } \\
\text { Cfsm } \\
\text { In. } \\
\text { Ac-ft }\end{array}$ & $\begin{array}{r}1.428 \\
46.1 \\
331 \\
16 \\
0.036 \\
0.04 \\
2.830\end{array}$ & $\begin{array}{r}724.5 \\
24.2 \\
31 \\
9.5 \\
0.019 \\
0.02 \\
1,440\end{array}$ & $\begin{array}{r}328.7 \\
10.6 \\
13 \\
5.0 \\
0.0083 \\
0.01 \\
652\end{array}$ & $\begin{array}{r}151.5 \\
4.89 \\
6.1 \\
4.6 \\
0.0038 \\
0.004 \\
300\end{array}$ & $\begin{array}{r}210.6 \\
7.26 \\
7.7 \\
6.3 \\
0.0057 \\
0.006 \\
418\end{array}$ & $\begin{array}{r}1.987 .7 \\
64.1 \\
127 \\
7.7 \\
0.050 \\
0.06 \\
3.940\end{array}$ & $\begin{array}{r}2.564 .5 \\
85.5 \\
195 \\
8.5 \\
0.067 \\
0.07 \\
5,090\end{array}$ & $\begin{array}{r}873.8 \\
28.2 \\
87 \\
2.5 \\
0.022 \\
0.03 \\
1.730\end{array}$ & $\begin{array}{r}2220 \\
74.0 \\
209 \\
23 \\
0.058 \\
0.06 \\
4.400\end{array}$ & $\begin{array}{r}17.987 \\
580 \\
3,540 \\
39 \\
0.453 \\
0.52 \\
35,680\end{array}$ & $\begin{array}{r}17.962 \\
579 \\
2,510 \\
102 \\
0.452 \\
0.52 \\
35,630 \\
\end{array}$ & $\begin{array}{r}25.121 \\
837 \\
4,170 \\
79 \\
0.654 \\
0.73 \\
49,830 \\
\end{array}$ \\
\hline $\begin{array}{l}\text { Cal yr } \\
\text { vtr yr }\end{array}$ & & $\begin{array}{cl}\text { zal } & 64,7 \\
\text { cal } & 71,5\end{array}$ & & $\begin{array}{l}177 \\
196\end{array}$ & $\begin{array}{l}\operatorname{Max} \quad 2, \\
\operatorname{Max}\end{array}$ & $\begin{array}{l}\text { Min } \\
\text { Min }\end{array}$ & $\begin{array}{l}3.0 \\
2.5\end{array}$ & $\begin{array}{l}0.138 \\
0.153\end{array}$ & $\begin{array}{l}\text { In. } \\
\text { In. }\end{array}$ & $\begin{array}{l}1.88 \\
2.08 \\
\end{array}$ & $\begin{array}{l}\mathrm{ft} \\
\mathrm{ft}\end{array}$ & $\begin{array}{l}400 \\
, 900 \\
\end{array}$ \\
\hline
\end{tabular}




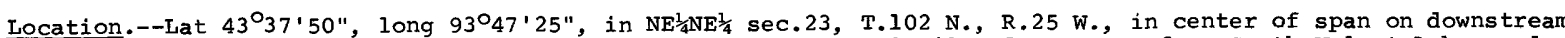
side of highway bridge, 2 miles upstream from Brush Creek, 3 miles downstream from South walnut Lake, and 5 miles northeast of Bricelyn.

Drainage area. $--132 \mathrm{sq} \mathrm{mi}$.

Records available.--March 1951 to September 1968. Prior to October 1957, published as East Fork Blue Earth River near Bricelyn.

Gage.-Wire-weight gage read twice daily. Datum of gage is $1,131.86$ ft above mean sea level, datum of 1929 (Minnesota state Highway Department bench mark).

Average discharge.--17 years, 38.0 cfs $(27,510$ acre-ft per year).

Extremes.--Maximum discharge during year, 469 cfs July 27 (gage height, $9.47 \mathrm{ft}$ ); no flow on many days.

1951-68: Maximum discharge, 1,320 cfs Apr. 7, 1951 (gage height, 10.68 ft, from graph based on gage readings); maximum gage height, $11.70 \mathrm{ft}$ Apr. 7, 1965; no flow on many days.

Remarks.--Records good except those for winter months, which are fair.

DISCHARGE, IN CFS, WATER YEAR OCTOBER 1967 TO SEPTEMBER 1968

\begin{tabular}{|c|c|c|c|c|c|c|c|c|c|c|c|c|}
\hline DAY & OCT & NOV & $\mathrm{DEC}$ & JAN & $\mathrm{FEB}$ & MAR & APR & MAY & JUN & JUL & AUG & SEP \\
\hline $\begin{array}{l}1 \\
2 \\
3 \\
4 \\
5\end{array}$ & & & & & & & $\begin{array}{l}0 \\
0 \\
0 \\
.1 \\
.1\end{array}$ & $\begin{array}{l}48 \\
38 \\
27 \\
22 \\
18\end{array}$ & $\begin{array}{l}23 \\
24 \\
24 \\
23 \\
19\end{array}$ & $\begin{array}{l}290 \\
276 \\
260 \\
244 \\
226\end{array}$ & $\begin{array}{l}345 \\
316 \\
296 \\
281 \\
263\end{array}$ & $\begin{array}{l}53 \\
49 \\
42 \\
46 \\
47\end{array}$ \\
\hline $\begin{array}{r}6 \\
7 \\
8 \\
9 \\
10\end{array}$ & & & & & & & $\begin{array}{l}.1 \\
.1 \\
.2 \\
.2 \\
.2\end{array}$ & $\begin{array}{l}15 \\
12 \\
10 \\
9.5 \\
10\end{array}$ & $\begin{array}{r}16 \\
13 \\
11 \\
62 \\
121\end{array}$ & $\begin{array}{l}207 \\
191 \\
173 \\
162 \\
156\end{array}$ & $\begin{array}{l}264 \\
307 \\
416 \\
443 \\
443\end{array}$ & $\begin{array}{l}47 \\
47 \\
49 \\
52 \\
58\end{array}$ \\
\hline $\begin{array}{l}11 \\
12 \\
13 \\
14 \\
15\end{array}$ & & & & & & & $\begin{array}{r}.3 \\
.5 \\
.6 \\
.9 \\
1.2\end{array}$ & $\begin{array}{l}8.8 \\
7.8 \\
6.9 \\
7.5 \\
6.6\end{array}$ & $\begin{array}{l}232 \\
280 \\
283 \\
274 \\
258\end{array}$ & $\begin{array}{l}149 \\
145 \\
140 \\
135 \\
131\end{array}$ & $\begin{array}{l}430 \\
400 \\
375 \\
355 \\
330\end{array}$ & $\begin{array}{l}60 \\
58 \\
54 \\
49 \\
44\end{array}$ \\
\hline $\begin{array}{l}16 \\
17 \\
18 \\
19 \\
20\end{array}$ & & & & & & & $\begin{array}{l}1.7 \\
2.5 \\
3.5 \\
5.2 \\
7.3\end{array}$ & $\begin{array}{l}6.9 \\
6.3 \\
5.7 \\
6.3 \\
6.9\end{array}$ & $\begin{array}{l}241 \\
229 \\
207 \\
191 \\
167\end{array}$ & $\begin{array}{r}128 \\
124 \\
115 \\
106 \\
98\end{array}$ & $\begin{array}{l}310 \\
295 \\
274 \\
258 \\
243\end{array}$ & $\begin{array}{r}42 \\
64 \\
94 \\
132 \\
156\end{array}$ \\
\hline $\begin{array}{l}21 \\
22 \\
23 \\
24 \\
25\end{array}$ & & & & & & & $\begin{array}{l}11 \\
16 \\
25 \\
38 \\
65\end{array}$ & $\begin{array}{l}6.3 \\
5.7 \\
5.4 \\
5.1 \\
4.5\end{array}$ & $\begin{array}{l}149 \\
131 \\
137 \\
130 \\
158\end{array}$ & $\begin{array}{r}84 \\
72 \\
159 \\
258 \\
381\end{array}$ & $\begin{array}{l}222 \\
200 \\
187 \\
154 \\
131\end{array}$ & $\begin{array}{l}180 \\
204 \\
221 \\
233 \\
243\end{array}$ \\
\hline $\begin{array}{l}26 \\
27 \\
28 \\
29 \\
30 \\
31\end{array}$ & & ----- & & & ----- & & $\begin{array}{r}88 \\
90 \\
84 \\
71 \\
58 \\
\end{array}$ & $\begin{array}{l}7.8 \\
10 \\
13 \\
16 \\
18 \\
20\end{array}$ & $\begin{array}{r}218 \\
264 \\
296 \\
310 \\
299 \\
\end{array}$ & $\begin{array}{l}462 \\
466 \\
439 \\
418 \\
395 \\
373\end{array}$ & $\begin{array}{r}114 \\
100 \\
87 \\
74 \\
64 \\
58\end{array}$ & $\begin{array}{r}237 \\
224 \\
219 \\
215 \\
211 \\
\end{array}$ \\
\hline $\begin{array}{l}\text { Total } \\
\text { Mean } \\
\text { Max } \\
\text { Min } \\
\text { Cfsm } \\
\text { In. }\end{array}$ & $\begin{array}{l}0 \\
0 \\
0 \\
0 \\
0 \\
0\end{array}$ & $\begin{array}{l}0 \\
0 \\
0 \\
0 \\
0 \\
0\end{array}$ & $\begin{array}{l}0 \\
0 \\
0 \\
0 \\
0 \\
0\end{array}$ & $\begin{array}{l}0 \\
0 \\
0 \\
0 \\
0 \\
0\end{array}$ & $\begin{array}{l}0 \\
0 \\
0 \\
0 \\
0 \\
0\end{array}$ & $\begin{array}{l}0 \\
0 \\
0 \\
0 \\
0 \\
0\end{array}$ & $\begin{array}{r}570.7 \\
19.0 \\
90 \\
0 \\
0.144 \\
0.16\end{array}$ & $\begin{array}{r}391 \\
12.6 \\
48 \\
4.5 \\
0.095 \\
0.11\end{array}$ & $\begin{array}{r}4.790 \\
160 \\
310 \\
11 \\
1.21 \\
1.35 \\
\end{array}$ & $\begin{array}{r}6963 \\
225 \\
466 \\
72 \\
1.70 \\
1.96\end{array}$ & $\begin{array}{r}8035 \\
259 \\
443 \\
58 \\
1.96 \\
2.26\end{array}$ & $\begin{array}{r}3.430 \\
114 \\
243 \\
42 \\
0.864 \\
0.97\end{array}$ \\
\hline $\begin{array}{l}\text { Cal ye } \\
\text { Wtr ye }\end{array}$ & $\begin{array}{l}\text { 7: } \\
\text { 8: }\end{array}$ & $\begin{array}{l}\text { Total } \\
\text { Total }\end{array}$ & $\begin{array}{l}19,113.8 \\
24,179.7\end{array}$ & $\begin{array}{l}\text { Mean } \\
\text { Mean }\end{array}$ & $\begin{array}{l}52.4 \\
66.1\end{array}$ & $\begin{array}{l}722 \\
466\end{array}$ & $\begin{array}{ll}\operatorname{Min} & 0 \\
\operatorname{Min} & 0\end{array}$ & $\begin{array}{l}\text { Cfsm } \\
\text { Cfsm }\end{array}$ & $\begin{array}{l}0.397 \\
0.501\end{array}$ & $\begin{array}{l}5.39 \\
6.81\end{array}$ & & \\
\hline
\end{tabular}


5-3200. Blue Earth River near Rapidan, Minn.

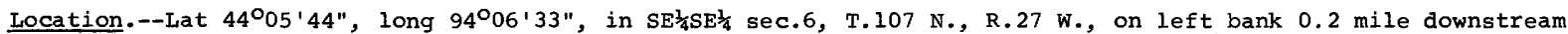

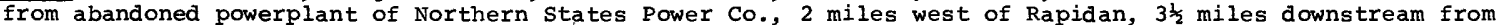
Watonwan River, and $7 \frac{3}{4}$ miles upstream from Lesueur River.

Drainage area..--2,430 sq $\mathrm{mi}$, approximately.

Records available.--July 1909 to November 1910 (no winter records), October 1939 to September 1945 , July 1949 to September 1968. Published as "at Rapidan Mills" 1909-10.

Gage.-Digital water-stage recorder. Datum of gage is 808.80 ft above mean sea level, adjustment of 1912 . July 20, 1909, to Apr. 28, 1910, chain gage at site a quarter mile upstream at different datum. Apr. 29 to Nov. 12, 1910, staff gage at site $800 \mathrm{ft}$ upstream at different datum. Oct. 4 to Nov. 14, 1939 , staff gage and Nov. 15, 1939 to June 1, 1964 graphic water-stage recorder at present site and datum.

Average discharge.--25 years $(1939-45,1949-68), 778$ cfs $(563,200$ acre-ft per year).

Extremes.--Maximum discharge during year, 3,160 cfs sept. 25 (gage height, 5.04 ft); minimum daily, 14 cfs Jan. 8-14; minimum gage height, 1.13 ft Jan. 11.

1909-10, 1939-45, 1949-68: Maximum discharge, 43,100 cfs Apr. 9, 1965 (gage height, 21.36 ft, from floodmark); minimum, 6.9 cfs oct. 12, 1955 (gage height, $1.04 \mathrm{ft}$ ).

Remarks.--Records good except those for winter months, which are fair. Flow regulated by Rapidan Reservoir. Rapidan Reservoir gates destroyed during April 1965 flood and not replaced. Capacity reduced to an undetermined figure. Regulation discontinued with closing of Northern states Power Co. plant on Dec. 31 , 1966.

OISCHARGE, IN CUBIC FEET PER SECOND, WATER YEAR OCTOBER 1967 TO SFPTEMBER 1968

\begin{tabular}{|c|c|c|c|c|c|c|c|c|c|c|c|c|}
\hline OAY & OCT & NOV & OEC & JAN & FEB & MAR & $A P R$ & MAY & JUN & JUL & AUG & SEP \\
\hline $\begin{array}{l}1 \\
2 \\
3 \\
4 \\
5\end{array}$ & $\begin{array}{l}60 \\
59 \\
57 \\
56 \\
55\end{array}$ & $\begin{array}{l}68 \\
70 \\
72 \\
69 \\
70\end{array}$ & $\begin{array}{l}54 \\
60 \\
58 \\
59 \\
59\end{array}$ & $\begin{array}{l}26 \\
26 \\
22 \\
20 \\
18\end{array}$ & $\begin{array}{l}34 \\
36 \\
38 \\
39 \\
40\end{array}$ & $\begin{array}{l}29 \\
29 \\
30 \\
34 \\
38\end{array}$ & $\begin{array}{r}101 \\
96 \\
98 \\
100 \\
96\end{array}$ & $\begin{array}{l}281 \\
253 \\
233 \\
216 \\
199\end{array}$ & $\begin{array}{l}160 \\
156 \\
150 \\
145 \\
139\end{array}$ & $\begin{array}{l}2.100 \\
1.680 \\
1.360 \\
1.150 \\
1.010\end{array}$ & $\begin{array}{l}2.600 \\
2.230 \\
2.010 \\
1.810 \\
1.530\end{array}$ & $\begin{array}{l}272 \\
261 \\
249 \\
237 \\
226\end{array}$ \\
\hline $\begin{array}{r}6 \\
7 \\
8 \\
9 \\
10\end{array}$ & $\begin{array}{l}55 \\
62 \\
61 \\
63 \\
63\end{array}$ & $\begin{array}{l}65 \\
61 \\
63 \\
68 \\
65\end{array}$ & $\begin{array}{l}58 \\
63 \\
67 \\
73 \\
73\end{array}$ & $\begin{array}{l}16 \\
15 \\
14 \\
14 \\
14\end{array}$ & $\begin{array}{l}40 \\
40 \\
39 \\
38 \\
38\end{array}$ & $\begin{array}{l}40 \\
42 \\
46 \\
60 \\
58\end{array}$ & $\begin{array}{l}89 \\
90 \\
90 \\
87 \\
85\end{array}$ & $\begin{array}{l}185 \\
173 \\
165 \\
153 \\
147\end{array}$ & $\begin{array}{l}130 \\
125 \\
120 \\
124 \\
261\end{array}$ & $\begin{array}{l}900 \\
840 \\
798 \\
756 \\
734\end{array}$ & $\begin{array}{l}1.250 \\
1.160 \\
1.090 \\
1.130 \\
1.280\end{array}$ & $\begin{array}{l}223 \\
230 \\
243 \\
248 \\
302\end{array}$ \\
\hline $\begin{array}{l}11 \\
12 \\
13 \\
14 \\
15\end{array}$ & $\begin{array}{l}65 \\
64 \\
65 \\
64 \\
64\end{array}$ & $\begin{array}{l}63 \\
62 \\
62 \\
60 \\
60\end{array}$ & $\begin{array}{l}74 \\
71 \\
46 \\
48 \\
52\end{array}$ & $\begin{array}{l}14 \\
14 \\
14 \\
14 \\
15\end{array}$ & $\begin{array}{l}37 \\
35 \\
34 \\
34 \\
33\end{array}$ & $\begin{array}{l}58 \\
6 t \\
80 \\
82 \\
88\end{array}$ & $\begin{array}{l}86 \\
86 \\
82 \\
88 \\
87\end{array}$ & $\begin{array}{l}140 \\
135 \\
130 \\
127 \\
124\end{array}$ & $\begin{array}{l}1.300 \\
1.720 \\
1.470 \\
1.480 \\
1.330\end{array}$ & $\begin{array}{l}740 \\
663 \\
645 \\
593 \\
584\end{array}$ & $\begin{array}{l}1.340 \\
1.280 \\
1.190 \\
1.110 \\
1.020\end{array}$ & $\begin{array}{l}344 \\
355 \\
332 \\
304 \\
284\end{array}$ \\
\hline $\begin{array}{l}16 \\
17 \\
18 \\
19 \\
20\end{array}$ & $\begin{array}{l}62 \\
60 \\
58 \\
59 \\
57\end{array}$ & $\begin{array}{l}59 \\
59 \\
50 \\
60 \\
60\end{array}$ & $\begin{array}{l}52 \\
57 \\
60 \\
58 \\
60\end{array}$ & $\begin{array}{l}16 \\
16 \\
17 \\
17 \\
18\end{array}$ & $\begin{array}{l}32 \\
31 \\
30 \\
29 \\
28\end{array}$ & $\begin{array}{r}96 \\
110 \\
135 \\
170 \\
135\end{array}$ & $\begin{array}{r}85 \\
90 \\
93 \\
93 \\
131\end{array}$ & $\begin{array}{l}119 \\
116 \\
119 \\
117 \\
112\end{array}$ & $\begin{array}{r}1,180 \\
1,070 \\
883 \\
744 \\
654\end{array}$ & $\begin{array}{l}548 \\
495 \\
444 \\
430 \\
470\end{array}$ & $\begin{array}{l}929 \\
847 \\
783 \\
739 \\
684\end{array}$ & $\begin{array}{r}279 \\
351 \\
578 \\
961 \\
1,560\end{array}$ \\
\hline $\begin{array}{l}21 \\
22 \\
23 \\
24 \\
25\end{array}$ & $\begin{array}{l}59 \\
55 \\
57 \\
57 \\
56\end{array}$ & $\begin{array}{l}60 \\
59 \\
45 \\
61 \\
63\end{array}$ & $\begin{array}{l}62 \\
44 \\
40 \\
45 \\
46\end{array}$ & $\begin{array}{l}19 \\
20 \\
22 \\
25 \\
25\end{array}$ & $\begin{array}{l}28 \\
27 \\
27 \\
26 \\
26\end{array}$ & $\begin{array}{l}171 \\
120 \\
140 \\
135 \\
145\end{array}$ & $\begin{array}{l}134 \\
154 \\
258 \\
278 \\
331\end{array}$ & $\begin{array}{lll}1 & 1 & 1 \\
1 & 1 & 1 \\
1 & 0 & 6 \\
1 & 02 \\
1 & 0 & 0\end{array}$ & $\begin{array}{l}572 \\
496 \\
454 \\
440 \\
437\end{array}$ & $\begin{array}{r}431 \\
374 \\
768 \\
1.440 \\
2.260\end{array}$ & $\begin{array}{l}644 \\
606 \\
547 \\
508 \\
459\end{array}$ & $\begin{array}{l}2,020 \\
2,050 \\
2,200 \\
2,700 \\
3,100\end{array}$ \\
\hline $\begin{array}{l}26 \\
27 \\
28 \\
29 \\
30 \\
31\end{array}$ & $\begin{array}{l}59 \\
58 \\
59 \\
68 \\
69 \\
70\end{array}$ & $\begin{array}{r}48 \\
28 \\
49 \\
50 \\
54 \\
\end{array}$ & $\begin{array}{l}40 \\
35 \\
35 \\
34 \\
32 \\
30\end{array}$ & $\begin{array}{l}26 \\
27 \\
29 \\
30 \\
31 \\
33\end{array}$ & $\begin{array}{r}26 \\
27 \\
28 \\
28 \\
\\
\end{array}$ & $\begin{array}{l}127 \\
132 \\
126 \\
118 \\
115 \\
108\end{array}$ & $\begin{array}{r}437 \\
436 \\
374 \\
330 \\
305 \\
\end{array}$ & $\begin{array}{l}113 \\
114 \\
113 \\
123 \\
133 \\
147\end{array}$ & $\begin{array}{r}593 \\
1,430 \\
2,150 \\
2,430 \\
2,430 \\
\end{array}$ & $\begin{array}{l}2.590 \\
2,330 \\
2.400 \\
2.810 \\
3.030 \\
2.960\end{array}$ & $\begin{array}{l}424 \\
391 \\
343 \\
312 \\
293 \\
278\end{array}$ & $\begin{array}{r}3,030 \\
2,630 \\
2,240 \\
2,030 \\
2,230 \\
\end{array}$ \\
\hline $\begin{array}{l}\text { TOTAL } \\
\text { MEAN } \\
\text { MAX } \\
\text { MIN } \\
\text { CFSM } \\
\text { IN. } \\
\text { AC-FT }\end{array}$ & $\begin{array}{r}1.876 \\
60.5 \\
70 \\
55 \\
.02 \\
.03 \\
3.720\end{array}$ & $\begin{array}{r}1.770 \\
59.7 \\
72 \\
28 \\
.02 \\
.03 \\
3.550\end{array}$ & $\begin{array}{r}1.645 \\
53.1 \\
74 \\
30 \\
.02 \\
.03 \\
3.260\end{array}$ & $\begin{array}{r}627 \\
20.2 \\
33 \\
14 \\
.008 \\
.01 \\
1.240\end{array}$ & $\begin{array}{r}948 \\
32.7 \\
40 \\
26 \\
.01 \\
.01 \\
1.880\end{array}$ & $\begin{array}{r}2.863 \\
92.4 \\
171 \\
29 \\
.04 \\
.04 \\
5.680\end{array}$ & $\begin{array}{r}4.890 \\
163 \\
437 \\
82 \\
.07 \\
.07 \\
9.700\end{array}$ & $\begin{array}{r}4.516 \\
146 \\
281 \\
100 \\
.06 \\
.07 \\
8.960\end{array}$ & $\begin{array}{r}24.773 \\
826 \\
2.430 \\
120 \\
.34 \\
.38 \\
49,140\end{array}$ & $\begin{array}{r}38.333 \\
1,237 \\
3,030 \\
374 \\
.51 \\
.59 \\
76,030\end{array}$ & $\begin{array}{r}30.817 \\
994 \\
2.600 \\
278 \\
.41 \\
.47 \\
61.120\end{array}$ & $\begin{array}{r}32.069 \\
1.069 \\
3.100 \\
223 \\
.44 \\
.49 \\
63.610\end{array}$ \\
\hline
\end{tabular}

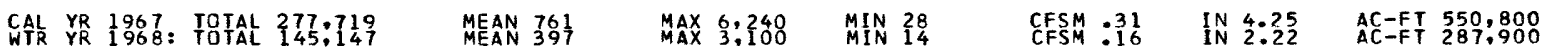


5-3205. Le Sueur River near Rapidan, Minn.

Location.--Lat $44^{\circ} 06^{\prime} 40^{\prime \prime}$, long $94^{\circ} 02^{\prime} 28^{\prime \prime}$, in sw $\frac{1}{4}$ sec.35, T.108 N., R. 27 w., on right bank 600 ft downstream

from highway bridge, 1.8 miles northeast of Rapidan, and 2.3 miles upstream from mouth.

Drainage area.--1, $100 \mathrm{sq} \mathrm{mi}$, approximately.

Records available.--October 1939 to September 1945, July 1949 to September 1968.

Gage.--Water-stage recorder. Datum of gage is $775.76 \mathrm{ft}$ above mean sea level, datum of 1929 . Prior to Nov. 15, 1939, staff gage at same site and datum.

Average discharge. --25 years, $389 \mathrm{cfs}(281,600$ acre-ft per year).

Extremes.--Maximum discharge during year, 10,900 cfs July 26 (gage height, $13.54 \mathrm{ft}$ ); minimum daily, 6.4 cfs Jan. 17,18

1939-45, 1949-68: Maximum discharge, 24,700 cfs Apr. 8, 1965 (gage height, 22.10 ft, from floodmark); maximum gage height, 22.72 ft May 22, 1960 (from floodmark); minimum daily discharge, 1.6 cfs Feb. 9-25, 1959; minimum gage height, 1.59 ft Nov. 20, 1965.

Remarks.--Records are fair. Records of chemical analyses for the water year 1968 are published in Part 2 of this report.

DISCHARGE, IN CFS, WATER YEAR OCTOBER 1967 TO SEPTEMBER 1968

\begin{tabular}{|c|c|c|c|c|c|c|c|c|c|c|c|c|}
\hline DAY & $\mathrm{OCT}$ & NOV & DEC & JAN & $\mathrm{F} E \mathrm{~B}$ & MAR & APR & MAY & JUN & JUL & AUG & SEP \\
\hline $\begin{array}{l}1 \\
2 \\
3 \\
4 \\
5\end{array}$ & $\begin{array}{l}28 \\
32 \\
29 \\
28 \\
26\end{array}$ & $\begin{array}{l}31 \\
34 \\
34 \\
34 \\
33\end{array}$ & $\begin{array}{l}29 \\
29 \\
29 \\
29 \\
30\end{array}$ & $\begin{array}{l}13 \\
12 \\
12 \\
11 \\
11\end{array}$ & $\begin{array}{l}9.0 \\
9.2 \\
9.3 \\
9.4 \\
9.5\end{array}$ & $\begin{array}{l}9.9 \\
10 \\
11 \\
12 \\
13\end{array}$ & $\begin{array}{l}51 \\
48 \\
50 \\
50 \\
45\end{array}$ & $\begin{array}{l}230 \\
212 \\
195 \\
182 \\
174\end{array}$ & $\begin{array}{l}150 \\
148 \\
155 \\
167 \\
164\end{array}$ & $\begin{array}{r}1.410 \\
1.220 \\
1.030 \\
876 \\
732\end{array}$ & $\begin{array}{l}2.730 \\
2.330 \\
2.020 \\
1.770 \\
1.650\end{array}$ & $\begin{array}{l}370 \\
332 \\
305 \\
297 \\
355\end{array}$ \\
\hline $\begin{array}{r}6 \\
7 \\
8 \\
9 \\
10\end{array}$ & $\begin{array}{l}28 \\
30 \\
30 \\
30 \\
28\end{array}$ & $\begin{array}{l}31 \\
31 \\
28 \\
28 \\
31\end{array}$ & $\begin{array}{l}31 \\
31 \\
33 \\
33 \\
32\end{array}$ & $\begin{array}{c}10 \\
9.5 \\
9.0 \\
8.5 \\
8.0\end{array}$ & $\begin{array}{l}9.5 \\
9.4 \\
9.3 \\
9.2 \\
9.2\end{array}$ & $\begin{array}{l}14 \\
16 \\
18 \\
20 \\
22\end{array}$ & $\begin{array}{l}41 \\
41 \\
41 \\
40 \\
37\end{array}$ & $\begin{array}{l}162 \\
146 \\
137 \\
126 \\
120\end{array}$ & $\begin{array}{l}148 \\
130 \\
118 \\
200 \\
254\end{array}$ & $\begin{array}{l}512 \\
466 \\
350 \\
301 \\
285\end{array}$ & $\begin{array}{l}1.510 \\
5.790 \\
6.120 \\
5.800 \\
7.000\end{array}$ & $\begin{array}{r}380 \\
395 \\
509 \\
1190 \\
1310\end{array}$ \\
\hline $\begin{array}{l}11 \\
12 \\
13 \\
14 \\
15\end{array}$ & $\begin{array}{l}28 \\
27 \\
24 \\
23 \\
24\end{array}$ & $\begin{array}{l}31 \\
31 \\
32 \\
32 \\
31\end{array}$ & $\begin{array}{l}32 \\
30 \\
29 \\
29 \\
28\end{array}$ & $\begin{array}{l}7.6 \\
7.5 \\
7.2 \\
6.8 \\
6.6\end{array}$ & $\begin{array}{l}9.1 \\
9.1 \\
9.0 \\
8.8 \\
8.7\end{array}$ & $\begin{array}{l}24 \\
25 \\
26 \\
30 \\
35\end{array}$ & $\begin{array}{l}36 \\
35 \\
33 \\
37 \\
36\end{array}$ & $\begin{array}{l}114 \\
110 \\
103 \\
100 \\
96\end{array}$ & $\begin{array}{r}436 \\
816 \\
1.040 \\
1.100 \\
1.030\end{array}$ & $\begin{array}{r}438 \\
522 \\
588 \\
1.500 \\
1.880\end{array}$ & $\begin{array}{l}7.050 \\
5.650 \\
4.360 \\
3.450 \\
2.830\end{array}$ & $\begin{array}{r}1.19 C \\
1.04 C \\
912 \\
780 \\
654\end{array}$ \\
\hline $\begin{array}{l}16 \\
17 \\
18 \\
19 \\
20\end{array}$ & $\begin{array}{l}24 \\
28 \\
24 \\
23 \\
22\end{array}$ & $\begin{array}{l}32 \\
32 \\
31 \\
31 \\
31\end{array}$ & $\begin{array}{l}28 \\
28 \\
27 \\
26 \\
25\end{array}$ & $\begin{array}{l}6.5 \\
6.4 \\
6.4 \\
6.5 \\
6.6\end{array}$ & $\begin{array}{l}8.6 \\
8.5 \\
8.4 \\
8.2 \\
8.2\end{array}$ & $\begin{array}{l}40 \\
48 \\
60 \\
70 \\
75\end{array}$ & $\begin{array}{l}36 \\
36 \\
37 \\
38 \\
64\end{array}$ & $\begin{array}{l}103 \\
129 \\
265 \\
230 \\
192\end{array}$ & $\begin{array}{l}954 \\
918 \\
768 \\
582 \\
460\end{array}$ & $\begin{array}{l}2.000 \\
1.700 \\
1.490 \\
1.400 \\
1.270\end{array}$ & $\begin{array}{l}2.480 \\
2.200 \\
2.000 \\
1.800 \\
1.720\end{array}$ & $\begin{array}{r}558 \\
700 \\
1.140 \\
1.890 \\
2.090\end{array}$ \\
\hline $\begin{array}{l}21 \\
22 \\
23 \\
24 \\
25\end{array}$ & $\begin{array}{l}22 \\
22 \\
23 \\
29 \\
27\end{array}$ & $\begin{array}{l}33 \\
33 \\
32 \\
34 \\
32\end{array}$ & $\begin{array}{l}24 \\
22 \\
21 \\
20 \\
19\end{array}$ & $\begin{array}{l}6.7 \\
6.8 \\
7.0 \\
7.2 \\
7.4\end{array}$ & $\begin{array}{l}8.2 \\
8.2 \\
8.3 \\
8.4 \\
8.6\end{array}$ & $\begin{array}{l}77 \\
76 \\
75 \\
74 \\
73\end{array}$ & $\begin{array}{r}70 \\
96 \\
286 \\
485 \\
642\end{array}$ & $\begin{array}{l}174 \\
155 \\
139 \\
118 \\
108\end{array}$ & $\begin{array}{l}370 \\
305 \\
258 \\
237 \\
234\end{array}$ & $\begin{array}{r}1.080 \\
870 \\
1.820 \\
3.430 \\
5.910\end{array}$ & $\begin{array}{l}1.700 \\
1.490 \\
1.340 \\
1.200 \\
1.050\end{array}$ & $\begin{array}{l}2.150 \\
2.200 \\
2.670 \\
3.320 \\
3.450\end{array}$ \\
\hline $\begin{array}{l}26 \\
27 \\
28 \\
29 \\
30 \\
31\end{array}$ & $\begin{array}{l}26 \\
28 \\
28 \\
30 \\
32 \\
32\end{array}$ & $\begin{array}{r}30 \\
31 \\
31 \\
30 \\
28 \\
\end{array}$ & $\begin{array}{l}18 \\
17 \\
16 \\
15 \\
14 \\
13\end{array}$ & $\begin{array}{l}7.6 \\
7.8 \\
8.0 \\
8.2 \\
8.4 \\
8.8\end{array}$ & $\begin{array}{r}8.8 \\
9.0 \\
9.2 \\
9.6 \\
-0--\end{array}$ & $\begin{array}{l}71 \\
70 \\
68 \\
66 \\
56 \\
56\end{array}$ & $\begin{array}{r}672 \\
522 \\
390 \\
328 \\
265 \\
-\end{array}$ & $\begin{array}{l}108 \\
108 \\
110 \\
124 \\
143 \\
148\end{array}$ & $\begin{array}{r}305 \\
758 \\
1.310 \\
1.580 \\
1.570\end{array}$ & $\begin{array}{r}10.000 \\
9.300 \\
7.320 \\
5.640 \\
4.210 \\
3.360\end{array}$ & $\begin{array}{l}894 \\
750 \\
624 \\
516 \\
444 \\
395\end{array}$ & $\begin{array}{r}3.280 \\
2.800 \\
2.480 \\
2.270 \\
2.060 \\
\end{array}$ \\
\hline $\begin{array}{l}\text { Total } \\
\text { Mean } \\
\text { Max } \\
\text { Min } \\
\text { Cfsm } \\
\text { In. } \\
\text { Ac-ft }\end{array}$ & $\begin{array}{r}835 \\
26.9 \\
32 \\
22 \\
0.024 \\
0.03 \\
1,660\end{array}$ & $\begin{array}{r}943 \\
31.4 \\
34 \\
28 \\
0.029 \\
0.03 \\
1,870\end{array}$ & $\begin{array}{r}787 \\
25.4 \\
33 \\
13 \\
0.023 \\
0.03 \\
1,560\end{array}$ & $\begin{array}{r}256.0 \\
8.26 \\
13 \\
6.4 \\
0.0075 \\
0.009 \\
508\end{array}$ & $\begin{array}{r}257.9 \\
8.89 \\
9.6 \\
8.2 \\
0.0081 \\
0.009 \\
512\end{array}$ & $\begin{array}{r}1.340 .9 \\
43.3 \\
75 \\
9.9 \\
0.039 \\
0.05 \\
2,660\end{array}$ & $\begin{array}{r}4.588 \\
153 \\
672 \\
33 \\
0.139 \\
0.16 \\
9,100\end{array}$ & $\begin{array}{r}4.561 \\
147 \\
265 \\
96 \\
0.134 \\
0.15 \\
9,050\end{array}$ & $\begin{array}{r}15.665 \\
556 \\
1,580 \\
118 \\
0.505 \\
0.56 \\
33,050\end{array}$ & $\begin{array}{r}73,010 \\
2,355 \\
10,000 \\
285 \\
2.14 \\
2.47 \\
144,800\end{array}$ & $\begin{array}{r}80.663 \\
2,602 \\
7,050 \\
395 \\
2.37 \\
2.73 \\
160,000\end{array}$ & $\begin{array}{r}43,077 \\
1,436 \\
3,450 \\
297 \\
1.31 \\
1.46 \\
85,440\end{array}$ \\
\hline $\begin{array}{ll}\text { Cal yr } \\
\text { wtr yr }\end{array}$ & $\begin{array}{l}967: \\
968:\end{array}$ & $\begin{array}{l}194 \\
226\end{array}$ & $\begin{array}{l}3 \\
3.8\end{array}$ & $\begin{array}{ll}\text { lean } & 533 \\
\text { lean } & 620\end{array}$ & $\begin{array}{l}\operatorname{Max} \\
\operatorname{Max}\end{array}$ & $\begin{array}{r}8,690 \\
10,000\end{array}$ & $\begin{array}{lr}\operatorname{Min} & 13 \\
\operatorname{Min} & 6.4\end{array}$ & $\begin{array}{l}\text { Cfsm } \\
\text { Cfsm }\end{array}$ & $\begin{array}{l}0.485 \\
0.564\end{array}$ & $\begin{array}{ll}\text { In. } & 6.58 \\
\text { In. } & 7.67\end{array}$ & $\begin{array}{l}A c-f t \\
A c-f t\end{array}$ & $\begin{array}{l}385,900 \\
450,100\end{array}$ \\
\hline
\end{tabular}

Peak discharge (base, 1,300 efs)

\begin{tabular}{|c|c|r|c||c|c|c|c|}
\hline Date & Time & $\begin{array}{c}\text { Gage } \\
\text { height }\end{array}$ & Discharge & Date & Time & $\begin{array}{c}\text { Gage } \\
\text { height }\end{array}$ & Discharge \\
\hline $6-29$ & 2200 & 5.04 & 1,610 & $8-11$ & 0100 & 11.17 & 7,420 \\
$7-16$ & 0800 & 5.67 & 2,030 & $9-10$ & 0200 & 4.60 & 1,350 \\
$7-26$ & 1030 & 13.54 & 10,900 & $9-25$ & 1200 & 7.52 & 3,470 \\
\hline
\end{tabular}


5-3250. Minnesota River at Mankato, Minn.

Location.--Lat $44^{\circ} 10^{\prime} 10^{\prime \prime}$, long $94^{\circ} 00^{\prime} 15^{\prime \prime}$, in sec.7, T.108 N., R.26 W., on left bank at downstream side of Main street Bridge in Mankato, 1.8 miles downstream from Blue Earth River and at mile 106.4 upstream from Mississippi River.

Drainage area. $--14,900 \mathrm{sq} \mathrm{mi}$, approximately.

Records available.--May 1903 to september 1968 (no winter records 1904, 1906-10, 1918-29). Monthly discharge only for some periods, published in wSP 1308. Published as "near Mankato" $1903-21$.

Gage.--Digital water-stage recorder. Datum of gage is 747.92 ft above mean sea level, datum of 1929 . Prior to Aug. 6, 1910, staff gage and Aug. 6, 1910, to Oct. 19, 1921, chain gage, at site 1.8 miles upstream at datum 6.4 ft higher. Mar. 15, 1922, to Nov. 30, 1924, chain gage at present site and datum. Mar. 18, 1925, to Dec. 2, 1964, graphic water-stage recorder at present site and datum.

Average discharge.--47 years (1905, 1910-17, 1929-68), 2,494 cfs (1,806,000 acre-ft per year).

Extremes:--Maximum discharge during year, 15,800 cfs July 27 (gage height, 14.65 ft); minimum, $76 \mathrm{cfs}$ Nov. 27 (gage height, $1.84 \mathrm{ft}$ ).

1903-68: Maximum discharge, 94,100 cfs Apr. 10, 1965 (gage height, 29.09 ft); minimum observed, 26 cfs Aug. 4, 1934 Max

Remarks.--Records good except those for winter months, which are fair. Records of suspended sediment loads for the water year 1968 are published in Part 2 of this report.

DISCHARGE, IN CUBIC FEET PER SECOND, WATER YEAR OCTOBER 1967 TO SFPTEMBER 1968

\begin{tabular}{|c|c|c|c|c|c|c|c|c|c|c|c|c|}
\hline DAY & $\mathrm{OCT}$ & NOV & DEC & JAN & $F E B$ & MAR & APR & MAY & JUN & JUL & AUG & SEP \\
\hline $\begin{array}{l}1 \\
2 \\
3 \\
4 \\
5\end{array}$ & $\begin{array}{l}264 \\
260 \\
269 \\
256 \\
244\end{array}$ & $\begin{array}{l}322 \\
325 \\
322 \\
325 \\
323\end{array}$ & $\begin{array}{l}292 \\
295 \\
289 \\
313 \\
364\end{array}$ & $\begin{array}{l}150 \\
140 \\
130 \\
120 \\
110\end{array}$ & $\begin{array}{l}133 \\
135 \\
135 \\
138 \\
139\end{array}$ & $\begin{array}{l}136 \\
140 \\
144 \\
150 \\
157\end{array}$ & $\begin{array}{l}948 \\
843 \\
801 \\
811 \\
759\end{array}$ & $\begin{array}{l}1,730 \\
1.620 \\
1.540 \\
1,450 \\
1.340\end{array}$ & $\begin{array}{l}874 \\
885 \\
927 \\
990 \\
990\end{array}$ & $\begin{array}{l}4,030 \\
3,490 \\
3,000 \\
2.680 \\
2.420\end{array}$ & $\begin{array}{r}10.800 \\
9.780 \\
8.560 \\
7.220 \\
5.840\end{array}$ & $\begin{array}{l}1,320 \\
1,240 \\
1,190 \\
1,180 \\
1,210\end{array}$ \\
\hline $\begin{array}{r}5 \\
7 \\
8 \\
9 \\
10\end{array}$ & $\begin{array}{l}240 \\
264 \\
274 \\
282 \\
260\end{array}$ & $\begin{array}{l}318 \\
311 \\
310 \\
322 \\
327\end{array}$ & $\begin{array}{l}308 \\
328 \\
334 \\
345 \\
350\end{array}$ & $\begin{array}{r}102 \\
96 \\
89 \\
89 \\
88\end{array}$ & $\begin{array}{l}139 \\
140 \\
140 \\
140 \\
139\end{array}$ & $\begin{array}{l}163 \\
180 \\
198 \\
210 \\
225\end{array}$ & $\begin{array}{l}822 \\
874 \\
811 \\
790 \\
769\end{array}$ & $\begin{array}{l}1.220 \\
1,160 \\
1.120 \\
1.080 \\
1.030\end{array}$ & $\begin{array}{r}937 \\
874 \\
843 \\
937 \\
1.290\end{array}$ & $\begin{array}{l}2.210 \\
2.040 \\
1.890 \\
1.760 \\
1.650\end{array}$ & $\begin{array}{r}5,080 \\
12,000 \\
12,300 \\
11.200 \\
11,500\end{array}$ & $\begin{array}{l}1,230 \\
1,270 \\
1,510 \\
1,960 \\
2,260\end{array}$ \\
\hline $\begin{array}{l}11 \\
12 \\
13 \\
14 \\
15\end{array}$ & $\begin{array}{l}260 \\
269 \\
271 \\
275 \\
279\end{array}$ & $\begin{array}{l}327 \\
332 \\
330 \\
327 \\
323\end{array}$ & $\begin{array}{l}355 \\
358 \\
303 \\
236 \\
250\end{array}$ & $\begin{array}{r}87 \\
88 \\
91 \\
96 \\
100\end{array}$ & $\begin{array}{l}138 \\
138 \\
137 \\
135 \\
135\end{array}$ & $\begin{array}{l}24 C \\
260 \\
300 \\
330 \\
355\end{array}$ & $\begin{array}{r}811 \\
948 \\
1,050 \\
1.090 \\
990\end{array}$ & $\begin{array}{l}969 \\
916 \\
843 \\
822 \\
811\end{array}$ & $\begin{array}{l}2.380 \\
3.610 \\
3.680 \\
3.680 \\
3.430\end{array}$ & $\begin{array}{l}1.760 \\
1,730 \\
1,860 \\
2.270 \\
2,680\end{array}$ & $\begin{array}{r}11.400 \\
9.450 \\
7.640 \\
6.330 \\
5.380\end{array}$ & $\begin{array}{l}2,280 \\
2,160 \\
1,990 \\
1,800 \\
1,620\end{array}$ \\
\hline $\begin{array}{l}16 \\
17 \\
18 \\
19 \\
20\end{array}$ & $\begin{array}{l}278 \\
285 \\
285 \\
362 \\
478\end{array}$ & $\begin{array}{l}329 \\
342 \\
351 \\
345 \\
338\end{array}$ & $\begin{array}{l}270 \\
285 \\
295 \\
290 \\
300\end{array}$ & $\begin{array}{l}103 \\
108 \\
110 \\
112 \\
115\end{array}$ & $\begin{array}{l}134 \\
132 \\
132 \\
131 \\
128\end{array}$ & $\begin{array}{l}405 \\
480 \\
660 \\
700 \\
723\end{array}$ & $\begin{array}{r}916 \\
948 \\
927 \\
874 \\
1,000\end{array}$ & $\begin{array}{l}822 \\
759 \\
927 \\
958 \\
885\end{array}$ & $\begin{array}{l}3,060 \\
2,840 \\
2,570 \\
2,340 \\
2,180\end{array}$ & $\begin{array}{l}3.050 \\
2.930 \\
2.640 \\
2.540 \\
2.460\end{array}$ & $\begin{array}{r}4.730 \\
4.200 \\
3.740 \\
3.310 \\
3.160\end{array}$ & $\begin{array}{l}1.540 \\
1.980 \\
2.720 \\
3.740 \\
4.380\end{array}$ \\
\hline $\begin{array}{l}21 \\
22 \\
23 \\
24 \\
25\end{array}$ & $\begin{array}{l}357 \\
303 \\
289 \\
312 \\
302\end{array}$ & $\begin{array}{l}344 \\
342 \\
319 \\
323 \\
338\end{array}$ & $\begin{array}{l}280 \\
220 \\
230 \\
251 \\
250\end{array}$ & $\begin{array}{l}117 \\
118 \\
120 \\
121 \\
125\end{array}$ & $\begin{array}{l}128 \\
128 \\
128 \\
128 \\
129\end{array}$ & $\begin{array}{r}700 \\
696 \\
601 \\
853 \\
1.65 C\end{array}$ & $\begin{array}{l}1,040 \\
1,220 \\
1,760 \\
1.850 \\
1,970\end{array}$ & $\begin{array}{l}822 \\
801 \\
769 \\
738 \\
696\end{array}$ & $\begin{array}{l}2.060 \\
1.900 \\
1.790 \\
1.760 \\
1.760\end{array}$ & $\begin{array}{l}2.180 \\
1,870 \\
2.640 \\
5.260 \\
8,170\end{array}$ & $\begin{array}{l}2.940 \\
2.670 \\
2.430 \\
2.260 \\
2.080\end{array}$ & $\begin{array}{r}4.920 \\
5.860 \\
7.980 \\
10.200 \\
11.400\end{array}$ \\
\hline $\begin{array}{l}26 \\
27 \\
28 \\
29 \\
30 \\
31\end{array}$ & $\begin{array}{l}286 \\
289 \\
286 \\
312 \\
330 \\
325\end{array}$ & $\begin{array}{r}313 \\
170 \\
269 \\
281 \\
291 \\
\end{array}$ & $\begin{array}{l}230 \\
205 \\
190 \\
180 \\
170 \\
160\end{array}$ & $\begin{array}{l}125 \\
127 \\
129 \\
130 \\
131 \\
132\end{array}$ & $\begin{array}{r}129 \\
130 \\
130 \\
131 \\
\end{array}$ & $\begin{array}{l}1,210 \\
1.240 \\
1,130 \\
1,060 \\
1,020 \\
1,010\end{array}$ & $\begin{array}{r}2,180 \\
2,190 \\
2,130 \\
2,000 \\
1,860 \\
\end{array}$ & $\begin{array}{l}738 \\
769 \\
769 \\
811 \\
822 \\
822\end{array}$ & $\begin{array}{r}1.840 \\
2.590 \\
3.670 \\
4.260 \\
4.350 \\
\end{array}$ & $\begin{array}{l}13,000 \\
15,600 \\
15,200 \\
14,100 \\
12,500 \\
11.400\end{array}$ & $\begin{array}{l}1.890 \\
1.730 \\
1.570 \\
1.460 \\
1.400 \\
1.350\end{array}$ & $\begin{array}{r}10,800 \\
9,170 \\
7.590 \\
6.590 \\
6.110 \\
\end{array}$ \\
\hline $\begin{array}{l}\text { TOTAL } \\
\text { MEAN } \\
\text { YAX } \\
\text { MIN } \\
\text { CFSM } \\
\text { IN. } \\
\text { AC-FT }\end{array}$ & $\begin{array}{r}9.055 \\
292 \\
478 \\
240 \\
.02 \\
.02 \\
17.960\end{array}$ & $\begin{array}{r}9.539 \\
318 \\
351 \\
170 \\
.02 \\
.02 \\
18.920\end{array}$ & $\begin{array}{r}8,466 \\
273 \\
358 \\
160 \\
.02 \\
.02 \\
16.790\end{array}$ & $\begin{array}{r}3.499 \\
113 \\
15 \mathrm{C} \\
87 \\
.008 \\
.009 \\
6.940\end{array}$ & $\begin{array}{r}3.379 \\
134 \\
140 \\
128 \\
.009 \\
.01 \\
7.690\end{array}$ & $\begin{array}{r}16.666 \\
538 \\
1.240 \\
136 \\
.04 \\
.04 \\
33.060\end{array}$ & $\begin{array}{r}35,982 \\
1.199 \\
2.190 \\
759 \\
.08 \\
.09 \\
71.370\end{array}$ & $\begin{array}{r}30,559 \\
986 \\
1.730 \\
696 \\
.07 \\
.08 \\
60,610\end{array}$ & $\begin{array}{r}65,297 \\
2.177 \\
4.350 \\
843 \\
.15 \\
.16 \\
129.500\end{array}$ & $\begin{array}{r}151.010 \\
4.871 \\
15,600 \\
1.650 \\
.33 \\
.38 \\
299.500\end{array}$ & $\begin{array}{r}175.400 \\
5.658 \\
12.300 \\
1.350 \\
.38 \\
.44 \\
347.900\end{array}$ & $\begin{array}{r}119,200 \\
3,973 \\
11,400 \\
1.180 \\
.27 \\
.30 \\
236,400\end{array}$ \\
\hline
\end{tabular}

CAL YR 1967 TOTAL 1.014 .268 MEAN 2.779 MAX 18.300 MIN 160 CFSM . 19 IN 2.53 AC-FT 2.012.000

WTR YR 1968: TJTAL 628,552 MEAN 1.717 MAX 15.6C0 MIN $87 \quad$ CFSM .12 $\quad$ IN $1.57 \quad$ AC-FT 1.247 .000 
5-3300. Minnesota River near Jordan, Minn.

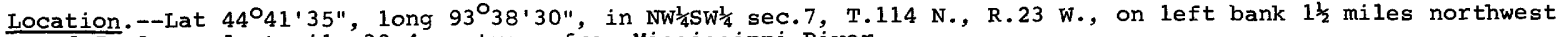
of Jordan and at mile' 39.4 upstream from Mississippi River.

Drainage area. $--16,200 \mathrm{sq} \mathrm{mi}$, approximate $1 \mathrm{y}$.

Records available.--September 1934 to September 1968. Prior to Oct. 1, 1966, pub1:shed as "near Carver, Minn".

Gage.--Digital water-stage recorder. Datum of gage is $690.00 \mathrm{ft}$ above mean sea leve1, datum of 1929 . Auxiliary water-stage recorder $2 \frac{3}{4}$ miles downstream at same datum. Prior to Oct. 1, 1966, graphic water-stage recorder

$2 \frac{3}{4}$ miles downstream with auxiliary chain gage at present site and same datum.

Average discharge.--34 years, 3,151 cfs $(2,281,000$ acre-ft per year).

Extremes.--Maximum discharge during year, 15,700 cfs Aug. 11, 12; maximum gage height, 20.03 ft Aug 11;

minimum daily discharge, 185 cfs Jan. 14-17; minimum gage height, 3.89 ft Nov. 29, 30, Dec. 23, 24, 265 (back-

1934-68: Maximum discharge, 117,000 cfs Apr. 11, 1965; maximum gage height, 34.37 ft Apr. 12, 1965

water from Mississippi River); minimum discharge, 79 cfs Nov. 17, 1955; minimum gage height, 2.66 ft

Nov. 22, 1935 .

Remarks.--Records fair. Records of chemical analyses for the water year 1968 are published in Part 2 of this report.

DISCHARGE, IN CUBIC FEET PER SECOND, WATER YEAR OCTORER 1967 TO SEPTEMBER 1968

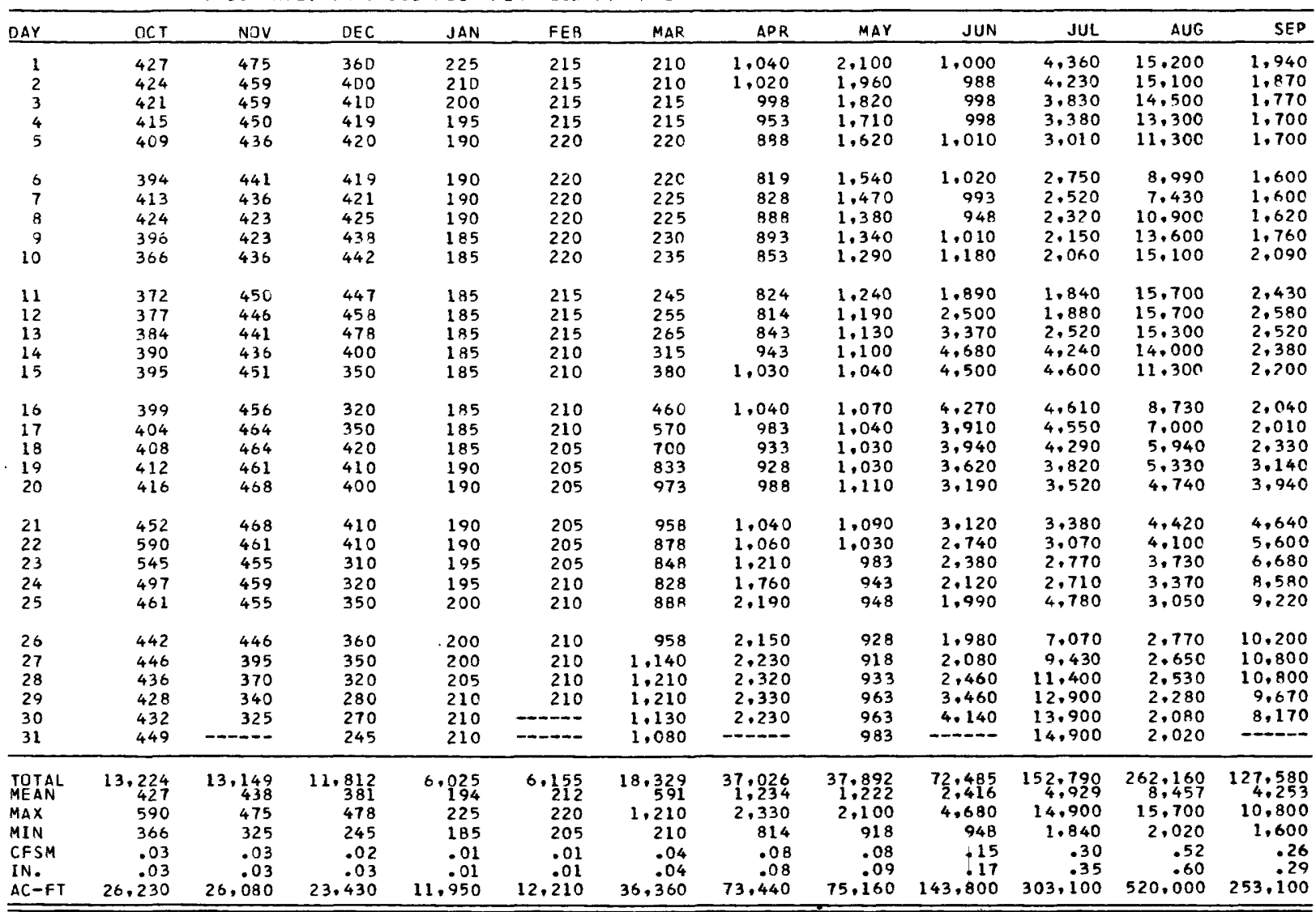

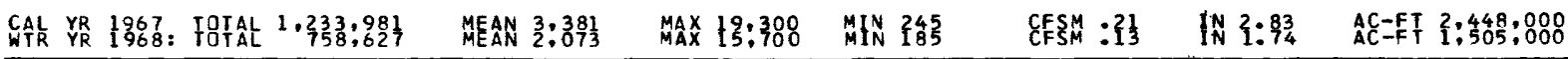


5-3309. Nine Mile Creek at Bloomington, Minn.

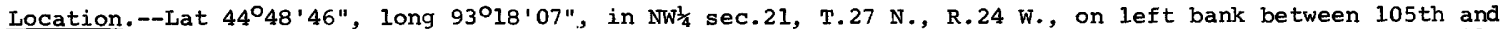
106 th street in Bloomington, Minn., 1.2 miles downstream from bridge on old shakopee Road and 2.1 miles upstream from mouth.

Records available.--January 1963 to September 1968.

Gage.--Digital water-stage recorder. Altitude of gage is 731 ft (from topographic map). Prior to May 16, 1963, staff gage $30 \mathrm{ft}$ upstream at datum $0.81 \mathrm{ft}$ higher. May 16, 1963, to Dec. 13, 1965, graphic water-stage recorder at present site and datum.

Extremes.--Maximum discharge during year, 298 cfs June 13, July 14; maximum gage height, 3.72 ft June 13; minimum daily discharge, $1.7 \mathrm{cfs}$ Feb. 16 to Mar. 4.

1963-68: Maximum discharge, 535 cfs Apr. 8, 1965 (gage height, 4.32 ft); minimum daily, 1.2 cfs Feb. 24, 1965; minimum gage height, $1.28 \mathrm{ft}$ oct. 14, 1966.

Remarks.--Records good except those for winter months and those for period of no gage-height record, which are fair.

DISCHARGE, IN CUBIC FEET PER SECOND, WATER YEAR OCTOBER 1967 TO SEPTEMBER 1968

\begin{tabular}{|c|c|c|c|c|c|c|c|c|c|c|c|c|}
\hline DAY & $\mathrm{OCT}$ & NOV & $D E C$ & JAN & FEB & MAR & APR & MAY & JUN & JIU & AUG & SEP \\
\hline $\begin{array}{l}1 \\
2 \\
3 \\
4 \\
5\end{array}$ & $\begin{array}{l}5.0 \\
5.4 \\
5.1 \\
5.2 \\
5.4\end{array}$ & $\begin{array}{l}5.0 \\
4.7 \\
4.6 \\
4.5 \\
4.4\end{array}$ & $\begin{array}{l}4.7 \\
5.1 \\
4.7 \\
4.7 \\
4.4\end{array}$ & $\begin{array}{l}2.3 \\
2.2 \\
2 \cdot 1 \\
2 \cdot 1 \\
2.1\end{array}$ & $\begin{array}{l}1.9 \\
1.9 \\
1.9 \\
1.9 \\
1.9\end{array}$ & $\begin{array}{l}1.7 \\
1.7 \\
1.7 \\
1.7 \\
1.8\end{array}$ & $\begin{array}{l}9.6 \\
10 \\
13 \\
24 \\
19\end{array}$ & $\begin{array}{l}12 \\
10 \\
10 \\
8.0 \\
7.9\end{array}$ & $\begin{array}{l}34 \\
29 \\
22 \\
17 \\
18\end{array}$ & $\begin{array}{l}29 \\
24 \\
21 \\
17 \\
15\end{array}$ & $\begin{array}{l}27 \\
27 \\
24 \\
23 \\
22\end{array}$ & $\begin{array}{l}6.4 \\
6.5 \\
10 \\
13 \\
9.2\end{array}$ \\
\hline $\begin{array}{r}6 \\
7 \\
9 \\
9 \\
10\end{array}$ & $\begin{array}{l}5.3 \\
17 \\
12 \\
9.1 \\
5.2\end{array}$ & $\begin{array}{l}4.5 \\
4.4 \\
4.7 \\
4.6 \\
4.7\end{array}$ & $\begin{array}{l}4.4 \\
4.5 \\
4.7 \\
4.9 \\
4.7\end{array}$ & $\begin{array}{l}2.0 \\
2.0 \\
2.0 \\
2.0 \\
2.0\end{array}$ & $\begin{array}{l}1.9 \\
1.9 \\
1.9 \\
1.9 \\
1.9\end{array}$ & $\begin{array}{l}1.8 \\
1.9 \\
2.0 \\
2.0 \\
2.1\end{array}$ & $\begin{array}{l}12 \\
11 \\
14 \\
13 \\
11\end{array}$ & $\begin{array}{l}7.0 \\
10 \\
15 \\
12 \\
8.5\end{array}$ & $\begin{array}{l}14 \\
12 \\
14 \\
18 \\
33\end{array}$ & $\begin{array}{l}14 \\
11 \\
9.6 \\
8.6 \\
7.6\end{array}$ & $\begin{array}{l}22 \\
21 \\
20 \\
13 \\
16\end{array}$ & $\begin{array}{l}7.3 \\
6.3 \\
12 \\
19 \\
20\end{array}$ \\
\hline $\begin{array}{l}11 \\
12 \\
13 \\
14 \\
15\end{array}$ & $\begin{array}{l}5.1 \\
5.0 \\
5.1 \\
5.0 \\
4.9\end{array}$ & $\begin{array}{l}4.7 \\
4.7 \\
4.5 \\
4.5 \\
4.5\end{array}$ & $\begin{array}{l}4.7 \\
5.1 \\
4.5 \\
4.5 \\
4.4\end{array}$ & $\begin{array}{l}2.0 \\
2.0 \\
2.0 \\
2.0 \\
1.9\end{array}$ & $\begin{array}{l}1.9 \\
1.8 \\
1.8 \\
1.8 \\
1.8\end{array}$ & $\begin{array}{l}2.1 \\
2.2 \\
2.2 \\
2.3 \\
2.3\end{array}$ & $\begin{array}{l}11 \\
12 \\
12 \\
21 \\
18\end{array}$ & $\begin{array}{l}6.7 \\
6.4 \\
6.5 \\
11 \\
17\end{array}$ & $\begin{array}{r}97 \\
74 \\
93 \\
123 \\
117\end{array}$ & $\begin{array}{l}9.0 \\
27 \\
54 \\
104 \\
102\end{array}$ & $\begin{array}{r}11 \\
9.4 \\
8.8 \\
8.8 \\
9.6\end{array}$ & $\begin{array}{r}12 \\
9.3 \\
7.9 \\
7.0 \\
7.3\end{array}$ \\
\hline $\begin{array}{l}16 \\
17 \\
19 \\
19 \\
20\end{array}$ & $\begin{array}{l}5.3 \\
5.8 \\
6.1 \\
6.4 \\
6.9\end{array}$ & $\begin{array}{l}4.9 \\
4.9 \\
5.1 \\
4.9 \\
4.9\end{array}$ & $\begin{array}{l}4.4 \\
4.5 \\
4.6 \\
4.6 \\
4.6\end{array}$ & $\begin{array}{l}1.9 \\
1.9 \\
1.9 \\
1.9 \\
1.9\end{array}$ & $\begin{array}{l}1.7 \\
1.7 \\
1.7 \\
1.7 \\
1.7\end{array}$ & $\begin{array}{l}2.4 \\
2.4 \\
23 \\
37 \\
25\end{array}$ & $\begin{array}{r}11 \\
9.9 \\
9.5 \\
9.6 \\
43\end{array}$ & $\begin{array}{l}40 \\
43 \\
36 \\
25 \\
17\end{array}$ & $\begin{array}{r}111 \\
104 \\
92 \\
76 \\
65\end{array}$ & $\begin{array}{r}118 \\
114 \\
98 \\
83 \\
69\end{array}$ & $\begin{array}{r}10 \\
8.8 \\
7.9 \\
8.8 \\
7.9\end{array}$ & $\begin{array}{l}7.7 \\
23 \\
45 \\
40 \\
32\end{array}$ \\
\hline $\begin{array}{l}21 \\
22 \\
23 \\
24 \\
25\end{array}$ & $\begin{array}{l}6.2 \\
6.9 \\
9.5 \\
9.7 \\
9.5\end{array}$ & $\begin{array}{l}4.9 \\
4.8 \\
4.7 \\
5.2 \\
5.1\end{array}$ & $\begin{array}{l}4.4 \\
4.1 \\
4.0 \\
3.9 \\
3.8\end{array}$ & $\begin{array}{l}1.9 \\
1.9 \\
1.9 \\
1.9 \\
1.9\end{array}$ & $\begin{array}{l}1.7 \\
1.7 \\
1.7 \\
1.7 \\
1.7\end{array}$ & $\begin{array}{l}15 \\
15 \\
20 \\
28 \\
31\end{array}$ & $\begin{array}{l}38 \\
22 \\
47 \\
52 \\
40\end{array}$ & $\begin{array}{l}15 \\
13 \\
9.5 \\
21 \\
36\end{array}$ & $\begin{array}{l}74 \\
69 \\
67 \\
61 \\
53\end{array}$ & $\begin{array}{l}55 \\
47 \\
50 \\
40 \\
35\end{array}$ & $\begin{array}{l}7.9 \\
7.9 \\
7.9 \\
7.9 \\
7.9\end{array}$ & $\begin{array}{l}24 \\
39 \\
60 \\
58 \\
51\end{array}$ \\
\hline $\begin{array}{l}26 \\
27 \\
28 \\
29 \\
30 \\
31\end{array}$ & $\begin{array}{l}5.8 \\
5.0 \\
4.7 \\
8.3 \\
7.6 \\
6.3\end{array}$ & $\begin{array}{r}4.5 \\
4.4 \\
4.4 \\
4.4 \\
4.2 \\
\\
\end{array}$ & $\begin{array}{l}3.6 \\
3.4 \\
3.2 \\
3.0 \\
2.8 \\
2.6\end{array}$ & $\begin{array}{l}1.9 \\
1.9 \\
1.9 \\
1.9 \\
1.9 \\
1.9\end{array}$ & $\begin{array}{r}1.7 \\
1.7 \\
1.7 \\
1.7 \\
\end{array}$ & $\begin{array}{c}30 \\
30 \\
34 \\
27 \\
16 \\
9.5\end{array}$ & $\begin{array}{l}25 \\
17 \\
14 \\
13 \\
14 \\
----\end{array}$ & $\begin{array}{l}54 \\
60 \\
59 \\
47 \\
30 \\
29\end{array}$ & $\begin{array}{r}63 \\
55 \\
52 \\
45 \\
36 \\
\end{array}$ & $\begin{array}{l}31 \\
30 \\
22 \\
19 \\
23 \\
26\end{array}$ & $\begin{array}{l}6.8 \\
6.8 \\
6.8 \\
6.4 \\
6.4 \\
6.8\end{array}$ & $\begin{array}{r}40 \\
23 \\
16 \\
14 \\
12 \\
\end{array}$ \\
\hline $\begin{array}{l}\text { TOTAL } \\
\text { MEAN }\end{array}$ & 209.88 & $\begin{array}{r}140.3 \\
4.68\end{array}$ & 131.55 & $61: 9 \frac{1}{1}$ & 51.79 & 374.8 & 575.6 & $\begin{array}{r}682.5 \\
22.0\end{array}$ & $\begin{array}{r}1.738 \\
57.9\end{array}$ & $1.3 \frac{12}{42} \cdot \frac{8}{3}$ & $\begin{array}{r}389.9 \\
12.6\end{array}$ & $\begin{array}{r}637 \cdot 9 \\
21: 3\end{array}$ \\
\hline $\begin{array}{l}\text { MAX } \\
\text { MIN } \\
\text { AC-FT }\end{array}$ & $\begin{array}{r}17 \\
4.7 \\
416\end{array}$ & $\begin{array}{l}5.2 \\
4.2 \\
278\end{array}$ & $\begin{array}{l}5.1 \\
2.6 \\
261\end{array}$ & $\begin{array}{l}2.3 \\
1.9 \\
121\end{array}$ & $\begin{array}{l}1.9 \\
1.7 \\
103\end{array}$ & $\begin{array}{r}37 \\
1.7 \\
743\end{array}$ & $\begin{array}{r}52 \\
9.5 \\
1,140\end{array}$ & $\begin{array}{r}60 \\
6.4 \\
1.350\end{array}$ & $\begin{array}{r}123 \\
12 \\
3.450\end{array}$ & $\begin{array}{r}118 \\
7.6 \\
2.600\end{array}$ & $\begin{array}{r}27 \\
6.4 \\
773\end{array}$ & $\begin{array}{r}60 \\
6.3 \\
1.270\end{array}$ \\
\hline
\end{tabular}

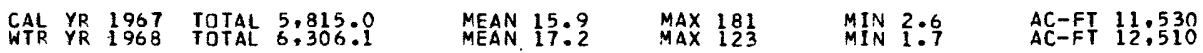




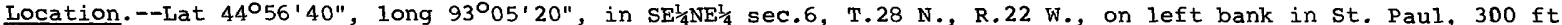
upstream from Robert street Bridge, 6 miles downstream from Minnesota River, and at mile 839.3 upstream from Ohio River.

Drainage area. $--36,800 \mathrm{sq} \mathrm{mi}$, approximately.

Records available.--Water years 1867-69, 1872-92 (annual maximum), Marc's 1892 to September 1968 (prior to 1901, fragmentary during some winters). Records prior to March 1892, published in the 19th Annual Report, Part 4 , have been found to be unreliable and should not be used. Monthly discharge only for some periods, published in WSP 1308. Gage-height records (winter records incomplete) collected at same site since 1866 are contained in reports of U.S. Weather Bureau, War Department and Mississippi River Commission.

Gage.-Water-stage recorder. Datum of gage is 684.16 ft above mean sea level, adjustment of 1912 . Prior to Mar. 18, 1925, staff or chain gage at several sites within $300 \mathrm{ft}$ of present site at same datum. Mar. 18 , 1925, to Mar. 10, 1933, water-stage recorder and Mar. 11, 1933, to Sept. 14, 1939, staff gage, at present site and datum. Since september 1938, auxiliary water-stage recorder 5.4 miles downstream.

Average discharge.--70 years (1894-95, 1896-97, 1900-1968), 10,080 cfs (adjusted for diversion).

Extremes.--Maximum discharge during year, 27,300 cfs June 16 (gage height, 5.72 ft); minimum daily, 1, 220 cfs Nov. 28 .

1867-69, 1872-1968: Maximum discharge, 171,000 cfs Apr. 16, 1965 (gage height, 26.01 ft, from floodmark).

1897, 1917-68: Minimum daily discharge, 632 cfs Aug. 26, 1934.

Maximum stage known since at least 1851, that of Apr. 16, 1965. Flood of Apr. 11, 1870 reached a'stage of $19.4 \mathrm{ft}$ (discharge, $100,000 \mathrm{cfs}$ ).

Remarks:--Records good. Records of water temperatures and chemical analysis for the water year 1968 are published in Part 2 of this report. Slight regulation except during extreme floods by reservoirs on headwaters and by powerplants. Beginning July 20, 1938, sewage from Minneapolis and St. Paul, which formerly entered above station, was diverted to a sewage-disposal plant, thence to river below station. Figures of daily discharge do not include this diversion.

Cooperation.--Records of Mississippi River at Twin City lock and dam computed and furnished by Ford Motor Co. Gage-height record at South St. Paul furnished by Corps of Engineers. Diversion through sewage disposal plant furnished by Minneapolis-st. Paul Sanitary District.

DISCHARGE, IN CUBIC FEET PER SECOND, WATER YEAR OCTOBER 1967 TO SEPTEMBER 1968

\begin{tabular}{|c|c|c|c|c|c|c|c|c|c|c|c|c|}
\hline DAY & OCT & NOV & DEC & JAN & FEB & MAR & APR & MAY & JUN & JUL & AUG & SEP \\
\hline $\begin{array}{l}1 \\
2 \\
3 \\
4 \\
5\end{array}$ & $\begin{array}{l}2.650 \\
2.990 \\
2.240 \\
3.060 \\
2.630\end{array}$ & $\begin{array}{l}3.220 \\
3.440 \\
3.410 \\
3.650 \\
3.420\end{array}$ & $\begin{array}{r}2.250 \\
-3.570 \\
2.800 \\
3.150 \\
3.150\end{array}$ & $\begin{array}{l}2.110 \\
2.200 \\
1.790 \\
2.320 \\
2.140\end{array}$ & $\begin{array}{l}2.940 \\
2.920 \\
2.820 \\
2.760 \\
2.840\end{array}$ & $\begin{array}{l}2.380 \\
2.460 \\
2.410 \\
2.510 \\
2.800\end{array}$ & $\begin{array}{l}6.510 \\
6.540 \\
6.080 \\
6.140 \\
6.850\end{array}$ & $\begin{array}{l}17.700 \\
16.200 \\
15.300 \\
14.300 \\
12.900\end{array}$ & $\begin{array}{l}9.570 \\
9.740 \\
9.500 \\
9.820 \\
9.500\end{array}$ & $\begin{array}{l}19.400 \\
18.400 \\
17.300 \\
16.200 \\
15800\end{array}$ & $\begin{array}{l}22.700 \\
22.200 \\
21.500 \\
20.300 \\
19.200\end{array}$ & $\begin{array}{l}4.900 \\
5.220 \\
4.940 \\
4.970 \\
4.930\end{array}$ \\
\hline $\begin{array}{r}6 \\
7 \\
8 \\
9 \\
10\end{array}$ & $\begin{array}{l}2.460 \\
2.540 \\
2.840 \\
3.560 \\
3.060\end{array}$ & $\begin{array}{l}3.240 \\
3.200 \\
3.140 \\
2.780 \\
3.210\end{array}$ & $\begin{array}{l}3.110 \\
3.570 \\
3.470 \\
3.580 \\
3.310\end{array}$ & $\begin{array}{l}2.110 \\
2.170 \\
2.340 \\
2.180 \\
2.130\end{array}$ & $\begin{array}{l}2.940 \\
2.810 \\
2.870 \\
2.900 \\
3.090\end{array}$ & $\begin{array}{l}2.600 \\
3.100 \\
3.250 \\
4.120 \\
4.180\end{array}$ & $\begin{array}{l}5.930 \\
5.800 \\
6.170 \\
6.680 \\
7.170\end{array}$ & $\begin{array}{l}11,400 \\
11000 \\
10600 \\
10400 \\
10600\end{array}$ & $\begin{array}{r}9.240 \\
9.630 \\
9.340 \\
9.000 \\
10.600\end{array}$ & $\begin{array}{l}15.900 \\
14.900 \\
14.200 \\
13.800 \\
12.700\end{array}$ & $\begin{array}{l}17.000 \\
14.600 \\
13.100 \\
16.600 \\
19.100\end{array}$ & $\begin{array}{l}4.920 \\
4.820 \\
4.930 \\
4.600 \\
5.380\end{array}$ \\
\hline $\begin{array}{l}11 \\
12 \\
13 \\
14 \\
15\end{array}$ & $\begin{array}{l}3.400 \\
2.810 \\
3.040 \\
2.500 \\
2.950\end{array}$ & $\begin{array}{l}3.750 \\
2.910 \\
3.580 \\
3.580 \\
3.290\end{array}$ & $\begin{array}{l}3.270 \\
3.390 \\
2.990 \\
2.920 \\
2.590\end{array}$ & $\begin{array}{l}2.390 \\
2.260 \\
2.420 \\
2.370 \\
2.400\end{array}$ & $\begin{array}{l}2.450 \\
2.800 \\
3.210 \\
2.340 \\
2.260\end{array}$ & $\begin{array}{l}4.970 \\
4.850 \\
4.790 \\
4.940 \\
5.230\end{array}$ & $\begin{array}{r}7.850 \\
10100 \\
9.820 \\
9.300 \\
9.990\end{array}$ & $\begin{array}{r}10.200 \\
9.950 \\
9.960 \\
10.700 \\
10600\end{array}$ & $\begin{array}{l}14.000 \\
18.000 \\
20.900 \\
23.600 \\
25.500\end{array}$ & $\begin{array}{l}11.400 \\
10800 \\
11.700 \\
12800 \\
15400\end{array}$ & $\begin{array}{l}20600 \\
20800 \\
20900 \\
20200 \\
13600\end{array}$ & $\begin{array}{l}5.790 \\
5.490 \\
5.490 \\
5.510 \\
4.720\end{array}$ \\
\hline $\begin{array}{l}16 \\
17 \\
18 \\
19 \\
20\end{array}$ & $\begin{array}{l}2.720 \\
3.430 \\
3.100 \\
3.730 \\
3.490\end{array}$ & $\begin{array}{l}3.220 \\
3.020 \\
3.070 \\
3.500 \\
3.430\end{array}$ & $\begin{array}{l}2.190 \\
2.270 \\
3.380 \\
3.340 \\
3.280\end{array}$ & $\begin{array}{l}2.390 \\
2.460 \\
2.500 \\
2.490 \\
2.560\end{array}$ & $\begin{array}{l}2.120 \\
2.160 \\
1.750 \\
2.300 \\
2.100\end{array}$ & $\begin{array}{l}5.650 \\
6.030 \\
6.440 \\
6.940 \\
6.580\end{array}$ & $\begin{array}{l}8.940 \\
9.860 \\
8440 \\
8.890 \\
9.130\end{array}$ & $\begin{array}{r}10.800 \\
10.300 \\
9.860 \\
10.400 \\
10.900\end{array}$ & $\begin{array}{l}25.500 \\
25.000 \\
24.700 \\
23.600 \\
22.200\end{array}$ & $\begin{array}{l}16.300 \\
16.400 \\
16.300 \\
17.000 \\
16.300\end{array}$ & $\begin{array}{r}16.300 \\
13.100 \\
11.400 \\
10300 \\
3.670\end{array}$ & $\begin{array}{l}5020 \\
5800 \\
6.110 \\
6.720 \\
7.430\end{array}$ \\
\hline $\begin{array}{l}21 \\
22 \\
23 \\
24 \\
25\end{array}$ & $\begin{array}{l}2.150 \\
3.080 \\
2.970 \\
3.830 \\
3.570\end{array}$ & $\begin{array}{r}3.390 \\
3.300 \\
3.120 \\
3.200 \\
2.760\end{array}$ & $\begin{array}{l}3.080 \\
2.030 \\
1.820 \\
2.310 \\
2.660\end{array}$ & $\begin{array}{l}2.390 \\
2.620 \\
2.450 \\
2.720 \\
2.800\end{array}$ & $\begin{array}{l}1.980 \\
2.120 \\
2.160 \\
2.220 \\
1.920\end{array}$ & $\begin{array}{l}6.920 \\
6.580 \\
6.240 \\
6.740 \\
7.230\end{array}$ & $\begin{array}{l}10.300 \\
11.600 \\
13.000 \\
14.800 \\
15.300\end{array}$ & $\begin{array}{l}10.400 \\
10.200 \\
10.100 \\
10.200 \\
10.500\end{array}$ & $\begin{array}{l}21.400 \\
21.100 \\
20.500 \\
19.900 \\
13.800\end{array}$ & $\begin{array}{l}15.800 \\
16.200 \\
14.100 \\
13.100 \\
12.600\end{array}$ & $\begin{array}{l}8.420 \\
8.090 \\
7.890 \\
7.680 \\
6.600\end{array}$ & $\begin{array}{r}8.440 \\
9.140 \\
11.400 \\
15.300 \\
17.700\end{array}$ \\
\hline $\begin{array}{l}26 \\
27 \\
28 \\
29 \\
30 \\
31\end{array}$ & $\begin{array}{l}3.060 \\
3.260 \\
3.260 \\
3.480 \\
3.150 \\
3.290\end{array}$ & $\begin{array}{l}2.890 \\
1.870 \\
1.220 \\
1.510 \\
1700\end{array}$ & $\begin{array}{l}3.180 \\
2.640 \\
2.580 \\
2.820 \\
2.660 \\
2.170 \\
\end{array}$ & $\begin{array}{l}2.790 \\
2.570 \\
2.910 \\
2.900 \\
2.800 \\
3.040\end{array}$ & $\begin{array}{r}2.190 \\
2.490 \\
2.340 \\
2.210 \\
\\
\end{array}$ & $\begin{array}{l}7.410 \\
6.860 \\
6.950 \\
7.080 \\
6.960 \\
6.930\end{array}$ & $\begin{array}{r}15.900 \\
16.300 \\
16.600 \\
17.500 \\
18.000\end{array}$ & $\begin{array}{l}9.540 \\
9.900 \\
8.840 \\
9930 \\
9850 \\
9.100\end{array}$ & $\begin{array}{l}17.900 \\
17.300 \\
17.000 \\
16.600 \\
17.700\end{array}$ & $\begin{array}{l}14.300 \\
16.300 \\
17.700 \\
19.600 \\
20.900 \\
21.900\end{array}$ & $\begin{array}{l}6.420 \\
5.470 \\
5.070 \\
5.200 \\
4.990 \\
5.180\end{array}$ & $\begin{array}{l}19900 \\
20.300 \\
20300 \\
20.100 \\
19500 \\
-\end{array}$ \\
\hline $\begin{array}{l}\text { Tota } 1 \\
\text { Mean } \\
(f) \\
\text { Mean } \neq \\
\text { Max } \\
\text { Min } \\
\text { Cfsm } \neq \\
\text { In. } \neq\end{array}$ & $\begin{array}{r}94,300 \\
3,042 \\
+322 \\
3,364 \\
3,830 \\
2,150 \\
0.091 \\
0.10 \\
\end{array}$ & $\begin{array}{r}91,020 \\
3,034 \\
+300 \\
3,334 \\
3,750 \\
1,220 \\
0.091 \\
0.10 \\
\end{array}$ & $\begin{array}{r}88,530 \\
2,856 \\
+293 \\
3,149 \\
3,580 \\
1,820 \\
0.086 \\
0.10 \\
\end{array}$ & $\begin{array}{r}75,720 \\
2,442 \\
+289 \\
2,731 \\
3,040 \\
1,790 \\
0.074 \\
0.09 \\
\end{array}$ & $\begin{array}{r}72,010 \\
2,483 \\
+284 \\
2,767 \\
3,210 \\
1,750 \\
0.075 \\
0.08 \\
\end{array}$ & $\begin{array}{r}162,130 \\
5,230 \\
+313 \\
5,543 \\
7,410 \\
2,380 \\
0.151 \\
0.17 \\
\end{array}$ & $\begin{array}{r}305,490 \\
10,180 \\
+319 \\
10,500 \\
18,000 \\
5,800 \\
0.285 \\
0.32 \\
\end{array}$ & $\begin{array}{r}339,630 \\
10,960 \\
+342 \\
11,300 \\
17,700 \\
8,840 \\
0.307 \\
0.35 \\
\end{array}$ & $\begin{array}{r}507,140 \\
16,900 \\
+378 \\
17,280 \\
25,500 \\
9,000 \\
0.470 \\
0.52 \\
\end{array}$ & $\begin{array}{r}485,500 \\
15,660 \\
+387 \\
16,050 \\
21,900 \\
10,800 \\
0.436 \\
0.50 \\
\end{array}$ & $\begin{array}{r}418,180 \\
13,490 \\
+381 \\
13,870 \\
22,700 \\
4,990 \\
0.377 \\
0.43 \\
\end{array}$ & $\begin{array}{r}267,770 \\
8,926 \\
+362 \\
9,288 \\
20,300 \\
4,600 \\
0.252 \\
0.28 \\
\end{array}$ \\
\hline er & 2 & $\operatorname{Max}$ & $\begin{array}{l}52,100 \\
25,500\end{array}$ & $\operatorname{lin} 1,2$ & $\begin{array}{ll}20 & \text { Mean } \\
20 & \text { Mean }\end{array}$ & $\begin{array}{r}10,700 \\
7,944\end{array}$ & $\begin{array}{l}\text { Meant } \\
\text { Meant }\end{array}$ & $\begin{array}{r}11,030 \\
8,275\end{array}$ & $\neq \neq$ & $\begin{array}{l}\text { In. } \neq \\
\text { In. } \neq\end{array}$ & $\begin{array}{l}.06 \\
.04\end{array}$ & \\
\hline
\end{tabular}

f Diversion, equivalent in cubic feet per second, through sewage-disposal plant.

f Adjusted for diversion.

Note.--Stage-fall discharge relation affected by indefinite slope throughout the year. 
5-3362. Glaisby Brook near Kettle River, Minn.

Location.--Lat $46^{\circ} 27^{\prime} 19^{\prime \prime}$, long $92^{\circ} 51^{\prime} 34^{\prime \prime}$, in SEl/ NW/ sec.22, T.46 N., R. 20 W., on left bank 20 ft upstream from bridge No. 2468 on State Highways 27 and $73,1.0$ mile upstream from mouth, and $2.4 \mathrm{miles}$ south of Kettle River.

Records available.--October 1959 to September 1968.

Gage.--Water-stage recorder. Altitude of gage is 1,105 ft (from topographic map).

Average discharqe.--9 years, 19.5 cfs.

Extremes.--Maximum discharge during year, 270 cfs Apr. 24 (gage height, 4.75 ft); minimum daily, 0.1 cfs Feb. 4 , $5,6,7$.

1959-68: Maximum discharge, 813 cfs Apr. 18, 1965 (gage height, 8.42 ft); minimum, 0.1 cfs Aug. 3, 1960, July 9, 1961, Aug. 8, Sept. 1, 1963, July 24, 25, 26, 1964, Feb. 4, 5, 6, 7, 1968.

Remarks.--Records good except those for winter months, which are fair.

DISCHARGE, IN CUBIC FEET PER SECOND, WATER YEAR OCTOBER 1967 TO SEPTEMBER 1968

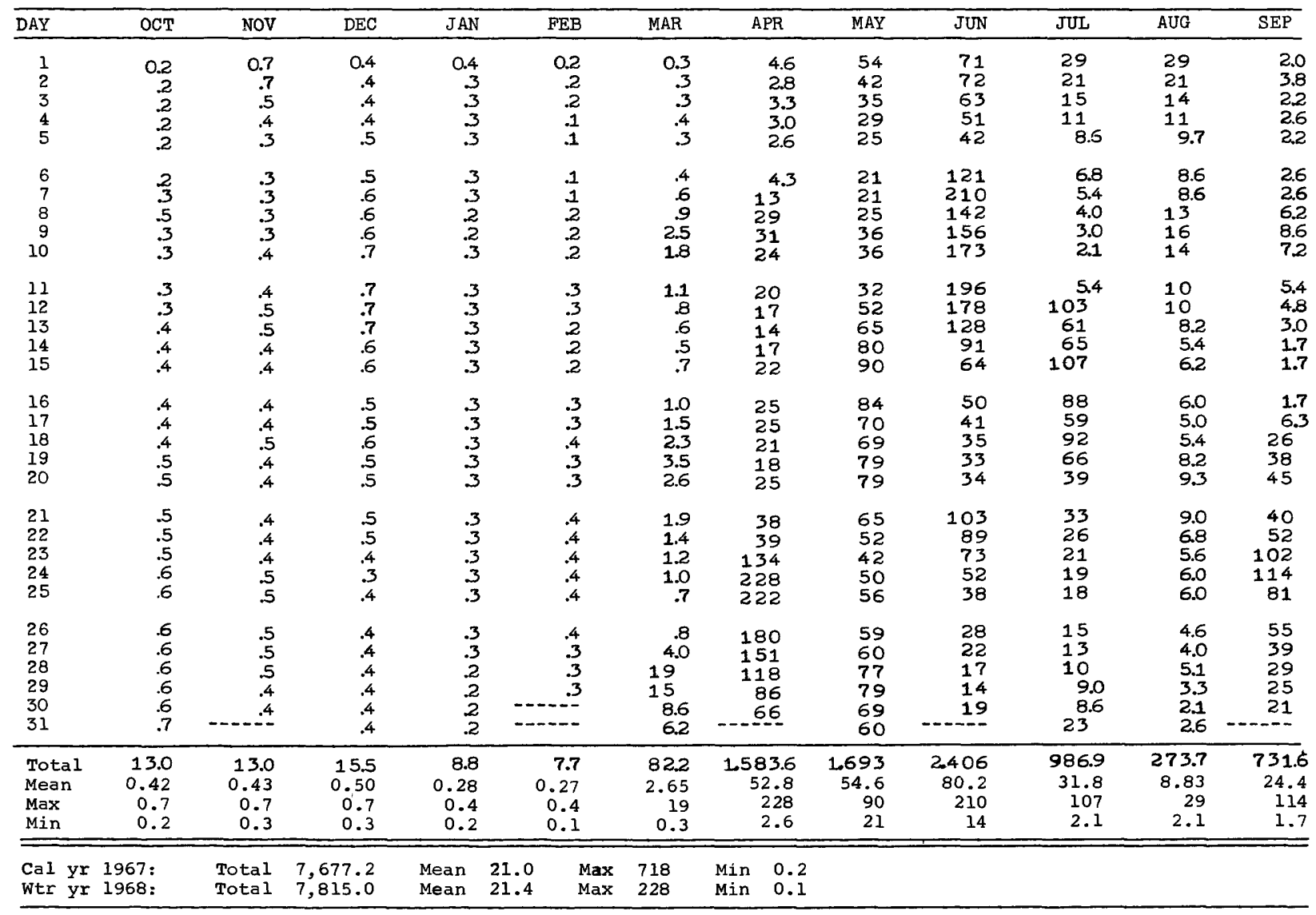


5-3367. Kettle River below Sandstone, Minn.

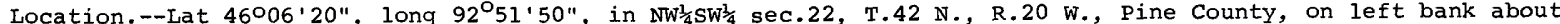
$900 \mathrm{ft}$ downstream from abandoned powerplant dam, on Sandstone Federal Correctional Institution property,

1.8 miles south of Sandstone.

Records available.--October 1967 to September 1968.

Gage.--Digital water-stage recorder. Altitude of gage is $930 \mathrm{ft}$ (from topographic map).

Extremes.--Maximum discharge during year, 5,180 cfs June 22 (gage height, $8.56 \mathrm{ft}$ ); minimum daily, $99 \mathrm{cfs}$ Feb. 19 to Mar. 1.

Remarks.--Records fair. Records of Chemical analyses for the water year 1968 are published in Part 2 of this report.

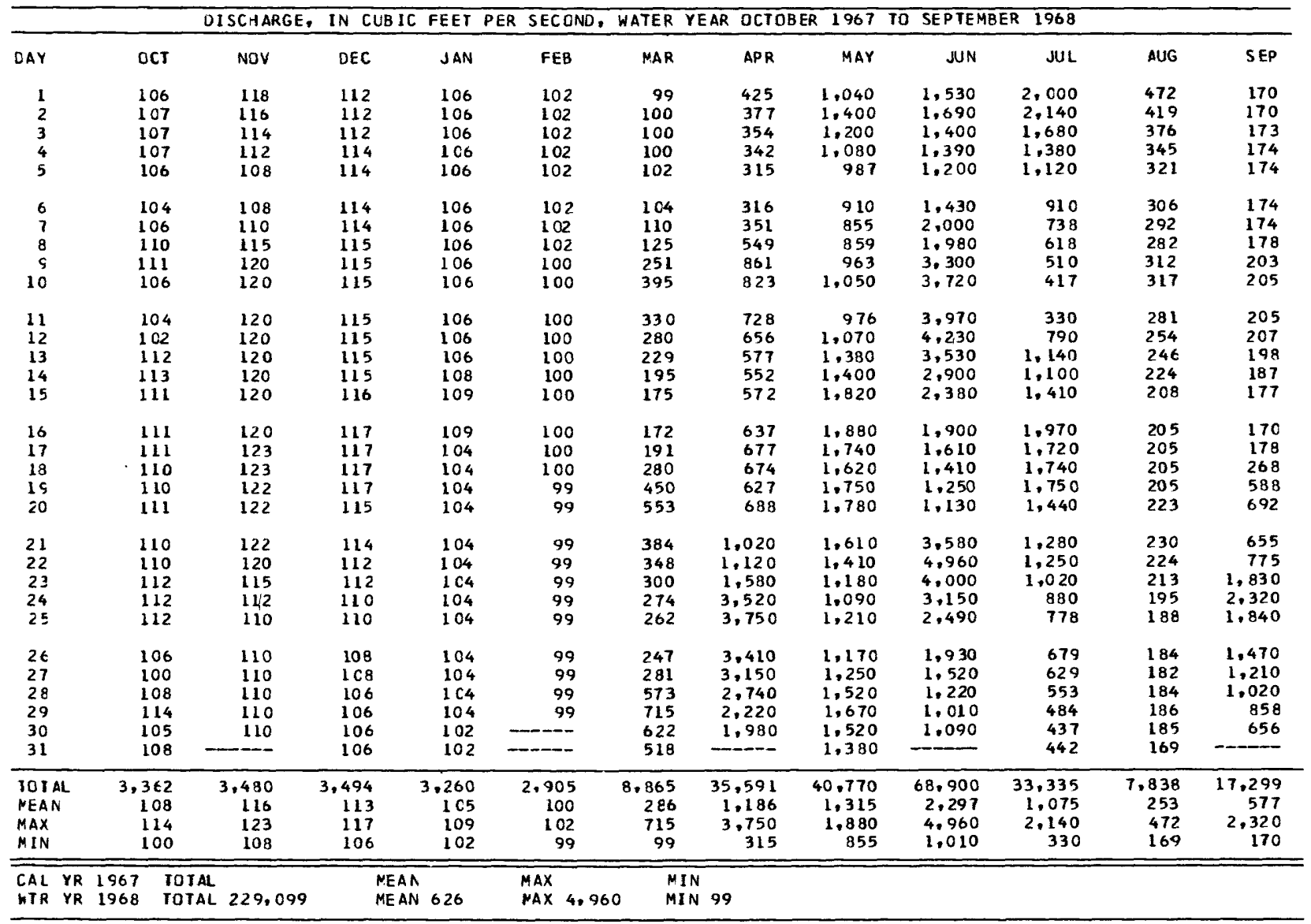


5-3385. Snake River near Pine City, Minn.

Location.--Lat $45^{\circ} 50^{\prime} 30^{\prime \prime}$, long $92^{\circ} 56^{\prime} 00^{\prime \prime}$, in SE $\frac{1}{4} \mathrm{NW}^{\frac{1}{4}}$ sec.26, T.39 N., R.21 W., on left bank at site of former powerplant and dam, half a mile downstream from Cross Lake and $1 \frac{1}{2}$ miles northeast of Pine City.

Drainage area. $--958 \mathrm{sq} \mathrm{mi}$.

Records available.--June 1913 to September 1917, July 1951 to september 1968.

Gage.-Digital water-stage recorder. Datum of gage is $919.00 \mathrm{ft}$ above mean sea level, datum of 1929 . June 25 , 1913 , to sept. 30 , 1917, staff gage at site 500 ft downstream at different datum. July 1 to Oct. 28 , 1951, staff gage and oct. 29, 1951 to May 10, 1966, graphic water-stage recorder at present site and datum.

Average discharge.--21 years, $556 \mathrm{cfs}$.

Extremes.--Maximum discharge during year, 3, 200 cfs June 26 (gage height, 5.95 ft); minimum, 35 cfs Oct. 6 (gage height, $2.78 \mathrm{ft}$ )

1913-17, 1951-68: Maximum discharge 11, 500 cfs April 18, 1965 (gage height, 9.56 ft); minimum, 5.5 cfs oct. 1, 1964 (gage height, $2.57 \mathrm{ft}$ ).

A discharge measurement of 12,500 cfs was made May 9, 1950.

Remarks.--Records good except those for winter months, which are fair.

DISCHARGE, IN CUBIC FEET PER SECOND, WATER YEAR OCTOBER 1967 TO SEPTEMBER 1968

\begin{tabular}{|c|c|c|c|c|c|c|c|c|c|c|c|c|}
\hline DAY & $\mathrm{OCT}$ & NaV & DEC & JAN & FEB & MAR & APR & MAY & JUN & JUL & AUG & SEP \\
\hline $\begin{array}{l}1 \\
2 \\
3 \\
4 \\
5\end{array}$ & $\begin{array}{l}46 \\
50 \\
48 \\
59 \\
49\end{array}$ & $\begin{array}{l}35 \\
89 \\
89 \\
93 \\
81\end{array}$ & $\begin{array}{l}78 \\
78 \\
78 \\
78 \\
78\end{array}$ & $\begin{array}{l}66 \\
65 \\
64 \\
63 \\
63\end{array}$ & $\begin{array}{l}87 \\
86 \\
87 \\
81 \\
80\end{array}$ & $\begin{array}{l}52 \\
52 \\
52 \\
58 \\
76\end{array}$ & $\begin{array}{l}328 \\
282 \\
250 \\
296 \\
280\end{array}$ & $\begin{array}{r}1,410 \\
1,240 \\
1,070 \\
888 \\
750\end{array}$ & $\begin{array}{l}1.470 \\
1.430 \\
1.400 \\
1.370 \\
1.270\end{array}$ & $\begin{array}{l}2.590 \\
2.640 \\
2.770 \\
2.840 \\
2,790\end{array}$ & $\begin{array}{l}341 \\
341 \\
313 \\
299 \\
273\end{array}$ & $\begin{array}{l}133 \\
116 \\
115 \\
129 \\
121\end{array}$ \\
\hline $\begin{array}{r}6 \\
7 \\
8 \\
9 \\
10\end{array}$ & $\begin{array}{l}38 \\
49 \\
80 \\
74 \\
64\end{array}$ & $\begin{array}{l}71 \\
71 \\
74 \\
85 \\
89\end{array}$ & $\begin{array}{l}81 \\
85 \\
85 \\
89 \\
94\end{array}$ & $\begin{array}{l}63 \\
64 \\
64 \\
65 \\
67\end{array}$ & $\begin{array}{l}79 \\
78 \\
74 \\
70 \\
67\end{array}$ & $\begin{array}{l}100 \\
123 \\
153 \\
171 \\
221\end{array}$ & $\begin{array}{l}247 \\
256 \\
316 \\
311 \\
393\end{array}$ & $\begin{array}{l}622 \\
568 \\
593 \\
575 \\
572\end{array}$ & $\begin{array}{r}1.140 \\
978 \\
919 \\
997 \\
1.160\end{array}$ & $\begin{array}{l}2.610 \\
2.330 \\
1.990 \\
1.590 \\
1.240\end{array}$ & $\begin{array}{l}248 \\
230 \\
273 \\
279 \\
279\end{array}$ & $\begin{array}{r}113 \\
94 \\
101 \\
111 \\
104\end{array}$ \\
\hline $\begin{array}{l}11 \\
12 \\
13 \\
14 \\
15\end{array}$ & $\begin{array}{l}62 \\
55 \\
93 \\
68 \\
78\end{array}$ & $\begin{array}{r}100 \\
108 \\
97 \\
93 \\
74\end{array}$ & $\begin{array}{l}97 \\
97 \\
90 \\
83 \\
80\end{array}$ & $\begin{array}{l}69 \\
73 \\
76 \\
75 \\
75\end{array}$ & $\begin{array}{l}64 \\
60 \\
57 \\
55 \\
53\end{array}$ & $\begin{array}{l}280 \\
325 \\
333 \\
321 \\
310\end{array}$ & $\begin{array}{l}471 \\
494 \\
440 \\
498 \\
449\end{array}$ & $\begin{array}{l}608 \\
658 \\
635 \\
678 \\
719\end{array}$ & $\begin{array}{l}1.390 \\
1.600 \\
1.760 \\
1.890 \\
1.870\end{array}$ & $\begin{array}{l}994 \\
832 \\
736 \\
802 \\
818\end{array}$ & $\begin{array}{l}273 \\
242 \\
224 \\
184 \\
167\end{array}$ & $\begin{array}{r}109 \\
101 \\
97 \\
101 \\
96\end{array}$ \\
\hline $\begin{array}{l}16 \\
17 \\
18 \\
19 \\
20\end{array}$ & $\begin{array}{l}76 \\
93 \\
76 \\
61 \\
96\end{array}$ & $\begin{array}{l}85 \\
95 \\
97 \\
78 \\
78\end{array}$ & $\begin{array}{l}79 \\
80 \\
81 \\
81 \\
80\end{array}$ & $\begin{array}{l}76 \\
78 \\
78 \\
78 \\
81\end{array}$ & $\begin{array}{l}52 \\
52 \\
52 \\
52 \\
52\end{array}$ & $\begin{array}{l}288 \\
269 \\
311 \\
383 \\
408\end{array}$ & $\begin{array}{l}435 \\
442 \\
429 \\
404 \\
514\end{array}$ & $\begin{array}{l}833 \\
892 \\
910 \\
984 \\
991\end{array}$ & $\begin{array}{l}1.810 \\
1.690 \\
1.530 \\
1.350 \\
1.230\end{array}$ & $\begin{array}{l}807 \\
793 \\
802 \\
769 \\
717\end{array}$ & $\begin{array}{l}201 \\
167 \\
148 \\
162 \\
162\end{array}$ & $\begin{array}{r}91 \\
139 \\
181 \\
182 \\
211\end{array}$ \\
\hline $\begin{array}{l}21 \\
22 \\
23 \\
24 \\
25\end{array}$ & $\begin{array}{r}55 \\
55 \\
64 \\
116 \\
108\end{array}$ & $\begin{array}{l}80 \\
79 \\
77 \\
78 \\
79\end{array}$ & $\begin{array}{l}80 \\
81 \\
80 \\
80 \\
80\end{array}$ & $\begin{array}{l}77 \\
80 \\
82 \\
80 \\
81\end{array}$ & $\begin{array}{l}52 \\
52 \\
52 \\
52 \\
52\end{array}$ & $\begin{array}{l}389 \\
359 \\
342 \\
316 \\
305\end{array}$ & $\begin{array}{r}684 \\
787 \\
1.040 \\
1.180 \\
1.330\end{array}$ & $\begin{array}{r}1,010 \\
998 \\
938 \\
898 \\
835\end{array}$ & $\begin{array}{l}1.770 \\
2.210 \\
2.600 \\
2.890 \\
3.070\end{array}$ & $\begin{array}{l}699 \\
613 \\
561 \\
510 \\
470\end{array}$ & $\begin{array}{l}148 \\
158 \\
144 \\
162 \\
125\end{array}$ & $\begin{array}{r}239 \\
398 \\
1,050 \\
1,790 \\
2,260\end{array}$ \\
\hline $\begin{array}{l}26 \\
27 \\
28 \\
29 \\
30 \\
31\end{array}$ & $\begin{array}{l}64 \\
64 \\
53 \\
74 \\
81 \\
81\end{array}$ & $\begin{array}{r}80 \\
81 \\
81 \\
81 \\
78 \\
\end{array}$ & $\begin{array}{l}76 \\
73 \\
72 \\
72 \\
71 \\
70\end{array}$ & $\begin{array}{l}81 \\
82 \\
82 \\
83 \\
83 \\
83\end{array}$ & $\begin{array}{r}52 \\
52 \\
52 \\
52 \\
\\
\end{array}$ & $\begin{array}{l}283 \\
313 \\
356 \\
370 \\
375 \\
396\end{array}$ & $\begin{array}{l}1.520 \\
1.640 \\
1.700 \\
1.690 \\
1.560\end{array}$ & $\begin{array}{r}847 \\
896 \\
976 \\
1,110 \\
1,260 \\
1,370\end{array}$ & $\begin{array}{l}3,170 \\
3.140 \\
2,930 \\
2,590 \\
2,540 \\
-\end{array}$ & $\begin{array}{l}438 \\
438 \\
385 \\
327 \\
306 \\
355\end{array}$ & $\begin{array}{r}100 \\
96 \\
92 \\
90 \\
88 \\
107\end{array}$ & $\begin{array}{r}2,390 \\
2,350 \\
2,210 \\
2,000 \\
1,730 \\
\end{array}$ \\
\hline $\begin{array}{l}\text { TOTAL } \\
\text { MEAN }\end{array}$ & $\begin{array}{r}2.130 \\
68.7\end{array}$ & $\begin{array}{r}2.526 \\
84.2\end{array}$ & $\begin{array}{r}2.507 \\
80.9\end{array}$ & $2+297$ & $\begin{array}{r}1.806 \\
62.3\end{array}$ & $\begin{array}{r}8.090 \\
261\end{array}$ & $\begin{array}{r}20,666 \\
689\end{array}$ & $\begin{array}{r}27.334 \\
882\end{array}$ & $\begin{array}{r}55,164 \\
1,839\end{array}$ & $\begin{array}{r}36.562 \\
1.179\end{array}$ & 6.116 & 18.862 \\
\hline $\begin{array}{l}\text { MAX } \\
\text { MIN } \\
\text { CFSM } \\
\text { IN. }\end{array}$ & $\begin{array}{r}116 \\
38 \\
.07 \\
.08\end{array}$ & $\begin{array}{r}108 \\
71 \\
.09 \\
.10\end{array}$ & $\begin{array}{r}.97 \\
70 \\
.08 \\
.10\end{array}$ & $\begin{array}{r}83 \\
63 \\
.08 \\
.09\end{array}$ & $\begin{array}{r}87 \\
52 \\
.07 \\
.07\end{array}$ & $\begin{array}{r}408 \\
52 \\
.27 \\
.31\end{array}$ & $\begin{array}{r}1.700 \\
247 \\
.72 \\
.80\end{array}$ & $\begin{array}{r}1.410 \\
568 \\
.92 \\
1.06\end{array}$ & $\begin{array}{r}3.170 \\
919 \\
1.92 \\
2.14\end{array}$ & $\begin{array}{r}2.840 \\
306 \\
1.23 \\
1.42\end{array}$ & $\begin{array}{r}341 \\
88 \\
.21 \\
.24\end{array}$ & $\begin{array}{r}2.390 \\
91 \\
.66 \\
.73\end{array}$ \\
\hline
\end{tabular}

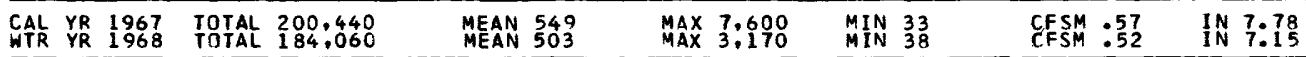




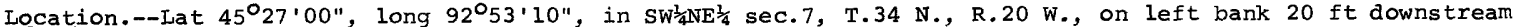
from highway bridge and 4.5 miles northwest of Lindstrom.

Records available.--July 1965 to September 1968. Records for January 1949 to July 1965 at site $6.5 \mathrm{miles}$ upstream, published as "near stacy", not equivalent owing to increased drainage area and Minnesota Game and Fish reservoir between sites.

Gage.--Water-stage recorder. Altitude of gage is $850 \mathrm{ft}$ (from topographic map).

Extremes.--Maximum discharge during year, 335 cfs June 22 (gage height, 5.86 ft); minimum, 11 cfs Aug. 28-31 (gage height, $2.10 \mathrm{ft}$ ).

1965-68: Maximum discharge, 508 cfs Apr. 14, 1967 (gage height, 6.93 ft); minimum 7.9 cfs Sept. 11, 1965 (gage height, $2.07 \mathrm{ft}$ ).

Remarks.--Records good except those for winter months, which are fair. Some regulation by Minnesota Game and Fish Wildife Refuge ponds above the station. At high stages a small part of $f$ low discharges into the Rum River and coon Creek basins from West Arm of Coon Lake and South Coon Lake, respectively.

DISCHARGE, IN CUBIC FEET PER SECOND, WATER YEAR OCTOBER 1967 TO SEPTEMBER 1968

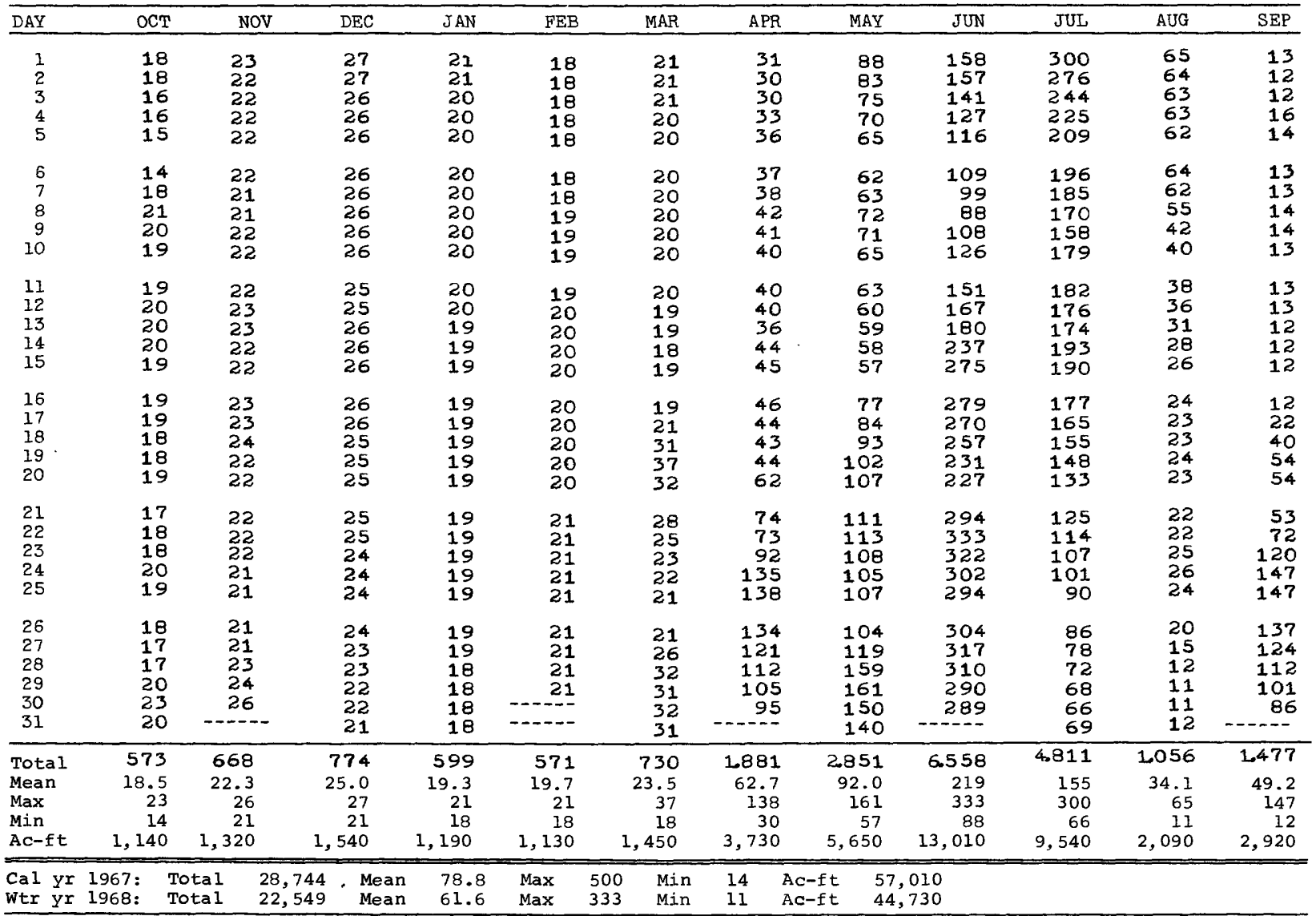


5-3405. St. Croix River at st. Croix Falls, Wis.

Location.--Lat $45^{\circ} 24^{\prime} 30^{\prime \prime}$, long 92\%38'45", in NW/ sec.30, T.34 N., R.18 W., on left bank 1 , 800 ft downstream from powerplant of Northern states Power Co., in st. Croix Falls, and at mile 52.2.

Drainage area. $--5,930 \mathrm{sq} \mathrm{mi}$, approximately.

Records available.--January 1902 to september 1968 in reports of Geological Survey. Prior to January 1910, monthly discharge only, published in WSP 1308. Prior to October 1939, published as "near st. Croix Falls."

Gage.-Digital water-stage recorder. Datum of gage is $690.47 \mathrm{ft}$ above mean sea level, adjustment of 1912 . Prior to July 1905, gage heights and discharge measurements were used to determine flow. July 1905 to February 1940, records were computed from power generation at the st Croix Falls powerplant 1 , 800 ft upstream. Mar. 16, 1940, to Nov. 30, 1963, graphic water-stage recorder at present site and datum.

Average discharge. --66 years, 4, $070 \mathrm{cfs}$.

Extremes.--Maximum discharge during year, 19,700 cfs June 23, 24 (gage height, 9.49 ft); minimum daily, 1,030 cfs Nov. 28

1902-68: Maximum discharge, 54,900 cfs May 8, 1950 (gage height, 25.19 ft); minimum daily, 75 cfs July $17,1910$.

Remarks.--Records good except Nov. 1-7, June 30 to July 18, which are fair. Flow regulated by powerplant upstream.

DISCHARGE, IN CUBIC FEET PER SECOND, WATER YEAR OCTOBER 1967 TO SEPTEMBER 1968

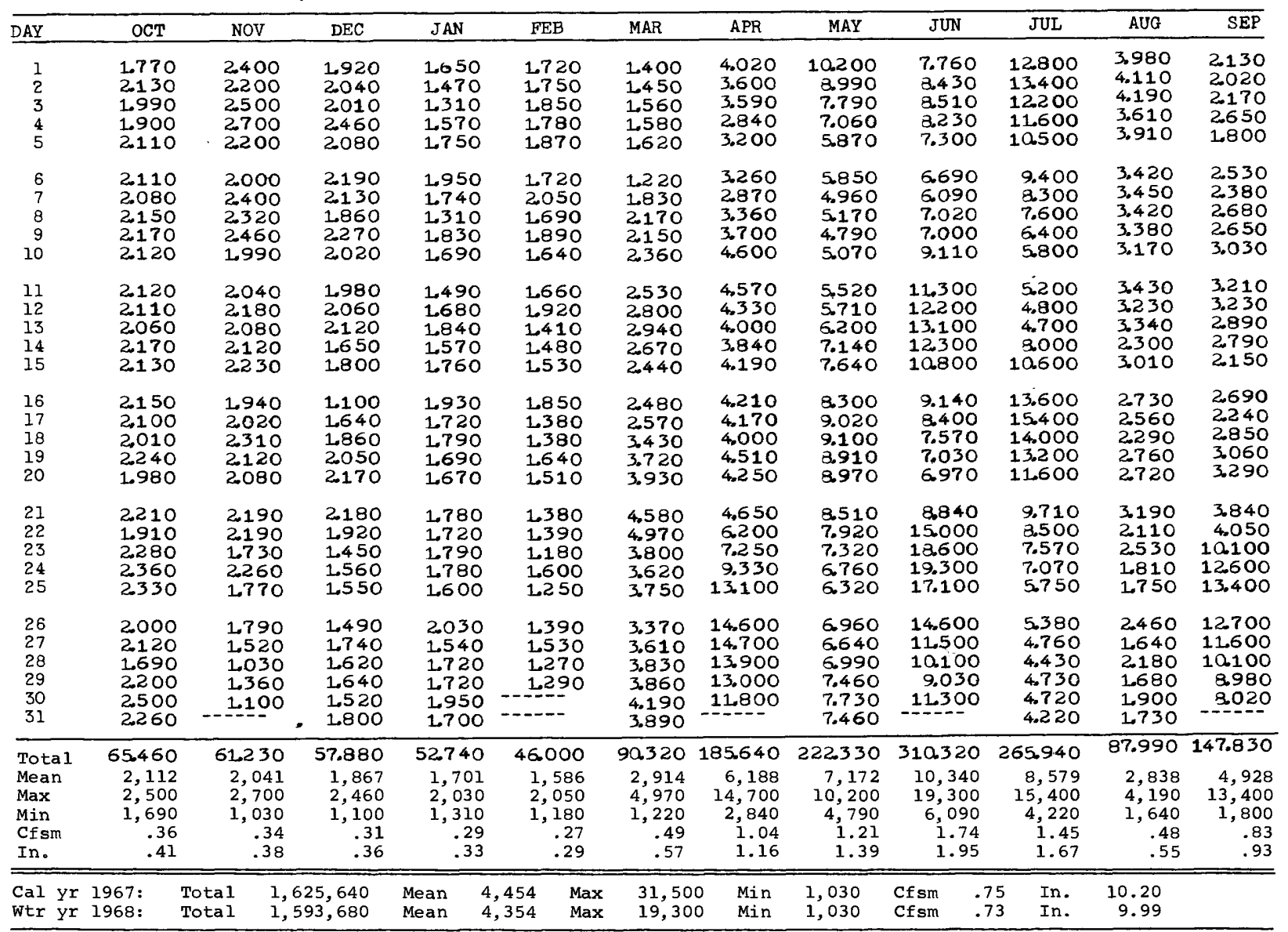


Location.--Lat $44^{\circ} 44^{\prime} 45^{\prime \prime}$, long $92^{\circ} 48^{\prime} 00^{\prime \prime}$, in sec.9, T.26 N., R. 20 W., on left bank at Prescott, 200 ft downstream from st. Croix River, $300 \mathrm{ft}$ south of Chicago, Burlington \& Quincy Railroad bridge, $800 \mathrm{ft}$ south of stream from St. Croix River, $300 \mathrm{ft}$ south of Chicago, Burlington \& Quinc
bridge on U.S. Highway 10, and at mile 811.4 upstream from ohio River.

Drainage area. $--44,800 \mathrm{sq} \mathrm{mi}$, approximately.

Records available.--June 1928 to september 1968.

Gage.--Water-stage recorder. Datum of gage is $600.00 \mathrm{ft}$ above mean sea level, adjustment of 1912 (levels by Corps of Engineers). Prior to Aug. 2, 1932, staff gage at railroad bridge 300 ft upstream at following datums: June 3, 1928, to sept. 30, 1929, 69.27 ft higher; Oct. 1, 1929, to sept. 30, 1930 , 67.68 ft higher; Oct. 1, 1930, to Aug. 1, 1932, 69.28 ft higher. Aug. 2, 1932, to oct. 30, 1938, water-stage recorder at present site at datum $69.28 \mathrm{ft}$ higher. Auxiliary water-stage recorder 10.7 miles downstream from base gage.

Average discharge. -40 years, $15,330 \mathrm{cfs}$.

Extremes:--Maximum discharge during year, 40,200 cfs June 26 (gage height, $79.44 \mathrm{ft}$ ); minimum daily, 3, 200 cfs Dec. 1; minimum gage height, $74.54 \mathrm{ft}$ Mar. 7.

1928-68: Maximum discharge 228,000 cfs Apr. 18, 1965 (gage height, 93.11 ft); minimum daily, 1,380 cfs. July 13, 1940; minimum gage height, $65.08 \mathrm{ft}$ Aug. 29, 1934, present datum.

Remarks.--Records good. Some regulation by reservoirs, navigation dams, and powerplants at low and medium stages. Flood flow not materially affected by artificial storage.

DISCHARGE, IN CUBIC FEET PER SECOND, WATER YEAR OCTOBER 1967 TO SEPTEMBER 1968

\begin{tabular}{|c|c|c|c|c|c|c|c|c|c|c|c|c|}
\hline DAY & OCT & NOV & $\mathrm{DEC}$ & JAN & FEB & MAR & APR & MAY & JUN & JUL & AUG & SEP \\
\hline $\begin{array}{l}1 \\
2 \\
3 \\
4 \\
5\end{array}$ & $\begin{array}{l}4.700 \\
5.600 \\
5.900 \\
5.600 \\
6.000\end{array}$ & $\begin{array}{l}6.300 \\
6.400 \\
6.200 \\
6.100 \\
7.200\end{array}$ & $\begin{array}{l}3.200 \\
3.500 \\
5.400 \\
5.600 \\
6.000\end{array}$ & $\begin{array}{l}4.300 \\
4.900 \\
4.800 \\
4.400 \\
4.200\end{array}$ & $\begin{array}{l}6.200 \\
5.900 \\
5.900 \\
5.700 \\
5.400\end{array}$ & $\begin{array}{l}4.700 \\
5.000 \\
4.800 \\
5.100 \\
6.700\end{array}$ & $\begin{array}{l}12.300 \\
12.400 \\
12.200 \\
11.100 \\
10700\end{array}$ & $\begin{array}{l}31.300 \\
30.000 \\
27.600 \\
24.600 \\
23.200\end{array}$ & $\begin{array}{l}19.900 \\
20100 \\
19.600 \\
19.300 \\
19.500\end{array}$ & $\begin{array}{l}32.500 \\
34.200 \\
33.800 \\
33.100 \\
32.200\end{array}$ & $\begin{array}{l}\text { OO } \\
00 \\
00 \\
00 \\
00\end{array}$ & $\begin{array}{l}8.970 \\
9.420 \\
9.420 \\
9.120 \\
9.520\end{array}$ \\
\hline $\begin{array}{r}6 \\
7 \\
8 \\
9 \\
10\end{array}$ & $\begin{array}{l}5.500 \\
5.300 \\
7.100 \\
6.900 \\
7.300\end{array}$ & $\begin{array}{l}6.500 \\
7.000 \\
5.300 \\
6.300 \\
6.100\end{array}$ & $\begin{array}{l}6.200 \\
6.500 \\
6.400 \\
6.500 \\
6.100\end{array}$ & $\begin{array}{l}4.300 \\
4.300 \\
4.300 \\
4.300 \\
4.500\end{array}$ & $\begin{array}{l}6.600 \\
4.700 \\
5.400 \\
6.700 \\
4.000\end{array}$ & $\begin{array}{l}5.900 \\
5.700 \\
7.500 \\
8.600 \\
3.600\end{array}$ & $\begin{array}{r}11200 \\
10700 \\
10.000 \\
9.740 \\
11.100\end{array}$ & $\begin{array}{l}20800 \\
19.100 \\
13.900 \\
13.300 \\
17.400\end{array}$ & $\begin{array}{l}19.400 \\
13.100 \\
17.300 \\
18.500 \\
18.800\end{array}$ & $\begin{array}{l}30800 \\
29.800 \\
28.200 \\
25200 \\
22.400\end{array}$ & $\begin{array}{l}23.200 \\
20.300 \\
20.800 \\
21000 \\
22.600\end{array}$ & $\begin{array}{l}3.920 \\
9.590 \\
9.170 \\
9.440 \\
9.680\end{array}$ \\
\hline $\begin{array}{l}11 \\
12 \\
13 \\
14 \\
15\end{array}$ & $\begin{array}{l}4.500 \\
5.500 \\
6.400 \\
7.000 \\
6.100\end{array}$ & $\begin{array}{l}6.200 \\
6.200 \\
5.700 \\
6.100 \\
6.300\end{array}$ & $\begin{array}{l}6.700 \\
6.800 \\
6.500 \\
6.200 \\
5.100\end{array}$ & $\begin{array}{l}4.500 \\
4.500 \\
4.800 \\
4.900 \\
4.900\end{array}$ & $\begin{array}{l}5.300 \\
5.500 \\
6.400 \\
5.400 \\
5.200\end{array}$ & $\begin{array}{l}3.500 \\
3.100 \\
3.600 \\
9.100 \\
9.900\end{array}$ & $\begin{array}{l}13.100 \\
16.400 \\
17.200 \\
15.700 \\
14.300\end{array}$ & $\begin{array}{l}17.200 \\
17.700 \\
17.700 \\
13.300 \\
20.000\end{array}$ & $\begin{array}{l}22.800 \\
27.700 \\
31.200 \\
36.100 \\
33.500\end{array}$ & $\begin{array}{l}500 \\
300 \\
900 \\
500 \\
300\end{array}$ & $\begin{array}{l}23.000 \\
23.400 \\
24.000 \\
24.000 \\
22.400\end{array}$ & $\begin{array}{r}9.850 \\
10.600 \\
11.800 \\
11.200 \\
10600\end{array}$ \\
\hline $\begin{array}{l}16 \\
17 \\
18 \\
19 \\
20\end{array}$ & $\begin{array}{l}6.800 \\
6.600 \\
6.400 \\
5.700 \\
6.300\end{array}$ & $\begin{array}{l}6.400 \\
6.900 \\
7.000 \\
4.500 \\
6.200\end{array}$ & $\begin{array}{l}4.900 \\
4.900 \\
4.700 \\
5.600 \\
6.000\end{array}$ & $\begin{array}{l}5.100 \\
5.500 \\
5.500 \\
5.100 \\
5.000\end{array}$ & $\begin{array}{l}5.000 \\
5.100 \\
5.000 \\
4.400 \\
5.100\end{array}$ & $\begin{array}{r}9.600 \\
9.700 \\
12.200 \\
14.100 \\
12.400\end{array}$ & $\begin{array}{l}14.900 \\
15.100 \\
15.400 \\
16.000 \\
16.500\end{array}$ & $\begin{array}{l}21.700 \\
20.900 \\
20.400 \\
20.400 \\
21.000\end{array}$ & $\begin{array}{l}38600 \\
33.100 \\
37.400 \\
36.700 \\
35.000\end{array}$ & $\begin{array}{l}27.900 \\
30.000 \\
31.600 \\
32.200 \\
31.700\end{array}$ & $\begin{array}{l}21.200 \\
19.200 \\
16.400 \\
15.000 \\
13.200\end{array}$ & $\begin{array}{r}9.930 \\
10.800 \\
11.900 \\
11.700 \\
11.200\end{array}$ \\
\hline $\begin{array}{l}21 \\
22 \\
23 \\
24 \\
25\end{array}$ & $\begin{array}{l}6.000 \\
5.100 \\
5.500 \\
6.100 \\
7.600\end{array}$ & $\begin{array}{l}8100 \\
6.800 \\
3.900 \\
5.500 \\
5.600\end{array}$ & $\begin{array}{l}6.800 \\
5.100 \\
5.400 \\
4.800 \\
4.900\end{array}$ & $\begin{array}{l}5.000 \\
5.300 \\
5.100 \\
4.800 \\
5.000\end{array}$ & $\begin{array}{l}4.700 \\
4.500 \\
4.500 \\
4.700 \\
4.600\end{array}$ & $\begin{array}{l}10.900 \\
10500 \\
11.700 \\
12.200 \\
13.200\end{array}$ & $\begin{array}{l}17.000 \\
17.700 \\
19.600 \\
22.200 \\
24.900\end{array}$ & $\begin{array}{l}21.200 \\
20.700 \\
20.000 \\
19.400 \\
20100\end{array}$ & $\begin{array}{l}35.400 \\
34.700 \\
35.700 \\
33.000 \\
39.100\end{array}$ & $\begin{array}{l}31.400 \\
29.400 \\
27.000 \\
25.000 \\
23.000\end{array}$ & $\begin{array}{l}11.400 \\
11800 \\
11.200 \\
11.200 \\
10.400\end{array}$ & $\begin{array}{l}13.300 \\
16.900 \\
22.200 \\
24.800 \\
27.500\end{array}$ \\
\hline $\begin{array}{l}26 \\
27 \\
28 \\
29 \\
30 \\
31\end{array}$ & $\begin{array}{l}6.200 \\
7.400 \\
5.600 \\
7.600 \\
8.600 \\
6.400\end{array}$ & $\begin{array}{l}6.000 \\
4.600 \\
4.300 \\
4.400 \\
4.400\end{array}$ & $\begin{array}{l}3.800 \\
4.900 \\
4.900 \\
5.300 \\
4.800 \\
4.700\end{array}$ & $\begin{array}{l}5.000 \\
5.600 \\
5.600 \\
5.500 \\
5.600 \\
5.600\end{array}$ & $\begin{array}{r}4.700 \\
4.700 \\
4.600 \\
4.600\end{array}$ & $\begin{array}{l}14.100 \\
13.000 \\
12.500 \\
12.200 \\
12.500 \\
120000\end{array}$ & $\begin{array}{r}27.200 \\
30.200 \\
32.900 \\
32.900 \\
32.200\end{array}$ & $\begin{array}{l}19.800 \\
18.300 \\
16.600 \\
16.500 \\
18.400 \\
19.200\end{array}$ & $\begin{array}{l}39.800 \\
38.200 \\
35.100 \\
33.300 \\
32.000\end{array}$ & $\begin{array}{l}23.000 \\
24.200 \\
25.300 \\
24.900 \\
25.100 \\
27.700\end{array}$ & $\begin{array}{r}10.500 \\
10.000 \\
9.420 \\
9.310 \\
8.560 \\
3.860\end{array}$ & $\begin{array}{l}31.200 \\
32.300 \\
32.200 \\
31.600 \\
30.400 \\
-\end{array}$ \\
\hline $\begin{array}{l}\text { Total } \\
\text { Mean } \\
\text { Max } \\
\text { Min } \\
\text { Cfsm } \\
\text { In. }\end{array}$ & $\begin{array}{r}193,300 \\
6,235 \\
8,600 \\
4,500 \\
0.139 \\
0.16\end{array}$ & $\begin{array}{r}179,500 \\
5,983 \\
8,100 \\
3,900 \\
0.134 \\
0.15\end{array}$ & $\begin{array}{r}168,200 \\
5,426 \\
6,800 \\
3,200 \\
0.121 \\
0.14\end{array}$ & $\begin{array}{r}52,200 \\
4,910 \\
5,600 \\
4,200 \\
0.110 \\
0.13\end{array}$ & $\begin{array}{r}150,500 \\
5,190 \\
6,700 \\
4,000 \\
0.116 \\
0.12\end{array}$ & $\begin{array}{r}299,600 \\
9,665 \\
14,100 \\
4,700 \\
0.216 \\
0.25\end{array}$ & $\begin{array}{r}522,840 \\
17,430 \\
32,900 \\
9,740 \\
0.389 \\
0.43\end{array}$ & $\begin{array}{r}636,700 \\
20,540 \\
31,300 \\
16,500 \\
0.458 \\
0.52\end{array}$ & $\begin{array}{r}893,900 \\
29,800 \\
39,800 \\
17,300 \\
0.665 \\
0.74\end{array}$ & $\begin{array}{r}848,300 \\
27,360 \\
34,200 \\
19,800 \\
0.611 \\
0.70\end{array}$ & $\begin{array}{r}555,750 \\
17,930 \\
27,400 \\
8,660 \\
0.400 \\
0.46\end{array}$ & $\begin{array}{r}465,230 \\
15,510 \\
32,300 \\
8,920 \\
0.346 \\
0.39\end{array}$ \\
\hline $\begin{array}{ll}\text { Cal yr } \\
\text { Ntr yr }\end{array}$ & 1968: & , & 20 & & $\operatorname{Max}$ & 300 & $\begin{array}{l}\text { Min } \\
\text { Min }\end{array}$ & 00 Cfsm & 0.309 & $\begin{array}{l}\text { In. } \\
\text { In. }\end{array}$ & & \\
\hline
\end{tabular}


5-3538. Straight River near Faribault, Minn.

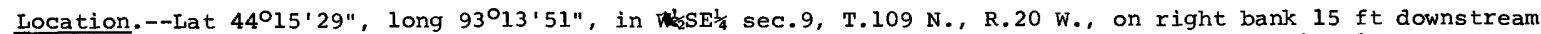
from highway bridge, 2.8 miles upstream from Falls Creek and 3.2 miles southeast of Faribault.

Records_available.--October 1965 to september 1968.

Gage.--Water-stage recorder. Altitude of gage is 1,035 ft (from topographic map).

Extremes.--Maximum discharge during year, 1,930 cfs July 13 (gage height, 7.57 ft); minimum daily, 11 cfs Feb. 18 to Mar. 1; minimum gage height, $3.74 \mathrm{ft}$ Nov, 27.

1965-68: Maximum discharge, 3,590 cfs June 16, 1967 (gage height 9.83 ft); minimum daily, that of Feb. 18 to Mar. 1, 1968; minimum gage height, that of Nov. 27, 1967.

Remarks.--Records good except for winter months, which are fair. Records of chemical analyses and suspended sediment loads for the water year 1968 are published in Part 2 of this report.

DISCHARGE, IN CFS, WATER YEAR OCTOBER 1967 TO SEPTEMBER 1968

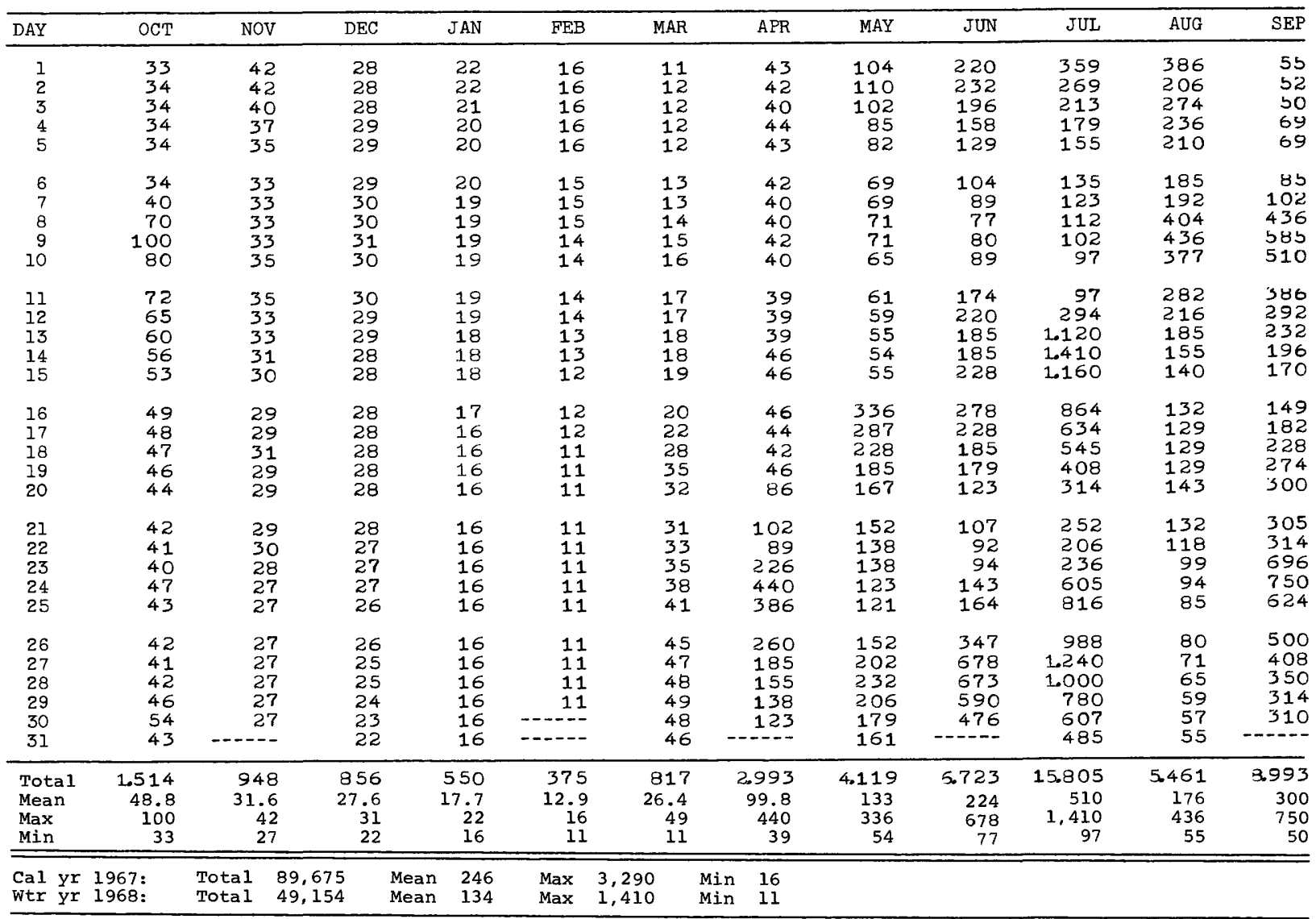


5-3552. Cannon River at Welch, Minn.

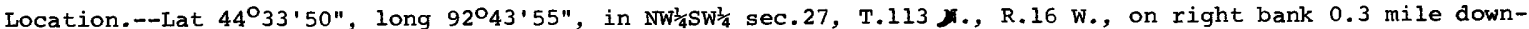
stream from highway bridge at Welch and 1.8 miles upstream from Belle Creek.

Drainage area. $--1,320 \mathrm{sq} \mathrm{mi}$, approximately.

Records available.--June 1909 to January 1914 (no winter records 1909-11), November 1930 to September 1968.

Gage.--Digital water-stage recorder. Datum of gage is $699.16 \mathrm{ft}$ above mean sea level, datum of 1929 . Prior to Nov. 11, 1930, chain gage on highway bridge at site 0.3 mile upstream at datum 3.00 ft lower. Nov. 11, 1930 , to Oct. 11, 1938, graphic water-stage recorder at site 0.3 mile upstream at present datum. Oct. 12 , 1938 to May 24, 1964, graphic water-stage recorder at same site and datum.

Average discharge. -39 years $(1911-13,1931-68), 477 \mathrm{cfs}$.

Extremes.--Maximum discharge during year, 3,380 cfs July 14 (gage height, 6.75 ft); minimum daily, 75 cfs Nov. 29; minimum gage height, $1.39 \mathrm{ft}$ Nov. 29.

1909-14, 1930-68: Maximum discharge, 36,100 cfs Apr. 8, 1965 (gage height, 14.01 ft); minimum, 2.5 cfs

Jan. 3, 1950 (gage height, $0.06 \mathrm{ft}$, backwater from ice)

Maximum stage known, $17.1 \mathrm{ft}$, present datum, in April 1888, from floodmark at mill about 2,400 ft upstream.

Remarks.--Records good except those for winter months, which are fair. Diurnal fluctuation caused by powerplants above station to Dec. 31,1966 when the powerplants were shut down.

UISCHARGE, IN CUBIC FEET PER SECONU, WATEK YEAR OCTOBER 1967 TO SEPTEMBER 1968

\begin{tabular}{|c|c|c|c|c|c|c|c|c|c|c|c|c|}
\hline UAY & ULT & NuV & $D E C$ & JAN & FEB & MAR & APR & MAY & JUN & JUL & ALG & SEP \\
\hline $\begin{array}{l}1 \\
4 \\
3 \\
4 \\
5\end{array}$ & $\begin{array}{l}138 \\
138 \\
138 \\
138 \\
130\end{array}$ & $\begin{array}{l}184 \\
182 \\
182 \\
176 \\
168\end{array}$ & $\begin{array}{l}100 \\
140 \\
148 \\
150 \\
150\end{array}$ & $\begin{array}{l}115 \\
114 \\
113 \\
116 \\
110\end{array}$ & $\begin{array}{l}109 \\
109 \\
109 \\
109 \\
109\end{array}$ & $\begin{array}{l}118 \\
120 \\
1<3 \\
125 \\
1<8\end{array}$ & $\begin{array}{l}125 \\
155 \\
135 \\
178 \\
105\end{array}$ & $\begin{array}{l}305 \\
290 \\
280 \\
246 \\
\angle 36\end{array}$ & $\begin{array}{l}498 \\
474 \\
474 \\
466 \\
419\end{array}$ & $\begin{array}{l}737 \\
598 \\
522 \\
470 \\
419\end{array}$ & $\begin{array}{r}1.040 \\
875 \\
850 \\
728 \\
683\end{array}$ & $\begin{array}{l}263 \\
246 \\
236 \\
330 \\
315\end{array}$ \\
\hline $\begin{array}{r}6 \\
7 \\
8 \\
9 \\
10\end{array}$ & $\begin{array}{l}136 \\
130 \\
171 \\
168 \\
165\end{array}$ & $\begin{array}{l}153 \\
150 \\
135 \\
153 \\
155\end{array}$ & $\begin{array}{l}150 \\
158 \\
158 \\
160 \\
163\end{array}$ & $\begin{array}{l}110 \\
110 \\
109 \\
109 \\
100\end{array}$ & $\begin{array}{l}110 \\
110 \\
110 \\
110 \\
110\end{array}$ & $\begin{array}{l}134 \\
148 \\
189 \\
197 \\
189\end{array}$ & $\begin{array}{l}155 \\
152 \\
203 \\
158 \\
146\end{array}$ & $\begin{array}{l}218 \\
221 \\
259 \\
230 \\
221\end{array}$ & $\begin{array}{l}374 \\
348 \\
323 \\
363 \\
337\end{array}$ & $\begin{array}{l}185 \\
381 \\
367 \\
515 \\
280\end{array}$ & $\begin{array}{l}625 \\
569 \\
571 \\
065 \\
710\end{array}$ & $\begin{array}{l}291 \\
259 \\
287 \\
478 \\
670\end{array}$ \\
\hline $\begin{array}{l}11 \\
12 \\
13 \\
14 \\
15\end{array}$ & $\begin{array}{l}310 \\
001 \\
484 \\
270 \\
261\end{array}$ & $\begin{array}{l}156 \\
168 \\
176 \\
168 \\
136\end{array}$ & $\begin{array}{l}160 \\
160 \\
150 \\
148 \\
140\end{array}$ & $\begin{array}{l}108 \\
108 \\
108 \\
108 \\
108\end{array}$ & $\begin{array}{l}110 \\
110 \\
110 \\
110 \\
110\end{array}$ & $\begin{array}{l}181 \\
168 \\
100 \\
173 \\
181\end{array}$ & $\begin{array}{l}155 \\
135 \\
134 \\
170 \\
145\end{array}$ & $\begin{array}{r}215 \\
209 \\
192 \\
195 \\
1.220\end{array}$ & $\begin{array}{l}363 \\
389 \\
407 \\
419 \\
374\end{array}$ & $\begin{array}{r}260 \\
593 \\
710 \\
\angle, 100 \\
\angle, 770\end{array}$ & $\begin{array}{l}670 \\
548 \\
528 \\
434 \\
393\end{array}$ & $\begin{array}{l}674 \\
593 \\
510 \\
454 \\
400\end{array}$ \\
\hline $\begin{array}{l}16 \\
17 \\
18 \\
19 \\
20\end{array}$ & $\begin{array}{l}367 \\
497 \\
243 \\
184 \\
205\end{array}$ & $\begin{array}{l}140 \\
140 \\
146 \\
116 \\
140\end{array}$ & $\begin{array}{l}148 \\
150 \\
149 \\
149 \\
146\end{array}$ & $\begin{array}{l}108 \\
108 \\
108 \\
108 \\
108\end{array}$ & $\begin{array}{l}110 \\
110 \\
111 \\
112 \\
111\end{array}$ & $\begin{array}{l}181 \\
183 \\
213 \\
269 \\
263\end{array}$ & $\begin{array}{l}150 \\
160 \\
160 \\
155 \\
200\end{array}$ & $\begin{array}{r}1,640 \\
330 \\
\angle 63 \\
230 \\
\angle 15\end{array}$ & $\begin{array}{r}367 \\
407 \\
1,050 \\
780 \\
535\end{array}$ & $\begin{array}{r}2.130 \\
1.610 \\
1.380 \\
1.190 \\
977\end{array}$ & $\begin{array}{l}423 \\
390 \\
352 \\
470 \\
825\end{array}$ & $\begin{array}{l}370 \\
400 \\
498 \\
535 \\
526\end{array}$ \\
\hline $\begin{array}{l}21 \\
24 \\
23 \\
24 \\
25\end{array}$ & $\begin{array}{l}146 \\
140 \\
100 \\
162 \\
190\end{array}$ & $\begin{array}{l}145 \\
146 \\
147 \\
147 \\
143\end{array}$ & $\begin{array}{l}150 \\
130 \\
128 \\
127 \\
127\end{array}$ & $\begin{array}{l}108 \\
108 \\
108 \\
108 \\
108\end{array}$ & $\begin{array}{l}113 \\
113 \\
113 \\
113 \\
113\end{array}$ & $\begin{array}{l}118 \\
190 \\
181 \\
178 \\
183\end{array}$ & $\begin{array}{l}259 \\
240 \\
333 \\
482 \\
543\end{array}$ & $\begin{array}{l}250 \\
308 \\
352 \\
359 \\
385\end{array}$ & $\begin{array}{l}746 \\
518 \\
446 \\
404 \\
396\end{array}$ & $\begin{array}{l}850 \\
728 \\
683 \\
755 \\
870\end{array}$ & $\begin{array}{l}062 \\
486 \\
436 \\
427 \\
367\end{array}$ & $\begin{array}{r}589 \\
820 \\
1,520 \\
1,340 \\
1,270\end{array}$ \\
\hline $\begin{array}{l}\angle 6 \\
27 \\
\angle 8 \\
29 \\
30 \\
31\end{array}$ & $\begin{array}{l}140 \\
150 \\
150 \\
176 \\
202 \\
184\end{array}$ & $\begin{array}{r}130 \\
115 \\
90 \\
75 \\
85 \\
\end{array}$ & $\begin{array}{l}125 \\
123 \\
120 \\
119 \\
117 \\
116\end{array}$ & $\begin{array}{l}108 \\
108 \\
108 \\
108 \\
109 \\
109\end{array}$ & $\begin{array}{r}124 \\
115 \\
116 \\
117 \\
- \\
\end{array}$ & $\begin{array}{l}180 \\
203 \\
.221 \\
195 \\
175 \\
183\end{array}$ & $\begin{array}{r}393 \\
506 \\
450 \\
430 \\
352 \\
\end{array}$ & $\begin{array}{l}431 \\
470 \\
494 \\
518 \\
502 \\
470\end{array}$ & $\begin{array}{r}482 \\
683 \\
830 \\
850 \\
805 \\
\end{array}$ & $\begin{array}{l}1,090 \\
2,230 \\
1,780 \\
1,410 \\
1,230 \\
1,220\end{array}$ & $\begin{array}{l}337 \\
319 \\
305 \\
207 \\
277 \\
273\end{array}$ & $\begin{array}{r}1,140 \\
988 \\
855 \\
760 \\
697 \\
\end{array}$ \\
\hline $\begin{array}{l}\text { TUTAL } \\
\text { MEAN } \\
\text { MAX } \\
\text { MIN } \\
\text { CFSM } \\
\text { IN. }\end{array}$ & $\begin{array}{r}6,776 \\
219 \\
001 \\
138 \\
.17 \\
.19\end{array}$ & $\begin{array}{r}4.395 \\
147 \\
184 \\
75 \\
.11 \\
.12\end{array}$ & $\begin{array}{r}4,375 \\
141 \\
163 \\
100 \\
.11 \\
.12\end{array}$ & $\begin{array}{r}3,378 \\
109 \\
115 \\
108 \\
.08 \\
.10\end{array}$ & $\begin{array}{r}3,228 \\
111 \\
117 \\
109 \\
.08 \\
.09\end{array}$ & $\begin{array}{r}0,561 \\
179 \\
263 \\
118 \\
.14 \\
.16\end{array}$ & $\begin{array}{r}7,443 \\
248 \\
593 \\
134 \\
.19 \\
.21\end{array}$ & $\begin{array}{r}11,954 \\
386 \\
1,640 \\
192 \\
.29 \\
.34\end{array}$ & $\begin{array}{r}15,327 \\
511 \\
1.050 \\
323 \\
.39 \\
.43\end{array}$ & $\begin{array}{r}31,046 \\
1,061 \\
2,770 \\
266 \\
.76 \\
.87\end{array}$ & $\begin{array}{r}16,541 \\
.534 \\
1.040 \\
273 \\
.40 \\
.47\end{array}$ & $\begin{array}{r}18,314 \\
610 \\
1,520 \\
236 \\
.46 \\
.52\end{array}$ \\
\hline
\end{tabular}


5-3730. South Fork Zumbro River near Rochester, Minn.

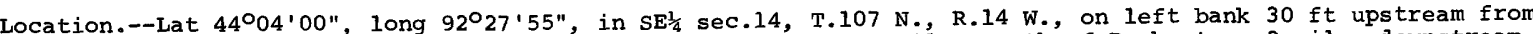
ford, a quarter of a mile downstream from sewage plant, 1.6 miles north of Rochester, 2 miles downstream from Cascade Creek, and $2 \frac{1}{2}$ miles downstream from Silver Lake Dam.

Drainage area. $--304 \mathrm{sq} \mathrm{mi}$

Records available.--January 1952 to September 1968

Gage.--Digital water-stage recorder. Datum of gage is $949.56 \mathrm{ft}$ above mean sea level, datum of 1929 . Prior to July 31, 1967, graphic water-stage recorder at same site and datum.

Average discharge.--16 years, $117 \mathrm{cfs}$.

Extremes.--Maximum discharge during year, 1,570 cfs June 27 (gage height, 7.18 ft); minimum, 17 cfs Jan. 1, $4,8,9$, Feb. 21 (gage height, $1.68 \mathrm{ft}$ ).

1952-68: Maximum discharge, 19,600 cfs Mar. 1, 1965 (gage height, 19.12 ft, from floodmark); minimum,

$8.4 \mathrm{cfs}$ Dec. 7, 1955.

Flood of July 21, 1951 reached a stage of about $17.5 \mathrm{ft}$, from information by sewage plant superintendent.

This is the highest known stage outside the period of record since at least 1908.

Remarks.--Records good. Records of chemical analyses for the water year 1968 are published in Part 2 of this report. Slight regulation at times from silver Lake and at very low flows from sewage-plant effluent.

DISC4ARGE, IN CUBIC FEET PER SECOND, WAIER YEAR DCTOBER 1967 TO SEPTEMBER 1968

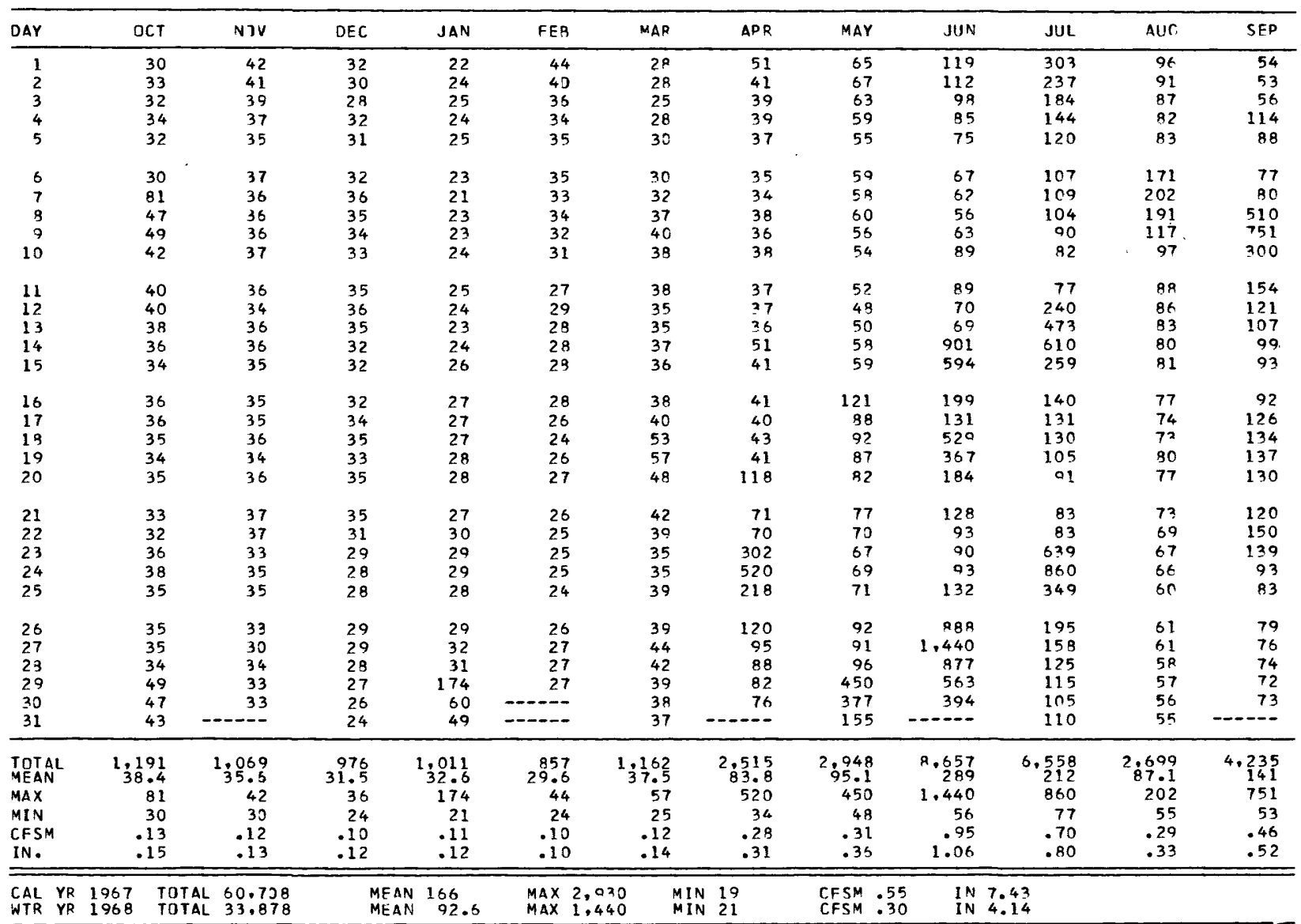

Peak discharge (base, 1,000 cfs)

\begin{tabular}{|c|c|c|c||c|c|c|c|}
\hline Date & Time & $\begin{array}{c}\text { Gage } \\
\text { height }\end{array}$ & Discharge & Date & Time & $\begin{array}{c}\text { Gage } \\
\text { height }\end{array}$ & Discharge \\
\hline $6-14$ & 1700 & 6.45 & 1,240 & $7-23$ & 0845 & 6.12 & 1,020 \\
$6-27$ & 0915 & 7.18 & 1,570 & $9-8$ & 1045 & 5.96 & 1,040 \\
\hline
\end{tabular}


5-3740. Zumbro River at Zumbro Falls, Minn.

Location.--Lat $44^{\circ} 17^{\prime} 12^{\prime \prime}$, long $92^{\circ} 25^{\prime} 56^{\prime \prime}$, in sec.36, T.110 N., R.14 W., on left bank in Zumbro Falls, 1, O00 ft downstream from Spring Creek, 0.7 mile upstream from bridge on U.S. Highway 63 , and 6.3 miles downstream from North Fork.

Drainage area. $--1,130 \mathrm{sq} \mathrm{mi}$, approximately.

Records available.--June 1909 to September 1917, April to November 1929, March 1930 to September 1968. Monthly discharge only for some periods, published in wSP 1308.

Gage.--Digital water-stage recorder. Datum of gage is $811.26 \mathrm{ft}$ above mean sea level, datum of 1929 . Prior to Nov. 11, 1933, chain gage on bridge $800 \mathrm{ft}$ downstream at same datum. Nov. 11, 1933 to May 28, 1964, graphic water-stage recorder at present site and datum.

Average discharge.--46 years $(1909-17,1930-68), 475$ cfs.

Extremes.--Maximum discharge during year, 5, 320 cfs July 27 (gage height, 13.27 ft); minimum, 28 cfs Nov. 27 (gage height, $5.96 \mathrm{ft}$ ).

1909-17, 1929-68: Maximum discharge, 35,900 cfs July 22, 1951 (gage height, 30.80 ft, from floodmark); minimum, 27 cfs Jan. 12, 1935; minimum gage height, 5.96 ft Nov. 27, 1967.

Flood of April 1888 reached stage of about $30.5 \mathrm{ft}$ at present site or $29.7 \mathrm{ft}$ original site. Flood in 1859 is known to have exceeded that of 1888 (gage height, not determined).

Remarks.--Records good except those for winter months, which are fair. Diurnal fluctuation caused by powerplant above station.

DISCHARGE, IN CUBIC FEET PER SECOND, WATER YFAR OCTOBER 1967 TO SEPTEMBER 1968

\begin{tabular}{|c|c|c|c|c|c|c|c|c|c|c|c|c|}
\hline DAY & $\mathrm{OCT}$ & NOV & DEC & JAN & FEB & MAR & $A P R$ & MAYY & JUN & JUL & AUG & SER \\
\hline $\begin{array}{l}1 \\
2 \\
3 \\
4 \\
5\end{array}$ & $\begin{array}{l}82 \\
125 \\
152 \\
149 \\
136\end{array}$ & $\begin{array}{l}176 \\
164 \\
176 \\
171 \\
34\end{array}$ & $\begin{array}{l}382 \\
233 \\
121 \\
214 \\
274\end{array}$ & $\begin{array}{r}67 \\
66 \\
110 \\
130 \\
152\end{array}$ & $\begin{array}{r}230 \\
235 \\
140 \\
68 \\
78\end{array}$ & $\begin{array}{r}177 \\
163 \\
78 \\
270 \\
434\end{array}$ & $\begin{array}{r}84 \\
181 \\
248 \\
247 \\
151\end{array}$ & $\begin{array}{l}355 \\
296 \\
206 \\
249 \\
157\end{array}$ & $\begin{array}{l}559 \\
311 \\
425 \\
709 \\
330\end{array}$ & $\begin{array}{l}981 \\
888 \\
734 \\
564 \\
472\end{array}$ & $\begin{array}{l}789 \\
704 \\
636 \\
430 \\
456\end{array}$ & $\begin{array}{l}120 \\
120 \\
306 \\
558 \\
391\end{array}$ \\
\hline $\begin{array}{r}6 \\
7 \\
8 \\
9 \\
10\end{array}$ & $\begin{array}{l}166 \\
190 \\
404 \\
160 \\
136\end{array}$ & $\begin{array}{l}137 \\
329 \\
449 \\
399 \\
198\end{array}$ & $\begin{array}{r}290 \\
283 \\
198 \\
134 \\
88\end{array}$ & $\begin{array}{r}195 \\
94 \\
64 \\
121 \\
128\end{array}$ & $\begin{array}{l}102 \\
100 \\
205 \\
200 \\
150\end{array}$ & $\begin{array}{r}148 \\
87 \\
83 \\
84 \\
80\end{array}$ & $\begin{array}{r}83 \\
78 \\
83 \\
134 \\
138\end{array}$ & $\begin{array}{r}97 \\
196 \\
274 \\
181 \\
242\end{array}$ & $\begin{array}{l}339 \\
336 \\
299 \\
193 \\
345\end{array}$ & $\begin{array}{l}459 \\
408 \\
410 \\
399 \\
242\end{array}$ & $\begin{array}{l}462 \\
472 \\
457 \\
586 \\
298\end{array}$ & $\begin{array}{r}520 \\
352 \\
774 \\
1,570 \\
1,520\end{array}$ \\
\hline $\begin{array}{l}11 \\
12 \\
13 \\
14\end{array}$ & $\begin{array}{l}136 \\
119 \\
227 \\
135\end{array}$ & $\begin{array}{r}121 \\
39 \\
111 \\
221\end{array}$ & $\begin{array}{l}112 \\
193 \\
203 \\
202\end{array}$ & $\begin{array}{l}136 \\
143 \\
122 \\
108\end{array}$ & $\begin{array}{r}71 \\
66 \\
178 \\
140\end{array}$ & $\begin{array}{r}77 \\
138 \\
103 \\
92\end{array}$ & $\begin{array}{r}150 \\
136 \\
131 \\
83\end{array}$ & $\begin{array}{r}184 \\
94 \\
203 \\
251\end{array}$ & $\begin{array}{l}282 \\
259 \\
427 \\
719\end{array}$ & $\begin{array}{r}350 \\
1.000 \\
2.620 \\
4.050\end{array}$ & $\begin{array}{l}252 \\
495 \\
359 \\
309\end{array}$ & $\begin{array}{r}1,010 \\
925 \\
540 \\
434\end{array}$ \\
\hline 15 & 81 & 137 & 186 & 71 & 136 & 79 & 95 & 260 & 802 & 2.930 & 319 & 488 \\
\hline $\begin{array}{l}16 \\
17 \\
18 \\
19 \\
20\end{array}$ & $\begin{array}{r}82 \\
131 \\
172 \\
221 \\
139\end{array}$ & $\begin{array}{r}140 \\
137 \\
128 \\
33 \\
157\end{array}$ & $\begin{array}{r}140 \\
82 \\
143 \\
194 \\
229\end{array}$ & $\begin{array}{l}176 \\
125 \\
230 \\
137 \\
102\end{array}$ & $\begin{array}{r}143 \\
147 \\
69 \\
68 \\
116\end{array}$ & $\begin{array}{r}134 \\
81 \\
128 \\
271 \\
247\end{array}$ & $\begin{array}{l}163 \\
166 \\
141 \\
238 \\
373\end{array}$ & $\begin{array}{r}1,060 \\
809 \\
589 \\
275 \\
434\end{array}$ & $\begin{array}{r}366 \\
332 \\
1,050 \\
1,070 \\
637\end{array}$ & $\begin{array}{r}1.610 \\
1.220 \\
1.030 \\
1.010 \\
660\end{array}$ & $\begin{array}{l}353 \\
246 \\
142 \\
398 \\
924\end{array}$ & $\begin{array}{l}473 \\
398 \\
608 \\
708 \\
548\end{array}$ \\
\hline $\begin{array}{l}21 \\
22 \\
23 \\
24 \\
25\end{array}$ & $\begin{array}{r}134 \\
79 \\
193 \\
263 \\
159\end{array}$ & $\begin{array}{l}230 \\
205 \\
168 \\
124 \\
178\end{array}$ & $\begin{array}{r}184 \\
141 \\
112 \\
67 \\
61\end{array}$ & $\begin{array}{r}73 \\
84 \\
182 \\
184 \\
179\end{array}$ & $\begin{array}{r}135 \\
111 \\
105 \\
262 \\
71\end{array}$ & $\begin{array}{r}385 \\
259 \\
224 \\
91 \\
178\end{array}$ & $\begin{array}{l}210 \\
282 \\
382 \\
561 \\
382\end{array}$ & $\begin{array}{l}482 \\
422 \\
283 \\
268 \\
199\end{array}$ & $\begin{array}{l}623 \\
462 \\
336 \\
373 \\
437\end{array}$ & $\begin{array}{l}451 \\
646 \\
596 \\
737 \\
958\end{array}$ & $\begin{array}{l}476 \\
436 \\
459 \\
253 \\
138\end{array}$ & $\begin{array}{r}650 \\
930 \\
1,680 \\
1,020 \\
678\end{array}$ \\
\hline $\begin{array}{l}26 \\
27 \\
28 \\
29 \\
30 \\
31\end{array}$ & $\begin{array}{r}121 \\
120 \\
117 \\
84 \\
205 \\
210\end{array}$ & $\begin{array}{r}85 \\
164 \\
179 \\
300 \\
412 \\
\end{array}$ & $\begin{array}{r}60 \\
61 \\
140 \\
190 \\
185 \\
138\end{array}$ & $\begin{array}{r}178 \\
113 \\
78 \\
160 \\
190 \\
230\end{array}$ & $\begin{array}{r}70 \\
199 \\
175 \\
176 \\
\\
\end{array}$ & $\begin{array}{r}275 \\
431 \\
293 \\
280 \\
191 \\
92\end{array}$ & $\begin{array}{r}211 \\
132 \\
122 \\
232 \\
284 \\
\end{array}$ & $\begin{array}{l}164 \\
589 \\
585 \\
636 \\
711 \\
529\end{array}$ & $\begin{array}{r}714 \\
2,150 \\
2.430 \\
1.560 \\
1.230 \\
\end{array}$ & $\begin{array}{r}942 \\
3,640 \\
2,440 \\
1,260 \\
1,090 \\
793\end{array}$ & $\begin{array}{l}215 \\
265 \\
238 \\
278 \\
216 \\
169\end{array}$ & $\begin{array}{r}832 \\
761 \\
559 \\
267 \\
415 \\
\\
\end{array}$ \\
\hline METAL & $\begin{array}{r}4,828 \\
156\end{array}$ & $\begin{array}{r}5.632 \\
188\end{array}$ & $\begin{array}{r}5,240 \\
169\end{array}$ & $4 \cdot 12 \frac{28}{133}$ & $\begin{array}{r}3.946 \\
136\end{array}$ & $\begin{array}{r}5.653 \\
182\end{array}$ & $5.90 \frac{1}{197}$ & $11 \cdot 280$ & $20, \frac{105}{670}$ & $\begin{array}{r}35: 590 \\
1: 148\end{array}$ & $12 \cdot 230$ & $20, \frac{155}{672}$ \\
\hline $\begin{array}{l}\text { MAX } \\
\text { MIN }\end{array}$ & $\begin{array}{r}404 \\
79\end{array}$ & $\begin{array}{r}449 \\
83\end{array}$ & $\begin{array}{r}382 \\
60\end{array}$ & $\begin{array}{r}230 \\
64\end{array}$ & $\begin{array}{r}262 \\
66\end{array}$ & $\begin{array}{r}434 \\
77\end{array}$ & $\begin{array}{r}561 \\
78\end{array}$ & $\begin{array}{r}1,060 \\
94\end{array}$ & $\begin{array}{r}2,430 \\
193\end{array}$ & $\begin{array}{r}4,050 \\
242\end{array}$ & $\begin{array}{l}924 \\
138\end{array}$ & $\begin{array}{r}1,680 \\
120\end{array}$ \\
\hline $\begin{array}{l}\text { CFSM } \\
\text { IN. }\end{array}$ & $\begin{array}{l}.14 \\
.16\end{array}$ & $\begin{array}{r}.17 \\
.19 \\
\end{array}$ & $\begin{array}{r}15 \\
117 \\
\end{array}$ & $\begin{array}{r}.12 \\
.14\end{array}$ & $\begin{array}{l}.12 \\
.13\end{array}$ & $\begin{array}{r}16 \\
.19 \\
\end{array}$ & $\begin{array}{r}17 \\
.19\end{array}$ & $\begin{array}{l}.32 \\
.37\end{array}$ & $\begin{array}{l}.59 \\
.66\end{array}$ & $\begin{array}{l}1.02 \\
1.17\end{array}$ & $\begin{array}{r}.35 \\
.40\end{array}$ & $\begin{array}{r}.59 \\
.66\end{array}$ \\
\hline
\end{tabular}

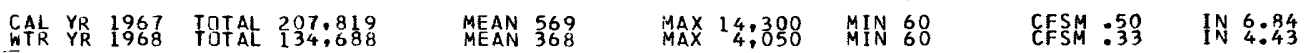


5-3760. North Fork Whitewater River near Elba, Minn.

(Hydrologic bench-mark station)

Location.--Lat $44^{\circ} 05^{\prime} 30^{\prime \prime}$, long $92^{\circ} 03^{\prime} 57^{\prime \prime}$, in sec.7, T.107 N., R.10 W., Winona County, on left bank 2.3 mile upstream from Middle Fork, 2.4 miles west of Elba, and 3.5 miles upstream from confluence with south Fork.

Drainage area.--101 sq $\mathrm{mi}$.

Records available.--May 1939 to september 1941, July 1967 to september 1968.

Gage.--Digital water-stage recorder. Datum of gage is $769.60 \mathrm{ft}$ above mean sea level, datum of 1929 . Oct. 12 , 1939, to sept. 30, 1941, graphic water-stage recorder at site 600 ft downstream at same datum. Prior to Oct. 12, 1939, nonrecording gage at site 2 miles downstream at different datum.

Extremes.--1967: Maximum discharge during period July 1 to september $30,103 \mathrm{cfs}$ Aug. 15 (gage height, $3.32 \mathrm{ft}$ ); minimum daily, $13 \mathrm{cfs} \mathrm{July} 25$ to Aug. 5 .

1967-68: Maximum discharge during year, 880 cfs sept. 13 (gage height, 6.63 ft); minimum, 11 cfs Feb. 21 (gage height, $2.37 \mathrm{ft}$ ).

1939-41, 1967-68: Maximum discharge, 2,730 cfs July 11, 1940 (gage height, 6.13 ft), may have been exceeded sept. 15, 1941; minimum, 11 cfs Feb. 21 (gage height, $2.37 \mathrm{ft}$ ).

Remarks.--Records good. Records of chemical analyses and suspended sediment loads for the water year 1967 and 1968 are published in part 2 of this report.

DISCHARGE, IN CUBIC FEET PER SECOND, July to September 1967

\begin{tabular}{|c|c|c|c|c|c|c|c|c|c|c|c|c|}
\hline DAY & $\mathrm{OCT}$ & NOV & $\mathrm{DEC}$ & JAN & F'EB & MAR & APR & MAY & JUN & JUL & AUG & SEP \\
\hline $\begin{array}{l}1 \\
2 \\
3 \\
4 \\
5\end{array}$ & & & & & & & & & & $\begin{array}{l}21 \\
20 \\
20 \\
19 \\
19\end{array}$ & $\begin{array}{l}13 \\
13 \\
13 \\
13 \\
13\end{array}$ & $\begin{array}{l}14 \\
15 \\
14 \\
14 \\
14\end{array}$ \\
\hline $\begin{array}{r}6 \\
7 \\
8 \\
9 \\
10\end{array}$ & & & & & & & & & & $\begin{array}{l}19 \\
18 \\
18 \\
18 \\
18\end{array}$ & $\begin{array}{l}16 \\
16 \\
15 \\
15 \\
15\end{array}$ & $\begin{array}{l}14 \\
14 \\
14 \\
14 \\
14\end{array}$ \\
\hline $\begin{array}{l}11 \\
12 \\
13 \\
14 \\
15\end{array}$ & & & & & & & & & & $\begin{array}{l}17 \\
16 \\
16 \\
15 \\
15\end{array}$ & $\begin{array}{l}15 \\
15 \\
14 \\
14 \\
25\end{array}$ & $\begin{array}{l}14 \\
14 \\
15 \\
16 \\
14\end{array}$ \\
\hline $\begin{array}{l}16 \\
17 \\
18 \\
19 \\
20\end{array}$ & & & & & & & & & & $\begin{array}{l}15 \\
15 \\
15 \\
14 \\
15\end{array}$ & $\begin{array}{l}16 \\
15 \\
15 \\
15 \\
15\end{array}$ & $\begin{array}{l}14 \\
13 \\
14 \\
14 \\
14\end{array}$ \\
\hline $\begin{array}{l}21 \\
22 \\
23 \\
24 \\
25\end{array}$ & & & & & & & & & & $\begin{array}{l}15 \\
14 \\
14 \\
14 \\
13\end{array}$ & $\begin{array}{l}15 \\
15 \\
15 \\
15 \\
15\end{array}$ & $\begin{array}{l}14 \\
14 \\
14 \\
14 \\
14\end{array}$ \\
\hline $\begin{array}{l}26 \\
27 \\
28 \\
29 \\
30 \\
31\end{array}$ & & ------ & & & ----- & & ------ & & ----- & $\begin{array}{l}13 \\
13 \\
13 \\
13 \\
13 \\
13\end{array}$ & $\begin{array}{l}16 \\
15 \\
15 \\
15 \\
15 \\
14\end{array}$ & $\begin{array}{r}13 \\
13 \\
13 \\
14 \\
14 \\
\end{array}$ \\
\hline Tota 1 & & & & & & & & & & 491 & 466 & 420 \\
\hline $\begin{array}{l}\text { Mean } \\
\text { Max } \\
\text { Min } \\
\text { Cfsm } \\
\text { In. }\end{array}$ & & & & & & & & & & $\begin{array}{r}15.8 \\
21 \\
13 \\
0.156 \\
0.18\end{array}$ & $\begin{array}{r}15.0 \\
25 \\
13 \\
0.149 \\
0.17\end{array}$ & $\begin{array}{r}14.0 \\
16 \\
13 \\
0.139 \\
0.15\end{array}$ \\
\hline
\end{tabular}


5-3760. North Fork Whitewater River near Elba, Minn.--Continued

CISCHARGE, IN CUBIC FEET PER SECOND, WATER YEAR OCTOBER 1967 TO SEPTEMBER 1968

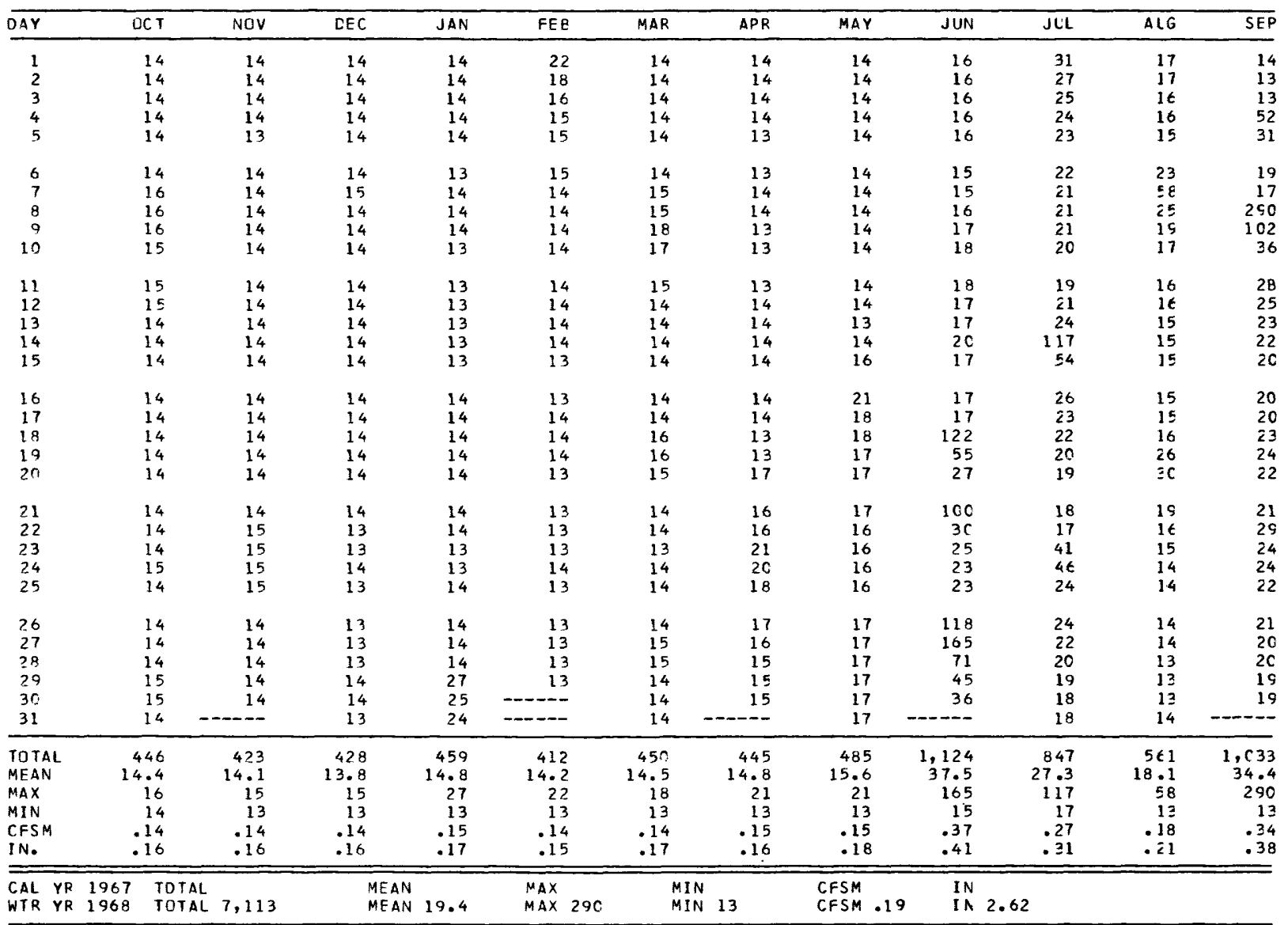


5-3765. South Fork Whitewater River near Altura, Minn.

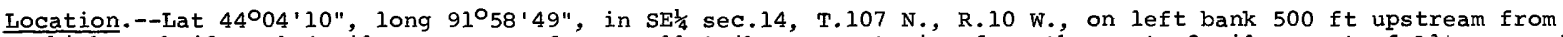
highway bridge, 1.4 miles upstream from small tributary entering from the west, 2 miles west of Altura, and 2.4 miles upstream from Keefer Creek.

Drainage area. $--76.8 \mathrm{sq} \mathrm{mi}$.

Records available.--October 1939 to september 1968.

Gage.--Water-stage recorder. Datum of gage is $761.80 \mathrm{ft}$ above mean sea level, adjustment of 1912 (levels by Corps of Engineers).

Average discharge. -29 years, $27.2 \mathrm{cfs}$.

Extremes.--Maximum discharge during year, 391 cfs June 21 (gage height, 3.53 ft); minimum, 5.7 cfs Jan. 23 (gage height, $0.76 \mathrm{ft}$ ).

1939-68: Maximum discharge, 5,460 cfs Aug. 31, 1947 (gage height, 10.61 ft); minimum, 3.8 cfs Mar. 24, 1940; minimum gage height, 0.23 ft Mar. 12, 1965.

Remarks.--Records fair.

DISCHARGE, IN CUBIC FEET PER SECOND, WATER YEAR OCTOBER 1967 TO SEPTEMBER 1968

\begin{tabular}{|c|c|c|c|c|c|c|c|c|c|c|c|c|}
\hline DAY & OCT & NOV & $\mathrm{DEC}$ & JAN & FEB & MAR & APR & MAY & JUN & JUL & AUG & SEP \\
\hline $\begin{array}{l}1 \\
2 \\
3 \\
4 \\
5\end{array}$ & $\begin{array}{ll}1 & 1 \\
1 & 1 \\
1 & 1 \\
1 & 1 \\
1 & 1\end{array}$ & $\begin{array}{ll}1 & 1 \\
1 & 1 \\
1 & 1 \\
1 & 1 \\
11\end{array}$ & $\begin{array}{l}10 \\
10 \\
10 \\
10 \\
10\end{array}$ & $\begin{array}{l}11 \\
11 \\
11 \\
12 \\
12\end{array}$ & $\begin{array}{l}8.5 \\
7.6 \\
7.5 \\
7.2 \\
7.3\end{array}$ & $\begin{array}{r}11 \\
9.9 \\
9.0 \\
8.7 \\
7.3\end{array}$ & $\begin{array}{ll}1 & 0 \\
1 & 0 \\
1 & 1 \\
1 & 1 \\
1 & 1\end{array}$ & $\begin{array}{l}14 \\
14 \\
13 \\
13 \\
12\end{array}$ & $\begin{array}{l}23 \\
24 \\
22 \\
21 \\
20\end{array}$ & $\begin{array}{l}54 \\
47 \\
45 \\
37 \\
34\end{array}$ & $\begin{array}{l}50 \\
46 \\
51 \\
62 \\
64\end{array}$ & $\begin{array}{l}23 \\
22 \\
25 \\
61 \\
28\end{array}$ \\
\hline $\begin{array}{r}6 \\
7 \\
8 \\
9 \\
10\end{array}$ & $\begin{array}{l}11 \\
13 \\
12 \\
12 \\
12\end{array}$ & $\begin{array}{ll}1 & 1 \\
11 & 1 \\
11 & 1 \\
11 \\
11\end{array}$ & $\begin{array}{ll}1 & 0 \\
1 & 1 \\
10 & 0 \\
10 \\
10\end{array}$ & $\begin{array}{l}14 \\
15 \\
15 \\
16 \\
17\end{array}$ & $\begin{array}{l}7.1 \\
7.0 \\
7.2 \\
7.4 \\
7.6\end{array}$ & $\begin{array}{l}7.3 \\
7.7 \\
8.3 \\
9.1 \\
8.8\end{array}$ & $\begin{array}{ll}1 & 1 \\
1 & 1 \\
1 & 1 \\
1 & 1 \\
11\end{array}$ & $\begin{array}{l}12 \\
12 \\
12 \\
12 \\
12\end{array}$ & $\begin{array}{l}19 \\
18 \\
18 \\
25 \\
28\end{array}$ & $\begin{array}{l}32 \\
32 \\
32 \\
28 \\
26\end{array}$ & $\begin{array}{l}65 \\
40 \\
28 \\
22 \\
20\end{array}$ & $\begin{array}{l}20 \\
18 \\
37 \\
32 \\
24\end{array}$ \\
\hline $\begin{array}{l}11 \\
12 \\
13 \\
14 \\
15\end{array}$ & $\begin{array}{l}12 \\
12 \\
11 \\
11 \\
11\end{array}$ & $\begin{array}{l}11 \\
11 \\
11 \\
11 \\
11\end{array}$ & $\begin{array}{l}10 \\
10 \\
10 \\
10 \\
10\end{array}$ & $\begin{array}{l}16 \\
15 \\
14 \\
13 \\
12\end{array}$ & $\begin{array}{l}8.0 \\
8.4 \\
8.8 \\
9.2 \\
9.2\end{array}$ & $\begin{array}{l}9.0 \\
9.3 \\
9.5 \\
9.8 \\
10\end{array}$ & $\begin{array}{ll}1 & 1 \\
1 & 1 \\
1 & 1 \\
1 & 1 \\
1 & 2\end{array}$ & $\begin{array}{l}12 \\
12 \\
13 \\
14 \\
19\end{array}$ & $\begin{array}{l}28 \\
28 \\
26 \\
44 \\
42\end{array}$ & $\begin{array}{l}25 \\
26 \\
32 \\
52 \\
32\end{array}$ & $\begin{array}{l}18 \\
17 \\
17 \\
16 \\
14\end{array}$ & $\begin{array}{l}19 \\
18 \\
17 \\
16 \\
16\end{array}$ \\
\hline $\begin{array}{l}16 \\
17 \\
18 \\
19 \\
20\end{array}$ & $\begin{array}{l}11 \\
11 \\
11 \\
11 \\
11\end{array}$ & $\begin{array}{ll}1 & 1 \\
1 & 1 \\
1 & 1 \\
1 & 1 \\
1 & 1\end{array}$ & $\begin{array}{l}10 \\
10 \\
11 \\
11 \\
11\end{array}$ & $\begin{array}{l}12 \\
12 \\
11 \\
11 \\
10\end{array}$ & $\begin{array}{l}8.8 \\
9.2 \\
10 \\
9.8 \\
9.8\end{array}$ & $\begin{array}{l}10 \\
10 \\
12 \\
12 \\
10\end{array}$ & $\begin{array}{l}12 \\
12 \\
12 \\
12 \\
16\end{array}$ & $\begin{array}{l}35 \\
30 \\
21 \\
20 \\
20\end{array}$ & $\begin{array}{r}30 \\
27 \\
108 \\
65 \\
39\end{array}$ & $\begin{array}{l}27 \\
24 \\
22 \\
19 \\
17\end{array}$ & $\begin{array}{l}18 \\
17 \\
17 \\
18 \\
20\end{array}$ & $\begin{array}{l}16 \\
18 \\
21 \\
23 \\
21\end{array}$ \\
\hline $\begin{array}{l}21 \\
22 \\
23 \\
24 \\
25\end{array}$ & $\begin{array}{l}11 \\
11 \\
12 \\
12 \\
12\end{array}$ & $\begin{array}{l}11 \\
11 \\
11 \\
11 \\
11\end{array}$ & $\begin{array}{l}10 \\
10 \\
10 \\
10 \\
10\end{array}$ & $\begin{array}{r}9.8 \\
9.4 \\
9.0 \\
9.9 \\
10\end{array}$ & $\begin{array}{ll}1 & 0 \\
1 & 1 \\
1 & 3 \\
12 \\
1 & 1\end{array}$ & $\begin{array}{l}9.9 \\
9.6 \\
9.6 \\
9.6 \\
9.6\end{array}$ & $\begin{array}{l}15 \\
15 \\
21 \\
32 \\
23\end{array}$ & $\begin{array}{l}18 \\
17 \\
16 \\
16 \\
16\end{array}$ & $\begin{array}{l}70 \\
34 \\
30 \\
49 \\
38\end{array}$ & $\begin{array}{l}20 \\
32 \\
73 \\
63 \\
36\end{array}$ & $\begin{array}{l}16 \\
14 \\
13 \\
28 \\
30\end{array}$ & $\begin{array}{l}20 \\
23 \\
24 \\
22 \\
21\end{array}$ \\
\hline $\begin{array}{l}26 \\
27 \\
28 \\
29 \\
30 \\
31\end{array}$ & $\begin{array}{l}12 \\
12 \\
11 \\
12 \\
12 \\
12\end{array}$ & $\begin{array}{r}11 \\
11 \\
11 \\
11 \\
11 \\
\end{array}$ & $\begin{array}{ll}1 & 0 \\
1 & 0 \\
1 & 0 \\
1 & 1 \\
1 & 1 \\
1 & 1 \\
\end{array}$ & $\begin{array}{c}9.1 \\
8.3 \\
7.9 \\
18 \\
11 \\
9.5 \\
\end{array}$ & $\begin{array}{r}11 \\
10 \\
10 \\
10 \\
- \\
-\end{array}$ & $\begin{array}{l}9.6 \\
10 \\
10 \\
10 \\
9.9 \\
9.9 \\
\end{array}$ & $\begin{array}{r}18 \\
16 \\
16 \\
15 \\
14 \\
\end{array}$ & $\begin{array}{l}19 \\
22 \\
21 \\
24 \\
28 \\
22 \\
\end{array}$ & $\begin{array}{r}103 \\
142 \\
88 \\
71 \\
61 \\
\end{array}$ & $\begin{array}{l}31 \\
47 \\
62 \\
52 \\
50 \\
53 \\
\end{array}$ & $\begin{array}{l}30 \\
29 \\
28 \\
27 \\
25 \\
25\end{array}$ & $\begin{array}{r}21 \\
20 \\
20 \\
19 \\
19 \\
\end{array}$ \\
\hline $\begin{array}{l}\text { Total } \\
\text { Mean } \\
\text { Max } \\
\text { Min } \\
\text { Cfsm } \\
\text { In. } \\
\end{array}$ & $\begin{array}{r}356 \\
11.5 \\
13 \\
11 \\
0.150 \\
0.17 \\
\end{array}$ & $\begin{array}{r}330 \\
11.0 \\
11 \\
11 \\
0.143 \\
0.16\end{array}$ & $\begin{array}{r}317 \\
10.2 \\
11 \\
10 \\
0.133 \\
0.15 \\
\end{array}$ & $\begin{array}{r}371.9 \\
12.0 \\
18 \\
7.9 \\
0.156 \\
0.18 \\
\end{array}$ & $\begin{array}{r}263.6 \\
9.09 \\
13 \\
7.0 \\
0.118 \\
0.13 \\
\end{array}$ & $\begin{array}{r}296.4 \\
9.56 \\
12 \\
7.3 \\
0.124 \\
0.14 \\
\end{array}$ & $\begin{array}{r}413 \\
13.8 \\
32 \\
10 \\
0.180 \\
0.20 \\
\end{array}$ & $\begin{array}{r}541 \\
17.4 \\
35 \\
12 \\
0.227 \\
0.26 \\
\end{array}$ & $\begin{array}{r}1.341 \\
44.7 \\
142 \\
18 \\
0.582 \\
0.65 \\
\end{array}$ & $\begin{array}{r}1.162 \\
37.5 \\
73 \\
17 \\
0.488 \\
0.56 \\
\end{array}$ & $\begin{array}{r}885 \\
28.5 \\
65 \\
13 \\
0.371 \\
0.43 \\
\end{array}$ & $\begin{array}{r}684 \\
22.8 \\
61 \\
16 \\
0.297 \\
0.33\end{array}$ \\
\hline $\begin{array}{ll}\text { Cal yr } \\
\text { wtr }\end{array}$ & 8: & $\begin{array}{l}\text { Total } \\
\text { Total }\end{array}$ & $\begin{array}{l}489 \\
960.9\end{array}$ & $\begin{array}{l}\text { Mean } \\
\text { Mean }\end{array}$ & $\begin{array}{r}.5 \\
.0\end{array}$ & $\begin{array}{r}1,46 \\
14\end{array}$ & $\begin{array}{l}\operatorname{Min} \\
\operatorname{Min}\end{array}$ & $\begin{array}{l}9.4 \\
7.0\end{array}$ & $\begin{array}{l}\mathrm{sm} \\
\mathrm{sm}\end{array}$ & $\begin{array}{l}\text { In. } \\
\text { In. }\end{array}$ & $\begin{array}{l}5.56 \\
3.37\end{array}$ & \\
\hline
\end{tabular}

Peak discharge (base, $400 \mathrm{cfs}$ ).-- No peak above base. 
5-3785. Mississippi River at Winona, Minn.

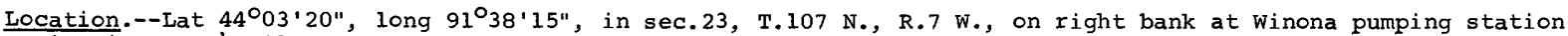
in Winona, $9 \frac{1}{2}$ miles upstream from Trempealeau River and at mile 725.7 upstream from the Ohio River.

Drainage area. $--59,200 \mathrm{sq} \mathrm{mi}$, approximately.

Records available.--June 1928 to September 1968. Gage-height records collected in this vicinity since 1878 are contained in reports of Mississippi River Commission.

Gage.--Water-stage recorder. Datum of gage is $639.64 \mathrm{ft}$ above mean sea level, datum of 1929 , June 10 , 1928 to Apr. 15, 1931, staff gage at site $800 \mathrm{ft}$ upstream. Prior to Oct. 1, 1929, at datum 0.20 ft higher and Oct. 1, 1929, to Apr. 15, 1931, at datum 0.12 ft lower. Apr. 16, 1931, to Nov. 12, 1934, staff gage at present site and datum. Since Mar. 31, 1937, auxiliary water-stage recorder 2.7 miles upstream at tailwater of navigation dam $5 \mathrm{~A}$.

Average discharge. --40 years, $24,920 \mathrm{cfs}$.

Extremes.--Maximum discharge during year, 75,000 cfs June 27 (gage height, 9.97 ft); minimum daily, 5, 000 cfs Dec. 17; minimum gage height, $4.83 \mathrm{ft}$ Dec. 17.

1928-68: Maximum discharge, 268, 000 cfs Apr. 19, 1965 (gage height, 20.77 ft, from floodmark); minimum, 2, 250 cfs Dec. 29, 1933 (gage height, $-1.18 \mathrm{ft}$ ); minimum gage height, $-3.38 \mathrm{ft} \mathrm{Aug.} 31$, 1934

Remarks.--Records good. Records of chemical analyses for the water year 1968 are published in Part 2 of this report. Some regulation by reservoirs, navigation dams, and powerplants at low and medium stages. Flood flow not materially affected by artificial storage.

Cooperation.--Gage-height record at dam 5A furnished by Corps of Engineers.

DISCHARGE, IN CUBIC FEET PER SECOND, WATER YEAR OCTOBER 1967 TO SEPTEMBER 1968

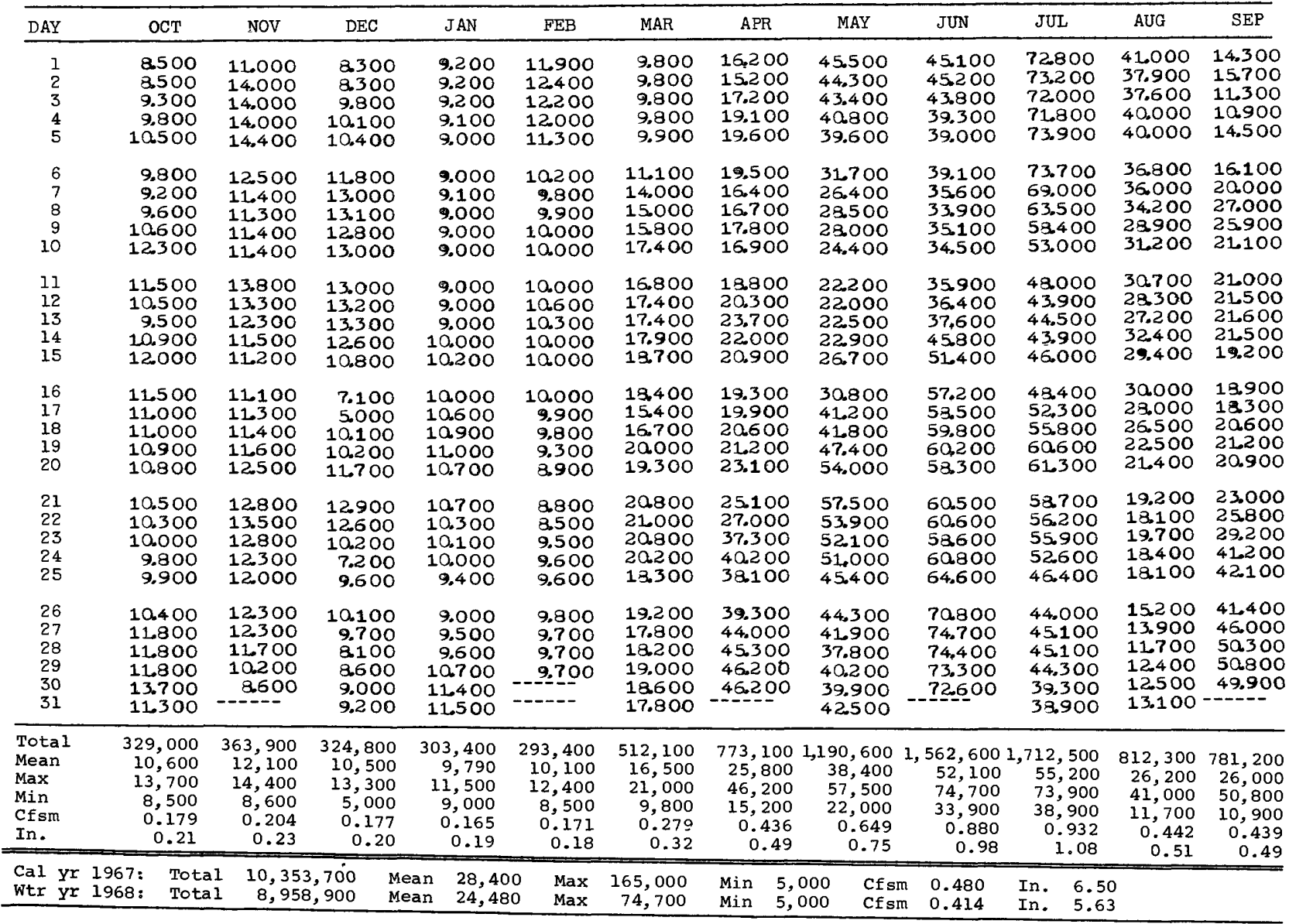


5-3840. Root River near Lanesboro, Minn.

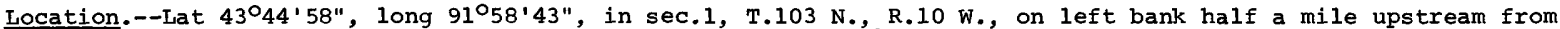
highway bridge, $1 \frac{1}{4}$ miles upstream from South Branch, and $2 \frac{1}{2}$ miles northeast of Lanesboro.

Drainage area. $--615 \mathrm{sq} \mathrm{mi}$.

Records available.--February to November 1910, February 1911 to September 1914, July 1915 to September 1917, August 1940 to september 1968. Published as North Branch Root River near Lanesboro, 1910-17.

Gage.-Digital water-stage recorder. Datum of gage is $791.84 \mathrm{ft}$ above mean sea level, adjustment of 1912 . Prior to Oct. 1, 1917, chain gage at site half a mile downstream at datum about $1.5 \mathrm{ft}$ higher. Aug. 2 , 1940 to May 19, 1966, graphic water-stage recorder at present site and datum.

Average discharge.--33 years $(1911-14,1915-17,1940-68), 314$ cfs.

Extremes.--Maximum discharge during year, 1,790 cfs July 23 (gage height, 4.76 ft); minimum, 54 cfs Dec. 15 (gage height, $0.80 \mathrm{ft}$ )

1910-17, 1940-68: Maximum discharge 22,100 cfs Mar. 29, 1962 (gage height, 16.11 ft); maximum gage height, $17.83 \mathrm{ft}$ Mar. 1, 1965, from floodmark, backwater from ice; minimum discharge, 29 cfs Aug. 27,1949 (gage height, $1.08 \mathrm{ft}$ ); minimum gage height, $0.80 \mathrm{ft}$ Dec. 15, 1967.

Remarks.--Records good except those for winter months, which are fair. Diurnal fluctuation at times during medium and low flow caused by powerplant above station. Records of suspended sediment loads for the water year 1968 are published in Part 2 of this report.

OISCHARGE, IN CUBIC FEET PER SECOND, WATER YEAR OCTOBER 1967 TO SEPTEMBER 1968

\begin{tabular}{|c|c|c|c|c|c|c|c|c|c|c|c|c|}
\hline DAY & OCT & NOV & DEC & JAN & FEB & MAR & APR & MAY & JUN & JUL & ALG & SEP \\
\hline $\begin{array}{l}1 \\
2 \\
3 \\
4 \\
5\end{array}$ & $\begin{array}{l}84 \\
84 \\
84 \\
83 \\
83\end{array}$ & $\begin{array}{l}99 \\
99 \\
97 \\
95 \\
93\end{array}$ & $\begin{array}{l}93 \\
93 \\
92 \\
90 \\
90\end{array}$ & $\begin{array}{l}80 \\
80 \\
80 \\
80 \\
80\end{array}$ & $\begin{array}{l}190 \\
155 \\
145 \\
125 \\
110\end{array}$ & $\begin{array}{r}88 \\
82 \\
115 \\
100 \\
90\end{array}$ & $\begin{array}{l}107 \\
106 \\
108 \\
108 \\
108\end{array}$ & $\begin{array}{l}140 \\
130 \\
123 \\
115 \\
110\end{array}$ & $\begin{array}{l}237 \\
216 \\
194 \\
176 \\
162\end{array}$ & $\begin{array}{l}417 \\
350 \\
306 \\
276 \\
251\end{array}$ & $\begin{array}{l}2 C 4 \\
1 \subseteq C \\
178 \\
1 \in 8 \\
1 \in C\end{array}$ & $\begin{array}{r}90 \\
89 \\
89 \\
330 \\
250\end{array}$ \\
\hline $\begin{array}{r}6 \\
7 \\
8 \\
9 \\
10\end{array}$ & $\begin{array}{r}84 \\
94 \\
102 \\
106 \\
110\end{array}$ & $\begin{array}{l}92 \\
92 \\
92 \\
93 \\
93\end{array}$ & $\begin{array}{l}90 \\
92 \\
52 \\
91 \\
90\end{array}$ & $\begin{array}{l}80 \\
80 \\
80 \\
80 \\
80\end{array}$ & $\begin{array}{r}105 \\
100 \\
96 \\
92 \\
94\end{array}$ & $\begin{array}{l}100 \\
115 \\
105 \\
110 \\
120\end{array}$ & $\begin{array}{l}108 \\
108 \\
108 \\
110 \\
110\end{array}$ & $\begin{array}{l}108 \\
108 \\
113 \\
114 \\
112\end{array}$ & $\begin{array}{l}150 \\
139 \\
132 \\
143 \\
171\end{array}$ & $\begin{array}{l}229 \\
221 \\
220 \\
267 \\
188\end{array}$ & $\begin{array}{l}155 \\
155 \\
151 \\
147 \\
144\end{array}$ & $\begin{array}{l}168 \\
144 \\
160 \\
166 \\
147\end{array}$ \\
\hline $\begin{array}{l}11 \\
12 \\
13 \\
14 \\
15\end{array}$ & $\begin{array}{r}102 \\
97 \\
95 \\
53 \\
91\end{array}$ & $\begin{array}{l}93 \\
93 \\
91 \\
91 \\
93\end{array}$ & $\begin{array}{l}89 \\
88 \\
86 \\
85 \\
85\end{array}$ & $\begin{array}{l}80 \\
80 \\
80 \\
81 \\
82\end{array}$ & $\begin{array}{r}100 \\
98 \\
115 \\
100 \\
85\end{array}$ & $\begin{array}{l}137 \\
135 \\
128 \\
122 \\
120\end{array}$ & $\begin{array}{l}109 \\
108 \\
109 \\
112 \\
118\end{array}$ & $\begin{array}{l}112 \\
112 \\
112 \\
115 \\
165\end{array}$ & $\begin{array}{l}17 C \\
155 \\
144 \\
195 \\
221\end{array}$ & $\begin{array}{l}175 \\
170 \\
185 \\
224 \\
219\end{array}$ & $\begin{array}{l}138 \\
136 \\
130 \\
124 \\
119\end{array}$ & $\begin{array}{l}132 \\
123 \\
124 \\
116 \\
108\end{array}$ \\
\hline $\begin{array}{l}16 \\
17 \\
18 \\
19 \\
27\end{array}$ & $\begin{array}{l}90 \\
88 \\
87 \\
87 \\
86\end{array}$ & $\begin{array}{l}92 \\
93 \\
92 \\
91 \\
91\end{array}$ & $\begin{array}{l}85 \\
83 \\
82 \\
81 \\
81\end{array}$ & $\begin{array}{l}83 \\
84 \\
85 \\
86 \\
87\end{array}$ & $\begin{array}{l}82 \\
80 \\
78 \\
77 \\
76\end{array}$ & $\begin{array}{l}125 \\
136 \\
131 \\
148 \\
142\end{array}$ & $\begin{array}{l}116 \\
116 \\
116 \\
116 \\
126\end{array}$ & $\begin{array}{l}742 \\
526 \\
376 \\
295 \\
268\end{array}$ & $\begin{array}{l}184 \\
184 \\
312 \\
361 \\
261\end{array}$ & $\begin{array}{l}213 \\
194 \\
175 \\
160 \\
147\end{array}$ & $\begin{array}{r}118 \\
117 \\
119 \\
116 \\
1115\end{array}$ & $\begin{array}{l}103 \\
106 \\
118 \\
123 \\
132\end{array}$ \\
\hline $\begin{array}{l}21 \\
22 \\
23 \\
24 \\
25\end{array}$ & $\begin{array}{l}87 \\
86 \\
87 \\
88 \\
87\end{array}$ & $\begin{array}{l}91 \\
91 \\
89 \\
94 \\
93\end{array}$ & $\begin{array}{l}80 \\
80 \\
80 \\
80 \\
80\end{array}$ & $\begin{array}{l}88 \\
89 \\
89 \\
88 \\
88\end{array}$ & $\begin{array}{l}76 \\
75 \\
75 \\
75 \\
75\end{array}$ & $\begin{array}{l}123 \\
111 \\
105 \\
103 \\
107\end{array}$ & $\begin{array}{l}211 \\
202 \\
288 \\
409 \\
356\end{array}$ & $\begin{array}{l}240 \\
217 \\
196 \\
182 \\
174\end{array}$ & $\begin{array}{l}202 \\
177 \\
168 \\
165 \\
177\end{array}$ & $\begin{array}{r}148 \\
171 \\
743 \\
1,170 \\
1,100\end{array}$ & $\begin{array}{l}113 \\
1 C 6 \\
102 \\
101 \\
100\end{array}$ & $\begin{array}{l}127 \\
141 \\
147 \\
137 \\
128\end{array}$ \\
\hline $\begin{array}{l}26 \\
27 \\
28 \\
29 \\
30 \\
31\end{array}$ & $\begin{array}{l}87 \\
88 \\
88 \\
93 \\
99 \\
98\end{array}$ & $\begin{array}{r}93 \\
93 \\
93 \\
93 \\
93 \\
\\
\end{array}$ & $\begin{array}{l}80 \\
80 \\
80 \\
80 \\
80 \\
80\end{array}$ & $\begin{array}{r}89 \\
88 \\
86 \\
200 \\
350 \\
230\end{array}$ & $\begin{array}{r}75 \\
75 \\
78 \\
80 \\
-\square \\
\end{array}$ & $\begin{array}{l}108 \\
109 \\
113 \\
111 \\
110 \\
110\end{array}$ & $\begin{array}{r}259 \\
214 \\
188 \\
167 \\
152 \\
\end{array}$ & $\begin{array}{l}184 \\
202 \\
218 \\
241 \\
280 \\
260\end{array}$ & $\begin{array}{r}286 \\
1,030 \\
1,220 \\
759 \\
530 \\
\end{array}$ & $\begin{array}{l}553 \\
384 \\
311 \\
2 \in 6 \\
237 \\
218\end{array}$ & $\begin{array}{l}95 \\
94 \\
92 \\
\varepsilon 9 \\
89 \\
90\end{array}$ & $\begin{array}{r}121 \\
117 \\
115 \\
117 \\
116 \\
\end{array}$ \\
\hline $\begin{array}{l}\text { TOTAL } \\
\text { MEAN } \\
\text { MAX } \\
\text { MIN } \\
\text { CFSM } \\
\text { IN. }\end{array}$ & $\begin{array}{r}2,818 \\
90.9 \\
110 \\
83 \\
.15 \\
.17\end{array}$ & $\begin{array}{r}2,788 \\
92.9 \\
99 \\
89 \\
.15 \\
.17\end{array}$ & $\begin{array}{r}2,638 \\
85.1 \\
93 \\
80 \\
.14 \\
.16\end{array}$ & $\begin{array}{r}3,113 \\
100 \\
350 \\
80 \\
.16 \\
.19\end{array}$ & $\begin{array}{r}2,787 \\
96.1 \\
190 \\
75 \\
.16 \\
.17\end{array}$ & $\begin{array}{r}3,553 \\
115 \\
148 \\
82 \\
.19 \\
.21\end{array}$ & $\begin{array}{r}4,673 \\
156 \\
409 \\
106 \\
.25 \\
.28\end{array}$ & $\begin{array}{r}6,390 \\
206 \\
742 \\
108 \\
.34 \\
.39\end{array}$ & $\begin{array}{r}8,621 \\
287 \\
1,220 \\
132 \\
.47 \\
.52\end{array}$ & $\begin{array}{r}9,828 \\
317 \\
1,170 \\
147 \\
.52 \\
.59\end{array}$ & $\begin{array}{r}2.955 \\
128 \\
264 \\
89 \\
.21 \\
.24\end{array}$ & $\begin{array}{r}4, C 84 \\
136 \\
330 \\
89 \\
.22 \\
.25\end{array}$ \\
\hline $\begin{array}{ll}\text { CAL } & \text { YR } \\
\text { WTR } & \text { YR }\end{array}$ & $\begin{array}{l}1967 \\
1968\end{array}$ & $\begin{array}{l}113,7 \\
\mathrm{~L} \quad 55,2\end{array}$ & & $\begin{array}{ll} & 312 \\
N & 151\end{array}$ & $\begin{array}{l}\text { MAX } \\
\text { MAX }\end{array}$ & & $\begin{array}{ll}N & 80 \\
N & 75\end{array}$ & $\begin{array}{l}\text { CFSM } \\
\text { CFSM }\end{array}$ & $\begin{array}{l}\text { IN } \\
\text { IN }\end{array}$ & & & \\
\hline
\end{tabular}

Peak discharge (base 3,500 cfs).--No peak above base. 
5-3845. Rush Creek near Rushford, Minn.

Location.--Lat $43^{\circ} 50^{\prime} 00^{\prime \prime}$, long $91^{\circ} 46^{\prime} 40^{\prime \prime}$, on line between secs. 3 and 10, T.104 N., R.8 W., on downstream side near center of span of highway bridge, 1 $\frac{1}{2}$ miles northwest of Rushford and 3 miles upstream from mouth.

Drainaqe area.--129 sq $\mathrm{mi}$.

Records available.--August 1942 to September 1968.

Gage.--Wire-weight gage read twice daily. Datum of gage is 735.00 ft above mean sea level, adjustment of 1912 .

Prior to June 14, 1950, water-stage recorder at site $100 \mathrm{ft}$ upstream; at datum 5 ft higher, Aug. 5, 1942 ,

to Oct. 27, 1945; at datum $3 \mathrm{ft} \mathrm{higher,} \mathrm{Oct.} \mathrm{28,} \mathrm{1945,} \mathrm{to} \mathrm{Aug.} \mathrm{3,} \mathrm{1949;} \mathrm{at} \mathrm{present} \mathrm{datum,} \mathrm{Aug.} \mathrm{4,} \mathrm{1949,}$

to June 13, 1950. June 14, 1950, to Aug. 26, 1964, chain gage at present site and datum.

Average discharge. --26 years, $53.8 \mathrm{cfs}$.

Extremes.--Maximum discharge during year, 370 cfs July 23 (gage height, 3.50 ft); minimum daily, 30 cfs Dec.14, 20-29, Mar. 3, May 10-13.

1942-68: Maximum discharge, $11,600 \mathrm{cfs}$ Mar. 26, 1950 (gage height 13.54 ft, from floodmark), from rating curve extended above $1,400 \mathrm{cfs}$ on basis of contracted-opening measurements at gage heights 11.0 and 13.5 ft; minimum, 17 cfs May 22, 1959; minimum gage height, 0.93 ft June 16, 17, $18,1965$.

Flood of June 28, 29, 1942 reached a discharge of 11,000 cfs (by slope-area measurement of peak flow).

Remarks.--Records fair.

DISCHARGE, IN CUBIC FEET PER SECOND, WATER YEAR OCTOBER 1967 TO SEPTEMBER 1968

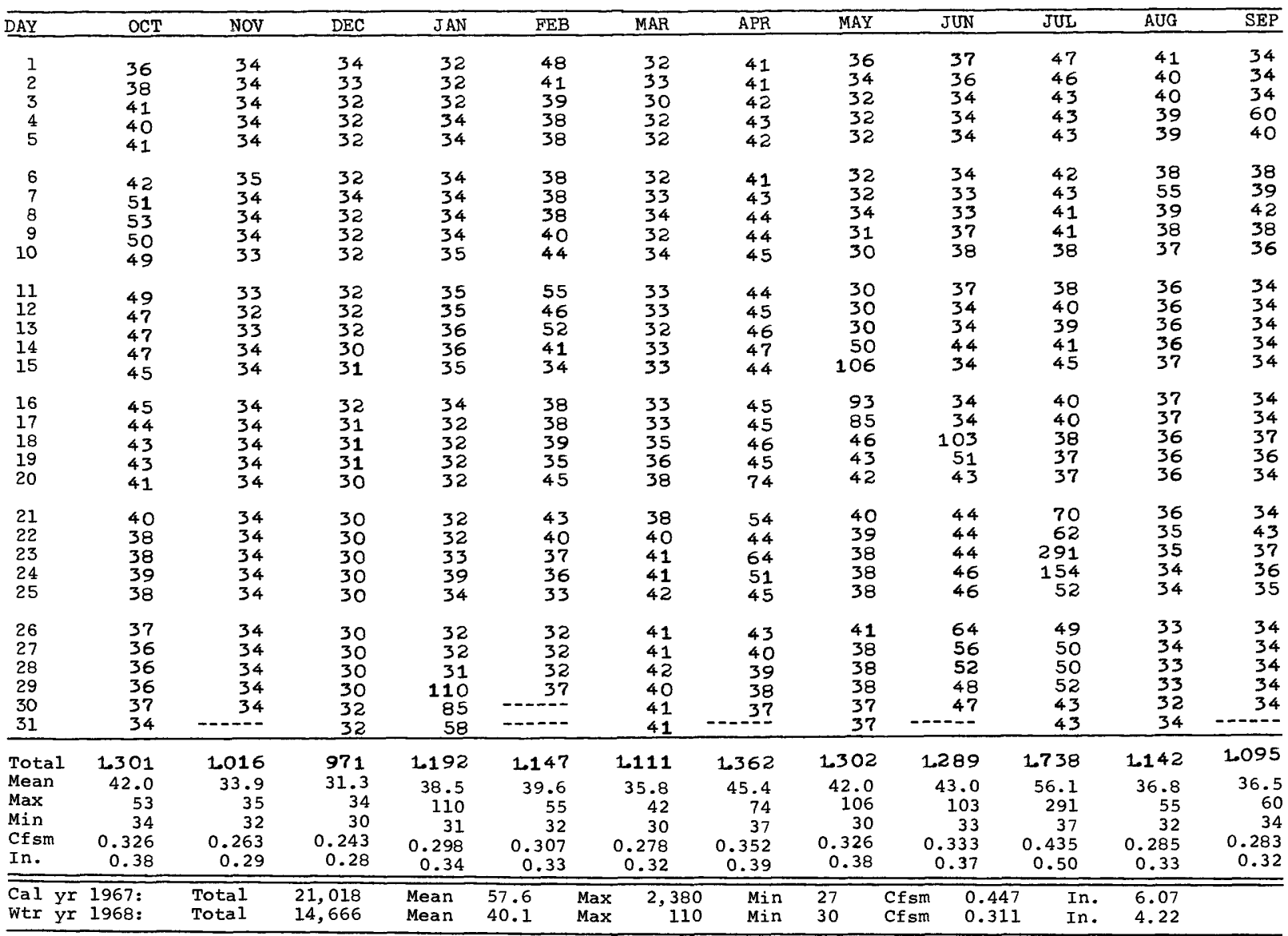


5-3850. Root River near Houston, Minn.

Location.--Lat 43046'05", long 91035'11", in sec.32, T.104 N., R.6 W., on right bank 1 mile west of Houston and $2 \frac{1}{2}$ miles upstream from south Fork.

Drainage area.--1, $270 \mathrm{sq} \mathrm{mi}$, approximately.

Records available.--May 1909 to September 1917, May to November 1929, March 1930 to September 1968. Monthly discharge only for some periods, published in WSP 1308 .

Gage.--Water-stage recorder. Datum of gage is $671.86 \mathrm{ft}$ above mean sea level, datum of 1929 . May 28 , 1909 , to sept. 30, 1917, staff gage at site $1 \frac{1}{2}$ miles downstream at different datum. May 4, 1929, to sept. 27, 1933, chain gage at present site and datum.

Average discharge.--46 years $(1909-17,1930-68), 639$ cfs.

Extremes.--Maximum discharge during year 3,210 cfs May 16 (gage height, 5.53 ft); minimum daily, 226 cfs Apr. 6. 1909-17, 1929-68: Maximum discharge, 37, 000 cfs Apr. 1, 1952 (gage height, 13.90 ft); maximum gage height, 18.32 ft Mar. 2, 1965 (backwater from ice); minimum discharge, 65 cfs Dec. 26, 1933, Feb. 25, 1935.

Remarks.--Records good except those for winter months, which are fair. slight diurnal fluctuation at low flows caused by powerplants above station. Records for suspended sediment loads for water year 1968 are published in Part 2 of this report.

DISCHARGE, IN CUBIC FEET PER SECOND, WATER YEAR OCTOBER 1967 TO SEPTEMBER 1968

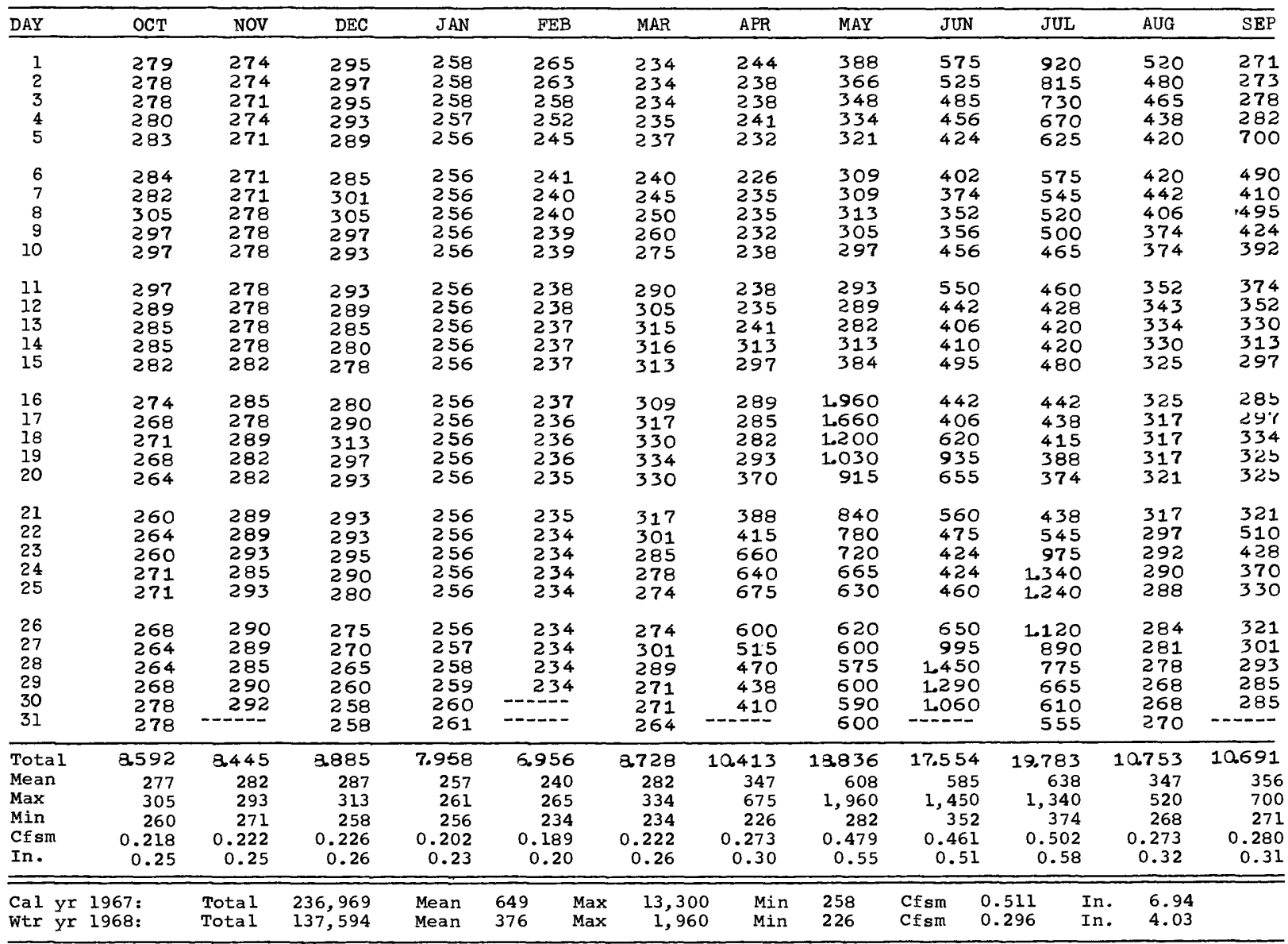

Peak discharge (base, 5,000 cfs).--No peak above base. 
5-3855. South Fork Root River near Houston, Minn.

Location.--Lat $43^{\circ} 44^{\prime} 19^{\prime \prime}$, long 91033'50", in NE $\frac{1}{4} \mathrm{SW}^{\frac{1}{4}}$ sec.9, T.103 N., R.6 W., on left bank 50 ft downstream from bridge on State Highway 76 , half a mile upstream from Badger Creek and $1 \frac{1}{2}$ miles south of Houston.

Drainage area. $--275 \mathrm{sq} \mathrm{mi}$.

Records available.--January 1953 to September 1968.

Gage.--Water-stage recorder. Datum of gage is $680.41 \mathrm{ft}$ above mean sea level, datum of 1929 .

Average discharge. --15 years, $120 \mathrm{cfs}$.

Extremes.--Maximum discharge during year, $768 \mathrm{cfs} J u n e ~ 18$ (gage height, $6.25 \mathrm{ft}$ ); maximum gage height, 10.86 ft Jan. 29, (from highwater mark, backwater from ice); minimum discharge $60 \mathrm{cfs}$ oct. 1 (gage height, 1.23 $\mathrm{ft}$ ).

953-68. Maximum discharge 8,420 cfs Mar. 29, 1962 (gage height, 13.35 ft); maximum gage height, 13.74

ft Mar. 26, 1961 (backwater from ice); minimum discharge, 11 cfs Nov. 28, 1961 (gage height, 1.47 ft); minimum gage height, $0.85 \mathrm{ft}$ Aug. 17, 1967.

Remarks.--Records good except those for winter months, which are fair. Recoro for chemical analyses for the water year 1968 are published in Part 2 of this report.

DISCHARGE, IN CUBIC FEET PER SECOND, WATER YEAR OCTOBER 1967 TO SEPTEMBER 1968

\begin{tabular}{|c|c|c|c|c|c|c|c|c|c|c|c|c|}
\hline$\widehat{\mathrm{DAY}}$ & OCT & NOV & $\mathrm{DEC}$ & JAN & FEB & MAR & APR & MAY & JUN & JUL & AUG & SEP \\
\hline $\begin{array}{l}1 \\
2 \\
3 \\
4 \\
5\end{array}$ & $\begin{array}{l}65 \\
68 \\
69 \\
66 \\
67\end{array}$ & $\begin{array}{l}72 \\
72 \\
72 \\
70 \\
70\end{array}$ & $\begin{array}{l}69 \\
69 \\
68 \\
68 \\
68\end{array}$ & $\begin{array}{l}64 \\
64 \\
64 \\
64\end{array}$ & $\begin{array}{r}250 \\
145 \\
110 \\
92 \\
83\end{array}$ & $\begin{array}{l}69 \\
71 \\
73 \\
74 \\
74\end{array}$ & $\begin{array}{l}68 \\
68 \\
69 \\
77 \\
72\end{array}$ & $\begin{array}{l}74 \\
72 \\
71 \\
70 \\
70\end{array}$ & $\begin{array}{l}77 \\
74 \\
73 \\
72 \\
69\end{array}$ & $\begin{array}{lll}1 & 30 \\
1 & 1 & 9 \\
1 & 1 & 0 \\
1 & 03 \\
1 & 0 & 1\end{array}$ & $\begin{array}{l}79 \\
78 \\
77 \\
77 \\
76\end{array}$ & $\begin{array}{l}71 \\
70 \\
69 \\
88 \\
86\end{array}$ \\
\hline $\begin{array}{r}6 \\
7 \\
8 \\
9 \\
10\end{array}$ & $\begin{array}{l}68 \\
78 \\
85 \\
78 \\
75\end{array}$ & $\begin{array}{l}70 \\
70 \\
70 \\
70 \\
70\end{array}$ & $\begin{array}{l}67 \\
68 \\
68 \\
68 \\
67\end{array}$ & $\begin{array}{l}63 \\
63 \\
63 \\
63 \\
63\end{array}$ & $\begin{array}{l}80 \\
76 \\
72 \\
71 \\
69\end{array}$ & $\begin{array}{l}72 \\
72 \\
75 \\
83 \\
89\end{array}$ & $\begin{array}{l}71 \\
68 \\
70 \\
68 \\
68\end{array}$ & $\begin{array}{l}69 \\
70 \\
74 \\
71 \\
69\end{array}$ & $\begin{array}{r}68 \\
68 \\
68 \\
71 \\
114\end{array}$ & $\begin{array}{l}96 \\
96 \\
95 \\
92 \\
91\end{array}$ & $\begin{array}{l}76 \\
86 \\
78 \\
77 \\
77\end{array}$ & $\begin{array}{l}78 \\
77 \\
98 \\
85 \\
76\end{array}$ \\
\hline $\begin{array}{l}11 \\
12 \\
13 \\
14 \\
15\end{array}$ & $\begin{array}{l}74 \\
74 \\
76 \\
74 \\
68\end{array}$ & $\begin{array}{l}70 \\
68 \\
68 \\
68 \\
68\end{array}$ & $\begin{array}{l}68 \\
68 \\
66 \\
65 \\
64\end{array}$ & $\begin{array}{l}63 \\
63 \\
63 \\
63 \\
63\end{array}$ & $\begin{array}{l}68 \\
67 \\
67 \\
66 \\
65\end{array}$ & $\begin{array}{l}79 \\
74 \\
72 \\
72 \\
71\end{array}$ & $\begin{array}{l}68 \\
67 \\
68 \\
74 \\
72\end{array}$ & $\begin{array}{l}68 \\
68 \\
65 \\
72 \\
74\end{array}$ & $\begin{array}{r}82 \\
70 \\
68 \\
135 \\
87\end{array}$ & $\begin{array}{l}89 \\
85 \\
91 \\
91 \\
90\end{array}$ & $\begin{array}{l}72 \\
69 \\
68 \\
66 \\
68\end{array}$ & $\begin{array}{l}73 \\
72 \\
71 \\
69 \\
69\end{array}$ \\
\hline $\begin{array}{l}16 \\
17 \\
18 \\
19 \\
20\end{array}$ & $\begin{array}{l}70 \\
71 \\
71 \\
71 \\
71\end{array}$ & $\begin{array}{l}68 \\
68 \\
68 \\
68 \\
67\end{array}$ & $\begin{array}{l}66 \\
67 \\
68 \\
68 \\
68\end{array}$ & $\begin{array}{l}63 \\
63 \\
63 \\
63 \\
63\end{array}$ & $\begin{array}{l}65 \\
64 \\
64 \\
63 \\
63\end{array}$ & $\begin{array}{l}71 \\
71 \\
74 \\
78 \\
72\end{array}$ & $\begin{array}{l}69 \\
70 \\
68 \\
67 \\
82\end{array}$ & $\begin{array}{r}360 \\
149 \\
106 \\
100 \\
95\end{array}$ & $\begin{array}{r}74 \\
72 \\
436 \\
212 \\
103\end{array}$ & $\begin{array}{r}81 \\
101 \\
89 \\
83 \\
80\end{array}$ & $\begin{array}{l}71 \\
70 \\
69 \\
71 \\
71\end{array}$ & $\begin{array}{l}69 \\
75 \\
76 \\
75 \\
74\end{array}$ \\
\hline $\begin{array}{l}21 \\
22 \\
23 \\
24 \\
25\end{array}$ & $\begin{array}{l}67 \\
66 \\
66 \\
73 \\
75\end{array}$ & $\begin{array}{l}68 \\
68 \\
68 \\
68 \\
68\end{array}$ & $\begin{array}{l}68 \\
67 \\
66 \\
65 \\
65\end{array}$ & $\begin{array}{l}64 \\
64 \\
64 \\
65 \\
65\end{array}$ & $\begin{array}{l}63 \\
63 \\
63 \\
63 \\
63\end{array}$ & $\begin{array}{l}72 \\
70 \\
70 \\
69 \\
70\end{array}$ & $\begin{array}{r}84 \\
76 \\
119 \\
113 \\
97\end{array}$ & $\begin{array}{l}91 \\
87 \\
84 \\
82 \\
81\end{array}$ & $\begin{array}{r}87 \\
78 \\
146 \\
260 \\
439\end{array}$ & $\begin{array}{r}86 \\
102 \\
249 \\
197 \\
112\end{array}$ & $\begin{array}{l}70 \\
69 \\
68 \\
68 \\
68\end{array}$ & $\begin{array}{r}70 \\
141 \\
262 \\
113 \\
91\end{array}$ \\
\hline $\begin{array}{l}26 \\
27 \\
28 \\
29 \\
30 \\
31\end{array}$ & $\begin{array}{l}73 \\
73 \\
73 \\
74 \\
76 \\
73 \\
\end{array}$ & $\begin{array}{r}68 \\
67 \\
66 \\
67 \\
68 \\
\end{array}$ & $\begin{array}{l}65 \\
65 \\
64 \\
64 \\
64 \\
64 \\
\end{array}$ & $\begin{array}{r}65 \\
66 \\
68 \\
300 \\
460 \\
370 \\
\end{array}$ & $\begin{array}{r}64 \\
65 \\
67 \\
68 \\
\end{array}$ & $\begin{array}{l}68 \\
72 \\
75 \\
70 \\
69 \\
72 \\
\end{array}$ & $\begin{array}{r}87 \\
81 \\
79 \\
78 \\
77 \\
\end{array}$ & $\begin{array}{l}82 \\
81 \\
81 \\
95 \\
79 \\
77 \\
\end{array}$ & $\begin{array}{r}260 \\
248 \\
185 \\
157 \\
143 \\
\end{array}$ & $\begin{array}{l}93 \\
91 \\
86 \\
83 \\
81 \\
81 \\
\end{array}$ & $\begin{array}{l}69 \\
69 \\
72 \\
69 \\
68 \\
70 \\
\end{array}$ & $\begin{array}{r}86 \\
82 \\
80 \\
79 \\
78 \\
\end{array}$ \\
\hline $\begin{array}{l}\text { Total } \\
\text { Mean } \\
\text { Max } \\
\text { Min } \\
\text { Cfsm } \\
\text { In. }\end{array}$ & $\begin{array}{r}2.228 \\
71.9 \\
85 \\
65 \\
0.261 \\
0.30\end{array}$ & $\begin{array}{r}2.063 \\
68.8 \\
72 \\
66 \\
0.250 \\
0.28\end{array}$ & $\begin{array}{r}2.06 b \\
66.6 \\
69 \\
64 \\
0.242 \\
0.28\end{array}$ & $\begin{array}{r}2.916 \\
94.1 \\
460 \\
63 \\
0.342 \\
0.39\end{array}$ & $\begin{array}{r}2.279 \\
78.6 \\
250 \\
63 \\
0.286 \\
0.31\end{array}$ & $\begin{array}{r}2.263 \\
73.0 \\
89 \\
68 \\
0.265 \\
0.31\end{array}$ & $\begin{array}{r}2295 \\
76.5 \\
119 \\
67 \\
0.278 \\
0.31\end{array}$ & $\begin{array}{r}2787 \\
89.9 \\
360 \\
65 \\
0.327 \\
0.38\end{array}$ & $\begin{array}{r}4.096 \\
137 \\
439 \\
68 \\
0.498 \\
0.55\end{array}$ & $\begin{array}{r}3.174 \\
102 \\
249 \\
80 \\
0.371 \\
0.43\end{array}$ & $\begin{array}{r}2.236 \\
72.1 \\
86 \\
66 \\
0.262 \\
0.30\end{array}$ & $\begin{array}{r}2603 \\
86.8 \\
262 \\
69 \\
0.316 \\
0.35\end{array}$ \\
\hline $\begin{array}{l}\text { Cal yr } \\
\text { wtr yr }\end{array}$ & $\begin{array}{l}1967: \\
1968:\end{array}$ & $\begin{array}{l}\text { Total } \\
\text { Total }\end{array}$ & $\begin{array}{l}45,715 \\
31,005\end{array}$ & $\begin{array}{l}\text { Mean } \\
\text { Mean }\end{array}$ & $\begin{array}{r}125 \\
84.7\end{array}$ & $\begin{array}{l}\operatorname{Max} \\
\operatorname{Max}\end{array}$ & $\begin{array}{l}\text { Min } \\
\text { Min }\end{array}$ & $\begin{array}{l}60 \\
63\end{array}$ & $\begin{array}{l}\text { Cfsm } \\
\text { Cfsm }\end{array}$ & $\begin{array}{l}0.455 \\
0.308\end{array}$ & $\begin{array}{l}6.18 \\
4.19\end{array}$ & \\
\hline
\end{tabular}


5-4570. Cedar River near Austin, Minn.

Location.--Lat $43^{\circ} 38^{\prime} 10^{\prime \prime}$, long $92^{\circ}$ ' $^{\prime} 20^{\prime \prime}$, in NE $\frac{1}{4}$ SE $\frac{1}{4}$ sec.15, T.102 N., R. 18 W., on left bank 200 ft upstream from abandoned powerhouse, 500 ft downstream from highway bridge, 1.1 miles downstream from Turtle Creek, and 1.1 miles south of Austin.

Drainage area. $--425 \mathrm{sq} \mathrm{mi}$.

Rècords available.--May 1909 to september 1914, October 1944 to September 1968.

Gage.-Digital water-stage recorder. Datum of gage is $1,162.10 \mathrm{ft}$ above mean sea 1 evel, datum of 1929 . May 1909 to April 1912, staff gage in tailwater of powerplant 200 ft downstream at datum 3.1 ft lower. May 1912 to september 1914, chain gage on highway bridge 500 ft downstream at datum 1.1 ft $10 w e r$. october 1914 to May 18, 1964, graphic water-stage recorder at present site and datum.

Average discharge.--29 years, 171 cfs.

Extremes.--Maximum discharge during year, 2,920 cfs July 24 (gage height, 8.78 ft); minimum, 36 cfs Feb. 23 (gage height, $2.15 \mathrm{ft}$ ).

1909-14, 1944-68: Maximum discharge, 9,530 cfs Mar. 29, 1962 (gage height, $17.18 \mathrm{ft}$ ) ; maximum gage height, $18.87 \mathrm{ft} \mathrm{Mar.} \mathrm{1,} 1965$ (from floodmark, backwater from ice); no flow for several days in 1911.

Remarks.--Records good except those for period of backwater from aquatic growth and those for winter periods, which are fair.

DISCHARGE, IN CUBIC FEET PER SECOND, WATER YEAR OCTOBER 1967 TO SEPTEMBER 1968

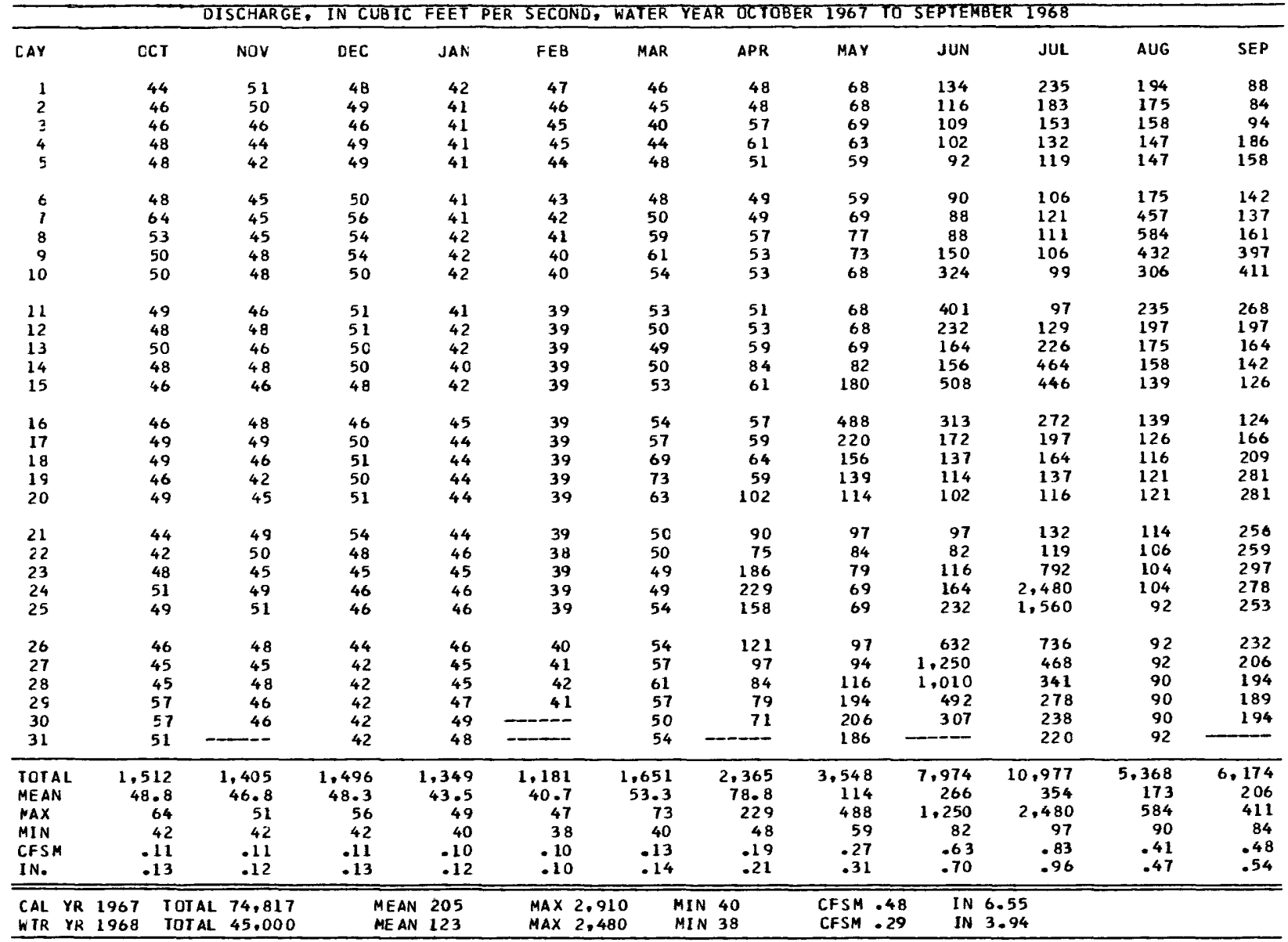


5-4760. West Fork Des Moines River at Jackson, Minn.

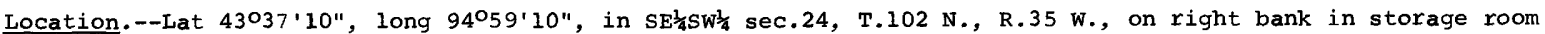
of city powerplant in Jackson.

Drainaqe area. $--1,220 \mathrm{sq} \mathrm{mi}$, approximately.

Records available.--May 1909 to December 1913, August 1930 to september 1968 (winter records incomplete prior to 1936). Published as Des Moines River at Jackson, 1909-13, as Des Moines River near Jackson, 1930-35, and as West Fork Des Moines River near Jackson, 1936-44.

Gage.--Water-stage recorder. Datum of gage is 1,287.75 ft above mean sea level, datum of 1929 . May 31 , 1909 , to Dec. 20, 1913, staff gage at site $0.6 \mathrm{mile}$ downstream at datum 0.99 ft lower. Aug. 22 , 1930, to

Sept. 30, 1944, chain gage at site 7 miles upstream at datum $17.10 \mathrm{ft}$ higher. Oct. 1 , 1944, to Oct. 26, 1949, wire-weight gage at site $600 \mathrm{ft}$ upstream at datum $10.64 \mathrm{ft}$ higher. Oct. 27, 1949 to Dec. 15, 1965, water-stage recorder $200 \mathrm{ft}$ downstream at same datum.

Average discharge. --33 years $(1935-68), 260$ cfs.

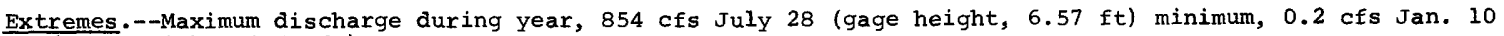
(gage height, $2.47 \mathrm{ft}$ ).

1909-13, 1930-68: Maximum discharge 9,530 cfs Apr. 9, 1965; maximum gage height, 18.62 ft Apr. 6, 1965, (from floodmark, backwater from ice); no flow at times.

Remarks.--Records good except those for winter months, which are fair. Regulation at times by Yankton, Long, Shetek, and Heron Lakes. Records of chemical analyses and suspended sediment loads for the water year 1968 are published in part 2 of this report.

DISCHARGE, IN CFS, WATER YEAR OCTOBER 1967 TO SEPTEMBER 1968

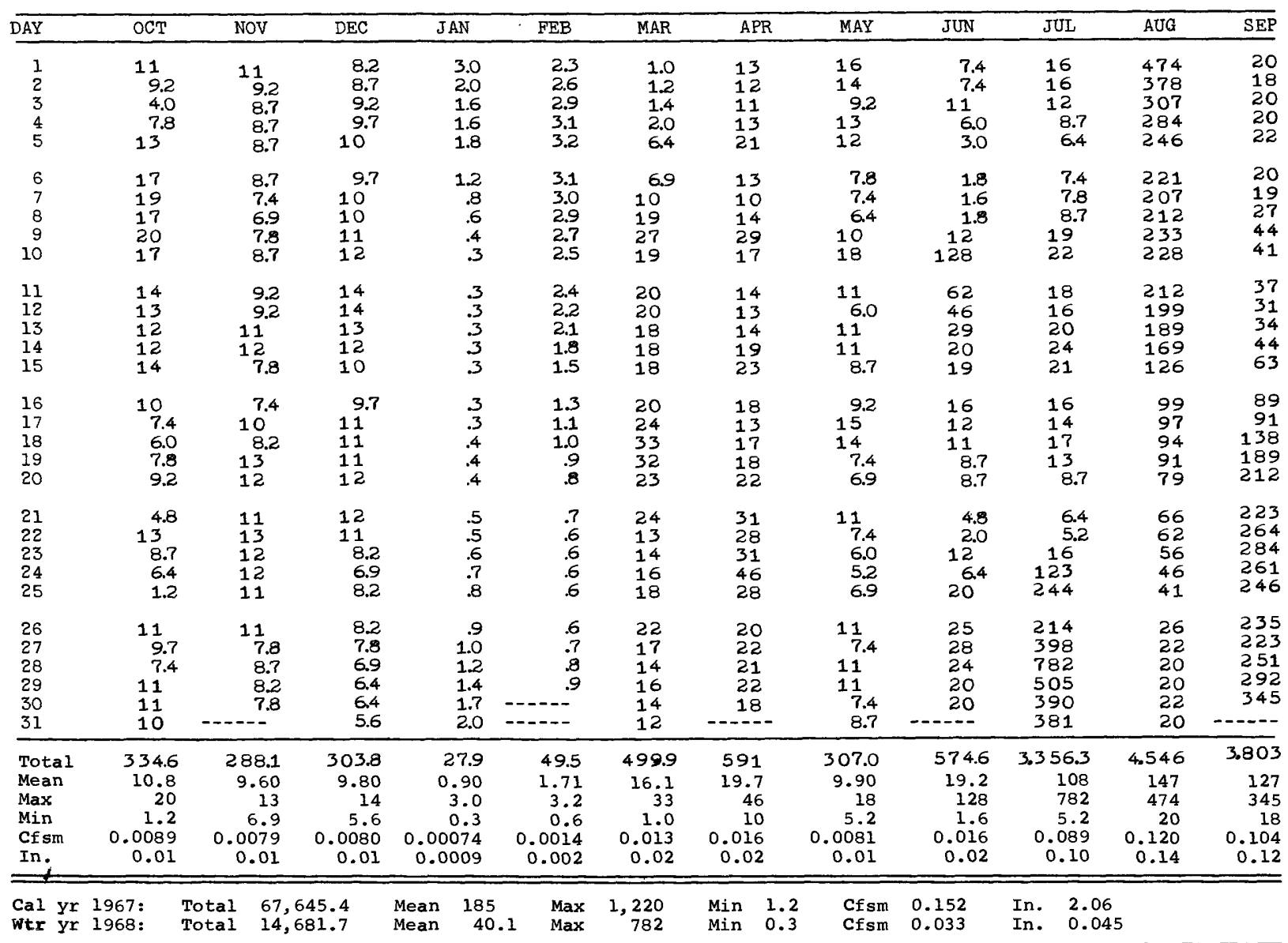

Peak discharge (base, 500 cf's)

\begin{tabular}{|c|c|c|c||c|c|c|c|}
\hline Date & Time & $\begin{array}{c}\text { Gage } \\
\text { height }\end{array}$ & Discharge & Date & Time & $\begin{array}{c}\text { Gage } \\
\text { height }\end{array}$ & Discharge \\
\hline $7-28$ & 0900 & 6.57 & 854 & $8-1$ & 0600 & 5.51 & 505 \\
& & & & & & & \\
\hline
\end{tabular}


As the number of streams on which streamflow information is likely to be desired far exceeds the number of stream-gaging stations feasible to operate at one time, the Geological survey collects limited streamflow data at sites other than stream-gaging stations. When limited streamflow data are collected on a systematic basis over a period of years for use in hydrologic analyses, the site at which the data are collected is called a partial-record station. Data collected at these partial-record stations are usable in low-flow or flood-flow analyses, depending on the type of data collected. In addition, discharge measurements are made at other sites not included in the partial-record program. These measurements are generally made in times of drought or flood to give better areal coverage to those events. Those measurements and others collected for some special reason are called measurements at miscellaneous sites.

Records collected at partial-record stations are presented in two tables. The first is a table of discharge measurements at low-flow partial-record stations and the second is a table of annual maximum stage and discharge at crest-stage stations. Discharge measurements made at miscellaneous sites for both low flow and high flow are given in a third table.

Low-flow partial-record stations

Measuring of streamflow in the area covered by this report made at low-flow partial-record stations are given in the following table. Most of these measurements were made during periods of base flow when streamflow is primarily from ground-water storage. These measurements, when correlated with the simultaneous discharge of a nearby stream where continuous records are available, will give a picture of the low-flow potentiality of the stream. The column headed "Period of record" shows the water years in which measurements were made at the same, or practically the same, site.

Discharge measurements made at low-flow partial-record stations during water year 1968

\begin{tabular}{|c|c|c|c|c|c|c|}
\hline \multirow[b]{2}{*}{ Station No. } & \multirow[b]{2}{*}{ Station name } & \multirow[b]{2}{*}{ Location } & \multirow{2}{*}{$\begin{array}{l}\text { Drainage } \\
\text { area } \\
(\mathrm{sq} \mathrm{mi})\end{array}$} & \multirow{2}{*}{$\begin{array}{l}\text { Period } \\
\text { of } \\
\text { record }\end{array}$} & \multicolumn{2}{|c|}{ Measurements } \\
\hline & & & & & Date & $\begin{array}{c}\text { Dizcharge } \\
\text { (cfs) }\end{array}$ \\
\hline \multicolumn{7}{|c|}{ Crow wing River basin } \\
\hline $5-2427$ & $\begin{array}{l}\text { Little sand Lake } \\
\text { Outlet near } \\
\text { Dorset, Minn. }\end{array}$ & $\begin{array}{l}\mathrm{NE}^{\frac{1}{4}} \text { sec. } 36, \mathrm{~T} .141 \mathrm{~N} ., \mathrm{R} .34 \mathrm{~W} ., \frac{1}{1 / 2} \text { mile } \\
\text { below Little Sand Lake and } 3 \text { miles } \\
\text { northeast of Dorset }\end{array}$ & a 74 & $\left|\begin{array}{r}1930-417 \\
1942 \\
1956-67\end{array}\right|$ & $\begin{array}{r}10-31-67 \\
11-20-67 \\
12-18-67 \\
1-22-68 \\
2-19-68 \\
3-18-68 \\
4-22-68 \\
5-20-68 \\
6-17-68 \\
7-22-68 \\
8-26-68 \\
9-30-68\end{array}$ & $\begin{array}{l}5.22 \\
10.4 \\
21.4 \\
19.7 \\
20.4 \\
30.3 \\
36.6 \\
45.2 \\
56.6 \\
52.4 \\
40.5 \\
33.2\end{array}$ \\
\hline
\end{tabular}

a Approximately.

$\neq$ operated as à continuous-record gaging station. 
The following table contains annual maximum discharges for crest-stage stations. A crest-stage gage is a device which will register the peak stage occurring between inspections of the gage. A stage-discharge relation for each gage is developed from discharge measurements made by indirect measurements of peak flow or by current meter. The date of the maximum discharge is not always certain but is usually determined by comparison with nearby continuous-record stations, weather records, or local inquiry. Only the maximum discharge for each water year is given. Information on some lower floods may have been obtained but is not published herein. The years given in the period of record represent water years for which the annual maximum has been determined.

Annual maximum discharge at crest-stage partial-record stations

\begin{tabular}{|c|c|c|c|c|c|c|c|}
\hline \multirow[b]{2}{*}{ Station No. } & \multirow[b]{2}{*}{ Station name } & \multirow[b]{2}{*}{ Location } & \multirow[b]{2}{*}{$\begin{array}{l}\text { Drainage } \\
\text { area } \\
(\mathrm{sq} \mathrm{mi})\end{array}$} & \multirow[b]{2}{*}{$\begin{array}{l}\text { Period } \\
\text { of } \\
\text { record }\end{array}$} & \multicolumn{3}{|c|}{ Annual maximum } \\
\hline & & & & & Date & $\begin{array}{l}\text { Gage } \\
\text { height } \\
\text { (feet) }\end{array}$ & $\begin{array}{l}\text { Dis- } \\
\text { charge } \\
\text { (cfs) }\end{array}$ \\
\hline \multicolumn{8}{|c|}{ Streams tributary to Lake superior } \\
\hline $4-0113.7$ & $\begin{array}{l}\text { Little Devil Track } \\
\text { River near Grand } \\
\text { Marais, Minn. }\end{array}$ & $\begin{array}{l}\text { NW/ } \frac{1}{4} \text { sec. } 9, \text { T. } 61 \text { N., R. } 1 \text { 'E., at culvert } \\
\text { on County Highway } 12 \text {, } 1 \frac{1}{2} \text { miles above } \\
\text { mouth, and } 2 \frac{1}{2} \text { miles north of Grand } \\
\text { Marais. }\end{array}$ & 7.49 & $1961-68$ & $4-26-68$ & 16.56 & 92 \\
\hline $4-0113.9$ & $\begin{array}{l}\text { Little Devil Track } \\
\text { River tributary } \\
\text { near Grand Marais, } \\
\text { Minn. }\end{array}$ & $\begin{array}{l}\mathrm{SE}^{\frac{1}{4}} \mathrm{SE}^{\frac{1}{4}} \text { sec. } 4 \text {, T. } 61 \mathrm{~N} ., \mathrm{R} .1 \mathrm{E} ., \text { at } \\
\text { culvert on County Highway } 55,0.2 \mathrm{mile} \\
\text { above mouth, and } 2.8 \mathrm{miles} \text { north of } \\
\text { Grand Marais. }\end{array}$ & 0.47 & $1966-68$ & $6-14-68$ & 10.09 & $(t)$ \\
\hline $4-0131$ & $\begin{array}{l}\text { Lake Superior trib- } \\
\text { utary near Tacon- } \\
\text { ite Harbor, Minn. }\end{array}$ & $\begin{array}{l}\text { SW } \frac{1}{4} S E \frac{1}{4} \text { sec. } 20 \text {, T. } 58 \text { N., R. } 5 \text { W., at cul- } \\
\text { vert on U.S. Highway } 61,0.2 \text { mile } \\
\text { above mouth, and } 3.7 \text { miles southwest } \\
\text { of Taconite Harbor. }\end{array}$ & 1.56 & $1964-68$ & $4-25-68$ & 7.20 & 33 \\
\hline $4-0132$ & $\begin{array}{l}\text { Caribou River near } \\
\text { Little Marais, } \\
\text { Minn. }\end{array}$ & $\begin{array}{l}\text { NW } \frac{1}{4} S E \frac{1}{4} \text { sec. } 36, \text { T. } 58 \text { N., R. } 6 \mathrm{~W} . \text {, at } \\
\text { culvert on U.S. Highway } 61 \text {, } 0.2 \text { mile } \\
\text { above mouth, and } 5.2 \text { miles northeast } \\
\text { of Little Marais. }\end{array}$ & 22.8 & $1961-68$ & $6-9-68$ & 12.14 & 378 \\
\hline $4-0151.5$ & $\begin{array}{l}\text { Crow Creek near } \\
\text { Silver Creek, } \\
\text { Minn. }\end{array}$ & $\begin{array}{l}\text { SW } \frac{1}{4} \mathrm{SW} \frac{1}{4} \text { sec. } 23 \text {, T. } 54 \text { N., R. } 10 \text { W. , at } \\
\text { culvert on County Highway } 3,2.3 \text { miles } \\
\text { northeast of Silver Creek, and } 4.0 \\
\text { miles above mouth. }\end{array}$ & 1.07 & $1960-68$ & $4-24-68$ & 9.65 & 51 \\
\hline $4-0152$ & $\begin{array}{l}\text { Encampment River } \\
\text { tributary at } \\
\text { Silver Creek, } \\
\text { Minn. }\end{array}$ & $\begin{array}{l}\text { NE } \frac{1}{4} S E \frac{1}{4} \text { sec. } 33 \text {, T. } 54 \text { N., R. } 10 \text { W., at } \\
\text { culvert on County Highway } 3,0.3 \text { mile } \\
\text { north of Silver Creek, and } 1.4 \text { miles } \\
\text { above mouth. }\end{array}$ & .96 & $1960-68$ & $7-12-68$ & 11.49 & 245 \\
\hline $4-0152.5$ & $\begin{array}{l}\text { Silver Creek trib- } \\
\text { utary near Two } \\
\text { Harbors, Minn. }\end{array}$ & $\begin{array}{l}\text { SW }{ }^{\frac{1}{4} \mathrm{NE}} \frac{1}{4} \text { sec. } 16, \text { T. } 53 \mathrm{~N} ., \mathrm{R} .10 \mathrm{~W} . \text {, at } \\
\text { culvert on County Highway } 3 \text {, } 1.0 \text { mile } \\
\text { above mouth, and } 4.5 \text { miles northeast } \\
\text { of Two Harbors. }\end{array}$ & 3.72 & $1965-68$ & $7-12-68$ & 14.41 & 1,020 \\
\hline $4-0153$ & $\begin{array}{l}\text { Little stewart } \\
\text { River near Two } \\
\text { Harbors, Minn. }\end{array}$ & $\begin{array}{l}\text { SE } \frac{1}{4} \mathrm{NE} \frac{1}{4} \text { sec. } 24 \text {, T. } 53 \mathrm{~N} ., \mathrm{R} .11 \mathrm{~W} . \text {, at } \\
\text { culvert on county highway, } 2.0 \mathrm{miles} \\
\text { above mouth, and } 2.7 \text { miles north of } \\
\text { Two Harbors. }\end{array}$ & 5.54 & $1960-68$ & $\begin{array}{r}9-7-64 \\
4-19-65 \\
10-18-65 \\
6-12-67 \\
4-24-68\end{array}$ & $\begin{array}{r}12.03 \\
\mathrm{a} 10.62 \\
\mathrm{a} 10.34 \\
10.78 \\
11.80\end{array}$ & $\begin{array}{r}\text { a } 260 \\
\text { a } 161 \\
\text { a } 139 \\
\text { a } 171 \\
243\end{array}$ \\
\hline $4-0153.6$ & $\begin{array}{l}\text { Lake superior trib- } \\
\text { utary at French } \\
\text { River, Minn. }\end{array}$ & 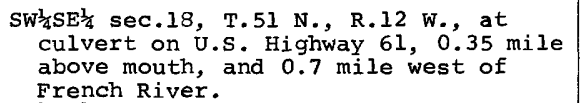 & 1.41 & $1964-68$ & $4-24-68$ & 19.72 & 109 \\
\hline $4-0153.7$ & $\begin{array}{l}\text { Talmadge River at } \\
\text { Duluth, Minn. }\end{array}$ & $\begin{array}{l}\mathrm{SE}^{\frac{1}{4} \mathrm{NE}} \frac{1}{4} \text { sec. } 24, \mathrm{~T} .51 \mathrm{~N} ., \mathrm{R} .13 \mathrm{~W} ., \text { at } \\
\text { culvert on U.S.Highway } 61 \text {, } 0.6 \mathrm{mile} \\
\text { above mouth, and } 0.5 \mathrm{mile} \text { northeast } \\
\text { of Duluth city limits. }\end{array}$ & 5.79 & $1964-68$ & $\begin{array}{l}4-19-65 \\
10-18-65 \\
4-24-68\end{array}$ & $\begin{array}{l}13.17 \\
12.61 \\
15.23\end{array}$ & $\begin{array}{l}187 \\
150 \\
(t)\end{array}$ \\
\hline $4-0154$ & $\begin{array}{l}\text { Miller Creek at } \\
\text { Duluth, Minn. }\end{array}$ & 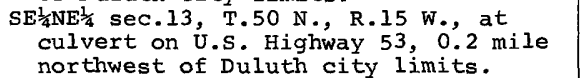 & 4.92 & $1960-68$ & $6-9-68$ & 15.74 & 167 \\
\hline $4-0177$ & $\begin{array}{l}\text { Mckinley Lake } \\
\text { tributary at } \\
\text { Mckinley, Minn. }\end{array}$ & $\begin{array}{l}\text { SW } \frac{1}{4} N^{\frac{1}{4}} \text { sec. } 18 \text {, T. } 58 \text { N., R. } 16 \mathrm{~W} \text {., at } \\
\text { culvert on State Highway } 135 \text { at west } \\
\text { edge of McKinley. }\end{array}$ & .42 & $1960-68$ & $\begin{array}{l}4-17-61 \\
8-21-68\end{array}$ & $\begin{array}{r}\mathrm{b} 9.84 \\
9.73\end{array}$ & $\begin{array}{r}27 \\
27\end{array}$ \\
\hline $4-0188$ & $\begin{array}{l}\text { East Two River } \\
\text { tributary at } \\
\text { Virginia, Minn. }\end{array}$ & $\begin{array}{l}\text { NE } \frac{1}{4} \mathrm{NE}^{\frac{1}{4}} \text { sec. } 12 \text {, T. } 58 \text { N., R. } 18 \mathrm{~W} . \text {, at } \\
\text { culvert on U.S. Highway } 169,0.2 \mathrm{mile} \\
\text { west of Virginia city limits, and } \\
1.1 \text { miles above mouth. }\end{array}$ & 4.26 & $1959-68$ & $8-21-68$ & 8.37 & 85 \\
\hline $4-0241$ & $\begin{array}{l}\text { Rock Creek near } \\
\text { Blackhoof, Minn. }\end{array}$ & $\begin{array}{l}\text { SW } \frac{1}{4} \mathrm{SE}^{\frac{1}{4}} \text { sec. } 21 \text {, T. } 47 \text { N., R. } 16 \text { W., at } \\
\text { culvert on State Highway } 23,4.0 \text { miles } \\
\text { above mouth, and } 4.4 \text { miles east of } \\
\text { Blackhoof. }\end{array}$ & 4.94 & $\begin{array}{l}1961-65 \\
1967-68\end{array}$ & $6-9-68$ & 24.7 & 1,110 \\
\hline $4-0241.1$ & $\begin{array}{l}\text { Rock Creek tribu- } \\
\text { tary near Black- } \\
\text { hoof, Minn. }\end{array}$ & $\begin{array}{l}\text { NE } \frac{1}{4} \mathrm{SE}^{\frac{1}{4}} \text { sec. } 21 \text {, T. } 47 \mathrm{~N} ., \mathrm{R} .16 \mathrm{~W} . \text {, at } \\
\text { culvert on State Highway } 23 \text {, } 0.1 \text { mile } \\
\text { above mouth, and } 4.5 \text { miles east of } \\
\text { Blackhoof. }\end{array}$ & .20 & $1961-68$ & $6-9-68$ & 12.73 & 27 \\
\hline $4-0242$ & $\begin{array}{l}\text { South Fork Nemadji } \\
\text { River near } \\
\text { Eolyoke, Minn. }\end{array}$ & 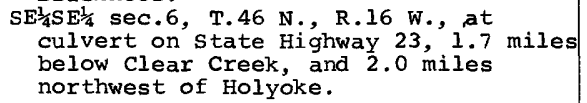 & 19.4 & $1961-68$ & $6-9-68$ & 12.71 & 730 \\
\hline
\end{tabular}

f Discharge not determined.

a Revised.

b Backwater from ice. 
Annual maximum discharge at crest-stage partial-record stations--continued

\begin{tabular}{|c|c|c|c|c|c|c|c|}
\hline \multirow[b]{2}{*}{ Station No. } & \multirow[b]{2}{*}{ Station name } & \multirow[b]{2}{*}{ Location } & \multirow[b]{2}{*}{$\begin{array}{l}\text { Drainage } \\
\text { area } \\
(\mathrm{sq} \mathrm{mi})\end{array}$} & \multirow[b]{2}{*}{$\begin{array}{l}\text { Period } \\
\text { of } \\
\text { record }\end{array}$} & \multicolumn{3}{|c|}{ Annua 1 maximum } \\
\hline & & & & & Date & $\begin{array}{l}\text { Gage } \\
\text { height } \\
\text { (feet) }\end{array}$ & $\begin{array}{r}\text { Dis- } \\
\text { charge } \\
(\mathrm{cfs})\end{array}$ \\
\hline \multicolumn{8}{|c|}{ Red River of the North basin } \\
\hline $5-0476$ & $\begin{array}{l}\text { West Branch Mus- } \\
\text { tinka River near } \\
\text { Graceville, Minn. }\end{array}$ & $\begin{array}{l}\text { NW } \frac{1}{4} N W^{\frac{1}{4}} \text { sec. } 22, \text { T. } 125 \text { N., R. R6 W., at } \\
\text { culverts on county highway, } 4.1 \text { miles } \\
\text { north of Graceville. }\end{array}$ & 56.7 & $1964-68$ & 1968 & (c) & $<5$ \\
\hline $5-0477$ & $\begin{array}{l}\text { West Branch Mus- } \\
\text { tinka River trib- } \\
\text { utary near Grace- } \\
\text { ville, Minn. }\end{array}$ & $\begin{array}{l}\text { NE } \frac{1}{4} \mathrm{NW} \frac{1}{4} \text { sec. } 28 \text {, T. } 125 \mathrm{~N} ., \mathrm{R} .45 \mathrm{~W} ., \text { at } \\
\text { culvert on county highway, } 0.6 \mathrm{mile} \\
\text { northeast of Graceville. }\end{array}$ & 3.37 & $1964-68$ & $\begin{array}{c}4-13-64 \\
9-30-65 \\
4-17-66 \\
1968\end{array}$ & $\begin{array}{l}8.76 \\
6.99 \\
7.53 \\
\text { (c) }\end{array}$ & $\begin{array}{l}96 \\
10 \\
27 \\
<3\end{array}$ \\
\hline $5-0492$ & $\begin{array}{l}\text { Eighteenmile Creek } \\
\text { near Wheaton, } \\
\text { Minn. }\end{array}$ & $\begin{array}{l}\text { On west quarter of line between secs. } \\
24 \text { and } 25 \text {, T. } 127 \text { N., R.47 W., at cul- } \\
\text { vert on County Highway } 67,1.4 \text { miles } \\
\text { above mouth, and } 2.0 \text { miles southwest } \\
\text { of Wheaton. }\end{array}$ & 68.5 & $1965-68$ & $4-22-68$ & 4.90 & $(t)$ \\
\hline $5-0608$ & $\begin{array}{l}\text { Buffalo River near } \\
\text { Callaway, Minn. }\end{array}$ & $\begin{array}{l}\text { SW } \frac{1}{4} \text { SW } \frac{1}{4} \text { sec. } 17 \text {, T. } 141 \text { N., R.41 W., at } \\
\text { culvert on U.S. Highway } 59,2.7 \text { miles } \\
\text { north of Callaway. }\end{array}$ & 49.9 & $1960-68$ & $6-30-68$ & 10.06 & 81 \\
\hline $5-0612$ & $\begin{array}{l}\text { Whisky Creek at } \\
\text { Barnesville, } \\
\text { Minn. }\end{array}$ & 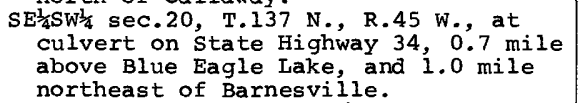 & 25.3 & $\left|\begin{array}{r}1961-64 \\
1965-66 \neq \\
1967-68\end{array}\right|$ & $\begin{array}{r}6-14-67 \\
4-8-68\end{array}$ & $\begin{array}{l}4.90 \\
4.94\end{array}$ & $\begin{array}{l}159 \\
164\end{array}$ \\
\hline $5-0614$ & $\begin{array}{l}\text { Hay Creek above } \\
\text { Downer, Minn. }\end{array}$ & $\begin{array}{l}\text { NW } \frac{1}{4} \mathrm{NW}^{\frac{1}{4}} \text { sec. } 30 \text {, T. } 138 \mathrm{~N} ., \mathrm{R}_{.} .45 \mathrm{~W} \text {., at } \\
\text { culvert on county road, } 3.1 \text { miles east } \\
\text { of Downer. }\end{array}$ & 5.81 & $1961-68$ & $6-10-68$ & 6.30 & 35 \\
\hline $5-0622.8$ & $\begin{array}{l}\text { Wild Rice River } \\
\text { tributary near } \\
\text { Bagley, Minn. }\end{array}$ & $\begin{array}{l}\text { SW } \frac{1}{4} \text { NW } \frac{1}{4} \text { sec. } 21 \text {, T. } 146 \text { N., R. } 37 \text { W., at } \\
\text { culvert on State Highway } 92,5.0 \text { miles } \\
\text { south of Bagley. }\end{array}$ & 3.34 & $1961-68$ & $4-20-68$ & 8.06 & 28 \\
\hline $5-0624.7$ & $\begin{array}{l}\text { Marsh River tribu- } \\
\text { tary near Mahno- } \\
\text { men, Minn. }\end{array}$ & $\begin{array}{l}\mathrm{SE} \frac{1}{4} \mathrm{SW} \frac{1}{4} \text { sec. } 36 \text {, T. } 145 \mathrm{~N} ., \mathrm{R} .43 \mathrm{~W} \text {., at } \\
\text { culvert on State Highway } 31 \text {, a quarter } \\
\text { mile above mouth, and } 5 \frac{1}{4} \text { miles west } \\
\text { of Mahnomen. }\end{array}$ & 6.57 & $1961-68$ & $3-25-68$ & 9.18 & 25 \\
\hline $5-0627$ & $\begin{array}{l}\text { Wild Rice River } \\
\text { tributary near } \\
\text { Twin Valley, } \\
\text { Minn. }\end{array}$ & 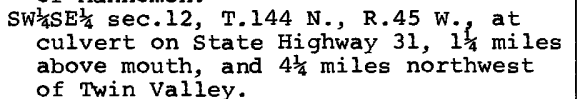 & 2.25 & $1961-68$ & $3-24-68$ & $\mathrm{~b} 13.32$ & 61 \\
\hline $5-0628$ & $\begin{array}{l}\text { Coon Creek near } \\
\text { Twin Valley, } \\
\text { Minn. }\end{array}$ & $\begin{array}{l}\text { NE } \frac{1}{4} \text { NE } \frac{1}{4} \text { sec. } 26 \text {, T. } 144 \text { N., R. } 45 \text { w., at } \\
\text { bridge on County Highway } 28,1.1 \text { miles } \\
\text { above mouth, and } 4.0 \text { miles west of } \\
\text { Twin valley. }\end{array}$ & 32.1 & $1962-68$ & $3-24-68$ & $\mathrm{~b} 9.59$ & 44 \\
\hline $5-0632$ & $\begin{array}{l}\text { South Branch wild } \\
\text { Rice River near } \\
\text { Ogema, Minn. }\end{array}$ & $\begin{array}{l}\mathrm{SE}^{\frac{1}{4}} \mathrm{SE} \frac{1}{4} \mathrm{sec} .11 \text {, T. } 142 \mathrm{~N} ., \mathrm{R} .42 \mathrm{~W} . \text {, at } \\
\text { culvert on county highway, } 2 \mathrm{miles} \\
\text { northwest of Ogema. }\end{array}$ & 6.50 & $1963-68$ & $6-30-68$ & 7.30 & 34 \\
\hline $5-0736$ & $\begin{array}{l}\text { South Branch Battle } \\
\text { River at North- } \\
\text { ome, Minn. }\end{array}$ & $\begin{array}{l}\text { NE } \frac{1}{4} \text { sec. } 25 \text {, T. } 151 \text { N., R. } 29 \text { w., at cul- } \\
\text { vert on U.S. Highway } 71 \text {, three- } \\
\text { quarters of a mile west of Northome, } \\
\text { and } 3 \text { miles above Battle Lake. }\end{array}$ & 3.19 & $1960-68$ & $4-7-68$ & 13.90 & 36 \\
\hline $5-0737.5$ & $\begin{array}{l}\text { South Branch } \\
\text { Cormorant River } \\
\text { tributary near } \\
\text { Blackduck, Minn. }\end{array}$ & $\begin{array}{l}\text { NW } \frac{1}{4} N W^{\frac{1}{4}} \text { sec. } 32 \text {, T. } 150 \mathrm{~N} \text {., R. } 30 \mathrm{~W} . \text {, at } \\
\text { culvert on County Highway } 304,3 \text { miles } \\
\text { above mouth, and } 3 \frac{1}{4} \text { miles north of } \\
\text { Blackduck. }\end{array}$ & 4.45 & $1960-68$ & $4-7-68$ & 12.53 & 39 \\
\hline $5-0738$ & $\begin{array}{l}\text { Perry Creek near } \\
\text { Shooks, Minn. }\end{array}$ & $\begin{array}{l}\text { NW } \frac{1}{4} S W^{\frac{1}{4}} \text { sec. } 30, \text { T. } 151 \text { N., R. } 30 \text { W., at } \\
\text { culvert on State Highway } 72,5 \text { miles } \\
\text { west of Shooks. }\end{array}$ & 2.41 & $1960-68$ & $6-10-68$ & 6.03 & 15 \\
\hline $5-0766$ & $\begin{array}{l}\text { Red Lake River } \\
\text { tributary near } \\
\text { Thief River Falls, } \\
\text { Minn. }\end{array}$ & $\begin{array}{l}\text { SW } \frac{1}{4} S^{\frac{1}{4}} \text { sec. } 8, \text { T. } 153 \mathrm{~N} ., \mathrm{R} .43 \mathrm{~W} . \text {, at } \\
\text { culvert on County Highway } 7,0.5 \mathrm{mile} \\
\text { above mouth, and } 3.1 \text { miles south of } \\
\text { Thief River Falls. }\end{array}$ & - & $1962-68$ & $6-7-68$ & 5.85 & 30 \\
\hline $5-0781$ & $\begin{array}{l}\text { Lost River at } \\
\text { Gonvick, Minn. }\end{array}$ & $\begin{array}{l}\text { NE } \frac{1}{4} \text { NE } \frac{1}{4} \text { sec. } 16 \text {, T. } 149 \text { N., R. } 38 \text { w., at } \\
\text { culvert on county highway, a half mile } \\
\text { south of Gonvick, and } 3 \text { miles below } \\
\text { Pine Lake. }\end{array}$ & 30.9 & $1960-68$ & $3-28-68$ & d7. .14 & 60 \\
\hline $5-0781.8$ & $\begin{array}{l}\text { Lost River tribu- } \\
\text { tary near Clear- } \\
\text { brook, Minn. }\end{array}$ & $\begin{array}{l}\text { NW } \frac{1}{4} \text { sec. } 13 \text {, T. } 148 \text { N., R. } 38 \text { W., at cul- } \\
\text { vert on county highway, } 3 \frac{1}{2} \text { miles } \\
\text { south of Clearbrook. }\end{array}$ & 1.79 & $1960-68$ & $4-20-68$ & 10.01 & 63 \\
\hline $5-0782$ & $\begin{array}{l}\text { Lost River tribu- } \\
\text { tary at clear- } \\
\text { brook, Minn. }\end{array}$ & 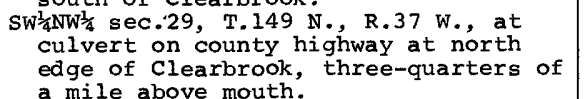 & 3.05 & $1960-68$ & $3-28-68$ & $\mathrm{~b} 9.99$ & 30 \\
\hline $5-0784$ & $\begin{array}{l}\text { Clearwater River } \\
\text { tributary near } \\
\text { Plummer, Minn. }\end{array}$ & $\begin{array}{l}\mathrm{SE} \frac{1}{4} \mathrm{SE} \frac{1}{4} \text { sec. } 22 \text {, T. } 151 \mathrm{~N} . \text {, R. } 43 \mathrm{~W} . \text {, at } \\
\text { culvert on county highway, } 1 \frac{1}{4} \text { miles } \\
\text { above mouth, and } 5 \frac{1}{2} \text { miles southwest } \\
\text { of plummer. }\end{array}$ & 1.17 & $1961-68$ & $7-16-68$ & e7. 36 & 41 \\
\hline
\end{tabular}

4 Discharge not determined.

f Operated as a continuous-record gaging station.

$<$ Less than.

b Backwater from ice.

c Peak stage did not reach bottom of gage.

d Backwater from debris.

e Backwater from aquatic growth. 
Annual maximum discharge at crest-stage partial-record stations--continued

\begin{tabular}{|c|c|c|c|c|c|c|c|}
\hline \multirow[b]{2}{*}{ Station No. } & \multirow[b]{2}{*}{ Station name } & \multirow[b]{2}{*}{ Location } & \multirow[b]{2}{*}{$\begin{array}{c}\text { Drainage } \\
\text { area } \\
\text { (sq } \mathrm{mi})\end{array}$} & \multirow[b]{2}{*}{$\begin{array}{l}\text { Period } \\
\text { of } \\
\text { record }\end{array}$} & \multicolumn{3}{|c|}{ Annua 1 maximum } \\
\hline & & & & & Date & $\begin{array}{c}\text { Gage } \\
\text { height } \\
\text { (feet) }\end{array}$ & \begin{tabular}{|c|}
$\begin{array}{c}\text { Dis- } \\
\text { charge } \\
(c f s)\end{array}$ \\
\end{tabular} \\
\hline \multicolumn{8}{|c|}{ Lake of the Woods basin } \\
\hline $5-1283$ & $\begin{array}{l}\text { Pike River near } \\
\text { Gilbert, Minn. }\end{array}$ & 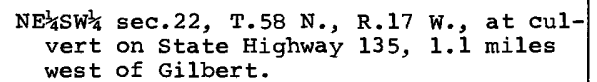 & - & $1966-68$ & $8-21-68$ & 8.11 & $(t)$ \\
\hline $5-1287$ & $\begin{array}{l}\text { Pike River tribu- } \\
\text { tary near Wahl- } \\
\text { sten, Minn. }\end{array}$ & $\begin{array}{l}\text { SW } \frac{1}{4} \mathrm{SW} \frac{1}{4} \text { sec.32, T.61 N., R.15 W., at cul- } \\
\text { vert on State Highway } 135,1.2 \text { miles } \\
\text { south of Wahlsten, and } 2.7 \text { miles above }\end{array}$ & - & $1961-68$ & $6-8-68$ & 7.08 & 62 \\
\hline $5-1297.1$ & $\begin{array}{l}\text { Johnson Creek near } \\
\text { Britt, Minn. }\end{array}$ & $\begin{array}{l}\text { mouth. } \\
\text { NW } \frac{1}{4} N E^{\frac{1}{4}} \text { sec. } 28, \text { T. } 60 \mathrm{~N} ., \text { R.18 W., at cul- } \\
\text { vert adjacent to U.S. Highway } 53 \text {, } 0.6 \\
\text { mile below Sand Lake, and } 5.9 \text { miles }\end{array}$ & - & $\left|\begin{array}{l}1961-64 \\
1966-68\end{array}\right|$ & $6-14-68$ & 7.46 & 22 \\
\hline $5-1303$ & $\begin{array}{l}\text { Boriin zreek near } \\
\text { Chisholm, Minn. }\end{array}$ & $\begin{array}{l}\text { west of Britt. } \\
\text { SE } \frac{1}{4} \text { SE } \frac{1}{4} \text { sec. } 9, \text { T. } 59 \text { N., R. } 20 \text { w., at cul- } \\
\text { vert on State Highway } 73,1.2 \text { miles } \\
\text { above mouth, and } 7.8 \text { miles north of } \\
\text { Chisholm. }\end{array}$ & 13.7 & $1959-68$ & $9-18-68$ & 12.02 & 132 \\
\hline \multicolumn{8}{|c|}{ Split Hand Creek basin } \\
\hline $5-2150$ & $\begin{array}{l}\text { Smith Creek near } \\
\text { Hill City, Minn. }\end{array}$ & $\begin{array}{l}\text { SE } \frac{1}{4} N W^{\frac{1}{4}} \text { sec. } 13 \text {, T. } 53 \text { N., R. } 26 \text { W., at } \\
\text { culvert on U.S. Highway 169, } 6 \frac{1}{2} \text { miles } \\
\text { north of Hili City. }\end{array}$ & 5.06 & $1961-68$ & $4-23-68$ & 3.71 & 16 \\
\hline \multicolumn{8}{|c|}{ Swan River basin } \\
\hline $5-2167$ & $\begin{array}{l}\text { ' ' Brien Creek near } \\
\text { Nashwauk, Minn. } \\
\text { Swan River tribu- } \\
\text { tary at Warba, } \\
\text { Minn. }\end{array}$ & $\begin{array}{l}\text { NE } \frac{1}{4} \mathrm{NE} \frac{1}{4} \text { sec. } 33, \mathrm{~T} .57 \mathrm{~N} ., \mathrm{R} .22 \mathrm{~W} . \text {, at } \\
\text { culvert on U.S. Highway } 169 \text {, } 1.5 \text { miles } \\
\text { east of Nashwauk, and } 3.0 \text { miles above } \\
\text { Welcome Creek. } \\
\text { NW } \frac{1}{4} \text { sec. } 34 \text {, T. } 54 \text { N., R. } 23 \text { W., at culvert } \\
\text { on U.S. Highway 2, three-quarters of a } \\
\text { mile above mouth, and } 1 \text { mile southeast } \\
\text { of Warba. }\end{array}$ & 8.26 & $1959-68$ & $6-6-68$ & d 9.76 & 94 \\
\hline \multicolumn{8}{|c|}{ Bluff Creek basin } \\
\hline $5-2177$ & $\begin{array}{l}\text { Bluff Creek near } \\
\text { Jacobson, Minn. }\end{array}$ & $\begin{array}{l}\text { SW } \frac{1}{4} \mathrm{NW}^{\frac{1}{4}} \text { sec. } 8, \text { T. } 52 \mathrm{~N} ., \mathrm{R}_{2} 23 \mathrm{~W} \text {, at cul- } \\
\text { vert on State Highway } 34 \text {, } 1 \frac{1}{4} \text { miles } \\
\text { west of Jacobson. }\end{array}$ & 2.95 & $1961-68$ & 1968 & (c) & $<15$ \\
\hline \multicolumn{8}{|c|}{ Crow Wing River basin } \\
\hline $5-2441$ & $\begin{array}{l}\text { Kitten Creek near } \\
\text { Sebeka, Minn. } \\
\text { Cat River near } \\
\text { Nimrod, Minn. }\end{array}$ & 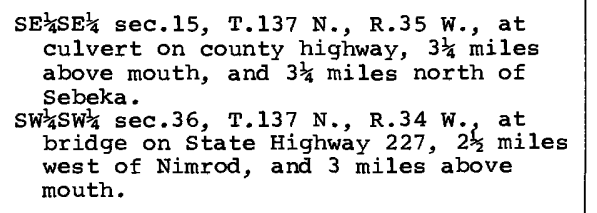 & 9.34 & $1961-68$ & $6-10-68$ & 10.45 & 160 \\
\hline \multicolumn{8}{|c|}{ Platte River basin } \\
\hline $5-2678$ & $\begin{array}{l}\text { Big Mink Creek } \\
\text { tributary near } \\
\text { Lastrup, Minn. } \\
\text { Hillman Creek near } \\
\text { Pierz, Minn. }\end{array}$ & 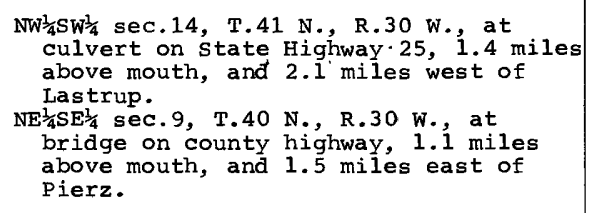 & 52.6 & $1961-68$ & $6-29-68$ & 8.29 & 190 \\
\hline \multicolumn{8}{|c|}{ Sauk River basin } \\
\hline $5-2703.1$ & $\begin{array}{l}\text { Sauk River tribu- } \\
\text { tary at Spring } \\
\text { Hill, Minn. } \\
\text { Sauk River tribu- } \\
\text { tary near St. } \\
\text { Martin, Minn. }\end{array}$ & 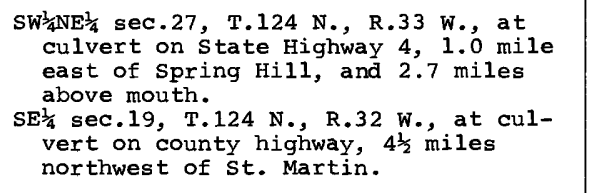 & 6.42 & $\begin{array}{r}1960 \\
1962-68\end{array}$ & $6-10-68$ & 9.78 & 82 \\
\hline
\end{tabular}

$f$ Discharge not determined.

$<$ Less than.

c Peak stage did not reach bottom of gage.

d Backwater from debris. 
Annual maximum discharge at crest-stage partial-record stations--continued

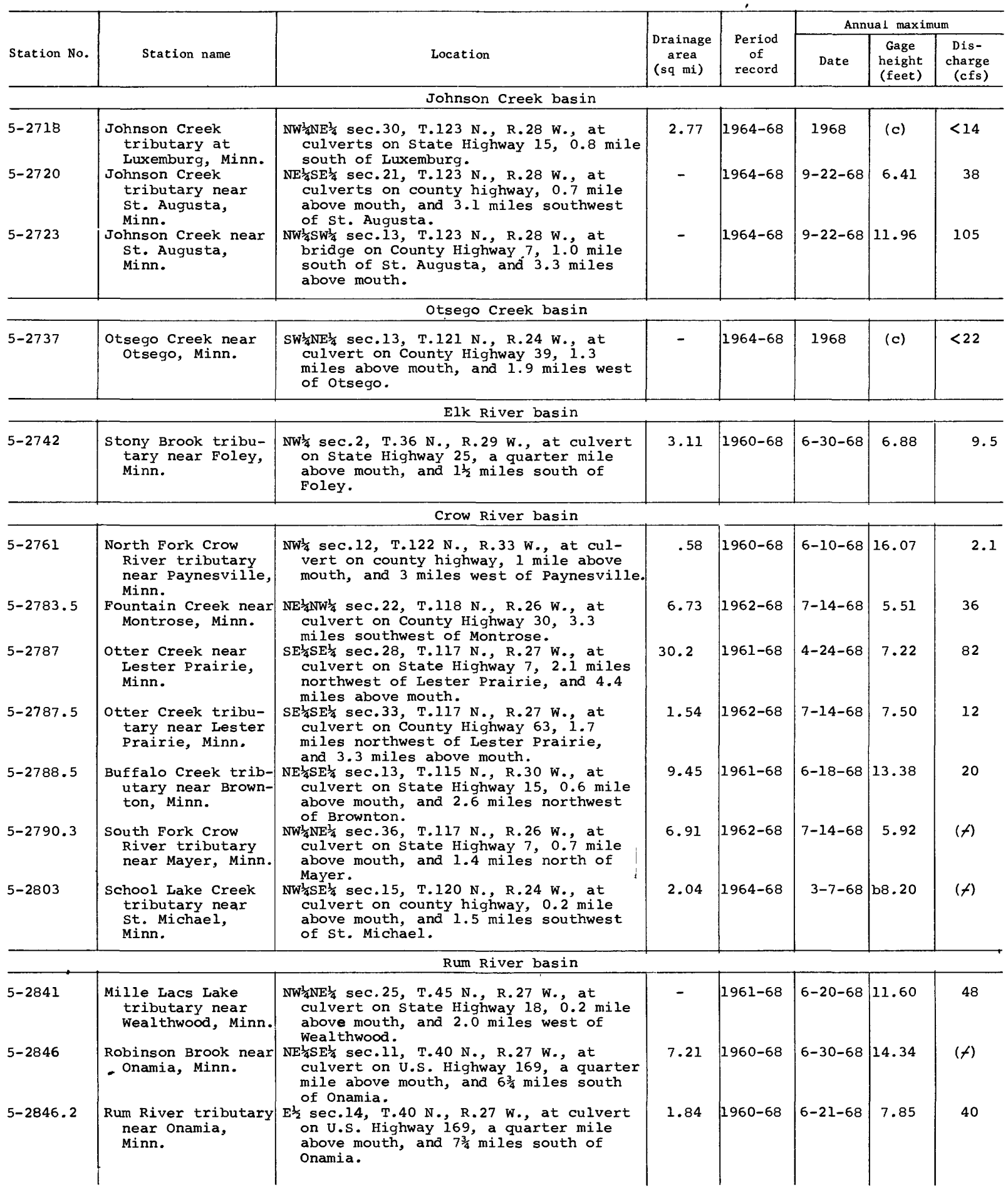

$f$ Discharge not determined.

$<$ Less than.

b Backwater from ice.

c Peak stage did not reach bottom of gage. 
Annual maximum discharge at crest-stage partial-record stations--continued

\begin{tabular}{|c|c|c|c|c|c|c|c|}
\hline \multirow[b]{2}{*}{ Station No. } & \multirow[b]{2}{*}{ Station name } & \multirow[b]{2}{*}{ Location } & \multirow[b]{2}{*}{$\begin{array}{l}\text { Drainage } \\
\text { area } \\
(\mathrm{sq} \mathrm{mi})\end{array}$} & \multirow[b]{2}{*}{$\begin{array}{l}\text { Period } \\
\text { of } \\
\text { record }\end{array}$} & \multicolumn{3}{|c|}{ Annual maximum } \\
\hline & & & & & Date & $\begin{array}{l}\text { Gage } \\
\text { height } \\
\text { (feet) }\end{array}$ & $\begin{array}{l}\text { Dis- } \\
\text { charge } \\
\text { (cfs) }\end{array}$ \\
\hline \multicolumn{8}{|c|}{ Rum River basin--continued } \\
\hline $5-2849.2$ & $\begin{array}{l}\text { Stanchfield Creek } \\
\text { tributary near } \\
\text { Day, Minn. }\end{array}$ & $\begin{array}{l}\text { NW } \frac{1}{4} \mathrm{SE} \frac{1}{4} \text { sec. } 13 \text {, T. } 37 \mathrm{~N} ., \mathrm{R} .25 \mathrm{~W} . \text {, at } \\
\text { culvert on County Highway } 60 \text {, } 0.5 \mathrm{mile} \\
\text { above mouth, and } 1.5 \mathrm{miles} \text { southwest } \\
\text { of Day. }\end{array}$ & 1.26 & $1961-68$ & $9-23-68$ & 6.51 & 41 \\
\hline \multicolumn{8}{|c|}{ Minnesota River basin } \\
\hline $5-2991$ & $\begin{array}{l}\text { Lazarus creek } \\
\text { tributary near } \\
\text { Canby, Minn. }\end{array}$ & $\begin{array}{l}\text { NE } \frac{1}{4} N W \frac{1}{4} \text { sec. } 6 \text {, T. } 114 \text { N., R. } 45 \text { W. , at cul- } \\
\text { vert on State Highway } 68,2.7 \text { miles } \\
\text { west of Canby, and } 4.2 \text { miles above } \\
\text { mouth. }\end{array}$ & a2.97 & $1960-68$ & 1968 & (c) & $<5$ \\
\hline $5-3012$ & $\begin{array}{l}\text { Minnesota River } \\
\text { tributary near } \\
\text { Montevideo, Minn. }\end{array}$ & $\begin{array}{l}\mathrm{SW}_{\frac{1}{4} \mathrm{SE}} \frac{1}{4} \mathrm{sec} .16, \mathrm{~T} .117 \mathrm{~N} ., \mathrm{R} .41 \mathrm{~W} . \text {, at } \\
\text { culvert on U.S. Highway } 212 \text {, } 1 \text { mile } \\
\text { above mouth, and } 3 \frac{3}{4} \text { miles west of } \\
\text { Montevideo. }\end{array}$ & 0.54 & $1960-68$ & 1968 & (c) & $<5$ \\
\hline $5-3029.7$ & $\begin{array}{l}\text { Lake Emily tribu- } \\
\text { tary near Star- } \\
\text { buck, Minn. }\end{array}$ & $\begin{array}{l}\text { NW } \frac{1}{4} \text { sec. } 27, \text { T.124 N., R. } 39 \text { W., at cul- } \\
\text { vert on State Highway } 29,6 \frac{1}{4} \text { miles } \\
\text { south of Starbuck. }\end{array}$ & .13 & $1962-68$ & 1968 & (c) & $<2$ \\
\hline $5-3034.5$ & $\begin{array}{l}\text { Hassel Creek near } \\
\text { Clontarf, Minn. }\end{array}$ & $\begin{array}{l}\text { NW } \frac{1}{4} \mathrm{SE}^{\frac{1}{4}} \text { sec. } 4 \text {, T. } 122 \mathrm{~N} ., \mathrm{R} .39 \mathrm{~W} . \text {, at } \\
\text { culvert on State Highway } 29 \text {, a quarter } \\
\text { mile above Lake Hassei, and } 5 \frac{1}{4} \text { miles } \\
\text { east of Clontarf. }\end{array}$ & 4.03 & $1962-68$ & $\begin{array}{r}7-19-62 \\
6-3-63 \\
10-20-63 \\
5-23-65 \\
3-12-66 \\
3-26-67 \\
3-19-68\end{array}$ & $\begin{array}{r}11.92 \\
8.41 \\
9.70 \\
7.86 \\
\mathrm{~b} 8.69 \\
\mathrm{~b} 10.89 \\
\mathrm{~b} 8.26\end{array}$ & $\begin{array}{r}177 \\
44 \\
81 \\
30 \\
38 \\
56 \\
28\end{array}$ \\
\hline $5-3052$ & $\begin{array}{l}\text { Spring Creek near } \\
\text { Montevideo, Minn. }\end{array}$ & 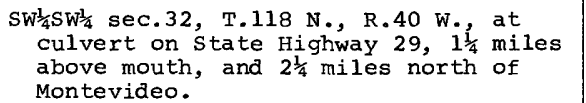 & 16.3 & $1959-68$ & $6-20-68$ & 12.60 & 17 \\
\hline $5-3112$ & $\begin{array}{l}\text { North Branch Yellow } \\
\text { Medicine River } \\
\text { near Ivanhoe, } \\
\text { Minn. }\end{array}$ & $\begin{array}{l}\text { NW } \frac{1}{4} \text { sec. } 2 \text {, T. } 111 \mathrm{~N} ., \mathrm{R} .46 \mathrm{~W} . \text {, at culvert } \\
\text { on State Highway 19, } 5 \frac{1}{4} \text { miles west of } \\
\text { Ivanhoe. }\end{array}$ & 15.2 & $1960-68$ & $\begin{array}{r}4-13-60 \\
7-1-62 \\
7-26-63 \\
4-21-64 \\
4-8-65 \\
3-10-66 \\
6-15-67 \\
7-26-68\end{array}$ & $\begin{array}{r}13.98 \\
13.66 \\
14.30 \\
12.60 \\
\mathrm{~b} 15.89 \\
\mathrm{~b} 14.28 \\
14.17 \\
12.70\end{array}$ & $\begin{array}{r}\text { a228 } \\
\text { a168 } \\
288 \\
25 \\
431 \\
19 \\
540 \\
31\end{array}$ \\
\hline $5-3112.5$ & $\begin{array}{l}\text { North Branch Yellow } \\
\text { Medicine River } \\
\text { tributary near } \\
\text { wilno, Minn. }\end{array}$ & $\begin{array}{l}\mathrm{SE} \frac{1}{4} \mathrm{NE} \frac{1}{4} \sec .33, \mathrm{~T} .113 \mathrm{~N} ., \mathrm{R} .45 \mathrm{~W} . \text {, at } \\
\text { culvert on U.S. Highway } 75 \text {, } 1 \frac{1}{2} \text { miles } \\
\text { above mouth, and } 4 \frac{1}{2} \text { miles northwest } \\
\text { of Wilno. }\end{array}$ & .33 & $1960-68$ & $7-26-68$ & 10.64 & 56 \\
\hline $5-3113$ & $\begin{array}{l}\text { North Branch Yellow } \\
\text { Medicine River } \\
\text { tributary near } \\
\text { Porter, Minn. }\end{array}$ & $\begin{array}{l}E^{\frac{1}{2}} \text { sec. } 16 \text {, T. } 113 \mathrm{~N} ., \mathrm{R}^{4} 45 \mathrm{~W} \text {., at culvert } \\
\text { on U.S. Highway } 75,6 \frac{1}{4} \mathrm{miles} \text { southwest } \\
\text { of Porter. }\end{array}$ & 1.46 & $1960-68$ & $7-26-68$ & 14.23 & 64 \\
\hline $5-3138$ & $\begin{array}{l}\text { Chetomba Creek } \\
\text { tributary near } \\
\text { Blomkest, Minn. }\end{array}$ & $\begin{array}{l}\text { SW } \frac{1}{4} \text { SW } \frac{1}{4} \text { sec. } 35, \text { T. } 118 \text { N., R. R5 W., at } \\
\text { culvert on U.S. Highway } 71,2 \frac{3}{4} \text { miles } \\
\text { northwest of Blomkest. }\end{array}$ & .79 & $1959-68$ & $3-18-68$ & 6.76 & 33 \\
\hline $5-3149$ & $\begin{array}{l}\text { Redwood River at } \\
\text { Ruthton, Minn. }\end{array}$ & $\begin{array}{l}N W^{\frac{1}{4}} \mathrm{NW} \frac{1}{4} \text { sec. } 11 \text {, T. } 108 \mathrm{~N} ., \text { R. } 44 \text { W., at } \\
\text { culvert on State Highway } 23,0.1 \text { mile } \\
\text { northeast of Ruthton. }\end{array}$ & 5.90 & $1959-68$ & $7-26-68$ & 14.52 & 187 \\
\hline $5-3152$ & $\begin{array}{l}\text { Prairie Ravine nean } \\
\text { Marsha11, Minn. }\end{array}$ & $\begin{array}{l}\text { SE } \frac{1}{4} \text { NE } \frac{1}{4} \text { sec. } 20, T .112 \mathrm{~N} ., \mathrm{R} .41 \mathrm{~W} ., \text { at } \\
\text { culvert on U.S. Highway } 59,2.7 \text { miles } \\
\text { north of Marshall. }\end{array}$ & 5.63 & $\begin{array}{l}1959-64 A \\
1965-68\end{array}$ & $6-10-68$ & 6.37 & 41 \\
\hline $5-3165.5$ & $\begin{array}{l}\text { West Fork Beaver } \\
\text { Creek near } \\
\text { Olivia, Minn. }\end{array}$ & $\begin{array}{l}\text { SW } w^{\frac{1}{4}} \mathrm{SW}^{\frac{1}{4}} \text { sec. } 14, \text { T.116 N., R.35 W., at } \\
\text { culvert on U.S. Highway } 71,5 \frac{1}{2} \text { miles } \\
\text { northwest of Olivia. }\end{array}$ & 9.71 & $1959-68$ & $7-14-68$ & 3.18 & $(t)$ \\
\hline $5-3166.9$ & $\begin{array}{l}\text { Spring Creek trib- } \\
\text { utary near } \\
\text { sleepy Eye, Minn. }\end{array}$ & $\begin{array}{l}\text { NW } \frac{1}{4} \text { sec. } 25, \text { T. } 111 \text { N., R. } 33 \mathrm{~W} \text {, at cul- } \\
\text { vert on county highway, } 0.1 \text { mile above } \\
\text { mouth, and } 7 \frac{1}{2} \text { miles north of sleepy } \\
\text { Eye. }\end{array}$ & - & $1966-68$ & $\begin{array}{r}3-3-66 \\
7-8-67 \\
7-26-68 \\
9-22-68\end{array}$ & $\begin{array}{r}55.49 \\
5.45 \\
3.97 \\
3.97\end{array}$ & $\begin{array}{l}40 \\
62 \\
20 \\
20\end{array}$ \\
\hline $5-3167$ & $\begin{array}{l}\text { Spring Creek near } \\
\text { sleepy Eye, Minn. }\end{array}$ & 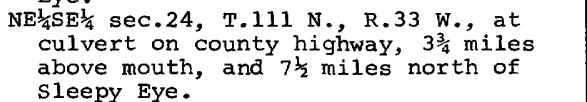 & 30.0 & $1959-68$ & $7-26-68$ & 11.89 & 241 \\
\hline $5-3168$ & $\begin{array}{l}\text { Cottonwood River } \\
\text { tributary near } \\
\text { Balaton, Minn. }\end{array}$ & $\begin{array}{l}\text { NW } \frac{1}{4} N W \frac{1}{4} \sec .19 \text {, T. } 109 \text { N., R. } 42 \text { W., at } \\
\text { culvert on U.S. Highway } 14,4 \frac{1}{4} \text { miles } \\
\text { west of Balaton. }\end{array}$ & 0.50 & $1959-68$ & $7-26-68$ & 4.89 & 8.6 \\
\hline $5-3168 \cdot 5$ & $\begin{array}{l}\text { Meadow Creek trib- } \\
\text { utary near Mar- } \\
\text { shall, Minn. }\end{array}$ & 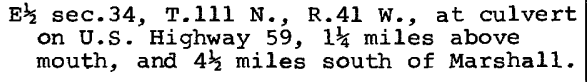 & 0.54 & $1961-68$ & $7-26-68$ & 13.52 & $(t)$ \\
\hline
\end{tabular}

t Discharge not determined.

F Operated as a continuous-record gaging station.

$<$ Less than

a Revised.

b Backwater from ice.

c Peak stage did not reach bottom of gage. 
Annual maximum discharge at crest-stage partial-record stations--continued

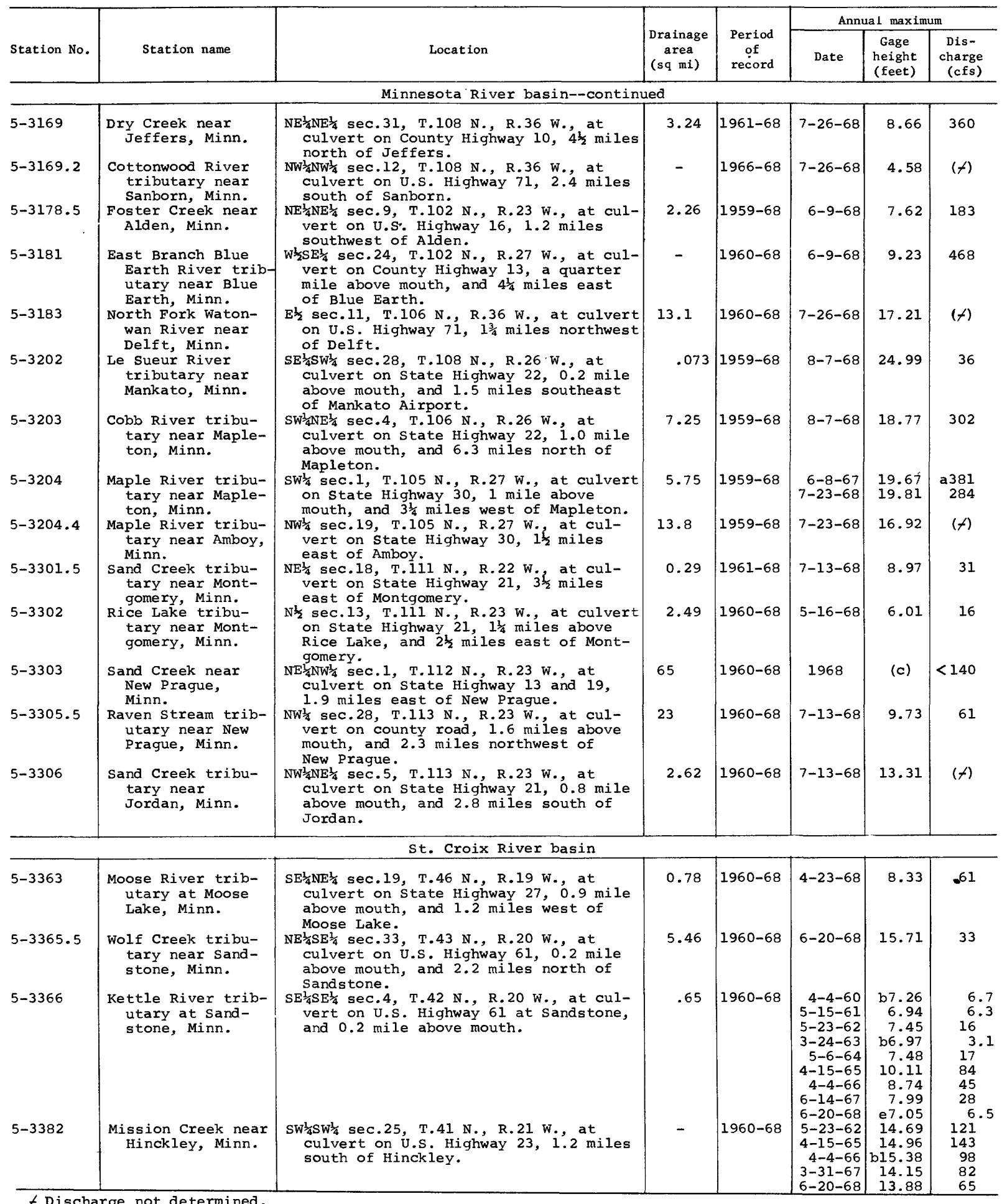

$f$ Discharge not determined.

$<$ Less than.

a Revised.

b Backwater from ice.

c Peak stage did not reach bottom of gage.

e Backwater from aquatic growth. 
Annual maximum discharge at crest-stage partial-record stations--continued

\begin{tabular}{|c|c|c|c|c|c|c|c|}
\hline \multirow[b]{2}{*}{ Station No. } & \multirow[b]{2}{*}{ Station name } & \multirow[b]{2}{*}{ Location } & \multirow[b]{2}{*}{$\begin{array}{l}\text { Drainage } \\
\text { area } \\
(\mathrm{sq} \mathrm{mi})\end{array}$} & \multirow[b]{2}{*}{$\begin{array}{l}\text { Period } \\
\text { of } \\
\text { record }\end{array}$} & \multicolumn{3}{|c|}{ Annual maximum } \\
\hline & & & & & Date & $\begin{array}{l}\text { Gage } \\
\text { height } \\
\text { (feet) }\end{array}$ & $\begin{array}{l}\text { Dis- } \\
\text { charge } \\
\text { (cfs) }\end{array}$ \\
\hline \multicolumn{8}{|c|}{ Vermillion River basin } \\
\hline $5-3459$ & $\begin{array}{l}\text { Vermillion River } \\
\text { tributary near } \\
\text { Hastings, Minn. }\end{array}$ & $\begin{array}{l}\text { NE } \frac{1}{4} \mathrm{SE}_{4}^{\frac{1}{4}} \text { sec. } 35, \mathrm{~T} .115 \mathrm{~N} ., \mathrm{R} .18 \mathrm{~W} \text {, at } \\
\text { culvert on county highway, } 2.0 \text { miles } \\
\text { above mouth, and } 4.1 \text { miles west of } \\
\text { Hastings. }\end{array}$ & 14.3 & $1960-68$ & 1968 & (c) & $<1$ \\
\hline \multicolumn{8}{|c|}{ Cannon River basin } \\
\hline $5-3527$ & $\begin{array}{l}\text { Turtle Creek trib- } \\
\text { utary near Pratt, } \\
\text { Minn. }\end{array}$ & $\begin{array}{l}\text { NW } \frac{1}{4} \text { sec.8, T. } 106 \mathrm{~N}, \mathrm{R}^{\mathrm{I}} 19 \mathrm{~W} \text {., at culvert } \\
\text { on U.S. Highway } 218,1 \text { mile above } \\
\text { mouth, and } 1 \frac{1}{2} \text { miles southeast of Pratt. }\end{array}$ & - & $1960-68$ & $7-23-68$ & 16.71 & 70 \\
\hline $5-3528$ & $\begin{array}{l}\text { Turtle Creek trib- } \\
\text { utary near steele } \\
\text { Center, Minn. }\end{array}$ & $\begin{array}{l}\text { NW } \frac{1}{4} \mathrm{NW}^{\frac{1}{4}} \text { sec. } 11 \text {, T. } 106 \mathrm{~N} \text {., R. } 20 \mathrm{~W} \text {., at } \\
\text { culvert on township road, } 1 \frac{1}{2} \text { miles } \\
\text { above mouth, and } 1 \frac{1}{2} \text { miles northeast } \\
\text { of steele center. }\end{array}$ & - & $1960-68$ & $7-23-68$ & 5.35 & 45 \\
\hline $5-3551$ & $\begin{array}{l}\text { Little Cannon River } \\
\text { tributary near } \\
\text { Kenyon, Minn. }\end{array}$ & $\begin{array}{l}\text { SE } \frac{1}{4} \text { sec. } 9 \text {, T.ll0 N., R. } 18 \text { W., at culvert } \\
\text { on State Highway } 56 \text {, a quarter mile } \\
\text { above mouth, and } 5 \text { miles north of } \\
\text { Kenyon. }\end{array}$ & 2.02 & $1960-68$ & $5-15-68$ & 20.84 & 1,040 \\
\hline $5-3551 \cdot 5$ & $\begin{array}{l}\text { Pine Creek near } \\
\text { Cannon Falls, } \\
\text { Minn. }\end{array}$ & 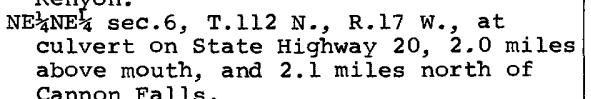 & 20.2 & $1960-68$ & $5-15-68$ & 4.92 & 412 \\
\hline $5-3551.8$ & $\begin{array}{l}\text { Cannon River trib- } \\
\text { utary near Mies- } \\
\text { ville, Minn. }\end{array}$ & $\begin{array}{l}\text { SW } \frac{1}{4} S^{\frac{1}{4}} \text { sec.9, T. } 113 \mathrm{~N} ., \text { R. } 17 \text { W., at } \\
\text { culvert on state Highway } 50,2.9 \text { miles } \\
\text { west of Miesville. }\end{array}$ & .086 & $1960-68$ & $5-15-68$ & 15.38 & $(t)$ \\
\hline $5-3552.3$ & $\begin{array}{l}\text { Cannon River trib- } \\
\text { utary near Welch, } \\
\text { Minn. }\end{array}$ & 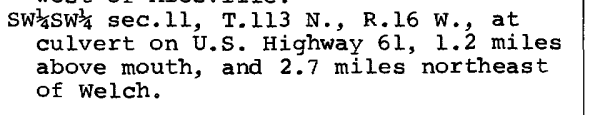 & - & $1960-68$ & $5-15-68$ & 10.15 & 48 \\
\hline \multicolumn{8}{|c|}{ Zumbro River basin } \\
\hline $5-3733.5$ & $\begin{array}{l}\text { Zumbro River trib- } \\
\text { utary near South } \\
\text { Troy, Minn. }\end{array}$ & 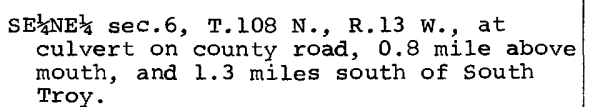 & 0.16 & $1962-68$ & $6-25-68$ & 8.15 & 26 \\
\hline $5-3737$ & $\begin{array}{l}\text { North Fork Zumbro } \\
\text { River tributary } \\
\text { near wanamingo, } \\
\text { Minn. }\end{array}$ & $\begin{array}{l}\mathrm{SE} \frac{1}{4} \mathrm{SE} \frac{1}{4} \text { sec. } 32 \text {, T. } 110 \mathrm{~N} ., \mathrm{R} .17 \text { W., at } \\
\text { culvert on County Highway } 1,3 \frac{1}{4} \text { miles } \\
\text { above mouth, and } 4 \frac{1}{4} \text { miles southwest } \\
\text { of Wanamingo. }\end{array}$ & 9.36 & $1960-68$ & $7-15-68$ & 10.38 & 350 \\
\hline $5-3739$ & $\begin{array}{l}\text { Trout Brook trib- } \\
\text { utary near Good- } \\
\text { hue, Minn. }\end{array}$ & 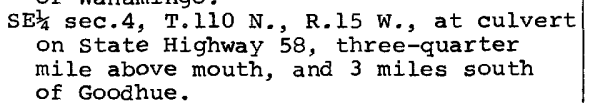 & .41 & $1960-68$ & $8-19-68$ & 7.04 & $(t)$ \\
\hline $5-3744$ & $\begin{array}{l}\text { Long creek near } \\
\text { Potsdam, Minn. }\end{array}$ & $\begin{array}{l}\text { At quarter corner on north line of sec. } \\
8 \text {, T. } 108 \mathrm{~N} ., \mathrm{R} .12 \mathrm{~W} . \text {, at culvert on } \\
\text { county highway, } 2.6 \mathrm{miles} \text { northeast } \\
\text { of Potsdam. }\end{array}$ & - & $1966-68$ & $\begin{array}{r}3-4-66 \\
6-15-67 \\
6-25-68\end{array}$ & $\begin{array}{l}17.39 \\
19.75 \\
15.96\end{array}$ & $\begin{array}{l}228 \\
408 \\
137\end{array}$ \\
\hline \multicolumn{8}{|c|}{ East Indian Creek basin } \\
\hline $5-3758$ & $\begin{array}{l}\text { East Indian Creek } \\
\text { tributary No. } 1 \\
\text { near Weaver, } \\
\text { Minn. }\end{array}$ & $\begin{array}{l}\text { NW } \frac{1}{4} \text { SE } \frac{1}{4} \text { sec. } 23 \text {, T. } 109 \text { N., R. } 10 \text { W., at } \\
\text { culvert on County Highway } 14,0.3 \text { mile } \\
\text { above mouth, and } 2.5 \text { miles northwest } \\
\text { of Weaver. }\end{array}$ & 0.21 & $1962-68$ & $6-21-68$ & 10.77 & 34 \\
\hline \multicolumn{8}{|c|}{ Garvin Brook basin } \\
\hline $5-3782.9$ & $\begin{array}{l}\text { Straight Valley } \\
\text { Creek near } \\
\text { Altura, Minn. } \\
\text { Straight Valley } \\
\text { Creek near Rol- } \\
\text { lingstone, Minn. }\end{array}$ & 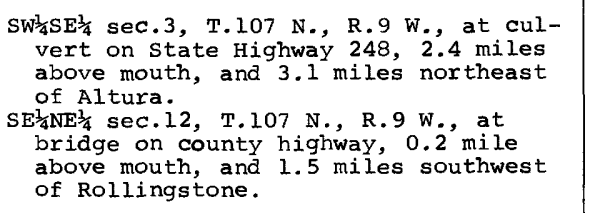 & 5.16 & $1966-68$ & $7-14-68$ & 12.55 & 373 \\
\hline \multicolumn{8}{|c|}{ Root River basin } \\
\hline $5-3836$ & $\begin{array}{l}\text { North Branch Root } \\
\text { River tributary } \\
\text { near stewart- } \\
\text { ville, Minn. }\end{array}$ & $\begin{array}{l}\text { Near center sec. } 36, \text { T. } 105 \mathrm{~N} ., \mathrm{R} .14 \mathrm{~W} . \text {, } \\
\text { at culvert on State Highway } 30,2.0 \\
\text { miles east of Stewartville, and } 2.3 \\
\text { miles above mouth. }\end{array}$ & .73 & $\begin{array}{r}1958 \\
1959-647 \\
1965-68\end{array} \mid$ & $6-13-68$ & 5.26 & 12 \\
\hline
\end{tabular}

t Discharge not determined.

$\neq$ Operated as a continuous-record gaging station.

$<$ Less than.

c Peak stage did not reach bottom of gage. 
Annual maximum discharge at crest-stage partial-record stations--continued

\begin{tabular}{|c|c|c|c|c|c|c|c|}
\hline \multirow[b]{2}{*}{ Station No. } & \multirow[b]{2}{*}{ Station name } & \multirow[b]{2}{*}{ Location } & \multirow[b]{2}{*}{$\begin{array}{l}\text { Drainage } \\
\text { area } \\
(\mathrm{sq} \mathrm{mi})\end{array}$} & \multirow[b]{2}{*}{$\begin{array}{l}\text { Period } \\
\quad \text { of } \\
\text { record }\end{array}$} & \multicolumn{3}{|c|}{ Annua 1 maximum } \\
\hline & & & & & Date & $\begin{array}{l}\text { Gage } \\
\text { height } \\
\text { (feet) }\end{array}$ & $\begin{array}{l}\text { Dis- } \\
\text { charge } \\
\text { (cfs) }\end{array}$ \\
\hline \multicolumn{8}{|c|}{ Root River basin--continued } \\
\hline $5-3837$ & $\begin{array}{l}\text { Mill Creek tribu- } \\
\text { tary near Chat- } \\
\text { field, Minn. }\end{array}$ & $\begin{array}{l}\text { SW-1/4W-1/4 sec. } 14 \text {, T. } 105 \mathrm{~N} ., \mathrm{R} .12 \mathrm{~W} . \text {, at } \\
\text { culvert on county highway, } 0.8 \text { mile } \\
\text { above mouth, and } 4.5 \text { miles northwest } \\
\text { of Chatfield. }\end{array}$ & 2.36 & $1959-68$ & $4-23-68$ & 13.45 & $(t)$ \\
\hline $5-3837.2$ & $\begin{array}{l}\text { Mill Creek near } \\
\text { Chatfield, Minn. }\end{array}$ & $\begin{array}{l}\text { SE } \frac{1}{4} N W \frac{1}{4} \text { sec. } 23 \text {, T. } 105 \mathrm{~N} ., \mathrm{R} .12 \mathrm{~W} \text {, at } \\
\text { bridge on county highway, } 3.4 \mathrm{miles} \\
\text { northwest of Chatfield, and } 4.8 \mathrm{miles}\end{array}$ & 22.4 & $1962-68$ & $\begin{array}{r}6-9-63 \\
3-12-64 \\
3-26-67 \\
6-26-68\end{array}$ & $\begin{array}{r}12.39 \\
\mathrm{~b} 10.06 \\
13.98 \\
10.76\end{array}$ & $\begin{array}{r}1,030 \\
137 \\
1,550\end{array}$ \\
\hline $5-3838 \cdot 5$ & $\begin{array}{l}\text { Bear Creek near } \\
\text { Grand Meadow, } \\
\text { Minn. }\end{array}$ & 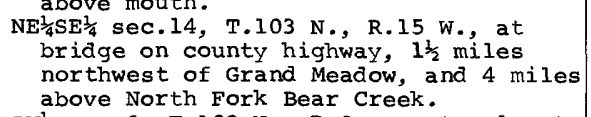 & 13.6 & $1962-68$ & $\begin{array}{l}6-26-68 \\
6-26-68\end{array} \mid$ & $\begin{array}{l}10.76 \\
16.98\end{array}$ & $\begin{array}{l}535 \\
520\end{array}$ \\
\hline $5-3841$ & $\begin{array}{l}\text { Trout Creek tribu- } \\
\text { tary near Lanes- } \\
\text { boro, Minn. }\end{array}$ & $\begin{array}{l}\text { SW } \frac{1}{4} \text { sec. } 6, \mathrm{~T} .102 \mathrm{~N} ., \mathrm{R} .9 \mathrm{~W} . \text {, at culvert } \\
\text { on county highway, three-quarter mile } \\
\text { above mouth, and } 4 \text { miles south of } \\
\text { Lanesboro. }\end{array}$ & 4.08 & $1959-68$ & $5-15-68$ & 14.68 & 236 \\
\hline $5-3841 \cdot 5$ & $\begin{array}{l}\text { Root River tribu- } \\
\text { tary near Whalan, } \\
\text { Minn. }\end{array}$ & 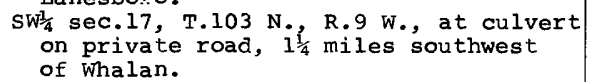 & .30 & $1959-68$ & $5-15-68$ & 7.15 & 24 \\
\hline $5-3842$ & $\begin{array}{l}\text { Whalan Creek near } \\
\text { Whalan. } 1 j \mathrm{mn} .\end{array}$ & $\begin{array}{l}\text { SE } \frac{1}{4} \text { sec. } 21, \text { T.103 N., R.9 W., at bridge } \\
\text { on county highway, } 1 \frac{3}{4} \text { miles southeast } \\
\text { of Whalan, and } 2 \frac{1}{4} \text { miles above mouth. }\end{array}$ & 7.85 & $1959-68$ & $5-15-68$ & 19.76 & 1,170 \\
\hline $5-3843$ & $\begin{array}{l}\text { Big Springs }:=\text { ik } \\
\text { near Arendahl, } \\
\text { Minn. }\end{array}$ & $\begin{array}{l}\mathrm{NE}^{\frac{1}{4} \mathrm{SE}} \frac{1}{4} \text { sec. } 7, \mathrm{~T} .104 \mathrm{~N} ., \mathrm{R} .9 \mathrm{~W} ., \text { at cul- } \\
\text { vert on State Highway } 250,2.0 \text { miles } \\
\text { west of Arendahl. }\end{array}$ & .14 & $1959-68$ & $6-18-68$ & 9.63 & 28 \\
\hline $5-3844$ & $\begin{array}{l}\text { Pine Creek nea. } \\
\text { Arendahl, Minn. }\end{array}$ & $\begin{array}{l}\mathrm{SE}^{\frac{1}{4} \mathrm{NE}} \frac{1}{4} \text { sec. } 3 \text {, T.104 N., R.9 W., at } \\
\text { bridge on County Highway } 25,1.3 \text { miles } \\
\text { northeast of Arendahl, and } 4.9 \text { miles } \\
\text { above Hemingway Creek. }\end{array}$ & 28.1 & $1959-68$ & $6-18-68$ & 11.96 & 458 \\
\hline \multicolumn{8}{|c|}{ Iowa River basin } \\
\hline $5-4570.8$ & $\begin{array}{l}\text { Rose Creek tribu- } \\
\text { tary near Dexter, } \\
\text { Minn. }\end{array}$ & $\begin{array}{l}\mathrm{SE}_{\frac{1}{4}}^{\frac{1}{4}} \mathrm{SW}^{\frac{1}{4}} \text { sec. } 22 \text {, T. } 103 \mathrm{~N} ., \mathrm{R} .16 \mathrm{~W} . \text {, at } \\
\text { culvert on county highway, } 1 \frac{3}{4} \mathrm{miles} \\
\text { above mouth, and } 2 \frac{1}{4} \text { miles southwest }\end{array}$ & 1.20 & $1962-68$ & $6-26-68$ & 7.06 & 19 \\
\hline $5-4589.5$ & $\begin{array}{l}\text { Shell Rock River } \\
\text { tributary near } \\
\text { Albert Lea, Minn. }\end{array}$ & $\begin{array}{l}\text { SW } \frac{1}{4} S E \frac{1}{4} \text { sec. } 25 \text {, T. } 103 \text { N., R. } 22 \text { W., at } \\
\text { culvert on State Highway } 13 \text {, 0.4 mile } \\
\text { above mouth, and } 2.4 \text { miles northwest } \\
\text { of Albert Lea city limits. }\end{array}$ & - & $1960-68$ & $\begin{array}{r}6-23-60 \\
3-25-61 \\
5-11-62 \\
7-18-63 \\
4-6-65 \\
3-31-66 \\
6-8-67 \\
8-6-68\end{array}$ & $\begin{array}{r}17.77 \\
\mathrm{~b} 18.51 \\
18.58 \\
17.80 \\
\mathrm{~b} 20.25 \\
17.90 \\
18.97 \\
23.17\end{array}$ & $\begin{array}{r}20 \\
33 \\
40 \\
21 \\
58 \\
23 \\
50 \\
158\end{array}$ \\
\hline \multicolumn{8}{|c|}{ Des Moines River basin } \\
\hline $5-4747 \cdot 5$ & $\begin{array}{l}\text { Beaver Creek trib- } \\
\text { utary near slay- } \\
\text { ton, Minn. }\end{array}$ & $\begin{array}{l}\text { NW } \frac{1}{4} N W^{\frac{1}{4}} \text { sec. } 17 \text {, T. } 106 \text { N., R. } 41 \text { W. } \text { at } \\
\text { culvert on State Highway } 30,2 \frac{1}{4} \text { miles } \\
\text { west of slayton, and } 2 \frac{3}{4} \text { miles above } \\
\text { mouth. }\end{array}$ & 2.67 & $1961-68$ & $9-21-68$ & - & $\mathrm{f} 40$ \\
\hline $5-4747.6$ & $\begin{array}{l}\text { Beaver Creek trib- } \\
\text { utary above } \\
\text { slayton, Minn. }\end{array}$ & 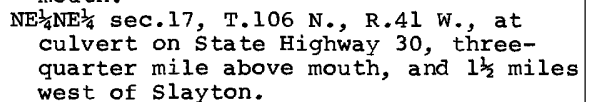 & .97 & $1961-68$ & $7-30-68$ & 16.87 & $(t)$ \\
\hline $5-4754$ & $\begin{array}{l}\text { Warren Lake trib- } \\
\text { utary near Win- } \\
\text { dom, Minn. }\end{array}$ & 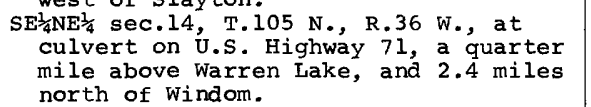 & 1.38 & $1960-68$ & $7-26-68$ & 6.93 & 104 \\
\hline $5-4758$ & $\begin{array}{l}\text { West Fork Des } \\
\text { Moines River } \\
\text { tributary near } \\
\text { Jackson, Minn. }\end{array}$ & $\begin{array}{l}\text { NW } \frac{1}{4} \mathrm{SE}^{\frac{1}{4}} \text { sec. } 27 \text {, T. I03 N., R. } 35 \text { W., at } \\
\text { culvert on county highway, three- } \\
\text { quarter mile above mouth, and } 5 \frac{1}{2} \text { miles } \\
\text { north of Jackson. }\end{array}$ & 1.42 & $1960-68$ & 1968 & (c) & $<3$ \\
\hline $5-4759$ & $\begin{array}{l}\text { West Fork Des } \\
\text { Moines River } \\
\text { tributary near } \\
\text { Lakefield, Minn. }\end{array}$ & 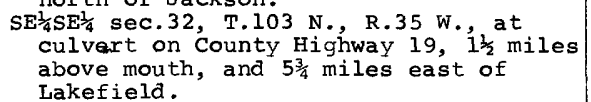 & 4.52 & $1960-68$ & 1968 & (c) & $<16$ \\
\hline $5-4760.1$ & $\begin{array}{l}\text { Nelson Creek at } \\
\text { Jackson, Minn. }\end{array}$ & $\begin{array}{l}\text { NW/4NW/4 sec. } 25 \text {, T. } 102 \text { N., R. } 35 \text { W., in } \\
\text { flume spillway at intersection of } \\
\text { U.S. Highways } 16 \text { and } 71 \text {, at south edge } \\
\text { of Jackson. }\end{array}$ & 6.8 & $\begin{array}{r}1959 \\
1964-68\end{array}$ & 1968 & (c) & $<120$ \\
\hline
\end{tabular}

t Discharge not determined.

$<$ Less than.

b Backwater from ice.

c Peak stage did not reach bottom of gage.

f Estimated; gage height unknown. 
Annual maximum discharge at crest-stage partial-record stations--continued

\begin{tabular}{|c|c|c|c|c|c|c|c|}
\hline \multirow[b]{2}{*}{ Station No. } & \multirow[b]{2}{*}{ Station name } & \multirow[b]{2}{*}{ Location } & \multirow[b]{2}{*}{$\begin{array}{l}\text { Drainage } \\
\text { area } \\
(\mathrm{sq} \mathrm{mi})\end{array}$} & \multirow[b]{2}{*}{$\begin{array}{l}\text { Period } \\
\text { of } \\
\text { record }\end{array}$} & \multicolumn{3}{|c|}{ Annual maximum } \\
\hline & & & & & Date & $\begin{array}{l}\text { Gage } \\
\text { height } \\
\text { (feet) }\end{array}$ & $\begin{array}{l}\text { Dis- } \\
\text { charge } \\
\text { (cfs) }\end{array}$ \\
\hline \multicolumn{8}{|c|}{ Des Moines River basin--continued } \\
\hline $5-4761$ & $\begin{array}{l}\text { Story Brook near } \\
\text { Petersburg, Minn. } \\
\text { East Fork Des } \\
\text { Moines River } \\
\text { tributary near } \\
\text { Dunnell, Minn. }\end{array}$ & 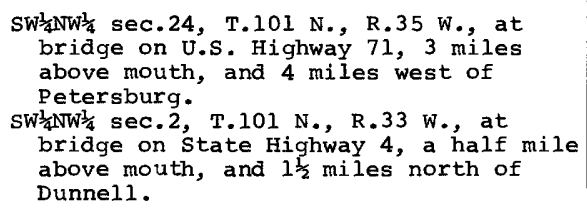 & 25.2 & $1960-68$ & $6-10-68$ & 14.55 & $<27$ \\
\hline \multicolumn{8}{|c|}{ Big Sioux River basin } \\
\hline $6-4829.5$ & $\begin{array}{l}\text { Mound Creek near } \\
\text { Hardwick, Minn. }\end{array}$ & 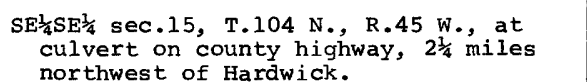 & 2.77 & $1959-68$ & 1968 & (c) & $<5$ \\
\hline $6-4829.6$ & $\begin{array}{l}\text { Mound Creek tribu- } \\
\text { tary at Hardwick, } \\
\text { Minn. }\end{array}$ & $\begin{array}{l}\mathrm{SE}^{\frac{1}{4}} \text { sec. } 34, \mathrm{~T} .104 \mathrm{~N} \text {., R. } 45 \text { W. , at cul- } \\
\text { vert on U.S. Highway } 75 \text {, a half mile } \\
\text { above mouth, and } 1 \text { mile southwest of } \\
\text { Hardwick. }\end{array}$ & .23 & $1959-68$ & $4-3-68$ & 6.22 & $(t)$ \\
\hline $6-4830.5$ & $\begin{array}{l}\text { Rock River tribu- } \\
\text { tary near } \\
\text { Luverne, Minn. }\end{array}$ & $\begin{array}{l}\text { NE } \frac{1}{4} \text { sec.10, T. } 101 \text { N., R.45 W., at cul- } \\
\text { vert on U.S. Highway } 75,5.8 \text { miles } \\
\text { south of Luverne. }\end{array}$ & .20 & $1959-68$ & 1968 & (c) & $<22$ \\
\hline $6-4832.1$ & $\begin{array}{l}\text { North Branch } \\
\text { Kanaranzi Creek } \\
\text { tributary near } \\
\text { Lismore, Minn. } \\
\text { North Branch } \\
\text { Kanaranzi Creek } \\
\text { tributary near } \\
\text { Wilmont, Minn. }\end{array}$ & 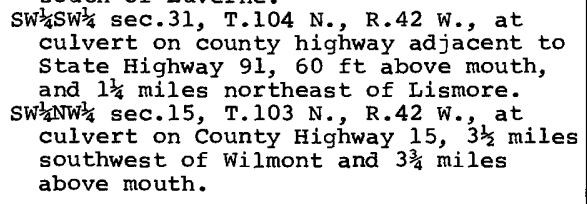 & .18 & $1959-68$ & $7-30-68$ & 17.46 & $(f)$ \\
\hline \multicolumn{8}{|c|}{ Little Sioux River basin } \\
\hline $6-6035.2$ & $\begin{array}{l}\text { Little Sioux River } \\
\text { tributary near } \\
\text { spafford, Minn. }\end{array}$ & $\begin{array}{l}N^{\frac{1}{4}} \mathrm{NE}^{\frac{1}{4}} \text { sec. } 27, \mathrm{~T} .102 \mathrm{~N} ., \mathrm{R} .38 \mathrm{~W} ., \text { at } \\
\text { culvert on U.S. Highway } 16,0.4 \mathrm{mile} \\
\text { west of Spafford, and a half mile } \\
\text { above mouth. }\end{array}$ & 4.06 & $1959-68$ & $6-24-68$ & 6.43 & 16 \\
\hline $6-6035.3$ & $\begin{array}{l}\text { Little Sioux River } \\
\text { near Spafford, } \\
\text { Minn. }\end{array}$ & $\begin{array}{l}\text { NE } \frac{1}{4} \mathrm{NE} \frac{1}{4} \text { sec. } 34 \text {, T.102 N., R. } 37 \text { W., at } \\
\text { bridge on county highway, } 1.6 \text { miles } \\
\text { below Jackson County ditch No. } 11 \text {, } \\
\text { and } 5.8 \text { miles east of Spafford. }\end{array}$ & - & $1962-68$ & $\begin{array}{r}4-6-62 \\
7-18-63 \\
5-23-64 \\
4-6-65 \\
3-14-66 \\
6-15-67 \\
4-23-68\end{array}$ & $\begin{array}{r}\mathrm{b} 9.23 \\
7.66 \\
6.80 \\
\mathrm{~b} 11.08 \\
\mathrm{~b} 8.33 \\
8.52 \\
5.92\end{array}$ & $\begin{array}{r}595 \\
118 \\
65 \\
2,700 \\
136 \\
310 \\
32\end{array}$ \\
\hline
\end{tabular}

f Discharge not determined.

$<$ Less than.

b Backwater from ice.

c Peak stage did not reach bottom of gage. 
Measurements of streamflow at points other than gaging stations or partial-record stations are given in the following table. Those that are measurements of base flow are designated by an asterisk (*).

Discharge measurements made at miscellaneous sites during water year. 1968

\begin{tabular}{|c|c|c|c|c|c|c|}
\hline \multirow[b]{2}{*}{ Stream } & \multirow[b]{2}{*}{ Tributary to } & \multirow[b]{2}{*}{ Location } & \multirow{2}{*}{$\begin{array}{l}\text { Drainage } \\
\text { area } \\
(\mathrm{sq} \mathrm{mi})\end{array}$} & \multirow{2}{*}{$\begin{array}{c}\text { Measured } \\
\text { previously } \\
\text { (water } \\
\text { years) }\end{array}$} & \multicolumn{2}{|c|}{ Measurements } \\
\hline & & & & & Date & $\begin{array}{c}\text { Discharge } \\
\text { (cfs) }\end{array}$ \\
\hline \multicolumn{7}{|c|}{ Streams tributary to Lake superior } \\
\hline Beaver River & Lake superior & $\begin{array}{l}\text { Sw } \frac{1}{4} \text { sec. } 12 \text {, T. } 55 \text { N., R. } 7 \text { W., Lake County, } \\
\text { at bridge on State Highway } 61 \text { in } \\
\text { Beaver Bay, Minn. }\end{array}$ & 126 & $\begin{array}{l}1911-14 \\
1928-31 \neq\end{array}$ & $\begin{array}{r}4-10-68 \\
4-25-68 \\
5-8-68 \\
6-7-68 \\
7-18-68 \\
8-14-68\end{array}$ & $\begin{array}{l}493 \\
825 \\
252 \\
327 \\
221 \\
* 7.21\end{array}$ \\
\hline Midway River & $\begin{array}{l}\text { st. Louis } \\
\text { River }\end{array}$ & 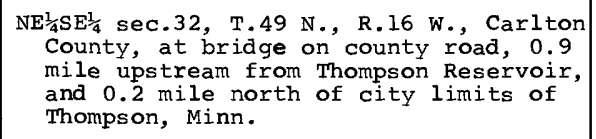 & - & - & $9-3-68$ & 15.1 \\
\hline \multicolumn{7}{|c|}{ Red River of the North basin } \\
\hline $\begin{array}{l}\text { Otter Tail } \\
\text { River }\end{array}$ & $\begin{array}{l}\text { Red River of } \\
\text { the North }\end{array}$ & $\begin{array}{l}\text { NW/ sec. } 19 \text {, T. } 137 \text { N., R. } 39 \text { W., Otter } \\
\text { Tail County, at bridge on county road, } \\
1 \text { mile north of Luce, Minn. }\end{array}$ & - & - & $\begin{array}{r}10-25-67 \\
6-6-68 \\
8-22-68\end{array}$ & $\begin{array}{l}28.2 \\
171 \\
60.3\end{array}$ \\
\hline $\begin{array}{l}\text { Otter Tail } \\
\text { River }\end{array}$ & $\begin{array}{l}\text { Red River of } \\
\text { the North }\end{array}$ & $\begin{array}{l}S^{\frac{1}{2}} \text { sec. } 20, \text { T. } 137 \text { N., R.39 W., Otter Tail } \\
\text { County, at bridge on County Highway } 60 \\
1 \text { mile east of Luce, Minn. }\end{array}$ & - & - & $\begin{array}{r}6-5-68 \\
8-21-68\end{array}$ & $\begin{array}{r}186 \\
55.6\end{array}$ \\
\hline $\begin{array}{l}\text { Otter Tail } \\
\text { River }\end{array}$ & $\begin{array}{l}\text { Red River of } \\
\text { the North }\end{array}$ & $\begin{array}{l}\text { NW } \frac{1}{4} N W^{-1} \frac{1}{4} \text { sec. } 34, \text { T. } 137 \text { N., R. } 39 \text { W., Otter } \\
\text { Tail County, at bridge on county road, } \\
2.2 \text { miles southeast of Luce, Minn. }\end{array}$ & - & - & $\begin{array}{r}10-25-67 \\
6-5-68 \\
8-21-68\end{array}$ & $\begin{array}{l}37.6 \\
220 \\
40.0\end{array}$ \\
\hline Toad River & $\begin{array}{l}\text { Otter Tail } \\
\text { River }\end{array}$ & 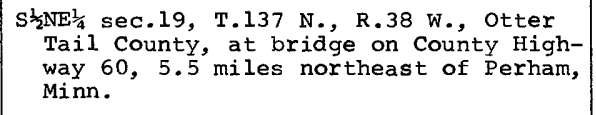 & - & - & $\begin{array}{r}10-25-67 \\
6-4-68 \\
8-21-68\end{array}$ & $\begin{array}{l}7.70 \\
25.4 \\
12.6\end{array}$ \\
\hline Toad River & $\begin{array}{l}\text { otter Tail } \\
\text { River }\end{array}$ & $\begin{array}{l}\text { On line between secs. } 29 \text { and } 32, \mathrm{~T} .137 \mathrm{~N} ., \\
\mathrm{R} .38 \mathrm{~W} . \text {, Otter Tail County, at bridge } \\
\text { on Sounty Road } 8,4.2 \text { miles northeast } \\
\text { of Perham, Minn. }\end{array}$ & - & - & $\begin{array}{r}10-25-67 \\
6-4-68 \\
8-21-68\end{array}$ & $\begin{array}{l}8.99 \\
31.4 \\
9.74\end{array}$ \\
\hline $\begin{array}{l}\text { Otter Tail } \\
\text { River }\end{array}$ & $\begin{array}{c}\text { Red River of } \\
\text { the North }\end{array}$ & 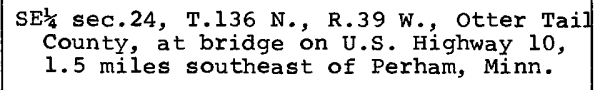 & - & - & $\begin{array}{r}10-24-67 \\
6-5-68 \\
8-14-68\end{array}$ & $\begin{array}{l}42.1 \\
269 \\
87.2\end{array}$ \\
\hline $\begin{array}{l}\text { Otter Tail } \\
\text { River }\end{array}$ & $\begin{array}{c}\text { Red River of } \\
\text { the North }\end{array}$ & $\begin{array}{l}\text { On line between secs. } 7 \text { and } 18, \mathrm{~T} .135 \mathrm{~N} . \text {, } \\
\mathrm{R} .38 \mathrm{w} ., \text { otter Tail County, at bridge } \\
\text { on County Highway } 14,5 \text { miles east of } \\
\text { Richville, Minn. }\end{array}$ & - & - & $\begin{array}{r}10-24-67 \\
6-5-68 \\
8-14-68\end{array}$ & $\begin{array}{l}52.3 \\
299 \\
97.8\end{array}$ \\
\hline Willow Creek & $\begin{array}{l}\text { Otter Tail } \\
\text { River }\end{array}$ & $\begin{array}{l}\text { On line between secs. } 15 \text { and } 16, \text { T.135 N., } \\
\text { R. } 38 \text { W., Otter Tail County, } 5 \text { miles } \\
\text { west of New York Mills, Minn. }\end{array}$ & - & - & $\begin{array}{r}6-7-68 \\
8-14-68\end{array}$ & $\begin{array}{l}3.14 \\
\star .30\end{array}$ \\
\hline Dead River & $\begin{array}{l}\text { otter Tail } \\
\text { River }\end{array}$ & $\begin{array}{l}\text { On line between secs. } 2 \text { and } 3, \mathrm{~T} .134 \mathrm{~N} . \text {, } \\
\mathrm{R} .40 \mathrm{~W} . \text {, Otter Tail County, } 1 \text { mile } \\
\text { southwest of Basswood, Minn. }\end{array}$ & - & - & $\begin{array}{r}6-6-68 \\
8-22-68\end{array}$ & $\begin{array}{l}30.6 \\
33.4\end{array}$ \\
\hline $\begin{array}{l}\text { Otter Tail } \\
\text { River }\end{array}$ & $\begin{array}{l}\text { Red River of } \\
\text { the North }\end{array}$ & 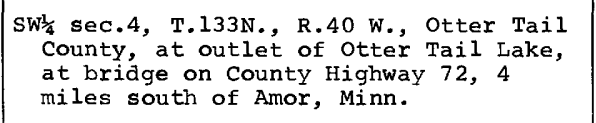 & - & - & $\begin{array}{r}6-6-68 \\
8-22-68\end{array}$ & $\begin{array}{l}370 \\
218\end{array}$ \\
\hline $\begin{array}{l}\text { Otter Tail } \\
\text { River }\end{array}$ & $\begin{array}{l}\text { Red River of } \\
\text { the North }\end{array}$ & $\begin{array}{l}E_{\frac{1}{2} S^{\frac{1}{4}}} \text { sec. } 29, \text { T. } 134 \mathrm{~N} ., \mathrm{R} .41 \mathrm{~W} . \text {, Otter } \\
\text { Tail County, at bridge on County High- } \\
\text { way } 35,200 \mathrm{ft} \text { upstream from West Lost } \\
\text { Lake, and } 2 \text { miles west of Phelps, Minn. }\end{array}$ & - & - & $\begin{array}{r}6-6-68 \\
8-22-68\end{array}$ & $\begin{array}{l}398 \\
182\end{array}$ \\
\hline $\begin{array}{c}\text { Pelican River } \\
\text { tributary }\end{array}$ & Pelican River & $\begin{array}{l}\text { Lat } 46^{\circ} 46^{\prime} 52^{\prime \prime}, \text { long } 95^{\circ} 48^{\prime} 01^{\prime \prime} \text {, in SE } \frac{1}{4} \\
\text { sec.12, T. } 138 \text { N., R. } 41 \text { W. Becker } \\
\text { County, at culvert on county road, } 100 \\
\text { ft upstream from Detroit Lake, } 0.8 \text { mile } \\
\text { north of Sucker Creek, and } 3.2 \text { miles } \\
\text { southeast of Detroit Lakes, Minn. }\end{array}$ & - & - & $\begin{array}{r}7-26-68 \\
8-14-68 \\
9-5-68 \\
9-30-68\end{array}$ & $\begin{array}{l}.05 \\
0.01 \\
.25\end{array}$ \\
\hline
\end{tabular}




\begin{tabular}{|c|c|c|c|c|c|c|}
\hline \multirow{2}{*}{ Stream } & \multirow[b]{2}{*}{ Tributary to } & \multirow[b]{2}{*}{ Location } & \multirow{2}{*}{$\begin{array}{l}\text { Drainage } \\
\text { area } \\
\text { (sq mi) }\end{array}$} & \multirow{2}{*}{$\mid \begin{array}{c}\text { Mea sured } \\
\text { previous ly } \\
\text { (water } \\
\text { years) }\end{array}$} & \multicolumn{2}{|c|}{ Measurements } \\
\hline & & & & & Date & $\begin{array}{c}\text { Discharge } \\
\text { (cfs) }\end{array}$ \\
\hline \multicolumn{7}{|c|}{ Red River of the North basin--continued } \\
\hline Sucker Creek & Pelican River & $\begin{array}{l}\text { Lat } 46^{\circ} 46^{\prime} 25^{\prime \prime} \text {, long } 95^{\circ} 48^{\prime} 23^{\prime \prime} \text {, in NW/ } \\
\text { sec.13, T.138 N., R. } 41 \text { W. , Becker } \\
\text { County, at culvert on county road, } \\
200 \mathrm{ft} \text { upstream from Detroit Lake and } \\
3.5 \mathrm{miles} \text { southeast of Detroit Lakes, } \\
\text { Minn. }\end{array}$ & - & - & $\begin{array}{r}7-26-68 \\
8-14-68 \\
9-5-68 \\
9-30-68\end{array}$ & $\begin{array}{r}2.63 \\
1.14 \\
.80 \\
1.20\end{array}$ \\
\hline $\begin{array}{c}\text { Pelican River } \\
\text { tributary }\end{array}$ & Pelican River & $\begin{array}{l}\text { Lat } 46^{\circ} 47^{\prime} 02^{\prime \prime} \text {, long } 95^{\circ} 53^{\prime} 07^{\prime \prime} \text {, in NE } \frac{1}{2} \\
\text { sec.8, T.138 N., R. } 41 \text { W. Becker } \\
\text { County, at State Fish Hatchery, on } \\
\text { left downstream abutment of bridge } \\
\text { over outlet from rearing ponds, 1, 000 } \\
\text { ft north of Muskrat Lake outlet and } \\
3 \text { miles southwest of Detroit Lakes, } \\
\text { Minn. }\end{array}$ & - & - & $\begin{array}{r}6-12-68 \\
7-26-68 \\
8-15-68 \\
9-6-68\end{array}$ & $\begin{array}{l}.60 \\
.41 \\
.24 \\
.25\end{array}$ \\
\hline $\begin{array}{l}\text { Monson Lake } \\
\text { Outlet }\end{array}$ & Pelican River & $\begin{array}{l}\text { Lat } 46^{\circ} 47^{\prime} 15^{\prime \prime}, \text { long } 95^{\circ} 53^{\prime} 30^{\prime \prime} \text {, in NW/ } \\
\text { sec.8, T.138 N., R. } 41 \text { W., Becker } \\
\text { County, } 50 \text { ft upstream from culvert, } \\
100 \mathrm{ft} \text { downstream from Monson Lake, } \\
800 \text { ft upstream from Lake Sallie, and } \\
3 \text { miles southwest of Detroit Lakes, } \\
\text { Minn. }\end{array}$ & - & - & $\begin{array}{r}6-13-68 \\
7-26-68 \\
8-15-68 \\
9-6-68\end{array}$ & $\begin{array}{l}.35 \\
.06 \\
0 \\
0\end{array}$ \\
\hline $\begin{array}{l}\text { Fox Lake } \\
\text { Outlet }\end{array}$ & Pelican River & 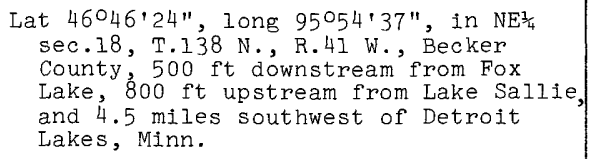 & - & - & $\begin{array}{r}6-14-68 \\
7-26-68 \\
8-15-68 \\
9-6-68\end{array}$ & $\begin{array}{r}1.27 \\
.36 \\
.10 \\
0\end{array}$ \\
\hline Pelican River & $\begin{array}{l}\text { Otter Tail } \\
\text { River }\end{array}$ & 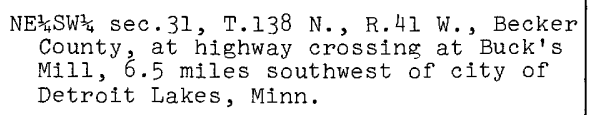 & 123 & $1942-50 \neq$ & $\begin{array}{r}8-8-68 \\
8-16-68 \\
9-5-68\end{array}$ & $\begin{array}{l}25 \cdot 5 \\
15 \cdot 0 \\
6 \cdot 24\end{array}$ \\
\hline Pelican River & $\begin{array}{l}\text { Otter Tail } \\
\text { River }\end{array}$ & 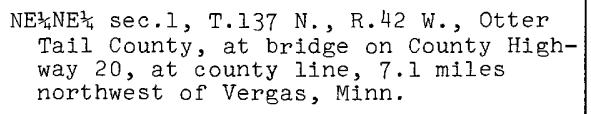 & - & - & $\begin{array}{r}7-25-68 \\
8-8-68\end{array}$ & $\begin{array}{l}47.3 \\
26.1\end{array}$ \\
\hline Wild Rice River & $\begin{array}{l}\text { Red River of } \\
\text { the North }\end{array}$ & $\begin{array}{l}\text { On line between sec.13, T.144 N., R. } 46 \\
\text { W., and sec.18, T.144 N., R. } 45 \text { W., } \\
\text { Norman County, at bridge on County } \\
\text { Highway } 24,0.3 \text { mile south of State } \\
\text { Highway } 31 \text {, and } 3.2 \text { miles southeast of } \\
\text { Ada, Minn. }\end{array}$ & - & $\left|\begin{array}{l}1945-51 \\
1965-67\end{array}\right|$ & $4-3-68$ & 139 \\
\hline $\begin{array}{l}\text { South Branch } \\
\text { Wild Rice } \\
\text { River }\end{array}$ & $\begin{array}{l}\text { Wild Rice } \\
\text { River }\end{array}$ & $\begin{array}{l}\text { On line between secs. } 8 \text { and } 9, \mathrm{~T} .142 \mathrm{~N} . \\
\text { R. } 45 \mathrm{~W} \text {. Clay County, at bridge on } \\
\text { County Road } 63,5.5 \text { miles northeast of } \\
\text { Felton, Minn. }\end{array}$ & - & $1959-67$ & $\begin{array}{r}4-3-68 \\
4-25-68\end{array}$ & $\begin{array}{l}16.1 \\
27.4\end{array}$ \\
\hline $\begin{array}{l}\text { State ditch } \\
\text { No. } 45\end{array}$ & $\begin{array}{l}\text { Wild Rice } \\
\text { River }\end{array}$ & $\begin{array}{l}\text { On line between secs. } 15 \text { and } 16 \text {, T.141 N., } \\
\text { R. } 46 \text { W. Clay County, at culvert on } \\
\text { State Highway } 9,3 \text { miles south of } \\
\text { Felton, Minn. }\end{array}$ & - & $1959-67$ & $\begin{array}{r}4-3-68 \\
4-25-68\end{array}$ & $\begin{array}{l}3.23 \\
6.53\end{array}$ \\
\hline Moose River & Thief River & $\begin{array}{l}\mathrm{NE}^{\frac{1}{4} \mathrm{SE}} \frac{1}{4} \mathrm{sec} .36, \mathrm{~T} .158 \mathrm{~N} ., \mathrm{R} .40 \mathrm{~W} ., \text { Mar- } \\
\text { shall County, at bridge on State High- } \\
\text { way } 89 \text {, about } 3.5 \text { miles northeast of } \\
\text { Gatzke, Minn. }\end{array}$ & - & $1964-66$ & $\begin{array}{l}6-12-68 \\
7-17-68\end{array}$ & $\begin{array}{l}505 \\
974\end{array}$ \\
\hline Mud River & Thief River & $\begin{array}{l}\text { At intersection of secs.13, } 14,23 \text {, and } \\
24, \mathrm{~T} .156 \mathrm{~N} \text {. R. R } 40 \mathrm{~W} . \text { Marshall County, } \\
\text { at bridge on State Highway } 89,6 \text { miles } \\
\text { west of Grygla, Minn. }\end{array}$ & - & 1966 & $7-17-68$ & 967 \\
\hline Two Rivers & $\begin{array}{c}\text { Red River of } \\
\text { the North }\end{array}$ & 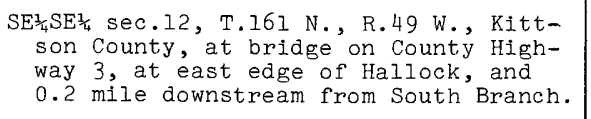 & 625 & $\mid \begin{array}{r}1911-14 \neq \\
1929-30 \neq \\
1941-43 \neq \\
1967\end{array}$ & $6-12-68$ & 1,370 \\
\hline Roseau River & $\begin{array}{c}\text { Red River of } \\
\text { the North }\end{array}$ & $\begin{array}{l}\text { SW } \frac{1}{4} \text { sec. } 13 \text {, T.162 N., R. } 40 \text { W., Roseau } \\
\text { County, at bridge on State Highway } 11 \text {, } \\
\text { at Roseau, Minn. }\end{array}$ & - & $\mid \begin{array}{c}1911-14 \neq \\
1943 \\
1967\end{array}$ & $\begin{array}{l}4-12-68 \\
6-11-68 \\
6-13-68\end{array}$ & $\begin{array}{r}150 \\
4,320 \\
2,930\end{array}$ \\
\hline
\end{tabular}

F Operated as a continuous-record gaging station. 
Discharge measurements made at miscellaneous sites during water year 1968

\begin{tabular}{|c|c|c|c|c|c|c|}
\hline \multirow[b]{2}{*}{ Stream } & \multirow[b]{2}{*}{ Tributery to } & \multirow[b]{2}{*}{ Locetion } & \multirow{2}{*}{$\begin{array}{l}\text { Drainage } \\
\text { erea } \\
(\mathrm{sq} \mathrm{mf})\end{array}$} & \multirow{2}{*}{$\begin{array}{c}\text { Measured } \\
\text { previous ly } \\
\text { (water } \\
\text { years) }\end{array}$} & \multicolumn{2}{|c|}{ Measurements } \\
\hline & & & & & Date & $\begin{array}{l}\text { Discharge } \\
\text { (cfs) }\end{array}$ \\
\hline \multicolumn{7}{|c|}{ Lake of the Woods basin } \\
\hline Isabella River & $\begin{array}{c}\text { South Kawish- } \\
\text { Iwi River }\end{array}$ & $\begin{array}{l}\mathrm{NW}_{\frac{1}{4} \mathrm{NE}}^{\frac{1}{4}} \text { sec.6, T. } 6 \text { i N., R.9 W. , Lake } \\
\text { County, } 200 \text { ft upstream from Bald } \\
\text { Eagle Lake, } 0.5 \text { mile upstream from } \\
\text { Snake River, and } 14.5 \text { miles northwest } \\
\text { of Isabella, Minn. }\end{array}$ & 341 & $\mid \begin{array}{c}1953-61 \neq \\
1967\end{array}$ & $\begin{array}{r}2-2-68 \\
5-28-68 \\
9-24-68\end{array}$ & $\begin{array}{l}* 61.9 \\
892 \\
* 381\end{array}$ \\
\hline $\begin{array}{l}\text { South Kawishiwi } \\
\text { River }\end{array}$ & $\begin{array}{l}\text { Kawishiwi } \\
\text { RIver }\end{array}$ & 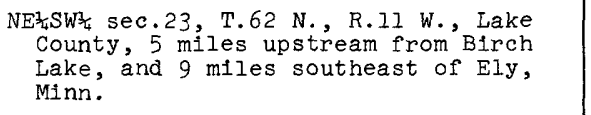 & - & $\left|\begin{array}{c}1952-61 \neq \\
1967\end{array}\right|$ & $3-26-68$ & 117 \\
\hline Stony River & $\begin{array}{c}\text { South Kawish- } \\
\text { Iw1 River }\end{array}$ & $\begin{array}{l}\mathrm{NW}_{\frac{1}{4} \mathrm{NW}} \mathrm{N}_{4} \text { sec. } 17 \text {, T. } 60 \mathrm{~N} \text {. , R. } 10 \text { W. , Lake } \\
\text { County, } 275 \text { ft downstream from Slate } \\
\text { Lake and bridge on State Highway I, II } \\
\text { miles upstream from Birch Lake, and } \\
12.8 \text { miles northwest of Isabelia, Minn. }\end{array}$ & 180 & $\left|\begin{array}{c}1953-65 \neq \\
1967\end{array}\right|$ & $\begin{array}{r}2-1-68 \\
5-28-68 \\
9-24-68\end{array}$ & $\begin{array}{l}* 15.8 \\
352 \\
* 226\end{array}$ \\
\hline $\begin{array}{l}\text { Little Fork } \\
\text { River }\end{array}$ & Rainy River & 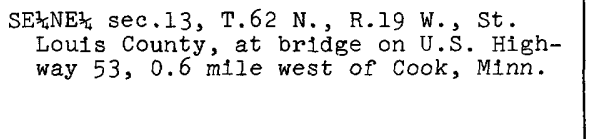 & - & $\left|\begin{array}{r}1950, \\
1958-67\end{array}\right|$ & $\begin{array}{r}4-9-68 \\
5-16-68 \\
6-11-68 \\
8-15-68 \\
9-19-68\end{array}$ & $\begin{array}{l}233 \\
108 \\
417 \\
* .47 \\
125\end{array}$ \\
\hline Big Fork River & Rainy River & $\begin{array}{l}\text { Sec.12, T.149 N., R. } 47 \text { W., Itasca County, } \\
\text { at bridge on County Highway } 29 \text {, at } \\
\text { Dora Lake, Minn. }\end{array}$ & - & $1965-67$ & $\begin{array}{r}11-2-67 \\
2-27-68 \\
4-30-68 \\
6-4-68 \\
8-2-68 \\
9-16-68\end{array}$ & $\begin{array}{l}61.5 \\
* 10.1 \\
303 \\
131 \\
* 49.2 \\
145\end{array}$ \\
\hline $\begin{array}{l}\text { East Branch } \\
\text { Warroad River }\end{array}$ & Warroad River & $\begin{array}{l}\mathrm{NE}_{\frac{1}{4} \mathrm{SW}} \mathrm{s}_{4} \mathrm{sec} .5, \mathrm{~T} .162 \mathrm{~N} . \text {, R. } 36 \mathrm{~W} . \text {, Roseau } \\
\text { County, at bridge on County Highway } 5 \text {, } \\
\text { 1.5 miles south of Warroad, Minn. }\end{array}$ & - & - & $6-13-68$ & 368 \\
\hline Warroad River & $\begin{array}{l}\text { Lake of the } \\
\text { Woods }\end{array}$ & $\begin{array}{l}\text { SW } \frac{1}{4} \mathrm{SE}_{\frac{1}{4}} \text { sec. } 29 \text {, T.I62 N., R. } 36 \text { W., Roseau } \\
\text { County, at bridge on State Highway II } \\
\text { in Warroad, Minn. }\end{array}$ & - & - & $6-13-68$ & 1,740 \\
\hline \multicolumn{7}{|c|}{ Mississippi River main stem } \\
\hline $\begin{array}{l}\text { Mississippi } \\
\text { River }\end{array}$ & Gulf of Mexico & $\begin{array}{l}\mathrm{N}^{\frac{1}{2}} \text { sec. I3, T. I55 N., R. } 26 \mathrm{~W} \text {. , Itasca } \\
\text { County, at dam at outlet of Pokegama } \\
\text { Lake, } 3.5 \text { miles northwest of Grand } \\
\text { Rapids, Minn. }\end{array}$ & 3,265 & $\left|\begin{array}{l}1929-30 \\
1944-45 \\
1948-55 \\
1957-67\end{array}\right|$ & $\begin{array}{r}I-26-68 \\
8-6-68\end{array}$ & $\begin{array}{r}809 \\
1,430\end{array}$ \\
\hline \multicolumn{7}{|c|}{ Crow Wing River basin } \\
\hline Leaf River & $\begin{array}{l}\text { Crow Wing } \\
\text { River }\end{array}$ & $\begin{array}{l}\text { W/2 sec. } 22 \text {, T.I34 N., R. } 38 \text { W. Otter Tail } \\
\text { County, between West and East Leaf } \\
\text { Lakes, at culvert just upstream from } \\
\text { State Road I08, and } 4 \text { miles east of } \\
\text { Ottertail, Minn. }\end{array}$ & - & - & $\begin{array}{r}6-7-68 \\
8-14-68\end{array}$ & $\begin{array}{l}9.96 \\
2.14\end{array}$ \\
\hline Oak Creek & Leaf River & $\begin{array}{l}\mathrm{SE}_{\frac{1}{4}} \mathrm{SE}_{\frac{1}{4}} \text { sec. } 26 \text {, T. } 134 \mathrm{~N} ., \mathrm{R} .36 \mathrm{~W} . \text {, otter } \\
\text { Tall County, at bridge on county road, } \\
4 \mathrm{miles} \text { southwest of Wadena, Minn. }\end{array}$ & - & - & $10-18-67$ & $* .53$ \\
\hline Oak Creek & Leaf River & 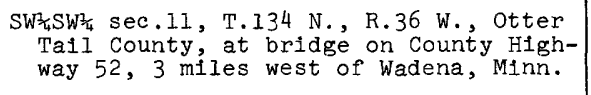 & - & - & $10-18-67$ & $* 1.04$ \\
\hline Oak Creek & Leaf River & $\begin{array}{l}\mathrm{SW}_{\frac{1}{4} \mathrm{NE} \mathrm{Z}_{4}} \text { sec. } 34, \mathrm{~T} .135 \mathrm{~N} ., \mathrm{R} .36 \mathrm{~W} . \text {, Otter } \\
\text { Tall County, at bridge on U.S. Highway } \\
10,3.5 \text { miles northwest of Wadena, } \\
\text { Minn. }\end{array}$ & - & - & $10-18-67$ & $* 1.94$ \\
\hline Leaf River & $\begin{array}{l}\text { Crow Wing } \\
\text { River }\end{array}$ & $\begin{array}{l}\mathrm{NE}_{\frac{1}{4}} \mathrm{NE}_{\frac{1}{4}} \text { sec. } 35 \text {, T. } 135 \mathrm{~N} ., \mathrm{R} .36 \mathrm{~W} . \text {, Otter } \\
\text { Tail County, at bridge on County High- } \\
\text { way } 75,2 \text { miles northwest of Wadena, } \\
\text { Minn. }\end{array}$ & - & - & $10-18-67$ & $* 29.9$ \\
\hline $\begin{array}{l}\text { Leaf River } \\
\text { tributary }\end{array}$ & Leaf River & $\begin{array}{l}N W \frac{1}{4} N_{1 / 4} \text { sec. } 34, \text { T. } 135 \mathrm{~N} ., \mathrm{R} .35 \mathrm{~W} . \text {, Wadena } \\
\text { County, at bridge on county road, } 2 \\
\text { miles northeast of Wadena, Minn. }\end{array}$ & - & - & $10-18-67$ & $* 5.27$ \\
\hline
\end{tabular}

F Operated as a continuous-record gaging station. 
Discharge measurements made at miscellaneous sites during water year 1968

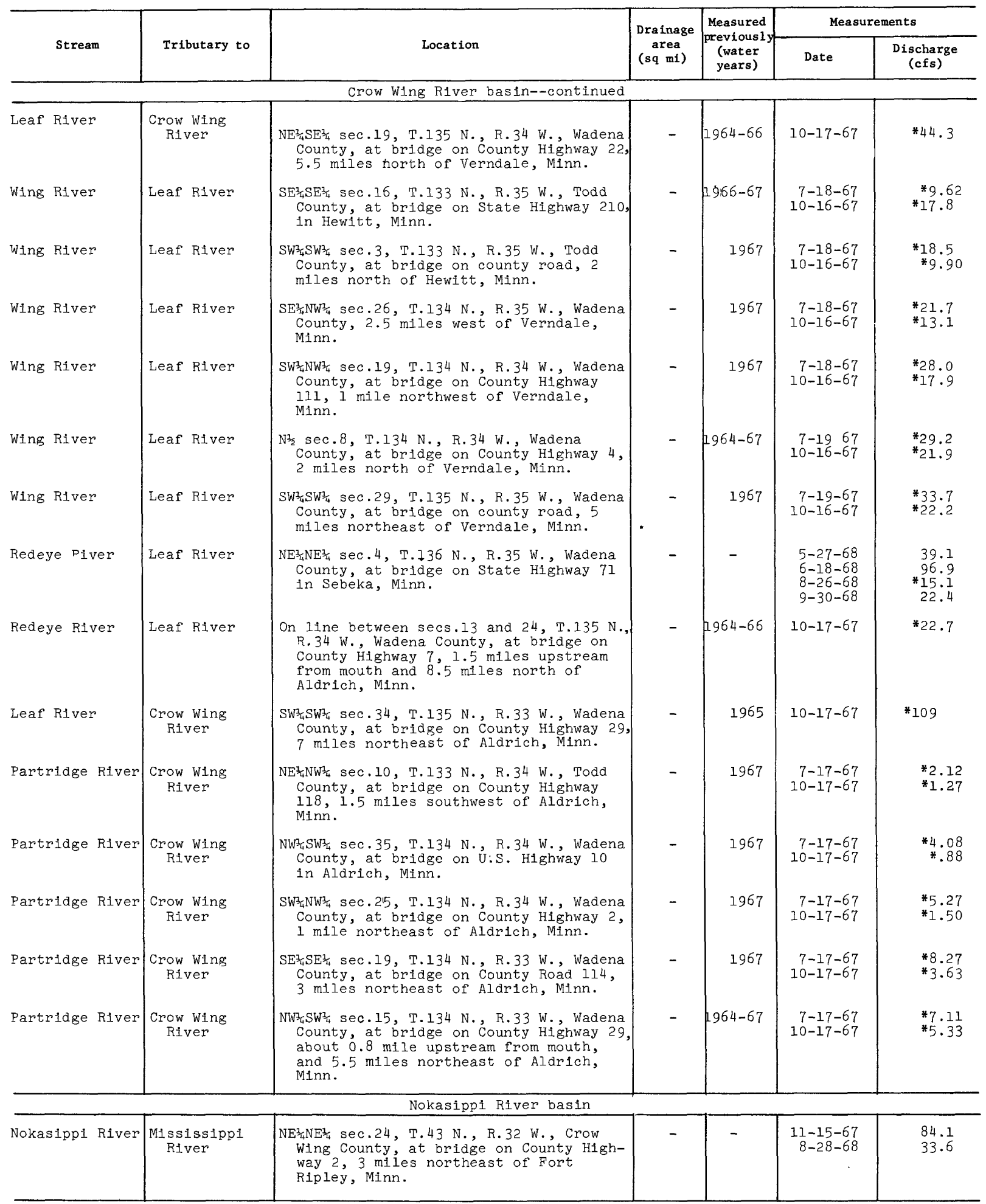

* Base flow. 
Discharge measurements made at miscellaneous sites during water year 1968

\begin{tabular}{|c|c|c|c|c|c|c|}
\hline \multirow{2}{*}{ Stream } & \multirow{2}{*}{ Tributary to } & \multirow[b]{2}{*}{ Location } & \multirow{2}{*}{$\begin{array}{l}\text { Drainage } \\
\text { area } \\
(\mathrm{sq} \text { mi) }\end{array}$} & \multirow{2}{*}{$\begin{array}{c}\text { Measured } \\
\text { previous ly } \\
\text { (water } \\
\text { years) }\end{array}$} & \multicolumn{2}{|c|}{ Measurements } \\
\hline & & & & & Date & $\begin{array}{c}\text { Discharga } \\
(\mathrm{cfs})\end{array}$ \\
\hline \multicolumn{7}{|c|}{ Elk River basin } \\
\hline Elk River & $\begin{array}{l}\text { Mississippi } \\
\text { River }\end{array}$ & $\begin{array}{l}\text { NWt } N^{\frac{1}{4}} \text { sec. } 6 \text {, T. } 129 \mathrm{~N} \text {, R. } 29 \text { W. Morri- } \\
\text { son County, at bridge on County High- } \\
\text { way } 13 \text {, } 1 \text { mile upstream from mouth, } \\
\text { and } 3 \text { miles north of Little Falls, } \\
\text { Minn. }\end{array}$ & - & - & $8-28-68$ & $* 3.50$ \\
\hline \multicolumn{7}{|c|}{ Swan River basin } \\
\hline Swan River & $\begin{array}{l}\text { Mississippi } \\
\text { River }\end{array}$ & $\begin{array}{l}\text { NW }{ }^{\frac{1}{4} N W / 4} \text { sec.l, T. } 128 \text { N., R. } 30 \text { W. Morri- } \\
\text { son County, at bridge on State Highway } \\
238 \text {, } 1.5 \text { miles upstream from mouth and } \\
3 \text { miles southwest of Little Falls, } \\
\text { Minn. }\end{array}$ & - & - & $8-28-68$ & $* 6.03$ \\
\hline \multicolumn{7}{|c|}{ Spunk Creek basin } \\
\hline Spunk Creek & $\begin{array}{l}\text { Mississippi } \\
\text { River }\end{array}$ & 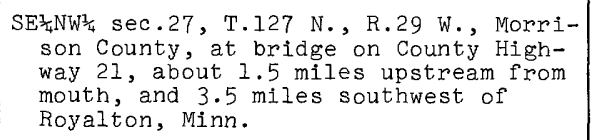 & - & $=-$ & $8-28-68$ & $* 2.83$ \\
\hline \multicolumn{7}{|c|}{ Sauk River basin } \\
\hline Ashley Creek & Sauk River & $\begin{array}{l}\text { NW/ } \text { SE }_{\frac{1}{4}} \text { sec. } 29 \text {, T. } 127 \text { N., R. } 34 \text { W. , Todd } \\
\text { County, at bridge on County Highway } \\
\text { Il, } 3 \text { miles north of Sauk Centre, Minn. }\end{array}$ & - & - & $8-28-68$ & *3.59 \\
\hline Sauk River & $\begin{array}{l}\text { Mississippi } \\
\text { River }\end{array}$ & 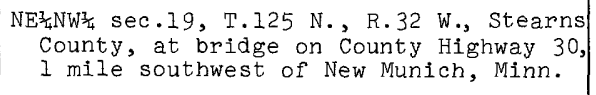 & - & - & $8-28-68$ & $* 22.6$ \\
\hline \multicolumn{7}{|c|}{ Mississippi River main stem } \\
\hline $\begin{array}{l}\text { Mississippi } \\
\text { River }\end{array}$ & Guzf of Mexico & 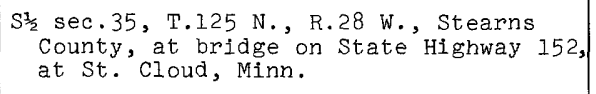 & - & 1967 & $7-26-68$ & 6,530 \\
\hline \multicolumn{7}{|c|}{ Elk River basin } \\
\hline Mayhew Creek & Elk River & 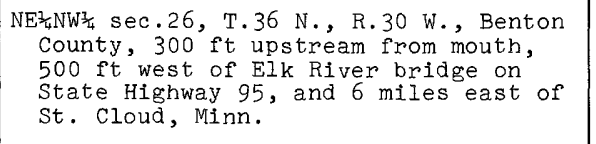 & - & - & $8-27-68$ & $* .52$ \\
\hline Elk River & $\begin{array}{l}\text { Mississippi } \\
\text { River }\end{array}$ & $\begin{array}{l}\mathrm{NE}_{\frac{1}{4}} \mathrm{NW} \frac{1}{4} \mathrm{sec} .26 \text {, T. } 36 \mathrm{~N} . \text { R. } 30 \text { W. Benton } \\
\text { County, at bridge on State Highway 95, } \\
6 \text { miles east of St. Cloud, Minn. }\end{array}$ & - & - & $8-27-68$ & $* 3.04$ \\
\hline Battle Brook & $\begin{array}{l}\text { St. Francis } \\
\text { River }\end{array}$ & $\begin{array}{l}\text { NW } \frac{1}{4} \mathrm{SW} \frac{1}{4} \text { sec. } 31 \text {, T. } 35 \mathrm{~N} \text {. R. } 26 \text { W. , Sher- } \\
\text { burne County, at outiet of Elk Lake, } \\
2.7 \text { miles northwest of Zimmerman, Minn. }\end{array}$ & - & $1965-67$ & $\begin{array}{r}10-25-67 \\
11-15-67 \\
12-13-67 \\
1-22-68 \\
2-21-68 \\
3-22-68 \\
4-15-68 \\
5-17-68 \\
6-24-68 \\
7-31-68 \\
8-30-68\end{array}$ & $\begin{array}{c}7.26 \\
7.30 \\
7.67 \\
* 7.10 \\
* 6.71 \\
* 7.94 \\
9.24 \\
13.1 \\
7.70 \\
7.86 \\
5.12\end{array}$ \\
\hline \multicolumn{7}{|c|}{ Mississippi River main stem } \\
\hline $\begin{array}{l}\text { Mississippi } \\
\text { River }\end{array}$ & Gulf of Mexico & $\begin{array}{l}\text { SEz sec. } 34 \text {, T. } 33 \text { N., R. } 26 \text { W. , Sherburne } \\
\text { County, on left bank in town of Elk } \\
\text { River, and at mile } 884.6 \text { above onio } \\
\text { River. }\end{array}$ & 14,500 & $\mid \begin{array}{l}915-56 \neq \\
1966-67\end{array}$ & $\begin{array}{r}6-25-68 \\
9-5-68\end{array}$ & $\begin{array}{r}13,100 \\
3,300\end{array}$ \\
\hline \multicolumn{7}{|c|}{ Crow River basin } \\
\hline Buffalo Creek & $\begin{array}{l}\text { South Fork } \\
\text { Crow River }\end{array}$ & $\begin{array}{l}\text { Near center sec.13, T. } 175 \text { N., R. } 28 \text { W., } \\
\text { NcLeod County, at bridge on County } \\
\text { Highway 75, at Glencoe, Minn. }\end{array}$ & - & 1947 & $6-14-68$ & 318 \\
\hline
\end{tabular}

* Base flow.

f Operated as a continuous-record gaging station. 
Discharge measurements made at miscellaneous sites during water year 1968

\begin{tabular}{|c|c|c|c|c|c|c|}
\hline \multirow[b]{2}{*}{ Stream } & \multirow[b]{2}{*}{ Tributary to } & \multirow[b]{2}{*}{ Location } & \multirow{2}{*}{$\begin{array}{l}\text { Drainage } \\
\text { area } \\
(\mathrm{sq} \mathrm{mi})\end{array}$} & \multirow{2}{*}{$\begin{array}{c}\text { Measured } \\
\text { previousiy } \\
\text { (water } \\
\text { years) }\end{array}$} & \multicolumn{2}{|c|}{ Measurements } \\
\hline & & & & & Date & $\begin{array}{l}\text { Discharge } \\
\text { (cfs) }\end{array}$ \\
\hline \multicolumn{7}{|c|}{ Rum River basin } \\
\hline Rum River & $\begin{array}{l}\text { Mississippi } \\
\text { River }\end{array}$ & $\begin{array}{c}\mathrm{NE}^{\frac{1}{4} \mathrm{SE}^{\frac{1}{4}}} \text { sec. } 6 \text {, T. } 41 \mathrm{~N} ., \mathrm{R} .26 \mathrm{~W} . \text {, Mille } \\
\text { Lacs County, at bridge on U.S. Highway } \\
\text { l69, } 0.5 \text { mile south of Onamia, Minn. }\end{array}$ & - & - & $8-27-68$ & * 155 \\
\hline Rum River & $\begin{array}{l}\text { Mississippi } \\
\text { River }\end{array}$ & $\begin{array}{l}\mathrm{NW}^{\frac{1}{4} \mathrm{NW}} \mathrm{N}_{4} \text { sec. } 24, \mathrm{~T} .38 \mathrm{~N} ., \mathrm{R} .27 \mathrm{~W} ., \mathrm{Mille} \\
\text { Lacs County, at bridge on County High- } \\
\text { way } 9,2 \text { miles north of Milaca, Minn. }\end{array}$ & - & - & $8-27-68$ & *176 \\
\hline $\begin{array}{l}\text { West Branch } \\
\text { Rum River }\end{array}$ & Rum River & $\begin{array}{l}\mathrm{NE}^{\frac{1}{4}} \text { sec. } 33 \text {, T. } 36 \mathrm{~N} . \text {, R. } 26 \mathrm{~W} . \text {, Mille Lacs } \\
\text { County, at bridge on U.S. Highway } 169, \\
400 \mathrm{ft} \text {, upstream from mouth, in Prince- } \\
\text { ton, Minn. }\end{array}$ & - & $\begin{array}{l}1961 \\
1965\end{array}$ & $8-27-68$ & $* 11.4$ \\
\hline Rum River & $\begin{array}{l}\text { Mississippi } \\
\text { River }\end{array}$ & 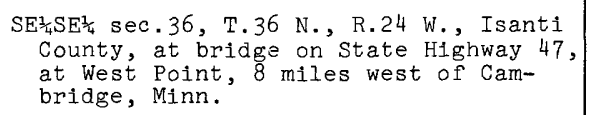 & - & $\left|\begin{array}{c}1958-62 \\
1965 \\
1967\end{array}\right|$ & $\begin{array}{l}3-30-67 \\
3-31-67\end{array}$ & $\begin{array}{r}696 \\
1,510\end{array}$ \\
\hline $\begin{array}{l}\text { Stanchfield } \\
\text { Creek }\end{array}$ & Rum River & $\begin{array}{l}\text { SW } W_{4}^{1} S^{\frac{1}{4}} \text { sec. } 11 \text {, T. } 36 \mathrm{~N} ., \mathrm{R} .24 \mathrm{~W} . \text {, Isanti } \\
\text { County, at bridge on County Highway } 32, \\
1.5 \text { miles upstream from mouth, and } 3 \\
\text { miles northeast of Walbo, Minn. }\end{array}$ & - & 1965 & $8-27-68$ & $* 8.55$ \\
\hline Rum River & $\begin{array}{l}\text { Mississippi } \\
\text { River }\end{array}$ & $\begin{array}{l}\text { W/2 sec. } 30, T .35 \mathrm{~N} ., \mathrm{R} .23 \text { W., Isanti } \\
\text { County, at bridge on County Highway } 5 \text {, } \\
0.8 \text { mile west of Isanti, Minn. }\end{array}$ & - & $\mid \begin{array}{r}1958-60 \\
1962 \\
1965 \\
1967\end{array}$ & $\begin{array}{l}3-30-67 \\
3-31-67\end{array}$ & $\begin{array}{r}959 \\
2,250\end{array}$ \\
\hline Cedar Creek & Rum River & $\begin{array}{l}\text { SW } \frac{1}{4} \text { SW } \frac{1}{4} \text { sec. } 32 \text {, T. } 32 \mathrm{~N} ., \mathrm{R} .24 \mathrm{~W} . \text {, Anoka } \\
\text { County, at bridge on county road, } 0.5 \\
\text { mile upstream from mouth and } 7 \text { miles } \\
\text { north of Anoka, Minn. }\end{array}$ & - & 1965 & $8-27-68$ & $* 21.4$ \\
\hline \multicolumn{7}{|c|}{ Bassett Creek basin } \\
\hline Bassett Creek & $\begin{array}{l}\text { Mississippi } \\
\text { River }\end{array}$ & $\begin{array}{l}\mathrm{W}^{\frac{1}{2}} \text { sec. } 28, \mathrm{~T} .118 \mathrm{~N}, \mathrm{R} .21 \mathrm{~W} \text {., Hennepin } \\
\text { County, at bridge on County Highway } 66 \\
\text { in Golden Valley, Minn., and } 0.2 \text { mile } \\
\text { west of underpass on State Highway } 100 \text {. }\end{array}$ & - & $1963-67$ & $\begin{array}{r}10-13-67 \\
11-16-67 \\
3-28-68 \\
5-20-68 \\
6-14-68 \\
9-9-68 \\
9-30-68\end{array}$ & $\begin{array}{c}3.01 \\
1.10 \\
6.40 \\
13.8 \\
60.5 \\
3.52 \\
4.97\end{array}$ \\
\hline $\begin{array}{l}\text { North Fork } \\
\text { Bassett Creek }\end{array}$ & Bassett Creek & $\begin{array}{l}\text { NWt/ sec. } 21 \text {, T. } 118 \mathrm{~N} ., \mathrm{R} .21 \mathrm{~W} . \text {, Hennepin } \\
\text { County, at culvert on } 34 \mathrm{th} \text { Ave. North } \\
\text { at Crystal, Minn., and } 0.8 \mathrm{mile} \text { up- } \\
\text { stream from mouth. }\end{array}$ & - & $1963-67$ & $\begin{array}{r}3-28-68 \\
4-17-68 \\
5-20-68 \\
8-1-68 \\
9-9-68 \\
9-30-68\end{array}$ & $\begin{array}{r}1.98 \\
.45 \\
1.60 \\
.92 \\
.06 \\
1.51\end{array}$ \\
\hline $\begin{array}{l}\text { South Fork } \\
\text { Bassett Creek }\end{array}$ & Bassett Creek & $\begin{array}{l}\text { Near center of } W^{\frac{1}{2}} \text { sec. I9, T. } 29 \text { N., R. } 24 \\
\text { W. Hennepin County, at culvert on } \\
\text { Olsen Highway, Golden Valley, Minn. } \\
\text { and } 0.2 \text { mile east of State Highway loo. }\end{array}$ & - & $1963-67$ & $\begin{array}{r}10-13-67 \\
11-16-67 \\
3-28-68 \\
4-17-68 \\
5-20-68 \\
8-2-68 \\
9-9-68 \\
9-30-68\end{array}$ & $\begin{array}{l}1.50 \\
1.24 \\
2.26 \\
1.50 \\
1.26 \\
1.98 \\
2.08 \\
1.05\end{array}$ \\
\hline Bassett Creek & $\begin{array}{l}\text { Mississippi } \\
\text { River }\end{array}$ & 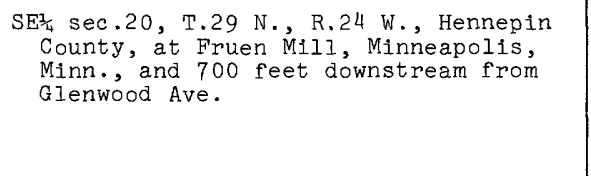 & - & $\left|\begin{array}{r}1952 \\
1954-55 \\
1963-67\end{array}\right|$ & $\begin{array}{r}3-28-68 \\
4-17-68 \\
5-20-68 \\
6-14-68 \\
8-2-68 \\
9-9-68 \\
9-30-68\end{array}$ & $\begin{array}{c}16.8 \\
5.44 \\
15.9 \\
165 \\
24.5 \\
11.0 \\
8.44\end{array}$ \\
\hline \multicolumn{7}{|c|}{ Mississippi River main stem } \\
\hline $\begin{array}{l}\text { Mississippi } \\
\text { River }\end{array}$ & Gulf of Mexico & $\begin{array}{l}\text { At lower St. Anthony Falls lock and dam, } \\
\text { Hennepin County, at Minneapolis, Minn., } \\
\text { and } 10 \text { miles upstream from Minnesota } \\
\text { River. }\end{array}$ & - & 1967 & $5-2-68$ & 13,600 \\
\hline
\end{tabular}

* Base flow. 
Discharge measurements made at miscellaneous sites during water year 1968

\begin{tabular}{|c|c|c|c|c|c|c|}
\hline \multirow{2}{*}{ Stream } & \multirow{2}{*}{ Tributary to } & \multirow{2}{*}{ Location } & \multirow{2}{*}{$\begin{array}{l}\text { Drainage } \\
\text { area } \\
(\mathrm{sq} \mathrm{mi})\end{array}$} & \multirow{2}{*}{$\begin{array}{c}\text { Measured } \\
\text { previous1y. } \\
\text { (water } \\
\text { years) }\end{array}$} & \multicolumn{2}{|c|}{ Measurements } \\
\hline & & & & & Date & $\begin{array}{c}\text { Discharge } \\
\text { (cfs) }\end{array}$ \\
\hline \multicolumn{7}{|c|}{ Mississippi River main stem--continued } \\
\hline $\begin{array}{l}\text { Mississippi } \\
\text { River }\end{array}$ & Gulf of Mexico & $\begin{array}{l}\text { At Washington Ave, and Dartmouth Ave. } \\
\text { bridges, Hennepin County, in Minne- } \\
\text { apolis, Minn., and } 9 \text { miles upstream } \\
\text { from Minnesota River. }\end{array}$ & & $\begin{array}{r}1912 \\
1953-54 \\
1957 \\
1963-67\end{array} \mid$ & $\begin{array}{r}5-2-68 \\
6-19-68\end{array}$ & $\begin{array}{l}12,900 \\
20,100\end{array}$ \\
\hline $\begin{array}{l}\text { Mississippi } \\
\text { River }\end{array}$ & Gulf of Mexico & $\begin{array}{l}\text { Below lock and dam No. 1, Hennepin } \\
\text { County, between Minneapolis and St. } \\
\text { Paul, Minn., } 4 \text { miles upstream from } \\
\text { Minnesota River. }\end{array}$ & 19,700 & $\mid \begin{array}{r}1935, \\
1938-39, \\
1941, \\
1945-50, \\
1954, \\
1959, \\
1961-67\end{array}$ & $\begin{array}{l}11-3-67 \\
9-18-68\end{array}$ & $\begin{array}{l}3,200 \\
4,630\end{array}$ \\
\hline \multicolumn{7}{|c|}{ Minnesota River basin } \\
\hline $\begin{array}{l}\text { Little Minne- } \\
\text { sota River } \\
\text { tributary }\end{array}$ & $\begin{array}{l}\text { Little Minne- } \\
\text { sota River }\end{array}$ & 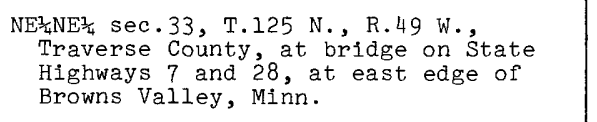 & - & - & $4-1-68$ & .23 \\
\hline Minnesota River & $\begin{array}{l}\text { Mississippi } \\
\text { River }\end{array}$ & 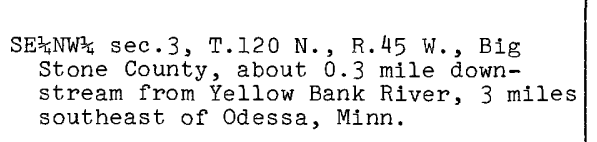 & - & - & $4-3-68$ & 10.8 \\
\hline $\begin{array}{l}\text { Lac qui Parle } \\
\text { River }\end{array}$ & $\begin{array}{l}\text { Minnesota } \\
\text { River }\end{array}$ & $\begin{array}{l}\text { SW } \frac{1}{4} N^{1} \frac{1}{4} \text { sec. } 14 \text {, T. } 114 \text { N., R. } 45 \text { W. Y Yellow } \\
\text { Medicine County, at bridge on State } \\
\text { Highway } 68,2.4 \text { miles southeast of } \\
\text { Canby, Minn. }\end{array}$ & - & $1965-66$ & $4-9-68$ & 1.73 \\
\hline $\begin{array}{l}\text { Lac qui Parle } \\
\text { River }\end{array}$ & $\begin{array}{l}\text { Minnesota } \\
\text { River }\end{array}$ & $\begin{array}{l}\text { On line between secs. } 21 \text { and } 22, \text { T. I15 N., } \\
\text { R. } 44 \text { W. Yellow Medicine County, at } \\
\text { bridge on County Highway D } 8,5.7 \text { miles } \\
\text { northwest of St. Leo, Minn. }\end{array}$ & - & - & $5-9-68$ & .91 \\
\hline $\begin{array}{l}\text { Lac qui Parle } \\
\text { River }\end{array}$ & $\begin{array}{l}\text { Minnesota } \\
\text { River }\end{array}$ & $\begin{array}{l}\mathrm{SE}_{\frac{1}{4} \mathrm{SW}} \frac{\mathrm{m}}{4} \mathrm{sec} .22 \text {, T. } 117 \mathrm{~N} ., \mathrm{R} .43 \mathrm{~W} . \text {, Lac } \\
\text { qui Parle County, at bridge on county } \\
\text { road, } 0.5 \text { mile upstream from West } \\
\text { Branch, and } 0.5 \text { mile east of Dawson, } \\
\text { Minn. }\end{array}$ & - & - & $4-5-68$ & 4.81 \\
\hline Florida Creek & $\begin{array}{l}\text { West Branch } \\
\text { Lac qui } \\
\text { Parle River }\end{array}$ & $\begin{array}{l}\text { On line between secs. } 8 \text { and } 17, \mathrm{~T} .116 \mathrm{~N} . \text {, } \\
\text { R. } 45 \mathrm{w} . \text {, Lac qui Parle County, at } \\
\text { bridge on county highway, } 12 \text { miles } \\
\text { southwest of Dawson, Minn. }\end{array}$ & - & 1966 & $4-5-68$ & 1.92 \\
\hline $\begin{array}{l}\text { West Branch } \\
\text { Lac qui Parle } \\
\text { River }\end{array}$ & Lac qui Parle & $\begin{array}{l}\text { Near center of sec.17, T.117 N., R. } 44 \mathrm{~W} ., \\
\text { Lac qui Parle County, at bridge on U. } \\
\text { S. Highway } 75,4.5 \text { miles south of } \\
\text { Madison, Minn. }\end{array}$ & - & $\left|\begin{array}{l}1948-49 \\
1966-67\end{array}\right|$ & $\begin{array}{l}4-5-68 \\
5-2-68\end{array}$ & $\begin{array}{l}2.10 \\
25.9\end{array}$ \\
\hline $\begin{array}{l}\text { West Branch } \\
\text { Lac qui Parle } \\
\text { River }\end{array}$ & $\begin{array}{l}\text { Lac qui Parle } \\
\text { River }\end{array}$ & 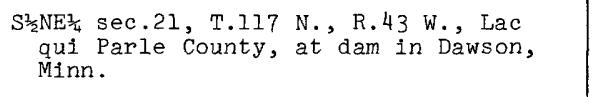 & - & - & $5-2-68$ & 30.1 \\
\hline $\begin{array}{c}\text { Minnesota River } \\
\text { tributary }\end{array}$ & $\begin{array}{l}\text { Minnesota } \\
\text { River }\end{array}$ & $\begin{array}{l}\text { SE } \frac{1}{4} \mathrm{SW}^{\frac{1}{4}} \text { sec. } 27 \text {, T. } 117 \mathrm{~N} ., \mathrm{R} .40 \mathrm{~W} . \text {, Chip- } \\
\text { pewa County, at bridge on County High- } \\
\text { way 15, } 2.1 \text { miles northeast of WegdahI, } \\
\text { Minn. }\end{array}$ & - & - & $10-17-67$ & 0 \\
\hline $\begin{array}{c}\text { Minnesota River } \\
\text { tributary }\end{array}$ & $\begin{array}{l}\text { Minnesota } \\
\text { River }\end{array}$ & $\begin{array}{l}\text { SW } \frac{1}{4} \mathrm{NE}^{\frac{1}{4}} \text { sec. } 34 \text {. T. } 116 \mathrm{~N} ., \mathrm{R} .39 \mathrm{~W} . \text {, Chip- } \\
\text { pewa County, at bridge on County High- } \\
\text { way } 5 \text { at Granite Falls, Minn. }\end{array}$ & - & - & $10-17-67$ & 0 \\
\hline $\begin{array}{l}\text { Yellow Medicine } \\
\text { River }\end{array}$ & $\begin{array}{l}\text { Minnesota } \\
\text { River }\end{array}$ & $\begin{array}{l}\text { On line between secs. } 13 \text { and } 14, \mathrm{~T} .113 \mathrm{~N} . \\
\text { R. } 44 \mathrm{~W} \text {, Lincoln County, at bridge on } \\
\text { county highway, } 3.5 \text { miles southeast of } \\
\text { Porter, Minn. }\end{array}$ & - & 1966 & $4-9-68$ & $3 \cdot 37$ \\
\hline Mud Creek & $\begin{array}{l}\text { Yellow Medi- } \\
\text { cine River }\end{array}$ & $\begin{array}{l}\text { On line between secs. } 28 \text { and } 29 \text {, T.114 N., } \\
\text { R. } 43 \mathrm{~W} ., \text { Yellow Medicine County, at } \\
\text { bridge on County Highway } 11,3.5 \text { miles } \\
\text { north of Taunton, Minn. }\end{array}$ & - & 1966 & $5-8-68$ & .19 \\
\hline $\begin{array}{l}\text { Yellow Medicine } \\
\text { River }\end{array}$ & $\begin{array}{l}\text { Minnesota } \\
\text { River }\end{array}$ & $\begin{array}{l}\text { On line between secs. } 7 \text { and } 18, \mathrm{~T} .114 \mathrm{~N} ., \\
\text { R. } 41 \text { W., Yellow Medicine County, at } \\
\text { bridge on County Highway } 18,4.3 \text { miles } \\
\text { west of Hanley Falls, Minn. }\end{array}$ & - & $-965-66$ & $4-9-68$ & 20.9 \\
\hline
\end{tabular}


Discharge measurements made at miscellaneous sites during water year 1968

\begin{tabular}{|c|c|c|c|c|c|c|}
\hline \multirow[b]{2}{*}{ Stream } & \multirow[b]{2}{*}{ Tributary to } & \multirow[b]{2}{*}{ Location } & \multirow{2}{*}{$\begin{array}{l}\text { Drainage } \\
\text { area } \\
(\mathrm{sq} \mathrm{mi})\end{array}$} & \multirow{2}{*}{$\begin{array}{c}\text { Measured } \\
\text { Previously } \\
\text { (water } \\
\text { years) }\end{array}$} & \multicolumn{2}{|c|}{ Measurements } \\
\hline & & & & & Date & $\begin{array}{c}\text { Discharge } \\
\text { (cfs) }\end{array}$ \\
\hline \multicolumn{7}{|c|}{ Minnesota River basin--continued } \\
\hline Hawk Creek & $\begin{array}{l}\text { Minnesota } \\
\text { River }\end{array}$ & $\begin{array}{l}\mathrm{NE}^{\frac{1}{4} \mathrm{SE}} \frac{1}{4} \text { sec. } 18, \mathrm{~T} .119 \mathrm{~N} ., \mathrm{R} .35 \mathrm{~W} . \text {, Kandi- } \\
\text { yohi County, at bridge on County High- } \\
\text { way } 5,2.8 \text { miles west of Willmar, Minn. }\end{array}$ & - & - & $10-17-67$ & 0 \\
\hline Hawk Creek & $\begin{array}{c}\text { Minnesota } \\
\text { River }\end{array}$ & $\begin{array}{l}\text { SW } \frac{1}{4} \mathrm{SE}_{1} / \mathrm{sec} .16, \mathrm{~T} .115 \mathrm{~N} ., \mathrm{R} .38 \mathrm{~W} . \text {, Ren- } \\
\text { ville County, at bridge on County } \\
\text { Highway } 52,3.5 \text { miles southeast of } \\
\text { Minnesota Falls, Minn. }\end{array}$ & - & - & $10-17-67$ & $4 \cdot 36$ \\
\hline $\begin{array}{c}\text { Minnesota River } \\
\text { tributary }\end{array}$ & $\begin{array}{c}\text { Minnesota } \\
\text { River }\end{array}$ & 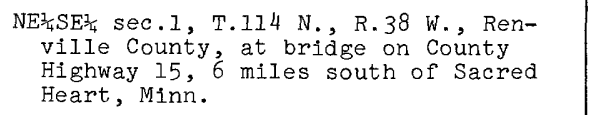 & - & - & $10-16-67$ & 0 \\
\hline $\begin{array}{l}\text { Sacred Heart } \\
\text { Creek }\end{array}$ & $\begin{array}{l}\text { Minnesota } \\
\text { River }\end{array}$ & 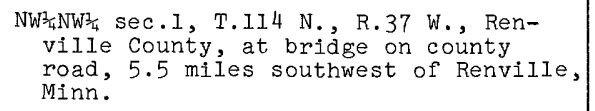 & - & - & $10-17-67$ & 0 \\
\hline Minnesota River & $\begin{array}{l}\text { Mississippi } \\
\text { River }\end{array}$ & $\begin{array}{l}\text { SW } \frac{1}{4} N_{\frac{1}{4}} \text { sec. } 30, \text { T. } 114 \text { N., R. } 36 \text { W., Red- } \\
\text { wood County, at bridge on County High- } \\
\text { way } 6,4 \text { miles north of Delhi, Minn. }\end{array}$ & - & - & $\begin{array}{r}5-9-68 \\
8-14-68 \\
9-16-68\end{array}$ & $\begin{array}{l}470 \\
176 \\
79.0\end{array}$ \\
\hline Redwood River & $\begin{array}{c}\text { Minnesota } \\
\text { River }\end{array}$ & $\begin{array}{l}\text { On Iine between secs. } 25 \text { and } 36 \text {, T. } 110 \mathrm{~N} \text {. } \\
\text { R. } 43 \text { w. Lyon County, at bridge on } \\
\text { county highway, } 1.2 \text { miles southwest } \\
\text { of Russeli, Minn. }\end{array}$ & - & $1966-67$ & $10-12-67$ & .62 \\
\hline Coon Creek & Redwood River & $\begin{array}{l}\text { On Iine between secs. } 23 \text { and } 24, \mathrm{~T} .110 \mathrm{~N} \text {. } \\
\text { R. } 43 \mathrm{w} . \text {, Lyon County, at bridge on } \\
\text { county highway, } 1 \text { mile west of Russell, } \\
\text { Minn. }\end{array}$ & - & $1966-67$ & $10-12-67$ & 0 \\
\hline $\begin{array}{l}\text { West Fork } \\
\text { Beaver Creek }\end{array}$ & Beaver Creek & $\begin{array}{l}\mathrm{NE}_{\frac{1}{4} \mathrm{SE}^{\frac{1}{4}}} \text { sec.18, T.116 N., R. } 35 \mathrm{~W} . \text {, Ren- } \\
\text { vilie County, at bridge on County } \\
\text { Highway } 1,4 \text { miles north of Danube, } \\
\text { Minn. }\end{array}$ & - & - & $10-17-67$ & 0 \\
\hline Beaver Creek & $\begin{array}{c}\text { Minnesota } \\
\text { River }\end{array}$ & $\begin{array}{l}\mathrm{NE}^{\frac{1}{4} \mathrm{NW}^{3} / 4} \text { sec.22, T.II3 N., R. } 35 \mathrm{~W} \text {, Ren- } \\
\text { vilie County, at bridge on county } \\
\text { highway in Beaver Falls, Minn. }\end{array}$ & - & $1966-67$ & $10-11-67$ & 7.08 \\
\hline $\begin{array}{l}\text { Little Rock } \\
\text { Creek }\end{array}$ & $\begin{array}{c}\text { Minnesota } \\
\text { River }\end{array}$ & $\begin{array}{l}\mathrm{NW}^{\frac{1}{4}} \mathrm{NW}^{\frac{1}{4}} \text { sec. } 30 \text {, T. III N., R. } 31 \text { W., Nicol- } \\
\text { let County, at bridge on County High- } \\
\text { way } 21 \text {, I0 miles southeast of Fairfax, } \\
\text { Minn. }\end{array}$ & - & $1966-67$ & $10-11-67$ & 0 \\
\hline$\underset{\text { Creek }}{\text { Eight mile }}$ & $\begin{array}{c}\text { Minnesota } \\
\text { River }\end{array}$ & $\begin{array}{l}\text { SE } \frac{1}{4} \text { SWh sec. } 29 \text {, T. } 111 \text { N., R. } 31 \text { W., Nicol- } \\
\text { let County, at bridge on County High- } \\
\text { way } 21,2.7 \text { miles west of St. George, } \\
\text { Minn. }\end{array}$ & - & - & $10-17-67$ & 0 \\
\hline $\begin{array}{c}\text { Minnesota River } \\
\text { tributary }\end{array}$ & $\begin{array}{l}\text { Minnesota } \\
\text { River }\end{array}$ & $\begin{array}{l}\text { SW } \frac{1}{4} \mathrm{SE}^{\frac{1}{4}} \text { sec. } 6 \text {, T.IIO N., R. } 30 \text { W., NicoI- } \\
\text { let County, at bridge on County High- } \\
\text { way } 21,3 \text { miles southwest of KIossner, } \\
\text { Minn. }\end{array}$ & - & - & $10-17-67$ & 0 \\
\hline $\begin{array}{l}\text { Cottonwood } \\
\text { River }\end{array}$ & $\begin{array}{l}\text { Minnesota } \\
\text { River }\end{array}$ & $\begin{array}{l}\text { On line between secs. } 11 \text { and } 12 \text {, T. } 110 \mathrm{~N} . \\
\text { R. } 40 \text { w. Lyon County, } 4.6 \text { miles north- } \\
\text { east of Amiret, Minn. }\end{array}$ & - & - & $9-4-68$ & *I. 51 \\
\hline $\begin{array}{l}\text { Cottonwood } \\
\text { River }\end{array}$ & $\begin{array}{c}\text { Minnesota } \\
\text { River }\end{array}$ & $\begin{array}{l}\text { On line between secs.l and } 2, \text { T. I09 N., } \\
\text { R. } 38 \text { w., Redwood County, at bridge on } \\
\text { county road, } 3.8 \text { miles north of Revere, } \\
\text { Minn. }\end{array}$ & - & - & $9-5-68$ & $* 1.75$ \\
\hline $\begin{array}{l}\text { Cottonwood } \\
\text { River }\end{array}$ & $\begin{array}{c}\text { Minnesota } \\
\text { River }\end{array}$ & 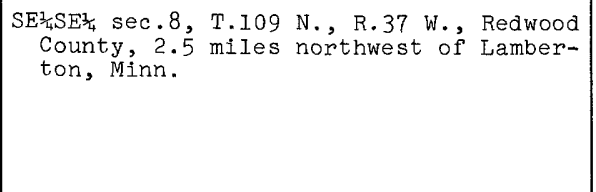 & - & $1966-67$ & $\begin{array}{r}10-13-67 \\
4-8-68 \\
5-10-68 \\
6-20-68 \\
7-9-68 \\
8-16-68 \\
9-20-68\end{array}$ & $\begin{array}{l}20.19 \\
7.24 \\
1.21 \\
1.30 \\
23.6 \\
31.1\end{array}$ \\
\hline
\end{tabular}


Discharge measurements made at miscellaneous sites during water year 1968

\begin{tabular}{|c|c|c|c|c|c|c|}
\hline \multirow[b]{2}{*}{ Stream } & \multirow[b]{2}{*}{ Tributary to } & \multirow[b]{2}{*}{ Location } & \multirow{2}{*}{$\begin{array}{l}\text { Drainage } \\
\text { area } \\
\text { (sq mi) }\end{array}$} & \multirow{2}{*}{$\begin{array}{c}\text { Measured } \\
\text { previous Iy } \\
\text { (water } \\
\text { years) }\end{array}$} & \multicolumn{2}{|c|}{ Measurements } \\
\hline & & & & & Date & $\begin{array}{l}\text { Discharge } \\
\text { (cfs) }\end{array}$ \\
\hline \multicolumn{7}{|c|}{ Minnesota River basin--continued } \\
\hline $\begin{array}{l}\text { Dutch Charley } \\
\text { Creek }\end{array}$ & $\begin{array}{l}\text { Cottonwood } \\
\text { River }\end{array}$ & $\begin{array}{l}\text { On line between sec. } 30, \mathrm{~T} .109 \mathrm{~N} ., \mathrm{R} .36 \\
\text { W., and sec. } 25 \text {, T.log N., R. } 37 \text { W. } \\
\text { Redwood County, at bridge on County } \\
\text { Highway } 15,2 \text { miles east of Lamberton, } \\
\text { Minn. }\end{array}$ & - & - & $9-5-68$ & *7.86 \\
\hline $\begin{array}{l}\text { Cottonwood } \\
\text { River }\end{array}$ & $\begin{array}{l}\text { Minnesota } \\
\text { River }\end{array}$ & $\begin{array}{l}\text { On line between secs. } 26 \text { and } 35, \text { T. } 109 \\
\text { N., R. } 36 \text { W., Redwood County, at bridge } \\
\text { on County Highway 15, } 0.5 \text { mile west of } \\
\text { Sanborn, Minn. }\end{array}$ & - & - & $9-5-68$ & *14.1 \\
\hline Mound Creek & $\begin{array}{l}\text { Cottonwood } \\
\text { River }\end{array}$ & $\begin{array}{l}\mathrm{NW}^{\frac{1}{4}} \mathrm{NW} / \frac{1}{4} \mathrm{sec} .4, \mathrm{~T} .108 \mathrm{~N} ., \mathrm{R} .35 \mathrm{~W} ., \text { Brown } \\
\text { County, at bridge on County Highway } 2, \\
5 \text { miles southwest of Springfield, } \\
\text { Minn. }\end{array}$ & - & - & $9-5-68$ & *3.16 \\
\hline Coal Mine Creek & $\begin{array}{l}\text { Cottonwood } \\
\text { River }\end{array}$ & $\begin{array}{l}\text { On line between secs. } 22 \text { and } 27, \mathrm{~T} .109 \mathrm{~N} ., \\
\mathrm{R} .35 \mathrm{~W} . \text {, Brown County, at bridge on } \\
\text { county road, } 3 \text { miles southwest of } \\
\text { Springfield, Minn. }\end{array}$ & - & - & $9-5-68$ & *1. 37 \\
\hline $\begin{array}{l}\text { Cottonwood } \\
\text { River }\end{array}$ & $\begin{array}{l}\text { Minnesota } \\
\text { River }\end{array}$ & $\begin{array}{l}\text { On line between secs.21 and } 22, \mathrm{~T} .109 \mathrm{~N} . \\
\text { R. } 33 \mathrm{~W} \text {. Brown County, at bridge on } \\
\text { county highway, } 0.2 \text { mile north of } \\
\text { Leavenworth, Minn. }\end{array}$ & - & $1966-67$ & $\begin{array}{r}10-11-67 \\
4-8-68 \\
5-9-68 \\
7-9-68 \\
8-16-68 \\
9-20-68\end{array}$ & $\begin{array}{c}5.82 \\
48.6 \\
31.4 \\
8.51 \\
162 \\
131\end{array}$ \\
\hline $\begin{array}{l}\text { Sleepy Eye } \\
\text { Creek }\end{array}$ & $\begin{array}{l}\text { Cottonwood } \\
\text { River }\end{array}$ & $\begin{array}{l}\text { On line between secs. } 8 \text { and } 9, \text { T. } 109 \mathrm{~N} ., \\
\text { R. } 33 \mathrm{~W} \text {. Brown County, at bridge on } \\
\text { county highway, } 1.8 \text { miles southeast of } \\
\text { Cobden, Minn. }\end{array}$ & - & $1966-67$ & $\begin{array}{r}10-I 1-67 \\
4-8-68 \\
5-9-68 \\
6-7-68 \\
7-9-68 \\
8-16-68 \\
9-20-68\end{array}$ & $\begin{array}{c}7.87 \\
19.0 \\
17.4 \\
6.89 \\
8.84 \\
66.2 \\
37.1\end{array}$ \\
\hline $\begin{array}{l}\text { Sleepy Eye } \\
\text { Creek }\end{array}$ & $\begin{array}{l}\text { Cottonwood } \\
\text { River }\end{array}$ & 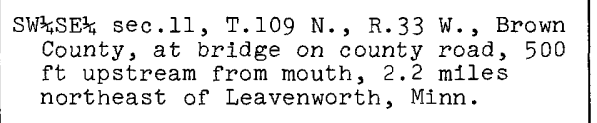 & - & - & $9-6-68$ & $* 18.0$ \\
\hline $\begin{array}{l}\text { Cottonwood } \\
\text { River }\end{array}$ & $\begin{array}{l}\text { Minnesota } \\
\text { River }\end{array}$ & $\begin{array}{l}\text { NE } \frac{1}{4} N W_{\frac{1}{4}} \text { sec. } 3, \text { T.109 N., R. } 32 \text { W., Brown } \\
\text { County, at bridge on County Highway 10, } \\
2.5 \text { miles southeast of Sleepy Eye, } \\
\text { Minn. }\end{array}$ & - & - & $9-6-68$ & $* 66.5$ \\
\hline $\begin{array}{l}\text { Little Cotton- } \\
\text { wood River }\end{array}$ & $\begin{array}{l}\text { Minnesota } \\
\text { River }\end{array}$ & $\begin{array}{l}\text { On Iine between secs. } 9 \text { and } 10, \text { T. } 108 \mathrm{~N} ., \\
\text { R. } 33 \text { w. Brown County, at bridge on } \\
\text { County Highway } 8,3.8 \text { miles south of } \\
\text { Leavenworth, Minn. }\end{array}$ & - & - & $9-6-68$ & $* 13.7$ \\
\hline $\begin{array}{l}\text { Little Cotton- } \\
\text { wood River }\end{array}$ & $\begin{array}{l}\text { Minnesota } \\
\text { R1ver }\end{array}$ & $\begin{array}{l}\mathrm{N}^{\frac{1}{2}} \text { sec.19, T.109 N., R. } 29 \text { W., Blue Earth } \\
\text { County, at bridge on County Highway } \\
\text { 105, } 2.5 \text { miles upstream from mouth, } \\
\text { and } 2.5 \text { miles west of Cambria, M1nn. }\end{array}$ & - & - & $9-6-68$ & $* 35.0$ \\
\hline $\begin{array}{l}\text { Little Cotton- } \\
\text { wood River }\end{array}$ & $\begin{array}{l}\text { Minnesota } \\
\text { River }\end{array}$ & $\begin{array}{l}\text { Near center of sec.17, T.109 N., R.29 W., } \\
\text { Blue Earth County, at bridge on State } \\
\text { Highway } 68,0.5 \text { mile south of Court- } \\
\text { land, Minn. }\end{array}$ & - & 1966 & $\begin{array}{r}10-11-67 \\
4-22-68 \\
5-21-68 \\
6-24-68 \\
8-6-68 \\
9-9-68\end{array}$ & $\begin{array}{l}6.68 \\
26.0 \\
12.4 \\
10.3 \\
454 \\
108\end{array}$ \\
\hline $\begin{array}{c}\text { Minnesota River } \\
\text { tributary }\end{array}$ & $\begin{array}{l}\text { Minnesota } \\
\text { River }\end{array}$ & $\begin{array}{l}N^{\frac{1}{4}} N_{W} \frac{1}{4} \text { sec. } 15, \text { T. } 109 \text { N., R. } 29 \text { W. , Nicol- } \\
\text { let County, at bridge on county road, } \\
2.3 \text { miles southeast of Courtland, Minn. }\end{array}$ & - & - & $10-17-67$ & 0 \\
\hline $\begin{array}{l}\text { Swan Lake } \\
\text { Outlet }\end{array}$ & $\begin{array}{l}\text { Minnesota } \\
\text { River }\end{array}$ & $\begin{array}{l}\mathrm{NW}_{4, \mathrm{SW}}^{\frac{1}{4}} \text { sec.5, T.109 N., R.28 W. , Nicol- } \\
\text { let County, at bridge on U.S. Highway } \\
\text { 14, } 2 \text { miles west of N1collet, Minn. }\end{array}$ & - & - & $10-17-67$ & 0 \\
\hline $\begin{array}{l}\text { Swan Lake } \\
\text { Outlet }\end{array}$ & $\begin{array}{c}\text { Minnesota } \\
\text { River }\end{array}$ & $\begin{array}{l}\text { On line between secs. } 27 \text { and } 28 \text {, T.log N. } \\
\text { R. } 28 \text { W. N1collet County, at culvert } \\
\text { on county highway, } 4.5 \text { miles south of } \\
\text { Nicollet, Minn. }\end{array}$ & - & $1966-67$ & $10-11-67$ & $* 59$ \\
\hline
\end{tabular}


Discharge measurements made at miscellaneous sites during water year 1968

\begin{tabular}{|c|c|c|c|c|c|c|}
\hline \multirow{2}{*}{ Stream } & \multirow{2}{*}{ Tributary to } & \multirow[b]{2}{*}{ Location } & \multirow{2}{*}{$\begin{array}{l}\text { Drainage } \\
\text { area } \\
(\mathrm{sq} \mathrm{mi})\end{array}$} & \multirow{2}{*}{$\begin{array}{c}\text { Measured } \\
\text { previous 1y } \\
\text { (water } \\
\text { years) }\end{array}$} & \multicolumn{2}{|c|}{ Measurements } \\
\hline & & & & & Date & $\begin{array}{l}\text { Discharge } \\
\text { (cfs) }\end{array}$ \\
\hline \multicolumn{7}{|c|}{ Minnesota River basin--continued } \\
\hline $\begin{array}{l}\text { Minnesota } \\
\text { River }\end{array}$ & $\begin{array}{l}\text { Mississippi } \\
\text { River }\end{array}$ & $\begin{array}{l}E^{\frac{1}{2}} \text { sec. } 33 \text {, T. } 109 \mathrm{~N} ., \text { R. } 28 \mathrm{~W} ., \text { Blue Earth } \\
\text { County, at highway bridge, a quarter } \\
\text { of a mile northeast of Judson, Minn., } \\
\text { and } 11 \text { miles upstream from Blue Earth } \\
\text { River. }\end{array}$ & 11,200 & $\mid \begin{array}{c}1938-50= \\
1967\end{array}$ & $\begin{array}{r}4-23-68 \\
9-9-68\end{array}$ & $\begin{array}{r}1,160 \\
480\end{array}$ \\
\hline $\begin{array}{c}\text { Minnesota River } \\
\text { tributary }\end{array}$ & $\begin{array}{l}\text { Minnesota } \\
\text { River }\end{array}$ & $\begin{array}{l}\mathrm{NW}_{\frac{1}{4}} \mathrm{NW} / \frac{1}{4} \mathrm{sec} .15, \mathrm{~T} .108 \mathrm{~N}, \mathrm{R} .27 \mathrm{~W} ., \mathrm{Nicol}- \\
\text { let County, at bridge on County High- } \\
\text { way } 71,2.7 \text { miles west of Mankato, } \\
\text { Minn. }\end{array}$ & - & - & $10-17-67$ & 0 \\
\hline $\begin{array}{l}\text { Blue Earth } \\
\text { River }\end{array}$ & $\begin{array}{l}\text { Minnesota } \\
\text { River }\end{array}$ & 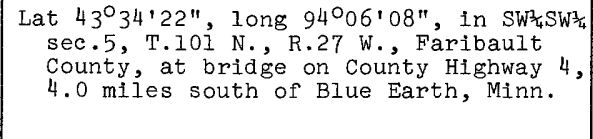 & - & - & $\begin{array}{r}4-18-68 \\
5-24-68 \\
7-1-68 \\
8-8-68 \\
9-11-68\end{array}$ & $\begin{array}{l}* 6.39 \\
12.4 \\
229 \\
73.7 \\
* 6.30\end{array}$ \\
\hline Center Creek & $\begin{array}{l}\text { Blue Earth } \\
\text { River }\end{array}$ & $\begin{array}{l}\text { Lat } 43^{\circ} 43^{\prime} 50^{\prime \prime}, \text { long } 94^{\circ} 12^{\prime} 26^{\prime \prime} \text {, on line } \\
\text { between secs. } 16 \text { and } 17 \text {, T. } 103 \mathrm{~N} \text {., R. } \\
28 \text { W., Faribault County, at bridge on } \\
\text { county road, } 2 \text { miles upstream from } \\
\text { mouth, } 1.5 \text { miles east of Huntley, } \\
\text { Minn. }\end{array}$ & - & - & $\begin{array}{r}4-18-68 \\
5-24-68 \\
7-1-68 \\
8-8-68 \\
9-11-68\end{array}$ & $\begin{array}{l}* 6.73 \\
* 4.98 \\
32.7 \\
20.0 \\
13.5\end{array}$ \\
\hline Elm Creek & $\begin{array}{l}\text { Blue Earth } \\
\text { River }\end{array}$ & $\begin{array}{l}\text { Lat } 43^{\circ} 45^{\prime} 02^{\prime \prime}, \text { long } 94^{\circ} 12^{\prime} 32^{\prime \prime} \text {, in } \mathrm{NE}_{\frac{1}{4}} \mathrm{SE}^{\frac{1}{4}} \\
\text { sec.5, T.103 N., R. } 28^{\mathrm{W}} \mathrm{W} \text {, Faribault } \\
\text { County, at bridge on county road, } 0.7 \\
\text { miles upstream from mouth, } 1.2 \text { miles } \\
\text { southwest of Winnebago, Minn. }\end{array}$ & - & - & $\begin{array}{r}4-18-68 \\
5-24-68 \\
7-1-68 \\
8-7-68 \\
9-11-68\end{array}$ & $\begin{array}{l}* 2.37 \\
* 1.75 \\
21.6 \\
20.4 \\
3.85\end{array}$ \\
\hline Watonwan River & $\begin{array}{l}\text { Blue Earth } \\
\text { River }\end{array}$ & $\begin{array}{l}\text { Lat } 44^{\circ} 03^{\prime} 53^{\prime \prime}, \text { long } 94^{\circ} 35^{\prime} 22^{\prime \prime} \text {, on line } \\
\text { between secs. } 19 \text { and } 20 \text {, T.107 N., R. } 31 \\
\text { W. , Watonwan County, at bridge on } \\
\text { County Highway 14, } 0.7 \text { mile west of } \\
\text { La Salle, Minn. }\end{array}$ & - & - & $\begin{array}{r}4-17-68 \\
5-22-68 \\
7-1-68 \\
8-6-68 \\
9-11-68\end{array}$ & $\begin{array}{l}11 \cdot 9 \\
* 4 \cdot 72 \\
10.7 \\
132 \\
56.9\end{array}$ \\
\hline St. James Creek & $\begin{array}{c}\text { Watonwan } \\
\text { River }\end{array}$ & 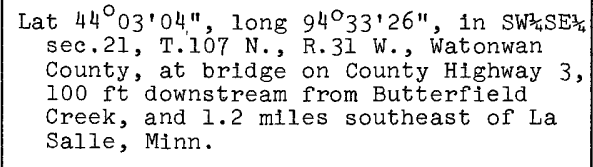 & - & - & $\begin{array}{r}4-12-68 \\
5-22-68 \\
7-1-68 \\
8-6-68 \\
9-11-68\end{array}$ & $\begin{array}{l}* 5.90 \\
* 5.42 \\
20.4 \\
57.8 \\
35.3\end{array}$ \\
\hline $\begin{array}{l}\text { South Fork } \\
\text { Watonwan } \\
\text { River }\end{array}$ & Watonwan Riven & $\begin{array}{l}\text { Lat } 44^{\circ} 02^{\prime} 33^{\prime \prime} \text {, long } 94^{\circ} 27^{\prime} 42^{\prime \prime} \text {, in W/2 sec. } \\
29 \text {, T. } 107 \mathrm{~N} \text {., R. } 30 \mathrm{~W} \text {., Watonwan County, } \\
\text { at bridge on County Highway } 116,0.4 \\
\text { mile upstream from mouth, and } 1.6 \text { mile } \\
\text { west of Madelia, Minn. }\end{array}$ & - & - & $\begin{array}{r}4-17-68 \\
5-22-68 \\
7-1-68 \\
8-7-68 \\
9-11-68\end{array}$ & $\begin{array}{l}* 3.80 \\
* 2.33 \\
8.18 \\
50.7 \\
19.2\end{array}$ \\
\hline Watonwan River & $\begin{array}{l}\text { Blue Earth } \\
\text { River }\end{array}$ & 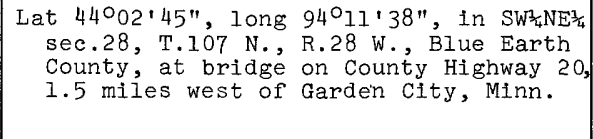 & 812 & $\left|\begin{array}{r}1940-45 \neq \\
1953 \\
1960-61\end{array}\right|$ & $\begin{array}{r}4-17-68 \\
5-22-68 \\
6-28-68 \\
8-7-68 \\
9-11-68\end{array}$ & $\begin{array}{l}32.4 \\
* 28.5 \\
132 \\
342 \\
162\end{array}$ \\
\hline Le Sueur River & $\begin{array}{l}\text { Blue Earth } \\
\text { River }\end{array}$ & 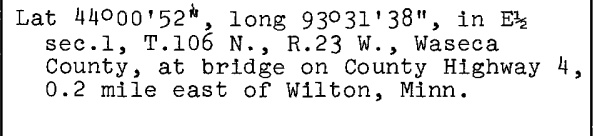 & - & - & $\begin{array}{r}4-18-68 \\
5-27-68 \\
7-2-68 \\
8-8-68 \\
9-12-68\end{array}$ & $\begin{array}{l}* 6.01 \\
60.7 \\
103 \\
1,180 \\
66.4\end{array}$ \\
\hline Cobb River & Le Sueur River & $\begin{array}{l}\text { Lat } 44^{\circ} 02^{\prime} 50^{\prime \prime}, \text { long } 94^{\circ} 00^{\prime} 00^{\prime \prime}, \mathrm{SE}_{\frac{1}{4}} \mathrm{NW}^{\prime} \frac{\mathrm{t}}{4} \\
\text { sec. } 30 \text {, T. } 107 \mathrm{~N} \text {., R. } 26 \mathrm{~W} \text {, Blue Earth } \\
\text { County, at bridge on County Highway 16, } \\
4.5 \text { miles northeast of Good Thunder, } \\
\text { Minn. }\end{array}$ & - & - & $\begin{array}{r}4-12-68 \\
5-23-68 \\
6-28-68 \\
8-7-68 \\
9-10-68\end{array}$ & $\begin{array}{l}* 2.19 \\
15.0 \\
181 \\
1,400 \\
384\end{array}$ \\
\hline Maple River & Le Sueur River & $\begin{array}{l}\text { Lat } 44^{\circ} 03^{\prime} 54^{\prime \prime}, \text { long } 94^{\circ} 01 \text { ' } 32 \text { ", on line } \\
\text { between secs.13 and } 24 \text {, T.107 N. R. R. } \\
\text { W., Blue Earth County, at bridge on } \\
\text { County Highway } 35,2 \text { miles southeast } \\
\text { of Rapidan, Minn. }\end{array}$ & - & - & $\begin{array}{r}4-17-68 \\
5-23-68 \\
6-27-68 \\
8-7-68 \\
9-10-68\end{array}$ & $\begin{array}{l}* 8.15 \\
* 15.2 \\
323 \\
416 \\
136\end{array}$ \\
\hline Warren Creek & $\begin{array}{c}\text { Minnesota } \\
\text { River }\end{array}$ & $\begin{array}{l}\mathrm{NW}_{4} \mathrm{NW}_{\frac{1}{4}} \text { sec. } 18, \mathrm{~T} .108 \mathrm{~N} ., \mathrm{R} .26 \mathrm{~W} ., \mathrm{Bl} \text {, } \\
\text { Earth County, at culvert under rail- } \\
\text { road tracks at mouth, at Mankato, Minn. }\end{array}$ & - & - & $4-24-68$ & 3.18 \\
\hline
\end{tabular}

* Base flow.

F Operated as a continuous-record gaging station. 


\begin{tabular}{|c|c|c|c|c|c|c|}
\hline \multirow[b]{2}{*}{ Stream } & \multirow{2}{*}{ Tributary to } & \multirow[b]{2}{*}{ Location } & \multirow{2}{*}{$\begin{array}{l}\text { Drainage } \\
\text { area } \\
(\mathrm{sq} \mathrm{mi})\end{array}$} & \multirow{2}{*}{$\begin{array}{c}\text { Measured } \\
\text { previous ly } \\
\text { (water } \\
\text { years) }\end{array}$} & \multicolumn{2}{|c|}{ Measurements } \\
\hline & & & & & Date & $\begin{array}{l}\text { Discharge } \\
\text { (cfs) }\end{array}$ \\
\hline \multicolumn{7}{|c|}{ Minnesota River basin--continued } \\
\hline$\underset{\text { JA }}{\text { Judicial ditch }}$ & $\begin{array}{c}\text { South Branch } \\
\text { Rush River }\end{array}$ & $\begin{array}{l}\mathrm{NW}^{\frac{1}{4}} \mathrm{NW}^{\frac{1}{4}} \text { sec.19, T.11l N., R. } 28 \mathrm{~W} . \text {, Nicol- } \\
\text { let County, at bridge on County High- } \\
\text { way } 4,1,000 \text { ft upstream from County } \\
\text { ditch No. } 9,9.5 \text { miles above mouth, and } \\
2 \text { miles west of New Sweden, Minn. }\end{array}$ & - & $1964-67$ & $\begin{array}{r}10-11-67 \\
11-13-67 \\
12-11-67 \\
3-18-68 \\
4-22-68 \\
5-21-68 \\
6-24-68 \\
7-11-68 \\
8-6-68\end{array}$ & $\begin{array}{r}.66 \\
* .28 \\
* .01 \\
2.49 \\
1.90 \\
1.07 \\
2.97 \\
.74 \\
34.4\end{array}$ \\
\hline Sand Creek & $\begin{array}{l}\text { Minnesota } \\
\text { River }\end{array}$ & $\begin{array}{l}\text { SW } \frac{1}{4} \text { SW } \frac{1}{4} \text { sec. } 29 \text {, T. } 115 \mathrm{~N} ., \text { R. } 23 \mathrm{~W} . \text {, scott } \\
\text { County, at bridge on county road, I } \\
\text { mile upstream from mouth, and } 4.5 \\
\text { miles north of Jordan, Minn. }\end{array}$ & - & - & $3-26-68$ & $12 \cdot 3$ \\
\hline Carver Creek & $\begin{array}{l}\text { Minnesota } \\
\text { River }\end{array}$ & 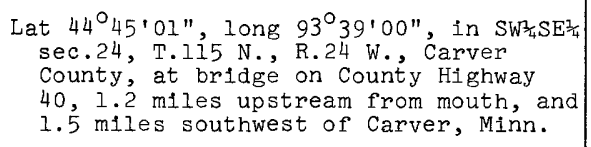 & - & - & $3-26-68$ & 5.45 \\
\hline $\begin{array}{c}\text { Minnesota River } \\
\text { tributary }\end{array}$ & $\begin{array}{l}\text { Minnesota } \\
\text { River }\end{array}$ & $\begin{array}{l}\mathrm{NE}_{\frac{1}{4}} \mathrm{NE}_{4}^{\frac{1}{4}} \text { sec. } 19 \text {, T. } 115 \mathrm{~N}, \mathrm{R}: 23 \mathrm{~W} \text {, Carver } \\
\text { County, at culvert on county road, } 900 \\
\text { ft upstream from mouth, in Carver, } \\
\text { Minn. }\end{array}$ & - & - & $3-26-68$ & .29 \\
\hline $\begin{array}{c}\text { Minnesota River } \\
\text { tributary }\end{array}$ & $\begin{array}{l}\text { Minnesota } \\
\text { River }\end{array}$ & $\begin{array}{l}\text { NE } \frac{1}{4} S W \frac{1}{4} \text { sec. } 17 \text {, T. } 115 \text { N., R. } 23 \text { W. Carver } \\
\text { County, at culvert on county road, } 300 \\
\text { ft upstream from mouth, and } 0.4 \text { mile } \\
\text { north of Carver, Minn. }\end{array}$ & - & - & $3-26-68$ & .44 \\
\hline Minnesota River & $\begin{array}{l}\text { Mississippi } \\
\text { River }\end{array}$ & $\begin{array}{l}\mathrm{SE}_{4}^{1} \mathrm{SW}_{\frac{1}{4}} \text { sec.9, T.II5 N., R. } 23 \mathrm{~W} . \text {, Carver } \\
\text { County, } 2200 \mathrm{ft} \text { upstream from State } \\
\text { Highway } 41 \text { bridge at south edge of } \\
\text { Chaska, Minn. }\end{array}$ & - & - & $3-26-68$ & 921 \\
\hline Chaska Creek & $\begin{array}{l}\text { Minnesota } \\
\text { River }\end{array}$ & $\begin{array}{l}\mathrm{NE}^{\frac{1}{4}} \mathrm{NE}^{\frac{1}{4}} \text { sec. } 8, \mathrm{~T} .115 \mathrm{~N} ., \mathrm{R} .23 \mathrm{~W} . \text {, Carver } \\
\text { County, at bridge on U.S. Highway } 212 \\
\text { in Chaska, Minn., and } 1 \text { mile upstream } \\
\text { from mouth. }\end{array}$ & - & 1967 & $\begin{array}{l}3-19-68 \\
6-14-68\end{array}$ & 303 \\
\hline Chaska Creek & $\begin{array}{l}\text { Minnesota } \\
\text { River }\end{array}$ & $\begin{array}{l}\text { NW } \frac{1}{4} \text { SW } \frac{1}{4} \text { sec.9, T.115 N., R. } 23 \text { W. , Carver } \\
\text { County, at mouth, } 500 \text { ft upstream from } \\
\text { bridge over Minnesota River on State } \\
\text { Highway } 41 \text { in Chaska, Minn. }\end{array}$ & - & - & $3-26-68$ & .67 \\
\hline $\begin{array}{l}\text { Gifford Lake } \\
\text { Outlet }\end{array}$ & $\begin{array}{l}\text { Minnesota } \\
\text { River }\end{array}$ & $\begin{array}{l}\mathrm{NE}_{\frac{1}{4} \mathrm{SE}}^{\frac{1}{4}} \mathrm{sec} .9, \mathrm{~T} .115 \mathrm{~N} ., \mathrm{R} .23 \mathrm{~W} . \text {, Scott } \\
\text { County, at mouth, } 0.6 \text { miles east of } \\
\text { Chaska and } 3 \text { miles west of Shakopee, } \\
\text { Minn. }\end{array}$ & - & - & $3-26-68$ & .74 \\
\hline $\begin{array}{l}\text { East Chaska } \\
\text { Creek }\end{array}$ & $\begin{array}{l}\text { Minnesota } \\
\text { River }\end{array}$ & $\begin{array}{l}\text { SW } 1 \text { SW } \frac{3}{4} \text { sec. } 4 \text {, T.115 N., R. } 23 \text { W., Carver } \\
\text { County, at bridge on U.S. Highway } 212 \\
\text { at Chaska, Minn., and } 1 \text { mile upstream } \\
\text { from mouth. }\end{array}$ & - & 1967 & $\begin{array}{l}3-26-68 \\
6-14-68\end{array}$ & $\begin{array}{l}* 1.19 \\
59.8\end{array}$ \\
\hline $\begin{array}{c}\text { Minnesota River } \\
\text { tributary }\end{array}$ & $\begin{array}{l}\text { Minnesota } \\
\text { River }\end{array}$ & $\begin{array}{l}\text { NW } \frac{1}{4} S W \frac{1}{4} \text { sec. } 3, \text { T. } 115 \mathrm{~N} ., \mathrm{R} .23 \mathrm{~W} . \text {, Carver } \\
\text { County, } 0.8 \text { mile northeast of Chaska, } \\
\text { Minn. }\end{array}$ & - & - & $3-26-68$ & .36 \\
\hline $\begin{array}{c}\text { Minnesota River } \\
\text { tributary }\end{array}$ & $\begin{array}{l}\text { Minnesota } \\
\text { River }\end{array}$ & $\begin{array}{l}\mathrm{NE}^{\frac{1}{4} \mathrm{SW}} \frac{1}{4} \mathrm{sec} .3, \mathrm{~T} .115 \mathrm{~N} ., \mathrm{R} .23 \mathrm{~W} . \text {, Carver } \\
\text { County, } 1 \text { mile northeast of Chaska, } \\
\text { Minn. }\end{array}$ & - & - & $3-26-68$ & 1.34 \\
\hline $\begin{array}{c}\text { Minnesota River } \\
\text { tributary }\end{array}$ & $\begin{array}{l}\text { Minnesota } \\
\text { River }\end{array}$ & $\begin{array}{l}\text { SW } \frac{1}{4} \mathrm{NW}^{\frac{1}{4}} \text { sec.2, T. } 115 \mathrm{~N} ., \mathrm{R} .23 \mathrm{~W} . \text {, Carver } \\
\text { County, }{ }^{2} .2 \text { miles northwest of Shako- } \\
\text { pee, and } 2.2 \text { miles northeast of } \\
\text { Chaska, Minn. }\end{array}$ & - & - & $3-26-68$ & 3.71 \\
\hline $\begin{array}{l}\text { Nyssen Lake } \\
\text { Outlet }\end{array}$ & $\begin{array}{l}\text { Minnesota } \\
\text { River }\end{array}$ & $\begin{array}{l}\text { NW/ } \frac{1}{4} \mathrm{SE}_{\frac{1}{4}} \text { sec. } 2, \mathrm{~T} .115 \mathrm{~N} ., \mathrm{R} .23 \mathrm{~W} . \text {, Scott } \\
\text { County, } 1.2 \text { miles upstream from High- } \\
\text { way } 169 \text { bridge at north edge of Shako- } \\
\text { pee, Minn. }\end{array}$ & - & - & $3-26-68$ & .60 \\
\hline
\end{tabular}




\begin{tabular}{|c|c|c|c|c|c|c|}
\hline \multirow[b]{2}{*}{ Stream } & \multirow[b]{2}{*}{ Tributary to } & \multirow[b]{2}{*}{ Location } & \multirow{2}{*}{$\begin{array}{l}\text { Drainage } \\
\text { area } \\
(\mathrm{sq} \mathrm{mi})\end{array}$} & \multirow{2}{*}{$\begin{array}{c}\text { Measured } \\
\text { previous } 1 \text { y } \\
\text { (water } \\
\text { years) }\end{array}$} & \multicolumn{2}{|c|}{ Measurements } \\
\hline & & & & & Date & $\begin{array}{c}\text { Discharge } \\
\text { (cfs) }\end{array}$ \\
\hline \multicolumn{7}{|c|}{ Minnesota River basin--continued } \\
\hline $\begin{array}{c}\text { Minnesota River } \\
\text { tributary }\end{array}$ & $\begin{array}{l}\text { Minnesota } \\
\text { River }\end{array}$ & $\begin{array}{l}\mathrm{SE}^{\frac{1}{4} \mathrm{NE}^{\frac{1}{4}} \text { sec. }}{ }^{2}, \mathrm{~T} .115 \mathrm{~N} ., \mathrm{R} .23 \mathrm{~W} . \text {, Carver } \\
\text { County, } 0.7 \text { mile upstream from U.S. } \\
\text { Highway } 169 \text {, and } 3.0 \text { miles northeast } \\
\text { of Chaska, Minn. }\end{array}$ & - & - & $3-26-68$ & 4.03 \\
\hline $\begin{array}{c}\text { Minnesota River } \\
\text { tributary }\end{array}$ & $\begin{array}{c}\text { Minnesota } \\
\text { River }\end{array}$ & $\begin{array}{l}\mathrm{NW}_{4} \mathrm{NE}^{\frac{1}{4}} \text { sec. } 6 \text {, T.115 N., R. } 22 \text { W., Scott } \\
\text { County, } 0.1 \text { mile downstream from U.S. } \\
\text { Highway } 169 \text {, at north edge of Shakopee, } \\
\text { Minn. }\end{array}$ & - & - & $3-26-68$ & 1.71 \\
\hline $\begin{array}{c}\text { Minnesota River } \\
\text { tributary }\end{array}$ & $\begin{array}{l}\text { Minnesota } \\
\text { River }\end{array}$ & $\begin{array}{l}\mathrm{SE}_{\frac{1}{4}}^{1} \mathrm{SW}^{\frac{1}{4}} \mathrm{sec} \cdot 32, \mathrm{~T} .116 \mathrm{~N} ., \mathrm{R} .22 \mathrm{~W} . \text {, Scott } \\
\text { County, } 0.9 \text { mile east of Shakopee, } \\
\text { Minn. }\end{array}$ & - & - & $3-26-68$ & 3.24 \\
\hline Minnesota River & $\begin{array}{l}\text { Mississippi } \\
\text { River }\end{array}$ & $\begin{array}{l}\text { On line between sec.5, T.115 N., R.22 W., } \\
\text { Scott County; and sec. } 32, \text { T.116 N., R. } \\
\text { R. } 22 \text { W. Hennepin County, } 2.2 \text { miles } \\
\text { downstream from U.S. Highway } 169 \\
\text { bridge at north edge of Shakopee, Minn. }\end{array}$ & - & - & $3-26-68$ & 954 \\
\hline $\begin{array}{c}\text { Rice Lake } \\
\text { Outlet }\end{array}$ & $\begin{array}{l}\text { Minnesota } \\
\text { River }\end{array}$ & $\begin{array}{l}\mathrm{NE}_{\frac{1}{4} \mathrm{SE}} \frac{1}{4} \text { sec. } 32, \mathrm{~T} .116 \mathrm{~N} ., \mathrm{R} .22 \mathrm{~W} . \text {, Henne- } \\
\text { pin County, } 1.2 \text { miles east of Shakopee, } \\
\text { and } 2.0 \text { miles south of Eden Prairie, } \\
\text { Minn. }\end{array}$ & - & - & $3-26-68$ & 8.26 \\
\hline $\begin{array}{l}\text { Grass Lake } \\
\text { Outlet }\end{array}$ & $\begin{array}{l}\text { Minnesota } \\
\text { River }\end{array}$ & $\begin{array}{l}\text { SE } \frac{1}{4} \mathrm{SE}_{4} \text { sec. } 32, \mathrm{~T} .116 \mathrm{~N} ., \mathrm{R} .22 \mathrm{~W} . \text {, Henne- } \\
\text { pin County, } 1.5 \text { miles east of Shakopee, } \\
\text { and } 2.2 \text { miles south of Eden Prairie, } \\
\text { Minn. }\end{array}$ & - & - & $3-26-68$ & 3.69 \\
\hline $\begin{array}{c}\text { Minnesota River } \\
\text { tributary }\end{array}$ & $\begin{array}{l}\text { Minnesota } \\
\text { River }\end{array}$ & $\begin{array}{l}\mathrm{NW}^{\frac{1}{4}} \mathrm{NW}^{\frac{1}{4}} \mathrm{sec} \cdot 3 \text {, T. } 115 \mathrm{~N} ., \mathrm{R} .22 \mathrm{~W} . \text {, Scott } \\
\text { County, at mouth, } 2.6 \text { miles east of } \\
\text { Shakopee, Minn. }\end{array}$ & $-\cdot$ & - & $3-26-68$ & $\cdot 32$ \\
\hline $\begin{array}{c}\text { Minnesota River } \\
\text { tributary }\end{array}$ & $\begin{array}{l}\text { Minnesota } \\
\text { River }\end{array}$ & 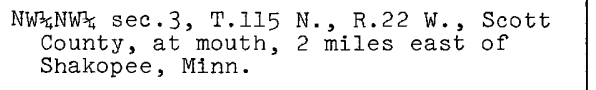 & - & - & $3-26-68$ & .83 \\
\hline Purgatory Creek & $\begin{array}{l}\text { Minnesota } \\
\text { River }\end{array}$ & $\begin{array}{c}\mathrm{NE}_{\frac{1}{4}} \mathrm{SE}^{\frac{1}{4}} \mathrm{sec} .36 \text {, T. } 116 \mathrm{~N} ., \mathrm{R} .22 \mathrm{~W} . \text {, Henne- } \\
\text { pln County, at bridge on county road, } \\
0.4 \text { mile upstream from mouth, and } 4 \\
\text { miles southeast of Eden Prairie, Minn. }\end{array}$ & - & - & $3-26-68$ & 6.84 \\
\hline $\begin{array}{c}\text { Fisher Iake } \\
\text { Outlet }\end{array}$ & $\begin{array}{l}\text { Minnesota } \\
\text { River }\end{array}$ & $\begin{array}{l}\text { NW } \frac{1}{4} S_{W}^{\frac{1}{4}} \text { sec. } 6, \text { T. } 115 \mathrm{~N} ., \text { R. } 21 \text { W. , Scott } \\
\text { County, at culvert on County Highway } \\
25,3 \text { miles northwest of Savage, Minn. }\end{array}$ & - & - & $3-26-68$ & 6.52 \\
\hline Eagle Creek & $\begin{array}{l}\text { Minnesota } \\
\text { River }\end{array}$ & $\begin{array}{l}\text { SE } \frac{1}{4} N^{\frac{1}{4}} \text { sec. } 7 \text {, T. } 115 \text { N., R. } 22 \text { W. Scott } \\
\text { County, at mouth, and } 1.8 \text { miles north- } \\
\text { west of Savage, Minn. }\end{array}$ & - & - & $3-26-68$ & 11.0 \\
\hline Minnesota River & $\begin{array}{l}\text { Mississippi } \\
\text { River }\end{array}$ & $\begin{array}{l}\mathrm{NE}_{\frac{1}{4}} \mathrm{NE}^{\frac{1}{4}} \mathrm{sec} .8, \mathrm{~T} .115 \mathrm{~N} ., \mathrm{R} .21 \mathrm{~W} ., \text { Scott } \\
\text { County, } 0.8 \mathrm{mile} \text { upstream from bridge } \\
\text { on County Road } 31,1.2 \text { miles northwest } \\
\text { of Savage, Minn. }\end{array}$ & - & - & $3-27-68$ & 1,140 \\
\hline $\begin{array}{c}\text { Minnesota River } \\
\text { tributary }\end{array}$ & $\begin{array}{l}\text { Minnesota } \\
\text { River }\end{array}$ & $\begin{array}{l}\mathrm{SE}_{\frac{1}{4} \mathrm{NE}^{\frac{1}{4}}} \text { sec. } 8 \text {, T. } 115 \mathrm{~N} ., \mathrm{R} .21 \mathrm{~W} . \text {, Scott } \\
\text { County, at mouth, and } 1 \text { mile northwest } \\
\text { of Savage, Minn. }\end{array}$ & - & - & $3-27-68$ & 12.6 \\
\hline Credit River & $\begin{array}{l}\text { Minnesota } \\
\text { River }\end{array}$ & $\begin{array}{l}\text { SE } \frac{1}{4} \text { SW sec. } 31 \text {, T. } 27 \text { N. R. R. } 24 \text { W. Scott } \\
\text { County, at bridge on first city street } \\
\text { south of State Highway } 101 \text { in Savage, } \\
\text { Minn., and } 0.6 \text { mile upstream from } \\
\text { mouth. }\end{array}$ & - & - & $3-27-68$ & 6.22 \\
\hline Credit River & $\begin{array}{l}\text { Minnesota } \\
\text { River }\end{array}$ & $\begin{array}{l}\text { SW/ } \mathrm{N}_{4}^{\frac{1}{4}} \text { sec. } 31, \mathrm{~T} .27 \text { N., R. } 24 \text { W., Scott } \\
\text { County, at mouth, at Savage, Minn. }\end{array}$ & - & - & $3-27-68$ & 1.05 \\
\hline $\begin{array}{l}\text { Minnesota River } \\
\text { tributary }\end{array}$ & $\begin{array}{l}\text { Minnesota } \\
\text { River }\end{array}$ & $\begin{array}{l}\mathrm{SE}_{\frac{1}{4} \mathrm{NE}} \frac{\mathrm{m}}{4} \mathrm{sec} .28, \mathrm{~T} .27 \mathrm{~N}, \mathrm{R} .24 \mathrm{~W} . \text {, Dakota } \\
\text { County, at culvert just upstream from } \\
\text { Interstate Highway } 35 \mathrm{~W} \text {, on right bank, } \\
1.7 \text { miles northeast of Savage, Minn. }\end{array}$ & - & - & $3-27-68$ & 11.5 \\
\hline
\end{tabular}


Discharge measurements made at miscellaneous sites during water year 1968

\begin{tabular}{|c|c|c|c|c|c|c|}
\hline \multirow{2}{*}{ Stream } & \multirow{2}{*}{ Tributary to } & \multirow[b]{2}{*}{ Location } & \multirow{2}{*}{$\begin{array}{l}\text { Drainage } \\
\text { area } \\
(\mathrm{sq} \mathrm{mi})\end{array}$} & \multirow{2}{*}{$\begin{array}{c}\text { Measured } \\
\text { previousiy } \\
\text { (water } \\
\text { years) }\end{array}$} & \multicolumn{2}{|c|}{ Measurements } \\
\hline & & & & & Date & $\begin{array}{l}\text { Discharge } \\
\text { (cfs) }\end{array}$ \\
\hline \multicolumn{7}{|c|}{ Minnesota River basin--continued } \\
\hline $\begin{array}{l}\text { Black Dog Lake } \\
\text { upper outlet }\end{array}$ & $\begin{array}{l}\text { Minnesota } \\
\text { River }\end{array}$ & $\begin{array}{l}\text { SW } W^{\frac{1}{4} N W^{2}} \text { sec. } 27 \text {, T. } 27 \text { N., R. } 20 \text { W. , Dakota } \\
\text { County, } 0.2 \text { mile downstream from In- } \\
\text { terstate Highway } 35 \mathrm{~W} \text { bridge, in Burns- } \\
\text { ville, Minn. }\end{array}$ & - & - & $3-27-68$ & 314 \\
\hline Minnesota River & $\begin{array}{l}\text { Mississippi } \\
\text { River }\end{array}$ & $\begin{array}{l}\text { NW }_{4} \mathrm{SE}_{4}^{1} \text { sec. } 13 \text {, T. } 27 \mathrm{~N} ., \mathrm{R} .24 \mathrm{~W} \text {, Dakota } \\
\text { County, } 400 \text { ft upstream from State } \\
\text { Highway } 36 \text {, at northeast edge of } \\
\text { Burnsville, Minn. }\end{array}$ & - & - & $3-27-68$ & 1,460 \\
\hline $\begin{array}{c}\text { Nine Mile } \\
\text { Creek }\end{array}$ & $\begin{array}{c}\text { Minnesota } \\
\text { River }\end{array}$ & 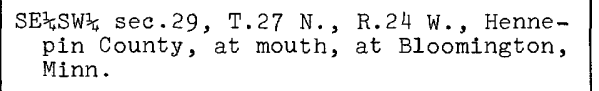 & - & - & $3-27-68$ & $\mathrm{~b} 47 \cdot 3$ \\
\hline \multicolumn{7}{|c|}{ Mississippi River main stem } \\
\hline $\begin{array}{l}\text { Mississippi } \\
\text { River }\end{array}$ & Gulf of Mexico & At Hastings, Dakota County, Minn. & 37,100 & $\mid \begin{array}{r}1929, \\
1931-39, \\
1945-48, \\
1950, \\
1953-57, \\
1959-67\end{array}$ & $6-27-68$ & 17,500 \\
\hline \multicolumn{7}{|c|}{ St. Croix River basin } \\
\hline Crooked Creek & $\begin{array}{l}\text { St. Croix } \\
\text { River }\end{array}$ & $\begin{array}{l}\mathrm{NE}_{\frac{1}{4}} \mathrm{NE}^{\frac{1}{4}} \text { sec. } 30, \mathrm{~T} .4 \mathrm{I} \text { N., R. } 17 \mathrm{~W} \text {, Pine } \\
\text { County, at bridge on State Highway } 48 \text {, } \\
2.8 \text { miles upstream from mouth, and } 19 \\
\text { miles east of Hinckley. Minn. }\end{array}$ & - & $1966-67$ & $\begin{array}{r}10-19-67 \\
4-16-68 \\
5-14-68 \\
6-16-68 \\
7-24-68 \\
8-26-68\end{array}$ & $\begin{array}{r}* 10.6 \\
67.3 \\
196 \\
69.0 \\
* 47.2 \\
* 17.2\end{array}$ \\
\hline Sand Creek & $\underbrace{\text { Croix }}_{\text {River }}$ & $\begin{array}{l}\text { NW } \frac{1}{4} \text { SW } \frac{1}{4} \text { sec. } 13 \text {, T. } 40 \mathrm{~N} ., \mathrm{R} .18 \mathrm{~W} . \text {, Pine } \\
\text { County, at bridge on St. Croix State } \\
\text { Park Road, } 2.5 \text { miles upstream from } \\
\text { mouth, and } 13 \text { miles southeast of } \\
\text { Hinckley, Minn. }\end{array}$ & - & $1966-67$ & $\begin{array}{r}10-23-67 \\
4-16-68 \\
5-14-68 \\
6-18-68 \\
7-24-68 \\
8-26-68\end{array}$ & $\begin{array}{l}* 12.9 \\
83.5 \\
223 \\
96.9 \\
* 74.2 \\
* 25.3\end{array}$ \\
\hline Kettle River & $\begin{array}{l}\text { St. Croix } \\
\text { River }\end{array}$ & $\begin{array}{l}\text { NW } \frac{1}{4} N^{\frac{1}{4}} \text { sec. } .16 \text {, T. } 45 \text { N., R. } 20 \text { W. Pine } \\
\text { County, at bridge on County Highway } 46 \\
2.5 \text { miles west of Sturgeon Lake, Minn. }\end{array}$ & - & $1965-67$ & $\begin{array}{r}10-24-67 \\
4-17-68 \\
5-15-68 \\
6-20-68 \\
7-23-68 \\
8-27-68\end{array}$ & $\begin{array}{l}* 7.77 \\
235 \\
823 \\
437 \\
* 263 \\
* 23.0\end{array}$ \\
\hline Moose River & Kettle River & $\begin{array}{l}\text { NW } \frac{1}{4} \mathrm{SW}^{\frac{1}{4}} \text { sec. } 14, \mathrm{~T} .45 \mathrm{~N} ., \mathrm{R} .20 \mathrm{~W} . \text {, Pine } \\
\text { County, at bridge on County Highway } 46 \\
\text { at Sturgeon Lake, Minn., and } 1.5 \text { miles } \\
\text { upstream from mouth. }\end{array}$ & - & $1965-67$ & $\begin{array}{r}10-23-67 \\
4-17-68 \\
5-15-68 \\
6-19-68 \\
7-23-68 \\
8-27-68\end{array}$ & $\begin{array}{l}* 13.7 \\
148 \\
292 \\
250 \\
* 141 \\
* 37.1\end{array}$ \\
\hline Kettle River & St. Crolx & 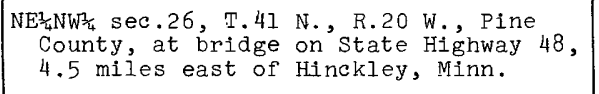 & - & $1966-67$ & $6-20-68$ & 2,050 \\
\hline Knife River & Snake River & $\begin{array}{l}\mathrm{SE}_{\frac{1}{4} \mathrm{SE}} \frac{1}{4} \text { sec. } 27, \mathrm{~T} .40 \mathrm{~N}, \mathrm{R}, 24 \mathrm{~W} ., \text { Kanabec } \\
\text { County, at bridge on County Highway } 77, \\
2.5 \text { miles north of Mora, Minn. }\end{array}$ & - & $1966-67$ & $\begin{array}{r}10-24-67 \\
4-17-68 \\
5-15-68 \\
6-19-68 \\
7-24-68 \\
8-28-68\end{array}$ & $\begin{array}{l}* 2.11 \\
62.9 \\
113 \\
126 \\
* 25.5 \\
* 4.04\end{array}$ \\
\hline Snake River & $\begin{array}{l}\text { St. Croix } \\
\text { River }\end{array}$ & 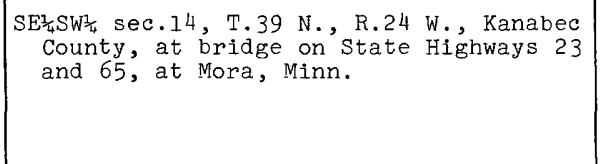 & - & $1966-67$ & $\begin{array}{r}10-24-67 \\
4-17-68 \\
5-15-68 \\
6-19-68 \\
7-24-68 \\
8-24-68\end{array}$ & $\begin{array}{l}* 38.4 \\
269 \\
639 \\
417 \\
* 214 \\
* 41.2\end{array}$ \\
\hline $\begin{array}{l}\text { Groundhouse } \\
\text { River }\end{array}$ & Snake River & 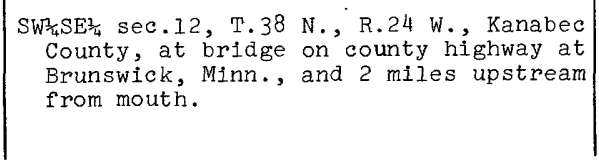 & - & $1966-67$ & $\begin{array}{r}10-24-67 \\
4-18-68 \\
5-16-68 \\
6-19-68 \\
7-24-68 \\
8-27-68\end{array}$ & $\begin{array}{l}* 7.77 \\
50.3 \\
105 \\
87.2 \\
* 27.4 \\
* 8.66\end{array}$ \\
\hline
\end{tabular}

* Base flow.

b May not represent total flow. 
Discharge measurements made at miscellaneous sites during water year 1967

\begin{tabular}{|c|c|c|c|c|c|c|}
\hline \multirow{2}{*}{ Stream } & \multirow{2}{*}{ Tributary to } & \multirow[b]{2}{*}{ Location } & \multirow{2}{*}{$\begin{array}{l}\text { Drainage } \\
\text { area } \\
\text { (sq } \mathrm{mi})\end{array}$} & \multirow{2}{*}{$\begin{array}{c}\text { Measured } \\
\text { previous ly } \\
\text { '(water } \\
\text { 'years) }\end{array}$} & \multicolumn{2}{|c|}{ Measurements } \\
\hline & & & & & Date & $\begin{array}{l}\text { Discharge } \\
\quad(\mathrm{cfs})\end{array}$ \\
\hline \multicolumn{7}{|c|}{ St. Croix River basin--continued } \\
\hline Rock Creek & St. Croix & $\begin{array}{l}\mathrm{NW}_{4} \mathrm{NE}_{4} \text { sec. } 7, \mathrm{~T} .37 \mathrm{~N} ., \mathrm{R} .20 \mathrm{~W} . \text {, Chisago } \\
\text { County, at bridge on County Highway } 3 \text {, } \\
4.5 \text { miles northeast of Rush City, } \\
\text { Minn. }\end{array}$ & - & 1967 & $9-9-68$ & $* 4.37$ \\
\hline Rush Creek & St. Croix & $\begin{array}{l}\mathrm{NW}_{4} \mathrm{NW}^{\frac{3}{4}} \text { sec. } 31, \mathrm{~T} .37 \mathrm{~N} ., \text { R. } 20 \text { W., Chisago } \\
\text { County, at bridge on County Highway } \\
56,3.5 \text { miles southeast of Rush City, } \\
\text { Minn. }\end{array}$ & - & 1967 & $9-9-68$ & *9.75 \\
\hline $\begin{array}{l}\text { North Branch } \\
\text { Sunrise River }\end{array}$ & Sunrise River & $\begin{array}{l}\text { On line between secs. } 18 \text { and } 19, \mathrm{~T} .35 \mathrm{~N} . \\
\text { R. } 20 \text { W. Chisago County, at bridge on } \\
\text { State Highway } 95,4 \text { miles east of } \\
\text { North Branch, Minn. }\end{array}$ & - & - & $9-9-68$ & *29.5 \\
\hline $\begin{array}{l}\text { St. Croix } \\
\text { River }\end{array}$ & $\begin{array}{l}\text { Mississippi } \\
\text { River }\end{array}$ & At Prescott, Pierce County, Wis. & 7,650 & $\left|\begin{array}{r}1928+30 \\
1932-39 \\
1946-48 \\
1950 \\
1953-57 \\
1959-67\end{array}\right|$ & $6-27-68$ & 17,600 \\
\hline \multicolumn{7}{|c|}{ Vermillion River basin } \\
\hline $\begin{array}{l}\text { Vermillion } \\
\text { River }\end{array}$ & $\begin{array}{l}\text { Mississippi } \\
\text { River }\end{array}$ & $\begin{array}{l}\mathrm{SE}_{4} \mathrm{SW}_{4} \text { sec. } 33 \text {, T. } 115 \text { N., R.l7 W., Dakota } \\
\text { County, at highway bridge in Hastings, } \\
0.7 \text { mile upstream from mill dam, and } \\
3 \text { miles upstream from Vermillion } \\
\text { Slough. }\end{array}$ & 195 & $\left|\begin{array}{l}1942-47 \neq \\
1966-67\end{array}\right|$ & $5-16-68$ & 131 \\
\hline \multicolumn{7}{|c|}{ Cannon River basin } \\
\hline Straight River & Cannon River & $\begin{array}{l}\text { SE } \frac{1}{4} \text { sec.9, T. } 107 \mathrm{~N} ., \text { R. } 20 \mathrm{~W} . \text { Steele } \\
\text { County, at bridge on West Bridge } \\
\text { Street, in Owatonna, Minn. }\end{array}$ & - & $1966-67$ & $\begin{array}{r}10-31-67 \\
4-30-68 \\
5-27-68 \\
7-2-68 \\
8-5-68 \\
9-12-68\end{array}$ & $\begin{array}{r}* 22.0 \\
57.6 \\
77.9 \\
123 \\
79.1 \\
88.3\end{array}$ \\
\hline Crane Creek & Straight River & $\begin{array}{l}\mathrm{SE}_{4}^{\frac{1}{4} N W / 4} \text { sec. } 20, \mathrm{~T} .108 \mathrm{~N} ., \mathrm{R} .20 \mathrm{~W} . \text {, Steele } \\
\text { County, at culvert on service road, } 1 \\
\text { mile northwest of Clinton Falls, Minn. }\end{array}$ & - & $1965-67$ & $\begin{array}{r}10-30-67 \\
11-20-67 \\
12-19-67 \\
1-23-68 \\
2-22-68 \\
3-25-68 \\
5-1-68 \\
5-28-68 \\
7-2-68 \\
8-5-68 \\
9-12-68\end{array}$ & $\begin{array}{r}2.18 \\
1.16 \\
2.00 \\
2.75 \\
1.74 \\
3.17 \\
18.0 \\
67.5 \\
52.8 \\
195 \\
76.6\end{array}$ \\
\hline Cannon River & $\begin{array}{l}\text { Mississippi } \\
\text { River }\end{array}$ & $\begin{array}{l}\mathrm{SW}_{4} \mathrm{NE} \frac{1}{4} \mathrm{sec} .30 \text {, T.IIO N., R. } 20 \text { W. Rice } \\
\text { County, at mili dam upstream from } \\
\text { bridge on State Highway } 3 \text {, in Fari- } \\
\text { bault, Minn. }\end{array}$ & - & $1965-67$ & $\begin{array}{r}10-30-67 \\
11-20-67 \\
12-19-67 \\
1-22-68 \\
2-22-68 \\
3-25-68 \\
5-1-68 \\
5-28-68 \\
7-3-68 \\
8-2-68 \\
9-12-68\end{array}$ & $\begin{array}{c}11.7 \\
3.67 \\
11.9 \\
* 7.51 \\
11.9 \\
20.7 \\
31.0 \\
66.5 \\
50.4 \\
222 \\
81.4\end{array}$ \\
\hline Prairie Creek & Cannon River & $\begin{array}{l}\text { On line between secs. } 21 \text { and } 28, \mathrm{~T} .112 \mathrm{~N} \text {. } \\
\text { R. } 18 \text { W. Goodhue County, at bridge on } \\
\text { State Highway } 19,4.5 \text { miles southwest } \\
\text { of Cannon Falls, Minn. }\end{array}$ & - & $1966-67$ & $\begin{array}{r}10-31-67 \\
4-19-68 \\
5-13-68 \\
7-5-68 \\
8-5-68 \\
8-26-68\end{array}$ & $\begin{array}{r}* 8.16 \\
* 7.37 \\
* 6.16 \\
8.24 \\
14.7 \\
12.3\end{array}$ \\
\hline $\begin{array}{l}\text { Little Cannon } \\
\text { River }\end{array}$ & Cannon River & $\begin{array}{l}\mathrm{SW}_{\frac{3}{4}} \text { sec. } 25, \mathrm{~T} .112 \mathrm{~N} . \text {, R. } 18 \mathrm{~W} ., \text { Goodhue } \\
\text { County, at bridge on county highway, } \\
2.5 \text { miles south of Cannon Falls, Minn. }\end{array}$ & - & $1966-67$ & $\begin{array}{r}10-31-67 \\
4-19-68 \\
5-13-68 \\
7-5-68 \\
8-8-68 \\
8-26-68\end{array}$ & $\begin{array}{c}* 14.5 \\
* 5.83 \\
* 11.4 \\
* 23.9 \\
29.4 \\
* 21.0\end{array}$ \\
\hline
\end{tabular}

* Base flow.

f Operated asia continuous-record gaging station. 
Discharge measurements made at miscellaneous sites during water year 1968

\begin{tabular}{|c|c|c|c|c|c|c|}
\hline \multirow[b]{2}{*}{ Streeam } & \multirow[b]{2}{*}{ Tributary to } & \multirow[b]{2}{*}{ Location } & \multirow{2}{*}{$\begin{array}{l}\text { Drainage } \\
\text { area } \\
\text { (sq } \mathrm{mi} \text { ) }\end{array}$} & \multirow{2}{*}{$\begin{array}{c}\text { Measured } \\
\text { previous } 1 \text { y } \\
\text { (water } \\
\text { years) }\end{array}$} & \multicolumn{2}{|c|}{ Measurements } \\
\hline & & & & & Date & $\begin{array}{l}\text { Discharge } \\
\text { (cfs) }\end{array}$ \\
\hline \multicolumn{7}{|c|}{ Cannon River basin--continued } \\
\hline Belle Creek & Cannon River & $\begin{array}{l}\text { SE }^{\frac{1}{4}} \text { sec. } 4, \text { T. } 112 \text { N., R. } 16 \text { W., Goodhue } \\
\text { County, at bridge on county highway, } \\
2 \text { miles north of Vasa, Minn. }\end{array}$ & - & $1966-67$ & $\begin{array}{r}4-19-68 \\
5-13-68 \\
7-5-68 \\
8-8-68 \\
9-10-68\end{array}$ & $\begin{array}{l}0 \\
* 5.54 \\
13.0 \\
14.7 \\
19.1\end{array}$ \\
\hline \multicolumn{7}{|c|}{ Zumbro River basin } \\
\hline $\begin{array}{l}\text { South Fork } \\
\text { Zumbro River }\end{array}$ & Zumbro River & 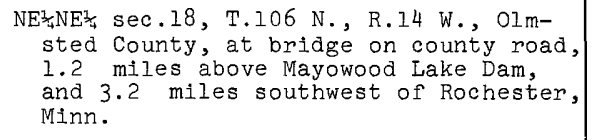 & - & - & $3-22-68$ & 13.0 \\
\hline \multicolumn{7}{|c|}{ Burns Valley Creek basin } \\
\hline $\begin{array}{l}\text { Burns Valley } \\
\text { Creek }\end{array}$ & $\begin{array}{l}\text { Mississippi } \\
\text { River }\end{array}$ & $\begin{array}{l}\text { SEt sec. } 35 \text {, T. } 107 \mathrm{~N} . \text {, R. } 7 \text { W., Winona } \\
\text { County, at bridge on County Highway } \\
17 \text {, at southeast edge of Winona, Minn. }\end{array}$ & - & 1967 & $4-22-68$ & 6.41 \\
\hline \multicolumn{7}{|c|}{ Pleasant Valley Creek basin } \\
\hline $\begin{array}{l}\text { Pleasant Valley } \\
\text { Creek }\end{array}$ & $\begin{array}{l}\text { Mississippi } \\
\text { River }\end{array}$ & $\begin{array}{l}\text { On line between sec. } 36, \mathrm{~T} .107 \mathrm{~N} . \mathrm{R} .7 \mathrm{~W} . \\
\text { and sec.l, T. } 106 \mathrm{~N} \text {., R. } 7 \mathrm{~W} \text {, Winona } \\
\text { County, at bridge on County Highway 15, } \\
\text { at southeast edge of Winona, Minn. }\end{array}$ & - & 1967 & $4-22-68$ & $7 \cdot 59$ \\
\hline \multicolumn{7}{|c|}{ Mississippi River main stem } \\
\hline $\begin{array}{l}\text { Mississippi } \\
\text { River }\end{array}$ & Gulf of Mexico & $\begin{array}{l}\text { At lock and dam No. } 8 \text {, Vernon County, } \\
\text { near Genoa, Wis. }\end{array}$ & - & $1966-67$ & $7-23-68$ & 61,600 \\
\hline \multicolumn{7}{|c|}{ Des Moines River basin } \\
\hline $\begin{array}{l}\text { West Fork Des } \\
\text { Moines River }\end{array}$ & $\begin{array}{l}\text { Des Moines } \\
\text { River }\end{array}$ & $\begin{array}{l}\text { Near center of sec. } 20 \text {, T. } 105 \mathrm{~N} ., \text { R. } 38 \mathrm{~W} \text {.' } \\
\text { Cottonwood County, at outlet of Talcot } \\
\text { Lake, } 3.2 \text { miles northeast of Dundee, } \\
\text { Minn. }\end{array}$ & - & $1963-67$ & $\begin{array}{r}10-12-67 \\
11-15-67 \\
12-11-67 \\
1-9-68 \\
2-12-68 \\
3-11-68 \\
3-19-68 \\
4-8-68 \\
5-10-68 \\
7-10-68 \\
8-21-68 \\
9-19-68\end{array}$ & $\begin{array}{r}0 \\
* .36 \\
* .33 \\
* .38 \\
* .34 \\
* .26 \\
116 \\
.78 \\
.36 \\
9.86 \\
29.3 \\
74.6\end{array}$ \\
\hline \multicolumn{7}{|c|}{ Big Sioux River basin } \\
\hline Rock River & $\begin{array}{l}\text { Big Sioux } \\
\text { River }\end{array}$ & 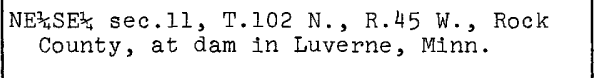 & 440 & $1911-14 \neq$ & $\begin{array}{r}10-12-67 \\
9-18-68\end{array}$ & $\begin{array}{l}5.07 \\
10.6\end{array}$ \\
\hline
\end{tabular}

* Base flow.

F Operated as a continuous-record gaging station. 
United States Department of the Interior Geological Survey - Water Resources Division

\author{
WATER RESOURCES DATA \\ FOR \\ MINNESOTA
}

1968

Part 2. Water Quality Records

Prepared in cooperation with

Minnesota Department of Conservation, Division of Waters, Soils, and Minerals Corps of Engineers, U. S. Army

Copies of this report may be obtained from

District Chief, Water Resources Division

U.S. Geological Survey

1033 Post Office Building

St. Paul, Minnesota 55101 


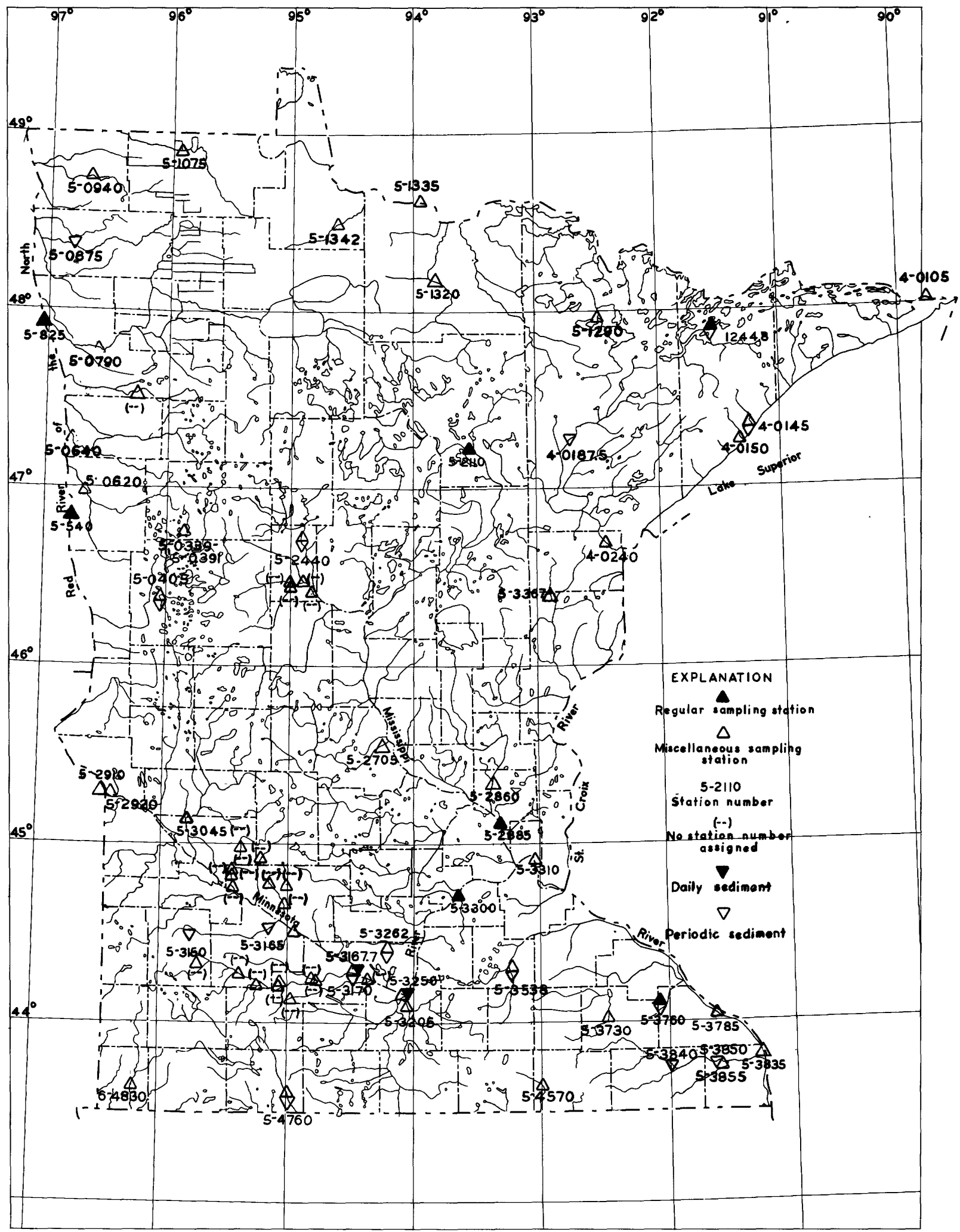

Figure 1.-- Map showing location of water-quality stations in Minnesota. 
CONTENTS

Part 2. Water Quality Records

[Symbols after station name designate type of data: c, chemical;

$t$, water temperature; $s$, sediment]

Page

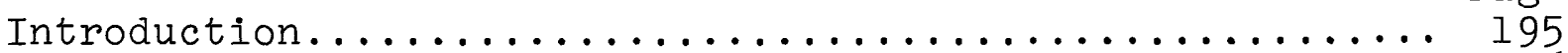

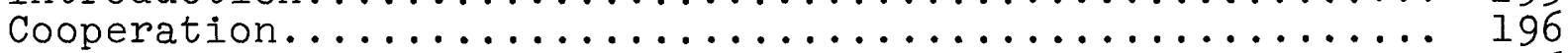

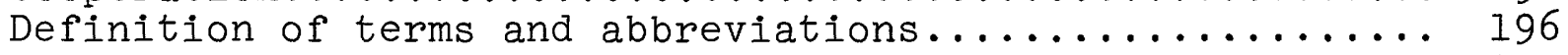

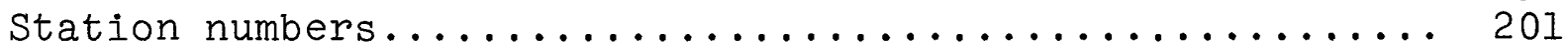

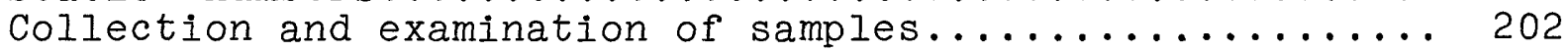

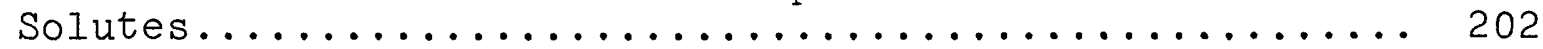

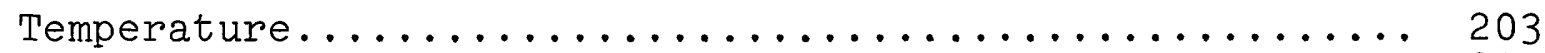

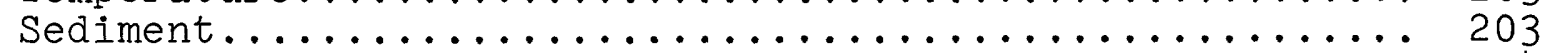

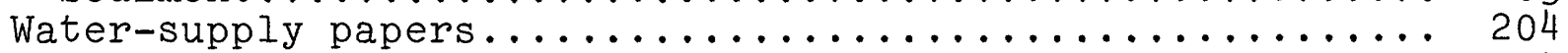

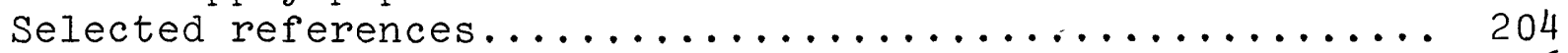

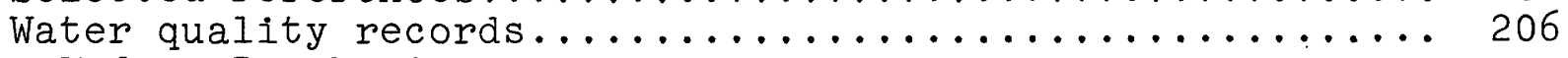

Hudson Bay basin

Lake Winnipeg:

Red River of the North basin

Red River of the North at Fargo, N. Dak. (ct)....... 206

Red River of the North at Grand Forks,

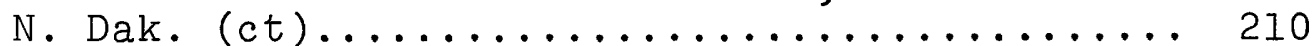

Lake of the Woods basin

Namakan River:

Basswood River:

Kawishiwi River near Ely (cst)........... 214

Upper Mississippi River basin

Mississippi River:

Mississippi River near Anoka (c)................. 218

Minnesota River basin

Whetstone River near Big Stone City, S. Dak. (c).... 220

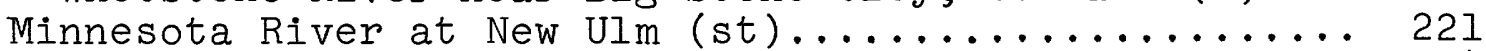

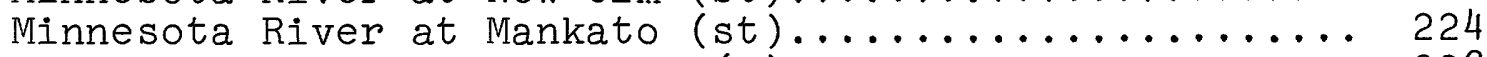

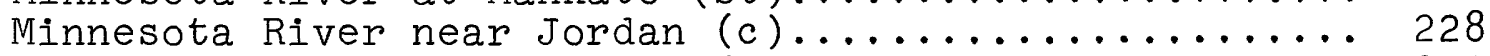

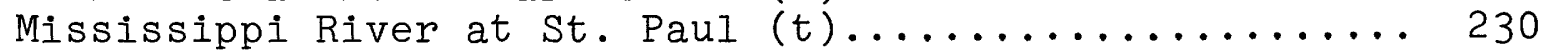

Whitewater River basin

North Fork Whitewater River near Elba (cs)......... 232

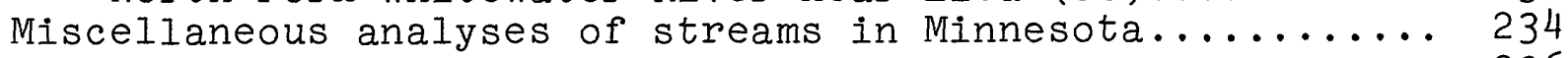

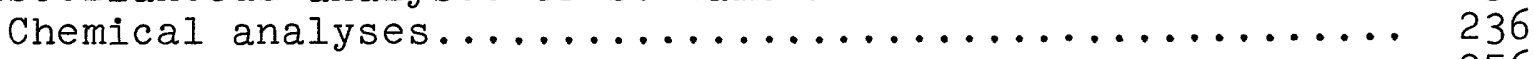

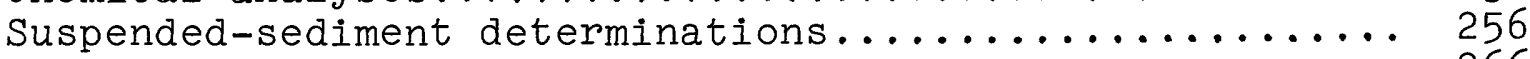

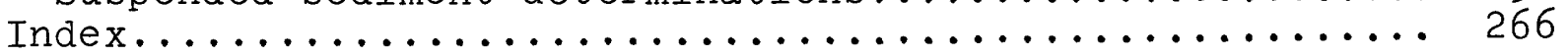


Figure 1. Map showing locations of water-quality stations in Minnesota................ 192 


\section{WATER RESOURCES DATA FOR MINNESOTA, 1968}

Part 2. Water Quality Records

\section{INTRODUCTION}

Water-resources investigations of the U.S. Geological Survey include the collection of water quality data on the chemical and physical characteristics of surface- and groundwater supplies of the Nation. These data for the 1968 water year for the quality of surface waters in Minnesota are presented in this report. Data for a few water quality stations in bordering states are also included. The data were collected by the Water Resources Division of the U.S. Geological Survey under the direction of, Charles R. Collier, District Chief.

Water quality information is presented for chemical quality, water temperatures, and fluvial sediment. Chemical quality includes concentrations of individual dissolved constituents and certain properties or characteristics such as hardness, sodium-adsorption-ratio, specific conductance, and $\mathrm{pH}$. Water-temperature data represent once-daily observations except for stations where a continuous temperature recorder furnishes information from which daily minimums and maximums are obtained. Fluvial-sediment information is given for suspended-sediment discharges and concentrations and for particle-size distribution of suspended sediment and bed material.

The Geological Survey, from 1941 through 1965, published an annual series of water-supply papers, "Quality of Surface Waters of the United States," which contained the chemical quality, temperature, and suspended-sediment data of the water. Each volume covered an area whose boundaries coincided with those of certain natural drainage areas. The records for Minnesota are contained in Parts 4, 5, and 6 of the watersupply paper series. (See table 3, p. .) These publications are available in most public libraries. Beginning with the 1964 water year, water quality records for surface water obtained by the Geological Survey were published in a new series of annual releases on a state boundary basis. This report is primarily for local and immediate use, and its distribution is limited. The records will be published in Geological Survey water-supply papers.

Prior to the 1968 water year, data for chemical constituents and concentration of suspended sediment were reported 
in parts per million (ppm) and water temperatures were reported in degrees Fahrenheit $\left({ }^{\circ} \mathrm{F}\right)$. In October 1967 the U.S. Geological Survey began to use the metric system; data for chemical constituents and concentrations of suspended sediment are now reported in milligrams per liter (mg/l) and water temperatures are given in degrees Celsius (centigrade, ${ }^{\circ} \mathrm{C}$ ). In waters with a density of $1.000 \mathrm{~g} / \mathrm{ml}$ (grams per miliiliter), parts per million and milligrams per liter can be considered equal. In waters with a density greater than $1.000 \mathrm{~g} / \mathrm{ml}$, values in parts per million should be multiplied by the density to convert to milligrams per liter. To convert temperatures in degrees Fahrenheit to degrees Celsius, subtract $32^{\circ}$ and divide by 1.8. (See section "Definition of Terms and Abbreviations" for further information.)

\section{COOPERATION}

Most of the records for Minnesota were obtained as a part of a cooperative program with the Minnesota Department of Conservation, Division of Waters, Soils, and Minerals, Eugene R. Gere, Director. Some records for the Red River of the North, which borders the State on the west, were obtained at the request of other Federal agencies as a part of the program of the U.S. Department of the Interior for development of the Missouri River basin.

\section{DEFINITION OF TERMS AND ABBREVIATIONS}

The terms and abbreviations of water-quality and hydrologic data, as used in the text and tabular data of this report, are defined below:

Acre-foot $(a c-f t)$ is a quantity of water required to cover 1 acre to a depth of $I$ foot and is equivalent to 43,560 cubic feet or 325,851 gallons.

Biochemical oxygen demand (BOD) is the amount of oxygen required by bacteria while stablizing decomposable organic matter under aerobic conditions.

Cfs-day is the volume of water represented by a flow of 1 cubic foot per second for 24 hours. It is equivalent to 86,400 cubic feet, 1.983471 acre-feet, or 646,317 gallons.

Chemical oxygen demand (COD) indicates the quantity of oxidizable compounds present in a water and will vary with water compositions, concentration of reagent, temperature, period of contact, and other factors. 
Coliform organisms are a group of bacteria used as an indicator of the sanitary quality of the water. The number of coliform colonies per 100 milliters is determined by the delayed incubation membrane filter method.

Cubic foot per second (cfs) is the rate of discharge through a cross-sectional area of 1 square foot of a stream at an average velocity of $I$ foot per second.

Discharge, in its simplest concept, means outflow; therefore, the use of this term is not restricted as to course or location. In this report it represents the total fluids measured in the stream.

Mean discharge is the arithmetic mean of individual daily mean discharges during a specific period.

Instantaneous discharge is the discharge at time of sampling. If this discharge is reported instead of the daily mean, the heading of the discharge column is "Discharge (cfs)."

Drainage area of a stream above a specified location is that area, measured in a horizontal plane, enclosed by a topographic divide from which direct surface runoff from precipitation normally drains by gravity into the river above the specified point.

Drainage basin is a part of the surface of the earth that is occupied by a drainage system, which consists of a surface stream or body of impounded surface water together with all tributary surface streams and bodies of impounded surface water.

Gaging station is a particular site on a stream, canal, lake, or reservoir where systematic observations of gage height or discharge are obtained. When used in connection with a discharge record, the term is applied only to those gaging stations where a continuous record of discharge is obtained.

Hardness of water is the property of water attributable to the presence of alkaline earths and is expressed as equivalent calcium carbonate $\left(\mathrm{CaCO}_{3}\right)$. Hardness is a physical-chemical characteristic, not a substance.

Hydrologic bench-mark station is one that provides hydrologic data for a basin in which the hydrologic regimes will likely be governed solely by natural conditions. Data collected at a bench-mark station may be used to separate effects 
of natural from manmade changes in other basins which have been developed and in which the physiography, climate, and geology are similar to those in the undeveloped bench-mark basin.

Methylene blue active substance (MBAS) is a measure of apparent detergents. This determination depends on the formation of a blue color when methylene blue dye reacts with synthetic detergent compounds.

Milligrams per liter $(\mathrm{mg} / \mathrm{l})$ is a unit for expressing the concentration of chemical constituents in solution. Miligrams per liter represents the weight of solute per unit volume of water. Milligrams per liter may be converted to milliequivalents per liter by multiplying by the factors in table 1 , page. Concentration of suspended sediment expressed in milligrams per liter is based on the weight of sediment in a liter of water-sediment mixture. Sediment corcentrations that are expressed in parts per million may be converted to milligrams per liter by using the factors in table 2, page.

Most probable number (MPN) is computed from probability analysis based on the number of positive findings of coliform group organisms resulting from multiple-dilation decimal dilutions. (Standard Methods, 12th edition, p. 604)

Partial-record station is a particular site where limited data are collected systematically over a period of years for use in hydrologic analyses.

Particle size is the diameter, in millimeters ( $\mathrm{mm}$ ), of suspended sediment or bed material determined by sieve and sedimentation methods.

Particle size classification, used in this report agrees close with recommendations made by the American Geophysical Union Subcommittee on Sediment Terminology (Lane and others, 1947, p. 937). The classification is as follows:

$\begin{array}{ll}\text { Clay: } & \text { Smaller than } 0.004 \mathrm{~mm} . \\ \text { Silt: } & \text { Between } 0.004 \text { and } 0.062 \mathrm{~mm} \text {. } \\ \text { Sand: } & \text { Between } 0.062 \text { and } 2.0 \mathrm{~mm} . \\ \text { Gravel: } & \text { Between } 2.0 \text { and } 64.0 \mathrm{~mm} .\end{array}$

The particle size distributions given in this report are not necessarily representative of the particle sizes of sediment in transport in the natural stream. Most of the organic matter is removed and the sample is subjected to mechanical and 
chemical dispersion before analysis of the silt and clay.

Sediment is solid material that originates mostly from disintegrated rocks and is transported by, suspended in, or deposited from water; it includes chemical and biochemical precipitates and decomposed organic material such as humus. The quantity, characteristics, and cause of the occurrence of sediment in streams are influenced by environmental factors. Some major factors are degree of slope, length of slope, soil characteristics, land usage, and quantity and intensity of precipitation.

Sediment discharge is the rate at which dry weight of sediment passes a section of a stream or is the quantity of sediment, as measured by dry weight, or by volume, that is discharged in a given time.

Solute is any substance derived from the atmosphere, vegetation, soil, or rocks and is dissolved in water.

Specific conductance is a measure of the ability of a water to conduct an electrical current and is expressed in micromhos per centimeter at $25^{\circ} \mathrm{C}$. Because the specific conductance is related to the number and specific chemical types of ions in solution, it can be used for approximating the dissolved-solids content in the water. Commonly, the amount of dissolved solids (in milligrams per liter) is about 65 percent of the specific conductance (in micromhos). This relation is not constant from stream to stream or from well to well, and it may even vary in the same source with changes in the composition of the water.

Sodium adsorption ratio (SAR) is the expression of relative activity of sodium ions in exchange reactions with soil and is an index of sodium or alkali hazard to the soil. This ratio should be known especially for water used for irrigating farmland.

Streamflow is the discharge that occurs in a natural channel. Although the term "discharge" can be applied to the flow of a canal, the word "streamflow" uniquely describes the discharge in a surface stream course. The term "streamflow" is more general than "runof'f." Streamflow may be applied to discharge whether or not it is affected by diversion or regulation.

Suspended sediment is the sediment that at any given time is maintained in suspension by the upward components of turbulent currents or that exists in suspension as a colloid. 
Table 1.--Factors for conversion of chemical constituents in milligrams per liter to milliequivalents per liter

Ion
Multi-

ply by

Aluminum $\left(\mathrm{Al}^{+3}\right)_{1} \ldots \ldots$

Barium $\left(\mathrm{Ba}^{+2}\right) \ldots \ldots$.

Bicarbonate $\left(\mathrm{HCO}_{3}-\mathbf{i}\right)$

Bromide $\left(\mathrm{Br}^{-1}\right)$

Calcium $(\mathrm{Ca}+2)$

........ .04990

Carbonate $\left(\mathrm{CO}_{3}-2 \mathrm{j} \ldots . . .0333\right.$

Chloride $\left(\mathrm{Cl}^{-1}\right) \ldots \ldots . .02821$

Chromium $(\mathrm{Cr}+6) \ldots \ldots$........ .11539

Cobalt $\left(\mathrm{Co}^{+2}\right) \ldots \ldots \ldots . \quad .03394$

Copper $\left.\left(\mathrm{Cu}^{+2}\right) \ldots \ldots \ldots \ldots\right) \quad .03148$

Cyanide $\left.\left(\mathrm{CN}^{-1}\right) \ldots \ldots \ldots\right) \quad .03844$

Fluoride $\left(\mathrm{F}^{-1}\right) \ldots \ldots \ldots .0 .05264$

Hydrogen $\left(\mathrm{H}^{+1}\right) \ldots \ldots \ldots . .96209$

Hydroxide $\left(\mathrm{OH}^{-1}\right) \ldots \ldots . \quad .05880$
Ion

Iodide $\left(I^{-1}\right) \ldots \ldots 0.00788$

Iron $(\mathrm{Fe}+3)$

Lead $(\mathrm{Pb}+2)$

Lithium ( $\mathrm{Li}+1 j$

Magnesium $\left(\mathrm{Mg}^{+2}\right) \ldots . \quad .08226$

Manganese $\left(\mathrm{Mn}^{+2}\right) \ldots .03640$

Nickel $\left(\mathrm{Ni}^{+2}\right) \ldots . .03406$

Nitrate $\left(\mathrm{NO}_{3}-1 \mathrm{j}\right) . . .01613$

Nitrite $\left(\mathrm{NO}_{2}{ }^{-1}\right) \ldots .02174$

Phosphate $\left(\mathrm{PO}^{-3}\right) . \quad .03159$

Potassium $\left(\mathrm{K}^{+1}\right) \ldots .02557$

Sodium $\left(\mathrm{Na}^{+1}\right) \ldots . .04350$

Strontium $\left(\mathrm{Sr}^{+2}\right) \ldots \quad .02283$

Sulfate $\left(\mathrm{SO}^{-2}\right) \ldots . \quad .02082$

Zinc $\left(\mathrm{Zn}^{+2}\right) \ldots \ldots . \quad .03060$

Table 2.--Factors for conversion of sediment concentration in parts per million to milligrams per liter* (All values calculated to three significant figures)

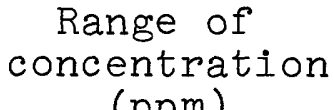
(ppm)

$0-15,900$
$16,000-46,800$
$46,900-76,500$
$76,600-105,000$
$106,000-133,000$
$134,000-159,000$
$160,000-185,000$
$186,000-210,000$
$211,000-233,000$
$234,000-256,000$
$257,000-279,000$
$280,000-300,000$
$301,000-321,000$

Multiply by

1.00

1.02

1.04

1.06

1.08

1.10

1.12

1.14

1.16

1.18

1.20

1.22

1.24
Range of concentration (ppm)
Multiply by

1.26

1.28

1. 30

1. 32

1.34

1.36

1.38

1.40

1.42

1.44

1.46

1.48

1.50

*Based on water density of $1.000 \mathrm{~g} / \mathrm{ml}$ and sediment density of $2.65 \mathrm{~g} / \mathrm{cc}$. 
Thermograph is a thermometer that continuously and automatically records, on a chart, the water temperature of a stream. "Temperature recorder" is the term used to indicate the location of the thermograph.

Time-weighted average is computed by multiplying the number of days in the sampling period by the concentrations of individual constituents for the corresponding period and dividing the sum of the products by the total number of days. A time-weighted average represents the composition of water that would be contained in a vessel or reservoir that had received equal quantities of water from the stream each day for the water year.

Tons per acre-foot indicates the dry weight of dissolved solids in l acre-foot of water. It is computed by multiplying the concentration in milligrams per liter by 0.00136 .

Tons per day is the quantity of a substance in solution or suspension that passes a stream section during a 24-hour period.

Water year in Geological Survey reports dealing with surface water supply is the 12-month period, October 1 through September 30. The water year is designated by the calendar year in which it ends and which includes 9 of the 12 months. Thus, the year ending September 30, 1968, is called the "1968 water year."

Weighted average is used in this report to indicate discharge-weighted average. It is computed by multiplying the discharge for a sampling period by the concentrations of individual constituents for the corresponding period and dividing the sum of the products by the sum of the discharges. A discharge-weighted average approximates the composition of water that would be found in a reservoir containing all the water passing a given location during the water year after thorough mixing in the reservoir.

\section{STATION NUMBERS}

A station number has been assigned as an added means of identification for each stream location where regular measurements of streamflow and determinations of water quality have been made. The numbers have been assigned to conform with the standard downstream order of listing gaging stations. The numbering system consists of 2 digits followed by a hyphen and 
a 6-digit number. The notation to the left of the hyphen identifies the Part or hydrologic region used by the Geological Survey for reporting hydrologic data. The number to the right of the hyphen represents the position of the location in the standard downstream order listing the stations within each of the parts. The assigned numbers are in numerical order but are not consecutive. They are so selected from the complete 6-digit-number scale that intervening numbers will be available for future assignments to new locations. The identification number for each station in this report is printed to the left of the station name and contains only the essential digits. For example, the number is printed as 5-3310 for a station whose complete identification number is $05-3310.00$.

\section{COLLECTION AND EXAMINATION OF SAMPLES}

Water samples for analyses usually are collected at or near points on streams where gaging stations are maintained by the U.S. Geological Survey for measurement of water discharge. Discharge records for streams in Minnesota are contained in Part 1 of this report. Most of these records are used in conjunction with the computations of the chemical constituents and sediment loads in this report.

Data on the quality of surface water were collected daily at some sites and less frequently at other sites; the locations of the sites are shown on the map on page

\section{Solutes}

The methods of collecting and analyzing water samples for determining the kinds and concentrations of solutes are described by Rainwater and Thatcher (1960). One sample can define adequately the water quality at a given time if the mixture of solutes throughout the stream cross section is homogeneous. However, the concentration of solutes at different locations in the cross section may vary widely with different rates of water discharge depending on the source of material and the turbulence and the mixing of the stream. Some streams must be sampled at several verticals across the channel to determine accurately the solute load.

The daily chemical quality data in this report generally represent equal-volume composites for 2- to 30-day periods; the composite periods are selected on the basis of specific conductance of the daily samples and fluctuation of water dìscharge. 
Water temperatures were measured at most of the water quality stations. For daily stations, the water temperatures were taken at about the same time each day in order that the data would not reflect normal variations in water temperature. Most large streams have a small diurnal variation in water temperature; small, shallow streams may have a daily range of several degrees and may follow closely the changes in air temperature.

At stations where thermographs are located, the records consist of maximum and minimum temperatures for each day and the monthly averages of maximum daily and minimum daily temperatures.

\section{Sediment}

At some stations, suspended-sediment samples were collected daily with depth-integrating cable-suspended samplers from a fixed sampling point. A hand sampler was used at many stations during periods of low flow. Depth-integrated samples were collected periodically at many verticals in the cross section to determine the ratio of the cross sectional distribution of the concentration of suspended sediment to the daily sampling verticals.

During periods of high or rapidly changing flow, samples were taken twice or more often throughout the day at most stations. For periods when no samples were collected, daily loads of suspended sediment were estimated on the basis of water discharge, sediment concentrations observed immediately before and after the periods, and suspended-sediment loads for other periods of similar discharge.

At other stations, suspended-sediment samples were collected periodically at many verticals in the stream cross section. Although data collected periodically may represent conditions only at the time of observations, such data are useful in establishing seasonal relations between quality and streamflow in predicting long-term sediment-discharge characteristics of the stream.

In addition to the records of the quantities of suspended sediment, records of periodic measurements of the particle size distribution of the suspended sediment and bed material are included. 


\section{WATER-SUPPLY PAPERS}

Table 3 below, shows the annual series of water-supply papers that give information on quality of surface waters in Minnesota. Data for streams in the St. Lawrence River basin (northeastern Minnesota) are given in Part 4. Those for streams in the Hudson Bay, Upper Mississippi River, and Missouri River basins are given in Parts 5 and 6.

Table 3.--Water-supply paper numbers and parts, water years, 1941-65

Water Parts Parts Parts Parts Water Parts Parts Parts Parts

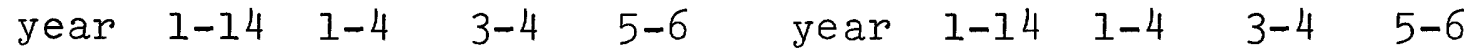

\begin{tabular}{|c|c|c|c|c|c|c|c|c|c|}
\hline 1941 & 942 & --- & --- & -- & 1954 & --- & 1350 & --- & 1351 \\
\hline 1942 & 950 & --- & --- & -- & 1955 & -- & 1400 & -- & 1401 \\
\hline 1943 & 970 & --- & --- & -- & 1956 & -- & 1450 & --- & 1451 \\
\hline 1944 & 1022 & -- & --- & -- & 1957 & -- & 1520 & --- & 152 \\
\hline 1945 & 1030 & --- & --- & --- & 1958 & -- & 1571 & & 757 \\
\hline 946 & 1050 & -- & --- & -- & 1959 & -- & --- & 1642 & 164 \\
\hline 47 & 1102 & -- & --- & --- & 1960 & -- & -- & 1742 & 174 \\
\hline 48 & -- & 1132 & --- & 1132 & 1961 & --- & -- & 1882 & 188 \\
\hline & --- & 1162 & --- & 1162 & 1962 & -- & -- & 1942 & 3 \\
\hline & --- & 1186 & -- & 1187 & 1963 & -- & --- & 1948 & 19 \\
\hline & -- & 1197 & --- & 1198 & 1964 & -- & -- & Al955 & A19 \\
\hline & -- & 1250 & --- & 1251 & 1965 & -- & -- & Al962 & A196 \\
\hline 1 & -- & 1290 & --- & 1291 & & & & & \\
\hline
\end{tabular}

A In preparation.

\section{SELECTED REFERENCES}

American Public Health Association, and others, 1965, Standard methods for the examination of water and wastewater, 12th ed.: Am. Public Health Assoc., New York, 769 p.

Benedict, P. C., 1948, Determination of the suspended-sediment discharge of streams, in Federal Interagency Sedimentation Conference, Ist, Denver, Colo., May 6-8, 1947, Proc.: Washington, D. C., U.S. Bur. Reclamation, p. 55-67.

Colby, B. R., 1963, Fluvial sediments--a summary of source, transportation, deposition, and measurement of sediment discharge: U.S. Geol. Survey Bull. 1181-A, $47 \mathrm{p}$. 
Colby, B. R., and Hubbell, D. W., 1961, Simplified methods for computing total sediment discharge with the modified Einstein procedure: U.S. Geol. Survey Water-Supply Paper $1593,17 \mathrm{p}$.

Hem, J. D., 1959, Study and interpretation of the chemical characteristics of natural water: U.S. Geol. Survey Water-Supply Paper 1473, 269 p.

Lane, E. W., and others, 1947, Report of subcommittee on sediment terminology: Am. Geophys. Union Trans., v. 28, no. 6, p. 936-938.

Langbein, W. B., and Iseri, K. T., 1960, General introduction and hydrologic definitions: U.S. Geol. Survey WaterSupply Paper 1541-A, 29 p.

Love, S. K., and Benedict, P. C., 1948, Discharge and sediment loads in the Boise River drainage basin, Idaho, 1939-40: U.S. Geol. Survey Water-Supply Paper 1048, $150 \mathrm{p}$.

Rainwater, F. H., and Thatcher, L. I., 1960, Methods for collection and analysis of water samples: U.S. Geol. Survey Water-Supply Paper 1454, $301 \mathrm{p}$.

U.S. Inter-Agency Committee on Water Resources, Subcommittee on Sedimentation, A study of methods used in measurement and analysis of sediment loads in streams. Published by the St. Anthony Falls Hydraulic Laboratory, Minneapolis, Minn.

1957, The development and calibration of visual accumulation tube: Rept. 11.

1957, Some fundamentals of particle size analysis: Rept. 12 .

1959, Federal Inter-agency sedimentation instruments and reports: Rept. AA.

1961, The single stage sampler for suspended sediment: Rept. 13.

1963, Determinations of fluvial sediment discharge: Rept. 14.

1963, A summary of the work of the Inter-agency sedimentation project: Rept. S. 
LOCATION.--Lat $46^{\circ} 51^{\prime} 40^{\prime \prime}$, long $96^{\circ} 47^{\prime} 00^{\prime \prime}$, in NW $\frac{1}{4} \mathrm{NE}^{\frac{1}{4}}$ sec.18, T.139 N., R.48 W., Cass County, at gaging station at city waterplant on $4 \mathrm{th} \mathrm{St}$. S. in Fargo, 25 miles upstream from mouth of Sheyenne River, and at mile 453 . DRAINAGE AREA. $--6,800 \mathrm{sq}$ mi, approximately. RECORDS AVAILABLE.-Chemical analyses: October 1955 to September 1968.

Water temperatures: October 1955 to September 1968.

EXTREMES, 1967-68.--Dissolved solids: Maximum, $409 \mathrm{mg} / 1$ Apr. 25 to May 18; minimum, $251 \mathrm{mg} / 1$ Sept. 1-25.

Hardness: Maximum, $304 \mathrm{mg} / 1$ Feb. 1-29; minimum, 214 mg/1 Dec. 1-25, Sept. 26-30.

Specific conductance: Maximum daily, 694 micromos May 1; minimum daily, 306 micromhos Dec. 3.

Water temperatures: Maximum, $26^{\circ} \mathrm{C}$ on several days during July; minimum, $2^{\circ} \mathrm{C}$ on many days during November to March.

CHEMICAL ANALYSES IN MILLIGRAMS PER LITER, WATER YEAR OCTOBER 1967 TO SEPTEMBER 1968

\begin{tabular}{|c|c|c|c|c|c|c|c|c|c|c|c|c|}
\hline & $\begin{array}{l}\text { MEAN } \\
\text { DIS- } \\
\text { CHARGE }\end{array}$ & SIL & $\begin{array}{l}\text { TOTAL } \\
\text { IRDN }\end{array}$ & $\begin{array}{l}\text { CAL- } \\
\text { CIUM }\end{array}$ & $\begin{array}{l}\text { MAG- } \\
\text { NE- } \\
\text { SIUM }\end{array}$ & SogIuM & $\begin{array}{l}\text { PO- } \\
\text { TAS- } \\
\text { SI UM }\end{array}$ & $\begin{array}{l}\text { RICAR- } \\
\text { RONATE }\end{array}$ & $\begin{array}{l}\text { CAR- } \\
\text { BONATE }\end{array}$ & SULFATE & $\begin{array}{l}\text { CHLO- } \\
\text { RIDE }\end{array}$ & $\begin{array}{l}\text { FLUO- } \\
\text { RIDF } \\
\text { (F) }\end{array}$ \\
\hline & (CFS) & $S(02)$ & $(F E)$ & $(C A)$ & $(M G)$ & (NA) & $(K)$ & $(\mathrm{HCO} 3)$ & $\left(\mathrm{CO}_{3}\right)$ & $(\operatorname{Sn} 4)$ & $(C L)$ & (F) \\
\hline
\end{tabular}

OCT .

\begin{tabular}{|c|c|c|c|c|c|c|c|c|c|c|c|c|}
\hline $01-27$ & 167 & -- & -- & - & -- & 10 & -- & 273 & 0 & 36 & -- & -- \\
\hline $28 \ldots$ & 208 & 2.7 & .13 & 46 & 31 & 10 & 4.9 & 273 & 2 & 29 & 4.4 & .3 \\
\hline $29-31$ & 164 & -- & - & - & - & 10 & -- & 278 & 0 & 31 & -- & -- \\
\hline \multicolumn{13}{|l|}{ Nov. } \\
\hline $01-30$ & 164 & - & - & -- & -- & 10 & - & 278 & 0 & 31 & -- & -- \\
\hline \multicolumn{13}{|l|}{ DEC. } \\
\hline $26 . .$. & 148 & 11 & .05 & 38 & 37 & 14 & 4.9 & 262 & 9 & 48 & 5.0 & .3 \\
\hline $27-31$ & 137 & - & -- & - & - & .13 & - & 328 & $n$ & 40 & - & -- \\
\hline \multicolumn{13}{|l|}{ JAN. } \\
\hline $\begin{array}{l}01-31 \\
F E R\end{array}$ & 137 & -- & - & - & -- & 13 & -- & 328 & 0 & 40 & -- & -- \\
\hline FEB & 135 & -- & -- & - & -- & 14 & - & 344 & 0 & 36 & -- & -- \\
\hline \multirow{2}{*}{\multicolumn{13}{|c|}{$\begin{array}{l}\text { MAR. } \\
01-25\end{array}$}} \\
\hline & 342 & -- & -- & -- & -- & 17 & -- & 283 & 0 & 62 & -- & -- \\
\hline $26 \ldots$ & 496 & 11 & .08 & 45 & 26 & 15 & 4.8 & 207 & 0 & 68 & 6.1 & .1 \\
\hline \multirow{2}{*}{\multicolumn{13}{|c|}{ APR. }} \\
\hline & & & & & & & & & & & & \\
\hline $01-11$ & 416 & -- & - & -- & -- & 14 & -- & 216 & 0 & 65 & -- & -- \\
\hline $12-17$ & 598 & -- & -- & - & - & 20 & -- & 220 & 0 & 101 & -- & - \\
\hline $18-24$ & 463 & -- & - & - & - & 22 & - & 228 & 0 & 118 & -- & - \\
\hline $25-30$ & 675 & -- & -- & - & -- & 25 & -- & 254 & 0 & 114 & -- & - \\
\hline \multicolumn{13}{|l|}{ MAY } \\
\hline $01-18$ & 675 & - & -- & - & - & 25 & - & 254 & 0 & 114 & -- & - \\
\hline $19 \ldots$ & 772 & 7.8 & .03 & 52 & 34 & 25 & 2.0 & 256 & 0 & 106 & 8.6 & .2 \\
\hline \multirow{2}{*}{\multicolumn{13}{|c|}{ JUNE }} \\
\hline & & & & & & & & & & & & \\
\hline $01-30$ & 649 & -- & - & -- & -- & 20 & -- & 257 & 0 & 77 & -- & - \\
\hline \multicolumn{13}{|l|}{ JULY } \\
\hline $01-08$ & 578 & - & - & -- & - & 13 & - & 243 & 0 & 45 & -- & - \\
\hline $09-31$ & 386 & -- & -- & -- & - & 10 & - & 252 & 0 & 30 & -- & - \\
\hline \multicolumn{13}{|l|}{ AUG. } \\
\hline $01-31$ & 223 & -- & -- & -- & -- & 10 & - & 252 & 0 & 27 & - & -- \\
\hline \multicolumn{13}{|l|}{ SEPT. } \\
\hline $01-25$ & 141 & -- & - & - & - & 8.9 & -- & 250 & 0 & 29 & - & - \\
\hline $26-30$ & 233 & 5.9 & -- & 41 & 27 & 10 & 4.1 & 248 & 0 & 35 & 4.0 & \\
\hline
\end{tabular}


5-0540. RED RIVER OF THE NORTH AT FARGO, N. DAK.--Continued

EXTREMES, 1955-68.--Dissolved solids (1955-58, 1959-68): Maximum, 650 mg/1 May 6-9, 1958; minimum, 174 mg/1 Dec. 1-2, 1955 .

Hardness: Maximum, $420 \mathrm{mg} / 1$ May 6-9, 1958; minimum, $118 \mathrm{mg} / 1$ Apr. 6-17, 1962.

Specific conductance: Maximum daily, 960 micromhos May 6, 1958 ; minimum daily, 223 micromhos Apr. 11, 1962. Water temperatures: Maximum, $28^{\circ} \mathrm{C}$ on several days in 1957,1960 , and 1964 ; minimum, $1^{\circ} \mathrm{C}$ on many days in 1956 and 1959 .

REMARKS,--Values reported for sodium (Na) are determined by analysis and do not include potassium (K). Daily samples for chemical ana1ysis composited by discharge. Water temperature, measured in waterp1ant, modified slight1y in transit.

CHEMICAL ANALYSES IN MILLIGRAMS PER LITER, WATER YEAP OCTOBER 1967 TO SEPTEMBER 1968

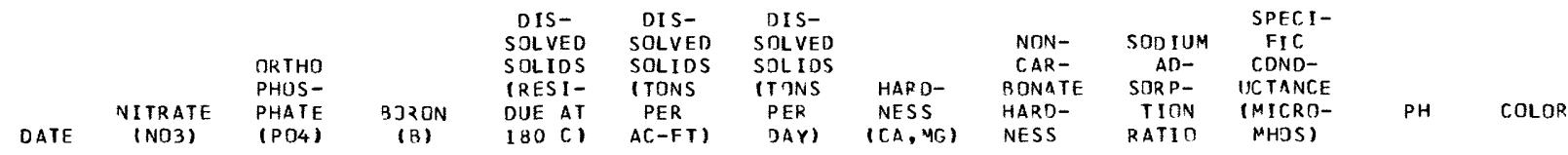

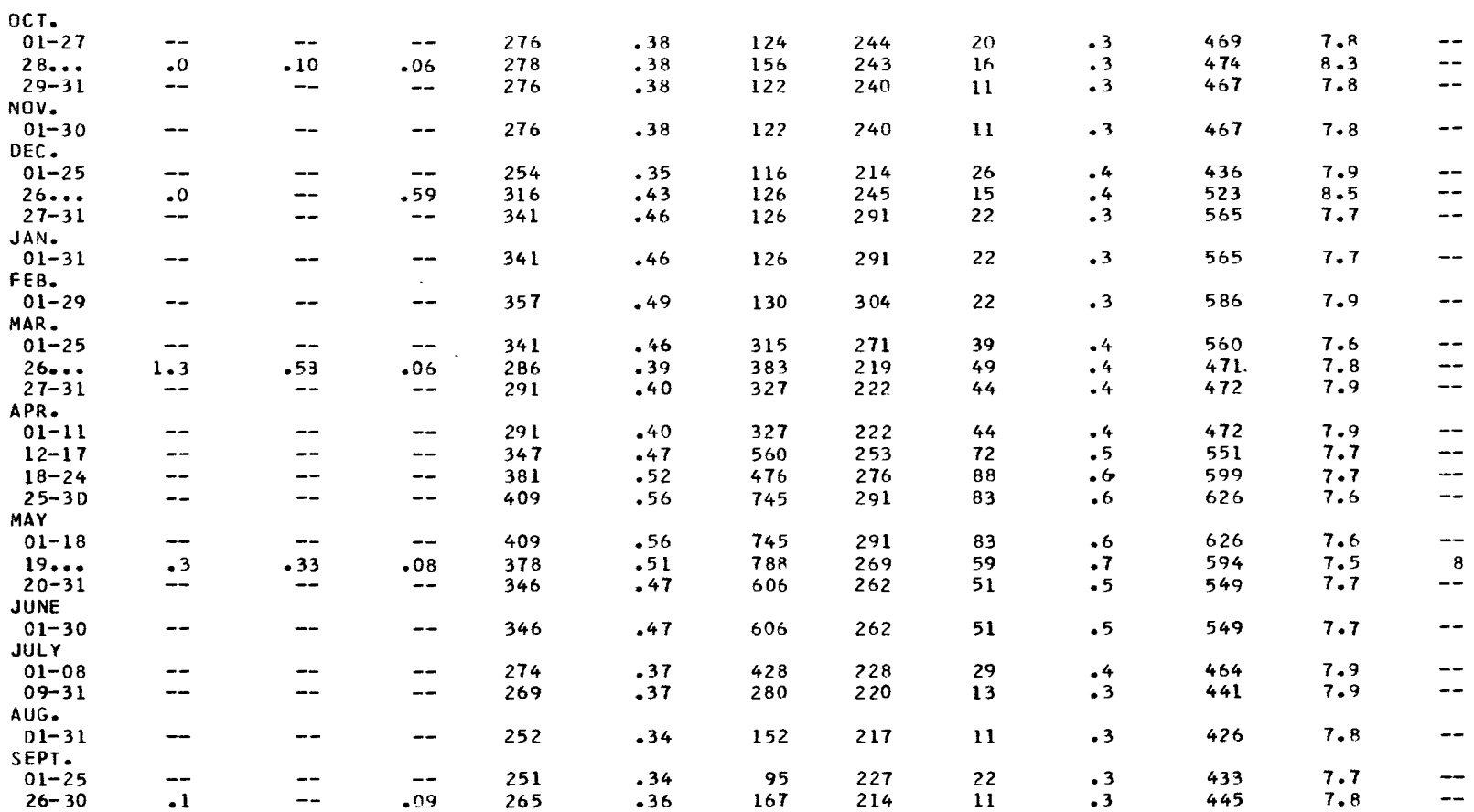


5-0540. RED RIVER OF THE NORTH AT FARGO, N. DAK.--Continued SPECIFIC CONDUCTANCE (MICROMHOS AT $25^{\circ} \mathrm{C}$ ), WATER YEAR OCTOBER 1967 TO SEPTEMBER 1968

DAY OCTOBER NOVEMBER DECEMBER JANUARY FEBRUARY MARCH APRIL MAY JUNE JULY AUEUST SEPTEMBER

\begin{tabular}{|c|c|c|c|c|c|c|c|c|c|c|c|c|}
\hline $\begin{array}{l}1 \ldots \ldots \\
2 \ldots \ldots \\
3 \ldots \ldots \\
4 \ldots \ldots \\
5 \ldots \ldots\end{array}$ & $\begin{array}{l}496 \\
496 \\
489 \\
501 \\
529\end{array}$ & $\begin{array}{l}462 \\
459 \\
459 \\
459 \\
462\end{array}$ & $\begin{array}{l}419 \\
347 \\
306 \\
307 \\
401\end{array}$ & $\begin{array}{l}551 \\
551 \\
556 \\
556 \\
567\end{array}$ & $\begin{array}{l}594 \\
586 \\
577 \\
586 \\
584\end{array}$ & $\begin{array}{l}585 \\
586 \\
588 \\
582 \\
572\end{array}$ & $\begin{array}{l}443 \\
451 \\
449 \\
476 \\
503\end{array}$ & $\begin{array}{l}694 \\
632 \\
678 \\
690 \\
690\end{array}$ & $\begin{array}{l}541 \\
533 \\
528 \\
530 \\
530\end{array}$ & $\begin{array}{l}465 \\
436 \\
464 \\
472 \\
466\end{array}$ & $\begin{array}{l}405 \\
400 \\
39 B \\
412 \\
400\end{array}$ & $\begin{array}{l}427 \\
423 \\
425 \\
425 \\
425\end{array}$ \\
\hline $\begin{array}{c}6 \ldots \ldots \\
7 \ldots \ldots \\
8 \ldots \ldots \\
10 \ldots \ldots\end{array}$ & $\begin{array}{l}546 \\
527 \\
469 \\
439 \\
439\end{array}$ & $\begin{array}{l}462 \\
460 \\
460 \\
460 \\
459\end{array}$ & $\begin{array}{l}375 \\
396 \\
402 \\
380 \\
415\end{array}$ & $\begin{array}{l}576 \\
567 \\
564 \\
564 \\
573\end{array}$ & $\begin{array}{l}580 \\
576 \\
578 \\
589 \\
579\end{array}$ & $\begin{array}{l}567 \\
551 \\
539 \\
533 \\
537\end{array}$ & $\begin{array}{l}496 \\
471 \\
478 \\
486 \\
483\end{array}$ & $\begin{array}{l}668 \\
680 \\
640 \\
628 \\
620\end{array}$ & $\begin{array}{l}527 \\
525 \\
531 \\
538 \\
528\end{array}$ & $\begin{array}{l}458 \\
454 \\
454 \\
447 \\
442\end{array}$ & $\begin{array}{l}402 \\
401 \\
397 \\
408 \\
434\end{array}$ & $\begin{array}{l}437 \\
427 \\
427 \\
427 \\
425\end{array}$ \\
\hline $\begin{array}{l}11 \ldots \ldots \\
12 \ldots \ldots \\
13 \ldots \ldots \\
14 \ldots \ldots \\
15 \ldots \ldots\end{array}$ & $\begin{array}{l}443 \\
447 \\
449 \\
448 \\
449\end{array}$ & $\begin{array}{l}454 \\
459 \\
457 \\
463 \\
466\end{array}$ & $\begin{array}{l}440 \\
405 \\
417 \\
425 \\
428\end{array}$ & $\begin{array}{l}568 \\
568 \\
566 \\
564 \\
564\end{array}$ & $\begin{array}{l}563 \\
577 \\
589 \\
586 \\
588\end{array}$ & $\begin{array}{l}578 \\
611 \\
529 \\
522 \\
498\end{array}$ & $\begin{array}{l}479 \\
502 \\
470 \\
490 \\
57 B\end{array}$ & $\begin{array}{l}614 \\
620 \\
594 \\
604 \\
595\end{array}$ & $\begin{array}{l}528 \\
528 \\
535 \\
510 \\
580\end{array}$ & $\begin{array}{l}438 \\
439 \\
439 \\
450 \\
433\end{array}$ & $\begin{array}{l}438 \\
440 \\
435 \\
448 \\
465\end{array}$ & $\begin{array}{l}419 \\
419 \\
425 \\
423 \\
421\end{array}$ \\
\hline $\begin{array}{l}16 \ldots \ldots \\
17 \ldots \ldots \\
18 \ldots \ldots \\
19 \ldots \ldots \\
20 \ldots \ldots\end{array}$ & $\begin{array}{l}452 \\
452 \\
446 \\
446 \\
446\end{array}$ & $\begin{array}{l}468 \\
473 \\
477 \\
477 \\
462\end{array}$ & $\begin{array}{l}358 \\
375 \\
42 B \\
420 \\
426\end{array}$ & $\begin{array}{l}577 \\
581 \\
575 \\
576 \\
582\end{array}$ & $\begin{array}{l}588 \\
580 \\
581 \\
578 \\
570\end{array}$ & $\begin{array}{l}487 \\
480 \\
531 \\
568 \\
651\end{array}$ & $\begin{array}{l}593 \\
593 \\
618 \\
602 \\
575\end{array}$ & $\begin{array}{l}605 \\
624 \\
621 \\
610 \\
605\end{array}$ & $\begin{array}{l}582 \\
576 \\
547 \\
561 \\
567\end{array}$ & $\begin{array}{l}430 \\
424 \\
424 \\
442 \\
455\end{array}$ & $\begin{array}{l}430 \\
409 \\
425 \\
422 \\
418\end{array}$ & $\begin{array}{l}414 \\
414 \\
420 \\
428 \\
430\end{array}$ \\
\hline $\begin{array}{l}21 \ldots \ldots \\
22 \ldots \ldots \\
23 \ldots \ldots \\
24 \ldots \ldots \\
25 \ldots \ldots\end{array}$ & $\begin{array}{l}449 \\
451 \\
455 \\
456 \\
458\end{array}$ & $\begin{array}{l}467 \\
462 \\
474 \\
472 \\
474\end{array}$ & $\begin{array}{l}483 \\
438 \\
482 \\
454 \\
380\end{array}$ & $\begin{array}{l}574 \\
587 \\
580 \\
580 \\
575\end{array}$ & $\begin{array}{l}568 \\
572 \\
565 \\
581 \\
579\end{array}$ & $\begin{array}{l}566 \\
556 \\
564 \\
508 \\
495\end{array}$ & $\begin{array}{l}594 \\
604 \\
576 \\
576 \\
584\end{array}$ & $\begin{array}{l}583 \\
580 \\
604 \\
595 \\
579\end{array}$ & $\begin{array}{l}538 \\
518 \\
507 \\
528 \\
533\end{array}$ & $\begin{array}{l}443 \\
434 \\
434 \\
434 \\
434\end{array}$ & $\begin{array}{l}418 \\
416 \\
409 \\
408 \\
405\end{array}$ & $\begin{array}{l}436 \\
411 \\
416 \\
426 \\
427\end{array}$ \\
\hline $\begin{array}{l}26 \ldots \ldots \\
27 \ldots \ldots \\
28 \ldots \ldots \\
29 \ldots \ldots \\
30 \ldots \ldots \\
31 \ldots \ldots\end{array}$ & $\begin{array}{l}460 \\
461 \\
464 \\
464 \\
464 \\
461\end{array}$ & $\begin{array}{l}481 \\
483 \\
496 \\
501 \\
496 \\
--\end{array}$ & $\begin{array}{l}438 \\
426 \\
486 \\
425 \\
442 \\
380\end{array}$ & $\begin{array}{l}584 \\
587 \\
598 \\
586 \\
579 \\
576\end{array}$ & $\begin{array}{l}583 \\
588 \\
585 \\
576 \\
-- \\
--\end{array}$ & $\begin{array}{l}469 \\
451 \\
479 \\
446 \\
419 \\
419\end{array}$ & $\begin{array}{r}528 \\
538 \\
532 \\
607 \\
666 \\
--\end{array}$ & $\begin{array}{l}593 \\
606 \\
595 \\
559 \\
556 \\
547\end{array}$ & $\begin{array}{l}517 \\
503 \\
511 \\
495 \\
453 \\
--\end{array}$ & $\begin{array}{l}436 \\
428 \\
425 \\
414 \\
409 \\
409\end{array}$ & $\begin{array}{l}408 \\
418 \\
414 \\
422 \\
414 \\
421\end{array}$ & $\begin{array}{l}437 \\
444 \\
444 \\
427 \\
426 \\
--\end{array}$ \\
\hline AVERAGE & 466 & 468 & 409 & 572 & 580 & 531 & 534 & 616 & 530 & 439 & 417 & 425 \\
\hline
\end{tabular}


5-0540. RED RIVER OF THE NORTH AT FARGO, N. DAK.--Continued

TEMPERATURE $\left({ }^{\circ} \mathrm{C}\right)$ OF WATER, WATER YEAR OCTOBER 1967 TO SEPTEMBER 1968

DAY

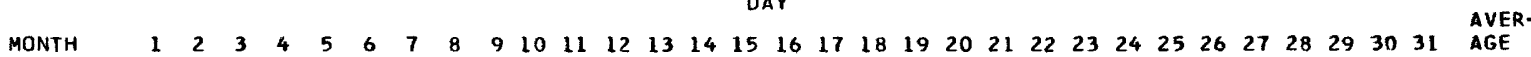

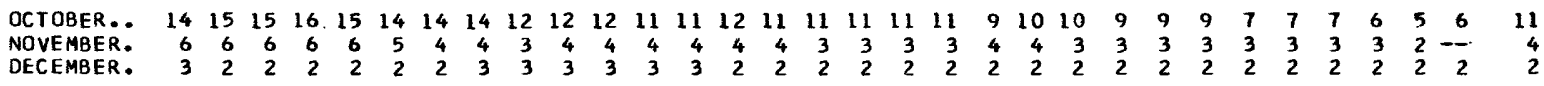
$\begin{array}{lllllllllllllllllllllllllllllllllllllllllllllll}\text { JANUARY.. } & 2 & 2 & 2 & 2 & 2 & 1 & 2 & 2 & 2 & 2 & 2 & 2 & 2 & 2 & 2 & 2 & 2 & 2 & 2 & 2 & 2 & 2 & 2 & 2 & 2 & 2 & 2 & 2 & 2 & 2 & 2 & 2\end{array}$

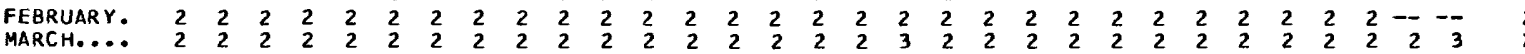

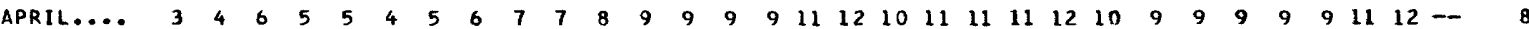

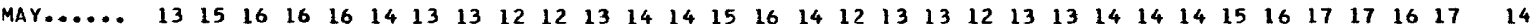

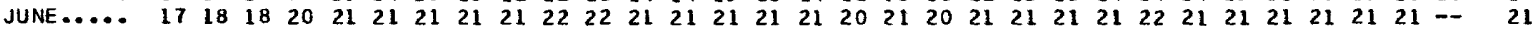

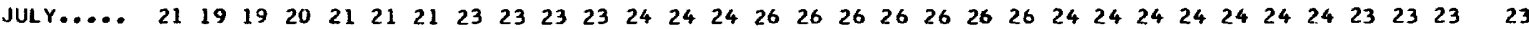

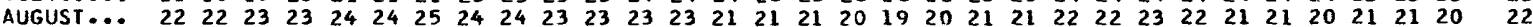

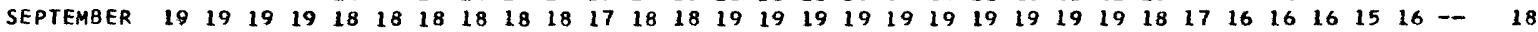


LOCATION.--Lat $47^{\circ} 56^{\prime} 28^{\prime \prime}$, long $97^{\circ} 02^{\prime} 40^{\prime \prime}$, in SW $\frac{1}{4} N E \frac{1}{4}$ sec.33, T.152 N., R.50 W. , Grand Forks County, at dam at Riverside Park in Grand Forks, 1,500 feet upstream from gaging station, 2 miles downstream from Red Lake River, and at mile 296.0

DRAINAGE AREA. $--30,100$ sq mi, approximately.

RECORDS AVAILABLE.--Chemical analyses: September 1956 to September 1968.

Water temperatures: October 1956 to September 1968.

EXTREMES, 1967-68.--Dissolved solids: Maximum, 471 mg/1 May 20-31; minimum, 259 mg/1 July $18-25$.

Hardness: Maximum, $328 \mathrm{mg} / 1$ May 20-31; minimum, $195 \mathrm{mg} / 1$ July 18-25.

Specific conductance: Maximum daily, 849 micromhos Mar. 16; minimum daily, 358 micromhos July 19.

Water temperatures: Maximum, $24^{\circ} \mathrm{C}$ Júly 15-17, Aug. 7 ; minimum, $2^{\circ} \mathrm{C}$ on many days during November to April.

CHEMICAL ANALYSES IN MILLIGRAMS PER LITER, WATER YEAR OCTOBER 1967 TO SEPTEMREP 196R

\begin{tabular}{|c|c|c|c|c|c|c|c|c|c|c|c|c|}
\hline & $\begin{array}{l}\text { MEAN } \\
\text { DIS- } \\
\text { CHARGE }\end{array}$ & IL & $\begin{array}{l}\text { TOTAL } \\
\text { IRON }\end{array}$ & $\begin{array}{l}\text { CAL- } \\
\text { CIUM }\end{array}$ & $\begin{array}{l}\text { MAG- } \\
\text { NE- } \\
\text { SIUM }\end{array}$ & SOOI UM & $\begin{array}{l}\text { PO- } \\
\text { TAS- } \\
\text { SIUM }\end{array}$ & $\begin{array}{l}\text { BICAR- } \\
\text { BONATE }\end{array}$ & $\begin{array}{l}\text { CAR- } \\
\text { BONATE }\end{array}$ & SULFATE & $\begin{array}{l}\text { CHLO- } \\
\text { RIDE }\end{array}$ & $\begin{array}{l}\text { FLUN- } \\
\text { RIDF }\end{array}$ \\
\hline & (CFS) & $(5102)$ & (FE) & (CA) & (MG) & (NA) & (k) & $(\mathrm{HCO} 3)$ & $([03)$ & $(504)$ & $(C L)$ & $(F)$ \\
\hline
\end{tabular}

ocT.

$01-26$

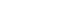

(MG)

(NA) IKI

$27 . .$.

782
749

$$
\begin{aligned}
& 782 \\
& 749 \\
& 707
\end{aligned}
$$

NOV.

01-30

$$
13
$$

\begin{tabular}{|c|c|c|}
\hline$-\sim$ & -- & -- \\
\hline & .10 & 51 \\
\hline- & - & -- \\
\hline-- & - & - \\
\hline
\end{tabular}

$\overline{24}$

$3.0 \quad 24$

241
252
269

01-31

JAN.

JAN.

FE 8 .

01-29

MAR.

01-18

$19-21$
$22-24$

25 ...

$26-31$

$A P R$.

O1-30

707

718

592

$--$

$--$

540

754

1650

2630

2600

2930

2930

01-18 1930

$\begin{array}{ll}01-18 & 1930 \\ 19 \ldots & 2200\end{array}$

$\begin{array}{ll}19 \ldots 3 & 2200 \\ 20-31 & 1780\end{array}$

JUNE

01-06

$07-08$

09-13

14-20

21-30

JuLY

$01-17$

$01-17$
$18-25$

26-31

AUG.

01-31

1370

4420
8260

8260
5720

3900

2930
7200

7200
3760

2160

SEPT.

01.30

1780

1540

-- $\quad-$

$$
-
$$$$
\text { - } \quad 269
$$

0

$$
\begin{aligned}
& 45 \\
& 40 \\
& 43
\end{aligned}
$$

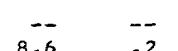$$
\text { -- } \quad--
$$$$
-
$$$$
--
$$$$
3>0
$$$$
--
$$$$
332
$$$$
\text { - } \quad 333
$$$$
12^{--}
$$$$
.0
$$$$
--
$$$$
--
$$$$
21
$$$$
--
$$$$
\begin{array}{ll}
-- & 318 \\
-- & 264 \\
-- & 219
\end{array}
$$$$
7.1
$$$$
219
$$$$
-
$$$$
.02
$$$$
47
$$$$
--
$$$$
\begin{array}{llll}
-- & -- & -- & -- \\
7.7 & .04 & 70 & 35
\end{array}
$$$$
--
$$$$
19
$$$$
-
$$$$
200
$$$$
\text { - } 2 n 0
$$$$
1.8
$$$$
276
$$$$
\begin{aligned}
& 280 \\
& 290
\end{aligned}
$$

$$
\begin{aligned}
& 27 \\
& 31 \\
& 31
\end{aligned}
$$$$
1.8
$$$$
\begin{array}{lll}
30 & -- & 298 \\
23 & -- & 276 \\
10 & -- & 184
\end{array}
$$$$
\text { -- }
$$$$
\begin{array}{lll}
-- & -- & -- \\
-- & -- & -
\end{array}
$$$$
\text { - }
$$$$
26
$$$$
--
$$$$
\begin{aligned}
& 220 \\
& 245
\end{aligned}
$$$$
17
$$$$
\begin{array}{r}
6.2 \\
8.7
\end{array}
$$$$
--
$$$$
250
$$$$
176
$$$$
210
$$$$
11
$$$$
221
$$$$
8 \cdot 5
$$$$
.03
$$$$
-
$$$$
4 \cdot 3
$$

0

75

65

60

77

77
93
74
74

10
10

$4 \cdot 3$

225
221

91
58
74
68
62
61

$\begin{array}{cc}13 & -3 \\ -- & - \\ -- & -- \\ -- & -- \\ -- & - \\ -- & -- \\ -- & -- \\ -- & -- \\ -- & - \\ -. & -- \\ 11 & \\ -- & -\end{array}$


EXTREMES, 1956-68.--Dissolved solids (1956-58, 1959-68): Maximum, 540 mg/1 Jan. 21, 1962; minimum, 191 mg/1 Mar. 24, 1966

Hardness: Maximum, $468 \mathrm{mg} / 1$ Dec. 29-31, 1958; minimum, $126 \mathrm{mg} / 1$ Apr. $12,1965$.

Specific conductance: Maximum daily, 976 micromhos Dec. 29-31, 1958; minimum daily, 278 micromhos Mar. 26, 1966.

Water temperatures: Maximum, $28^{\circ} \mathrm{C}$ July 19, 1964 ; minimum, freezing point on many days in 1967.

REMARKS.--Values reported for sodium (Na) are determined by ana1ysis and do not include potassium (K). Daily samples for chemical analysis composited by discharge.

CHEMICAL ANALYSES IN MILLIGRAMS PER LITER, WATFR YEAP OCTOBER 1967 TO SEPTEMBEP 1969

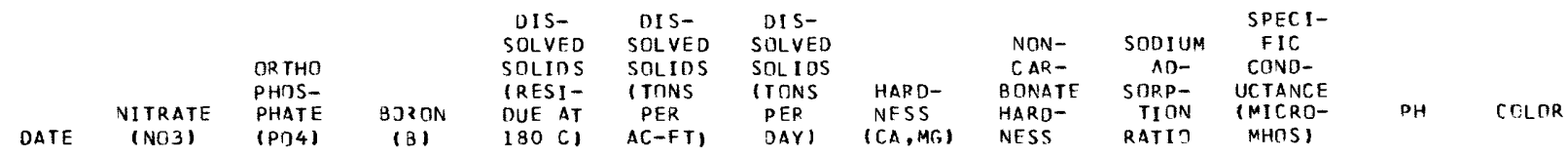

OCT.

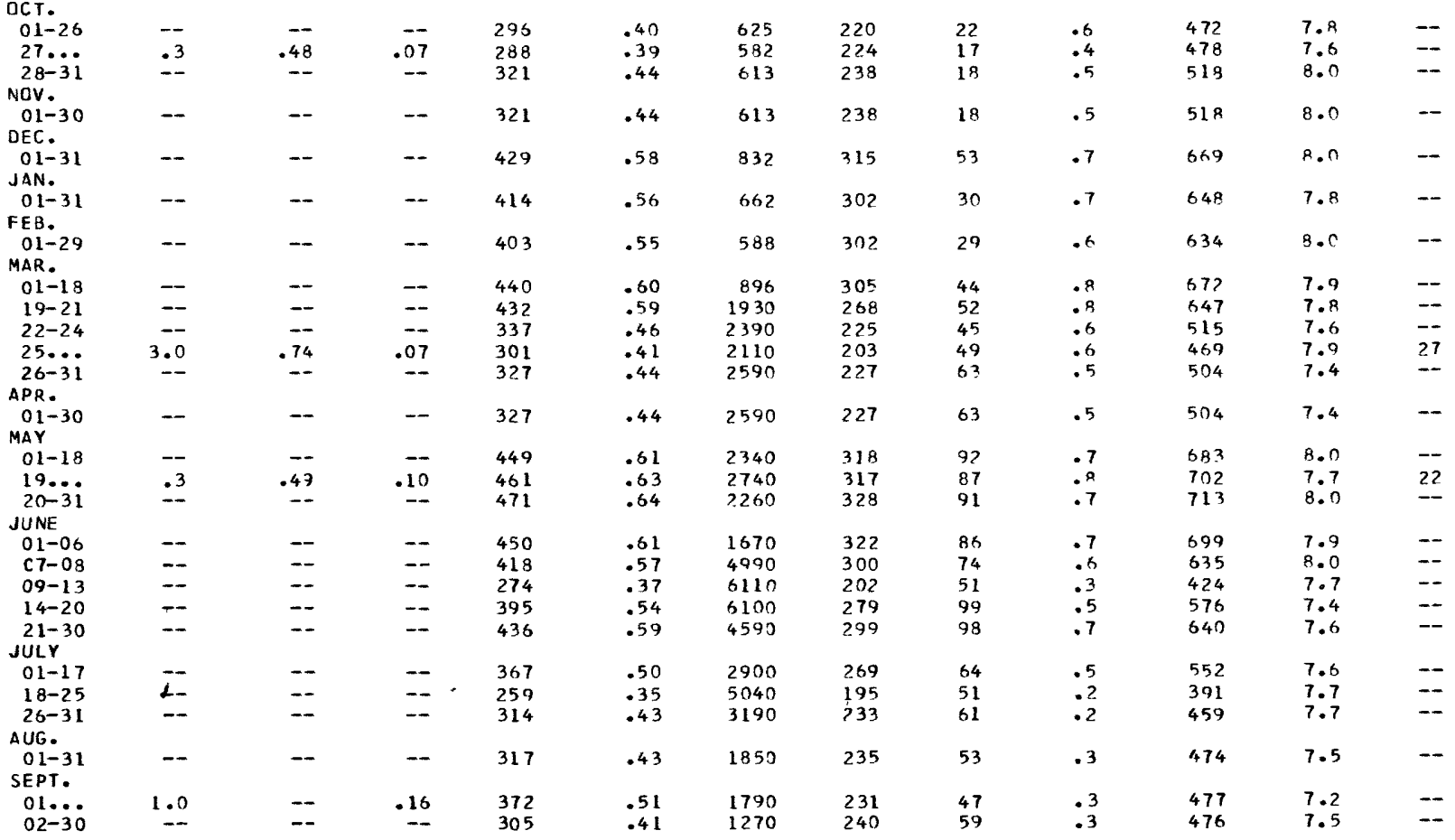


5-0825. RED RIVER OF THE NORTH AT GRAND FORKS, N. DAK.--Continued

SPECIFIC CONDUCTANCE (MICROMHOS AT $25^{\circ} \mathrm{C}$ ), WATER YEAR OCTOBER 1967 TO SEPTEMBER 1968

DAY OCTOBER NOVEMBER DECEMBER JANUARY FEBRUARY MARCH APRIL MAY JUNE JULY AUEUST SEPTEMBER

\begin{tabular}{|c|c|c|c|c|c|c|c|c|c|c|c|c|}
\hline $\begin{array}{l}1 \ldots \ldots \\
2 \ldots \ldots \\
3 \ldots \ldots \\
4 \ldots \ldots \\
5 \ldots \ldots\end{array}$ & $\begin{array}{l}409 \\
440 \\
454 \\
464 \\
459\end{array}$ & $\begin{array}{l}511 \\
494 \\
490 \\
486 \\
499\end{array}$ & $\begin{array}{l}567 \\
565 \\
56 B \\
589 \\
624\end{array}$ & $\begin{array}{l}654 \\
647 \\
652 \\
661 \\
657\end{array}$ & $\begin{array}{l}620 \\
620 \\
622 \\
615 \\
618\end{array}$ & $\begin{array}{l}670 \\
663 \\
663 \\
658 \\
659\end{array}$ & $\begin{array}{l}385 \\
412 \\
416 \\
453 \\
453\end{array}$ & $\begin{array}{l}664 \\
664 \\
649 \\
649 \\
652\end{array}$ & $\begin{array}{l}724 \\
716 \\
711 \\
706 \\
702\end{array}$ & $\begin{array}{l}609 \\
60 C \\
574 \\
532 \\
542\end{array}$ & $\begin{array}{l}49 C \\
490 \\
488 \\
487 \\
496\end{array}$ & $\begin{array}{l}420 \\
455 \\
444 \\
438 \\
434\end{array}$ \\
\hline $\begin{array}{c}6 \ldots \ldots \\
7 \ldots \ldots \\
8 \ldots \ldots \\
9 \ldots \ldots \\
10 \ldots \ldots\end{array}$ & $\begin{array}{l}456 \\
459 \\
459 \\
462 \\
453\end{array}$ & $\begin{array}{l}485 \\
505 \\
523 \\
507 \\
535\end{array}$ & $\begin{array}{l}613 \\
616 \\
673 \\
684 \\
667\end{array}$ & $\begin{array}{l}661 \\
657 \\
670 \\
678 \\
679\end{array}$ & $\begin{array}{l}622 \\
624 \\
624 \\
636 \\
636\end{array}$ & $\begin{array}{l}658 \\
661 \\
650 \\
648 \\
646\end{array}$ & $\begin{array}{l}472 \\
472 \\
497 \\
502 \\
505\end{array}$ & $\begin{array}{l}667 \\
690 \\
695 \\
690 \\
686\end{array}$ & $\begin{array}{l}641 \\
665 \\
616 \\
382 \\
381\end{array}$ & $\begin{array}{l}556 \\
556 \\
556 \\
545 \\
552\end{array}$ & $\begin{array}{l}493 \\
493 \\
486 \\
490 \\
468\end{array}$ & $\begin{array}{l}435 \\
446 \\
446 \\
441 \\
464\end{array}$ \\
\hline $\begin{array}{l}11 \ldots \ldots \\
12 \ldots \ldots \\
13 \ldots \ldots \\
14 \ldots \ldots \\
15 \ldots \ldots\end{array}$ & $\begin{array}{l}456 \\
465 \\
480 \\
511 \\
4 B 3\end{array}$ & $\begin{array}{l}497 \\
474 \\
471 \\
479 \\
502\end{array}$ & $\begin{array}{l}696 \\
737 \\
706 \\
715 \\
715\end{array}$ & $\begin{array}{l}675 \\
665 \\
674 \\
667 \\
660\end{array}$ & $\begin{array}{l}637 \\
632 \\
636 \\
636 \\
633\end{array}$ & $\begin{array}{l}623 \\
611 \\
609 \\
609 \\
670\end{array}$ & $\begin{array}{l}520 \\
557 \\
549 \\
553 \\
565\end{array}$ & $\begin{array}{l}700 \\
706 \\
695 \\
692 \\
690\end{array}$ & $\begin{array}{l}4 C 2 \\
448 \\
475 \\
495 \\
583\end{array}$ & $\begin{array}{l}554 \\
557 \\
554 \\
547 \\
539\end{array}$ & $\begin{array}{l}462 \\
462 \\
463 \\
459 \\
465\end{array}$ & $\begin{array}{l}441 \\
464 \\
478 \\
470 \\
467\end{array}$ \\
\hline $\begin{array}{l}16 \ldots \ldots \\
17 \ldots \ldots \\
18 \ldots \ldots \\
19 \ldots \ldots \\
20 \ldots \ldots\end{array}$ & $\begin{array}{l}456 \\
465 \\
478 \\
484 \\
472\end{array}$ & $\begin{array}{l}516 \\
538 \\
516 \\
505 \\
492\end{array}$ & $\begin{array}{l}70 B \\
703 \\
667 \\
659 \\
652\end{array}$ & $\begin{array}{l}672 \\
662 \\
665 \\
656 \\
643\end{array}$ & $\begin{array}{l}627 \\
627 \\
637 \\
633 \\
631\end{array}$ & $\begin{array}{l}849 \\
743 \\
674 \\
635 \\
630\end{array}$ & $\begin{array}{l}559 \\
553 \\
5 B 8 \\
5 B 6 \\
597\end{array}$ & $\begin{array}{l}688 \\
676 \\
700 \\
695 \\
692\end{array}$ & $\begin{array}{l}579 \\
594 \\
589 \\
608 \\
599\end{array}$ & $\begin{array}{l}530 \\
482 \\
416 \\
358 \\
364\end{array}$ & $\begin{array}{l}470 \\
473 \\
462 \\
468 \\
455\end{array}$ & $\begin{array}{l}451 \\
470 \\
461 \\
462 \\
458\end{array}$ \\
\hline $\begin{array}{l}21 \ldots \ldots \\
22 \ldots \ldots \\
23 \ldots \ldots \\
24 \ldots \ldots \\
25 \ldots \ldots\end{array}$ & $\begin{array}{l}477 \\
464 \\
470 \\
476 \\
478\end{array}$ & $\begin{array}{l}492 \\
531 \\
52 B \\
550 \\
520\end{array}$ & $\begin{array}{l}659 \\
656 \\
660 \\
660 \\
664\end{array}$ & $\begin{array}{l}633 \\
634 \\
634 \\
629 \\
623\end{array}$ & $\begin{array}{l}642 \\
644 \\
643 \\
651 \\
655\end{array}$ & $\begin{array}{l}629 \\
581 \\
516 \\
464 \\
469\end{array}$ & $\begin{array}{l}611 \\
618 \\
606 \\
609 \\
616\end{array}$ & $\begin{array}{l}704 \\
698 \\
714 \\
720 \\
720\end{array}$ & $\begin{array}{l}606 \\
637 \\
658 \\
650 \\
656\end{array}$ & $\begin{array}{l}369 \\
379 \\
401 \\
422 \\
434\end{array}$ & $\begin{array}{l}455 \\
437 \\
430 \\
427 \\
427\end{array}$ & $\begin{array}{l}455 \\
510 \\
4 B 4 \\
510 \\
531\end{array}$ \\
\hline $\begin{array}{l}26 \ldots \ldots \\
27 \ldots \ldots \\
28 \ldots \ldots \\
29 \ldots \ldots \\
30 \ldots \ldots \\
31 \ldots \ldots\end{array}$ & $\begin{array}{l}477 \\
464 \\
470 \\
471 \\
477 \\
489\end{array}$ & $\begin{array}{c}535 \\
555 \\
580 \\
538 \\
568 \\
--\end{array}$ & $\begin{array}{l}662 \\
664 \\
669 \\
680 \\
668 \\
650\end{array}$ & $\begin{array}{l}614 \\
614 \\
606 \\
605 \\
609 \\
636\end{array}$ & $\begin{array}{c}648 \\
655 \\
653 \\
659 \\
-- \\
--\end{array}$ & $\begin{array}{l}473 \\
461 \\
447 \\
403 \\
392 \\
380\end{array}$ & $\begin{array}{c}627 \\
625 \\
632 \\
642 \\
650 \\
--\end{array}$ & $\begin{array}{l}719 \\
720 \\
712 \\
70 B \\
708 \\
714\end{array}$ & $\begin{array}{l}649 \\
652 \\
644 \\
634 \\
6 C 6 \\
-\end{array}$ & $\begin{array}{l}441 \\
453 \\
464 \\
464 \\
477 \\
482\end{array}$ & $\begin{array}{l}431 \\
439 \\
444 \\
456 \\
463 \\
466\end{array}$ & $\begin{array}{c}515 \\
513 \\
496 \\
500 \\
500 \\
--\end{array}$ \\
\hline AVERAGE & 467 & 514 & 658 & $64 B$ & 635 & 594 & 547 & 692 & $6 c 0$ & 493 & 464 & 468 \\
\hline
\end{tabular}


5-0825. RED RIVER OF THE NORTH AT GRAND FORKS, N. DAK.--Continued

TEMPERATURE $\left({ }^{\circ} \mathrm{C}\right)$ OF WATER, WATER YEAR OCTOBER 1967 TO SEPTEMBER 1968

DAY

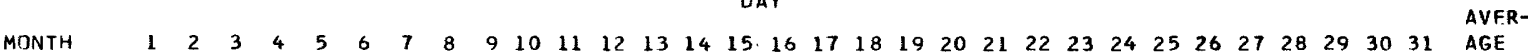

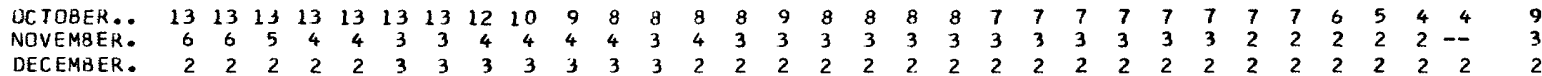

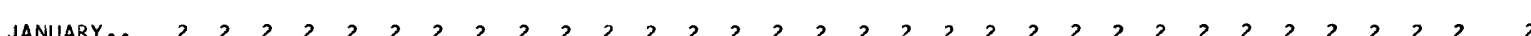

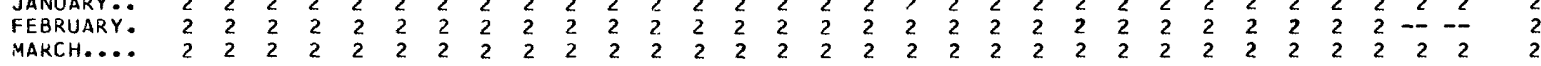

$\begin{array}{lllllllllllllllllllllllllllllllllllll}\text { APRIL.... } & 2 & 2 & 2 & 2 & 2 & 2 & 3 & 3 & 3 & 4 & 4 & 7 & 6 & 7 & 7 & 7 & 7 & 7 & 8 & 9 & 9 & 9 & 9 & 9 & 9 & 9 & 9 & 9 & 10 & 12 & -- & 6 & 6\end{array}$

$\begin{array}{lllllllllllllllllllllllllllllllllllll}\text { MAY...... } 10 & 12 & 13 & 13 & 13 & 13 & 12 & 12 & 11 & 11 & 11 & 11 & 11 & 12 & 14 & 13 & 13 & 12 & 10 & 10 & 11 & 12 & 12 & 12 & 14 & 15 & 15 & 15 & 15 & 16 & 16 & 12\end{array}$

JUNE..... $17171817191919191919181818181818181818192021 \quad 212121202020 \quad 2020-2 \quad 19$

$\begin{array}{lllllllllllllllllllllllllllllllllll}\text { JULY..... } & 20 & 18 & 18 & 18 & 18 & 20 & 22 & 23 & 22 & 22 & 23 & 22 & 23 & 23 & 24 & 24 & 24 & 23 & 23 & 23 & 23 & 23 & 23 & 23 & 22 & 22 & 22 & 22 & 22 & 22 & 22 & 22\end{array}$

$\begin{array}{lllllllllllllllllllllllllllllllll}\text { AUGUST... } & 21 & 21 & 21 & 21 & 22 & 23 & 24 & 23 & 22 & 22 & 23 & 22 & 21 & 21 & 21 & 21 & 18 & 18 & 18 & 18 & 18 & 18 & 18 & 19 & 19 & 18 & 18 & 18 & 19 & 19 & 19 & 20\end{array}$

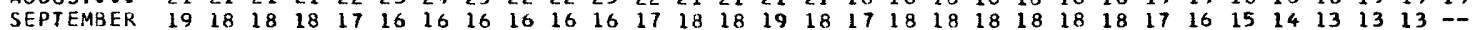


5-1244.8 KAWISHIWI RIVER NEAR ELY, MINN.

(Hydrologic bench-mark station)

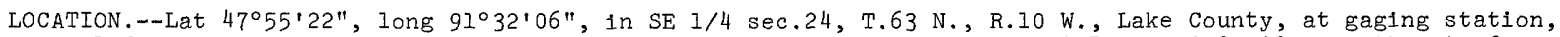
on left bank upstream from rapids, 2 miles upstream from South Kawishiwi River, 2.2 miles southwest of Fernberg Lookout Tower and 14 miles east of Ely. DRAINAGE AREA. --253 sq mi.

RECORDS AVAILABLE.--Chemical analyses: October 1967 to September 1968.

Water temperatures: July 1966 to september 1968.

CHEMICAL ANALYSES IN MILLIGRAMS PER LITER, WATER YEAR DCTOBER 1967 TO SEPTEMBER 1968

\begin{tabular}{|c|c|c|c|c|c|c|c|c|c|c|c|}
\hline DATE & $\begin{array}{l}\text { DIS- } \\
\text { CHARGE } \\
\text { (CFS) }\end{array}$ & $\begin{array}{l}\text { TEMP- } \\
\text { ERATURE } \\
\text { (DEG CI }\end{array}$ & $\begin{array}{l}\text { SILICA } \\
\text { (SID2) }\end{array}$ & $\begin{array}{l}\text { TOTAL } \\
\text { IRCN } \\
\text { (FE) }\end{array}$ & $\begin{array}{l}\text { CAL- } \\
\text { CIUM } \\
\text { (CA) }\end{array}$ & $\begin{array}{l}\text { MAG- } \\
\text { NE- } \\
\text { S IUM } \\
\text { (MG) }\end{array}$ & $\begin{array}{l}\text { SODIUM } \\
\text { (NA) }\end{array}$ & $\begin{array}{l}\text { PO- } \\
\text { TAS- } \\
\text { SIUM } \\
\text { (K) }\end{array}$ & $\begin{array}{l}\text { BICAR- } \\
\text { BONA TE } \\
\text { (HCO3) }\end{array}$ & $\begin{array}{l}\text { CAR- } \\
\text { BONATE } \\
\left(\mathrm{CO}_{3}\right)\end{array}$ & $\begin{array}{l}\text { ALKA- } \\
\text { LIAITY } \\
\text { AS } \\
\text { CACO } 3\end{array}$ \\
\hline $\begin{array}{l}\text { NOV. } \\
\text { O2 } 2 . . \\
\text { JAN. }\end{array}$ & 31 & 6 & 3.5 & .13 & 3.0 & 1.0 & 1.1 & .2 & 10 & 0 & $\varepsilon$ \\
\hline $\begin{array}{l}03 . . . \\
\text { FEB. }\end{array}$ & 33 & 1 & 4.0 & .14 & 4.0 & 1.5 & 1.6 & .8 & 19 & C & $1 t$ \\
\hline $\begin{array}{c}01 \ldots . . \\
27 \ldots \\
\text { MAR... }\end{array}$ & $\begin{array}{l}37 \\
29\end{array}$ & $\begin{array}{l}1 \\
1\end{array}$ & $\begin{array}{l}4.1 \\
4.1\end{array}$ & .10 & $\begin{array}{l}3.8 \\
3.8\end{array}$ & $\begin{array}{l}1.5 \\
1.5\end{array}$ & $\begin{array}{l}1.3 \\
1.5\end{array}$ & $\begin{array}{r}1.6 \\
.8\end{array}$ & $\begin{array}{l}18 \\
18\end{array}$ & $\begin{array}{l}0 \\
\mathrm{c}\end{array}$ & $\begin{array}{l}15 \\
15\end{array}$ \\
\hline$\underset{\text { MAY }}{26 \ldots}$ & 38 & 1 & 4.0 & .08 & 3.8 & 1.5 & 1.2 & .8 & 17 & c & 14 \\
\hline $\begin{array}{c}02 \ldots \\
29 \ldots \\
\text { JUNE }\end{array}$ & $\begin{array}{r}1030 \\
547\end{array}$ & 11 & $\begin{array}{l}3.3 \\
3.4\end{array}$ & $\begin{array}{l}.18 \\
.20\end{array}$ & $\begin{array}{l}3.2 \\
3.3\end{array}$ & $\begin{array}{l}1.3 \\
1.4\end{array}$ & $\begin{array}{r}.9 \\
1.2\end{array}$ & $\begin{array}{r}1.0 \\
.4\end{array}$ & $\begin{array}{l}13 \\
12\end{array}$ & $\begin{array}{l}0 \\
0\end{array}$ & $\begin{array}{l}11 \\
10\end{array}$ \\
\hline juL 19 & 1200 & 17 & 3.6 & .18 & 3.2 & 1.4 & .9 & .4 & 12 & 0 & 10 \\
\hline $\begin{array}{l}03 \ldots \\
A \cup G .\end{array}$ & 698 & 16 & 3.5 & .19 & 3.4 & 1.4 & 1.0 & .4 & 12 & c & 1C \\
\hline SEPT... & 204 & 22 & 2.8 & .13 & 3.7 & .1 .4 & 1.0 & .6 & 14 & c & 11 \\
\hline $11 \ldots$ & 100 & 17 & 3.6 & .08 & 3.7 & 1.5 & 1.0 & .4 & 13 & 0 & 11 \\
\hline
\end{tabular}


5-1244.8 KAWISHIWI RIVER NEAR ELY, MINN.--Continued

(Hydrologic bench-mark station)

EXTREMES, 1967-68.--Water temperatures: Maximum, $21^{\circ} \mathrm{C} \mathrm{July} 17$ to Aug. 19; minimum, freezing point on many days in February and March.

EXTREMES, 1966-68.--Water temperatures: Maximum, 24\% $\mathrm{C}$ Juy 24, 25, 1966; minimum, freezing point on many days during winter months.

REMARKS.--Recorder stopped Mar. 27 to Apr. 29; range in temperature, $1^{\circ} \mathrm{C}$ to $6^{\circ} \mathrm{C}$.

CHEMICAL ANALYSES IN MILLIGRAMS PER LITER, WATER YEAR DCTOBER 1967 TO SEPTEMBER 1968

\begin{tabular}{|c|c|c|c|c|c|c|c|c|c|c|c|}
\hline DATE & $\begin{array}{l}\text { SULFATE } \\
\text { (SO4) }\end{array}$ & $\begin{array}{l}\text { CHLO- } \\
\text { RIDE } \\
\text { (CL) }\end{array}$ & $\begin{array}{l}\text { FLUD- } \\
\text { RIDE } \\
\text { (F) }\end{array}$ & $\begin{array}{l}\text { NITRATE } \\
\text { (NO3) }\end{array}$ & $\begin{array}{l}\text { ORTHO } \\
\text { PHOS- } \\
\text { PHATE } \\
\text { (PO4) }\end{array}$ & $\begin{array}{l}\text { PHOS- } \\
\text { PHATE } \\
\text { (P04) }\end{array}$ & $\begin{array}{l}\text { TOTAL } \\
\text { ALUM- } \\
\text { INIUM } \\
\text { (AL) }\end{array}$ & $\begin{array}{c}\text { BORON } \\
\text { (B) }\end{array}$ & $\begin{array}{l}\text { OIS- } \\
\text { SOLVED } \\
\text { SOLIOS, } \\
\text { ISUM OF } \\
\text { CONSTI- } \\
\text { TUENTS ) }\end{array}$ & $\begin{array}{l}\text { DIS- } \\
\text { SOLVED } \\
\text { SCLICS } \\
\text { (TCAS } \\
\text { PER } \\
\text { AC-FT I }\end{array}$ & $\begin{array}{l}\text { CIS- } \\
\text { SCIVED } \\
\text { SDLICS } \\
\text { ITCAS } \\
\text { PER } \\
\text { CAY) }\end{array}$ \\
\hline
\end{tabular}

NDV.

$\begin{array}{llll}\text { NDV. } & & & \\ 02 & 4.5 & .3 & .0\end{array}$

$02 \ldots$

$03 .$.

FEB.

$01 \ldots$

4.5

- 0

- 4

$-$

.01
.17
.28
.20
.17
.26
.12
1.0
.31
.24
.16

$\begin{array}{ll}.5 & .02 \\ .5 & .01 \\ .4 & .01 \\ .5 & .00 \\ .4 & .00 \\ .6 & .04 \\ .0 & .03 \\ .9 & .04 \\ .8 & .03 \\ 1.0 & .01 \\ .7 & .04\end{array}$

20

.05

2. 87

6.0

.03

.03
.03

28

- 18

5. 26

MAR .

26...

MAY

O2...

JUNE

$19 .$.

$03 .$.

28

.06

4.69

$\begin{array}{lll}6.0 & .2 & .0\end{array}$

.07

27

.

2.85

$\begin{array}{ll}3.5 & .2\end{array}$

.02

27

- $\mathrm{Cb}$

4.91

.4

21

$.04 \quad 91.8$

$\begin{array}{llll}4.3 & .3 & .0 & .7\end{array}$

.04

21

- $\mathrm{C}$

56.1

AUG.

SEPT.

$4.3 \quad \cdot 3$

.7

.59

22

.05

123

$5.0 \quad .8$

.07

22

.$c 6$

77.3

$11 . .$.

5.0

.5

.10

.04

.05

19.8

24

.05

9.99 
(Hydrologic bench-mark station)

CHEMICAL ANALYSES IN MILLIGRAMS PER LItER, WATER YEAR OCTOBER 1967 TO SEPTEMBER 1968

\begin{tabular}{|c|c|c|c|c|c|c|c|}
\hline DATE & $\begin{array}{l}\text { HARO- } \\
\text { NESS } \\
(C A, M G)\end{array}$ & $\begin{array}{l}\text { NON- } \\
\text { CAR- } \\
\text { BONATE } \\
\text { HARD- } \\
\text { NESS }\end{array}$ & $\begin{array}{c}\text { SOCIUM } \\
\text { AD- } \\
\text { SORP- } \\
\text { TION } \\
\text { RATIO }\end{array}$ & $\begin{array}{l}\text { PERCENT } \\
\text { SODIUM }\end{array}$ & $\begin{array}{l}\text { SPECI - } \\
\text { FIC } \\
\text { COND- } \\
\text { UCTANCE } \\
\text { (MICRO- } \\
\text { MHOS) }\end{array}$ & $\mathbf{P H}$ & COLOF \\
\hline NOV. & 12 & 4 & .1 & 17 & 35 & 6.3 & 45 \\
\hline $\begin{array}{l}03 . . \\
\text { FEB. }\end{array}$ & 16 & 0 & .2 & 17 & 40 & 7.1 & 31 \\
\hline $\begin{array}{l}01 \ldots \\
27 \ldots\end{array}$ & $\begin{array}{l}16 \\
16\end{array}$ & 1 & $\begin{array}{l}.1 \\
.2\end{array}$ & $\begin{array}{l}14 \\
16\end{array}$ & $\begin{array}{l}40 \\
38\end{array}$ & $\begin{array}{l}7.0 \\
7.1\end{array}$ & $\begin{array}{l}24 \\
41\end{array}$ \\
\hline $\begin{array}{l}\text { MAR. } \\
26 \ldots \\
\text { MAY }\end{array}$ & 16 & 2 & .1 & 14 & 37 & 7.0 & $2 B$ \\
\hline $02 \ldots$ & $\begin{array}{l}13 \\
14\end{array}$ & $\begin{array}{l}2 \\
4\end{array}$ & .1 & $\begin{array}{l}12 \\
15\end{array}$ & $\begin{array}{l}31 \\
34\end{array}$ & $\begin{array}{l}6.6 \\
6.4\end{array}$ & $\begin{array}{l}50 \\
40\end{array}$ \\
\hline $\begin{array}{l}\text { JUNE } \\
19 . . . \\
\text { JULY }\end{array}$ & 14 & 4 & .1 & 12 & 31 & 6.4 & 50 \\
\hline $\begin{array}{l}03 \ldots \\
\text { AUG. }\end{array}$ & 14 & 4 & .1 & 13 & 32 & 6.4 & 50 \\
\hline SEPT... & 15 & 3 & .1 & 12 & 33 & 6.7 & 45 \\
\hline 11. & 15 & 4 & .1 & 12 & 35 & 6.7 & 40 \\
\hline
\end{tabular}

\section{RADIOCHEMICAL ANALYSES, WATER YEAR OCTOBER 1967 TO SEPTEMBER 1968}

Units of measurement: Uranium, micrograms per liter of water, radium as radium-226, in picocuries per liter of water; gross beta radiation as strontium-90-yttrium-90, in picocuries per liter of water; gross alpha radiation, as micrograms of uranium equivalent per liter of water.

\begin{tabular}{|c|c|c|c|c|c|c|c|c|c|}
\hline \multirow{2}{*}{\multicolumn{2}{|c|}{$\begin{array}{c}\text { Date } \\
\text { of } \\
\text { Collection }\end{array}$}} & \multicolumn{5}{|c|}{ Dissolved } & \multicolumn{3}{|c|}{ Suspended } \\
\hline & & $\begin{array}{l}\text { Uranium } \\
\text { (ug/1) }\end{array}$ & $\begin{array}{l}\text { Radium } \\
(\mathrm{pc} / 1)\end{array}$ & $\begin{array}{l}\text { Gross } \\
(\mathrm{pc} / 1)^{\circ}\end{array}$ & $\begin{array}{l}\text { Gross } \propto \\
(\operatorname{ug} U / 1)\end{array}$ & $\begin{array}{c}\text { Total } \\
\text { Dissolved } \\
\text { Solids } \\
(\mathrm{mg} / \mathrm{l})\end{array}$ & $\underset{(p c / 1)}{\operatorname{Gross}} \beta$ & $\begin{array}{l}\text { Gross } \propto \\
(\operatorname{ug} \mathrm{U} / 1)\end{array}$ & $\begin{array}{c}\text { Suspended } \\
\text { Sediments } \\
\text { (mg/1) }\end{array}$ \\
\hline ov. & $2,1967 \ldots$ & $<0.4$ & $<0.1$ & 5.1 & 0.7 & 34 & 1.6 & 0.7 & $<1$ \\
\hline ay & $2,1968 \ldots$ & $<.4$ & $<.1$ & 5.9 & $<.4$ & 34 & 2.1 & $<.4$ & 4 \\
\hline
\end{tabular}

PERIODIC DAILY SUSPENDED SEDIMENT, WATER YEAR OCTOBER 1967 TO SEPTEMBER 1968

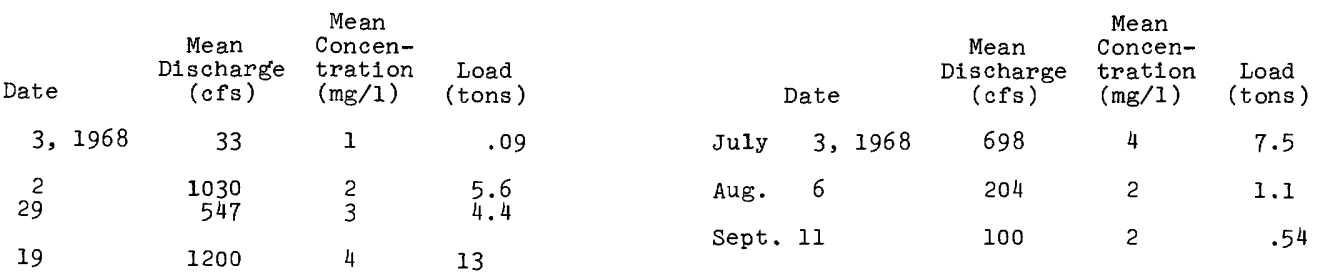


5-1244.8 KAWISHIWI RIVER NEAR ELY, MINN.--Continued

(Hydrologic bench-mark station)

TEMPERATURE $\left({ }^{\circ} \mathrm{C}\right)$ OF WATER, WATER YEAR OCTOBER 1967 TO SEPTEMBER 1968

(WATER-STAGE RECORDER WITH TEMPERATURE ATTACHMENT, CONTINUOUS ETHYL ALCOHOL-ACTUATED THERMOGRAPH)

Day

Month $\begin{array}{llllllllllllllllllllllllllllllll}1 & 2 & 3 & 4 & 5 & 6 & 7 & 8 & 9 & 10 & 11 & 12 & 13 & 14 & 15 & 16 & 17 & 18 & 19 & 20 & 21 & 22 & 23 & 24 & 25 & 26 & 27 & 28 & 29 & 30 & 31\end{array}$

Average

October

$\begin{array}{lllllllllllllllllllllllllllllllllllll}\text { Maximum } & 16 & 15 & 14 & 13 & 13 & 13 & 13 & 13 & 12 & 11 & 11 & 10 & 10 & 10 & 10 & 10 & 10 & 10 & 10 & 9 & 9 & 9 & 8 & 8 & 8 & 8 & 8 & 7 & 6 & 6 & 6 & 10\end{array}$

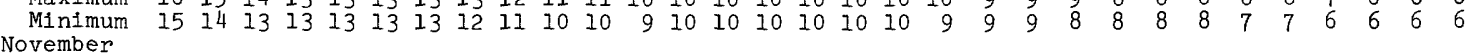

$\begin{array}{lllllllllllllllllllllllllllllllllllllllll}\text { Maximum } & 6 & 6 & 6 & 6 & 5 & 5 & 5 & 5 & 4 & 4 & 4 & 4 & 4 & 4 & 4 & 4 & 4 & 4 & 4 & 4 & 4 & 4 & 4 & 4 & 4 & 4 & 4 & 3 & 3 & 3 & -- \\ \text { Minimum } & 6 & 6 & 6 & 5 & 5 & 5 & 5 & 4 & 4 & 4 & 4 & 4 & 4 & 4 & 4 & 4 & 4 & 4 & 4 & 4 & 4 & 4 & 4 & 4 & 4 & 4 & 3 & 3 & 3 & 3 & --\end{array}$

December

Maximum

Minimum

January

Maximum

February

Maximum

Minimum

March

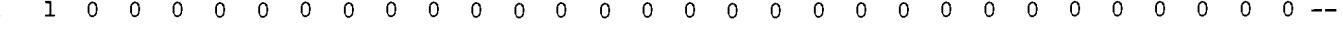

Maximum

April

Maximum

$\begin{array}{lllllllllllllllllllllllllllllllllllllll}3 & 3 & 3 & 3 & 3 & 3 & 3 & 3 & 3 & 3 & 3 & 2 & 2 & 2 & 2 & 2 & 2 & 2 & 2 & 2 & 2 & 1 & 1 & 1 & 1 & 1 & 1 & 1 & 1 & 1 & 1 \\ 3 & 3 & 3 & 3 & 3 & 3 & 3 & 3 & 3 & 3 & 2 & 2 & 2 & 2 & 2 & 2 & 2 & 2 & 2 & 2 & 1 & 1 & 1 & 1 & 1 & 1 & 1 & 1 & 1 & 1 & 1\end{array}$

$\begin{array}{lllllllllllllllllllllllllllllllllllllllll}1 & 1 & 1 & 1 & 1 & 1 & 1 & 1 & 1 & 1 & 1 & 1 & 1 & 1 & 1 & 1 & 1 & 1 & 1 & 1 & 1 & 1 & 1 & 1 & 1 & 1 & 1 & 1 & 1 & 1 & 1 \\ 1 & 1 & 1 & 1 & 1 & 1 & 1 & 1 & 1 & 1 & 1 & 1 & 1 & 1 & 1 & 1 & 1 & 1 & 1 & 1 & 1 & 1 & 1 & 1 & 1 & 1 & 1 & 1 & 1 & 1 & 1\end{array}$

$\begin{array}{llllllllllllllllllllllllllllllllllllll}1 & 1 & 0 & 0 & 0 & 0 & 0 & 0 & 0 & 0 & 0 & 0 & 0 & 0 & 0 & 0 & 0 & 0 & 0 & 0 & 0 & 0 & 0 & 0 & 0 & 0 & 0 & 0 & 0 & 0 & -- \\ 1 & 0 & 0 & 0 & 0 & 0 & 0 & 0 & 0 & 0 & 0 & 0 & 0 & 0 & 0 & 0 & 0 & 0 & 0 & 0 & 0 & 0 & 0 & 0 & 0 & 0 & 0 & 0 & 0 & 0 & --\end{array}$

$\begin{array}{lllllllllllllllllllllllllllllllllllllllllll}0 & 0 & 1 & 1 & 1 & 1 & 1 & 1 & 1 & 1 & 1 & 1 & 1 & 1 & 1 & 1 & 1 & 1 & 1 & 1 & 1 & 1 & 1 & 1 & 1 & 1 & -- & -- & -- & -- & -- \\ 0 & 0 & 0 & 1 & 1 & 1 & 1 & 1 & 1 & 1 & 1 & 1 & 1 & 1 & 1 & 1 & 1 & 1 & 1 & 1 & 1 & 1 & 1 & 1 & 1 & 1 & -- & -- & -- & -- & --\end{array}$ Minimum

May

$\begin{array}{lllllllllllllllllllllllllllllllllll}\text { Maximum } & 7 & 7 & 7 & 7 & 7 & 7 & 7 & 7 & 7 & 7 & 8 & 8 & 9 & 9 & 10 & 10 & 11 & 11 & 11 & 11 & 11 & 11 & 11 & 11 & 11 & 11 & 11 & 11 & 11 & 11 & 11\end{array}$ Minimum

June

$\begin{array}{llllllllllllllllllllllllllllllllllllllll}\text { Maximum } & 11 & 11 & 12 & 13 & 14 & 14 & 14 & 15 & 16 & 16 & 16 & 16 & 16 & 17 & 17 & 17 & 17 & 17 & 17 & 17 & 16 & 16 & 16 & 16 & 16 & 16 & 16 & 16 & 16 & 16 & -0\end{array}$

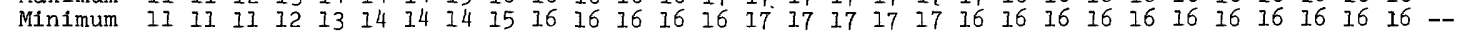
JuIy

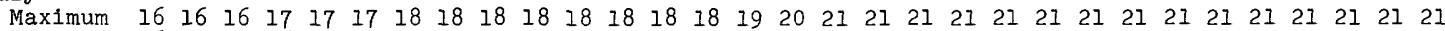
$\begin{array}{llllllllllllllllllllllllllllllllllll}\text { Minimum } & 16 & 16 & 16 & 16 & 17 & 17 & 17 & 18 & 18 & 18 & 18 & 18 & 18 & 18 & 18 & 19 & 20 & 21 & 21 & 21 & 21 & 21 & 21 & 21 & 21 & 21 & 21 & 21 & 21 & 21 & 21\end{array}$ August

$\begin{array}{llllllllllllllllllllllllllllllllll}\text { Maximum } & 21 & 21 & 21 & 21 & 21 & 21 & 21 & 21 & 21 & 21 & 21 & 21 & 21 & 21 & 21 & 21 & 21 & 21 & 21 & 20 & 20 & 20 & 20 & 20 & 20 & 20 & 19 & 19 & 19 & 19 & 19\end{array}$

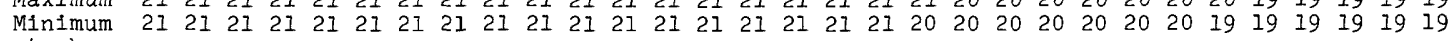

September

$\begin{array}{llllllllllllllllllllllllllllllllllllllllll}\text { Maximum } & 19 & 19 & 19 & 19 & 19 & 19 & 18 & 17 & 17 & 17 & 17 & 17 & 16 & 17 & 17 & 17 & 17 & 17 & 17 & 17 & 17 & 17 & 17 & 17 & 17 & 17 & 16 & 16 & 16 & 15 & -0\end{array}$

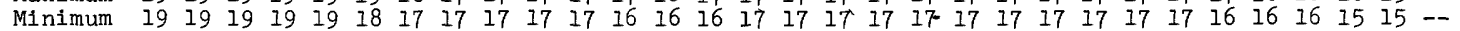

10 
LOCATION.--Lat 45 07'36", long 93 17'48", in SW/ sec.12, T.119 N., R.21 W., Hennepin County, at gaging station on right bank half a mile downstream from coon Creek, 1.5 miles downstream from hydroelectric plant of

Northern States Power Co. at Coon Rapids, 6.5 miles downstream from Anoka, and at mile 864.8 upstream from Ohio River.

\section{CHEMICAL ANALYSES IN MILLIGRAMS PER LITER, WATER YEAR OCTOBER 1967 TO SEPTEMBER 1968}

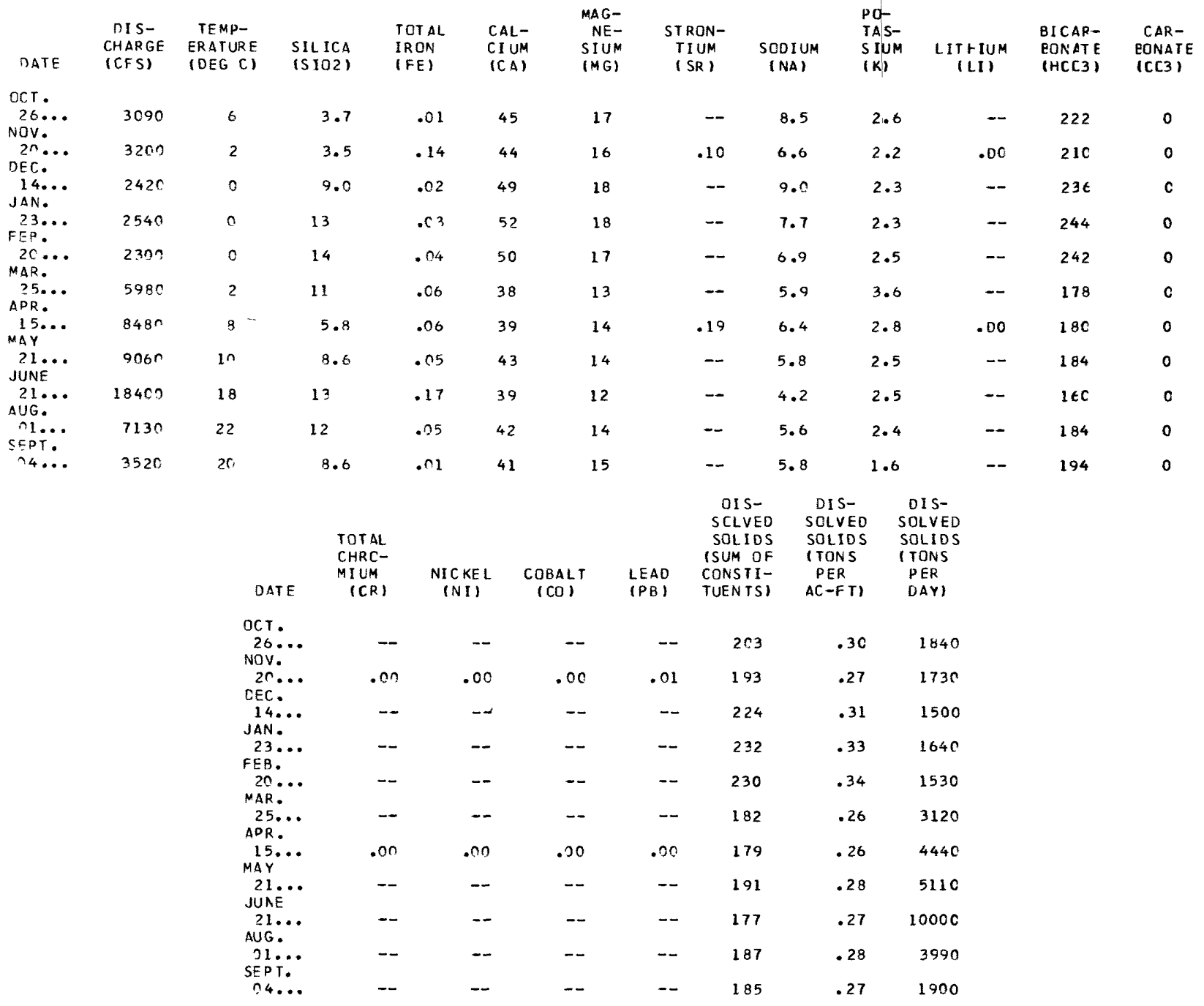


5-2885. MISSISSIPPI RIVER NEAR ANOKA, MINN.--Continued

DRAINAGE AREA.--19,100 sq mi, approximately.

RECORDS AVAILABLE.-Chemical analyses: August 1960 to September 1965 , October 1967 to September 1968.

Water temperatures: August 1960 to September 1963.

CHEMICAL ANALYSFS IN NILLIGRAMS PER LITER, WATER YEAR OCTOBER 1967 TO SEPTEMBER 1968

\begin{tabular}{|c|c|c|c|c|c|c|c|c|c|c|c|}
\hline$D \triangle T E$ & $\begin{array}{l}\text { ALKA- } \\
\text { LINITY } \\
\text { AS } \\
\text { CACO3 }\end{array}$ & $\begin{array}{l}\text { SULFATE } \\
\text { (SO4) }\end{array}$ & $\begin{array}{l}\text { CHLO- } \\
\text { RIDE } \\
\text { (CL) }\end{array}$ & $\begin{array}{l}\text { FLUO- } \\
\text { RIDE } \\
\text { (F) }\end{array}$ & $\begin{array}{l}\text { NITRATE } \\
\text { (NC3) }\end{array}$ & $\begin{array}{l}\text { CPTHO } \\
\text { PHCS - } \\
\text { PHATE } \\
\text { (P04) }\end{array}$ & $\begin{array}{l}\text { TOT AL } \\
\text { ALUM- } \\
\text { INIUM } \\
\text { (AL) }\end{array}$ & $\begin{array}{c}\text { BORON } \\
(B)\end{array}$ & $\begin{array}{l}\text { COPPER } \\
\text { (CU) }\end{array}$ & $\begin{array}{l}\text { ZIAC } \\
\text { IZNI }\end{array}$ & $\begin{array}{l}C A C- \\
N I C F \\
(C D)\end{array}$ \\
\hline \multicolumn{12}{|l|}{ пCT. } \\
\hline $\begin{array}{l}26 \ldots \\
\text { Nav. }\end{array}$ & 182 & 12. & 5.0 & .1 & .0 & .12 & .4 & .03 & -- & - & - \\
\hline$\underset{\text { DER. }}{29}$ & 172 & 12 & 4.2 & .1 & .1 & .07 & .2 & .03 & .00 & .00 & .00 \\
\hline $\begin{array}{l}14 \ldots \\
\text { JAN. }\end{array}$ & 193 & 14 & 5.8 & .1 & .7 & .10 & .4 & .02 & - & - & -- \\
\hline $\begin{array}{l}23 . . \\
\text { FEB. }\end{array}$ & $20 n$ & 13 & 4.2 & .1 & .9 & .24 & .3 & .08 & -- & - & - \\
\hline$\stackrel{2^{2}}{M \Delta R} \cdot$ & 198 & 13 & 4.6 & .1 & 1.6 & .46 & .3 & .02 & - & - & - \\
\hline $\begin{array}{l}25 \ldots \\
\triangle P R .\end{array}$ & 146 & 16 & 4.6 & .1 & 1.4 & .34 & .4 & .02 & - & - & - \\
\hline $\operatorname{MAY}_{\operatorname{MAY}}^{15}$ & 148 & 17 & 4.8 & .2 & .2 & .24 & .0 & .03 & .00 & .00 &. $\mathrm{co}$ \\
\hline JUNE & 151 & 21 & 3.9 & .2 & 1.1 & .33 & .0 & .03 & - & - & - \\
\hline $\begin{array}{l}21 \ldots \\
\triangle \cup G\end{array}$ & 131 & 21 & 3.7 & .3 & 1.6 & .31 & .8 & .06 & -- & -- & -- \\
\hline SEPT. & 151 & 14 & 4.6 & .2 & 1.3 & .36 & .8 & .02 & -- & - & - \\
\hline \multirow[t]{13}{*}{$44 \ldots$} & 159 & 13 & 3.4 & .2 & .5 & .39 & .6 & .04 & -- & - & -- \\
\hline & & DATE & $\begin{array}{l}\text { HARD- } \\
\text { NESS } \\
\text { (CA,MG) }\end{array}$ & $\begin{array}{l}\text { NON- } \\
\text { CAR- } \\
\text { BONATE } \\
\text { HARD- } \\
\text { NESS }\end{array}$ & $\begin{array}{c}\text { SOCIUM } \\
\text { AD- } \\
\text { SORP- } \\
\text { TION } \\
\text { RATIO }\end{array}$ & $\begin{array}{l}\text { PERCENT } \\
\text { SODIUM }\end{array}$ & $\begin{array}{l}\text { SPECI- } \\
\text { FIC } \\
\text { COND- } \\
\text { LCTANCE } \\
\text { (MICRO- } \\
\text { MHOS) }\end{array}$ & PH & $C O L D R$ & & \\
\hline & & $\begin{array}{l}\text { OCT } \\
26 . . . \\
\text { NOV. }\end{array}$ & $1 B 2$ & 0 & .3 & 9 & 365 & 7.8 & 4 & & \\
\hline & & DEC... & 177 & 5 & .2 & 7 & 357 & 7.4 & 8 & & \\
\hline & & $\begin{array}{l}14 \ldots . . \\
\text { JAN. }\end{array}$ & 196 & 3 & .3 & 9 & 387 & B.1 & 9 & & \\
\hline & & $\begin{array}{l}23 \ldots \\
\text { FEB. }\end{array}$ & 203 & 3 & .2 & 7 & 399 & 7.9 & 4 & & \\
\hline & & $\begin{array}{l}20 \ldots \\
\text { MAR. }\end{array}$ & 195 & $c$ & .2 & 7 & 393 & 8.0 & B & & \\
\hline & & $\begin{array}{c}25 \ldots . \\
\triangle P R .\end{array}$ & 148 & 2 & .2 & B & 316 & 7.6 & 18 & & \\
\hline & & $\operatorname{MAY}_{15}$ & 154 & 6 & .2 & 8 & $32 B$ & 7.7 & 8 & & \\
\hline & & $\underset{\text { JUNE }}{21 \ldots}$ & 164 & 13 & .2 & 7 & 335 & 7.9 & 22 & & \\
\hline & & $\begin{array}{l}21 \ldots \\
\text { AUG. }\end{array}$ & 146 & 15 & .2 & 6 & 298 & 7.9 & 50 & & \\
\hline & & $\begin{array}{l}\text { O1... } \\
\text { SEPT. }\end{array}$ & 160 & 10 & .2 & 7 & 321 & 7.7 & 25 & & \\
\hline & & $04 \ldots$ & 163 & 4 & .2 & 7 & $32 B$ & 7.5 & 4 & & \\
\hline
\end{tabular}


5-2910. WHETSTONE RIVER NEAR BIG STONE CITY, S. DAK.

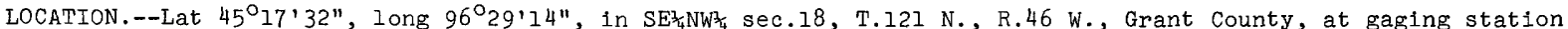
on right bank $20 \mathrm{ft}$ downstream from highway bridge, 1.5 miles west of Big Stone City, and 4.5 miles upstream from Big Stone Lake.

DRAINAGE AREA. $--389 \mathrm{sq} \mathrm{mi}$.

RECORDS AVAILABLE.--Chemical analyses: October 1967 to September 1968.

CHEMICAL ANALYSES IN MILLIGRAMS PER LITER, WATER YEAR OCTOBER 1967 TD SEPTEMBER 1968

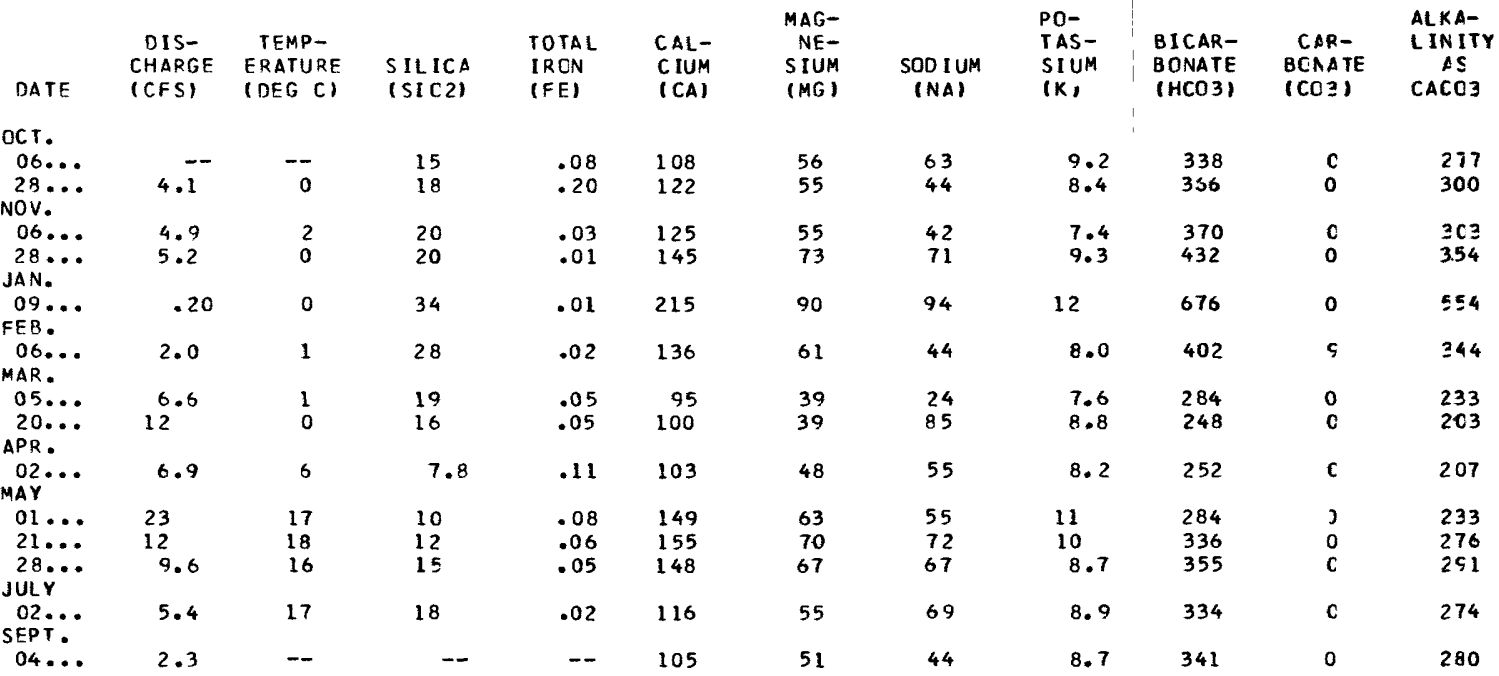

\begin{tabular}{|c|c|c|c|c|c|c|c|c|c|c|}
\hline DATE & $\begin{array}{l}\text { SULFATE } \\
\text { (SO } 4 \text { ) }\end{array}$ & $\begin{array}{l}\text { CHLO- } \\
\text { RIDE } \\
(\mathrm{CL})\end{array}$ & $\begin{array}{l}\text { FLUO- } \\
\text { RIDE } \\
\text { (F) }\end{array}$ & $\begin{array}{l}\text { NITRATE } \\
\text { (NO3) }\end{array}$ & $\begin{array}{l}\text { ORTHO } \\
\text { PHOS- } \\
\text { PHAT E } \\
\text { (PO4) }\end{array}$ & $\begin{array}{l}\text { TOT AL } \\
\text { ALUM- } \\
\text { INIUM } \\
\text { (AL) }\end{array}$ & $\begin{array}{c}\text { BORDN } \\
\text { (B) }\end{array}$ & $\begin{array}{l}\text { OI S- } \\
\text { SOLVED } \\
\text { SOL IDS } \\
\text { (SUM OF } \\
\text { CDNSTI- } \\
\text { TUENTS) }\end{array}$ & $\begin{array}{l}\text { DIS- } \\
\text { SOL VED } \\
\text { SOL IDS } \\
\text { ITOAS } \\
\text { PER } \\
\text { AC-FT I }\end{array}$ & $\begin{array}{l}\text { CIS- } \\
\text { SCLVED } \\
\text { SOL IDS } \\
\text { IICAS } \\
\text { PER } \\
\text { CAYI }\end{array}$ \\
\hline $\begin{array}{l}\text { OCT. } \\
06 \ldots \\
28 \ldots\end{array}$ & $\begin{array}{l}289 \\
263\end{array}$ & $\begin{array}{l}52 \\
30\end{array}$ & .3 & $\begin{array}{l}.1 \\
.2\end{array}$ & $\begin{array}{l}.45 \\
.26\end{array}$ & $=$ & $\begin{array}{l}.20 \\
.16\end{array}$ & $\begin{array}{l}759 \\
722\end{array}$ & $\begin{array}{l}1.11 \\
1.04\end{array}$ & $8 . \overline{47}$ \\
\hline $\begin{array}{l}\text { Nov. } \\
06 . . . \\
28 . . .\end{array}$ & $\begin{array}{l}266 \\
372\end{array}$ & $\begin{array}{l}28 \\
42\end{array}$ & $\begin{array}{l}.2 \\
.2\end{array}$ & .3 & $\begin{array}{l}.44 \\
.29\end{array}$ & $1 . \overline{2}$ & .14 & $\begin{array}{l}726 \\
946\end{array}$ & $\begin{array}{l}1.05 \\
1.36\end{array}$ & $\begin{array}{l}10.2 \\
14.3\end{array}$ \\
\hline $\begin{array}{l}\text { JAN. } \\
09 . . \\
\text { FE8. }\end{array}$ & 445 & 61 & .3 & .1 & .77 & .6 & .33 & 1290 & 1.84 & .73 \\
\hline MAR... & 328 & 23 & .2 & 4.4 & .33 & .9 & .15 & 841 & 1.23 & 5.05 \\
\hline $\begin{array}{c}05 \ldots \\
20 \ldots \\
\text { APR. }\end{array}$ & $\begin{array}{l}204 \\
277\end{array}$ & $\begin{array}{l}12 \\
62\end{array}$ & .2 & $12^{1.2}$ & $\begin{array}{c}.62 \\
6.1\end{array}$ & -6 & $\begin{array}{l}.10 \\
.26\end{array}$ & $\begin{array}{l}544 \\
729\end{array}$ & $\begin{array}{r}.78 \\
1.03\end{array}$ & $\begin{array}{l}10.3 \\
24.6\end{array}$ \\
\hline MAY $02 \ldots$ & 308 & 26 & .4 & .2 & .71 & .8 & .22 & 682 & 1.01 & 13.8 \\
\hline $\begin{array}{l}01 \ldots . \\
21 \ldots \\
28 \ldots\end{array}$ & $\begin{array}{l}463 \\
468 \\
430\end{array}$ & $\begin{array}{l}22 \\
34 \\
26\end{array}$ & $\begin{array}{l}.3 \\
.3 \\
.3\end{array}$ & $\begin{array}{l}.1 \\
.6 \\
.1\end{array}$ & $\begin{array}{l}.60 \\
.61 \\
.74\end{array}$ & .0 & $\begin{array}{l}.22 \\
.28 \\
.04\end{array}$ & $\begin{array}{l}914 \\
988 \\
938\end{array}$ & $\begin{array}{l}1.34 \\
1.47 \\
1.37\end{array}$ & $\begin{array}{l}61.2 \\
35.0 \\
26.2\end{array}$ \\
\hline $\begin{array}{l}\text { JULY } \\
02 \ldots . . \\
\text { SEPT. }\end{array}$ & 318 & 42 & .3 & .1 & 1.1 & $\cdot 3$ & .23 & 793 & 1.14 & 12.3 \\
\hline $04 \ldots$ & 253 & 24 & -- & .5 & - & -- & .19 & -- & .98 & 4.50 \\
\hline
\end{tabular}


5-3167.70. MINNESOTA RIVER AT NEW ULM, MINN.

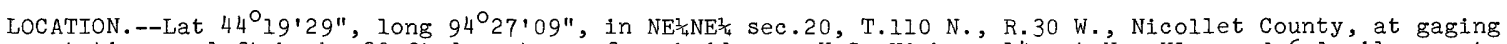
station on left bank, $30 \mathrm{ft}$ downstream from bridge on U.S. Highway 14 , at New Ulm, and 6.1 miles upstream from Cottonwood River.

DRAINAGE AREA. $--9,536 \mathrm{sq} \mathrm{mi}$ (at mouth of Cottonwood River).

RECORDS AVAILABLE. --Water temperatures: October 1967 to September 1968.

Sediment records: October 1967 to September 1968.

EXTREMES, 1967-68.--Water temperatures: Maximum, $26^{\circ} \mathrm{C}$ July 15, 16, Aug. 23; minimum, freezing point on many days.

Sediment concentrations: Maximum daily, $567 \mathrm{mg} / \mathrm{l}$ June 12; minimum daily, $10 \mathrm{mg} / 1 \mathrm{Mar} .22$.

Sediment loads: Maximum daily, 2,150 tons July 28; minimum daily, 7.6 tons Jan. 15.

TEMPERATURE $\left({ }^{\circ} \mathrm{C}\right)$ OF WATER, WATER YEAR OCTOBER 1967 TO SEPTEMBER 1968 (ONCE-DAILY MEASUREMENT, BETWEEN 0700 AND 1200)

DAY

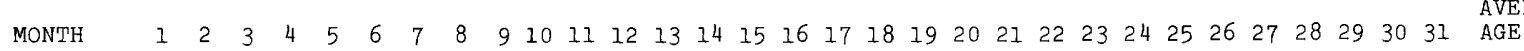

OCTOBER. . - - - - - - - - - - - - - - - - -

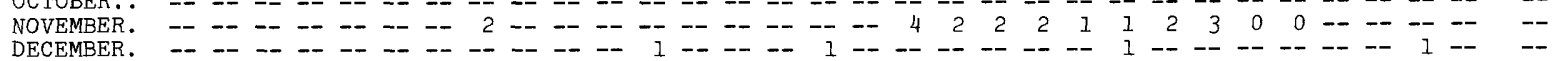

JANUARY.. -

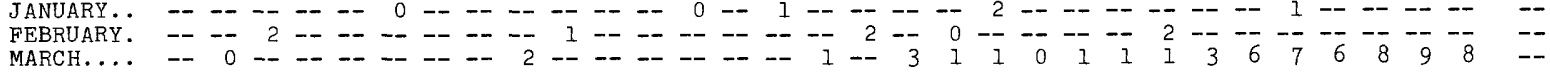

APRIL.... $\quad \begin{array}{rlllllllllllllllllllllllllllllllll}7 & 8 & 9 & 5 & 6 & 7 & 7 & 6 & 6 & 7 & 9 & 12 & 12 & 8 & 8 & 10 & 12 & 10 & 12 & 12 & 12 & 13 & 11 & 6 & 8 & 9 & 10 & 11 & 12 & 13 & -- & 9\end{array}$

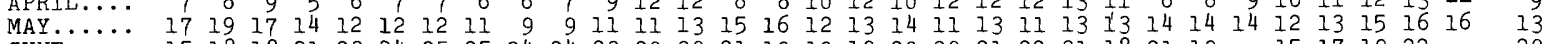

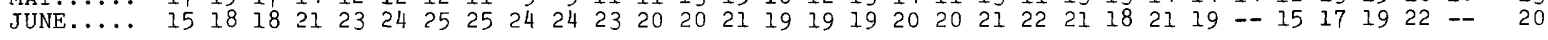

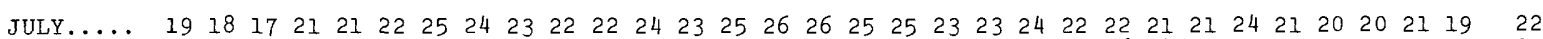

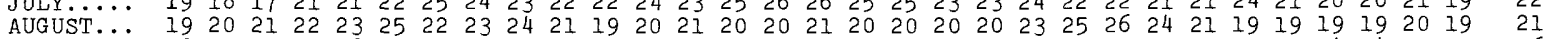

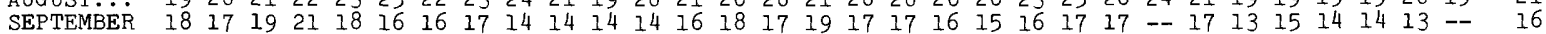

INSTANTANEOUS SUSPENDED SEDTMENT AND PARTICLE SIZE, WATTER YEAR OCTOBER 1967 TO SEPTIEMBER 1968

(METHODS OF ANALYSIS: B. BOTTOM WITHDRAWAL TUBE: C. CHFMICALLY DISPERSED: N. IN NATIVE WATER: P. PIPET: S. SIEVE: V. VISUAL ACCUMULATION TUBE: W. IN DISTILIEDD WATER)

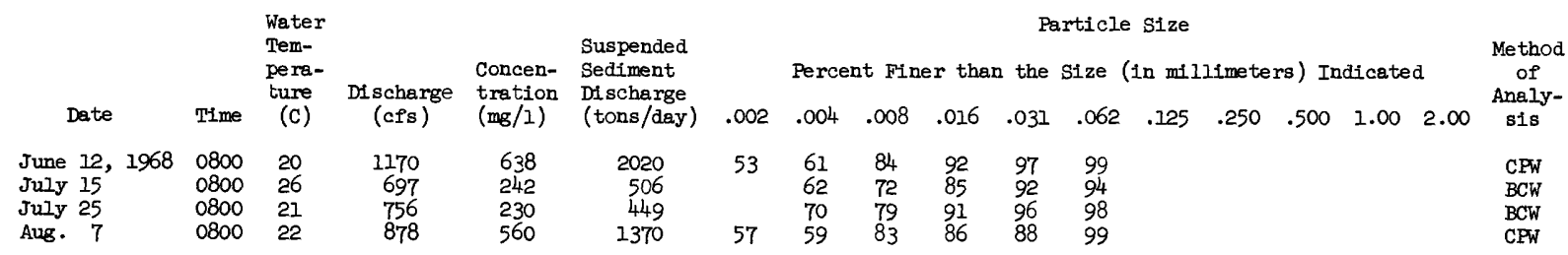

PARTICLE SIZE OF BED MATERTAL, WATER YRAR OCTOBER 1967 TO SEPTIEMBER 1968

(METHODS OF ANALYSIS; H' HYDROMETER: 0. OPTICAL ANALYZER: S. SIEVE: V. VISUAL ACCUMULATION TUBE)

Particle Size

\begin{tabular}{|c|c|c|c|c|c|c|c|c|c|c|c|c|c|c|c|}
\hline Date & Time & $\begin{array}{l}\text { No. of } \\
\text { Sampling } \\
\text { Points }\end{array}$ & $\begin{array}{l}\text { Discharge } \\
\text { (cfs) }\end{array}$ & 0.016 & 0.031 & 0.062 & $r$ than & the $\mathrm{Si}$ & $\begin{array}{l}\text { ze (in } \\
0.354\end{array}$ & millin & neters) & Indicat & 4.000 & 7.9 & $\begin{array}{l}\text { Method } \\
\text { of } \\
\text { Anelysis }\end{array}$ \\
\hline $\begin{array}{l}\text { July } \frac{11}{8}, 1968 \\
\text { Aug. }\end{array}$ & $\begin{array}{l}1605 \\
1545\end{array}$ & $\begin{array}{l}4 \\
4\end{array}$ & $\begin{array}{r}407 \\
1060\end{array}$ & & & $\begin{array}{l}8 \\
6\end{array}$ & $\begin{array}{l}13 \\
14\end{array}$ & $\begin{array}{l}24 \\
25\end{array}$ & $\begin{array}{l}33 \\
30\end{array}$ & & $\begin{array}{l}56 \\
46\end{array}$ & $\begin{array}{l}69 \\
58\end{array}$ & 82 & $\begin{array}{l}88 \\
82\end{array}$ & $\begin{array}{l}S \\
\text { S }\end{array}$ \\
\hline
\end{tabular}


5-3167.70. MINNESOTA RIVER AT NEW ULM, MINN.--Continued

SUSPENDED SEDIMENT, WATER YEAR OCTOBER 1967 TO SEPTEMBER 1968

OCTOBER

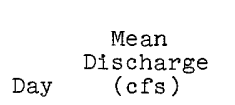

Mean

Concen-
tration

tration (tons

$(\mathrm{mg} / \mathrm{l})$ per day)

$128 \quad 130$

128
128

128

130
130

130

45
45
45
45
45

$\begin{array}{ll}128 & 130 \\ 128 & 130\end{array}$

128

130
130

130
130

45
45
45
46

$\begin{array}{ll}11 & 130 \\ 12 & 130 \\ 13 & 130\end{array}$

$\begin{array}{ll}14 & 131 \\ 15 & 132\end{array}$

130
130

130
130

130

$\begin{array}{lll}16 & 138 & 130 \\ 17 & 139 & 130\end{array}$

$\begin{array}{lll}17 & 139 & 130 \\ 18 & 140 & 130 \\ 19 & 160 & 130\end{array}$

$\begin{array}{lll}19 & 160 & 130 \\ 20 & 200 & 130\end{array}$

$21 \quad 160 \quad 130$

$\begin{array}{lll}21 & 160 & 130 \\ 22 & 140 & 130 \\ 23 & 138 & 130 \\ 24 & 138 & 130 \\ 25 & 128 & 130\end{array}$

25

128

130

$26 \quad 132 \quad 130$

$\begin{array}{lll}26 & 132 & 130 \\ 27 & 138 & 130 \\ 28 & 142 & 130\end{array}$

$29 \quad 143$

$30 \quad 149$

130
130

130

Total

1499

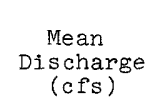

149
148

142
141

161

160

150
144
142
138
140

JANUARY

1
2
3
4
5
6
7
8
9
10
11
12
13
14
15
16
17
18
19
20
21
22
23
24
25
26
27
28
29
30
31

Total

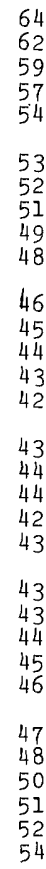$$
54
$$

29
28
27
28
26
26
26
24
22
20
18
16
16
10
7
10
15
19
23
29
29
29
30
30
31
32
32
34
34
35
36

771.6

$$
\begin{aligned}
& 170 \\
& 170 \\
& 170 \\
& 180 \\
& 180 \\
& 184 \\
& 184 \\
& 174 \\
& 164 \\
& 154 \\
& 144 \\
& 134 \\
& 134 \\
& 90 \\
& 67 \\
& \\
& 90 \\
& 130 \\
& 160 \\
& 200 \\
& 250 \\
& 250 \\
& 250 \\
& 250 \\
& 250 \\
& 250 \\
& 250 \\
& 250 \\
& 250 \\
& 250 \\
& 250 \\
& 250
\end{aligned}
$$

NOVEMBER
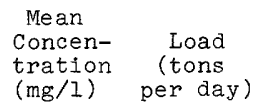

148
148
148
148
148

148

148
146
142

140
138

138
136

136
145
155

155

152
154

152

130
130
130
130
130

130

130
130

130

130
130

130

130

130

130
130

130

130
130

130

153

161
148

148
134

134
140

140
144

206

208

200

200

112

105

52
52
52
52

52
52

52
52
51
50
49

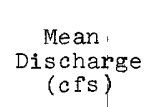

DECEMBER

$\begin{array}{lll}110 & 150 & 45 \\ 121 & 150 & 49 \\ 123 & 150 & 50 \\ 125 & 150 & 51 \\ 127 & 150 & 51\end{array}$

$\begin{array}{lll}125 & 150 & 51 \\ 123 & 150 & 50 \\ 128 & 150 & 52 \\ 134 & 150 & 54 \\ 136 & 150 & 55 \\ 144 & 150 & 58 \\ 146 & 156 & 61 \\ 123 & 150 & 50 \\ 107 & 140 & 40 \\ 116 & 100 & 31\end{array}$

$119 \quad 60$

128

130

136

60
70
90

110

130

136
114

109
114
99

150

157

160
160

$\begin{array}{ll}84 & 160 \\ 68 & 160 \\ 73 & 160 \\ 68 & 160 \\ 65 & 160 \\ 64 & 160\end{array}$

19
24
32

32

$$
48
$$$$
\begin{aligned}
& 55 \\
& 46 \\
& 46 \\
& 49 \\
& 43 \\
& 36 \\
& 29 \\
& 32 \\
& 29 \\
& 28 \\
& 28
\end{aligned}
$$

1638

1331

FEBRUARY

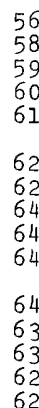

$\begin{array}{ll}240 & 36 \\ 240 & 38 \\ 230 & 37 \\ 200 & 3 \\ 161 & 27 \\ 161 & 27 \\ 161 & 27 \\ 161 & 28 \\ 161 & 28 \\ 161 & 28\end{array}$

36
38
37
32
27
27
27
28
28
28

161

161

161

130

28
27

27
27

22
18

108

108

140

190
190

18
17
22
29

29

190

200

220

220

29
31
32
34
36

220

220
220

220

--

$\frac{60}{--}$

36
36
36
36
36
--

831

MARCH

$\begin{array}{ll}220 & 36 \\ 220 & 36 \\ 220 & 36 \\ 220 & 36 \\ 220 & 36 \\ 150 & 27 \\ 120 & 24 \\ 110 & 27 \\ 110 & 33 \\ 100 & 38\end{array}$

$\begin{array}{lll}180 & 90 & 44 \\ 250 & 80 & 54 \\ 310 & 70 & 59 \\ 305 & 60 & 49 \\ 300 & 40 & 32\end{array}$

290

330

355
363

$\begin{array}{ll}14 & 11 \\ 21 & 19 \\ 35 & 34 \\ 58 & 57 \\ 18 & 17\end{array}$

$\begin{array}{lll}355 & 15 & 14 \\ 360 & 10 & 9.7 \\ 365 & 11 & 11 \\ 566 & 15 & 23 \\ 756 & 25 & 51\end{array}$

$794 \quad 26$

707

641
629

629
623

26
40
53
65
92
100

56
76
92
110
155
154

1445.7 
5-3167.70. MINNESOTA RIVER AT NEW ULM, MINN.--Continued

SUSPENDED SEDIMENT, WATER YEAR OCTOBER 1967 TO SEPTEMBER 1968

APRIL

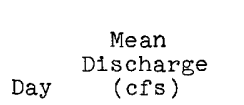

Mean

Concen- Load tration (tons Discharge (mg/l) per day)

102
100
93
62
35

152
138
113
78
45

58
65

52
95

95
126

163

139

135
110

102

132

122

140
125

123

130
130

130

152
168

1010

1120

1190

1210
1150

1050

152

172
157

157
192

156

Total

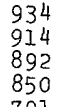

934
914
892
850

791

710

693

650

127
183

253

224
239

239
189

168

203
190

190
209

183
188

256
288

274
356
458

460

553

513
596

442

332

404
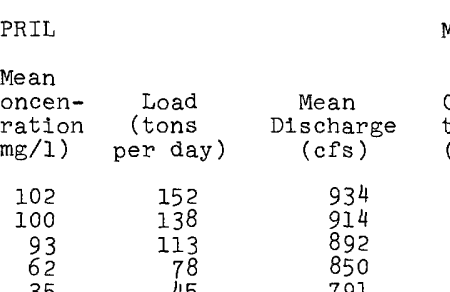

MAY

Mean

ration Load

JULY

$\begin{array}{ll}I & 704 \\ 2 & 714 \\ 3 & 742 \\ 4 & 732 \\ 5 & 700\end{array}$

138

120
124

124
117

133

262
231
248
231
251

217
164
151
119
126

125
102

107
96

107

98
102

102
93
119

119
230

227
245

273

305
250

220
206

272

258
226

189

240
288

227
238

238
266

193

170
176

148

118

110
110

96
82

82
100

85
65

65
94
110

110
100

95
118

118
120

130

134

157
124

133

140
247

130

130
123
160

160
151

151
148

135

UGUST

2170
1960
1350
930
611

506
878
1050
1030
728

237
147
112
125
133
114
495
275
200
196
195
183
184
185
182

487

487
420
424
340

252

211

180

144

144
171

135

$$
\begin{aligned}
& 135 \\
& 154
\end{aligned}
$$

133

121

148

161

161

185

152

158
163

143

139
180

168

157
147

5966

$672 \quad 195 \quad 354$

$\begin{array}{lll}672 & 195 & 354 \\ 503 & 183 & 249\end{array}$

440

353

249
219

176

145

194
522

285
258

230

234

175
155
163

163
148
157

135
108

101

94
96

170
197
207
196
218

95

116

111

$180 \quad 242$

427

1300

2150

1560

1260
1540

$\begin{array}{ll}174 & 221 \\ 162 & 250\end{array}$

$168 \quad 259$

156

118

104
109

117

93
116
Concen-

(mg/l) per day

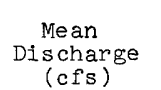

JUNE

$\begin{array}{lll}428 & 178 & 206 \\ 467 & 166 & 209 \\ 527 & 150 & 213 \\ 527 & 167 & 238 \\ 491 & 207 & 274 \\ 452 & 232 & 283 \\ 419 & 208 & 235 \\ 417 & 160 & 187 \\ 410 & 155 & 172 \\ 533 & 185 & 266 \\ 1040 & 369 & 1040 \\ 1170 & 567 & 1790 \\ 1110 & 318 & 953 \\ 1120 & 284 & 859 \\ 1070 & 263 & 760\end{array}$

942

$822 \quad 21$

$\begin{array}{ll}763 & 204 \\ 7 & 223\end{array}$

$\begin{array}{ll}728 & 257 \\ 718 & 247\end{array}$

686

656
653

679
710

714
690

690
700
696

710

240

235
225

203

197

134

105

118
114

125

539

453

459

505
479

444

416

397

372
378

258

196

223
214

240

13258

SEPTEMBER

$\begin{array}{rrrr}1390 & 168 & 218 & 99 \\ 778 & 164 & 211 & 93 \\ 408 & 162 & 269 & 118 \\ 314 & 160 & 261 & 113 \\ 219 & 164 & 257 & 114 \\ 156 & 162 & 267 & 117 \\ 1170 & 156 & 210 & 88 \\ 1060 & 150 & 238 & 96 \\ 584 & 222 & 230 & 138 \\ 385 & 292 & 186 & 147\end{array}$

275

240
226

212
188

$158 \quad 117$

117

17
34
4

174
344

392
347

431

672
950
1250

1250
1060

15553

Total

9325

$\begin{array}{rrr}730 & 112 & 221 \\ 467 & 167 & 211 \\ 371 & 217 & 217 \\ 270 & 205 & 149 \\ 247 & 195 & 130 \\ -- & -- & -\end{array}$

4369

740153
63319.3 
5-3250. MINNESOTA RIVER AT MANKATO, MINN.

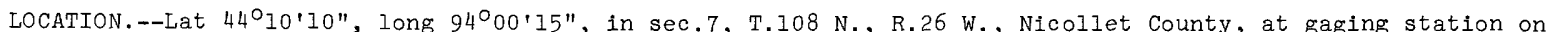
left bank at downstream side of Main Street Bridge in Mankato, 1.8 miles downstream from Blue Earth River, and at mile 106.4 upstream from Mississippi River.

DRAINAGE AREA. $-14,900 \mathrm{sq} \mathrm{mi}$, approximately.

RECORDS AVAILABLE. - Chemical analyses: October 1963 to August 1966.

Water temperatures: October 1967 to September 1968.

Sediment records: October 1967 to September 1968

EXTREMES, 1967-68,--Water temperatures: Maximum, $28^{\circ} \mathrm{C}$ July 28 ; minimum, freezing point on many days.

Sediment concentrations: Maximum daily, 2,850 mg/1 Aug. 7 ; minimum daily, $20 \mathrm{mg} / 1 \mathrm{Mar} .23$.

Sediment loads: Maximum dally, 90,200 tons Aug. 7; minimum daily, 14 tons Jan. 13, 14.

TEMPERATURE $\left({ }^{\circ} \mathrm{C}\right)$ OF WATER, WATER YEAR OCTOBER 1967 TO SEPTEMBER 1968

(ONCE-DAILY MEASUREMENT, BETWEEN 0700 AND 1200)

DAY

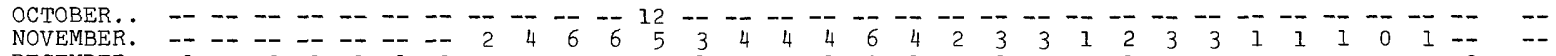

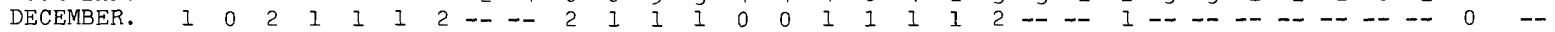

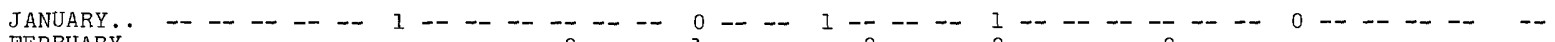

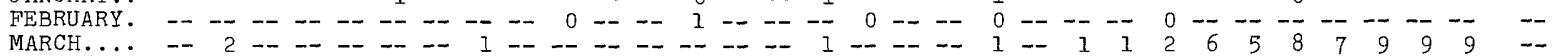

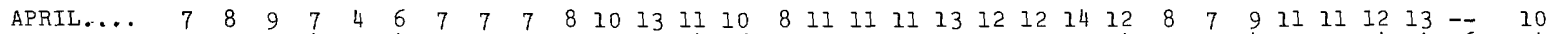

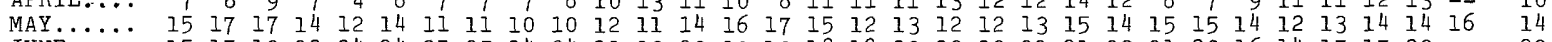

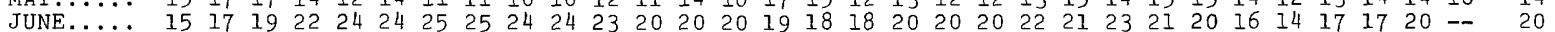

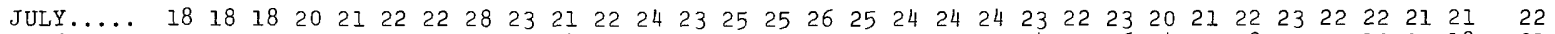

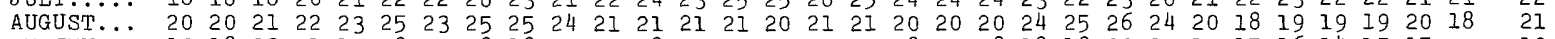

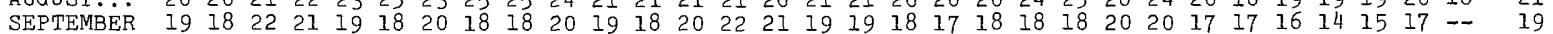

INSTANTANEOUS SUSPENDED SEDIMENT AND PARTICLE SIZE, WATER YEAR OCTOBER 1967 TO SEPTTEMBER 1968

(METHODS OF ANALYSIS: B. BOTTOM WITHDRAWAL TUBE: C. CHEMICALLY DISPERSED: N. IN NATIVE WATER: P. PIPET: S. SIEVE:

V. VISUAL ACCUMUTATION TUBE: W. IN DISTILLLED WATER)

\begin{tabular}{|c|c|c|c|c|c|c|c|c|c|c|c|c|c|c|c|c|}
\hline \multirow[b]{3}{*}{ Date } & \multirow[b]{3}{*}{ Time } & \multirow{3}{*}{$\begin{array}{l}\text { Water } \\
\text { Tem- } \\
\text { pera- } \\
\text { ture } \\
\text { (c) }\end{array}$} & \multirow[b]{3}{*}{$\begin{array}{c}\text { Discharge } \\
\text { (cf's) }\end{array}$} & \multirow{3}{*}{$\begin{array}{l}\text { Concen- } \\
\text { tration } \\
(\mathrm{mg} / \mathrm{l})\end{array}$} & \multirow{3}{*}{$\begin{array}{l}\text { Suspended } \\
\text { Sediment } \\
\text { Discharge } \\
\text { (tons/day) }\end{array}$} & \multicolumn{10}{|c|}{ Particle Size } & \multirow{3}{*}{$\begin{array}{l}\text { Method } \\
\text { of } \\
\text { Analy- } \\
\text { sis }\end{array}$} \\
\hline & & & & & & & Perce & at Fin & er than & the & Size ( & in $\mathrm{mil}$ & limeters) In & idicate & & \\
\hline & & & & & & .002 & .004 & .008 & .016 & .031 & .062 & .125 & $.250 \quad .500$ & 1.00 & 2.00 & \\
\hline June 29, 1968 & 1507 & 19 & 4310 & 560 & 6520 & 37 & 40 & 50 & $7 \varepsilon$ & 72 & 9 & 4 & 100 & & & VPI \\
\hline July 25 & 1958 & 23 & 9770 & 1710 & 45100 & 28 & 35 & 42 & 51 & 63 & 83 & 95 & 100 & & & VPWC \\
\hline July 26 & 1055 & 22 & 13400 & 1980 & 71600 & 38 & 41 & 58 & 62 & 80 & 87 & 95 & 100 & & & VPWC \\
\hline Aug. 8 & 1000 & 25 & 12700 & 1080 & 37000 & & 43 & 50 & 63 & 81 & 92 & 98 & 100 & & & VBWC \\
\hline
\end{tabular}

PARTICLE SIZE OF BED MATERLAL, WATER YFAR OCTOBER 1967 TO SEPTEMBER 1968

(METHODS OF ANALYSIS: H. HYDROMETER: O. OPTICAL ANALYZER; S. SIEVE: V. VISUAL ACCUMULATION IUBE)

Particle Size

\begin{tabular}{|c|c|c|c|c|c|c|c|c|c|c|c|c|c|c|}
\hline$D$ & Time & $\begin{array}{l}\text { No. of } \\
\text { Sampling } \\
\text { Points }\end{array}$ & $\begin{array}{l}\text { Discharge } \\
\text { (cfs) }\end{array}$ & 0.016 & Percent & Finer & than th & Size & (in $\mathrm{mt}$ & limet & ers) & dicated & 4.000 & $\begin{array}{l}\text { Method } \\
\text { of } \\
\text { Anelysis }\end{array}$ \\
\hline 1968 & $\begin{array}{l}1510 \\
1900\end{array}$ & $\begin{array}{l}5 \\
4\end{array}$ & $\begin{array}{r}1720 \\
\geq \geq 800\end{array}$ & & & $\begin{array}{l}6 \\
1\end{array}$ & $\begin{array}{r}26 \\
4\end{array}$ & $\begin{array}{l}71 \\
49\end{array}$ & $\begin{array}{l}86 \\
78\end{array}$ & & $\begin{array}{l}97 \\
96\end{array}$ & $\begin{array}{l}99 \\
98\end{array}$ & $\begin{array}{l}100 \\
100\end{array}$ & $\begin{array}{l}S \\
S\end{array}$ \\
\hline
\end{tabular}


5-3250. MINNESOTA RIVER AT MANKATO, MINN.--Continued

SUSPENDED SEDIMENT, WATER YEAR OCTOBER 1967 TO SEPTEMBER 1968 OCTOBER

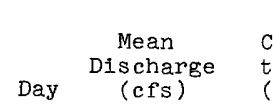

Mean
Concen-
tration NOVEMBER

DECEMBER

$$
\begin{aligned}
& 1 \\
& 2 \\
& 3 \\
& 4 \\
& 5
\end{aligned}
$$

Load

mg/l) per day)
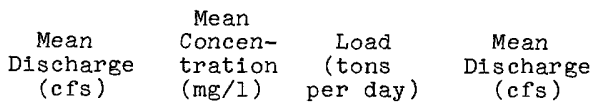

Mean

Concen- Load

tration (tons
(mg/l) per day

$\begin{array}{ll}264 & 100 \\ 260 & 100 \\ 269 & 100 \\ 256 & 100 \\ 244 & 100\end{array}$

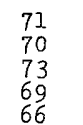

322

325
322

322
325

$140 \quad 122$

$140 \quad 123$

$\begin{array}{ll}140 & 123 \\ 140 & 122\end{array}$

$140 \quad 123$

292

295
289

289
313
304

$154 \quad 121$

$240 \quad 100$

$\begin{array}{lll}6 & 240 & 100 \\ 7 & 264 & 100 \\ 8 & 274 & 100\end{array}$

$\begin{array}{rll}9 & 282 & 100 \\ 10 & 269 & 100\end{array}$

$65 \quad 318$

318
311

310

322

$\begin{array}{ll}140 & 120 \\ 160 & 134\end{array}$

$180 \quad 151$

$140 \quad 122$

76
73

126

111

327
332

330

330
327
323

142

152

136

$141 \quad 124$

$\begin{array}{ll}271 & 100 \\ 275 & 100\end{array}$

73
74
75

151

124
132

329

342

75
77
77
137

153

150

149141

$147 \quad 137$

140
140

137
181

345
338

133

121

344
342

342
319

122

135
115

109
118

323
338

97
118

118
146

113
90

102

127

313

108

109
108

118

140

140

140
140

125
123

147

134

308

308
328
334

334

345
350

179

$\begin{array}{ll}170 & 144 \\ 162 & 133\end{array}$

$163 \quad 136$

$\begin{array}{ll}167 & 148 \\ 169 & 152\end{array}$

162 I5]

$171 \quad 162$

$\begin{array}{lll}355 & 183 & 17 \\ 358 & 160 & 155\end{array}$

$\begin{array}{lll}303 & 109 & 89 \\ 236 & 103 & 66 \\ 250 & 115 & 78\end{array}$

$270 \quad 109$

285

295

290

109
82

82
70
72

280
220

230

230
251
250

81
77
74
76
82

79
63
56
56
64

$\begin{array}{llr}313 & 145 & 123\end{array}$

$269 \quad 157 \quad 114$

281

291

153
175

114
116

137

9539

$-$

3676

J ANUARY

FEBRUARY

230

205

190

180
170

160

88
95
102
111
122
125

8466

--

MARCH

$\begin{array}{rr}1 & 150 \\ 2 & 140 \\ 3 & 130 \\ 4 & 120 \\ 5 & 110 \\ 6 & 102 \\ 7 & 96 \\ 8 & 89 \\ 9 & 89 \\ 10 & 88\end{array}$

$\begin{array}{ll}120 & 4 \\ 115 & 4 \\ 110 & 39 \\ 107 & 35 \\ 105 & 31\end{array}$

49
43
39
35
31
28
25
22
20
18

133

135
135

135
138

107
107
107
109
109

139

$\begin{array}{ll}139 & 110 \\ 140 & 110\end{array}$

$140 \quad 110$

$140 \quad 110$

139

109

$138 \quad 104$

138

137

135
135

99

93
85

134

132
132

132
131
128

77

70

54
57
62
65
72

15
17
18
20

77
84
92
97

22

128

128
128

128

$\lcm{129}$

66
77
97
29

38
39
39
41
41

136

140
144

144
150

157

$\begin{array}{ll}41 & 163 \\ 42 & 180\end{array}$

42

42
41

198
210
225

$\begin{array}{ll}72 & 26 \\ 69 & 26 \\ 63 & 24 \\ 58 & 23 \\ 53 & 22\end{array}$

39

37

31
28

240
260
300

300
330

355

25

24

27
34
44

405
480
600
700

723

124

112
112

109

103

43
39
39
38
36

60

601

1050

$\begin{array}{ll}97 & 34 \\ 87 & 31 \\ 78 & 27 \\ 74 & 26 \\ -- & -- \\ -- & --\end{array}$

1210

129
130

130

131

$--$

$846 \quad 3879$

-- $\quad 1042$

1130

1130
1060

1020

48

44

41

41
42

42

40

40
40

38

38
40

48
57
$6]$

21

21

22

23
26

27

28

32
36
36

Total 3499

-- 846

16666$$
119
$$

$\begin{array}{rr}47 & 89 \\ 36 & 68 \\ 20 & 32 \\ 32 & 74 \\ 51 & 144 \\ 111 & 363 \\ 113 & 378 \\ 97 & 296 \\ 92 & 263 \\ 114 & 314 \\ 142 & 387\end{array}$

- 3200 
5-3250. MINNESOTA RIVER AT MANKATO, MINN.--Continued

SUSPENDED SEDIMENT, WATER YEAR OCTOBER 1967 TO SEPTEMBER 1968

\begin{tabular}{|c|c|c|c|c|c|c|c|c|c|}
\hline & & APRIL & & & MAY & & \multicolumn{3}{|c|}{ JUNE } \\
\hline Day & $\begin{array}{l}\text { Mean } \\
\text { Discharge } \\
\text { (cfs) }\end{array}$ & $\begin{array}{l}\text { Mean } \\
\text { Concen- } \\
\text { tration } \\
(\mathrm{mg} / \mathrm{l})\end{array}$ & $\begin{array}{l}\text { Load } \\
\text { (tons } \\
\text { per day) }\end{array}$ & $\begin{array}{c}\text { Mean } \\
\text { Discharge } \\
\text { (cfs) }\end{array}$ & $\begin{array}{l}\text { Mean } \\
\text { Concen- } \\
\text { tration } \\
(\mathrm{mg} / \mathrm{l})\end{array}$ & $\begin{array}{l}\text { Load } \\
\text { (tons } \\
\text { per day) }\end{array}$ & $\begin{array}{c}\text { Mean } \\
\text { Discharge } \\
\quad(\text { cfs) }\end{array}$ & $\begin{array}{l}\text { Mean } \\
\text { Concen- } \\
\text { tration } \\
(\mathrm{mg} / \mathrm{l})\end{array}$ & $\begin{array}{l}\text { Load } \\
\text { (tons } \\
\text { per day) }\end{array}$ \\
\hline $\begin{array}{l}1 \\
2 \\
3 \\
4 \\
5\end{array}$ & $\begin{array}{l}948 \\
843 \\
801 \\
811 \\
759\end{array}$ & $\begin{array}{r}112 \\
96 \\
106 \\
119 \\
56\end{array}$ & $\begin{array}{l}287 \\
219 \\
229 \\
261 \\
115\end{array}$ & $\begin{array}{l}1730 \\
1620 \\
1540 \\
1450 \\
1340\end{array}$ & $\begin{array}{l}162 \\
142 \\
165 \\
184 \\
162\end{array}$ & $\begin{array}{l}757 \\
621 \\
686 \\
720 \\
586\end{array}$ & $\begin{array}{l}874 \\
885 \\
927 \\
990 \\
990\end{array}$ & $\begin{array}{r}124 \\
141 \\
137 \\
112 \\
98\end{array}$ & $\begin{array}{l}293 \\
337 \\
343 \\
299 \\
262\end{array}$ \\
\hline $\begin{array}{r}6 \\
7 \\
8 \\
9 \\
10\end{array}$ & $\begin{array}{l}822 \\
874 \\
811 \\
790 \\
769\end{array}$ & $\begin{array}{l}69 \\
81 \\
64 \\
65 \\
67\end{array}$ & $\begin{array}{l}153 \\
191 \\
140 \\
139 \\
139\end{array}$ & $\begin{array}{l}1220 \\
1160 \\
1120 \\
1080 \\
1030\end{array}$ & $\begin{array}{r}134 \\
123 \\
136 \\
102 \\
83\end{array}$ & $\begin{array}{l}441 \\
385 \\
411 \\
297 \\
231\end{array}$ & $\begin{array}{r}937 \\
874 \\
843 \\
937 \\
1290\end{array}$ & $\begin{array}{r}100 \\
101 \\
91 \\
113 \\
374\end{array}$ & $\begin{array}{r}253 \\
238 \\
207 \\
286 \\
1350\end{array}$ \\
\hline $\begin{array}{l}11 \\
12 \\
13 \\
14 \\
15\end{array}$ & $\begin{array}{r}811 \\
948 \\
1050 \\
1090 \\
990\end{array}$ & $\begin{array}{r}94 \\
124 \\
130 \\
126 \\
114\end{array}$ & $\begin{array}{l}206 \\
317 \\
368 \\
371 \\
305\end{array}$ & $\begin{array}{l}969 \\
916 \\
843 \\
822 \\
811\end{array}$ & $\begin{array}{r}81 \\
95 \\
96 \\
89 \\
111\end{array}$ & $\begin{array}{l}212 \\
235 \\
219 \\
198 \\
243\end{array}$ & $\begin{array}{l}2380 \\
3610 \\
3680 \\
3680 \\
3430\end{array}$ & $\begin{array}{r}1040 \\
1090 \\
790 \\
628 \\
457\end{array}$ & $\begin{array}{r}7030 \\
10600 \\
7850 \\
6240 \\
4230\end{array}$ \\
\hline $\begin{array}{l}16 \\
17 \\
18 \\
19 \\
20\end{array}$ & $\begin{array}{r}916 \\
948 \\
927 \\
874 \\
1000\end{array}$ & $\begin{array}{r}112 \\
106 \\
96 \\
99 \\
100\end{array}$ & $\begin{array}{l}277 \\
271 \\
240 \\
234 \\
270\end{array}$ & $\begin{array}{l}822 \\
759 \\
927 \\
958 \\
885\end{array}$ & $\begin{array}{l}113 \\
131 \\
132 \\
146 \\
141\end{array}$ & $\begin{array}{l}251 \\
268 \\
330 \\
378 \\
337\end{array}$ & $\begin{array}{l}3060 \\
2840 \\
2570 \\
2340 \\
2180\end{array}$ & $\begin{array}{l}397 \\
368 \\
314 \\
261 \\
231\end{array}$ & $\begin{array}{l}3280 \\
2820 \\
2180 \\
1650 \\
1360\end{array}$ \\
\hline $\begin{array}{l}21 \\
22 \\
23 \\
24 \\
25\end{array}$ & $\begin{array}{l}1040 \\
1220 \\
1760 \\
1850 \\
1970\end{array}$ & $\begin{array}{l}102 \\
151 \\
309 \\
294 \\
241\end{array}$ & $\begin{array}{r}286 \\
497 \\
1470 \\
1470 \\
1280\end{array}$ & $\begin{array}{l}822 \\
801 \\
769 \\
738 \\
696\end{array}$ & $\begin{array}{r}153 \\
164 \\
146 \\
94 \\
91\end{array}$ & $\begin{array}{l}340 \\
355 \\
303 \\
187 \\
171\end{array}$ & $\begin{array}{l}2060 \\
1900 \\
1790 \\
1760 \\
1760\end{array}$ & $\begin{array}{l}225 \\
220 \\
211 \\
184 \\
180\end{array}$ & $\begin{array}{r}1250 \\
1130 \\
1020 \\
874 \\
855\end{array}$ \\
\hline $\begin{array}{l}26 \\
27 \\
28 \\
29 \\
30 \\
31\end{array}$ & $\begin{array}{r}2180 \\
2190 \\
2130 \\
2000 \\
1860 \\
\end{array}$ & $\begin{array}{r}298 \\
244 \\
178 \\
191 \\
167 \\
--\end{array}$ & $\begin{array}{r}1750 \\
1440 \\
1020 \\
1030 \\
839 \\
--\end{array}$ & $\begin{array}{l}738 \\
769 \\
769 \\
811 \\
822 \\
822\end{array}$ & $\begin{array}{r}96 \\
87 \\
90 \\
93 \\
117 \\
110\end{array}$ & $\begin{array}{l}191 \\
181 \\
187 \\
204 \\
260 \\
244\end{array}$ & $\begin{array}{r}1840 \\
2590 \\
3670 \\
4260 \\
4350 \\
--\end{array}$ & $\begin{array}{r}176 \\
314 \\
660 \\
555 \\
438 \\
--\end{array}$ & $\begin{array}{r}874 \\
2280 \\
6540 \\
6380 \\
5140 \\
\end{array}$ \\
\hline \multirow[t]{2}{*}{ Total } & 35982 & -- & 15814 & 30559 & - & 10429 & 65297 & & 77451 \\
\hline & \multicolumn{3}{|c|}{ JULY } & \multicolumn{3}{|c|}{ AUGUST } & \multicolumn{3}{|c|}{ SEPTEMBER } \\
\hline $\begin{array}{l}1 \\
2 \\
3 \\
4 \\
5\end{array}$ & $\begin{array}{l}4030 \\
3490 \\
3000 \\
2680 \\
2420\end{array}$ & $\begin{array}{l}322 \\
259 \\
234 \\
203 \\
196\end{array}$ & $\begin{array}{l}3500 \\
2440 \\
1900 \\
1470 \\
1280\end{array}$ & $\begin{array}{r}10800 \\
9780 \\
8560 \\
7220 \\
5840\end{array}$ & $\begin{array}{l}432 \\
418 \\
408 \\
374 \\
336\end{array}$ & $\begin{array}{r}12600 \\
11000 \\
9430 \\
7290 \\
5300\end{array}$ & $\begin{array}{l}1320 \\
1240 \\
1190 \\
1180 \\
1210\end{array}$ & $\begin{array}{r}133 \\
99 \\
91 \\
87 \\
93\end{array}$ & $\begin{array}{l}474 \\
331 \\
292 \\
277 \\
304\end{array}$ \\
\hline $\begin{array}{r}6 \\
7 \\
8 \\
9 \\
10\end{array}$ & $\begin{array}{l}2210 \\
2040 \\
1890 \\
1760 \\
1650\end{array}$ & $\begin{array}{l}182 \\
153 \\
176 \\
158 \\
161\end{array}$ & $\begin{array}{r}1090 \\
843 \\
898 \\
751 \\
717\end{array}$ & $\begin{array}{r}5080 \\
12000 \\
12300 \\
11200 \\
11500\end{array}$ & $\begin{array}{r}369 \\
2850 \\
921 \\
569 \\
691\end{array}$ & $\begin{array}{r}5060 \\
90200 \\
30600 \\
17200 \\
21500\end{array}$ & $\begin{array}{l}1230 \\
1270 \\
1510 \\
1960 \\
2260\end{array}$ & $\begin{array}{l}102 \\
109 \\
241 \\
432 \\
306\end{array}$ & $\begin{array}{r}339 \\
374 \\
1000 \\
2290 \\
1870\end{array}$ \\
\hline $\begin{array}{l}11 \\
12 \\
13 \\
14 \\
15\end{array}$ & $\begin{array}{l}1760 \\
1730 \\
1860 \\
2270 \\
2680\end{array}$ & $\begin{array}{l}180 \\
201 \\
270 \\
412 \\
645\end{array}$ & $\begin{array}{r}855 \\
939 \\
1360 \\
2530 \\
4670\end{array}$ & $\begin{array}{r}11400 \\
9450 \\
7640 \\
6330 \\
5380\end{array}$ & $\begin{array}{l}487 \\
433 \\
402 \\
339 \\
252\end{array}$ & $\begin{array}{r}15000 \\
11000 \\
8290 \\
5790 \\
3660\end{array}$ & $\begin{array}{l}2280 \\
2160 \\
1990 \\
1800 \\
1620\end{array}$ & $\begin{array}{l}206 \\
213 \\
192 \\
164 \\
131\end{array}$ & $\begin{array}{r}1270 \\
1240 \\
1030 \\
797 \\
573\end{array}$ \\
\hline $\begin{array}{l}16 \\
17 \\
18 \\
19 \\
20\end{array}$ & $\begin{array}{l}3050 \\
2930 \\
2640 \\
2540 \\
2460\end{array}$ & $\begin{array}{l}521 \\
330 \\
293 \\
274 \\
232\end{array}$ & $\begin{array}{l}4290 \\
2610 \\
2090 \\
1880 \\
1540\end{array}$ & $\begin{array}{l}4730 \\
4200 \\
3740 \\
3310 \\
3160\end{array}$ & $\begin{array}{l}260 \\
241 \\
206 \\
179 \\
175\end{array}$ & $\begin{array}{l}3320 \\
2730 \\
2080 \\
1600 \\
1490\end{array}$ & $\begin{array}{l}1540 \\
1980 \\
2720 \\
3740 \\
4380\end{array}$ & $\begin{array}{l}136 \\
232 \\
319 \\
430 \\
397\end{array}$ & $\begin{array}{r}565 \\
1240 \\
2340 \\
4340 \\
4690\end{array}$ \\
\hline $\begin{array}{l}21 \\
22 \\
23 \\
24 \\
25\end{array}$ & $\begin{array}{l}2180 \\
1870 \\
2640 \\
5260 \\
8170\end{array}$ & $\begin{array}{r}200 \\
172 \\
555 \\
1310 \\
1310\end{array}$ & $\begin{array}{r}1180 \\
868 \\
4550 \\
18600 \\
28900\end{array}$ & $\begin{array}{l}2940 \\
2670 \\
2430 \\
2260 \\
2080\end{array}$ & $\begin{array}{l}157 \\
137 \\
134 \\
149 \\
127\end{array}$ & $\begin{array}{r}1250 \\
988 \\
879 \\
909 \\
713\end{array}$ & $\begin{array}{r}4920 \\
5860 \\
7980 \\
10200 \\
11400\end{array}$ & $\begin{array}{l}390 \\
427 \\
543 \\
708 \\
606\end{array}$ & $\begin{array}{r}5180 \\
6760 \\
11700 \\
19500 \\
18700\end{array}$ \\
\hline $\begin{array}{l}26 \\
27 \\
28 \\
29 \\
30 \\
31\end{array}$ & $\begin{array}{l}13000 \\
15600 \\
15200 \\
14100 \\
12500 \\
11400\end{array}$ & $\begin{array}{r}1800 \\
1230 \\
837 \\
686 \\
620 \\
508\end{array}$ & $\begin{array}{l}63200 \\
51800 \\
34400 \\
26100 \\
20900 \\
15600\end{array}$ & $\begin{array}{l}1890 \\
1730 \\
1570 \\
1460 \\
1400 \\
1350\end{array}$ & $\begin{array}{r}129 \\
129 \\
122 \\
110 \\
99 \\
107\end{array}$ & $\begin{array}{l}658 \\
603 \\
517 \\
434 \\
374 \\
390\end{array}$ & $\begin{array}{r}10800 \\
9170 \\
7590 \\
6590 \\
6110 \\
--\end{array}$ & $\begin{array}{r}478 \\
363 \\
270 \\
271 \\
281 \\
--\end{array}$ & $\begin{array}{r}13900 \\
8990 \\
5530 \\
4820 \\
4640 \\
--\end{array}$ \\
\hline Total & 151010 & - & 303751 & 175400 & -- & 272855 & 119200 & & 125356 \\
\hline
\end{tabular}


5-3300. MINNESOTA RIVER NEAR JORDAN, MINN.

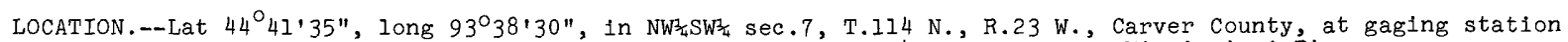
on left bank, 1.5 miles northwest of Jordan, and at mile 39.4 upstream from Mississippi River.

CHEMICAL ANALYSES IN MILLIGRAMS PER LITER, WATER YEAR OCTOBER 1967 TO.SEPTEMBER 1968

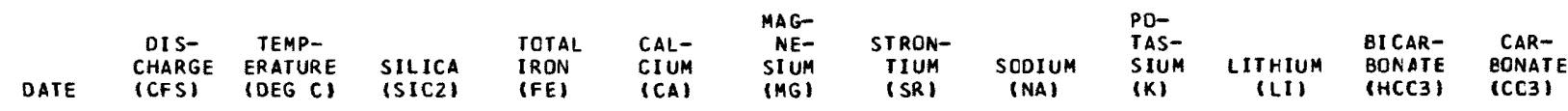

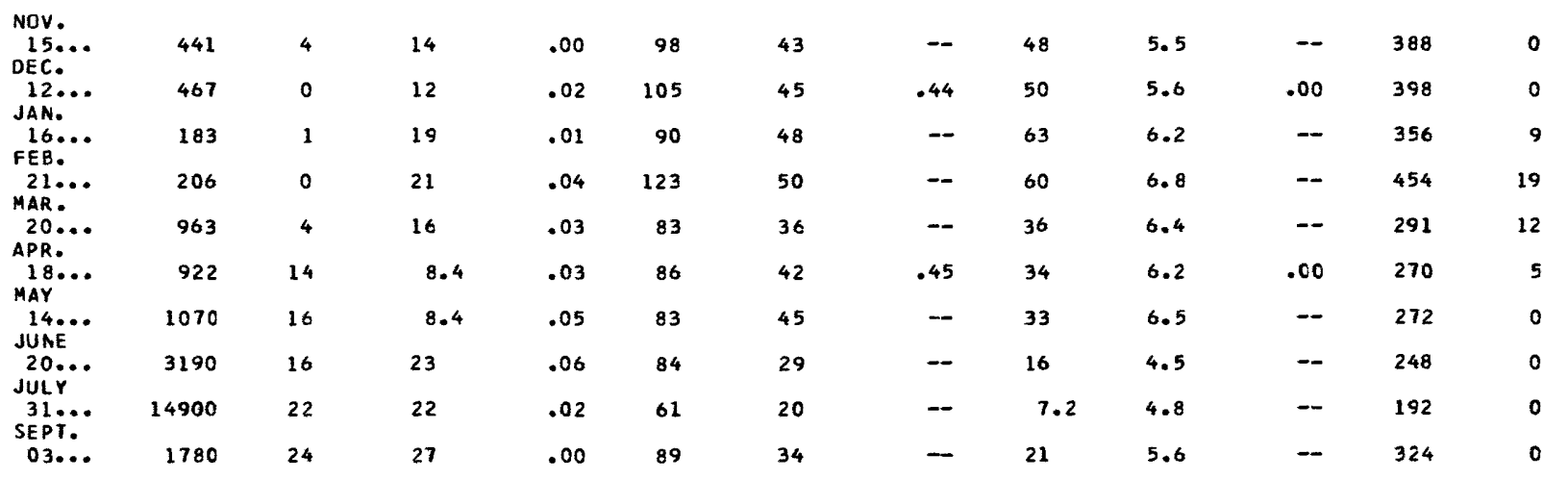

\begin{tabular}{|c|c|c|c|c|c|c|c|}
\hline DATE & $\begin{array}{l}\text { TOTAL } \\
\text { CHRO- } \\
\text { MIUN } \\
\text { ICR }\end{array}$ & $\begin{array}{c}\text { NICKEL } \\
\text { (NI) }\end{array}$ & $\begin{array}{l}\text { COBALT } \\
\text { (CO) }\end{array}$ & $\begin{array}{l}\text { LEAD } \\
\text { (PB) }\end{array}$ & $\begin{array}{l}\text { DIS- } \\
\text { SOLVED } \\
\text { SOLIOS } \\
\text { (SUM OF } \\
\text { CONSTI- } \\
\text { TUENTSI }\end{array}$ & $\begin{array}{l}\text { DIS- } \\
\text { SOLVED } \\
\text { SOLIDS } \\
\text { ITONS } \\
\text { PER } \\
\text { AC-FT I }\end{array}$ & $\begin{array}{l}\text { OIS- } \\
\text { SOLVED } \\
\text { SOL IDS } \\
\text { ITONS } \\
\text { PER } \\
\text { DAYI }\end{array}$ \\
\hline $\begin{array}{l}\text { NOV. } \\
15 . . \\
\text { DEC. }\end{array}$ & -- & -- & -- & -- & 590 & .84 & 733 \\
\hline JAN... & .00 & .00 & .00 & .01 & 620 & .87 & 806 \\
\hline $\begin{array}{l}16 . . \\
\text { FEB. }\end{array}$ & -- & -- & -- & -- & 638 & .89 & 324 \\
\hline $\begin{array}{l}21 . . \\
\text { MAR.. }\end{array}$ & -- & -- & -- & - & 736 & 1.05 & 429 \\
\hline $20 .$. & -- & -- & -- & -- & 513 & .74 & 1410 \\
\hline $18 .$. & .00 & .00 & .00 & .00 & 545 & .79 & 1450 \\
\hline $\begin{array}{l}14 \ldots \\
\text { JUNE }\end{array}$ & -- & -- & -- & -- & 537 & .78 & 1650 \\
\hline JULY $^{20 . . .}$ & -- & -- & -- & -- & 430 & .63 & 4010 \\
\hline $31 \ldots$ & -- & -- & -- & -- & 304 & .47 & 14000 \\
\hline $03 . .$. & -- & -- & - & -- & 470 & .66 & 2350 \\
\hline
\end{tabular}


5-3300. MINNESOTA RIVER NEAR JORDAN, MINN.--Continued

DRAINAGE AREA.--16,200 sq mi, approximately.

RECORDS AVAILABLE.--Chemical analyses: December 1962 to August 1966, November 1967 to September 1968.

CHEMICAL ANALYSES IN MILLIGRAMS PER LITER, WATER YEAR OCTOBER 1967 TO SEPTEMBER 1968

\begin{tabular}{|c|c|c|c|c|c|c|c|c|c|c|c|}
\hline DATE & $\begin{array}{l}\text { ALKA- } \\
\text { LINITY } \\
\text { AS } \\
\text { CACO3 }\end{array}$ & $\begin{array}{l}\text { SULFATE } \\
\text { (S04) }\end{array}$ & $\begin{array}{l}\text { CHLC- } \\
\text { RIDE } \\
(C L)\end{array}$ & $\begin{array}{l}\text { FLUC- } \\
\text { RIOE } \\
\text { (F) }\end{array}$ & $\begin{array}{l}\text { NI TRATE } \\
\text { (NO3) }\end{array}$ & $\begin{array}{l}\text { ORTHO } \\
\text { PHOS - } \\
\text { PHATE } \\
\text { (PO4) }\end{array}$ & $\begin{array}{l}\text { TOTAL } \\
\text { ALUM- } \\
\text { INI UM } \\
\text { (AL) }\end{array}$ & $\begin{array}{c}\text { BORON } \\
\text { (B) }\end{array}$ & $\begin{array}{l}\text { COPPER } \\
\text { (CU) }\end{array}$ & $\begin{array}{l}\text { ZINC } \\
(2 N)\end{array}$ & $\begin{array}{l}\text { CAD- } \\
\text { MIUM } \\
\text { (CC) }\end{array}$ \\
\hline $\begin{array}{l}\text { NOV. } \\
15 \ldots . \\
\text { DEC. }\end{array}$ & 318 & 156 & 34 & .3 & .2 & .38 & .3 & .14 & -- & -- & - \\
\hline $\begin{array}{l}12 \ldots \\
\text { JAN. }\end{array}$ & 326 & 169 & 34 & .2 & .9 & .46 & .1 & .15 & .00 & .00 & .00 \\
\hline $\begin{array}{l}16 \ldots \\
\text { FEB. }\end{array}$ & 307 & 168 & 52 & .2 & 5.1 & .64 & .3 & .17 & -- & - & -- \\
\hline $\begin{array}{l}21 \ldots \\
\text { MAR. }\end{array}$ & 404 & 179 & 46 & .3 & 5.8 & .46 & .3 & .18 & -- & -- & -- \\
\hline $\begin{array}{l}20 \ldots \\
\text { APR... }\end{array}$ & 258 & 153 & 25 & .3 & 1.7 & .84 & .2 & .13 & -- & -- & - \\
\hline$\underset{\text { MAY }}{18 \cdots}$ & 230 & 208 & 20 & $\cdot 3$ & $\cdot 1$ & .54 & .0 & $\cdot 14$ & .00 &. $\mathrm{CO}$ & .00 \\
\hline $\begin{array}{l}14 \ldots \\
\text { JUNE }\end{array}$ & 223 & 205 & 21 & .3 & .9 & .60 & .7 & .12 & -- & - & -- \\
\hline $20 \ldots$ & 203 & 108 & 14 & .4 & 28 & .59 & .7 & .07 & -- & - & -- \\
\hline SEPT. & 157 & 64 & 8.2 & .3 & 20 & .56 & .6 & .06 & -- & - & -- \\
\hline $03 \ldots$ & 266 & 109 & 19 & .4 & 5.1 & .65 & .7 & .10 & -- & - & - \\
\hline
\end{tabular}

\begin{tabular}{|c|c|c|c|c|c|c|c|}
\hline DATE & $\begin{array}{l}\text { HARD- } \\
\text { NESS } \\
\text { (CA, MG) }\end{array}$ & $\begin{array}{l}\text { NON- } \\
\text { CAR- } \\
\text { BONATE } \\
\text { HARD- } \\
\text { NESS }\end{array}$ & $\begin{array}{l}\text { SOOIUM } \\
\text { AO- } \\
\text { SORP- } \\
\text { IION } \\
\text { RAT IO }\end{array}$ & $\begin{array}{l}\text { PERCENT } \\
\text { SODIUM }\end{array}$ & $\begin{array}{l}\text { SPECI- } \\
\text { FIC } \\
\text { COND- } \\
\text { UCTANCE } \\
\text { (MICRO- } \\
\text { MHDSI }\end{array}$ & PH & COLOI \\
\hline $\begin{array}{l}\text { NOV. } \\
15 . . .\end{array}$ & 420 & 102 & 1.0 & 20 & 923 & 7.9 & 7 \\
\hline $\begin{array}{l}\text { DEC. } \\
12 . . \\
\text { JAN. }\end{array}$ & 446 & 120 & 1.0 & 19 & 966 & 7.7 & 7 \\
\hline $\begin{array}{l}16 \ldots \\
\text { FEB. }\end{array}$ & 423 & 116 & 1.3 & 24 & 997 & 8.4 & 4 \\
\hline $\begin{array}{l}21 \ldots . \\
\text { MAR. }\end{array}$ & 510 & 106 & 1.2 & 20 & 1140 & 8.5 & 6 \\
\hline$\underset{A P R}{20} .$. & 352 & 53 & .8 & 18 & 796 & 8.5 & 12 \\
\hline $\operatorname{MAY}_{\operatorname{MAY}}^{18 . .}$ & 389 & 159 & .7 & 16 & 846 & 8.3 & 4 \\
\hline JUNE & 389 & 166 & .7 & 15 & 838 & 7.8 & 9 \\
\hline JULY & 329 & 126 & .4 & 9 & 667 & 8.1 & 28 \\
\hline $\begin{array}{l}31 \ldots . . \\
\text { SEPT. }\end{array}$ & 232 & 75 & .2 & 6 & 481 & 7.6 & 20 \\
\hline $03 \ldots$ & 360 & 94 & .5 & 11 & 723 & 8.0 & 15 \\
\hline
\end{tabular}


5-3310. MISSISSIPPI RIVER AT ST. PAUL, MINN.

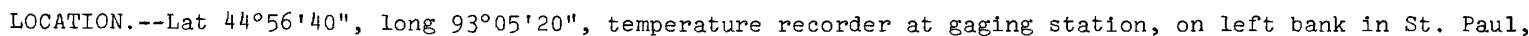
- Ramsey County, 300 feet upstream from Robert Street Bridge, 6 miles downstream from Minnesota River, and at mile 839.3 upstream from Ohio River.

DRAINAGE AREA.--36,800 square miles, approximately.

RECORDS AVAILABLE. - Water temperatures: October 1956 to september 1968.

EXTREMES, 1967-68.--Water temperatures: Maximum, $28^{\circ} \mathrm{C}$ July 18-20, Aug. 7-9; minimum, freezing point on many days during December to March.

EXTREMES, 1956-68.--Water temperatures: Maximum, $31^{\circ} \mathrm{C}$ July 24-28, 1964; minimum, freezing point on many days during winter months.

REMARKS.--Recorder stopped Feb. 10-13 and May 17-22.

TEMPERATURE $\left({ }^{\circ} \mathrm{C}\right)$ OF WATER, WATER YEAR OCTOBER 1967 TO SEPTEMBER 1968

(WATER-STAGE RECORDER WITH TEMPERATURE ATTACHMENT, CONTINUOUS ETHYL ALCOHOL-ACTUATED THERMOGRAPH)

Day

Month

$\begin{array}{lllllllllllllllllllllllllllllll}1 & 2 & 3 & 4 & 5 & 6 & 7 & 8 & 9 & 10 & 11 & 12 & 13 & 14 & 15 & 16 & 17 & 18 & 19 & 20 & 21 & 22 & 23 & 24 & 25 & 26 & 27 & 28 & 29 & 30 & 31\end{array}$

October

$\begin{array}{lllllllllllllllllllllllllllllllllll}\text { Maximum } & 16 & 17 & 17 & 17 & 19 & 18 & 18 & 17 & 16 & 15 & 14 & 14 & 13 & 13 & 13 & 13 & 13 & 13 & 13 & 13 & 13 & 12 & 13 & 13 & 12 & 12 & 12 & 11 & 11 & 11 & 11 & 14\end{array}$

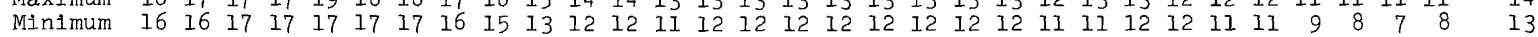

November

$\begin{array}{lrrrrrrrrrrrrrrrrrrrrrrrrrrrrrrrrrrrrrrrrr}\text { Maximum } & 10 & 10 & 10 & 9 & 9 & 9 & 7 & 8 & 8 & 7 & 8 & 8 & 8 & 7 & 7 & 7 & 7 & 7 & 7 & 7 & 7 & 7 & 7 & 6 & 6 & 6 & 6 & 6 & 6 & 6 & -- & 7\end{array}$

Minimum

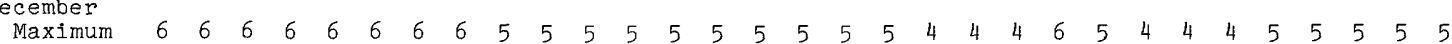

Minimum

January

Maximum

Minimum

February

Maximum

Minim

Maximum

Minimum

April

Maximum

Minimum

May

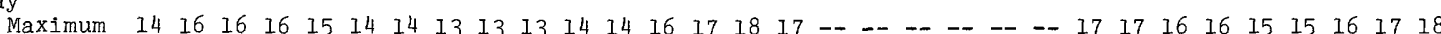

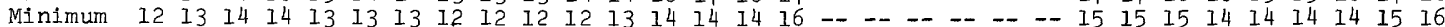

June

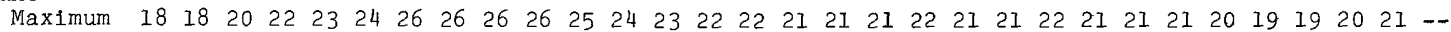

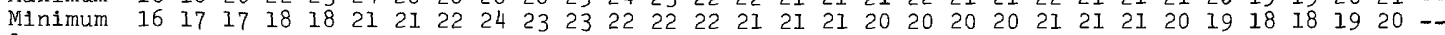

July

Maximum $20 \begin{array}{llllllllllllllllllllllllllllll}19 & 20 & 21 & 22 & 22 & 23 & 25 & 25 & 25 & 25 & 26 & 26 & 26 & 26 & 27 & 28 & 28 & 28 & 28 & 27 & 27 & 27 & 26 & 26 & 26 & 26 & 25 & 24 & 24 & 24\end{array}$

Minimum $19 \begin{array}{llllllllllllllllllllllllllllllll}19 & 19 & 19 & 19 & 21 & 19 & 21 & 22 & 22 & 22 & 23 & 23 & 25 & 25 & 24 & 25 & 26 & 26 & 27 & 26 & 24 & 24 & 25 & 25 & 24 & 24 & 24 & 24 & 23 & 23\end{array}$

August

Maximum $23 \quad 24 \quad 24 \quad 25 \quad 26 \quad 27 \quad 28 \quad 28 \quad 28 \quad 24 \quad 24 \quad 23 \quad 23 \quad 2322 \quad 23 \quad 22 \quad 22 \quad 22 \quad 2324 \quad 25 \quad 27 \quad 27 \quad 25 \quad 24 \quad 23 \quad 22 \quad 22 \quad 22 \quad 22$

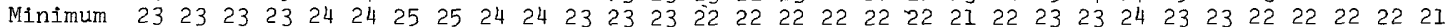

September

$\begin{array}{lllllllllllllllllllllllllllllllll}\text { Maximum } & 21 & 21 & 21 & 21 & 21 & 21 & 21 & 20 & 19 & 19 & 19 & 20 & 20 & 21 & 20 & 21 & 21 & 20 & 19 & 19 & 19 & 19 & 19 & 19 & 19 & 19 & 18 & 17 & 17 & 17 & \text {-- }\end{array}$

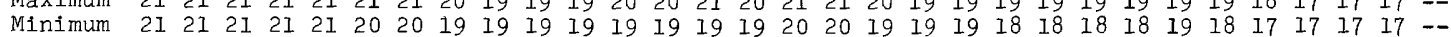


$$
\text { . }
$$ 
5-3760. NORTH FORK WHITEWATER RIVER NEAR ELBA, MINN.

(Hydrologic bench-mark station)

LOCATION.--Lat $44^{\circ} 05^{\prime} 30^{\prime \prime}$, long $92^{\circ} 03^{\prime} 57^{\prime \prime}$, in sec.7, T.107 N., R. I0 w., Winona County, at gaging station on left bank, 2.3 miles upstream from Middle Fork, 2.4 miles west of Elba, and 3.5 miles upstream from confluence with South Fork.

CHEMICAL ANALYSES IN MILLIGRAMS PER LITER, WATER YEAR OC TOBER 1967 TO SEPTEMBER 1968

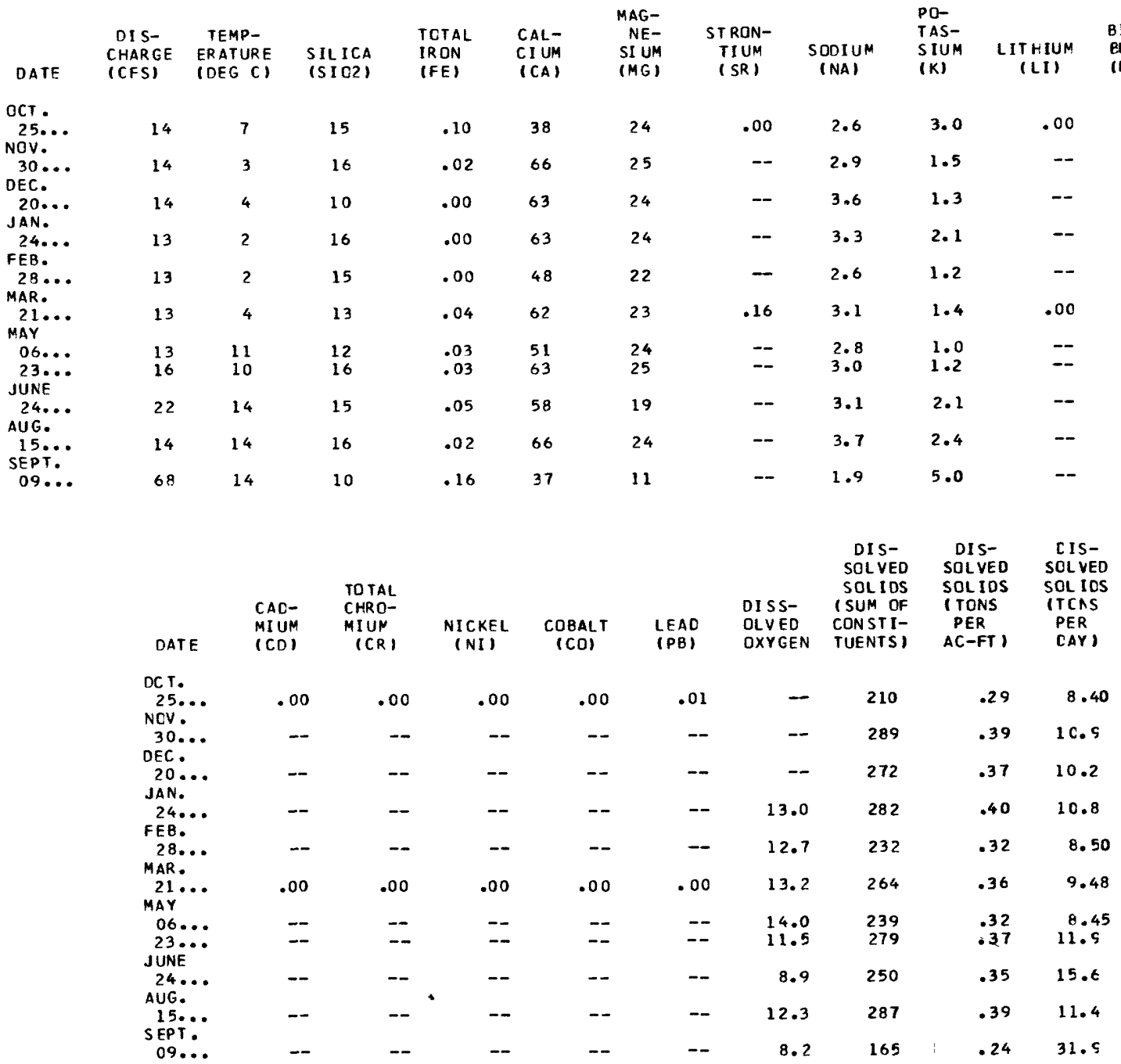

RADIOCHEMICAL ANALYSES, WATER YEAR OCTOBER 1967 TO SEPTEMBER 1968

Units of measurement: Uranium, micrograms per 1iter of water; radium as radium-226, in picocuries per liter of water; gross beta radiation as strontium-90-yttrium-90, in picocuries per liter of water; gross alpha radiation, as micrograms of uranium equivalent per liter of water.

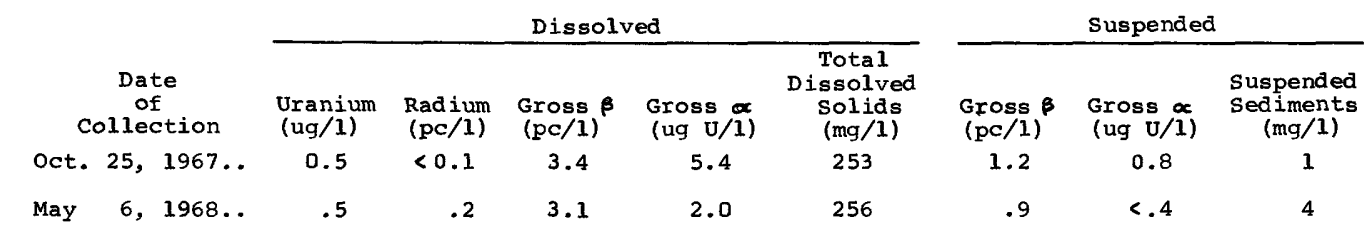

PESTICIDE ANALYSES, IN MICROGRAMS PER LITER, WATER YEAR OCTOBER 1967 TO SEPTEMBER 1968

\begin{tabular}{|c|c|c|c|c|c|c|c|c|c|c|}
\hline DATE & ALDRIN & DDD & $\mathrm{DDE}$ & $\mathrm{DDT}$ & DIELDRIN & ENDRIN & HEPTACHLOR & LINDANE & $2,4-D$ & $2,4,5-T$ \\
\hline $\begin{array}{l}\text { OCT. } \\
25 . . . \\
\text { May }\end{array}$ & 0.00 & 0.00 & 0.00 & 0.00 & 0.00 & 0.00 & 0.00 & 0.00 & & \\
\hline $6 \ldots$ & .00 & .00 & .00 & .00 & .00 & .00 & .00 & .00 & .00 & .00 \\
\hline
\end{tabular}


5-3760. NORTH FORK WHITEWATER RIVER. NEAR ELBA, MINN.--Continued

(Hydrologic bench-mark station)

DRAINAGE AREA.--101 sq mi.

RECORDS AVAILABLE.--Chemical analyses: August 1967 to september 1968.

CHEMICAL ANALYSES IN MILLIGRAMS PER LITER, WATER YEAR OC TOBER 1967 TO SEPTEMBER 1968

\begin{tabular}{|c|c|c|c|c|c|c|c|c|c|c|c|}
\hline DATE & $\begin{array}{l}\text { ALKA- } \\
\text { LINITY } \\
\text { AS } \\
\text { CACO3 }\end{array}$ & $\begin{array}{l}\text { SULFATE } \\
\text { (SO4) }\end{array}$ & $\begin{array}{l}\text { CHLO- } \\
\text { RIDE } \\
\text { (CL) }\end{array}$ & $\begin{array}{l}\text { FLUO- } \\
\text { RIDE } \\
\text { (F) }\end{array}$ & $\begin{array}{l}\text { NITRATE } \\
\text { (NO3) }\end{array}$ & $\begin{array}{l}\text { ORTHO } \\
\text { PHOS- } \\
\text { PHATE } \\
\text { (PO4) }\end{array}$ & $\begin{array}{l}\text { PHOS- } \\
\text { PHA TE } \\
\text { (PO4) }\end{array}$ & $\begin{array}{l}\text { TOTAL } \\
\text { ALUM- } \\
\text { INIUM } \\
\text { (AL) }\end{array}$ & $\begin{array}{l}\text { BORON } \\
(B)\end{array}$ & $\begin{array}{l}\text { CCPPER } \\
\text { (CU) }\end{array}$ & $\begin{array}{l}\operatorname{lng} \\
(2 N)\end{array}$ \\
\hline $\begin{array}{l}\text { OCT. } \\
25 . . \\
\text { NOV. }\end{array}$ & 182 & 12 & 1.7 & .1 & 4.4 & .16 & .26 & $\bullet 1$ & .01 & .00 & .00 \\
\hline $\begin{array}{l}30 \ldots . . \\
\text { DEC. }\end{array}$ & 261 & 13 & 1.8 & .0 & 5.9 & .11 & .17 & .4 & .00 & - & -- \\
\hline $\begin{array}{l}20 . . . \\
\text { JAN. }\end{array}$ & 254 & 12 & 2.2 & .0 & 3.3 & .13 & .38 & .1 & .00 & - & - \\
\hline $\begin{array}{l}24 \ldots \\
\text { FEB.. }\end{array}$ & 257 & 12 & 1.5 & .1 & 5.1 & .18 & .31 & .4 & .00 & - & - \\
\hline $\begin{array}{c}28 \ldots . . \\
\text { MAR. }\end{array}$ & 208 & 11 & 1.3 & .1 & 5.8 & .13 & .23 & .2 & .01 & - & - \\
\hline MAY $21 \cdots$ & 239 & 13 & 2.5 & .1 & 3.6 & .18 & .22 & .1 & .00 & .00 & .00 \\
\hline $\begin{array}{l}06 \ldots . . \\
23 \ldots \\
\text { JUNE }\end{array}$ & $\begin{array}{l}218 \\
253\end{array}$ & $\begin{array}{l}12 \\
13\end{array}$ & $\begin{array}{l}1.7 \\
2.0\end{array}$ & .2 & $\begin{array}{l}2.2 \\
4.0\end{array}$ & $\begin{array}{r}.07 \\
.12\end{array}$ & $\begin{array}{l}.52 \\
.22\end{array}$ & .0 & $\begin{array}{l}.00 \\
.00\end{array}$ & - & $=$ \\
\hline $\begin{array}{l}24 \ldots \\
\text { AUG... }\end{array}$ & 213 & 13 & 2.3 & .2 & 8.5 & .56 & .71 & .6 & .00 & - & - \\
\hline $\begin{array}{l}15 \ldots \\
\text { SEPT. }\end{array}$ & 256 & 12 & 2.6 & .2 & 5.1 & .23 & .34 & 1.8 & .00 & - & - \\
\hline 09 & 136 & 8.3 & 4.6 & .1 & 4.4 & 1.2 & 1.2 & .5 & .03 & - & -- \\
\hline
\end{tabular}

\begin{tabular}{|c|c|c|c|c|c|c|c|c|c|}
\hline DATE & $\begin{array}{l}\text { HARD- } \\
\text { NESS } \\
(C A, M G)\end{array}$ & $\begin{array}{l}\text { NON- } \\
\text { CAR- } \\
\text { BONATE } \\
\text { HARD- } \\
\text { NESS }\end{array}$ & $\begin{array}{l}\text { SOOIUM } \\
\text { AD- } \\
\text { SORP- } \\
\text { TICN } \\
\text { RATIO }\end{array}$ & $\begin{array}{l}\text { PERCENT } \\
\text { SODIUM }\end{array}$ & $\begin{array}{l}\text { SPECI- } \\
\text { FIC } \\
\text { COND- } \\
\text { UCTANCE } \\
\text { (MICRO- } \\
\text { MHOSI }\end{array}$ & PH & COLOR & $\begin{array}{c}\text { COLI- } \\
\text { FORM } \\
\text { ICOL- } \\
\text { ONIES } \\
\text { PER } \\
100 \mathrm{ML} \text { ) }\end{array}$ & $\begin{array}{l}\text { BIO- } \\
\text { CHEM- } \\
\text { ICAL } \\
\text { OXYGEN } \\
\text { OEMANO }\end{array}$ \\
\hline $\begin{array}{l}\text { OCT. } \\
25 \ldots . . \\
\text { NOV. }\end{array}$ & 192 & 10 & $\cdot 1$ & 3 & 377 & 7.7 & 2 & - & .6 \\
\hline $\begin{array}{l}30 \ldots . . \\
\text { DEC. }\end{array}$ & 269 & 8 & .1 & 2 & 492 & 8.0 & 2 & - & . $\epsilon$ \\
\hline $\begin{array}{l}20 \ldots . . \\
\text { JAN. }\end{array}$ & 257 & 3 & .1 & 3 & 467 & B. 4 & 3 & -- & 2.6 \\
\hline $\begin{array}{l}24 \ldots \\
\text { FEB. }\end{array}$ & 257 & 0 & .1 & 3 & 483 & B. 0 & 1 & - & .4 \\
\hline $\begin{array}{c}28 . . . \\
\text { MAR. }\end{array}$ & 211 & 3 & .1 & 3 & 399 & 8.0 & 2 & - & .4 \\
\hline$\underset{\text { MAY }}{21 \ldots}$ & 247 & 8 & .1 & 3 & 600 & 7.7 & 3 & 16 & $\cdot \varepsilon$ \\
\hline $\begin{array}{l}06 \ldots . . \\
23 \ldots \\
\text { JUNE }\end{array}$ & $\begin{array}{l}228 \\
258\end{array}$ & $\begin{array}{r}10 \\
5\end{array}$ & .1 & $\begin{array}{l}3 \\
2\end{array}$ & $\begin{array}{l}419 \\
485\end{array}$ & $\begin{array}{l}8.2 \\
8.2\end{array}$ & $\begin{array}{l}2 \\
3\end{array}$ & $\begin{array}{l}30 \\
14\end{array}$ & $\begin{array}{l}1.5 \\
1.1\end{array}$ \\
\hline $\begin{array}{l}24 \ldots . . \\
\text { AUG. }\end{array}$ & 223 & 10 & .1 & 3 & 438 & 8.0 & 80 & 200 & 1.2 \\
\hline $\begin{array}{l}15 \ldots . . \\
\text { SEPT.: } \\
09 . .\end{array}$ & 264 & 9 & $\begin{array}{l}.1 \\
.1\end{array}$ & 3 & $\begin{array}{l}491 \\
289\end{array}$ & $\begin{array}{l}7.4 \\
7.3\end{array}$ & 5 & - & $\begin{array}{r}.3 \\
1.7\end{array}$ \\
\hline
\end{tabular}

PERIODIC DAILY SUSPENDED SEDIMENT, WATER YEAR OCTOBER 1967 TO SEPTEMBER 1968

\begin{tabular}{|c|c|c|c|c|c|c|c|c|c|}
\hline & Date & $\begin{array}{l}\text { Mean } \\
\text { Discharge } \\
\quad(\text { cfs) }\end{array}$ & $\begin{array}{l}\text { Mean } \\
\text { Concen- } \\
\text { tration } \\
\text { (mg/l) }\end{array}$ & $\begin{array}{l}\text { Load } \\
\text { (tons) }\end{array}$ & & Date & $\begin{array}{c}\text { Mean } \\
\text { Discharge } \\
(\mathrm{cfs})\end{array}$ & $\begin{array}{l}\text { Mean } \\
\text { Concen- } \\
\text { tration } \\
(\mathrm{mg} / \mathrm{l})\end{array}$ & $\begin{array}{c}\text { Load } \\
\text { (tons) }\end{array}$ \\
\hline Oct. & 25,1967 & 15 & 84 & 3.4 & May & 22,1968 & 16 & 23 & 1.0 \\
\hline Nov. & 30 & 14 & 113 & 4.3 & Junè & 24 & 22 & 4.15 & 25 \\
\hline Mar. & 21,1968 & 13 & 9 & .3 & Aug. & 15 & 15 & 57 & 2.3 \\
\hline Apr. & 25 & 18 & 12 & .6 & Sept. & 9 & 68 & 773 & 142 \\
\hline
\end{tabular}


CHEMICAL ANALYSES IN MILLIGRAMS PER LITER, WATER YEAR OCTOBER 1967 TO SEPTEMBER 1968

\begin{tabular}{|c|c|c|c|c|c|c|c|c|c|c|c|c|}
\hline CATE & $\begin{array}{l}\text { DIS- } \\
\text { CHARGE } \\
\text { (CFS) }\end{array}$ & $\begin{array}{l}\text { TEMP- } \\
\text { ERATURE } \\
\text { (DEG C) }\end{array}$ & $\begin{array}{l}\text { SILICA } \\
\text { (SI } 02 \text { ) }\end{array}$ & $\begin{array}{l}\text { TCTAL } \\
\text { IRON } \\
\text { (FE) }\end{array}$ & $\begin{array}{l}\text { CAL- } \\
\text { CIUM } \\
\text { (CA) }\end{array}$ & $\begin{array}{l}\text { MAG- } \\
\text { NE- } \\
\text { SIUM } \\
\text { (MG) }\end{array}$ & $\begin{array}{l}\text { SOD IUM } \\
\text { (NA) }\end{array}$ & $\begin{array}{l}\text { PO- } \\
\text { TAS- } \\
\text { SIUM } \\
\text { (K) }\end{array}$ & $\begin{array}{l}\text { BICAR- } \\
\text { BONATE } \\
\text { (HCO3) }\end{array}$ & $\begin{array}{l}\text { CAR- } \\
\text { BONATE } \\
\text { (C.03) }\end{array}$ & $\begin{array}{c}\text { ALKA- } \\
\text { LINITY } \\
\text { AS } \\
\text { CACO3 }\end{array}$ & $\begin{array}{l}\text { SULFATE } \\
\text { (SO4) }\end{array}$ \\
\hline
\end{tabular}

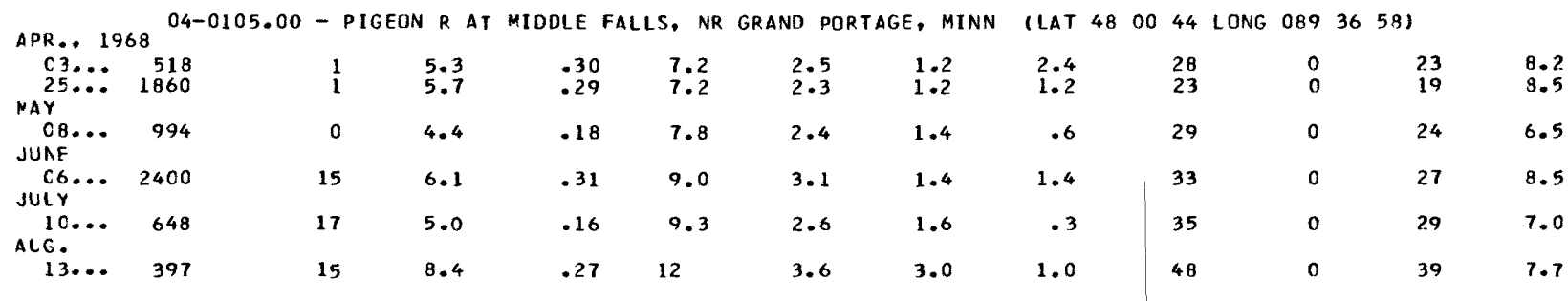

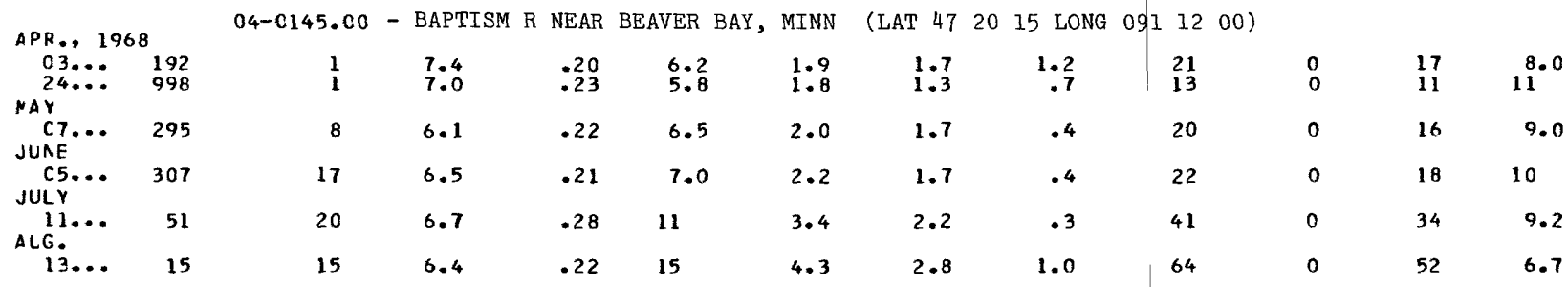

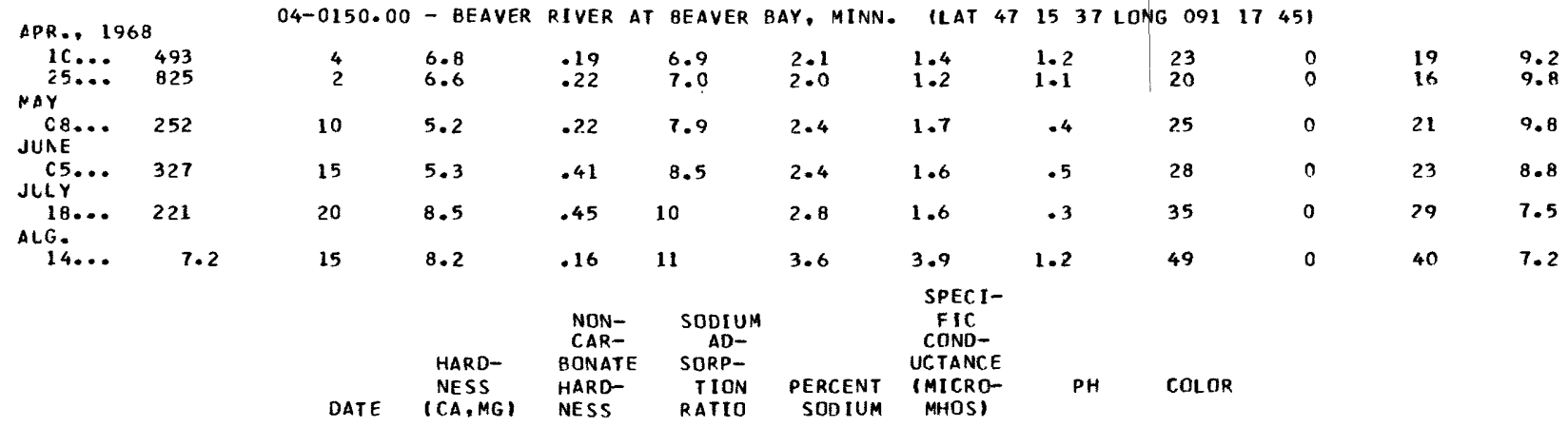

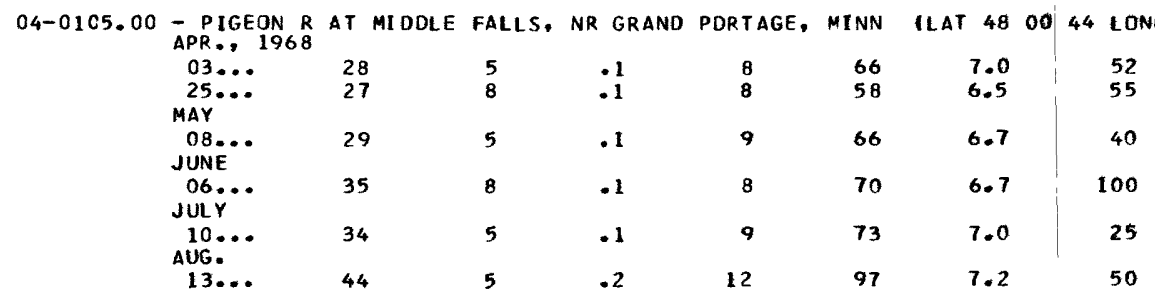

04-0145 ${ }_{A}^{00}$ - BAPTISM R NEAR BEAVER BAY, MINN (LAT 472015 LONG $\left.091 \quad 1200\right)$

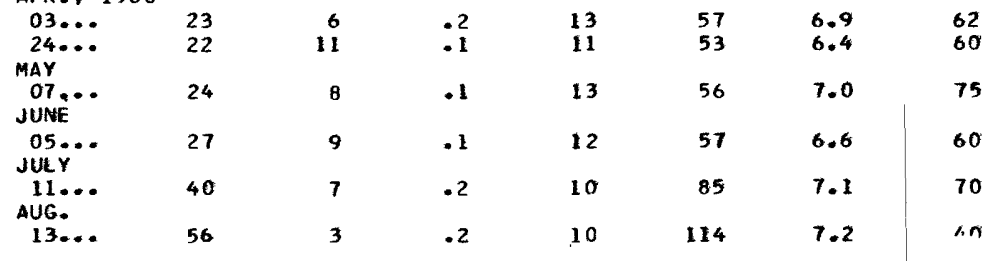

04-0150.00- BEAVER RIVER AT BEAVER BAY. MINN. (LAT 471537 LONG O91 I745)

\begin{tabular}{|c|c|c|c|c|c|c|c|}
\hline $\begin{array}{l}10 \ldots \\
25 \ldots\end{array}$ & $\begin{array}{l}26 \\
26\end{array}$ & ${ }_{10}^{7}$ & .1 & $\begin{array}{r}10 \\
9\end{array}$ & $\begin{array}{l}59 \\
56\end{array}$ & $\begin{array}{l}7.0 \\
6.5\end{array}$ & $\begin{array}{r}58 \\
100\end{array}$ \\
\hline $\begin{array}{l}\text { MAY } \\
\text { O8_... }\end{array}$ & 30 & 9 & .1 & 11 & 66 & 7.1 & 75 \\
\hline $\begin{array}{l}\text { JUNE } \\
\text { OS... } \\
\text { JULY }\end{array}$ & 31 & 8 & .1 & 10 & 62 & 6.7 & 100 \\
\hline $\begin{array}{l}18 \ldots \\
\text { AUG. }\end{array}$ & 37 & 8 & .1 & 9 & 68 & 7.5 & 100 \\
\hline $14 \ldots$ & -43 & 3 & .3 & 16 & 97 & 7,1 & 40 \\
\hline
\end{tabular}


CHEMICAL ANALYSES IN MILLIGRAMS PER LITER, WATER YEAR OCTOBER 1967 TO SEPTEMBER 1968

\begin{tabular}{|c|c|c|c|c|c|c|c|c|c|c|c|}
\hline & & & & & & & & & $\begin{array}{c}\text { DIS- } \\
\text { SOLVFD }\end{array}$ & $\begin{array}{c}\text { DIS- } \\
\text { SOLVED }\end{array}$ & $\begin{array}{c}\text { DIS- } \\
\text { SOLVEO }\end{array}$ \\
\hline & CHLO- & FLUO- & & $\begin{array}{l}\text { ORTHO } \\
\text { PHOS- }\end{array}$ & PHOS- & $\begin{array}{l}\text { TOTAL } \\
\text { ALUM- }\end{array}$ & & DISS- & $\begin{array}{l}\text { SOLIDS } \\
\text { ISUM OF }\end{array}$ & $\begin{array}{l}\text { SOLIDS } \\
\text { ITONS }\end{array}$ & $\begin{array}{l}\text { SOL IOS } \\
\text { ITONS }\end{array}$ \\
\hline CATE & $\begin{array}{l}R \perp D E \\
(C L)\end{array}$ & $\begin{array}{l}R \text { IDE } \\
\text { (F) }\end{array}$ & $\begin{array}{l}\text { NITRATE } \\
\text { (NO3) }\end{array}$ & $\begin{array}{l}\text { PHATE } \\
\text { (P04) }\end{array}$ & $\begin{array}{l}\text { PHATE } \\
\text { (PO4) }\end{array}$ & $\begin{array}{l}\text { INIUM } \\
\text { (AL) }\end{array}$ & $\begin{array}{c}\text { BORON } \\
\text { (A) }\end{array}$ & $\begin{array}{l}\text { OLVED } \\
\text { OXYGEN }\end{array}$ & $\begin{array}{l}\text { CONSTI- } \\
\text { TUENTSI }\end{array}$ & $\begin{array}{c}\text { PER } \\
A C-F T)\end{array}$ & $\begin{array}{l}\text { PER } \\
\text { DAY) }\end{array}$ \\
\hline
\end{tabular}

\begin{tabular}{|c|c|c|c|c|c|c|c|c|c|c|c|}
\hline APR . & $\begin{array}{l}04-0105.00- \\
1968\end{array}$ & PIGEON R & AT MI DDLE & FALLS. & NR GRAND & PORTAGE. & MINN ILAT & 480044 & LONG O89 & & \\
\hline $\begin{array}{l}03 \ldots \\
25 \ldots \\
N A Y\end{array}$ & $\begin{array}{r}1.1 \\
.6\end{array}$ & .1 & $\begin{array}{l}1.1 \\
1.0\end{array}$ & $\begin{array}{l}.35 \\
.05\end{array}$ & -- & $\begin{array}{l}.6 \\
.9\end{array}$ & $\begin{array}{l}.03 \\
.13\end{array}$ & -- & $\begin{array}{l}44 \\
41\end{array}$ & $\begin{array}{l}.07 \\
.09\end{array}$ & $\begin{array}{l}76.9 \\
326\end{array}$ \\
\hline JUAE & .6 & .1 & .5 & .02 & -- & .8 & .04 & -- & 40 & .07 & 137 \\
\hline $\begin{array}{l}\text { C6... } \\
\text { JULY }\end{array}$ & .5 & .1 & 1.1 & .06 & - & 1.0 & .05 & -- & 49 & .10 & 492 \\
\hline $\begin{array}{l}10 . \cdots \\
\text { AUG. }\end{array}$ & 1.6 & .1 & .4 & .04 & -- & 3.3 & .00 & -- & 49 & .08 & 98.0 \\
\hline $13 \ldots$ & 1.8 & .5 & .5 & .04 & -- & .9 & .03 & $=-$ & 63 & .11 & 83.6 \\
\hline
\end{tabular}

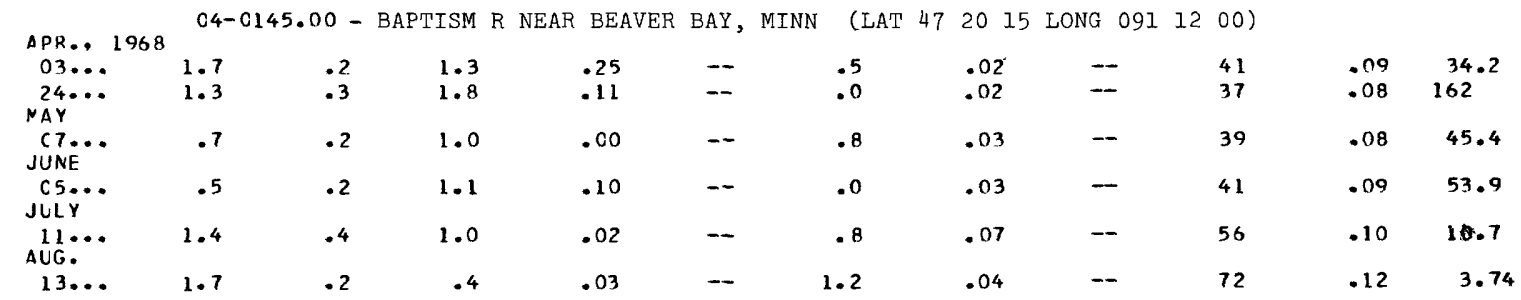

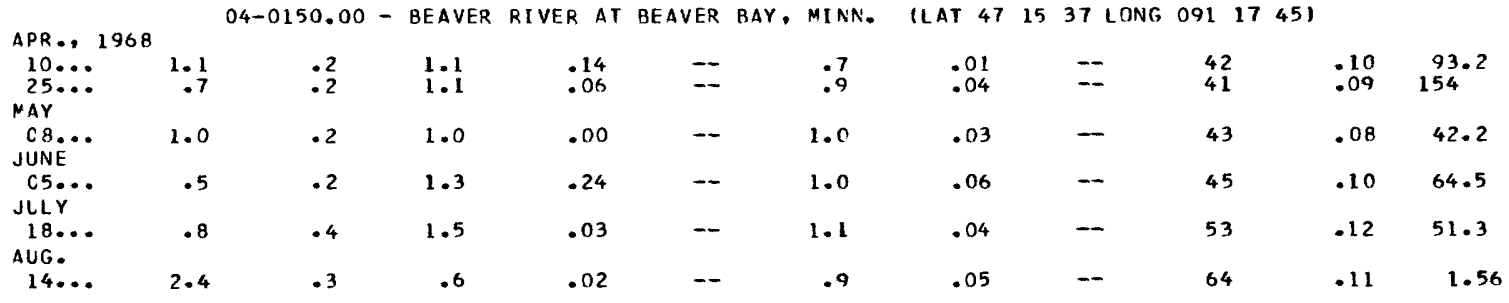




\begin{tabular}{|c|c|c|c|c|c|c|c|c|c|c|c|c|}
\hline ATE & $\begin{array}{l}\text { DIS- } \\
\text { CHARGE } \\
\text { (CFS) }\end{array}$ & $\begin{array}{l}\text { TEMP- } \\
\text { ERATURE } \\
\text { (DEG C) }\end{array}$ & $\begin{array}{l}\text { SILICA } \\
\text { (SIOZ) }\end{array}$ & $\begin{array}{l}\text { TOTAL } \\
\text { IRON } \\
\text { (FE) }\end{array}$ & $\begin{array}{l}\text { CAL- } \\
\text { CIUM } \\
\text { (CA) }\end{array}$ & $\begin{array}{l}\text { MAG- } \\
\text { NE- } \\
\text { SIUM } \\
(M G)\end{array}$ & $\begin{array}{l}\text { SOD IUM } \\
\text { (NA) }\end{array}$ & $\begin{array}{l}\text { PO- } \\
\text { TAS- } \\
\text { SIUM } \\
\text { (K) }\end{array}$ & $\begin{array}{l}\text { BICAR- } \\
\text { BONATE } \\
\text { (HCO3) }\end{array}$ & $\begin{array}{l}\text { CAR- } \\
\text { BONATE } \\
\text { (C03) }\end{array}$ & $\begin{array}{l}\text { ALKA- } \\
\text { LINITY } \\
\text { AS } \\
\text { CACOB }\end{array}$ & $\begin{array}{l}\text { SULFATE } \\
\text { (S04) }\end{array}$ \\
\hline
\end{tabular}

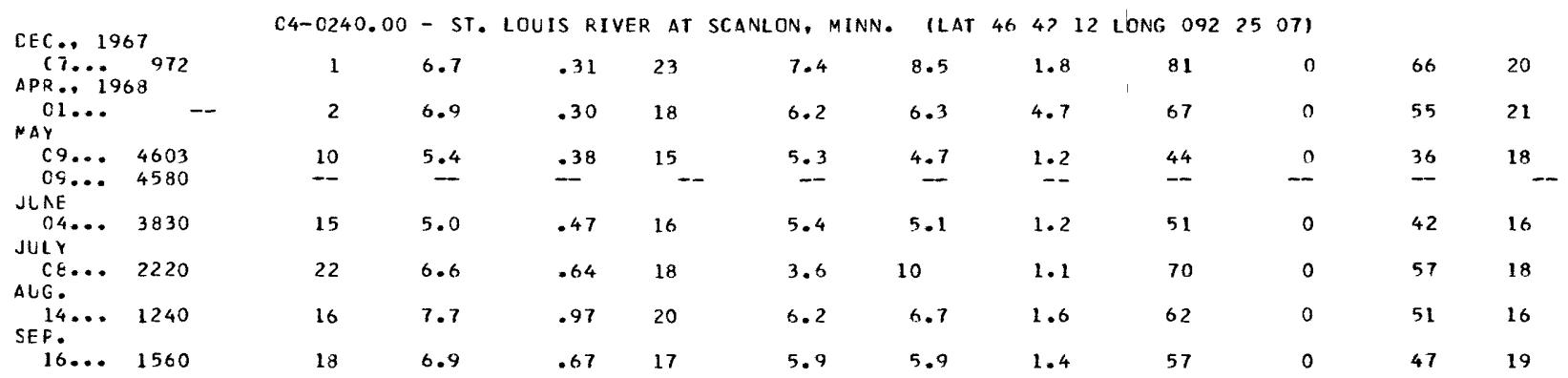

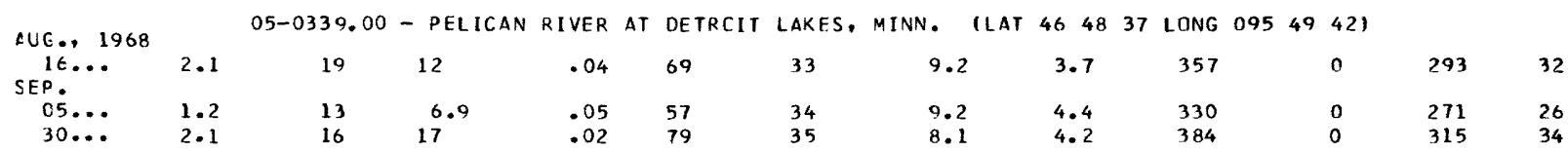

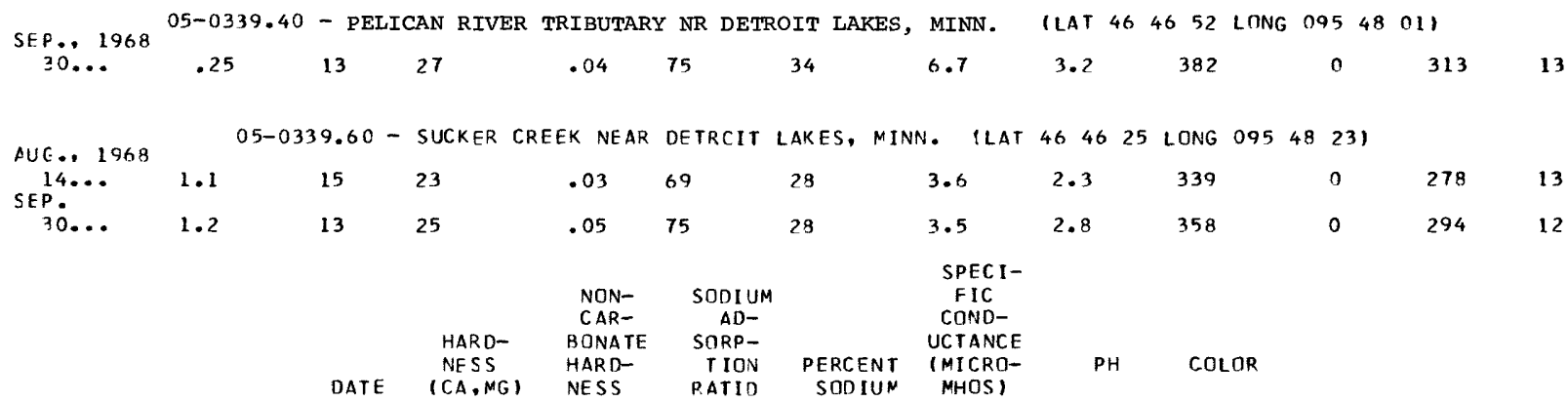

04-0240.00-ST. LOUIS RIVER AT SCANLON, MINN. (LAT 464212 LONG 0922507 )

\begin{tabular}{|c|c|c|c|c|c|c|c|}
\hline $\begin{array}{l}\text { DEC . } 1967 \\
\text { O7... } \\
\text { APR . } 1968\end{array}$ & 88 & 22 & .4 & 17 & 230 & 7.5 & 84 \\
\hline $01 \ldots$ & 71 & 16 & .3 & 15 & 181 & 7.1 & 90 \\
\hline $\begin{array}{l}\text { MAY } \\
\text { O9.... } \\
\text { JUNE }\end{array}$ & 58 & 22 & .3 & 14 & 140 & 6.7 & 75 \\
\hline $05 \ldots$ & $\overline{61}$ & $\overline{19}$ &.-3 & -15 & 135 & $-\overline{6}$ & 175 \\
\hline $\begin{array}{l}\text { JULY } \\
08 \ldots .\end{array}$ & 59 & 1 & .6 & 26 & 170 & 6.9 & 100 \\
\hline $\begin{array}{l}\text { AUG. } \\
14 \ldots . \\
\text { SEP. }\end{array}$ & 75 & 25 & .3 & 16 & 178 & 6.7 & 80 \\
\hline $16 \ldots$ & 66 & 19 & .3 & 16 & 161 & 7.4 & 120 \\
\hline
\end{tabular}

05-0339,00 - PELICAN RIVER AT DETROIT LAKES, MINN. (LAT 464837 LONG 0954942 )

\begin{tabular}{|c|c|c|c|c|c|c|}
\hline $\begin{array}{l}16 \ldots \\
\text { SEP. }\end{array}$ & 309 & 16 & .2 & 6 & 582 & 8.0 \\
\hline $\begin{array}{l}05 \ldots \\
30 \ldots\end{array}$ & $\begin{array}{l}283 \\
340\end{array}$ & $\begin{array}{l}12 \\
25\end{array}$ & .2 & $\begin{array}{l}7 \\
5\end{array}$ & $\begin{array}{l}544 \\
622\end{array}$ & $\begin{array}{l}8.1 \\
7.7\end{array}$ \\
\hline
\end{tabular}

05-0339.40 - PELICAN RIVER TRIBUTARY NR DETROIT LAKES, MINN. (LAT 464652 LONG 0954801 ) $\begin{array}{llllllll}30 \ldots & 328 & 15 & .2 & 4 & 591 & 7.6 & 2\end{array}$

05-0339.60 - SUCKER CREEK NEAR CETROIT LAKES, MINN. (LAT 464625 LONG 0954823 )

$\begin{array}{cccccccc}14 \ldots . \ldots & 285 & 8 & .1 & 3 & 516 & 8.2 & 5 \\ \text { SEP. } & & & & & & & \\ 30 \ldots & 302 & 9 & .1 & 2 & 546 & 7.7 & 2\end{array}$


CHEMICAL ANALYSES IN MILIIGRAMS PER LITER, WATER YEAR OCTOBER 1967 TO SEPTEMBER 1968

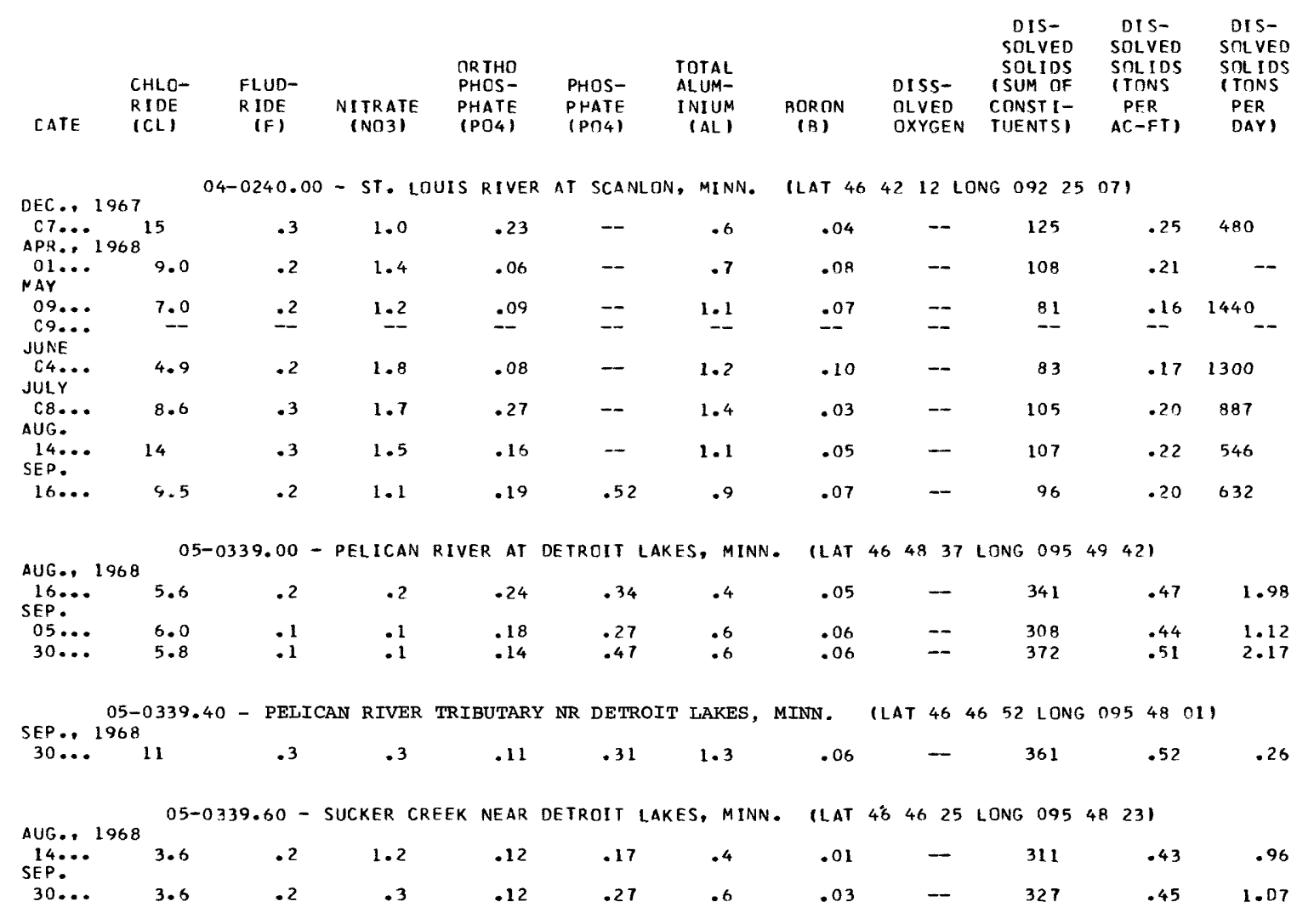


CHEMICAL ANALYSES IN MILLIGRAMS PER LITER, WATER YEAR OCTOBER 1967 TO SEPTEMBER 1968

\begin{tabular}{|c|c|c|c|c|c|c|c|c|c|c|c|c|}
\hline CATE & $\begin{array}{l}\text { DIS- } \\
\text { CHARGE } \\
\text { (CFS) }\end{array}$ & $\begin{array}{l}\text { TEMP- } \\
\text { ERATURE } \\
\text { (DEG C) }\end{array}$ & $\begin{array}{l}\text { SILICA } \\
\text { (SIO2) }\end{array}$ & $\begin{array}{l}\text { TCTAL } \\
\text { IRON } \\
\text { (FE) }\end{array}$ & $\begin{array}{l}\text { CAL- } \\
\text { CIUM } \\
\text { (CA) }\end{array}$ & $\begin{array}{l}\text { MAG- } \\
\text { NE- } \\
\text { SIUM } \\
\text { (MG) }\end{array}$ & $\begin{array}{l}\text { SOD I UM } \\
\text { (NA) }\end{array}$ & $\begin{array}{l}\text { PO- } \\
\text { TAS- } \\
\text { SIUM } \\
(\mathrm{K})\end{array}$ & $\begin{array}{l}\text { BICAR- } \\
\text { BONATE } \\
\text { IHCO3) }\end{array}$ & $\begin{array}{l}\text { CAR- } \\
\text { BONATE } \\
\text { (CO3) }\end{array}$ & $\begin{array}{l}\text { ALKA- } \\
\text { LINITY } \\
\text { AS } \\
\text { CACO3 }\end{array}$ & $\begin{array}{c}\text { SULFATE } \\
\text { (S04) }\end{array}$ \\
\hline
\end{tabular}

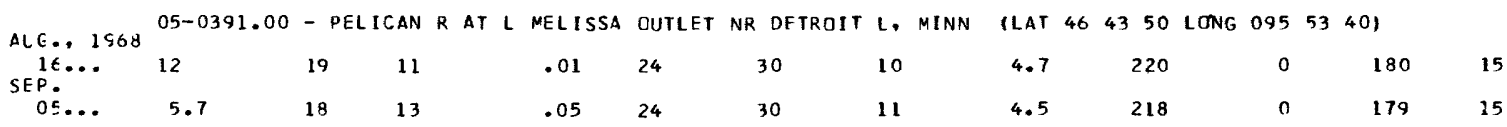

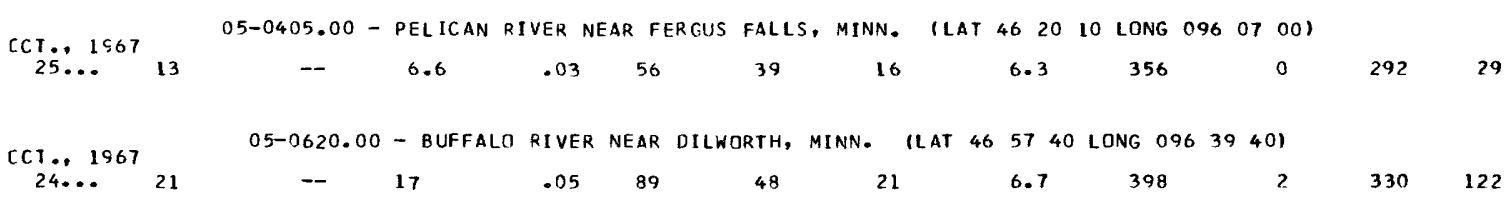

CCI., 1967

$24 \ldots 21$

05-0640.00 - WILO RICE RIVER AT HENDRUM, MINN. ILAT 471605 LONG 0964750 )

$\begin{array}{lllllllllll}6 & 16 & .15 & 71 & 34 & 20 & 4.7 & 348 & 5 & 294 & 54\end{array}$

05-0790.00 - RED LAKE RIVER AT CRCOKSTON, MINN. (LAT 474632 LONG 09636301

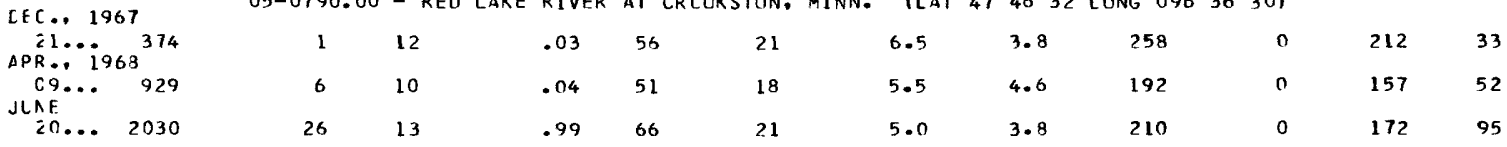

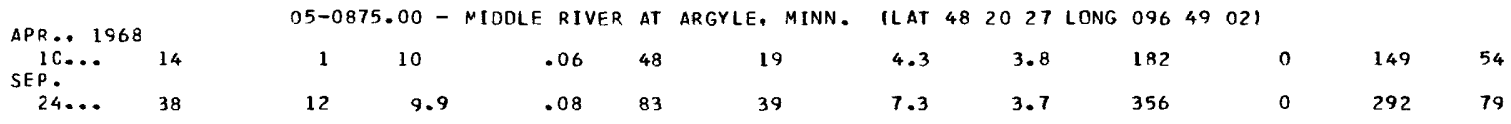

05-0940.00 - SOUTH BRANCH TWC RIVERS AT LAKE BPONSON, MINN. ILAT 484350 LONG 096 3950 I

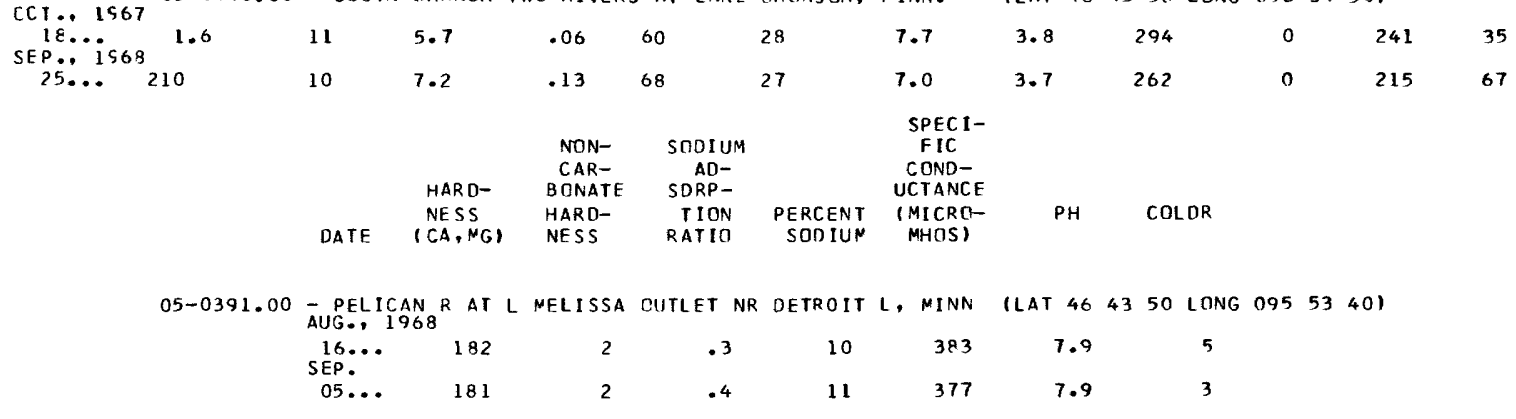

05-0405.00 - PELICAN RIVER NEAR FERGUS FALLS, MINN. ILAT 462010 LONG 0960700 )

$\begin{array}{llllllll}25 \ldots & 3 C O & B & .4 & 10 & 592 & 8.1 & 17\end{array}$

05-0620.00 - BUFFALO RIVER NEAR DILWORTH, MINN. ILAT 465740 LONG 09639401

$\begin{array}{lllllll}24 \ldots & 420 & 90 & .4 & 10 & 800 & 8.4\end{array}$

05-0640.00 $0 \overline{197}$ WILD RICE RIVER AT HENDRUM, MINN. (LAT 471605 LONG 0964750 )

$\begin{array}{llllllll}24 \ldots & 317 & 23 & .5 & 12 & 633 & 8.4 & 21\end{array}$

05-0790.00 - RED LAKE RIVER AT CRCOKSTDN. MINN. ILAT 474632 LONG 09636301

$\begin{array}{llllllll}21 \ldots . .0 & 226 & 14 & .2 & 6 & 448 & 8.0 & 13 \\ \begin{array}{l}\text { APR... } 1968 \\ \text { O9... }\end{array} & 202 & 44 & .2 & 5 & 399 & 7.5 & 26 \\ \text { JUNE } & 251 . . & 79 & .1 & 4 & 500 & 7.3 & 45\end{array}$

05-0875.00 - MIDDLE RIVER AT ARGYLE. MINN. (LAT 482027 LONG 096 4902 )

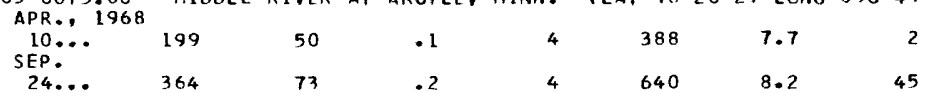

05-0940.00 - SOUTH BRANCH TWC RIVERS AT LAKE BRONSON. MINN. ILAT 484350 LONG 096 3950 I

\begin{tabular}{|c|c|c|c|c|c|c|c|}
\hline $\begin{array}{l}18 \ldots \\
\text { SEP... } 1968\end{array}$ & 264 & 23 & $\cdot 2$ & 6 & 505 & 7.5 & 37 \\
\hline $25 \ldots$ & 280 & 66 & .2 & 5 & 510 & 7.7 & 50 \\
\hline
\end{tabular}


CHEMICAL ANALYSES IN MILLIGRAMS PER LITER, WATER YEAR OCTOBER 1967 TO SEPTEMBER 1968

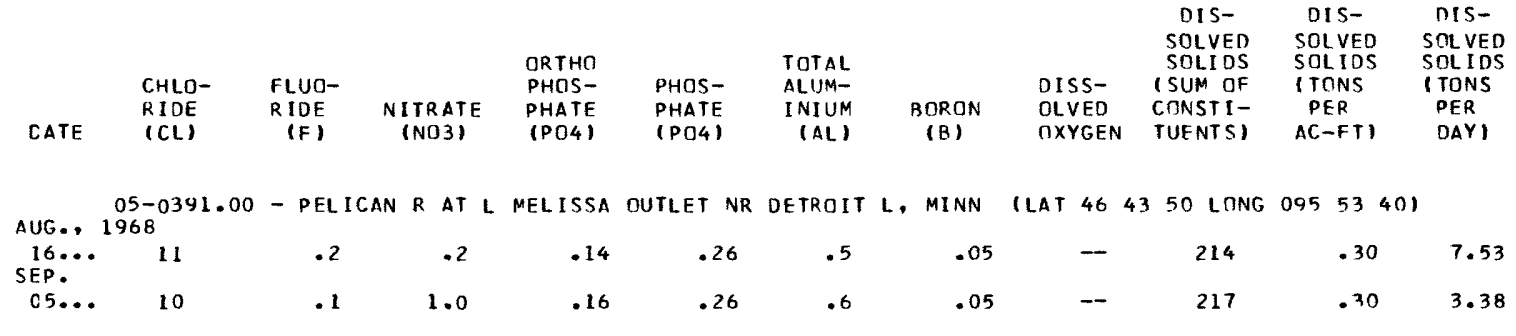

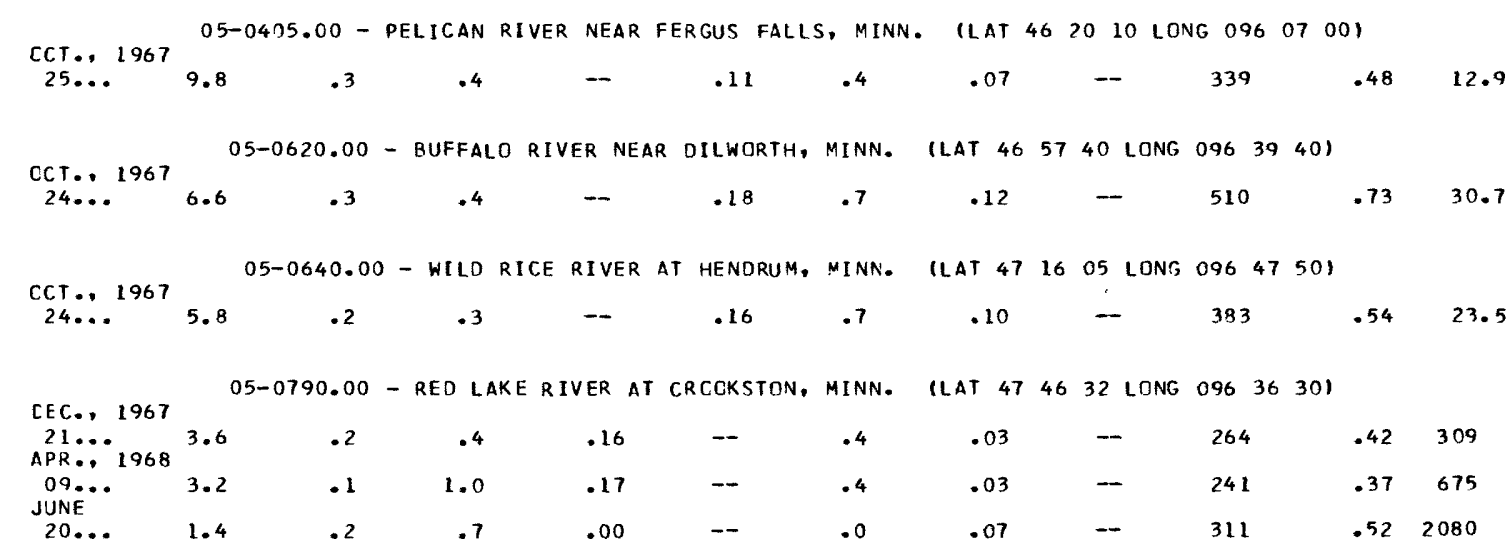

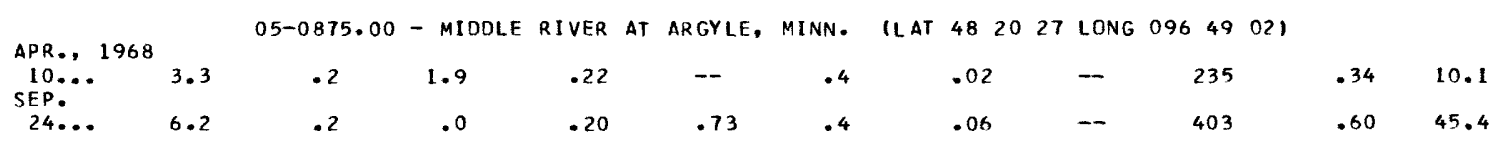
05-0940.00 - SOUTH BRANCH TWO RIVERS AT LAKE BRONSON. MINN.
(LAT 484350 LONG O96 3950 ) $\begin{array}{rrrrrrrrrrrrrrr}18 \ldots . . .1968 & 5.2 & .2 & .4 & - & .43 & .6 & .05 & .- & 292 & .43 & 1.38 \\ 25 . . . & 4.8 & .2 & .2 & .12 & .44 & .4 & .13 & - & 315 & .47 & 197 & \end{array}$ 


\begin{tabular}{|c|c|c|c|c|c|c|c|c|c|c|c|c|}
\hline$E$ & $\begin{array}{l}\text { DIS- } \\
\text { CHARGE } \\
\text { (CFS) }\end{array}$ & $\begin{array}{l}\text { TEMP- } \\
\text { ERATURE } \\
\text { (DEG C) }\end{array}$ & $\begin{array}{l}\text { SILICA } \\
\text { (SIO2) }\end{array}$ & $\begin{array}{l}\text { TOTAL } \\
\text { IRON } \\
\text { (FE) }\end{array}$ & $\begin{array}{l}\text { CAL- } \\
\text { CIUM } \\
\text { (CA) }\end{array}$ & $\begin{array}{l}\text { MAG- } \\
\text { NE- } \\
\text { SIUM } \\
\text { (MG) }\end{array}$ & $\begin{array}{l}\text { SODIUM } \\
\text { (NA) }\end{array}$ & $\begin{array}{l}\text { PO- } \\
\text { TAS- } \\
\text { SIUM } \\
(K)\end{array}$ & $\begin{array}{l}\text { BICAR- } \\
\text { BONATE } \\
\text { (HCO3) }\end{array}$ & $\begin{array}{l}\text { CAR- } \\
\text { BONATE } \\
\text { (CO3) }\end{array}$ & $\begin{array}{l}\text { ALKA- } \\
\text { LINI TY } \\
\text { AS } \\
\text { CACO3 }\end{array}$ & $\begin{array}{l}\text { SULFATE } \\
\left(\mathrm{SO}^{2}\right)\end{array}$ \\
\hline
\end{tabular}

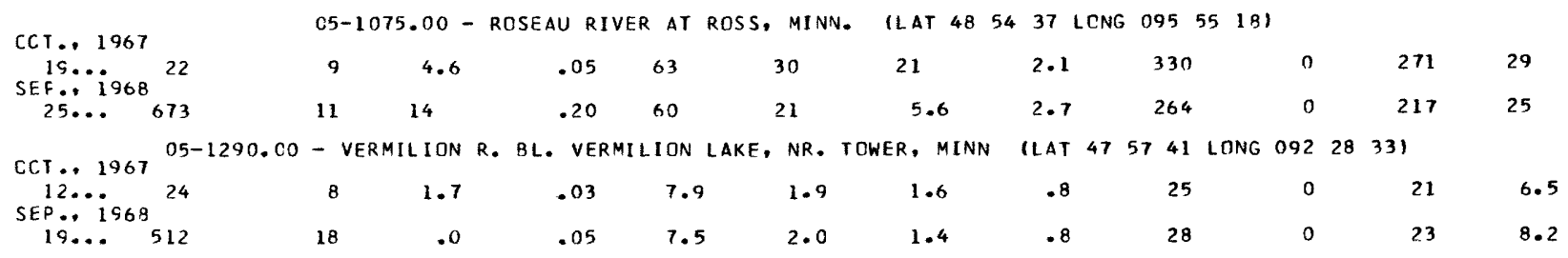

$\begin{aligned} \text { SEF. } & 1968 \\ 12 . & 449\end{aligned}$

05-1320.00 - BIG FORK RIVER AT BIG FALLS, MINN

\section{LONG 09348001}

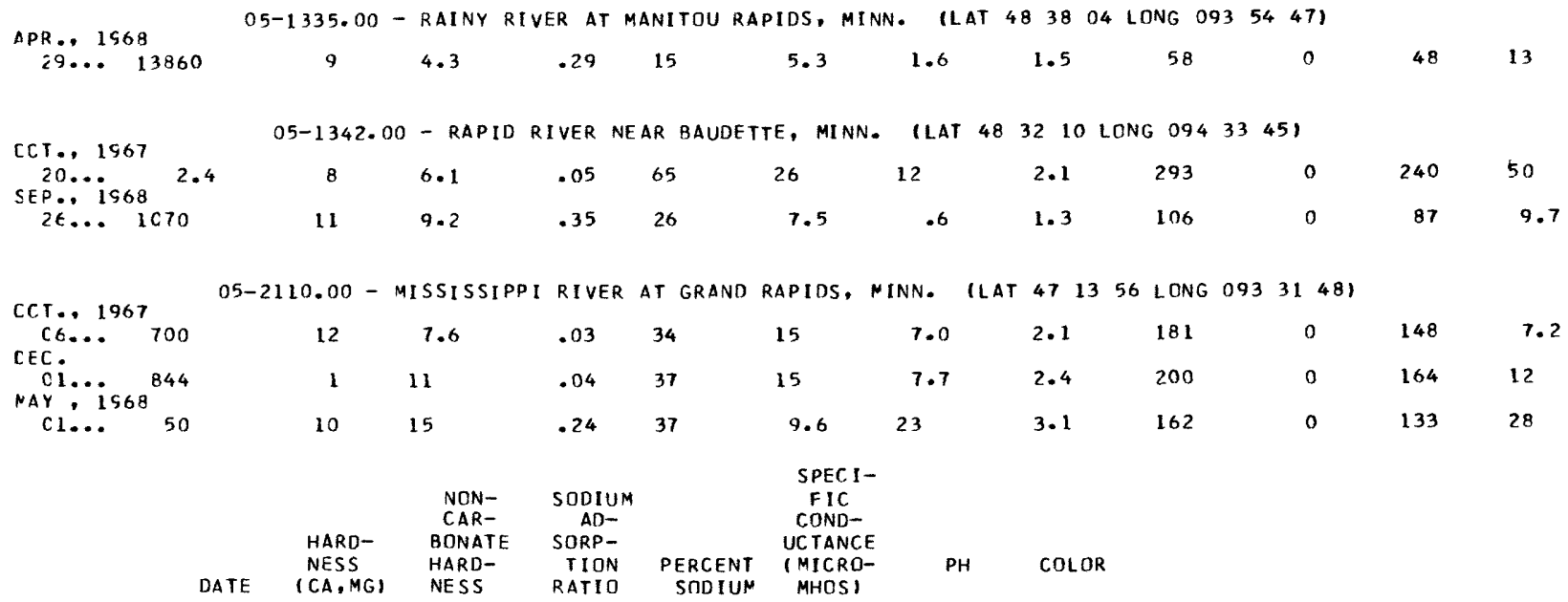

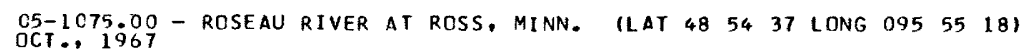

\begin{tabular}{|c|c|c|c|c|c|c|}
\hline $\begin{array}{l}19 \ldots \\
\text { SEP., } 1968\end{array}$ & 280 & 9 & .5 & 14 & 572 & 7.6 \\
\hline $25 \ldots$ & 235 & 19 & .2 & 5 & 423 & 8.1 \\
\hline
\end{tabular}

05-1290.00 - VERMILION R. BL. VERMILION LAKE, NR. TOWER, MINN (LAT 475741 LONG 0922833 )

\begin{tabular}{|c|c|c|c|c|c|c|}
\hline${ }_{\text {SEP... }}^{12 \ldots 968}$ & 28 & 7 & .1 & 11 & 63 & 6.5 \\
\hline $19 \ldots$ & 27 & 4 & .1 & 10 & 65 & 7.2 \\
\hline
\end{tabular}

05-1320.00- BIG FORK RIVER AT BIG FALLS, MINN. (LAT 4812 OO LONG 09348001
SEP. 1968

$\begin{array}{llllllll}12 \ldots & 92 & 8 & -1 & 5 & 170 & 6.5 & 130\end{array}$

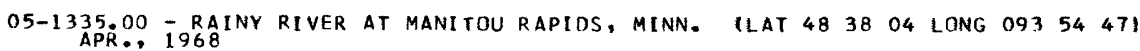
$\begin{array}{llllllll}29 . . & 60 & 12 & .1 & 5 & 119 & 6.9 & 125\end{array}$

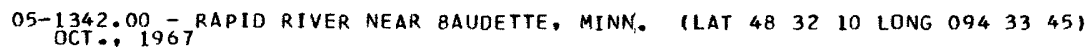

\begin{tabular}{|c|c|c|c|c|c|c|c|}
\hline SEP.:. 1968 & 269 & 29 & .3 & 9 & 516 & 8.2 & 34 \\
\hline $26 \ldots$ & 96 & 8 & .0 & 1 & 168 & 7.6 & 170 \\
\hline
\end{tabular}

05-2110.00 - MISSISSIPPI RIVER AT GRAND RAPIDS, MINN. (LAT 471356 LONG 0933148 )

$\begin{array}{rrrrrrrr}\text { O6... } & 146 & 0 & .3 & 9 & 295 & 7.7 & 7 \\ \text { DEC... } & 155 & 0 & .3 & 10 & 330 & 7.8 & 8 \\ \begin{array}{llll}\text { MAY } 1968 \\ 01 . . .\end{array} & 132 & 0 & .9 & 27 & 362 & 7.0 & 30\end{array}$


CHEMICAL ANALYSES IN MILLIGRAMS PER LITER, WATER YEAR OCTOBER 1967 TO SEPTEMBER 1968

\begin{tabular}{|c|c|c|c|c|c|c|c|c|c|c|c|}
\hline & & & & OR THO & & TOTAL & & & $\begin{array}{l}\text { OIS- } \\
\text { SOLVED } \\
\text { SOLIOS }\end{array}$ & $\begin{array}{l}\text { OIS- } \\
\text { SOLVFO } \\
\text { SOLIOS }\end{array}$ & $\begin{array}{l}\text { OIS- } \\
\text { SOLVFD } \\
\text { SOLIOS }\end{array}$ \\
\hline & $\begin{array}{l}\text { CHLO- } \\
\text { RIDE }\end{array}$ & $\begin{array}{l}\text { FLUD- } \\
\text { RIDE }\end{array}$ & NI TRATE & $\begin{array}{l}\text { PHOS- } \\
\text { PHATF }\end{array}$ & $\begin{array}{l}\text { PHOS- } \\
\text { PHATE }\end{array}$ & $\begin{array}{l}\text { ALUM- } \\
\text { I NIUM }\end{array}$ & BORON & $\begin{array}{l}\text { DI SS- } \\
\text { OLVED }\end{array}$ & $\begin{array}{l}\text { I SUM OF } \\
\text { CONST I- }\end{array}$ & $\begin{array}{l}\text { ITONS } \\
\text { PER }\end{array}$ & $\begin{array}{l}1 \text { TONS } \\
\text { PFR }\end{array}$ \\
\hline CATE & $(C L)$ & (F) & (NO3) & (P04) & (P04) & $(A L)$ & (B) & OXYGEN & TUENTS I & $A C-F T)$ & DAYI \\
\hline
\end{tabular}

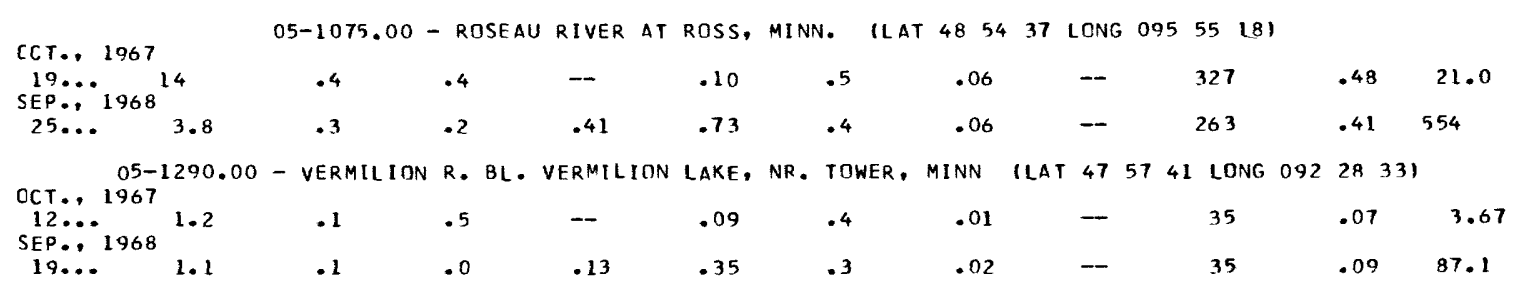
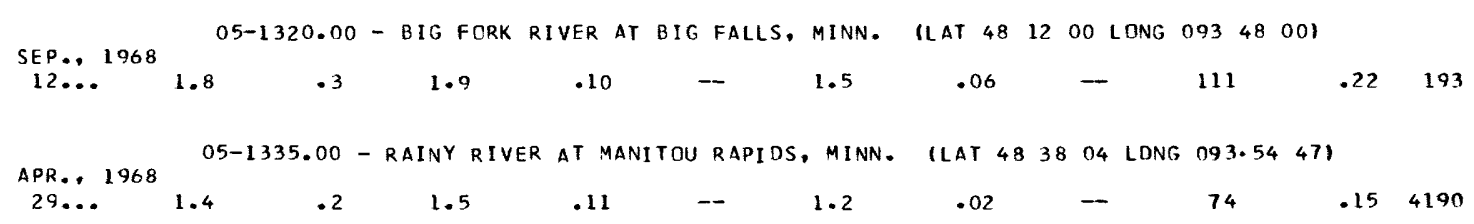

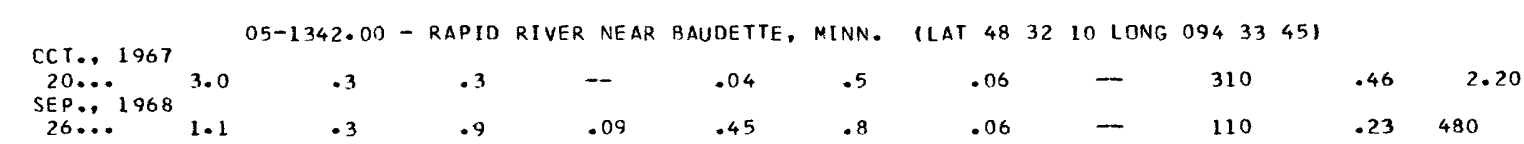

\begin{tabular}{|c|c|c|c|c|c|c|c|c|c|c|c|c|}
\hline OCT., 1967 & $05-2110.00$ & - & MISSISSIPP I & RIVER AT & GRAND & RAPIDS, MINN. & ILAT & 47 & 1356 & LONG $093 \quad 31$ & 481 & \\
\hline $\begin{array}{l}\text { C } 6 \ldots . . \\
\text { DEC. }\end{array}$ & 1.6 & .1 & - 4 & - & .10 & .4 & .03 & & - & 165 & .24 & 335 \\
\hline $\begin{array}{l}01 \ldots \\
\text { MAY }, 1968\end{array}$ & 2.0 & $\cdot 2$ & $\cdot 3$ & .22 & - & $\cdot 3$ & .02 & & - & 187 & .29 & $48 B$ \\
\hline $01 \ldots$ & 15 & $\cdot 2$ & .4 & 1.1 & -- & .0 & .06 & & - & 212 & .30 & 30.2 \\
\hline
\end{tabular}


CHEMICAL ANALYSES IN MILLIGRAMS PER LITER, WATER YEAR OCTOBER 1967 TO SEPTEMBER 1968

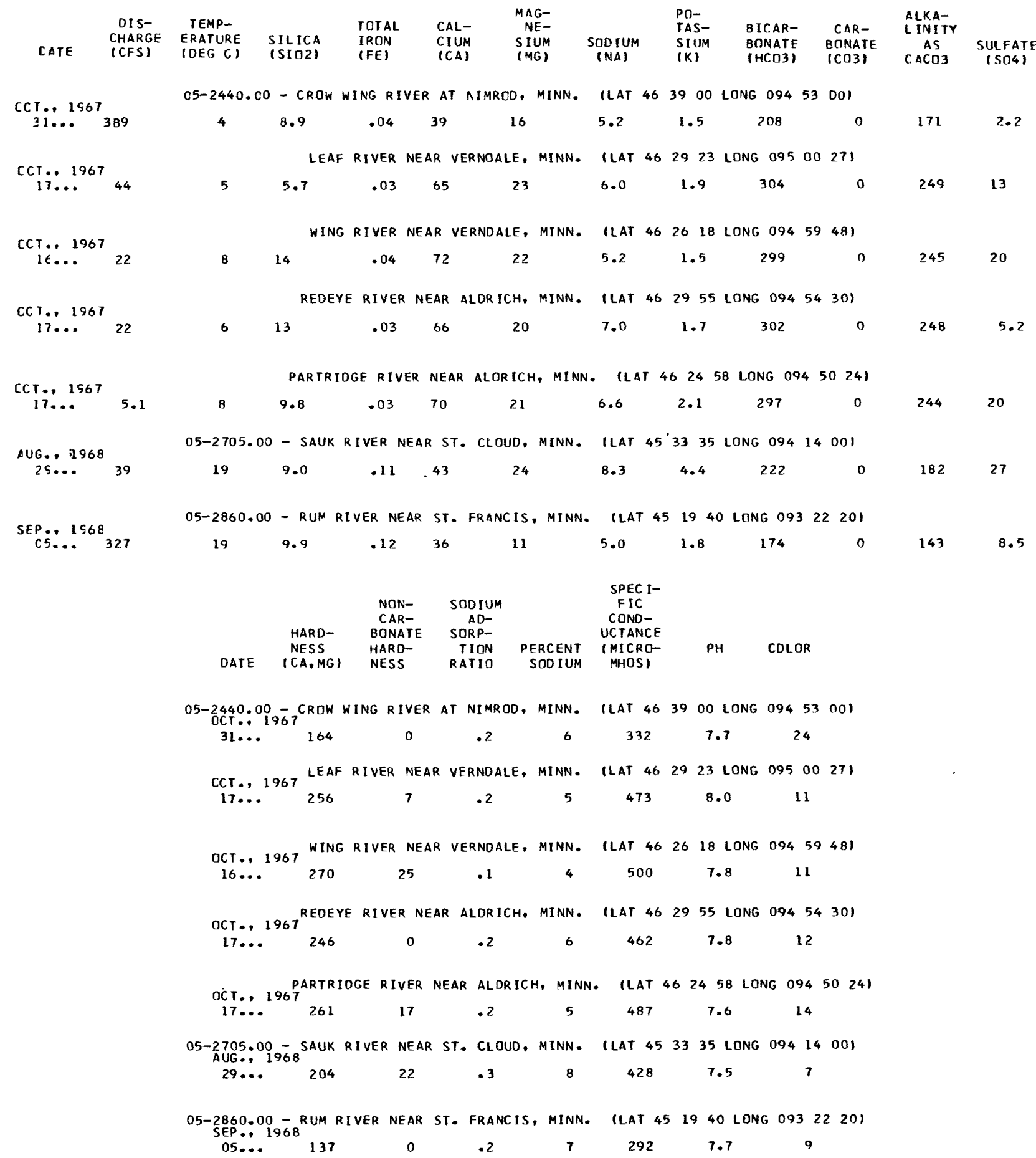


CHEMICAI ANALYSES IN MILIIGRAMS PER LITER, WATER YEAR OCTOBER 1967 TO SEPTEMBER 1968

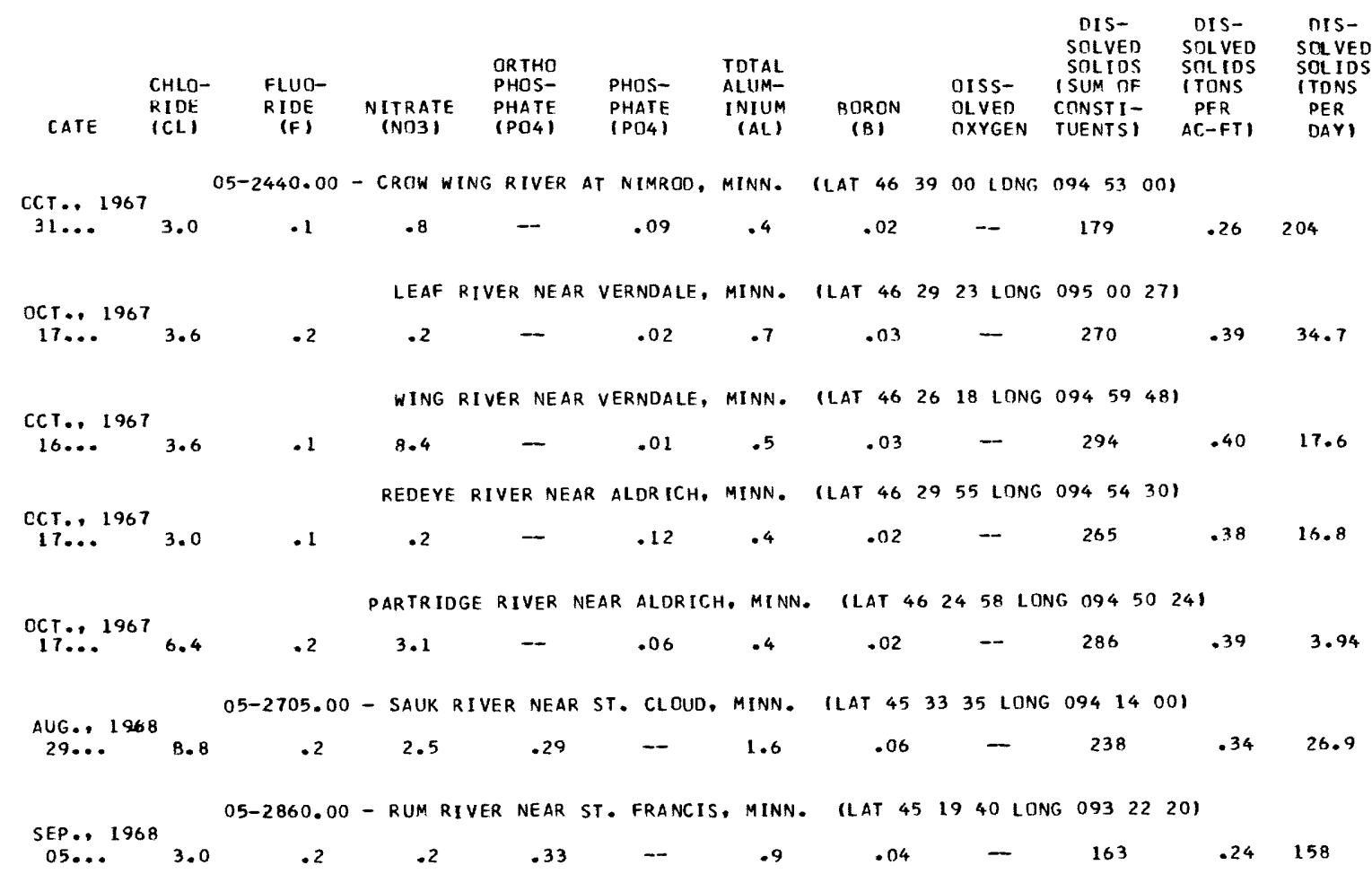




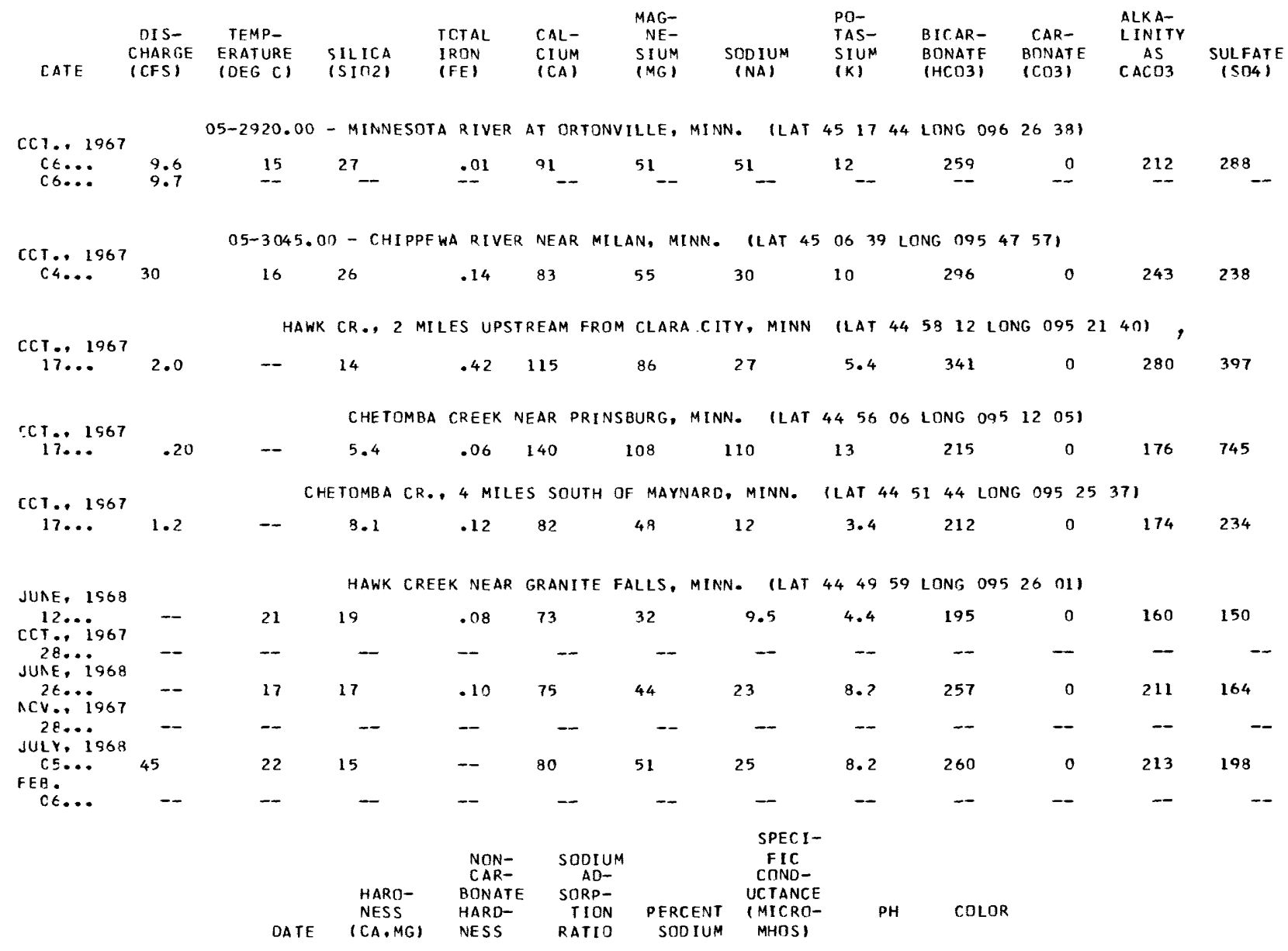

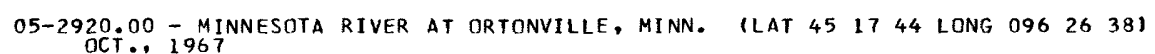

$\begin{array}{lrrrrrrr}06 \ldots & 437 & 225 & 1.1 & 20 & 9 B 2 & 8.2 & 8 \\ 28 . . . & - & - & -1 & -2 & -- & -1\end{array}$

05-3045.00- CHIPPEWA RIVER NEAR MILAN. MINN. ILAT 450639 LONG 09547571

\begin{tabular}{lllllll|l}
$04 \ldots$ & 431 & 188 & .6 & 13 & 877 & 7.7 & 12
\end{tabular}

OCTAWK CR 19672 MILES UPSTREAM FROM CLARA CITY, MINN ILAT 445812 LONG O95 21401

$\begin{array}{llllllll}17 . . . & 640 & 360 & .5 & 8 & 1160 & 7.9 & 9\end{array}$

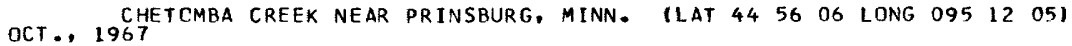

$\begin{array}{llllllll}17 \ldots & 794 & 618 & 1.7 & 23 & 1710 & 7.4 & 11\end{array}$

CHETOMBA CR., 4 MILES SOUTH OF MAYNARD, MINN. (LAT 445144 LONG 0952537 )

$\begin{array}{llllllll}C C T \ldots & 1967 & & & & & & \\ 17 . . . & 402 & 228 & .3 & 6 & 780 & 8.0 & 5\end{array}$

JUNE, 1968 HAWK CREEK NEAR GRANITE FALLS, MINN. ILAT 444959 LONG 09526011

\begin{tabular}{|c|c|c|c|c|c|c|c|}
\hline $12 \ldots$ & 311 & 151 & .2 & 6 & 626 & 7.9 & 23 \\
\hline $12 \ldots$ & - & - & - & -- & 620 & - & \\
\hline $26 \ldots$ & 366 & 155 & .5 & 12 & 774 & .8 & 27 \\
\hline $\begin{array}{l}26 \\
\text { JULY }\end{array}$ & -- & - & - & - & 950 & - & - \\
\hline $05 \ldots$ & 409 & 196 & .5 & 11 & 840 & 7.4 & 0 \\
\hline 05 & -- & -- & -- & - & 830 & - & \\
\hline
\end{tabular}


CHEMICAL ANALYSES IN MILLIGRAMS PER LITER, WATER YEAR OCTOBER 1967 TO SEPTEMBER 1968

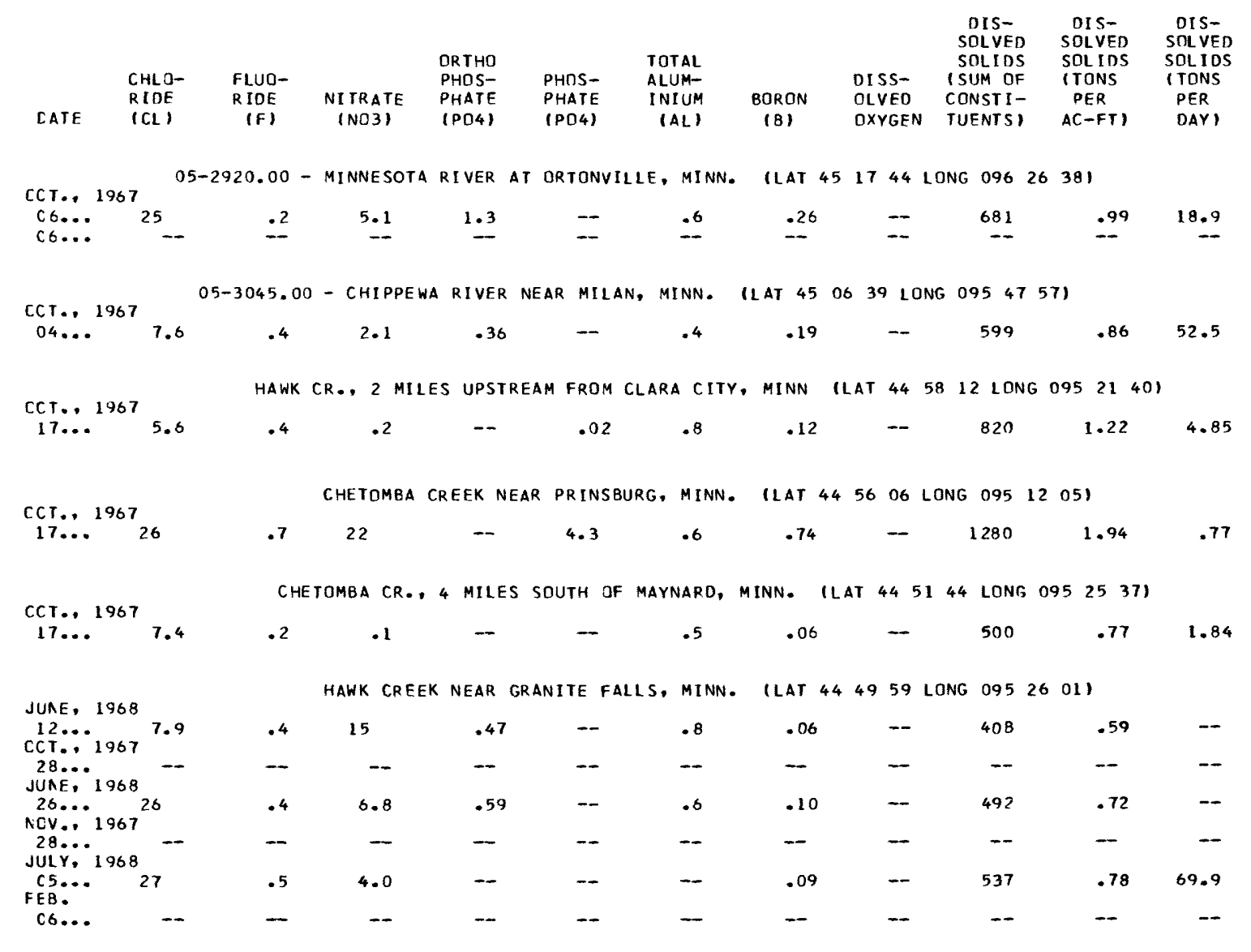




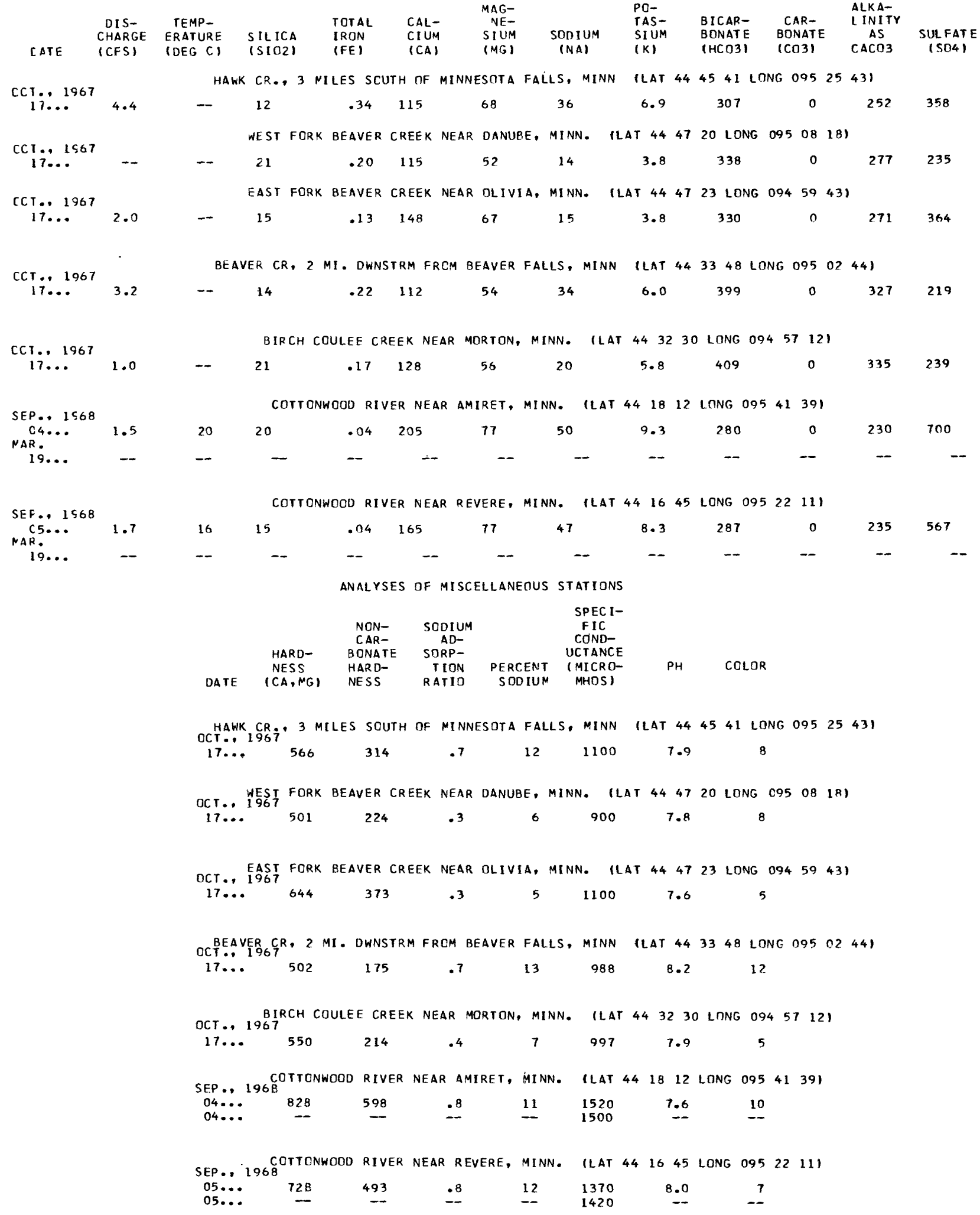


CHEMICAL ANALYSES IN MILLIGRAMS PER LITER, WATER YEAR OCTOBER 1967 TO SEPTEMBER 1968

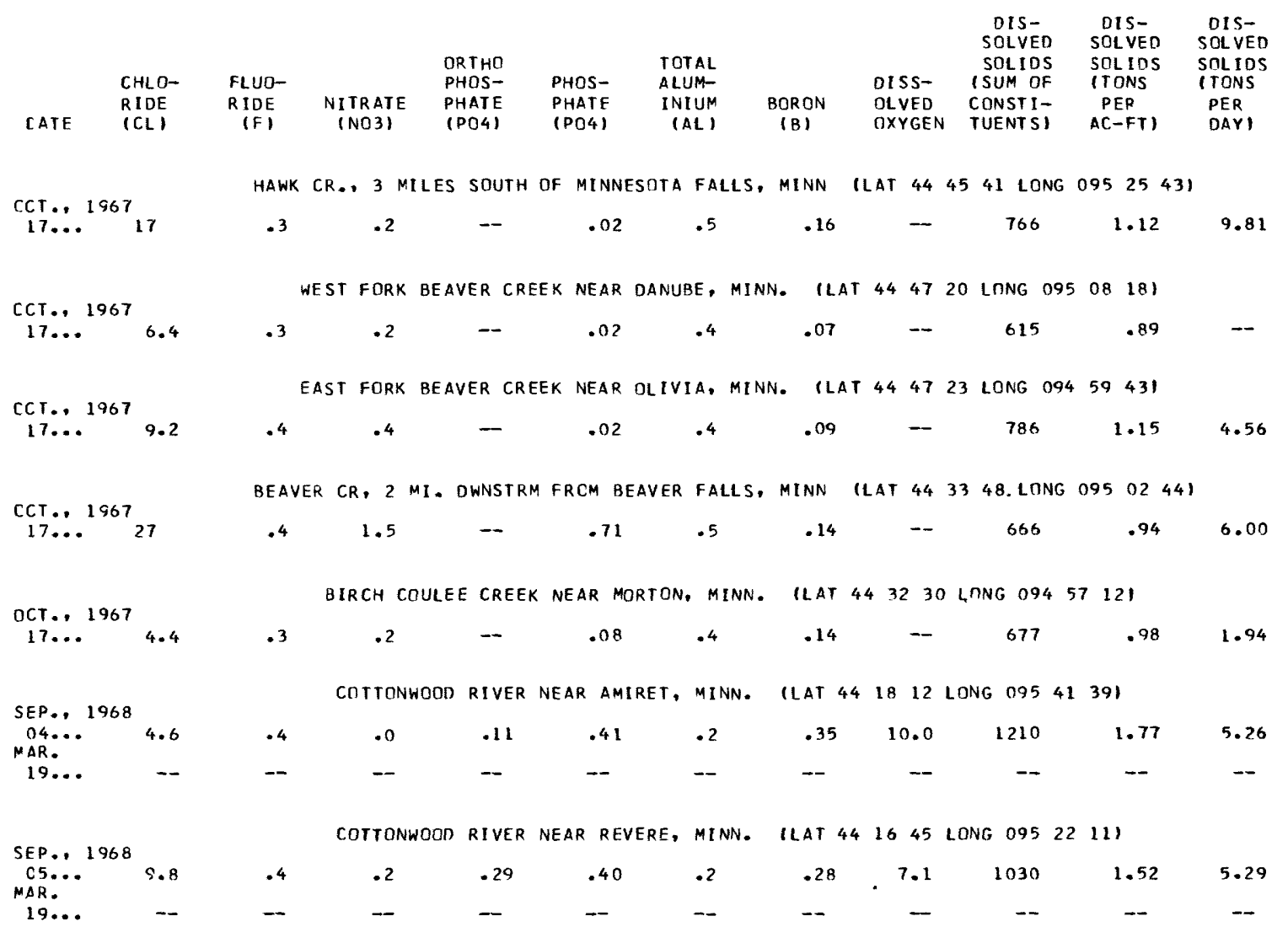




\begin{tabular}{|c|c|c|c|c|c|c|c|c|c|c|c|c|}
\hline САТE & $\begin{array}{l}\text { DIS- } \\
\text { CHARGE } \\
\text { (CFS) }\end{array}$ & $\begin{array}{l}\text { TEMP- } \\
\text { ERATURE } \\
\text { (DEG C) }\end{array}$ & $\begin{array}{l}\text { SIL ICA } \\
\text { (SIO2) }\end{array}$ & $\begin{array}{l}\text { TOT AL } \\
\text { IRON } \\
\text { (FE) }\end{array}$ & $\begin{array}{l}\text { CAL- } \\
\text { CIUM } \\
\text { (CA) }\end{array}$ & $\begin{array}{l}\text { MAG- } \\
\text { NE- } \\
\text { SIUM } \\
\text { (MG) }\end{array}$ & $\begin{array}{l}\text { SODIUM } \\
\text { (NA) }\end{array}$ & $\begin{array}{l}\text { PO- } \\
\text { TAS- } \\
\text { SIUM } \\
(K)\end{array}$ & $\begin{array}{l}\text { B ICAR- } \\
\text { BONATE } \\
\text { (HCD3) }\end{array}$ & $\begin{array}{l}\text { CAR- } \\
\text { BONATE } \\
\text { (CO3) }\end{array}$ & $\begin{array}{l}\text { ALKA- } \\
\text { LINITY } \\
\text { AS } \\
\text { CACD3 }\end{array}$ & $\begin{array}{c}\text { SULFATE } \\
\text { (S04) }\end{array}$ \\
\hline
\end{tabular}

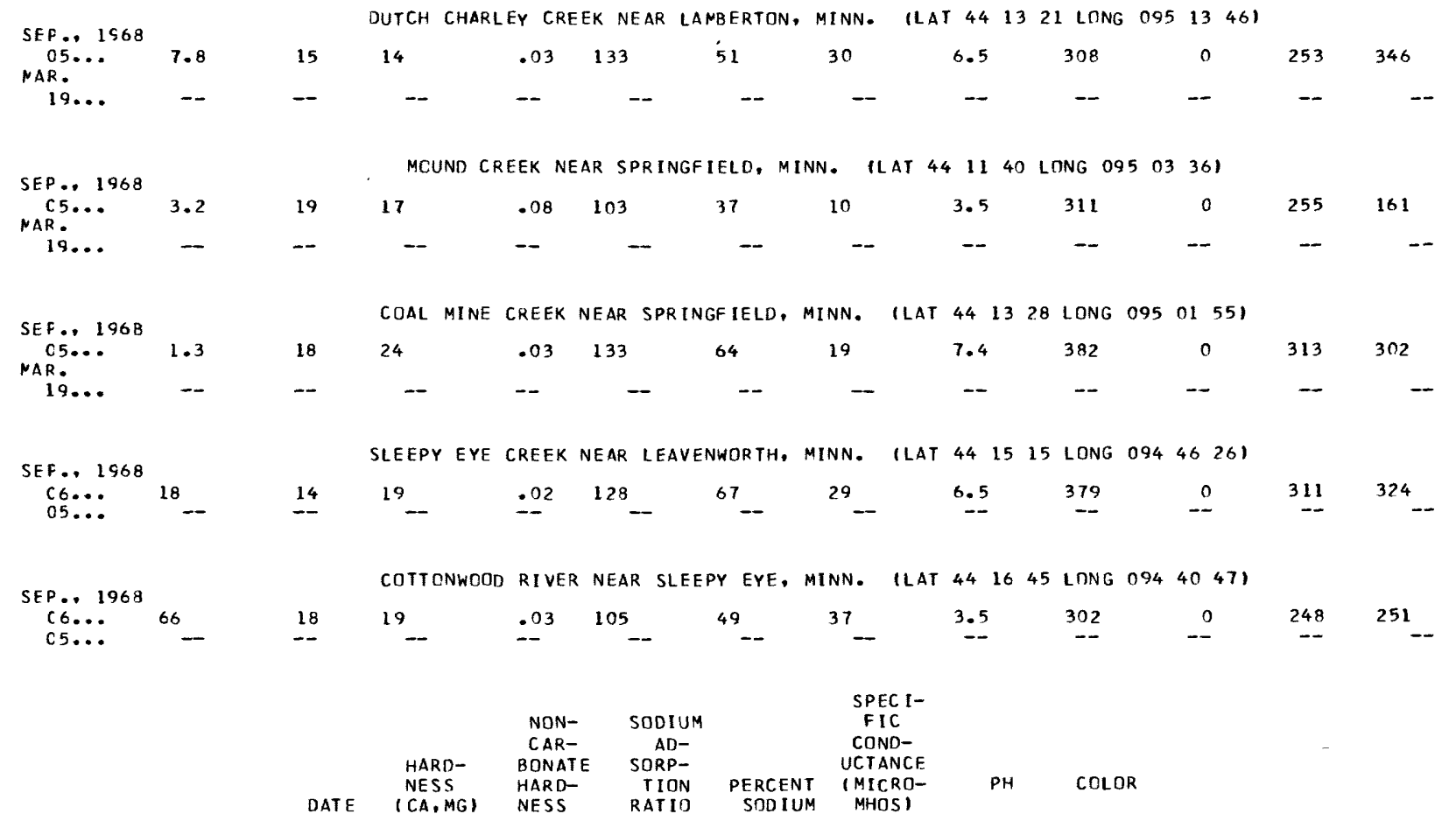

DUTCH CHARLEY CREFK NEAR LAMBERTON, MINN. (LAT 441321 LONG $095 \quad 1346$ ) SEP. DUTCH

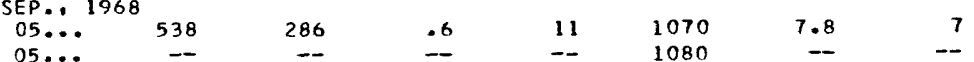

SEP.. 1969 MQUND CREEK NEAR SPRINGFIELD, MINN. (LAT 441140 LONG 0950336 )

$\begin{array}{cccccccc}05 \ldots . & 409 & 154 & .2 & 5 & 758 & 7.9 & 3 \\ 05 \ldots & - & - & - & -- & 740 & -- & -\end{array}$

COAL MINE CREEK NEAR SPRINGFIELD, MINN. (LAT 441328 LONG 0950155 ) SEP., 1968

$\begin{array}{crrrrrrr}05 \ldots . . & 592 & 279 & .3 & 6 & 1080 & 7.9 & 10 \\ 05 \ldots & - & -3 & - & -- & 1080 & -\end{array}$

SLEEPY EYE CREEK NEAR LEAVENWORTH, MINN. (IAT 441515 LONG O94 46 26)

$\begin{array}{llllllll}06 & \ldots & 592 & 281 & .5 & 9 & 1110 & 7.9\end{array}$

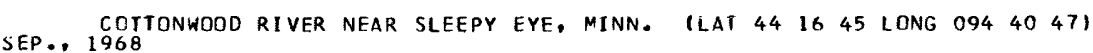

$\begin{array}{rrrrrrrr}06 \ldots & 464 & 216 & .7 & 15 & 969 & \mathrm{~B} .1 & 2 \\ 06 \ldots & -1 & - & -2 & -2 & 980 & - & -2\end{array}$ 
CHEMICAL ANAIYSES IN MILIIGRAMS PER LITER, WATER YEAR OCTOBER 1967 .TO SEPTEMBER 1968

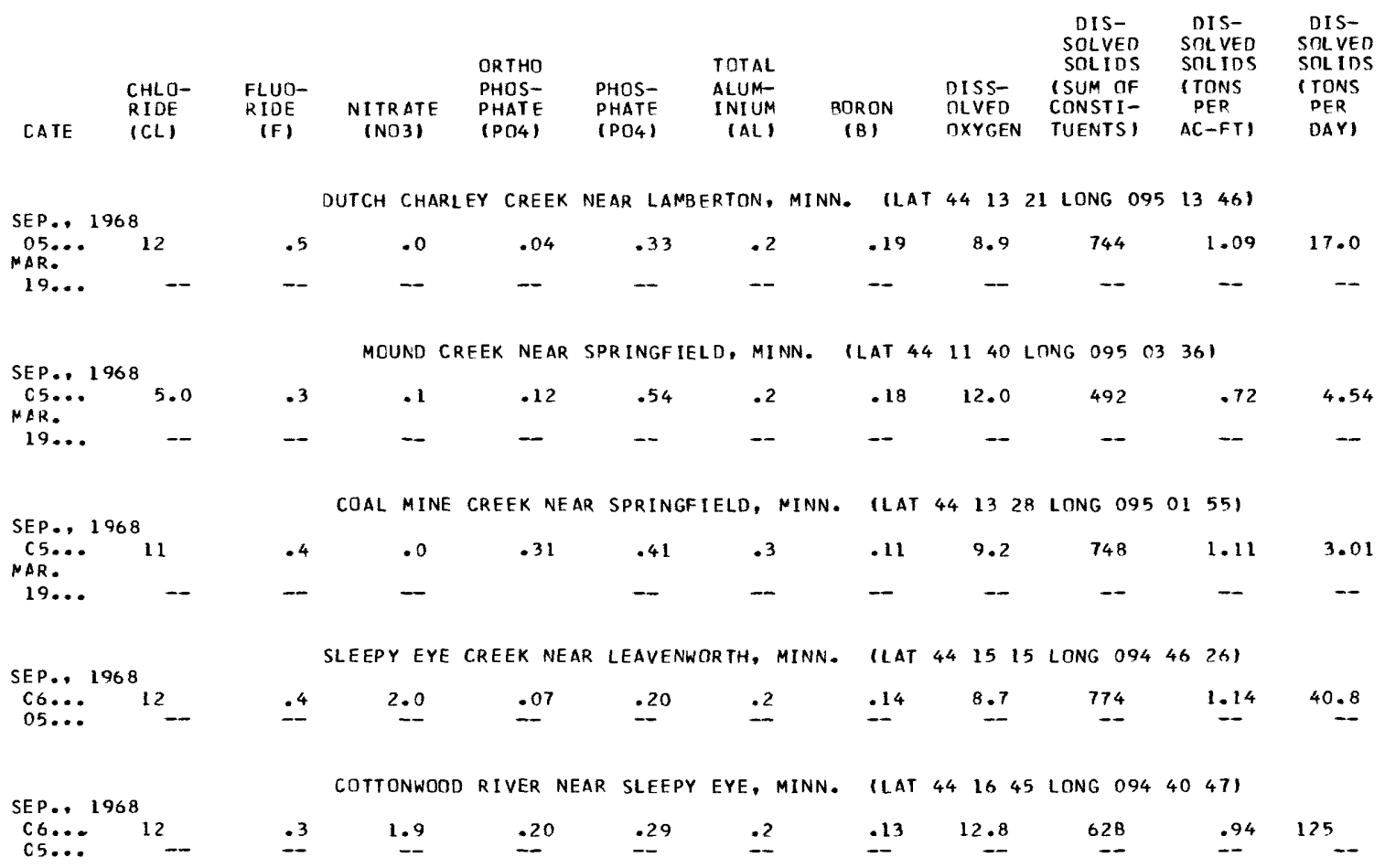




\begin{tabular}{|c|c|c|c|c|c|c|c|c|c|c|c|c|}
\hline CATE & $\begin{array}{l}\text { DIS- } \\
\text { CHARGE } \\
\text { (CFS) }\end{array}$ & $\begin{array}{l}\text { TEMP- } \\
\text { ERATURE } \\
\text { (DEG C) }\end{array}$ & $\begin{array}{l}\text { SILICA } \\
(S I 02)\end{array}$ & $\begin{array}{l}\text { TOTAL } \\
\text { IRON } \\
\text { (FE) }\end{array}$ & $\begin{array}{l}\text { CAL- } \\
\text { CIUM } \\
\text { (CA) }\end{array}$ & $\begin{array}{l}\text { MAG- } \\
\text { NE- } \\
\text { SIUM } \\
\text { (MG) }\end{array}$ & $\begin{array}{l}\text { SODIUM } \\
\text { (NA) }\end{array}$ & $\begin{array}{l}\text { PO- } \\
\text { IAS- } \\
\text { SIUM } \\
\text { (K) }\end{array}$ & $\begin{array}{l}\text { BICAR- } \\
\text { BONATE } \\
\text { (HCD3) }\end{array}$ & $\begin{array}{l}\text { CAR- } \\
\text { BONATE } \\
\text { (CD3) }\end{array}$ & $\begin{array}{l}\text { ALKA- } \\
\text { LINITY } \\
\text { AS } \\
\text { CACD3 }\end{array}$ & $\begin{array}{l}\text { SULFATE } \\
\text { (S04) }\end{array}$ \\
\hline
\end{tabular}

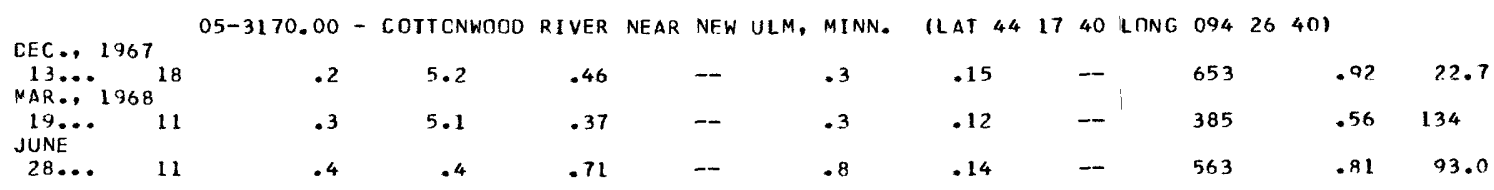

\begin{tabular}{|c|c|c|c|c|c|c|c|c|c|c|c|c|c|c|}
\hline E P., & & LIT TLE & COTTONWOOO & RIVER & NEAR & LEA & DRTH, & MINN. & ILAT & 44 & 10 & 19 LONG & 094 & 47551 \\
\hline $\begin{array}{l}C 6 \ldots \\
05 \ldots\end{array}$ & $\begin{array}{c}8.4 \\
--\end{array}$ & -3 & 3.1 & - OR & & .22 & .2 & .47 & & -- & & $\begin{array}{r}604 \\
--\end{array}$ & & $\begin{array}{l}.88 \\
--\end{array}$ \\
\hline
\end{tabular}

LITTLE COTTONWOOD RIVER NEAR CAMBRIA, MINN. (LAT 441413 LONG 0942139 )

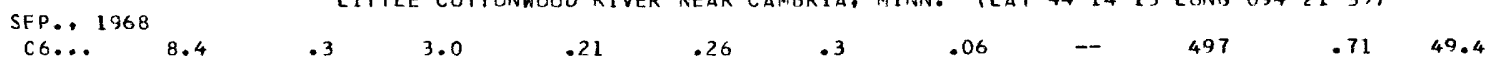

MINNEUPA CREEK NEAR MANKATO, MINN. (LAT 440912 LONG 0940458 )

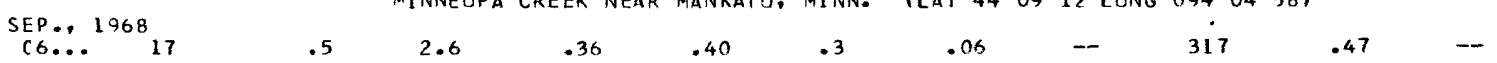

05-3205.00 - LE SUEUR RIVER NEAR RAPIDAN. MINN. (LAT 440640 LONG $094022 B)$

CCT.. 1967

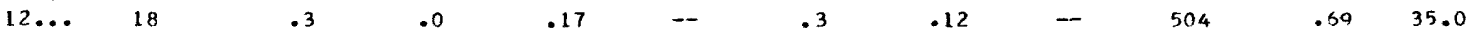

05-3262.00 - JUDICIAL DITCH 1-A NEAR NEW SWEDEN, MINN. (LAT 452500 LONG D94 15 OOI

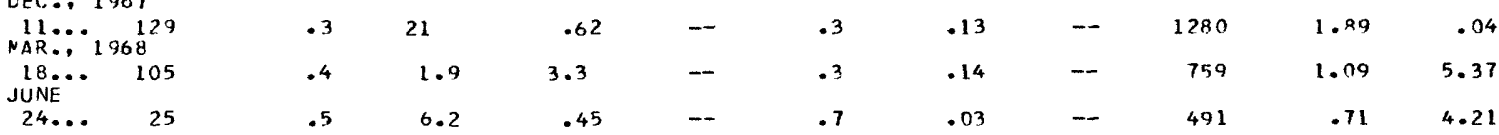

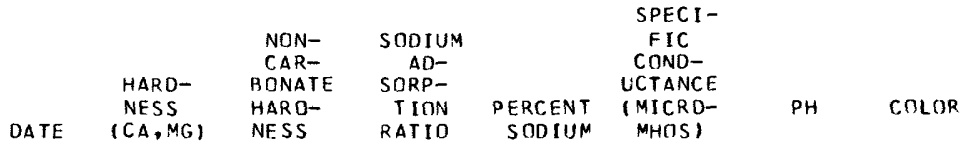

05-3170.00-COTTCNhOOD RIVER NEAR NEN ULM, MINN. (LAT 441740 LNNG 0942640 )

\begin{tabular}{|c|c|c|c|c|c|c|c|}
\hline $\begin{array}{l}13 \ldots \\
\text { MAR.. } 1968\end{array}$ & 483 & 152 & .8 & 16 & 997 & 7.8 & 4 \\
\hline JUNE & 265 & 102 & .6 & 16 & 612 & 7.5 & 8 \\
\hline $28 \ldots$ & 408 & 227 & .5 & 11 & 835 & 7.9 & 12 \\
\hline
\end{tabular}

LITTLE COTTONWOCO RIVER NEAR LEAVENWORTH, MINN. ILAT 441019 LANG 0944755 I

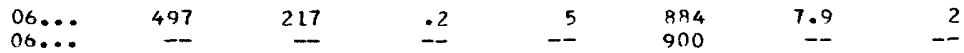

LITTLE COTTONWOOD RIVER NEAR CAMBRIA. MINN. (LAT 441413 LCNG 0942139

$\begin{array}{llllllll}06 \ldots & 414 & 143 & .2 & 5 & 754 & 7.6\end{array}$

SEP., 1968 INNEOPA CREEK NEAR MANKATO. MINN. ILAT 440912 LONG 9940458 )

$\begin{array}{llllllll}06 \ldots & 258 & 63 & 3 & 8 & 538 & 7.5 & 10\end{array}$

05-32 C5. 00 $19 \overline{6}$ LE SUEUR RIVER NFAR RAPIDAN, MINN. (LAT 440640 LONG 09402281

$\begin{array}{llllllll}12 \ldots & 375 & 30 & .9 & 18 & 796 & 8.0 & 3\end{array}$

05-3262.00 - JUDICIAL DITCH 1-A NEAR NEW SWFOEN. MINN. (LAT 4525 OO LONG N94 15 NO)

$\begin{array}{rrrrrrrr}11 \ldots & 861 & 430 & 1.7 & 22 & 1850 & 7.9 & 17 \\ \begin{array}{l}\text { MAR... } 1968 \\ 18 \ldots\end{array} & 413 & 180 & 2.0 & 32 & 1210 & 7.9 & 27 \\ \begin{array}{l}\text { JUNE } \\ 24 \ldots\end{array} & 401 & 152 & .4 & 9 & 781 & 8.2 & 21\end{array}$


CHEMICAL ANALYSES IN MILLIGRAMS PER IITER, WATER YEAR OCTOBER 1967 TO SEPTEMBER 1968

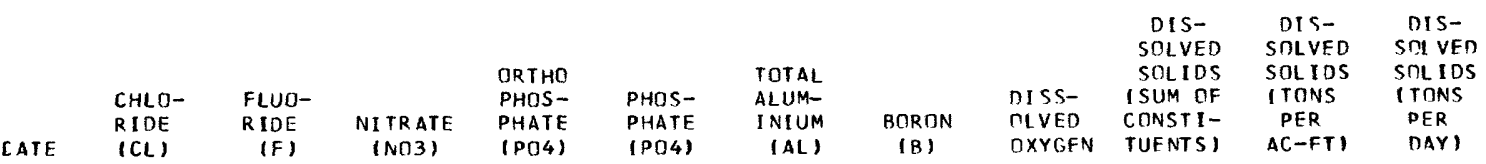

CEC.. 1967

\begin{tabular}{|c|c|c|c|c|c|c|c|c|c|c|c|c|}
\hline NAR 12. & $i_{1968} 12$ & 1 & 13 & .04 & 111 & 50 & 42 & 5.4 & 403 & 0 & 331 & 209 \\
\hline $\begin{array}{l}19 . . \\
\text { JUNE }\end{array}$ & $=120$ & 1 & 10 & .04 & 65 & 25 & 23 & 4.6 & 199 & 0 & 163 & 143 \\
\hline $28 \ldots$ & 57 & 21 & $17^{\circ}$ & .09 & 94 & 42 & 23 & 6.2 & 221 & 0 & 181 & 258 \\
\hline
\end{tabular}

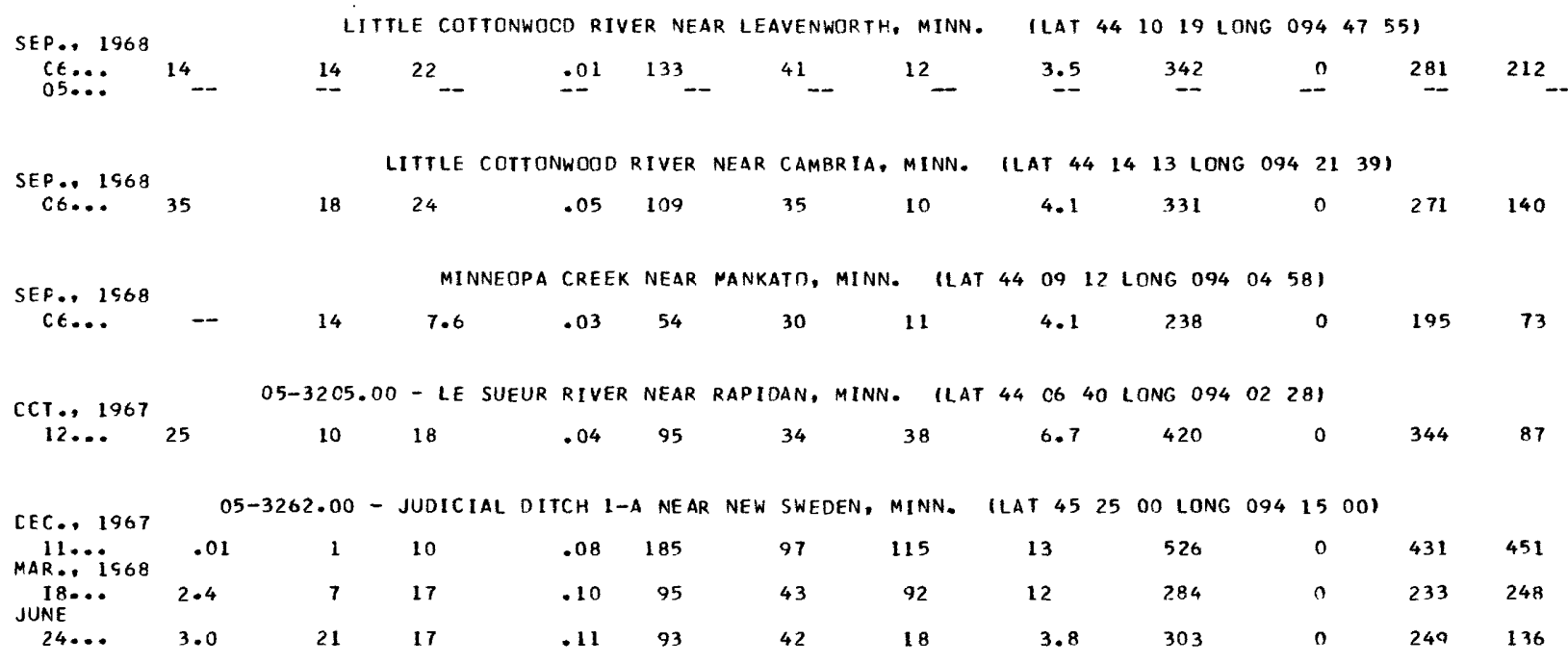




\begin{tabular}{|c|c|c|c|c|c|c|c|c|c|c|c|c|}
\hline & $\begin{array}{l}\text { DIS- } \\
\text { CHARGE } \\
\text { (CFS) }\end{array}$ & $\begin{array}{l}\text { TEMP- } \\
\text { ERATURE } \\
\text { (DEG C ) }\end{array}$ & $\begin{array}{l}\text { SILICA } \\
\text { (SIO2) }\end{array}$ & $\begin{array}{l}\text { TOT AL } \\
\text { IRON } \\
\text { IFEI }\end{array}$ & $\begin{array}{l}\text { CAL- } \\
\text { CIUM } \\
\text { (CA) }\end{array}$ & $\begin{array}{l}\text { MAG- } \\
\text { NE- } \\
\text { SIUM } \\
\text { (MG) }\end{array}$ & $\begin{array}{l}\text { SODIUM } \\
\text { (NA) }\end{array}$ & $\begin{array}{l}\text { PO- } \\
\text { TAS- } \\
\text { SIUM } \\
\text { (K) }\end{array}$ & $\begin{array}{l}\text { BICAR- } \\
\text { BONATE } \\
\text { (HCD3) }\end{array}$ & $\begin{array}{l}\text { CAR- } \\
\text { BONATE } \\
\text { (CO3) }\end{array}$ & $\begin{array}{l}\text { ALKA- } \\
\text { LINITY } \\
\text { AS } \\
\text { CACO3 }\end{array}$ & $\begin{array}{l}\text { SULFATE } \\
\text { (SO4) }\end{array}$ \\
\hline
\end{tabular}

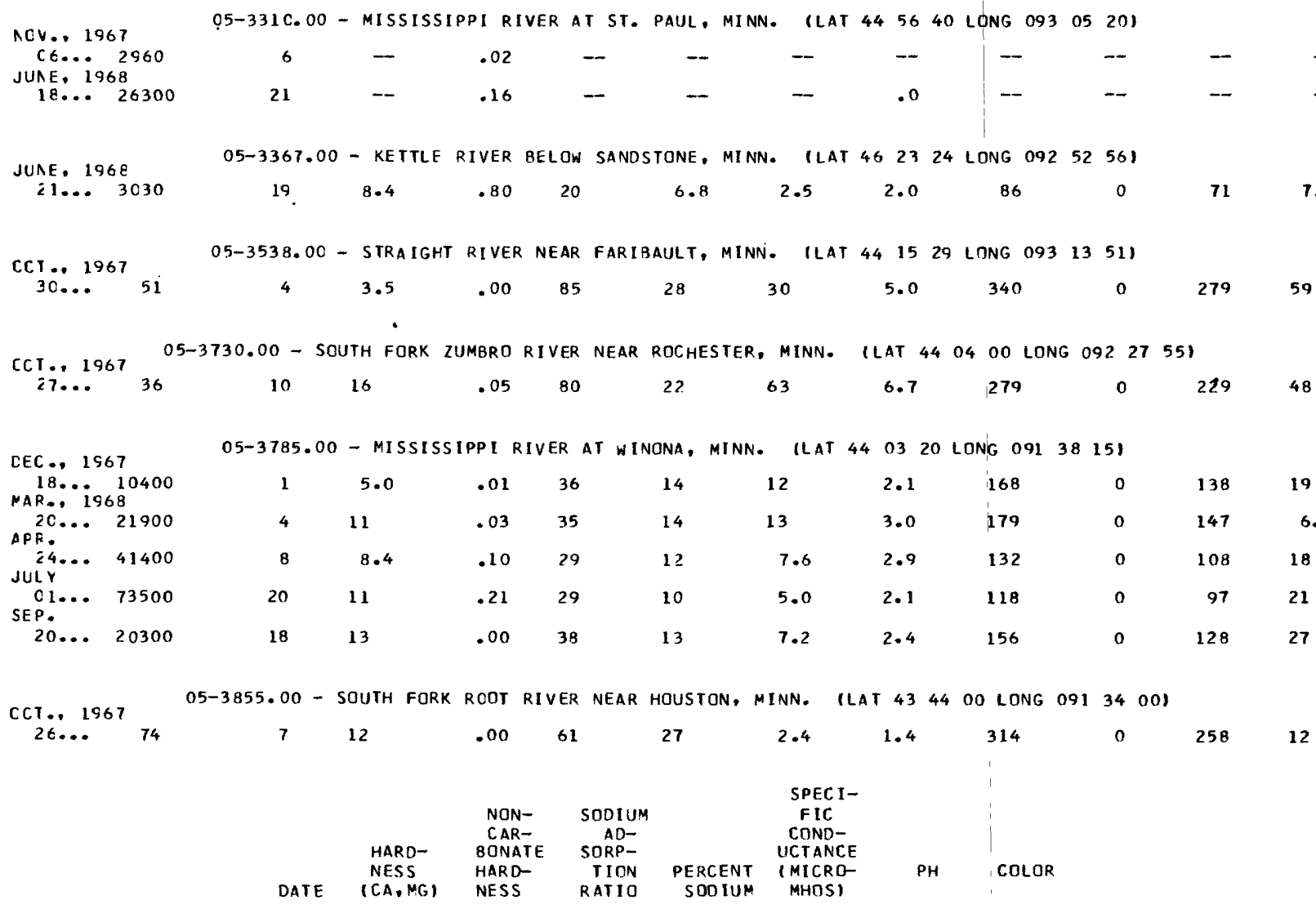

05-3310.00 - MISSISSIPPI RIVER AT ST. PAUL. MINN. (LAT 445640 LONG 0930520 )

11 ...

MAR 1968

$18 . .$.

05-3367.00 - KETtLe RIVER BelOW SANDSTONE. MINN. (LAT 462324 LONG 0925256 ) JUNE, 1968
$21 \ldots$
7
.1
6
152
\begin{tabular}{l|l}
7.9 & 100
\end{tabular}

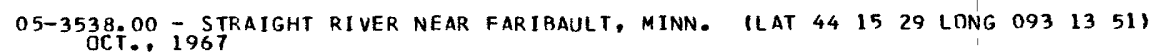

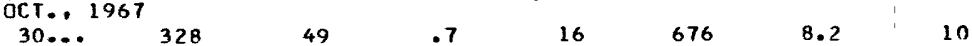

05-3730.00 OCT. SOUTH FORK ZUMBRO RIVER NEAR ROCHESTER. MINN. (LAT 440400 LONG 0922755 ) \begin{tabular}{lllllll|l}
$27 . .$. & 288 & 59 & 1.6 & 31 & 833 & 8.2 & 12
\end{tabular}

05-3785.00- MISSISSIPPI RIVER AT WINONA, MINN. ILAT 440320 LONG O91 38151

\begin{tabular}{|c|c|c|c|c|c|c|c|}
\hline MAR.: 1968 & 148 & 10 & .4 & 15 & 334 & 7.5 & 13 \\
\hline $\begin{array}{l}20 \ldots \\
\text { APR. }\end{array}$ & 143 & 0 & .5 & 16 & 348 & 7.8 & 18 \\
\hline $24 \ldots$ & 120 & 12 & .3 & 12 & 276 & 7.6 & 12 \\
\hline $01 \ldots$ & 114 & 17 & .2 & 9 & 248 & 7.9 & 55 \\
\hline $\begin{array}{c}\text { SEP. } \\
20 . .\end{array}$ & 148 & 19 & .3 & 9 & 324 & 7.3 & 10 \\
\hline
\end{tabular}


CHEMICAL ANALYSES IN MILLIGRAMS PER LITER, WATER YEAR OCTOBER 1967 TO SEPTEMBER 1968

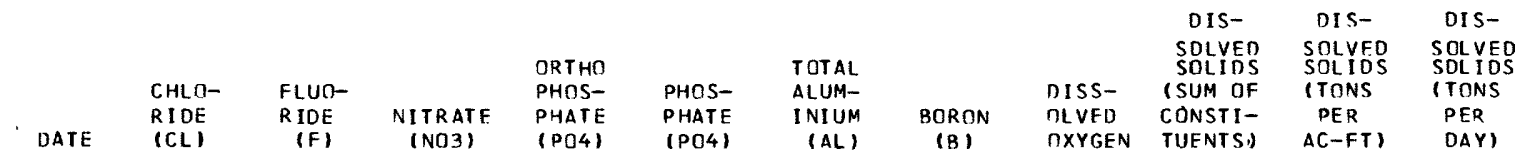

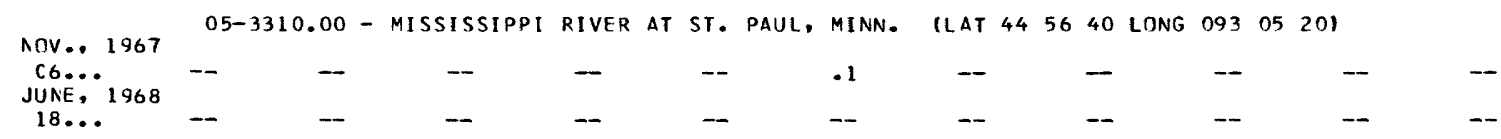

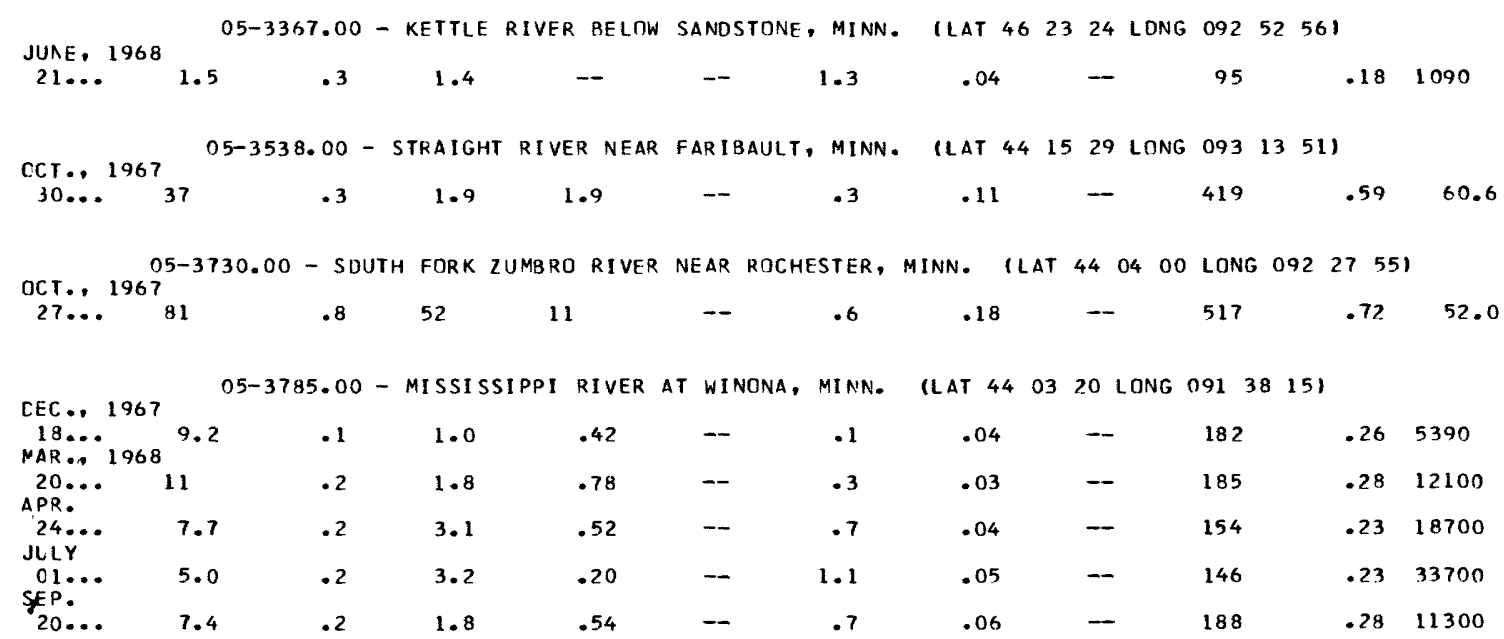

05-3855.00 - SOUTH FORK ROOT RIVER NEAR HOUSTON, MINN. ILAT 4344 OO LONG O91 34 OOI

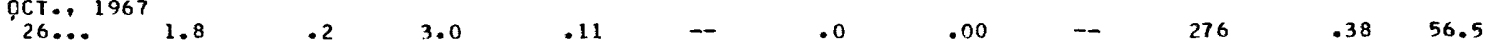




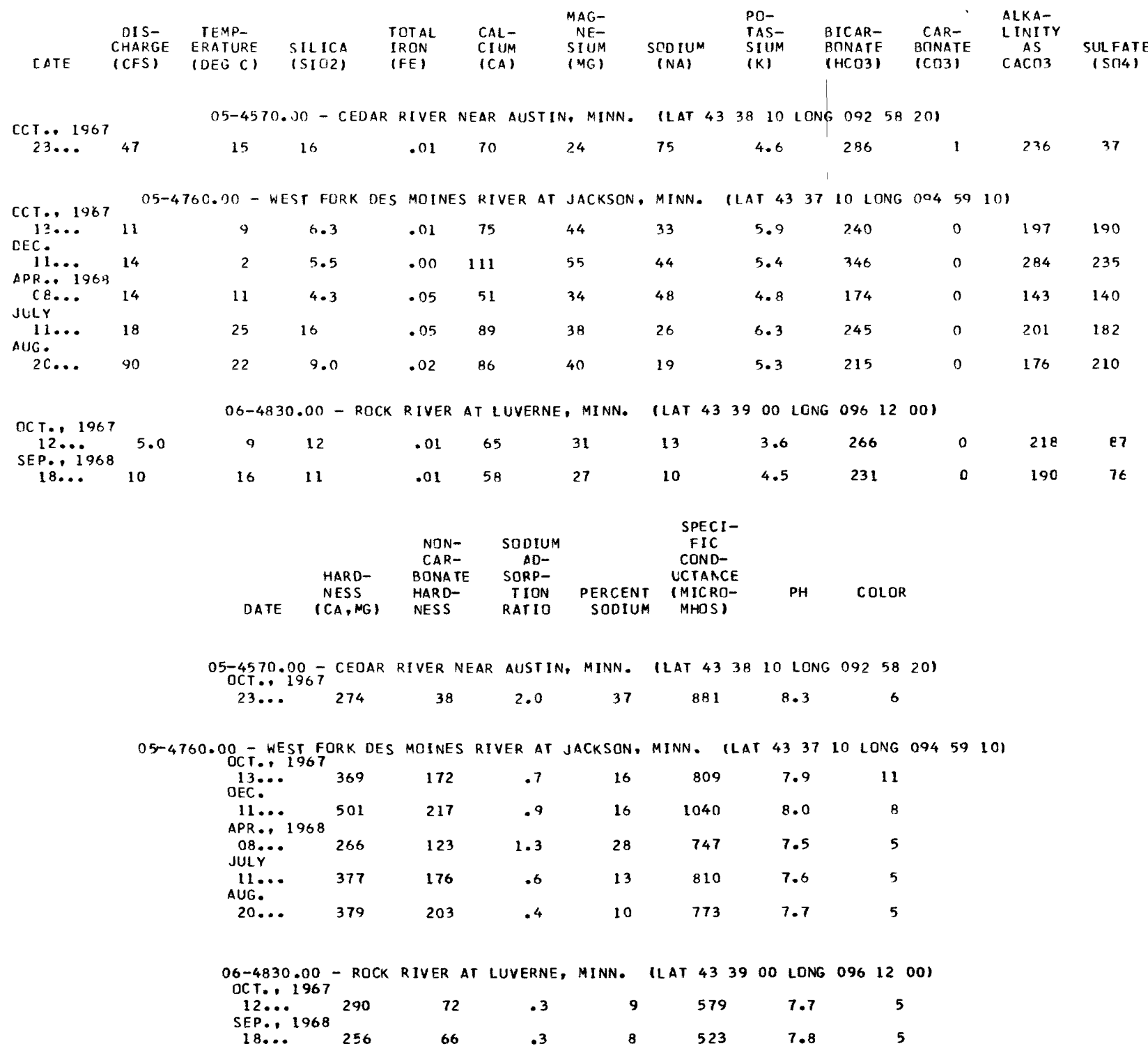


CHEMICAL ANALYSES IN MILLIGRAMS PER LITER, WATER YEAR OCTOBER 1967 TO SEPTEMBER 1968

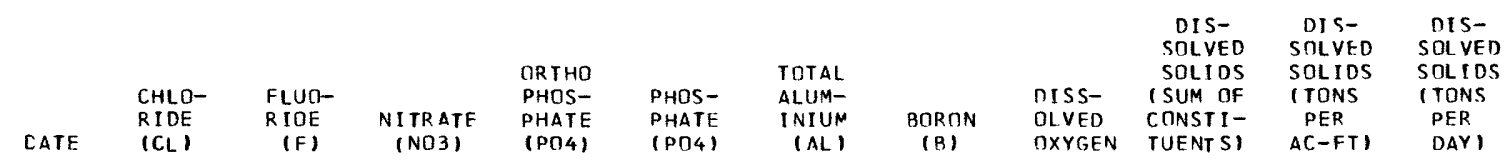

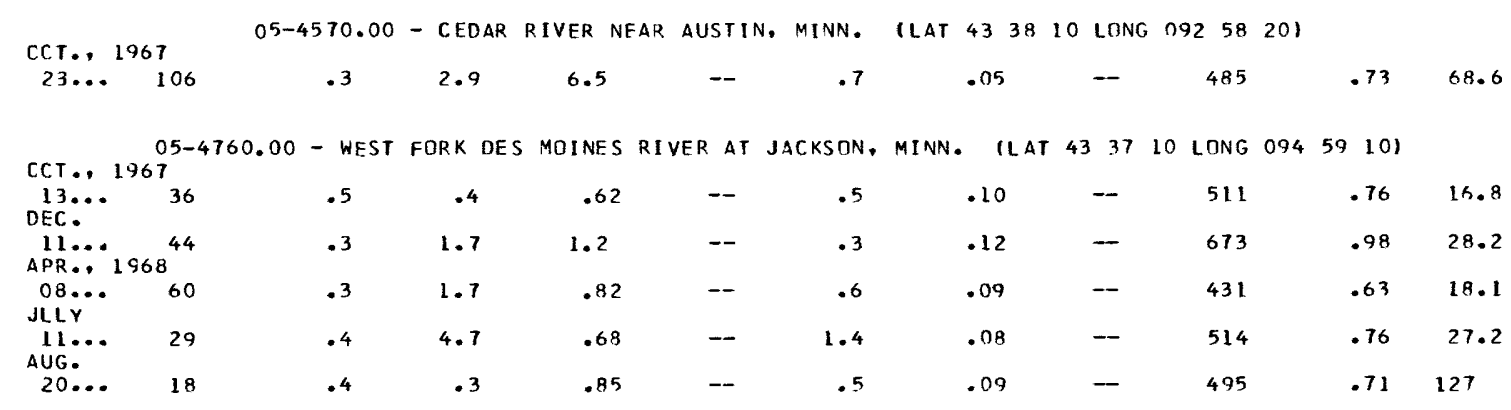

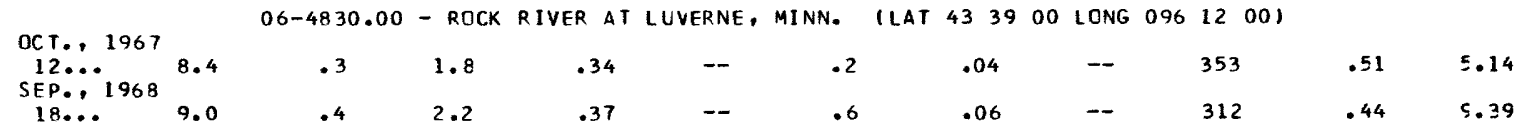


4-0145. BAPTISM RIVER NEAR BEAVER BAY, MINN.

PERIODIC DAILY SUSPENDED SEDIMENT, WATER YEAR OCTOBER 1967 TO SEPTEMBER-1968

\begin{tabular}{|c|c|c|c|c|c|c|c|c|c|c|}
\hline \multirow[b]{2}{*}{ Dec. } & \multicolumn{2}{|l|}{ Date } & $\begin{array}{c}\text { Mean } \\
\text { Discharge } \\
\text { (cfs) }\end{array}$ & $\begin{array}{l}\text { Mean } \\
\text { Concen- } \\
\text { tration } \\
(\mathrm{mg} / \mathrm{l})\end{array}$ & $\begin{array}{l}\text { Load } \\
\text { (tons) }\end{array}$ & & Date & $\begin{array}{c}\text { Mean } \\
\text { Discharge } \\
\text { (cfs) }\end{array}$ & $\begin{array}{l}\text { Mean } \\
\text { Concen- } \\
\text { tration } \\
(m g / 1)\end{array}$ & $\begin{array}{l}\text { Load } \\
\text { (tons) }\end{array}$ \\
\hline & 6 , & 1967 & 28 & 2 & .15 & $\begin{array}{l}\text { June } \\
\text { June }\end{array}$ & $\frac{5}{6} \cdot 1968$ & $\begin{array}{l}312 \\
630\end{array}$ & $\begin{array}{l}2 \\
7\end{array}$ & $12^{1.7}$ \\
\hline Jan. & 9, & 1968 & 10 & 3 & .08 & $\begin{array}{l}\text { June } \\
\text { June }\end{array}$ & 17 & $\begin{array}{l}658 \\
582\end{array}$ & $\begin{array}{l}7 \\
3\end{array}$ & $\begin{array}{l}12 \\
4.7\end{array}$ \\
\hline Feb. & 6 & & 13 & 2 & .07 & $\begin{array}{l}\text { June } \\
\text { June }\end{array}$ & $\begin{array}{l}15 \\
16\end{array}$ & $\begin{array}{l}516 \\
430\end{array}$ & $\begin{array}{l}3 \\
4\end{array}$ & $\begin{array}{l}4.2 \\
4.6\end{array}$ \\
\hline Mar. & 5 & & 7.4 & 3 & .06 & June & 17 & 367 & 4 & 4.0 \\
\hline Mar. & 30 & & 554 & 18 & 27 & June & 18 & 305 & 3 & 2.5 \\
\hline Mar. & 31 & & 478 & 10 & 13 & $\begin{array}{l}\text { June } \\
\text { June }\end{array}$ & $\begin{array}{l}19 \\
20\end{array}$ & $\begin{array}{l}265 \\
212\end{array}$ & $\begin{array}{l}2 \\
1\end{array}$ & $\begin{array}{r}1.4 \\
.57\end{array}$ \\
\hline Apr. & I & & 326 & 6 & 5.3 & June & $2 I$ & 221 & 3 & 1.8 \\
\hline Apr. & 2 & & 270 & 5 & 3.6 & Tune & 22 & 248 & 4 & 2.7 \\
\hline Apr. & 3 & & 192 & 3 & 1.6 & June & 23 & 421 & 5 & 5.7 \\
\hline Apr. & 4 & & 158 & 4 & 1.7 & June & 24 & 376 & 4 & 4.1 \\
\hline Apr. & 5 & & 155 & 13 & 5.4 & June & 25 & 284 & 3 & $2 \cdot 3$ \\
\hline Apr. & 6 & & 161 & 24 & 10 & June & 26 & 216 & 3 & 1.7 \\
\hline Apr. & $\begin{array}{l}7 \\
8\end{array}$ & & 188 & 18 & 9.1 & June & 27 & 180 & 2 & .97 \\
\hline Apr. & 8 & & 412 & 36 & 44 & June & 28 & 161 & 2 & .87 \\
\hline Apr. & $\begin{array}{r}9 \\
10\end{array}$ & & $\begin{array}{l}440 \\
412\end{array}$ & & 58 & June & 29 & 152 & 2 & $\begin{array}{r}.82 \\
78\end{array}$ \\
\hline $\begin{array}{l}\text { Apr. } \\
\text { Apr. }\end{array}$ & $\begin{array}{l}10 \\
11\end{array}$ & & $\begin{array}{l}412 \\
412\end{array}$ & $\begin{array}{l}40 \\
42\end{array}$ & $\begin{array}{l}51 \\
47\end{array}$ & June & 30 & 144 & 2 & .78 \\
\hline Apr. & 12 & & 487 & 40 & 53 & July & 1 & 155 & 2 & .84 \\
\hline Apr. & 13 & & 478 & 38 & 49 & July & 2 & 164 & 2 & .89 \\
\hline Apr. & 14 & & 554 & 34 & 51 & July & 3 & 146 & 2 & .79 \\
\hline Apr. & 15 & & 421 & 18 & 20 & July & 4 & 167 & 2 & .90 \\
\hline Apr. & 16 & & 449 & 10 & 12 & July & 11 & 67 & 5 & .90 \\
\hline Apr. & 22 & & 896 & 20 & 48 & July & 15 & 421 & 10 & 11 \\
\hline Apr. & 24 & & 1060 & 14 & 40 & July & 16 & 312 & 5 & 4.2 \\
\hline Apr. & 25 & & 734 & 8 & 16 & July & 17 & 277 & 4 & 3.0 \\
\hline Apr. & 26 & & 610 & 3 & 4.9 & July & 18 & 221 & 4 & 2.4 \\
\hline $\begin{array}{l}\text { Apr. } \\
\text { Apr. }\end{array}$ & $\begin{array}{l}27 \\
28\end{array}$ & & $\begin{array}{l}630 \\
610\end{array}$ & $\begin{array}{l}1 \\
1\end{array}$ & $\begin{array}{l}1.7 \\
1.6\end{array}$ & July & 19 & 208 & 7 & 3.9 \\
\hline Apr. & 29 & & 620 & $\bar{I}$ & 1.7 & Aug. & 13 & 14 & 6 & .23 \\
\hline Apr. & 30 & & 601 & 1 & 1.6 & Aug. & 22 & 2480 & 59 & 416 \\
\hline & & & & & & Aug. & 23 & 2380 & 39 & 251 \\
\hline May & $I$ & & 525 & 3 & 4.2 & Aug. & 24 & 2100 & 13 & 74 \\
\hline May & 2 & & 458 & 3 & 3.7 & Aug. & 25 & I 370 & 7 & 26 \\
\hline May & 3 & & 385 & 2 & 2.1 & Aug. & 26 & 924 & 6 & 15 \\
\hline May & 4 & & 421 & 2 & 2.3 & Aug. & 27 & 620 & 6 & 10 \\
\hline May & 5 & & 358 & 4 & 3.9 & Aug. & 28 & 458 & 4 & 4.9 \\
\hline May & 6 & & 305 & 3 & 2.5 & Aug. & 29 & 319 & 3 & 2.6 \\
\hline May & 7 & & 291 & 2 & 1.6 & Aug. & 30 & 232 & 2 & 1.3 \\
\hline May & 8 & & 305 & 2 & 1.6 & Aug. & 31 & 192 & 2 & 1.0 \\
\hline May & 9 & & 319 & 2 & 1.7 & & & & & \\
\hline May & 10 & & 284 & 1 & .77 & Sept. & 1 & 200 & 4 & 2.2 \\
\hline May & 11 & & 243 & 1 & .66 & Sept. & 2 & 176 & 4 & 1.9 \\
\hline May & 12 & & 227 & 1 & .61 & Sept. & 3 & 149 & 2 & $0^{\circ}$ \\
\hline May & 13 & & $\begin{array}{l}212 \\
216\end{array}$ & $\begin{array}{l}2 \\
3\end{array}$ & $1 \cdot \frac{1}{7}$ & Sept. & 4 & 505 & 12 & 18 \\
\hline May & 14 & & 216 & 3 & 1.7 & Sept. & 5 & 600 & 12 & 19 \\
\hline May & $\begin{array}{l}15 \\
16\end{array}$ & & $\begin{array}{l}265 \\
630\end{array}$ & 2 & $\frac{1.4}{7}$ & Sept. & 6 & 690 & 11 & 20 \\
\hline May & $\begin{array}{l}16 \\
17\end{array}$ & & $\begin{array}{l}630 \\
706\end{array}$ & 10 & 17 & Sept. & 7 & 750 & 6 & 12 \\
\hline May & $\begin{array}{l}17 \\
18\end{array}$ & & $\begin{array}{l}706 \\
610\end{array}$ & $\begin{array}{l}4 \\
2\end{array}$ & $\begin{array}{l}7.6 \\
3.3\end{array}$ & Sept. & 8 & 730 & 5 & 9.8 \\
\hline May & $\begin{array}{l}18 \\
19\end{array}$ & & $\begin{array}{l}610 \\
468\end{array}$ & $\begin{array}{l}2 \\
3\end{array}$ & $3 \cdot 3$ & Sept. & 9 & 580 & 5 & 7.8 \\
\hline May & $\begin{array}{l}19 \\
20\end{array}$ & & $\begin{array}{l}468 \\
385\end{array}$ & 3 & 3.8 & Sept. & 10 & 370 & 6 & 6.0 \\
\hline May & $\begin{array}{l}20 \\
21\end{array}$ & & $\begin{array}{l}385 \\
312\end{array}$ & 3 & 3.1 & Sept. & 11 & 230 & 8 & 5.0 \\
\hline May & $\begin{array}{l}21 \\
22\end{array}$ & & $\begin{array}{l}312 \\
259\end{array}$ & 3 & 2.5 & Sept. & 12 & 140 & 5 & 1.9 \\
\hline May & $\begin{array}{l}22 \\
23\end{array}$ & & $\begin{array}{l}259 \\
265\end{array}$ & 2 & 1.4 & Sept. & 13 & 105 & 2 & .57 \\
\hline May & $\begin{array}{l}23 \\
24\end{array}$ & & 265 & 2 & 1.4 & Sept. & 17 & 170 & 9 & 4.1 \\
\hline May & 24 & & 277 & 4 & 3.0 & Sept. & 18 & 770 & 18 & 37 \\
\hline May & 25 & & 238 & 3 & 1.9 & Sept. & 19 & 660 & 8 & 14 \\
\hline May & 26 & & 255 & 4.8 & 3.3 & Sept. & 20 & 500 & 4 & 5.4 \\
\hline May & 27 & & 1030 & 44 & 122 & Sept. & 21 & 310 & 4 & 3.3 \\
\hline May & 28 & & 1060 & 13 & 3 & Sept & 22 & 230 & 4 & 2.5 \\
\hline May & 29 & & 896 & 5 & 12 & Sept: & 23 & 210 & 3 & 1.7 \\
\hline May & 30 & & 668 & 6 & 11 & Sept. & 24 & 175 & 4 & 1.9 \\
\hline May & 31 & & 534 & 7 & 10 & $\begin{array}{l}\text { Sept. } \\
\text { Sept. }\end{array}$ & $\begin{array}{l}25 \\
26\end{array}$ & $\begin{array}{l}140 \\
120\end{array}$ & $\begin{array}{l}4 \\
3\end{array}$ & $\begin{array}{l}1.5 \\
.97\end{array}$ \\
\hline June & 1 & & 582 & 9 & 14 & Sept. & 27 & 105 & 3 & .85 \\
\hline June & $\frac{2}{3}$ & & 534 & 5 & 7.2 & Sept. & 28 & 95 & 4 & 1.0 \\
\hline June & 3 & & 430 & 3 & $3 \cdot 5$ & Sept. & 29 & 90 & 5 & 1.2 \\
\hline June & 4 & & 367 & 2 & 2.0 & Sept. & 30 & 85 & 5 & 1.1 \\
\hline
\end{tabular}


4-0187.5. ST. IOUIS RIVER AT FORBES, MINN.

PERIODIC DAILY SUSPENDED SEDIMENT, WATER YEAR OCTOBER 1967 TO SEPTEMBER 1968

\begin{tabular}{|c|c|c|c|c|c|c|c|c|c|}
\hline & Date & $\begin{array}{c}\text { Mean } \\
\text { Discharge } \\
\text { (cfs) }\end{array}$ & $\begin{array}{l}\text { Mean } \\
\text { Concen- } \\
\text { tration } \\
(\mathrm{mg} / \mathrm{l})\end{array}$ & $\begin{array}{l}\text { Load } \\
\text { (tons) }\end{array}$ & & Date & $\begin{array}{c}\text { Mean } \\
\text { Discharge } \\
\text { (cfs) }\end{array}$ & $\begin{array}{l}\text { Mean } \\
\text { Concen- } \\
\text { tration } \\
(\mathrm{mg} / \mathrm{l})\end{array}$ & $\begin{array}{l}\text { Load } \\
\text { (tons) }\end{array}$ \\
\hline $\begin{array}{l}\text { Jan. } \\
\text { Jan. }\end{array}$ & $\underset{31}{9,} 1968$ & $\begin{array}{l}44 \\
38\end{array}$ & $\begin{array}{l}4 \\
4\end{array}$ & $\begin{array}{r}0.47 \\
.41\end{array}$ & $\begin{array}{l}\text { June } \\
\text { June } \\
\text { June }\end{array}$ & $\begin{array}{l}14,1968 \\
15 \\
16\end{array}$ & $\begin{array}{l}2480 \\
2630 \\
2700\end{array}$ & $\begin{array}{l}47 \\
61 \\
72\end{array}$ & $\begin{array}{l}315 \\
433 \\
525\end{array}$ \\
\hline Feb. & 29 & 37 & 2 & .20 & $\begin{array}{l}\text { June } \\
\text { June }\end{array}$ & $\begin{array}{l}17 \\
18\end{array}$ & $\begin{array}{l}2710 \\
2650\end{array}$ & $\begin{array}{l}57 \\
41\end{array}$ & $\begin{array}{l}417 \\
293\end{array}$ \\
\hline Apr. & $\begin{array}{l}3 \\
4\end{array}$ & $\begin{array}{l}430 \\
400\end{array}$ & $\begin{array}{r}18 \\
8\end{array}$ & 21 & June & 19 & 2470 & 41 & 273 \\
\hline $\begin{array}{l}\text { Apr. } \\
\text { Apr. }\end{array}$ & $\begin{array}{l}4 \\
5\end{array}$ & $\begin{array}{l}400 \\
390\end{array}$ & $\begin{array}{r}8 \\
12\end{array}$ & $\begin{array}{l}8.6 \\
13\end{array}$ & June & 20 & 2240 & $\begin{array}{l}52 \\
43\end{array}$ & $\begin{array}{l}314 \\
232\end{array}$ \\
\hline Apr. & 6 & 360 & 11 & 11 & June & 22 & 1820 & 25 & 123 \\
\hline Apr. & 7 & 350 & 9 & 8.5 & June & 23 & 1770 & 28 & 134 \\
\hline Apr. & 8 & 385 & 11 & 11 & June & 24 & 1750 & 35 & 165 \\
\hline Apr. & 9 & 450 & 10 & 12 & June & 25 & 1680 & 32 & 145 \\
\hline Apr. & 10 & 515 & 10 & 14 & June & 26 & 1580 & 26 & 111 \\
\hline Apr. & 11 & 550 & 15 & 22 & June & 27 & 1490 & 22 & 88 \\
\hline Apr. & 12 & 543 & 16 & 23 & June & 28 & 1340 & 20 & 72 \\
\hline Apr. & 13 & 515 & 8 & 11 & June & 29 & 1260 & 36 & 122 \\
\hline $\begin{array}{l}\text { Apr. } \\
\text { Apr. }\end{array}$ & $\begin{array}{l}14 \\
15\end{array}$ & $\begin{array}{l}504 \\
498\end{array}$ & $\begin{array}{r}8 \\
11\end{array}$ & $\begin{array}{l}11 \\
15\end{array}$ & June & 30 & 1200 & 72 & 233 \\
\hline Apr. & 16 & 592 & 9 & 14 & July & 1 & 1200 & 22 & 71 \\
\hline Apr. & 17 & 750 & 13 & 26 & July & 2 & 1130 & 23 & 70 \\
\hline Apr. & 18 & 794 & 32 & 69 & July & 3 & 1060 & 23 & 66 \\
\hline Apr. & 19 & 776 & 21 & 44 & JuIy & 4 & 1010 & 11 & 30 \\
\hline Apr. & 20 & 765 & 15 & 31 & July & 5 & 957 & 8 & 21 \\
\hline Apr. & 21 & 816 & 14 & 31 & $\mathrm{July}$ & 6 & 881 & 9 & 21 \\
\hline Apr. & 22 & 888 & 20 & 48 & July & 7 & 809 & 13 & 28 \\
\hline Apr. & 23 & 1120 & 32 & 97 & July & 8 & 614 & 11 & 18 \\
\hline Apr. & 24 & 1360 & 40 & 147 & July & 9 & 702 & 5 & 9.5 \\
\hline Apr. & 25 & 1440 & 39 & 152 & July & 10 & 636 & 6 & 10 \\
\hline Apr. & 26 & 1570 & 40 & 170 & July & 11 & 585 & 5 & 7.9 \\
\hline Apr. & 30 & 2140 & 50 & 288 & $\begin{array}{l}\text { July } \\
\text { July }\end{array}$ & $\begin{array}{l}12 \\
13\end{array}$ & $\begin{array}{l}512 \\
540\end{array}$ & $\begin{array}{l}5 \\
6\end{array}$ & $\begin{array}{l}6.9 \\
8.7\end{array}$ \\
\hline May & 1 & 2220 & 85 & 509 & JuIy & 14 & 529 & 5 & 7.1 \\
\hline May & 2 & 2310 & 72 & 449 & July & 15 & 508 & 11 & 15 \\
\hline May & 3 & 2340 & 82 & 518 & July & 16 & 508 & 6 & 8.2 \\
\hline May & 4 & 2340 & 55 & 347 & JuIy & 17 & 501 & 6 & 8.1 \\
\hline May & 5 & 2290 & 45 & 278 & & & & & \\
\hline May & 6 & 2170 & 39 & 228 & Aug. & 22 & 384 & 11 & 11 \\
\hline May & 7 & 2030 & 31 & 170 & Aug. & 23 & 709 & 38 & 73 \\
\hline May & 8 & 1920 & 24 & 124 & Aug. & 24 & 1350 & 61 & 222 \\
\hline May & 9 & 1820 & 22 & 108 & Aug. & 25 & 1650 & 69 & 307 \\
\hline May & 10 & 1700 & 70 & 321 & Aug. & 26 & 1720 & 35 & 162 \\
\hline May & 11 & 1560 & 68 & 286 & Aug. & 27 & 1800 & 50 & 243 \\
\hline May & 12 & 1450 & 20 & 78 & Aug. & 28 & 1820 & 49 & 241 \\
\hline May & 13 & 1280 & 13 & 45 & Aug. & 29 & 1750 & 44 & 208 \\
\hline May & 14 & 1240 & 14 & 47 & Aug. & 30 & 1580 & 37 & 158 \\
\hline May & 15 & 1070 & 8 & 23 & Aug. & 31 & 1360 & 31 & 114 \\
\hline May & 16 & 1030 & 9 & 25 & & & & & \\
\hline May & 17 & 1010 & 12 & 33 & Sept. & 1 & 1220 & 44 & 145 \\
\hline May & 18 & 976 & 12 & 32 & Sept. & 2 & 1090 & 18 & 53 \\
\hline May & 19 & 953 & 8 & 21 & Sept & 3 & 968 & 14 & 37 \\
\hline May & 20 & 907 & 14 & 34 & Sept. & 4 & 888 & 13 & 31 \\
\hline May & 21 & 794 & 8 & 17 & Sept. & 7 & 813 & 12 & 26 \\
\hline May & 22 & 854 & 13 & 30 & Sept. & 8 & 820 & 11 & 24 \\
\hline May & 23 & 813 & 14 & 31 & Sept. & 9 & 816 & 9 & 20 \\
\hline May & 24 & 783 & 6 & 13 & Sept. & 10 & 791 & 10 & 21 \\
\hline May & 25 & 742 & 6 & 12 & Sept. & 11 & 754 & 9 & 18 \\
\hline May & 26 & 706 & 7 & 13 & Sept. & 12 & 713 & 7 & 13 \\
\hline May & 27 & 761 & 7 & 14 & Sept. & 13 & 568 & 6 & 9.2 \\
\hline May & 28 & 846 & 23 & 52 & Sept. & 24 & 605 & 6 & 9.8 \\
\hline May & 29 & 892 & 16 & 38 & $\begin{array}{l}\text { Sept. } \\
\text { Sept. }\end{array}$ & $\begin{array}{l}15 \\
16\end{array}$ & $\begin{array}{l}573 \\
530\end{array}$ & $\begin{array}{r}9 \\
15\end{array}$ & $\begin{array}{l}14 \\
21\end{array}$ \\
\hline June & 4 & 1040 & 11 & 31 & Sept. & 17 & 513 & 12 & 17 \\
\hline June & 5 & 934 & 9 & 23 & Sept. & 18 & 581 & 8 & 13 \\
\hline June & 6 & 1020 & 9 & 25 & Sept. & 19 & 672 & 7 & 13 \\
\hline June & 7 & 1060 & 10 & 29 & Sept. & 20 & 658 & 7 & 12 \\
\hline June & 8 & 1200 & 22 & 71 & Sept. & 21 & 634 & 6 & 10 \\
\hline June & 9 & 1470 & 22 & 87 & Sept. & 26 & 573 & 5 & 7.8 \\
\hline June & 10 & 1620 & 28 & - 122 & Sept. & 27 & 458 & 4 & 4.9 \\
\hline June & 11 & 1900 & 71 & 364 & Sept. & 28 & 519 & 4 & 5.6 \\
\hline June & 12 & 2070 & 39 & 218 & Sept. & 29 & 606 & 5 & 8.2 \\
\hline June & 13 & 2180 & 35 & 206 & & & & & \\
\hline
\end{tabular}


5-0405. PELICAN RIVER NEAR FERGUS FALLS, MINN.

PERIODIC DAILY SUSPENDED SEDIMENT, WATER YEAR OCTOBER 1967 TO SEPTEMBER 1968

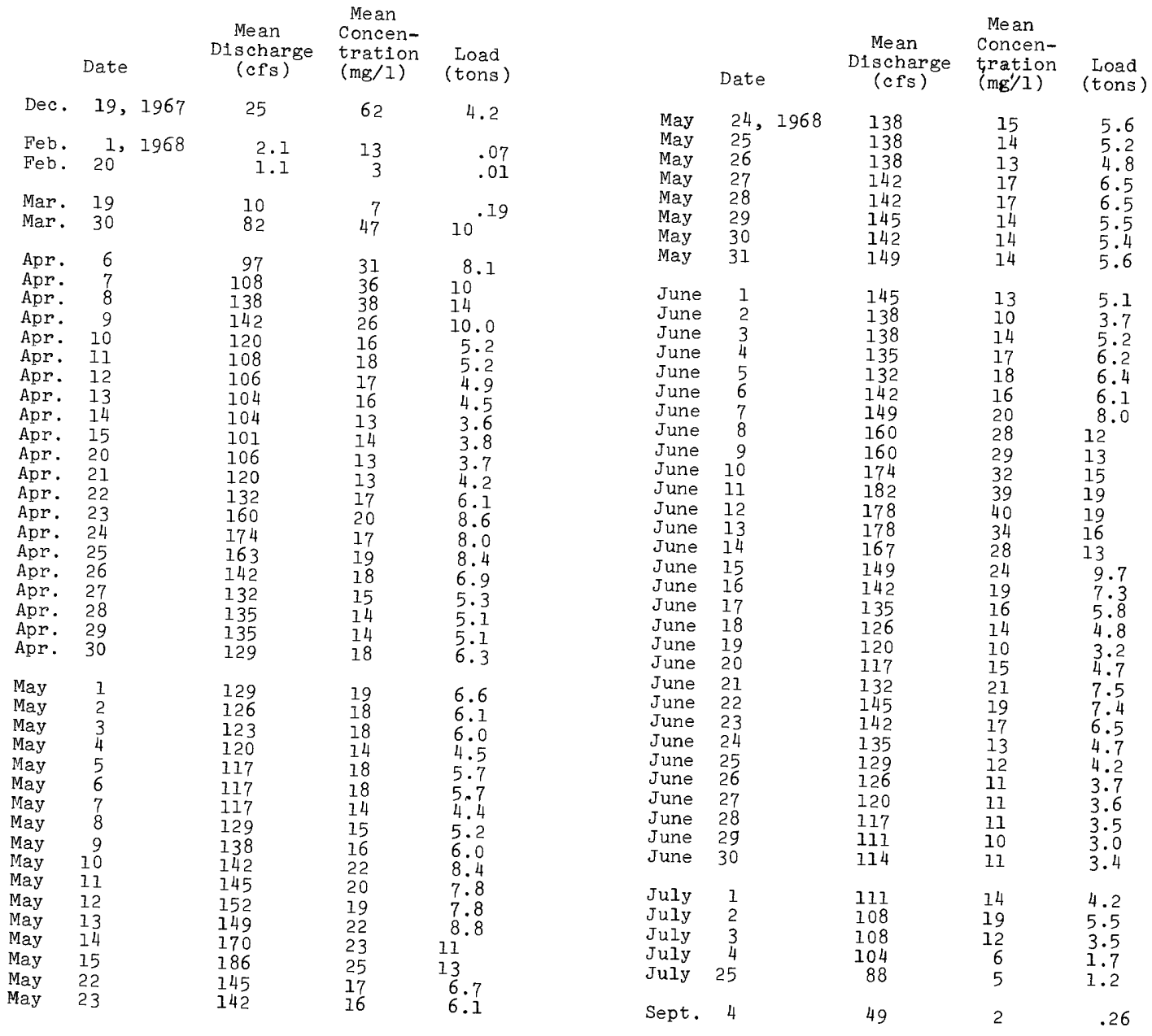


5-0875. MIDDLE RIVER NEAR ARGYLE, MINN.

PERIODIC DAILY SUSPENDED SEDIMENT, WATER YEAR OCTOBER 1967 TO SEPTEMBER 1968

\begin{tabular}{ccc} 
& \multicolumn{3}{c}{ Mean } \\
Mean & Concen- \\
Discharge & tration & Load \\
(cfs) & $(\mathrm{mg} / \mathrm{l})$ & (tons)
\end{tabular}

$\begin{array}{ccc} & \text { Mean } & \\ \text { Mean } & \text { Concen- } & \\ \text { Discharge } & \text { tration } & \text { Load } \\ (\mathrm{cfs}) & (\mathrm{mg} / \mathrm{l}) & \text { (tons) }\end{array}$

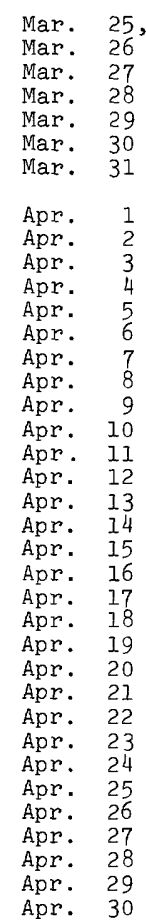

20
65
130
160
170
150
100

$\begin{array}{ll}10 & .54 \\ 12 & 2.1 \\ 25 & 8.8 \\ 45 & 19 \\ 46 & 21 \\ 45 & 18 \\ 40 & 11\end{array}$

$\begin{array}{rrr}60 & 32 & 5.2 \\ 40 & 17 & 1.8 \\ 32 & 11 & .95 \\ 35 & 8 & .76 \\ 40 & 6 & .65 \\ 34 & 7 & .64 \\ 26 & 9 & .63 \\ 20 & 13 & .70 \\ 17 & 18 & .83 \\ 15 & 23 & .93 \\ 13 & 25 & .88 \\ 12 & 19 & .62 \\ 11 & 11 & .33 \\ 11 & 15 & .45 \\ 12 & 16 & .52 \\ 14 & 10 & .38 \\ 13 & 8 & .28 \\ 11 & 8 & .24 \\ 10 & 10 & .27 \\ 10 & 12 & .32 \\ 11 & 11 & .33 \\ 13 & 8 & .28 \\ 17 & 13 & .60 \\ 20 & 22 & .2 \\ 22 & 15 & .89 \\ 19 & 16 & .82 \\ 15 & 18 & .73 \\ 10 & 14 & .38 \\ 7.0 & 10 & .12 \\ 5.5 & 8 & \end{array}$

$\begin{array}{lr}\text { May } & 1 \\ \text { May } & 2 \\ \text { May } & 3 \\ \text { May } & 4 \\ \text { May } & 5 \\ \text { May } & 6 \\ \text { May } & 7 \\ \text { May } & 8 \\ \text { May } & 9 \\ \text { May } & 10 \\ \text { May } & 11 \\ \text { May } & 12 \\ \text { May } & 13 \\ \text { May } & 14 \\ \text { May } & 15 \\ \text { May } & 16\end{array}$

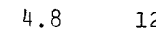

$4.8 \quad 12$

4. 0

3.8

$3.8 \quad 12$

3.8
4.5

4.7

4.3

4.3
3.7
4.0

4.3

4.6
4.9

4.913

4.412

$\begin{array}{ll}3.8 & 16 \\ 4.4 & 21\end{array}$

24,1968

June
June 26

June 27

June 27
June 28

June 29

7
6
6
6
5
4
4
4

$\begin{array}{ll}22 & 4.3 \\ 19 & 3.4 \\ 18 & 3.1 \\ 19 & 3.2 \\ 19 & 2.8 \\ 20 & 2.5 \\ 25 & 2.9\end{array}$

June 30

2.9

June 9

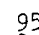

95
280
502

June I0

June 12

June 13

June 14

June 15

June 16

June 17

June 18

June 19

June 20

June 21

June 23

$\begin{array}{rr}93 & 27 \\ 120 & 91 \\ 136 & 184 \\ 113 & 200 \\ 125 & 286 \\ 83 & 238 \\ 62 & 162 \\ 65 & 126 \\ 54 & 63 \\ 58 & 44 \\ 36 & 19 \\ 29 & 12 \\ 28 & 9.5 \\ 24 & 6.5 \\ 21 & 4.8\end{array}$

$\begin{array}{ll}\text { JuIy } & 1 \\ \text { JuIy } & 2 \\ \text { JuIy } & 3 \\ \text { JuIy } & 4 \\ \text { JuIy } & 5\end{array}$

43

196

$\begin{array}{lll}\text { July } & 5 & 231 \\ \text { July } & 6 & 189\end{array}$

July

July

July 9

JuIy I0

July 11

July 21

July 22

July 23

July 24

July 25

July 26

$\begin{array}{ll}\text { JuIy } & 27 \\ \text { JuIy } & 28\end{array}$

July 29

July 30

July 31

Aug. 1

Aug. 3

Aug. 4

Aug.

Aug. 6

Aug. 7

Aug.
Aug. $\quad 9$

Aug. 10

Aug. 11

Aug. 12

Aug. 13

Aug. 20

Aug. 26

Aug. 27

Aug. $\quad 28$

Aug. 29

Aug. 30

Aug. $3 I$

$\begin{array}{ll}22 & 2.6 \\ 30 & 3 .\end{array}$

Sept. 1

Sept. 2

Sept. 3

Sept. 4

Sept. 5

Sept.

Sept. 7

Sept. 8

Sept. 10

Sept. 11

Sept. 12

Sept. 13

Sept. 14

Sept. 15

$\begin{array}{ll}9.5 & \text { Sept. } 16 \\ .5 & \text { Sept. } 24\end{array}$

Sept. 25

23.

22

14

8.7
5.6

3.6

3.1

1.9

162

95
48

29

20

15

6.0

6.6

5.2

5.2
4.7

4.1

3.6
3.5

2.9

2. 8

3.4

2.9
3.6

2.4

2.6

2. 4

1.7

4.6

4.8

3.9

3.4
2.8

2.5
2.5

2. 5

2.3
2.7

2.7
2.3

1. 8

1.9

1.7
2.4

2. 8

2. 7

2. 3

2.4
2.2

1. 7

5.8 
5-2440. CROW WING RIVER AT NIMROD, MINN.

PERIODIC DAILY SUSPENDED SEDIMENT, WATER YEAR OCTOBER 1967 TO SEPTEMBER 1968

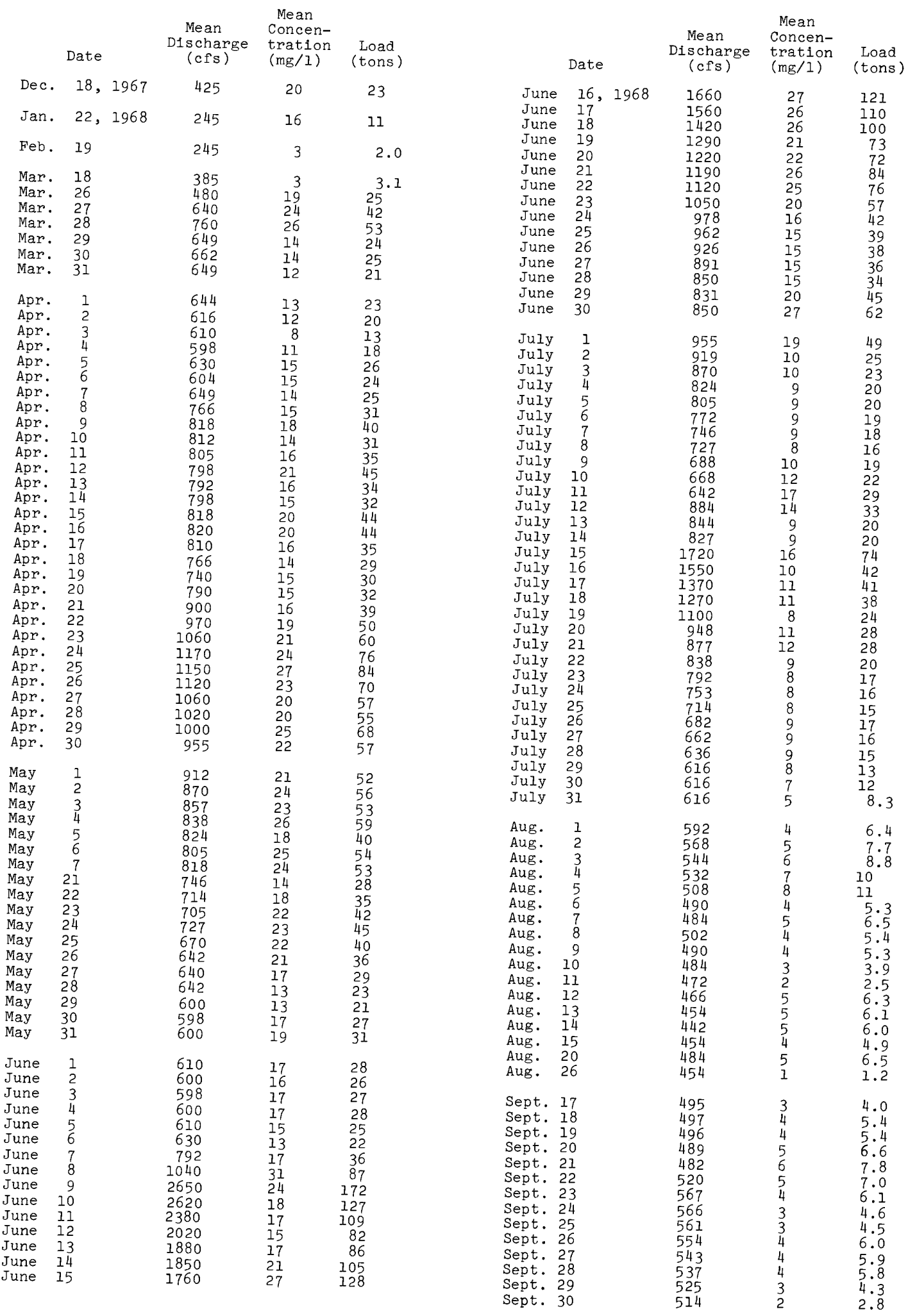


5-3150. REDWOOD RIVER AT MARSHALL, MINN.

PERIODIC DAILY SUSPENDED SEDIMENT, WATER YEAR OCTOBER 1967 TO SEPTEMBER 1968

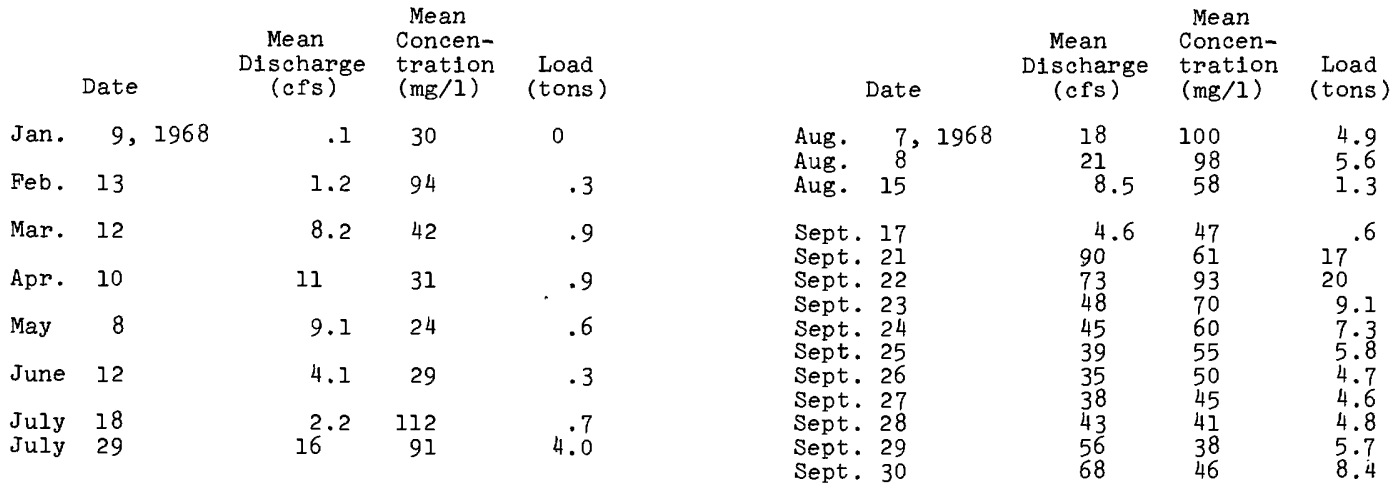

5-3165. REDWOOD RIVER NEAR REDWOOD FALLS, MINN.

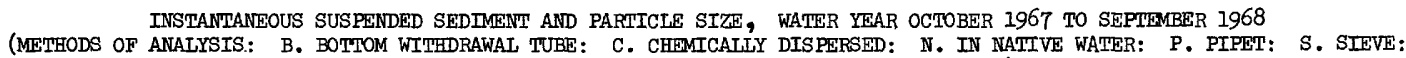
v. VISUAL ACCUMULATION TUBE: W. IN DISTILLED WATER)

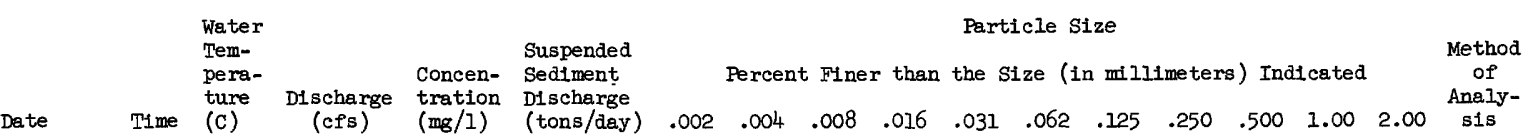

Feb. 12, 1968 162

Mer. 13

Mer. 27

Apr. 10

1625

May 8

1330

$\begin{array}{rc}54 & .26 \\ 8 & .30 \\ 26 & 2.4 \\ 59 & 8.3 \\ 46 & 3.2 \\ & \\ 162 & 10 \\ 122 & 3.6 \\ 245 & 177 \\ 299 & 169 \\ 240 & 97 \\ & \\ 75 & 11 \\ 135 & 6.6 \\ 107 & 29\end{array}$

$\begin{array}{lllll}77 & 78 & 84 & 87 & 90\end{array}$

BWC 
5-3262. JUDICIAL DITCH NO. 1-A NEAR NEW SWEDEN, MINN.

INSTANTANEOUS SUSPENDED SEDIMENT AND PARTICIE SIZE, WATER YEAR OCTOBER 1967 TO SEPTEMBER 1968

(METHODS OF ANALYSIS: B. BOTTOM WITHDRAWAL TUBE: C. CHEMICALLY DISPERSED: N. IN NATIVE WATER: P. PIPET: S. SIEVE:

V. VISUAL ACCUMULATION TUBE: W. IN DISTILIED WATER)

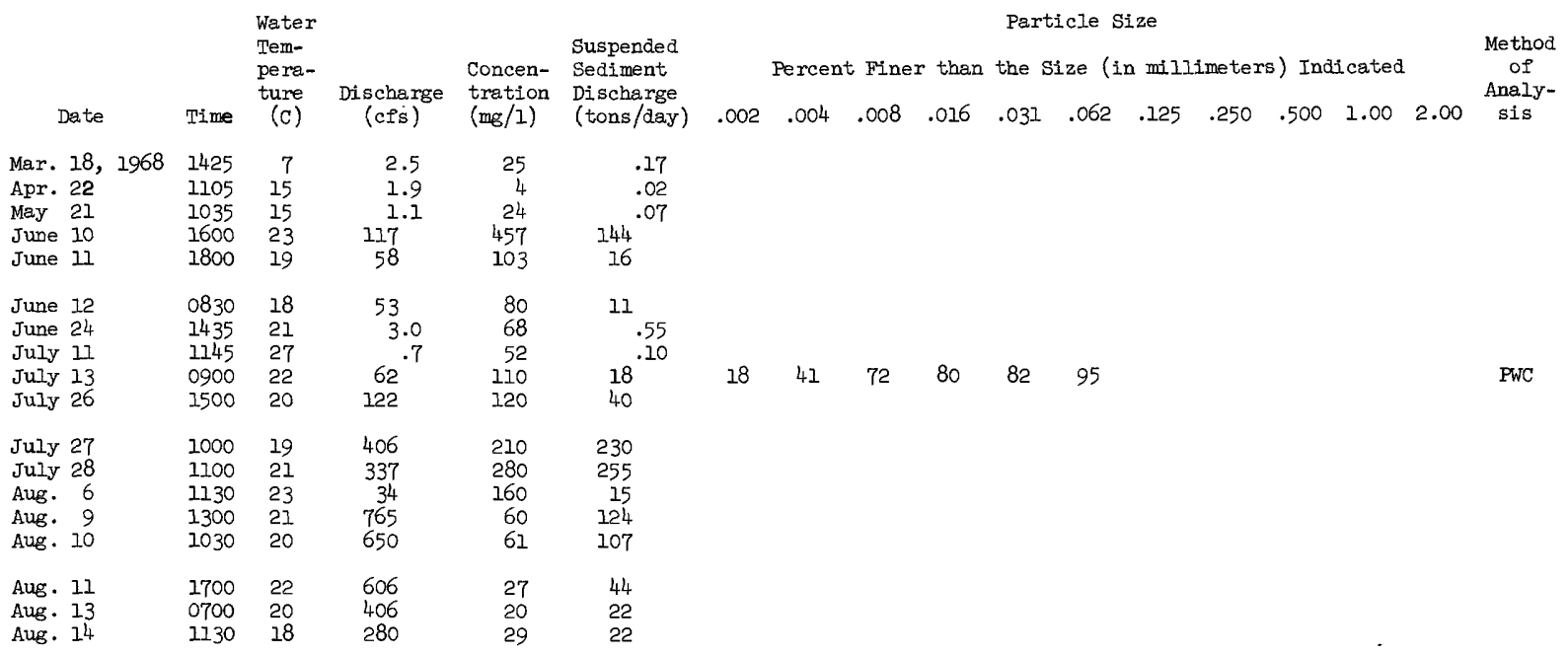

PARTICLE SIZE OF BED MATERIAL, WATER YEAR OCTOBER 1967 TO.SEPTEMBER 1968

(METHOD OF ANALYSIS: H. HYDROMETER: O. OPTICAL ANAIJZER: S. SIEVE: V. VISUAL ACCUMULATION TUBE)

Particle Size

\begin{tabular}{|c|c|c|c|c|c|c|c|c|c|c|c|c|c|c|}
\hline ate & Time & $\begin{array}{l}\text { No. of } \\
\text { Sampling } \\
\text { Points }\end{array}$ & $\begin{array}{c}\text { Discharge } \\
\text { (cf's) }\end{array}$ & 0.016 & ercent & Finer $t$ & han the & $\begin{array}{l}\text { Size } \\
0.25\end{array}$ & (in $\mathrm{mi}$ ) & Iimete & rs) Ind & icated & 4.000 & $\begin{array}{l}\text { Me thod } \\
\text { of } \\
\text { Analysis }\end{array}$ \\
\hline July 11, 1968 & 1145 & 2 & 0.7 & & & 11 & 19 & 30 & 36 & & 50 & 63 & & $\mathrm{~S}$ \\
\hline
\end{tabular}

5-3538. STRAIGHT RIVER NEAR FARIBAULT, MINN.

PERIODIC DAILY SUSPENDED SEDIMENT, WATER YEAR OCTOBER 1967 TO SEPTEMBER 1968

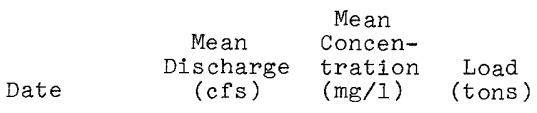

\begin{tabular}{|c|c|c|c|c|}
\hline Dec. & 19,1967 & 28 & 39 & 2.9 \\
\hline Jan. & 22,1968 & 16 & 1 & 0 \\
\hline Feb. & 22 & 11 & 10 & 0.3 \\
\hline Mar. & 25 & 41 & 5 & 0.6 \\
\hline $\begin{array}{l}\text { Apr. } \\
\text { Apr. } \\
\text { Apr. } \\
\text { Apr. } \\
\text { Apr. }\end{array}$ & $\begin{array}{l}23 \\
24 \\
25 \\
26 \\
27\end{array}$ & $\begin{array}{l}223 \\
440 \\
386 \\
260 \\
185\end{array}$ & $\begin{array}{r}452 \\
328 \\
143 \\
102 \\
59\end{array}$ & $\begin{array}{r}288 \\
390 \\
149 \\
72 \\
29\end{array}$ \\
\hline $\begin{array}{l}\text { May } \\
\text { May } \\
\text { May } \\
\text { May } \\
\text { May } \\
\text { May } \\
\text { May } \\
\text { May } \\
\text { May } \\
\text { May } \\
\text { May } \\
\text { May }\end{array}$ & $\begin{array}{l}1 \\
16 \\
17 \\
18 \\
19 \\
20 \\
21 \\
27 \\
28 \\
29 \\
30 \\
31\end{array}$ & $\begin{array}{l}104 \\
340 \\
287 \\
228 \\
185 \\
167 \\
152 \\
202 \\
232 \\
206 \\
179 \\
161\end{array}$ & $\begin{array}{r}22 \\
1210 \\
295 \\
124 \\
89 \\
100 \\
85 \\
189 \\
158 \\
198 \\
190 \\
211\end{array}$ & $\begin{array}{r}6.2 \\
1110 \\
228 \\
76 \\
44 \\
45 \\
35 \\
103 \\
99 \\
110 \\
92 \\
92\end{array}$ \\
\hline $\begin{array}{l}\text { June } \\
\text { June } \\
\text { June } \\
\text { June }\end{array}$ & $\begin{array}{r}1 \\
2 \\
3 \\
24\end{array}$ & $\begin{array}{l}220 \\
232 \\
196 \\
143\end{array}$ & $\begin{array}{l}214 \\
188 \\
154 \\
376\end{array}$ & $\begin{array}{r}127 \\
118 \\
81 \\
145\end{array}$ \\
\hline $\begin{array}{l}\text { July } \\
\text { July } \\
\text { July }\end{array}$ & $\begin{array}{r}3 \\
12 \\
13\end{array}$ & $\begin{array}{r}213 \\
294 \\
1120\end{array}$ & $\begin{array}{l}217 \\
864 \\
694\end{array}$ & $\begin{array}{r}125 \\
857 \\
4620\end{array}$ \\
\hline
\end{tabular}

$\begin{array}{cc}\text { Mean } & \text { Concen- } \\ \text { Discharge tration Load }\end{array}$

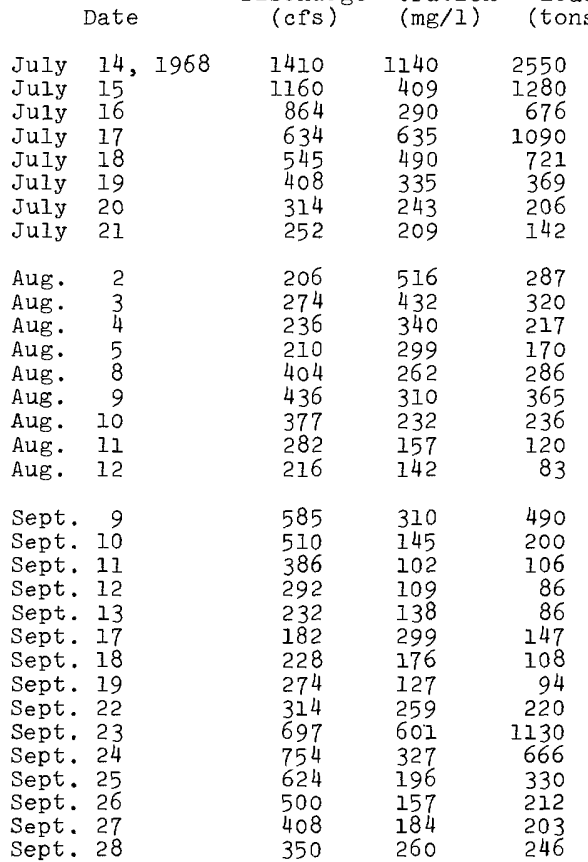


PERIODIC DAILY SUSPENDED SEDIMENT, WATER YEAR OCTOBER 1967 TO SEPTEMBER 1968

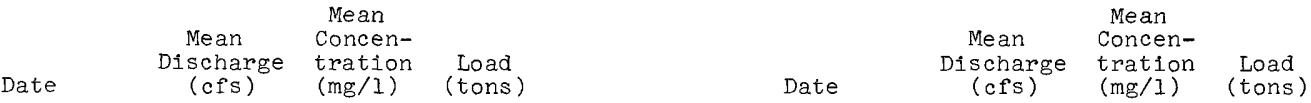

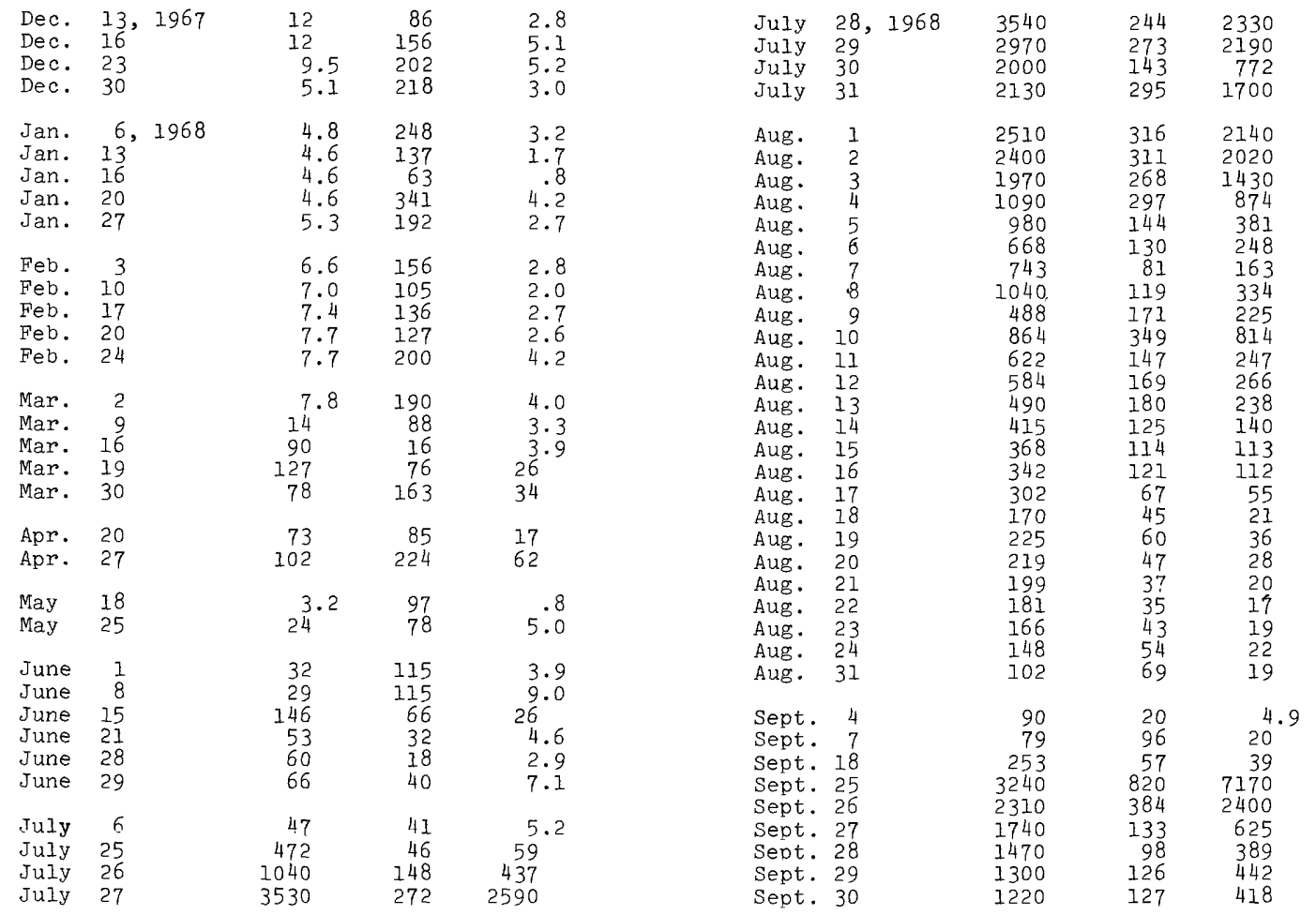

PARTICIE SIZE OF BED MATERIAL, WATER YEAR OCTOBER 1967 TO SEPTEMBER 1968

(METHOD OF ANALYSIS: H. HYDROMETER: O. OPTICAL ANALYZER: S. STEVE: V. VISUAL ACCUMULATION TUBE)

Particle Size

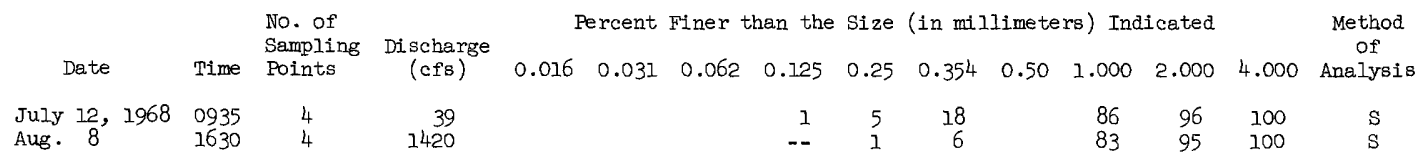

5-3840. ROOT RIVER NEAR LANESBORO, MINN.

PERIODIC DAILY SUSPENDED SEDIMENT, WATER YEAR OCTOBER 1967 TO SEPTEMBER 1968

\begin{tabular}{|c|c|c|c|c|c|c|c|c|}
\hline Date & $\begin{array}{c}\text { Mean } \\
\text { Discharge } \\
\text { (cfs) }\end{array}$ & $\begin{array}{l}\text { Mean } \\
\text { Concen- } \\
\text { tration } \\
(\mathrm{mg} / \mathrm{l})\end{array}$ & $\begin{array}{l}\text { Load } \\
\text { (tons) }\end{array}$ & & Date & $\begin{array}{c}\text { Mean } \\
\text { Discharge } \\
\text { (cfs) }\end{array}$ & $\begin{array}{l}\text { Mean } \\
\text { Concen- } \\
\text { tration } \\
(\mathrm{mg} / \mathrm{l})\end{array}$ & $\begin{array}{l}\text { Load } \\
\text { (tons) }\end{array}$ \\
\hline Dec. 19, 1967 & 81 & 15 & 3.3 & $\begin{array}{l}\text { June } \\
\text { June }\end{array}$ & $\frac{11}{18}, 1968$ & $\begin{array}{l}170 \\
312\end{array}$ & $\begin{array}{l}1290 \\
1030\end{array}$ & $\begin{array}{l}592 \\
868\end{array}$ \\
\hline 22,1968 & 89 & 29 & 7.0 & $\begin{array}{l}\text { June } \\
\text { June }\end{array}$ & $\begin{array}{l}26 \\
27\end{array}$ & $\begin{array}{r}286 \\
1030\end{array}$ & $\begin{array}{r}685 \\
1270\end{array}$ & $\begin{array}{r}576 \\
3530\end{array}$ \\
\hline Feb. & 75 & 10 & 2.0 & $\begin{array}{l}\text { June } \\
\text { June }\end{array}$ & $\begin{array}{l}28 \\
29\end{array}$ & $\begin{array}{r}1220 \\
759\end{array}$ & $\begin{array}{l}578 \\
400\end{array}$ & $\begin{array}{r}1950 \\
820\end{array}$ \\
\hline Mar. & 148 & 13 & 5.2 & June & 30 & 530 & 360 & 515 \\
\hline $\begin{array}{ll}\text { Apr. } & 23 \\
\text { Apr. } & 24 \\
\text { Apr. } & 25\end{array}$ & $\begin{array}{l}288 \\
409 \\
356\end{array}$ & $\begin{array}{l}263 \\
382 \\
182\end{array}$ & $\begin{array}{l}204 \\
422 \\
175\end{array}$ & $\begin{array}{l}\text { July } \\
\text { July }\end{array}$ & $\begin{array}{l}23 \\
24\end{array}$ & $\begin{array}{r}743 \\
1170\end{array}$ & $\begin{array}{l}2073 \\
2160\end{array}$ & $\begin{array}{l}4560 \\
6960\end{array}$ \\
\hline 16 & 742 & 5020 & 10100 & Aug. & 13 & 130 & 102 & 36 \\
\hline $\begin{array}{l}\text { May } \\
\text { May } \\
\text { May }\end{array}$ & $\begin{array}{l}508 \\
376 \\
240\end{array}$ & $\begin{array}{r}2290 \\
700 \\
170\end{array}$ & $\begin{array}{r}3390 \\
711 \\
110\end{array}$ & $\begin{array}{l}\text { Sept } \\
\text { Sept }\end{array}$ & $\begin{array}{r}4 \\
\cdot \quad 10\end{array}$ & $\begin{array}{l}330 \\
147\end{array}$ & $\begin{array}{r}491 \\
98\end{array}$ & $\begin{array}{r}437 \\
39\end{array}$ \\
\hline
\end{tabular}


5-3850. ROOT RIVER NEAR HOUSTON, MINN.

PERIODIC DAILY SUSPENDED SEDIMENT, WATER YEAR OCTOBER 1967 TO SEPTEMBER 1968

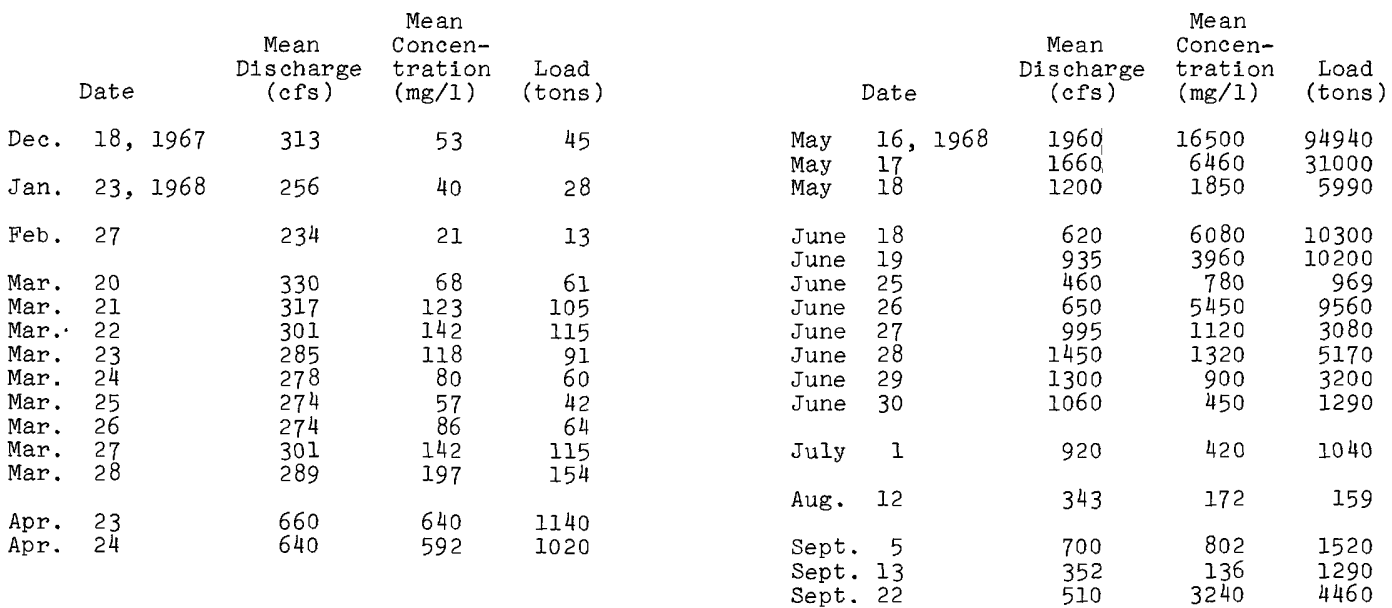

INSTANTANEOUS SUSPENDED SEDTMENT AND PARTICTE SIZE, WATTER YEAR OCTOBER 1967 TO SEPTEMBER 1968

(METHODS OF ANALYSIS: B. BOTTOM WITHDRAWAL TUBE: C. CHEMICALLY DISPERSED: N. IN NATIVE WATER: P. PIPET: S. SIEVE: V. VISUAI ACCUMULATION TUBE: W. IN DISTILIED WATER)

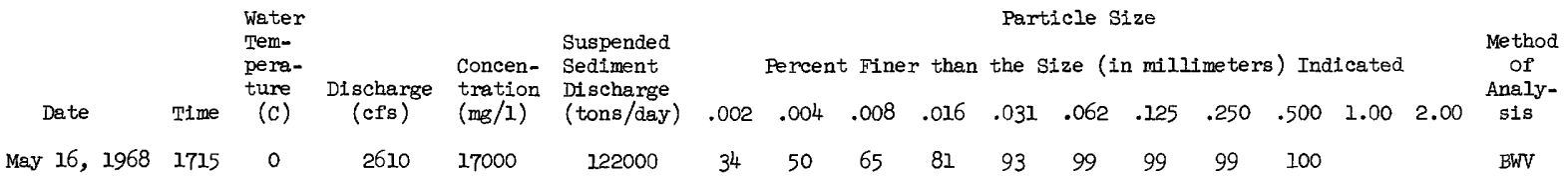

5-4760. WEST FORK DES MOINES RIVER AT JACKSON, MINN.

PERIODIC DAILY SUSPENDED SEDIMENT, WATER YEAR OCTOBER 1967 TO SEPTEMBER 1968

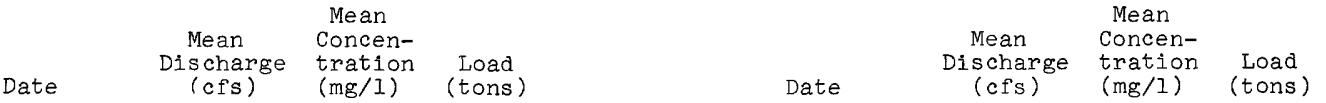

$\begin{array}{lcccc}\text { Jan. } & 9,1968 & .4 & 40 & .04 \\ \text { Feb. } & 12 & 2.2 & 21 & .12 \\ \text { Mar. } & 11 & 20 & 22 & 1.2 \\ \text { Apr. } & 8 & 14 & 58 & 2.2 \\ & & 18 & 26 & 1.3 \\ \text { May } & 10 & 1.8 & 17 & .08 \\ \text { June } & 6 & 18 & 132 & 6.4 \\ & & 505 & 114 & 155 \\ \text { July } & 11 & 390 & 104 & 110 \\ \text { July } & 29 & 381 & 97 & 100 \\ \text { July } & 30 & & & \\ \text { July } & 31 & & & \end{array}$

$\begin{array}{lrrrr}\text { Aug. } & 1,1968 & 474 & 140 & 179 \\ \text { Aug. } & 2 & 378 & 121 & 123 \\ \text { Aug. } & 3 & 307 & 134 & 152 \\ \text { Aug. } & 4 & 284 & 150 & 115 \\ \text { Aug. } & 5 & 246 & 102 & 68 \\ \text { Aug. } & 6 & 221 & 74 & 44 \\ \text { Aug. } & 7 & 207 & 64 & 36 \\ \text { Aug. } & 8 & 212 & 61 & 35 \\ \text { Aug. } & 9 & 233 & 60 & 38 \\ \text { Aug. } 20 & 79 & 77 & 16 \\ & & & & \\ \text { Sept. } 19 & 189 & 49 & 25 \\ \text { Sept. } 23 & 284 & 42 & 32 \\ \text { Sept. } 24 & 261 & 48 & 34 \\ \text { Sept. } 30 & 345 & 52 & 48\end{array}$


Part 1. Surface Water Records

Accuracy of field data and computed results....... Page

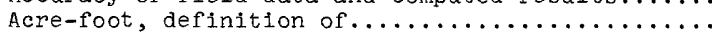

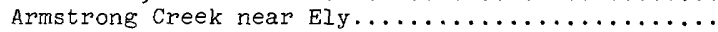

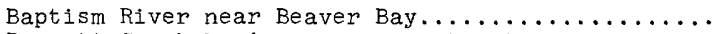
Bassett Creek basin, measurements at miscellaneous sites in.................... 180

Basswood River near Winton................. 77

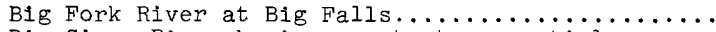

Big Sioux River basin, crest-stage partialrecord stations in.

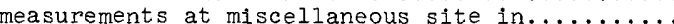

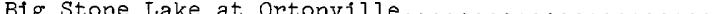

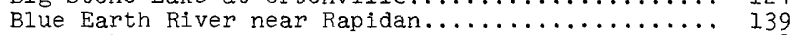

East Branch, near Bricelyn.................. 138

Bluff Creek basin, crest-stage partialrecord stations in................... 168

Bois de Sioux River near white Rock, S. Dak....... ${ }_{3} 37$

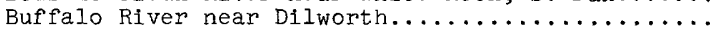

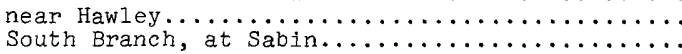

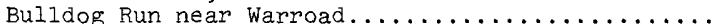

Burgo Creek near Ely..................... 74

Burns Valley Creek basin, measurement at

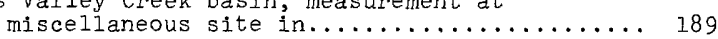

Burntside River near Ely.................. 71

Cannon River at welch................... 152

Cannon River basin, crest-stage partial-

record stations in................... 172

gaging-station records in.................. $151-152$

measurements at miscellaneous sites in...... 188-189

Cedar River near Austin..................... 163

Cfs-day, definition of $\ldots \ldots \ldots \ldots \ldots \ldots \ldots \ldots \ldots \ldots,{ }_{3}$

Chippewa River near Milan................... 130

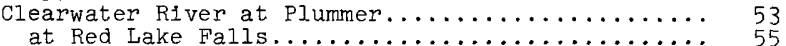

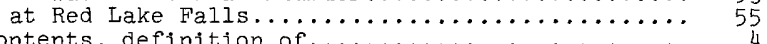

Contents, definition of $\ldots \ldots \ldots \ldots \ldots \ldots \ldots \ldots \ldots \ldots \ldots \ldots \ldots \ldots$
Control, definition of $\ldots \ldots \ldots \ldots \ldots \ldots \ldots$

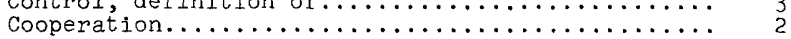

Cottonwood River near New Uim..................... 137

Crow River at Rockford........................ 118

Middle Fork, near Spicer........................ 116

South Fork, near Mayer................. 117

Crow River basin, crest-stage partial-

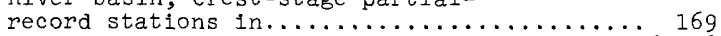

gaging-station records in...................

measurement at miscellaneous site in......... 179

Crow wing River at Nimrod................ 108

Crow Wing River basin, crest-stage partial-

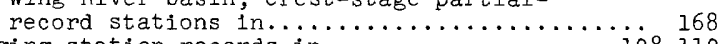

gaging-station records in.............. 108-110

low-flow partial-record station in........... 165

measurement at miscellaneous site in............ $177-178$

Cubic feet per second per square mile,

definition of.

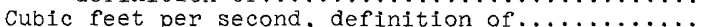

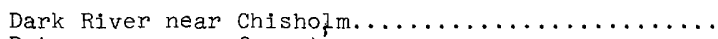

Deta, accuracy of ...: $: \ldots \ldots \ldots \ldots \ldots \ldots \ldots \ldots$.

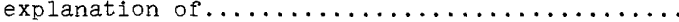

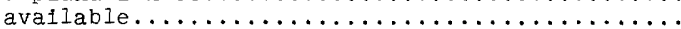

Des Moines River, west Fork at Jackson............ 164

Des Moines River basin, crest-stage partialrecord stations in.................. 173-174

gaging-station records in................ 164

measurements at miscellaneous site in......... 189

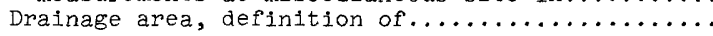

East Branch County ditch No. 14 near Detroit Lakes 28

East Indian Creek basin, crest-stage partial-

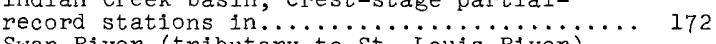

East Swan River (tributary to St. Louis River)

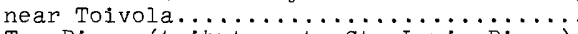

East Two River (tributary to St. Louis River) near Iron Junction................... 18

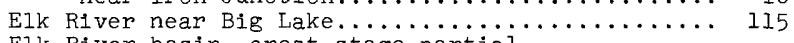

Elk River basin, crest-stage partial-

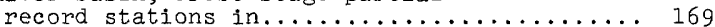

gaging-station records in................ 113-115

measurements at miscellaneous site in............ 179

Elk River basin (north of Little Falls), measurements at miscellaneous site in.......
Gage height, definition of $\ldots \ldots \ldots \ldots \ldots \ldots \ldots$ Page

Gaging-station, definttion of ..................

Garvin Brook basin, crest-stage partial-

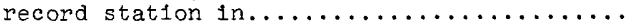

Glaisby Brook near Kettle River.............. 145

Gull Lake near Brainerd................... 109

Gull River at Gull Lake Dam, near Brainerd...... 110

Hydrologic bench-mark station, definttion of.....

Introduction. ...................... I

Iowa River basin, crest-stage partial-

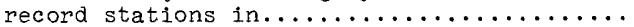

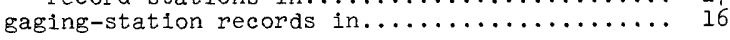

Johnson Creek basin, crest-stage partial-

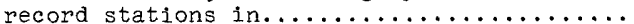

Kawishiwi River near Ely $\ldots \ldots \ldots \ldots \ldots \ldots \ldots$

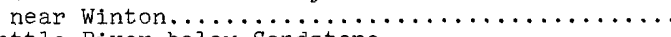

70
146

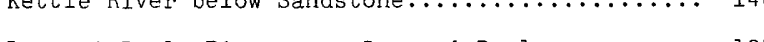

Lac qui Parle River near Lac qui Parle........... 128
Lake of the Woods basin, crest-stage partiaj-

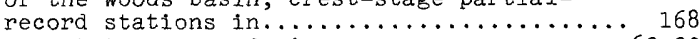

gaging-station records in..............6.69-90

measurements at miscellaneous sites in........ 177

Lake Superior, streams tributary to, crest-

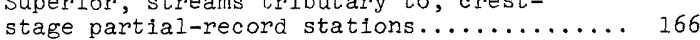

gaging-station records................ 12-22

measurement at miscellaneous sites in........ 175

Lakes and reservoirs:

Big Stone Lake at Ortonville............. 124

Gull Lake near Brainerd....................... 109

Leech Lake at Federal Dam................. 93

Lower Red Lake near Red Lake.............. 48

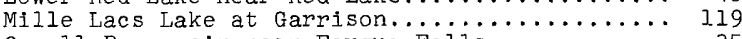

Orwell Reservoir near Fergus Fails............

Pelican Lake near Pequot Lakes....................... 107

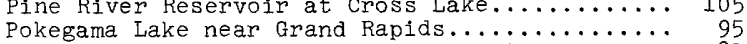

Rainy Lake near Fort Francis, Ontario.......... 81

St. Clair Lake near Detroit Lakes........... ${ }^{29}$

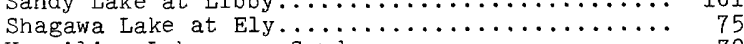

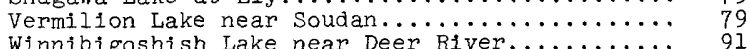

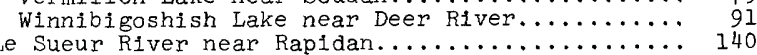

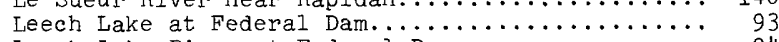

Leech Lake River at Federal Dam.................... 94

Little Minnesota River near Peever, S. Dak........ 122

Little Sioux River basin, crest-stage partialrecord stations in................... 174

Long Lake outlet near Detroit Lakes............... ${ }_{2} 6$

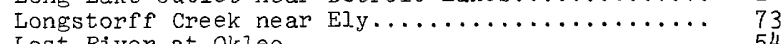

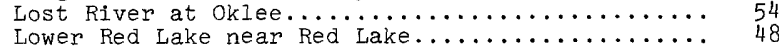

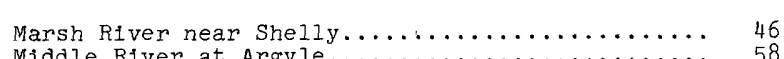

Middle River at Argyle.................... 58

Mille Lacs Lake at Garrison................. 119

Minnesota River at Mankato................... 141

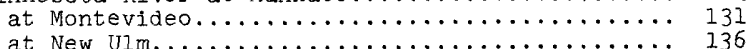

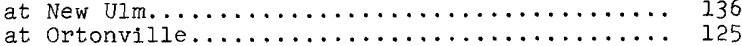

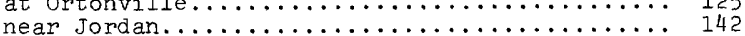

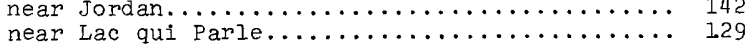

Minnesota River basin, crest-stage partial-

record stations in.................. 170-171

gaging-station records in............... 122-143

measurements at miscellaneous sites in...... 181-187

Mississippi River at Aitkin.................. 104

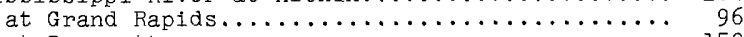

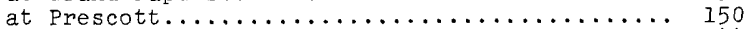

at St. Paul........................... 144

at Winnibigoshish Dam, near Deer River........ 92 at Winona........................................... 158 measurements at miscellaneous sites in.. i77,i79, I80,

near Anoka........................... 181,187

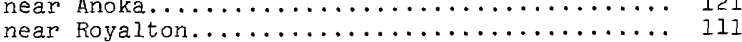

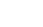

(1)

.

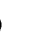

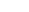


Namakan River at outlet of Lac la Croix, Ontario..

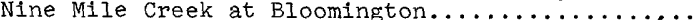

Nokasippi River basin, measurements at

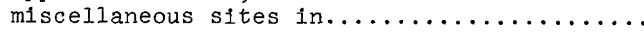

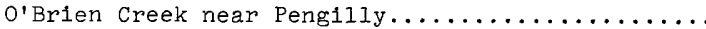

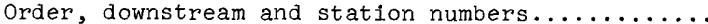

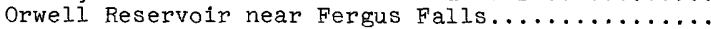

ot sego Creek basin, crest-stage partial-

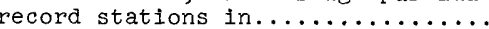

otter Tail River, below Orwell Dam, near

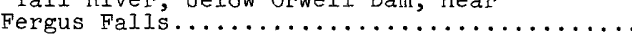

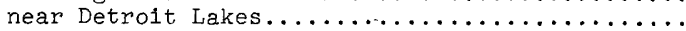

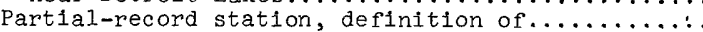

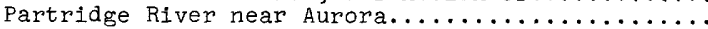

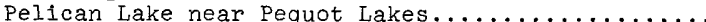

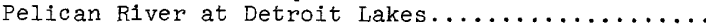

at Detroit Lake Outlet, near Detroit Lakes......

at Lake Melissa Outlet, near Detroit Lakes......

at Muskrat Lake Outlet, near Detroit Lakes......

at Sallie Lake Outlet, near Detroit Lakes.......

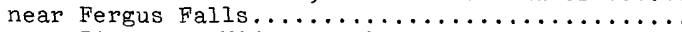

Pigeon River, at Middie Falis, near Grand Portage.

Pine River at Cross Lake Dam, at Cross Lake.......

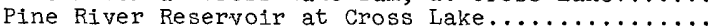

Platte River basin, crest-stage partial-

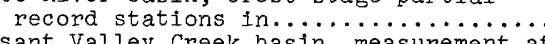

Pleasant Valley Creek basin, measurement at

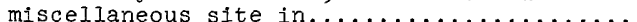

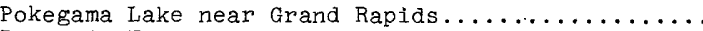

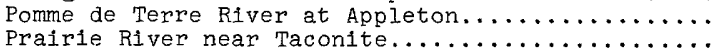

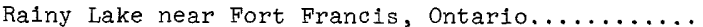

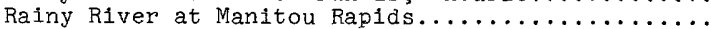

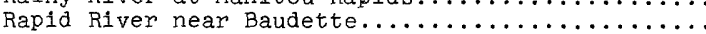

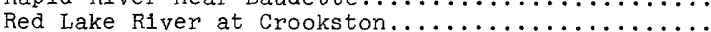

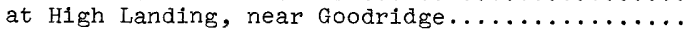

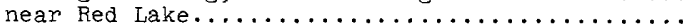

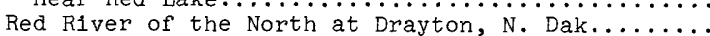

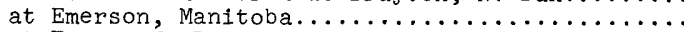

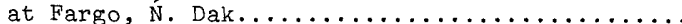

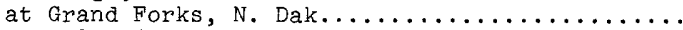

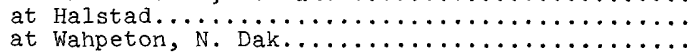

Red River of the North basin, crest-stage

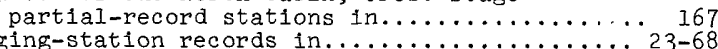

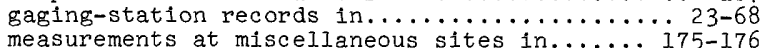

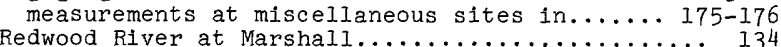

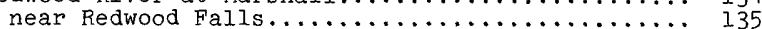

Reservoirs: See lakes and reservoirs.

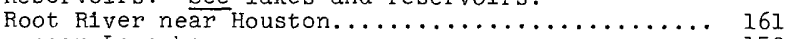

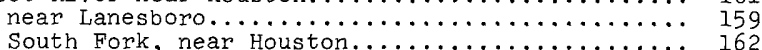

Root River basin, crest-stage partial-

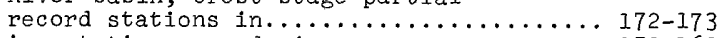

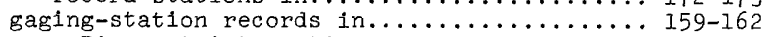

Roseau River at international boundary,

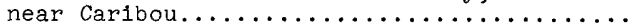

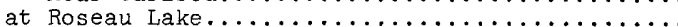

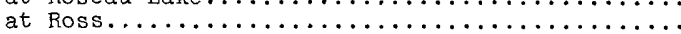

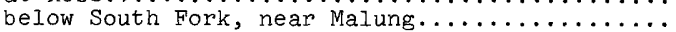

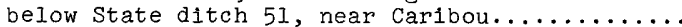

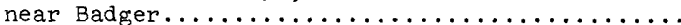

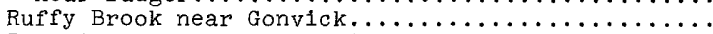

Rum River near St. Francis........................ 120

Rum River basin, crest-stage partial-

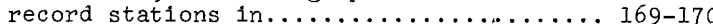

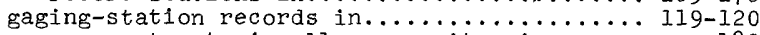

measurements at miscellaneous sites in........ 180

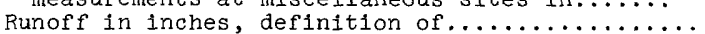

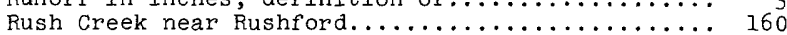

St. Clair Lake near Detroit Lakes............. 29

St. Clair Lake Outlet near Detroit Lakes............. 30

St. Croix River at St. Croix Falls, Wis............. 149

St. Croix River basin, crest-stage partiai-

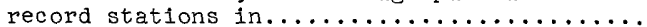

St. Croix River basin, gaging-station

Page

records in......................... 145-149

measurements at misceijaneous sites in...... 187-188

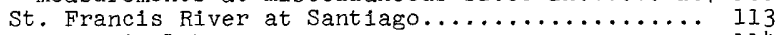

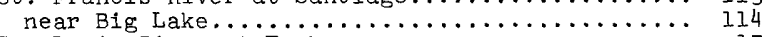

St. Louis River at Forbes............... 17

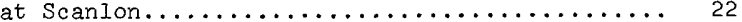

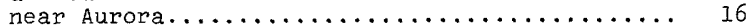

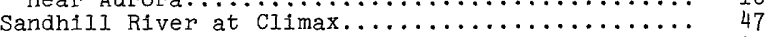

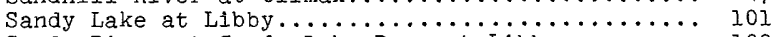

Sandy River at Sandy Lake Dam, at Libby........ 102

Sauk River near St. Cloud...................... 112

Sauk River basin, crest-stage partial-

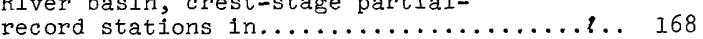

measurements at miscellaneous sites in....... I79

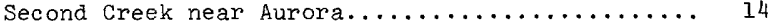

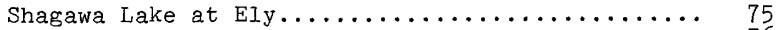

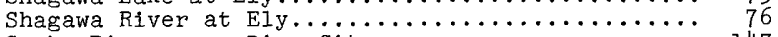

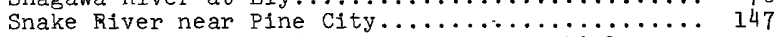

Split Hand Creek basin, crest-stage partial-

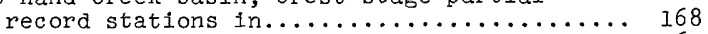

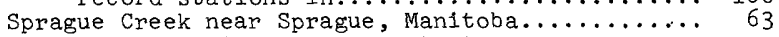

Spunk Creek basin, measurement at

miscellaneous site in..............

Stage-discharge relation, definition of ........ 3

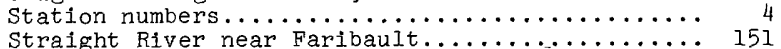

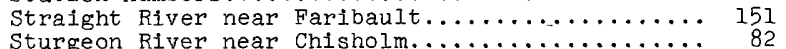

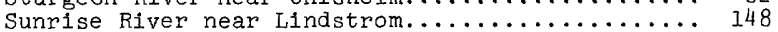

Swan River (tributary to Mississippi River)

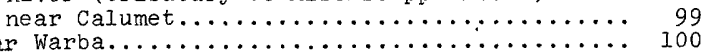

near Warba..... Ma River basin (tribury to Mississippi

River) crest-stage partial-record

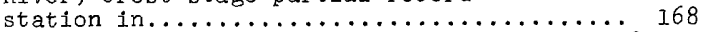

gaging-station records in.................

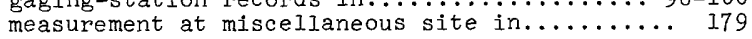

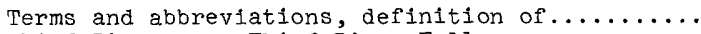

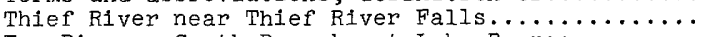

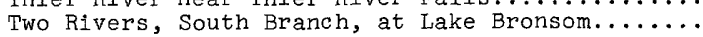

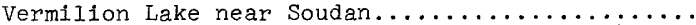

Vermilion River (tributary to Namakan River)

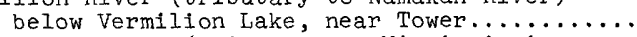

Vermililon River (tributary to Mississippi

River) basin, crest-stage partial-

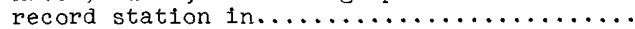

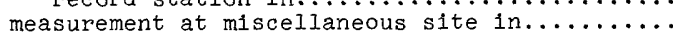

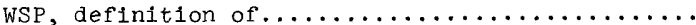

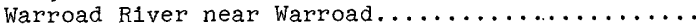

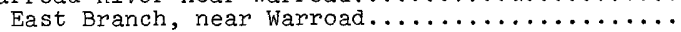

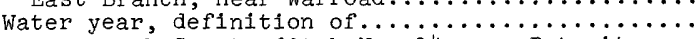

West Branch County ditch No. is near Detroit

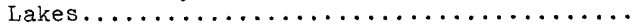

West Swan River (tributary to St. Louis River)

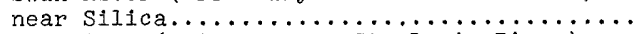

West Two River (tributary to St. Louis River)

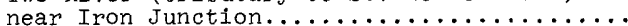

Whetstone River near Big Stone City, S. Dak.....

Whitewater River, North Fork, near Elba.........

South Fork, near Altura................. 157

Whitewater River basin, gaging-station

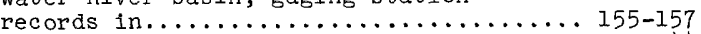

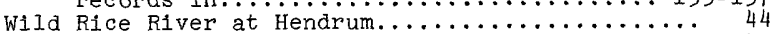

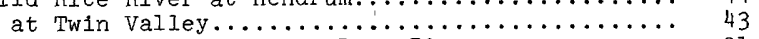

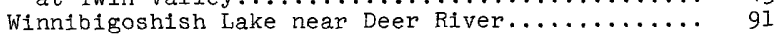

Yellow Bank River near Odessa................ 126

Yellow Medicine River near Granite Fails........ 133

South Branch, at Minneota.................. 132

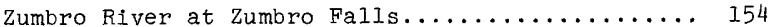

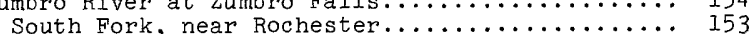

Zumbro River basin, crest-stage partial-

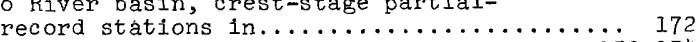

gaging-station records in............... I53-154

measurement at miscellaneous site in........ 189 
Part 2. Water-Quality Records

Baptism River near Beaver Bay............234-235 Page Beaver Creek, 2 miles downstream from

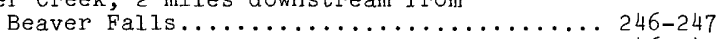

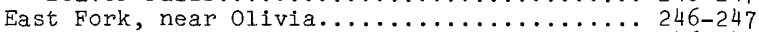

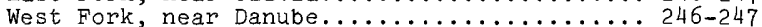

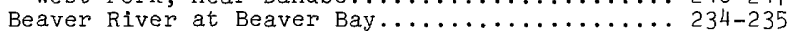

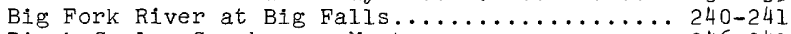

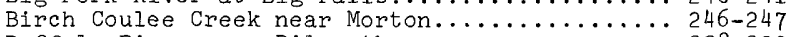
Buffalo River near Dilworth............... 238-239

Cedar River near Austin................ 254-255

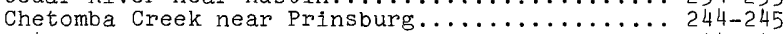

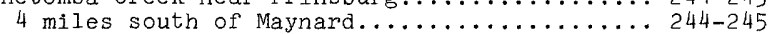

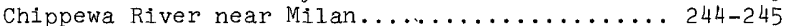

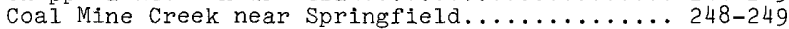

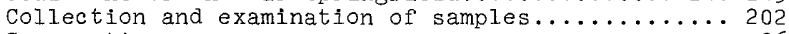

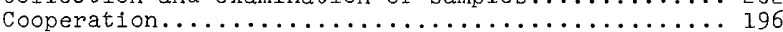

Cottonwood River near Amiret..............246-247

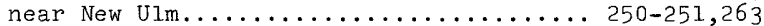

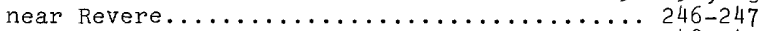

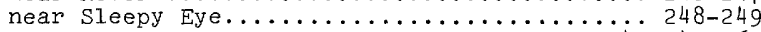

Crow Wing River at Nimrod.............. 242-243,260

Definition of terms and abbreviations........... 196 Des Moines River, West Fork, at Jackson.... 2 $2 \dot{5} 4-255,264$ Dutch Charley Creek near Lamberton.......... 248-249

Hawk Creek near Granite Falls.............. 244-245

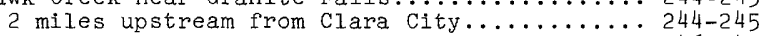

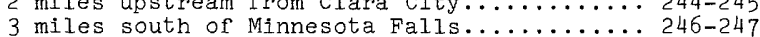

Introduction.

195

Judicial ditch No. 1-A near New Sweden.... 250-251,262

Kawishiwi River near Ely............... 214-217

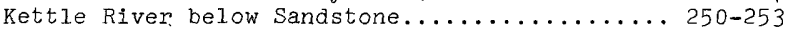

Leaf River near Verndale................ 242-243

Le Sueur River near Rapidan................ 250-251

Little Cottonwood River near Cambria.......... 250-251

Little Cottonwood River near Levenworth..........250-251

Middle River at Argyle............. 238-239,259

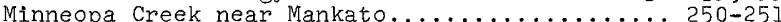

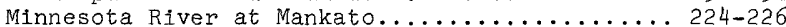

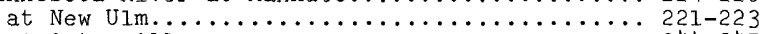

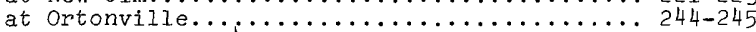

near Jordan........................ 228-229

Mississippi River at Grand Rapids........... 240-241

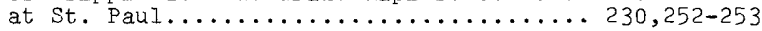

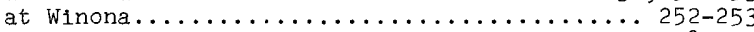

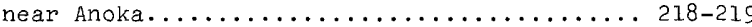

Mound Creek near Springfield........... 248-249

Partridge River near Aldridge........... 242-243 Pelican River at Detroit Lakes................ 236-237 at Melissa Lake Outlet, near Detroit Lakes... 238-239 near Fergus Falls................ 238-239,258 tributary near Detroit Lakes............ 236-237 Pigeon River at Middle Falls, near Grand

portage........................234-235

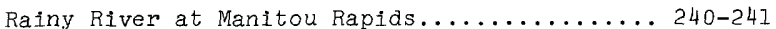

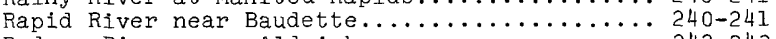

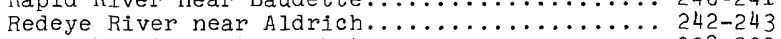

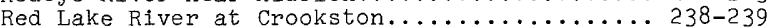

Red River of the North at Fargo, N. Dak....... 206-209

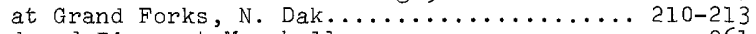

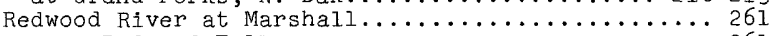

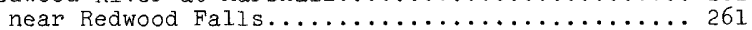

Rock River at Luverne................. 254-255

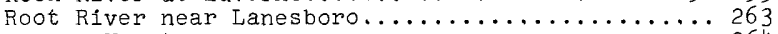

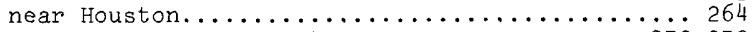

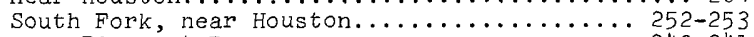

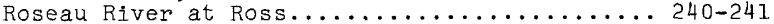
Rum River near St. Francis.............. 242-243

St. Louis River at Forbes................ 257

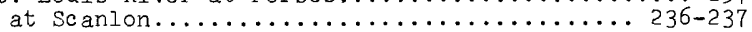

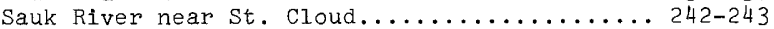

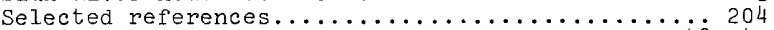
Sleepy Eye Creek near Leavenworth.............. 248 4 . 249

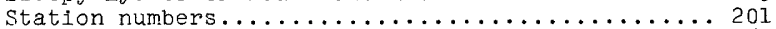
Straight River near Faribault......... 252-253,262 Sucker Creek near Detroit Lakes............. 236-237

Two Rivers, South Branch, at Lake Bronscn.... 238-239

Vermilion River below Vermilion Lake, near Tower....................... 240-241

Water-supply papers..................... 204 Whetstone River near Big Stone City, S. Dak...... 220 Whitewater River, North Fork, near Elba..... 232-233 Wild Rice River at Hendrum............. 238-239

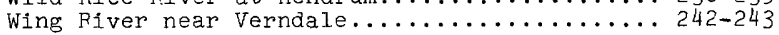

Zumbro River, South Fork, near Rochester.... . 252-253 